\title{
Fungal diversity notes 1387-1511: taxonomic and phylogenetic contributions on genera and species of fungal taxa
}

\author{
Saranyaphat Boonmee ${ }^{1,2}$. Dhanushka N. Wanasinghe ${ }^{3,4,5} \cdot$ Mark S. Calabon $^{1,2} \cdot$ Naruemon Huanraluek $^{1}$. \\ Sajini K. U. Chandrasiri ${ }^{1,2}$. Gareth E. B. Jones ${ }^{6}$. Walter Rossi ${ }^{7}$. Marco Leonardi ${ }^{7}$. Sanjay K. Singh ${ }^{8}$. \\ Shiwali Rana ${ }^{8} \cdot$ Paras N. Singh $^{8}$. Deepak K. Maurya ${ }^{8} \cdot$ Ajay C. Lagashetti $^{8} \cdot$ Deepika Choudhary $^{8}$. \\ Yu-Cheng Dai ${ }^{9}$. Chang-Lin Zhao ${ }^{10}$. Yan-Hong Mu ${ }^{11,12}$. Hai-Sheng Yuan ${ }^{11}$. Shuang-Hui He ${ }^{9}$. \\ Rungtiwa Phookamsak ${ }^{3,4,5,13} \cdot$ Hong-Bo Jiang ${ }^{1,2,3} \cdot$ María P. Martín $^{14} \cdot$ Margarita Dueñas $^{14} \cdot$ M. Teresa Telleriaa $^{14}$. \\ Izabela L. Kałucka ${ }^{15} \cdot$ Andrzej M. Jagodziński $^{16} \cdot$ Kare Liimatainen $^{17} \cdot$ Diana S. Pereira ${ }^{18}$ • Alan J. L. Phillips ${ }^{18}$. \\ Nakarin Suwannarach ${ }^{19,20}$. Jaturong Kumla ${ }^{19,20}$. Surapong Khuna ${ }^{19,20}$. Saisamorn Lumyong ${ }^{19,20,21}$. \\ Tarynn B. Potter ${ }^{22} \cdot$ Roger G. Shivas ${ }^{22,23}$. Adam H. Sparks ${ }^{22,24}$. Niloofar Vaghefi' ${ }^{22}$ Mohamed A. Abdel-Wahab ${ }^{25}$. \\ Faten A. Abdel-Aziz ${ }^{25} \cdot$ Guo-Jie $\mathrm{Li}^{26} \cdot$ Wen-Fei Lin ${ }^{27}$. Upendra Singh ${ }^{28} \cdot$ Rajendra P. Bhatt $^{28} \cdot$ Hyang Burm Lee $^{29}$. \\ Thuong T. T. Nguyen ${ }^{29}$. Paul M. Kirk ${ }^{30}$. Arun Kumar Dutta ${ }^{31,32} \cdot K_{\text {Krishnendu Acharya }}^{32} \cdot$ V. Venkateswara Sarma ${ }^{33}$. \\ M. Niranjan ${ }^{33,34} \cdot$ Kunhiraman C. Rajeshkumar $^{8} \cdot$ Nikhil Ashtekar $^{8} \cdot$ Sneha $^{3}$ Lad $^{8} \cdot$ Nalin N. Wijayawardene $^{35}$. \\ Darbe J. Bhat ${ }^{36} \cdot$ Rong-Ju Xu $^{1,2,3}$. Subodini N. Wijesinghe ${ }^{1,2} \cdot$ Hong-Wei Shen ${ }^{1,2,37} \cdot$ Zong-Long Luo $^{37}$. \\ Jing-Yi Zhang ${ }^{1,2,38} \cdot$ Phongeun Sysouphanthong ${ }^{1,2,39} \cdot$ Naritsada Thongklang ${ }^{1,2} \cdot$ Dan-Feng Bao ${ }^{1,37,40}$. \\ Janith V. S. Aluthmuhandiram ${ }^{1,2,41}$. Jafar Abdollahzadeh ${ }^{42}$. Alireza Javadi ${ }^{43}$. Francesco Dovana ${ }^{44}$. \\ Muhammad Usman ${ }^{45}$. Abdul Nasir Khalid ${ }^{45}$. Asha J. Dissanayake ${ }^{46}$. Anusha Telagathoti ${ }^{47} \cdot$ Maraike Probst $^{47}$. \\ Ursula Peintner ${ }^{47}$. Isaac Garrido-Benavent ${ }^{48}$. Lilla Bóna ${ }^{49} \cdot$ Zsolt Merényi $^{50}$ - Lajos Boros $^{51}$ - Bratek Zoltán ${ }^{49}$. \\ J. Benjamin Stielow ${ }^{52,53}$. Ning Jiang ${ }^{54}$. Cheng-Ming Tian ${ }^{54}$. Esmaeil Shams ${ }^{55}$. Farzaneh Dehghanizadeh $^{56}$. \\ Adel Pordel ${ }^{57} \cdot$ Mohammad Javan-Nikkhah $^{55}$. Teodor T. Denchev ${ }^{58}$. Cvetomir M. Denchev ${ }^{58} \cdot$ Martin Kemler $^{59}$. \\ Dominik Begerow ${ }^{59}$. Chun-Ying Deng ${ }^{60} \cdot$ Emma Harrower $^{61}$. Tohir Bozorov ${ }^{62}$. Tutigul Kholmuradova ${ }^{63}$. \\ Yusufjon Gafforov ${ }^{63}$. Aziz Abdurazakov ${ }^{63,64}$. Jian-Chu Xu'3,4,5,13 . Peter E. Mortimer ${ }^{3,4}$. Guang-Cong Ren ${ }^{1,2}$. \\ Rajesh Jeewon ${ }^{66}$. Sajeewa S. N. Maharachchikumbura ${ }^{46}$. Chayanard Phukhamsakda ${ }^{67}$. Ausana Mapook ${ }^{1}$. \\ Kevin D. Hyde ${ }^{1,3,19,20,65}$
}

Received: 9 May 2021 / Accepted: 10 August 2021 / Published online: 7 December 2021 C MUSHROOM RESEARCH FOUNDATION 2021

\begin{abstract}
This article is the 13th contribution in the Fungal Diversity Notes series, wherein 125 taxa from four phyla, ten classes, 31 orders, 69 families, 92 genera and three genera incertae sedis are treated, demonstrating worldwide and geographic distribution. Fungal taxa described and illustrated in the present study include three new genera, 69 new species, one new combination, one reference specimen and 51 new records on new hosts and new geographical distributions. Three new genera, Cylindrotorula (Torulaceae), Scolecoleotia (Leotiales genus incertae sedis) and Xenovaginatispora (Lindomycetaceae) are introduced based on distinct phylogenetic lineages and unique morphologies. Newly described species are Aspergillus lannaensis, Cercophora dulciaquae, Cladophialophora aquatica, Coprinellus punjabensis, Cortinarius alutarius, C. mammillatus, C. quercoflocculosus, Coryneum fagi, Cruentomycena uttarakhandina, Cryptocoryneum rosae, Cyathus uniperidiolus, Cylindrotorula indica, Diaporthe chamaeropicola, Didymella azollae, Diplodia alanphillipsii, Dothiora coronicola, Efibula rodriguezarmasiae, Erysiphe salicicola, Fusarium queenslandicum, Geastrum gorgonicum, G. hansagiense, Helicosporium sexualis, Helminthosporium chiangraiensis, Hongkongmyces kokensis, Hydrophilomyces hydraenae, Hygrocybe boertmannii, Hyphoderma australosetigerum, Hyphodontia yunnanensis, Khaleijomyces umikazeana, Laboulbenia divisa, Laboulbenia triarthronis, Laccaria populina, Lactarius pallidozonarius, Lepidosphaeria strobelii, Longipedicellata megafusiformis,
\end{abstract}

Communicated by Jian-Kui Liu.

Extended author information available on the last page of the article 
Lophiotrema lincangensis, Marasmius benghalensis, M. jinfoshanensis, M. subtropicus, Mariannaea camelliae, Melanographium smilaxii, Microbotryum polycnemoides, Mimeomyces digitatus, Minutisphaera thailandensis, Mortierella solitaria, Mucor harpali, Nigrograna jinghongensis, Odontia huanrenensis, O. parvispina, Paraconiothyrium ajrekarii, Parafuscosporella niloticus, Phaeocytostroma yomensis, Phaeoisaria synnematicus, Phanerochaete hainanensis, Pleopunctum thailandicum, Pleurotheciella dimorphospora, Pseudochaetosphaeronema chiangraiense, Pseudodactylaria albicolonia, Rhexoacrodictys nigrospora, Russula paravioleipes, Scolecoleotia eriocamporesi, Seriascoma honghense, Synandromyces makranczyi, Thyridaria aureobrunnea, Torula lancangjiangensis, Tubeufia longihelicospora, Wicklowia fusiformispora, Xenovaginatispora phichaiensis and Xylaria apiospora. One new combination, Pseudobactrodesmium stilboideus is proposed. A reference specimen of Comoclathris permunda is designated. New host or distribution records are provided for Acrocalymma fici, Aliquandostipite khaoyaiensis, Camarosporidiella laburni, Canalisporium caribense, Chaetoscutula juniperi, Chlorophyllum demangei, C. globosum, C. hortense, Cladophialophora abundans, Dendryphion hydei, Diaporthe foeniculina, D. pseudophoenicicola, D. pyracanthae, Dictyosporium pandanicola, Dyfrolomyces distoseptatus, Ernakulamia tanakae, Eutypa flavovirens, E. lata, Favolus septatus, Fusarium atrovinosum, F. clavum, Helicosporium luteosporum, Hermatomyces nabanheensis, Hermatomyces sphaericoides, Longipedicellata aquatica, Lophiostoma caudata, L. clematidisvitalbae, Lophiotrema hydei, L. neoarundinaria, Marasmiellus palmivorus, Megacapitula villosa, Micropsalliota globocystis, M. gracilis, Montagnula thailandica, Neohelicosporium irregulare, N. parisporum, Paradictyoarthrinium diffractum, Phaeoisaria aquatica, Poaceascoma taiwanense, Saproamanita manicata, Spegazzinia camelliae, Submersispora variabilis, Thyronectria caudata, T. mackenziei, Tubeufia chiangmaiensis, T. roseohelicospora, Vaginatispora nypae, Wicklowia submersa, Xanthagaricus necopinatus and Xylaria haemorrhoidalis. The data presented herein are based on morphological examination of fresh specimens, coupled with analysis of phylogenetic sequence data to better integrate taxa into appropriate taxonomic ranks and infer their evolutionary relationships.

Keywords 72 new taxa One new combination $\cdot$ One reference specimen $\cdot 51$ new records $\cdot$ Agaricomycetes . Ascomycota $\cdot$ Bartheletiomycetes $\cdot$ Basidiomycota $\cdot$ Dothideomycetes $\cdot$ Eurotiomycetes $\cdot$ Exobasidiomycetes $\cdot$ Laboulbeniomycetes $\cdot$ Leotiomycetes $\cdot$ Mortierellomycetes $\cdot$ Mortierellomycota $\cdot$ Mucoromycetes $\cdot$ Mucoromycota .

Phylogeny $\cdot$ Sordariomycetes $\cdot$ Taxonomy

\section{Table of contents}

Ascomycota Caval.-Sm.

Dothideomycetes O.E. Erikss \& Winka

Botryosphaeriales C.L. Schoch et al.

Botryosphaeriaceae Theiss. \& P. Syd.

1387. Diplodia alanphillipsii Abdollahz. \& A. Javadi, sp. nov. (Contributed by Jafar Abdollahzadeh and Alireza Javadi)

\section{Capnodiales Woron.}

Dissoconiaceae Crous \& de Hoog

1388. Chaetoscutula juniperi E. Müll., new record (Contributed by Isaac Garrido-Benavent)

Dothideales Lindau (=Neocelosporiales Crous)

Dothioraceae Chevall.

1389. Dothiora coronicola Dissanayake, Camporesi \& K.D. Hyde, sp. nov. (Contributed by Asha J. Dissanayake and Erio Camporesi)
Dyfrolomycetales K.L. Pang, K.D. Hyde \& E.B.G. Jones

Pleurotremataceae Walt. Watson (=Dyfrolomycetaceae K.D. Hyde et al.)

1390. Dyfrolomyces distoseptatus M. Niranjan \& V.V. Sarma, new geographical record (Contributed by Mark S. Calabon)

Jahnulales K.L. Pang, Abdel-Wahab, El-Shar., E.B.G. Jones \& Sivichai

Aliquandostipitaceae Inderbitzin

1391. Aliquandostipite khaoyaiensis Inderbitzin, new record (Contributed by Mark S. Calabon)

Minutisphaerales Raja, Oberlies, Shearer \& A.N. Mill.

Minutisphaeraceae Raja, Oberlies, Shearer \& A.N. Mill. 1392. Minutisphaera thailandensis R.J. Xu, Boonmee \& K.D. Hyde, sp. nov. (Contributed by Rong-Ju Xu)

Pleosporales Luttr. ex M.E. Barr

Acrocalymmaceae Crous \& Trakun. 
1393. Acrocalymma fici Crous \& Trakun., new habitat and geographical record (Contributed by Mark S. Calabon)

Dictyosporiaceae Boonmee \& K.D. Hyde

1394. Dictyosporium pandanicola Tibpromma \& K.D. Hyde, new record (Contributed by Dan-Feng Bao)

Camarosporidiellaceae Wanas., Wijayaw., Crous \& K.D. Hyde

1395. Camarosporidiella laburni (Pers.) Wanas., Bulgakov, Camporesi \& K.D. Hyde, new record (Contributed by Dhanushka Wanasinghe, Yusufjon Gafforov and Aziz Abdurazakov)

Cryptocoryneaceae A. Hashim. \& Kaz. Tanaka 1396. Cryptocoryneum rosae Wanas. \& K.D. Hyde, sp. nov. (Contributed by Dhanushka Wanasinghe)

Didymellaceae Gruyter, Aveskamp \& Verkley (= Microsphaeropsidaceae Qian Chen et al.)

1397. Didymella azollae Shams E., Dehghanizadeh F. \& Pordel A., sp. nov. (Contributed by Esmaeil Shams, Farzaneh Dehghanizadeh, Adel Pordel and Mohammad Javan-Nikkhah)

Didymosphaeriaceae Munk

1398. Montagnula thailandica Mapook \& K.D. Hyde, new record (Contributed by Saranyaphat Boonmee and Naruemon Huanraluek)

1399. Paraconiothyrium ajrekarii S. Rana \& S.K. Singh, sp. nov. (Contributed by Sanjay K. Singh and Shiwali Rana) 1400. Spegazzinia camelliae N. Suwannarach, J. Kumla \& S. Lumyong, new record (Contributed by Saranyaphat Boonmee)

\section{Hermatomycetaceae Locq.}

1401. Hermatomyces nabanheensis Tibpromma, Bhat \& K.D. Hyde, new record (Contributed by Jing-Yi Zhang)

1402. Hermatomyces sphaericoides Koukol \& G. Delgado, new record (Contributed by Saranyaphat Boonmee and Naruemon Huanraluek)

Lentitheciaceae Y. Zhang ter, C.L. Schoch, J. Fourn., Crous \& K.D. Hyde

1403. Poaceascoma taiwanense Tennakoon, C.H. Kuo \& K.D. Hyde, new geographical record (Contributed by Saranyaphat Boonmee)

Lindgomycetaceae K. Hiray. et al.

1404. Hongkongmyces kokensis Boonmee, Huanraluek, Chandrasirie \& K.D. Hyde, sp. nov. (Contributed by Saranyaphat Boonmee, Naruemon Huanraluek and Sajini K.U. Chandrasiri)
1405. Xenovaginatispora Boonmee, Huanraluek \& K.D. Hyde, gen. nov. (Contributed by Saranyaphat Boonmee) 1406. Xenovaginatispora phichaiensis Boonmee, Huanraluek \& K.D. Hyde, sp. nov. (Contributed by Saranyaphat Boonmee)

Longipedicellataceae Phukhams., Bhat \& K.D. Hyde 1407. Longipedicellata aquatica W. Dong, H. Zhang \& K.D. Hyde, new record (Contributed by Saranyaphat Boonmee and Sajini K.U. Chandrasiri)

1408. Longipedicellata megafusiformis Chandrasiri, Boonmee \& K.D. Hyde, sp. nov. (Contributed by Saranyaphat Boonmee and Sajini K.U. Chandrasiri)

1409. Submersispora variabilis $W$. Dong, H. Zhang \& K.D. Hyde, new record (Contributed by Saranyaphat Boonmee and Naruemon Huanraluek)

Lophiostomataceae Sacc.

1410. Lophiostoma caudatum Fabre, new host and geographical record (Contributed by Janith Vishvakeerthi and Yusufjon Gafforov)

1411. Lophiostoma clematidis-vitalbae Andreasen, Jaklitsch \& Voglmayr, new host and geographical record (Contributed by Janith Vishvakeerthi and Yusufjon Gafforov) 1412. Vaginatispora nypae Jayasiri, E.B.G. Jones \& K.D. Hyde, new record (Contributed by Dan-Feng Bao)

Lophiotremataceae K. Hiray. \& Kaz.

1413. Lophiotrema hydei J.F. Zhang, J.K. Liu \& Z.Y. Liu, new host record (Contributed by Dhanushka Wanasinghe) 1414. Lophiotrema lincangensis Wanas. \& K.D. Hyde, sp. nov. (Contributed by Dhanushka Wanasinghe)

1415. Lophiotrema neoarundinariae Y. Zhang ter, Kaz. Tanaka \& K.D. Hyde, new host record (Contributed by Dhanushka Wanasinghe)

Macrodiplodiopsidaceae Voglmayr, Jaklitsch \& Crous 1416. Pseudochaetosphaeronema chiangraiense Wijesinghe, Boonmee \& K.D. Hyde, sp. nov. (Contributed by Subodini N. Wijesinghe)

Massarinaceae Munk

1417. Helminthosporium chiangraiense Boonmee, Huanraluek \& K.D. Hyde, sp. nov. (Contributed by Saranyaphat Boonmee and Naruemon Huanraluek)

Nigrogranaceae Jaklitsch \& Voglmayr

1418. Nigrograna jinghongensis Wanas. \& K.D. Hyde, sp. nov. (Contributed by Dhanushka Wanasinghe)

Occultibambusaceae D.Q. Dai \& K.D. Hyde 
1419. Seriascoma honghense H.B. Jiang, Phookamsak \& K.D. Hyde, sp. nov. (Contributed by Rungtiwa Phookamsak and Hong-Bo Jiang)

Paradictyoarthriniaceae Doilom, Ariyaw., Bhat \& K.D. Hyde

1420. Paradictyoarthrinium diffractum Matsush., new record (Contributed by Saranyaphat Boonmee and Naruemon Huanraluek)

Phaeoseptaceae S. Boonmee, Thambug. \& K.D. Hyde 1421. Pleopunctum thailandicum J.Y. Zhang, Y.Z. Lu \& K.D. Hyde, sp. nov. (Contributed by Jing-Yi Zhang)

Phaeosphaeriaceae M.E. Barr

1422. Phaeosphaeriopsis aloes Crous \& Y. Marín, new host and new geographical record (Contributed by Dhanushka Wanasinghe)

Pleosporaceae Nitschke

1423. Comoclathris permunda Thambug., Camporesi \& K.D. Hyde, reference specimen (Contributed by Dhanushka Wanasinghe, Yusufjon Gafforov and Aziz Abdurazakov)

\section{Testudinaceae Arx}

1424. Lepidosphaeria strobelii A.C. Lagashetti, D. Choudhary \& S.K. Singh, sp. nov. (Contributed by A.C. Lagashetti and S.K. Singh)

Tetraplosphaeriaceae Kaz. Tanaka \& K. Hiray

1425. Ernakulamia tanakae Rajeshkumar \& K.D. Hyde, new record (Contributed by Saranyaphat Boonmee and Naruemon Huanraluek)

Thyridariaceae Q. Tian \& K.D. Hyde

1426. Thyridaria aureobrunnea Boonmee, Huanraluek \& K.D. Hyde, sp. nov. (Contributed by Saranyaphat Boonmee)

Torulaceae Corda

1427. Cylindrotorula Rajeshkumar, Wijayaw. \& Bhat, gen. nov. (Contributed by Kunhiraman C. Rajeshkumar, Nikhil Ashtekar, Sneha Lad, Nalin N. Wijayawardene and Darbe J. Bhat)

1428. Cylindrotorula indica Rajeshkumar, Wijayaw., Bhat, N. Ashtekar \& S. Lad, sp. nov. (Contributed by Kunhiraman C. Rajeshkumar, Nikhil Ashtekar, Sneha Lad, Nalin N. Wijayawardene and Darbe J. Bhat)

1429. Dendryphion hydei J.F. Li, Phookamsak \& Jeewon, new record (Contributed by Hong-Wei Shen)

1430. Torula lancangjiangensis H.W. Shen, S. Boonmee, Z.L. Luo \& K.D. Hyde, sp. nov. (Contributed by Hong-Wei Shen)
1431. Torula mackenziei Jun F. Li, Phook. \& K.D. Hyde, new record (Contributed by Hong-Wei Shen)

Wicklowiaceae Ariyaw. \& K.D. Hyde

1432. Wicklowia fusiformispora Boonmee, Huanraluek \& K.D. Hyde, sp. nov. (Contributed by Saranyaphat Boonmee and Naruemon Huanraluek)

1433. Wicklowia submersa Boonmee, Sorvongxay \& K.D. Hyde, new geographical record (Contributed by Saranyaphat Boonmee and Naruemon Huanraluek)

Tubeufiales Boonmee \& K.D. Hyde

Tubeufiaceae M.E. Barr

1434. Helicosporium luteosporum Y.Z. Lu, Boonmee \& K.D. Hyde, new record (Contributed by Saranyaphat Boonmee)

1435. Helicosporium sexuale Boonmee, sp. nov. (Contributed by Saranyaphat Boonmee)

1436. Neohelicosporium irregulare Y.Z. Lu, J.C. Kang \& K.D. Hyde, new record (Contributed by Saranyaphat Boonmee)

1437. Neohelicosporium parvisporum Y.Z. Lu, J.C. Kang \& K.D. Hyde, new record (Contributed by Mark S. Calabon) 1438. Tubeufia chiangmaiensis Boonmee \& K.D. Hyde, new record (Contributed by Saranyaphat Boonmee)

1439. Tubeufia longihelicospora Boonmee \& K.D. Hyde, sp. nov. (Contributed by Saranyaphat Boonmee)

1440. Tubeufia roseohelicospora Y.Z. Lu, Boonmee \& K.D. Hyde, new record (Contributed by Saranyaphat Boonmee)

Pleosporales, genus incertae sedis

1441. Megacapitula villosa J.L. Chen \& Tzean, new geographical record (Contributed by Saranyaphat Boonmee)

Eurotiomycetes O.E. Erikss. \& Winka

Chaetothyriales M.E. Barr

Herpotrichiellaceae Munk

1442. Cladophialophora abundans $P$. Feng, V.A. Vicente, Najafz., van den Ende, Stielow, Badali, Boeger \& de Hoog, new geographical record (Contributed by Mark S. Calabon) 1443. Cladophialophora aquatica M.S. Calabon, Boonmee, E.B.G. Jones \& K.D. Hyde, sp. nov. (Contributed by Mark S. Calabon)

Eurotiales G.W. Martin ex Benny \& Kimbr.

Aspergillaceae Link (= Monascaceae J. Schröt.)

1444. Aspergillus lannaensis N. Suwannarach, S. Khuna \& S. Lumyong, sp. nov. (Contributed by Nakarin Suwannarach, Surapong Khuna and Saisamorn Lumyong) 
Sclerococcales Réblová, Unter. \& W. Gams

Dactylosporaceae Bellem. \& Hafellner (=Sclerococcaceae Réblová et al.)

1445. Pseudobactrodesmium stilboideum (R.F. Castañeda \& G.R.W. Arnold) M.S. Calabon, Boonmee, E.B.G. Jones, K.D. Hyde, comb. nov. (Contributed by Mark S. Calabon)

Laboulbeniomycetes Engl.

Laboulbeniales Lindau

Laboulbeniaceae G. Winter 1446. Hydrophilomyces hydraenae W. Rossi \& M. Leonardi, sp. nov. (Contributed by Walter Rossi and Marco Leonardi)

1447. Laboulbenia divisa W. Rossi \& M. Leonardi, sp. nov. (Contributed by Walter Rossi and Marco Leonardi)

1448. Laboulbenia triarthronis W. Rossi \& M. Leonardi, sp. nov. (Contributed by Walter Rossi and Marco Leonardi) 1449. Mimeomyces digitatus W. Rossi \& M. Leonardi, sp. nov. (Contributed by Walter Rossi and Marco Leonardi) 1450. Synandromyces makranczyi W. Rossi \& M. Leonardi, sp. nov. (Contributed by Walter Rossi and Marco Leonardi)

Leotiomycetes O.E. Erikss. \& Winka

Erysiphales Warm.

Erysiphaceae Tul. \& C. Tul.

1451. Erysiphe salicicola Hyang B. Lee, P.M. Kirk \& T.T.T. Nguyen, sp. nov. (Contributed by Hyang Burm Lee, Thuong T.T. Nguyen and Paul M. Kirk)

Leotiomycetes O.E. Erikss. \& Winka

Leotiales Korf \& Lizoň

Leotiales, genus incertae sedis

1452. Scolecoleotia H.B. Jiang, Phookamsak \& K.D. Hyde, gen. nov. (Contributed by Rungtiwa Phookamsak and HongBo Jiang)

1453. Scolecoleotia eriocamporesi H.B. Jiang, Phookamsak \& K.D. Hyde, sp. nov. (Contributed by Rungtiwa Phookamsak and Hong-Bo Jiang)

Sordariomycetes O.E. Erikss. \& Winka

Diaporthales Nannf.

Coryneaceae Corda (= Pseudovalsaceae M.E. Barr) 1454. Coryneum fagi C.M. Tian \& N. Jiang, sp. nov. (Contributed by Ning Jiang and Cheng-Ming Tian)
Diaporthaceae Höhn. ex Wehm.

1455. Diaporthe chamaeropicola D.R.S. Pereira \& A.J.L. Phillips, sp. nov. (Contributed by Diana S. Pereira and Alan J.L. Phillips)

1456. Diaporthe foeniculina (Sacc.) Udayanga \& Castl., new record (Contributed by Diana S. Pereira and Alan J.L. Phillips)

1457. Diaporthe pseudophoenicicola R.R. Gomes, C. Glienke \& Crous, new record (Contributed by Diana $S$. Pereira and Alan J.L. Phillips)

1458. Diaporthe pyracanthae L. Santos \& A. Alves, new record (Contributed by Diana S. Pereira and Alan J.L. Phillips)

1459. Phaeocytostroma yomensis Boonmee, Chandrasiri \& K.D. Hyde, sp. nov. (Contributed by Saranyaphat Boonmee and Sajini K.U. Chandrasiri)

Fuscosporellales Jing Yang, Bhat \& K.D. Hyde

Fuscosporellaceae Jing Yang, Bhat \& K.D. Hyde

1460. Parafuscosporella nilotica Abdel-Aziz, sp. nov. (Contributed by Mohamed A. Abdel-Wahab and Faten A. Abdel-Aziz)

Hypocreales Lindau

Nectriaceae Tul. \& C. Tul.

1461. Fusarium atrovinosum L. Lombard \& Crous, new record (Contributed by Tarynn B. Potter, Roger G. Shivas, Adam H. Sparks and Niloofar Vaghefi)

1462. Fusarium clavum J.W. Xia, L. Lombard, Sand.-Den., X.G. Zhang \& Crous, new record (Contributed by Tarynn B. Potter, Roger G. Shivas, Adam H. Sparks and Niloofar Vaghefi)

1463. Fusarium queenslandicum T.B. Potter, A.H. Sparks, Vaghefi \& R.G. Shivas, sp. nov. (Contributed by Tarynn B. Potter, Roger G. Shivas, Adam H. Sparks and Niloofar Vaghefi)

1464. Mariannaea camelliae N. Suwannarach \& J. Kumla, sp. nov. (Contributed by Nakarin Suwannarach and Jaturong Kumla)

1465. Thyronectria caudata (Desm.) Seeler, new record (Contributed by Dhanushka Wanasinghe, Yusufjon Gafforov and Aziz Abdurazakov)

Pleurotheciales Réblová \& Seifert

Pleurotheciaceae Réblová \& Seifert

1466. Phaeoisaria aquatica Z.L. Luo, X.J. Su \& K.D. Hyde, new record (Contributed by Saranyaphat Boonmee) 1467. Phaeoisaria synnematicus P.N. Singh \& S.K. Singh, sp. nov. (Contributed by P.N. Singh and S.K. Singh) 
1468. Pleurotheciella dimorphospora H.B. Jiang, Phookamsak \& K.D. Hyde, sp. nov. (Contributed by Rungtiwa Phookamsak)

\section{Pseudodactylariales Crous}

\section{Pseudodactylariaceae Crous}

1469. Pseudodactylaria albicolonia $\mathrm{R} . J . \mathrm{Xu}$, Boonmee \& K.D. Hyde, sp. nov. (Contributed by Rong-Ju Xu)

Savoryellales Boonyuen, Suetrong, Sivichai, K.L. Pang \& E.B.G. Jones

Savoryellaceae Jaklitsch \& Réblová

1470. Canalisporium caribense (Hol.-Jech. \& Mercado) Nawawi \& Kuthub., new record (Contributed by Saranyaphat Boonmee and Sajini K.U. Chandrasiri)

1471. Rhexoacrodictys nigrospora Boonmee, D.F. Bao \& K.D. Hyde, sp. nov. (Contributed by Saranyaphat Boonmee)

Lasiosphaeriaceae Nannf.

1472. Cercophora dulciaquae M.S. Calabon, E.B.G. Jones \& K.D. Hyde, sp. nov. (Contributed by Mark S. Calabon)

Torpedosporales E.B.G. Jones, Abdel-Wahab \& K.L. Pang

Juncigenaceae E.B.G. Jones, Abdel-Wahab \& K.L. Pang 1473. Khaleijomyces umikazeanus Abdel-Wahab, sp. nov. (Contributed by Mohamed A. Abdel-Wahab and Faten A. Abdel-Aziz)

Xylariales Nannf.

Diatrypaceae Nitschke

1474. Eutypa flavovirens Nitschke, new record (Contributed by Subodini N. Wijesinghe)

1475. Eutypa lata (Pers.) Tul. \& C. Tul., new record (Contributed by Subodini N. Wijesinghe)

Xylariaceae Tul. \& C. Tul.

1476. Xylaria apiospora M. Niranjan \& V.V. Sarma, sp. nov. (contribution by M. Niranjan and V. Venkateswara Sarma)

1477. Xylaria haemorrhoidalis Berk. \& Broome, new record (contribution by M. Niranjan and V. Venkateswara Sarma)

Xylariales genus incertae sedis

1478. Melanographium smilacis Boonmee, Huanraluek \& K.D. Hyde, sp. nov. (contribution by Saranyaphat Boonmee and Naruemon Huanraluek)

Basidiomycota R.T. Moore Agaricomycetes Doweld
Agaricales Underw.

Agaricaceae Chevall. 1479. Chlorophyllum demangei (Pat.) Z.W. Ge \& Zhu L. Yang, new record (contribution by Phongeun Sysouphanthong and Naritsada Thongklang)

1480. Chlorophyllum globosum (Mossebo) Vellinga, new record (contribution by Phongeun Sysouphanthong and Naritsada Thongklang)

1481. Chlorophyllum hortense (Murrill) Vellinga, new record (contribution by Phongeun Sysouphanthong and Naritsada Thongklang)

1482. Micropsalliota globocystis Heinem., new record (contribution by Phongeun Sysouphanthong and Naritsada Thongklang)

1483. Micropsalliota gracilis Heinem., new record (contribution by Phongeun Sysouphanthong and Naritsada Thongklang)

1484. Xanthagaricus necopinatus Iqbal Hosen, T.H. Li, \& G.M. Gates, new record (contribution by Phongeun Sysouphanthong and Naritsada Thongklang)

Amanitaceae E.-J. Gilbert

1485. Saproamanita manicata (Berk. \& Broome) Redhead, Vizzini, Drehmel \& Contu, new record (contribution by Nakarin Suwannarach and Surapong Khuna)

Cortinariaceae R. Heim ex Pouzar

1486. Cortinarius alutarius Kałucka \& Liimat., sp. nov. (Contributed by Izabela L. Kałucka, Andrzej M. Jagodziński and Kare Liimatainen)

1487. Cortinarius mammillatus Kałucka, Kytöv., Niskanen \& Liimat., sp. nov. (Contributed by Izabela L. Kałucka, Andrzej M. Jagodziński and Kare Liimatainen)

1488. Cortinarius quercoflocculosus Kałucka \& Liimat., sp. nov. (Contributed by Izabela L. Kałucka, Andrzej M. Jagodziński and Kare Liimatainen)

Hydnangiaceae Gäum. \& C.W. Dodge

1489. Laccaria populina Dovana, sp. nov. (Contributed by Francesco Dovana)

Hygrophoraceae Lotsy

1490. Hygrocybe boertmannii U. Singh \& R.P. Bhatt, sp. nov. (Contributed by Upendra Singh)

Marasmiaceae Roze ex Kühner

1491. Marasmius benghalensis A.K. Dutta \& K. Acharya, sp. nov. (Contributed by Arun Kumar Dutta and Krishnendu Acharya)

1492. Marasmius jinfoshanensis Chun Y. Deng \& Gafforov, sp. nov. (Contributed by Chun Y. Deng, Emma Harrower, 
Tohir Bozorov, Tutigul Kholmuradova and Yusufjon Gafforov)

1493. Marasmius subtropicus A.K. Dutta \& K. Acharya, sp. nov. (Contributed by Arun Kumar Dutta and Krishnendu Acharya)

\section{Mycenaceae Overeem}

1494. Cruentomycena uttarakhandina U. Singh \& R.P. Bhatt, sp. nov. (Contributed by Upendra Singh)

Nidulariaceae Dumort.

1495. Cyathus uniperidiolus P.N. Singh \& S.K. Singh, sp. nov. (Contributed by P.N. Singh and Sanjay K. Singh)

Omphalotaceae Bresinsky

1496. Marasmiellus palmivorus (Sharples) Desjardin, new record (Contributed by Nakarin Suwannarach and Saisamorn Lumyong)

Psathyrellaceae Vilgalys, Moncalvo \& Redhead

1497. Coprinellus punjabensis Usman \& Khalid, sp. nov. (Contributed by Muhammad Usman and Abdul Nasir Khalid)

Geastrales K. Hosaka \& Castellano

\section{Geastraceae Corda}

1498. Geastrum gorgonicum M.P. Martín, M. Dueñas \& Telleria, sp. nov. (Contributed by María P. Martín, Margarita Dueñas and M. Teresa Telleria)

1499. Geastrum hansagiense Bóna, Merényi, Boros, Stielow \& Bratek, sp. nov. (Contributed by Lilla Bóna, Zsolt Merényi, Lajos Boros, J. Benjamin Stielow and Bratek Zoltán)

\section{Schizoporaceae Jülich}

1500. Hyphodontia yunnanensis C.L. Zhao \& Y.C. Dai, sp. nov. (Contributed by Chang-Lin Zhao and Yu-Cheng Dai)

\section{Thelephoraceae Chevall.}

1501. Odontia huanrenensis Y.H. Mu, H.S. Yuan \& Y.C. Dai, sp. nov. (Contributed by Yan-Hong Mu, Hai-Sheng Yuan and Yu-Cheng Dai)

1502. Odontia parvispina Y.H. Mu, H.S. Yuan \& Y.C. Dai, sp. nov. (Contributed by Yan-Hong Mu, Hai-Sheng Yuan and Yu-Cheng Dai)

Bartheletiomycetes Thines

Polyporales Gäum.

Hyphodermataceae Jülich
1503. Hyphoderma australosetigerum $M$. Dueñas, Telleria \& M.P. Martín, sp. nov. (Contributed by Margarita Dueñas, M. Teresa Telleria and María P. Martín)

Irpicaceae Spirin \& Zmitr.

1504. Efibula rodriguezarmasiae Telleria, M. Dueñas, Beltrán-Tej., Melo, Salcedo \& M.P. Martín, sp. nov. (Contributed by M. Teresa Telleria, Margarita Dueñas and María P. Martín)

Phanerochaetaceae Jülich

1505. Phanerochaete hainanensis S.H. He \& Y.C. Dai, sp. nov. (Contributed by Shuang-Hui He and Yu-Cheng Dai)

Polyporaceae Fr. ex Corda

1506. Favolus septatus J.L. Zhou \& B.K. Cui, new record (Contributed by Deepak K. Maurya and Sanjay K. Singh)

Russulales Kreisel ex P.M. Kirk, P.F. Cannon \& J.C. David

Russulaceae Losty

1507. Lactarius pallidozonarius G.J. Li \& W.F. Lin, sp. nov. (Contributed by Guo-Jie Li and Wen-Fei Lin)

1508. Russula paravioleipes G.J. Li \& W.F. Lin, sp. nov. (Contributed by Guo-Jie Li and Wen-Fei Lin)

Microbotryomycetes R. Bauer et al.

Microbotryales R. Bauer \& Oberw.

Microbotryaceae R.T. Moore

1509. Microbotryum polycnemoides T. Denchev, Denchev, Kemler \& Begerow, sp. nov. (Contributed by Teodor T. Denchev, Cvetomir M. Denchev, Martin Kemler and Dominik Begerow)

Mortierellomycota Tedersoo et al.

Mortierellomycetes Doweld

Mortierellales Caval.-Sm.

Mortierellaceae A. Fisch.

1510. Mortierella solitaria Telagathoti, M. Probst \& Peintner, sp. nov. (Contributed by Anusha Telagathoti, Maraike Probst and Ursula Peintner)

Mucoromycota Doweld

Mucoromycetes Doweld

Mucorales Dumort.

Mucoraceae Fr. 
1511. Mucor harpali Hyang B. Lee, P.M. Kirk \& T.T.T. Nguyen, sp. nov. (Contributed by Hyang Burm Lee, Thuong T.T. Nguyen and Paul M. Kirk)

\section{Introduction}

The Fungal Diversity Notes series provides evidence of a largescale wave of species discovery, the rapid description of these novel species, and the implication of many yet undescribed taxa (Hyde et al. 2020c). These notes show that in countries where mycological studies are taking place, there is a high diversity of undiscovered fungi that was previously not apparent (Hyde et al. 2018b). The series provides an outlet for publishing new species, new records and new combinations.

From the first to 13th series of the Fungal Diversity Notes 1-1511, a total of 1511 taxa were included with 11 new families, 115 new genera and 1068 new species (Ariyawansa et al. 2015a; Liu et al. 2015a; Li et al. 2016a; Hyde et al. 2016, 2017, 2019, 2020a; Tibpromma et al. 2017b, 2018; Wanasinghe et al. 2018; Phookamsak et al. 2017a, 2019; Yuan et al. 2020; this study). Nineteen phyla have been accepted in the kingdom of Fungi following an updated outline of fungi and fungus-like taxa in Wijayawardene et al. (2020). The Fungal Diversity Notes Series largest contribution to diversity can be found in the phyla of Ascomycota and Basidiomycota.

The newly published records are also important for species documentation in order to provide comprehensive understanding of their taxonomic and phylogenetic relationships with up to date sequence data (Hyde et al. 2020a; Chethana et al. 2021). The new records will also benefit future studies which cover critical fields including fungal diagnostics and identification, economics and environmental management (Chethana et al. 2021). Several contemporary webpages provide data on fungal taxa from different habitats and regions, including descriptions, illustrations, phylogenetic trees, notes and the current number of accepted species (Pem et al. 2019a; Bundhun et al. 2020; Calabon et al. 2020b; Wijesinghe et al. 2021).

As mycologists strive to accurately define species with an ever-increasing amount of supporting evidence, new records will help determine the boundaries of a species (Hyde et al. 2020a; Chethana et al. 2021; Lucking et al. 2021). When an abundance of evidence including: full descriptions, illustrations and plates, molecular data and material examined are provided, the taxa can be described and accepted as a novel species. In cases where new species become less distinct with further data, those species can then be synonymized. The data also provides new distribution and host records.

The aim of this paper is to provide thorough descriptions of 72 new taxa, one new combination, one reference specimen and 51 new records with supporting taxonomic and phylogenetic evidence. A compilation of notes on new taxonomic contributions, distribution and host records are provided under each fungal taxon.

\section{Materials and methods}

Materials and methods follow the recent Fungal Diversity Notes (Hyde et al. 2020b). Fresh specimens were collected from Africa, America, Asia and Europe (i.e., Austria, Cape Verde, Chile, China, Ecuador, Egypt, Hungary, India, Iran, Italy, Japan, Pakistan, Peru, Poland, Portugal, Republic of Costa Rica, Republic of Korea, Russia, Spain, Thailand and USA). Microscopic characters, illustration photo-plates and molecular phylogenetic analyses were performed as previously described in recent publications (Phookamsak et al. 2019; Senanayake et al. 2020; Yuan et al. 2020).

\section{Taxonomy}

\section{Ascomycota Caval.-Sm.}

Notes: The latest updated account of Ascomycota see Wijayawardene et al. (2020).

\section{Dothideomycetes O.E. Erikss \& Winka.}

Notes: We follow the latest treatments and updated accounts of Dothideomycetes as available in Hongsanan et al. (2020a, b).

Botryosphaeriales C.L. Schoch et al.

Notes: Botryosphaeriales was introduced with a single family Botryosphaeriaceae (Schoch et al. 2006). In the last decade, several researchers have extensively contributed to the taxonomy and phylogeny of species within the Botryosphaeriales (Liu et al. 2012; Slippers et al. 2013; Yang et al. 2017b; Phillips et al. 2019; Zhang et al. 2021). The most recent phylogenetic analyses on Botryosphaeriales, recognized six families: Aplosporellaceae, Botryosphaeriaceae, Melanopsaceae, Phyllostictaceae, Planistromellaceae and Saccharataceae (Phillips et al. 2019; Hongsanan et al. 2020b).

\section{Botryosphaeriaceae Theiss. \& P. Syd.}

Notes: So far 22 genera have been characterized within Botryosphaeriaceae (Hongsanan et al. 2020b). To identify Botryosphaeriaceae genera and species, along with morphology, it is necessary to use sequence data of SSU, ITS, LSU, TEF1- $\alpha$ and $\beta$-tubulin loci (Phillips et al. 2013; Dissanayake et al. 2016; Wu et al. 2021). Herein a new species of Diplodia is introduced based on morphology and DNA 
fingerprinting patterns along with ITS and TEF1- $\alpha$ sequence data.

\section{Diplodia Fr.}

Diplodia was introduced by Montagne (1834) based on the type species D. mutila. Diplodia species are pathogens, endophytes or saprobes on a wide range of woody hosts (Phillips et al. 2012; Zhang et al. 2021). Pathogenic species are associated with different disease symptoms such as twig blight, canker, die-back, gummosis and fruit rot (Abdollahzadeh 2015). More than 1000 species have been listed in Index Fungorum, MycoBank and Species Fungorum databases. Sequences data are available for a limited number of species. In this study, Diplodia alanphillipsii is introduced as a new species (Figs. 1 and 2).

Diplodia alanphillipsii Abdollahz. \& A. Javadi, sp. nov.

Index Fungorum number: IF557831; Facesoffungi number: FoF 09953; Fig. 1

Etymology: Named after Dr. Alan J.L. Phillips for his extensive, qualitative and valuable research on the systematics of the Botryosphaeriaceae.

Holotype: IRAN 14273F

On twigs of Citrus sp. Sexual morph Undetermined. Asexual morph Conidiomata pycnidial, solitary or clustered, immersed in the host or partially erumpent, globose, dark brown to black, ostiolate, apapillate, unilocular, thickwalled with outer dark brown layers and inner thin-walled hyaline textura angularis. Paraphyses not observed. Conidiophores reduced to conidiogenous cells or with a supporting cell. Conidiogenous cells hyaline, thin-walled, smooth, cylindrical, swollen at the base, discrete, enteroblastic, annellidic, indeterminate, often proliferating internally giving rise to periclinal thickenings or proliferating percurrently forming 2-3 annellations. Conidia (17-)20-22(-26 )$\times(8-) 10-11(-13.5) \mu \mathrm{m}(\mathrm{av} . \pm \mathrm{SD}=21.5 \pm 1.5 \times 10.5 \pm 0$. $8 \mu \mathrm{m}, 1 / \mathrm{w}$ ratio $=2.2 \pm 0.2$ ), aseptate, ovoid, widest in the middle, obtuse at the apex, truncate or rounded at the base, initially hyaline, becoming dark brown before release from the pycnidium, moderately thick-walled, externally smooth, roughened on the inner surface.

Material examined: IRAN, Hormozgan Province, Minab, Hajikhademi, on twigs of Citrus sp., 3 March 2007, J. Abdollahzadeh and A. Javadi (IRAN 14273F, holotype), extype living culture, IRAN 1508C = CBS 124713; Rodan, on twigs of Mangifera indica, 3 March 2007, J. Abdollahzadeh and A. Javadi, IRAN 1509C; on twigs of Citrus sp., 3 March 2007, J. Abdollahzadeh and A. Javadi, IRAN 1574C = CBS 124712.

GenBank numbers: IRAN 1508C: ITS $=$ KF890208, TEF1- $\alpha=$ KF890190; IRAN 1509C: ITS $=$ KF890209, TEF1 $-\alpha=$ KF890191; IRAN 1574C: ITS $=$ MT258875; TEF1- $\alpha=$ MT270153.
Notes: Diplodia alanphillipsii is close to D. citricarpa with 4 base pairs differences in ITS ( 3 substitutions, 1 deletion/insertion) and one in TEF1- $\alpha$ (substitution), but can be distinguished due to the relatively smaller conidia when compared to D. citricarpa $(22-25 \times 9-10 \mu \mathrm{m})$ (Yang et al. 2017b). These two species are also differentiated based on the ISSR (data not shown) and rep-PCR fingerprinting patterns (Abdollahzadeh and Zolfaghari 2014). Sequences generated for D. citricarpa were deposited in GenBank: IRAN 1578C = CBS 124715 (ITS: KF890207; TEF1- $\alpha$ : KF890189); IRAN 1511C (ITS: KF890206; TEF1- $\alpha$ : KF890188); IRAN 1510C (ITS: MT258874; TEF1- $\alpha$ : MT270152).

\section{Capnodiales Woron.}

Notes: The latest updated account of Capnodiales includes 19 families and 243 genera (Wijayawardene et al. 2020).

\section{Dissoconiaceae Crous \& de Hoog}

Notes: Dissoconiaceae is a family within Capnodiales (Dothideomycetes) that was introduced by Crous et al. (2009) to accommodate the genera Dissoconium and Ramichloridium. The number of genera within the Dissoconiaceae has increased to four after Pseudoveronaea was introduced, and Uwebraunia, a former synonym of Dissoconium was reinstated, to accommodate taxa of a distinct clade within the latter genus ( $\mathrm{Li}$ et al. 2012). The new genus Globoramichloridium has also been incorporated (Marín-Félix et al. 2019a). In general, species in this family produce pseudothecial ascomata, which are immersed, globose, unilocular, papillate, and ostiolate with a periphysate canal (Crous et al. 2009). Pseudoparaphyses are absent and bitunicate asci are of a fasciculate manner. Asci contain eight spores, which are usually ellipsoid to fusoid with 1 septum, hyaline, and with or without a gelatinous sheath. In the asexual cycle, these fungi produce subcylindrical, subulate or lageniform to cylindrical conidiophores and solitary conidia, which are often pale olivaceous-brown, smooth or coarsely verrucose, ellipsoid to obclavate or globose, and 0-1-septate. Species in this family are mainly saprobic and pathogens of plants; some cause sooty blotch and flyspeck on their hosts and are widespread across different landmasses (Diaz Arias et al. 2010; Chen et al. 2014). From a phylogenetic perspective, we followed the two most comprehensive assessments of Dissoconiaceae based on multi-locus sequence data (Crous et al. 2009; Marín-Félix et al. 2019a) to demonstrate that the monotypic Chaetoscutula (Müller 1958) belongs to this family.

\section{Chaetoscutula E. Müll.}

Notes: Chaetoscutula is a monotypic genus based on Chaetoscutula juniperi E. Müll (Müller 1958). It grows on 


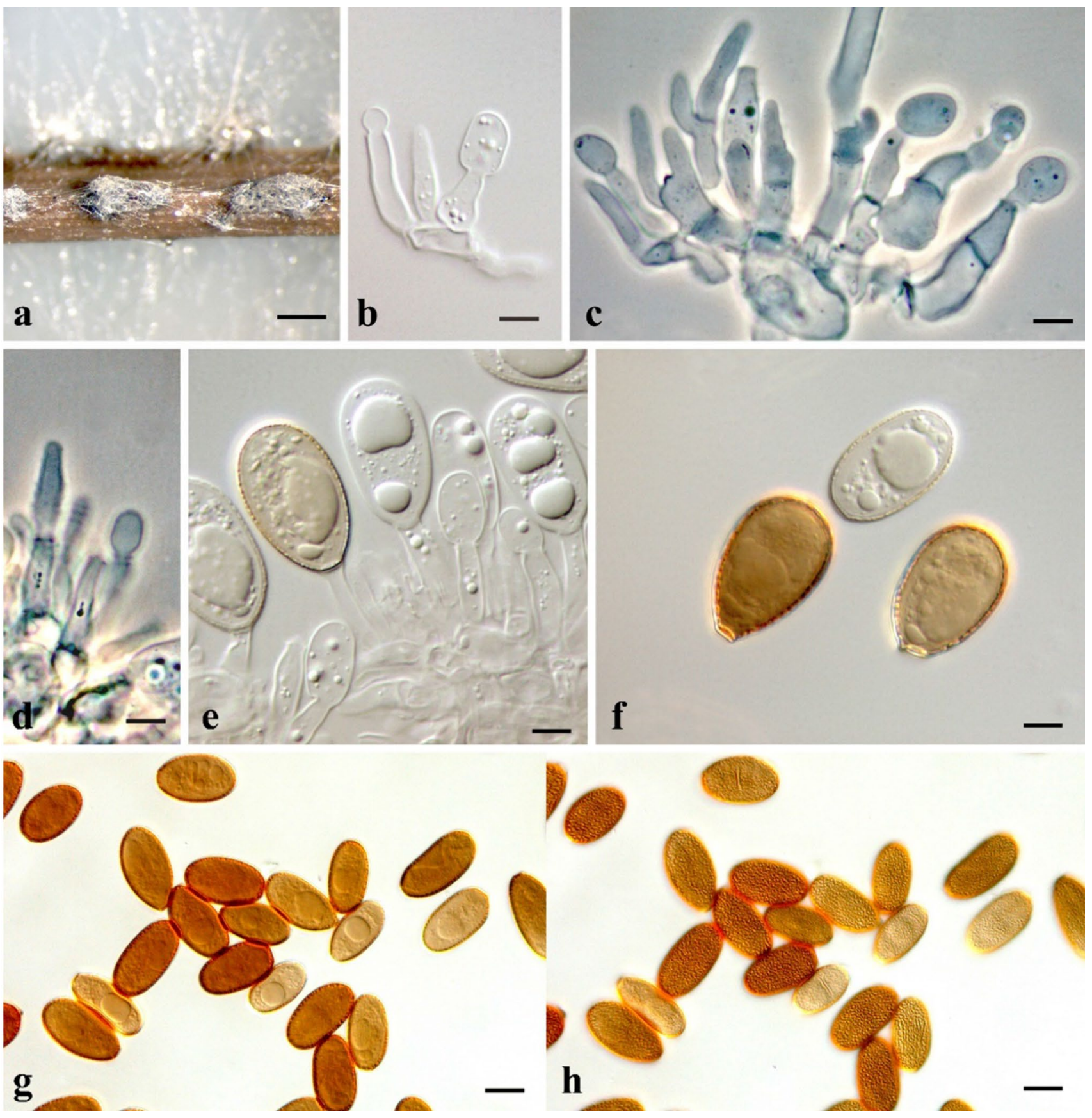

Fig. 1 Diplodia alanphillipsii (IRAN 14273F, holotype). a Conidiomata on pine needles in culture. b Hyaline immature conidia developing on conidiogenous cells. c, d Conidiogenous cells with periclinal

Juniperus spp. (Cupressaceae) and, although the mycelium is usually inconspicuous, this fungus produces tiny black hemispherical ascomata, solitary or gregarious, between adjacent leaves of the plant. The absence of signs of damage to the host suggests that the species is saprobic. The ascomata surface is characterized by displaying more or less dense, long and gnarled setae. Asci are bitunicate, 8-spored, and spores are cylindrical to clavate, two-celled, at first

thickenings or annellations. e Brown aseptate conidia on conidiogenous cells. f Mature brown conidia. $\mathbf{g}$, $\mathbf{h}$ Mature conidia in two different focal planes. Scale bars: $\mathbf{a}=500 \mu \mathrm{m}, \mathbf{b}-\mathbf{f}=5 \mu \mathrm{m}, \mathbf{g}, \mathbf{h}=10 \mu \mathrm{m}$

hyaline and then slightly brownish when mature. The genus was formerly placed within Schizothyriaceae, and later in Saccardiaceae (Müller and von Arx 1962; von Arx and Müller 1975) based on morphological comparisons. Recently, Tian et al. (2014) transferred Chaetoscutula to Pseudoperisporiaceae on the basis of the anatomical similarities to species in Pododimeria, a genus included in that family that also grows on Juniperus leaves (Müller 1958). In this 


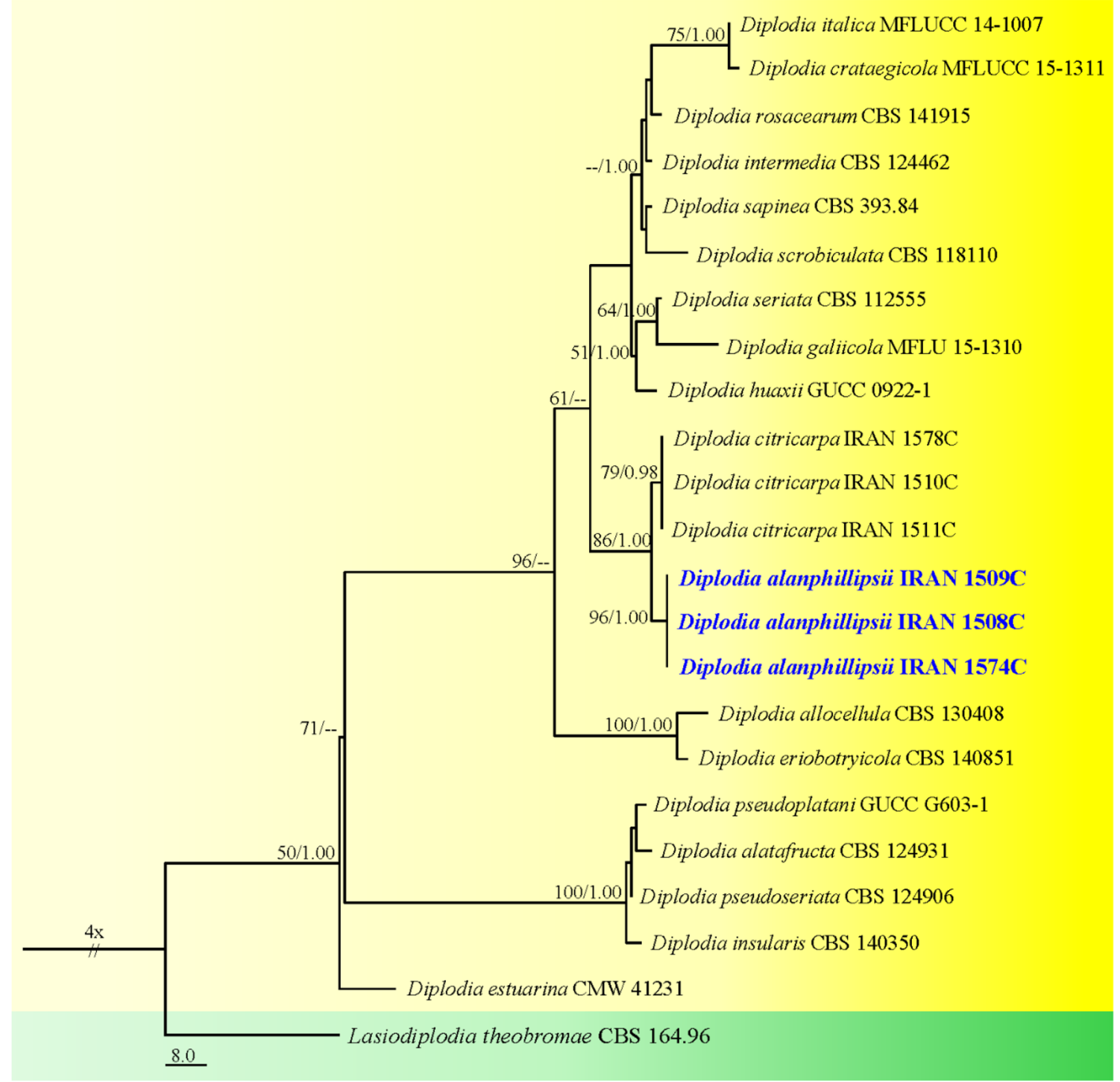

Fig. 2 Phylogram generated Maximum parsimony analysis based on combined ITS and TEF1- $\alpha$ sequence data of Diplodia species. The tree is rooted with Lasiodiplodia theobromae (CBS 164.96). The scale bar represents the expected number of changes per site. The MP analysis resulted one of the 36 equally most parsimonious trees $(\mathrm{TL}=246, \mathrm{CI}=0.87, \mathrm{HI}=0.13, \mathrm{RI}=0.83)$. Bootstrap values for max-

study, we transfer Chaetoscutula to Dissoconiaceae based on phylogenetic results (Fig. 4) of novel sequence data from three regions of the nuclear ribosomal DNA: the ITS, LSU and SSU.

Chaetoscutula juniperi E. Müll., Sydowia 12(1-6): 191 (1959) [1958]

Index Fungorum number: IF294737; Facesoffungi number: FoF 03694; Fig. 3

Saprobic on leaves of Juniperus spp. Sexual morph Ascomata growing in the contact zone between two adjacent imum parsimony (MPBS, left) based on 1000 pseudoreplicates equal to or greater than $50 \%$ are indicated at the nodes and posterior probabilities from Bayesian analysis (BYPP, right) equal to or greater than 0.95 are indicated at the nodes. The isolates of new species characterized in this study are in blue bold

leaves, solitary or gregarious, superficial, hemispherical, 146-200 $\mu \mathrm{m}$ diam. $(\bar{x}=179 \mu \mathrm{m}, \mathrm{n}=5)$, dark brown to black, and always ornamented with more or less dense, long and gnarled setae, $80-128 \times 3.5-4.4 \mu \mathrm{m}(\bar{x}=99 \times 3.9 \mu \mathrm{m}, \mathrm{n}=8)$ and with a thickened wall up to $2 \mu \mathrm{m}$. Shorter, lighter and blunt developing setae interspersed among the former. Peridium thin of dark brown, 4-9 $\mu \mathrm{m}$ wide, thick-walled cells of textura angularis. Ostiole inconspicuous. Hamathecium not distinguishable, but hyaline gelatinous matrix is discernible among asci. Asci $50-84 \times 20-30 \mu \mathrm{m}(\bar{x}=66 \times 25 \mu \mathrm{m}, \mathrm{n}=7)$, 8 -spored, bitunicate, arranged in parallel and originating 

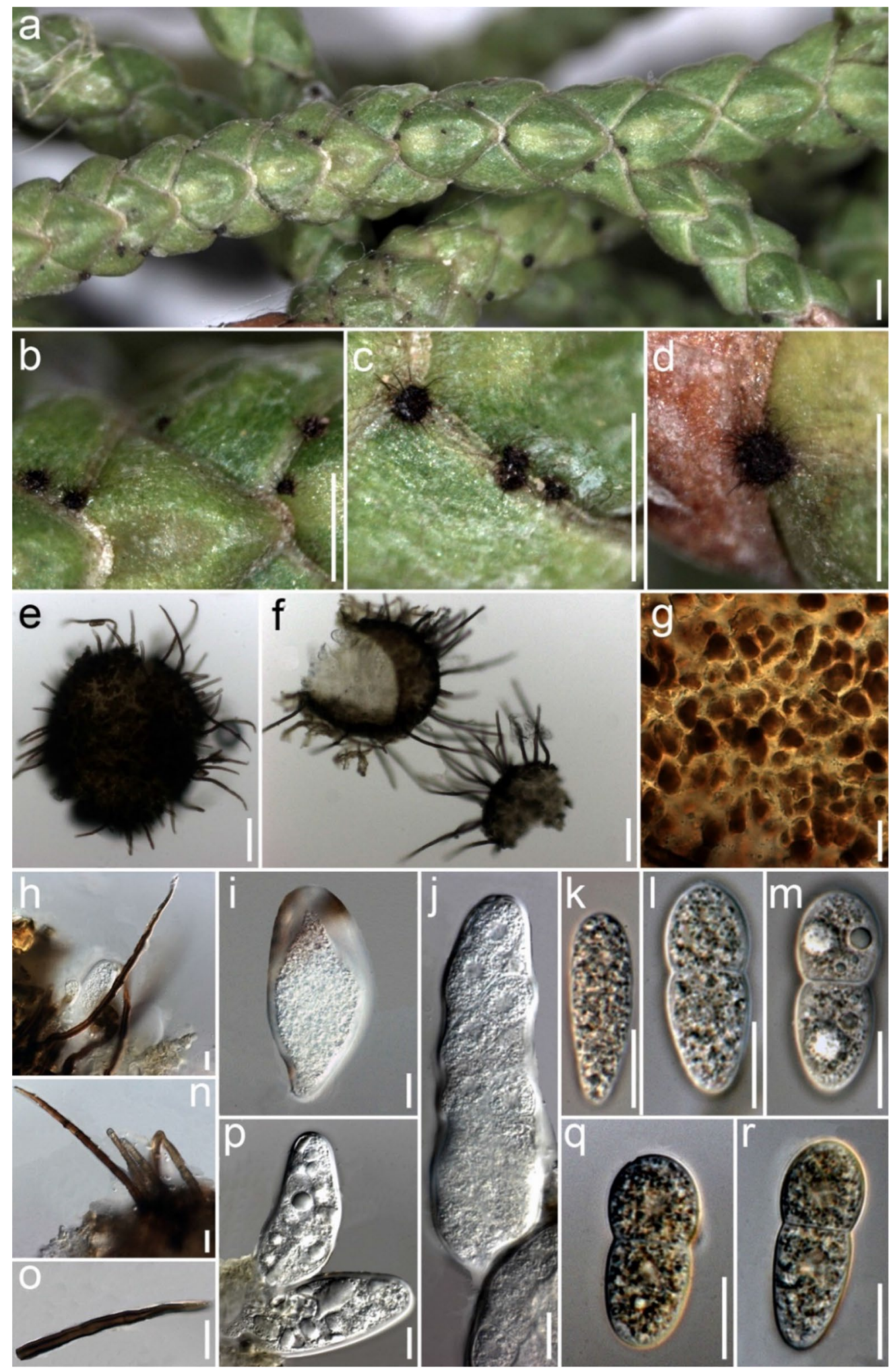

Fig. 3 Chaetoscutula juniperi (MA-90552, new record). a-d Ascomata on leaves of Juniperus phoenicea. e, f Ascomata immersed in water showing their hemispherical shape and their surface with dense, long and gnarled setae. g Peridium cells in surface view. $\mathbf{h}, \mathbf{n}, \mathbf{o}$ Setae. $\mathbf{i}, \mathbf{j}, \mathbf{p}$ Asci in several developmental stages. $\mathbf{k}-\mathbf{m}$, $\mathbf{q}, \mathbf{r}$ Ascospores in several developmental stages. Scale bars: a$\mathbf{d}=0.5 \mathrm{~mm}, \mathbf{e}, \mathbf{f}=50 \mu \mathrm{m}, \mathbf{g}-\mathbf{r}=10 \mu \mathrm{m}$ 
from a basal layer of hyaline, subcylindrical, broadly ellipsoid to obpyriform, loosely interwoven hyphae. Ascospores $23-28 \mu \mathrm{m}$ long $(\bar{x}=25.5 \mu \mathrm{m}, \mathrm{n}=10)$, cylindrical to clavate, 1 -septate, constricted at the septum, heteropolar, smaller cell width of $8.3-10.8 \mu \mathrm{m}(\bar{x}=9.6 \mu \mathrm{m}, \mathrm{n}=10)$, larger cell width of 9.7-11.5 $\mu \mathrm{m}(\bar{x}=10.5 \mu \mathrm{m}, \mathrm{n}=10)$, rounded at both extremes, first hyaline and then slightly brownish when mature, not surrounded by a distinct gelatinous sheath. Asexual morph Undetermined.

Material examined: SPAIN, Comunitat Valenciana, Valencia Province, Racó d'Ademús, Vallanca, on leaves of Juniperus phoenicea growing along the Bohílgues River margin, 40³'50.08" N 1'20'50.88" W, 958 msl, 19 August 2019, I. Garrido-Benavent, IGB756 (Real Jardín Botánico herbarium, MA-90552); Alacant Province, Vall d'Ebo, Corrals de Pego, entrance to the Barranc de l'Infern, on leaves of J. phoenicea, 38 $48^{\circ} 23.59^{\prime \prime} \mathrm{N} 0^{\circ} 08^{\prime 2} 23.41^{\prime \prime} \mathrm{W}, 377 \mathrm{msl}, 29$ December 2019, I. Garrido-Benavent, IGB857 (MA-92235); Castelló Province, Vistabella del Maestrat, between Alt de l'Asevar and El Chaparral, on leaves of J. sabina in a forest of Pinus sylvestris, $40^{\circ} 15^{\prime} 55.94^{\prime \prime} \mathrm{N} 0^{\circ} 22^{\prime} 57.52^{\prime \prime} \mathrm{W}, 1624$ msl, 21 August 2014, I. Garrido-Benavent, IGB793 (MA90553); Pina de Montalgrao, on leaves of J. phoenicea, 4001'16.47" N 0³9'24.86" W, 1034 msl, 21 August 2014, I. Garrido-Benavent, IGB794 (MA-90554); Castilla y León, Soria Province, Abejar, Sierra de Cabrejas, road to Calatañazor, on leaves of $J$. thurifera, $41^{\circ} 46^{\prime} 37.50^{\prime \prime} \mathrm{N} 2^{\circ} 46^{\prime} 41.20^{\prime \prime}$ W, 1161 msl, 17 March 2014, I. Garrido-Benavent, IGB795 (MA-90641); Aragón, Teruel Province, La Puebla de Valverde, Corral de la Ceja, on leaves of J. sabina, 40 $12^{\prime} 41.65^{\prime \prime}$ N 057'36.61" W, 1276 msl, 17 March 2014, I. GarridoBenavent, IGB796 (MA-90556); Castilla-La Mancha, Albacete Province, Férez, Montes de Aguas Calientes, on leaves of J. phoenicea, 38 $25^{\circ} 24.82^{\prime \prime} \mathrm{N} \mathrm{1}{ }^{\circ} 52^{\prime} 40.46^{\prime \prime} \mathrm{W}$, $501 \mathrm{msl}, 27$ December 2019, I. Garrido-Benavent, IGB835 (MA-92233); Murcia, Moratalla, between Benízar and Casicas del Portal, on leaves of J. phoenicea, $38^{\circ} 14^{\prime} 49.05^{\prime \prime} \mathrm{N}$ 1 $59^{\prime} 59.85^{\prime \prime}$ W, 1170 msl, 27 December 2019, I. GarridoBenavent, IGB845 (MA-92234); Ibidem, El Sabinar, close to Cortijo de la Leona, road to Letur, on leaves of J. thurifera, 38 $8^{\circ} 13^{\prime} 01.38^{\prime \prime}$ N 2 $2^{\circ} 08^{\prime} 07.61^{\prime \prime} \mathrm{W}, 1194$ msl, I. GarridoBenavent, IGB842.

GenBank numbers: ITS $=$ MN756704, LSU $=$ MN756705, $\mathrm{SSU}=\mathrm{MN756706}$ (all sequences obtained from the voucher MA-90552).

Notes: The morphological description of Iberian Chaetoscutula juniperi (Fig. 3) specimens that we provide here is in the agreement with the original species description (Müller 1958). There are, however, subtle differences in the size of setae and spores, which in our specimens tend to be slightly larger than previously described. Tian et al. (2014) provided the second and most recent description of the species based on material collected by E. Müller in France (collection S-F225899). Although the authors claimed that the studied ascomata "sometimes" displayed setae, the ascomata represented in the photographic plate (Tian et al. 2014; Fig. 1d) and the associated text description do not seem to match well with the concept of $C$. juniperi. Either in the present description or the one provided in the original publication (Müller 1958), C. juniperi has always been characterized as forming setae on the surface of ascomata; besides, ascomata were described as hemispherical, whereas Tian et al. (2014) described it as globose to subglobose and with much smaller ascomata (see Tian et al. 2014; Fig. 1e, f). A reevaluation of the collection S-F225899 must be conducted to ascertain whether it corresponds to the true C. juniperi or to other species growing on the same substrate (Juniperus leaves) such as Pododimeria gallica and Seynesiella juniperi, which share some microscopic characters with $C$. juniperi and cooccurred in the type collection (ZT Myc 60709).

Data provided in the present work increase the range of known Juniperus species to which Chaetoscutula juniperi is associated, as we found it on J. sabina and J. phoenica (Müller 1958), but also on J. thurifera, a tree species up to $20 \mathrm{~m}$ tall that is mainly distributed at high altitude regions in the inner Iberian Peninsula. Müller (1958) found this fungus growing on leaves of $J$. chinensis in the botanical garden of Munich. From a geographic perspective, the species appears to be present in France, Italy, Germany and Switzerland according to Müller (1958), Spain (present study) and possibly also in Canada (Annotated checklist for larger fungi on vancouver island 2020; http://azkurs.org/annotated-check list-for-larger-fungi-on-vancouver-island.html?page $=3$ ), despite the fact that there are no associated metadata to the Canadian reference. In the Global Biodiversity Information Facility (GBIF) database, there is an additional Canadian specimen collected in 1963 occurring on J. communis, as well as eight observation records of this fungus in Scotland. Finally, our three-loci phylogeny (Fig. 4) revealed Chaetoscutula as a new member of Dissoconiaceae, thus widening the spectrum of morphological variation of ascomata that characterizes this family. The phylogenetically closest genus is Pseudoveronaea. Only the asexual state of the two species included in the latter genus, $P$. ellipsoidea and P. obclavata is known ( $\mathrm{Li}$ et al. 2012), so that it is currently not possible to conduct a comprehensive morphological comparison between Chaetoscutula and Pseudoveronaea.

Dothideales Lindau (= Neocelosporiales Crous)

Notes: Latest updated accounts of Dothideales includes four families and 24 genera (Hongsanan et al. 2020a). 


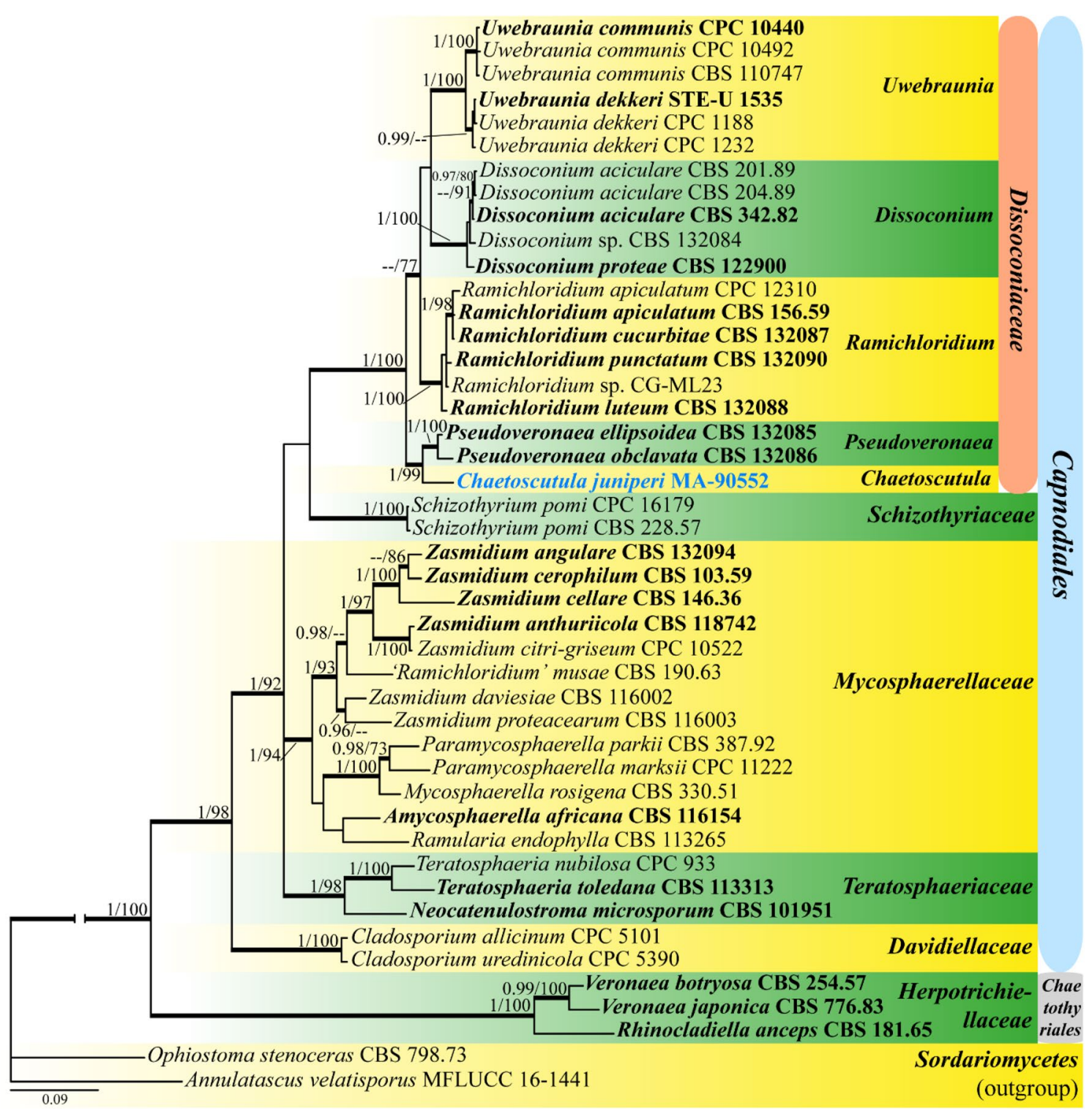

Fig. 4 Phylogram depicting the evolutionary relationships of Chaetoscutula and related clades in Dissoconiaceae (Capnodiales) based on a three-locus dataset (ITS, LSU and SSU). Species sampling was based on $\mathrm{Li}$ et al. (2012). The alignment matrix consisted of $2220 \mathrm{bp}$ and inferred substitution models were GTR $+\Gamma$ (ITS1 + ITS2), $\mathrm{K} 80+\mathrm{I}+\Gamma$ (5.8S, SSU), and GTR $+\mathrm{I}+\Gamma$ (LSU). The represented topology is obtained under a Bayesian framework with MrBayes v.3.2.6. Posterior

\section{Dothideaceae Chevall.}

Notes: Chevallier (1826) introduced Dothideaceae as 'Dothideae', and later Fuckel (1869) designated Dothidea as the type genus with $D$. gibberulosa (Ach.) Fr. as the type species. Recently, Dothideaceae was treated with 15 genera by Thambugala et al. (2014a). Dothideaceae is characterized by immersed to erumpent or superficial, uni or multiloculate ascostromata, 8- or polyspored, bitunicate asci and hyaline or brown, transversely septate and sometimes muriform ascospores (Thambugala et al. 2014a).
Probabilities (PP) are represented on branches leading to nodes. Bootstrap support values obtained in a complementary Maximum Likelihood analysis (MLBS, right) with RAxML using 1000 pseudoreplicates are provided after the BYPP values (left). Branches in bold had MLBS equal or greater than 70\% and BYPP equal or greater than 0.95 . For each terminal, the species name and the voucher/herbarium code are indicated, and type strains are in bold and new isolate is in blue

\section{Dothiora Fr.}

Notes: Dothiora was introduced by Fries (1849) with D. pyrenophora as the type species. Dothiora (based on D. pyrenophora) produces a Dothichiza asexual morph in culture (Crous and Groenewald 2017). Species of Dothiora are commonly isolated from dead branches of woody hosts (Sivanesan 1984), while Crous and Groenewald (2016) also reported some species from dead leaves and fruits of diverse hosts, indicating that it is a saprobe, possibly acting as a weak pathogen on stressed plant tissues. 

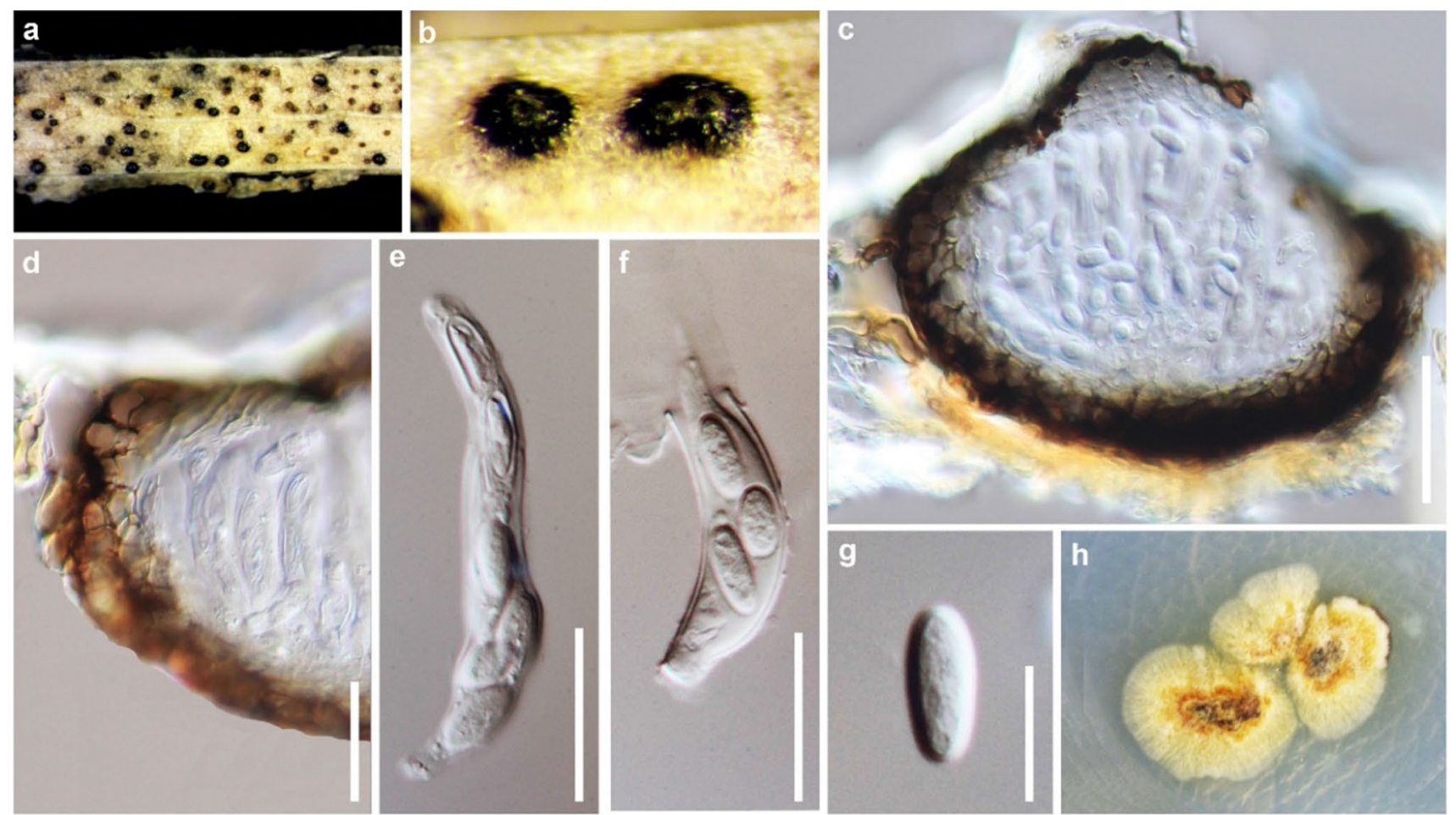

Fig. 5 Dothiora coronicola (MFLU 16-1110, holotype). a, b Ascomata on host surface. c Section through the ascoma. d Peridium. e, f Asci. g Ascospore. $\mathbf{h}$ Culture on PDA. Scale bars: $\mathbf{c}, \mathbf{d}=100 \mu \mathrm{m}, \mathbf{e}, \mathbf{f}=50 \mu \mathrm{m}, \mathbf{g}=20 \mu \mathrm{m}$

Dothiora coronicola Dissanayake, Camporesi \& K.D. Hyde, sp. nov.

Index Fungorum number: IF556815; Facesoffungi number: FoF 06509; Fig. 5

Etymology: In reference to the host Coronilla, and cola meaning loving.

Holotype: MFLU 16-1110

Saprobic on dead aerial branch of Coronilla emerus L. Sexual morph Ascomata 215-430×240-285 $\mu$ m, immersed or erumpent through the epidermis, solitary or clustered, globose, brown to black, with single locules. Peridium 39-76 $\mu \mathrm{m}$ wide, two-layered, outer layer composed of dark brown or brown, thick-walled cells of textura angularis, inner layer composed of hyaline, thin-walled cells of textura angularis.

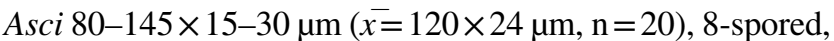
bitunicate, fissitunicate, cylindro-clavate, pedicellate, apically rounded, with a small ocular chamber. Ascospores $21-25 \times 8-11 \mu \mathrm{m}(\bar{x}=23 \times 10 \mu \mathrm{m}, \mathrm{n}=30)$, bi-seriate to multiseriate, hyaline, aseptate, fusoid to ovoid, one end narrower than the other, smooth-walled with granular contents, lacking a mucilaginous sheath. Asexual morph Undetermined.

Culture characteristics: Colonies on PDA reaching $2 \mathrm{~cm}$ after 14 days at $25{ }^{\circ} \mathrm{C}$, mycelium velvety and moderately fluffy with an irregular margin, surface initially white and later turning dark yellow from the middle of the colony and dark grey in reverse.
Material examined: ITALY, Province of Forlì-Cesena, near Passo delle Forche- Galeata, on dead aerial branch of Coronilla emerus (Fabaceae), 30 March 2016, E. Camporesi, IT 2894 (MFLU 16-1110, holotype), ex-type living culture, MFLUCC 17-1007.

GenBank numbers: ITS $=$ MZ571206, LSU = MZ571207.

Notes: We were able to obtain a culture from a single conidium. In the phylogenetic analysis, Dothiora coronicola forms a distinct lineage basal to $D$. buxi, $D$. cactacearum, $D$. coronillae and D. spartii with 96\% MLBS support (Fig. 6). However, this taxon can be differentiated from above mentioned species as follows. Dothiora buxi has polysporous asci with 32 ascospores in an ascus, while $D$. coronicola has asci with only eight ascospores. Dothiora buxi also has pale brown ascospores, whereas $D$. coronicola has hyaline ascospores (Fig. 5). The placement of D. buxi within the Dothiora was proven by molecular data (Hyde et al. 2016). Morphological variations of $D$. coronicola and D. cactacearum cannot be compared as the latter composed only an asexual morph. Dothiora coronicola can be easily distinguished from $D$. coronillae as the latter comprised with cylindro-clavate asci (Hyde et al. 2017). Both D. coronicola and $D$. spartii are morphologically comparable but phylogenetically they cluster in two different places (Fig. 6).

Dyfrolomycetales K.L. Pang, K.D. Hyde \& E.B.G. Jones 


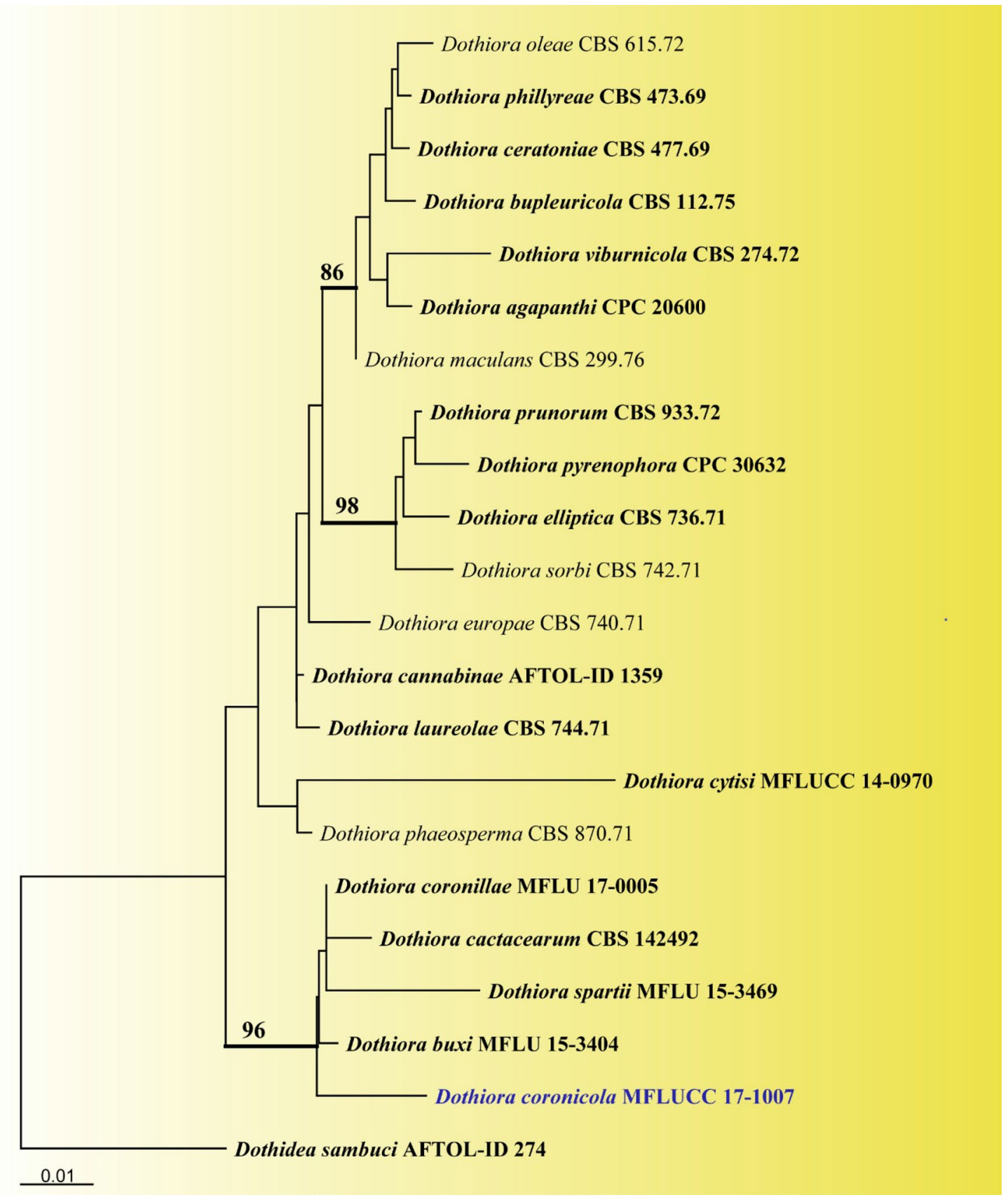

Fig. 6 Phylogram generated from maximum likelihood analysis based on combined LSU and ITS sequence data representing Dothiora coronicola (MFLUCC 17-1007) and related species. The scale bar indicates 0.01 changes. The tree is rooted to Dothidea sambuci (AFTOL-ID 274). Related sequences were taken from Hongsanan et al. (2020a). Twenty-two sequences are included in the analysis which comprise 1396 characters after alignment. The best RAxML tree with a final likelihood value of -3690.034105 is presented.

Notes: Dyfrolomycetales was introduced by Pang et al. (2013) based on the collection of marine fungi Dyfrolomyces tiomanensis from Tioman Island, Malaysia. Only the single family Pleurotremataceae was accepted in this order with three associated
The matrix had 195 distinct alignment patterns, with 5.69\% undetermined characters or gaps. Estimated base frequencies are as follows: $\mathrm{A}=0.251756, \mathrm{C}=0.223630, \mathrm{G}=0.280088, \mathrm{~T}=0.244526$; substitution rates $\mathrm{AC}=1.042605, \mathrm{AG}=2.446647, \mathrm{AT}=1.739048, \mathrm{CG}=0.584297$, $\mathrm{CT}=5.850014, \mathrm{GT}=1.000000$; gamma distribution shape parameter $\alpha=0.020014$. Bootstrap values for maximum likelihood (MLBS) equal to or greater than $70 \%$ are indicated at the nodes. Type and extype strains are in bold and the newly generated sequence is in blue

genera Dyfrolomyces, Melomastia and Pleurotrema. The latest treatment of the order follows Hongsanan et al. (2020b).

Pleurotremataceae Walt. Watson (=Dyfrolomycetaceae K.D. Hyde et al.) 
Notes: Pleurotremataceae, typified by Pleurotrema with Pleurotrema polysemum, was introduced by Watson (1929) and accepted as a monotypic genus Pleurotrema within the order Chaetosphaeriales. Pleurotremataceae was assigned to Xylariales based on the non fissitunicate ascus character by Barr (1994) with five genera included: Daruvedia, Melomastia, Phomatospora, Pleurotrema and Saccardoella. The taxonomic placement of Pleurotremataceae was revised by various authors (Hyde 1992; Hawksworth et al. 1995; Tsui et al. 1998; Kirk et al. 2008; Suetrong et al. 2009; Lumbsch and Huhndorf 2010; Hu et al. 2010b; Hyde et al. 2013; Pang et al. 2013; Senanayake et al. 2016). Maharachchikumbura et al. (2015) resulting in it being accepted as Pleurotrema in Pleurotremataceae and placing it under Chaetosphaeriales, Sordariomycetes. Maharachchikumbura et al. (2016) synonymized Dyfrolomycetaceae under Pleurotremataceae and excluded it from Sordariomycetes based on morphology of the Pleurotrema polysemum isotype. This family comprises three genera characterized by perithecial, ostiolate, glabrous, ovoid to subglobose ascomata, clavate to cylindrical, bitunicate, short pedicellate asci, with a J-apical ring, and hyaline, ellipsoidal to cylindrical, multi-distoseptate, guttulate ascospores that are uni-seriate arranged in the asci (Hongsanan et al. 2020b).

Dyfrolomyces K.D. Hyde, K.L. Pang, Alias, Suetrong \& E.B.G. Jones

Notes: Dyfrolomyces was introduced by Pang et al. (2013) to accommodate taxa observed on unidentified mangrove wood in Malaysia. Pang et al. (2013) transferred the three marine Saccardoella species to Dyfrolomyces viz. D. mangrovei, D. marinosporus and D. rhizophorae based on ascomatal structure and three-loci phylogenetic analysis. Ten species are included in the genus which are characterized by the presence of relatively large, immersed, globose or subglobose, clypeate, ostiolate, papillate ascomata, bitunicate, fissitunicate, cylindrical asci and broadly fusiform, symmetrical, hyaline, septate ascospores, with or without a mucilaginous sheath (Pang et al. 2013; Dayarathne et al. 2020; Hongsanan et al. 2020b).

Dyfrolomyces distoseptatus M. Niranjan \& V.V. Sarma, in Hongsanan et al., Fungal Diversity 105:73 (2020)

Index Fungorum number: IF556726; Facesoffungi number: FoF 06625; Fig. 7

Saprobic on submerged decaying wood in a freshwater habitat. Sexual morph Ascomata $600-800 \times 350-530 \mu \mathrm{m}$ $(\bar{x}=682 \times 433 \mu \mathrm{m}, \mathrm{n}=10)$, perithecial, immersed in periderm, erumpent neck with pseudoparaphyses, clypeate, ostiolate, papillate. Peridium 30-40 $\mu \mathrm{m}$, with two strata, outer thick, carbonaceous and inner brown and hyaline cells of textura angularis. Peridium comprising of dark pigmented cells of textura angularis. Hamathecium pseudoparaphyses, filamentous, septate, unbranched, 2-4 $\mu \mathrm{m}$ wide, long, dense, longer than asci.
Asci thin walled and quickly evanescent, unable to observed. Ascospores $17-26 \times 3.5-6 \mu \mathrm{m}(\bar{x}=23 \times 5 \mu \mathrm{m}, \mathrm{n}=30)$, uniseriate, hyaline, 1-2-distoseptate in early stages, usually 3-distoseptate, with a large guttules at each cell, fusoid, acute ends, apical ends slightly bent. Asexual morph Undetermined.

Culture characteristics: Conidia germinating on malt extract agar (MEA) within $24 \mathrm{~h}$. Germ tubes produced from the basal and apical cell of conidia. Colonies growing on MEA, reaching $25-30 \mathrm{~mm}$ in 2 weeks at $25^{\circ} \mathrm{C}$, colony circular, entire to filiform edge, dry, surface rough, with dense mycelium, grayish brown in top view, reverse dark brown.

Material examined: THAILAND, Tak Province, Tha Sing Yang, Ban Mae Ja Wang on submerged decaying wood in a freshwater river, 17 October 2019, N. Padaruth, CC51 (MFLU 21-0121, new geographical record), living culture, MFLUCC 21-0102.

GenBank numbers: ITS =MT864349, LSU = MT860427.

Notes: Dyfrolomyces distoseptatus was introduced by Hongsanan et al. (2020b) isolated from an unidentified decaying twig in India. Our new isolate, Dyfrolomyces distoseptatus MFLUCC 21-0102 clustered with D. distoseptatus strain NFCCI: 4377 with 95\% MPBS, 96\% MLBS, 1.00 BYPP (Fig. 8). Dyfrolomyces distoseptatus clustered with D. sinensis and D. phetchaburiensis, however, D. distoseptatus differs having 2-3 septate ascospores with acute ends, while $D$. sinensis and D. phetchaburiensis have 6-7 septate and 1-10 septate ascospores, respectively (Hyde et al. 2017, 2018a). Unlike other species (viz. D. aquatica, D. mangrovei, D. marinosporus, D. rhizophorae and D. thailandicus), D. distoseptatus lacks a gelatinous sheath around the ascospores (Hyde 1992; Tsui et al. 1998; Pang et al. 2013; Hongsanan et al. 2020b). In addition, Dyfrolomyces distoseptatusis strains (MFLUCC 21-0102 and NFCCI: 4377) clustered with D. thamplaensis, however, D. distoseptatusis differs in having longer ascospores (17-26.5×3.5-6.5 $\mu \mathrm{m})$ whereas D. thamplaensis has shorter ascospores $(9.5-23.5 \times 5-6.5 \mu \mathrm{m})$ (Fig. 7). A comparison of the ITS nucleotides indicates that our strain of $D$. distoseptatus is largely similar to strain NFCCI: 4377 based on DNA sequence (Jeewon and Hyde 2016). Therefore, we identify our new collection as $D$. distoseptatus which is the new record from a freshwater habitat in Thailand. Since the ascal morphology was not included in the observed substrates, recollection of the specimen is recommended.

Jahnulales K.L. Pang, Abdel-Wahab, El-Shar., E.B.G. Jones \& Sivichai

Notes: Jahnulales was introduced by Pang et al. (2002) to accommodate three genera Aliquandostipite, Jahnula and Patescospora. We follow the latest treatment and updated accounts of Jahnulales in Hongsanan et al. (2020b). The order includes Aliquandostipitaceae (seven genera) and Manglicolaceae (one genus) which are commonly isolated from submerged decaying wood. 


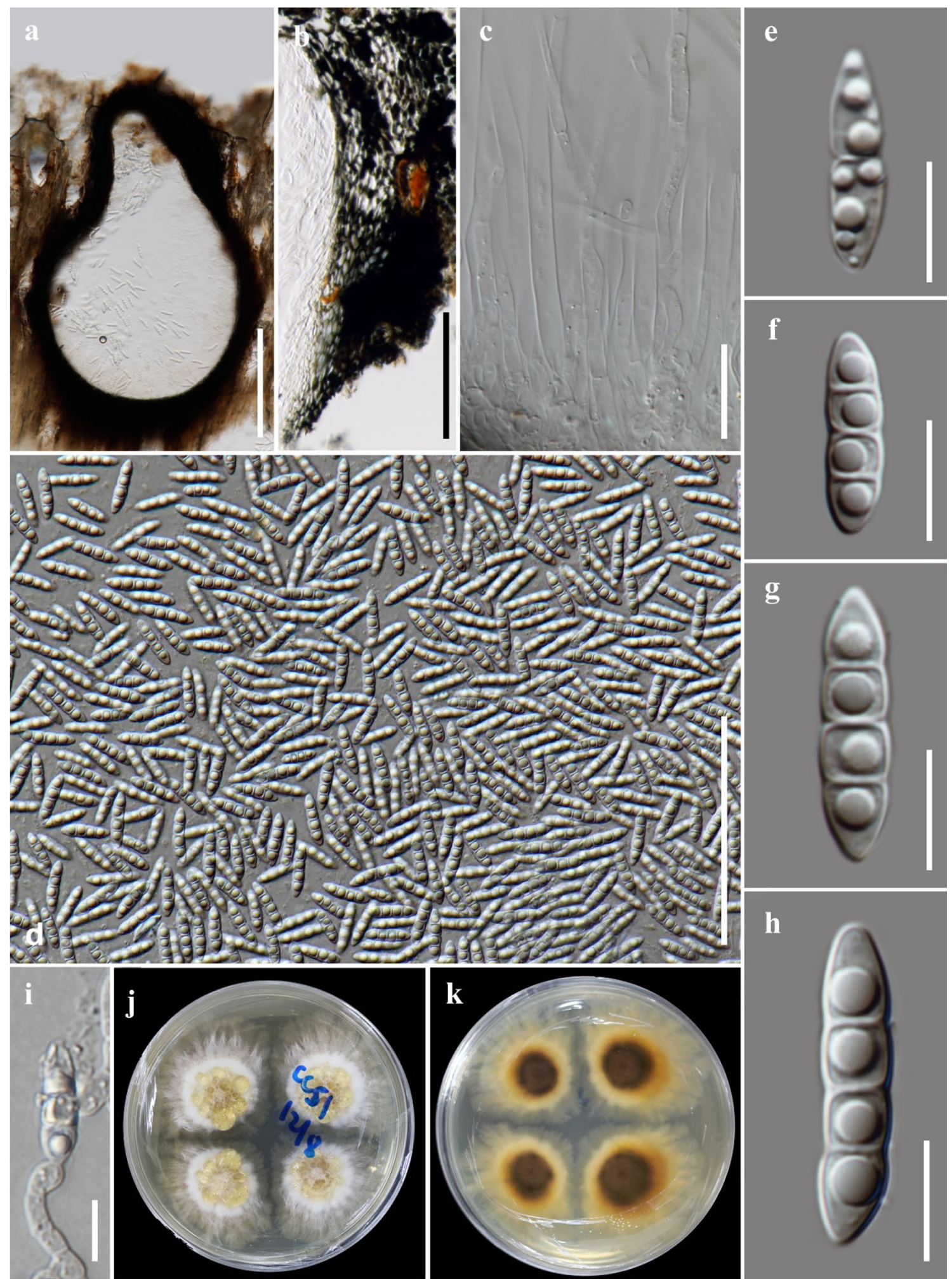

Fig. 7 Dyfrolomyces distoseptatus (MFLU 21-0121, new geographical record). a Vertical section of ascoma. b Peridium. c Pseudoparaphyses. d-h Ascospores (Note: Asci thin walled and quickly eva- nescent, unable to observe). $\mathbf{i}$ Germinated ascospore. $\mathbf{j}, \mathbf{k}$ Culture on MEA from surface and reverse. Scale bars: $\mathbf{a}=200 \mu \mathrm{m}, \mathbf{b}=100 \mu \mathrm{m}$, $\mathbf{c}=20 \mu \mathrm{m}, \mathbf{d}-\mathbf{i}=10 \mu \mathrm{m}$ 


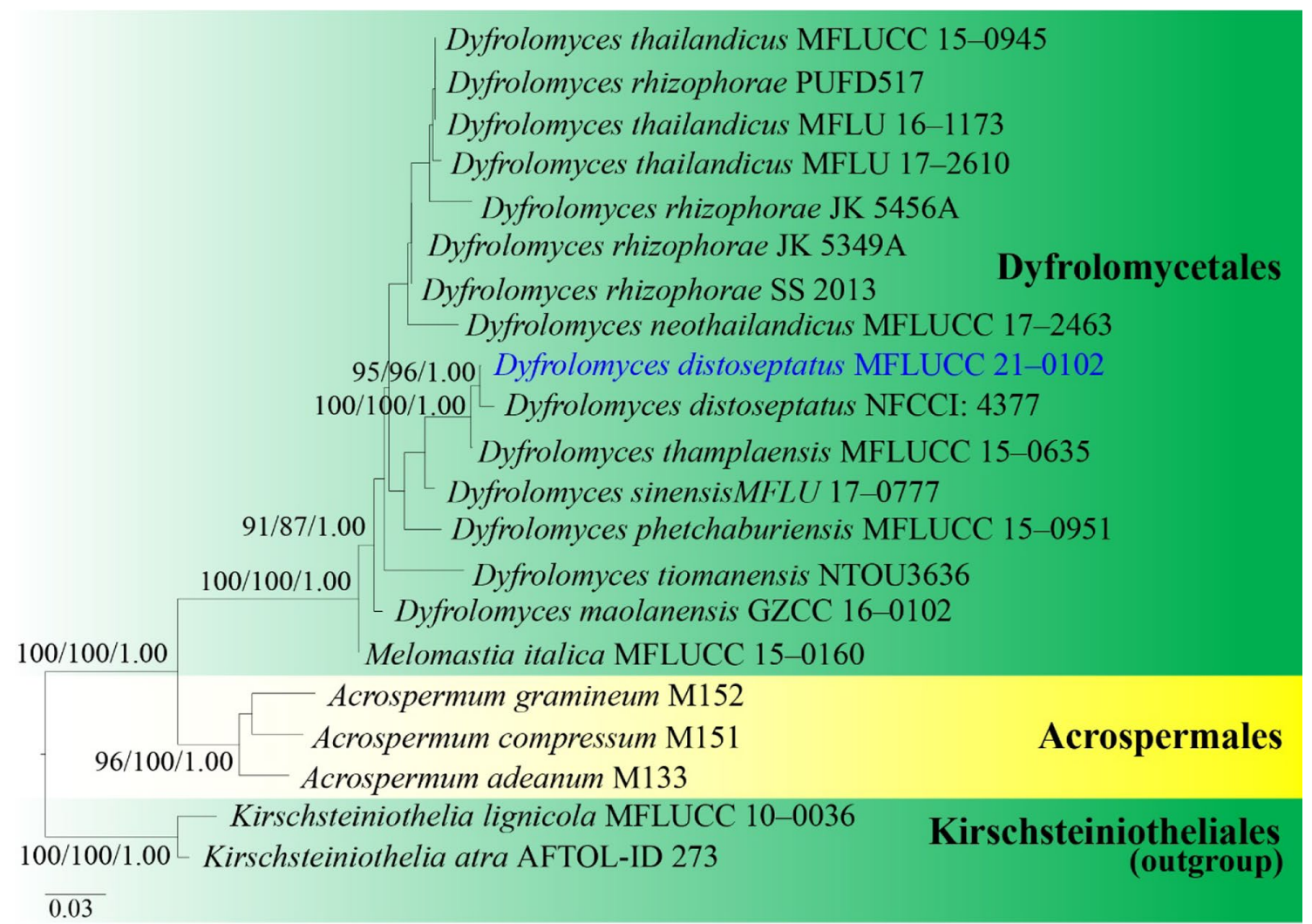

Fig. 8 Phylogram generated from maximum likelihood analysis based on combined LSU, SSU and ITS sequence data representing the species of Dyfrolomycetales and Acrospermales. Related sequences are taken from Hongsanan et al. (2020b). Kirschsteiniothelia lignicola (MFLUCC 10-0036) and $K$. atra (AFTOL-ID 273) are used as the outgroup taxa. Twenty-one taxa are included in the combined analyses which comprised 3532 characters (1359 characters for LSU, 1669 characters for SSU, 504 characters for ITS) after alignment. The best scoring RAxML tree with a final likelihood value of -10567.325091 is presented. The matrix had 700 distinct alignment patterns, with $44.47 \%$ of undeter-

\section{Aliquandostipitaceae Inderbitzin}

Notes: Inderbitzin et al. (2001) introduced Aliquandostipitaceae that are characterized by the widest hyphae reported in the ascomycetes, and the formation of both sessile and stalked ascomata side by side on the substrate. Seven genera, Aliquandostipite, Brachiosphaera, Jahnula, Megalohypha, Neojahnula, Pseudojahnula and Xylomyces are accepted in Aliquandostipitaceae (Hongsanan et al. 2020b).

\section{Aliquandostipite Inderbitzin}

Notes: Aliquandostipite was introduced to accommodate two species, Aliquandostipite khaoyaiensis and A. sunyatsenii based on SSU sequence data (Inderbitzin et al. 2001). Aliquandostipite includes six accepted species which are supported by both morphology and phylogenetic analysis (Suetrong et al. 2011; Liu et al. 2015a, Species Fungorum 2021).

Aliquandostipite khaoyaiensis Inderbitzin, Am. J. Bot. 88: 54 (2001) mined characters or gaps. Estimated base frequencies were as follows: $\mathrm{A}=0.242587, \mathrm{C}=0.243215, \mathrm{G}=0.300854, \mathrm{~T}=0.213344$; substitution rates: $\mathrm{AC}=1.335920, \mathrm{AG}=2.320033, \mathrm{AT}=0.575696, \mathrm{CG}=1.101975$, $\mathrm{CT}=5.314102$, GT $=1.000000$; gamma distribution shape parameter $\alpha=0.248862$. The MP analysis resulted a single most parsimonious tree $(\mathrm{TL}=1145, \mathrm{CI}=0.851, \mathrm{RI}=0.803, \mathrm{RC}=0.683, \mathrm{HI}=0.149)$. Bootstrap support values for ML and MP equal to or greater than 70\% and BYPP equal to or greater than 0.95 are given above the nodes. The newly generated sequence is in blue

Index Fungorum number: IF483979; Facesoffungi number: FoF 09158; Fig. 9

Saprobic on submerged wood. Sexual morph Ascomata 340-415 $\mu \mathrm{m}$ high, 250-290 $\mu \mathrm{m}$ diam., hyaline to pale brown, becoming dark brown with age, scattered, superficial, sometimes seated in a pseudostroma, globose or subglobose, sessile, membranous, papillate, ostiolate. Ostioles 100-135 $\mu \mathrm{m}$ apically lined by elongated cells. Peridium $30-40 \mu \mathrm{m}$ thick, membranous, composed of thin-walled, pale brown, compressed cells of textura angularis. Pseudoparaphyses 2-5 $\mu \mathrm{m}$ diam., numerous, sparsely branched, hyaline, septate. Asci $155-200 \times 35-65 \mu \mathrm{m}(\bar{x}=175 \times 50 \mu \mathrm{m}, \mathrm{n}=20)$, 8 -spored, bitunicate, fissitunicate, clavate, thickened at apex, sessile or short pedicellate, with a well-developed ocular chamber. Ascospores 55-70 $\times 15-25 \mu \mathrm{m}(\bar{x}=65 \times 19 \mu \mathrm{m}$, $\mathrm{n}=30$ ), variably arranged in asci, oval, hyaline to pale brown, 1-septate, deeply constricted at the septum, asymmetric, upper cell shorter and wider than lower cell, guttulate, straight or curved, thin-walled, smooth, sheathed. 


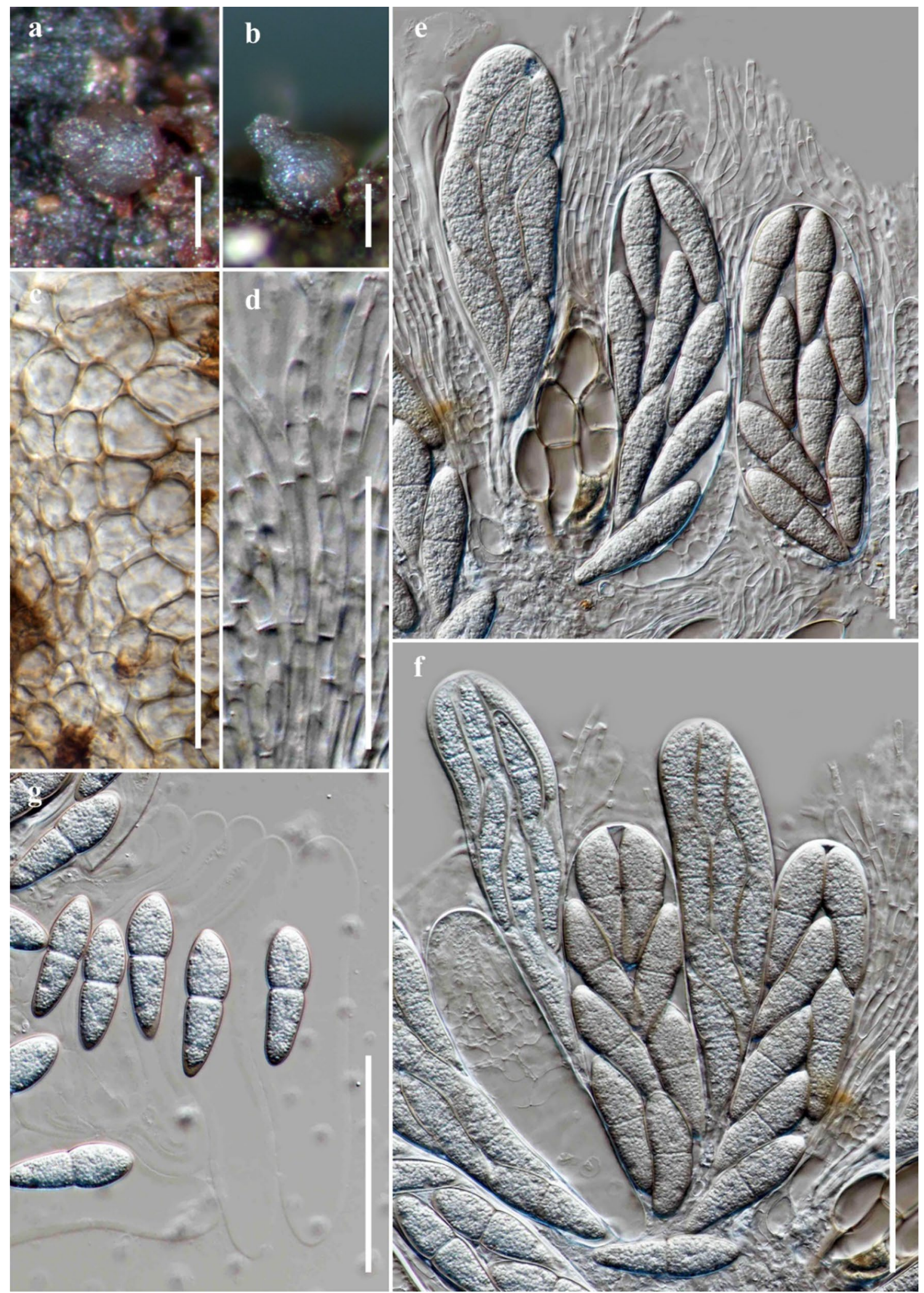

Fig. 9 Aliquandostipite khaoyaiensis (MFLU 21-0125, new record). a, b Ascomata superficial on host surface. c Peridium cells. d Pseudoparaphyses. e, f Asci. $\mathbf{g}$ Ascospores. Scale bars: $\mathbf{a}, \mathbf{b}, \mathbf{e}, \mathbf{f}=200 \mu \mathrm{m}, \mathbf{c}, \mathbf{g}=100 \mu \mathrm{m}, \mathbf{d}=20 \mu \mathrm{m}$ 
Sheath first appressed to the ascospore wall, expanding and detaching from the polar regions when mounted in water, becoming balloon-like at the two poles, finally surrounding the entire ascospore. Asexual morph Undetermined.

Culture characteristics: Conidia germinating on malt extract agar (MEA) within $24 \mathrm{~h}$. Germ tubes produced from the basal and apical cells of conidia. Colonies growing on MEA, reaching $20-25 \mathrm{~mm}$ in 2 weeks at $25^{\circ} \mathrm{C}$, colony circular, entire edge, dry, surface rough, with dense mycelium, pale yellow from above, dark brown from below

Material examined: THAILAND, Phitsanulok Province, Wang Thong, Kaeng Sopha stream, on submerged wood, 25 July 2019, S. Boonmee, ISAN100 (MFLU 21-0125, new record), living culture, MFLUCC 21-0106.

GenBank numbers: ITS $=$ MT864350, LSU $=$ MT860428, SSU $=$ MT $860445, \quad$ TEF $1-\alpha=$ MT 873577, $\mathrm{RPB} 2=\mathrm{MT} 873578$.
Notes: Aliquandostipite khaoyaiensis has been recorded from Costa Rica (Raja et al. 2005), Thailand (Campbell et al. 2007) and U.S.A. (Raja et al. 2009). Our isolate MFLUCC 21-0106 resembles the generic description of the species except it lacks stalked ascomata. Furthermore, A. khaoyaiensis (MFLUCC 21-0106) has shorter ascospores compared to A. khaoyaiensis UBC F13875, the holotype isolated from Khao Yai National Park, Thailand $(55-70 \times 15-25 \mu \mathrm{m}$ vs. $39-52 \times 16-23 \mu \mathrm{m})$. In the phylogenetic tree, our new isolate clustered with other strains of A. khaoyaiensis and A. siamensis with 99\% MPBS, 0.99 BYPP support (Fig. 10), which appears to be conspecific. Aliquandostipite siamensis is distinct from A. khaoyaiensis in having dimorphic, hyaline or brown ascospores without a sheath while the latter produces monomorphic, pale brown ascospores with welldeveloped sheaths (Inderbitzin et al. 2001; Pang et al. 2002; Campbell et al. 2007). We therefore report our collection as

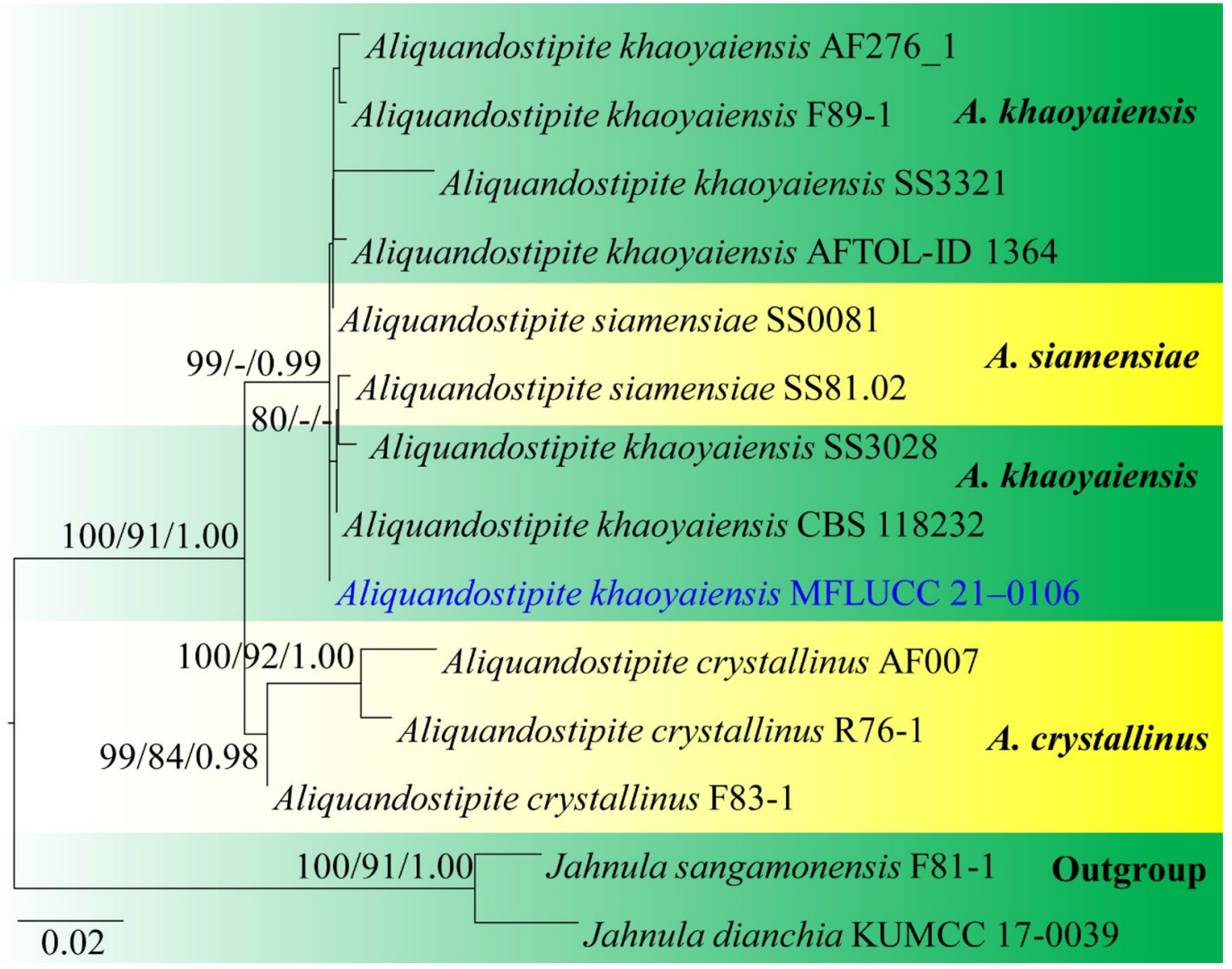

Fig. 10 Phylogram generated from maximum likelihood analysis based on combined LSU, SSU, ITS, TEF1- $\alpha$ and RPB2 sequence data representing the species of Aliquandostipite. Related sequences are taken from Suetrong et al. (2011). Jahnula sangamonensis (F81-1) and J. dianchia (KUMCC 17-0039) in Aliquandostipitaceae (Jahnulales) were used as the outgroup taxa. Fourteen taxa are included in the combined analyses which comprised 4306 characters (1006 characters for LSU, 1005 characters for SSU, 587 characters for ITS, 726 characters for TEF1- $\alpha$, 982 characters for RPB2) after alignment. The best scoring RAxML tree with a final likelihood value of -8403.913612 is presented. The matrix had 301 distinct alignment patterns, with $51.74 \%$ of undetermined characters or gaps. The MP analysis resulted a single most parsimonious tree $(\mathrm{TL}=442, \mathrm{CI}=0.986, \mathrm{RI}=0.977, \mathrm{RC}=0.964$, $\mathrm{HI}=0.014)$. Bootstrap support values for ML and MP equal to or greater than $75 \%$ and BYPP equal to or greater than 0.95 are given above the nodes. The newly generated sequence is in blue 
a fourth record of A. khaoyaiensis from submerged wood in Thailand (Fig. 9).

Minutisphaerales Raja, Oberlies, Shearer \& A.N. Mill.

Notes: Minutisphaerales comprises a single family Minutisphaeraceae to accommodate the monotypic genus Minutisphaera (Raja et al. 2015). We follow the latest treatment and update accounts of Minutisphaerales in Bao et al. (2019a).

Minutisphaeraceae Raja, Oberlies, Shearer \& A.N. Mill.

Notes: Minutisphaeraceae comprises single genus Minutisphaera and its members commonly found as saprobic on submerged wood in freshwater habitat (Ferrer et al. 2011; Raja et al. 2015; Bao et al. 2019a). The family is characterized by globose to subglobose, erumpent to superficial, dark pigmented ascomata, obpyriform to obclavate asci, oblong, clavate to broadly fusiform, septate, hyaline to pale brown ascospores, with or without a gelatinous sheath and filamentous appendages (Raja et al. 2015).

\section{Minutisphaera Shearer, A.N. Mill. \& A. Ferrer}

Notes: A monotypic genus Minutisphaera was introduced by Ferrer et al. (2011) and is typified by Minutisphaera fimbriatispora. Only five species are presently listed in this genus (Bao et al. 2019a; Index Fungorum 2021). Minutisphaera thailandensis is introduced as the sixth species in this genus.

Minutisphaera thailandensis R.J. Xu, Boonmee \& K.D. Hyde, sp. nov.

Index Fungorum number: IF558547; Facesoffungi number: FoF 09943; Fig. 11

Etymology: The specific epithet "thailandensis" referring to the country from which the species was collected.

Holotype: MFLU 21-0094

Saprobic on submerged wood, submerged in freshwater habitats. Sexual morph Ascomata 80-108 $\mu \mathrm{m}$ high, 95-101 $\mu \mathrm{m}$ diam., immersed to erumpent, solitary, scattered, globose to subglobose, dark brown to black, ostiolate. Peridium thin, soft, composed of brown to dark brown cells of textura angularis. Hamathecium pseudoparaphyses not seen. Asci $40-56 \times 15-27 \mu \mathrm{m}(\bar{x}=47 \times 21 \mu \mathrm{m}, \mathrm{n}=10), 8$-spored, bitunicate, obpyriform to broadly clavate, slightly curved, rounded at the apex, sessile to obtuse at the base. Ascospores $21-27 \times 5-8 \mu \mathrm{m}(\bar{x}=24 \times 7 \mu \mathrm{m}, \mathrm{n}=20), 2-3$-seriate overlapping, ovoid to obovoid, apex wider, narrower towards the lower and rounded end, 1-septate, slightly above the middle, constricted at the septum, hyaline, surrounded by prominent mucilaginous sheath, sheath constricted the septum, with long filamentous appendages separating out of the sheath, numerous granular contents, smooth-walled. Asexual morph Undetermined.
Culture characteristics: Ascospores germinating on MEA within $12 \mathrm{~h}$. Colonies on MEA, reaching $5 \mathrm{~cm}$ diam. after 4 weeks at room temperature, mycelium dense, brown to dark brown on surface, dark brown to black in reverse with entire edge.

Material examined: THAILAND, Chiang Rai Province, Mueang, Ban Nang Lae (99 $52^{\prime} 52.93^{\prime \prime}$ E $\left.20^{\circ} 3^{\prime} 2.52^{\prime \prime} \mathrm{N}\right)$, on submerged wood in freshwater stream, 27 May 2020, R.J. Xu, MD-39 (MFLU 21-0094, holotype), ex-type living culture, MFLUCC 21-0072.

GenBank numbers: ITS $=$ MZ493355, LSU $=$ MZ493343, SSU $=$ MZ493342.

Notes: Multigene phylogenetic analysis indicates our new taxon consistently clustered with Minutisphaera aquaticum with 100\% MLBS, 1.00 BYPP support (Fig. 12). However, M. thailandensis (MFLUCC 21-0072) significantly differs from M. aquaticum in term of the ascospore features including having a septum located in the upper cell and possessing long filament appendages (Fig. 11). In addition, $M$. thailandensis shares similarly characterized ascomata and ascospores with some species, namely $M$. fimbriatispora and M. parafimbriatispora but they differ in shape and size (Ferrer et al. 2011; Raja et al. 2015). Furthermore, M. thailandensis differs from M. aspera and M. japonica in both shape and pigmentation of the ascospores (Raja et al. 2013, 2015). Therefore, Minutisphaera thailandensis is introduced as a new species based on its distinct morphology and phylogenetic evidence.

Pleosporales Luttr. ex M.E. Barr

Notes: We follow the latest updated account of Pleosporales included in Hongsanan et al. (2020a).

\section{Acrocalymmaceae Crous \& Trakun}

Notes: Acrocalymmaceae was introduced by Trakunyingcharoen et al. (2014) to accommodate the monotypic genus Acrocalymma (Wijayawardene et al. 2017, 2020; Hongsanan et al. 2020a), and establishment was supported by additional evidence using divergence estimates (Liu et al. 2017; Hongsanan et al. 2020a). We follow the latest updated account of Acrocalymmaceae included in Mortimer et al. (2021).

\section{Acrocalymma Alcorn \& J.A.G. Irwin}

Notes: Alcorn and Irwin (1987) introduced Acrocalymma with A. medicaginis as the type species. Acrocalymma medicaginis, a root pathogen, was linked by Shoemaker et al. (1991) as the asexual morph of Massarina walkeri, but based on the study of Trakunyingcharoen et al. (2014), the two species are phylogenetically distinct, resulting in the new combination, A. walkeri. In addition, Rhizopycnis vaga, the type species of Rhizopycnis, clustered in Acrocalymma sensu stricto and hence, Rhizopycnis has been treated as a 

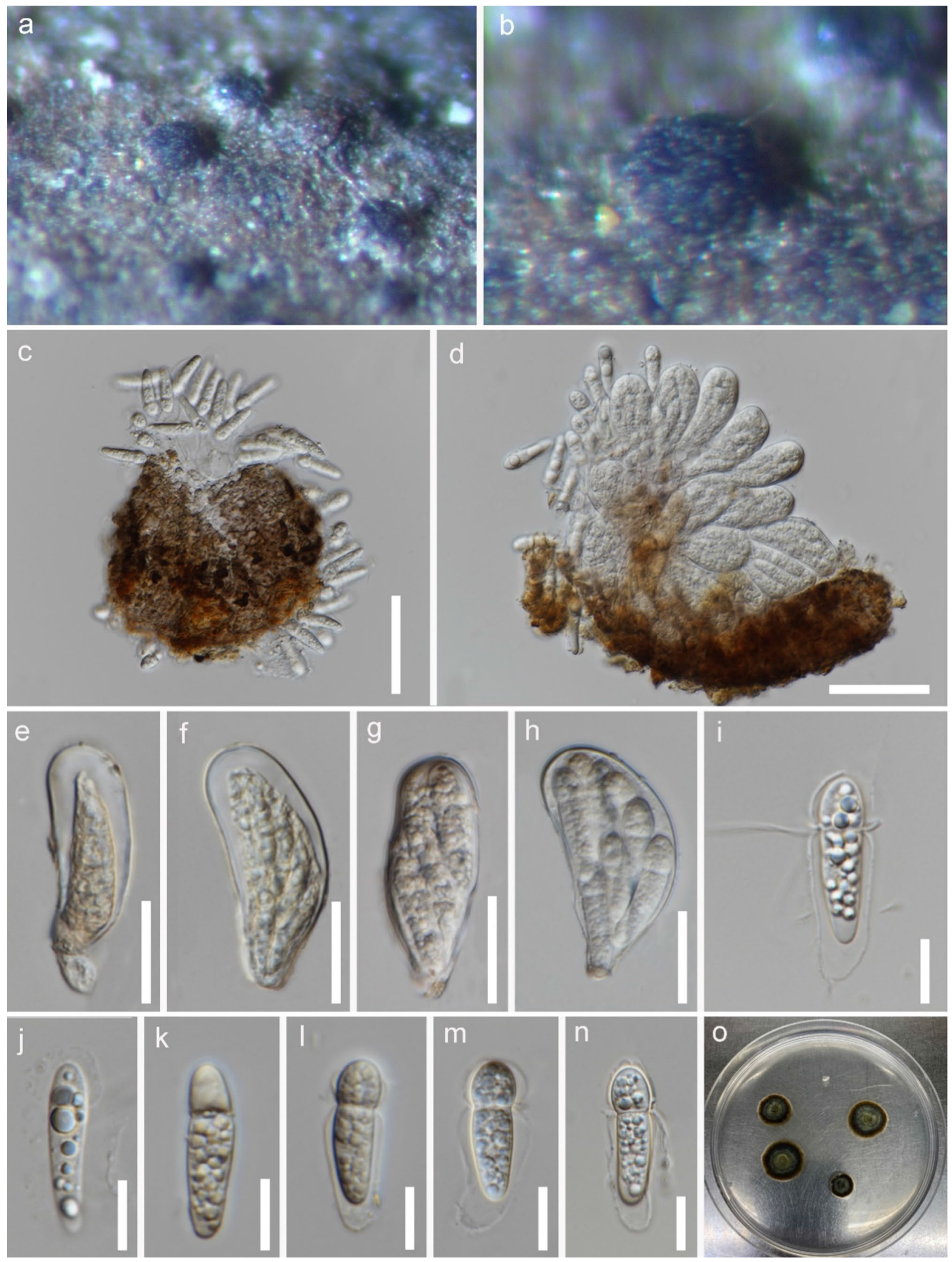

Fig. 11 Minutisphaera thailandensis (MFLU 21-0094, holotype). a, b Ascomata on submerged wood. c, d Squash of ascoma. e-h Asci. i-n Ascospores. o Culture on PDA. Scale bars: $\mathbf{c}, \mathbf{d}=50 \mu \mathrm{m}, \mathbf{e}-\mathbf{h}=20 \mu \mathrm{m}, \mathbf{i}-\mathbf{n}=10 \mu \mathrm{m}$ 


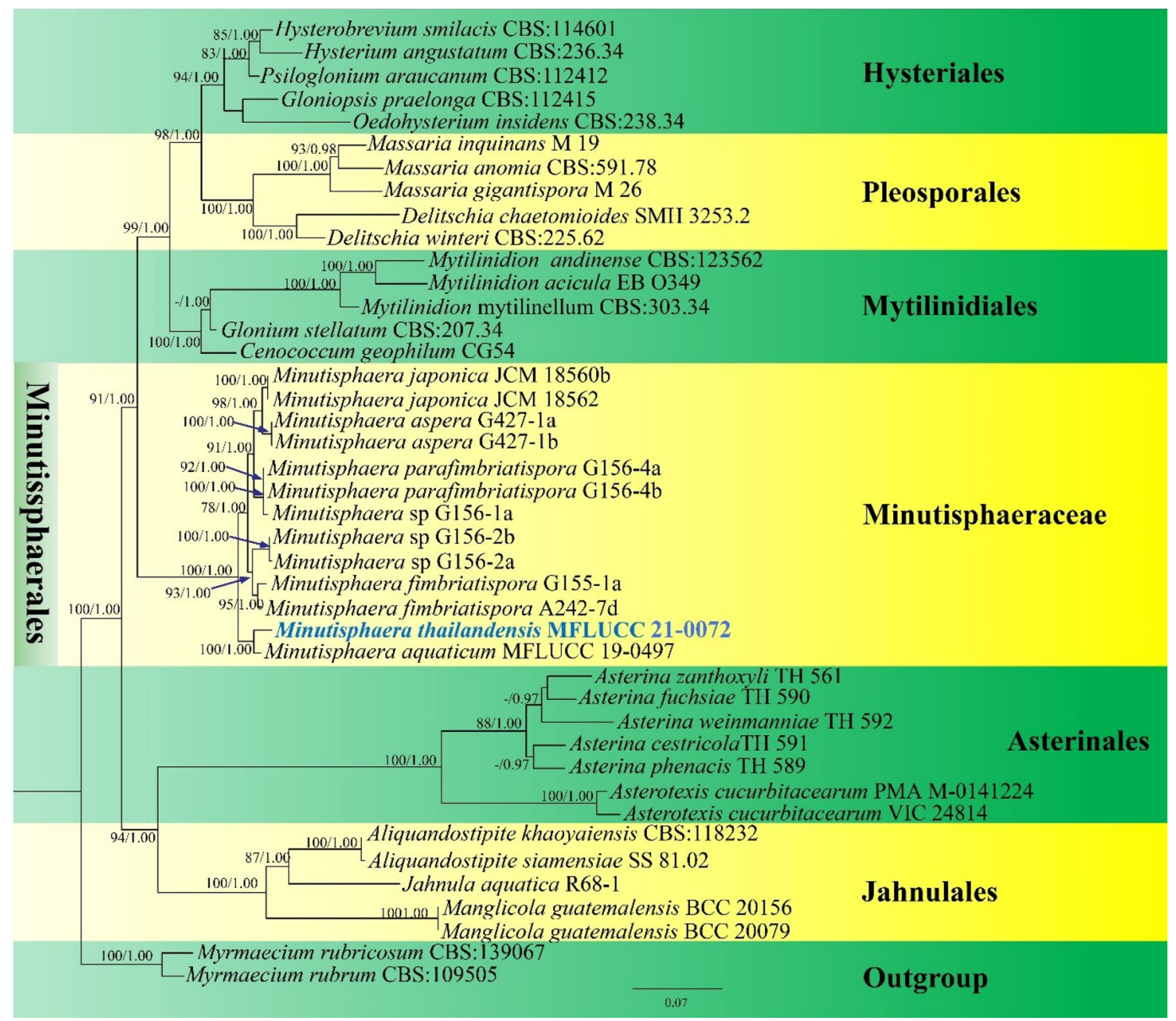

Fig. 12 Phylogram generated from maximum likelihood analysis based on combined LSU, SSU and ITS sequence data representing the species of Minutisphaera in Minutisphaeraceae. Myrmaecium rubricosum CBS139067 and M. rubrum CBS109505 are selected as the outgroup taxa. The best RAxML tree with a final likelihood value of -17637.597976 is presented. RAxML analysis yielded 1292 distinct alignment patterns and $38.19 \%$ of undetermined characters or gaps. Estimated base frequencies were as follows: $\mathrm{A}=0.254974$,

synonym of Acrocalymma (Trakunyingcharoen et al. 2014). Eleven species are currently known in this genus (Index Fungorum 2021). The most recent treatment for Acrocalymma is in Mortimer et al. (2021). In this study, Acrocalymma fici is reported as a new habitat and geographical record from Thailand.

Acrocalymma fici Crous \& Trakun., IMA Fungus 5(2): 405 (2014)

Index Fungorum number: IF810838; Facesoffungi number: FoF 09155; Fig. 13

Saprobic on submerged decaying wood. Sexual morph Undetermined. Asexual morph Conidiomata

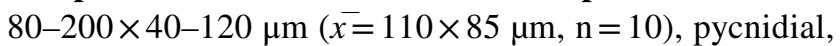

$\mathrm{C}=0.223826, \mathrm{G}=0.282377, \mathrm{~T}=0.238823$, with substitution rates $\mathrm{AC}=1.255015, \quad \mathrm{AG}=2.734543, \quad \mathrm{AT}=1.149276, \quad \mathrm{CG}=1.117855$, $\mathrm{CT}=6.544858, \mathrm{GT}=1.000000$; gamma distribution shape parameter alpha $=0.206169$. Bootstrap support values for maximum likelihood (MLBS, left) equal to or greater than $70 \%$ is given above the nodes. Bayesian posterior probabilities (BYPP, right) equal to or greater than 0.95 are given above the nodes. Ex-type strains are in bold and newly generated sequence is in blue

globose to subglobose, erumpent, separate but aggregated in clusters, sub-hyaline with dark brown to black region around ostiole. Pycnidial wall 41-55 $\mu \mathrm{m}$ diam., 3-6 layers of hyaline to subhyaline cells of textura angularis. Conidiophores reduced to conidiogenous cells. Conidiogenous cells $4-15 \times 2-5 \mu \mathrm{m}(\bar{x}=7 \times 3 \mu \mathrm{m}, \mathrm{n}=20)$, ampulliform to doliiform, hyaline, smooth, 5-12 $\times 3-5 \mu \mathrm{m}$, with inconspicuous percurrent proliferation visible at apex. Conidia $12-15 \times 2-3 \mu \mathrm{m}(\bar{x}=13 \times 3 \mu \mathrm{m})$, hyaline, smooth, guttulate, cylindrical, with subobtuse at the apex, acutely tapered at base to a small flattened central scar, 0-1-septate, not constricted at septum, with flaring mucoid apical appendage, 2-5 $\mu \mathrm{m}$ wide, visible in water mounts. 


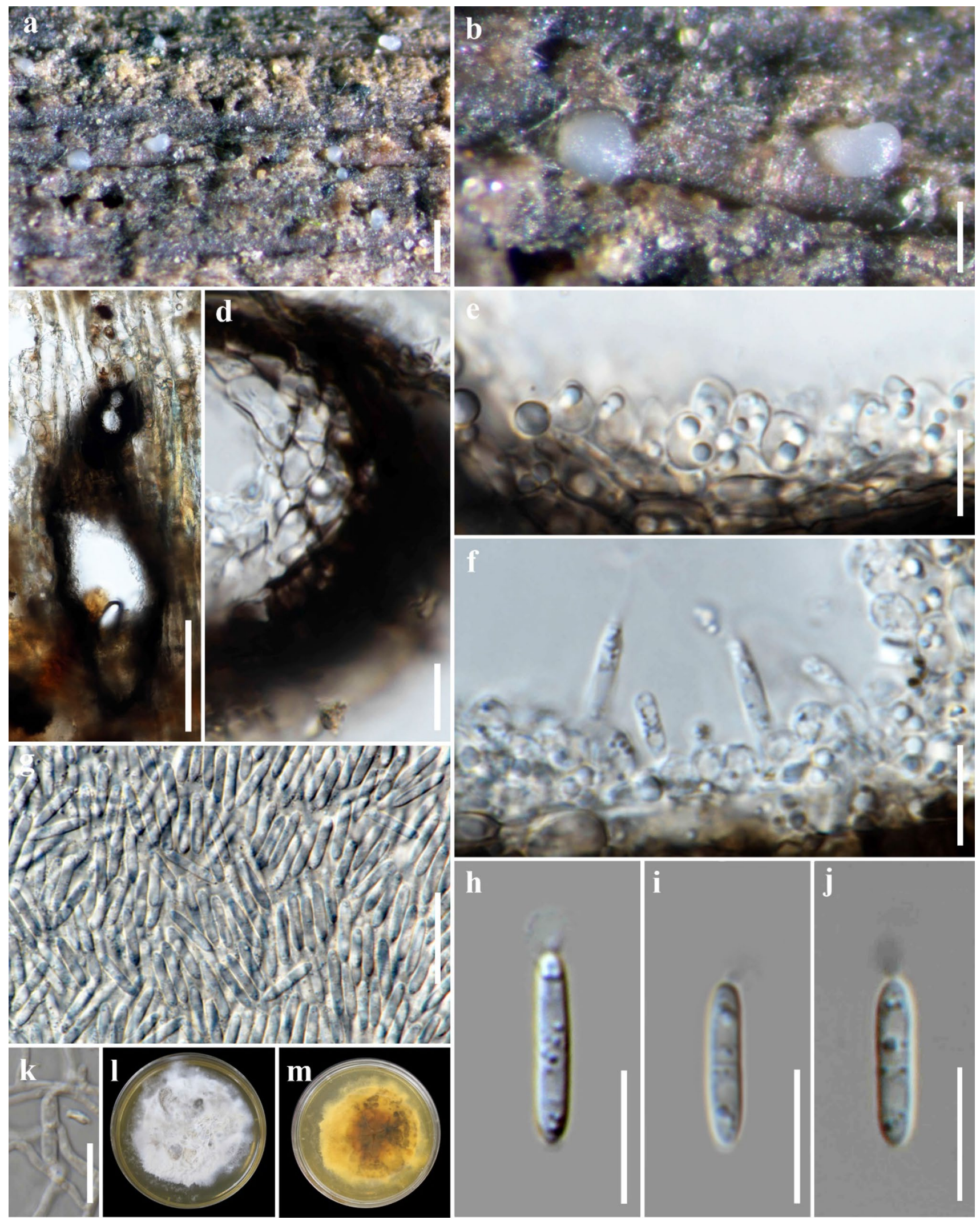

Fig. 13 Acrocalymma fici (MFLU 21-0124, new habitat and geographical record). a, b Appearance of erumpent fruiting bodies on host substrate. c Section through conidioma. d Section through pycnidial wall. e, f Immature and mature conidia attached to conidiog- enous cells. $\mathbf{g}-\mathbf{j}$ Conidium. $\mathbf{k}$ Germinated conidium $\mathbf{l}, \mathbf{m}$ Culture on MEA (upper and lower view). Scale bars: $\mathbf{a}=500 \mu \mathrm{m}, \mathbf{b}=200 \mu \mathrm{m}$, $\mathbf{c}=100 \mu \mathrm{m}, \mathbf{d}, \mathbf{f}, \mathbf{h}-\mathbf{k}=10 \mu \mathrm{m}, \mathbf{e}=5 \mu \mathrm{m}, \mathbf{g}=20 \mu \mathrm{m}$ 
Culture characters: Conidia germinated on MEA within $24 \mathrm{~h}$. Colonies on MEA reaching 4-5 cm after 4 weeks at room temperature, colonies irregular, medium dense, flat with smooth and filiform margins; white to smoke-grey in top view, reverse yellowish orange in center and yellowish in the outer region.

Material examined: THAILAND, Kanchanaburi Province, Sangkhla Buri, Liwo, on submerged decaying wood in a stream, 27 June 2019, N. Chaiwan, TFW5 (MFLU 21-0124, new habitat and geographical record), living culture, MFLUCC 21-0103.

GenBank numbers: ITS $=$ MT864351, LSU $=$ MT860429.

Notes: Acrocalymma fici, introduced by Trakunyingcharoen et al. (2014), was collected from Ficus sp. in a terrestrial habitat. Based on phylogenetic analysis of combined LSU and ITS sequence data, our isolate MFLUCC 21-0103 clustered with the ex-type strain of Acrocalymma fici (CBS 317.76 ) with 96\% MLBS and 97\% MPBS bootstrap support
(Fig. 14). Our isolate resembles A. fici in shape and size of the conidiomata, conidiogenous cells and conidia (Fig. 13). Moreover, there are no base pair differences of the ITS nucleotides. However, Acrocalymma fici strain CBS 317.76 was isolated from a terrestrial host, Ficus sp., while our isolate was collected from a freshwater habitat. Therefore, we report this isolate as a new record of $A$. fici from a freshwater habitat.

Dictyosporiaceae Boonmee \& K.D. Hyde

Notes: Dictyosporiaceae was established by Boonmee et al. (2016) with Dictyosporium as the type genus. Species in this family are cosmopolitan with a worldwide distribution. They are mainly found from decaying wood and plant litter in both terrestrial and aquatic habitats. Fifteen genera are currently listed in Dictyosporiaceae (Hongsanan et al. 2020a).

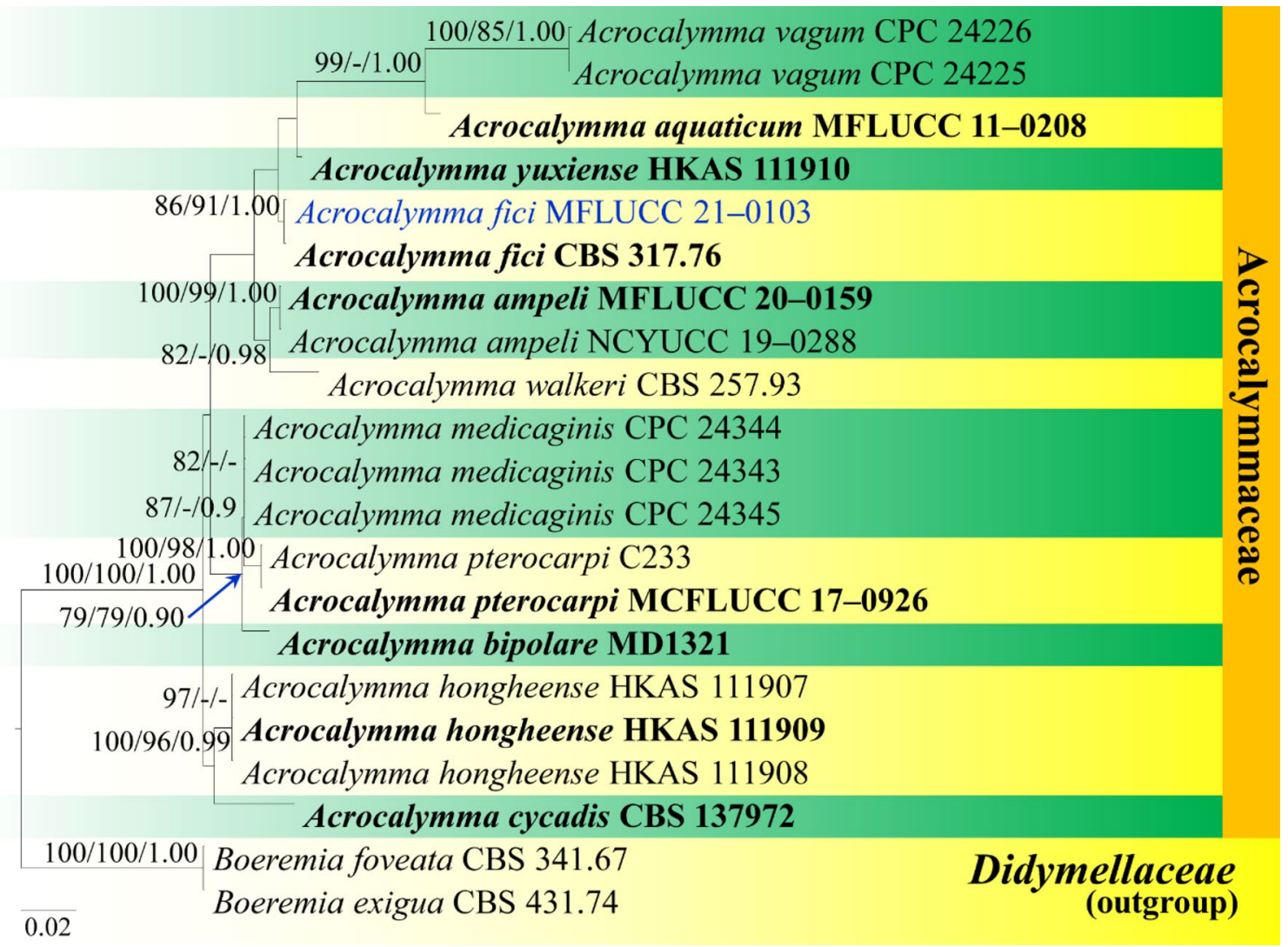

Fig. 14 Phylogenetic tree generated from maximum likelihood (ML) analysis based on combined LSU and ITS sequence data for the species from Acrocalymmaceae. Boeremia exigua (CBS 431.74) and Boeremia foveata (CBS 341.67) are used as the outgroup taxa. The dataset comprised 1997 characters after alignment including gaps $(\mathrm{LSU}=1308 \mathrm{bp}$ and ITS $=689 \mathrm{bp}$ ). The RAxML analysis of the combined dataset yielded a best scoring tree with a final ML optimization likelihood value of -4737.006546 . The matrix had 316 distinct alignment patterns, with $31.82 \%$ of undetermined characters or gaps. Estimated base frequencies were as follows: $\mathrm{A}=0.245559$, $\mathrm{C}=0.218996, \quad \mathrm{G}=0.276710, \mathrm{~T}=0.258736 ; \quad$ substitution rates: $\mathrm{AC}=1.502433, \quad \mathrm{AG}=1.688434, \quad \mathrm{AT}=1.833814, \quad \mathrm{CG}=0.473416$, $\mathrm{CT}=4.863789, \mathrm{GT}=1.000000$; gamma distribution shape parameter $\alpha=0.020000$. The MP analysis resulted a single most parsimonious tree $(\mathrm{TL}=391, \mathrm{CI}=0.831, \mathrm{RI}=0.875, \mathrm{RC}=0.727, \mathrm{HI}=0.169)$. Bootstrap support values for MLBS and MPBS equal to or greater than $75 \%$ BYPP equal to or greater than 0.95 are given above the nodes. Ex-type strains are in bold and newly generated sequence is in blue 


\section{Dictyosporium Corda}

Notes: Dictyosporium was established by Corda (1836) with a single species, Di. elegans Corda. Since then, many new species have been introduced to this genus (Boonmee et al. 2016; Tibpromma et al. 2018; Yang et al. 2018a; Hyde et al. 2020a). Currently, 83 species epithets are listed in Index Fungorum (2021) and only 60 species are accepted in Species Fungorum (2021). The genus is characterized by superficial, subglobose ascomata, bitunicate, cylindrical asci and hyaline, fusiform uniseptate ascospores with or without a sheath; sporodochial colonies, micronematous to macronematous conidiophores and cheiroid, digitate complanate conidia with several parallel rows of cells (Boonmee et al. 2016; Yang et al. 2018a). Species in Dictyosporium have been recorded worldwide from dead wood, decaying leaves and plant litter in terrestrial and aquatic habitats (Goh et al.1999; Ho et al. 2002; Pinnoi et al. 2006; Pinruan et al. 2007; Boonmee et al. 2016; Yang et al. 2018a; Hyde et al. 2020a).

Dictyosporium pandanicola Tibpromma \& K.D. Hyde, in Tibpromma et al., Fungal Diversity: 93: 20 (2018)

Index Fungorum number: IF554481; Facesoffungi number: FoF 04491; Fig. 15

Saprobic on submerged decaying wood. Sexual morph Undetermined. Asexual morph Colonies, punctiform, sporodochial, scattered, dark brown to black, glistening. Mycelium mostly immersed, composed of smooth, septate, branched, hyaline to pale brown hyphae. Conidiomata sporodochia on the natural substrate in small groups, dark

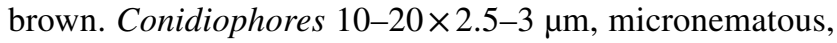
mononematous, cylindrical, subhyaline to pale brown, septate, smooth, sometimes reduced to conidiogenous cell. Conidiogenous cells holoblastic, integrated, indeterminate, subhyaline to pale brown. Conidia 40-48 $\times 20-25 \mu \mathrm{m}$ $(\bar{x}=44 \times 23 \mu \mathrm{m}, \mathrm{n}=35)$, solitary, oval to ellipsoid, cheiroid, smooth-walled, consisting of 5-6 arms, 9-13-euseptate in each arm, with a basal connecting cell, yellow-brown to brown with age, sometimes with a hyaline, globose, apical appendage.

Culture characters: Conidia germinated on PDA within $24 \mathrm{~h}$. Colonies on PDA reaching 2.5-3.5 cm diam., after 4 weeks at room temperature, colonies irregular, medium dense, white to yellow in top view. In reverse, yellow in the middle and white at the margin.

Material examined: THAILAND, Surat Thani Province, Sai Daeng, on decaying wood submerged in a small river, 2 October 2017, Z.L. Luo, B77 (MFLU 20-0425, new record), living culture, MFLUCC 18-0331.

GenBank numbers: ITS $=$ MZ490792, LSU $=$ MZ490775, TEF1- $\alpha=$ MZ501208.

Notes: In the phylogenetic analysis (Fig. 16), the new isolate of Dictyosporium pandanicola (MFLUCC 18-0331) clusters with Di. pandanicola (MFLU 16-1886) with high bootstrap support (100\% MLBS, 0.97 BYPP). In addition, there is only 1 base pair difference observed in the ITS region between these two strains. These two strains share some similar characters such as micronematous conidiophores, holoblastic conidiogenous cells and oval to ellipsoid, cheiroid, yellow-brown to brown conidia with 5-6 arms and overlapping conidia size $(40-48 \times 20-25 \mu \mathrm{m}$ vs. $30-50 \times 15-33 \mu \mathrm{m})$. However, conidia of MFLUCC 18-0331 sometimes have a hyaline, globose, apical appendage. While conidia of MFLU 16-1886 lack apical appendages. This difference in appendages may be because of the different habitats. MFLU 16-1886 was collected form a terrestrial habitat on Pandanus, while MFLUCC 18-0331 was collected from a freshwater habitat on submerged wood. Based on both morphology and molecular data, we consider the isolate MFLUCC 18-0331 and Di. pandanicola to be conspecific.

Tibpromma et al. (2018) discussed Dictyosporium pandanicola as being phylogenetically close to Di. strelitziae. The ITS blast result in NCBI GenBank showed that $D i$. pandanicola (MFLU 16-1886) is 99\% similar to Di. strelitziae (CBS 123359). We also obtained the same result and found that there are only $3 \mathrm{bp}$ of difference in the ITS region between Di. pandanicola and Di. strelitziae. Tibpromma et al. (2018) distinguished Di. pandanicola and Di. strelitziae by the apical appendage and number of arms and cells. However, in this study, we found that the conidia of the new isolate of Di. pandanicola (MFLUCC 18-0331) also has apical appendages (Fig. 15). Thus, future morpho-molecular studies are needed to confirm the relationship between $D i$. pandanicola and Di. strelitziae.

Camarosporidiellaceae Wanas., Wijayaw., Crous \& K.D. Hyde

Notes: Wanasinghe et al. (2017) introduced Camarosporidiellaceae to accommodate Camarosporidiella which are saprobic and sometimes can be endophytic or pathogenic and potentially opportunistic pathogens on leaves and woody materials. We follow the latest treatment of this family in Hongsanan et al. (2020a). In this study, we introduce a geographical record of Camarosporidiella laburni from Uzbekistan.

Camarosporidiella Wanas., Wijayaw., K.D. Hyde

Notes: Camarosporidiella is similar to other camarosporium-like genera in that distinguishing species and species identification based solely on morphology and plant host association can be problematic (Wanasinghe et al. 2017). Wanasinghe et al. (2017) recommended to use a combination of LSU, SSU, ITS and TEF1- $\alpha$ loci for investigating phylogenetic relationships of taxa in this genus. Currently there are 23 Camarosporidiella species accepted in Species Fungorum (2021). 

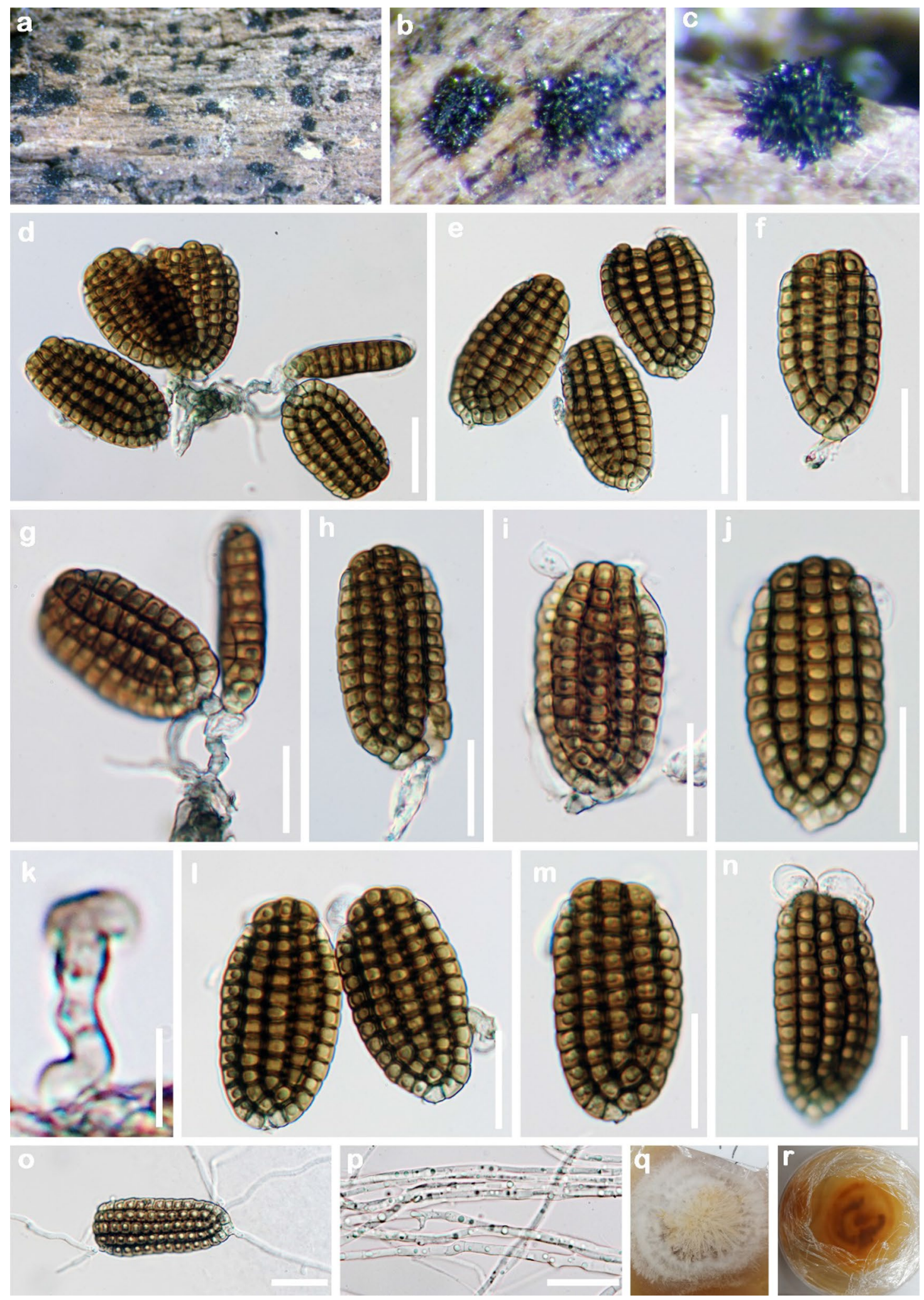

Fig. 15 Dictyosporium pandanicola (MFLU 20-0425, new record). a-c Colonies on wood. $\mathbf{d}-\mathbf{h}$ Conidiophores, conidiogenous cells and conidia. $\mathbf{i}-\mathbf{j}$, l-n Conidia. $\mathbf{k}$ Conidiophores. o Germinated conidia. $\mathbf{p}$
Mycelium. q, r Culture on MEA (upper and lower view). Scale bars: $\mathbf{d}-\mathbf{i}, \mathbf{k}-\mathbf{p}=20 \mu \mathrm{m}, \mathbf{k}=10 \mu \mathrm{m}$ 


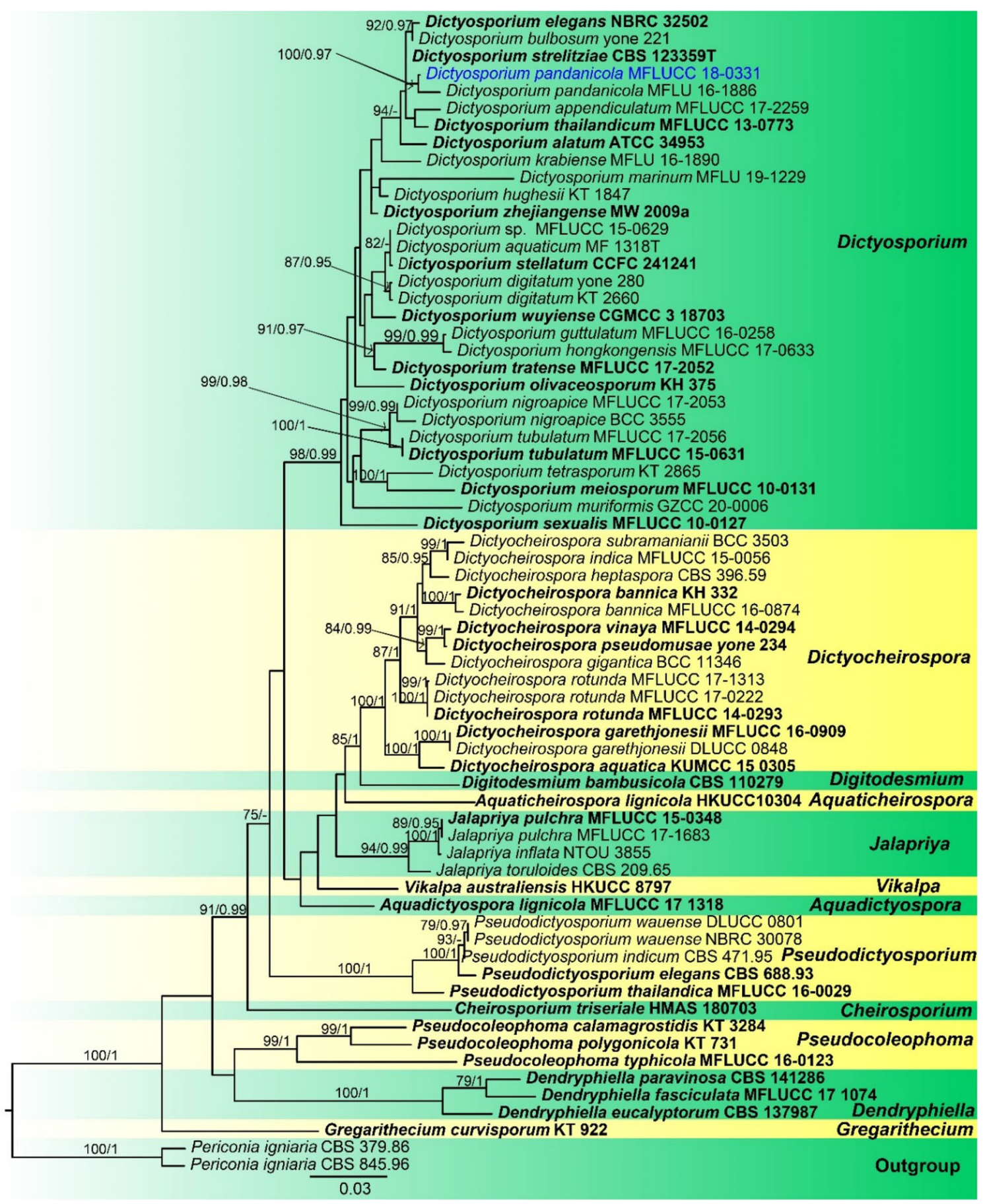

Fig. 16 Phylogenetic tree generated from maximum likelihood (ML) analysis based on combined LSU, ITS and TEF1- $\alpha$ sequence data for the species from Melanommataceae. Periconia igniaria (CBS 379.86 and CBS 845.96) are used as the outgroup taxa. The dataset comprised 2694 characters after alignment including gaps (LSU $=1245 \mathrm{bp}$; ITS $=537 \mathrm{bp}, \mathrm{TEF} 1-\alpha=912 \mathrm{bp}$ and RPB2 $=1006 \mathrm{bp}$ ). The RAxML analysis of the combined dataset yielded a best scoring tree with a final ML optimization likelihood value of -14912.416315 . The matrix had 871 distinct alignment patterns, with $36.52 \%$ undetermined characters or gaps. Estimated base frequencies were as follows: $\mathrm{A}=0.239281, \mathrm{C}=0.249660$, $\mathrm{G}=0.270492, \mathrm{~T}=0.240568 ;$ substitution rates $\mathrm{AC}=1.684833$, $\mathrm{AG}=3.636355, \quad \mathrm{AT}=2.551537, \quad \mathrm{CG}=0.785971, \quad \mathrm{CT}=9.189334$, GT $=1.000000$; gamma distribution shape parameter $\alpha=0.180969$. Support values for maximum likelihood (MLBS) above than $75 \%$ and Bayesian posterior probabilities (BYPP) greater than 0.95 are given at the nodes. Ex-type strains are in bold and newly generated sequence is in blue 
Camarosporidiella laburni (Pers.) Wanas., Bulgakov, Camporesi \& K.D. Hyde, Stud. Mycol. 87: 233 (2017)

Index Fungorum number: IF821952; Facesoffungi number: FoF 03540; Fig. 17

$\equiv$ Sphaeria laburni Pers., Observ. mycol. (Lipsiae) 1: 68 (1796)

Saprobic on woody branches. Sexual morph Ascomata $400-550 \mu \mathrm{m}$ high, $450-550 \mu \mathrm{m}$ diam. $(\bar{x}=485.5 \times 488 \mu \mathrm{m}$, $\mathrm{n}=10$ ), black, superficial to semi-immersed, confluent, gregarious, sometimes scattered beneath the host periderm or on decorticated wood, fully or partly erumpent, globose, uniloculate, ostiolate. Ostiole central, short, slightly sunken, minute and inconspicuous at the surface, smooth, ostiolar canal filled with hyaline cells. Peridium $30-50 \mu \mathrm{m}$ wide at the base, $60-90 \mu \mathrm{m}$ wide in sides, thick, comprising two layers, outermost layer heavily pigmented, thin-walled, comprising blackish to dark brown loosely packed cells of textura angularis, inner layer composed of pale brown to hyaline, cells towards the inside lighter, flattened, thickwalled cells of textura angularis. Hamathecium comprising numerous, $2-3 \mu \mathrm{m}(\mathrm{n}=30)$ wide, filamentous, branched septate, cellular pseudoparaphyses. Asci 150-220 $\times 12-16 \mu \mathrm{m}$ $(\bar{x}=178 \times 13 \mu \mathrm{m}, \mathrm{n}=30), 8$-spored, bitunicate, fissitunicate, cylindrical, short-pedicellate, apex rounded with a minute ocular chamber. Ascospores 24-30 $\times 10-13 \mu \mathrm{m}$ $(x=29 \times 11 \mu \mathrm{m}, \mathrm{n}=30)$, overlapping uni-seriate, muriform, mostly ellipsoidal, 6-7-transversely septate, with 1-2 longitudinal septa, deeply constricted at the middle septum, slightly constricted at remaining septa, initially hyaline, becoming pale brown at maturity, asymmetrical, slightly paler, conical and narrowly rounded at the end cells, lacking a mucilaginous sheath.

Material examined: UZBEKISTAN, Jizzakh Province, Zaamin District, Zaamin National Nature Park, river of Uriklisoy, Turkestan range of Pamir-Alay Mountains, on dead stem of Astragalus sp. (Fabaceae), 12 July 2019, Y. Gafforov YG-Z48-2 (TASM 6162, new record); ibid., on dead stems of unidentified plant, 14 July 2019, Y. Gafforov and A. Abdurazakov, YG-Z59-2 (TASM 6163).

GenBank numbers: ITS = MZ493295, MZ493296, LSU = MZ493309, MZ493310, SSU: MZ493281, MZ493282, TEF1- $\alpha=$ MZ508404, MZ508405.

Notes: Wanasinghe et al. (2017) introduced a combination species Camarosporidiella laburni from Italy based on the morphological features of its holomorph and multi-locus phylogeny. The species was previously established as Cucurbitaria laburni by De Notaris (1862) and detailed morphological studies were carried out by Green (1931) and Mirza (1968). This species was noted as clearly distinct from the remaining described species in Cucurbitaria (Wanasinghe et al. 2017). Multi-locus phylogeny demonstrated the species within Camarosporidiellaceae, distant from Cucurbitaria in Cucurbitariaceae (Wanasinghe et al. 2017). Camarosporidiella laburni is widely distributed in Europe and North America and host preferences are currently restricted to the family Fabaceae. Table 1 shows the updates on host species and geographic distribution of Camarosporidiella laburni and it indicates that this fungus is specific to Fabaceae hosts.

In this study, our new strains (TASM 6162 and TASM 6163) clustered with Camarosporidiella laburni strains MFLUCC 14-0885 and MFLUCC 14-0919 with 60\% MLBS support (Fig. 18). However, a nucleotide base comparison of ITS and TEF1- $\alpha$ demonstrated that our new strains are identical to Camarosporidiella laburni strains MFLUCC 14-0885 and MFLUCC 14-0919 (0/542 bp) of ITS, and $1 / 926$ bp $(0.1 \%)$ and $2 / 926$ bp $(0.1 \%)$ of TEF1- $\alpha)$, respectively. Therefore, we identified our new collections as $C$. laburni and the species is reported from Astragalus sp. in Uzbekistan for the first time (Fig. 17).

\section{Cryptocoryneaceae A. Hashim. \& Kaz. Tanaka}

Notes: Hashimoto et al. (2017) introduced Cryptocoryneaceae to accommodate the single genus Cryptocoryneum Fuckel. Cryptocoryneaceae is phylogenetically closely affiliated with Lophiotremataceae. We follow the latest treatment of this family in Hashimoto et al. (2017) and Hongsanan et al. (2020a). An updated phylogenetic tree for Cryptocoryneaceae is presented in Fig. 20. In this study, we introduce a new saprobic species Cryptocoryneum rosae, which collected on Rosa sp. in Yunnan Province, China.

\section{Cryptocoryneum Fuckel}

Notes: Cryptocoryneum was introduced based on C. fasciculatum (Fuckel 1865), however, its taxonomic status was contested by later studies (Hashimoto et al. 2016). Hashimoto et al. (2016) examined holotype specimens of $C$. fasciculatum, C. hysterioides, and Torula uniformis, and concluded that these species are conspecific. Thus, Hashimoto et al. (2016) designated Cryptocoryneum hysterioides as the type species. Most species of Cryptocoryneum have been reported on dead wood (Hashimoto et al. 2016), with some species rarely reported from leaf litter (Kirk 1983) or arthropod dung (Talbot 1952). This genus is only known from its asexual morph, and is characterized by stromatic sporodochia, monoblastic conidiogenous cells, cheiroid conidia and conidial arms developing downward from the cap cells (Schoknecht and Crane 1977; Hashimoto et al. 2016; Hongsanan et al. 2020a). Sixteen species are currently listed under Cryptocoryneum in Species Fungorum (2021) and only eight of these have been confirmed by DNA base evidence. 

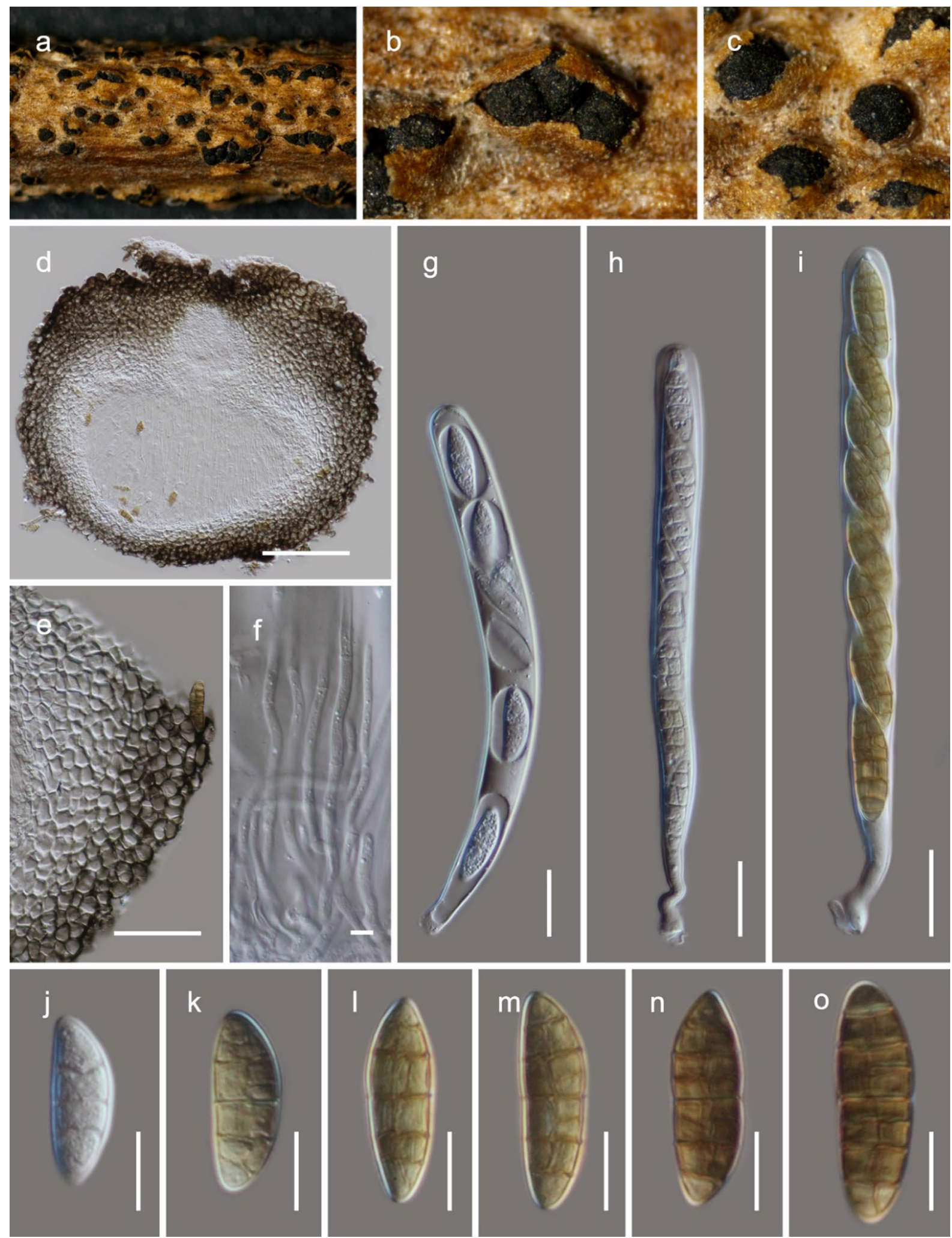

Fig. 17 Camarosporidiella laburni (TASM 6162, new record). a-c Appearance of ascomata on host surface. d Longitudinal section of an ascoma. e Section of the peridium. f Pseudoparaphyses. g-i Asci. j-o Ascospores. Scale bars: $\mathbf{d}=100 \mu \mathrm{m}, \mathbf{e}=50 \mu \mathrm{m}, \mathbf{f}=5 \mu \mathrm{m}, \mathbf{g}-$ $\mathbf{i}=20 \mu \mathrm{m}, \mathbf{j}-\mathbf{o}=10 \mu \mathrm{m}$ 
Table 1 Known hosts found for Camarosporidiella laburni

\begin{tabular}{|c|c|c|c|}
\hline Host species & Host Family & Country & References \\
\hline Astragalus sp. & Fabaceae & Uzbekistan & This study \\
\hline Chamaecytisus sp. & Fabaceae & Ukraine & Dudka et al. (2004) \\
\hline Cytisus alpinus & Fabaceae & Germany & Mirza (1968) \\
\hline Cytisus laburnum & Fabaceae & England & Dennis (1978) \\
\hline \multirow[t]{2}{*}{ Cytisus radiatus } & \multirow[t]{2}{*}{ Fabaceae } & Italy & Mirza (1968) \\
\hline & & Switzerland & Mirza (1968) \\
\hline \multirow[t]{2}{*}{ Laburnum alpinum } & \multirow[t]{2}{*}{ Fabaceae } & Denmark & Munk (1957) \\
\hline & & Sweden & Eriksson (1992) \\
\hline \multirow[t]{10}{*}{ Laburnum anagyroides } & \multirow[t]{10}{*}{ Fabaceae } & Denmark & Munk (1957) \\
\hline & & Germany & Spaulding (1961) \\
\hline & & Italy & Wanasinghe et al. (2017) \\
\hline & & Poland & Mulenko et al. (2008) \\
\hline & & Russia & Wanasinghe et al. (2017) \\
\hline & & Scotland & Foister (1961) \\
\hline & & Sweden & Eriksson (1992) \\
\hline & & Switzerland & Mirza (1968) \\
\hline & & Ukraine & Dudka et al. (2004) \\
\hline & & USA & Farr and Rossman (2021) \\
\hline \multirow[t]{2}{*}{ Laburnum sp. } & \multirow[t]{2}{*}{ Fabaceae } & United Kingdom & Cannon et al. (1985) \\
\hline & & Russia & Wanasinghe et al. (2017) \\
\hline Ononis tridentata & Fabaceae & Spain & Larios et al. (1988), Checa (2004) \\
\hline Ononis tridentata var. intermedia & Fabaceae & Spain & Unamuno (1941) \\
\hline Retama sphaerocarpa & Fabaceae & Spain & Checa (2004) \\
\hline
\end{tabular}

Cryptocoryneum rosae Wanas. \& K.D. Hyde, sp. nov.

Index Fungorum number: IF558583; Facesoffungi number: FoF 09944; Fig. 19

Etymology: The specific epithet "rosae" refers to the Rosa.

\section{Holotype: KUN-HKAS 115780}

Saprobic on dead wood of Rosa sp. Sexual morph Undetermined. Asexual morph Sporodochia 100-300(-450) $\mu \mathrm{m}$ diam., 20-55 $\mu \mathrm{m}$ high, pulvinate, often confluent, dark brown to black. Conidiophores arising from the stromatic cells, straight, simple, septate, hyaline to pale brown, smooth. Conidiogenous cells $5-7 \times 4-6 \mu \mathrm{m}(\bar{x}=5.8 \times 4.8 \mu \mathrm{m}$, $\mathrm{n}=20$ ), monoblastic, cylindrical, terminal, determinate, hyaline to pale brown. Conidia $30-50 \times 20-30 \mu \mathrm{m}$ $(\bar{x}=36 \times 27.5 \mu \mathrm{m}, \mathrm{n}=30), 1 / \mathrm{w} 1.2-1.6(x=1.3, \mathrm{n}=30)$, solitary, acrogenous, branched, cheiroid, with dark brown cap cells firmly united together, basal cells brown, cuneiform, smooth, thin-walled, with 4-12 arms $(\bar{x}=9$ arms, $\mathrm{n}=40)$. Arms $20-30(-37) \times 3.5-6 \mu \mathrm{m}(\bar{x}=26 \times 4.5 \mu \mathrm{m}$, $\mathrm{n}=40$ ), cylindrical, brown, branched at the base, smooth, (5)8-12-septate $(\bar{x}=9 \mu \mathrm{m}, \mathrm{n}=40)$.

Culture characteristics: Conidia germinating on PDA within $24 \mathrm{~h}$ and germ tubes arising from both end cells. Colonies on PDA, slow growing, reaching $2 \mathrm{~cm}$ diam. after 4 weeks of at room temperature, initially white becoming dark brown at maturity, slightly effuse, radially with an undulate edge, reverse blackish brown.

Material examined: CHINA, Yunnan, Diqing Autonomous Prefecture, Shangri-La, Xiaozhongdianzhen, $27.425825^{\circ} \mathrm{N}, 99.845329^{\circ} \mathrm{E}, 3195 \mathrm{msl}$, on dead wood of Rosa sp. (Rosaceae), 30 August 2020, GC Ren, NX25-1 (KUN-HKAS 115780, holotype), living culture (KUMUCC 21-0033), ibid., NX25-2 (KUN-HKAS 115781), living culture (KUMUCC 21-0034).

GenBank numbers: ITS = MZ493300, MZ493301, LSU = MZ493314, MZ493315; SSU = MZ493286, MZ493287, RPB2 = MZ508418, MZ508419, TEF1- $\alpha=$ MZ508409, MZ508410.

Notes: Cryptocoryneum rosae was collected from Uzbekistan on Rosa and representing the first report of a Cryptocoryneum species from Central Asia and Rosa sp. (Farr and Rossman 2021). The new fungus resembling Cryptocoryneum species well in its pulvinate sporodochia, monoblastic conidiogenous cells and acrogenous, branched, cheiroid, brown conidia with multiple arms (Fig. 19). In the phylogenetic analyses of concatenated SSU, LSU, ITS, TEF1- $\alpha$ and RPB2, C. rosae is related to C. akitaense and C. brevicondensatum with 99\% MLBS support (Fig. 20). Comparatively, $C$. rosae has shorter and narrower conidia $(\bar{x}=36.3 \times 27.5 \mu \mathrm{m})$ than $C$. akitaense $(\bar{x}=49.1 \times 38.6 \mu \mathrm{m})$ and $C$. brevicondensatum $(x=57.5 \times 34.2 \mu \mathrm{m})$. In 


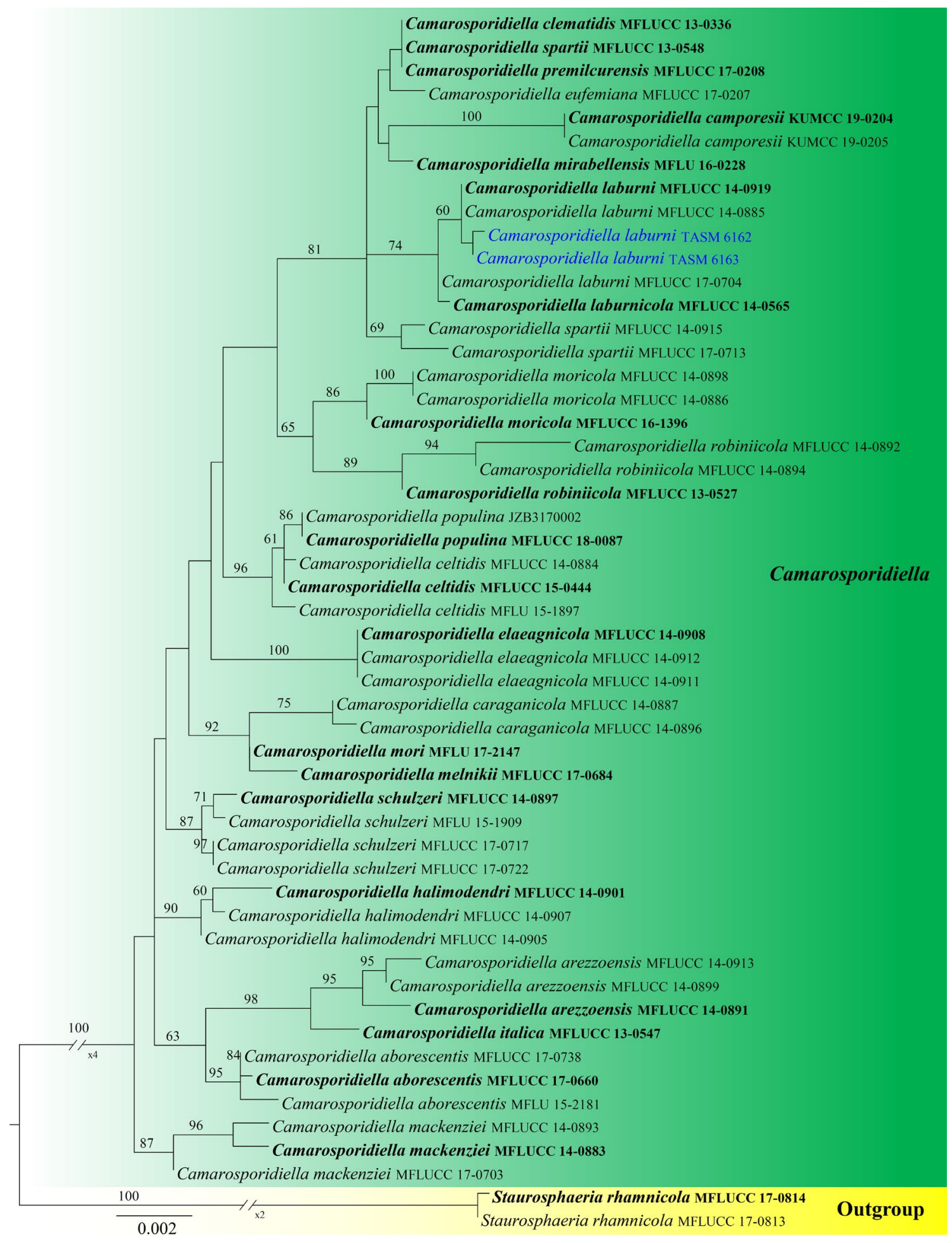

Fig. 18 Phylogram generated from the best scoring of the RAxML tree based on combined SSU, LSU, ITS, and TEF1- $\alpha$ sequence dataset to indicate Camarosporidiella and related species. Fifty-two strains are included in the combined analyses which comprise a total of 3309 characters. Staurosphaeria rhamnicola (MFLUCC 17-0813 and MFLUCC 17-0814) is selected as the outgroup taxon. The best RAxML tree with a final likelihood value of -6629.415262 is presented. RAxML analysis yielded 226 distinct alignment patterns and
$5.73 \%$ of undetermined characters or gaps. Estimated base frequencies were as follows: $\mathrm{A}=0.240666, \mathrm{C}=0.244239, \mathrm{G}=0.266553$, $\mathrm{T}=0.248542$, with substitution rates $\mathrm{AC}=1.544599, \mathrm{AG}=4.613774$, $\mathrm{AT}=3.7595, \mathrm{CG}=0.783994, \mathrm{CT}=9.045003, \mathrm{GT}=1.000000$; gamma distribution shape parameter alpha $=0.997372$. Bootstrap support values for maximum likelihood (MLBS, left) equal to or greater than $70 \%$ is given above the nodes. Ex-type strains are in bold and newly generated sequences are in blue 

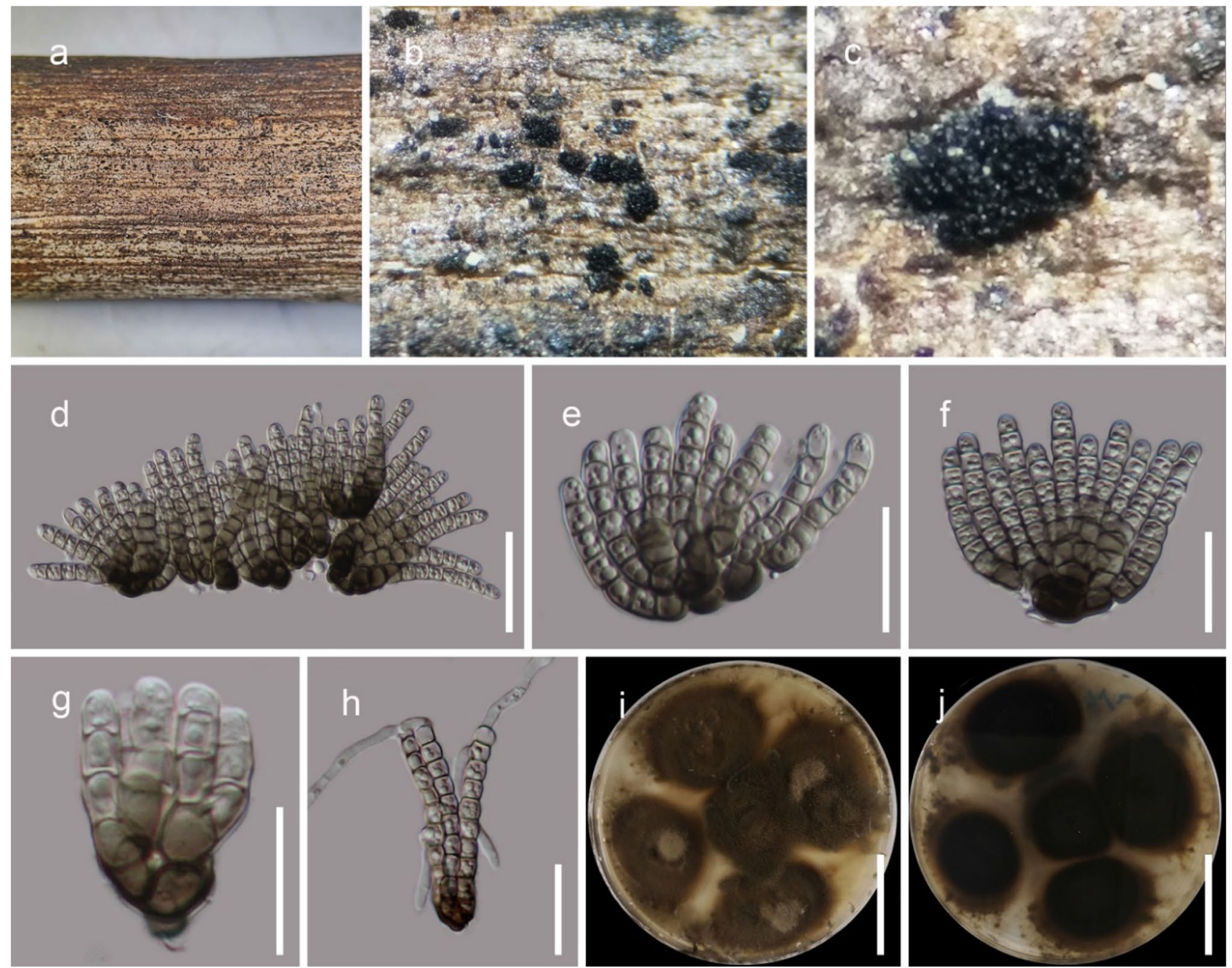

Fig. 19 Cryptocoryneum rosae (KUN-HKAS 115780, holotype). a-c Appearance of sporodochia on host surface. d-g Conidia. h Germinated conidium. $\mathbf{i}, \mathbf{j}$ Culture on PDA from surface and reverse. Scale bars: $\mathbf{d}=30 \mu \mathrm{m}, \mathbf{e}, \mathbf{f}, \mathbf{h}=20 \mu \mathrm{m}, \mathbf{g}=15 \mu \mathrm{m}, \mathbf{i}, \mathbf{j}=3 \mathrm{~cm}$

Cryptocoryneum, there were not many base pair differences between species in the ITS region (except $C$. condensatum). We observed, on the other hand, that there were greater differences in the TEF1- $\alpha$ and RPB2 gene regions. Between Cryptocoryneum rosae and $C$. akitaense, TEF1- $\alpha$ had a base pair variation of 3.2\% (29/898) and RPB2 had a variation of $4 \%$ (40/988). Between Cryptocoryneum rosae and C. brevicondensatum there were $2.9 \%(26 / 898)$ and $3.5 \%$ (34/988) base pair differences respectively in TEF1- $\alpha$ and RPB2 gene regions.

Didymellaceae Gruyter, Aveskamp \& Verkley (= Microsphaeropsidaceae Qian Chen, L. Cai \& Crous)

Notes: Didymellaceae has a wide host range and represents an important pathogenic, endophytic and saprobic species (Hyde et al. 2020b). Thirty-five genera are listed in this family (Hongsanan et al. 2020a).
Didymella Sacc. ex D. Sacc.

Notes: We follow the latest treatment and updated accounts of Didymella in Hongsanan et al. (2020a).

Didymella azollae E. Shams, F. Dehghanizadeh, A. Pordel \& M. Javan-Nikkhah, sp. nov.

Index Fungorum number: IF558550; Facesoffungi number: FoF 09945; Figs. 21, 22

Etymology: Name refers to the host plant species, Azolla filiculoides.

Holotype: IRAN $18020 \mathrm{~F}$

Endophytic from leave of Azolla filiculoides. Sexual morph Undetermined. Asexual morph Coelomycetous. Conidiomata 118-275 $\mu \mathrm{m}$ high, 80-200 $\mu \mathrm{m}$ diam., pycnidial, brown, solitary or confluent, globose, subglobose to pyriform, often with a short neck, glabrous or with some hyphal outgrows. Pycnidial wall pseudoparechymatous, 


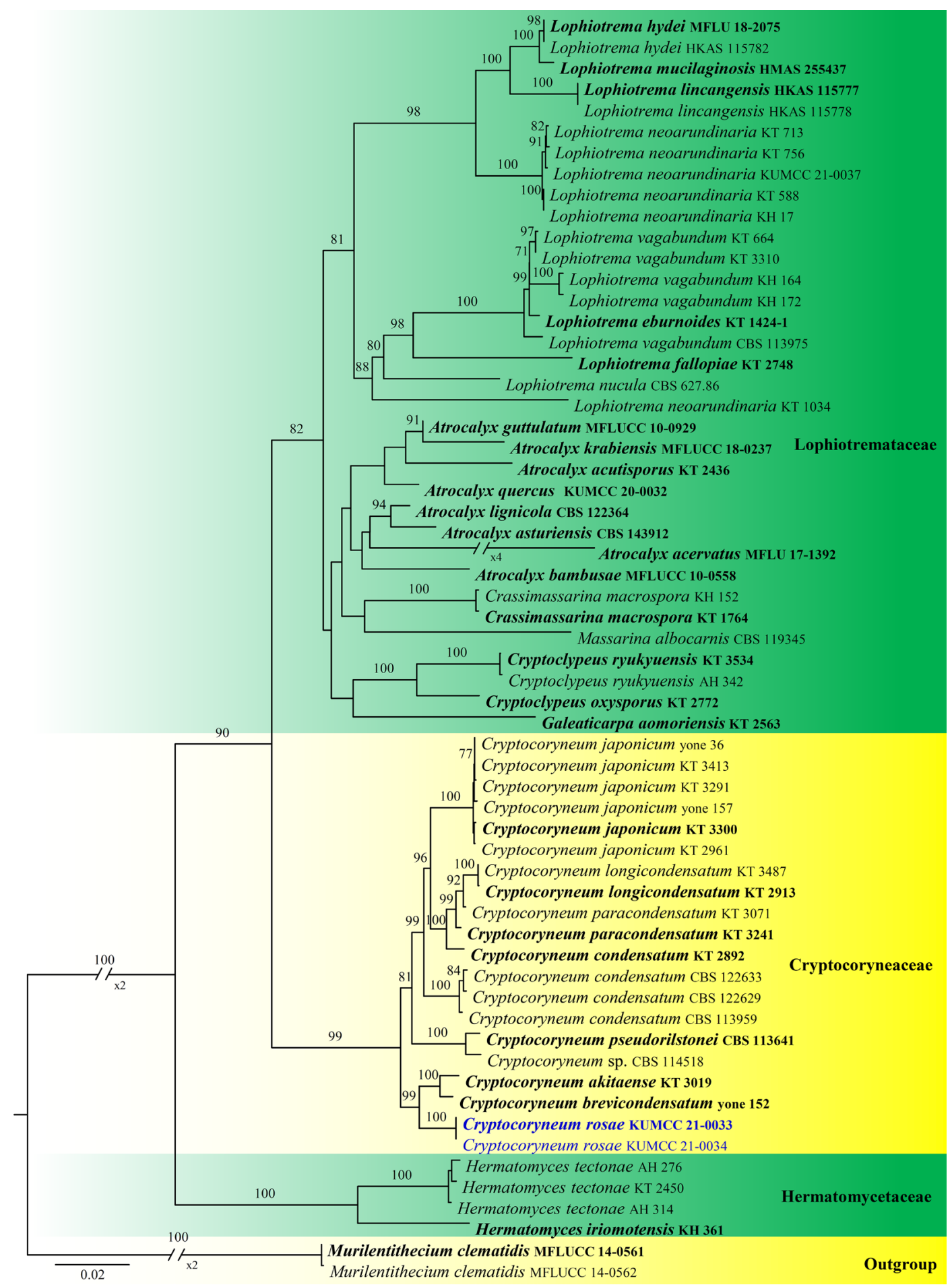

Fig. 20 Phylogram generated from the best scoring of the RAxML tree based on combined SSU, LSU, ITS, TEF1- $\alpha$ and RPB2 sequence dataset to indicate the new species in Cryptocoryneaceae. Sixty strains are included in the combined analyses which comprise a total of 4357 characters. Murilentithecium clematidis (MFLUCC 14-0561 and MFLUCC 14-0562) is selected as the outgroup taxon. The best RAxML tree with a final likelihood value of -24211.323363 is presented. RAxML analysis yielded 1272 distinct alignment patterns and
$6.17 \%$ of undetermined characters or gaps. Estimated base frequencies were as follows: $\mathrm{A}=0.246394, \mathrm{C}=0.256619, \mathrm{G}=0.267068$, $\mathrm{T}=0.229919$, with substitution rates $\mathrm{AC}=1.576531, \mathrm{AG}=4.38051$, $\mathrm{AT}=1.49611, \quad \mathrm{CG}=1.339907, \quad \mathrm{CT}=10.502267, \quad \mathrm{GT}=1.000000$; gamma distribution shape parameter alpha $=0.450773$. Bootstrap support values for maximum likelihood (MLBS, left) equal to or greater than $70 \%$ is given above the nodes. Ex-type strains are in bold and newly generated sequences are in blue 

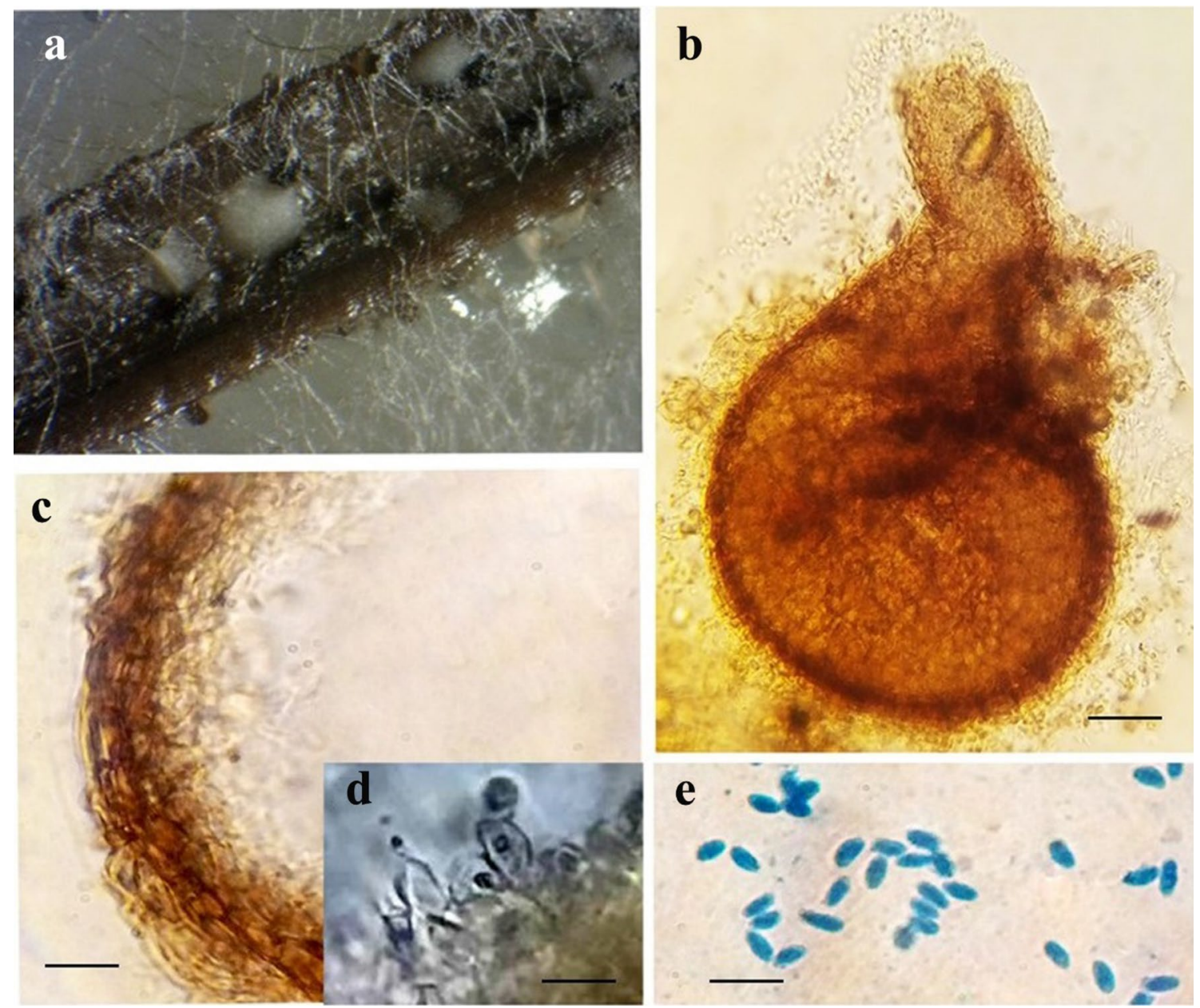

Fig. 21 Didymella azollae (IRAN 18020F, holotype). a Pycnidia on host. b Pycnidia. c Pycnidial wall. d Conidiogenous cells. e Conidia. Scale bars: $\mathbf{b}-\mathbf{e}=10 \mu \mathrm{m}$

comprising several layers of dark brown to lightly pigmented cells of textura angularis, outer layers 3-5 layers, 3-30 $\mu \mathrm{m}$ thick, pigmented, inner layers lightly pigmented. Conidiophores reduced to conidiogenous cells. Conidiogenous cells phialidic, hyaline, smooth, ampulliform to doliiform. Conidia $3-5 \times 1.5-2 \mu \mathrm{m}(\bar{x}=4.2 \times 2 \mu \mathrm{m}, \mathrm{n}=30)$, subglobose or ellipsoidal, hyaline, aseptate, smooth-walled.

Culture characteristics: Colonies on PDA, $40-48 \mathrm{~mm}$ diam. after 7 days, margin regular, densely covered by floccose aerial mycelia, greenish brown, reverse black (Fig. 22A). Colonies on OA, margin regular, floccose, aerial mycelia sparse, grayish brown, reverse black (Fig. 22B). Colonies on MEA, margin regular, floccose, buff, dense, reverse dark brown (Fig. 22C).

Material examined: IRAN, Guilan Province, endophytic from leave of Azolla filiculoides (Salviniaceae), 15 December 2016, E. Shams, (IRAN 18020F, holotype), ex-type living culture, IRAN 3058C.
GenBank numbers: ITS = MT514913, MT514914, MT514915, LSU = MT514910, MT514911, MT514912, TUB2 = MT512516, MT512517, MT512518.

Notes: Didymella azollae was isolated from Azolla filiculoides in Guilan, Iran. The characters of the conidia and colony (Figs. 21 and 22) fit the generic concept of Didymella (Chen et al. 2015). The species identified in the present study is closely related to Didymella dactylidis (CBS 124513) and D. rhei (CBS 109177) (Fig. 23). Didymella azollae differs from $D$. dactylidis in type of conidia (Chen et al. 2017). In pairwise nucleotide comparisons of Didymella azollae with the type strain of $D$. dactylidis (CBS 124513), there is a nucleotide difference of $0.75 \%$ (6 bp) in LSU (of 799 nucleotides altogether) and $1.37 \%$ (5 bp) nucleotide difference in ITS (of 435 nucleotides altogether). However, in the proteincoding region of TUB2, the nucleotide difference was $6.7 \%$ (20 bp) across 286 nucleotides. Didymella rhei differs from D. azollae by conidia size (3.5-) 5-8 $(-10.5) \times 1.5-3 \mu \mathrm{m})$. In pairwise nucleotide comparisons of Didymella azollae with 

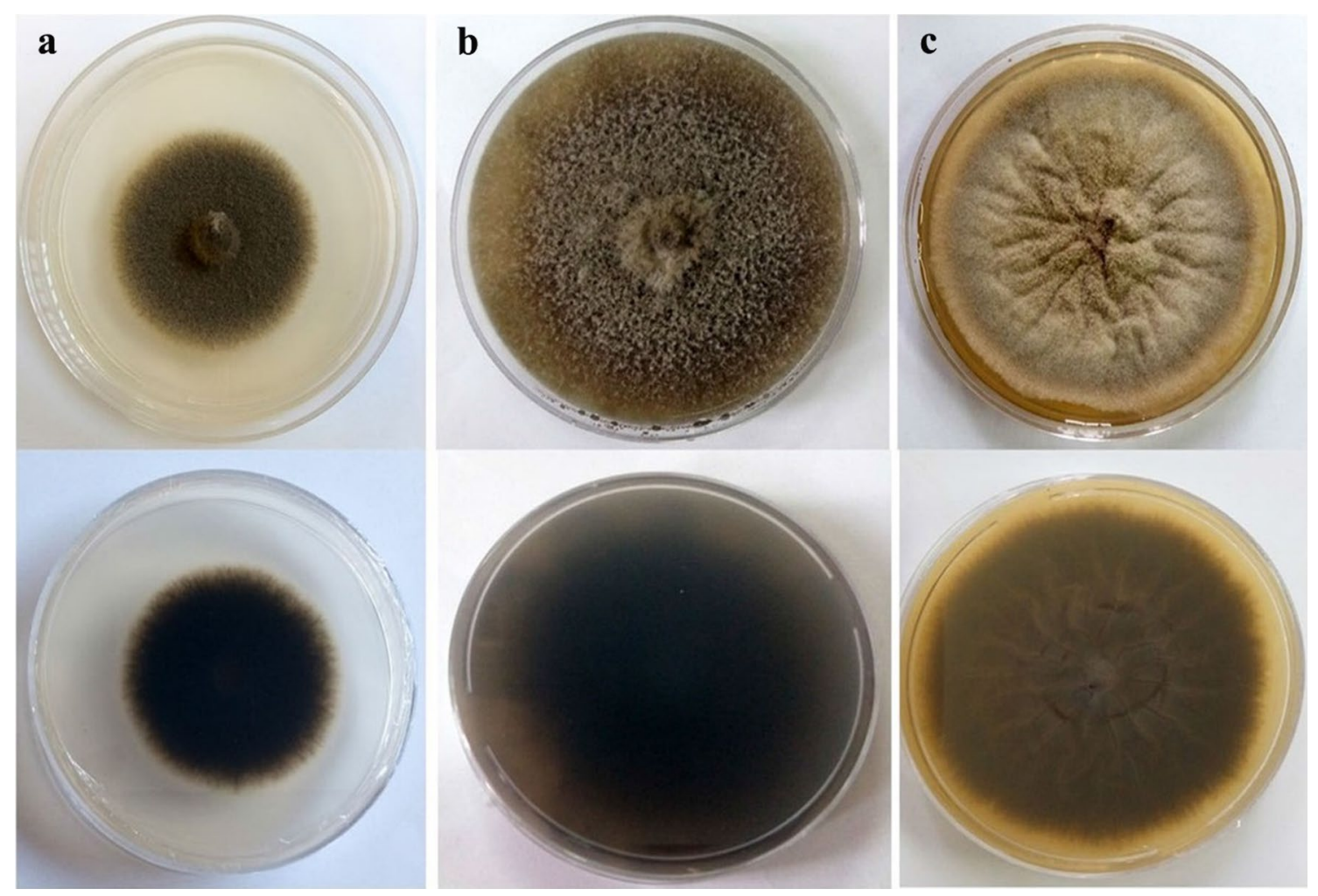

Fig. 22 Didymella azollae (IRAN 3058C, ex-type culture) cultures after 7 days at $25{ }^{\circ} \mathrm{C}$ on different media. a PDA. b OA. c MEA

the type strain of $D$. rhei (CBS 109177), there is a nucleotide difference of $0.85 \%$ ( $7 \mathrm{bp}$ ) in LSU (of 817 nucleotides altogether), $1.33 \%$ (6 bp) nucleotide difference in ITS (of 450 nucleotides altogether) and in the protein-coding region of TUB2, there is a large nucleotide difference of $6.7 \%$ (20 bp out of 286 nucleotides).

\section{Didymosphaeriaceae Munk}

Notes: Didymosphaeriaceae was established by Munk (1953) with Didymosphaeria Fuckel as the type genus. This family consists of 32 genera, which includes isolates from different habitats including terrestrial, aquatic, saprotrophic, parasitic, and hemibiotrophic (Hongsanan et al. 2020a). Didymosphaeriaceae is characterized by immersed, gregarious or scattered ascomata with two to three layered peridium composed of cells of textura angularis or textura intricate, septate hamathecium with or without trabeculate pseudoparaphyses. Asci bitunicate, fissitunicate, cylindrical or oblong, septate or muriform and pedicellate. Ascospores uni-seriate or biseriate, oblong, verruculose, with or without a gelatinous sheath. The asexual morphs were known as coelomycetous and hyphomycetous taxa (Ariyawansa et al. 2014a, 2015b). Based on phylogenetic analysis of ITS, LSU and $\beta$-tubulin sequence data, a novel species Paraconiothyrium ajrekarii is introduced. In addition, two new records, Montagnula thailandica and Spegazzinia camelliae are described as new record based on morphological and phylogenetic analyses.

\section{Montagnula Berl.}

Notes: We follow the latest treatment and updated accounts of Montagnula in Mapook et al. (2020). Although 44 epithets of Montagnula are listed in Species Fungorum (Index Fungorum 2021), only 17 species have been confirmed in Montagnula based on molecular data (Fig. 7).

Montagnula thailandica Mapook \& K.D. Hyde, in Mapook et al., Fungal Diversity 101: 35 (2020)

Index Fungorum number: IF557299; Facesofungi number: FoF 07792; Fig. 24

Saprobic on dead stems. Sexual morph Ascomata (333-)463-514×264-505 $\mu \mathrm{m}$, immersed to erumpent, scattered, gregarious to grouped, uni-loculate, globose to obpyriform, coriaceous, brown to dark brown, with papillate ostiole in the centre. Peridium (44-)60-81 $\mu \mathrm{m}$ wide, comprising several layers of thick-walled, brown to dark brown cells of textura angularis. Hamathecium comprising 1-2 $\mu \mathrm{m}$ wide, cylindrical to filiform, septate, branched, hyaline pseudoparaphyses. Asci $66-121 \times 6-11 \mu \mathrm{m}(\bar{x}=82 \times 10 \mu \mathrm{m}$, $\mathrm{n}=20$ ), 8 -spored, bitunicate, fissitunicate, elongate-clavate, slightly curved, long pedicel. Ascospores $14-17 \times 4-6 \mu \mathrm{m}$ ( $\overline{x=} 15 \times 5 \mu \mathrm{m}, \mathrm{n}=20$ ), overlapping $1-2$-seriate, broadly 


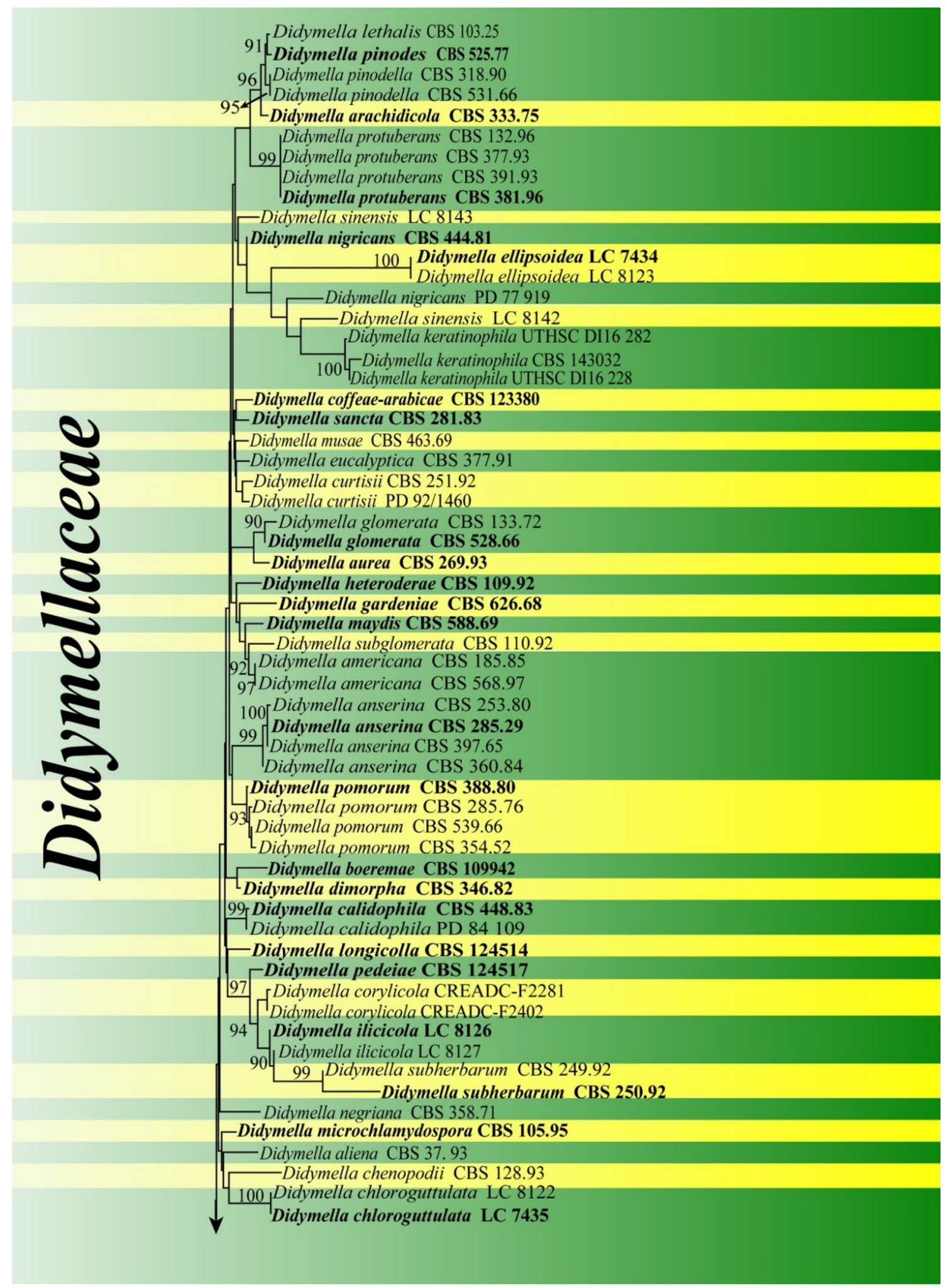

Fig. 23 Maximum Likelihood tree inferred by MEGA v.7 from the combined ITS, LSU, and TUB gene regions of 92 isolates. Bootstrap support values from ML equal to or greater than $90 \%$ are provided above or below the branches. Ex-type strains are in bold and novel species is shown in blue 


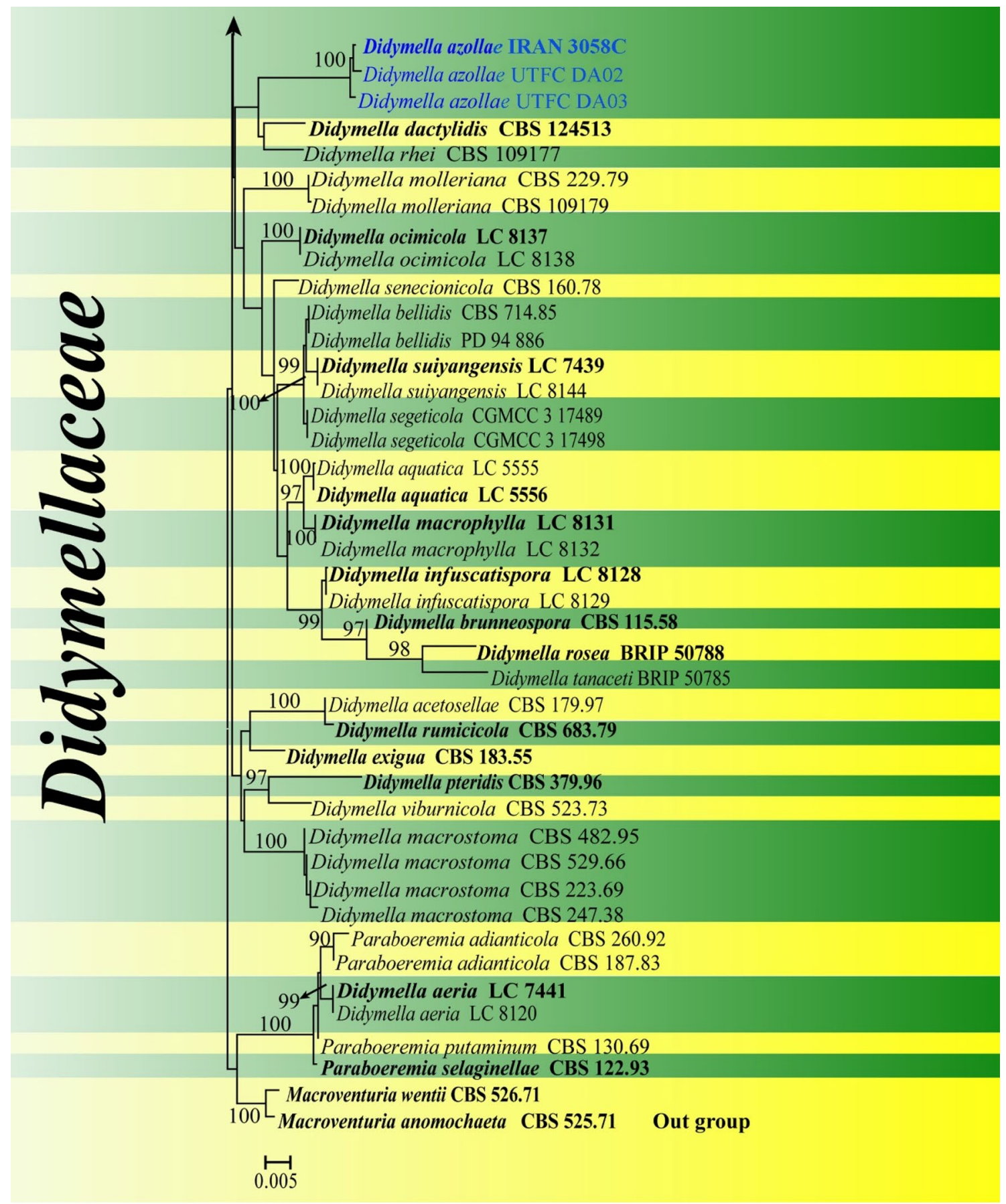

Fig. 23 (continued)

fusiform to ellipsoid, hyaline to pale brown when immature, becoming brown to dark brown when mature, 1-septate, constricted at the septum, slightly wider upper cell and tapering towards ends, slightly curved, with 2-4-guttulate, smoothwalled. Asexual morph Undetermined.
Culture characteristics: Ascospores germinating on PDA within $24 \mathrm{~h}$ at room temperature and germ tubes produced from both cells. Colonies on PDA circular, mycelium velvety with moderately fluffy, filamentous at margin, cultures grey with white from the centre of the colony on surface, darkened from reverse. 

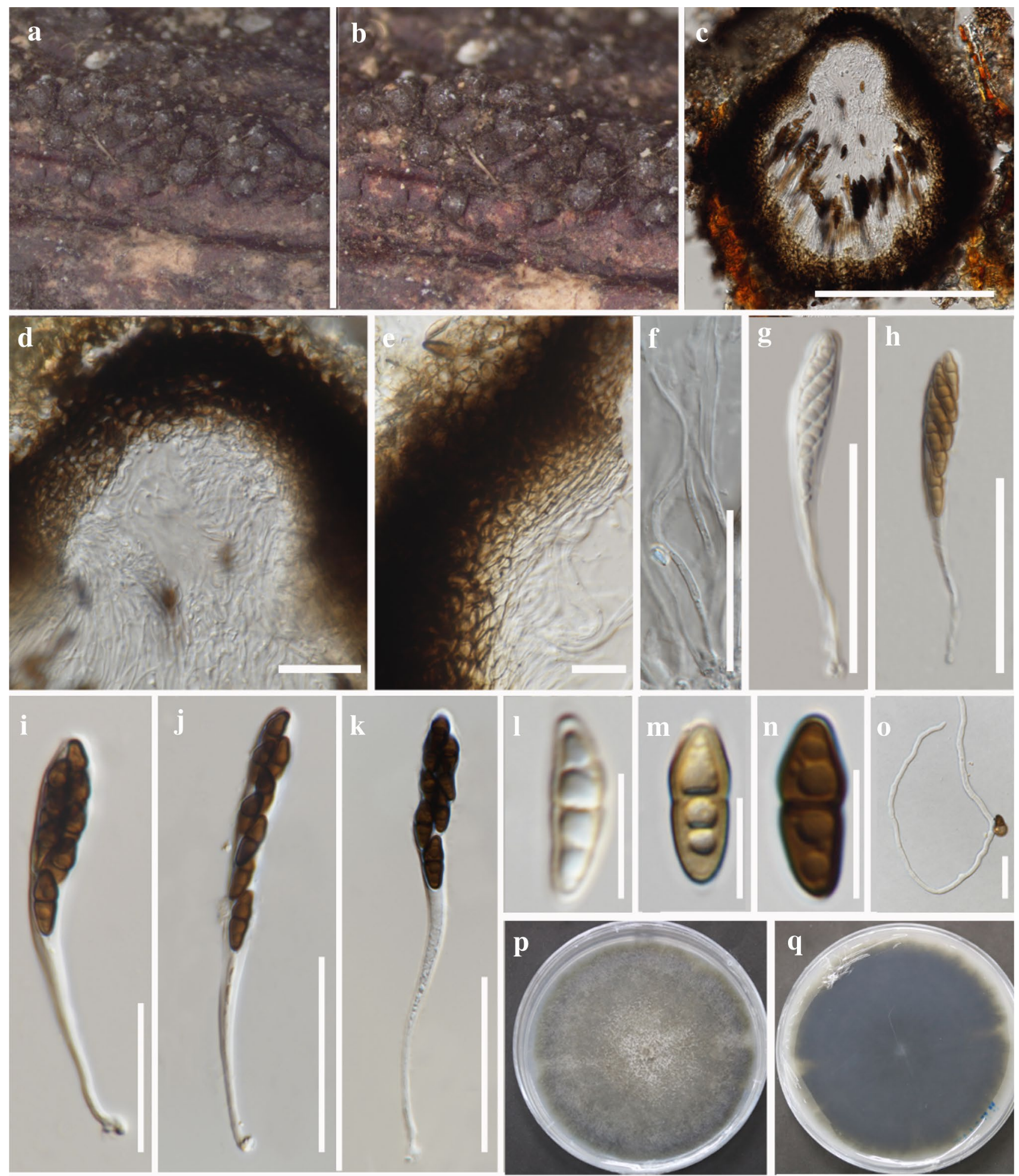

Fig. 24 Montagnula thailandica (MFLU 21-0052, new record). a, b Appearance of ascomata on woody substrate. c Section of ascoma through ostiole. d Section of ostiole. e Peridium. f Pseudoparaphy- ses. g-k Immature and mature asci. l-n Ascospores. o Germinated ascospore. $\mathbf{p}, \mathbf{q}$ Culture on PDA from surface and reverse. Scale bars: $\mathbf{c}=200 \mu \mathrm{m}, \mathbf{d}, \mathbf{e}=100 \mu \mathrm{m}, \mathbf{f}-\mathbf{k}=50 \mu \mathrm{m}, \mathbf{l}-\mathbf{n}=10 \mu \mathrm{m}, \mathbf{o}=20 \mu \mathrm{m}$ 
Material examined: THAILAND, Chiang Rai Province, Muang, Mae Yao, on dead stems of unidentified host, 23 September 2019, N. Huanraluek, MY04 (MFLU 21-0052, new record), living culture, MFLUCC 21-0075.

GenBank numbers: ITS = MZ538515, LSU = MZ538549, TEF1- $\alpha=$ MZ567092.

Notes: The new collection shares cluster and a phylogenetic affinity to the ex-type strain Montagnula thailandica (MFLUCC 17-1508) based on a combined LSU, ITS, SSU and TEF1- $\alpha$ sequence analysis but with low statistical support (Fig. 25). Comparisons of ITS, TEF1- $\alpha$ and LSU sequences show that our isolate MFLUCC 21-0075 differs from the ex-type strain M. thailandica (MFLUCC 17-1508) in $1 / 550 \mathrm{bp}(0.18 \%)$ of ITS and $7 / 907 \mathrm{bp}(0.77 \%)$ of TEF1- $\alpha$, while LSU has no base pair difference. In addition, our collection MFLUCC 21-0075 shares identical characters with M. thailandica (MFLUCC 17-1508) isolated by Mapook et al. (2020) from dead stems of Chromolaena odorata from Chiang Mai, Thailand. This is a second report of this species on dead stems as a new record from Thailand (Fig. 24).

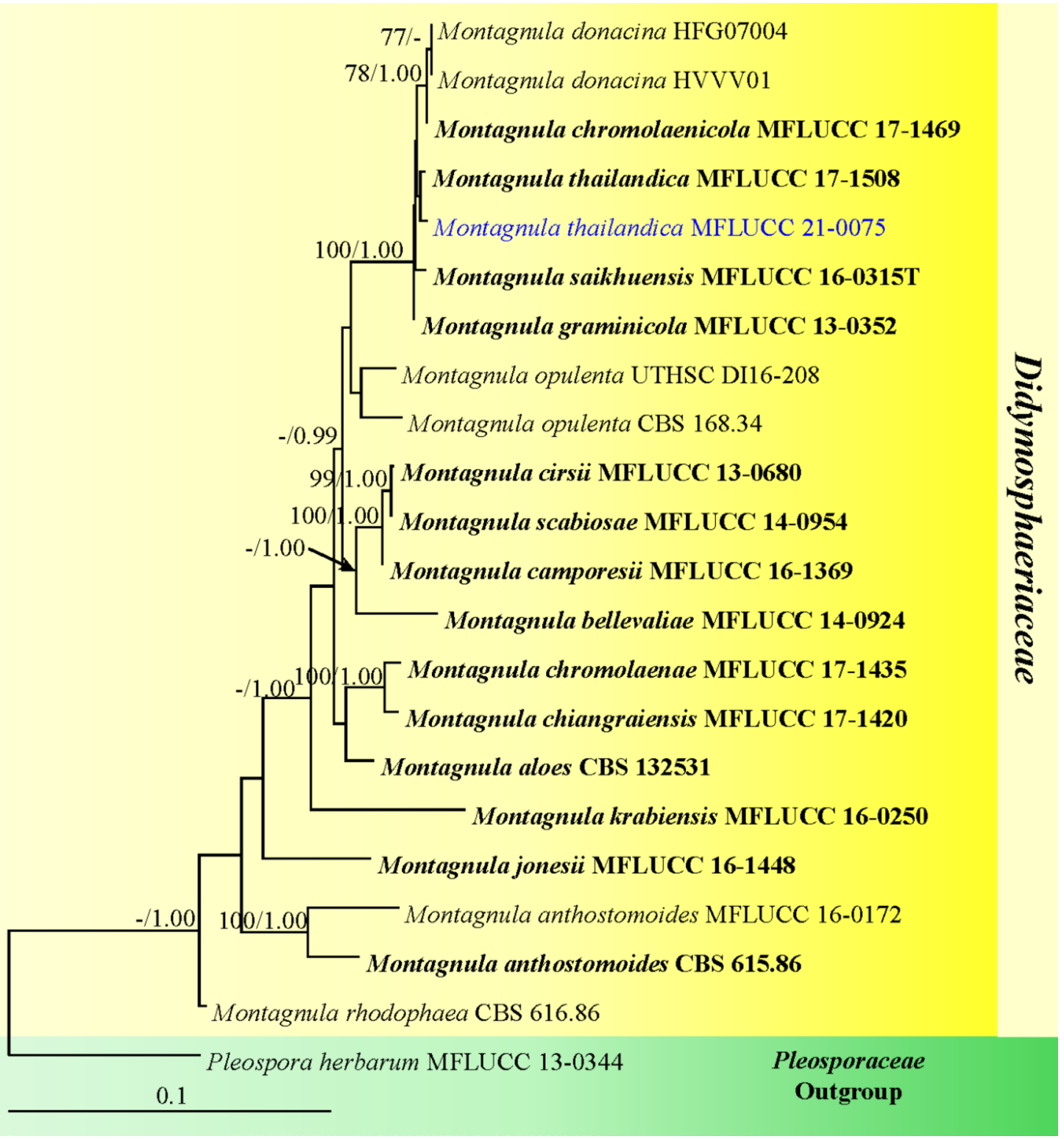

Fig. 25 Phylogram generated from maximum likelihood analysis based on combined LSU, ITS, SSU and TEF1- $\alpha$ sequence data of Montagnula taxa. The tree is rooted with Pleospora herbarum (MFLUCC 13-0344). Bootstrap support values for maximum likeli- hood (MLBS, left) equal to or greater than $70 \%$ is given above the nodes. Bayesian posterior probabilities (BYPP, right) equal to or greater than 0.95 are given above the nodes. Ex-type strains are in bold and newly generated sequence is in blue 


\section{Paraconiothyrium G.J.M. Verkley}

Notes: Paraconiothyrium, a close relative genus of Paraphaeosphaeria was introduced by Verkley et al. (2004) to accommodate four species viz. P. estuarinum, $P$. brasiliense, $P$. cyclothyrioides and $P$. fungicola. Whereas, the two mycoparasites Coniothyrium minitans and $C$. sporulosum were therefore transferred to Paraconiothyrium (as P. minitans) based on phylogeny. Verkley et al. (2014) transferred Paraconiothyrium minitans and P. sporulosum to Paraphaeosphaeria based on multigene phylogenetic analysis. Ariyawansa et al. (2020) demonstrated that Paraconiothyrium species are polyphyletic within Didymosphaeriaceae. Members of Paraconiothyrium are used as biocontrol agents, antibiotic producers and potential bioremediators. In this study, a new species Paraconiothyrium ajrekarii is introduced based on evidence of morphology and phylogenetic placement (Figs. 26 and 27).

Paraconiothyrium ajrekarii S. Rana \& S.K. Singh, sp. nov. Index Fungorum number: IF557844; Facesoffungi number: FoF 08020; Fig. 26

Etymology: Named after Late Professor S.L. Ajrekar, who was a distinguished mycologist and plant pathologist of India.

Holotype: AMH 10218

Color Codes Follow: Methuen Handbook of Colour (Kornerup and Wanscher 1978).

From leaf phylloplane of Mangifera indica L. (Anacardiaceae). Sexual morph Undetermined. Asexual morph Hyphae $1-4 \mu \mathrm{m}(\bar{x}=3 \mu \mathrm{m}, \mathrm{n}=15)$, hyaline to light olivaceous, smooth-walled. Chlamydospores 6-23.5 $\times 6-10.5 \mu \mathrm{m}$ $(\bar{x}=10.5 \times 8 \mu \mathrm{m}, \mathrm{n}=15)$, solitary or in chains, globose to sub globose, wall thickened and darkened, sub hyaline to light olivaceous to dark brown. Conidiomata up to $500 \times 300 \mu \mathrm{m}$ diam., pycnidial, deeply seated, produced in groups, globose to sub globose to oval, dark brown, sometimes with elongated ostiolar neck (up to $245 \times 84 \mu \mathrm{m}$ ). Peridium up to $33 \mu \mathrm{m}$, multi-layered, textura angularis, delicate, pale brown. Conidiophores reduced to conidiogenous cells, variable in shape and size. Conidiogenous cells 7.5-21 $\times 3-8.5 \mu \mathrm{m}(\bar{x}=12 \times 5 \mu \mathrm{m}, \mathrm{n}=15)$, short, phialidic, with a collarette, flask-shaped, elongated, ampuliform with swollen basal cell, smooth walled, hyaline. Conidia 3-6 $\times 1-4 \mu \mathrm{m}(\overline{x=} 5 \times 3 \mu \mathrm{m}, \mathrm{n}=30)$, subhyaline, light olivaceous to olivaceous brown, oval to broadly fusoid, rarely angular, aseptate, wall smooth, thickened and darkened.
Culture characteristics: Colonies on PDA reaching $34 \mathrm{~mm}$ diam. after 2 weeks, at $25^{\circ} \mathrm{C}$; floccose, circular, umbonate with smooth margins; sulcate on both sides; front colour pinkish white (7A2) to chalky white (7A1), reverse grayish brown at center, light yellow (4A4) towards margin. Colonies on V8 juice agar reaching $46 \mathrm{~mm}$ after 2 weeks, at $25^{\circ} \mathrm{C}$; circular, flat, slightly floccose, margin smooth and regular; front colour yellowish white (4A2), reverse grayish yellow (golden wheat) (4B5) and sulcate. Colonies on oat meal agar reaching $61 \mathrm{~mm}$ after 2 weeks, at $25^{\circ} \mathrm{C}$; circular, floccose, umbonate, sulcate, margins smooth; front colour orange white (6A2) to white (1A1), reverse light yellow (4A4) and sulcate. Colonies on PCA reaching $57 \mathrm{~mm}$ after 2 weeks, at $25^{\circ} \mathrm{C}$; circular, floccose, slightly raised, margins smooth, regular; front colour pale orange (5A3) to dull green (25E3), periphery beige (4C3), reverse dark green (28F6) to light yellow (4A4). Colonies on Czapek Dox agar (CDA) reaching $61 \mathrm{~mm}$ after 2 weeks at $25^{\circ} \mathrm{C}$; circular, slightly floccose, velvety, sulcate, margins smooth and regular; colour from front orange grey (5B2) in centre and reddish blond (5C3) towards periphery, reverse brownish orange (5C3) in centre and periphery, beaver (5F4) in middle, sulcate. Colonies on MEA reaching $53 \mathrm{~mm}$ after 2 weeks at $25^{\circ} \mathrm{C}$; circular, slightly floccose, margins smooth, regular; front colour white (1A1) in centre and dark blonde (5D4) towards periphery, reverse chocolate brown (6F3) in centre and clay (5D5) towards margins.

Material examined: INDIA, Himachal Pradesh, Kangra, Baijnath, Simbal, from leaf phylloplane of Magnifera indica L. (Anacardiaceae), 10 May 2019, S. Rana (AMH 10218, holotype), ex-type living culture, NFCCI 4810.

GenBank numbers: $\beta$-tubulin $=$ MT394161, ITS $=$ MT372906, LSU $=$ MT372905.

Notes: This current taxon is placed in Paraconiothyrium based on pycnidial conidiomata, phialidic conidiogenous cells producing aseptate, thick and smooth-walled, light olivaceous to olivaceous brown conidia (Verkley et al. 2004). Paraconiothyrium ajrekarii differs from other known species based on its phylogenetic as well as morphological analyses (Fig. 26). The combined $\beta$-tubulin, ITS and LSU phylogeny indicates that Paraconiothyrium ajrekarii forms a sister taxon with $P$. archidendri and P. magnoliae. Paraconiothyrium ajrekarii differs from $P$. archidendri based on its combination of characters such as dimensions of conidiomata and conidiogenous cells (Verkley et al. 2014). Paraconiothyrium ajrekarii has somewhat slender conidia in comparison to $P$. archidendri (Table 2). Conidiogenous cells in P. ajrekarii has collarette, flask shaped 

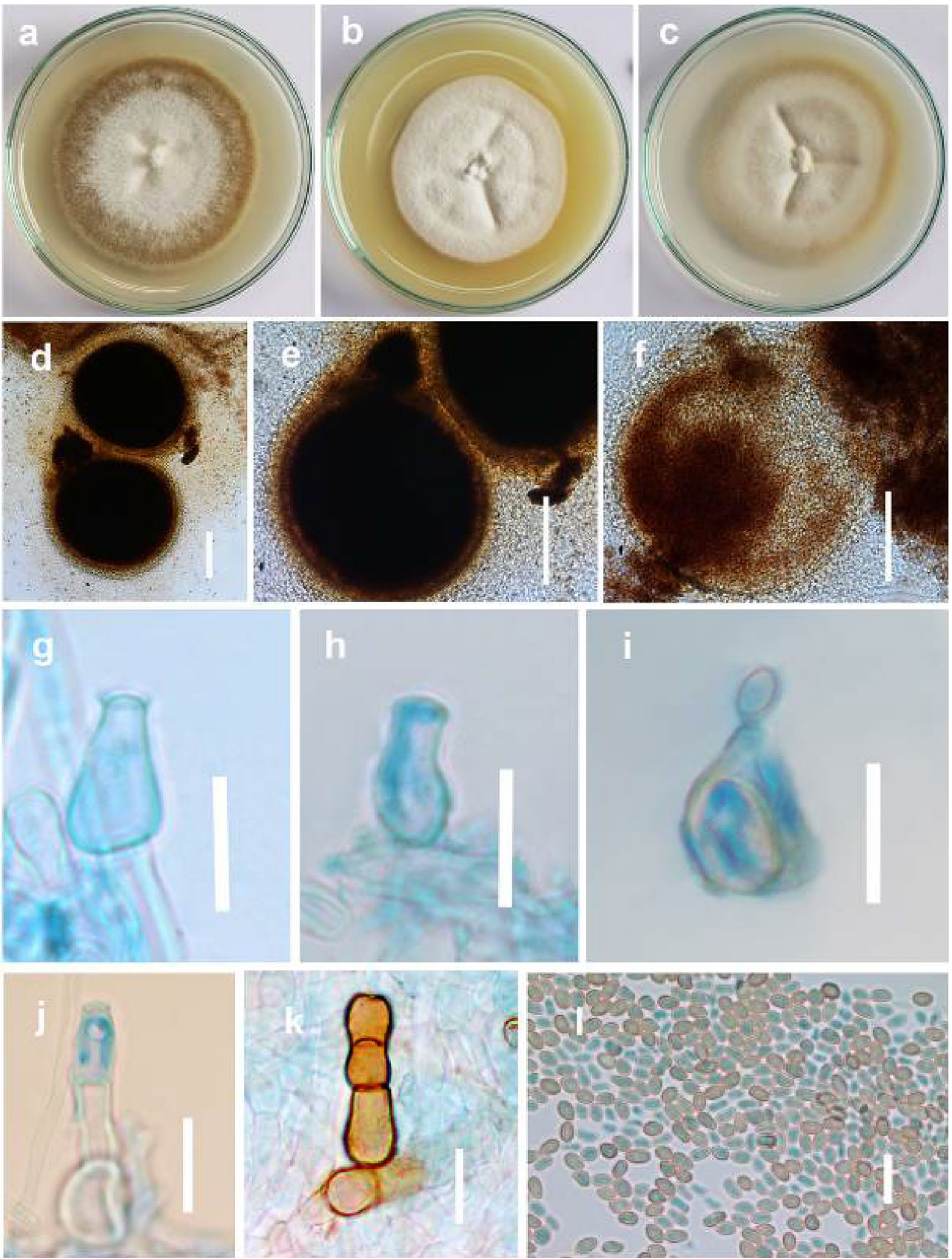

Fig. 26 Paraconiothyrium ajrekarii (AMH 10218, holotype). a-c Colonies from surface on various media after 2 weeks; a MEA, b V8 juice agar, $\mathbf{c}$ Czapek Dox agar. $\mathbf{d}$ Conidiomata. e, f Enlarged view of conidiomata. $\mathbf{g}, \mathbf{h}$ Conidiogenous cells showing distinct collarette rings. $\mathbf{i}, \mathbf{j}$ Condiogenous cells bearing developing conidia. $\mathbf{k}$ Chlamydospores. I Conidia. Scale bars: $\mathbf{d}-\mathbf{f}=100 \mu \mathrm{m}, \mathbf{g}-\mathbf{l}=10 \mu \mathrm{m}$ 


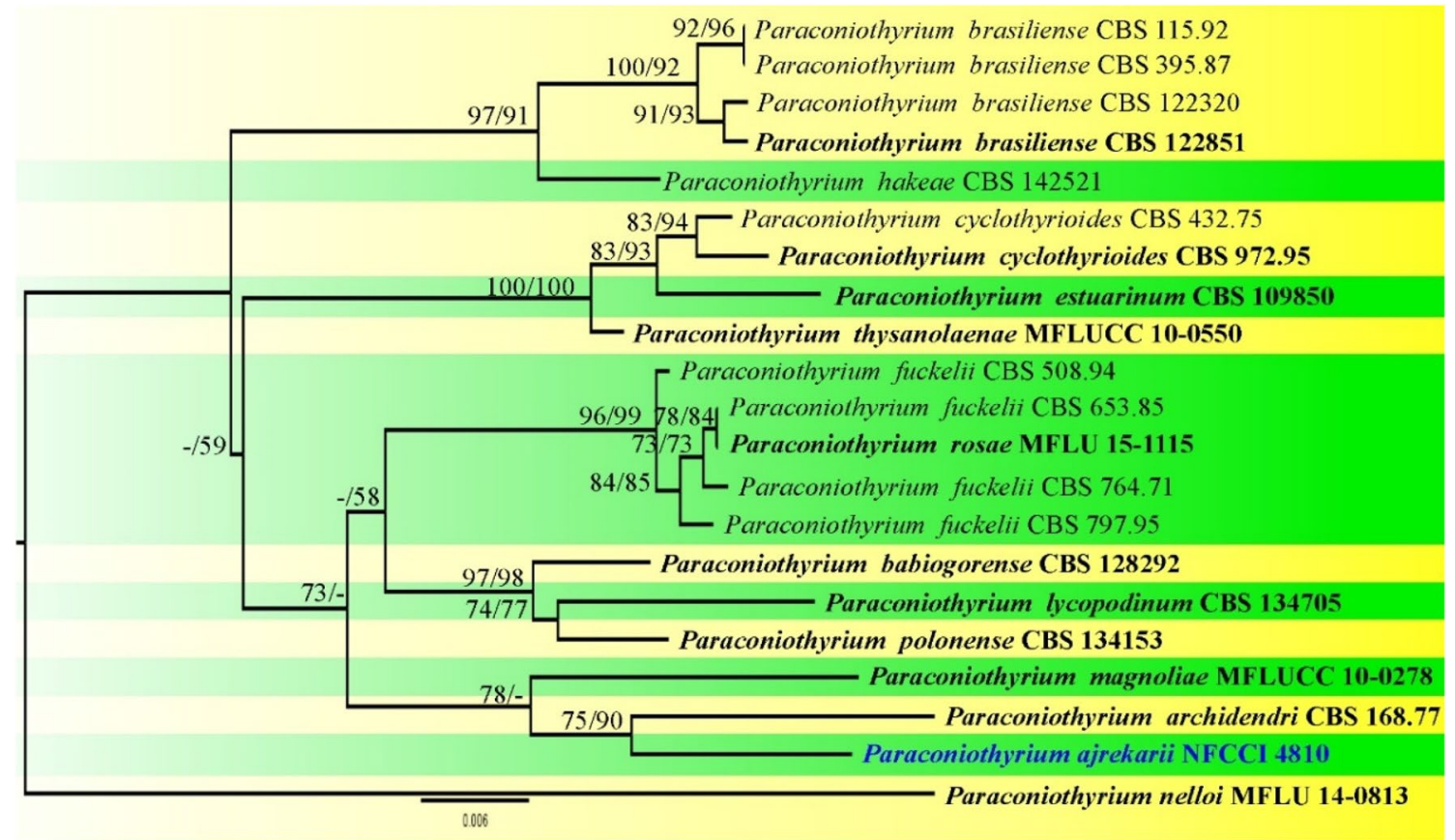

Fig. 27 Molecular phylogenetic analysis by maximum-likelihood (ML) method based on combined $\beta$-tubulin, LSU and ITS sequence data. Statistical supports are indicated next to each node, non-parametric maximum likelihood ultrafast-bootstrap (UFBS) values and SH-aLRT obtained from 1000 replicates using IQ-TREE and the $\mathrm{TIM} 3+\mathrm{F}+\mathrm{I}+\mathrm{G} 4$ model. Bootstrap values for maximum likelihood (MLBS) equal to or greater than $50 \%$ are labeled on the nodes. Twenty-one strains are included in the combined analyses which comprise 2393 sites of which 2089 were found to be conserved, 286 were the variable sites and included nearly 178 parsimony-informative sites. The optimized log-likelihood values of -5846.542 is presented. Estimated base frequencies are as follows: $\mathrm{A}=0.300$, $\mathrm{C}=0.183, \mathrm{G}=0.205, \mathrm{~T}=0.312$; substitution rates $\mathrm{AC}=2.10979$, $\mathrm{AG}=3.70012, \quad \mathrm{AT}=1.00000, \quad \mathrm{CG}=2.10979, \quad \mathrm{CT}=8.84516$, $\mathrm{GT}=1.00000$; gamma distribution shape parameter $\alpha=0.559$. New sequence data of Paraconiothyrium ajrekarii (AMH 10,218; NFCCI 4810) is highlighted in blue bold and all sequences from type specimen is in bold

Table 2 Morpho-taxonomic comparison of Paraconiothyrium ajrekarii sp. nov. with closest taxa

\begin{tabular}{|c|c|c|c|c|c|}
\hline Species & Host & Locality & Conidiogenous cells & Conidial septa and sizes & References \\
\hline $\begin{array}{l}\text { Paraconiothy- } \\
\text { rium ajrekarii } \\
\text { sp. nov }\end{array}$ & $\begin{array}{l}\text { Mangifera indica (Phyl- } \\
\text { loplane) }\end{array}$ & India & $\begin{array}{l}\text { Phialidic, with collarette, } \\
\text { flask shaped, }\end{array}$ & $\begin{array}{l}\text { Aseptate, } \\
3-6 \times 1-4 \mu \mathrm{m}\end{array}$ & This study \\
\hline P. archidendri & $\begin{array}{l}\text { Pithecellobium bigeminum } \\
\text { (Leaf spots) }\end{array}$ & Myanmar & $\begin{array}{l}\text { Holoblastic, occasionally } \\
\text { annellidic, discrete }\end{array}$ & $\begin{array}{l}\text { Aseptate, } \\
3.5-6 \times 2.5-3.5(-4) \mu \mathrm{m}\end{array}$ & Verkley et al. (2014) \\
\hline P. magnoliae & $\begin{array}{l}\text { Magnolia liliifera (Dead } \\
\text { leaves) }\end{array}$ & Thailand & N/A & N/A & Ariyawansa et al. (2014b) \\
\hline
\end{tabular}

N/A Data not available

with swollen basal cells as against blastic to annellidic in $P$. archidendri. However, Paraconiothyrium ajrekarii is an asexual morph and hence cannot be compared with morphological characters of $P$. magnoliae as it is reported to have only a sexual morph (Ariyawansa et al. 2014b). However, Paraconiothyrium ajrekarii is an asexual morph and cannot be compared with $P$. magnoliae reported to have only a sexual morph (Ariyawansa et al. 2014b). A megablast search using the ITS sequence data of $P$. ajrekarii revealed closest affinities with members of Paraconiothyrium, i.e. Paraconiothyrium archidendri CBS 168.77 ( similarity $=552 / 571 \mathrm{bp}, 96.67 \%$ ) and $P$. magnoliae MFLUCC
$10-0278$ ( similarity $=496 / 517 \mathrm{bp}, 95.94 \%$ ). In the phylogenetic analysis based on combined $\beta$-tubulin, LSU and ITS sequence data, Paraconiothyrium ajrekarii forms a distinct linage from P. archidendri with 75\% MLBS, 0.90 BYPP support (Fig. 27). Thus, we illustrated and described as a novel species.

\section{Spegazzinia Sacc.}

Notes: Spegazzinia was introduced by Saccardo (1880) with the type species $S$. ornata and is characterized as having two conidial types (a-conidia with the long spines and b-conidia with the shorter spines to smooth-walled). The 
genus was included in Didymospheriaceae based on molecular phylogenetic placement (Tanaka et al. 2015; Hongsanan et al. 2020a). We follow the latest treatment and updated members of Spegazzinia in Samarakoon et al. (2020). A new record, S. camelliae is described based on its morphological characteristics and phylogenetic evidence.

Spegazzinia camelliae N. Suwannarach, J. Kumla \& S. Lumyong, Phytotaxa 483(2): 120 (2021)

MycoBank number: MB837969; Facesoffungi number: FoF 09467; Fig. 28

Saprobic on decaying wood. Sexual morph Undetermined. Asexual morph Hyphomycetous. Colonies punctiform, effuse, scattered, dark brown to black on natural wood substrate. Mycelium slight raised on host surface, branched, septate, hyaline to pale brown. Conidiophores micronematous, mononematous, basauxic, slight erect, flexuous, simple, unbranched, intercalarily, pale brown. Conidiogenous cells indiscrete, indeterminate, terminal. Conidia 14-17 diam. $\mu \mathrm{m}$, holoblastic, globose to subglobose, 4-celled, cruciately septate and constricted at the septa, dentate at the margin, brown to dark brown, darker at the septa, faint septa at maturity, guttulate when immature, smooth-walled.

Culture characteristics: Conidium germinating on PDA within $24 \mathrm{~h}$ and germ tubes arising from terminal end. Colonies on PDA, slow growing, reaching $5 \mathrm{~cm}$ diam. after 1 month at room temperature, flat to slightly effuse, surface smooth, circular, radial striations and entire edges, olivaceous brown to dark brown, and sporulated conidia in cultures after 14 days. Mycelium superficial, partly immersed, composed of branched, septate, hyaline to pale brown, smooth-walled. Conidia 11-17 $\mu \mathrm{m}$ diam., globose to subglobose, 4-celled cruciate septate and constricted at the septa, dentate at the margin, brown to dark brown, darker at the septa, guttulate, smooth-walled.

Material examined: THAILAND, Phitsanulok Province, Wang Thong, Wang Nok Aen, on decaying wood, 25 July 2019, S. Boonmee, WNA03 (MFLU 21-0053, new record), living culture, MFLUCC 21-0076.

GenBank numbers: ITS = MZ538526, LSU = MZ538560, TEF1- $\alpha=$ MZ567102.

Notes: Spegazzinia camelliae was described as an endophytic isolate from living leaves of the Camellia sinensis plant in northern Thailand (Suwannarach et al. 2021). Based on phylogenetic analysis of a combined LSU, ITS and TEF1- $\alpha$ sequence dataset of Spegazzinia species, our strain MFLUCC 21-0076 clusters with the ex-type strain SDBRCMU328 with 88\% MLBS, 1.00 BYPP support (Fig. 29). When compared our strain was similar with the type specimen of S. camelliae (SDBR-CMU328), they are morphologically similar (Fig. 28). This is the first record of S. camelliae (MFLU 21-0053) as a saprobic strain on decaying wood.
Hermatomycetaceae Locq.

Notes: Hermatomycetaceae was introduced to accommodate a single genus Hermatomyces that is characterized by sporodochial conidiomata with one to two types of muriform and dark brown to black conidia (Locquin 1984; Hashimoto et al. 2017). We follow the latest treatments and updated accounts of Hermatomycetaceae in Hongsanan et al. (2020a).

\section{Hermatomyces Speg.}

Notes: Members of Hermatomyces are commonly recognized as saprobes on dead decaying or rotten plant substrates in terrestrial habitats (Koukol et al. 2018; Hyde et al. 2019; Jayasiri et al. 2019; Koukol and Delgado 2019; Nuankaew et al. 2019). Hermatomyces currently includes 28 epithets that are listed in Index Fungorum (2021). In this study, we provided on new host record for Hermatomyces nabanheensis from China and a new geographical record for $H$. sphaericoides from Thailand. The phylogenetic tree is presented in Fig. 32.

Hermatomyces nabanheensis Tibpromma, Bhat \& K.D. Hyde, Fungal Diversity 87: 39 (2017)

Index Fungorum number: IF552901; Facesoffungi number: FoF 03135; Fig. 30

Saprobic on dead rachis of Cyathea spinulosa. Sexual morph Undetermined. Asexual morph Hyphomycetes. Colonies on natural substrate dry, blackish brown, superficial, circular or oval, bird nest-like, doughnut-shaped, scattered, blackish brown, glistening, conidia readily liberated when disturbed. Mycelium 1.7-3.3 $\mu \mathrm{m}$ wide, superficial, composed of a network of branched, septate, hyaline to pale brown. Conidiophores are reduced to Conidiogenous cells, micronematous, short, hyaline to pale brown, arising from prostrate hyphae at the centre of circular colony. Conidiogenous cells $4-5.5(-8.5) \times 2.4-3.7$, holoblastic, monoblastic, integrated, terminal, cylindrical, hyaline to subhyaline. Conidia dimorphic, lenticular and cylindrical, thick-walled. Lenticular conidia 30-39 $\times 20-28 \mu \mathrm{m}$ $(\bar{x}=34 \times 23.5 \mu \mathrm{m}, \mathrm{n}=20)$, numerous, thick-walled, central cells dark brown, hyaline to pale brown peripheral cells with septa, forming a distinct ring on the outside. Cylindrical conidia $32-39 \times 19.5-27 \mu \mathrm{m}(\bar{x}=36 \times 24 \mu \mathrm{m}, \mathrm{n}=15) \mu \mathrm{m}$ in broadest part of lower cells, with 2 forked columns of $2-4$ cells, cylindrical, swollen, constricted at septa, subhyaline, upper part of terminal cells dark brown, granulate, rough.

Culture characteristics: Conidia germinated on PDA within $15 \mathrm{~h}$ at incubator at $25^{\circ} \mathrm{C}$. Germ tubes produced around conidia and were transferred to fresh PDA media. Pure cultures were incubated at $25^{\circ} \mathrm{C}$ and reaching $2.2 \mathrm{~cm}$ diam. in 10 days, circular, smooth surface, velvety and raised, gray to brown to gray from center to margin from 


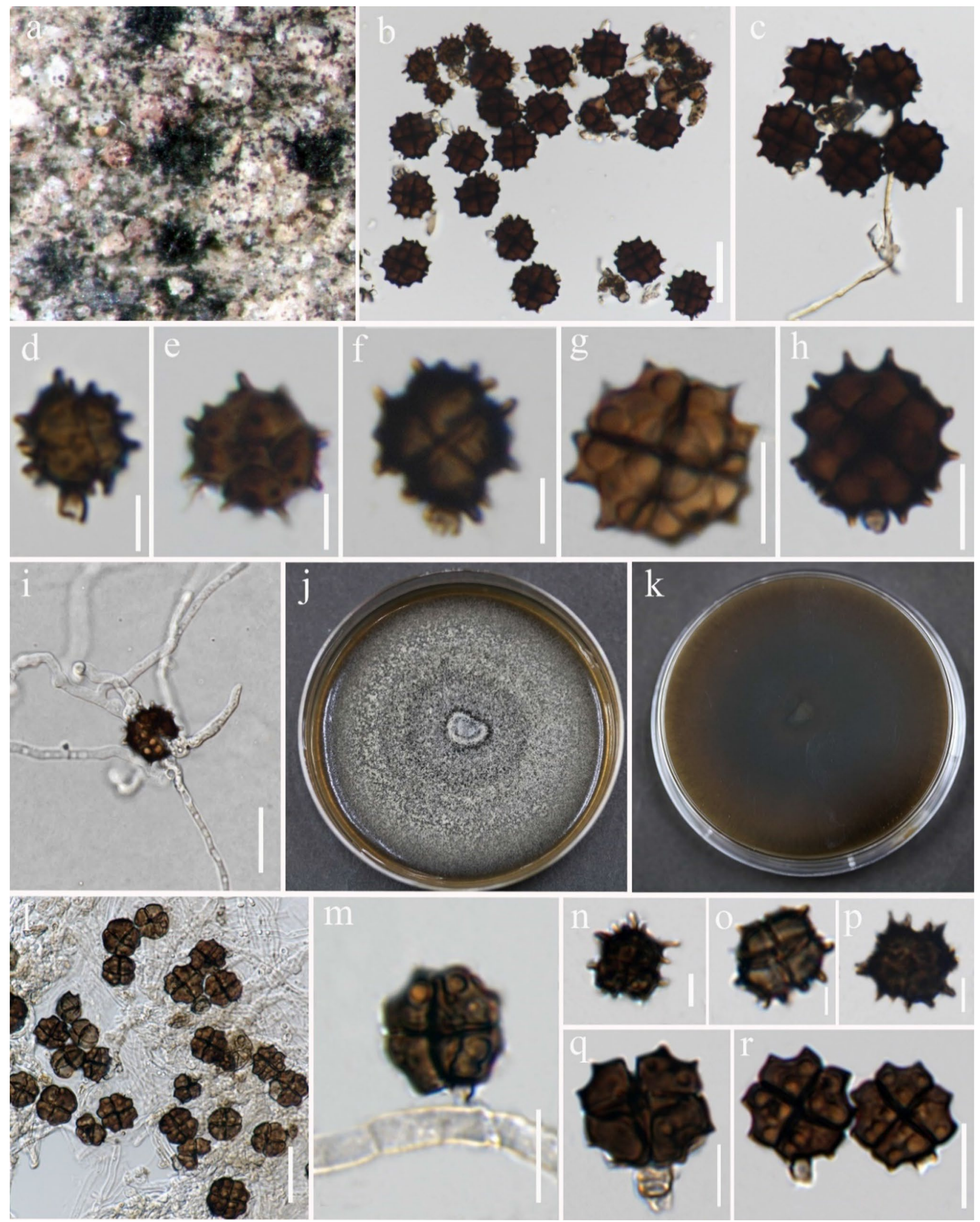

Fig. 28 Spegazzinia camelliae (MFLU 21-0053, new record). a Colonies on wood substrate. b, $\mathbf{c}$ Conidiophores and conidiogenous cells. d-h Conidia. i Germinated conidium. $\mathbf{j}$, $\mathbf{k}$ Culture on PDA from sur- face and reverse at 1 month. $\mathbf{l}-\mathbf{r}$ Conidia developing in culture. Scale bars: $\mathbf{b}-\mathbf{c}, \mathbf{i}, \mathbf{l}=20 \mu \mathrm{m}, \mathbf{d}-\mathbf{f}, \mathbf{n}-\mathbf{p}=5 \mu \mathrm{m}, \mathbf{g}-\mathbf{h}, \mathbf{m}, \mathbf{q}-\mathbf{r}=10 \mu \mathrm{m}$ 


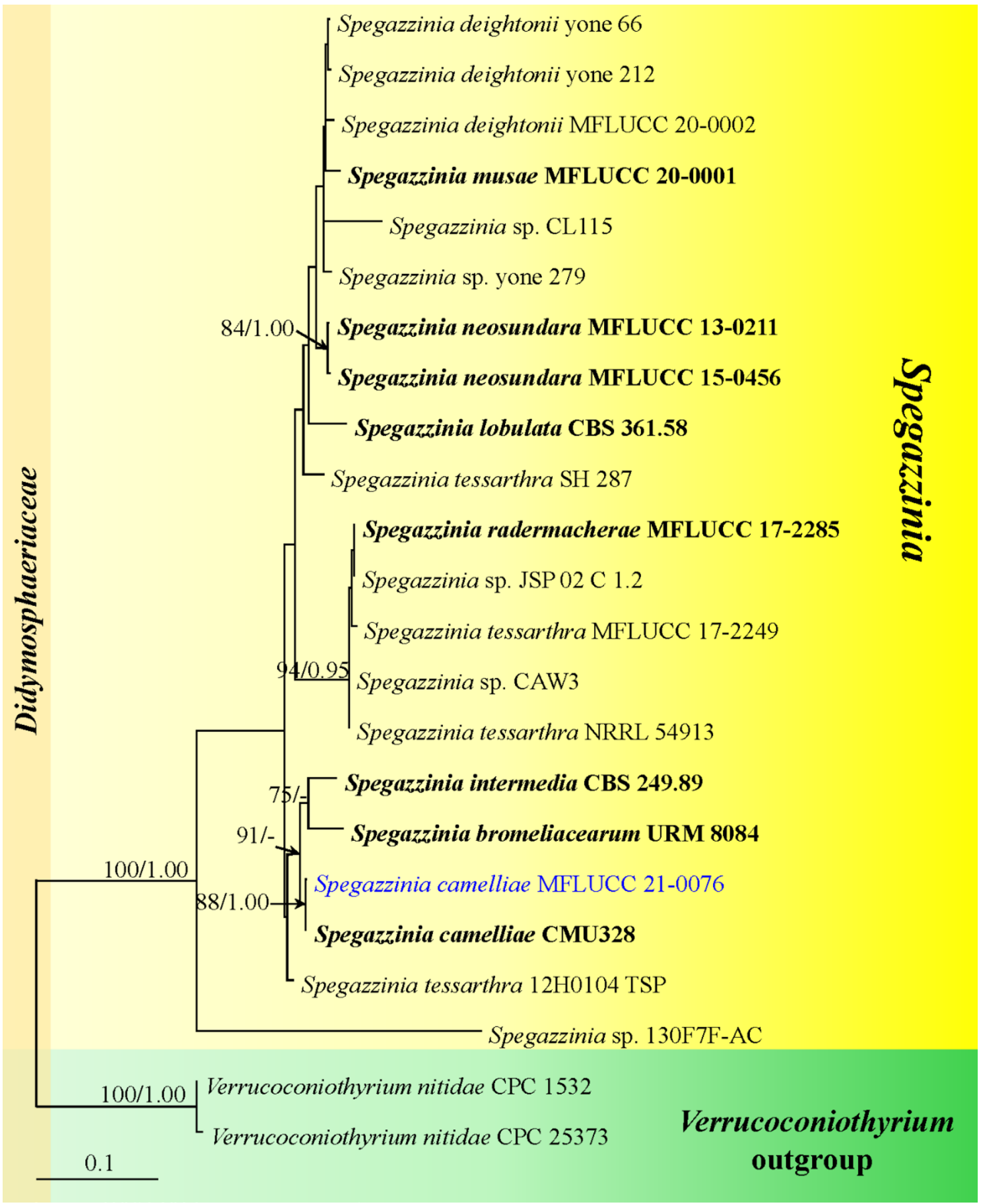

Fig. 29 Phylogram generated from maximum likelihood analysis based on combined LSU, ITS and TEF1- $\alpha$ sequence data of Spegazzinia taxa. Verrucoconiothyrium nitidae (CPC1532 and CPC25373) are selected as the outgroup taxa. Bootstrap support

the forward of culture, yellow-brown in the centre while white at the edge from the reverse of culture.

Material examined: CHINA, Guizhou Province, Zunyi, Chishui, Hushi, Chishui Alsophila Natural Reserve $\left(28^{\circ} 29^{\prime} 43^{\prime \prime} \mathrm{N} 106^{\circ} 0^{\prime} 24^{\prime \prime} \mathrm{E}\right)$, on dead rachis of Cyathea values for maximum likelihood (MLBS, left) equal to or greater than $70 \%$ is given above the nodes. Bayesian posterior probabilities (BYPP, right) equal to or greater than 0.95 are given above the nodes. Ex-type strains are in bold and newly generated sequence is in blue

spinulosa, 22 September 2019, J.Y. Zhang, C30 (MFLU 21-0025, new record), living culture, MFLUCC 21-0024.

GenBank numbers: ITS $=$ MZ198895, LSU $=$ MZ198897.

Notes: The phylogenetic analysis based on multigene phylogenetic analysis indicates that our new isolate 

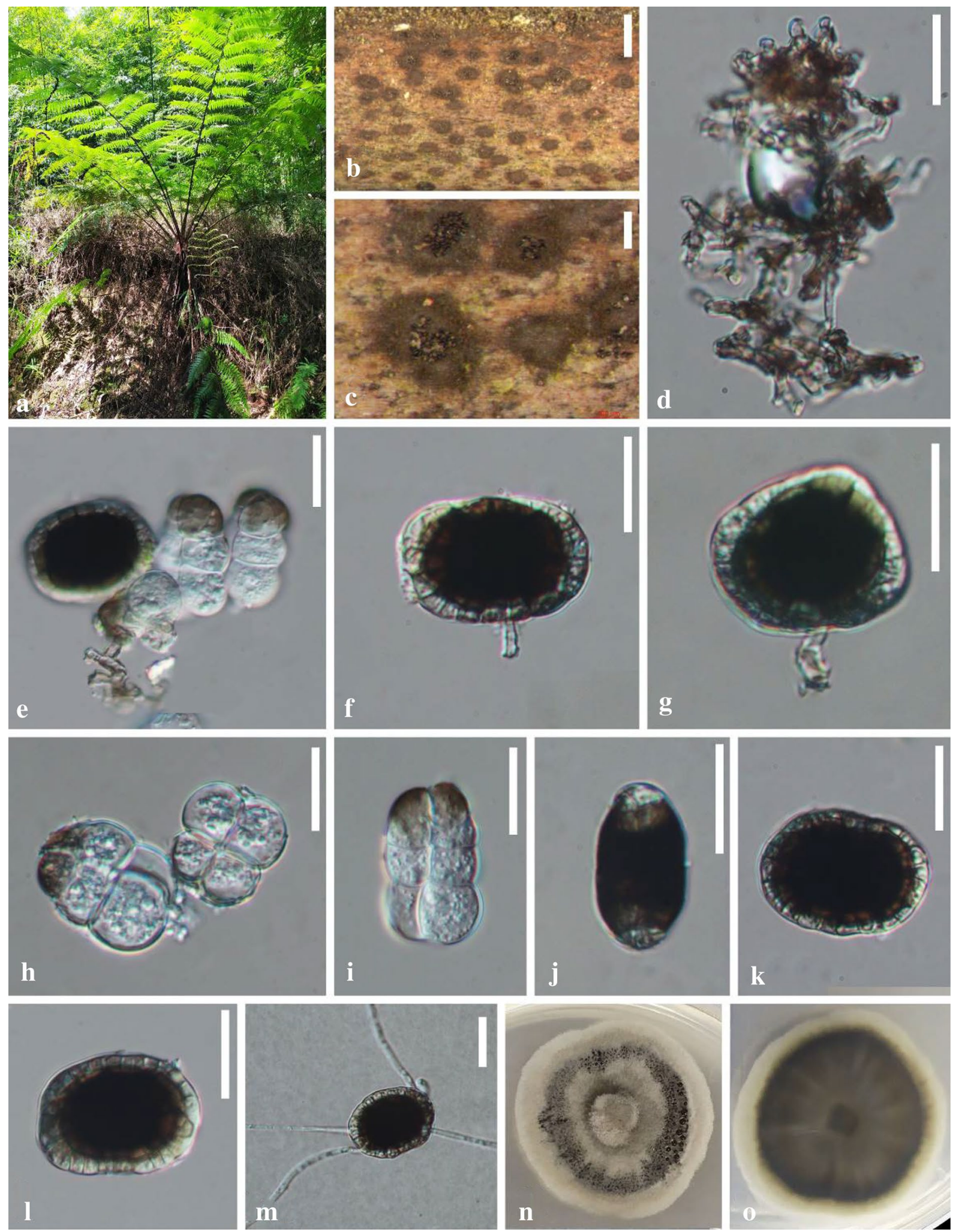

Fig. 30 Hermatomyces nabanheensis (MFLU 21-0025, new record). a Host. b, c Colonies on substrate. d Mycelium. e-g Conidia with conidiogenous cells. h, i Cylindrical conidia. j-l Lenticular conidia. $\mathbf{m}$ Germinated conidium. $\mathbf{n}, \mathbf{o}$ Culture on PDA from above and below. Scale bars: $\mathbf{b}=1000 \mu \mathrm{m}, \mathbf{c}=200 \mu \mathrm{m}, \mathbf{d}-\mathbf{m}=20 \mu \mathrm{m}$ 
clustered together with the ex-type strain Hermatomyces nabanheensis (KMUCC 16-0149) with 100\% MLBP, 1.00 BYPP support (Fig. 32). A comparison of base pair sequences between $H$. nabanheensis (KMUCC 16-0149; Hyde et al. 2017) and our isolate (MFLUCC 21-0024) showed that they differ by 3 bases in LSU and 5 bases in ITS. The new isolate is similar to H. nabanheensis (KMUCC 16-0149) in shape of colony, conidiophores, conidiogenous cells and conidia (Hyde et al. 2017; see Fig. 28). However, the new isolate differs from $H$. nabanheensis (HKAS96214) in having larger conidia (lenticular conidia $30-39 \times 20-28 \mu \mathrm{m}$ vs. $20.2-25 \times 16.6-20.7$; cylindrical conidia $30-37 \times 19.5-25 \mu \mathrm{m}$ vs. $15-26.8 \times 12.1-18.2$ ) (Fig. 30). Based on the evidence of phylogeny and morphological characteristics, we consider the new isolates conspecific to $H$. nabanheensis and this is the first record of $H$. nabanheensis from a fern (Cyathea spinulosa).

Hermatomyces sphaericoides Koukol \& G. Delgado, IMA Fungus 9(1): 122 (2018)

Index Fungorum number: IF824248; Facesoffungi number: FoF 09190; Fig. 31

Saprobic on decaying wood. Sexual morph Undetermined. Asexual morph Hyphomycetous. Colonies on the natural substrate superficial, dry, scattered to partly grouped, black. Mycelium 2-3 $\mu \mathrm{m}$ wide, superficial and partly immersed, composed of branched, septate, pale brown or brown, sparsely network hyphae. Conidiophores micronematous, mononematous, flexuous, inconspicuous, septate, hyaline to pale brown. Conidiogenous cells $4-6 \times 2-3 \mu \mathrm{m}$, holoblastic, monoblastic, integrated, terminal, determinate, cylindrical, pale brown. Conidia $25.5-32 \times 21-27.5 \mu \mathrm{m}$ $(\bar{x}=28.5 \times 25 \mu \mathrm{m}, \mathrm{n}=20)$, solitary, acrogenous, cheiroid, globose to subglobose, inwardly curved at the apex, arising from a basal cell, consisting 4-5 rows, row digitate, each row composed of 4-5(-6) cells, multi-septate, constricted at the septa, dark brown to black, smooth-walled.

Culture characteristics: Conidium germinating on PDA within $24 \mathrm{~h}$ and germ tubes arising from terminal end of conidium. Colonies on PDA, fast growing, reaching $30 \mathrm{~mm}$ diam. after 2 weeks at room temperature, flat, with slightly crenated to radially striated with lobate margin, with white-grey mycelium, pastel grey in middle towards dark brown, greenish brown at margin, dark green to brown in reverse view, not sporulating in cultures after 2 months.

Material examined: THAILAND, Phetchabun Province, Lom Sak, on decaying wood, 25 July 2019, S. Boonmee, LSP01 (MFLU 21-0054, new record), living culture, MFLUCC 21-0077.

GenBank numbers: ITS $=$ MZ538505, LSU $=$ MZ538539, TEF1- $\alpha=$ MZ567083.

Notes: Hermatomyces sphaericoides was described on dry rotten twig of an unidentified tree in Panama and shares similar conidial features with $H$. sphaericus, but it differs in conidial characteristic details in having dark brown to blackish brown and finely verruculose with an outer ring of peripheral cells (Koukol et al. 2018). Furthermore, phylogenetic analyses indicate that $H$. sphaericoides and $H$. sphaericus are distinct species. Our strain MFLUCC 21-0077 shows some similarities with $H$. sphaericoides in terms of conidial characteristics (Koukol et al. 2018). Multi-gene phylogenetic analysis of a combined ITS, LSU and TEF1- $\alpha$ dataset indicated that our isolate MFLUCC 21-0077 clustered together with $H$. sphaericoides isolates (CCF 5896, CCF 5907, CCF 5908 (ex-type) and KZP 470) with 100\% MLBS, 1.00 BYPP support (Fig. 32), whereas CCF 5895 forms a basal lineage. Therefore, we identify our collection as $H$. sphaericoides (Fig. 31) and it is reported here as a new geographical record from Thailand.

Lentitheciaceae Y. Zhang ter, C.L. Schoch, J. Fourn., Crous \& K.D. Hyde

Notes: We follow the latest treatments and the updated accounts of Lentitheciaceae in Hongsanan et al. (2020a). Based on phylogenetic analysis of a combined LSU, TEF1- $\alpha$, SSU and ITS sequences, the isolate is identified as Poaceascoma taiwanense and is a new geographical record from Thailand.

\section{Poaceascoma Phookamsak \& K.D. Hyde}

Notes: Poaceascoma was introduced by Phookamsak et al. (2015) to accommodate a taxon occurring on dead stems and roots of Digitaria sanguinalis (Poaceae) host in a terrestrial habitat. Poaceascoma is typified by P. helicoides Phookamsak \& K.D. Hyde and is characterized by semi-immersed to erumpent, sphaerical, short to long papilla, turf-like surrounded ascomata; bitunicate, fissitunicate, cylindrical asci and fasiculate, sometime spiral, filiform, multi-septate, hyaline ascospores (Phookamsak et al. 2015). Five species have been accepted and confirmed for Poaceascoma by phylogenetic evidence (Crous et al. 2020b; Hongsanan et al. 2020a; Index Fungorum 2021).

Poaceascoma taiwanense C.H. Kuo \& K.D. Hyde, Mycosphere 9(2): 297 (2018)

Index Fungorum number: IF554200; Facesoffungi number: FoF 04084; Fig. 33

Saprobic on submerged wood. Sexual morph Ascomata 117-200 $\mu \mathrm{m}$ high, 198-209 $\mu \mathrm{m}$ diam., immersed to erumpent when mature, solitary, scattered or sometimes grouped beneath the host tissues, globose, dark brown to black, coriaceous, ostiolate, papillate. Peridium 22-31 $\mu \mathrm{m}$ wide, with thick walls, composed of several layers of pseudoparenchymatous cells, outer layer comprising several layers of dark brown cells, arranged in a textura angularis, inner layer comprising several layers of 

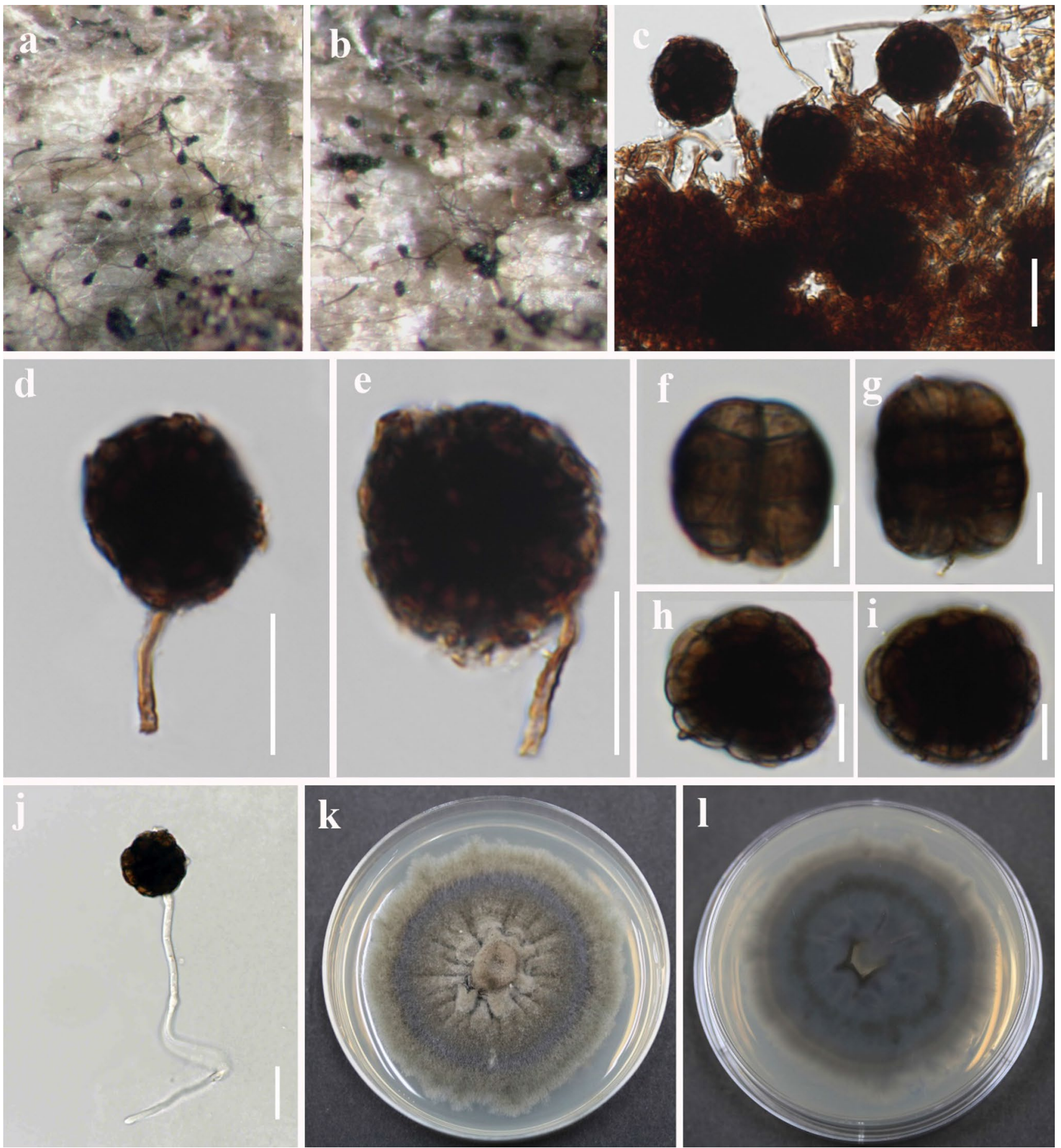

Fig. 31 Hermatomyces sphaericoides (MFLU 21-0054, new record). a, b Colonies on the natural substrate. c-e Conidiogenous cells. f-i Conidia. $\mathbf{j}$ Germinated conidium. $\mathbf{k}$, I Culture on PDA from surface and reverse at 1 month. Scale bars: $\mathbf{c}-\mathbf{e}, \mathbf{j}=20 \mu \mathrm{m}, \mathbf{f}-\mathbf{i}=10 \mu \mathrm{m}$

hyaline, flattened cells, arranged in a textura angularis to textura prismatica. Hamathecium composed of numerous, 1.5-2 $\mu \mathrm{m}$ wide, filamentous, broad, cellular pseudoparaphyses, with distinct septa, embedded in a mucilaginous matrix. Asci $63-77 \times 8.5-10 \mu \mathrm{m}(\bar{x}=70 \times 9.5 \mu \mathrm{m}, \mathrm{n}=10)$, 8 -spored, bitunicate, cylindrical clavate, short pedicellate, apically rounded with minute ocular chamber. Ascospores $42-54 \times 2.5-3 \mu \mathrm{m}(\bar{x}=49 \times 3 \mu \mathrm{m}, \mathrm{n}=10)$, fasciculate, scolecosporous, hyaline, elongate, cylindrical filiform, tapering towards the rounded ends, slightly curved, 6-7-septate, slightly constricted at the septa, smooth-walled. Asexual morph Undetermined. 


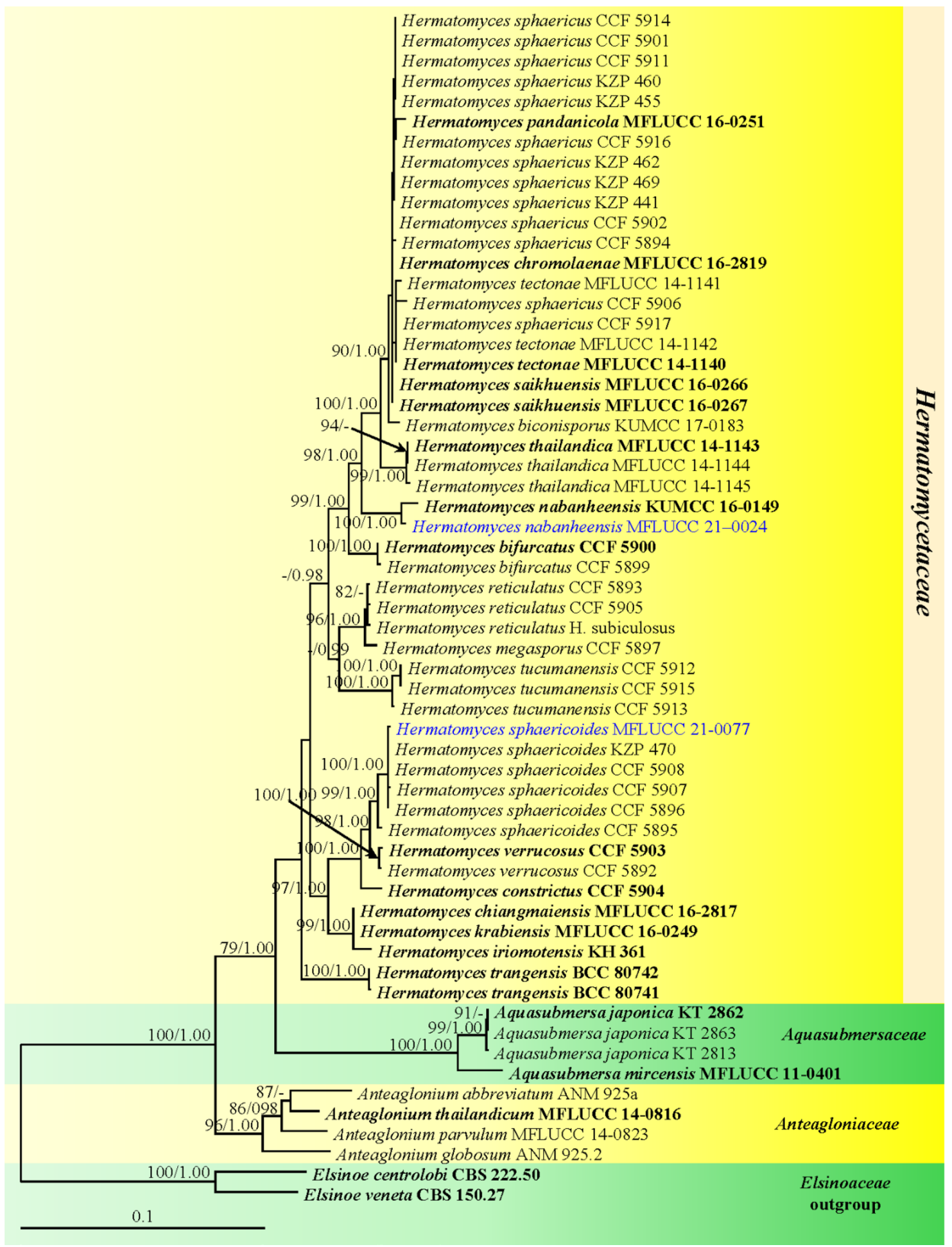

Fig. 32 Phylogram generated from maximum likelihood analysis based on combined ITS, LSU and TEF1- $\alpha$ sequence data of Hermatomyces taxa (Hermatomycetaceae) and closely related families Aquasubmersaceae and Anteagloniaceae. Elsinoe centrolobi CBS 222.50 and E. veneta CBS 150.27 are selected as the outgroup taxa.
Bootstrap support values for maximum likelihood (MLBS, left) equal to or greater than $70 \%$ is given above or below the nodes. Bayesian posterior probabilities (BYPP, right) equal to or greater than 0.95 are given above the nodes. Ex-type strains are in bold and newly generated sequences are in blue 

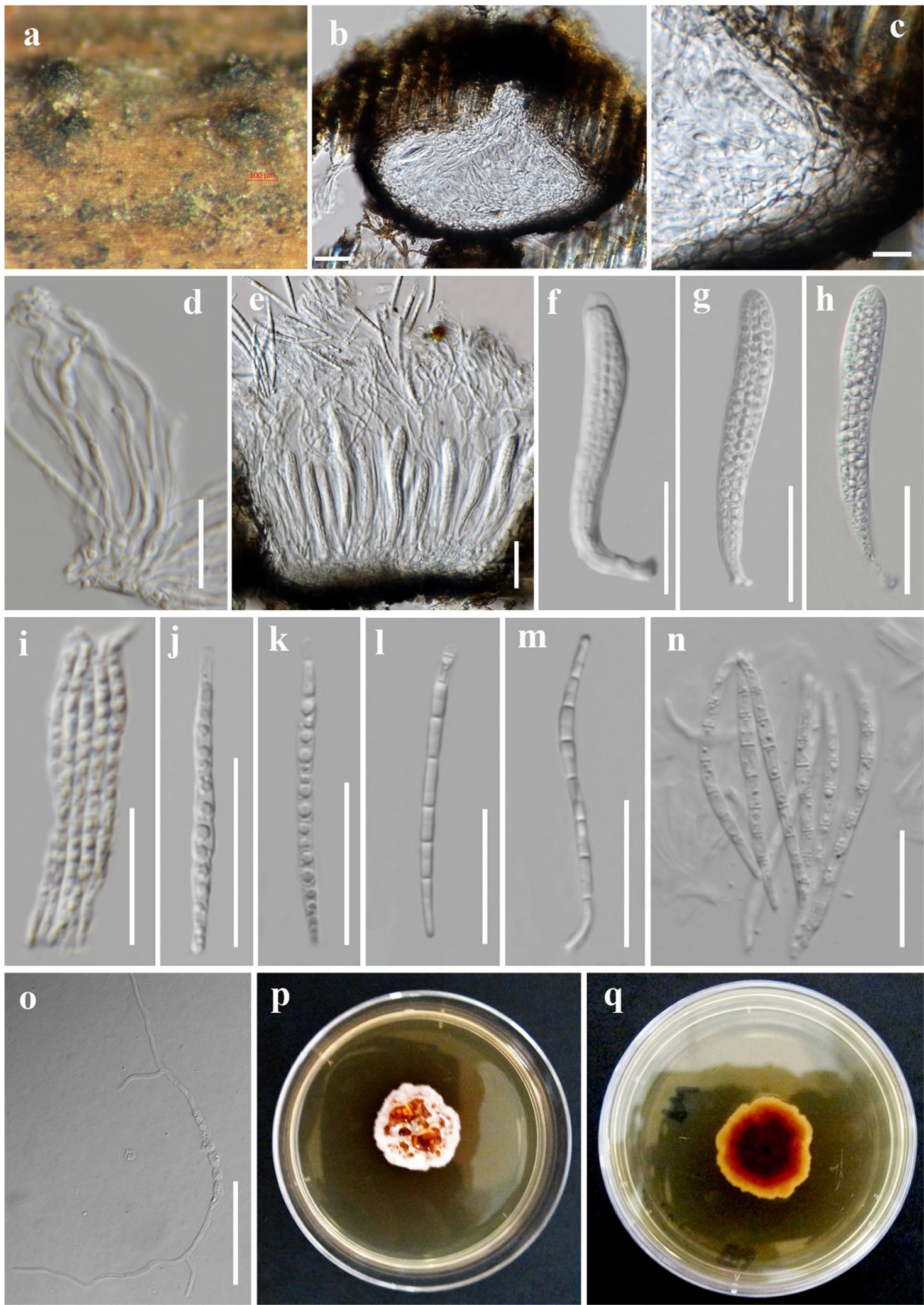

Fig. 33 Poaceascoma taiwanense (MFLU 21-0055, new record). a Ascomata immersed, aggregated, erumpent on the dead wood. b Longitudinal sections of ascomata. c Peridium. d Pseudoparaphyses. e-h Asci. i-n Ascospores. o Germinated ascospore. p, q Culture on MEA from surface and reverse at 1 month. Scale bars: $\mathbf{b}=50 \mu \mathrm{m}$, $\mathbf{c}-\mathbf{n}=20 \mu \mathrm{m}, \mathbf{o}=30 \mu \mathrm{m}$ 
Culture characteristics: Ascospores germinating on PDA within $24 \mathrm{~h}$ and germ tubes arising from both ends. Colonies on MEA reaching $20 \mathrm{~mm}$ after 1 month at room temperature, slightly effuse, dense, radially with undulate edge, surface white with forming the water drops on the mycelium, with orangish pigmented at the surface, reverse dark purple at the middle, radiating dark pink to yellowish orange towards the edge.

Material examined: THAILAND, Phayao Province, Pong, on submerged wood in a small freshwater stream, 26 March 2019, S. Boonmee, PY1 (MFLU 21-0055, new record), living culture, MFLUCC 21-0078.

GenBank numbers: ITS $=$ MZ538523, LSU $=$ MZ538557, TEF1- $\alpha=$ MZ567099.

Notes: Multigene phylogenetic analysis indicates that our strain MFLUCC 21-0078 is sister to the type strain P. taiwanense (MFLU 18-0083) with high levels of support (100\% MLBS, 1.00 BYPP (Fig. 34). A comparison of LSU and ITS nucleotide base pairs indicates that our strain is highly to $P$. taiwanense (3/842 bp differences $(0.35 \%)$ in LSU and $5 / 564$ bp differences $(0.89 \%)$ in ITS). Therefore, we identify our strain MFLUCC 21-0078 as P. taiwanense (Fig. 33). Our strain shares similar characteristics of the ascomata, asci and ascospores to P. taiwanense (MFLU 18-0083). Although, our strain differs in having 8-spored in ascus and 6-7-septate ascospores, while P. taiwanense (MFLU 18-0083) having 4-spored in ascus and 15-17-septate. However, LSU and ITS sequences indicated that they are conspecific (Hyde et al. 2018a; see Fig. 14). Therefore, P. taiwanense is reported from submerged wood in a freshwater habitat for the first time in Thailand. If we used protein coding genes, they may however prove to be distinctive species, as showed by the morphological evidence.

Lindgomycetaceae K. Hiray., Kaz. Tanaka \& Shearer

Notes: Lindgomycetaceae includes seven genera, Arundellina, Clohesyomyces, Hongkongmyces, Lindgomassariosphaeria, Lindgomyces (generic type), Lolia, Massariosphaeria and Neolindgomyces (Dong et al. 2020b; Wijayawardene et al. 2020). Most taxa are saprobes on submerged decaying wood in a freshwater habitat and some species (e.g. Hongkongmyces pedis have been found to cause human diseases (Tsang et al. 2014). We follow the latest treatment of this family in Dong et al. (2020b). An updated phylogenetic tree for Lindgomycetaceae is presented in Fig. 37. In this study, a new genus Xenovaginatispora with $X$. phichaiensis as a type species and a new species in Hongkongmyces are introduced.

Hongkongmyces C.C.C. Tsang et al.

Notes: Tsang et al. (2014) introduced Hongkongmyces which was isolated from biopsy tissues of an infected foot, resulting in the establishment of Lindgomycetaceae.
Currently, Hongkongmyces comprises sexual and asexual morphs of four species which are namely Hongkongmyces aquaticus, $H$. pedis, $H$. snookiorum and $H$. thailandica (Tsang et al. 2014; Hyde et al. 2017; Crous et al. 2018; Dong et al. 2020b). The new species of Hongkongmyces, H. kokensis is introduced here with morphology and phylogeny evidences.

Hongkongmyces kokensis Boonmee, Chandrasiri, Huanraluek \& K.D. Hyde, sp. nov.

Index Fungorum number: IF558535; Facesoffungi number: FoF 09946; Fig. 35

Etymology: The specific epithet "kokensis" refers to the Kok river.

\section{Holotype: MFLU 21-0056}

Saprobic on submerged decaying wood in the river. Sexual morph Undetermined. Asexual morph Coelomycetous. Conidiomata 261-347 $\mu \mathrm{m}$ high, 264-417 $\mu \mathrm{m}$ diam., pycnidial, scattered, semi-immersed or erumpent, subglobose or ellipsoidal, dark brown to black, coriaceous, ostiolate. Conidiomata wall 13-38 $\mu \mathrm{m}$ thick, composed of several layers of dark brown cells of textura angularis, difficult to distinguish from conidiogenous cells. Conidiophores reduced to conidiogenous cells. Conidiogenous cells $7-21 \times 3-12 \mu \mathrm{m}$ $(x=12 \times 6 \mu \mathrm{m}, \mathrm{n}=10)$, phialidic, determinate, cylindrical to subcylindrical, hyaline, thin-walled. Conidia $10-15 \times 8-14 \mu \mathrm{m}(\bar{x}=12 \times 10 \mu \mathrm{m}, \mathrm{n}=20)$, subglobose to obovoid, hyaline, aseptate, large guttule present when immature, lacking a mucilaginous sheath, smooth-walled.

Culture characteristics: Conidia germinated on PDA within $24 \mathrm{~h}$. Colonies on PDA reaching $3.5 \mathrm{~cm}$ diam. after 3 weeks at room temperature, circular, entire to slightly undulate edge, raised effuse, olivaceous brown to brown from the center towards margin, surrounded by light brown at the margin, dark brown at the reverse with surrounded by light brown at the margin, superficial and velvety mycelia.

Material examined: THAILAND, Chiang Rai Province, Muang, Mae Yao, Kok river, on submerged decaying wood in the river shore, 4 December 2019, S. Boonmee and K.S.U. Chandrasiri, N-KR4 (MFLU 21-0056, holotype), ex-type living culture, MFLUCC 21-0079; Chiang Rai Province, Wiang Pa Pao, Lao River, on submerged decaying wood in the river shore, 11 December 2019, S. Boonmee and K.S.U. Chandrasiri, LR4 (MFLU 21-0057), living culture, MFLUCC 21-0080; Phrae, Pong, Yom River, on submerged decaying wood in the river shore, 18 December 2019, S. Boonmee and K.S.U. Chandrasiri, YR21 (MFLU 21-0058), living culture, MFLUCC 21-0081.

GenBank number: LR4: ITS = MZ538507, LSU $=$ MZ538541, TEF $1-\alpha=$ MZ567085; N-KR4: ITS $=$ MZ538506, LSU $=$ MZ538540, TEF1 $-\alpha=$ MZ567084; YR21: $\quad$ ITS $=$ MZ538508, $\quad$ LSU $=$ MZ538542, TEF1- $\alpha=$ MZ567086. 


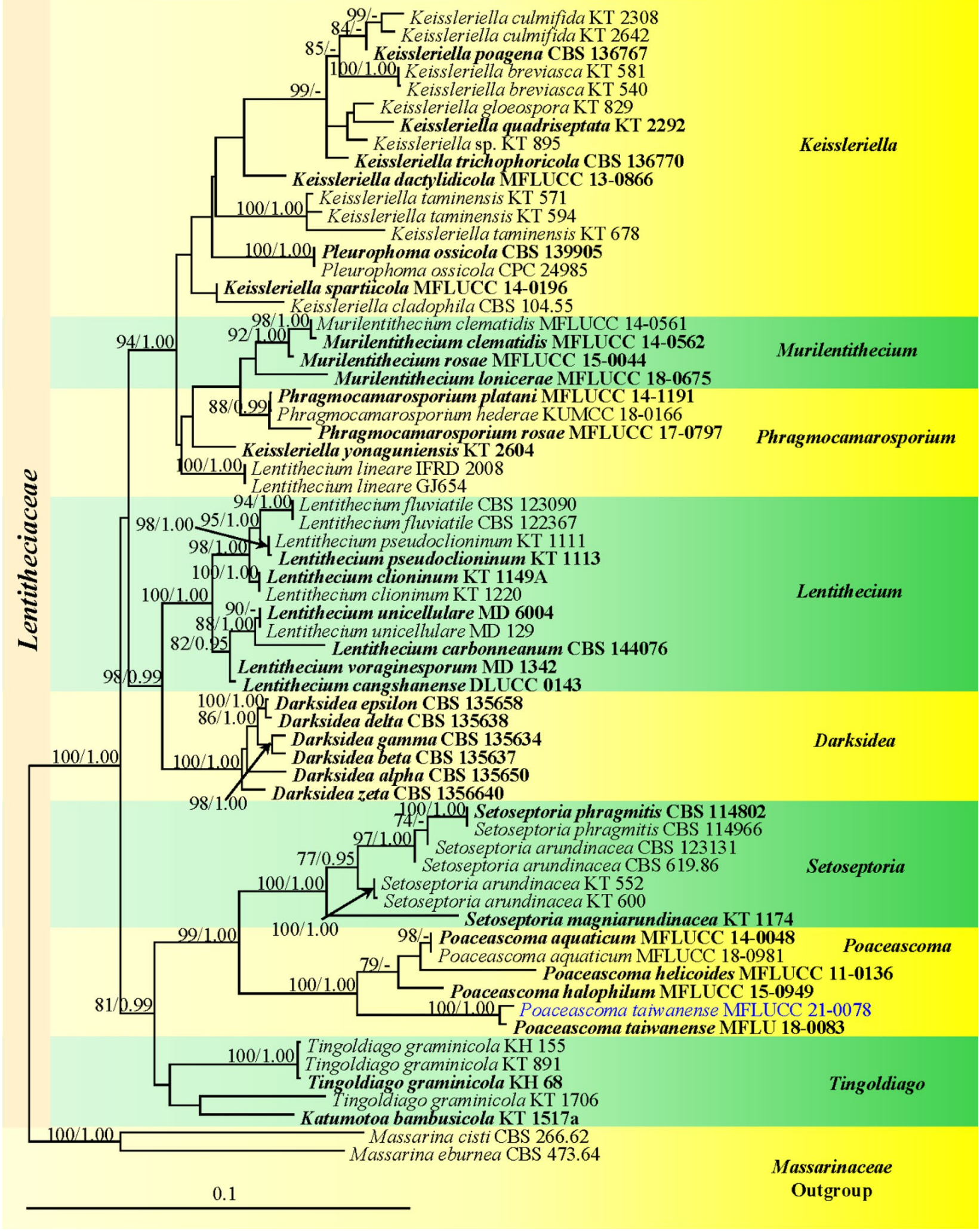

Fig. 34 Phylogram generated from maximum likelihood analysis based on combined LSU, TEF1- $\alpha$, SSU and ITS sequence data of Poaceascoma taxa and related genera in Lentitheciaceae. Massarina cisti CBS 266.62 and M. eburnea CBS 473.64 are selected as the outgroup taxa. Bootstrap support values for maximum likelihood
(MLBS, left) equal to or greater than $70 \%$ is given above the nodes. Bayesian posterior probabilities (BYPP, right) equal to or greater than 0.95 are given above the nodes. Ex-type strains are in bold and newly generated sequence is in blue 

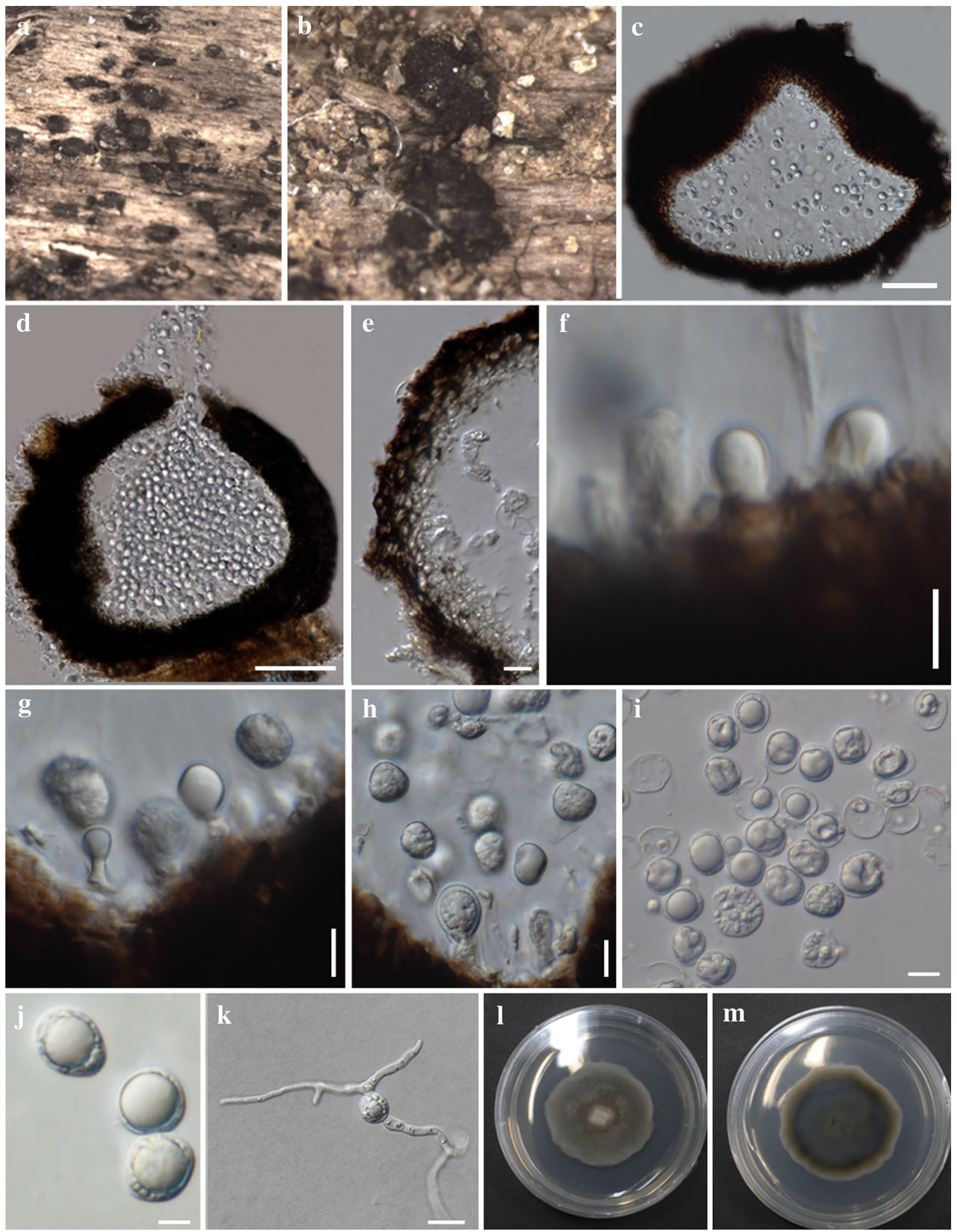

Fig. 35 Hongkongmyces kokensis (MFLU 21-0056, holotype). a, b Appearance of conidiomata on submerged decaying wood substrate. c, d Section through conidiomata. e Section of conidioma wall. $\mathbf{f}-\mathbf{h}$
Conidiogenous cells and conidia. i, $\mathbf{j}$ Conidia. k Germinated conidium. $\mathbf{l}, \mathbf{m}$ Culture on PDA ( $1=$ from above, $m=$ from below). Scale bars: $\mathbf{c}=50 \mu \mathrm{m}, \mathbf{d}=200 \mu \mathrm{m}, \mathbf{e}, \mathbf{g}, \mathbf{h}=20 \mu \mathrm{m}, \mathbf{f}, \mathbf{i}, \mathbf{k}=10 \mu \mathrm{m}, \mathbf{j}=5 \mu \mathrm{m}$ 
Notes: Multigene phylogenetic analysis of a combined LSU, ITS and TEF1- $\alpha$ sequence dataset indicated that Hongkongmyces kokensis forms a sister clade with the sexual species $H$. thailandica with 97\% MLBS and 1.00 BYPP support (Fig. 37). A comparison of LSU and ITS base pair shows that $H$. kokensis differs from $H$. thailandica with by 14/788 bp (1.77\%) of LSU and 86/994 bp (8.65\%) with 21 gaps of ITS. Furthermore, the morphological characters of $H$. kokensis and $H$. thailandica could not be directly compared as they represent as different morphs (Hyde et al. 2017). Hongkongmyces kokensis also shares some morphological features of conidiomata and conidia with $H$. aquaticus (Dong et al. 2020b), but they differ in shape and size of conidiomata and conidia (Fig. 35). However, phylogenetic analysis indicates that $H$. kokensis is a distinct species from $H$. aquaticus and $H$. thailandica. We therefore, introduce Hongkongmyces kokensis as a new species following the guidelines of Jeewon and Hyde (2016). The addition of protein coding gene data would likely strengthen the distinction of the new taxa.

Xenovaginatispora Boonmee, Huanraluek \& K.D. Hyde, gen. nov.

Index Fungorum number: IF558536; Facesoffungi number: FoF 09188.

Etymology: Named after its morphological similarity to Vaginatispora, but phylogenetically distinct.

Saprobic on submerged decaying wood. Sexual morph Ascomata immersed to semi-immersed, erumpent, solitary, scattered, globose to subglobose, dark brown, coriaceous to carbonaceous, ostiolate. Papilla slit-like, variable in shape, central, crest-like at the apex, with irregular pore-like opening. Peridium composed of several layers of dark brown cells of textura angularis, merged with the host tissues. Hamathecium comprising numerous, filamentous, rarely branched and anastomosed, hyaline, pseudoparaphyses, embedded in a gelatinous matrix. Asci 8 -spored, bitunicate, elongate ovoid to clavate, short pedicellate, knob-like or obtuse, slightly narrow, rounded at the apex. Ascospores 1-2-seriate, overlapping, broadly fusoid to fusiform, septate, slightly constricted at the septum, both cells at central septum wider, with a single large guttule towards the centre of each cell, surrounded by a prominent and thick-walled mucilaginous sheath with wavy edge, drawn out towards each end to forming tapering appendages. Asexual morph Undetermined.

Type species: Xenovaginatispora phichaiensis Boonmee, Huanraluek \& K.D. Hyde

Notes: Xenovaginatispora shares similarities with Vaginatispora taxa in having immersed to semi-immersed, erumpent, slit-like openings in the ascomata and broadly fusoid to fusiform, 1-septate, guttulate, surrounded by a prominent mucilaginous sheath, hyaline ascospores, which are variable in shape (Thambugala et al. 2015; Wanasinghe et al. 2016; Hashimoto et al. 2018; Hyde et al. 2019; Jayasiri et al. 2019). Phylogenetically, Xenovaginatispora belongs to Lindgomycetaceae, whereas Vaginatispora belongs to Lophiostomataceae (Thambugala et al. 2015). Xenovaginatispora forms a basal lineage to Neolindgomyces with high statistical support (93\% MLBS, 1.00 BYPP). Based on morphology and phylogenetic distinctions, we introduce our collection as a new genus with the monotypic species, Xenovaginatispora phichaiensis.

Xenovaginatispora phichaiensis Boonmee, Huanraluek \& K.D. Hyde, sp. nov.

Index Fungorum number: IF558537; Facesoffungi number: FoF 09191; Fig. 36

Etymology: The specific epithet "phichaiensis" refers to the Phichai District, Uttaradit Province, Thailand.

Holotype: MFLU 21-0059

Saprobic on submerged decaying wood in a freshwater stream. Sexual morph Ascomata 151-225 $\mu \mathrm{m}$ high, 187-211 $\mu \mathrm{m}$ diam., immersed to semi-immersed, erumpent, solitary, scattered, globose to subglobose, dark brown, coriaceous to carbonaceous, ostiolate. Papilla slit-like, variable in shape, central papilla, coriaceous, crest-like at the apex, with an irregular pore-like opening. Peridium 27-38 $\mu \mathrm{m}$ wide, composed of several layers of dark brown cells of textura angularis, merged with the host tissues. Hamathecium comprising numerous, 1.5-3.5 $\mu \mathrm{m}$ wide, filamentous, rarely branched and anastomosed, hyaline, pseudoparaphyses, embedded in a gelatinous matrix. Asci 98-115.5 ×22-28 $\mu \mathrm{m}(\bar{x}=107 \times 24 \mu \mathrm{m}, \mathrm{n}=15), 8$-spored, bitunicate, elongate ovoid to clavate, short pedicellate, with knob-like or obtuse, slightly narrow to rounded at the apex. Ascospores $35-42 \times 10-13 \mu \mathrm{m}(\bar{x}=39 \times 12 \mu \mathrm{m}, \mathrm{n}=10)$, overlapping 1-2-seriate, broadly fusoid to fusiform, 1 -septate, slightly constricted at the septum, both cells at central septum wider, with a single large guttule, $8-12 \mu \mathrm{m}$ diam., towards the centre of each cell, surrounded by a prominent and thick-walled mucilaginous sheath with wavy edge, drawn out towards each end to form tapering appendages.

Asexual morph Undetermined.

Culture characteristics: Ascospores germinating on PDA within $24 \mathrm{~h}$ and germ tubes arising from both ends. Colonies on MEA, slow growing, reaching $3 \mathrm{~cm}$ diam. after 1 month of incubation at room temperature, initially dull white to light grey becoming dark grey at maturity, slightly effuse, radially with an undulate edge, greyish, light brown with brown pigmented in media, no sporulating in culture after 60 days.

Material examined: THAILAND, Uttaradit Province, Phichai, Thasak, Chom Tok Village, on submerged decaying wood in a small freshwater stream, 29 December 2018, 

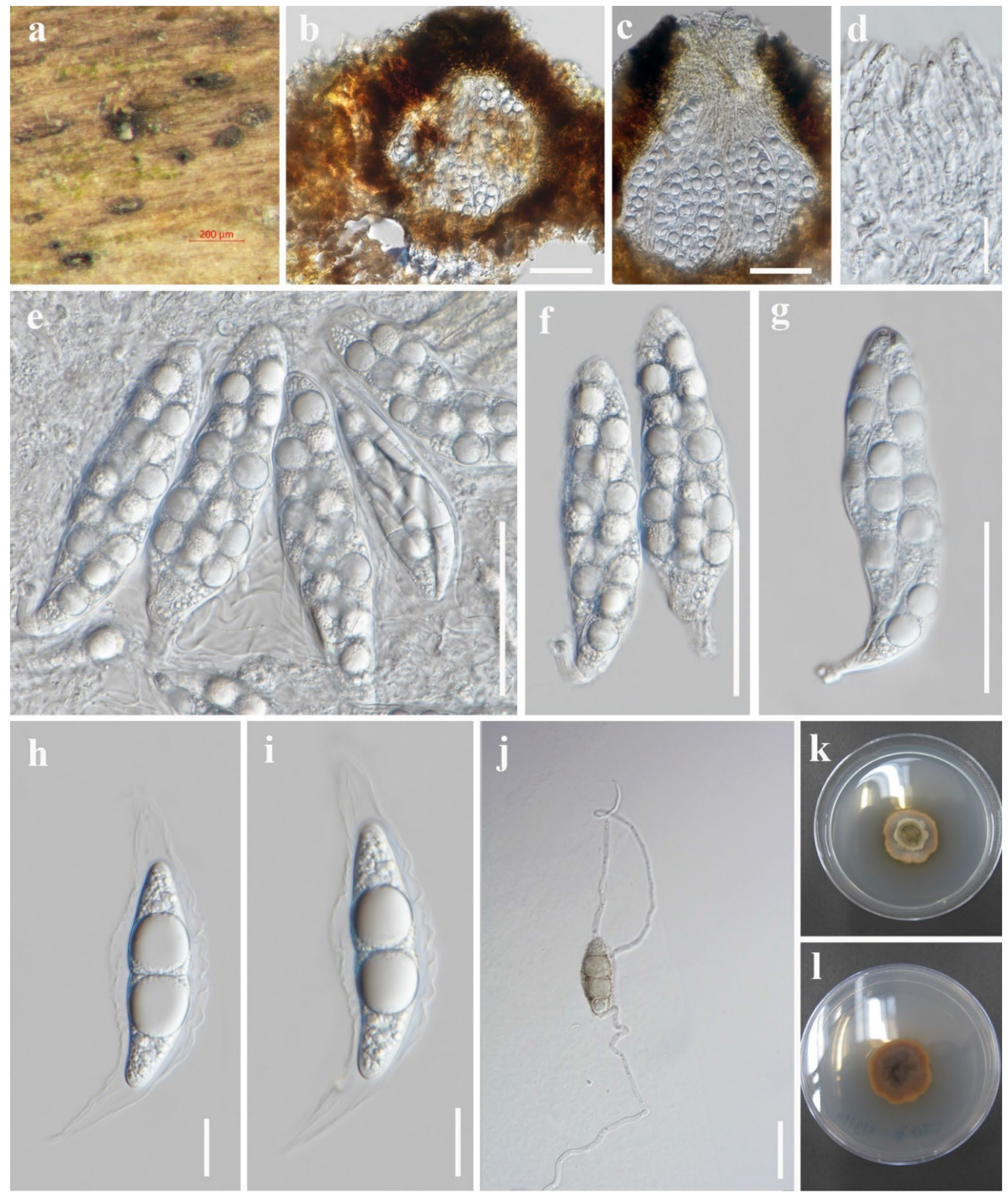

Fig. 36 Xenovaginatispora phichaiensis (MFLU 21-0059, holotype). a Appearance of ascomata. b Longitudinal sections of ascomata. c Ascomata with ostiole. d Pseudoparaphyses. e-g Asci. h, i Ascospores surrounded by mucilaginous sheath and distinct api- cal appendages. $\mathbf{j}$ Germinated ascospore. $\mathbf{k}, \mathbf{l}$ Culture on MEA at 1 month $(\mathbf{k}=$ from surface and $1=$ reverse). Scale bars: $\mathbf{b}=100 \mu \mathrm{m}, \mathbf{c}$, $\mathbf{d}=50 \mu \mathrm{m}, \mathbf{e}-\mathbf{g}, \mathbf{j}=20 \mu \mathrm{m}, \mathbf{h}-\mathbf{i}=10 \mu \mathrm{m}$ 
S. Boonmee, CTU8 (MFLU 21-0059, holotype), ex-type living culture, MFLUCC 21-0082.

GenBank numbers: ITS $=$ MZ538534, LSU $=$ MZ538569, SSU $=$ MZ538577, TEF1- $\alpha=$ MZ567110.

Notes: In the NCBI BLASTn search of LSU and SSU sequences, Xenovaginatispora phichaiensis MFLUCC 21-0082 is most similar to an undescribed taxon Pleosporales $\mathrm{sp}$. (F65-1) with $99.19 \%$ (LSU) and $99.70 \%$ (SSU) similarity. Unfortunately, we cannot compare the morphology in Shearer et al. (2009) as it lacks a description. Multigene phylogenetic analysis indicates that Xenovaginatispora phichaiensis clusters with the undescribed Pleosporales sp. (F65-1) and both of them form a subclade basal to Neolindgomyces species (Lindgomycetaceae) with 93\% MLBS, 1.00 BYPP support (Fig. 37). Xenovaginatispora phichaiensis differs from Neolindgomyces species (Jayasiri et al. 2019) in having characteristics such as elongate ovoid to clavate asci and broadly fusoid to fusiform, only 1-septate ascospores, with two distinct large guttules in both cells. Therefore, we introduce $X$. phichaiensis as a new species based on morphology and phylogenetic evidences (Figs. 36 and 37).

\section{Longipedicellataceae Phukhams., Bhat \& K.D. Hyde}

Notes: Longipedicellataceae was introduced to accommodate two genera Longipedicellata and Pseudoxylomyces, and the members of this family can be found in freshwater habitats (Phukhamsakda et al 2016). Recently, Dong et al. (2020b) included a new genus Submersispora in Longipedicellataceae, thus three genera are accepted in this family.

\section{Longipedicellata H. Zhang, K.D. Hyde \& Jian K. Liu}

Notes: Since Longipedicellata was introduced by Zhang et al. (2016a), there are only two species included i.e., Longipedicellata aptrootii and L. aquatica (Dong et al. 2020b). We follow the latest treatment and the updated accounts of Longipedicellata in Dong et al. (2020b). Based on morphological uniqueness and phylogenetic evidence, a new species, Longipedicellata fusiformispora is introduced and three new isolates of $L$. aquatica are reported.

Longipedicellata aquatica W. Dong, H. Zhang \& K.D. Hyde, in Dong et al., Fungal Diversity 105: 435 (2020)

Index Fungorum number: IF557915; Facesoffungi number: FoF 09257; Fig. 38

Saprobic on submerged decaying wood in the river. Sexual morph Ascomata 138-278 $\mu \mathrm{m}$ high, 148-276 $\mu \mathrm{m}$ diam., scattered, gregarious, immersed to, erumpent through the host surface, uni-loculate, sometimes bi-loculate, subglobose, dark brown to black, ostiolate. Peridium 15-25 $\mu \mathrm{m}$ thick, composed of several layers of brown to black cells of textura angularis. Hamathecium composed of sparse, anastomosed pseudoparaphyses, indistinctly septate. Asci $53-84 \times 17-32 \mu \mathrm{m}(\bar{x}=68 \times 23 \mu \mathrm{m}, \mathrm{n}=20)$,
8 -spored, bitunicate, broadly clavate to spatulate, thickness and rounded at the apex, with a minute ocular chamber, short to long pedicellate. Ascospores 20-25 $\times 7-10 \mu \mathrm{m}$ ( $\bar{x}=22 \times 8 \mu \mathrm{m}, \mathrm{n}=20$ ), 2-3-seriate overlapping, broadly fusiform to ellipsoidal, rounded at both ends, asymmetrical, hyaline, 1-septate, constricted at the septum, the upper cell slightly broader and shorter than the lower cell, with large distinctive guttule in each cell when immature, surrounded by thickened mucilaginous sheath, smooth-walled. Asexual morph: Undetermined.

Culture characteristics: Ascospores germinating on MEA within $12 \mathrm{~h}$ at room temperature. Colony reaching $3 \mathrm{~cm}$ diam., dense, entire to slightly undulate edge, raised, dark grey, fluffy with aerial mycelium, with a lobate at the centre from above, darkened from reverse.

Material examined: THAILAND, Chiang Mai, Mae Ai, Tha Ton, Kok River, on submerged decaying wood in the river shore, 9 December 2019, S. Boonmee and N. Huanraluek, N-KR7 (MFLU 21-0060, new record), living culture, MFLUCC 21-0083; N-KR11 (MFLU 21-0096), living culture, MFLUCC 21-0100; Chiang Rai Province, Wiang Pa Pao, Lao River, on submerged decaying wood in the river shore, 11 December 2019, S. Boonmee and K.S.U. Chandrasiri, LR6 (MFLU 21-0061), living culture, MFLUCC 21-0084.

GenBank numbers: LR6: ITS $=$ MZ538511, LSU $=$ MZ538545, TEF $1-\alpha=$ MZ567089; N-KR7: ITS $=$ MZ538510, LSU $=$ MZ538544, SSU $=$ MZ538571, TEF $1-\alpha=$ MZ567088; N-KR11: ITS $=$ MZ538509, LSU $=$ MZ538543, SSU $=$ MZ538570, TEF1- $\alpha=$ MZ567087.

Notes: Multigene phylogenetic analysis of a combined LSU, ITS, TEF1- $\alpha$ and SSU sequence dataset indicates that our three new collections (MFLUCC 21-0083, MFLUCC 21-0084 and MFLUCC 21-0100) grouped with the ex-type strain Longipedicellata aquatica MFLUCC 17-2334 and other isolates with 79\% MLBS, 0.97 BYPP; 79\% MLBS and $96 \%$ MLBS support clade, respectively (Fig. 41). The morphological characters of the three new collections such as dark and immersed ascomata with apical erumpent ostiole, broadly clavate to spatulate, long pedicellate asci and broadly fusiform to ellipsoidal, hyaline, 1-septate, ascospores with a large distinctive guttule and surrounded by a mucilaginous sheath (Fig. 38) are similar to the ex-type strain MFLUCC 17-2334 (Dong et al. 2020a). Therefore, our three new collections as new freshwater records from Thailand.

Longipedicellata megafusiformis Chandrasiri, Boonmee \& K.D. Hyde, sp. nov.

Index Fungorum number: IF558538; Facesoffungi number: FoF 09947; Fig. 39 


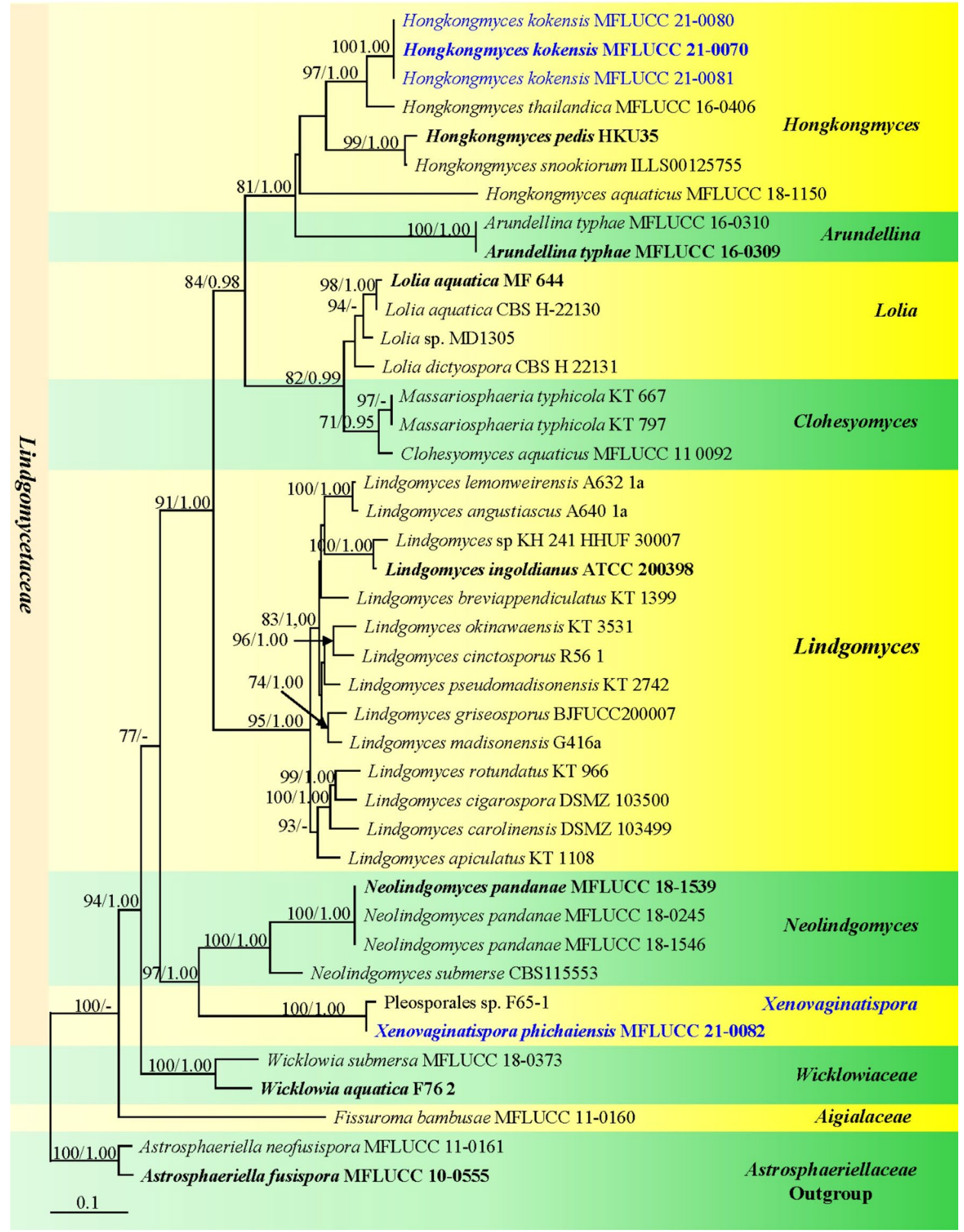

Fig. 37 Phylogram generated from maximum likelihood analysis based on combined LSU, SSU, ITS, and TEF1- $\alpha$ sequence data of new species and new genus in Lindgomycetaceae. Astrosphaeriella neofusispora MFLUCC 11-0161 and A. fusispora MFLUCC 10-0555 are selected as the outgroup taxa. Bootstrap support values for maxi- mum likelihood (MLBS, left) equal to or greater than $70 \%$ is given above the nodes. Bayesian posterior probabilities (BYPP, right) equal to or greater than 0.95 are given above the nodes. Ex-type strains are in bold and newly generated sequences are in blue 


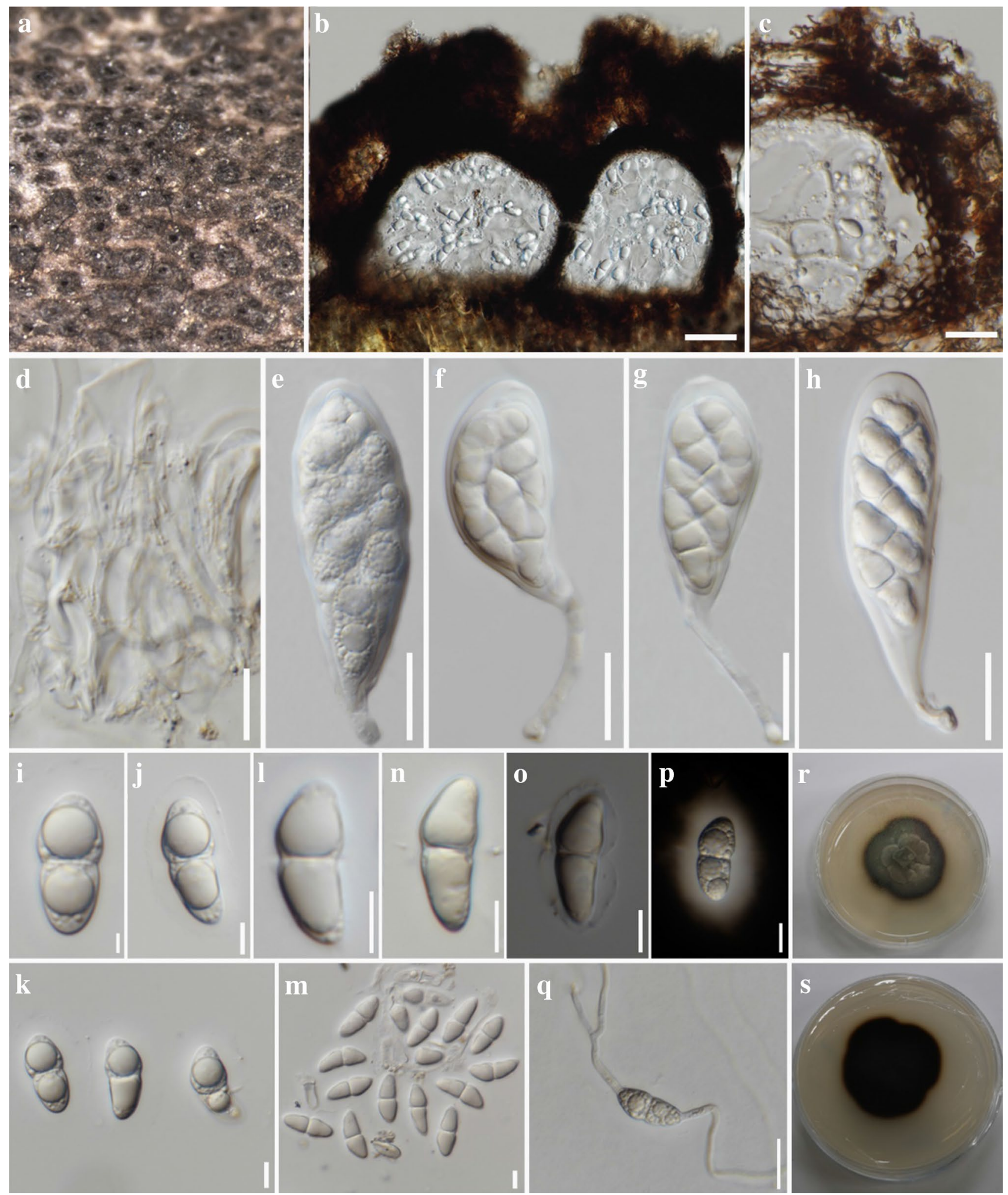

Fig. 38 Longipedicellata aquatica (MFLU 21-0060, new record). a Appearance of ascomata on submerged wood. b Section through ascomata. c Section of peridium. d Pseudoparaphyses. e-h Asci. i-n Ascospores. o Ascospore stained in Nigrosin. p Ascospore stained in
Indian Ink. q Geminated ascospore. $\mathbf{r}$, $\mathbf{s}$ Culture on MEA $(r=$ from above, $\mathrm{s}=$ from below). Scale bars: $\mathbf{b}=50 \mu \mathrm{m}, \mathbf{c}-\mathbf{h}, \mathbf{q}=20 \mu \mathrm{m}, \mathbf{i}-$ $\mathbf{p}=10 \mu \mathrm{m}$ 

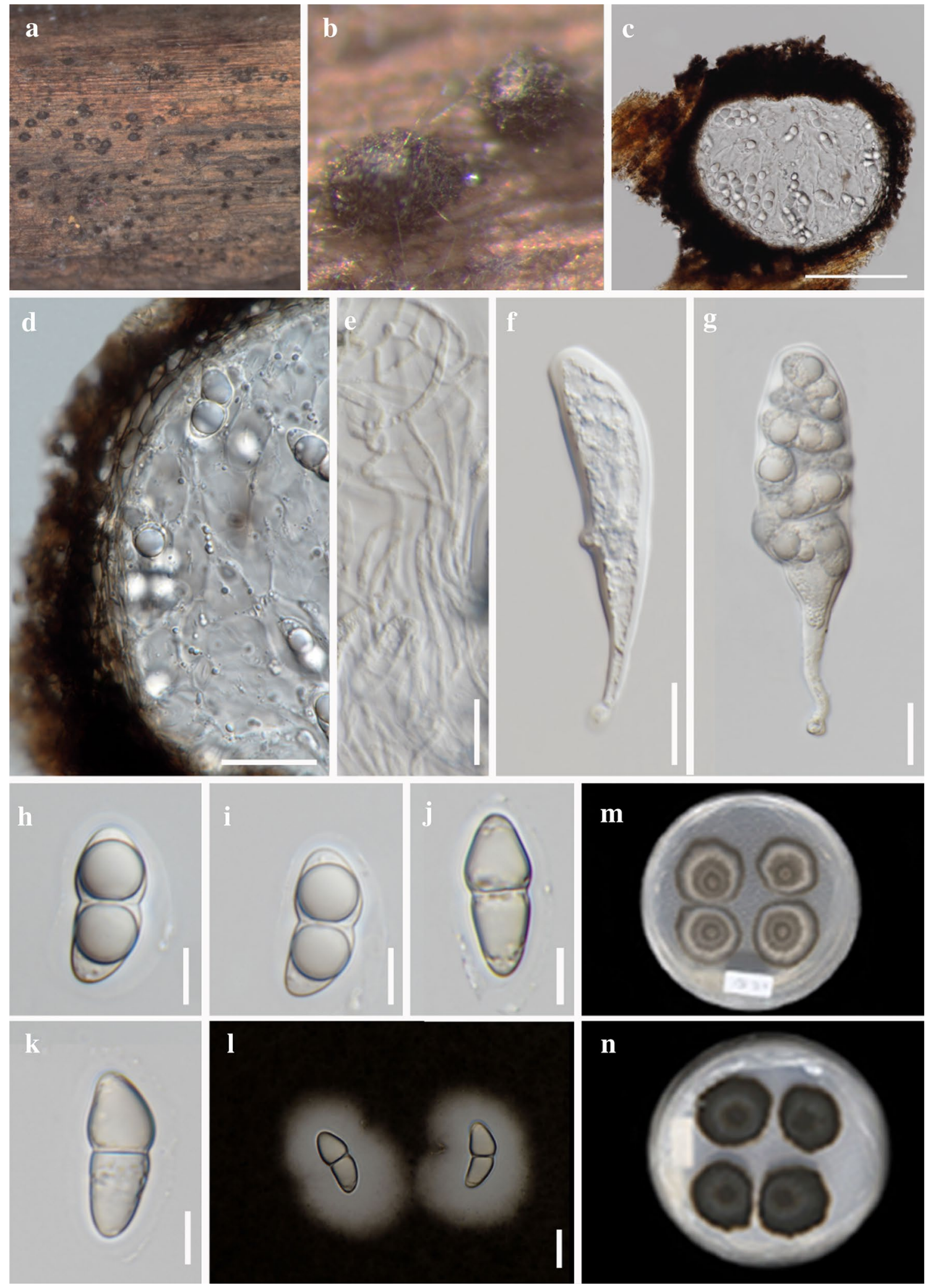

Fig. 39 Longipedicellata megafusiformis (MFLU 21-0062, holotype). a, b Appearance of ascomata on decaying submerged wood substrate. c Section through ascoma. d Section of peridium. e Pseudoparaphyses. f, $\mathbf{g}$ Asci. h-k Ascospores. $\mathbf{l}$ Ascospores stained in
Indian Ink showing a mucilaginous sheath. $\mathbf{m}, \mathbf{n}$ Culture on PDA $(\mathrm{m}=$ from above, $\mathrm{n}=$ from below $)$. Scale bars: $\mathbf{c}=100 \mu \mathrm{m}$, b$\mathbf{g}=20 \mu \mathrm{m}, \mathbf{h}-\mathbf{l}=10 \mu \mathrm{m}$ 
Etymology: The specific epithet "megafusiformis", in reference to the large and broad fusiform ascospores compared to other described species of Longipedicellata.

Holotype: MFLU 21-0062

Saprobic on decaying submerged wood in the river. Sexual morph Ascomata 153-204 $\mu \mathrm{m}$ high, 130-236 $\mu \mathrm{m}$ diam., solitary, scattered, semi-immersed to superficial, globose or subglobose, uni-loculate, black, with a bright ostiole. Peridium 20-30 $\mu \mathrm{m}$ thick, composed of several layers of brown to dark brown cells of textura angularis. Hamathecium comprising numerous, 1-1.5 $\mu \mathrm{m}$ wide, cylindrical filiform, sparsely branched, cellular pseudoparaphyses, embedded in gelatinous matrix. Asci $60-99 \times 12-26 \mu \mathrm{m}(\bar{x}=72 \times 17 \mu \mathrm{m}$, $\mathrm{n}=20$ ), 8 -spored, bitunicate, narrowly to broadly clavate. Ascospores $22-30 \times 7-16 \mu \mathrm{m}(\bar{x}=26 \times 11 \mu \mathrm{m}, \mathrm{n}=20)$, 2-3-seriate, ellipsoidal to fusiform, asymmetrical, upper cell wider, rounded at both ends, 1-septate, constricted at the septum, with a prominent guttule in both cells when immature, hyaline, surrounded by a large mucilaginous sheath, smooth-walled. Asexual morph Undetermined.

Culture characteristics: Ascospores germinating on water agar within $12 \mathrm{~h}$ at room temperature. Colonies growing on PDA, circular, dense, umbonate, edge entire in surface view and fimbriate in lateral view, opaque, with distinct colour zones, from periphery to middle dark grey, light grey, white and getting darker in the middle.

Material examined: THAILAND, Chiang Rai Province, Muang, Mae Yao, Kok River on decaying submerged wood in the river shore, 4 December 2019, S. Boonmee and S.K.U. Chandrasiri, SJ-KR4 (MFLU 21-0062, holotype), ex-type living culture, MFLUCC 21-0085.

GenBank numbers: ITS $=$ MZ538512, LSU $=$ MZ538546, TEF1- $\alpha=$ MZ567090.

Notes: The phylogenetic analysis of a combined LSU, ITS, TEF1- $\alpha$ and SSU sequence dataset indicates that Longipedicellata megafusiformis forms a distinct lineage basal to L. aquatica with 94\% MLBS, 1.00 BYPP support (Fig. 41). Longipedicellata megafusiformis is different from L. aptrootii and $L$. aquatica in terms of the ascomata features in having single loculate with a bright ostiole on the top view and the size of ascospores (Fig. 39). However, its mucilaginous sheath and septate are not significantly different from other Longipedicellata species. Longipedicellata megafusiformis has larger ascospores $(22-30 \times 7-16 \mu \mathrm{m})$ than other species such as $L$. aptrootii $(17.5-22 \times 8-8.5 \mu \mathrm{m})$ and $L$. aquatica (19-22 $\times 8.5-10.5 \mu \mathrm{m})$ (Dong et al. 2020b). Therefore, we introduce L. megafusiformis as a new species in Longipedicellata based on morphological and phylogenetic analyses in Longipedicellata.

Submersispora W. Dong, H. Zhang \& K.D. Hyde

Notes: Submersispora was introduced by Dong et al. (2020b) to accommodate a monotypic species Submersispora variabilis based on morphological characteristics and phylogenetic placement. The genus is characterized by freshwater inhabitants, slender semi-macronematous conidiophores and having variable in shaped chlamydospore-like conidia.

Submersispora variabilis W. Dong, H. Zhang \& K.D. Hyde, in Dong et al., Fungal Diversity 105: 440 (2020)

Index Fungorum number: IF557917; Facesofungi number: FoF 08720; Fig. 40

Saprobic on decaying submerged wood in the river. Sexual morph Undetermined. Asexual morph Colonies on natural substrate superficial, solitary, scattered, gregarious black. Mycelium immersed in natural substrate. Conidiophores rather short, 9-26 $\mu \mathrm{m}$ long, 2-4 $\mu \mathrm{m}$ wide, semimacronematous, mononematous, cylindrical, unbranched, septate, pale brown, thin and smooth-walled. Conidiogenous cells monoblastic, holoblastic, determinate, pale brown. Conidia $47-74 \times 50-79 \mu \mathrm{m}(\bar{x}=61 \times 57 \mu \mathrm{m}, \mathrm{n}=10)$, acrogenous, solitary, globose to subglobose, sometimes ellipsoidal to oblong, muriform, multi-septate, constricted at the septa, composed of angular cells, no septa when mature, brown to dark black, smooth-walled.

Culture characteristics: Conidia germinating on PDA within $12 \mathrm{~h}$ at room temperature. Colonies circular, reaching $2.5 \mathrm{~cm}$ in 3 weeks at room temperature, mycelium superficial, flat, slightly crenate edge, bright grey in surface, dark in reverse, with radially white grey arranges at the margin.

Material examined: THAILAND, Chiang Mai, Mae Ai District, Tha Ton, Kok River, on decaying submerged wood in the river shore, 9 December 2019, S. Boonmee and N. Huanraluek, N-KR15 (MFLU 21-0063, new record), living culture, MFLUCC 21-0086.

GenBank numbers: ITS = MZ538527, $\mathrm{LSU}=\mathrm{MZ538561}, \mathrm{SSU}=\mathrm{MZ538575}$, RPB2 $=\mathrm{MZ567114}$, TEF1- $\alpha=$ MZ567103.

Notes: Our new collection is a typical Submersispora species, and it shares similar morphology with Submersispora variabilis in the features of conidia (Dong et al. 2020b; see Fig. 67). The multigene phylogenetic analysis showed that our isolated MFLUCC 21-0086 clustered with the type species $S$. variabilis with 100\% MLBS, 1.00 BYPP support (Fig. 41). Therefore, we identify our collection (Fig. 40) as a new record for $S$. variabilis from Thailand.

\section{Lophiostomataceae Sacc.}

Notes: Lophiostomataceae was introduced by Saccardo (1883). The intergeneric relationships of the family have been well-studied by Thambugala et al. (2015), who provided a backbone tree for Lophiostomataceae and accepted 16 genera in Lophiostomataceae based on morphology and a multigene phylogenetic analysis. Eight additional genera have been introduced in this family (Wanasinghe et al. 2018; Hashimoto et al. 2018). Recently, Andreasen et al. (2021) 

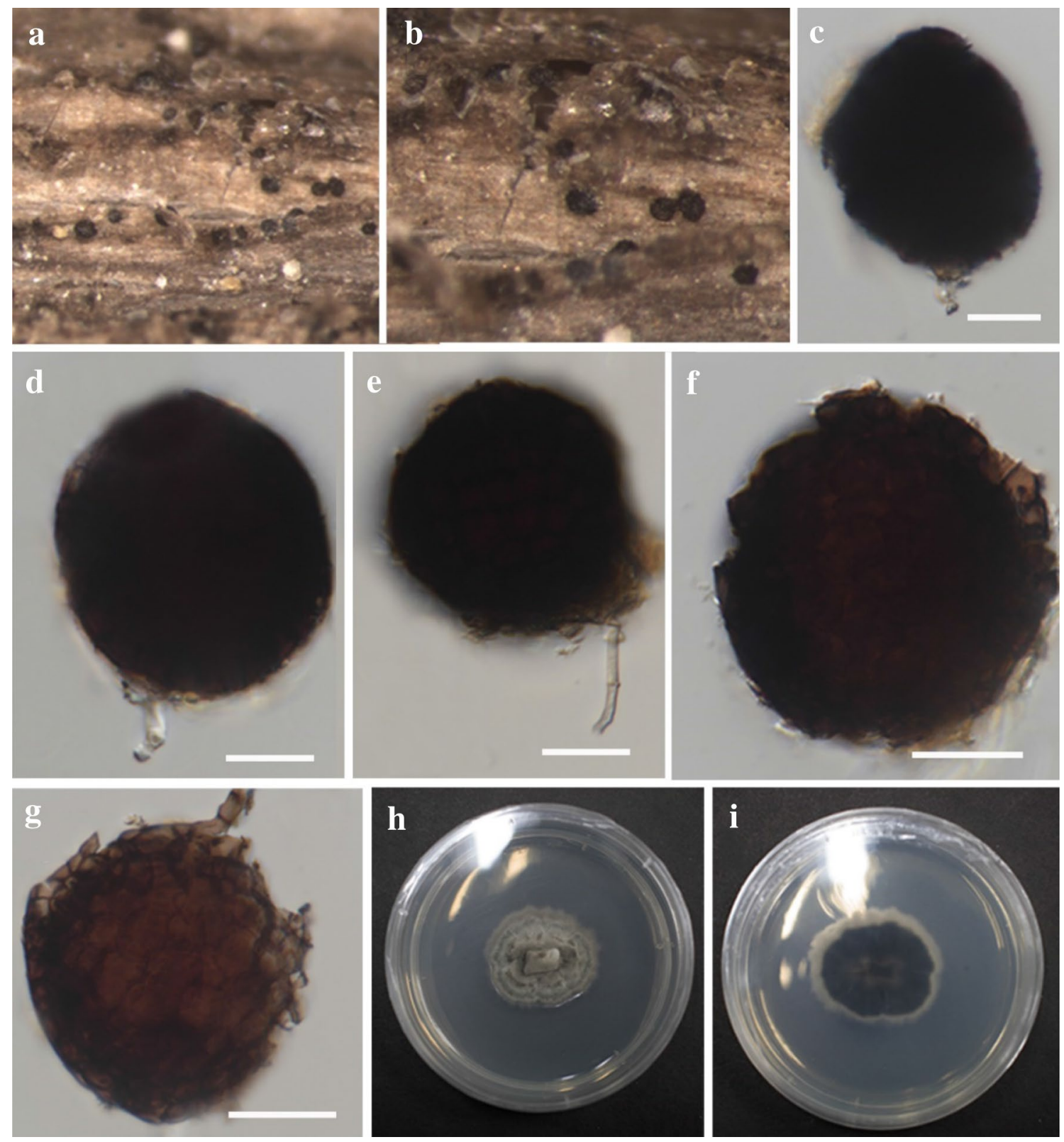

Fig. 40 Submersispora variabilis (MFLU 21-0063, new record). a, b Appearance of fungal colonies on woody substrate. c-e Conidiophores with conidia. f, $\mathbf{g}$ Close up of conidia. $\mathbf{h}, \mathbf{i}$ Culture on PDA from surface and reverse. Scale bars: $\mathbf{c}-\mathbf{e}=20 \mu \mathrm{m}, \mathbf{f}-\mathbf{g}=10 \mu \mathrm{m}$

synonymized 14 genera under Lophiostoma and accepted 11 genera in Lophiostomataceae. Members of this family are characterized by immersed to erumpent, carbonaceous to coriaceous ascomata with rounded or slit-like ostioles, mostly clavate asci, one to multi-septate, hyaline to dark brown ascospores with terminal appendages or mucilaginous sheaths (Thambugala et al. 2015; Hashimoto et al. 2018; Hongsanan et al. 2020a). Most species of Lophiostomataceae are reported as saprobes from terrestrial, freshwater, and marine environments. (Thambugala et al. 2015; Hashimoto et al. 2018; Bao et al. 2019b; Mapook et al. 2020; Hongsanan et al. 2020a).

\section{Lophiostoma Ces. \& De Not.}

Notes: Lophiostoma is a large genus included more 100 species (Hongsanan et al. 2020a). Members of Lophiostoma is characterized by laterally compressed or crest-like apices ascomata and common found saprobes on dead plants in aquatic and terrestrial habitats (Hirayama and Tanaka 2011; Thambugala et al. 2015). In this study, we provided on new host and geographical records for Lophiostoma caudatum and L. clematidis-vitalbae for Uzbekistan.

Lophiostoma caudatum Fabre, Annls Sci. Nat., Bot., sér. 6 9: 103 (1879) [1878] 
Fig. 41 Phylogram generated from maximum likelihood analysis based on combined LSU, ITS, TEF1- $\alpha$ and SSU sequence data of genera in Longipedicellataceae. The tree is rooted with Bambusicola bambusae (MFLUCC 11-0614). Bootstrap support values for maximum likelihood (MLBS, left) equal to or greater than $70 \%$ is given above the nodes. Bayesian posterior probabilities (BYPP, right) equal to or greater than 0.95 are given above the nodes. Ex-type strains are in bold and newly generated sequences are in blue

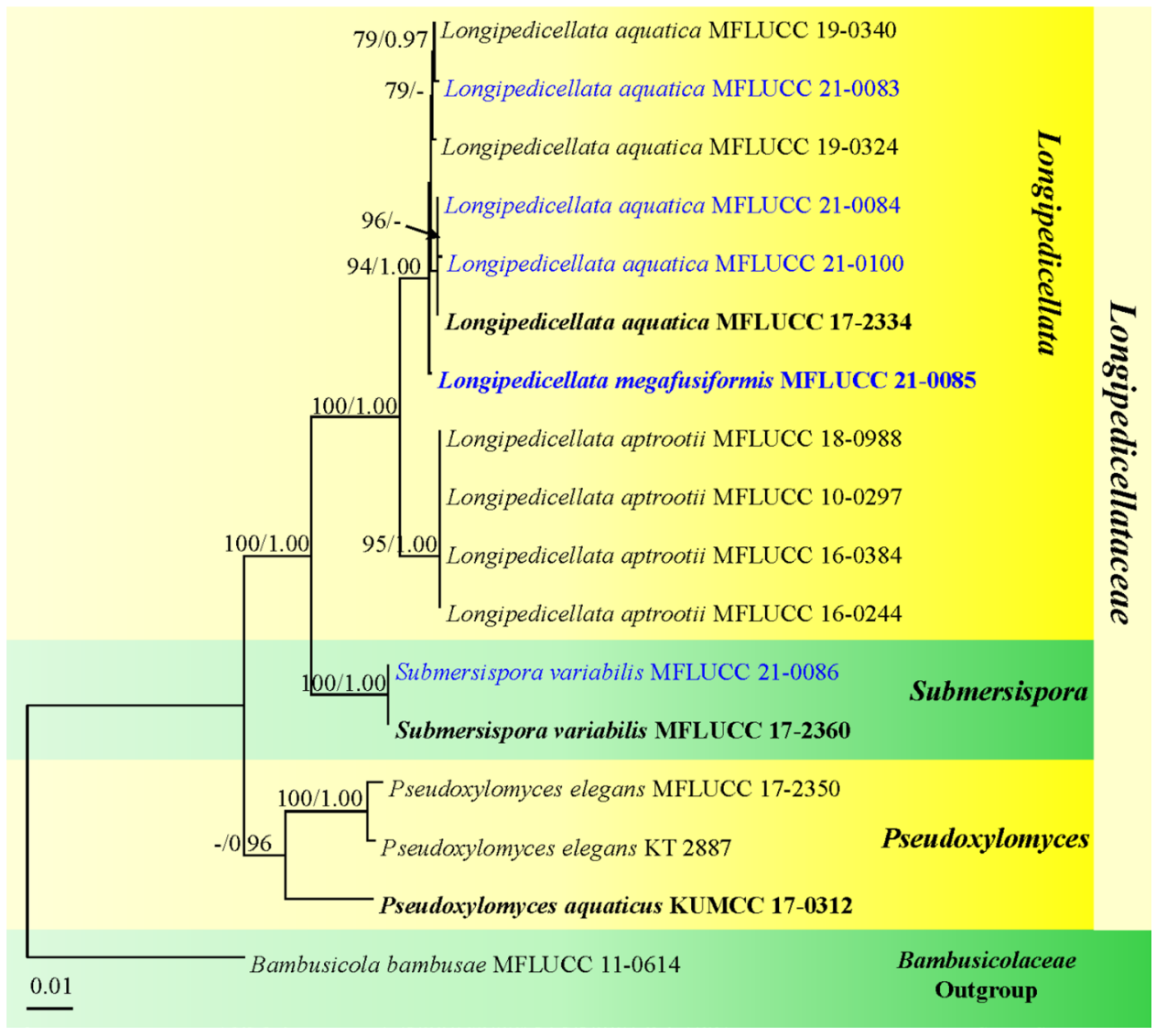

Index Fungorum number: IF185164; Facesoffungi number: FoF 00826; Fig. 42

= Sigarispora caudata (Fabre) Thambug., Qing Tian, Kaz. Tanaka \& K.D. Hyde, in Thambugala et al., Fungal Diversity 74: 241 (2015)

Saprobic on dead stem of Cousinia umbrosa in a terrestrial habitat. Sexual morph Ascomata 322-349 $\mu \mathrm{m}$ high, $191-251 \mu \mathrm{m}$ diam. $(\bar{x}=336 \times 211 \mu \mathrm{m}, \mathrm{n}=10)$, scattered, immersed to semi-immersed, papilla erumpent through host surface, flattened at the base, coriaceous, black, ostiolate. Ostiole $128-175 \times 35-39 \mu \mathrm{m}$ wide $(\bar{x}=146 \times 37 \mu \mathrm{m}$, $\mathrm{n}=5$ ), slit-like, central, with a crest-like apex and a porelike opening, elongated, irregular wall, filled with hyaline periphyses. Peridium $25-47 \mu \mathrm{m}$ wide $(\bar{x}=35 \mu \mathrm{m}, \mathrm{n}=13)$, wider at the apex and thinner at the base, with dark brown to lightly pigmented, thin-walled cells of textura angularis, cells towards the inside lighter and darker towards outside, merging with the host tissues. Hamathecium composed of numerous, $1-2 \mu \mathrm{m}$ wide, filamentous, septate, pseudoparaphyses. Asci $89-215 \times 12-13.5 \mu \mathrm{m}(\bar{x}=114 \times 13 \mu \mathrm{m}, \mathrm{n}=10)$, 8 -spored, bitunicate, fissitunicate, clavate, with an apically rounded and a bulbous pedicel. Ascospores 23-28 $\times 5-7 \mu \mathrm{m}$ $(\bar{x}=25 \times 6 \mu \mathrm{m}, \mathrm{n}=18$ ), biseriate, overlapping, brown to dark brown, fusiform with narrow, acute ends, usually 3-6 septate, not constricted at the central septum, guttulate, lacking a mucilaginous sheath, smooth-walled. Asexual morph Undetermined.

Material examined: UZBEKISTAN, Jizzakh Province, Zaamin District, Zaamin National Nature Park, river of Ettisuv, Turkestan range of Pamir-Alay Mountains, on dead stem of Cousinia umbrosa Bunge (Compositae), 11 July 2019, Y. Gafforov and T. Kholmuradova, YG-Z2-1 (TASM 6156, new host and geographical record).

GenBank Numbers: ITS $=$ MZ576205, LSU $=$ MZ577238, SSU $=$ MZ577240, TEF1- $\alpha=$ MZ605436.

Notes: In phylogenetic analysis of Lophiostoma, our strain (TASM 6156) clusters with the reliable strain Lophiostoma caudatum (KT 530) with 83\% MLBS, 0.99 BYPP (Fig. 44). However, our strain shares similar morphological characters of ascomata, asci and ascospores (Fig. 42) with $L$. caudatum (KT 530) (Thambugala et al. 2015). Pairwise comparison of the ITS, LSU, SSU and TEF1- $\alpha$ sequences of $L$. caudatum (TASM 6156) and L. caudatum (KT 530) reveals $0.61 \%$ in ITS, $0.11 \%$ in LSU and SSU, and $0.61 \%$ in TEF $1-\alpha$ are not significantly different. Therefore, $L$. caudatum is reported as a new host and geographical record on Cousinia umbrosa for Uzbekistan.

Lophiostoma clematidis-vitalbae Andreasen, Jaklitsch \& Voglmayr, in Andreasen et al., Persoonia 46: 251 (2021) 

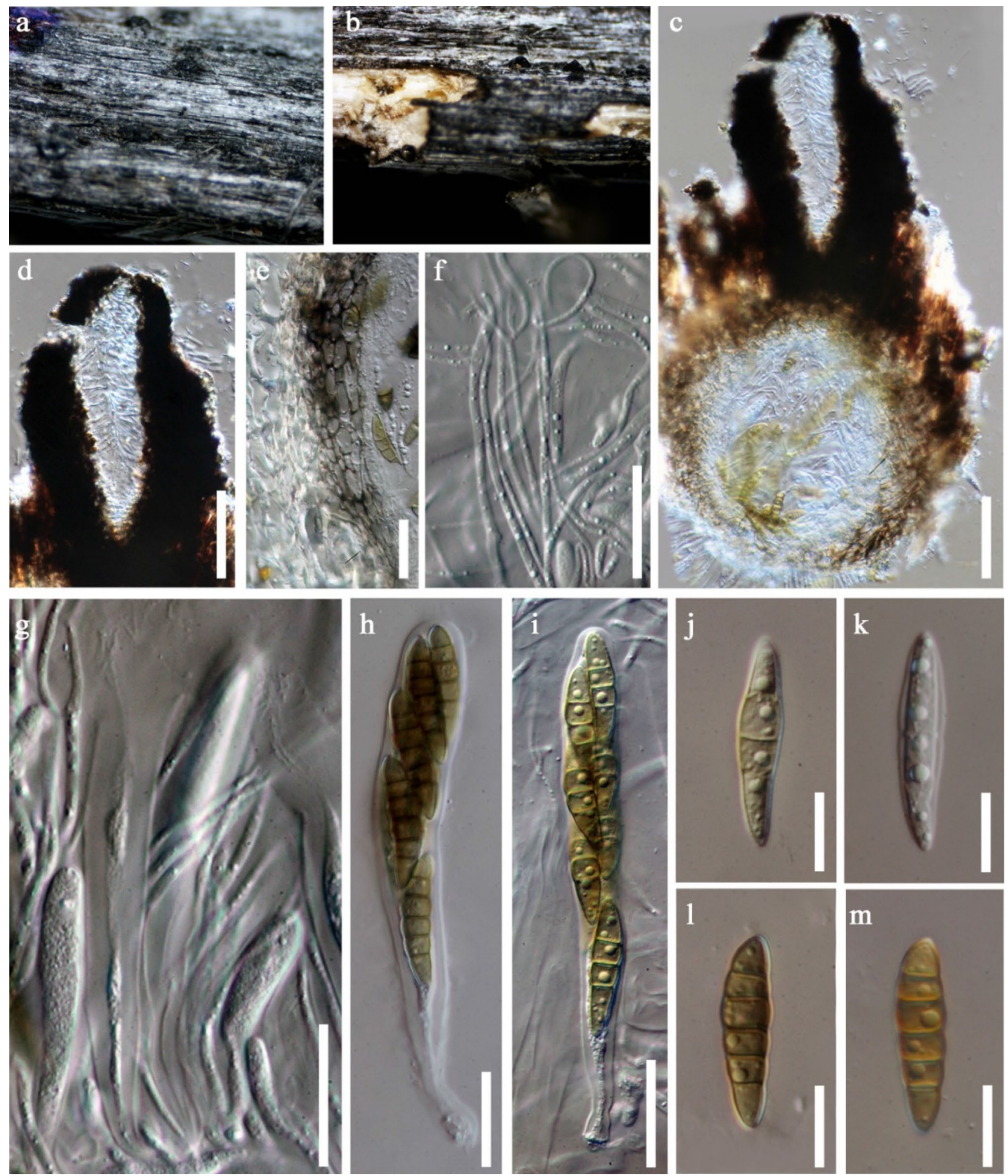

m

Fig. 42 Lophiostoma caudatum (TASM 6156, new host and geographical record). a, b Appearance of ascomata on host surface. c Vertical section of ascoma. d Ostiolar canal. e Peridium. f Pseudo- paraphyses. $\mathbf{g}-\mathbf{i}$ Asci. $\mathbf{j}-\mathbf{m}$ Ascospores. Scale bars: $\mathbf{c}, \mathbf{d}=50 \mu \mathrm{m}, \mathbf{e}-$ $\mathbf{i}=20 \mu \mathrm{m}, \mathbf{j}-\mathbf{m}=10 \mu \mathrm{m}$ 
Index Fungorum number: IF838978; Facesoffungi number: FoF 07294; Fig. 43

三 Sigarispora clematidis Phukhams. \& K.D. Hyde, in Phukhamsakda et al., Fungal Diversity 102: 53 (2020)

Saprobic on dead stem of unknown host. Sexual morph Ascomata 504-534 $\mu \mathrm{m}$ high, 372-473 $\mu \mathrm{m}$ diam. $(\bar{x}=523 \times 413 \mu \mathrm{m}, \mathrm{n}=10)$, solitary or aggregated, semiimmersed to superficial, with only black shiny ostioles visible, globose, coriaceous, dark brown to black, rough-walled, ostiolate. Ostioles $165-213 \times 25-98 \mu \mathrm{m}(x=193 \times 52 \mu \mathrm{m}$, $\mathrm{n}=5$ ), with a crest-like apex and a pore-like opening, central, elongated and laterally compressed, irregular wall, filled with hyaline periphyses. Peridium 62-82 $\mu \mathrm{m}$ wide $(\bar{x}=70 \mu \mathrm{m}, \mathrm{n}=20)$, wider at the apex, thinner at the base, with lightly pigmented dark brown to black, thick-walled cells of textura angularis, cell towards the inside lighter, somewhat flattened. Hamathecium composed of numerous, dense, 1.8-2.3 $\mu \mathrm{m}$ wide, filamentous, septate, pseudoparaphyses. Asci $93-111 \times 11-14 \mu \mathrm{m}(\bar{x}=101 \times 12 \mu \mathrm{m}, \mathrm{n}=30)$, 8 -spored, bitunicate, fissitunicate, clavate, with furcate pedicel, rounded at the apex, with an ocular chamber. Ascospores $19-25 \times 5-7 \mu \mathrm{m}(\bar{x}=23 \times 6 \mu \mathrm{m}, \mathrm{n}=50)$, bi-seriate or partially overlapping, broad fusiform, tapering towards the ends, initially hyaline, becoming yellowish brown at maturity, acute ends, mostly curved, 1-5 transversely eusepta, slightly constricted at the septa, cells above central septum swollen, a guttulate, with 5-9 $\mu \mathrm{m}$ long sheath drawn out to form polar appendages. Asexual morph Undetermined.

Material examined: UZBEKISTAN, Jizzakh Province, Zaamin District, Zaamin National Nature Park, river of Ettisuv, Turkestan range of Pamir-Alay Mountains, on dead stem of Lonicera sp. (Caprifoliaceae), 10 July 2019, Y. Gafforov and T. Kholmuradova YG-Z32-2 (TASM 6157, new host and geographical record).

GenBank accession numbers: ITS = MZ576206, $\mathrm{LSU}=\mathrm{MZ577239}$, SSU = MZ577241, TEF1- $\alpha=\mathrm{MZ605437,}$ RPB2 = MZ605438.

Notes: In phylogenetic analysis of Lophiostoma, our strains (TASM 6157) clusters with the ex-type strain Lophiostoma clematidis-vitalbae (MFLUCC 16-1368) with 0.98 BYPP (Fig. 44). Our strain (Fig. 43) shares similar ascomata, asci and ascospores features identical to the type strain $L$. clematidis-vitalbae (MFLUCC 16-1368) (Phukhamsakda et al. 2020). Therefore, L. clematidis-vitalbae is reported as a new host and geographical record on Cousinia umbrosa for Uzbekistan.

\section{Vaginatispora K.D. Hydes}

Notes: Vaginatispora was introduced by Hyde (1995) to accommodate Vaginatispora aquatica, which was previously placed in Massrinaceae. Thambugala et al. (2015) treated Vaginatispora as a separate genus in Lophiostomataceae with two new combinations, V. aquatica and V. fucklii.
Vaginatispora fucklii was later transferred to Neovaginatispora (Hashimoto et al. 2018). Six new species and a new combination $V$. armatispora were introduced in this genus (Wanasinghe et al. 2016; Devadatha et al. 2017; Hashimoto et al. 2018; Hyde et al. 2019; Jayasiri et al. 2019). Currently, nine species are included in this genus (Hashimoto et al. 2018; Hyde et al. 2019; Jayasiri et al. 2019). Vaginatispora species are characterized by depressed globose ascomata, immersed beneath a blackened neck, with a slot-like ostiole, numerous filamentous pseudoparaphyses, cylindrical to clavate asci and narrowly ellipsoidal, hyaline, 1-septate ascospores with a mucilaginous collar around its equator, having single large guttules in each cell, and a spreading papilionaceous sheath (Thambugala et al. 2015; Hashimoto et al. 2018; Hyde et al. 2020b).

Vaginatispora nypae Jayasiri, E.B.G. Jones \& K.D. Hyde, Mycosphere 10(1): 84 (2019)

Index Fungorum number: IF555558; Facesoffungi number: FoF 05264; Fig. 45

Saprobic on submerged decaying wood. Sexual morph Ascomata 250-420 $\mu \mathrm{m}$ high, 210-270 $\mu \mathrm{m}$ diam. $(\bar{x}=299 \times 247 \mu \mathrm{m}, \mathrm{n}=10)$, scattered to gregarious, immersed, coriaceous, dark brown to black, subglobose, ostiolate. Ostiole slit-like, elongated, with a pore-like opening, plugged by hyaline, filamentous hyphae, and occasionally lighter colour, periphysate. Peridium 30-60 $\mu \mathrm{m}$ wide, circular, symmetric, dark brown to black layers, somewhat flattened cells of textura angularis, inner stratum comprising hyaline cell layers of textura angularis. Hamathecium comprising numerous, filamentous, branched septate, pseudoparaphyses $1.5-2 \mu \mathrm{m}$ wide $(\bar{x}=1.8 \mu \mathrm{m}, \mathrm{n}=30)$. Asci 80-108 $\times 13-15 \mu \mathrm{m}(\bar{x}=94 \times 14.5 \mu \mathrm{m}, \mathrm{n}=20), 8$-spored, bitunicate, fissitunicate, cylindrical-clavate, short-pedicellate, apex rounded with a minute ocular chamber. Ascospores $26-29 \times 6-7 \mu \mathrm{m}(x=27 \times 6 \mu \mathrm{m}, \mathrm{n}=30)$, uni- to bi-seriate, overlapping, hyaline, fusiform with narrow, acute ends, 1-septate, slightly constricted at the septum, smoothwalled, guttulate, with a prominent mucilaginous sheath. Asexual morph Undetermined.

Culture characters: Conidia germinated on PDA within $24 \mathrm{~h}$. Colonies on PDA reaching 3-4 cm diam., after 4 weeks at room temperature, colonies irregular, medium dense, brown to grey in top view with pale brown edge. Lower surface dark brown with radially arranges margin.

Material examined: THAILAND, Yasothon Province, Thai Charoen District, Kham Phi, on decaying wood submerged in a River, 13 November 2018, D.F. Bao, B154 (MFLU 20-0424, new record), living culture, MFLUCC 20-0101.

GenBank numbers: ITS $=$ MZ490791, LSU $=$ MZ501207, SSU $=$ MZ490777, TEF1- $\alpha=$ MZ501207. 

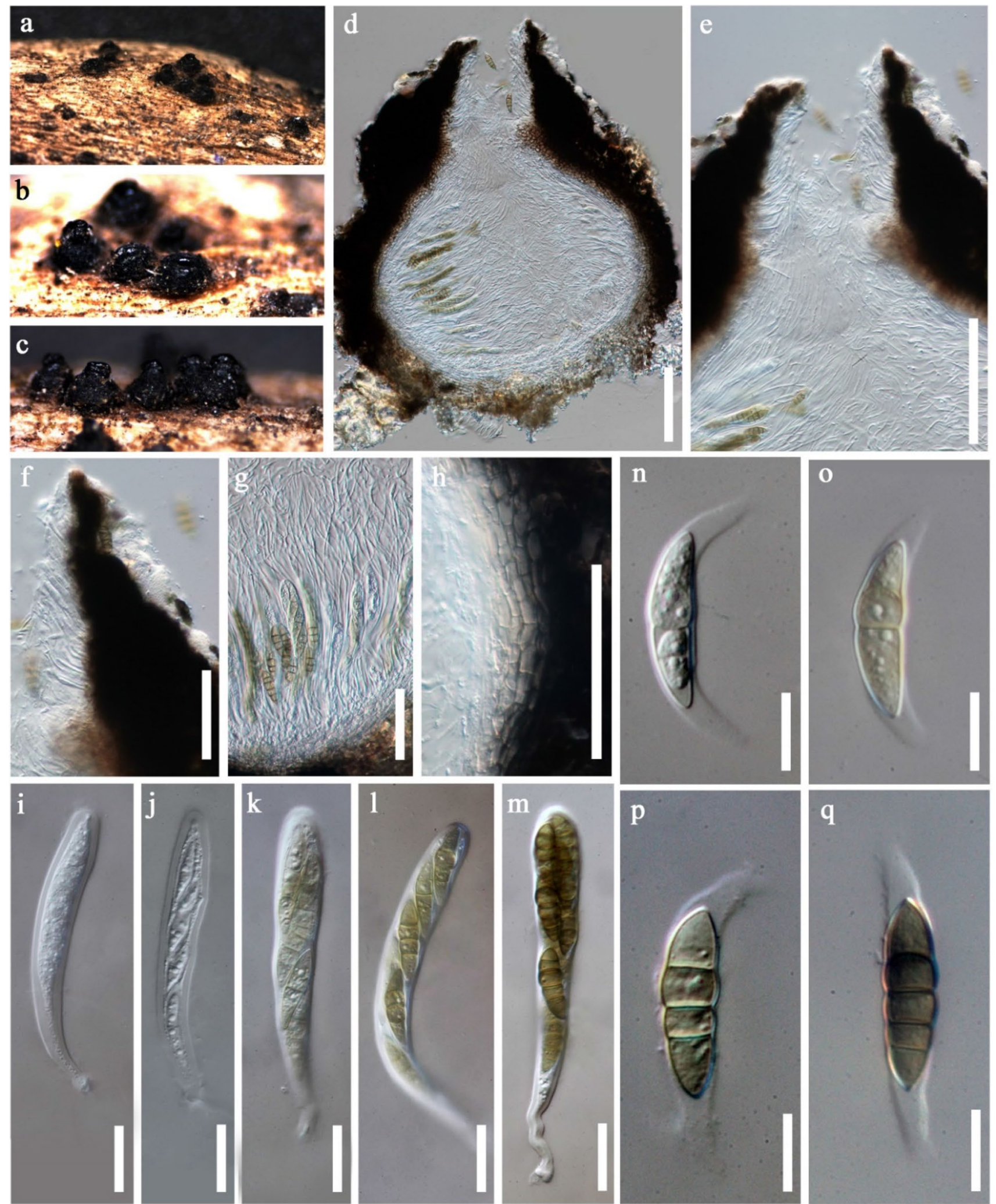

Fig. 43 Lophiostoma clematidis-vitalbae (TASM 6157, new host and geographical record). a-c Appearance of ascomata on host surface. d Vertical section of ascoma. e Ostiolar canal. f Periphyses at

ostiole. g Pseudoparaphyses. h Peridium. i-m Asci. n-q Ascospores. Scale bars: $\mathbf{d}, \mathbf{e}=100 \mu \mathrm{m}, \mathbf{f}-\mathbf{h}=50 \mu \mathrm{m}, \mathbf{i}-\mathbf{m}=20 \mu \mathrm{m}, \mathbf{n}-\mathbf{q}=10 \mu \mathrm{m}$ 


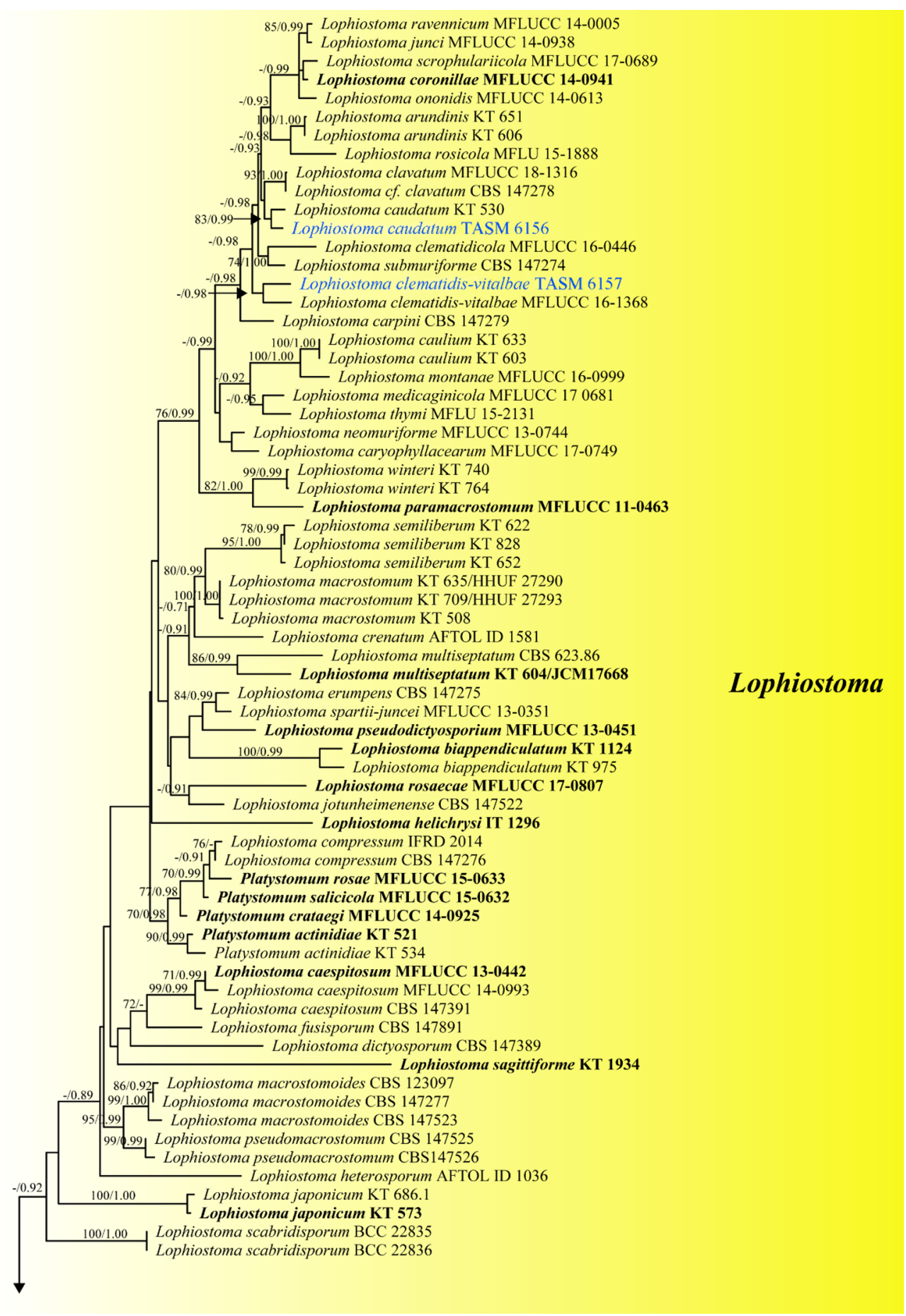

Fig. 44 Phylogram generated from maximum likelihood analysis based on combined SSU, LSU, ITS, TEF1- $\alpha$ and RPB2 sequence data for Lophiostomataceae. One hundred twelve strains are included in the combined analyses which comprise a total of 4199 characters. Teichospora rubriostiolata TR7 and Teichospora trabicola C134 are selected as the outgroup taxa. The best RAxML tree with a final likelihood value of - 30611.078207 is presented. RAxML analysis yielded 1567 distinct alignment patterns and $25.77 \%$ of undeter- mined characters or gaps. Estimated base frequencies were as follows: $\mathrm{A}=0.248812, \mathrm{C}=0.247504, \mathrm{G}=0.267733, \mathrm{~T}=0.235951$, with substitution rates $\mathrm{AC}=1.717311, \mathrm{AG}=4.965346, \mathrm{AT}=1.578028$, $\mathrm{CG}=1.454739, \mathrm{CT}=10.722777, \mathrm{GT}=1.000000$; gamma distribution shape parameter alpha $=0.639152$. Support values for maximum likelihood (MLBS, left) greater than $70 \%$ and Bayesian posterior probabilities (BYPP, right) greater than 0.90 are given at the nodes. Extype strains are in bold and newly generated sequences are in blue 


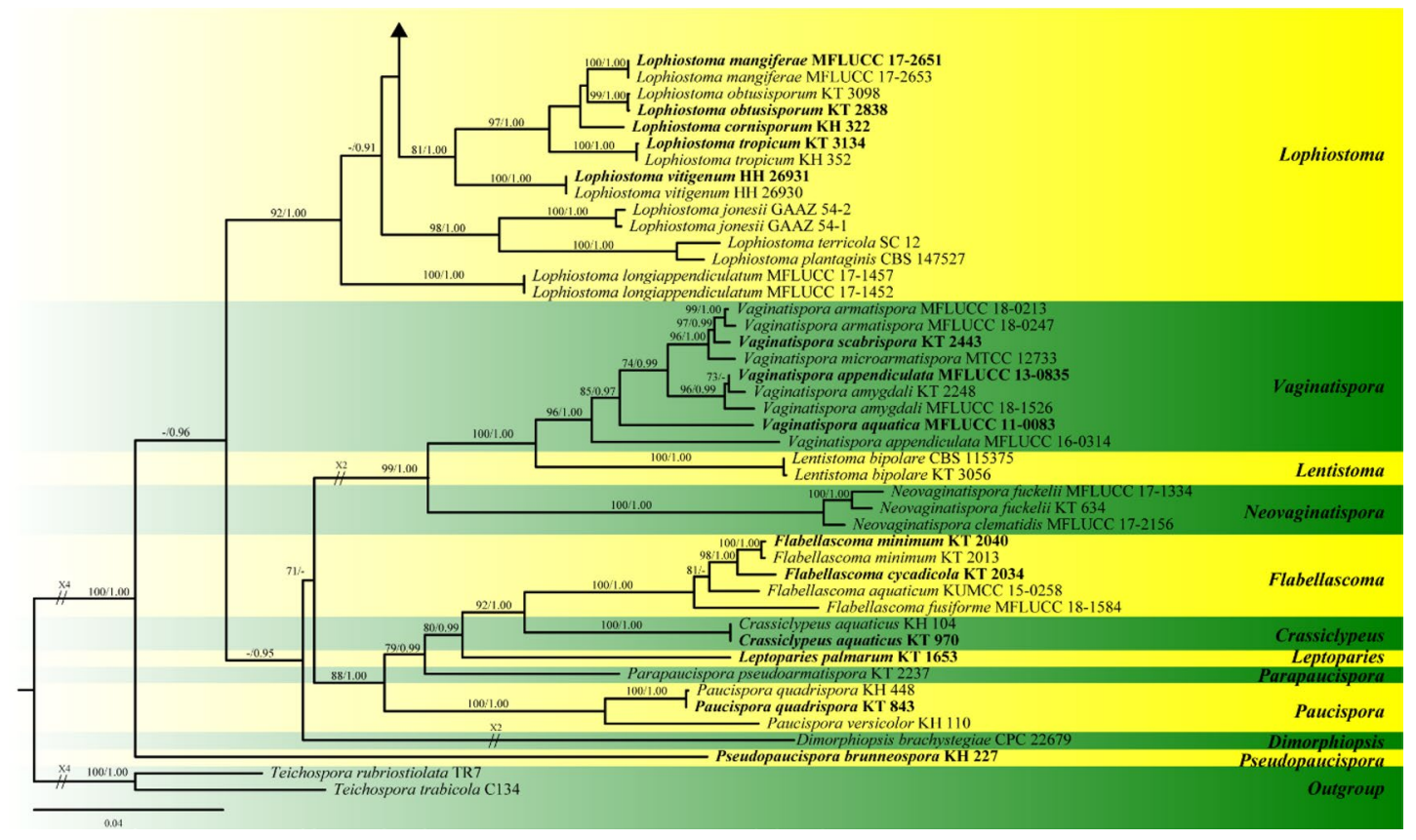

Fig. 44 (continued)

Notes: Vaginatispora nypae was introduced by Jayasiri et al. (2019), which was recorded from pericarp of fallen fruit of Nypa fruticans on terrestrial habitat in Thailand. Based on our phylogenetic analysis of combined LSU, SSU, ITS, TEF1- $\alpha$ and RPB2 sequence data, our isolate (MFLUCC 20-0101) clustered with the ex-type strain of Vaginatispora nypae (MFLUCC 18-1543) with $100 \%$ MLBS, 1.00 BYPP support (Fig. 46). Our isolate appears quite similar to $V$. nypae, in that they have similar shaped asci and ascospores. However, our isolate has larger asci $(80-108 \times 13-15 \mu \mathrm{m}$ vs. $75-85 \times 11-13 \mu \mathrm{m})$ and ascospores (26-29 $\times 6-7 \mu \mathrm{m}$ vs. $22-26 \times 10-13 \mu \mathrm{m})$, which may be explained by the different habitats (Fig. 45). Our isolate was collected from a freshwater habitat, while the strain of MFLUCC 18-1543 was collected from a terrestrial habitat. A comparison of the ITS and TEF1 $\alpha$ nucleotides of $V$. nypae (MFLUCC 18-0245) and a new strain (MFLUCC 20-0101) revealed $2 \mathrm{bp}(0.38 \%)$ and $6 \mathrm{bp}(0.6 \%)$ nucleotide differences respectively. This indicates that our new strain is $V$. nypae (Jeewon and Hyde 2016). Therefore, we introduce this isolate as a new record of $V$. nypae from a freshwater habitat.

\section{Lophiotremataceae K. Hiray. \& Kaz.}

Notes: Hirayama and Tanaka (2011) introduced Lophiotremataceae to accommodate Lophiotrema based on morphological characters and molecular phylogenetic analyses of LSU and SSU sequence data. Hashimoto et al. (2017) revised the family using morphological data and multigene phylogenetic analyses of ITS, LSU, RPB2, SSU and TEF1- $\alpha$. In the most recent outline of Dothideomycetes, Hongsanan et al. (2020a) accepted seven genera in this family viz. Atrocalyx, Crassimassarina, Cryptoclypeus, Galeaticarpa, Koordersiella, Lophiotrema and Pseudocryptoclypeus. An updated phylogenetic tree for Lophiotremataceae is presented in Fig. 50.

\section{Lophiotrema Sacc.}

Notes: Lophiotrema is one of the oldest genera in Pleosporales of which the species are characterized by immersed ascomata with a crest-like ostiolar neck and cylindrical asci (Hashimoto et al. 2017). Species in this genus are commonly found as saprobic on various plant species and plant-based substrates in terrestrial (Holm and Holm 1988), freshwater (Hyde and Aptroot 1998), and marine environments (Hongsanan et al. 2020a). Currently there are 38 species that are included in this genus but only eight species have been confirmed by molecular data (Hongsanan et al. 2020a). In this study, we introduce a new species and two new host records from Yunnan, China.

Lophiotrema hydei J.F. Zhang, J.K. Liu \& Z.Y. Liu, Phytotaxa 379(2): 172 (2018)

Index Fungorum number: IF555509; Facesoffungi number: FoF 05216; Fig. 47

Saprobic on wood of Rosa sp. Sexual morph: Ascomata $220-300 \times 200-250 \mu \mathrm{m}(x=261 \times 229 \mu \mathrm{m}, \mathrm{n}=10)$, solitary or gregarious, immersed to erumpent through host tissue, globose to subglobose, brown to dark brown, uni-loculate, glabrous, ostiolate. Ostiole mostly central. Peridium 20-40 $\mu \mathrm{m}$ wide, composed of angular cells, outer 

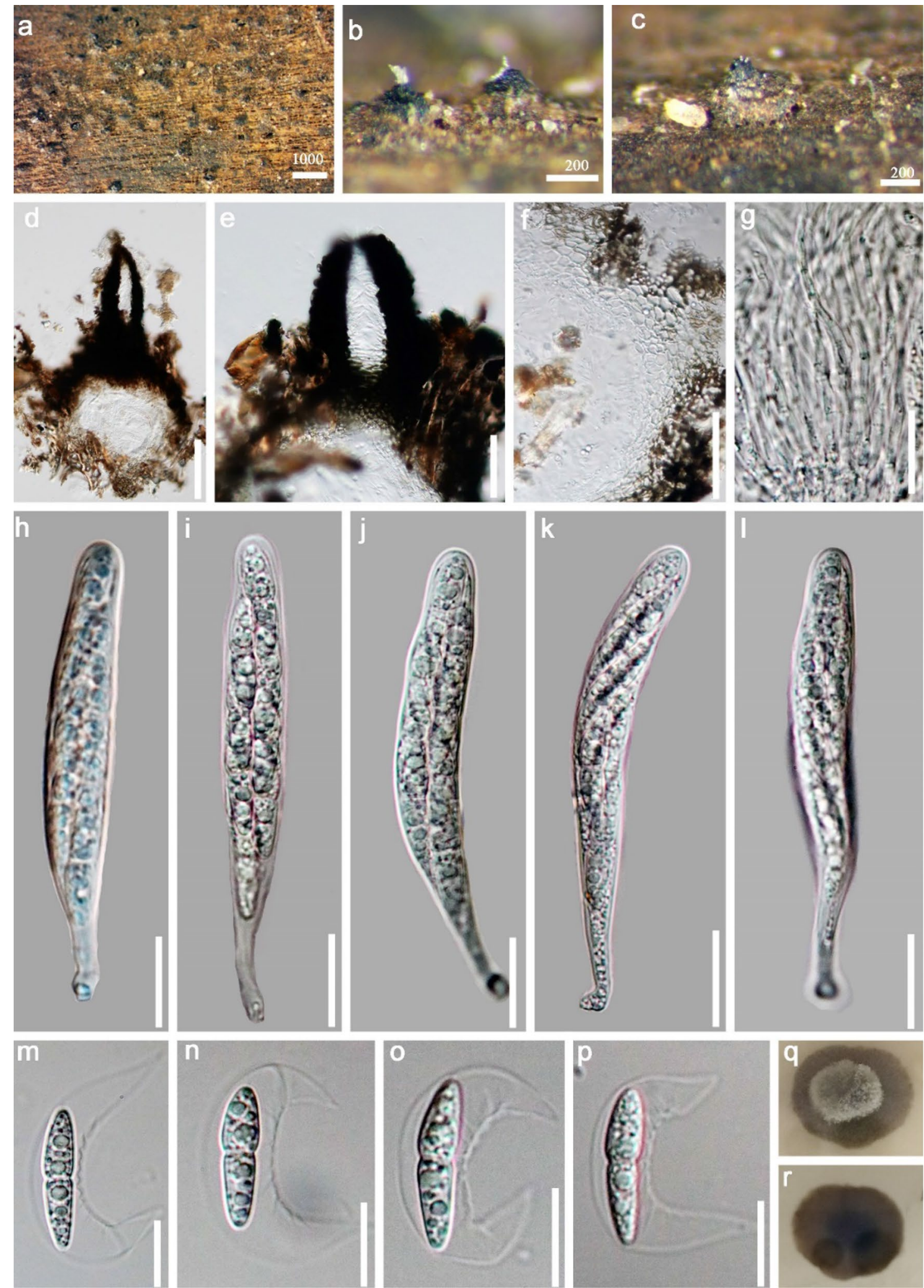

Fig. 45 Vaginatispora nypae (MFLU 20-0424, new record). a-c Ascomata on submerged wood. d Section of ascoma. e Ostiolar canal. f Peridium. g Pseudoparaphyses. h-l Asci. m-p Ascospore. q, r Cul- ture on MEA from upper and lower views. Scale bars: $\mathbf{a}=1000 \mu \mathrm{m}$, $\mathbf{b}, \mathbf{c}=200 \mu \mathrm{m}, \mathbf{d}=100 \mu \mathrm{m}, \mathbf{e}, \mathbf{f}=50 \mu \mathrm{m}, \mathbf{g}-\mathbf{l}=20 \mu \mathrm{m}, \mathbf{m}-\mathbf{p}=15 \mu \mathrm{m}$ 


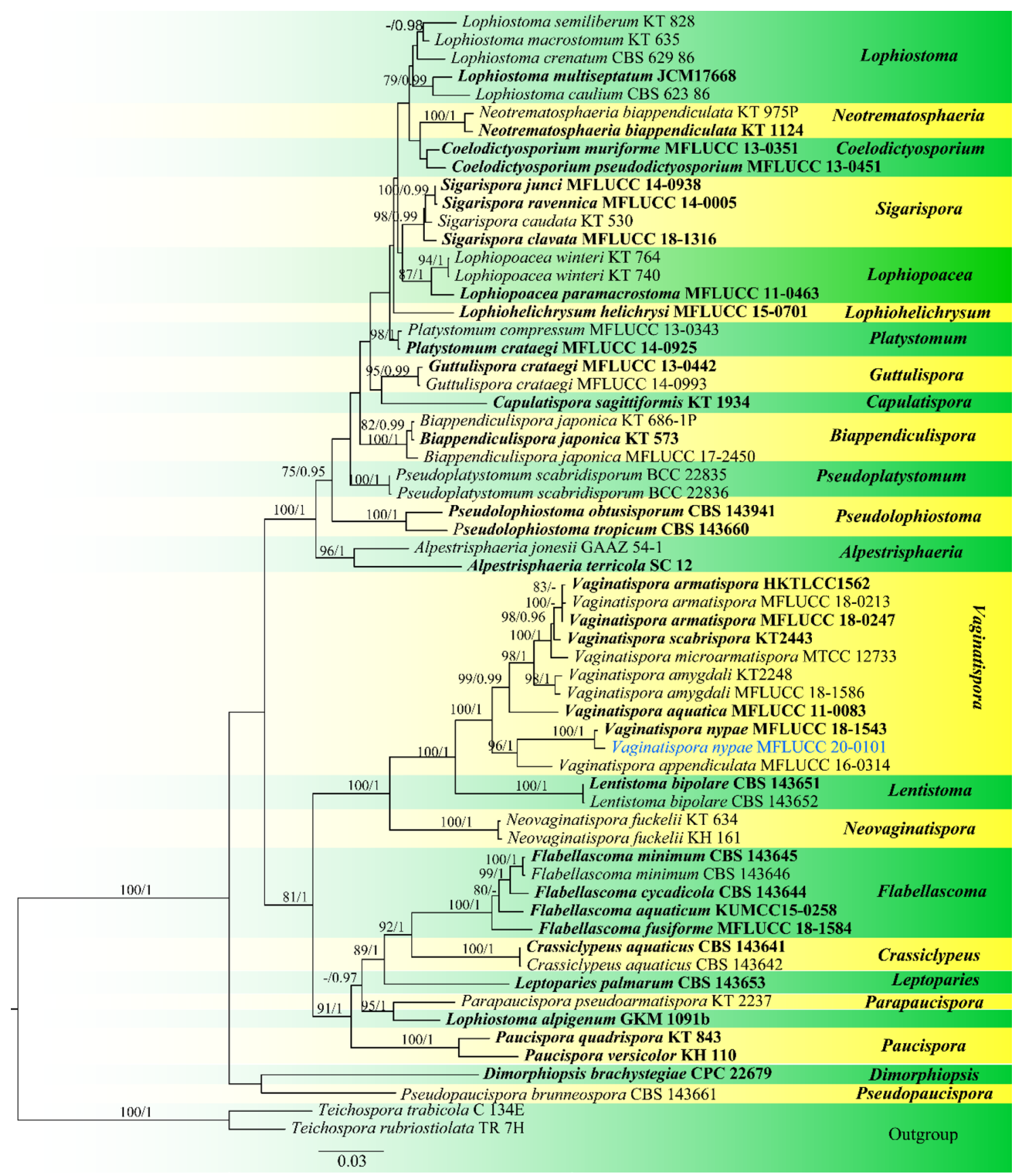

Fig. 46 Phylogenetic tree generated from maximum likelihood (ML) analysis based on combined LSU, SSU, ITS, TEF1- $\alpha$ and RPB2 sequence data for the species from Melanommataceae. Teichospora trabicola (C 134E) and T. rubriostiolata (TR 7) are used as the outgroup taxa. The dataset comprised 4379 characters after alignment including gaps $(\mathrm{LSU}=883 \mathrm{bp}, \mathrm{SSU}=966 \mathrm{bp}$, ITS $=523 \mathrm{bp}$, TEF $1-\alpha=1001$ bp and RPB2 $=1006 \mathrm{bp}$ ). The RAxML analysis of the combined dataset yielded a best scoring tree with a final ML optimization likelihood value of -26352.113239 . The matrix had 1530

layer, dark brown, thick-walled cells, inner layer, hyaline with thin-walled cells. Hamathecium composed of numerous, 1.5-2.5 $\mu \mathrm{m}$ wide, filamentous, septate, branched, distinct alignment patterns, with $26.20 \%$ undetermined characters or gaps. Estimated base frequencies were as follows: $A=0.248486$, $\mathrm{C}=0.247993, \quad \mathrm{G}=0.267004, \mathrm{~T}=0.236517 ;$ substitution rates $\mathrm{AC}=1.626883, \quad \mathrm{AG}=4.007531, \quad \mathrm{AT}=1.289884, \quad \mathrm{CG}=1.323552$, $\mathrm{CT}=8.595651, \mathrm{GT}=1.000000$; gamma distribution shape parameter $\alpha=0.180969$. Support values for maximum likelihood (MLBS, left) above than $75 \%$ and Bayesian posterior probabilities (BYPP, right) greater than 0.95 are given at the nodes. Ex-type strains are in bold and newly generated sequence is in blue

cellular pseudoparaphyses. Asci 80-120 $\times 10-13 \mu \mathrm{m}$ $(\bar{x}=105 \times 11 \mu \mathrm{m}, \mathrm{n}=20), 8$-spored, bitunicate, fissitunicate, cylindrical to cylindric-clavate, short pedicellate, 

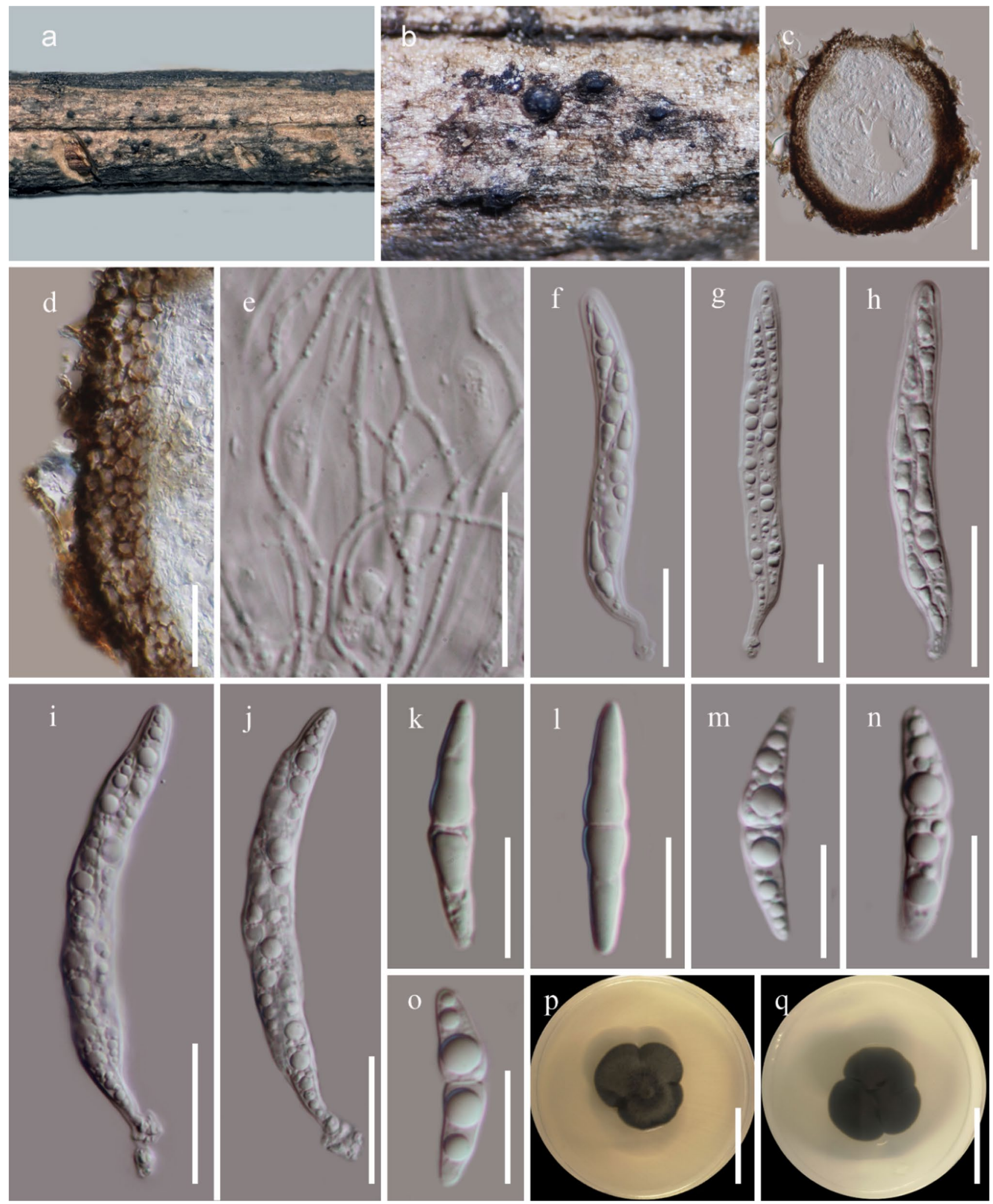

Fig. 47 Lophiotrema hydei (HKAS 115782, new host record). a, b Appearance of ascomata on host surface. c Longitudinal section of an ascoma. d Section of the peridium cells. e Pseudoparaphyses. $\mathbf{f}-\mathbf{j}$
Asci. $\mathbf{k}-\mathbf{o}$ Ascospores. $\mathbf{p}, \mathbf{q}$ Culture characteristic on PDA $(\mathbf{p}=$ from above, $\mathbf{q}=$ from below). Scale bars: $\mathbf{c}=100 \mu \mathrm{m}, \mathbf{d}, \mathbf{f}-\mathbf{j}=20 \mu \mathrm{m}$, $\mathbf{e}=5 \mu \mathrm{m}, \mathbf{k}-\mathbf{o}=10 \mu \mathrm{m}, \mathbf{p}, \mathbf{q}=10 \mathrm{~mm}$ 
apically rounded, with a minute ocular chamber. Ascospores 25-35 $\times 4-7 \mu \mathrm{m}(\bar{x}=31 \times 5.5 \mu \mathrm{m}, \mathrm{n}=30)$, overlapping biseriate, fusiform, hyaline, straight or slightly curved, 1-septate, the upper cell is longer than the lower cell, deeply constricted at the septum, narrower towards both ends, smooth-walled, guttulate. Asexual morph Undetermined.

Culture characteristics: Ascospores germinating on PDA within $24 \mathrm{~h}$ and germ tubes arising from both ends. Colonies on PDA, slow growing, reaching $2 \mathrm{~cm}$ diam. after 4 weeks of incubation at room temperature, initially white becoming blackish brown at maturity, slightly effuse, radially with an undulate edge, reverse blackish brown.

Material examined: CHINA, Yunnan, Diqing Autonomous Prefecture, Shangri-La, Xiaozhongdianzhen $\left(27.468883^{\circ} \mathrm{N} 99.845828^{\circ} \mathrm{E}\right), 2958 \mathrm{~m}$, on dead wood of Rosa sp. (Rosaceae), 30 August 2020, GC Ren, DQ33 (HKAS 115782, new host record), living culture could not maintain.

GenBank numbers: ITS $=$ MZ493297, LSU = MZ493311, SSU $=$ MZ493283, TEF1- $\alpha=$ MZ508406.

Notes: Zhang et al. (2018) introduced Lophiotrema hydei as a saprobe on herbaceous plant from Guizhou Province, China. In this study, we found a new strain that grouped with the ex-type strain of $L$. hydei in multi-gene phylogenetic analysis with 98\% MLBS support (Fig. 50). There were no nucleotide differences between these two strains in SSU, LSU, ITS and TEF1- $\alpha$. The asci of the new isolate are comparatively larger $(80-120 \times 10-13 \mu \mathrm{m}$ vs. $78-89(-99) \times 6.9-8.8 \mu \mathrm{m})$, but other features are similar in dimensions (Fig. 47). Therefore, we identify our collection as a new host record of Lophiotrema hydei on Rosa sp. from Yunnan Province, China.

Lophiotrema lincangensis Wanas. \& K.D. Hyde, sp. nov. Index Fungorum number: IF558584; Facesoffungi number: FoF 09948; Fig. 48

Etymology: The specific epithet "lincangensis" refers to the Lincang City, Yunnan Province, China where the type was collected.

\section{Holotype: HKAS 115777}

Saprobic on dead twigs of Fagaceae sp. Sexual morph: Ascomata 140-180 $\mu \mathrm{m}$ high, 120-160 $\mu \mathrm{m}$ diam. $(\bar{x}=158 \times 139 \mu \mathrm{m}, \mathrm{n}=10)$, solitary or gregarious, immersed to erumpent through host tissue, globose to subglobose, brown to dark brown, uni-loculate, glabrous, ostiolate. Ostiole mostly central, minute papilla. Peridium $15-30 \mu \mathrm{m}$ wide, composed of angular, pseudoparenchymatous cells, outer layer, dark brown, thick-walled cells, inner layer, hyaline with thin-walled cells. Hamathecium composed of numerous, 1.5-2 $\mu \mathrm{m}$ wide, filamentous, septate, cellular pseudoparaphyses. Asci $50-90 \times 6.5-9 \mu \mathrm{m}(\bar{x}=63.5 \times 7.5 \mu \mathrm{m}$, $\mathrm{n}=20$ ), 8 -spored, bitunicate, fissitunicate, cylindrical to cylindric-clavate, short pedicellate, apically rounded, with a minute ocular chamber. Ascospores 16-22 $\times 3-4 \mu \mathrm{m}$ $(\bar{x}=19.5 \times 3 \mu \mathrm{m}, \mathrm{n}=30$ ), overlapping bi-seriate, fusiform, hyaline, straight or slightly curved, one-celled when young and become inconspicuously 1-septate at maturation, the upper cell is longer than the lower cell, constricted at the septum, narrower towards both end cells, smooth-walled, guttules. Asexual morph Undetermined.

Culture characteristics: Ascospores germinating on PDA within $24 \mathrm{~h}$ and germ tubes arising from both ends. Colonies on PDA, slow growing, reaching $2 \mathrm{~cm}$ diam. after 4 weeks of incubation at room temperature, initially white becoming blackish brown at maturity, slightly effuse, radially with a smooth edge, blackish brown.

Material examined: CHINA, Yunnan, Lincang City, Linxiang, $24.883878^{\circ} \mathrm{N} 100.092519^{\circ} \mathrm{E}$, on dead twigs of Fagaceae sp., 10 August 2020, GC Ren, LC21-01 (HKAS 115777, holotype), ex-type living culture could not maintain; ibid. LC21-03 (HKAS 115778), living culture could not maintain.

GenBank numbers: ITS = MZ493298, MZ493299, LSU $=$ MZ493312, MZ493313, SSU $=$ MZ493284, MZ493285, RPB 2 = MZ508416, MZ508417, TEF1- $\alpha=$ MZ508407, MZ508408.

Notes: During our investigation on the woody-based microfungi in Yunnan, two isolates were recovered from Fagaceae hosts in Lincang City. Morphological characteristics such as asci and ascospores fit well within the species concept of Lophiotrema (Fig. 48). In our phylogenetic study, these new strains clustered with $L$. hydei and L. mucilaginosis with 100\% MLBS support (Fig. 50). Comparatively, our new collections had smaller asci and conidia than these two species (see notes of $L$. hydei). A nucleotide base comparison of ITS (513 bp) among our new strains, L. hydei and L. mucilaginosis reveals 23 bp (4.5\%) and 24 bp (4.7\%) base pair differences (Jeewon and Hyde 2016). The TEF1- $\alpha$ nucleotides ( $889 \mathrm{bp}$ ) comparison of the two species with our new strain reveals $29 \mathrm{bp}$ (3.3\%) and $24 \mathrm{bp}(2.7 \%)$ difference respectively. The RPB2 nucleotides comparison (953 bp) of with L. mucilaginosis and our new strains reveals $54 \mathrm{bp}$ (5.6\%) base pair differences. Whereas $L$. hydei is lacking of RPB2 sequence data.

Lophiotrema neoarundinariae Y. Zhang ter, Kaz. Tanaka \& K.D. Hyde [as 'neoarundinaria'], Stud. Mycol. 64: 97 (2009)

Index Fungorum number: IF836880; Facesoffungi number: FoF 09949; Fig. 49

$\equiv$ Didymosphaeria arundinariae Ellis \& Everh., N. Amer. Pyren. (Newfield): 732 (1892)

Saprobic on dead twigs of Prunus sp. Sexual morph: Ascomata 200-300 $\mu \mathrm{m}$ high, 150-200 $\mu \mathrm{m}$ diam. $(\bar{x}=248 \times 180.5 \mu \mathrm{m}, \mathrm{n}=10)$, solitary or gregarious, immersed to erumpent through host tissue, subglobose or obpyriform, brown to dark brown, ostiolate. Ostiole 

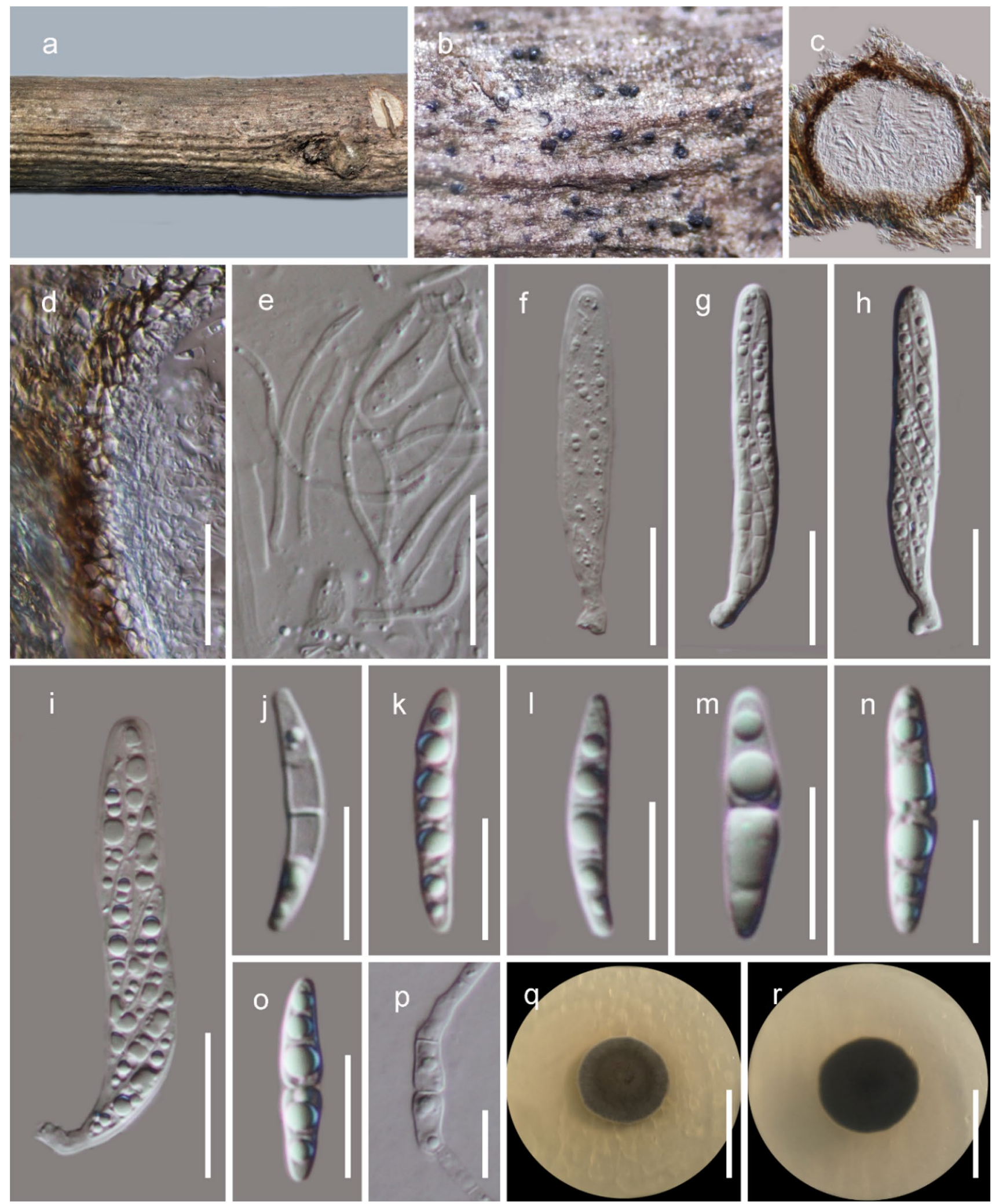

h
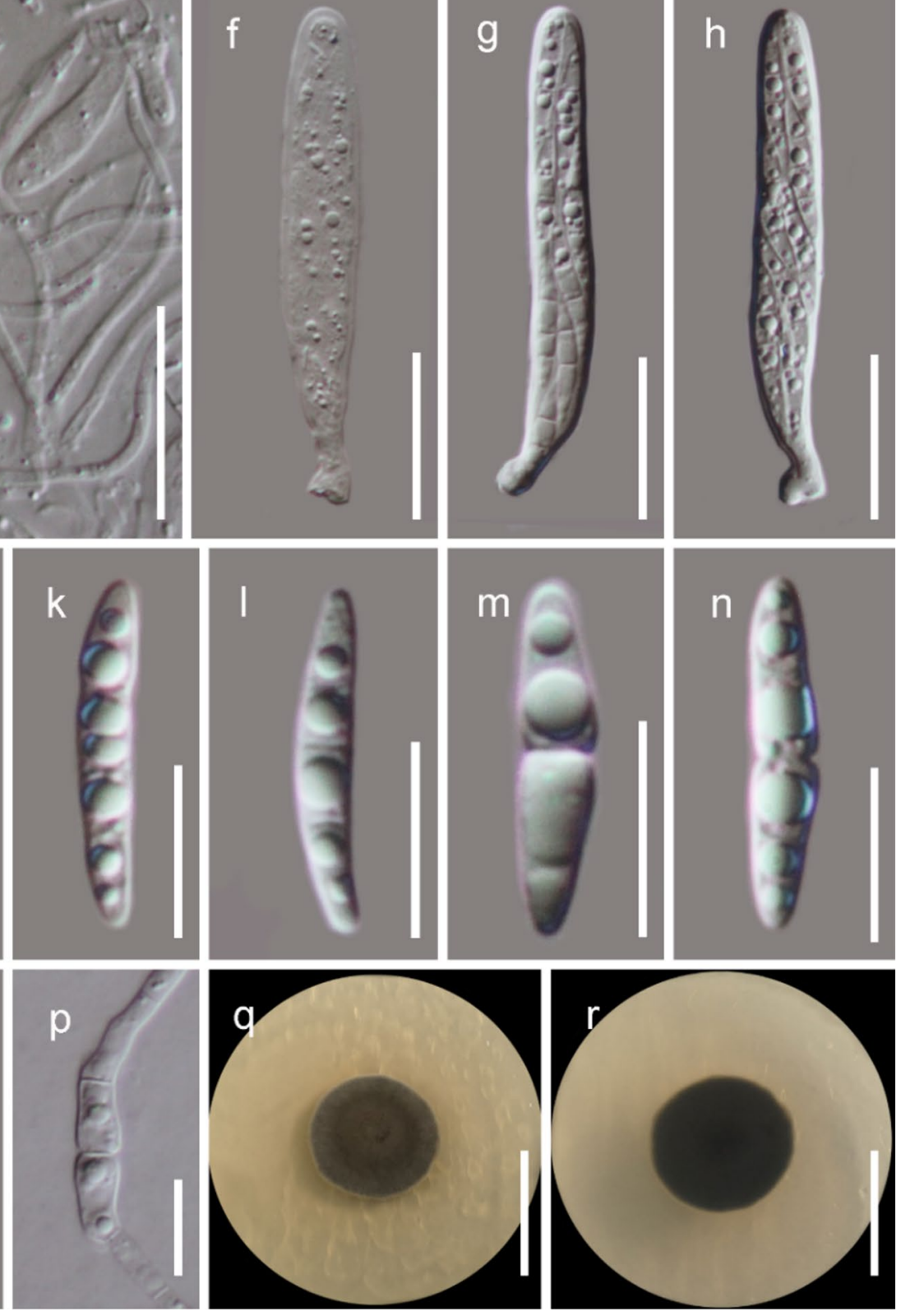

Fig. 48 Lophiotrema lincangensis (HKAS 115777, holotype). a, b Appearance of ascomata on host surface. c Longitudinal section of an ascoma. d Section of the peridium. e Pseudoparaphyses. $\mathbf{f}-\mathbf{i}$ Asci.

j-o Ascospores. p Germinated ascospore. q, $\mathbf{r}$ Culture characteristic on PDA ( $\mathbf{q}=$ from above, $\mathbf{r}=$ from below). Scale bars: $\mathbf{c}=100 \mu \mathrm{m}, \mathbf{d}$, $\mathbf{f}-\mathbf{i}=20 \mu \mathrm{m}, \mathbf{e}=5 \mu \mathrm{m}, \mathbf{j}-\mathbf{p}=10 \mu \mathrm{m}, \mathbf{q}, \mathbf{r}=10 \mathrm{~mm}$ 
50-80 $\mu \mathrm{m}$ long, 40-60 $\mu \mathrm{m}$ diam., carbonaceous, mostly central, minute papilla, with crest-like opening, filled with hyaline periphysate. Peridium $10-15 \mu \mathrm{m}$ wide at the base, 12-20 $\mu \mathrm{m}$ wide at sides, composed of flattened, angular, pseudoparenchymatous cells, outer layer, dark brown, thickwalled cells, inner layer, hyaline with thin-walled cells. Hamathecium composed of numerous, $1.5-2 \mu \mathrm{m}$ wide, filamentous, septate, branched, cellular pseudoparaphyses. Asci 80-120 $\times 8-9 \mu \mathrm{m}(\bar{x}=98 \times 8 \mu \mathrm{m}, \mathrm{n}=20), 8$-spored, bitunicate, fissitunicate, cylindrical, subsessile to short pedicellate, apically rounded, with a minute ocular chamber. Ascospores 20-30 $\times 3.5-4.5 \mu \mathrm{m}(\bar{x}=23 \times 4 \mu \mathrm{m}, \mathrm{n}=30)$, overlapping biseriate, fusiform, hyaline, straight or slightly curved, onecelled when young, becoming 3 -septate at maturation, the upper cell is longer than the lower cell, constricted at the septa, narrower towards both end cells, smooth-walled, with guttules. Asexual morph Undetermined.

Culture characteristics: Ascospores germinating on PDA within $24 \mathrm{~h}$ and germ tubes arising from both end cells. Colonies on PDA, reaching $4 \mathrm{~cm}$ diam. after 4 weeks of incubation at room temperature, initially white becoming dirty white to creamy, flattened, with a smooth edge, reverse dark brown, with pale grey margin.

Material examined: CHINA, Yunnan, Kunming, Xiaokong Mountain, $25.171311^{\circ} \mathrm{N} 102.703690^{\circ} \mathrm{E}$, on dead twigs of Prunus sp., 21 December 2019, CG Ren, KM007 HKAS 115779, new host record), living culture (KUMUCC 21-0037).

GenBank numbers: ITS = MZ493302, $\mathrm{LSU}=\mathrm{MZ493316,} \mathrm{SSU}=\mathrm{MZ493288,} \mathrm{RPB2}=\mathrm{MZ508420}$, TEF1- $\alpha=$ MZ508411.

Notes: Zhang et al. (2009) introduced Lophiotrema neoarundinariae comb. nov. to accommodate taxon which was previously described as Didymosphaeria arundinariae. Schoch et al. (2009a), Tanaka et al. (2009), Hirayama and Tanaka (2011) and Hashimoto et al. $(2016,2017)$ provided additional DNA based sequence data for this species. In this study, a newly collected taxon is identified as L. neoarundinariae based on multigene phylogenetic analysis and reported as a new host record on Prunus sp. from Yunnan, China (Figs. 49 and 50).

\section{Macrodiplodiopsidaceae Voglmayr, Jaklitsch \& Crous}

Notes: Macrodiplodiopsidaceae was introduced by Crous et al. (2015) to accommodate Macrodiplodiopsis and Pseudochaetosphaeronema in the suborder Massarineae. Tanaka et al. (2015) accepted the asexual genera Camarographium, Macrodiplodiopsis and Pseudochaetosphaeronema and Ariyawansa et al. (2015a) introduced Pseudomonodictys. However, Macrodiplodiopsis and Pseudochaetosphaeronema are currently accepted in Macrodiplodiopsidaceae (Wijayawardene et al. 2020). In this study, the sequences for phylogenetic analyses were downloaded from GenBank by following the latest treatment in Hyde et al. (2020b) and results of BLAST searches in NCBI. An updated phylogenetic tree is presented in Fig. 52. Our phylogenetic analysis is similar to that of Hyde et al. (2020b).

\section{Pseudochaetosphaeronema Punith.}

Notes: Pseudochaetosphaeronema was introduced by Punithalingam (1979) based on Pseudochaetosphaeronema larense. The whole genus was treated based on asexual morphology and sexual morphs were undetermined (Zhang et al. 2016b; Hyde et al. 2020b). The members of the genus can act as human pathogens, endophytes and saprobes (Ahmed et al. 2014; Zhang et al. 2016b; Hyde et al. 2020b). Six species epithets are listed in Index Fungorum (2021). In this study, we introduce a novel species of Pseudochaetosphaeronema collected from Thailand, and it is the first report of the sexual morph of the genus. The tree topology of our multigene phylogenetic analyses is similar to the latest analysis performed by Hyde et al. (2020b).

Pseudochaetosphaeronema chiangraiense Wijesinghe, Boonmee \& K.D. Hyde, sp. nov.

Index Fungorum number: IF558549; Facesofungi number: FoF 09950; Fig. 51

Etymology: The name reflects the location from which species was collected, Chiang Rai, Thailand.

Holotype: MFLU 21-0083

Saprobic on dead terrestrial branch of Tamarindus sp. Sexual morph Ascomata 190-255 $\mu \mathrm{m}$ high, 190-200 $\mu \mathrm{m}$ diam., $(\bar{x}=223 \times 197 \mu \mathrm{m}, \mathrm{n}=10)$, solitary, scattered, immersed, erumpent on host, uni-loculate, black, globose to subglobose. Peridium 13-17 $\mu \mathrm{m}$ wide, thin-walled, composed of several layers of small, brown to pale brown cells of textura angularis. Hamathecium comprising numerous, 2-4.5 $\mu \mathrm{m}$ wide, cellular, unbranched, pseudoparaphyses that are septate, without constrictions at the septa. Asci 50-110 $\times 15-30 \mu \mathrm{m}(\bar{x}=80 \times 18 \mu \mathrm{m}, \mathrm{n}=30), 8$-spored, bitunicate, fissitunicate, with obovoid, short distinct pedicel with rounded end, apex rounded with a minute ocular chamber. Ascospores $20-45 \times 7-8 \mu \mathrm{m}(\bar{x}=34 \times 7.5 \mu \mathrm{m}, \mathrm{n}=30)$, overlapping, 2-3-seriate, hyaline, fusiform, with pointed ends, 1 -septate at the center, constricted at the septa, guttulate, thick and smooth-walled. Asexual morph Undetermined.

Culture characteristics: Ascospores germinating on PDA within $24 \mathrm{~h}$. Germ tubes produced both sides of ascospore. Colonies on PDA reaching 1-2 cm diam. after 5 days in day light at $25{ }^{\circ} \mathrm{C}$, colonies medium dense, flat or effuse, from above, greenish gray in middle and pale brown at the margin, yellowish brown in reverse side.

Material examined: THAILAND, Chiang Rai Province, Nang Lae Village, on the dam of the pond near Lake Hill Resort, dead branch of Tamarindus sp. (Fabaceae),10 May 

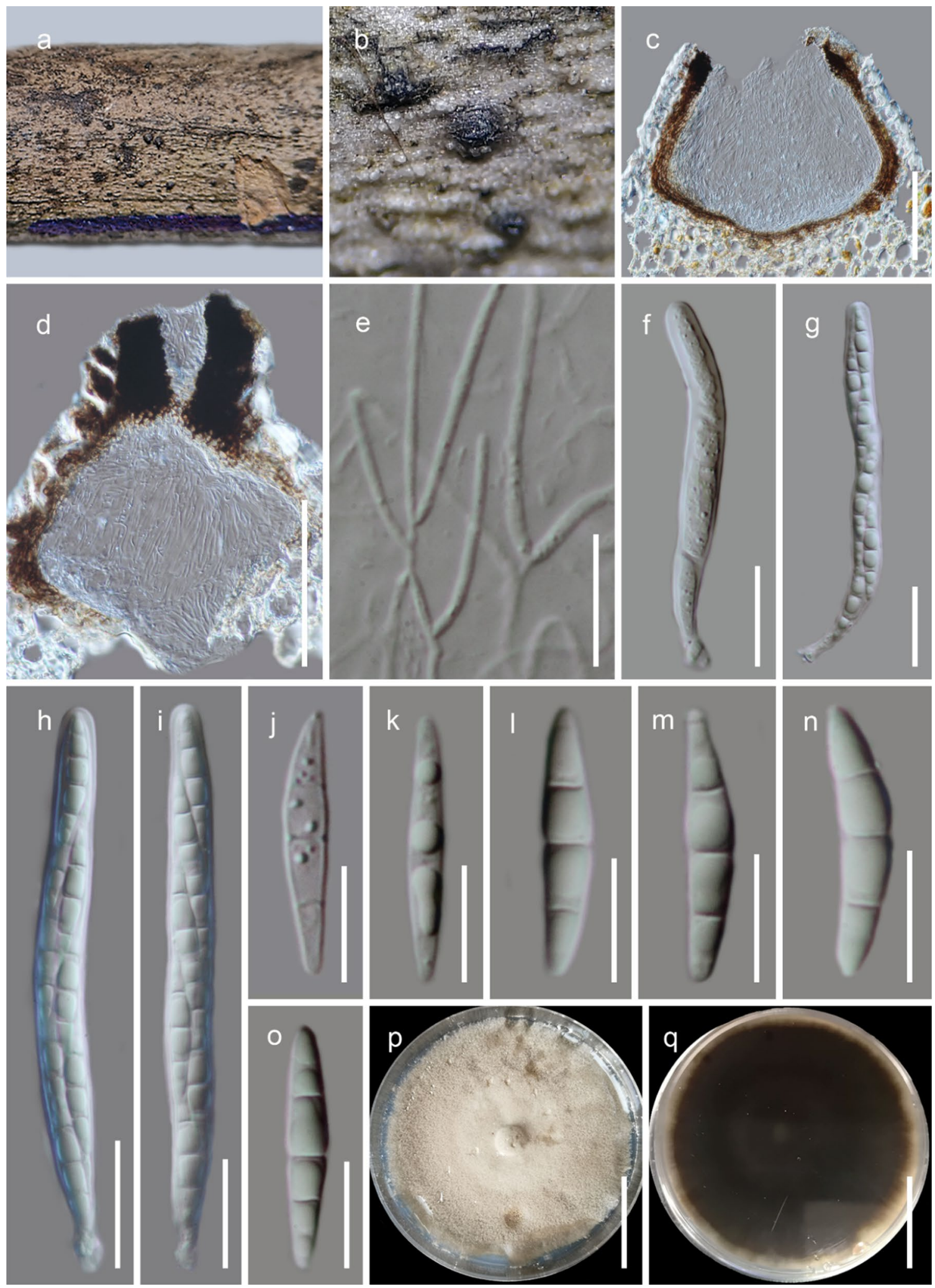

Fig. 49 Lophiotrema neoarundinariae (HKAS 115779, new record). a, b Appearance of ascomata on host surface. $\mathbf{c}$ Longitudinal section of an ascoma. d Section of peridium and papillate. e Pseu- doparaphyses. f-i Asci. $\mathbf{j}-\mathbf{o}$ Ascospores. p, q Culture characteristic on PDA ( $\mathbf{p}=$ from above, $\mathbf{q}=$ from below). Scale bars: $\mathbf{c}=100 \mu \mathrm{m}, \mathbf{d}$, $\mathbf{h}-\mathbf{i}=20 \mu \mathrm{m}, \mathbf{e}=5 \mu \mathrm{m}, \mathbf{j}-\mathbf{o}=10 \mu \mathrm{m}, \mathbf{p}, \mathbf{q}=10 \mathrm{~mm}$ 


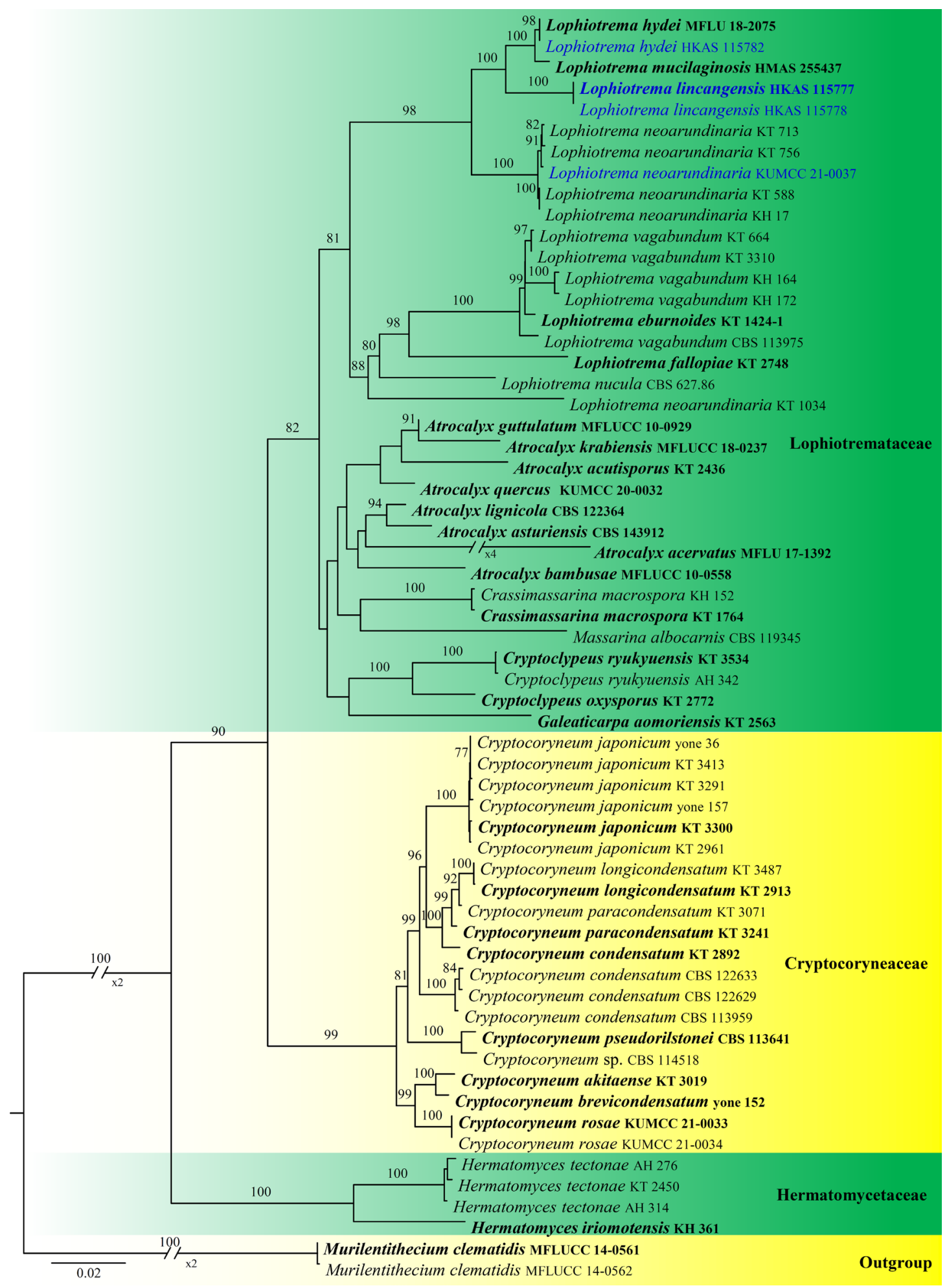

Fig. 50 Phylogram generated from maximum likelihood analysis based on combined SSU, LSU, ITS, TEF1- $\alpha$ and RPB2 sequence data to indicate the newly generated strains in Lophiotremataceae. Sixty strains are included in the combined analyses which comprise a total of 4357 characters. Murilentithecium clematidis (MFLUCC 14-0561 and MFLUCC 14-0562) is selected as the outgroup taxon. The best RAxML tree with a final likelihood value of -24211.323363 is presented. RAxML analysis yielded 1272 distinct alignment patterns and
$6.17 \%$ of undetermined characters or gaps. Estimated base frequencies were as follows: $\mathrm{A}=0.246394, \mathrm{C}=0.256619, \mathrm{G}=0.267068$, $\mathrm{T}=0.229919$, with substitution rates $\mathrm{AC}=1.576531, \mathrm{AG}=4.38051$, $\mathrm{AT}=1.49611, \quad \mathrm{CG}=1.339907, \quad \mathrm{CT}=10.502267, \quad \mathrm{GT}=1.000000$; gamma distribution shape parameter alpha $=0.450773$. Bootstrap support values for maximum likelihood (MLBS, left) equal to or greater than $70 \%$ is given above the nodes. Ex-type strains are in bold and newly generated sequences are in blue 

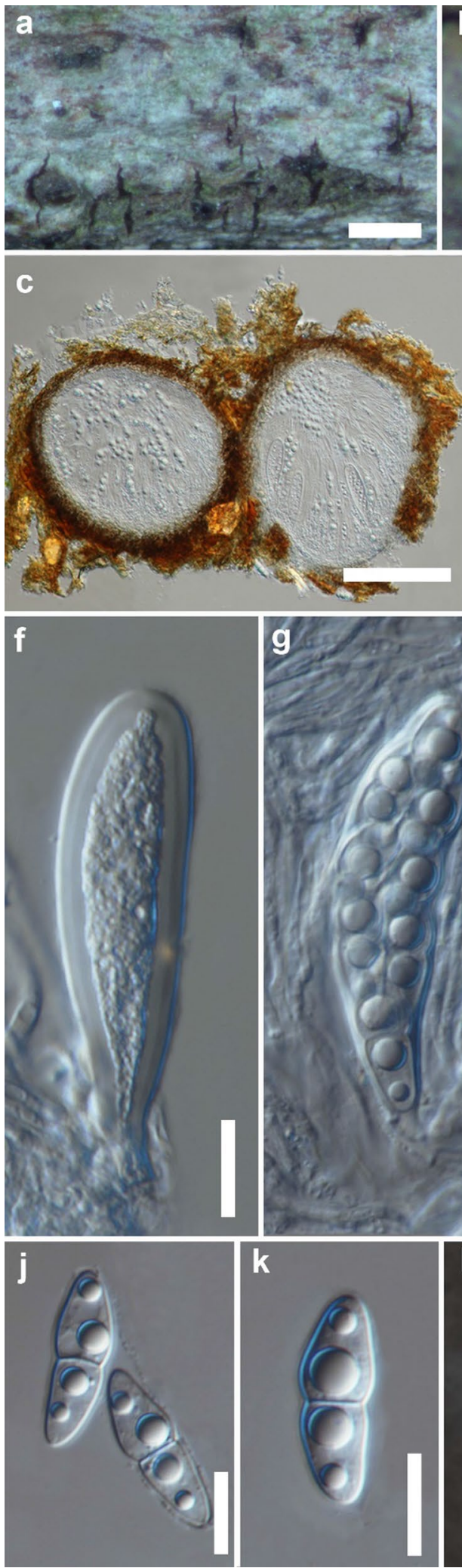
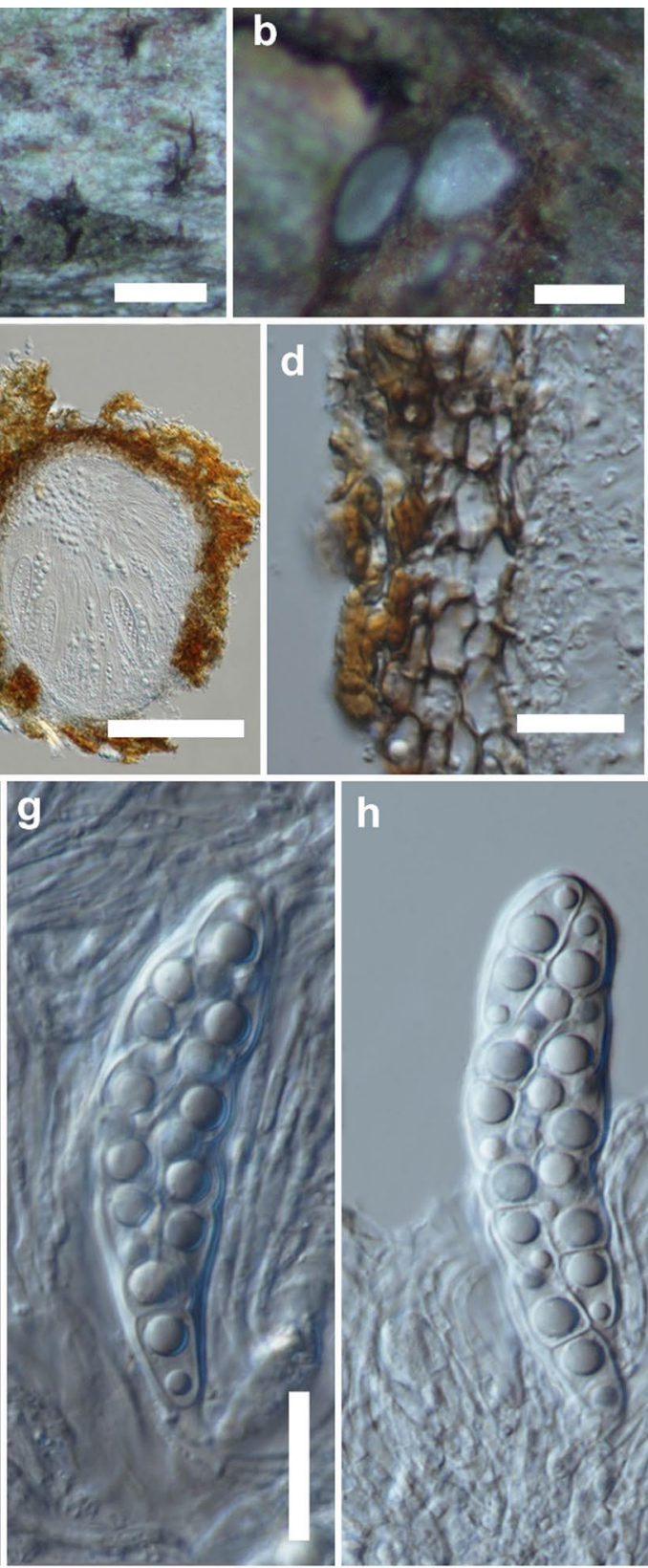

h
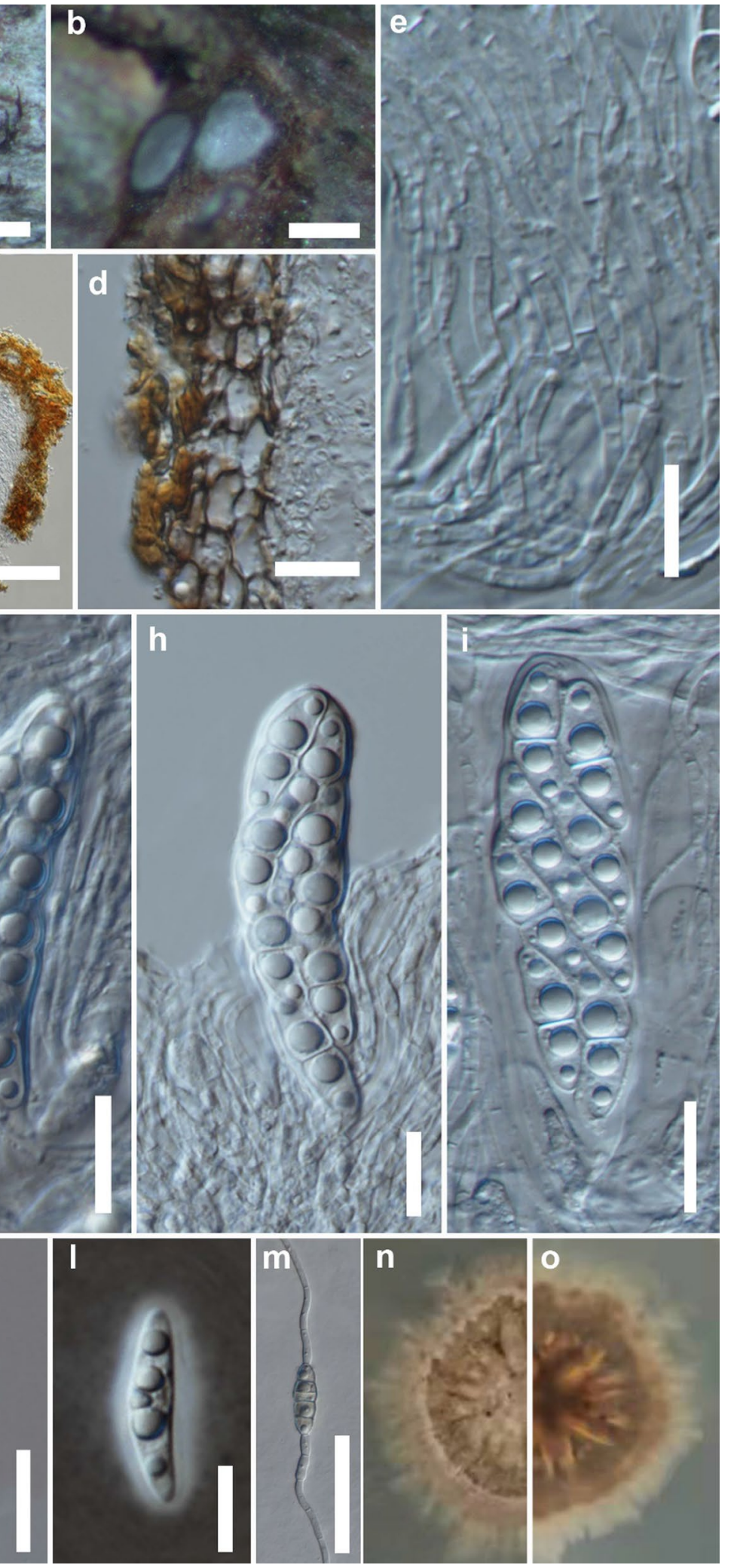

n

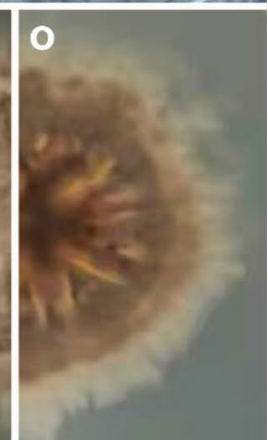

Fig. 51 Pseudochaetosphaeronema chiangraiense (MFLU 21-0083, holotype). a, b Appearance of ascostromata on host substrate. c Longitudinal section of ascomata. d Peridium e Pseudoparaphyses. f-i Asci. j-l Ascospores. m Germinated ascospore. $\mathbf{n}, \mathbf{o}$ Cultures on PDA from surface and reverse. Scale bars: Scale bars: $\mathbf{a}=500 \mu \mathrm{m}$, $\mathbf{b}=200 \mu \mathrm{m}, \mathbf{c}=100 \mu \mathrm{m}, \mathbf{m}=50 \mu \mathrm{m}, \mathbf{e}-\mathbf{k}=20 \mu \mathrm{m}, \mathbf{d}, \mathbf{l}=10 \mu \mathrm{m}$ 
2020, S.N. Wijesinghe, N2 (MFLU 21-0083, holotype), extype living culture, MFLUCC 21-0070.

GenBank numbers: ITS = MZ457923, LSU = MZ457922, TEF1- $\alpha=$ MZ476770.

Notes: In our DNA sequence analysis, all reported members of Pseudochaetosphaeronema and closest Camarographium strains are grouped together in Clade A in Macrodiplodiopsidaceae (Fig. 52). Pseudochaetosphaeronema chiangraiense is only known from its sexual morph (Fig. 51) and therefore, we cannot compare it with the existing species in this genus with respect to their asexual characteristics (Punithalingam 1979; Ahmed et al. 2015; Zhang et al. 2016b; Tibpromma et al. 2018; Jayasiri et al. 2019; Hyde et al. 2020b). The BLAST searches of ITS and LSU sequence data for our new isolate indicates high similarity to Camarographium koreanum (CBS 117,159) and TEF1- $\alpha$ sequence data indicates high similarity to Camarographium sp. (1 NV-2015). Therefore, we include these strains in our final multigene phylogenetic analysis and our novel isolate forms a distinct lineage with an unidentified Camarographium sp. (B45, B46) with 88\% MLBS support (Clade A; Fig. 52). According to the guidelines of Jeewon and Hyde (2016) we have analysed nucleotide differences within the rRNA gene region for further clarification. When comparing the ITS (ITS1-5.8S-ITS2) region between our isolate with the B45 and B46 strains, there were 48 and 43 bp (11.11\% and $9.95 \%$ ) difference respectively from 432 nucleotides in ITS. Also, in comparison of the LSU gene region of our strain with B45 and B46 strains, we have revealed (13/774) bp (1.67\%) difference and 16/785 bp (2.03\%) difference respectively. According to these phylogenetic results we conclude our isolate is a new species of Pseudochaetosphaeronema. We introduce the species as Pseudochaetosphaeronema chiangraiense collected from Thailand.

\section{Massarinaceae Munk}

Notes: We follow the latest treatment and updated accounts of Massarinaceae in Hongsanan et al. (2020a).

\section{Helminthosporium Link}

Notes: Helminthosporium includes mostly saprobes and pathogens on a wide range of hosts and has a worldwide distribution (Voglmayr and Jaklitsch 2017; Tian et al. 2017; Zhao et al. 2018a). Currently, an estimated 223 species are accepted for Helminthosporium in Hongsanan et al. (2020a). Zhao et al. (2018a) provided a recent account of Helminthosporium with several new isolates. In this study, we introduce $H$. chiangraiensis as a new species and provide a supporting phylogenetic tree.

Helminthosporium chiangraiense Boonmee, Huanraluek \& K.D. Hyde, sp. nov.
Index Fungorum number: IF558539; Facesoffungi number: FoF 09192; Fig. 53

Etymology: The specific epithet "chiangraiense" refers to it discover in Chiang Rai Province.

\section{Holotype: MFLU 21-0064}

Saprobic on dead twigs. Sexual morph Undetermined. Asexual morph Hyphomycetous. Colonies 230-584 $\mu \mathrm{m}$ diam., effuse, dark brown to black, hairy. Mycelium immersed, forming stroma-like aggregations of dark brown pseudoparenchymatous cells. Conidiophores $168-304.5 \times 5.5-12(\bar{x}=232 \times 9 \mu \mathrm{m}, \mathrm{n}=20)$, mononematous, macronematous, caespitose, erect, cylindrical, straight to slightly flexuous, wide at the base, unbranched, dark brown, with apical pore, multi-septate, with dark scars, smooth-walled. Conidiogenous cells mono- to polytretic, integrated, terminal and intercalary, cylindrical, cicatrized, with dark scars, with distinct pores. Conidia 141-207 $\times 14-22(\bar{x}=161 \times 19 \mu \mathrm{m}, \mathrm{n}=20)$, solitary, obclavate, straight to slightly curved, wider below than apex, truncate and with dark scar at base, upper cell tapering towards long apex, cylindrical, apically rostrate and pale brown, 9-13-distoseptate, smooth-walled. Conidial secession schizolytic.

Culture characteristics: Conidium germinating on PDA within $24 \mathrm{~h}$ and germ tubes produced from both ends. Colonies on PDA reaching $20 \mathrm{~mm}$ diam., in 7 days at room temperature, within a month covering the Petri dish, effuse, hairy, mycelium radiating outwards, fimbriate edge, dense, dark. Mycelium superficial and partly immersed, dark brown.

Material examined: THAILAND, Chiang Rai Province, Muang, Thasud, Mae Fah Lung University, Botanical garden, on dead twigs of unidentified plant, 14 June 2019, N. Huanraluek, CRB1 (MFLU 21-0064, holotype), ex-type living culture, MFLUCC 21-0087.

GenBank numbers: ITS $=$ MZ538504, LSU $=$ MZ538538.

Notes: Helminthosporium chiangraiense is phylogenetically related to $H$. leucadendri, but is clearly distinguished based on morphology and phylogeny. Phylogenetic analysis of the combined LSU, SSU and ITS sequence data of $\mathrm{Hel}$ minthosporium taxa, our strain (MFLUCC 21-0087) clusters with the ex-type strain H. leucadendri (CBS 135133) with moderate support (0.95 BYPP; Fig. 54). Sequence comparison for the ITS region between $H$. chiangraiense (MFLUCC 21-0087) and H. leucadendri (CBS 135133) showed a $13.38 \%$ (76/568 bp) base pair difference (Jeewon and Hyde 2016). Helminthosporium chiangraiense is distinct from $H$. leucadendri in colony features and conidial shape, size and number of distosepta (Quaedvlieg et al. 2013; Figs. 98 and 99). Therefore, we introduce Helminthosporium chiangraiense as a new species (Fig. 53). 


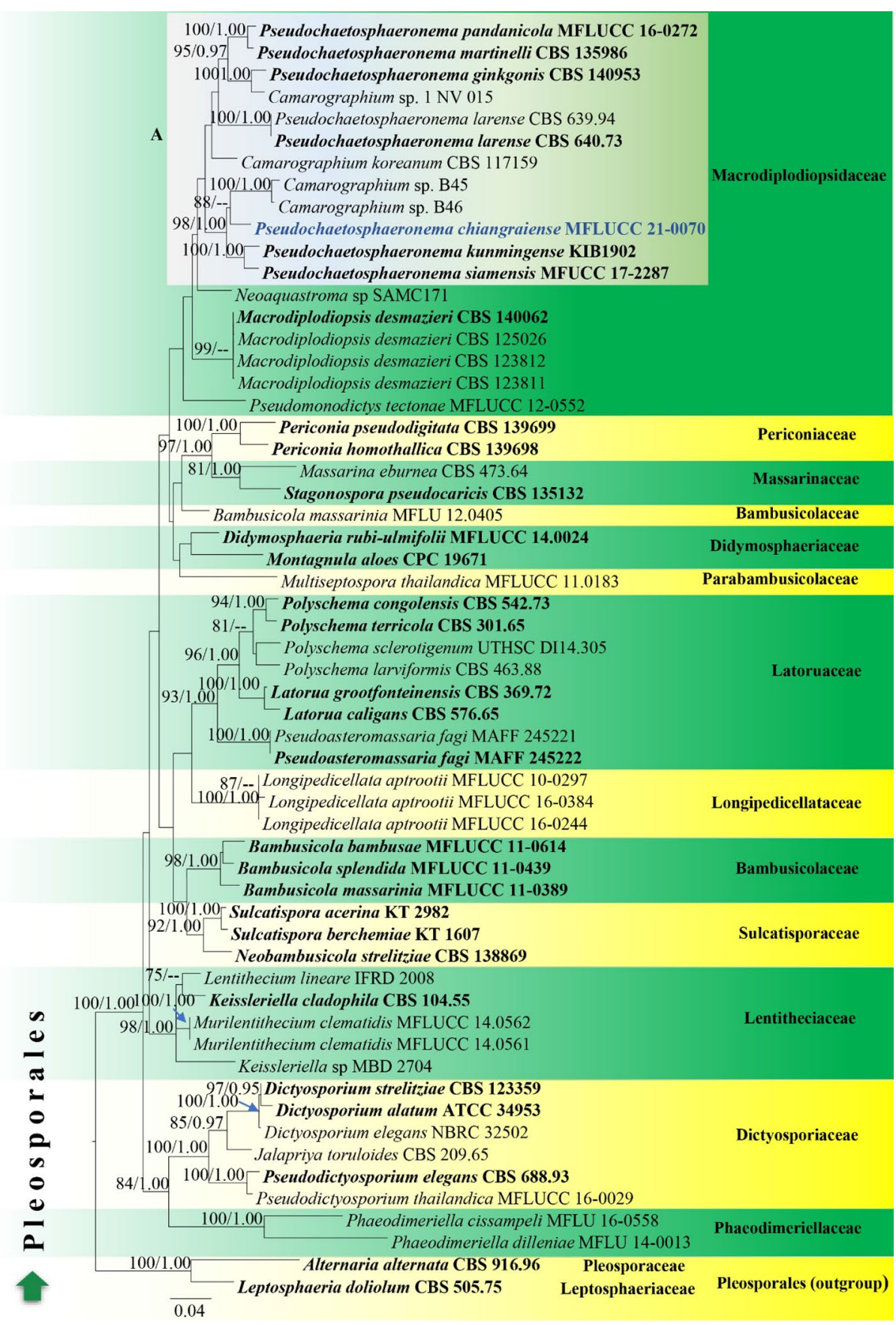

Fig. 52 Phylogram generated from maximum likelihood analysis based on combined LSU, SSU, ITS and TEF1- $\alpha$ sequence data representing suborder Massarineae in Pleosporales. Related sequences are taken from Hyde et al. (2020b) and additions according to the BLAST searches in NCBI. Fifty-eight strains are included in the combined analyses which comprised 3070 characters (798 characters for LSU, 961 characters for SSU, 469 characters for ITS, 842 characters for TEF1- $\alpha$ ) after alignment. Alternaria alternata (CBS 916.96) and Leptosphaeria doliolum (CBS 505.75) in Pleosporaceae and Leptosphaeriaceae respectively (Pleosporales) were used as the outgroup taxa. The best scoring RAxML tree with a final likelihood value of -18300.642497 is presented. The matrix had 1007 distinct alignment patterns, with $36.82 \%$ of undetermined characters or gaps. Estimated base frequencies were as follows: $\mathrm{A}=0.240587, \mathrm{C}=0.242773, \mathrm{G}=0.272823, \mathrm{~T}=0.243817$; substitution rates: $\mathrm{AC}=1.614921, \mathrm{AG}=3.258874, \mathrm{AT}=1.951323, \mathrm{CG}=1.249772, \mathrm{CT}=8.508461, \mathrm{GT}=1.000000$; gamma distribution shape parameter $\alpha=0.183977$. Bootstrap support values for ML equal to or greater than $75 \%$ are given above the nodes (left side). Bayesian posterior probabilities (BYPP) equal to or greater than 0.95 are given above the nodes (right side). Ex-type strains are in bold and newly generated sequence is in blue bold 


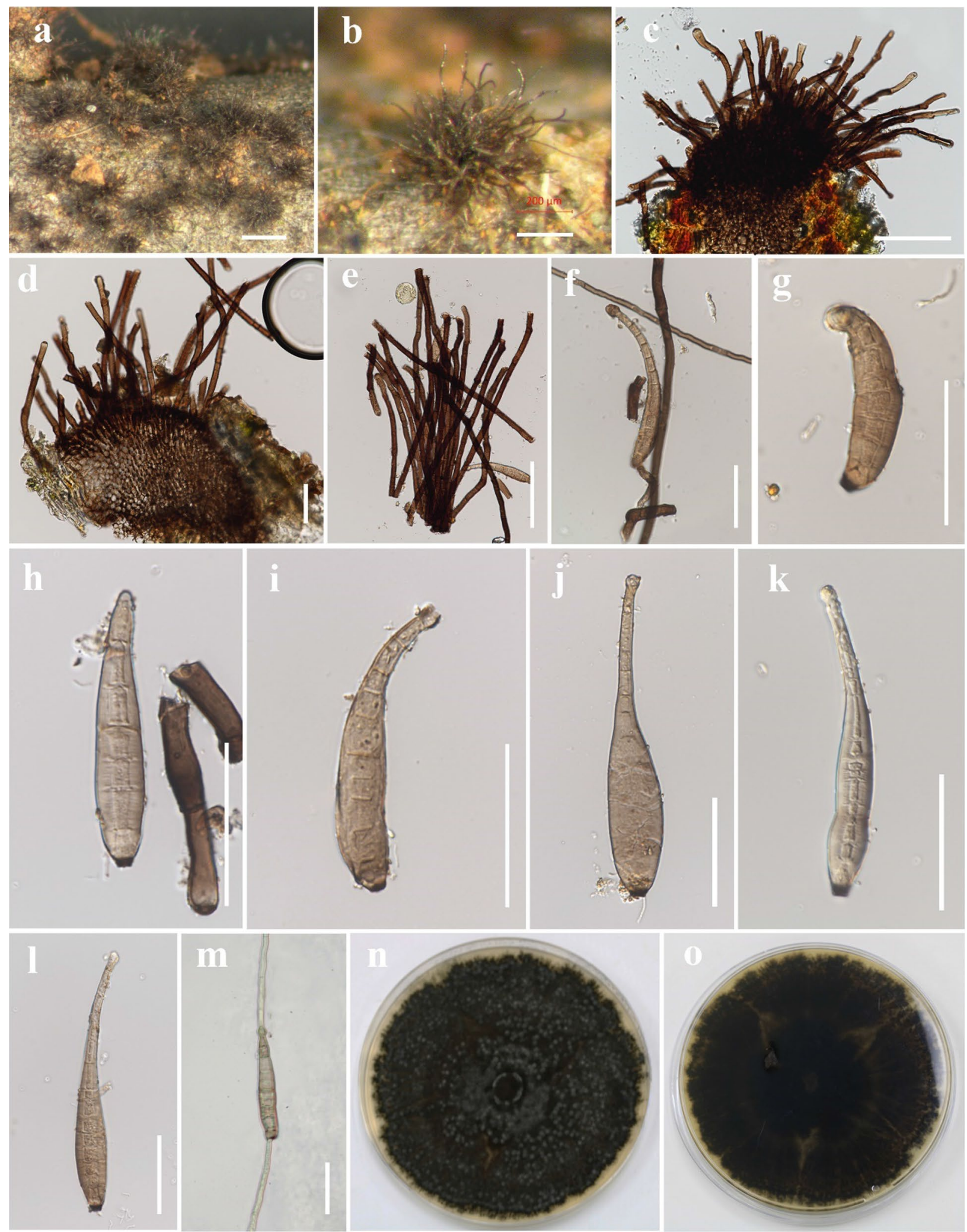

Fig. 53 Helminthosporium chiangraiense (MFLU 21-0064, holotype). a Colonies on natural substrate. b Punctiform conidioma. c Cross section of conidioma. d Cross section of conidioma cells. e Conidiophores. $\mathbf{f}$ Conidiophore with attached conidium. $\mathbf{g}-\mathbf{l}$ Conidia. m Germinated conidium. $\mathbf{n}$, o Culture on PDA from surface and reverse. Scale bars: $\mathbf{a}=500 \mu \mathrm{m}, \mathbf{b}=200 \mu \mathrm{m}, \mathbf{c}, \mathbf{e}=100 \mu \mathrm{m}, \mathbf{d}, \mathbf{f}-$ $\mathbf{m}=50 \mu \mathrm{m}$ 


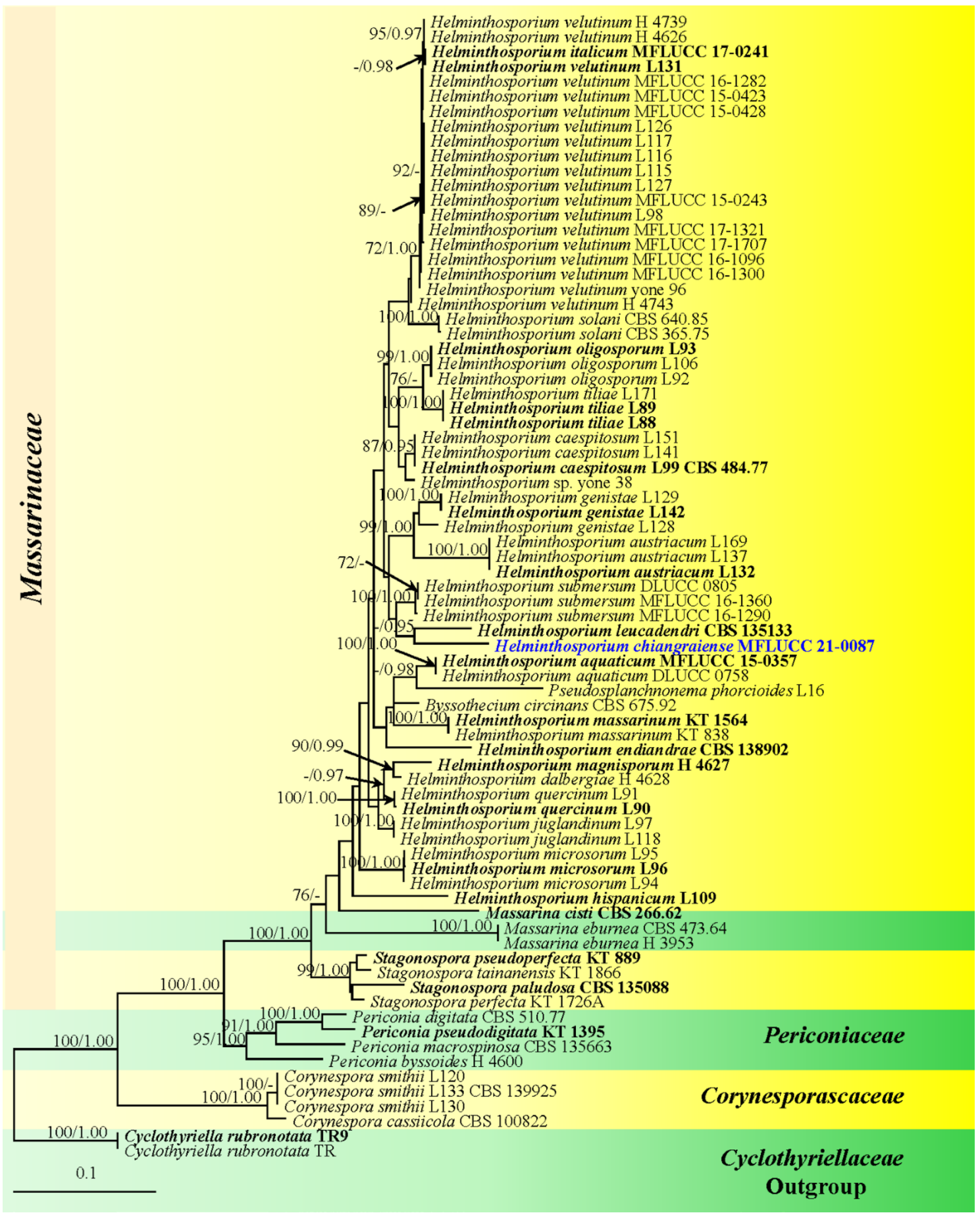

Fig. 54 Phylogram generated from maximum likelihood analysis based on combined LSU, SSU and ITS sequence data of $\mathrm{Hel}$ minthosporium taxa (Massarinaceae) and related families. Seventyseven strains are included in the combined analyses which comprise a total of 2567 characters. Cyclothyriella rubronotata (TR and TR9) are selected as the outgroup taxa. The best RAxML tree with a final likelihood value of - 11885.302979 is presented. RAxML analysis yielded 713 distinct alignment patterns and $23.08 \%$ of undetermined characters or gaps. Estimated base frequencies were as fol- lows: $\mathrm{A}=0.246572, \mathrm{C}=0.227244, \mathrm{G}=0.274051, \mathrm{~T}=0.252133$, with substitution rates $\mathrm{AC}=2.645156, \mathrm{AG}=3.449326, \mathrm{AT}=2.077592$, $\mathrm{CG}=1.025293, \mathrm{CT}=7.769520, \mathrm{GT}=1.000000$; gamma distribution shape parameter alpha $=0.143346$. Bootstrap support values for maximum likelihood (MLBS, left) equal to or greater than $70 \%$ is given above the nodes. Bayesian posterior probabilities (BYPP, right) equal to or greater than 0.95 are given above the nodes. Ex-type strains are in bold and newly generated sequence is in blue 
Nigrogranaceae Jaklitsch \& Voglmayr

Notes: Nigrogranaceae was erected by Jaklitsch and Voglmayr (2016) to accommodate three species of Nigrograna. The multigene phylogenetic analysis of a combined ITS, LSU, SSU, RPB2 and TEF1- $\alpha$ sequence data in Jaklitsch and Voglmayr (2016) revealed that Biatriospora marina (the type species of Biatriosporaceae) clusters with Nigrograna. Hence the latter study revamped Biatriosporaceae and established Nigrogranaceae. In addition, all the Biatriospora species were transferred in Nigrograna by Jaklitsch and Voglmayr (2016). However, it is unlikely that the strain of Biatriospora marina used in the analyses is this species and therefore Hongsanan et al. (2020a) maintained Biatriosporaceae as a distinct family. Currently Nigrogranaceae accommodates 16 species (both sexual and asexual morphs) with endophytic, human pathogenic and saprobic lifestyles (Hongsanan et al. 2020b; Zhang et al. 2020).

\section{Nigrograna Gruyter, Verkley \& Crous}

Notes: Nigrograna was introduced by de Gruyter et al. (2012) with $N$. mackinnonii as the type species. Jaklitsch and Voglmayr (2016) introduced three novel taxa (viz. $N$. mycophila, $N$. norvegica and $N$. obliqua) to the genus and also synonymized Melanomma fuscidulum (三 Sphaeria fuscidula) under Nigrograna as $N$. fuscidula. Currently the genus represents 16 species epithets in Index Fungorum (2021) which were recorded from a wide range of hosts in marine and terrestrial habitats (Hyde et al. 2017; Tibpromma et al. 2017b; Dayarathne et al. 2020). These existing taxa have also been confirmed by DNA sequence data in GenBank (Hongsanan et al. 2020b). Most of taxa in the genus Nigrograna exhibit saprobic lifestyles and also were recorded as human pathogens and endophytes with a cosmopolitan distribution (Kolař́ík 2018; Zhao et al. 2018b). Nigrograna is characterized by having black ascomata with clavate, short pedicellate asci and pale to chocolate brown, fusoid to narrowly ellipsoid, septate ascospores. Recently, Dayarathne et al. (2020) and Wanasinghe et al. (2020b) documented three taxonomic novelties (i.e. $N$. magnolia, $N$. samueliana and $N$. rhizophorae) in the genus. Nigrograna hydei and N. obtusispora are also included to the genus by Zhang et al. (2020).

Nigrograna jinghongensis Wanas. \& K.D. Hyde, sp. nov.

Index Fungorum number: IF558601; Facesoffungi number: FoF 09951; Fig. 55

Etymology: The specific epithet "jinghongensis" refers to the Jinghong City, Yunnan Province, China where the type was collected.

\section{Holotype: KUN-HKAS 115776}

Saprobic on woody litter. Sexual morph: Ascomata 300-400 $\mu \mathrm{m}$ high, $220-300 \mu \mathrm{m}$ diam. $(x=347 \times 259 \mu \mathrm{m}$, $\mathrm{n}=10$ ), perithecioid, solitary or gregarious, immersed to erumpent through host tissue, subglobose or obpyriform, uni-loculate, brown to dark brown, ostiolate. Ostiole mostly central. Peridium 10-15 $\mu \mathrm{m}$ wide at the base, $20-30 \mu \mathrm{m}$ wide in sides, composed of angular cells, outer layer, dark brown, thick-walled cells, inner layer, hyaline with thin-walled cells. Hamathecium composed of numerous, 1.5-2.5 $\mu \mathrm{m}$ wide, filamentous, septate pseudoparaphyses. Asci 60-90 $\times 7-9 \mu \mathrm{m}$ $(\bar{x}=77 \times 8 \mu \mathrm{m}, \mathrm{n}=20), 8$-spored, bitunicate, clavate to cylindric-clavate, short pedicellate, apically rounded, with a minute ocular chamber. Ascospores $12-15 \times 4-5.5 \mu \mathrm{m}$ ( $\bar{x}=14 \times 4.5 \mu \mathrm{m}, \mathrm{n}=30$ ), overlapping bi-seriate, ellipsoid, yellowish-brown to brown, 1-septate, slightly echinulate, guttulate. Asexual morph Undetermined.

Culture characteristics: Ascospores germinating on PDA within $24 \mathrm{~h}$ and germ tubes arising from both ends. Colonies on PDA, slow growing, reaching $2 \mathrm{~cm}$ diam. after 4 weeks of incubation at room temperature, initially white becoming dirty white to light grey at maturity, slightly effuse, radially with an undulate edge, greyish, reverse creamy.

Material examined: CHINA, Yunnan, Xishuangbanna Dai Autonomous Prefecture, Mengla County, Menglunzhen, $21.891084^{\circ} \mathrm{N} 101.305898^{\circ} \mathrm{E}$, on dead woody litter, 25 November 2020, D.N. Wanasinghe, DWX01-3 (KUN-HKAS 115776, holotype), ex-type living culture (KUMUCC 21-0035); ibid. DWX01-3-2 (KUN-HKAS 115775), living culture (KUMUCC 21-0036).

GenBank numbers: ITS = MZ493303, MZ493304, LSU $=$ MZ493317, MZ493318, SSU $=$ MZ493289, MZ493290, RPB2 = MZ508421, MZ508422, TEF1- $\alpha=$ MZ508412, MZ508413.

Notes: In the multigene phylogeny, Nigrograna jinghongensis (KUMUCC 21-0035 and KUMUCC 21-0036) clustered as a sister taxon to N. yasuniana and Nigrograna sp. (MFLUCC 17-2663) with 100\% MLBS support (Fig. 56). The sequence comparison between our new strain and $N$. yasuniana, ITS had $0.63 \%$, TEF1- $\alpha$ had $2.46 \%$ and RPB2 had $6.34 \%$ base pair variations. Nigrograna yasuniana was previously documented as an endophyte on Conceveiba guianensis (Euphorbiaceae) from Ecuador. Unfortunately, sufficient morphological data are not available for N. yasuniana to compare with our novel taxon. Based on its morphology and phylogenetic placement, we therefore, introduce Nigrograna jinghongensis as a new species (Fig. 55).

\section{Occultibambusaceae D.Q. Dai \& K.D. Hyde}

Notes: Occultibambusaceae is characterized by solitary, scattered, immersed, subglobose, dark brown, uni- or multiloculate ascostromata, bitunicate, fissitunicate, (6)-8-spored, shortly pedicellate, cylindrical to clavate asci, and 1-3-seriate, fusiform, sometimes asymmetric, hyaline or pale brown to dark brown, 1-3-septate ascospores with or without sheath (Doilom et al. 2016; Hyde et al. 2016; Jayasiri et al. 2016; Dai et al. 2017; Zhang et al. 2017; Tibpromma et al. 

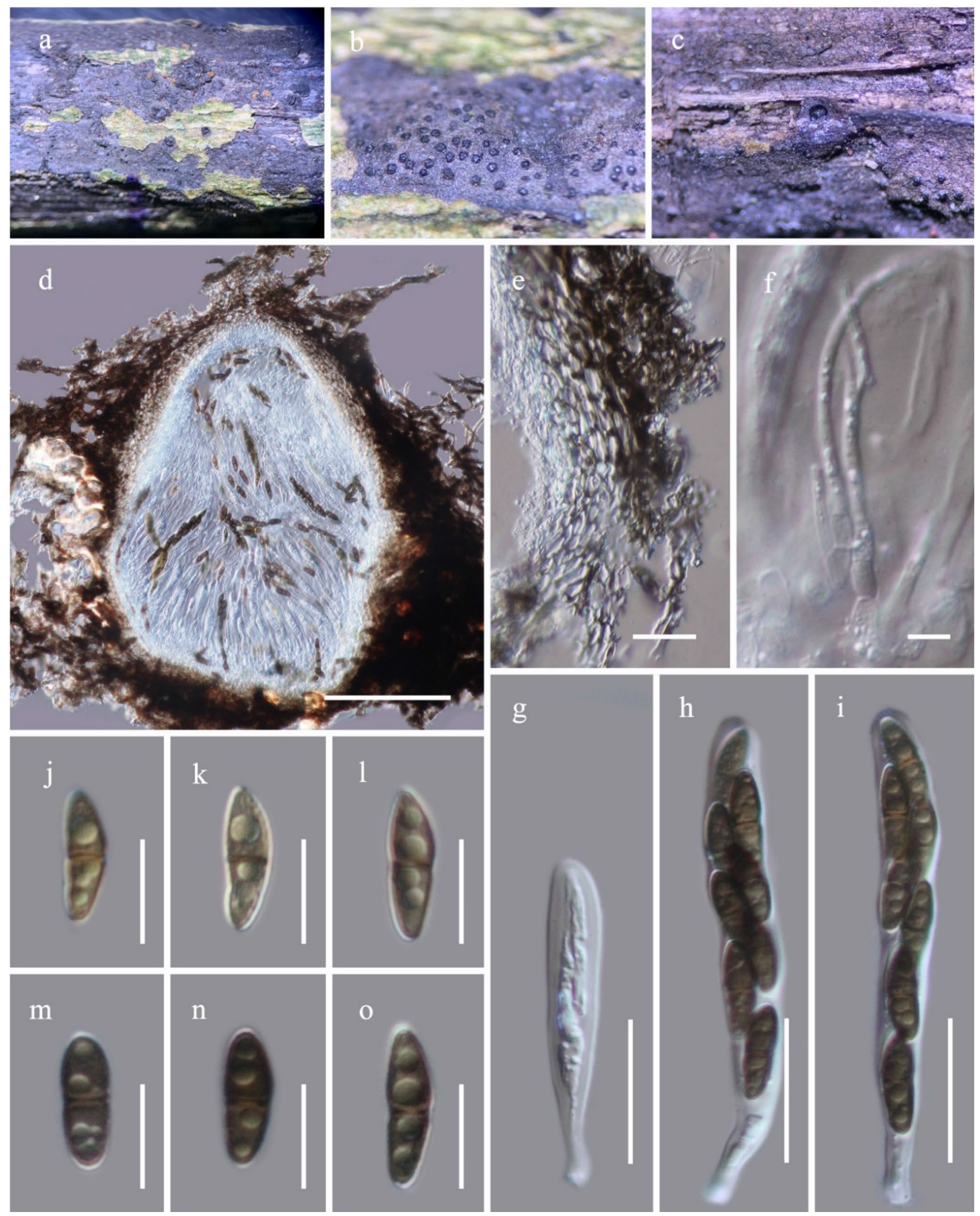

Fig. 55 Nigrograna jinghongensis (KUN-HKAS 115776, holotype). a-c Appearance of ascomata on host surface. d Longitudinal section of an ascoma. e Section of the peridium cells. f Pseudoparaphyses.

$\mathbf{g}-\mathbf{i}$ Asci. $\mathbf{j}-\mathbf{o}$ Ascospores. Scale bars: $\mathbf{d}=100 \mu \mathrm{m}, \mathbf{e}, \mathbf{g}-\mathbf{i}=20 \mu \mathrm{m}$, $\mathbf{f}=5 \mu \mathrm{m}, \mathbf{j}-\mathbf{o}=10 \mu \mathrm{m}$ 


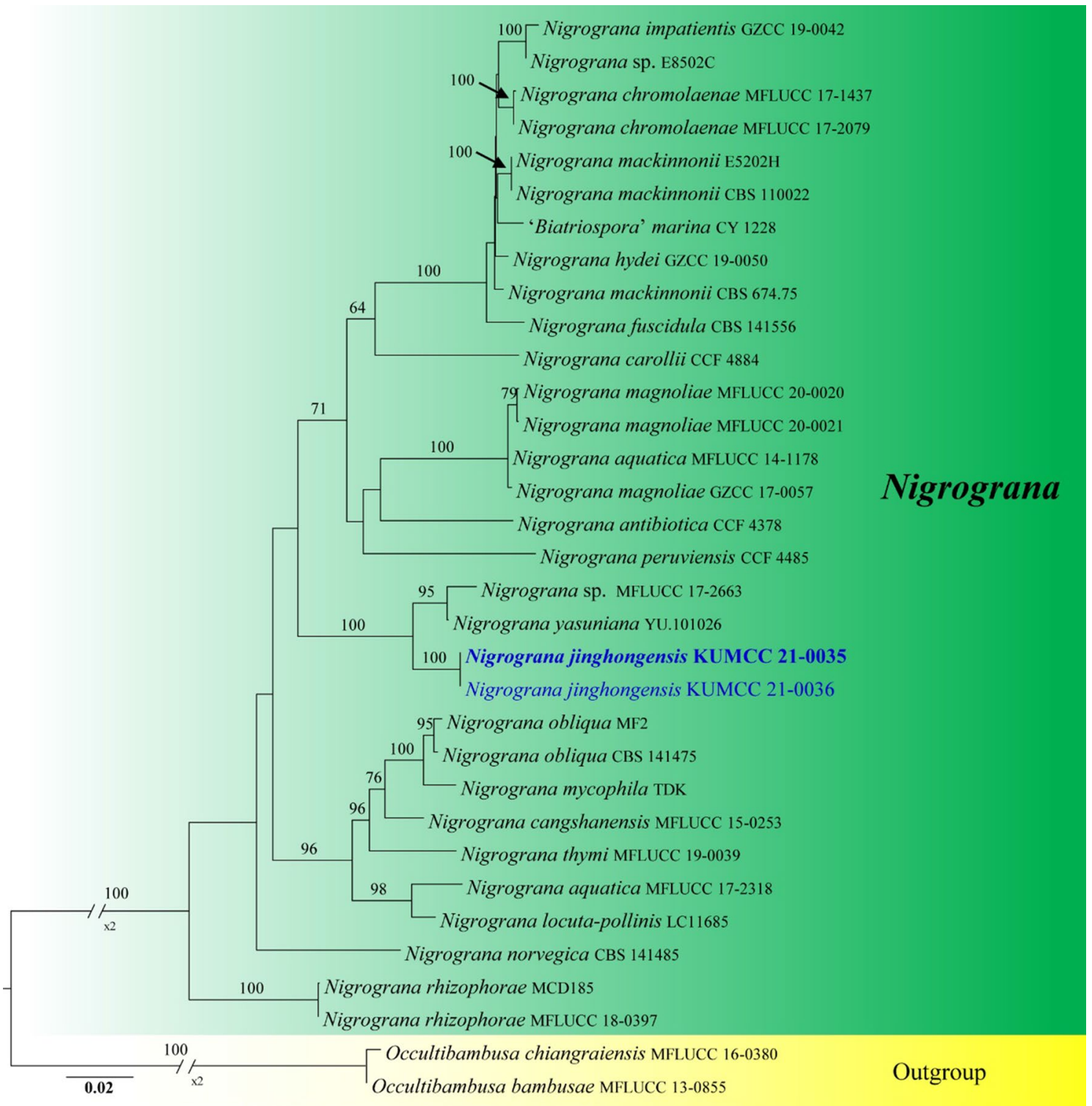

Fig. 56 Phylogram generated from the best scoring of the RAxML tree based on combined SSU, LSU, ITS, TEF1- $\alpha$ and RPB2 sequence dataset to indicate the new species Nigrograna jinghongensis and related species in Nigrogranaceae. Thirty-three strains are included in the combined analyses which comprise a total of 4372 characters. Occultibambusa bambusae (MFLUCC 13-0855) and O. chiangraiensis (MFLUCC 16-0380) are selected as the outgroup taxa. The best RAxML tree with a final likelihood value of -16022.187073 is presented. RAxML analysis yielded 1068 distinct alignment patterns

2018; Phookamsak et al. 2019; Rathnayaka et al. 2019; Dong et al. 2020b; Hongsanan et al. 2020a; Phukhamsakda et al. 2020). There are four genera accommodated in the family, viz. Brunneofusispora, Occultibambusa, Seriascoma and Versicolorisporium. Occultibambusa, Seriascoma and Versicolorisporium are reported to have coelomycetous asexual morphs (Hatakeyama et al. 2008; Dai et al. 2017), while and $26.01 \%$ of undetermined characters or gaps. Estimated base frequencies were as follows: $\mathrm{A}=0.24908, \mathrm{C}=0.245478, \mathrm{G}=0.267145$, $\mathrm{T}=0.238298$, with substitution rates $\mathrm{AC}=1.477319, \mathrm{AG}=4.369095$, $\mathrm{AT}=1.2688, \quad \mathrm{CG}=0.859595, \quad \mathrm{CT}=11.229756, \quad \mathrm{GT}=1.000000$; gamma distribution shape parameter alpha $=0.579103$. Bootstrap support values for maximum likelihood (MLBS, left) equal to or greater than $60 \%$ is given above the nodes. Ex-type strains are in bold and newly generated sequences are in blue

Neooccultibambusa forms chlamydospores in culture or exists as a hyphomycetous asexual morph (Doilom et al. 2016; Jayasiri et al. 2016; Hyde et al. 2018a). However, there have not been any asexual morphs of Brunneofusispora reported so far (Phookamsak et al. 2019; Phukhamsakda et al. 2020). 
Seriascoma Phookamsak, D.Q. Dai \& K.D. Hyde

Notes: Seriascoma is typified with Seriascoma didymospora. Two species are accommodated in the genus viz. $S$. didymospora and S. yunnanense (Hongsanan et al. 2020a; Species Fungorum 2021). The genus is characterized by solitary or gregarious, erumpent, subglobose or elongated, uni- or multi-loculate, coriaceous ascostromata embedded under a clypeus, bitunicate, fissitunicate, 8-spored, clavate asci with short to long furcate pedicel, and 1-3-seriate, fusiform, hyaline, asymmetric, 1-septate ascospores, slightly constricted at the septum, with or without a sheath (Dai et al. 2017; Rathnayaka et al. 2019). To date, asexual morphs of the genus have only been reported for $S$. didymospora which were characterized by eustromatic, solitary to gregarious, semi-immersed to erumpent, conical, black, uni-loculate conidiomata, enteroblastic, phialidic, determinate, cylindrical to ampulliform, hyaline, aseptate, smooth conidiogenous cells bearing oblong, hyaline, aseptate, smooth conidia (Dai et al. 2017). In the present study, we introduce a novel species in Seriascoma.

Seriascoma honghense H.B. Jiang, Phookamsak \& K.D. Hyde, sp. nov.

Index Fungorum number: IF558194; Facesoffungi number: FoF 09765; Fig. 57

Etymology: The specific epithet "honghense" refers to the collection site (Honghe County), of which the new species was collected.

Holotype: KUN-HKAS 112013

Saprobic on dead bamboo branches. Sexual morph Undetermined. Asexual morph Coelomycetous. Conidiomata pycnidial, 60-90 $\mu \mathrm{m}$ high, 320-510 $\mu \mathrm{m}$ diam., solitary to gregarious, immersed under cortex to superficial, raised, black, elongate-conical, 1- to multi-loculate, glabrous. Locules 45-300 $\mu \mathrm{m}$ diam., 30-70 $\mu \mathrm{m}$ high, clustered, arranged in rows, dark brown, subglobose to ampulliform. Peridium 12-20 $\mu \mathrm{m}$ thick, thin- to thick-walled, of unequal thickness, thick at the sides, thin at the base, composed of host and fungal tissue, with several layers of dark brown to brown, pseudoparenchymatous cells of textura angularis. Conidiophores reduced to conidiogenous cells. Conidiogenous cells 5.5-6.5 $\times 2-3 \mu \mathrm{m}(\bar{x}=6 \times 5 \mu \mathrm{m}, \mathrm{n}=20)$, enteroblastic, phialidic, determinate, discrete, subglobose or cylindrical to conical, hyaline, aseptate, smooth-walled. Conidia 4.5-5 $\times$ $2 \mu \mathrm{m}(\bar{x}=5 \times 2 \mu \mathrm{m}, \mathrm{n}=20)$, subglobose to oblong, hyaline, bi-guttulate, aseptate, smooth-walled.

Culture characteristics: Conidia germinating on PDA within $24 \mathrm{~h}$. Colonies growing fast on PDA, reaching $10 \mathrm{~mm}$ in 1 week at room temperature, under the normal light, cottony, circular, raised, grayish to dark brown from above and below. Mycelium superficial to immersed in media, with branched, septate, smooth hyphae.
Material examined: CHINA, Yunnan Province, Honghe Autonomous Prefecture, Honghe County, a roadside bamboo $\left(23^{\circ} 13^{\prime} 30.42^{\prime \prime} \mathrm{N}, 102^{\circ} 20^{\prime} 58.85^{\prime \prime} \mathrm{E}\right.$, altitude $1682 \mathrm{~m}$ ), on dead branches of bamboo in a terrestrial environment, 28 October 2020, H.B. Jiang, HONGHE008 (KUN-HKAS 112013, holotype); HMAS 249946 (isotype), ex-type living culture, KUMCC 21-0007.

GenBank numbers: ITS = MW981351, LSU = MW981347, SSU = MZ325471, RPB2 = MZ325473, TEF1- $\alpha=$ MZ325472.

Notes: Seriascoma honghense matches the typical characteristics of the coelomycetous asexual morph of Seriascoma (Fig. 57). However, it differs from Seriascoma didymospora in its multi-loculate, flat conidiomata and a thinner peridium. Based on the present phylogeny, S. honghense is determined to be a sister to $S$. didymospora with $98 \% \mathrm{ML}, 0.96 \mathrm{PP}$ statistical support (Fig. 58). Pairwise nucleotide comparison of ITS and TEF1- $\alpha$ also showed that $S$. honghense differs from $S$. didymospora in $36 / 483$ bp (7.45\%) and 26/947 bp $(2.75 \%)$, respectively.

Paradictyoarthriniaceae Doilom, Ariyaw., Bhat \& K.D. Hyde

Notes: Paradictyoarthriniaceae was introduced by Liu et al. (2015a) based on its unique morphology and phylogenetic placement. Members of this family include asexual and sexual morphs as saprobes in freshwater and terrestrial habitats (Matsushima 1996; Liu et al. 2015a, 2018; Wanasinghe et al. 2018). Only two genera have been identified, namely Paradictyoarthrinium and Xenomassariosphaeria are accommodated in this family (Wijayawardene et al. 2020).

\section{Paradictyoarthrinium Matsush.}

Notes: Matsushima (1996) introduced Paradictyoarthrinium to accommodate hyphomycetous taxon that occurs on a dead decaying spathe of Cocos nucifera from South Africa. The genus is characterized by superficial, gregarious, black, powdery fruiting bodies and macronematous conidiophores with unevenly dictyoseptate, muriform, subglobose to ellipsoidal dark brown conidia (Liu et al. 2015a). Presently, only four species have been reported in Paradictyoarthrinium (Index Fungorum 2021). In this study, we report Paradictyoarthrinium diffractum as a new record from a terrestrial habitat in Thailand.

Paradictyoarthrinium diffractum Matsush., Matsush. Mycol. Mem. 9:18 (1996)

Index Fungorum number: IF415849; Facesoffungi number: FoF 01854; Fig. 59

Saprobic on decaying wood. Sexual morph Undetermined. Asexual morph Colonies on natural substrate, 

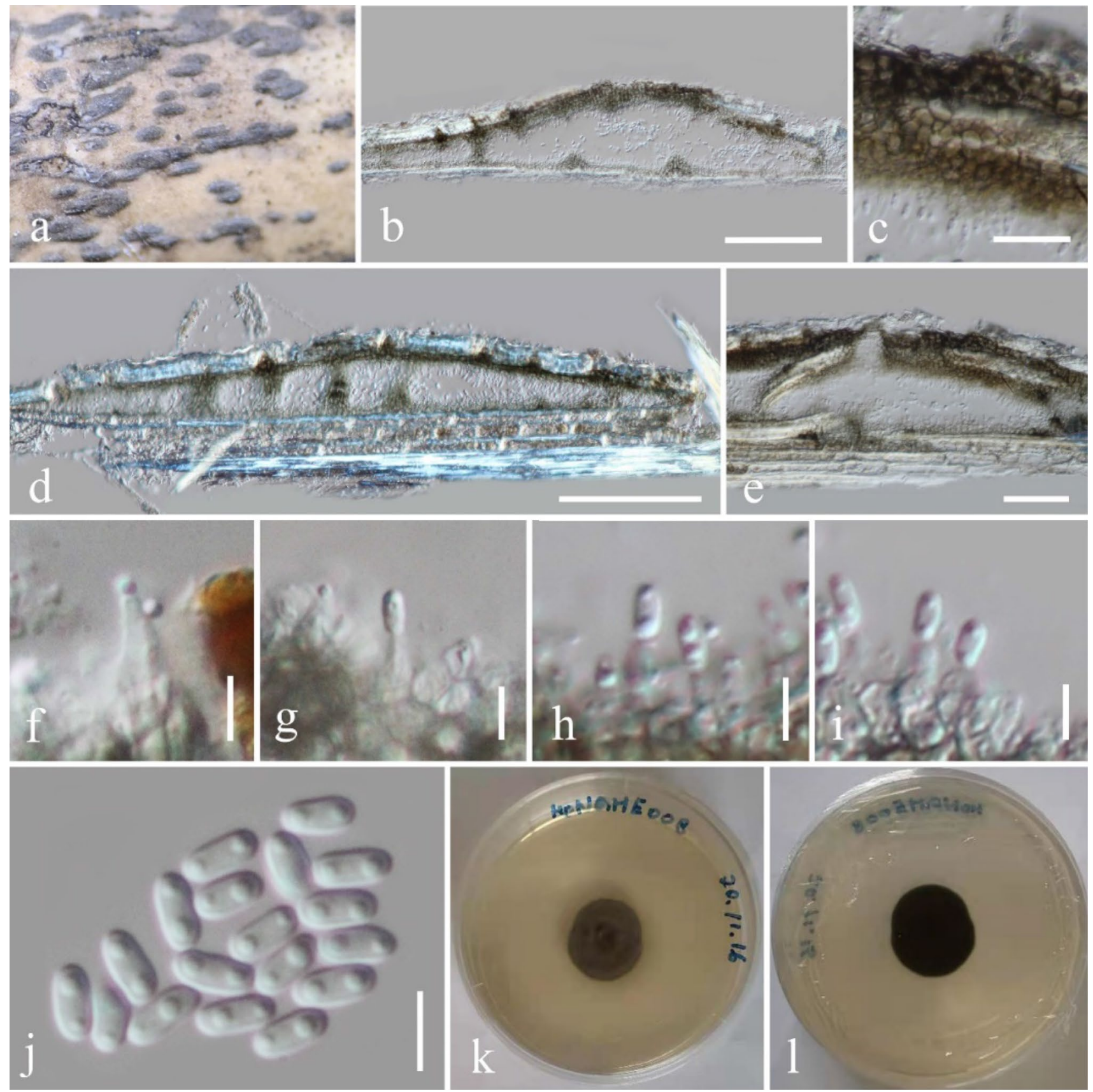

Fig. 57 Seriascoma honghense (KUN-HKAS 112013, holotype). a Conidiomata on surface of dead bamboo branches. b, d Vertical section of conidioma. $\mathbf{c}$ Wall of conidioma. e Ampulliform locule of

superficial, effuse, gregarious, scattered, black, shiny. Mycelium mostly superficial, partly immersed, composed of branched, septate, brown. Conidiophores 5-19 $\mu \mathrm{m}$ long, 4-5 $\mu \mathrm{m}$ wide, macronematous, erect to slightly curved, arising from hyphae. Conidiogenous cells blastic, integrated, terminal, determinate. Conidia $12-20 \times 11-19(x=15 \times 16 \mu \mathrm{m}$, $\mathrm{n}=10$ ), muriform, subglobose to irregular in shape, dark conidioma. $\mathbf{f}-\mathbf{i}$ Conidiogenous cells bearing conidia. $\mathbf{j}$ Conidia. $\mathbf{k}, \mathbf{l}$ Culture frontage and reverse. Scale bars: $\mathbf{b}, \mathbf{d}=100 \mu \mathrm{m}, \mathbf{e}=50 \mu \mathrm{m}$, $\mathbf{c}=20 \mu \mathrm{m}, \mathbf{f}-\mathbf{j}=5 \mu \mathrm{m}$

brown to black solitary, developing in branched chains, with 1-2 short chains, variable in shape and size.

Culture characteristics: Conidia germinating on PDA within $24 \mathrm{~h}$ and germ tubes produced around conidia. Colonies on PDA reaching $5 \mathrm{~cm}$ diam. after 4 weeks incubated at $25{ }^{\circ} \mathrm{C}$, superficial, dense and woolly, raised effuse, dull surface, velvety, olivaceous grey to dark grey, radiating outwards, entire edge. 


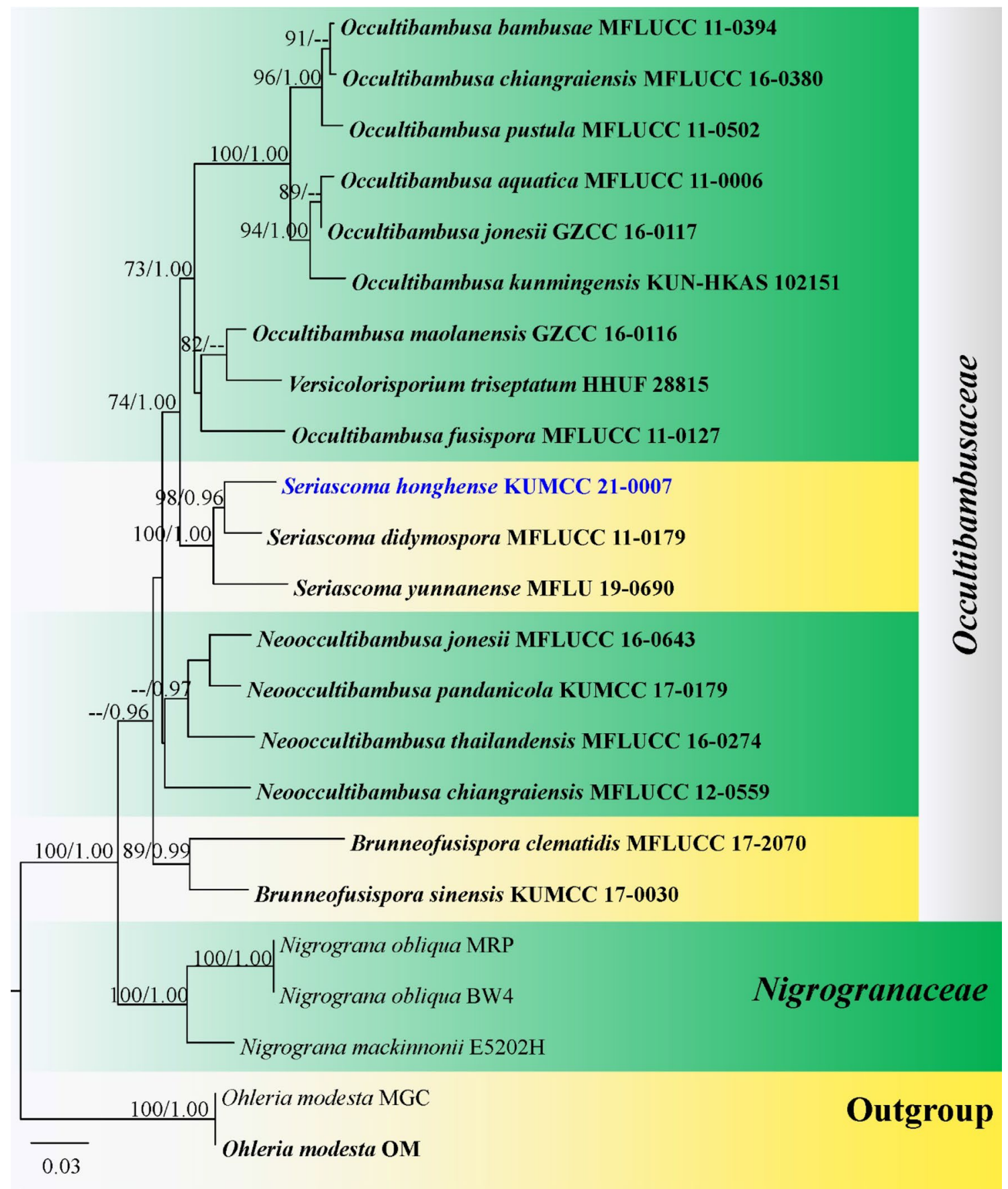

Fig. 58 Phylogram generated from maximum likelihood analysis based on combined ITS, LSU, SSU and TEF1- $\alpha$ sequence data. Twenty-three strains are included in the combined gene analyses comprising 3865 characters after alignment (643 characters for ITS, 853 characters for LSU, 1438 characters for SSU, 931 characters for TEF1- $\alpha$ ). Ohleria modesta (MGC and OM) is used as the outgroup taxon. The tree topology of the Bayesian analysis was similar to the maximum likelihood analysis. The best RAxML tree with a final likelihood value of -13136.916046 is presented. The matrix had 883 distinct alignment patterns, with $33.71 \%$ undetermined characters or gaps. Estimated base frequencies were as follows: $\mathrm{A}=0.241326$, $\mathrm{C}=0.251489, \quad \mathrm{G}=0.273232, \quad \mathrm{~T}=0.233953 ; \quad$ substitution rates $\mathrm{AC}=1.629497, \quad \mathrm{AG}=2.743632, \quad \mathrm{AT}=1.443415, \quad \mathrm{CG}=0.989741$, $\mathrm{CT}=7.278242, \mathrm{GT}=1.000000$; gamma distribution shape parameter $\alpha=0.150194$. Bootstrap values for maximum likelihood equal to or greater than $70 \%$ and Bayesian posterior probabilities equal or greater than 0.95 BYPP are placed above or below the branches. Ex-type strains are in bold and newly generated sequence is in blue 


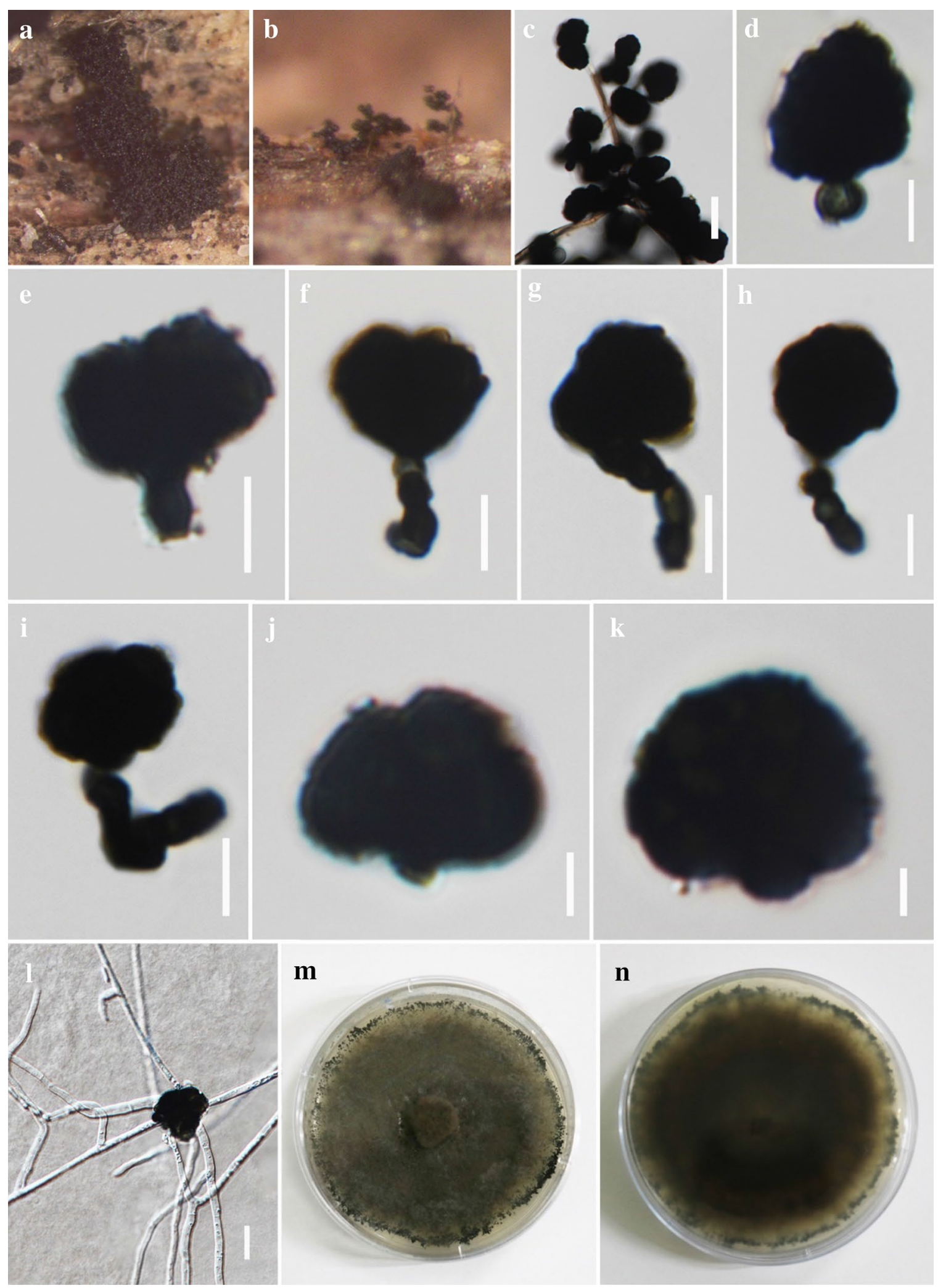

Fig. 59 Paradictyoarthrinium diffractum (MFLU 21-0065, new record). a, b Colonies on woody substrate. c Conidial masses arising from conidiogenous cells. $\mathbf{d}-\mathbf{i}$ Conidiogenous cells with attached conidia. $\mathbf{j}, \mathbf{k}$ Conidia. I Germinated conidium. $\mathbf{m}, \mathbf{n}$ Culture on PDA from surface and reverse. Scale bars: $\mathbf{c}=20 \mu \mathrm{m}, \mathbf{d}-\mathbf{i}, \mathbf{l}=10 \mu \mathrm{m}, \mathbf{j}$, $\mathbf{k}=5 \mu \mathrm{m}$ 
Material examined: THAILAND, Mukdahan Province, on decaying wood, 24 July 2019, S. Boonmee, WD10 (MFLU 21-0065, new record), living culture MFLUCC 21-0088.

GenBank numbers: ITS = MZ538520, LSU = $\mathrm{MZ538554,} \mathrm{SSU}=\mathrm{MZ538573}, \mathrm{RPB} 2=\mathrm{MZ567112}$, TEF1- $\alpha=$ MZ567097.

Notes: We collected a fungal strain from woody substrates in northeastern Thailand and it identified as Paradictyoarthrinium diffractum with the support of morphology (Fig. 59) and phylogenetic evidence (Fig. 60). Our isolate MFLUCC 21-0088 clustered among $P$. diffractum strains (MFLUCC 13-0466, MFLUCC 12-0557, 117F5C-AC, UFMG PEZEQ5, NFCCI-4665, KUMCC 19-0111, HUEFS 212651, BCC 8704, GUFCC 15514) with 82\% MLBS, 0.99 BYPP support. This is a new record of $P$. diffractum reported from northeastern Thailand. This may be a species complex.

\section{Phaeoseptaceae S. Boonmee, Thambug. \& K.D. Hyde}

Notes: Phaeoseptaceae (Pleosporales) was introduced as a monotypic family by Hyde et al. (2018a) with Phaeoseptum as the generic type. Members of Phaeoseptaceae are saprobic on dead wood in terrestrial and aquatic habitats (Hyde et al. 2018a; Liu et al. 2019; Phukhamsakda et al. 2020; Wanasinghe et al. 2020a). According to the treatment of Liu et al. (2019) and Phukhamsakda et al. (2020), we describe a novel species of Pleopunctum that is assigned to this family based on the evidence of phylogenetic analysis and morphological features.

\section{Pleopunctum N.G. Liu, K.D. Hyde \& J.K. Liu}

Notes: The hyphomycetous genus Pleopunctum was established in Phaeoseptaceae by Liu et al. (2019) to accommodate two species, namely, P. ellipticum (type species) and P. pseudoellipticum. Phukhamsakda et al. (2020) introduced the third Pleopunctum species from dead branches of Clematis sikkimensis. The genus is characterized by gregarious, superficial, brown colonies, mononematous, septate conidiophores, monoblastic conidiogenous cells, multi-septate, muriform and oval to ellipsoidal conidia (Liu et al. 2019; Phukhamsakda et al. 2020). In this study, morphological characteristics and multi-gene phylogenetic analysis of a combined LSU, ITS, SSU and TEF1- $\alpha$ sequence data reveals a new species of Pleopunctum from dead branches of unidentified plant collected in Thailand (Fig. 62).

Pleopunctum thailandicum J.Y. Zhang, Y.Z. Lu \& K.D. Hyde, sp. nov.

Index Fungorum number: IF558394; Facesoffungi number: FoF 09804; Fig. 61

Etymology: The specific epithet "thailandicum" reflects the country, where the specimen was collected, Thailand.

Holotype: MFLU 21-0043
Saprobic on dead branches of unidentified plant. Sexual morph Undetermined. Asexual morph Hyphomycetous. Colonies on natural substrate dry forming sporodochial conidiomata, superficial, scattered, gregarious, punctiform, glistening, oval. blackish brown. Mycelium immersed in the substrate, composed of septate, branched, hyaline to paleyellow. Conidiophores $2.5-5 \mu \mathrm{m}$ wide micronematous or macronematous, mononematous, cylindrical or truncate, erect, unbranched, hyaline to brown, sometimes reduced to conidiogenous cells. Conidiogenous cells, monoblastic, holoblastic, terminal, integrated, subspherical or ampulliform, hyaline. Conidia $29-38 \times 19-25 \mu \mathrm{m}(\bar{x}=34 \times 22 \mu \mathrm{m}$, $\mathrm{n}=25$ ), acrogenous, solitary, muriform, oval to ellipsoidal, multiseptate, slightly constricted at the septa, oval to ellipsoidal, thick-walled, smooth, brown to hyaline at upper and lower cells when immature, dark brown when mature, rounded at apex, truncate at base, sometimes with a hyaline, globose basal cell, 8-20 $\times 8.5-18.5 \mu \mathrm{m}(\bar{x}=13 \times 12 \mu \mathrm{m})$.

Culture characteristics: Conidia germinated on WA within $15 \mathrm{~h}$ at room temperature. Colonies reaching $15 \mathrm{~mm}$ at 2 weeks, convex, rough surface with edge entire, radiating outwards; reverse smooth, white to pale yellowish at the centre.

Material examined: THAILAND, Chiang Mai Province, Mae Taeng, Ki Lek, Chang Wat (1907'52.3" N 9845'35.7" E), on dead branches of unidentified plant, 8 August 2019, N. Wu, N17 (MFLU 21-0043, holotype); ex-type living culture, MFLUCC 21-0039.

GenBank numbers: ITS = MZ198894, LSU = MZ198896, TEF1- $\alpha$ : MZ172461.

Notes: The phylogenetic analysis reveals that a new species, Pleopunctum thailandicum belongs to Pleopunctum, where it is sister to P. clematidis with $100 \%$ MLBS, 1.00 BYPP support (Fig. 62). Pleopunctum thailandicum is similar to $P$. clematidis in having superficial and brown colonies, holoblastic, monoblastic conidiogenous cells and muriform, oval to ellipsoidal conidia (Liu et al. 2018; Phukhamsakda et al. 2020). However, $P$. thailandicum differs from $P$. clematidis by its black and larger conidia, and lacks dimorphic conidia (Fig. 61).

\section{Phaeosphaeriaceae M.E. Barr}

Notes: Members of Phaeosphaeriaceae are cosmopolitan and exhibit diverse life modes, mainly as saprobes, endophytes and pathogens of economically important plants as well humans (Phookamsak et al. 2014, 2017b; Hongsanan et al. 2020a). Apart from their cosmopolitan distribution, the family is morphologically and phylogenetically highly diverse. Thus, currently 83 genera are accommodated in this family (Hongsanan et al. 2020a). Species of Phaeosphaeriaceae are commonly found on monocotyledonous and herbaceous plants, as well as some other soft woody plants in both terrestrial and aquatic environments (Hongsanan 


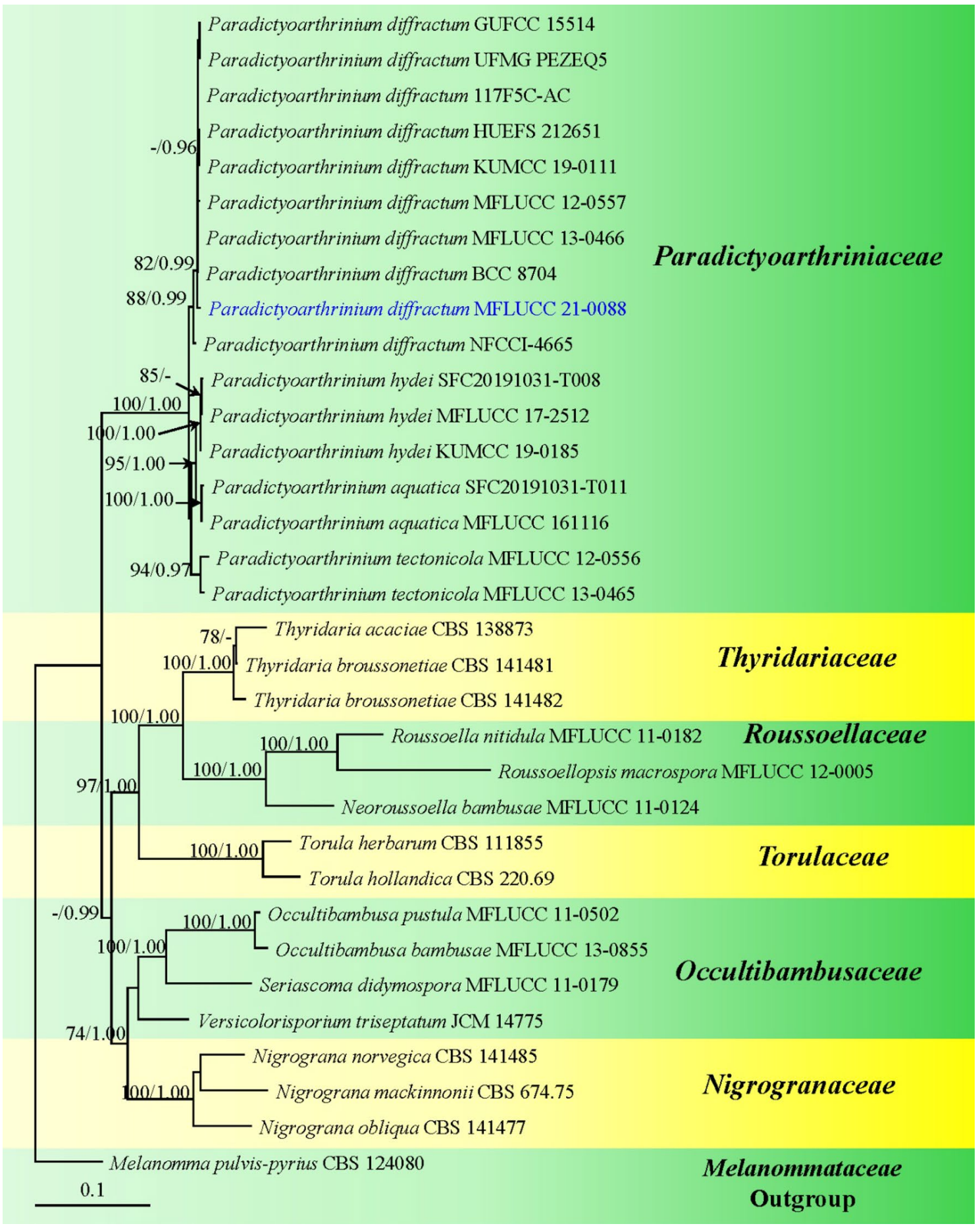

Fig. 60 Maximum likelihood phylogenetic tree based on a combined LSU, ITS, RPB2, SSU and TEF1- $\alpha$ sequence data of Paradictyoarthrinium taxa (Paradictyoarthriniaceae) and related families. The tree is rooted with Melanomma pulvis-pyrius (CBS 124080). Boot-

et al. 2020a). Some of the species in this family have an ability to produce secondary metabolites that are capable of antimicrobial activities (Mapook et al. 2020). strap support values for maximum likelihood (MLBS, left) equal to or greater than $70 \%$ is given above the nodes. Bayesian posterior probabilities (BYPP, right) equal to or greater than 0.95 are given above the nodes. Newly generated sequence is in blue
Phaeosphaeriopsis M.P.S. Câmara, M.E. Palm \& A.W. Ramaley

Notes: Phaeosphaeriopsis is a widely distributed group of phaeosphaeriacous fungi where species are defined primarily 

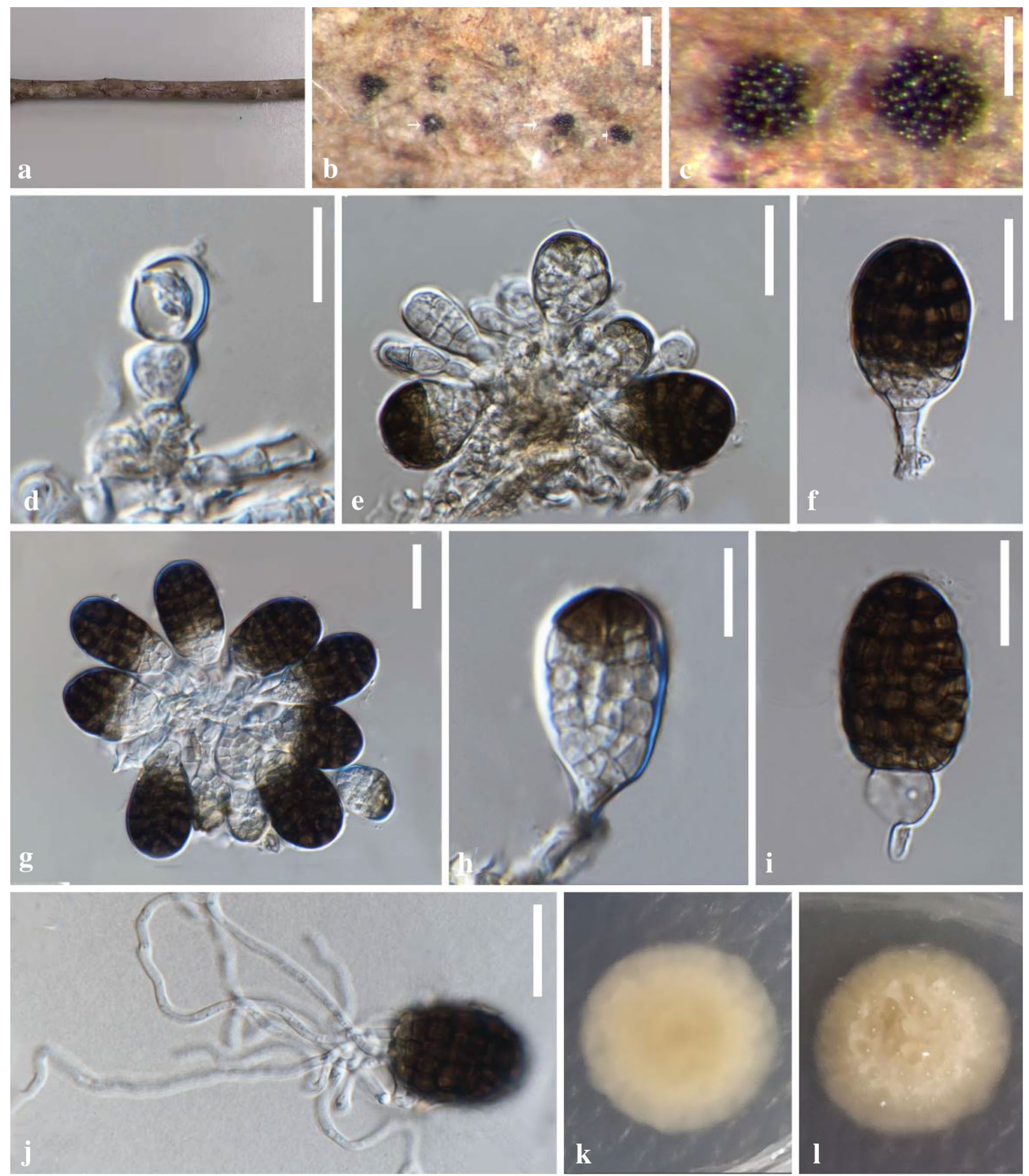

Fig. 61 Pleopunctum thailandcum (MFLU 21-0043, holotype). a-c Colonies on natural substrates. d, e Conidiophores with conidiogenous cells. $\mathbf{f}-\mathbf{h}$ Conidiogenous cells and conidia. i Conidium

based on immersed, subepidermal, globose to subglobose to pyriform ascomata, cylindric asci and vertically septate, punctate or verrucose ascospores with coniothyrium-like with basal hyaline cells. $\mathbf{j}$ Germinated conidium. $\mathbf{k}$, $\mathbf{l}$ Culture on PDA from surface and reverse. Scale bars: $\mathbf{b}=250 \mu \mathrm{m}, \mathbf{c}=100 \mu \mathrm{m}, \mathbf{e}-\mathbf{g}, \mathbf{i}$, $\mathbf{j}=20 \mu \mathrm{m}, \mathbf{d}, \mathbf{h}=10 \mu \mathrm{m}$

or phaeostagonospora-like asexual morphs (Câmara et al. 2003; Thambugala et al. 2014b). Currently, 17 species are accepted in this genus in Species Fungorum (2021). 


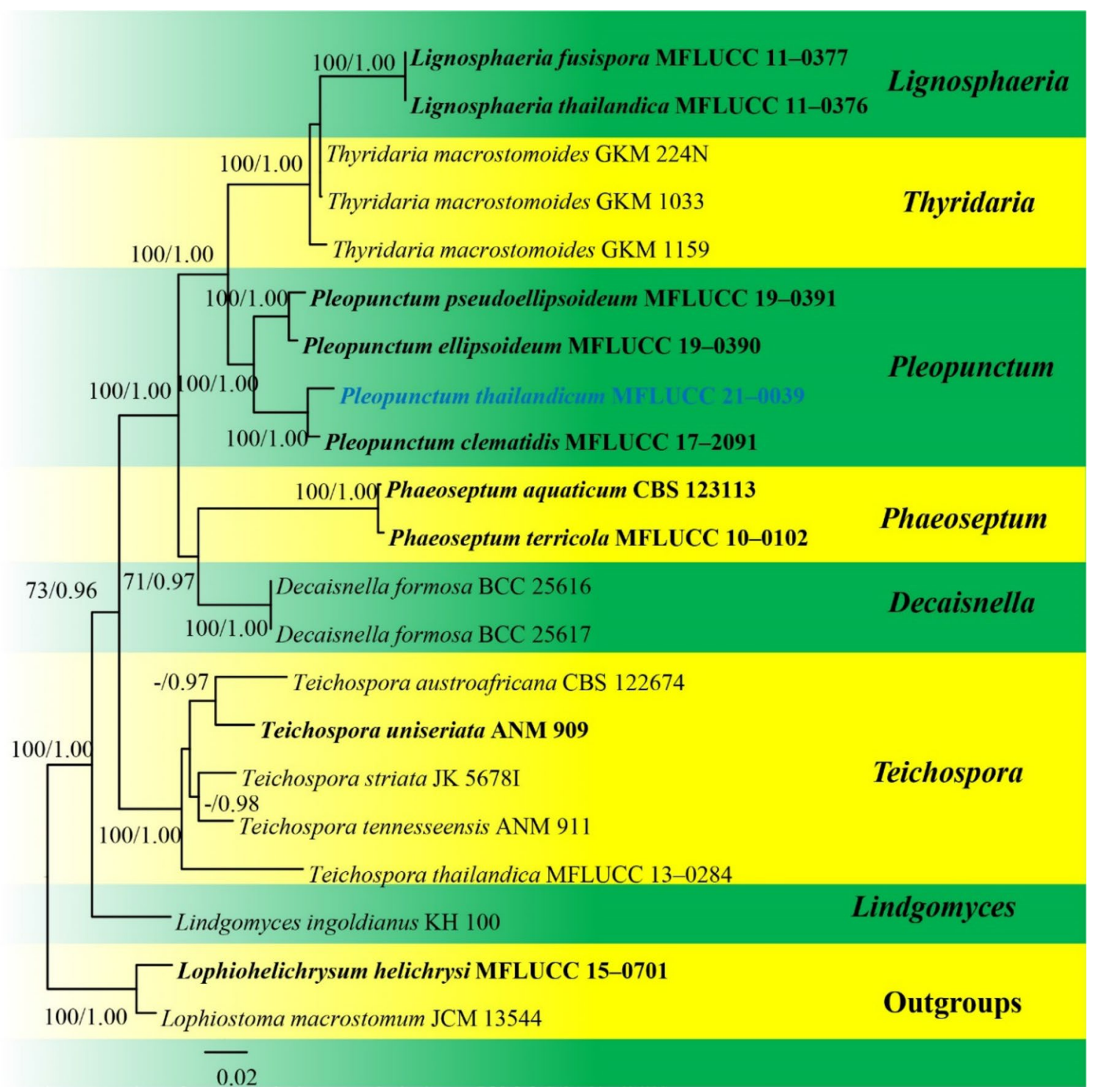

Fig. 62 Phylogram generated from Bayesian analysis based on combined LSU, SSU, ITS and TEF1- $\alpha$ sequence data. Twenty-one strains are included in the combined analyses which comprised 3338 characters (866 characters for LSU, 999 characters for SSU, 550 characters for ITS, 923 characters for TEF1- $\alpha$ ) after alignment. Tree topology of the maximum likelihood analysis is similar to the Bayesian analysis. The best RAxML tree with a final likelihood value of - 11657.841536 is presented. Estimated base frequencies were as

However, Thambugala et al. (2014b) synonymized Phaeosphaeriopsis musae under Phaeosphaeria musae and the accepted number of species in Phaeosphaeriopsis should therefore be 16 species. All Phaeosphaeriopsis species have available DNA sequence data for molecular comparisons, and the genus is monophyletic. The sexual morph of the majority of Phaeosphaeriopsis species is known and currently, P. agapanthi and P. aloes are known only from their coelomycetous asexual morph. Herein we report the sexual follows: $\mathrm{A}=0.239152, \mathrm{C}=0.258772, \mathrm{G}=0.277763, \mathrm{~T}=0.224314$; substitution rates $\mathrm{AC}=1.083541, \mathrm{AG}=2.347613, \mathrm{AT}=1.453520$, $\mathrm{CG}=1.144548, \mathrm{CT}=7.950168, \mathrm{GT}=1.000000$. Bootstrap support values for ML greater than $70 \%$ and Bayesian posterior probabilities greater than 0.95 are given near nodes respectively. The tree is rooted with Lophiohelichrysum helichrysi (MFLUCC 15-0701) and Lophiostoma macrostomum (JCM 13,544). Ex-type strains are in bold and newly generated sequence is in blue

morph of Phaeosphaeriopsis aloes from dead leaves of Yucca elephantipes in Yunnan, China.

Phaeosphaeriopsis aloes Crous \& Y. Marín, Marin-Felix et al., Stud. Mycol. 94: 61 (2019)

Index Fungorum number: IF829642; Facesoffungi number: FoF 09952; Fig. 63

Saprobic on dead leaves of Yucca elephantipes, visible as abundant black spots, scattered on entire leaf surface, Sexual 


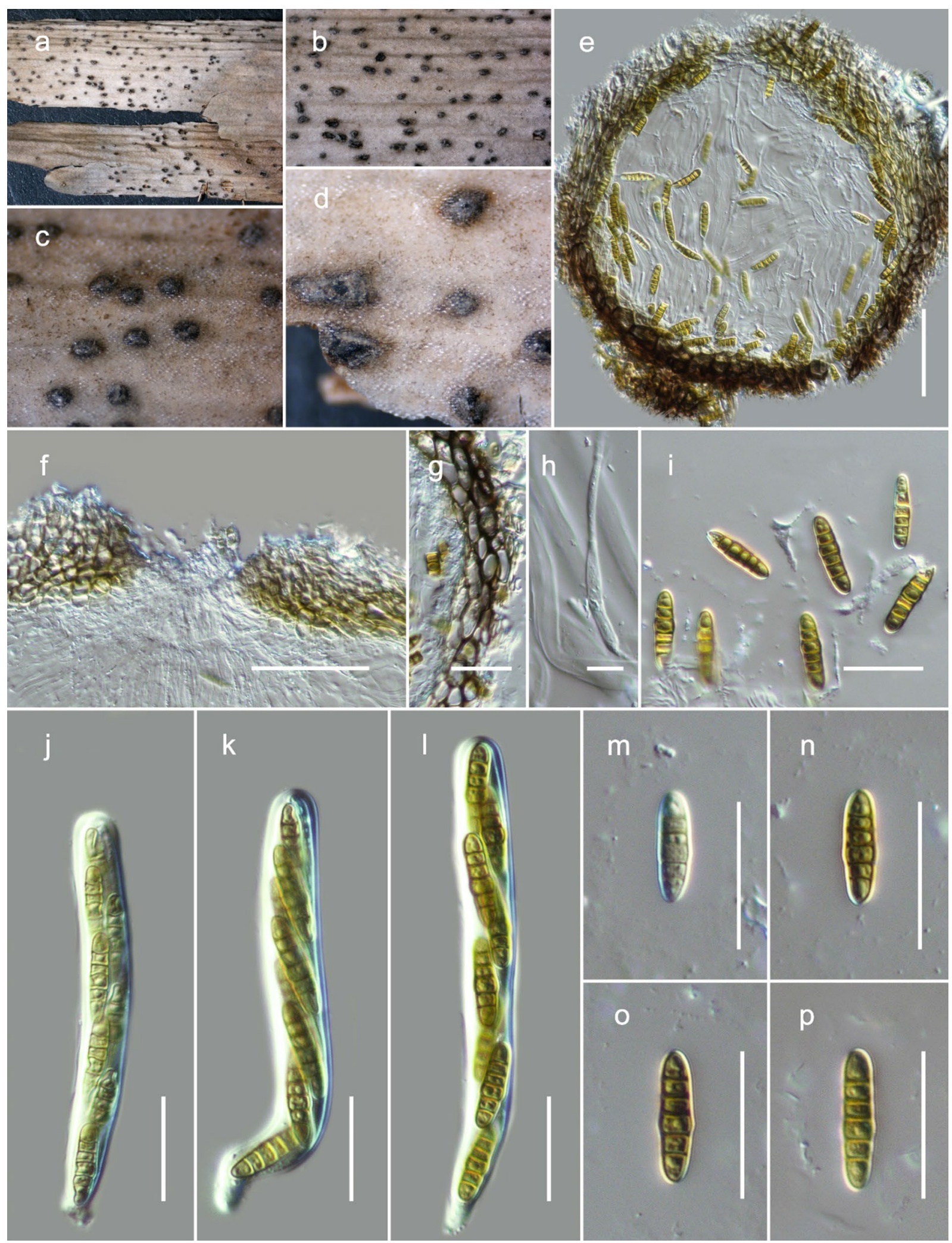

Fig. 63 Phaeosphaeriopsis aloes (KUN-HKAS 115783, new host and new geographical record). a-d Ascomata observed on host substrate. e Vertical section through an ascoma. f Close of an ostiole. $\mathbf{g}$
Cells of peridium. h Pseudoparaphyses. $\mathbf{j}-\mathbf{l}$ Asci. i, m-p Ascospores. Scale bars: d, $\mathbf{e}=50 \mu \mathrm{m}, \mathbf{g}, \mathbf{i}-\mathbf{p}=20 \mu \mathrm{m}, \mathbf{h}=10 \mu \mathrm{m}$ 
morph Ascomata 160-220 $\mu \mathrm{m}$ high, $140-180 \mu \mathrm{m}$ diam. $(\bar{x}=195.5 \times 169 \mu \mathrm{m}, \mathrm{n}=10)$, scattered, immersed, uniloculate, globose, black, papillate, ostiolate. Peridium $10-40 \mu \mathrm{m}$ wide, comprising two layers dark brown-walled cells of textura angularis, outer layer loosely arranged and inner layer compressed. Hamathecium of 1.5-2.5 $\mu \mathrm{m}$ wide, cellular, hyaline, septate, rarely branching, pseudoparaphyses anastomosing mostly above the asci, embedded in a mucilaginous matrix. Asci $80-100 \times 10-12 \mu \mathrm{m}(\bar{x}=93 \times 12 \mu \mathrm{m}$, $\mathrm{n}=20$ ), 8 -spored, bitunicate, fissitunicate, cylindrical, shortpedicellate, apically rounded, lacking an ocular chamber. Ascospores $16-22 \times 4-5 \mu \mathrm{m}(\bar{x}=19 \times 4.5 \mu \mathrm{m}, \mathrm{n}=25)$, overlapping biseriate, cylindrical, 3 -septate at young stage, becoming 5-septate when mature, without constriction or slightly constricted at the basal septum, fourth cell swollen, hyaline when immature, becoming yellowish brown at maturity, verrucose, prominent guttules in each cell, surrounded by a thick mucilaginous sheath. Asexual morph Coelomycetous (see Marin-Felix et al. 2019a, p. 61).

Material examined: CHINA, Yunnan Province, Heilongtan, Kunming Institute of Botany, $25.137711^{\circ} \mathrm{N}$ $102.745185^{\circ} \mathrm{E}$, on dead leaves of Yucca elephantipes (Asparagaceae), 2 February 2019, D.N. Wanasinghe, (KUNHKAS 115783, new host and new geographical record).

GenBank numbers: ITS = MZ493305; LSU = MZ493319; SSU $=$ MZ493291; TEF1- $\alpha=$ MZ508414.

Notes: Phaeosphaeriopsis aloes was introduced by Marin-Felix et al. (2019a) on leaves of Aloe sp. from USA, California. In this study we have acquired DNA from a sexual morph and in multi-gene phylogeny our strain and the ex-type strain of $P$. aloes (CBS 145,367) group in a wellsupported monophyletic clade (Fig. 64). Even though these two isolates are not derived from the same host or locality, there was only one bp difference in the comparison of the 518 nucleotides across the ITS regions. The TEF1- $\alpha$ gene region of CBS 145,367 is not available for further comparison. From these molecular perspectives, taxonomically, it would be wise to consider these two strains as belonging to a single species (Jeewon and Hyde 2016). The sexual morph is similar to Phaeosphaeriopsis agavensis, $P$. nolinae and $P$. obtusispora in terms of its ascospore characteristics (Câmara et al. 2003). These are however, not phylogenetically closely related (Fig. 64), therefore, we introduce our taxon as the sexual morph of Phaeosphaeriopsis aloes (Fig. 63). We could not manage to maintain a living culture as subsequent attempts to isolate failed, and hence a living culture is unavailable. Thus, we extracted DNA directly from the fruiting bodies.

\section{Pleosporaceae Nitschke}

Notes: Pleosporaceae is a species rich family that distributed worldwide, especially in tropical regions (Ariyawansa et al. 2015b). The species in this family are generally found as saprobes and endophytes on various plants, or opportunistic human, and plant pathogens (Hongsanan et al. 2020a). Hyphomycetous in the asexual morph and brown muriform ascospores in the sexual morph are typical of this family. Currently there are 23 accepted genera in Pleosporaceae. We follow the latest treatment of this family in Hongsanan et al. (2020a) and Wijayawardene et al. (2020).

\section{Comoclathris Clem.}

Notes: Comoclathris was introduced by Clements (1909) which is typified by Comoclathris lanata. The genus is accepted as a genus in Pleosporaceae (Hongsanan et al. 2020a; Wijayawardene et al. 2020). Currently there are 34 accepted species in this genus, but only 18 species have been confirmed by DNA based sequence data analyses (Hongsanan et al. 2020a). We report a new record of Comoclathris permunda on Achillea sp. in Uzbekistan for the first time.

Comoclathris permunda (Cooke) E. Müll., Monogr. Biol. Soc. Pakistan 8: 68 (1979) [1978]

Index Fungorum number: IF283403; Facesoffungi number: FoF 03231; Fig. 65

三Sphaeria permunda Cooke, Grevillea 5(no. 35): 111 (1877)

Saprobic on dead herbaceous branches. Sexual morph Ascomata $190-250 \mu \mathrm{m}$ high, $200-280 \mu \mathrm{m}$ diam. $(\bar{x}=226.5 \times 243 \mu \mathrm{m}, \mathrm{n}=10)$, semi immersed to erumpent, solitary, scattered, dark brown to black, coriaceous, cupulate when dry, with brown to reddish brown. Peridium 10-15 $\mu \mathrm{m}$ wide at the base, $15-30 \mu \mathrm{m}$ wide in sides, comprising two layers, outer layer heavily pigmented, thin-walled, comprising blackish to dark brown cells of textura angularis, inner layer composed of hyaline, thin-walled cells of textura angularis. Hamathecium comprising numerous, $2.5-4.5 \mu \mathrm{m}$ $(x=3 \mu \mathrm{m}, \mathrm{n}=20)$ wide, filamentous, branched, septate, pseudoparaphyses. Asci $80-120 \times 18-24 \mu \mathrm{m}(\bar{x}=103 \times 20 \mu \mathrm{m}$, $\mathrm{n}=20$ ), 8-spored, bitunicate, fissitunicate, cylindric-clavate, with a $10-15 \mu \mathrm{m}$ long pedicel, thick-walled at the apex, with a minute ocular chamber. Ascospores 20-30 $\times 8-12 \mu \mathrm{m}$ $(\bar{x}=25 \times 11 \mu \mathrm{m}, \mathrm{n}=30$ ), overlapping $1-2$-seriate, muriform, mostly ellipsoidal, $3-4$ transversely septate, with 1 vertical septum, constricted at the septa, initially hyaline, becoming brown at maturity, conically rounded at the ends, surrounded by 3-6 $\mu \mathrm{m}$ thick, hyaline, mucilaginous sheath. Asexual morph Undetermined.

Material examined: UZBEKISTAN, Jizzakh Province, Zaamin District, Zaamin National Nature Park, Ettisuv River, Turkestan range of Pamir-Alay Mountains, on dead aerial stem of Achillea sp. (Asteraceae), 11 July 2019, Yusufjon Gafforov, YG-Z12-1 (TASM 6159, KUN-HKAS 115786 , reference specimen).

GenBank numbers: ITS = MZ493294, LSU = MZ493308,

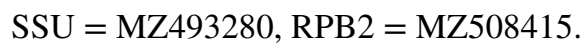




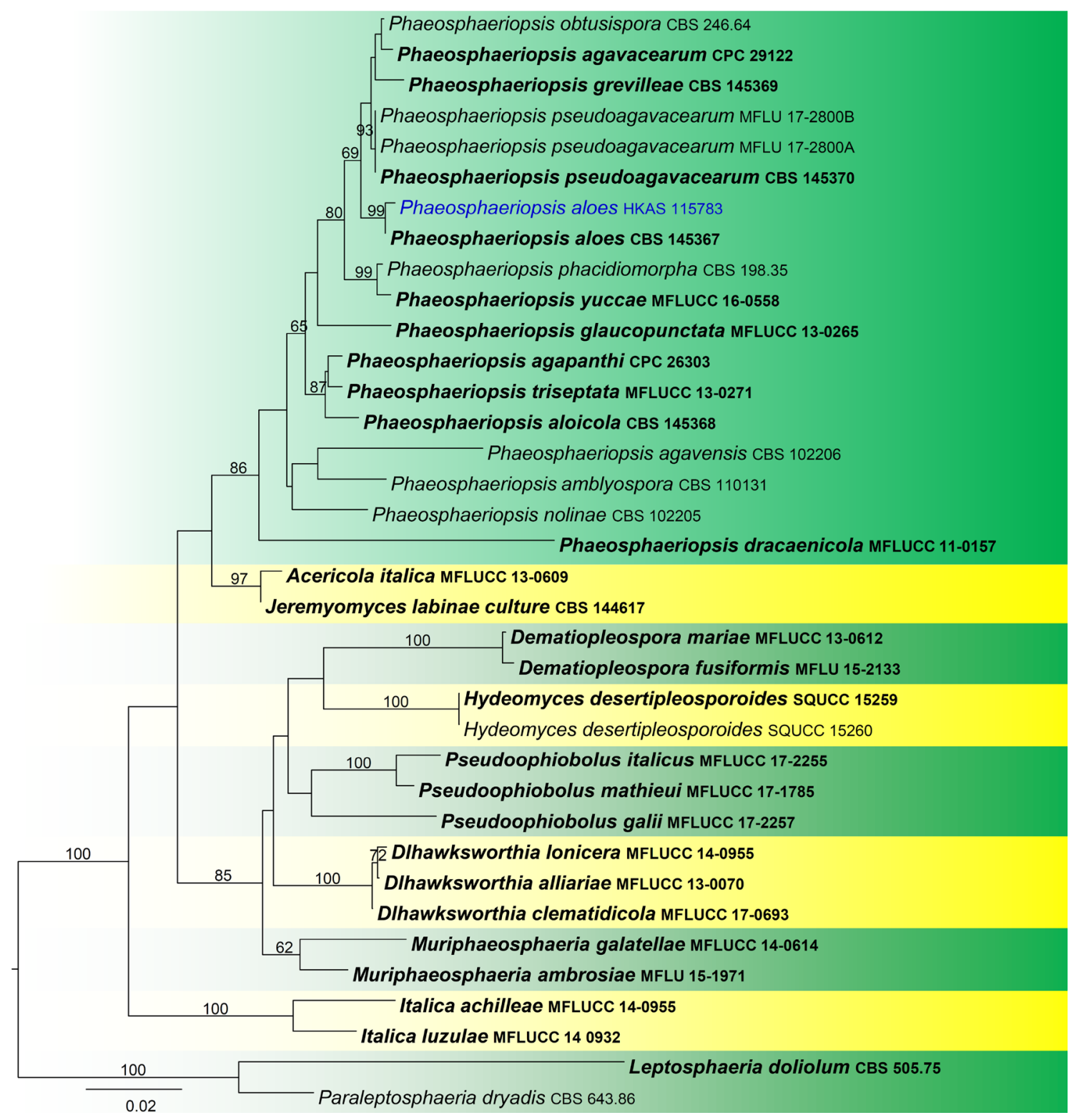

Fig. 64 Phylogram generated from the best scoring of the RAxML tree based on a combined SSU, LSU, TEF1- $\alpha$ and ITS sequence dataset to indicate the representative species in Phaeosphaeriaceae. Twenty-six strains are included in the combined analyses which comprise a total of 2930 characters. Leptosphaeria doliolum (CBS 505.75) and Paraleptosphaeria dryadis (CBS 643.86) are selected as the outgroup taxa. The best RAxML tree with a final likelihood value of -10260.911412 is presented. RAxML analysis yielded 541

Notes: Comoclathris permunda was introduced by Müller (1977) and the taxon was reported on different hosts from various countries (Table 3). Thambugala et al. (2017) collected a similar species to $C$. permunda (Müller 1977) in Italy on Phleum pratense and designated a reference specimen of this species. They also confirmed its phylogenetic affiliation in Comoclathris. Our new collection, TASM 6159 (from Uzbekistan), which fits with the original description distinct alignment patterns and $25.64 \%$ of undetermined characters or gaps. Estimated base frequencies were as follows: $\mathrm{A}=0.247998$, $\mathrm{C}=0.227405, \quad \mathrm{G}=0.266027, \quad \mathrm{~T}=0.258570 ;$ substitution rates $\mathrm{AC}=1.142059, \quad \mathrm{AG}=3.643753, \mathrm{AT}=2.176676, \quad \mathrm{CG}=0.693405$, $\mathrm{CT}=7.632890$, GT $=1.00$; gamma distribution shape parameter alpha $=0.572735$. Bootstrap support values for maximum likelihood (MLBS, left) equal to or greater than $60 \%$ are given above the nodes. Ex-type strains are in bold and newly generated sequence is in blue

of Comoclathris permunda (Müller 1977) and the description of Thambugala et al. (2017) in having solitary, dark brown to black ascomata that are cupulate when dry, cylindric-clavate, pedicellate asci with a minute ocular chamber, brown, ellipsoidal, 3-4 transversely septate, with 1 vertical septum ascospores with conically rounded ends (Fig. 65). In the phylogenetic analysis of combined SSU, LSU, ITS, and RPB2 sequence dataset to indicated that our new strain 

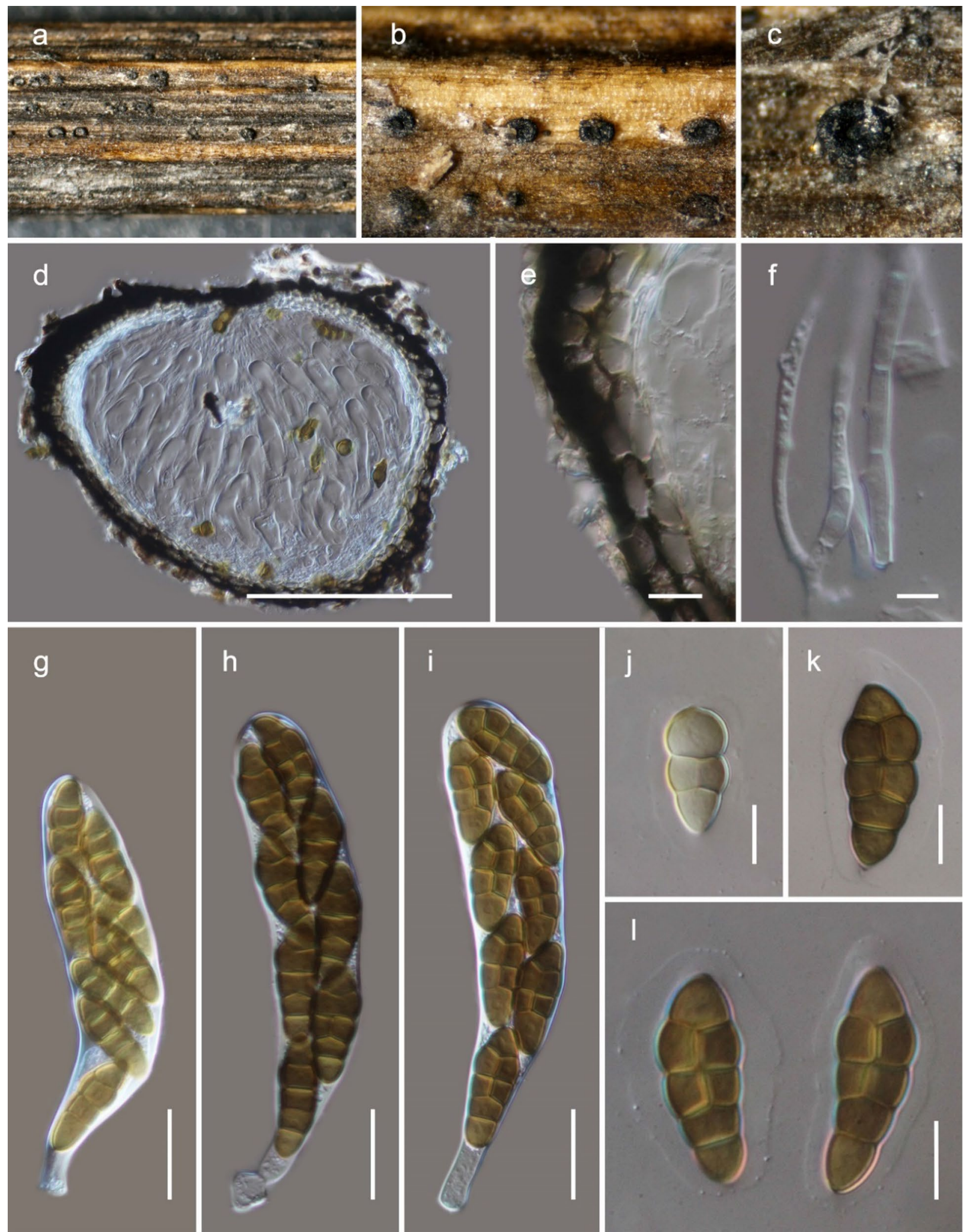

Fig. 65 Comoclathris permunda (TASM 6159, reference specimen). a-c Appearance of ascomata on host surface. d Longitudinal section of an ascoma. e Section of the peridium. $\mathbf{f}$ Pseudoparaphyses. g-i Asci. $\mathbf{j}-\mathbf{l}$ Ascospores surrounded by with a mucilaginous sheath. Scale bars: $\mathbf{d}=50 \mu \mathrm{m}, \mathbf{e}, \mathbf{j}-\mathbf{l}=10 \mu \mathrm{m}, \mathbf{f}=5 \mu \mathrm{m}, \mathbf{g}-\mathbf{i}=20 \mu \mathrm{m}$ 
Table 3 Known hosts were found for Comoclathris permunda and their distribution

\begin{tabular}{|c|c|c|c|}
\hline Plant host & Host family & Country & References \\
\hline Achillea sp. & Asteraceae & Uzbekistan & This study \\
\hline Amorpha fruticosa & Fabaceae & USA & Cooke (1983), Thambugala et al. (2017) \\
\hline Apiaceae sp. & Apiaceae & USA & Shoemaker and Babcock (1992) \\
\hline Aquilegia vulgaris & Ranunculaceae & Pakistan & Ahmad (1978), Ahmad et al. (1997) \\
\hline Arabis platysperma & Brassicaceae & California & Shoemaker and Babcock (1992) \\
\hline Arenaria drypideae & Caryophyllaceae & Turkey & Shoemaker and Babcock (1992) \\
\hline Artemisia sp. & Asteraceae & Pakistan & Ahmad (1978), Ahmad et al. (1997) \\
\hline Asclepias sp. & Apocynaceae & USA & French (1989) \\
\hline Atriplex halimus & Amaranthaceae & Portugal, Spain & Checa (2004) \\
\hline Boerhavia diffusa & Nyctaginaceae & Pakistan & Ahmad (1978), Ahmad et al. (1997) \\
\hline Bupleurum spinosum & Apiaceae & Portugal, Spain & Checa (2004) \\
\hline Cakile maritima & Brassicaceae & Portugal, Spain & Checa (2004) \\
\hline Castilleja miniata & Orobanchaceae & USA & Shoemaker and Babcock (1992) \\
\hline Chlorogalum pomeridianum & Asparagaceae & USA & Shoemaker and Babcock (1992) \\
\hline Chlorogalum sp. & Asparagaceae & USA & Thambugala et al. (2017) \\
\hline Eryngium campestre & Apiaceae & Portugal, Spain & Checa (2004) \\
\hline Heldreichia rotundifolia & Brassicaceae & Turkey & Shoemaker and Babcock (1992) \\
\hline Koeleria cristata & Poaceae & Canada & Shoemaker and Babcock (1992) \\
\hline Lactuca tenerrima & Asteraceae & Portugal, Spain & Checa (2004) \\
\hline Linum usitatissimum & Linaceae & USA & Thambugala et al. (2017) \\
\hline Penstemon gracilentus & Plantaginaceae & USA & French (1989) \\
\hline Phleum pratense & Poaceae & Italy & Thambugala et al. (2017) \\
\hline Polygonum amphibium & Polygonaceae & USA & Shoemaker and Babcock (1992) \\
\hline Prunus cerasus & Rosaceae & USA & Shoemaker and Babcock (1992) \\
\hline Sambucus racemosa var. microbotrys & Adoxaceae & USA & Shoemaker and Babcock (1992) \\
\hline Santolina chamaecyparissus & Asteraceae & Portugal, Spain & Checa (2004) \\
\hline Selinum papyraceum & Apiaceae & Pakistan & Ahmad (1978), Ahmad et al. (1997) \\
\hline Selinum tenuifolium & Apiaceae & Pakistan & Ahmad (1978), Ahmad et al. (1997) \\
\hline Senecio tournefortii var. granatensis & Asteráceas & Portugal, Spain & Checa (2004) \\
\hline Solanum dulcamara & Solanaceae & Portugal, Spain & Checa (2004) \\
\hline Stephanomeria tenuifolia & Asteraceae & USA & Shoemaker and Babcock (1992) \\
\hline Synthyris dissecta & Plantaginaceae & USA & Shoemaker and Babcock (1992) \\
\hline Thalictrum fendleri & Ranunculaceae & USA & Shoemaker and Babcock (1992) \\
\hline Wisteria chinensis & Fabaceae & Portugal, Spain & Checa (2004) \\
\hline
\end{tabular}

is monophyletic with Comoclathris permunda (MFLUCC 14-0974) with 100\% MLBS support (Fig. 66).

\section{Testudinaceae Arx}

Notes: Testudinaceae, currently consists of nine genera Angustospora, Halotestudina, Lepidosphaeria, Lojkania, Muritestudina, Neotestudina, Testudina, Ulospora and Verruculina (Hongsanan et al. 2020a). Presence of cleistothecioid ascomata, 1-septate or multi-septate, or muriform, brown, glabrous or ornamented ascospores are the characteristic features of all these genera (Hongsanan et al. 2020a). Different genera have been established under Testudinaceae based on the differences in size, shape and ornamentation of the ascospores (von Arx and Müller 1975; Hawksworth 1979). Recent phylogenetic studies have shown the close genetic relationship between genera of Testudinaceae (Hashimoto et al. 2017; Doilom et al. 2018; Hongsanan et al. 2020a).

Lepidosphaeria Parg.-Leduc

Notes: We follow the latest updated account of Lepidosphaeria in Hongsanan et al. (2020a). 


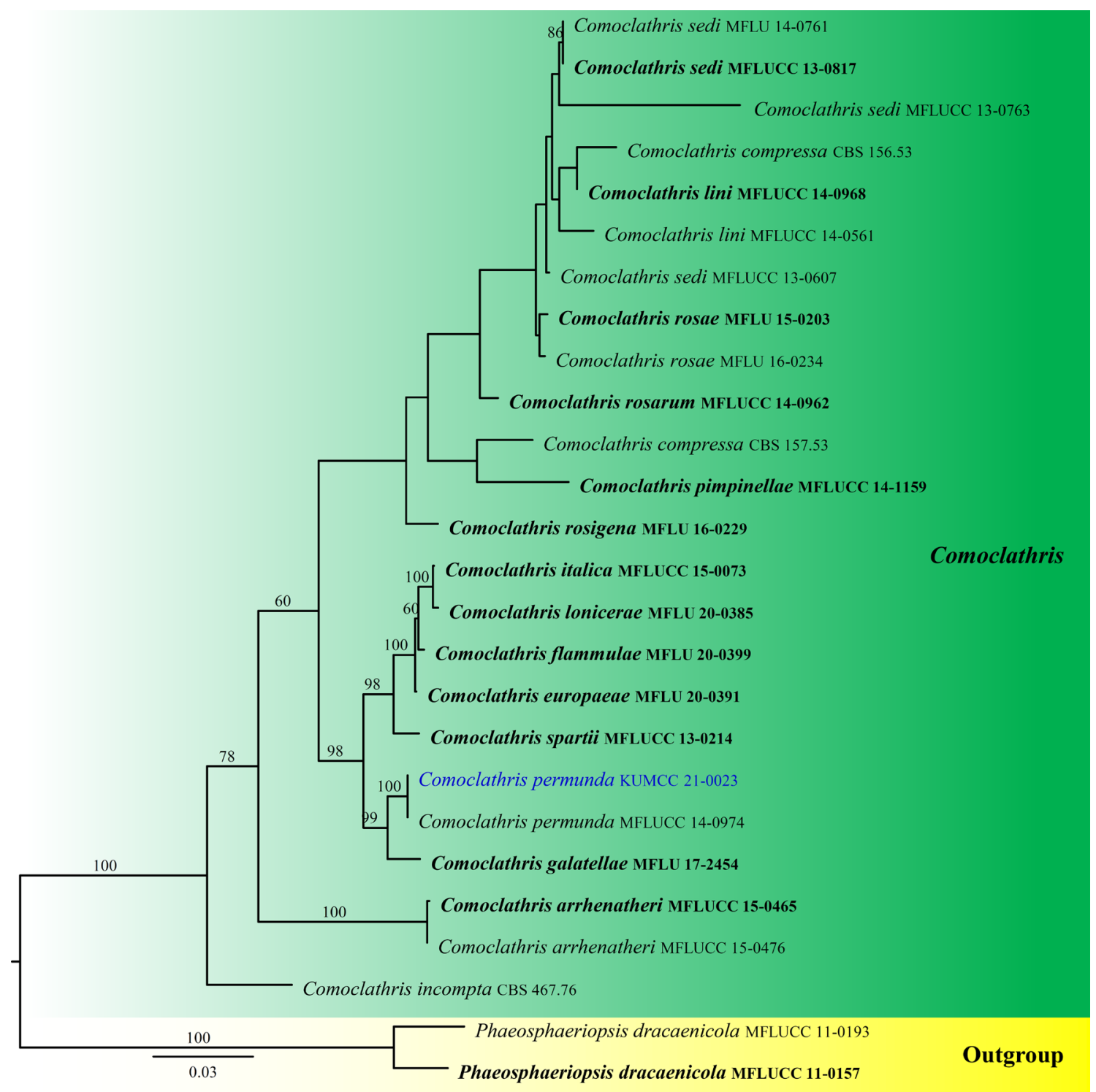

Fig. 66 Phylogram generated from the best scoring of the RAxML tree based on a combined SSU, LSU, ITS, and RPB2 sequence dataset to indicate the species in Comoclathris. Twenty-six strains are included in the combined analyses which comprise a total of 3318 characters. Phaeosphaeriopsis dracaenicola (MFLUCC 11-0157, MFLUCC 11-0193) is selected as the outgroup taxon. The best RAxML tree with a final likelihood value of -11070.111652 is presented. RAxML analysis yielded 691 distinct alignment patterns

Lepidosphaeria strobelii A.C. Lagashetti, D. Choudhary \& S.K. Singh, sp. nov.

MycoBank number: MB830723; Facesoffungi number: FoF 06120, Fig. 67

Etymology: specific epithet refers to Prof. Gary Strobel, MSU, USA to commemorate his immense contribution in the field of biology of endophytic fungi.

Holotype: AMH-10126 and $19.26 \%$ of undetermined characters or gaps. Estimated base frequencies were as follows: $\mathrm{A}=0.25654, \mathrm{C}=0.224566, \mathrm{G}=0.266202$, $\mathrm{T}=0.252692$, with substitution rates $\mathrm{AC}=1.928472, \mathrm{AG}=4.365502$, $\mathrm{AT}=1.451146, \quad \mathrm{CG}=1.060614, \quad \mathrm{CT}=9.003466, \quad \mathrm{GT}=1.000000$; gamma distribution shape parameter alpha $=0.483396$. Bootstrap support values for maximum likelihood (MLBS) equal to or greater than $60 \%$ is given above the nodes. Ex-type strains are in bold and newly generated sequence is in blue

Colour codes follow: Methuen Handbook of Colour (Kornerup and Wanscher 1978)

Isolated as an endophyte from Cinnemomum zeylanicum. Sexual morph Undetermined. Asexual morph Hyphae septate, hyaline, thin-walled, 1-4 $\mu \mathrm{m}$ wide, simple to branched, smooth-walled, consisting of series of inflated cells (chlamydospores), globulated and hyphae showed frequent anastomosis and spirally twisting. Hyphal cells, cylindrical, $7-19.5 \times 1.5-4 \mu \mathrm{m}(\bar{x}=12.5 \times 2 \mu \mathrm{m}, \mathrm{n}=30)$. 

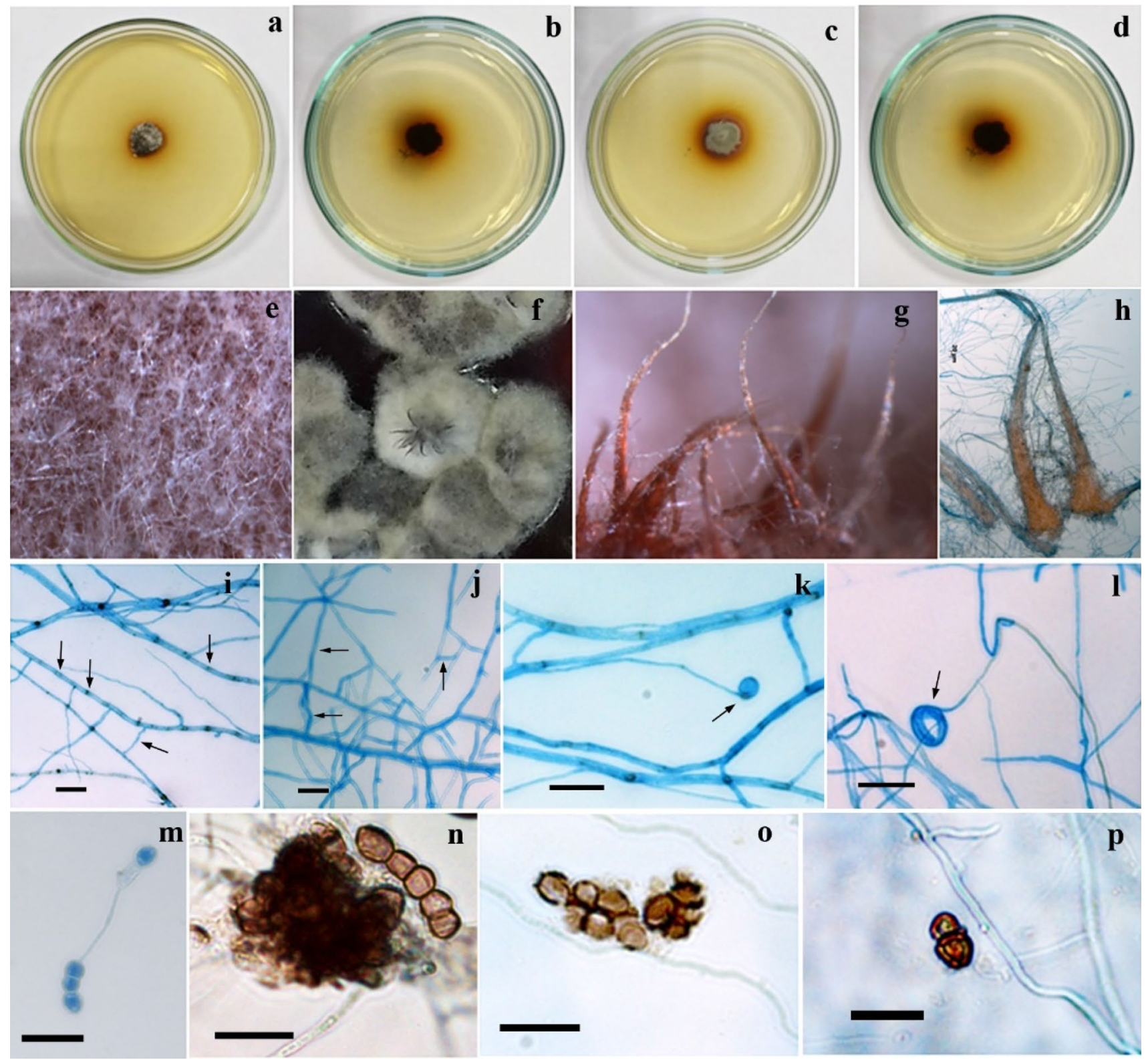

o

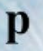

Fig. 67 Lepidosphaeria strobelii (AMH 10126, holotype; NFCCI 4579, ex-type culture). a, b Colonies morphology on potato dextrose agar (from surface and reverse views). c, $\mathbf{d}$ Colonies morphology on SDA, from surface and reverse views). e Stereoscopic view of colony growing on PDA. f, g Magnified view showing hyphal bundles. $\mathbf{h}$

Microscopic view of hyphal bundles. i Mycelia with septate hyphae (arrows). j Mycelia showing frequent anastomosis (arrows). k, l Mycelia with spiral twisting (arrows). m Young chlamydospores. $\mathbf{n}$ Mature chlamydospores in chain. o Mature chlamydospores in bunch. p Mature chlamydospore. Scale bars: $\mathbf{i}-\mathbf{p}=20 \mu \mathrm{m}$

Chlamydospores hyaline as well as light to dark olivaceous brown, observed frequently, terminal to intercalary, solitary, in branched chains and in bunches, with thickened and darkened wall, variable in shape, globose to cylindrical, $4.5-11 \times 5-9 \mu \mathrm{m}(\bar{x}=8.5 \times 7.5 \mu \mathrm{m}, \mathrm{n}=30)$.

Culture characteristics: Colonies growing slowly on PDA reaching to $12-14 \mathrm{~mm}$ diam. after 2 weeks at $25^{\circ} \mathrm{C}$; colonies from above grey (4D1), circular, raised, rocky, with slightly cottony aerial mycelium, synnematous, entire with irregular margin; colony from below, violet brown (10F8), sulcate, with diffusible red pigment. Colonies on Sabouraud Dextrose agar (SDA) reaching to 12-13 mm diam. after 2 weeks at $25^{\circ} \mathrm{C}$; colonies from above greyish green $(28 \mathrm{C} 3)$, circular, raised, velvety with smooth, entire and irregular margin; colony from below violet brown (10F8), sulcate and with diffusible red pigment. 
Material examined: INDIA, Karnataka, Belgaum $\left(15.8497^{\circ} \mathrm{N} 74.4977^{\circ} \mathrm{E}\right)$, on living leaves of Cinnemomum zeylanicum, 29 November 2011, S.K. Singh (AMH 10126, holotype), ex-type living culture, NFCCI 4579.

GenBank numbers: ITS $=$ MH790217, LSU $=$ MK749433.

Notes: The proposed a new species, Lepidosphaeria strobelii is phylogenetically distinct from L. nicotiae with $99 \%$ MLBS support (Fig. 68) based on maximum likelihood analysis of a combined ITS and LSU sequence dataset. A megablast analysis, the ITS sequence of $L$. strobelii showed $95.23 \%$ (419/440) similarity and 6 gaps (1\%) with L. nicotiae (CBS
559.71, Ex-type) and 96.08\% (441/459) similarity and 2 gaps (0\%) with $L$. nicotiae (1B0102). A comparison of the ITS sequence shows that $L$. strobelii differs from $L$. nicotiae, $21 / 441$ base positions (4.7\%) without gaps. Moreover, the source of isolation of these species are different. Type strain, L. nicotiae (CBS 559.71) was isolated from desert soil in Algeria and L. nicotiae (1B0102) was from totally suspended particles of air from Bangkok, Thailand. Whereas, L. strobelii (NFCCI 4579) was isolated as an endophyte from the leaves of Cinnemomum zeylanicum in India (Fig. 67). Morphologically, the new species could not be compared

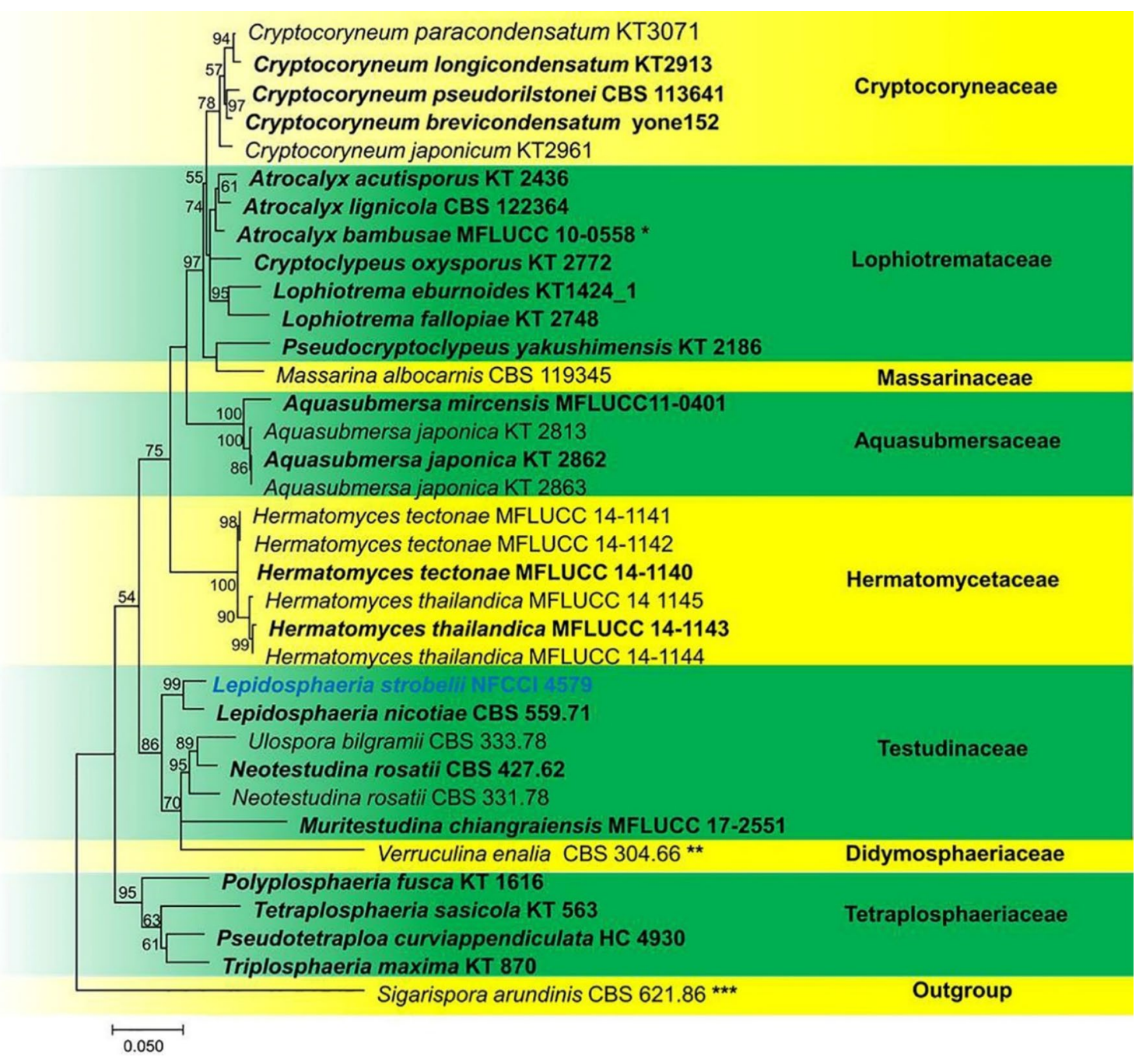

Fig. 68 Phylogram generated from maximum likelihood method for Lepidosphaeria strobelii (NFCCI 4579); using combined ITS and LSU sequence dataset. The evolutionary history was inferred based on the General Time Reversible model (Nei and Kumar 2000). The tree with the highest log likelihood (- 7009.2670) is shown. The percentage of trees in which the associated taxa clustered together is shown next to the branches. A discrete GAMMA distribution was used to model evolutionary rate differences among sites [5 categories $(+\mathrm{G}$, parameter $=0.5660)]$. The rate variation model allowed for some sites to be evolutionarily invariable $[(+\mathrm{I}), 58.2766 \%$ sites]. The tree is drawn to scale, with branch lengths measured in the number of substitutions per site. The analysis involved 35 nucleotide sequences. All positions with less than $85 \%$ site coverage were eliminated. That is fewer than $15 \%$ alignment gaps, missing data, and ambiguous bases were allowed at any position. There were a total of 1322 positions in the final dataset. Evolutionary analyses were conducted in MEGA7 (Kumar et al. 2016). Sigarispora arundinis (formerly known as Lophiostoma arundinis) was used as an outgroup. Ex-type strains are in bold and newly generated sequence is in blue 
with L. nicotiae. Lepidosphaeria nicotiae is characterized by its sexual morph, forming cleistothecial, black, globose to subglobose ascomata, elongate-clavate asci, with a long pedicel, embedded in filiform, hyphae-like pseudoparaphyses, and pale brown to brown, oblong, tuberculate, 1-septate, finely echinulate ascospores (Doilom et al. 2018). While the sexual morph of $L$. strobelii is undetermined.

Remarks:

*Atrocalyx bambusae is formerly known as Lophiotrema bambusae,

**Veruuculina enalia is formerly known as Didymosphaeria enalia and

***Sigarispora arundinis is formerly known as Lophiostoma arundinis.

Tetraplosphaeriaceae Kaz. Tanaka \& K. Hiray.

Notes: We follow the latest treatment and updated accounts of Tetraplosphaeriaceae in Hongsanan et al. (2020a).

\section{Ernakulamia Subram.}

Notes: The latest treatment and updated accounts of Ernakulamia were provided in Hyde et al. (2020a). Only four species are included in this genus, E. cochinensis (Subram.) Subram. (type species), E. krabiensis, E. tanakae and E. xishuangbannaensis (Dong et al. 2020b; Hyde et al. 2020b; Index Fungorum 2021) and all species have sequence data available in GenBank. Ernakulamia cochinensis and E. tanakae were found on decaying spathes of Cocos nucifera (Arecacceae) in India (Subramanian 1957, 1994; Hyde et al. 2020b), whereas Ernakulamia krabiensis was found on decaying pods septum of Acacia sp. (Fabaceae) in Thailand (Jayasiri et al. 2019). Unfortunately, Ernakulamia xishuangbannaensis was previously introduced as Polyplosphaeria xishuangbannaensis Tibpromma \& K.D. Hyde on dead leaves of Pandanus in Yunnan, China (Tibpromma et al. 2018). Multilocus phylogenetic analysis demonstrated P. xishuangbannaensis clustered within Ernakulamia (Dong et al. 2020b). Thus, the species was transferred to Ernakulamia as E. xishuangbannaensis by Dong et al. (2020b). In this study, Ernakulamia tanakae is reported for the first time on decaying wood of an unidentified host from Thailand. The updated phylogenetic analysis is presented (Fig. 70).

Ernakulamia tanakae Rajeshkumar \& K.D. Hyde, Fungal Diversity 100: 111 (2020)

Index Fungorum number: IF556700; Facesoffungi number: FoF 06338; Fig. 69

Saprobic on decaying wood. Sexual morph Undetermined. Asexual morph Hyphomycetous. Colonies effuse, superficial, dark brown to black, sparsely hyaline hyphal network. Conidiophores not seen. Conidiogenous cells monotretic, integrated. Conidia 35-48 $\times 22-37 \mu \mathrm{m}$ $(\bar{x}=42 \times 30 \mu \mathrm{m}, \mathrm{n}=10)$, dictyosporous, obconical, obovoid or subglobose, dark brown, multi-septate, thick-walled, with 3-5-appendages per conidium. Appendages 24-68 $\times 4-5 \mu \mathrm{m}$, cylindrical stiff, straight, apical or subapical, dark brown, 3-6-septate, smooth-walled.

Culture characteristics: Conidium germinating on PDA within $24 \mathrm{~h}$. Colonies on PDA reaching $3.5 \mathrm{~cm}$ diam. after 2 weeks at room temperature, circular, undulate edge, grey to dark grey, dense and raised on medium surface. Mycelium superficial, velvety, flossy.

Material examined: THAILAND, Phetchabun Province, Lom Sak, saprobic on decaying wood of unidentified host, 25 July 2019, S. Boonmee, LSP02 (MFLU 21-0066, new record), living culture, MFLUCC 21-0089.

GenBank numbers: ITS $=$ MZ538502, LSU $=$ MZ538536, TEF1- $\alpha=$ MZ567081.

Notes: Ernakulamia tanakae was introduced by Hyde et al. (2020a), which is reported as a second species found on the Cocos nucifera host from India as well as the type species E. cochinensis. All species of Ernakulamia are characterized by superficial, dictyosporous, with cylindrical stiff appendages and dark brown conidia (Fig. 69, Subramanian 1994; Jayasiri et al. 2019; Hyde et al. 2020b). Phylogenetic analysis placed our strain (MFLUCC 21-0089) close to the type strain (NFCCI 4615) and other isolates of E. tanakae (NFCCI 4616 and NFCCI 4617) (Fig. 70). Pairwise comparison of DNA sequences of LSU and ITS regions of these four strains reveals $2 / 872 \mathrm{bp}(0.23 \%)$ and $5 / 528 \mathrm{bp}(0.95 \%)$ very few base pair differences, which confirms that they are conspecific. Thus, a new geographical record of E. tanakae on decaying wood from Thailand is reported.

\section{Thyridariaceae Q. Tian \& K.D. Hyde}

Notes: We follow the latest treatment of Thyridariaceae in Mapook et al. (2020) and updated accounts of taxa in Hongsanan et al. (2020a).

\section{Thyridaria Sacc.}

Notes: Thyridaria was introduced by Saccardo (1875) which was typified by T. broussonetiae (Sacc.) Traverso, where T. incrustans Sacc. was synonymized (Barr 1990). Thyridaria comprises 52 species (Wijayawardene et al. 2020) which are widely distributed. Taxa in this genus are mostly saprobes on a diverse range of plant substrates (Hyde et al. 2013; Jaklitsch and Voglmayr 2016; Hongsanan et al. 2020a; Index Fungorum 2021). Only two species T. acaciae and T. broussonetiae have sequence data available in GenBank. We follow the latest treatment of Thyridaria in Jaklitsch and Voglmayr (2016) and updated accounts of taxa in Wijayawardene et al. (2020). 

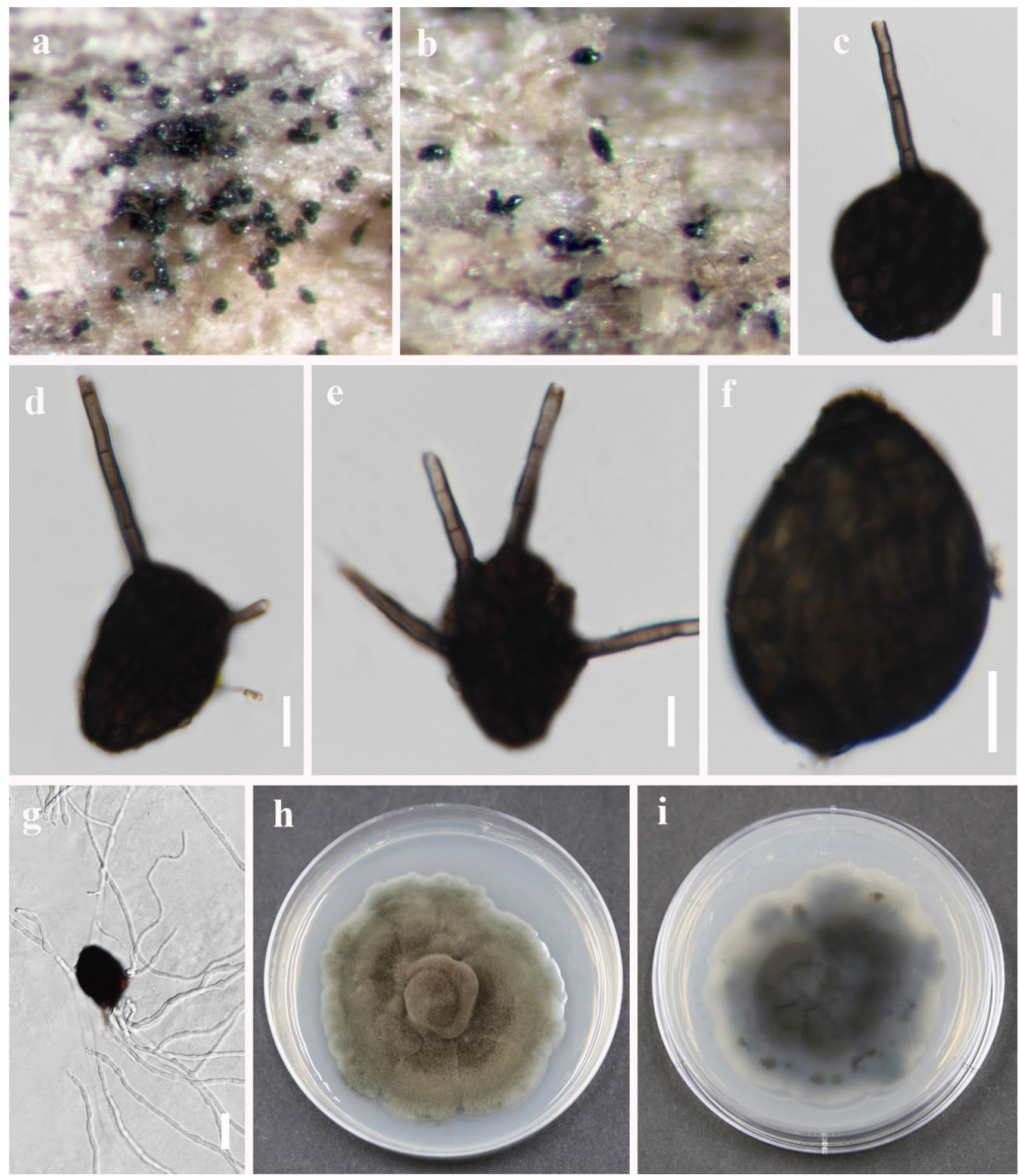

Fig. 69 Ernakulamia tanakae (MFLU 21-0066, new record). a, b Appearance of conidial masses on host substrate. c-f Mature conidia. $\mathbf{g}$ Germinated conidium. $\mathbf{h}, \mathbf{i}$ Culture on PDA from surface and reverse. Scale bars: $\mathbf{c}-\mathbf{f}=10 \mu \mathrm{m}, \mathbf{g}=20 \mu \mathrm{m}$ 


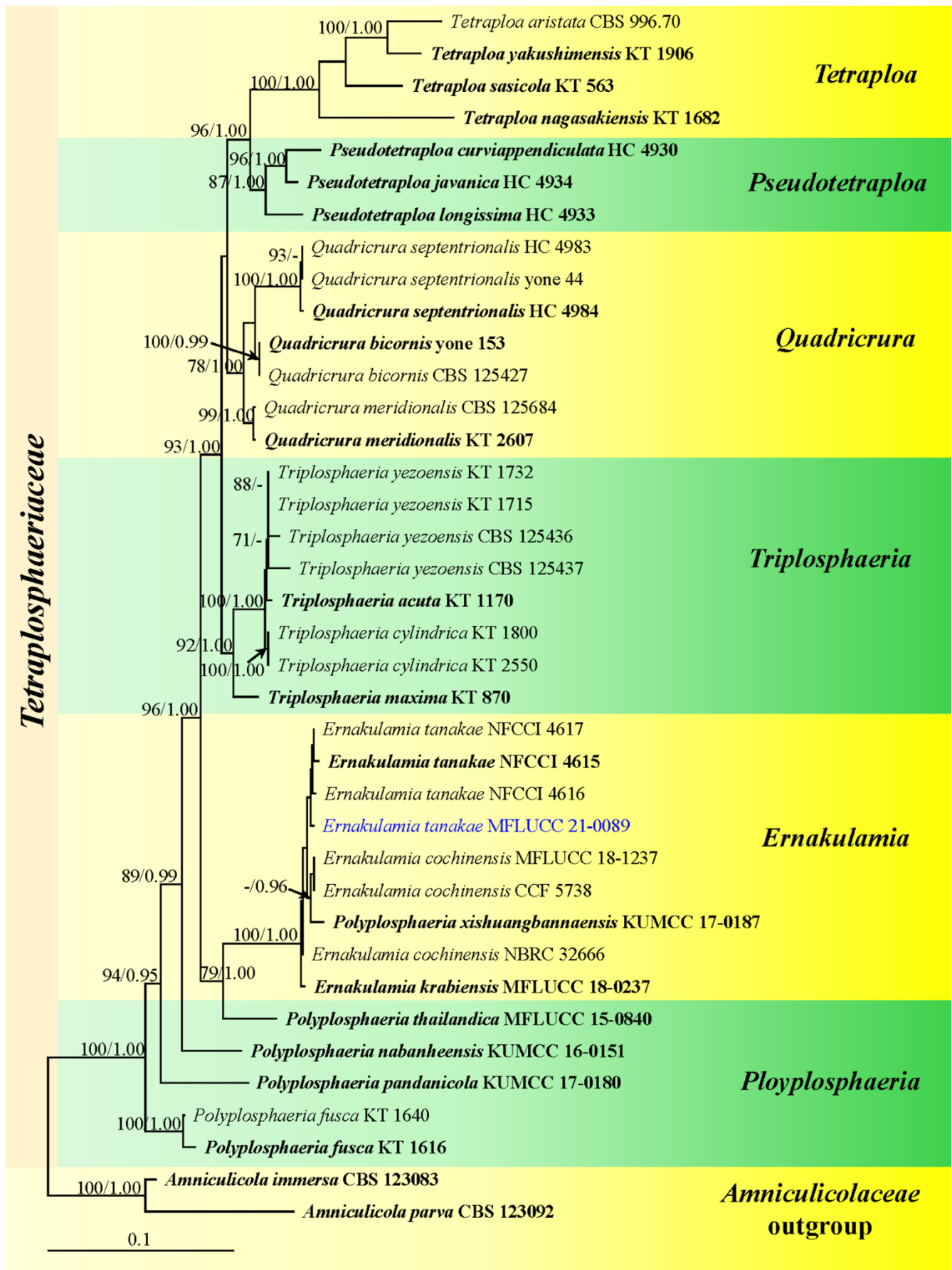

Fig. 70 Maximum likelihood phylogenetic tree based on a combined LSU and ITS sequence dataset of Ernakulamia taxa and related genera in Tetraplosphaeriaceae. Thirty-eight strains are included in the combined analyses which comprise a total of 1468 characters. Amniculicola immersa (CBS 12308) and A. parva (CBS 123092) are selected as the outgroup taxa. The best RAxML tree with a final likelihood value of -5624.746373 is presented. RAxML analysis yielded 413 distinct alignment patterns and $15 \%$ of undetermined characters or gaps. Estimated base frequencies were as follows: $\mathrm{A}=0.236603$,
$\mathrm{C}=0.245429, \mathrm{G}=0.293755, \mathrm{~T}=0.224212$, with substitution rates $\mathrm{AC}=2.551620, \quad \mathrm{AG}=2.407140, \quad \mathrm{AT}=1.683194, \quad \mathrm{CG}=0.618325$, $\mathrm{CT}=9.671311, \mathrm{GT}=1.000000$; gamma distribution shape parameter alpha $=0.164106$. Bootstrap support values for maximum likelihood (MLBS, left) equal to or greater than $70 \%$ are given above or below the nodes. Bayesian posterior probabilities (BYPP, right) equal to or greater than 0.95 are given above the nodes. Ex-type strains are in bold and newly generated sequence is in blue 
Thyridaria aureobrunnea Boonmee, Huanraluek \& K.D. Hyde, sp. nov.

Index Fungorum number: IF558540; Facesoffungi number: FoF 09193; Fig. 71

Etymology: The specific epithet 'aureobrunnea' refers to the golden brown to chestnut brown ascospores.

Holotype: MFLU 21-0067

Saprobic on decaying wood. Sexual morph Ascomata 248-294.5 $\mu \mathrm{m}$ high, $262-286.5 \mu \mathrm{m}$ diam. $(\bar{x}=330 \times 367 \mu \mathrm{m}$, $\mathrm{n}=4$ ), immersed, erumpent, aggregated, clustered, globose to subglobose, uniloculate, dark brown to black, central ostiole, with minute papilla. Ostiole, papillate, protruding from the center of the ascomata, with a pore-like opening, yellowish around apical pore when wet. Peridium $18 \times 33 \mu \mathrm{m}$ wide, comprising several layers of dark brown cells of textura angularis. Hamathecium comprising $1-2 \mu \mathrm{m}$ wide, cylindrical, filamentous, rarely branched, inconspicuously septate, anastomosed, hyaline pseudoparaphyses. Asci
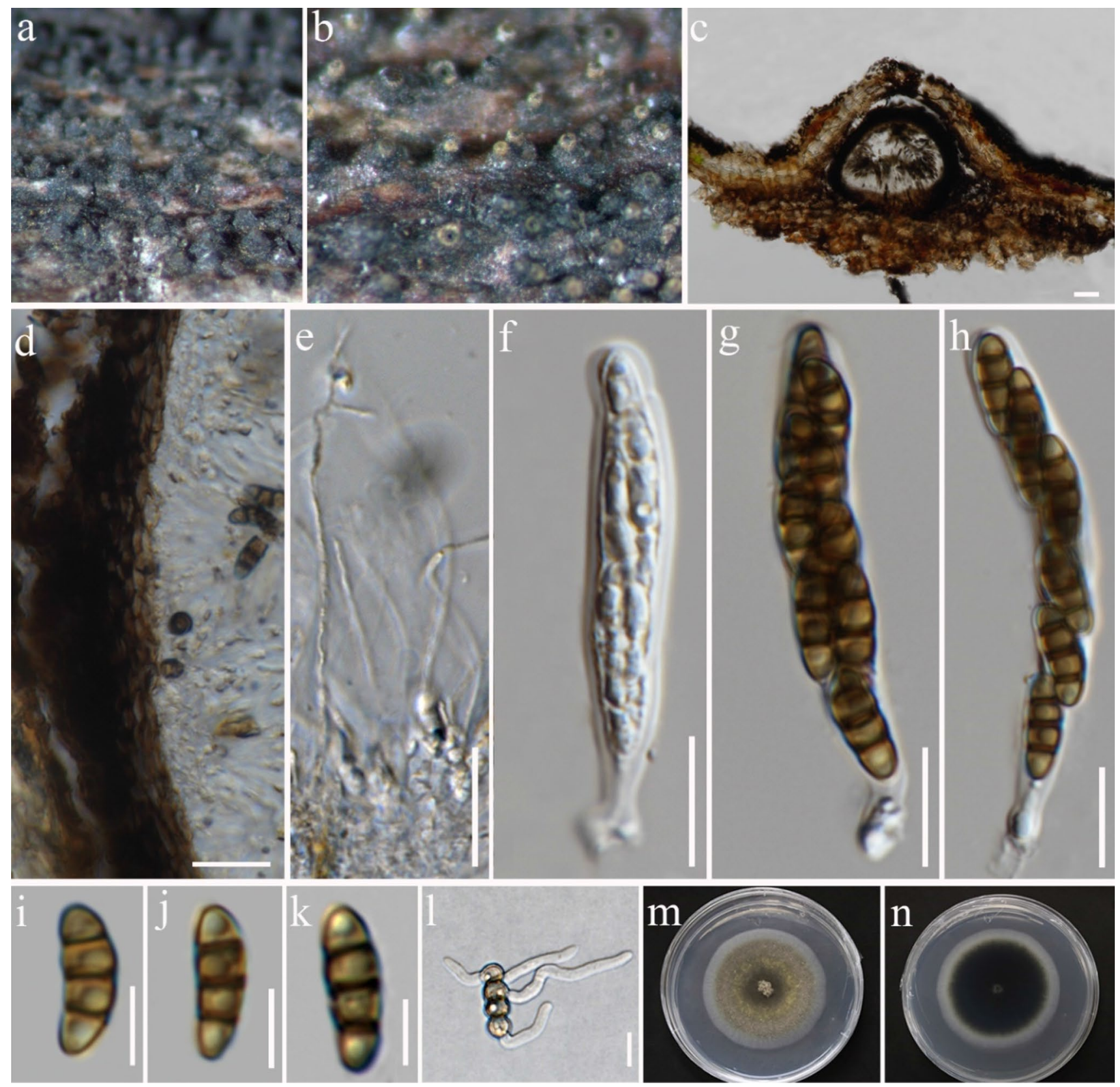

Fig. 71 Thyridaria aureobrunnea (MFLU 21-0067, holotype). a, b Ascomata on woody substrate. c Cross sections of ascoma. d Peridium. e Pseudoparaphyses. f-h Asci. i-k Ascospores. 1 Germinated ascospore. $\mathbf{m}, \mathbf{n}$ Culture on PDA from surface and reverse at 1 month. Scale bars: $\mathbf{c}=50 \mu \mathrm{m}, \mathbf{d}, \mathbf{e}=20 \mu \mathrm{m}, \mathbf{f}-\mathbf{h}=10 \mu \mathrm{m}, \mathbf{i}-\mathbf{l}=5 \mu \mathrm{m}$ 
45-61.5 ×7-8.5 $\mu \mathrm{m}(\bar{x}=52 \times 7.5 \mu \mathrm{m}, \mathrm{n}=10), 8$-spored, bitunicate, cylindric-clavate to clavate, slightly curved, bulbous pedicellate, apically rounded. Ascospores $10.5-13 \times 3.5-4.5 \mu \mathrm{m}(x=12 \times 4 \mu \mathrm{m}, \mathrm{n}=20)$, overlapping uni- to bi-seriate, ellipsoid to obovoid, slightly curved, golden brown to chestnut brown, 3-septate, strongly constricted and darkened at the septa, guttulate, smooth-walled. Asexual morph Undetermined.

Culture characteristics: Ascospore germinating on PDA within $24 \mathrm{~h}$ and germ tubes produced from each cell of the ascospores. Colonies on PDA reaching $3.5 \mathrm{~cm}$ diam. after 2 weeks at room temperature, circular, entire edge, olivaceous brown to brown from the center towards margin, white grey at the margin, dark brown at the reverse with surrounded by white grey at the margin. Mycelium superficial, velvety.

Material examined: THAILAND, Chiang Rai Province, Mae Yao, on decaying wood, 23 September 2019, N. Huanraluek, MY06 (MFLU 21-0067, holotype), ex-type living culture, MFLUCC 21-0090.

GenBank number: ITS $=$ MZ538528, LSU $=$ MZ538562.

Notes: In the BLASTn search of LSU and ITS sequences, Thyridaria aureobrunnea is most similar to T. broussonetiae (CBS 121895 and CBS 141481) with 99.18\% and $92.21 \%$ similarities, respectively. A combined LSU, ITS and TEF1- $\alpha$ sequence analysis indicates that $T$. aureobrunnea forms a distinct lineage basal to T. acaciae and $T$. broussonetiae (type species) with 100\% MLBS, 1.00 BYPP support (Fig. 72). Thyridaria aureobrunnea (Fig. 71) can be distinguished from the type species $T$. broussonetiae in morphological characteristics such as uniloculate ascomata, shapes and size of asci and ascospores (Jaklitsch and Voglmayr 2016). In addition, Thyridaria acaciae is known only as an asexual morph which is produced in culture (Crous et al. 2014b; Jaklitsch and Voglmayr 2016), and thus we cannot compare the morphology. Based on the highly supported distinct lineage and differences in DNA sequences, a new species, Thyridaria aureobrunnea is introduced.

\section{Torulaceae Corda}

Notes: Torulaceae was introduced by Corda in Sturm (1829) to accommodate Torula and typified by T. herbarum (Pers.) Link. Torulaceae forms a well-supported lineage in the order Pleosporales (Crous et al. 2015; Hyde et al. 2016, 2019, 2020a; Li et al. 2016b; Su et al. 2016, 2018). The family is circumscribed only by asexual morph characters with key distinguishing features such as; mostly immersed mycelium, erect, micro- or macronematous, straight or flexuous, subcylindrical conidiophores with or without apical branches, doliiform to ellipsoid or clavate, brown, smooth to verruculose, and mono- to polyblastic conidiogenous cells which in some cupulate after secession of conidia and subcylindrical, phragmosporous, acrogenous, brown, dry, and smooth to verrucose conidia which are often produced in branched chains (Li et al. 2016b; Hyde et al. 2019). Currently, Torulaceae includes six genera viz. Dendryphion, Neotorula, Rostriconidium, Rutola, Sporidesmioides and Torula (Crous et al. 2020a; Hongsanan et al 2020a). In this study, we propose a new genus Cylindrotorula within Torulaceae with evidence from morphology and multi-gene phylogenetic analyses (ITS, LSU, RPB2 and TEF1- $\alpha$ ). Cylindrotorula constitutes a well-supported distinct lineage (100\% MLBS, 1.00 BYPP) basal to Neotorula, Dendryphion and Torula.

Cylindrotorula Rajeshkumar, Wijayaw. \& Bhat, gen. nov. Index Fungorum number: IF557635; Facesoffungi number: FoF 08026

Etymology: Named after cylindrical, elongated, verruculose primary conidia, and conidial ornamentations similar to Torula species.

\section{Holotype: AMH 10228}

Saprobic on decaying Cocos nucifera spathe. Sexual morph Undetermined. Asexual morph Hyphomycetous. Conidiomata sporodochial, scattered, greyish, dark greyish brown or dark brown. Conidiophores micronematous, mononematous, reduced, cylindrical, smooth, erect, unbranched or rarely branched. Conidiogenous cells cylindrical or button-shaped, produce primary and secondary conidia. Primary conidia arising holoblastically, elongated, cylindrical, wavy or undulated or flexuous, smooth at base, verrucose above, pale to dark brown, unbranched, phragmo-septate. Secondary conidia two types, globose and cylindrical, verrucose or smooth, arising from primary conidia from intercalary, monotretic, non-cicatrized conidiogenous loci just below septa; cylindrical or finger shaped conidia slightly curved, aseptate, thin-walled, brown.

Type species: Cylindrotorula indica Rajeshkumar, Wijayaw., Bhat, N. Ashtekar \& S. Lad

Notes: The proposed new genus Cylindrotorula is similar to Alcornia, Parapericonia and Sporotretospora (Ellis 1976; Monteiro et al. 2017; Whitton et al. 2012). All these genera have been described solely on morphology and are yet to be investigated based on DNA sequence analyses. The conidiophores in Alcornia and Parapericonia are illustrated and interpreted as elongated, verruculose, flexuous and with a distinct basal constriction or zone of weakness. Sporotretophora and Alcornia are similar in arrangement of conidiophores, enteroblastic tretic conidiogenesis, and unicelled conidia, but Sporotretophora has monotretic conidiogenous cells and schizolytic secession of conidia (Whitton et al. 2012). A detailed micro-morphological study of Cylindroturula on natural substrates using SEM and compound microscopy revealed its holoblastic development of 


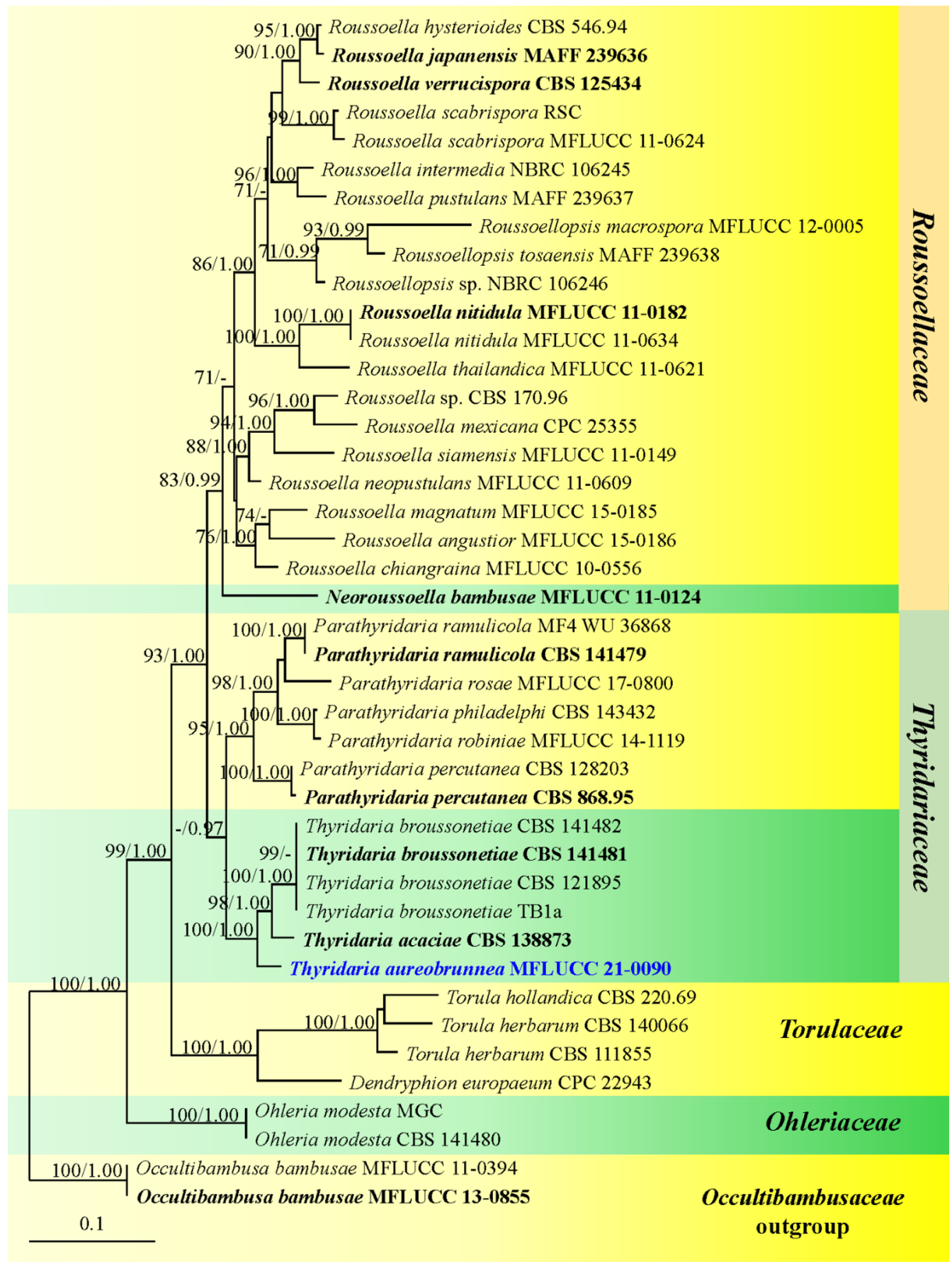

Fig. 72 Maximum likelihood phylogenetic tree based on a combined LSU, ITS and TEF1- $\alpha$ sequence data of Thyridaria taxa (Thyridariaceae) and related families. Forty-two strains are included in the combined analysis, which comprise a total of 2216 characters. Occultibambusa bambusae (MFLUCC 11-0394 and MFLUCC 13-0855) are selected as the outgroup taxa. The best RAxML tree with a final likelihood value of -12410.223662 is presented. RAxML analysis yielded 752 distinct alignment patterns and $19.90 \%$ of undetermined characters or gaps. Estimated base frequen- cies were as follows: $\mathrm{A}=0.235389, \mathrm{C}=0.270091, \mathrm{G}=0.280715$, $\mathrm{T}=0.213806$, with substitution rates $\mathrm{AC}=1.372292, \mathrm{AG}=2.751820$, $\mathrm{AT}=1.999735, \quad \mathrm{CG}=1.148507, \quad \mathrm{CT}=6.478337, \quad \mathrm{GT}=1.000000$; gamma distribution shape parameter alpha $=0.169795$. Bootstrap support values for maximum likelihood (MLBS, left) equal to or greater than $70 \%$ is given above or below the nodes. Bayesian posterior probabilities (BYPP, right) equal to or greater than 0.95 are given above the nodes. Ex-type strains are in bold and newly generated sequence is in blue 
cylindrical, elongated, flexuous, verrucose primary conidia from a reduced, sporodochial, micronematous, mononematous, septate, branched, or unbranched, erect conidiophores having an apical cylindrical or button-shaped conidiogenous cells (Figs. 73, 74 and 75). Secondary conidia develop monotretic and are of two types, (1) globose, verruculose, brown to dark brown and (2) finger-shaped or narrow cylindrical, thin-walled, smooth to minutely verrucose, with an obtuse tip and truncate base.

The monotretic, secondary conidial development segregates Cylindrotorula from the Parapericonia. Whereas monotretic to polytretic globose conidia with an integrated, rhexolytic, verruculose ornamentation is the key distinguishing character in Alcornia. The narrowly cylindrical, curved, thin-walled secondary conidia having monotretic conidial ontogeny seen in Cylindrotorula is absent in Alcornia, Parapericonia and Sporotretophora. A BLASTn search using ITS, LSU, RPB2 and TEF1- $\alpha$ sequence data in NCBI has shown about $90 \%$ similarity of the query sequences to Dendryphion and Neotorula strains. Dendryphion is characterized by polytretic conidiogenous cells with pores encircled by dark scars, and chains of brown, septate (didymo- or cheiro) conidia (Crous et al. 2014a). The phylogenetic analyses based on ITS, LSU, RPB2 and TEF1- $\alpha$ sequence data also formed a well-supported (100\% MLBS, 1.00 BYPP; Fig. 76) independent sister lineage sister to Neotorula and
Dendryphion within Torulaceae. Thus, based on morphological characteristics and phylogenetic analyses, we accommodate Cylindrotorula as new genus with the type species C. indica.

Cylindrotorula indica Rajeshkumar, Wijayaw., Bhat, N. Ashtekar \& S. Lad, sp. nov.

Index Fungorum number: IF557636; Facesoffungi number: FoF 08025; Figs. 73, 74, 75

Etymology: Named after India, the country where this fungus is native.

\section{Holotype: AMH 10228}

Saprobic on decaying Cocos nucifera spathe. Sexual morph Undetermined. Asexual morph Hyphomycetous. Conidiomata sporodochial, scattered, greyish, dark greyish brown or dark brown. Conidiophores micronematous, mononematous, reduced, cylindrical, smooth, erect, unbranched or rarely branched, $0-1$-septate, $3.5-12 \times 2.8-4 \mu \mathrm{m}$. Conidiogenous cells cylindrical or button-shaped, form primary and secondary conidia. Primary conidia arising holoblastically, elongated, cylindrical, wavy or undulated or flexuous, smooth at base, verruculose above, pale to dark-brown, unbranched, phragmo-septate, 5-10-septate, 68-125×3-4.5 $\mu \mathrm{m}$. Secondary conidia two types, globose and cylindrical or finger-shaped, arising from primary conidia at intercalary, monotretic, non-cicatrized conidiogenous loci, globose or
Fig. 73 Cylindrotorula indica (AMH 10228, holotype). a-d Sporodochial conidiomata on natural substrate. Scale bars: $\mathbf{a}-\mathbf{d}=100 \mu \mathrm{m}$
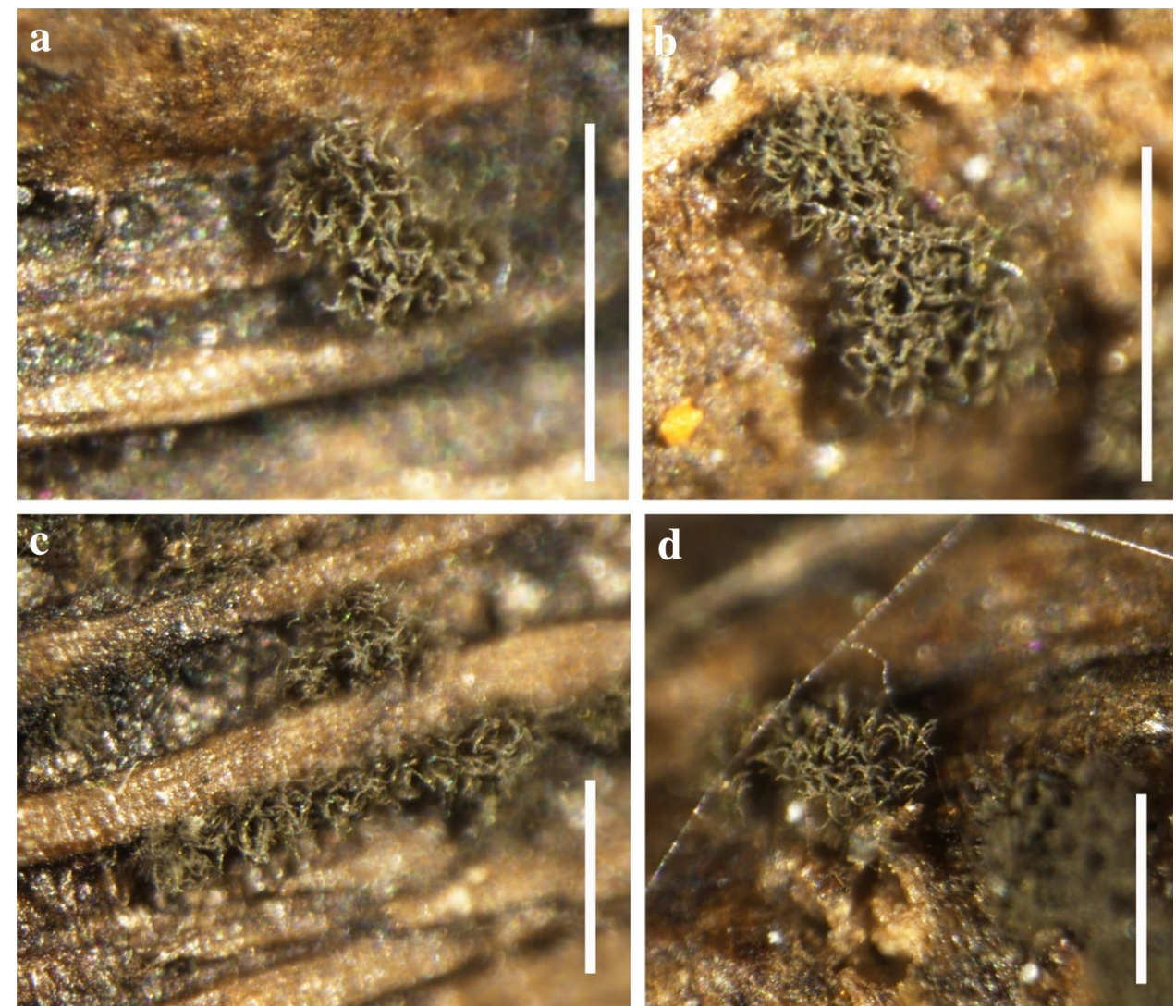

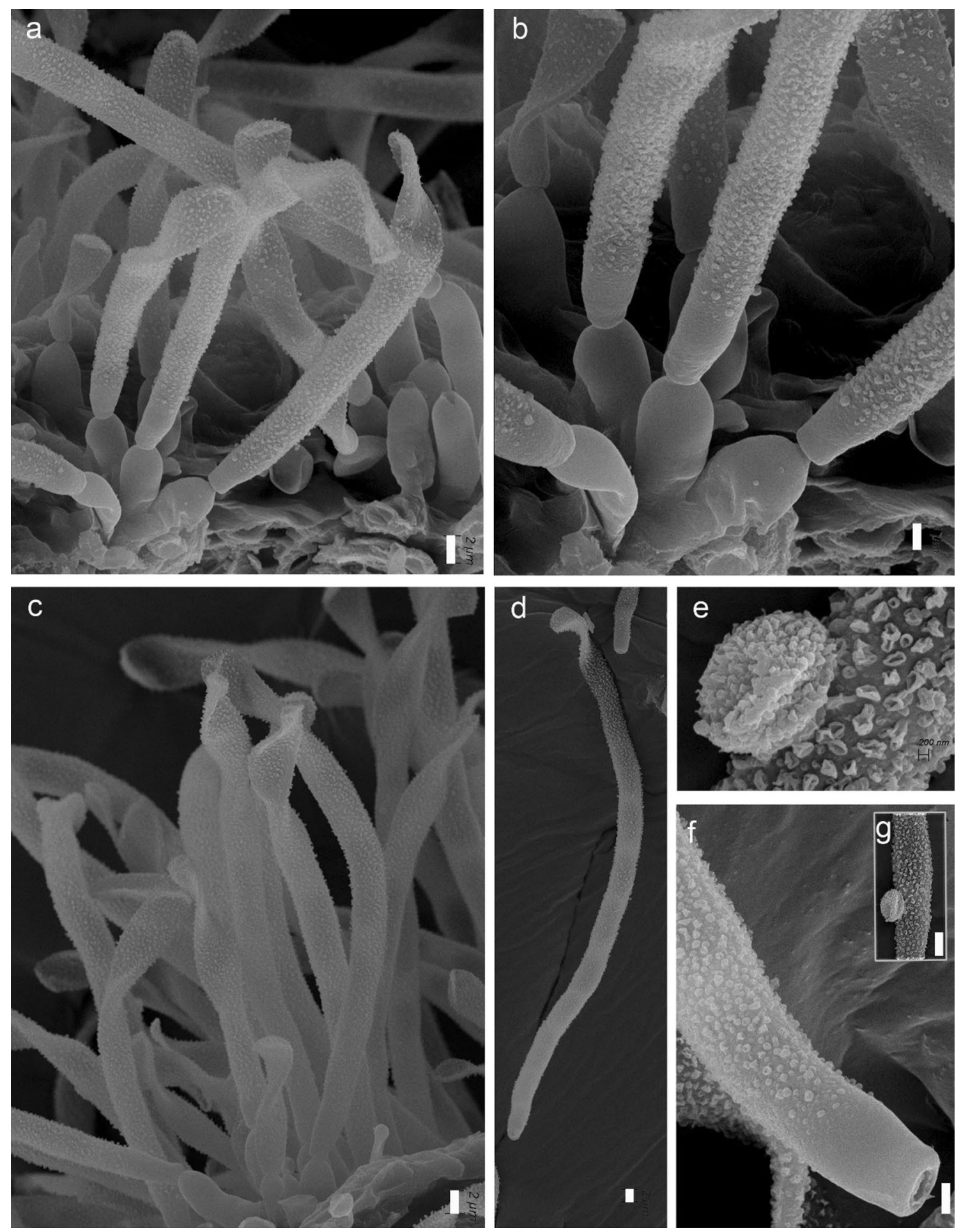

Fig. 74 Cylindrotorula indica (AMH 10228, holotype). a-c SEM of conidiomata showing conidiophores and primary conidia. d Cylindrical elongated primary conidium. e Globose secondary conidium.

f Base of primary conidium. g Secondary conidial development of primary conidia. Scale bars: $\mathbf{a}, \mathbf{c}, \mathbf{d}, \mathbf{g}=2 \mu \mathrm{m}, \mathbf{b}=1 \mu \mathrm{m}, \mathbf{e}=200 \mathrm{~nm}$, $\mathbf{f}=1 \mu \mathrm{m}$ 

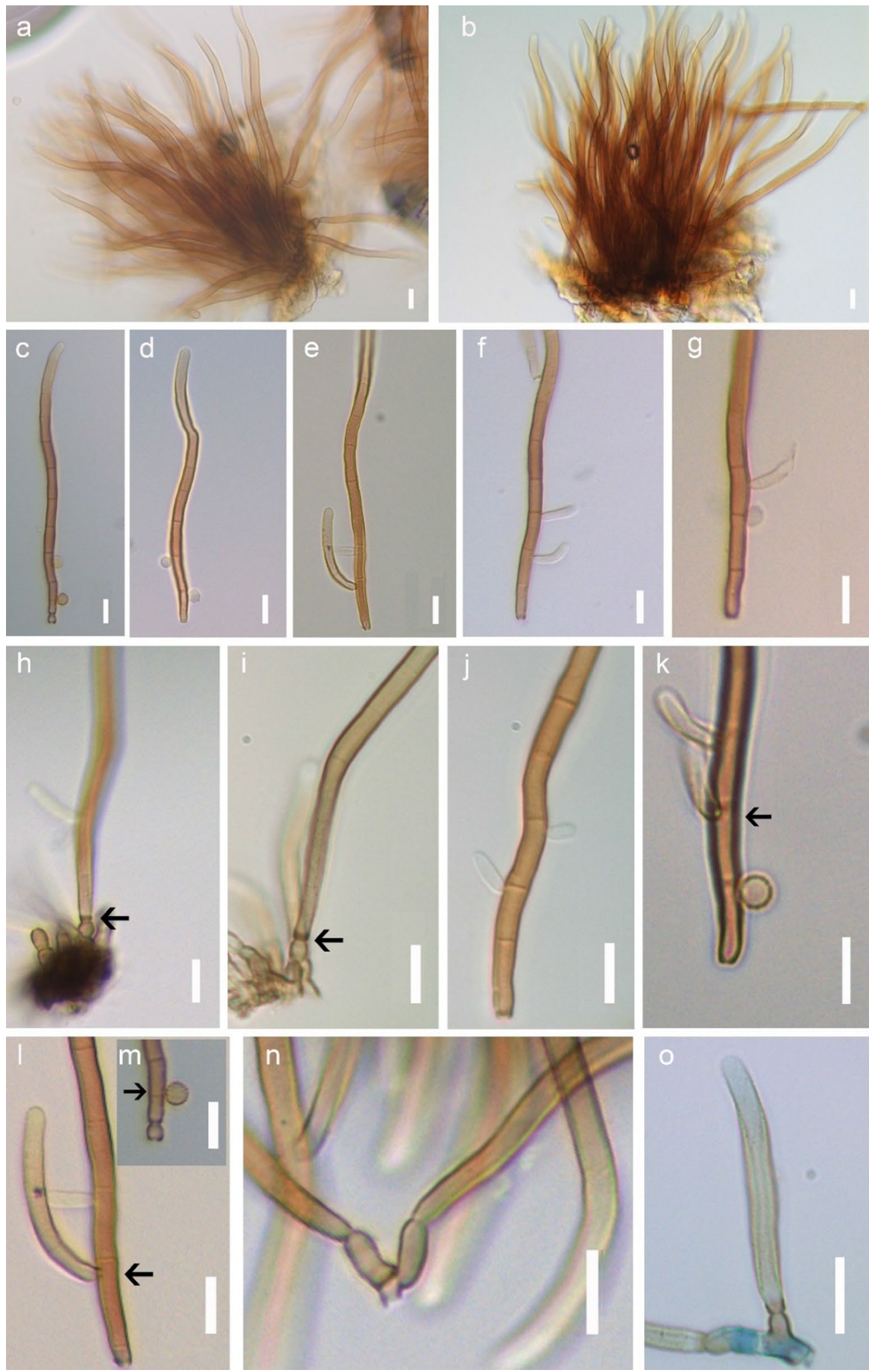

Fig. 75 Cylindrotorula indica (AMH 10228, holotype). a, b Sporodochial conidiomata. $\mathbf{c}-\mathbf{g}$ Primary conidia with monotretic secondary conidia. $\mathbf{h}$, i Dark thick hilum of primary conidia. $\mathbf{j}, \mathbf{k}$ Pleomorphic secondary conidia. l, m Monotretic secondary conidial development. $\mathbf{n}$, o Branched conidiophore with primary conidia. Scale bars: $\mathbf{a}-\mathbf{o}=10 \mu \mathrm{m}$ 


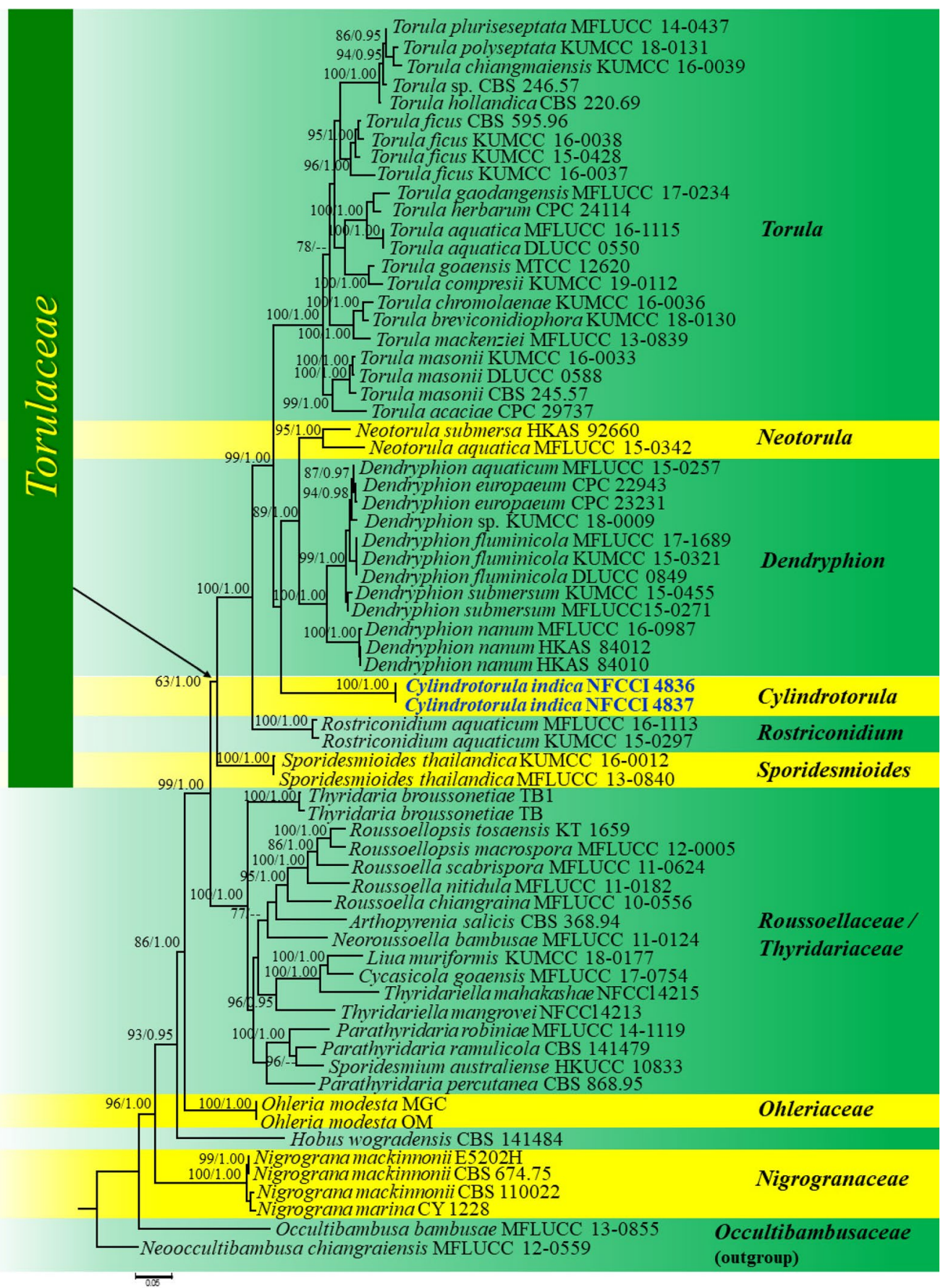

Fig. 76 Phylogram generated from maximum likelihood analysis based on LSU, RPB2, ITS, and TEF1- $\alpha$ sequence dataset representing Torulaceae and related families. Related sequences are taken from Hyde et al. (2019). Sixty-eight strains are included in the combined analyses which comprise 2807 characters and 517 characters for ITS after alignment. Neooccultibambusa chiangraiensis (MFLUCC 12-0559) and Occultibambusa bambusae (MFLUCC 12-0559) in Occultibambusaceae (Pleosporales) are used as the outgroup taxa. Single gene analyses were also performed to compare the topology and clade stability with combined gene analyses. Tree topology of the maximum likelihood analysis is similar to the Bayesian analysis. The best RAxML tree with a final likelihood values of -25944.916075 is presented. The matrix had 1201 distinct alignment patterns, with $23.66 \%$ undetermined characters or gaps. Estimated base frequencies were as follows: $\mathrm{A}=0.240116, \mathrm{C}=0.269729, \mathrm{G}=0.272735$, $\mathrm{T}=0.217421 ;$ substitution rates $\mathrm{AC}=1.567160, \mathrm{AG}=3.410853$, $\mathrm{AT}=1.595585, \quad \mathrm{CG}=0.809155, \quad \mathrm{CT}=7.747008, \quad \mathrm{GT}=1.000000$; gamma distribution shape parameter $\alpha=0.645809$. Bootstrap values for maximum likelihood (MLBS) equal to or greater than $60 \%$ and posterior probabilities (BYPP, right) equal to or greater than 0.95 (the rounding of values to 2 decimal places) from Bayesian inference analysis labeled on the nodes. The newly generated sequences are in blue bold 
elongated, slightly curved, aseptate, smooth, verrucose or verruculose, thin-walled, brown. Micrometry globose secondary conidia, $3.5-5 \times 3.5-5 \mu \mathrm{m}$, cylindrical or finger-shaped conidia, 9.5-30 $\times 2.5-3.5 \mu \mathrm{m}$.

Culture characteristics: Colonies on MEA at $25 \pm 2{ }^{\circ} \mathrm{C}$ reaching 30-35 mm diam. after 1 month; initially aerial mycelium white to brownish grey (4D2), velutinous, in old cultures brownish grey (4D2) with olive grey (4E4) margin; reverse dark grey (4F1). Soluble pigments and exudates absent. Colour codes and names used in descriptions are from Kornerup and Wanscher (1978).

Material examined: INDIA, Maharashtra, Thane $\left(19^{\circ} 13^{\prime} 13^{\prime \prime} \mathrm{N} 72^{\circ} 57^{\prime} 17^{\prime \prime} \mathrm{E}, \pm 23 \mathrm{msl}\right)$, on decaying spathe of Cocos nucifera (Arecacceae), July 2018, K.C. Rajeshkumar and S. Lad, AMH 10228 (holotype), ex-type living culture NFCCI 4836; ibid., NFCCI 4837.

GenBank numbers: NFCCI 4836: ITS $=$ MT339444, LSU $=$ MT339442, RPB2 = MT321490, TEF1 $\alpha=$ MT321492; NFCCI 4837: ITS $=$ MT339445, LSU $=$ MT339443, RPB2 $=$ MT321491, TEF1- $\alpha=$ MT321493.

\section{Dendryphion Wallr.}

Notes: Dendryphion was introduced by Wallroth (1833) and is typified by Dendryphion comosum Wallr. The genus is characterized by having erect, solitary, polytretic conidiophores, branched in upper part, forming septate, pigmented, thick-walled, finely roughened stipe and a distinct conidiogenous apparatus, with dark scars and catenate, in simple or branched chains of brown, septate (didymo- or cheiro) conidia (Crous et al. 2015; Su et al. 2016, 2018; Li et al. 2020a). Species of Dendryphion are saprobic on dead stems of herbaceous plants and decaying wood, and also grows on submerged decaying wood in freshwater habitats (Su et al. 2016, 2018; Li et al. 2020a). There are 85 Dendryphion epithets listed in Index Fungorum (2021) but only 36 species are accepted in Dendryphion (Species Fungorum 2021), and only seven species have DNA sequences available in the GenBank database.

Dendryphion hydei J.F. Li, Phookamsak \& Jeewon, PLoS ONE 15(2): e0228067 (2020)

Index Fungorum number: IF556746; Facesoffungi number: FoF 04574; Fig. 77

Saprobic on submerged wood in a freshwater habitat. Sexual morph Undetermined. Asexual morph Colonies on the substrate superficial, effuse, hairy, brown to dark brown. Mycelium partly immersed to superficial on the substrate, branched, septate, pale brown to brown hyphae. Conidiophores $338-428 \times 10-16 \mu \mathrm{m}(\bar{x}=383 \times 13 \mu \mathrm{m}, \mathrm{n}=15)$ (10-16 $\mu \mathrm{m}$ wide at the base, 5-7 $\mu \mathrm{m}$ wide at the apex branch) macronematous, mononematous, septate, straight or flexuous, cylindrical, thick-walled, simple branch on the top primary branches, pale brown to brown, unbranched part dark brown. Conidiogenous cells $5-9 \times 4-5 \mu \mathrm{m}(\bar{x}=7 \times 4 \mu \mathrm{m}$, $\mathrm{n}=25)$ polytretic, terminal, integrated, pale brown. Conidia 33-56 $(-108) \times 6-8 \mu \mathrm{m}(\bar{x}=44 \times 7 \mu \mathrm{m}, \mathrm{n}=30)$ single, dry, drum to globose, pale brown to brown, slightly paler at the end cells, bead-like chains, 4-13(-21)-septate, constricted at the septa, middle cells wider than the top and bottom. Conidial secession schizolytic.

Culture characteristics: Conidia germinated on the PDA within $24 \mathrm{~h}$, and germ tube produced from both ends. Colonies grow on the PDA, and after 2 weeks of incubation at room temperature, the diameter reaches about $4 \mathrm{~cm}$. Hyphae are initially white to light grey, and then gradually become grey to dark grey, with convex middle, flocculent, grey to dark grey, smooth edges, white, and flocculent, forming a white ring about one centimeter. Reverse light yellow-brown, uniform color, smooth.

Material examined: CHINA, Yunnan Province, Puer City, (100'37'59" E 22 $\left.36^{\circ} 36^{\prime \prime} \mathrm{N}, 919 \mathrm{msl}\right)$, on submerged wood, 30 April 2018, H.W. Shen, Q.S. Zhou and Z.L. Luo, S-2049 (HKAS 112706, new record), living culture, DLUCC 2049.

GenBank numbers: ITS = MW723060, $\mathrm{LSU}=\mathrm{MW} 879527, \quad \mathrm{SSU}=\mathrm{MW} 774583$, RPB2 = MW729781, TEF1- $\alpha$ : MW729786.

Notes: Dendryphion hydei was found on a branch litter of Bidens pilosa Linn. in Chiang Mai Province, Thailand (Li et al. 2020a). Phylogenetically, our isolate clustered with the ex-type strain of Dendryphion hydei (HKAS 97479) with low statistical support (Fig. 80). Our new isolate (Fig. 77) has some differences in size from the type species; it has longer conidiophores $(338-428 \times 10-16 \mu \mathrm{m}$ vs. $260-380 \times 7-14 \mu \mathrm{m})$ and conidia (33-56 (-108) $\times 6-8 \mu \mathrm{m}$ vs. (17-) $20-30(-35) \mu \mathrm{m}$ long $\times 4-7 \mu \mathrm{m})$, and has more septa (4-13 vs. $2-4)$. Based on morphological data and phylogenetic analysis, we report $D$. hyde $i$ as the first record in a freshwater habitat.

\section{Torula Pers.}

Notes: Torula was introduced by Persoon (1795) and is typified by $T$. herbarum. Members in this genus are hyphomycetous asexual morph and characterized by superficial dark colonies and branched chains, dark brown conidia (Crane and Schoknecht 1977; Crous et al. 2015, 2020a; Hyde et al. 2020b; Su et al. 2016; Li et al. 2017, 2020a). There are 543 Torula epithets are listed in Index Fungorum (2021), but only 49 species have morphological studies and 17 species have molecular data support (Hongsanan et al. 2020a). In this study, based on morphological and phylogenetic analyses, Torula lancangjiangensis is introduced as a new species and a new record of T. mackenziei from a freshwater habitat in China is reported.

Torula lancangjiangensis H.W. Shen, S. Boonmee, Z.L. Luo \& K.D. Hyde, sp. nov.

Index Fungorum number: IF558159; Facesoffungi number: FoF 09753; Fig. 78 

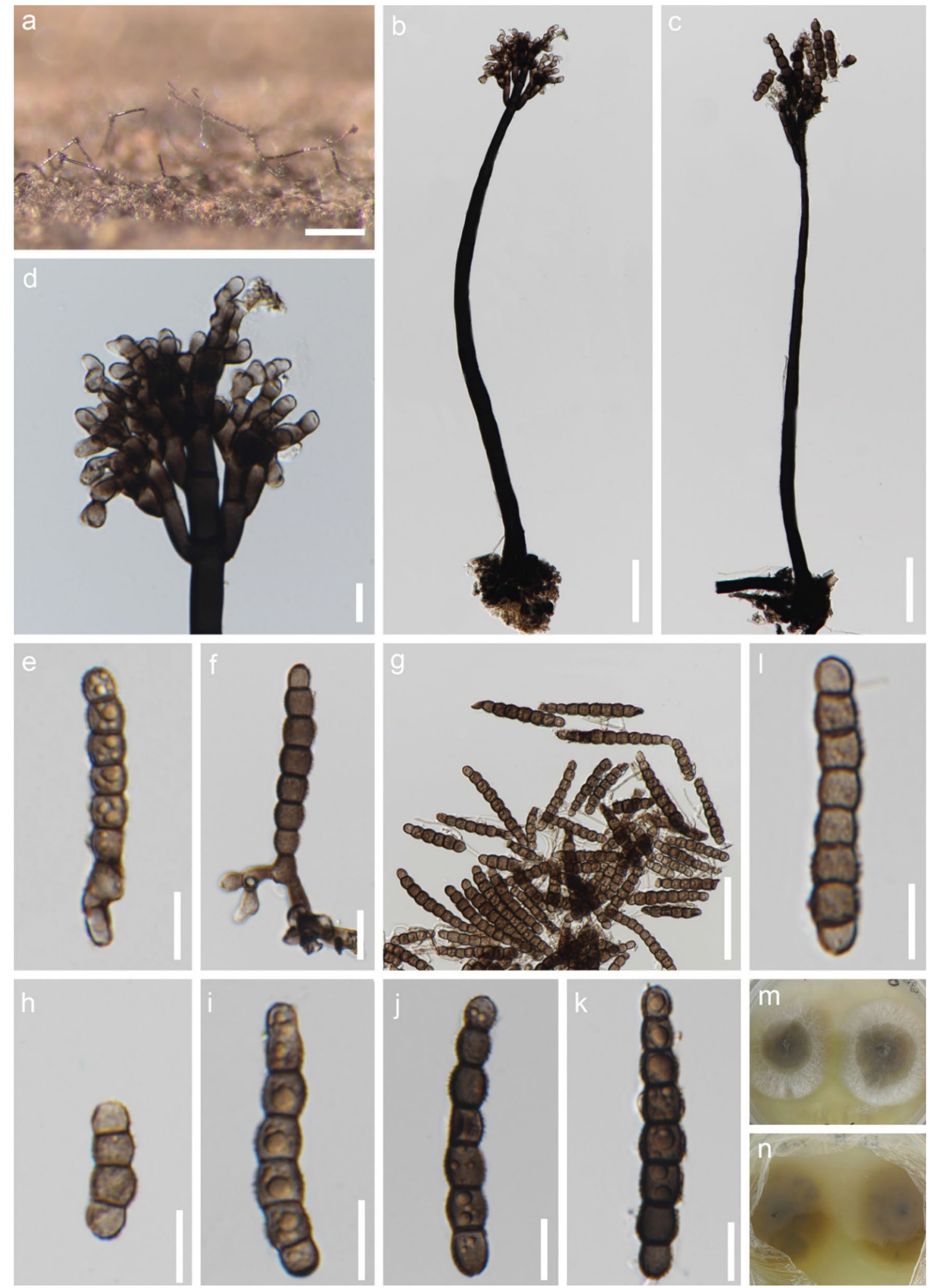

Fig. 77 Dendryphion hydei (HKAS 112706, new record). a Colonies on submerged wood. b-d Conidiophores with conidiogenous cells. e, f Conidiogenous cells with conidia. $\mathbf{g}-\mathbf{l}$ Conidia. $\mathbf{m}$, n Culture on PDA (surface and reverse). Scale bars: $\mathbf{a}=200 \mu \mathrm{m}$, b, $\mathbf{c}=50 \mu \mathrm{m}, \mathbf{d}-\mathbf{f}, \mathbf{h}-\mathbf{l}=10 \mu \mathrm{m}, \mathbf{g}=20 \mu \mathrm{m}$ 

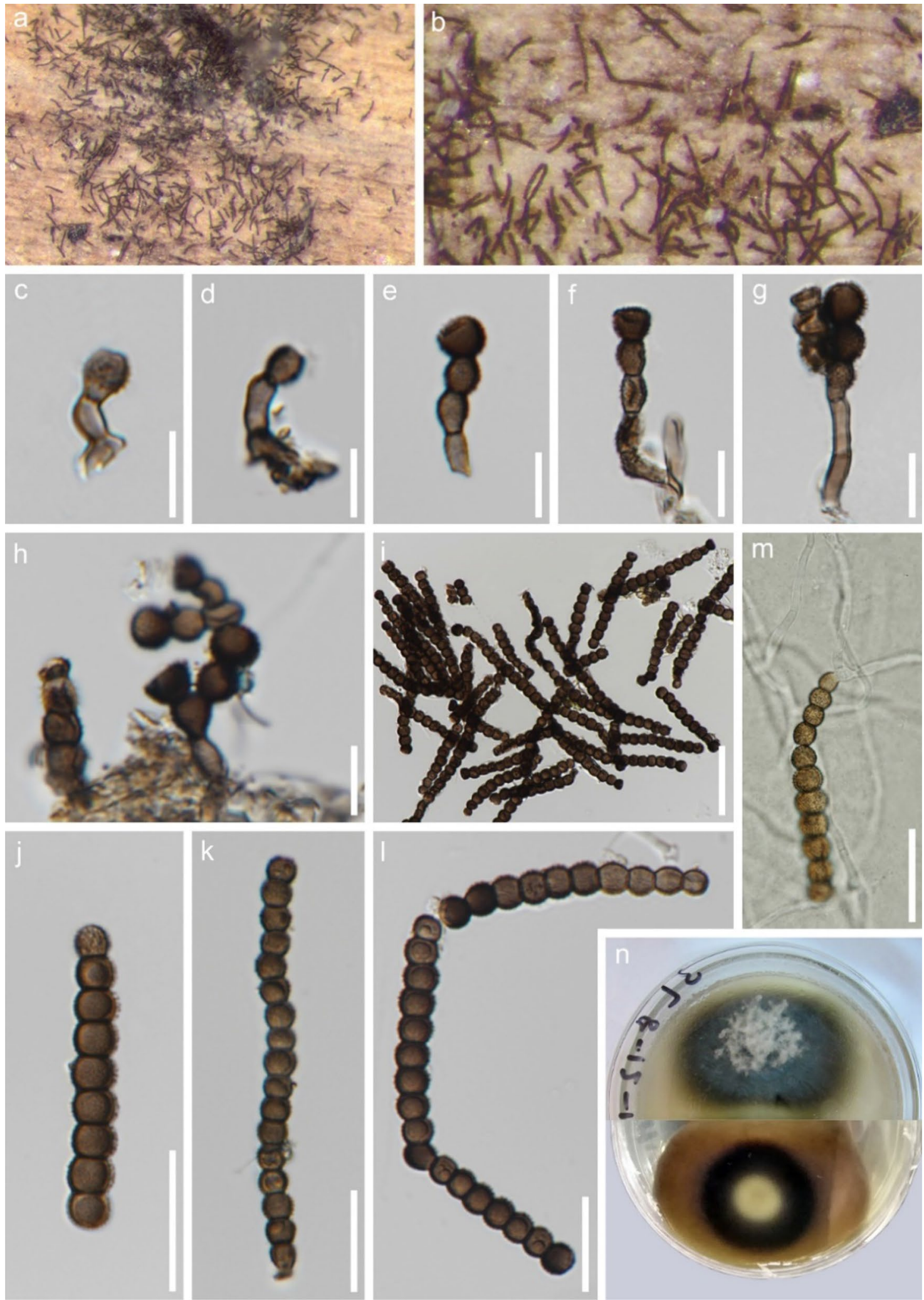

Fig. 78 Torula lancangjiangensis (HKAS 112709, holotype). a, b Colonies on submerged wood. c-f Conidiophores with conidiogenous cells. $\mathbf{g}$, h Conidiophores, conidiogenous cells with conidia. $\mathbf{i}-\mathbf{k}$
Conidia. $\mathbf{l}$ Conidia in chain. $\mathbf{m}$ Germinated conidium. $\mathbf{n}$ Culture on PDA from surface and reverse. Scale bars: $\mathbf{c}-\mathbf{m}=20 \mu \mathrm{m}$ 
Etymology: Referring to the collecting site from the Langcangjiang River in China.

Holotype: HKAS 112709

Saprobic on submerged wood in a freshwater habitat. Sexual morph Undetermined. Asexual morph Colonies sparse, hairy, dry, velvety, dark brown to black on substrate. Mycelium partly immersed to superficial on the substrate, septate, branched, light brown to brown hyphae. Conidiophores $5-15 \times 3-4 \mu \mathrm{m}(\bar{x}=10 \times 3 \mu \mathrm{m}, \mathrm{n}=25)$, macronematous to semi- macronematous, mononematous, erect, straight or slightly flexuous, without apical branches, light brown to brown, ellipsoid to subcylindrical, smooth-walled, 0-2-septate, with 1-2 doliiform to globose cells. Conidiogenous cells $5-7 \times 5-6 \mu \mathrm{m}(\bar{x}=6 \times 5 \mu \mathrm{m}, \mathrm{n}=25)$, polyblastic, integrated, terminal or intercalary, doliiform to subglobose, brown to dark brown, smooth to verrucose, thick-walled. Conidia (24-)45-74(-100) $\times 6-7(-8) \mu \mathrm{m}(\bar{x}=60 \times 7 \mu \mathrm{m}$, $\mathrm{n}=40$ ), acrogenous, catenated, composed of moniliform cells, brown to dark brown, 5-19-septate, constricted at the septa, doliiform to globose, most circular-drum form, with 1-2 cells black at the apex. Conidial secession schizolytic.

Culture characteristics: Conidia germinated on PDA within $24 \mathrm{~h}$, and germ tube produced from the conidial cells at both ends or in the middle. Colonies grow on the PDA, and after incubating at room temperature for 1 month, the diameter reaches to $3.5 \mathrm{~cm}$. Hyphae are initially light gray, turning dark grey at maturity, raised in the middle, flocculent, velvety, white, with edges smooth, light grey to light brown. Reverse light grey in the middle light gray to dark grey, smooth edges.

Material examined: CHINA, Yunnan Province, Xishuangbanna, (100 $42^{\prime} 04^{\prime \prime}$ E $\left.22^{\circ} 05^{\prime} 19^{\prime \prime} \mathrm{N}, 680 \mathrm{msl}\right)$, on submerged wood, 4 May 2018, H.W. Shen, Q.S. Zhou and Z.L. Luo, S-2043 (HKAS 112709, holotype, MFLU 21-0112, isotype), ex-type living culture, DLUCC 2043; THAILAND, Chiang Rai, Muang, Thasud, Mae Fah Lung University, Botanical garden, on dead twigs, 15 November 2018, Boonmee, B03 (MFLU 21-0076), living culture, MFLUCC 21-0099.

GenBank numbers: DLUCC 2043: ITS = MW723059, $\mathrm{LSU}=\mathrm{MW} 879526, \quad \mathrm{SSU}=\mathrm{MW} 774582$, RPB2 $=$ MW729780, TEF $1-\alpha=$ MW729785; B03: ITS $=$ MZ538529, LSU $=$ MZ538563, TEF $1-\alpha=$ MZ567104.

Notes: In a NCBI BLASTn search based on RPB2 sequences, the closest match of Torula lancangjiangensis was T. camporesii (KUMCC 19-0112; GenBank no. MN507404) with $97.67 \%$ similarity. A comparison of RPB2 nucleotide bases indicated that $T$. lancangensis differs from T. camporesii in 50/1055 bp (4.7\%). In addition, multigene phylogenetic analysis of a combined dataset of the LSU, SSU, TEF1- $\alpha$, RPB2, and ITS showed that T. lancangjiangensis and T. camporesii are close relatives with 95\% MLBS, 1.00 BYPP support (Fig. 80). Torula lancangjiangensis resembles $T$. camporesii in having ellipsoid to subcylindrical, multi-septate conidia; and doliiform to subglobose, brown to dark brown conidiogenous cells (Fig. 78). However, conidial cells of $T$. camporesii are wide in the middle and small at the ends, while the cells of T. lancangjiangensis are more uniform. Therefore, Torula lancangjiangensis is introduced as a new species.

Torula mackenziei J.F. Li, Phookamsak \& K.D. Hyde, Mycol Progress 16: 447-461 (2017)

Index Fungorum number: IF819537; Facesoffungi number: FoF 02714; Fig. 79

Saprobic on submerged wood in a freshwater habitat. Sexual morph Undetermined. Asexual morph Colonies on the substrate superficial, effuse, hairy, powdery, black. Mycelium immersed on the substrate, composed of septate, branched, smooth, light brown hyphae. Conidiophores $7-20(-48) \times 3-5 \mu \mathrm{m}(\bar{x}=13 \times 4 \mu \mathrm{m}, \mathrm{n}=15)$, macronematous, mononematous, solitary, erect, minutely verruculose, thickwalled, without apical branches, brown, ellipsoid to subglobose. Conidiogenous cells $5-7 \times 4-6 \mu \mathrm{m}(\bar{x}=6 \times 5 \mu \mathrm{m}$, $\mathrm{n}=15$ ), polyblastic, terminal, dark brown to black, paler at apex, smooth to minutely verruculose, thick-walled, globose to ellipsoid, discrete. Conidia 13-20 $\times 6-7 \mu \mathrm{m}$ $(\bar{x}=16 \times 7 \mu \mathrm{m}, \mathrm{n}=50)$ acrogenous, catenated, yellowish brown to dark brown, minutely verruculose, 3-5-septate, rounded at both ends, paler yellowish at apex, composed of moniliform cells, slightly constricted at septa, doliiform to globose, in beadlike chains, with conidial chains in branches. Conidial secession schizolytic.

Culture characteristics: Conidia germinated on PDA within $24 \mathrm{~h}$, and the germ tube produced by a certain conidial cell at either end or in the center. Colonies grow on PDA, and after incubating at room temperature for 1 month, the diameter reaches about $5 \mathrm{~cm}$. Hyphae are initially white to light grey, and then gradually become grey to brown, with convex middle, flocculent, gray to brown, smooth edges and dark grey; reverse light grey in the middle, dark gray to black, smooth edges.

Material examined: CHINA, Yunnan Province, Kunming City ( $103^{\circ} 08^{\prime} 59^{\prime \prime}$ E $\left.24^{\circ} 37^{\prime} 53^{\prime \prime} \mathrm{N}, 1790 \mathrm{msl}\right)$, on submerged wood, 13 May 2018, H.W. Shen and X. He, S-1837 (HKAS 112705, new record), living culture, DLUCC 1837.

GenBank numbers: $\mathrm{ITS}=\mathrm{MW} 723058$, LSU $=$ MW $879525, \quad S S U=M W 774581$, RPB2 = MW729779, TEF1- $\alpha:$ MW729784.

Notes: In the phylogenetic analyses our strain clustered with the type of Torula mackenziei (MFLU 16-2820) with 100\% ML, 1.00 BYPP support (Fig. 80). Torula mackenziei was collected from a dead branch of Bidens pilosa in Doi Mae Salong, Chiang Rai Province, Thailand (Li et al. 2017). Compared with T. mackenziei, our strain has larger conidiophores $(7-20 \times 3-5 \mu \mathrm{m}$ vs. $3-4.3 \times 3.4-3.7 \mu \mathrm{m})$, 

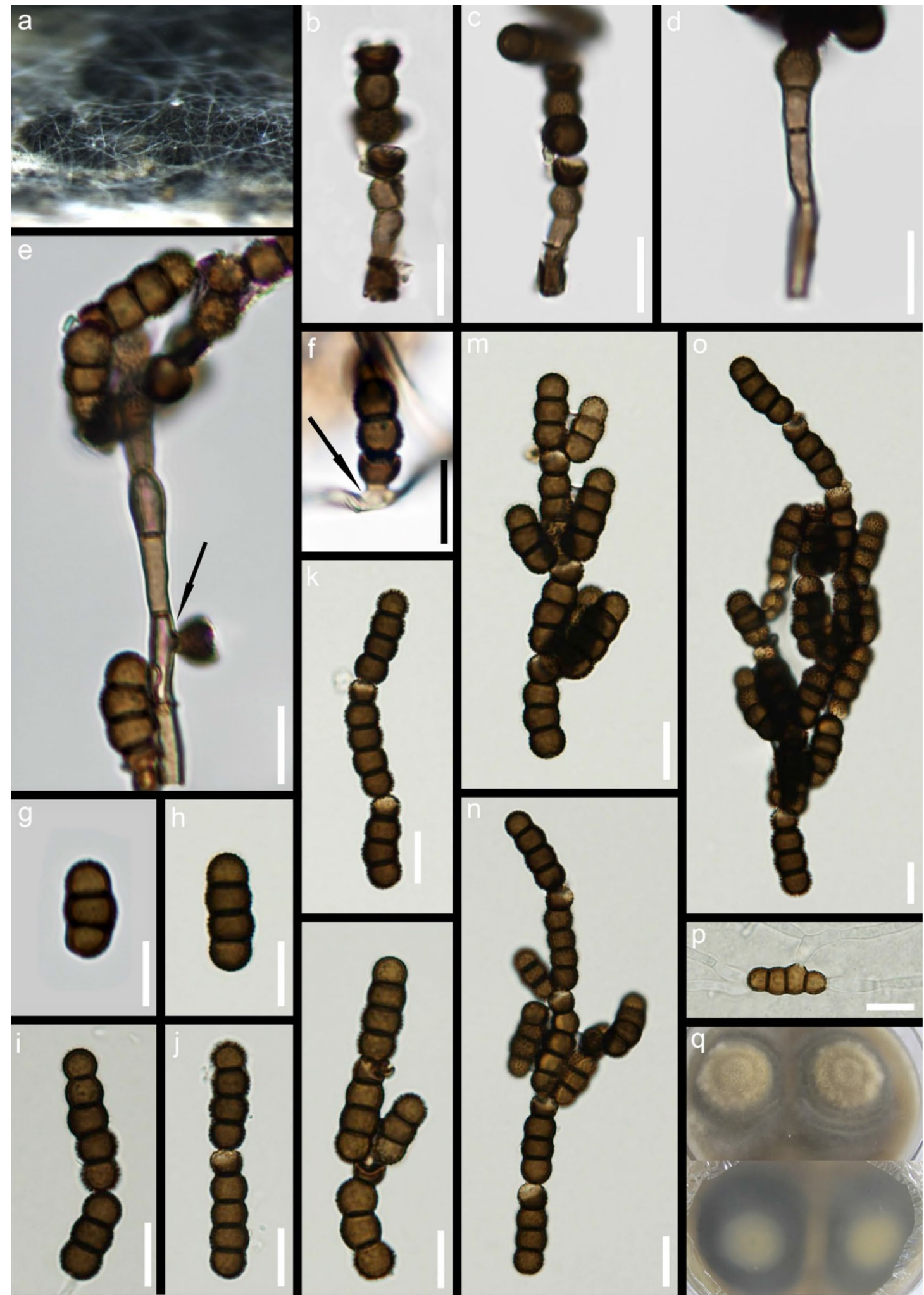

Fig. 79 Torula mackenziei (HKAS 112705, new record). a Colonies on submerged wood. b-f Conidiophores with conidiogenous cells and conidia. $\mathbf{g}, \mathbf{h}$ Conidia. $\mathbf{i}-\mathbf{k}$ Conidia in catenated chain. $\mathbf{l}-\mathbf{o}$ Branched chains of conidia. p Germinated conidium. q Culture on PDA from surface and reverse. Scale bars: $\mathbf{a}-\mathbf{p}=10 \mu \mathrm{m}$ 


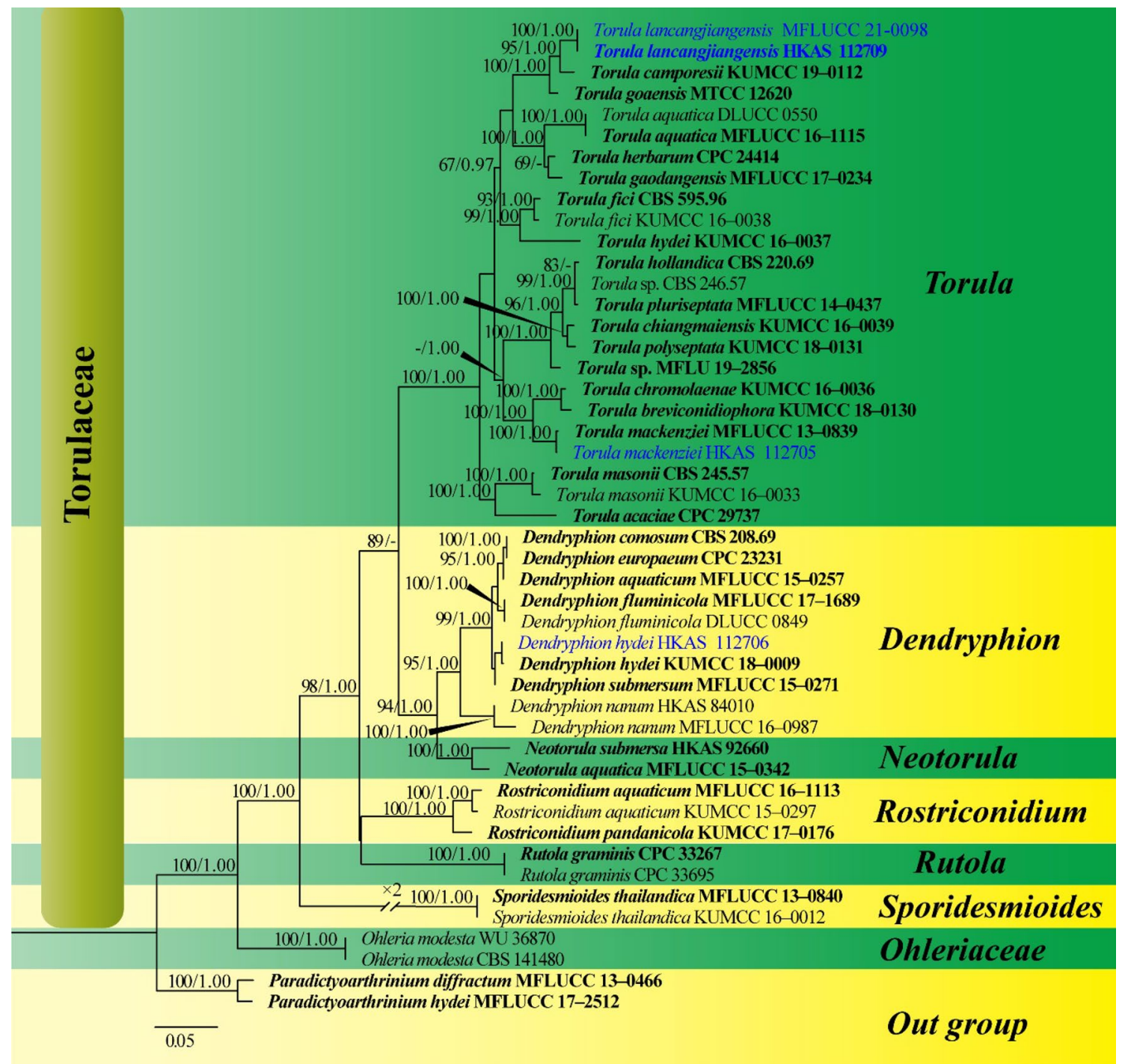

Fig. 80 Phylogram generated from maximum likelihood analysis based on combined LSU, SSU, TEF1- $\alpha$, RPB2 and ITS sequence dataset representing genera of Torulaceae and other related families in Pleosporales. The updated sequence dataset was derived from Hyde et al. (2020b) and Li et al. (2020a). Forty-seven strains are included in the combined analyses which comprise a total of 4193 characters. Paeadictyoarthrinium diffractun MFLUCC 13-0466 and P. hydei MFLUCC 17-2512 are selected as the outgroup taxa. The best RAxML tree with a final likelihood value of -20632.568415 is presented. RAxML analysis yielded 1256 distinct alignment patterns

conidiogenous cells $(5-7 \times 4-6 \mu \mathrm{m}$ vs. $4.3-4.7 \times 4-4.8 \mu \mathrm{m})$, and conidia $(13-20 \times 6-7 \mu \mathrm{m}$ vs. $9.4-18.5 \times 4.4-4.7 \mu \mathrm{m})$. Our strains were collected from submerged wood in a freshwater habitat in Yunnan, China (Fig. 79). We suspect that different growth habitats and substrates may lead to differences in sizes of the strains. In our study, we report the new collection as a new record in a freshwater habitat.

Wicklowiaceae Ariyaw. \& K.D. Hyde and $34.11 \%$ of undetermined characters or gaps. Estimated base frequencies were as follows: $\mathrm{A}=0.246275, \mathrm{C}=0.259573, \mathrm{G}=0.270969$, $\mathrm{T}=0.223183$, with substitution rates $\mathrm{AC}=1.544243, \mathrm{AG}=3.270041$, $\mathrm{AT}=1.437706, \quad \mathrm{CG}=0.944551, \quad \mathrm{CT}=7.854806, \quad \mathrm{GT}=1.000000$; gamma distribution shape parameter alpha $=0.178530$. Bootstrap support values for maximum likelihood (MLBS, left) equal to or greater than $70 \%$ is given above the nodes. Bayesian posterior probabilities (BYPP, right) equal to or greater than 0.95 are given above the nodes. Ex-type strains are in bold and newly generated sequences are in blue

Notes: We follow the latest treatment and updated accounts of Wicklowiaceae in Calabon et al. (2020a) and Hongsanan et al. (2020a).

\section{Wicklowia Raja, A. Ferrer \& Shearer}

Notes: Wicklowia was introduced by Raja et al. (2010) to accommodate the freshwater taxa and was typified by Wicklowia aquatica. Only three species are accepted in this genus (Raja et al. 2010; Boonmee et al. 2019; Calabon et al. 2020a; 
Index Fungorum 2021). In this study, Wicklowia fusiformispora is introduced as a new species and W. submersa is reported as a new record from northern Thailand.

Wicklowia fusiformispora Boonmee, Huanraluek \& K.D. Hyde, sp. nov.

Index Fungorum number: IF558541; Facesoffungi number: FoF 09954; Fig. 81

Etymology: The specific epithet "fusiformispora" refers to the fusiform shape of ascospores.

Holotype: MFLU 21-0068

Saprobic on decaying submerged wood in the river. Sexual morph Ascomata $212-244 \times 225-274 \mu \mathrm{m}$ $(x=231 \times 253 \mu \mathrm{m})$, immersed, erumpent when mature, ellipsoid on the host surface, scattered, gregarious, subglobose, uni-loculate, dark brown, ostiolate. Peridium 32-51 $\mu \mathrm{m}$ wide, thick-walled, composed of light brown to dark brown of pseudoparenchymatous cells of textura angularis. Hamathecium comprising 1-3 $\mu \mathrm{m}$ wide, numerous, cylindrical, filiform, branched, septate, hyaline pseudoparaphyses. Asci $78-151 \times 21-30 \mu \mathrm{m}(\bar{x}=115 \times 30 \mu \mathrm{m}, \mathrm{n}=10), 8$-spored, bitunicate, fissitunicate, cylindrical-clavate, apically thickened and rounded, with an ocular chamber when immature, sessile or short pedicellate. Ascospores 32-41 $\times 9-19 \mu \mathrm{m}$ ( $\bar{x}=36 \times 12 \mu \mathrm{m}, \mathrm{n}=20$ ), 2-3-seriate overlapping, fusiform to cylindrical-ellipsoid, with rounded ends, slightly inequilateral, asymmetrical, slightly curved, hyaline, 1-septate, constricted at the septum, guttulate when immature, surrounded by a thin mucilaginous sheath, smooth-walled. Asexual morph Undetermined.

Culture characteristics: Ascospores germinated on PDA within $12 \mathrm{~h}$ at room temperature. Colonies on MEA, reaching $15 \mathrm{~mm}$ diam. in 4 weeks at room temperature. Mycelium superficial, hairy, radiating outwards, effuse with wavy to fimbriate edge, dark brown.

Material examined: THAILAND, Chiang Mai Province, Mae Ai, Tha Ton, Kok River, on decaying submerged wood in the river shore, 9 December 2019, S. Boonmee, N-KR1 (MFLU 21-0068, holotype), ex-type living culture, MFLUCC 21-0091.

GenBank numbers: ITS $=$ MZ538533, LSU $=$ MZ538567,

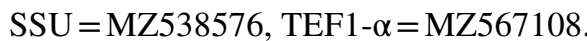

Notes: Wicklowia fusiformispora shares common characters with other described species in Wicklowia, but it differs from all the other species in terms of details regarding features including of shape and size of ascomata, asci and ascospores (Raja et al. 2010; Boonmee et al. 2019; Calabon et al. 2020a). Phylogenetic analysis based on a combined LSU and SSU sequence dataset indicates that $W$. fusiformispora clustered with W. phuketensis with 97\% MLBS, 1.00 BYPP support (Fig. 83). However, Wicklowia fusiformispora is distinct from $W$. phuketensis by its uni-loculate ascomata and has smaller asci $(78-151 \times 21-30 \mu \mathrm{m}$ vs.
86-288 $\times 22-32 \mu \mathrm{m})$ and ascospores $(32-41 \times 9-19 \mu \mathrm{m}$ vs. 34-43 $\times 9-12 \mu \mathrm{m}$ ) (see Calabon et al. 2020a, b; Fig. 2). A comparison of LSU sequences indicates that $W$. fusiformispora differs from $W$. aquatica in $27 / 850 \mathrm{bp}(3.17 \%), W$. phuketensis in $10 / 850 \mathrm{bp}(1.17 \%)$, and W. submersa $18 / 850$ $(2.11 \%)$. Sequence data of the ITS region of W. aquatica, $W$. phuketensis and W. submersa are not available in the GenBank for the comparison. We therefore, identify W. fusiformispora as a new species which collected from Kok River in northern Thailand (Fig. 81).

Wicklowia submersa Boonmee, Sorvongxay \& K.D. Hyde, Phytotaxa 411(1): 76 (2019)

Index Fungorum number: IF556334; Facesoffungi number: FoF 05994; Fig. 82

Saprobic on decaying wood submerged in a freshwater canal. Sexual morph Ascomata $93-186 \times 126-179 \mu \mathrm{m}$ $(\bar{x}=134 \times 151 \mu \mathrm{m})$, immersed, erumpent when mature, raising the host tissue, dark ellipsoid or slit-like, uni-loculate, subglobose, dark brown, scattered, gregarious, ostiolate. Peridium 20-24 $\mu \mathrm{m}$ wide, thick-walled, composed of dark brown of pseudoparenchymatous cells of textura angularis. Hamathecium comprising 1-2 $\mu \mathrm{m}$ wide, numerous, cylindrical, filiform, branched, septate, hyaline pseudoparaphyses. Asci $64-95 \times 21-28 \mu \mathrm{m}(\bar{x}=76 \times 24 \mu \mathrm{m}, \mathrm{n}=7), 8$-spored, bitunicate, fissitunicate, cylindrical-clavate, apically thickened and rounded, with an ocular chamber when immature, sessile or short pedicellate. Ascospores 24-28 $\times 7-15 \mu \mathrm{m}$ ( $\bar{x}=26 \times 9 \mu \mathrm{m}, \mathrm{n}=20$ ), 2-3-seriate, partially overlapping, ellipsoidal-oblong to broad fusiform, with rounded ends, slightly inequilateral, asymmetrical, upper cell wider, hyaline, 1-septate, constricted at the septum, guttulate when immature, surrounded by a distinctly mucilaginous sheath, smooth-walled. Asexual morph Undetermined.

Culture characteristics: Ascospores germinated on PDA within $12 \mathrm{~h}$. Colonies on MEA, reaching $0.5-1.5 \mathrm{~cm}$ diam., in 2 weeks at room temperature. Mycelium superficial, dark grey to brown, hairy, radiating outwards. effuse with wavy and dark brown edge.

Material examined: THAILAND, Uttaradit Province, Phichai, Thasak, Chom Tok Village, on decaying submerged wood in a freshwater canal, 29 December 2018, S. Boonmee, CTU11 (MFLU 21-0069, new record), living culture, MFLUCC 21-0092.

GenBank numbers: LSU = MZ538568, TEF1- $\alpha=$ MZ567109.

Notes: Wicklowia submersa was reported from a freshwater habitat collected in Krabi Province, southern Thailand (Boonmee et al. 2019). Our new isolate of W. submersa (MFLUCC 21-0092) was collected from Uttaradit Province in northern Thailand. Our new collection (Fig. 82) share similar characters of ascomata, asci and ascospores identical to the ex-type strain W. submersa (MFLUCC 18-0373). 

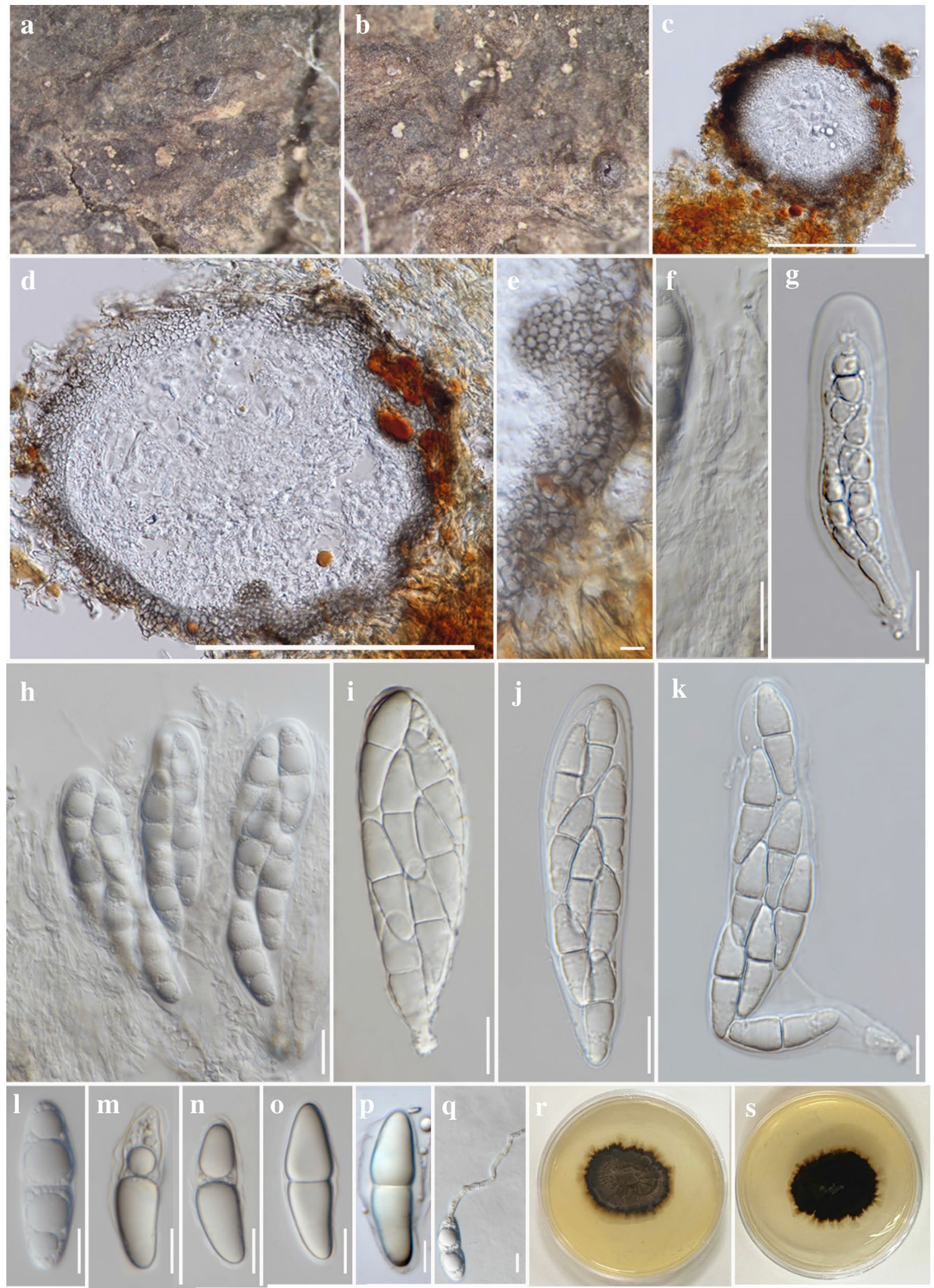

Fig. 81 Wicklowia fusiformispora (MFLU 21-0068, holotype). a, b Appearance of ascomata on decaying submerged wood. c, d Cross sections of ascomata. e Section of peridium. f Pseudoparaphyses. g-k
Asci. l-o Ascospores. p Ascospore stained in Nigrosine reagent. q Germinated ascospore. $\mathbf{r}, \mathbf{s}$ Culture on MEA from above and below. Scale bars: $\mathbf{c}, \mathbf{d}=200 \mu \mathrm{m}, \mathbf{e}-\mathbf{k}, \mathbf{q}=20 \mu \mathrm{m}, \mathbf{l}-\mathbf{p}=10 \mu \mathrm{m}$ 

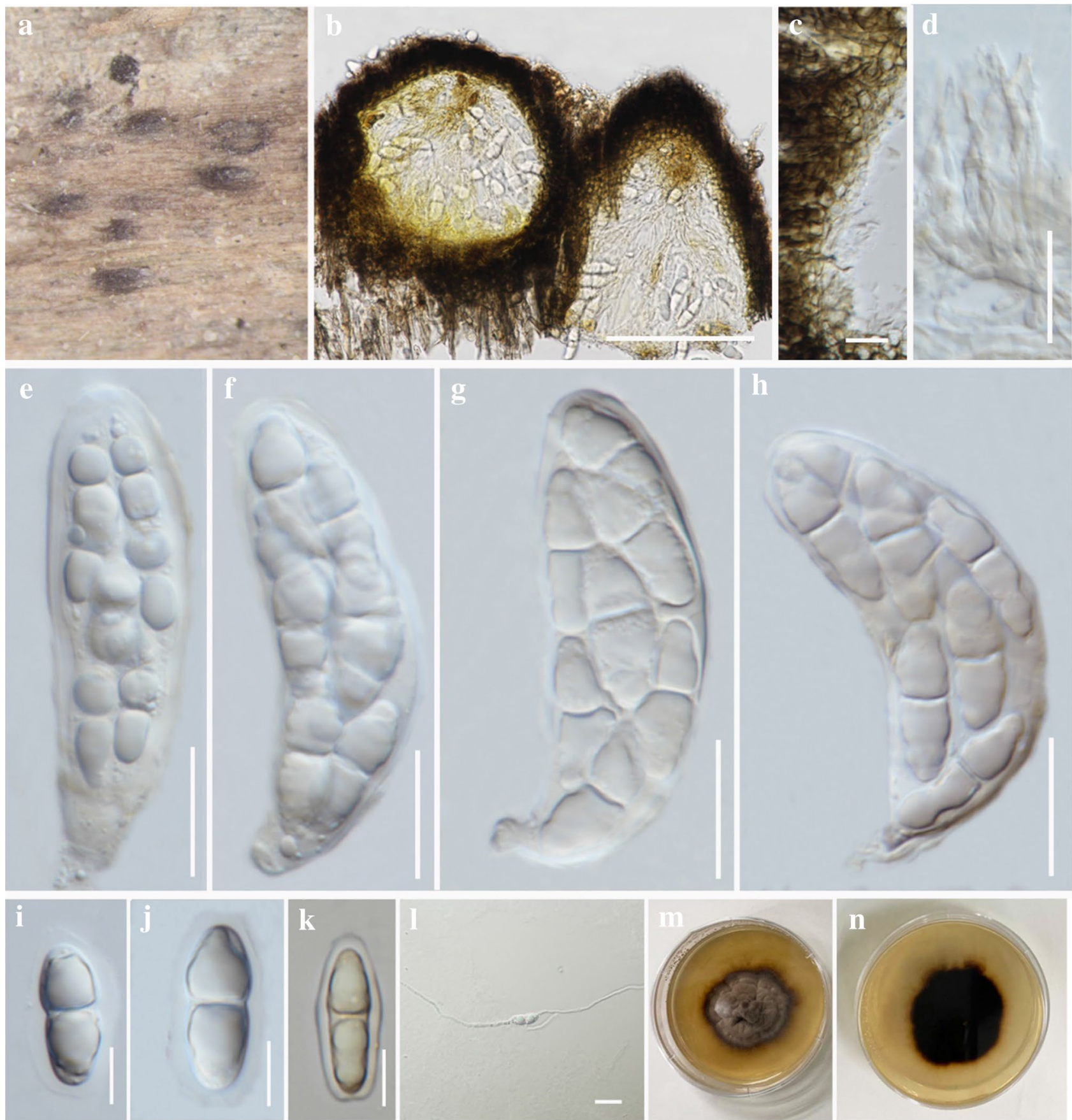

Fig. 82 Wicklowia submersa (MFLU 21-0069, new record). a Appearance of ascomata on substrate. $\mathbf{b}$ Cross section of ascomata. $\mathbf{c}$ Section of peridium. d Pseudoparaphyses. $\mathbf{e}-\mathbf{h}$ Asci. $\mathbf{i}-\mathbf{k}$ Ascospores ( $\mathrm{i}$ and $\mathrm{j}=$ ascospores mounted in water, $\mathrm{k}=$ ascospore stained in

Phylogenetic analysis indicates that our strain consistently clustered with the ex-type strain MFLUCC 18-0373 with 100\% MLBS, 1.00 BYPP support (Fig. 83). We therefore, report our isolate MFLUCC 21-0092 as a new record of $W$. submersa in Thailand.
Nigrosin reagent). l Germinated ascospore. $\mathbf{m}, \mathbf{n}$ Culture on MEA from surface and reverse. Scale bars: $\mathbf{b}=100 \mu \mathrm{m}, \mathbf{c}-\mathbf{h}, \mathbf{l}=20 \mu \mathrm{m}$, $\mathbf{i}-\mathbf{k}=10 \mu \mathrm{m}$

Tubeufiales S. Boonmee \& K.D. Hyde

Notes: Tubeufiales currently includes three families (i.e., Bezerromycetaceae, Tubeufiaceae and Wiesneriomycetaeae) with 55 genera and the latest updated accounts in Hongsanan et al. (2020b). 


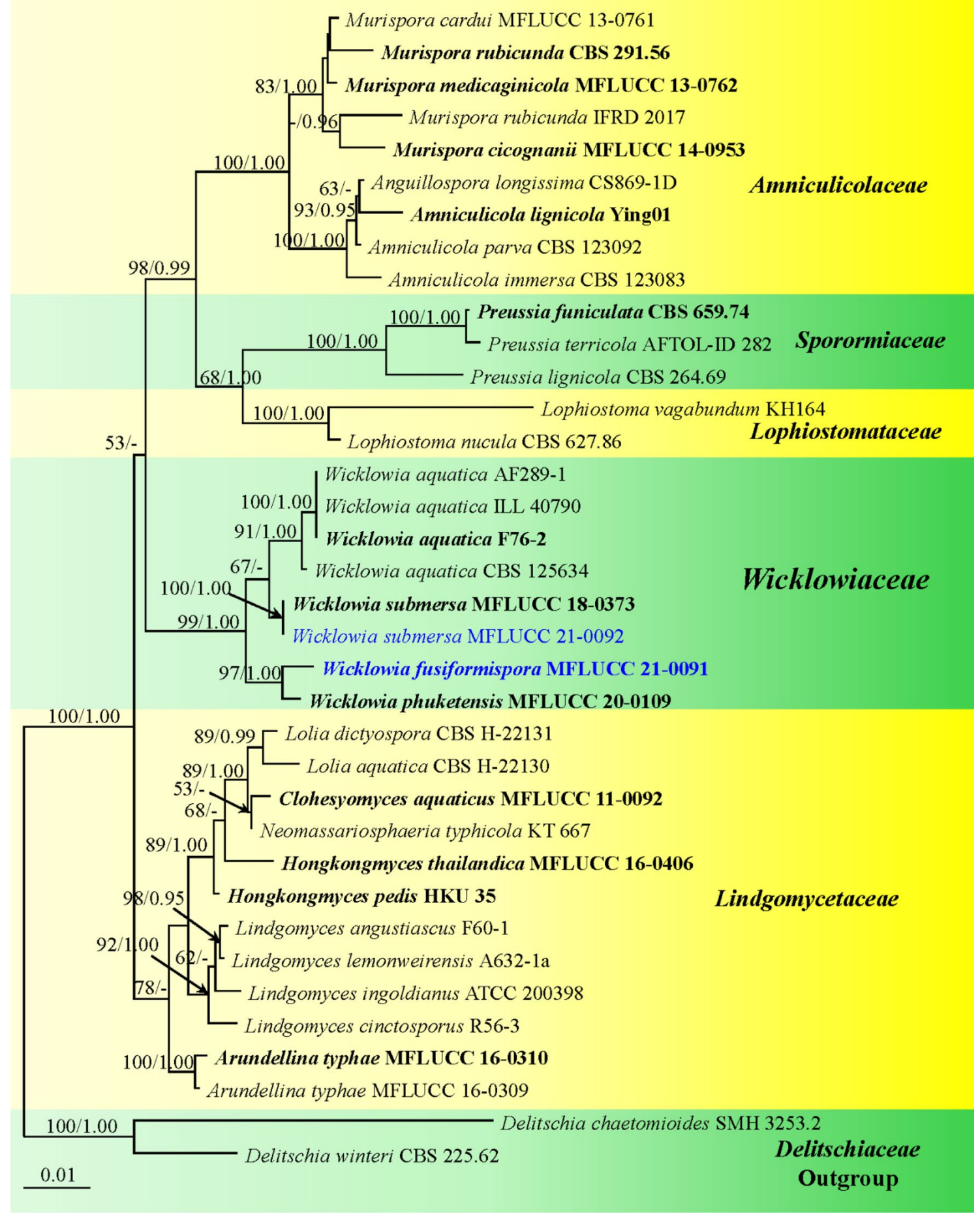

Fig. 83 Maximum likelihood phylogenetic tree based on a combined LSU and SSU sequence data of Wicklowia taxa (Wicklowiaceae) and related families. The tree is rooted with Delitschia chaetomioides (SMH 3253.2) and D. winteri (CBS 225.62). Bootstrap sup-

\section{Tubeufiaceae M.E. Barr}

Notes: We follow the latest treatments and updated accounts of Tubeufiaceae in Hongsanan et al. (2020b). Two new species and five new records from genera port values for maximum likelihood (MLBS, left) equal to or greater than $50 \%$ is given above the nodes. Bayesian posterior probabilities (BYPP, right) equal to or greater than 0.95 are given above the nodes. Ex-type strains are in bold and newly generated sequences are in blue

Helicosporium, Neohelicosporium and Tubuefia are reported in this study.

Helicosporium Nees 
Notes: Helicosporium comprises 101 species epithets (Index Fungorum 2021) but only 20 species are accepted in the genus (Species Fungorum 2021). Its members are hyphomycetous, helisporous, with multi-coil to loosely uncoiled, multi-septate, hyaline to pale brown and smooth-walled conidia (Boonmee et al. 2014; Lu et al. 2018b). One new species and one new record are provided for this genus.

Helicosporium luteosporum Y.Z. Lu, Boonmee \& K.D. Hyde, Phytotaxa 319(3): 248 (2017)

Index Fungorum number: IF552663; Facesoffungi number: FoF 02763; Figs. 84, 85
Saprobic on decaying wood. Sexual morph Undetermined. Asexual morph Hyphomycetous, helicosporous. Colonies effuse, yellow, shiny, with crowded by conidial masses. Mycelium composed of partly immersed to superficial, septate, branched, hyaline to brown hyphae. Conidiophores $142-370 \mu \mathrm{m}$ long, $5-8 \mu \mathrm{m}$ wide at base, micronematous, mononematous, arising from repent mycelium, tapering toward narrow subacute at apex, fuscous branching and brown at base, brownish to hyaline towards apex, septate, smooth-walled. Conidiogenous cells 3-7.5 $\times 2-3 \mu \mathrm{m}$, denticulate, holoblastic, monoblastic, bearing lateral minute denticles each with single conidium. Conidia 81-161 $\mu \mathrm{m}$ diam.,
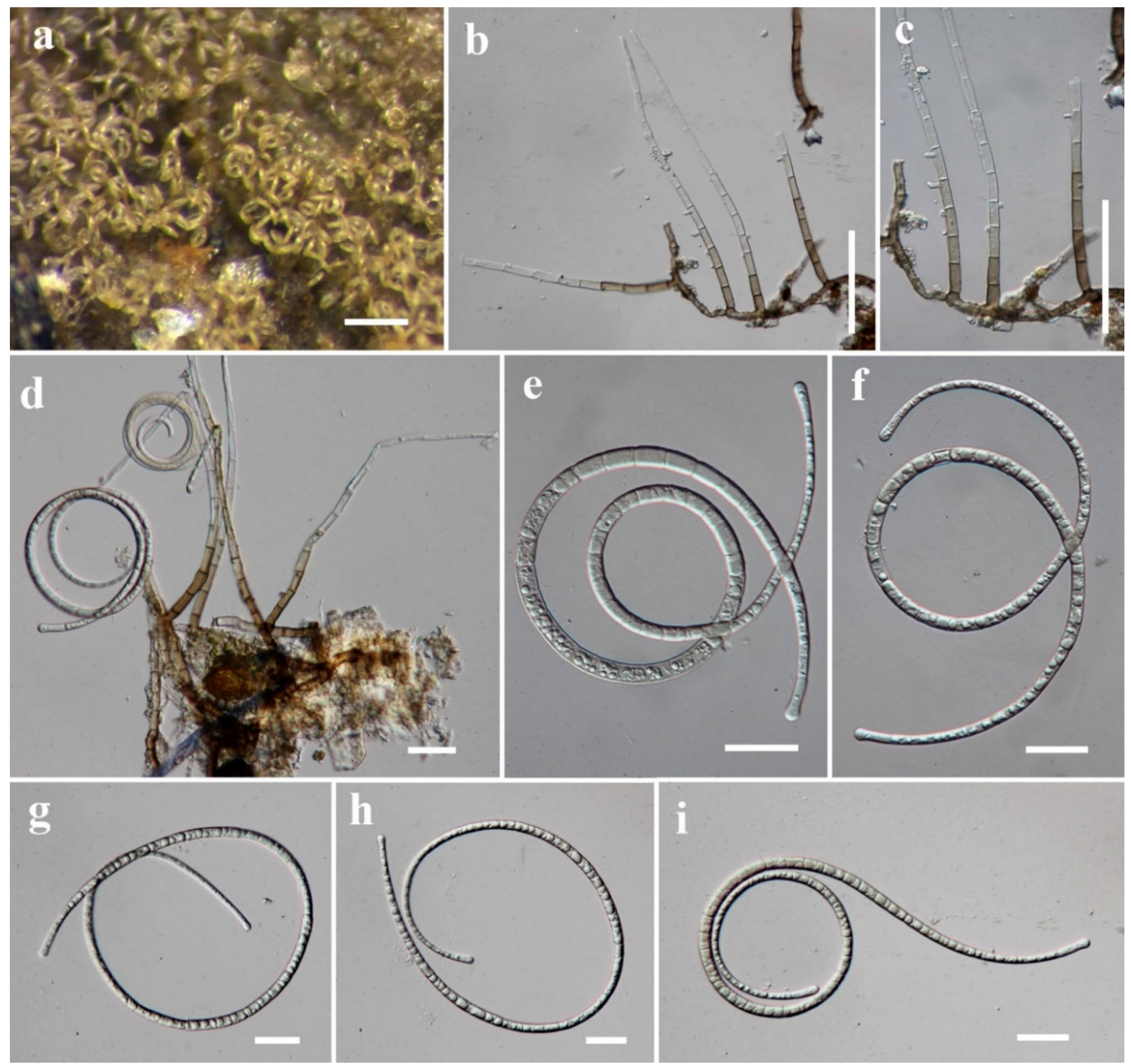

Fig. 84 Helicosporium luteosporum (MFLU 17-0502, new record). a Colony on decaying wood. b-d Conidiophores with lateral minute polyblastic denticles and attached conidia. $\mathbf{e}-\mathbf{i}$ Conidia. Scale bars: $\mathbf{a}=200 \mu \mathrm{m}, \mathbf{b}, \mathbf{c}=50 \mu \mathrm{m}, \mathbf{d}-\mathbf{i}=20 \mu \mathrm{m}$ 


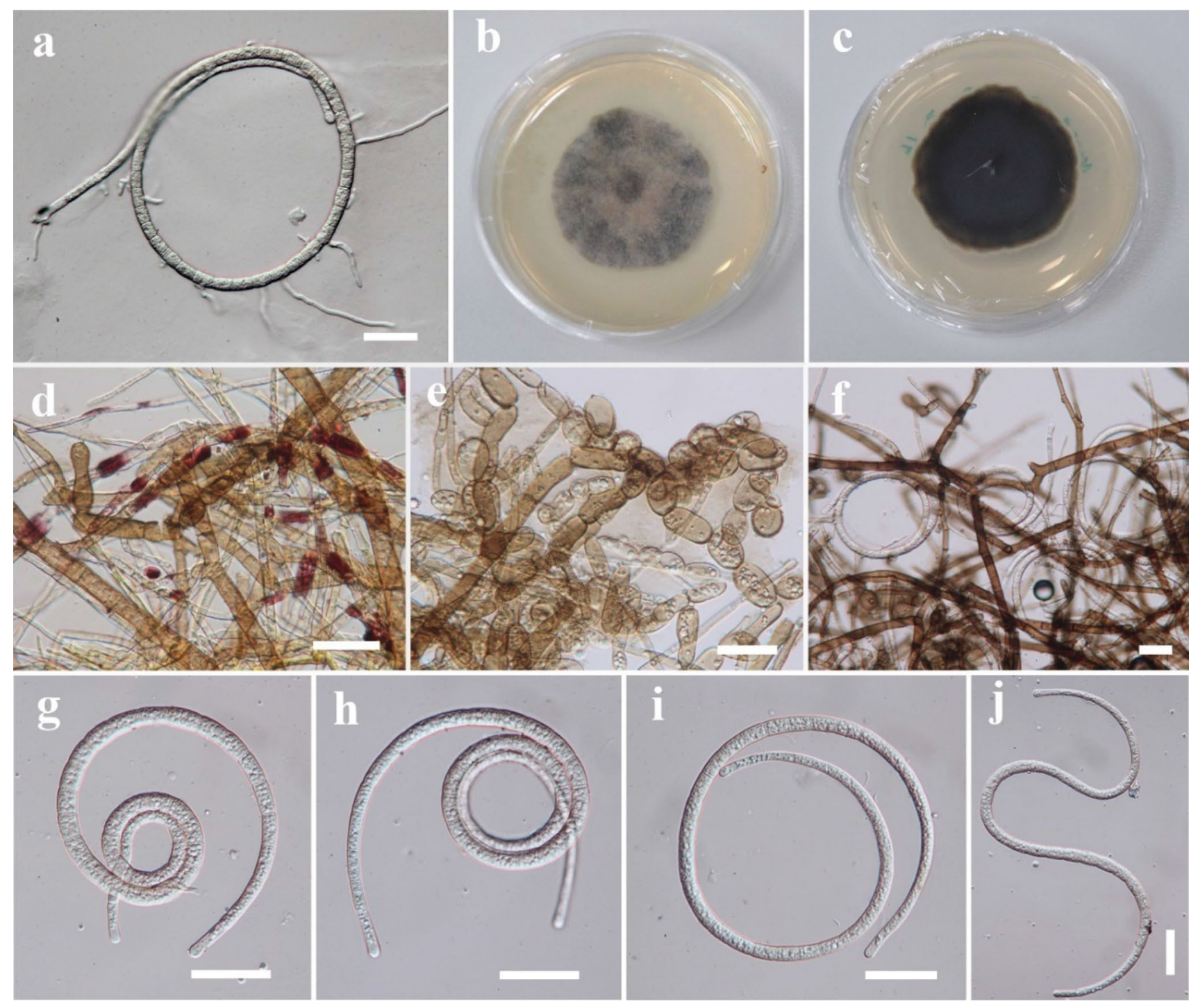

Fig. 85 Helicosporium luteosporum sporulation in culture (MFLUCC 16-1233). a Germinated conidium. b, c Culture on MEA from surface and reverse after 1 month. $\mathbf{d}-\mathbf{f}$ Mycelium and development of conidia in culture. $\mathbf{g}-\mathbf{j}$ Conidia. Scale bars: $\mathbf{a}, \mathbf{d}-\mathbf{j}=20 \mu \mathrm{m}$

with conidial filament 6-8 $\mu \mathrm{m}$ wide, with $634-674.5 \mu \mathrm{m}$ long, loosely coiled 1-21/2 times, becoming loosely uncoiled in water, rounded at apical ends, indistinctly multi-septate, not constricted at the septa, bright, hyaline to pale brown, guttulate, smooth-walled.

Culture characteristics: Ascospores germinating on MEA within $24 \mathrm{~h}$ and many germ tubes produced from conidium cells. Colonies growing on MEA slowly, less than $5 \mathrm{~mm}$ diam. in 7 days at room temperature, slightly raised-radially with entire edge, brownish-gray to pale brown. Mycelium developing superficial, composed of branched, septate, pale brown, reddish-brown to dark brown, with red pigmented hyphal filaments, conidial spores formed on hyphae in culture at 60 days. Conidia 55-112 $\mu \mathrm{m}$ diam., with conidial filament 7.5-8 $\mu \mathrm{m}$ wide, with 445-448 $\mu \mathrm{m}$ long, loosely coiled
$1-3 \frac{1}{2}$ times, becoming loosely uncoiled in water, rounded at apical ends, indistinctly multi-septate, not constricted at the septa, hyaline, with granular contents.

Material examined: THAILAND, Chanthaburi, Laem Sing, Ban Phlio, on decaying wood in a terrestrial habitat, 7 July 2016, S. Boonmee, NTP02-Ax (MFLU 17-0502, new record), living culture, MFLUCC 16-1233.

GenBank numbers: LSU $=$ KY873624.

Notes: Isolate (MFLUCC 16-1233) was identified as Helicosporium luteosporum based on phylogenetic analysis, but morphological illustrations were not provided (Brahamanage et al. 2017; Lu et al. 2017). A nucleotide base comparison of the LSU sequence reveals that our taxon is $100 \%$ similar to the ex-type strain H. luteosporum MFLUCC 16-0266 (Jeewon and Hyde 2016). Further, phylogenetic analyses of 
a combined ITS, LSU and TEF1- $\alpha$ sequence dataset indicates that our strain clusters with the H. luteosporum strain with 100\% MLBS, 1.00 BYPP support (Fig. 87). Therefore, we provide a detailed description, illustration and update phylogenetic tree for $H$. luteosporum (MFLUCC 16-1233) as a new geographical record in Thailand (Figs. 84 and 85).

Helicosporium sexuale Boonmee, Promputtha \& K.D. Hyde, sp. nov.

Index Fungorum number: IF558542; Facesoffungi number: FoF 09194; Fig. 86

Etymology: 'sexuale' referring to sexual morph.

Holotype: MFLU 21-0104

Saprobic on decaying wood. Sexual morph Ascomata 185-235 ×116-214 $\mu \mathrm{m}$, superficial, solitary, scattered, globose to subglobose, yellowish brown, shiny when fresh, with sparsely setae, minutely papilla, ostiolate. Setae 73-88 $\times 4-8 \mu \mathrm{m}$, stiff, tapering to an acute tip, septate, dark brown. Peridium 8-12 wide $\mu \mathrm{m}$, composed of 2-3layer cells of textura angularis, yellowish brown to hyaline cells, lacking pseudoparaphyses. Asci 54.5-76×11-20 $\mu \mathrm{m}$, 8 -spored, bitunicate, cylindric-clavate, rounded at apex, sessile. Ascospores 51.5-71 ×3-4 $\mu \mathrm{m}$, fasciculate, cylindrical, narrowly fusiform, subacute ends, straight to slightly curved, 9-11-spetate, not constricted at septa, hyaline, smoothwalled. Asexual morph Undetermined.

Culture characteristics: Ascospores germinating on MEA within $24 \mathrm{~h}$ and many germ tubes produced from ascospore cells. Colonies growing on MEA slowly, less than $5 \mathrm{~mm}$ diam. in 7 days at room temperature, slightly raised-radially with entire edge, brown to dark brown, asexual conidia not formed at 60 days.

Material examined: THAILAND, Chanthaburi Province, Laem Sing, Ban Phlio, on decaying wood in a terrestrial habitat, 7 July 2016, S. Boonmee, NTP15 (MFLU 21-0104, holotype), ex-type living culture, MFLUCC 16-1244.

GenBank numbers: ITS $=$ MZ538503, LSU $=$ MZ538537, RPB2 $=$ MZ567111, TEF1- $\alpha=$ MZ567082.

Notes: Phylogenetic analysis indicates that Helicosporium sexuale (MFLUCC 16-1244) is basal to $H$. vegetum strains (CBS 254.75, CBS 269.52, CBS 941.72, NBRC 303045 and NBRC 9014) with 96\% MLBS, 1.00 BYPP support (Fig. 87); the phylogeny also shows that it is a distinct species (Fig. 87). Helicosporium sexuale differed from $H$. vegetum and other species by the capacity to form its sexual morphs (Fig. 86). We therefore, introduce H. sexuale as a new sexual species in Helicosporium.

Neohelicosporium Y.Z. Lu, J.C. Kang \& K.D. Hyde

Notes: The latest treatments and updated accounts of Neohelicosporium followed herein are Lu et al. (2018a). Lu et al. (2018a) introduced Neohelicosporium to accommodate the taxa in having Helicosporium-like characters based on phylogenetic and morphological evidence, which is typified by Ne. parvisporum. Members of Neohelicosporium differ from Helicosporium in having acrogenous and/or acropleurogenous conidia developing from an integrated, sympodial conidiogenous cell while the latter has pleurogenous conidia and discrete, determinate conidiogenous cells ( $\mathrm{Lu}$ et al. 2018a). Four new isolates of Neohelicosporium irregulare and one new isolate of Neohelicosporium parvisporum are reported.

Neohelicosporium irregulare Y.Z. Lu, J.C. Kang \& K.D. Hyde, Fungal Diversity 92: 235 (2018)

Index Fungorum number: IF554871; Facesoffungi number: FoF 04739; Figs. 88, 89

Saprobic on submerged decaying wood. Sexual morph Undetermined. Asexual morph Hyphomycetous, helicosporous. Colonies effuse, white, shiny, with crowded by conidial masses. Mycelium composed of superficial and partly immersed, branched, anastomosing, septate, brown hyphae, smooth-walled. Conidiophores 116-229.5×6-8 $\mu \mathrm{m}$, micronematous, mononematous, arising from repent mycelium, tapering toward narrow at apex, widest at base, branched, septate, brown, smooth-walled. Conidiogenous cells holoblastic, mono- to polyblastic, terminal or intercalary, integrated, subhyaline, bearing lateral minute denticles each with single conidium. Conidia 36-53 $\mu \mathrm{m}$ diam., with conidial filament 3-4.5 $\mu \mathrm{m}$ wide, with 190.5-236 $\mu \mathrm{m}$ long, loosely coiled 1-3 times, becoming loosely uncoiled in water, rounded at apical ends, indistinctly multi-septate, not constricted at the septa, hyaline, with granular contents.

Culture characteristics: Conidium germinating on MEA within $24 \mathrm{~h}$ and many germ tubes produced from conidium cells. Colonies growing on MEA, reaching greater than $10 \mathrm{~mm}$ diam. in 7 days at room temperature, irregular, slightly raised, with edge undulate to fimbriate, dark brown. Mycelium developing superficial, partly immersed, composed of branched, septate, light brown to dark brown hyphal filaments, conidial spores formed on hyphae in culture at 60 days. Conidia 29-49 $\mu \mathrm{m}$ diam., conidial filament 4-5.5 $\mu \mathrm{m}$ wide, $167-208 \mu \mathrm{m}$ long, loosely coiled $1-3 \frac{1}{2}$ times, becoming loosely uncoiled in water, rounded at apical ends, indistinctly multi-septate, not constricted at the septa, hyaline to brown, with granular contents.

Material examined: THAILAND, Chiang Rai Province, Muang, Mae Yao, Huay Mae Sai, on submerged decaying wood in a small freshwater stream, 17 March 2016, S. Boonmee, HMS03 (MFLU 21-0097), living culture, MFLUCC 16-0721; HMS04 (MFLU 21-0098, new record) living culture, MFLUCC 16-0722; HMS05 (MFLU 21-0099), living culture, MFLUCC 16-0723; HMS17 (MFLU 21-0102), living culture, MFLUCC 16-0758.

GenBank numbers: HMS03: ITS $=$ MZ538516, LSU $=$ MZ538550, TEF $1-\alpha=$ MZ567093; HMS04: 

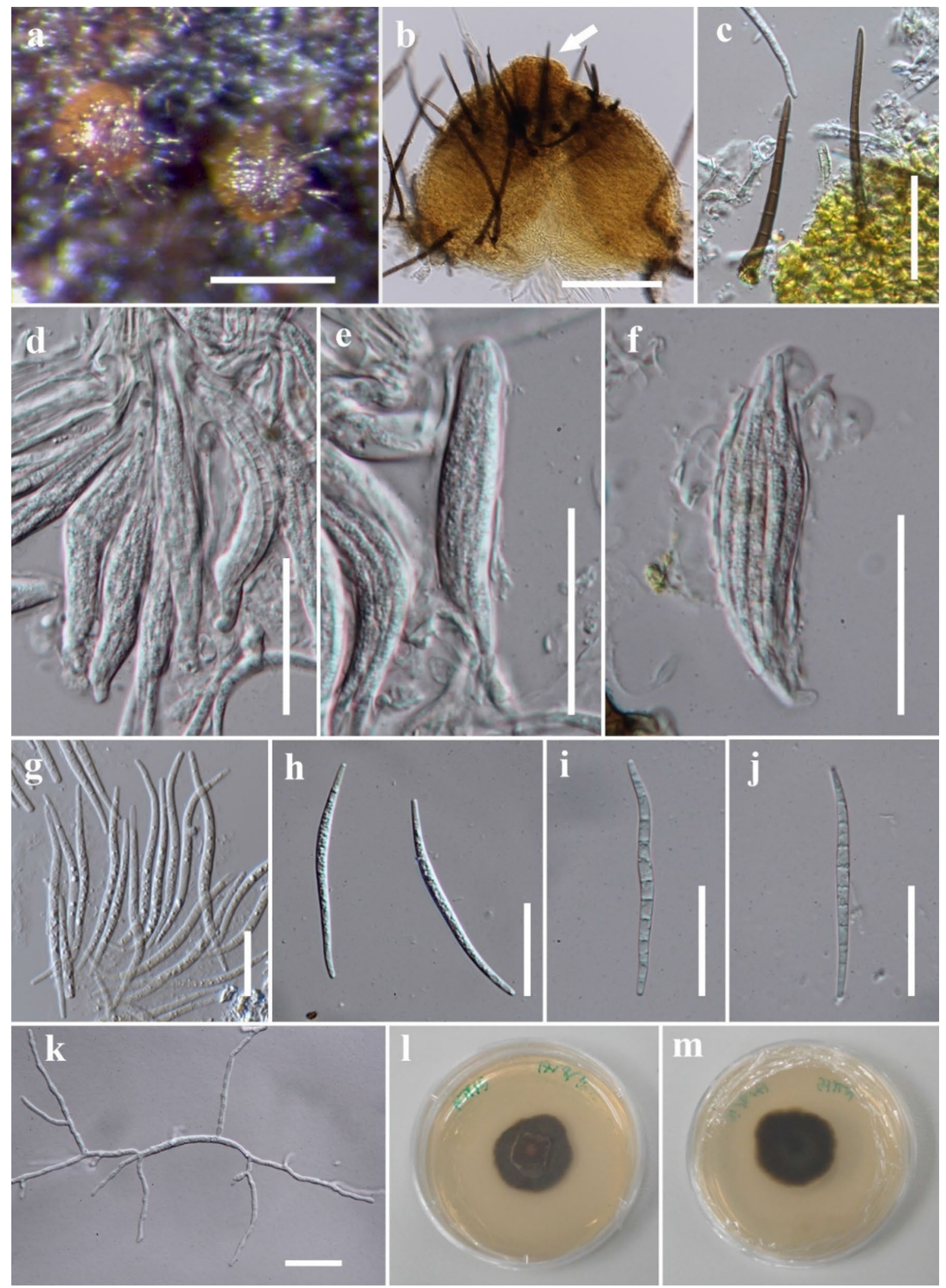

Fig. 86 Helicosporium sexuale (MFLU 21-0104, holotype). a Superficial ascomata on decaying wood substrate. b Squash mount of ascoma showing peridium. $\mathbf{c}$ Setae. $\mathbf{d}-\mathbf{f}$ Asci. $\mathbf{g}-\mathbf{j}$ Ascospores. $\mathbf{k}$
Germinated conidium. $\mathbf{l}, \mathbf{m}$ Culture on MEA from surface and reverse after 1 month. Scale bars: $\mathbf{a}=200 \mu \mathrm{m}, \mathbf{b}=100 \mu \mathrm{m}, \mathbf{c}-\mathbf{f}=40 \mu \mathrm{m}, \mathbf{g}$ $\mathbf{k}=20 \mu \mathrm{m}$ 


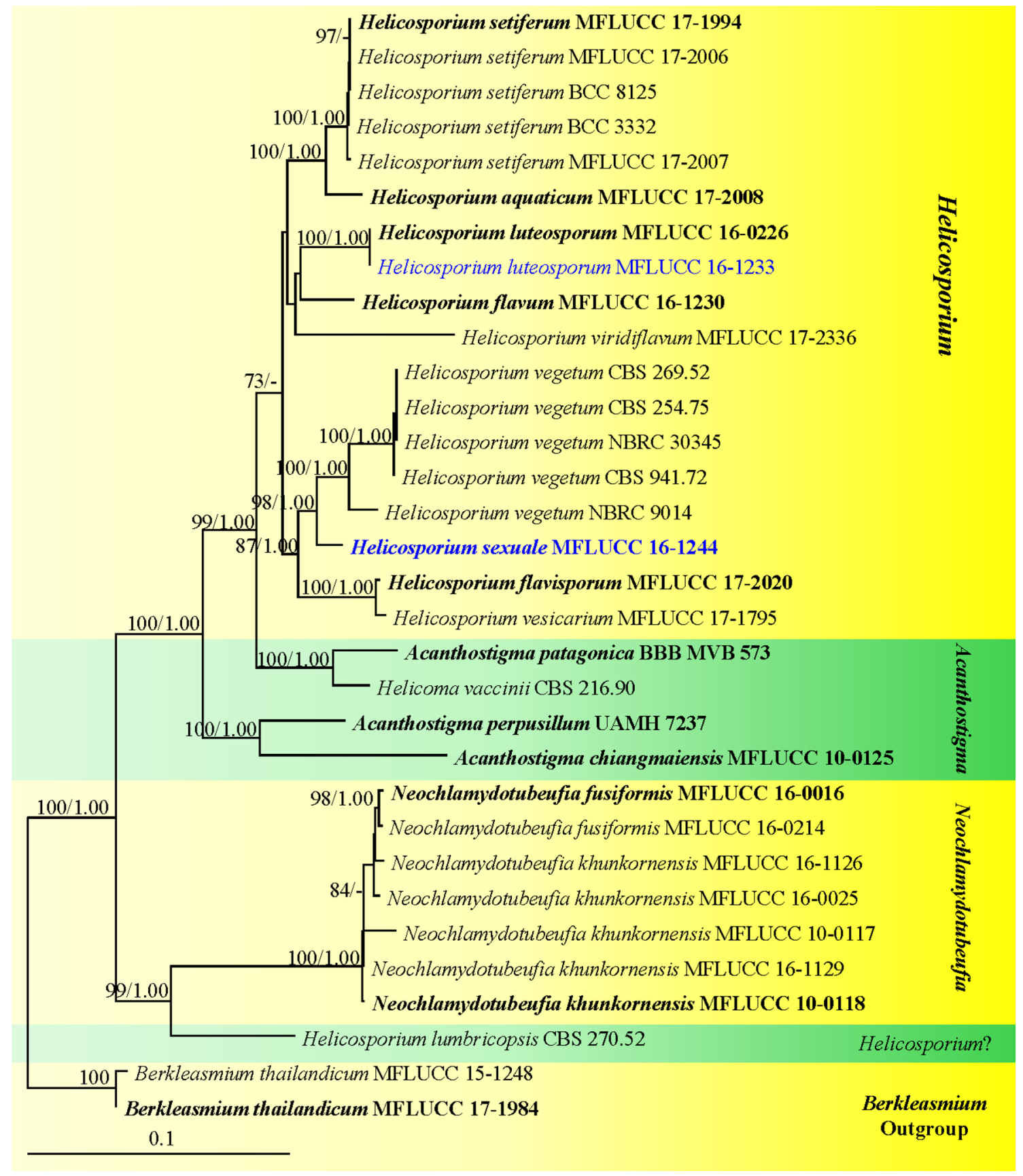

Fig. 87 Maximum likelihood phylogenetic tree based on a combined ITS, LSU and TEF1- $\alpha$ sequence data of Helicosporium taxa and related genera in Tubeufiaceae. Thirty-two taxa are included in the combined analyses which comprise a total of 2367 characters. Berkleasmium thailandicum (MFLUCC 15-1248 and MFLUCC 17-1984) are selected as the outgroup taxa. The best RAxML tree with a final likelihood value of -8690.594891 is presented. RAxML analysis yielded 609 distinct alignment patterns and $25.02 \%$ of undetermined characters or gaps. Estimated base frequencies were as fol- lows: $\mathrm{A}=0.231326, \mathrm{C}=0.266395, \mathrm{G}=0.273200, \mathrm{~T}=0.229079$, with substitution rates $\mathrm{AC}=1.159455, \mathrm{AG}=2.641204, \mathrm{AT}=1.962575$, $\mathrm{CG}=0.673701, \mathrm{CT}=9.846706, \mathrm{GT}=1.000000$; gamma distribution shape parameter alpha $=0.182397$. Bootstrap support values for maximum likelihood (MLBS, left) equal to or greater than $70 \%$ is given above the nodes. Bayesian posterior probabilities (BYPP, right) equal to or greater than 0.95 are given above the nodes. Ex-type strains are in bold and newly generated sequences are in blue 

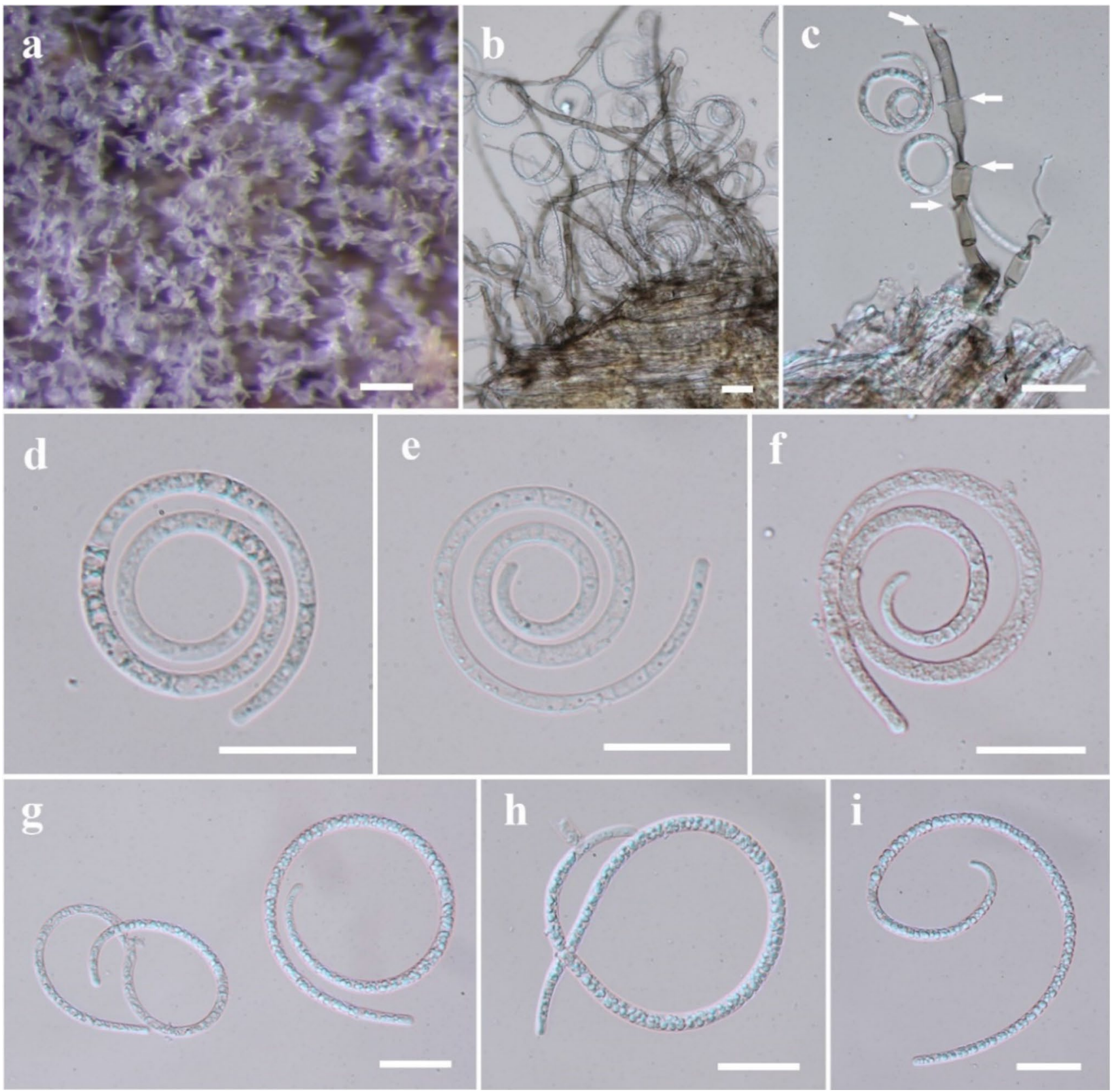

Fig. 88 Neohelicosporium irregulare (MFLU 21-0098, new record). a Colony on decaying wood. b, c Conidiophores with lateral minute polyblastic denticles and attached conidia. $\mathbf{d}-\mathbf{i}$ Conidia. Scale bars: $\mathbf{a}=100 \mu \mathrm{m}, \mathbf{b}-\mathbf{i}=20 \mu \mathrm{m}$

ITS $=$ MZ538517, LSU $=$ MZ538551, TEF1- $\alpha=$ MZ567094; HMS 05: ITS $=$ MZ538518, LSU $=$ MZ538552, TEF $1-\alpha=$ MZ567095; HMS17: ITS $=$ MZ538519, LSU $=$ MZ538553, TEF1- $\alpha=$ MZ567096.

Notes: Four new collections of Neohelicosporium irregulare (MFLUCC 16-0721, MFLUCC 16-0722, MFLUCC 16-0722 and MFLUCC 16-0758) were recovered from submerged decaying wood from Chiang Rai Province. They can be identified as Neohelicosporium since they formed typical effuse colonies, erect conidiophores and long filamentous, multi-septate with hyaline to light pigmented helicospores (Figs. 88 and 89). They share a close phylogenetic affinity to the ex-type strain Neohelicosporium irregulare (MFLUCC 17-1796) in the combined LSU, ITS and TEF1- $\alpha$ sequenced data, which confirmed that they are the same species (Fig. 91).

Neohelicosporium parvisporum Y.Z. Lu, J.C. Kang \& K.D. Hyde, Mycol. Progr. 17(5): 637 (2017)

Index Fungorum number: IF822056; Facesoffungi number: FoF 03571; Fig. 90 


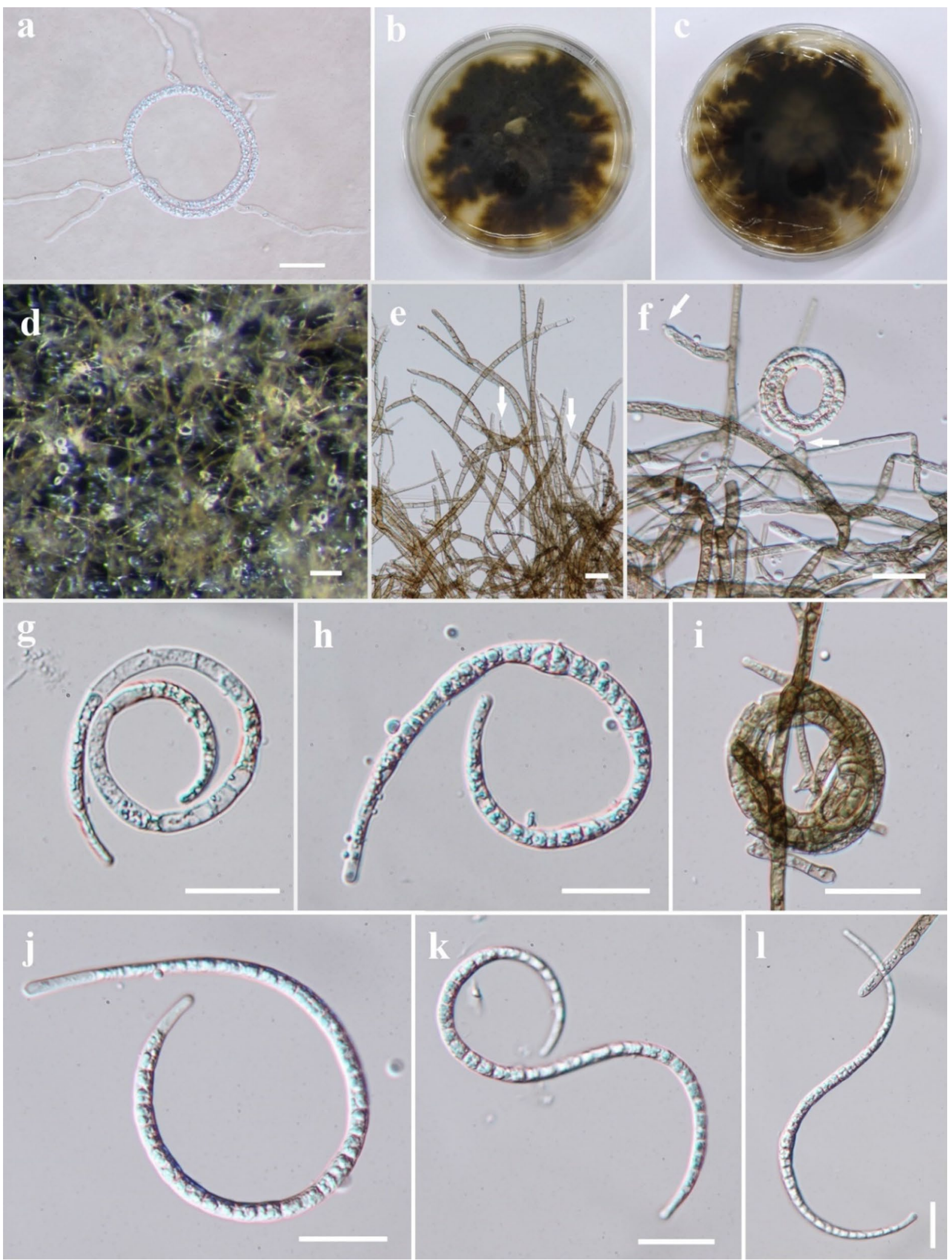

Fig. 89 Neohelicosporium irregulare sporulation in culture (MFLUCC 16-0722). a Germinated conidium. b, c Culture on MEA from surface and reverse. $\mathbf{d}-\mathbf{f}$ Mycelium and development of conidia in culture. $\mathbf{g}-\mathbf{l}$ Conidia. Scale bars: $\mathbf{a}, \mathbf{e}-\mathbf{l}=20 \mu \mathrm{m}, \mathbf{d}=100 \mu \mathrm{m}$ 

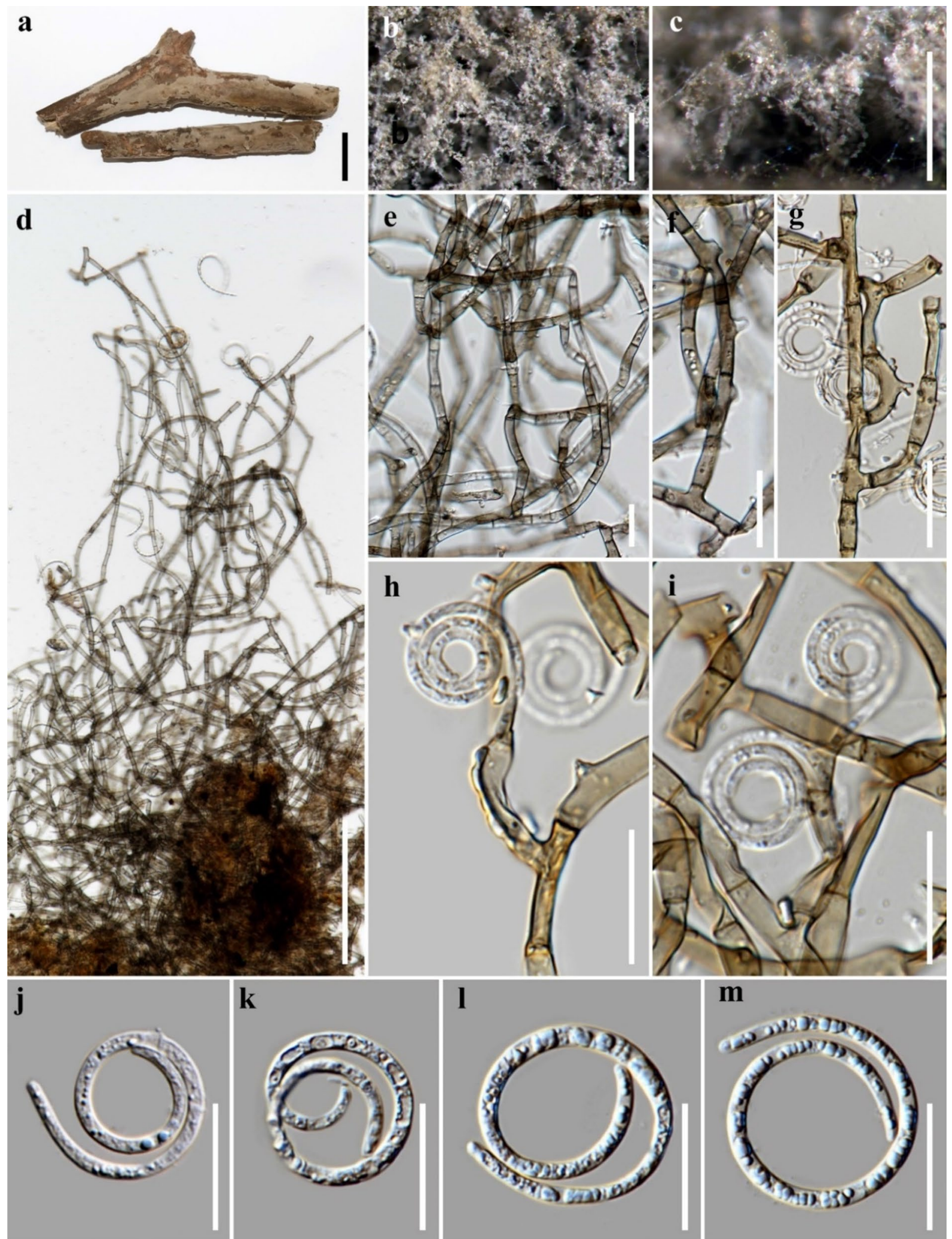

$\mathbf{m}$

Fig. 90 Neohelicosporium parvisporum (MFLU 21-0123, new record). a Substrate. b, c Colony on decaying wood. d-f Conidiophores with conidia. $\mathbf{g - i}$ Conidiogenous cells. $\mathbf{j}-\mathbf{m}$ Conidia. Scale bars: $\mathbf{a}=20 \mathrm{~mm}, \mathbf{b}, \mathbf{c}=500 \mu \mathrm{m}, \mathbf{d}=100 \mu \mathrm{m} ; \mathbf{e}-\mathbf{m}=20 \mu \mathrm{m}$ 


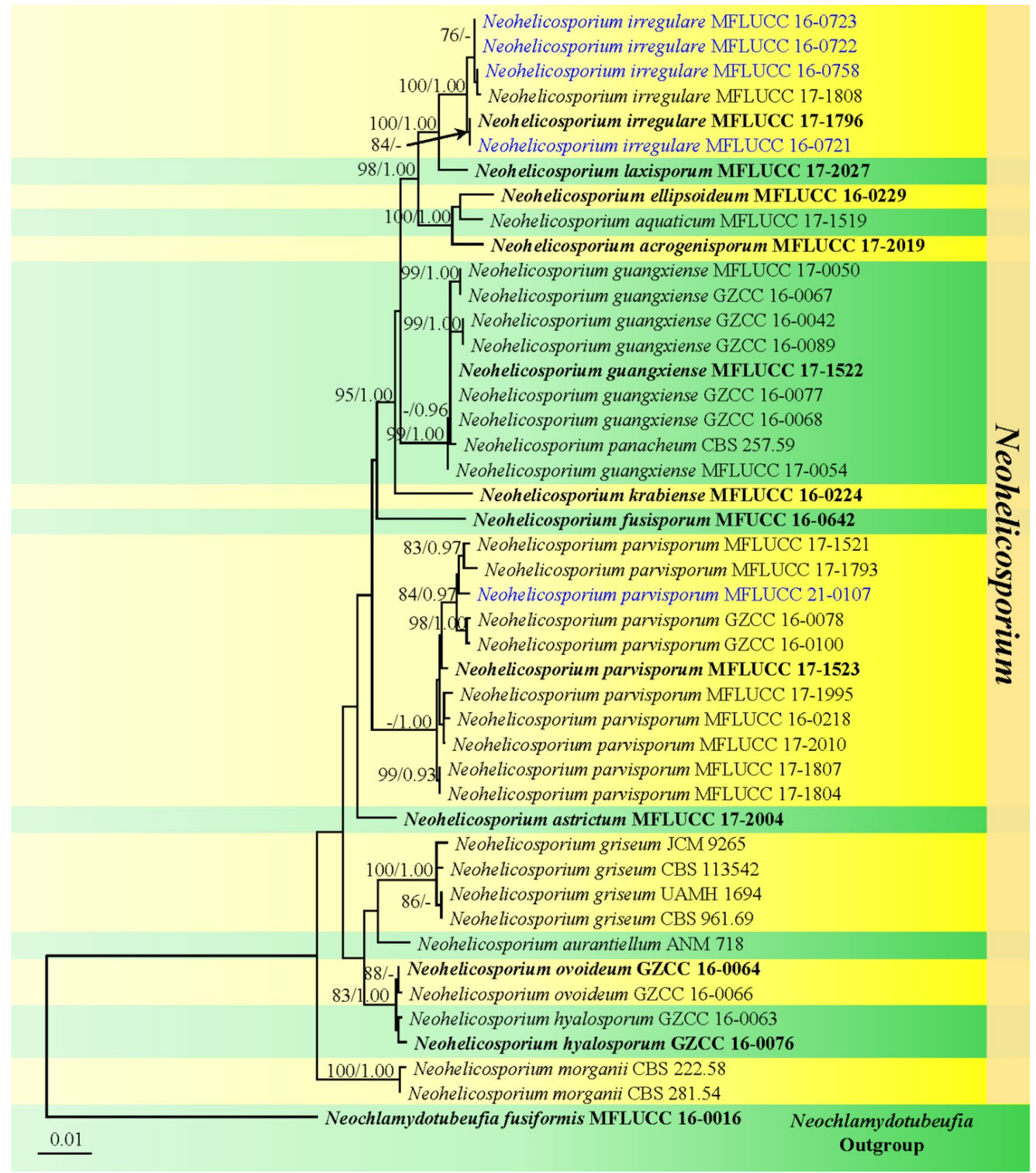

Fig. 91 Maximum likelihood phylogenetic tree based on a combined ITS, LSU and TEF1- $\alpha$ sequence data of Neohelicosporium taxa and related genera in Tubeufiaceae. Forty-five taxa are included in the combined analyses which comprise a total of 2348 characters. Neochlamydotubeufia fusiformis (MFLUCC 16-0016) is selected as the outgroup taxon. The best RAxML tree with a final likelihood value of - 5971.586719 is presented. RAxML analysis yielded 343 distinct alignment patterns and $12.46 \%$ of undetermined characters or gaps. Estimated base frequencies were as follows: $\mathrm{A}=0.230669$,

Saprobic on submerged decaying wood. Sexual morph Undetermined. Asexual morph Hyphomycetous, helicosporous. Colonies on the substrate superficial, effuse, gregarious, white to light pink. Mycelium composed of partly
$\mathrm{C}=0.271979, \mathrm{G}=0.266714, \mathrm{~T}=0.230637$, with substitution rates $\mathrm{AC}=0.718733, \quad \mathrm{AG}=2.140841, \quad \mathrm{AT}=1.942223, \quad \mathrm{CG}=0.669931$, $\mathrm{CT}=13.341832$, GT $=1.000000$; gamma distribution shape parameter alpha $=0.020000$. Bootstrap support values for maximum likelihood (MLBS, left) equal to or greater than $70 \%$ is given above the nodes. Bayesian posterior probabilities (BYPP, right) equal to or greater than 0.95 are given above the nodes. Ex-type strains are in bold and newly generated sequences are in blue

immersed, partly superficial, hyaline to pale brown, septate, abundantly branched hyphae, with masses of crowded, glistening conidia. Conidiophores up to $400 \mu \mathrm{m}$ long, 4-6 $\mu \mathrm{m}$ wide, micronematous, mononematous, flexuous, cylindrical, 
branched, septate, pale brown, smooth-walled. Conidiogenous cells 8-12 $\mu \mathrm{m}$ long, 2.5-5 $\mu \mathrm{m}$ wide, holoblastic, monoto polyblastic, integrated, intercalary, cylindrical, with denticles, pale brown, smooth-walled. Conidia 13.5-33 $\mu \mathrm{m}$ diam., conidial filament $2-4 \mu \mathrm{m}$ wide, $100-150 \mu \mathrm{m}$ long, tightly coiled 3-4 times, becoming loosely coiled in water, rounded at the tip, multi-septate, verrucose, guttulate, hyaline.

Culture characteristics: Conidia germinating on MEA and producing germ tubes within $12 \mathrm{~h}$. Colonies growing on PDA, circular, umbonate, surface rough and wrinkled, edge entire, reaching $10-15 \mathrm{~mm}$ in 2 weeks at $25^{\circ} \mathrm{C}$, pale brown to brown, mycelium superficial and partially immersed, branched, septate, hyaline to pale brown, smooth.

Material examined: THAILAND, Chiang Mai Province, Mushroom Research Center, on submerged decaying wood in a freshwater stream, 29 January 2019, S. Boonmee, SB18-3 (MFLU 21-0123, new record), living culture, MFLUCC 21-0107.

GenBank numbers: ITS = MT864353, LSU = MT860431.

Notes: Neohelicosporium parvisporum was introduced by Lu et al. (2018a), which was collected from a freshwater habitat. Based on phylogenetic analysis of combined ITS, LSU and TEF1- $\alpha$ sequence data showed that our isolate (MFLUCC 21-0107) clusters among isolates $N$. parvisporum without statistical support (Fig. 91). Our isolate has similar conidial and conidiophore morphology to $N$. parvisporum (MFLUCC 17-1523). However, our isolate has smaller conidiogenous cells $(8-12 \times 2.5-5$ vs. $14.5-22.5 \times 3.5-4.5 \mu \mathrm{m})$ and larger conidia (13.5-33×14-24.5 $\mu \mathrm{m})$ (Fig. 90). A comparison of the ITS nucleotides of $N$. parvisporum (MFLUCC 17-1523 = GZCC 16-0088) and the new strain (MFLUCC 21-0107) revealed 9/550 (1.64\%) nucleotide differences.

\section{Tubeufia Penz. \& Sacc.}

Notes: We follow the latest treatments and updated accounts in Lu et al. (2018b), Hyde et al. (2019) and Wijayawardene et al. (2020). The members of Tubeufia include sexual and asexual morphs and are widely distributed in tropical regions, and they are commonly can be found on dead wood in terrestrial and freshwater habitats (Lu et al. 2018b; Hyde et al. 2019). In this study, T. longiohelicospora is introduced and two species (T. chiangmaiensis and T. roseohelicospora) are reported as common species records on decaying wood.

Tubeufia chiangmaiensis Boonmee \& K.D. Hyde, Fungal Diversity 68: 248 (2014)

Index Fungorum number: IF550705; Facesoffungi number: FoF 00172; Figs. 92, 93

Saprobic on decaying wood. Sexual morph Ascomata 227.5-253 $\times 160-192 \mu \mathrm{m}$, superficial, seated on a subiculum, solitary, scattered, globose-subglobose to ovate, cream-white to yellowish when freshly, light brown to brown, collapsing when dry, slightly compressed subiculum hyphae, developing from ascomatal base onto substrate, with ambiguous ostiolate. Peridium 13-22.5 $\mu \mathrm{m}$ wide, composed of 2-3 layers of thick-walled, light brown cells of textura angularis. Hamathecium comprising 1-2 $\mu \mathrm{m}$ wide, numerous, septate, branched, hyaline pseudoparaphyses. Asci $116-123 \times 12-15.5 \mu \mathrm{m}(\bar{x}=118.5 \times 14 \mu \mathrm{m}, \mathrm{n}=10), 8$-spored, bitunicate, fissitunicate, elongate cylindrical to slightly clavate, apedicellate, thick-walled, rounded at apex, tapering towards narrow base, sessile. Asci $54.5-70 \times 3.5-5.5 \mu \mathrm{m}$ $(x=63 \times 5 \mu \mathrm{m}, \mathrm{n}=10)$, overlapping $2-3$-seriate, cylindricfusiform, tapering toward ends, 7-septate, slightly constricted at the medium septum, hyaline, smooth-walled. Asexual morph Hyphomycetous, helicosporous. Conidiophores semi-macronematous, mononematous, borne as lateral branches from superficial hyphae, pale brown to brown, septate, simple or branched, flexuous to geniculate. Conidiophores cells holoblastic, terminal or intercalary, dentate, hyaline, smooth. Conidia 26-54 $\mu$ m diam., conidial filament 4-6(-9) $\mu \mathrm{m}$ wide, (186-)265-309.5 $\mu \mathrm{m}$ long, loosely coiled $1-2^{1 / 2}$ times, becoming loosely uncoiled in water, rounded at apical ends, indistinctly multi-septate, not constricted at the septa, hyaline to brown, with granular contents.

Culture characteristics: Ascospore germinating on MEA within $24 \mathrm{~h}$ and many germ tubes produced from many cells. Colonies growing on MEA, reaching greater than $10 \mathrm{~mm}$ diam. in 7 days at room temperature, flat, sparsely hairy, radially striate, with fimbriate edge, dark brown, with yellowish pigmented in the medium after 30 days. Mycelium superficial, composed of branched, septate, brown to dark brown hyphal filaments, conidial spores formed on hyphae in culture at 60 days.

Material examined: THAILAND, Chiang Mai Province, Mae Taeng, Pa Pae, Mushroom Research Center, on decaying wood, 17 July 2015, S. Boonmee, MRC2 (MFLU 21-0103, new record), living culture, MFLUCC 16-0197.

GenBank numbers: ITS $=$ MZ538530, LSU $=$ MZ538564, TEF1- $\alpha=$ MZ567105.

Notes: Tubeufia chiangmaiensis was collected from Mushroom Research Center, Chiang Mai Province, Thailand. This is the third report for T. chiangmaiensis produced an asexual morph on culture (Fig. 93). Our isolate MFLUCC 16-0197 shares some similar morphological characters of sexual and asexual morphs (Figs. 93 and 94) to the type of T. chiangmaiensis MFLUCC 15-0514 and MFLUCC 17-01801 (Boonmee et al. 2014; Lu et al. 2018b). However, no outstanding differences were observed in the morphological comparison among three isolates (Fig. 97). Therefore, a record of T. chiangmaiensis on decaying wood, from Chiang Mai Province is reported. 

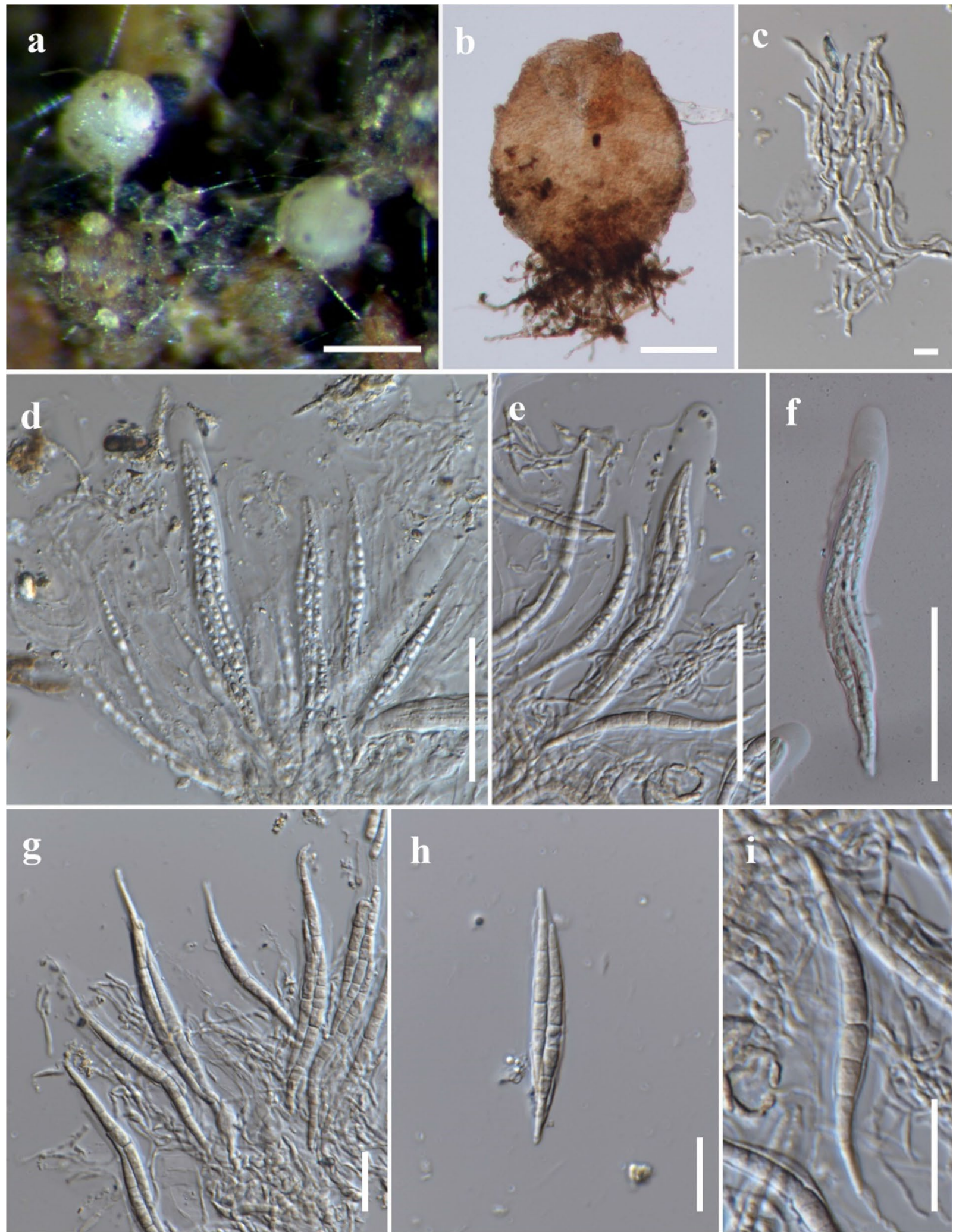

h

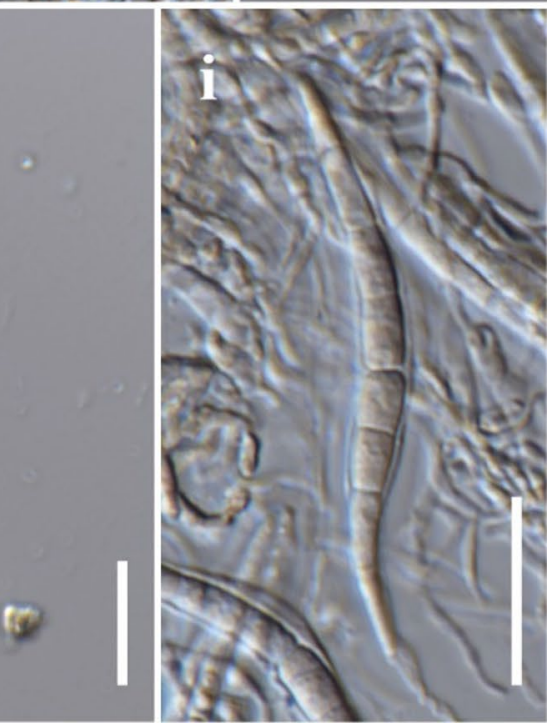

Fig. 92 Tubeufia chiangmaiensis (MFLU 21-0103, new record). a Superficial ascomata on decaying wood substrate. b Squash mount of ascoma showing peridium and subiculum hyphae at the base. c
Pseudoparaphyses. d-f Asci. $\mathbf{g}-\mathbf{i}$ Ascospores. Scale bars: $\mathbf{a}=200 \mu \mathrm{m}$, $\mathbf{b}=100 \mu \mathrm{m}, \mathbf{c}=10 \mu \mathrm{m}, \mathbf{d}-\mathbf{f}=50 \mu \mathrm{m}, \mathbf{g}-\mathbf{i}=20 \mu \mathrm{m}$ 


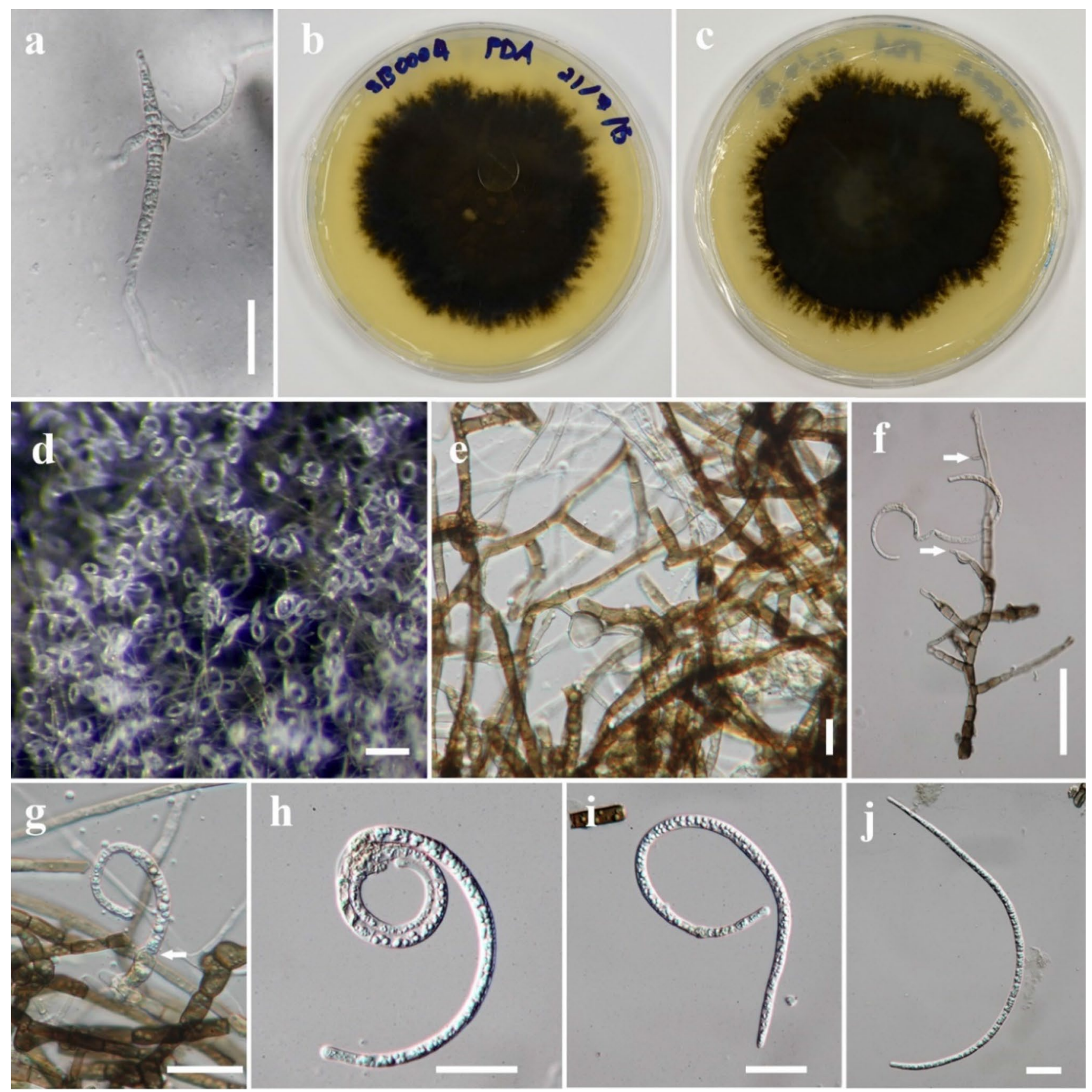

Fig. 93 Tubeufia chiangmaiensis sporulation in culture (MFLUCC 16-0197). a Germinated ascospore. b, c Culture on MEA from surface and reverse after 1 month. d-f Development of conidia and mycelium in culture. $\mathbf{g}-\mathbf{j}$ Conidia. Scale bars: $\mathbf{a}, \mathbf{f}=50 \mu \mathrm{m}$, $\mathbf{d}=100 \mu \mathrm{m}, \mathbf{e}=10 \mu \mathrm{m}, \mathbf{g}-\mathbf{j}=20 \mu \mathrm{m}$

Saprobic on submerged decaying wood. Sexual morph Undetermined. Asexual morph Hyphomycetous, helicosporous. Colonies on natural substrate effuse, superficial, shiny, hairy, hyaline to white of crowded conidiospore masses. Mycelium composed of superficial and partly immersed, branched at the base, septate, hyaline to brown hyphae. Conidiophores macronematous, thickwalled, branched at the base, septate, pale brown to brown, 

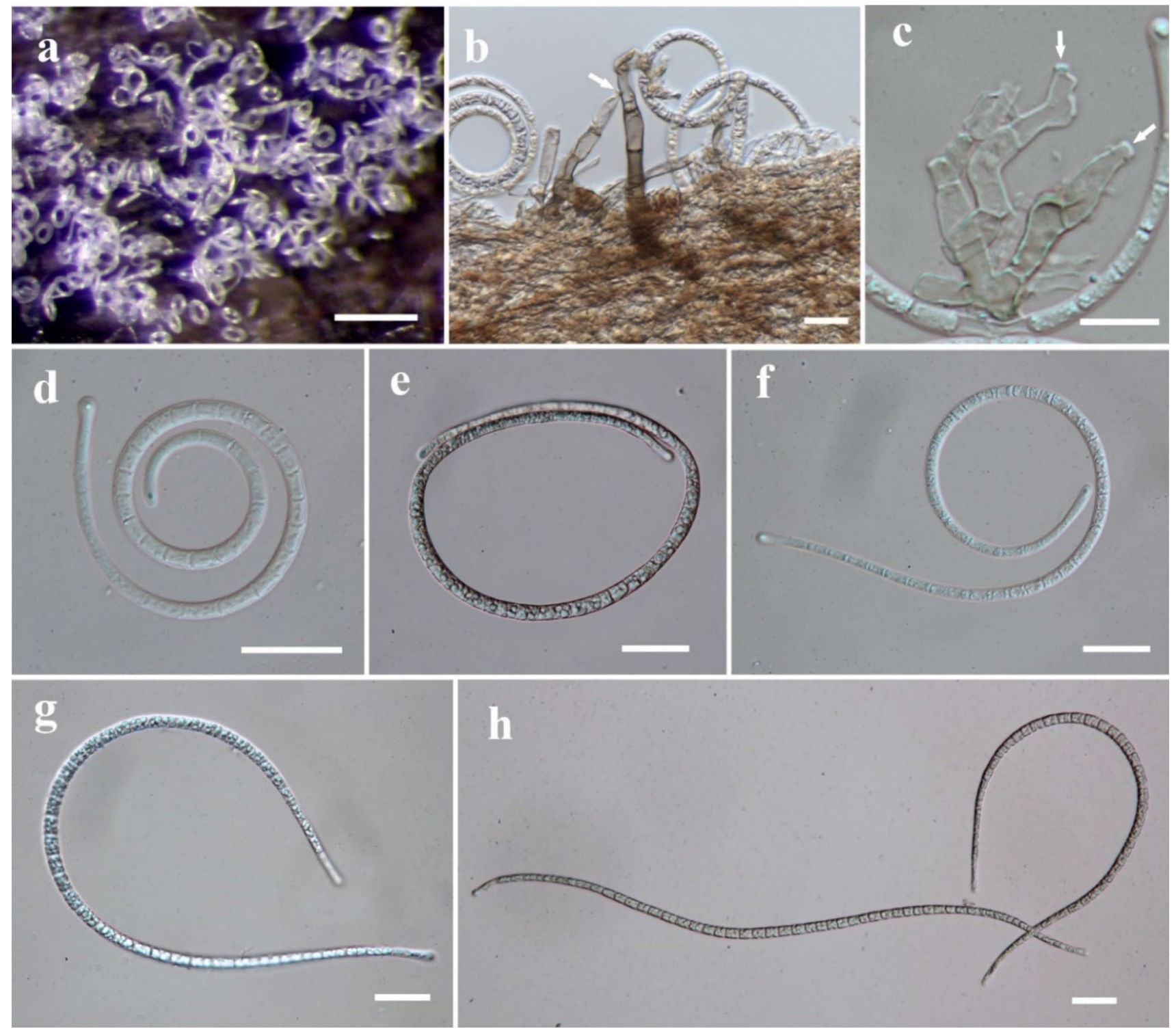

Fig. 94 Tubeufia longihelicospora (MFLU 21-0100, holotype). a Colony on decaying wood. b, c Conidiophores and lateral minute denticles. d-h Conidia. Scale bars: $\mathbf{a}=200 \mu \mathrm{m}, \mathbf{b}, \mathbf{c}=10 \mu \mathrm{m}, \mathbf{d}-\mathbf{h}=20 \mu \mathrm{m}$

smooth-walled. Conidiogenous cells monoblastic, terminal or intercalary, integrated, subhyaline, bearing lateral minute denticles. Conidia 36-52 $\mu \mathrm{m}$ diam., with conidial filament 4-5.5 $\mu \mathrm{m}$ wide, $252-360 \mu \mathrm{m}$ long, loosely coiled $1-3 \frac{1}{2}$ times, becoming loosely uncoiled in water, rounded at apical ends, multi-septate, 33-45-septate, slightly constricted at the septa, hyaline to subhyaline, with granular contents.

Culture characteristics: Conidium germinating on MEA within $24 \mathrm{~h}$ and many germ tubes produced from conidium cells. Colonies growing on MEA, reaching greater than $10 \mathrm{~mm}$ diam. in 7 days at room temperature, effuse, sparsely hairy, radially striate, slightly fimbriate edge, dark brown, with yellow brown pigmented in the medium after 30 days. Mycelium superficial, partly immersed, branched, septate, hyaline, brown to dark brown hyphal filaments, asexual conidia not formed at 60 days.

Material examined: THAILAND, Chiang Rai Province, Muang, Mae Yao, Huay Mae Sai, on submerged decaying wood in a small freshwater stream, 17 March 2016, S. Boonmee, HMS07 (MFLU 21-0100, holotype), ex-type living culture, MFLUCC 16-0753.

GenBank numbers: ITS $=$ MZ538531, LSU $=$ MZ538565, TEF1- $\alpha=$ MZ567106.

Notes: Tubeufia longihelicospora is introduced as a new species based on morphological characters and phylogenetic evidence. Our new taxon forms a separate lineage basal to $T$. brunnea, T. hechiensis and T. taiwanensis with 100\% MLBS, 1.00 BYPP support (Fig. 97). Tubeufia longihelicospora 


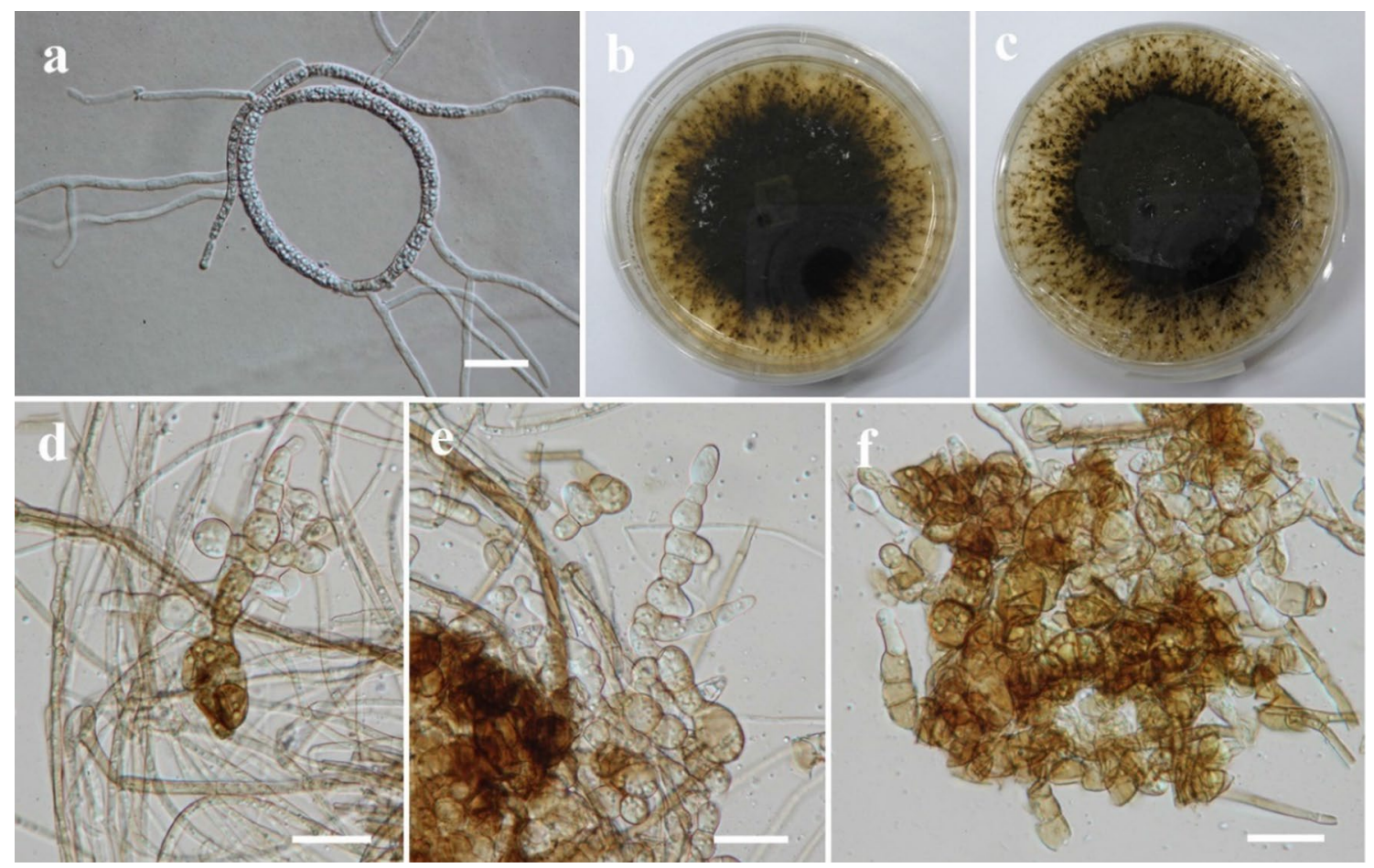

Fig. 95 Tubeufia longihelicospora (MFLUCC 16-0753, ex-type). a Germinated conidium. b, c Culture on MEA from surface and reverse after 1 month. d-f Mycelium and development on culture (Note: the formation of chlamydospores). Scale bars: $\mathbf{a}, \mathbf{d}-\mathbf{f}=20 \mu \mathrm{m}$

shares similar characteristics with $T$. hechiensis in having white colonies on natural woody substrates and multi-septate, hyaline, loosely coil conidia, but it differs in conidiophore and conidiogenous cell characters (Figs. 94 and 95). Furthermore, T. longihelicospora is distinguished from $T$. brunnea and T. taiwanensis by its macronematous, thickwalled, conidiophores and smaller, cylindrical filamentous conidia. A comparison of ITS nucleotide bases shows that T. longihelicospora differs from T. brunnea in $29 / 584 \mathrm{bp}$ (5.3\%), T. hechiensis in 28/546 bp (5.1\%) and T. taiwanensis in $28 / 546 \mathrm{bp}$ (5.1\%) following the guidelines of Jeewon and Hyde (2016).

Tubeufia roseohelicospora Y.Z. Lu, Boonmee \& K.D. Hyde, Fungal Diversity 80: 128 (2016)

Index Fungorum number: IF552222; Facesoffungi number: FoF 02362; Fig. 96

Saprobic on submerged decaying wood. Sexual morph Undetermined. Asexual morph Hyphomycetous, helicosporous. Colonies on wood substrate superficial, effuse, hyaline to white of crowded conidiospore masses. Mycelium composed of superficial and partly immersed, anastomosing, branched, septate, brown hyphae, smooth-walled. Conidiophores (19-)59-90(-158) ×3-7.5 $\mu \mathrm{m}$, macronematous, mononematous, erect, arising from repent mycelium, flexuous, branched, septate, brown, smooth-walled. Conidiogenous cells (8-) $18-40 \times 3-6 \mu \mathrm{m}$, holoblastic, mono- to polyblastic, terminal or intercalary, integrated, subhyaline, bearing lateral minute denticles each with single conidium. Conidia 20-44 $\mu \mathrm{m}$ diam., with conidial filament 3-6 $\mu \mathrm{m}$ wide, with $318-563 \mu \mathrm{m}$ long, loosely coiled $1 \frac{1}{2}-41 \frac{1}{2}$ times, becoming loosely uncoiled in water, rounded at apical ends, indistinctly multi-septate, not constricted at the septa, hyaline, guttulate, smooth-walled.

Culture characteristics: Conidium germinating on MEA within $24 \mathrm{~h}$ and many germ tubes produced from conidium cells. Colonies growing on MEA, reaching greater than $10 \mathrm{~mm}$ diam. in 7 days at room temperature, effuse, sparsely hairy, radially striate, with fimbriate edge, dark brown, with yellow brown pigmented in the medium after 45 days. Mycelium superficial, composed of branched, septate, brown to dark brown hyphal filaments, conidial spores formed on hyphae in culture at 60 days.

Material examined: THAILAND, Chiang Rai Province, Muang, Mae Yao, Huay Mae Sai, on submerged decaying wood in a small freshwater stream, 17 March 2016, S. Boonmee, HMS14 (MFLU 21-0101, new record), living culture, MFLUCC 16-0729.

GenBank numbers: ITS $=$ MZ538532, LSU $=$ MZ538566, TEF1- $\alpha=$ MZ567107.

Notes: Tubeufia roseohelicospora was introduced by Hyde et al. (2016) to accommodate a taxon that is characterized by tightly helicoid with light pink conidia. Phylogenetic analysis of the combined LSU, ITS and TEF1- $\alpha$ dataset 

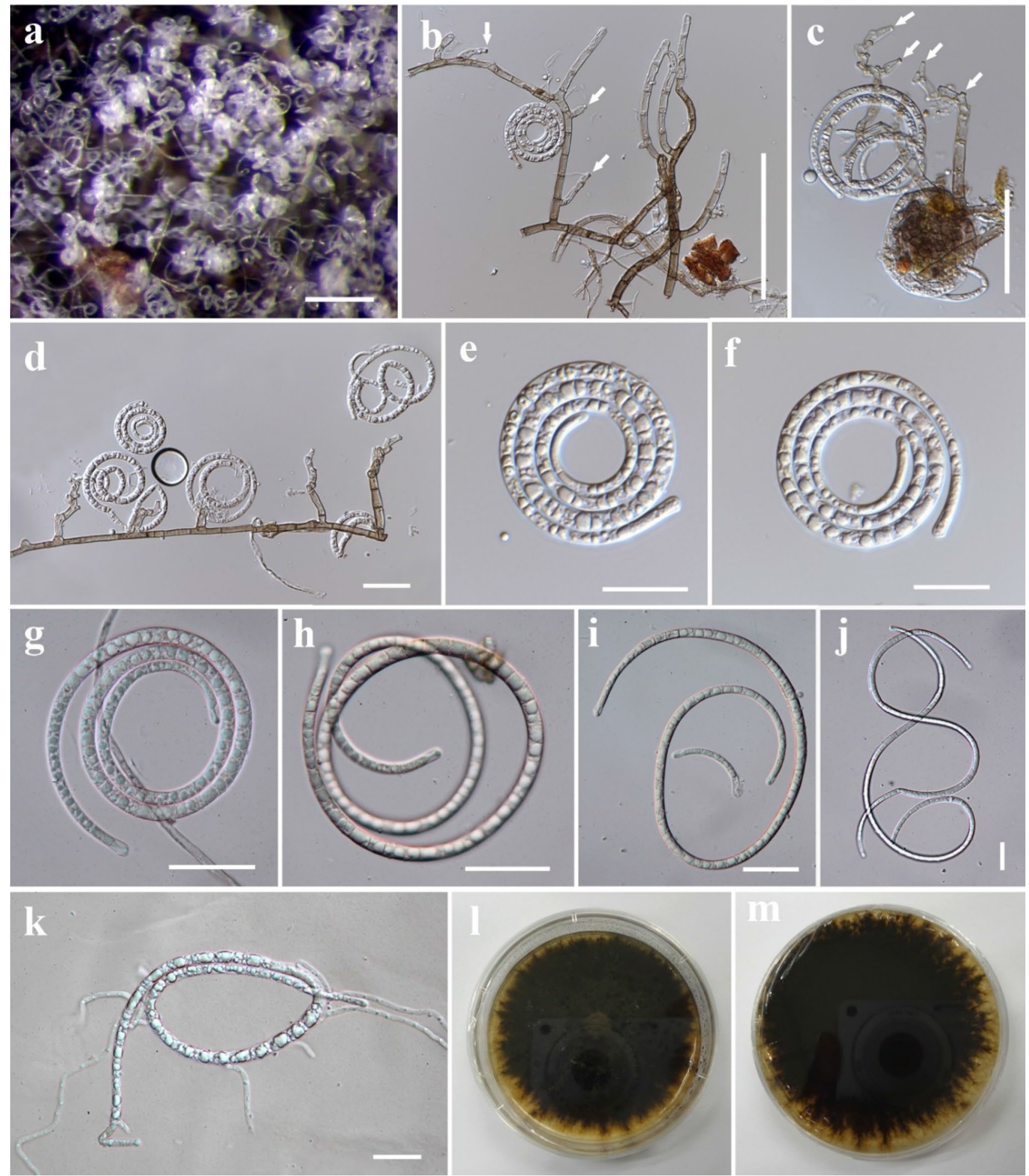

Fig. 96 Tubeufia roseohelicospora (MFLU 21-0101, new record). a Colony on decaying wood. b-d Conidiophores with lateral minute polyblastic denticles and attached conidia. $\mathbf{e}-\mathbf{j}$ Conidia. $\mathbf{k}$ Germinated conidium. 1, $\mathbf{m}$ Culture on MEA from surface and reverse after 1 month. Scale bars: $\mathbf{a}=200 \mu \mathrm{m}, \mathbf{b}, \mathbf{c}=50 \mu \mathrm{m}, \mathbf{d}-\mathbf{k}=20 \mu \mathrm{m}$ 


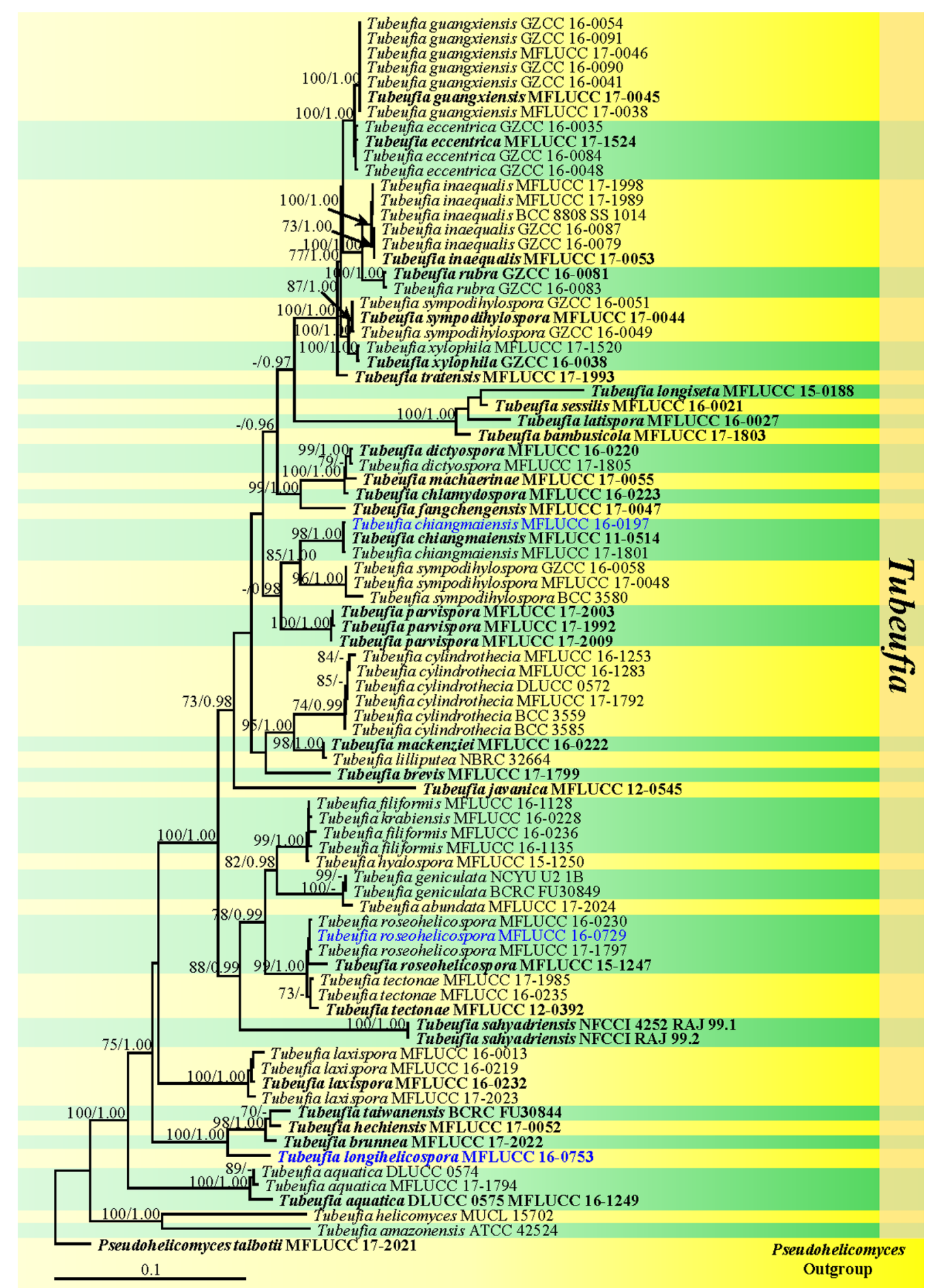

Fig. 97 Maximum likelihood phylogenetic tree based on a combined LSU, ITS and TEF1- $\alpha$ sequence dataset of Tubeufia taxa and related genera in Tubeufiaceae. Eighty-four strains are included in the combined analyses which comprise a total of 2395 characters. Pseudohelicomyces talbotii (MFLUCC 17-2021) is selected as the outgroup taxon. The best RAxML tree with a final likelihood value of -11543.960875 is presented. RAxML analysis yielded 680 distinct alignment patterns and $15.58 \%$ of undetermined characters or gaps. Estimated base frequencies were as follows: $\mathrm{A}=0.238955$,
$\mathrm{C}=0.251270, \mathrm{G}=0.256785, \mathrm{~T}=0.229079$, with substitution rates $\mathrm{AC}=0.670086, \quad \mathrm{AG}=4.164732, \quad \mathrm{AT}=3.398524, \quad \mathrm{CG}=0.528840$, $\mathrm{CT}=7.628422, \mathrm{GT}=1.000000$; gamma distribution shape parameter alpha $=0.148685$. Bootstrap support values for maximum likelihood (MLBS, left) equal to or greater than $70 \%$ is given above the nodes. Bayesian posterior probabilities (BYPP, right) equal to or greater than 0.95 are given above the nodes. Ex-type strains are in bold and newly generated sequences are in blue 
indicates that our strain MFLUCC 16-0721 is related to $T$. roseohelicospora isolates (MFLUCC 15-1247, MFLUCC 17-1797 and MFLUCC 17-2024) with 99\% MLBS, 1.00 BYPP support (Fig. 97). Furthermore, our taxon also shares similar morphological characters (Fig. 96) with the ex-type strain MFLUCC 15-1247 provided in Hyde et al. (2016). We therefore, identify our taxon as Tubeufia roseohelicospora a new record from Chiang Rai Province, Thailand.

\section{Pleosporales, genus incertae sedis}

\section{Megacapitula J.L. Chen \& Tzean}

Notes: Megacapitula was introduced to accommodate a single species $M$. villosa which was found on an unidentified fallen decayed petiole in Taiwan by Chen and Tzean (1993). There is no sequence data available. The genus is characterized by obclavate or ellipsoidal to obpyriform, darkly pigmented conidia, crowned with densely packed hairy, brown apical appendages (Chen and Tzean 1993; Delgado 2010). Prabhugaonkar and Bhat (2011) later provided ITS sequence for an isolate of $M$. villosa collected from dead fronds of palm Caryota urens (Arecaceae) in India. Phylogenetic analysis with only ITS sequence data indicated that $M$. villosa (GUFCC 15515) clustered with an unidentified endophyte strain P1802A and basal to other genera of Pleosporales (Prabhugaonkar and Bhat 2011). In addition, there are two unpublished sequences of M. villosa AL4 and cp053a that are available in GenBank databases but no morphological descriptions. In this study, we introduce the new record Megacapitula villosa which was isolated from submerged decaying wood in a small freshwater stream in Thailand and provide an updated phylogenetic tree for $\mathrm{Meg}$ acapitula (Fig. 100).

Megacapitula villosa J.L. Chen \& Tzean, Mycol. Res. 97(3): 347 (1993)

Index Fungorum number: IF359484; Facesoffungi number: FoF 09196; Figs. 98, 99

Saprobic on submerged decaying wood. Sexual morph Undetermined. Asexual morph Hyphomycetous. Mycelium superficial, partly immerged, composed of a loose network of repent, septate, branched, hyaline to light brown hyphae connected with conidia. Colonies on natural substrate effuse, solitary, dark brown to black. Conidiophores reduce to conidiogenous cells. Conidiogenous cells monoblastic, semi-macronematous, integrated, terminal or intercalary on mycelial extensions, branched, almost sessile. Conidia 139-321.5 $\times 42.5-94 \mu \mathrm{m}(\bar{x}=231 \times 77 \mu \mathrm{m}, \mathrm{n}=15)$, holoblastic, solitary, scattered, oblong to ovoid, obclavate, ellipsoidal or obpyriform, internally with bundles of narrow cylindrical hyphae, together emerging as brown apical appendages from the top, disappearing when dry, faintly septate, dark brown to black, smooth-walled.
Culture characteristics: Conidia germinating on malt extract agar (MEA) within $12 \mathrm{~h}$. Colonies growing on MEA, reaching $18 \mathrm{~mm}$ in 2 weeks at $28{ }^{\circ} \mathrm{C}$, low convex, round with scalloped from middle to margin, radiating, slightly wavy, greyish brown to dark brown in surface, dark brown to black in reverse. Mycelium superficial and partly immersed, branched, septate, with hyaline to dark brown, pigmented verrucose, rough-walled, conidia produced on mycelium in culture at 45 days, $117-314 \times 48-91 \mu \mathrm{m}(\bar{x}=213 \times 70 \mu \mathrm{m}$, $\mathrm{n}=10$ ), oblong to ovoid, obclavate, ellipsoidal or obpyriform, muriform, internally with bundles of narrow of cylindrical hyphal appendages emerging from the top, faintly septate, dark brown to black, smooth-walled.

Material examined: THAILAND, Chanthaburi Province, Laem Sing, on submerged decaying wood in a small freshwater stream, 7 July 2015, S. Boonmee, LS03 (MFLU 19-0257, new geographical record), living culture, MFLUCC 16-1231.

GenBank numbers: ITS $=$ MZ538513, LSU $=$ MZ538547, TEF1- $\alpha=$ MZ567115.

Notes: Megacapitula comprises a single species M. villosa as the type species (Wijayawardene et al. 2020; Index Fungorum 2021). Our new collection is a typical of Megacapitula, and it shares similar conidial morphology to Megacapitula villosa (PPH17E: ex-type species) from Taiwan (Chen and Tzean 1993), and strain GUFCC 15515 from India (Prabhugaonkar and Bhat 2011). Phylogenetic analysis of a combined LSU and ITS sequence dataset showed that our newly obtained strain MFLUCC 16-1231 clustered among Megacapitula villosa strains (AL4, cp053a), and basal to unidentified endophytic and saprobic strains with $100 \%$ MLBS and 1.00 BYPP support (Fig. 100). Comparison of ITS sequences showed that Megacapitula villosa (MFLUCC 16-1231) has $100 \%$ similarity with strain GUFCC 15,515. Two unidentified morphological strains AL4 and cp053a have only $1 / 459(0.21 \%)$ base pair different from our taxon. Therefore, we identify our collection as $M$. villosa isolated from submerged decaying wood in a small freshwater stream and it is reported here as a new geographical record from Thailand (Figs. 98 and 99). Megacapitula villosa is likely to comprise more than one species and may represent a distinct family.

\section{Eurotiomycetes O.E. Erikss. \& Winka}

\section{Chaetothyriales M.E. Barr}

Notes: Chaetothyriales was introduced by Barr (1987) based on the presence of periphysoids (apical paraphyses) in the ascomata and eight families were accepted in the order viz. Chaetothyriaceae, Coccodiniaceae, Herpotrichiellaceae, Metacapnodiaceae, Microtheliopsidaceae, Pyrenotrichaceae, Strigulaceae and Trichopeltidaceae. The 

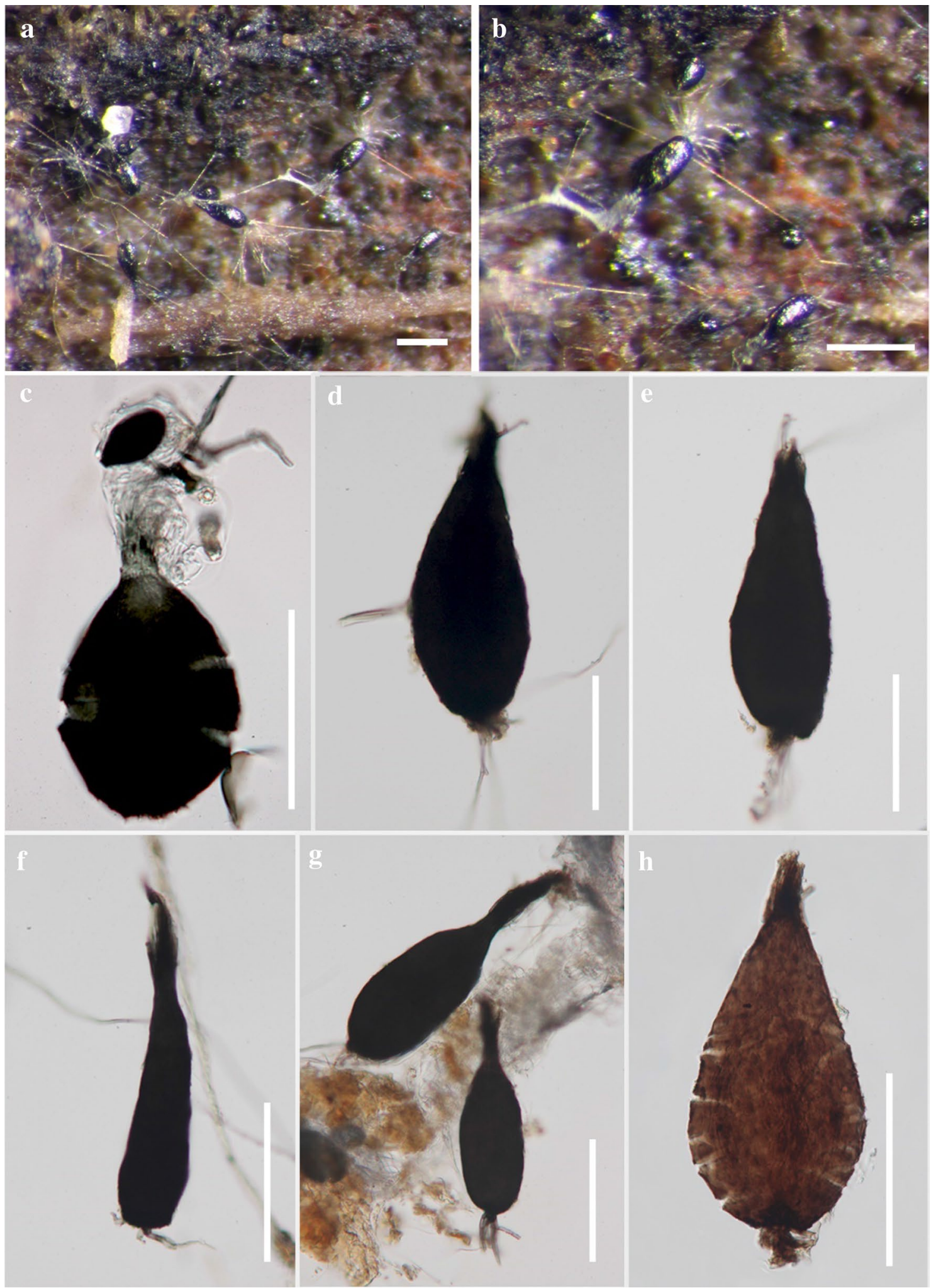

Fig. 98 Megacapitula villosa (MFLU 19-0257, new geographical record). a, b Appearance of conidia with hyphal-like appendages and habit on wood substrate. $\mathbf{c}$ Immature conidium with hyphal-like appendages. d-g Mature conidia with apical hyphal-like appendages. $\mathbf{h}$ Conidium changed to brown in lactoglycerol and composed of compressed prosenchyma cells. Scale bars: $\mathbf{a}, \mathbf{b}=200 \mu \mathrm{m}, \mathbf{c}-\mathbf{h}=100 \mu \mathrm{m}$ 


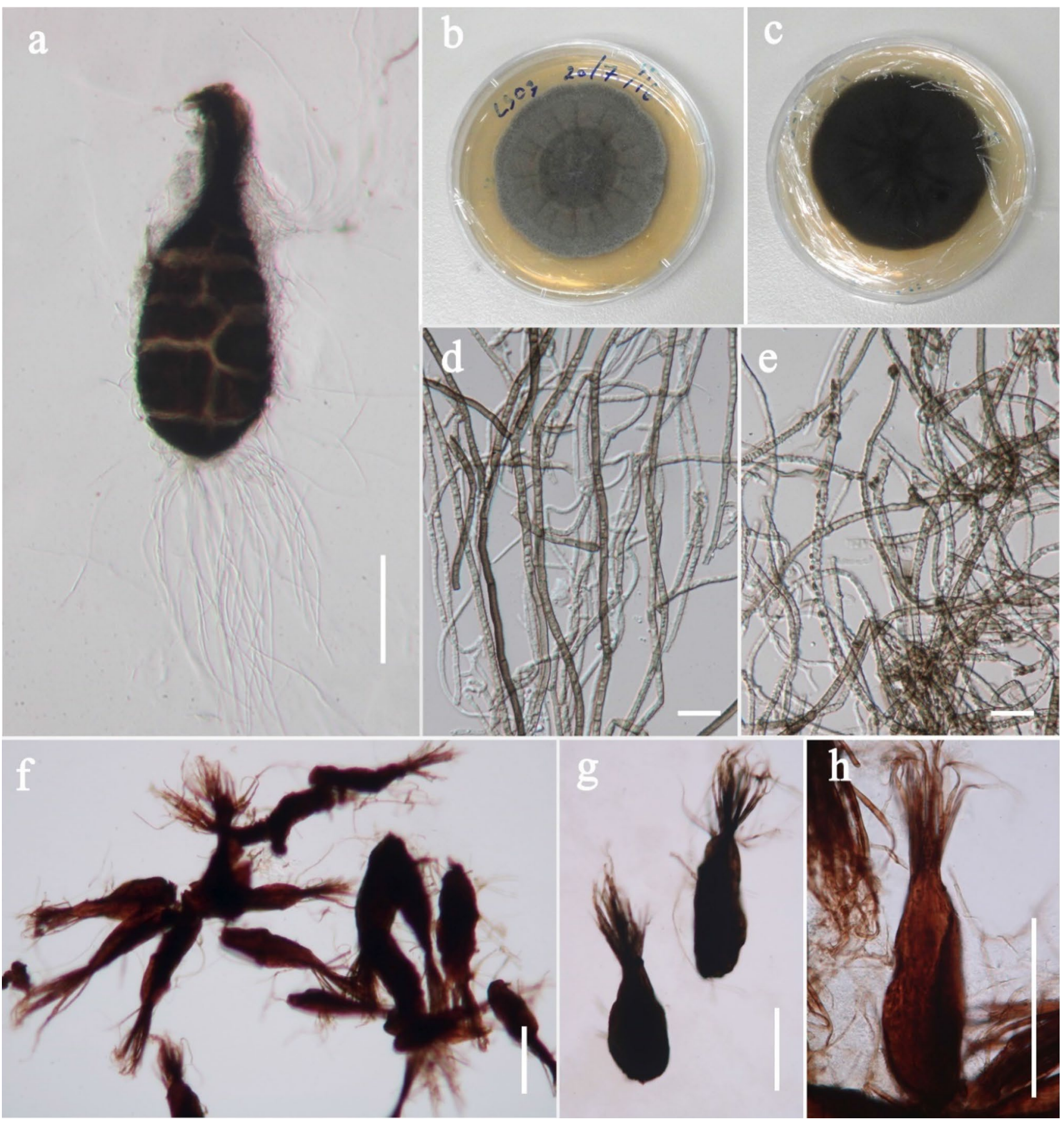

Fig. 99 Megacapitula villosa sporulated in culture (MFLUCC 16-1231). a Germinated conidium with germ tubes surrounded. b, c Cultures on MEA after 1 month. d, e Mycelium development in culture. $\mathbf{f}-\mathbf{h}$ Conidia. Scale bars: $\mathbf{a}, \mathbf{f}-\mathbf{h}=100 \mu \mathrm{m}, \mathbf{d}, \mathbf{e}=10 \mu \mathrm{m}$

latest classification of Wijayawardene et al. (2020), placed ten families in the order wherein Strelitzianaceae was synonymized as Trichomeriaceae, and Paracladophialophoraceae was added. Members of Chaetothyriales exhibit complex ecological variation and are common in tropical and temperate ecosystems (Chomnunti et al. 2012; Réblová et al. 2013; De Hoog 2014; Liu et al. 2015b; Hongsanan et al. 2016; Hyde et al. 2016; Teixeira et al. 2017; Dong et al. 2018).

\section{Herpotrichiellaceae Munk}

Notes: Munk (1953) established Herpotrichiellaceae that includes loculoascomycetes with small, superficial, inconspicuous, setose ascomata, bitunicate asci with a thickened 


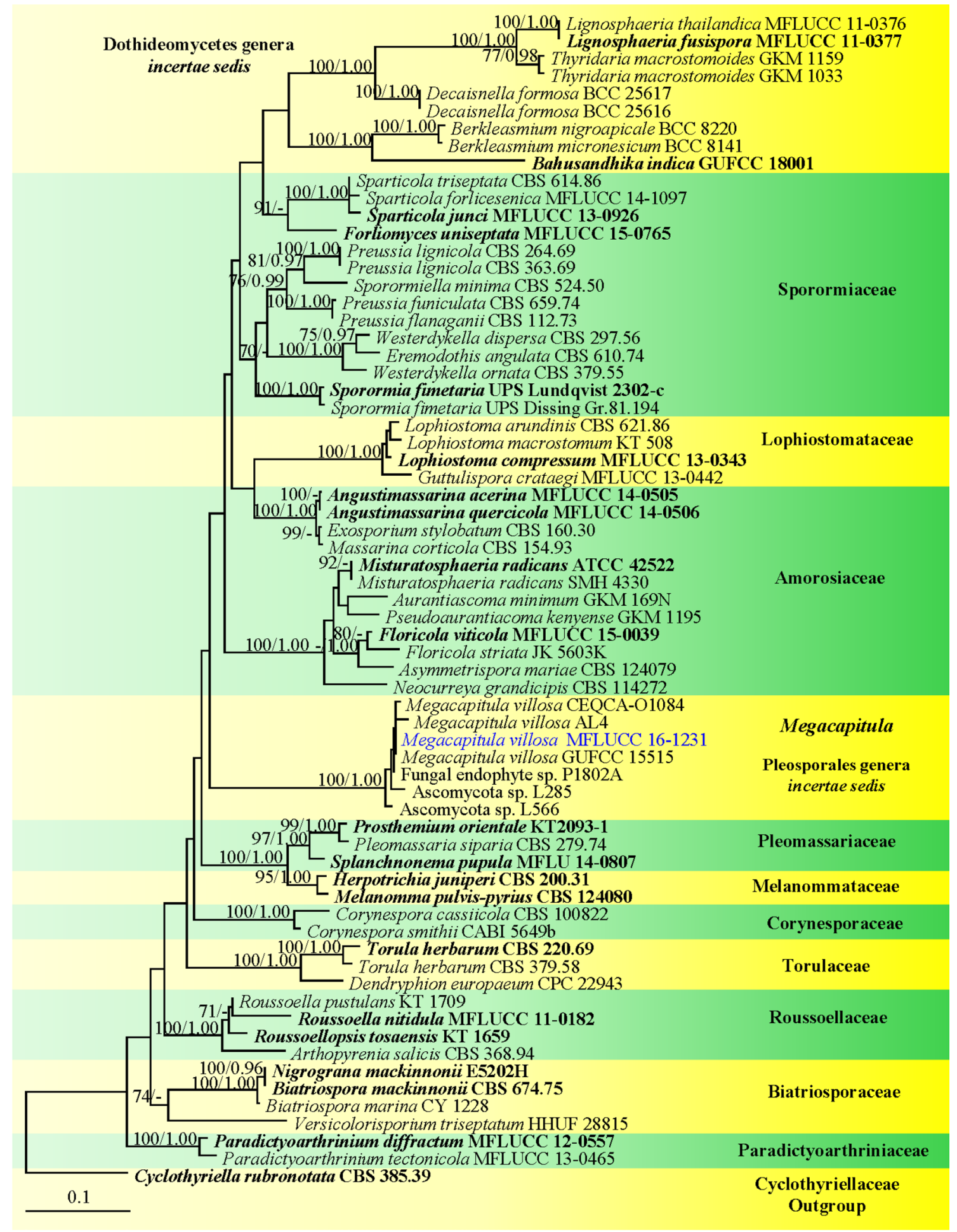

Fig. 100 Maximum likelihood phylogenetic tree based on a combined LSU and ITS sequence dataset of Megacapitula taxa and related families in Pleosporales. Sixty-seven strains are included in the combined analyses which comprise a total of 1456 characters. Cyclothyriella rubronotata CBS 385.39 is selected as the outgroup taxon. The best RAxML tree with a final likelihood value of -12939.878192 is presented. RAxML analysis yielded 723 distinct alignment patterns and $29.63 \%$ of undetermined characters or gaps. Estimated base fre- quencies were as follows: $\mathrm{A}=0.242721, \mathrm{C}=0.238518, \mathrm{G}=0.291320$, $\mathrm{T}=0.227442$, with substitution rates $\mathrm{AC}=1.943333, \mathrm{AG}=2.988826$, $\mathrm{AT}=2.324598, \quad \mathrm{CG}=1.416039, \quad \mathrm{CT}=8.575259, \quad \mathrm{GT}=1.000000$; gamma distribution shape parameter alpha $=0.245601$. Bootstrap support values for maximum likelihood (MLBS, left) equal to or greater than $70 \%$ is given above the nodes. Bayesian posterior probabilities (BYPP, right) equal to or greater than 0.95 are given above the nodes. Ex-type strains are in bold and newly generated sequence is in blue 
endotunica and greenish-grey to brown, septate ascospores. The genera Beriesiella, Capronia, Dictyotrichiella, Didymotrichiella and Herpotrichiella were included by Munk (1953) in Herpotrichiellaceae. Acanthostigmella and Polytrichiella were added by Barr $(1972,1977)$. In the latest outline of fungal classification of Wijayawardene et al. (2020), 16 genera: Aculeata, Brycekendrickomyces, Capronia, Cladophialophora, Exophiala, Fonsecaea, Marinophialophora, Melanoctona, Metulocladosporiella, Minimelanolocus, Phialophora, Pleomelogramma, Rhinocladiella, Sorocybe, Thysanorea and Veronaea were placed in the family. Members of Herpotrichiellaceae have a cosmopolitan distribution and are often found in extreme environments. Most species are saprobic in nature, others are opportunistic human and animal pathogens (Müller et al. 1987; Braun and Feiler 1995; Haase et al. 1999; Crous et al. 2007).

\section{Cladophialophora Borelli}

Notes: Cladophialophora, typified by C. carrionii (Trejos) de Hoog, Kwon-Chung \& McGinnis $(=C$. ajelloi Borelli), was introduced by Borelli (1980) to accommodate species exhibiting a cladosporium-like form of sporulation and phialophora-like conidiogenous cells (Borelli 1980; Badali et al. 2008; Bensch et al. 2012). Cladophialophora is characterized by 1-celled, globose to elongate, dry conidia arising through blastic, acropetal conidiogenesis, and arranged in branched chains, as melanized conidia with inconspicuous scars (Borelli 1980; Ho et al. 1999; De Hoog et al. 2000; Badali et al. 2008). Thirty-eight species constitute Cladophialophora and based on phylogenetic analysis, they are polyphyletic within Chaetothyriales. The generic type, $C$. carrionii and most species of this genus belong in Herpotrichiellaceae, but a few species are related to other chaetothyrialean families, such as Epibryaceae (e.g., C. humicola, C. minutissima) and Trichomeriaceae (C. modesta) (Gueidan et al. 2008, 2014). The genus occurs as opportunistic or pathogens of plants, animals and humans, and even as saprobes and endophytes (De Hoog et al. 2007; Crous et al. 2007; Badali et al. 2008; Feng et al. 2014).

Cladophialophora abundans P. Feng, V.A. Vicente, Najafz., van den Ende, Stielow, Badali, Boeger \& de Hoog, Mycol. Progr. 13(2): 386 (2013) [2014]

Index Fungorum number: IF803489; Facesoffungi number: FoF 09157; Fig. 101

Saprobic on submerged decaying wood in a freshwater. Sexual morph Undetermined. Asexual morph Hyphomycetous. Hyphae 2.5-4.5 $\mu \mathrm{m}$ wide, irregularly septate, straight or bent, smooth, thin-walled, hyaline to pale brown, guttulate, branched, with formation of hyphal strands, and differentiated ellipsoidal to cylindrical conidiophores with conidial chains. Conidiophores semi-macronematous, pale olivaceous to brown, septate, oblong to cylindrical.
Conidiogenous cells holoblastic, mono- or polyblastic, terminal or intercalary. Conidial chains forming laterally or terminally on undifferentiated hyphae, with profuse, strongly coherent conidial chains. Conidia 4.5-7 $\times 3.5-5.5 \mu \mathrm{m}$ $(\bar{x}=5.9 \times 4.5 \mu \mathrm{m}, \mathrm{n}=30)$, pale olivaceous to brown, smooth and thin-walled, globose, ovoidal and ellipsoidal, aseptate, guttulate; conidial scars slightly pigmented. Chlamydospores and yeast cells absent.

Culture characteristics: Colonies growing on MEA, reaching $30-35 \mathrm{~mm}$ in 2 weeks at $25^{\circ} \mathrm{C}$. Mycelia superficial, irregularly circular, with entire to undulate margin, flat, smooth, from above pale brown, from below dark brown. Sporulation produced in culture within 2 weeks.

Material examined: THAILAND, Phuket Province, Thalang District, Mai Khao, on submerged decaying wood in a mangrove habitat, 5 May 2019, M.S. Calabon, 0506HY3 (MFLU 21-0118, new geographical record), living culture, MFLUCC 21-0105.

GenBank numbers: ITS $=$ MT864354, LSU $=$ MT860432.

Notes: Cladophialophora abundans was introduced by Feng et al. (2014) to accommodate cladophialophora-like isolates from a Brazilian mangrove environment. The species was isolated from the thorn of Dioscorea multiflora and muddy burrows of the mangrove-land crab (Ucides cordatus) (Pie et al. 2011; Feng et al. 2014). The phylogenetic analysis of a combined LSU and ITS sequence data shows that $C$. abundans (MFLUCC 21-0105) clusters with the ex-type strain of $C$. abundans (CBS 126736) with 100\% MLBS, 100\% MPBS, 1.00 BYPP support (Fig. 103). Cladophialophora abundans (MFLUCC 21-0105; Fig. 101) resembles $C$. abundans when it comes to the shapes and sizes of conidia $(4.5-7 \times 3.5-5.5 \mu \mathrm{m}$ vs. $4.5-6 \times 2-3.5 \mu \mathrm{m})$ and hyphal width $(2.5-4.5 \mu \mathrm{m}$ vs. $2-3.5 \mu \mathrm{m})$. There are no base pair differences of the ITS nucleotides which indicates that the new strain is C. abundans (Jeewon and Hyde 2016). Thus, a record of $C$. abundans from Thailand is reported.

Cladophialophora aquatica M.S. Calabon, Boonmee, E.B.G. Jones \& K.D. Hyde, sp. nov.

Index Fungorum number: IF558644; Facesoffungi number: FoF 09153; Fig. 102

Etymology: Name reflects the aquatic habitat from where this species was collected.

Holotype: MFLU 21-0119

Saprobic on submerged decaying wood in a freshwater. Sexual morph Undetermined. Asexual morph Hyphomycetous. Hyphae 1.2-4 $\mu \mathrm{m}$ wide, septate, hyaline to lightly pigmented. Chlamydospores $8-13.5 \times 3.5-9 \mu \mathrm{m}(\bar{x}=10.5-6 \mu \mathrm{m}$, $\mathrm{n}=30$ ), numerous, mostly in chains, intercalary or solitary, globose to subglobose, smooth, olivaceous brown to dark brown.

Culture characteristics: Colonies growing on MEA, reaching 20-30 mm in 2 weeks at $25^{\circ} \mathrm{C}$. Mycelia superficial, 

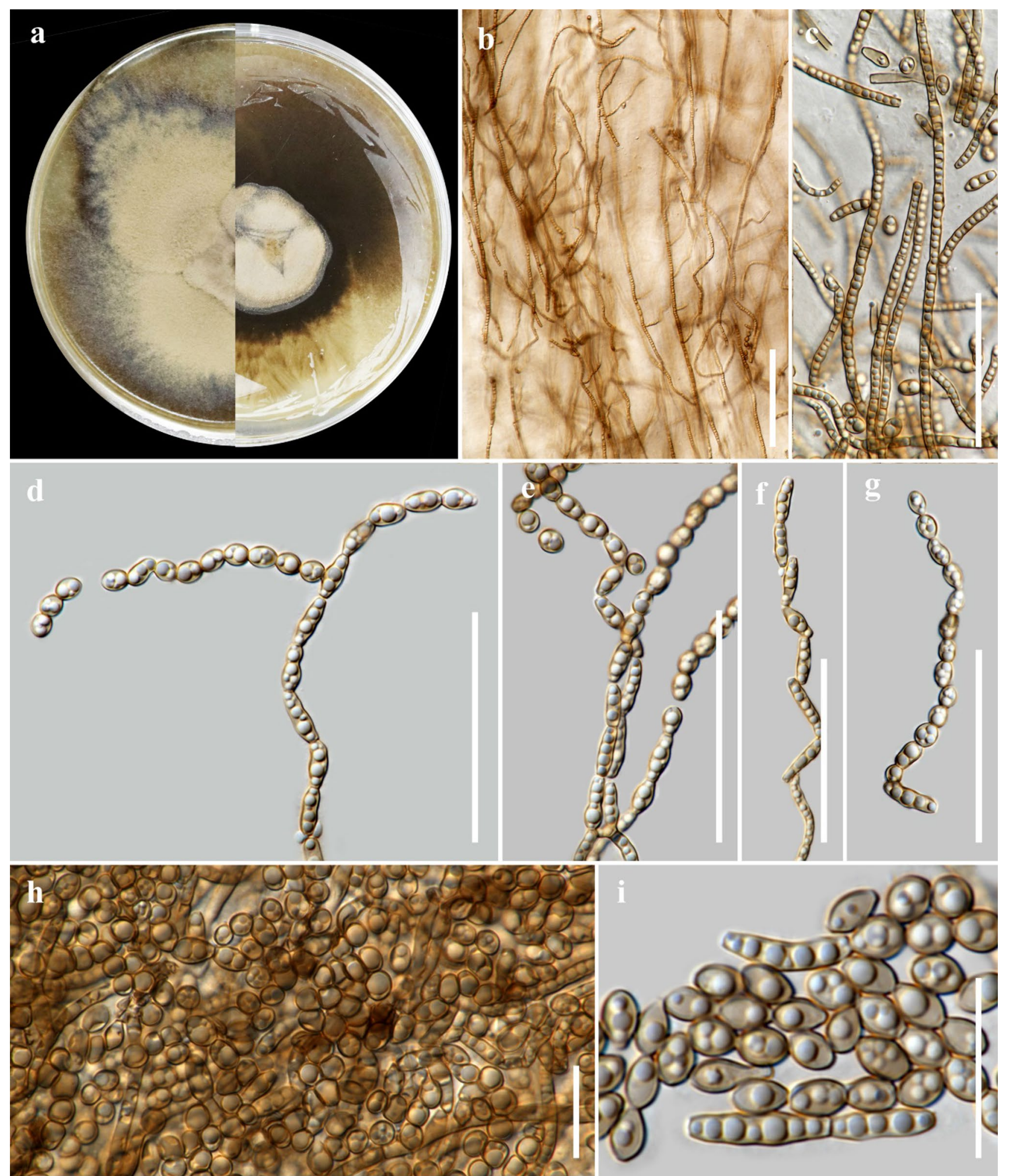

Fig. 101 Cladophialophora abundans (MFLUCC 21-0105, new geographical record). a Culture on MEA from surface and reverse. b, c Long strongly coherent conidial chains. $\mathbf{d}-\mathbf{g}$ Conidiophores and conidia. $\mathbf{h}$, i Conidia. Scale bars: $\mathbf{b}=100 \mu \mathrm{m}, \mathbf{c}-\mathbf{g}=50 \mu \mathrm{m}, \mathbf{h}, \mathbf{i}=20 \mu \mathrm{m}$ 

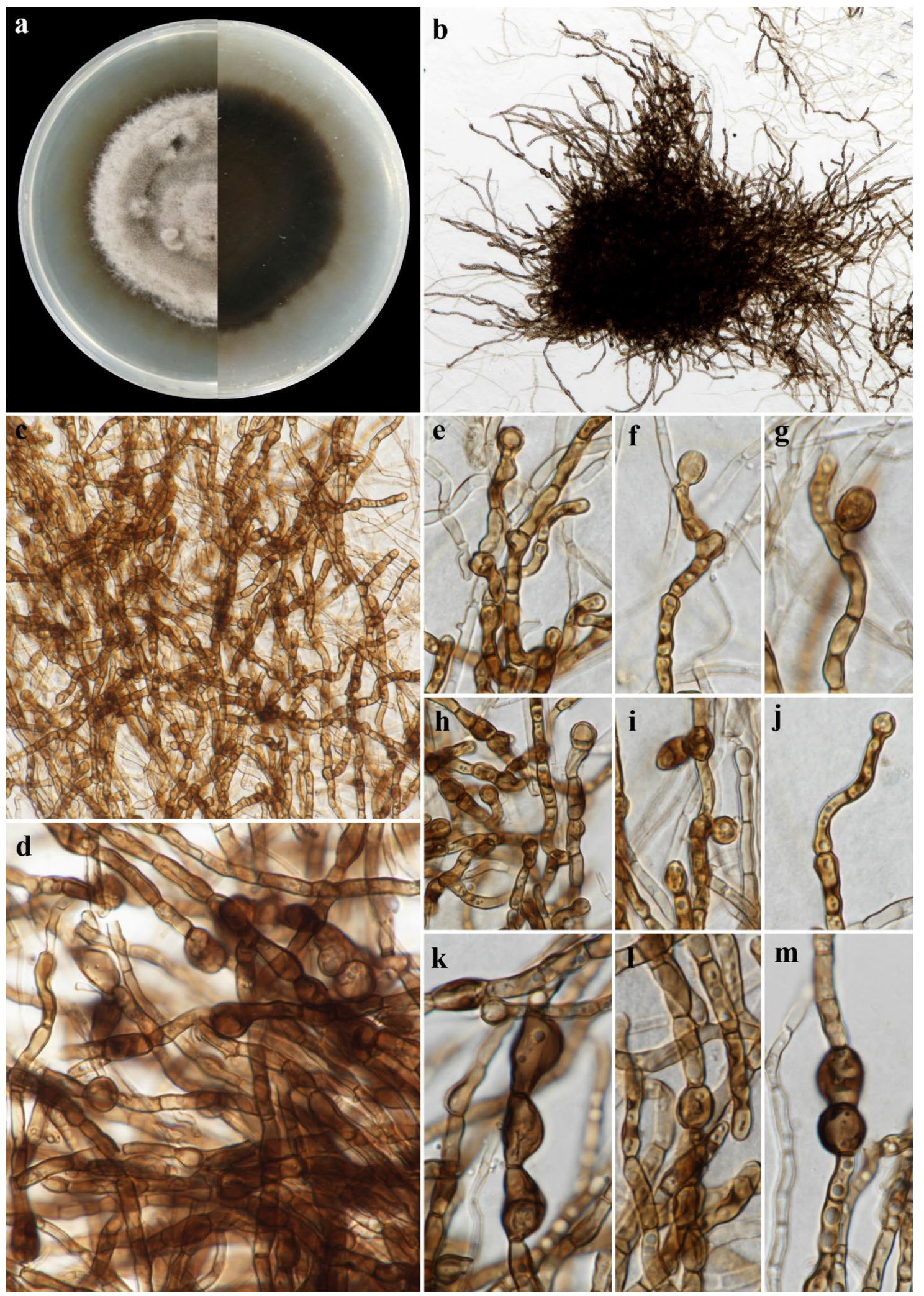

Fig. 102 Cladophialophora aquatica (MFLUCC 21-0108, ex-type). a Culture on MEA from surface and reverse. b-m Chlamydospores. Scale bars: $\mathbf{b}=300 \mu \mathrm{m}, \mathbf{c}, \mathbf{d}=50 \mu \mathrm{m}, \mathbf{e}-\mathbf{m}=20 \mu \mathrm{m}$ 
circular, with entire margin, flat, smooth, from above gray, from below dark brown. Chlamydospores produced in culture and induced with plant tissues within 30 days.

Material examined: THAILAND, Chiang Mai Province, Mushroom Research Center, on submerged decaying wood in a freshwater habitat, 11 February 2019, M.S. Calabon, MC03 (MFLU 21-0119, holotype), ex-type living culture, MFLUCC 21-0108.

GenBank numbers: ITS = MT864355, LSU = MT860433.

Notes: Unlike other species of Cladophialophora, C. aquatica did not produce conidial chains in culture but chlamydospores were prominent and numerous (Fig. 102). One strain of Cladophialophora immunda CBS 109797 and C. tortuosa produced chlamydospores in culture infrequently (Badali et al. 2008; Obase et al. 2016). In the phylogenetic analysis of LSU and ITS sequence data, Cladophialophora aquatica clustered with $C$. exuberans, $C$. matsushimae and $C$. mycetomatis with only 1.00 BYPP support (Fig. 103). Cladophialphora matsushimae differs in having conidial chains that resembles septate vegetative hyphae and subcylindrical conidia, ramoconidia, and microconidia (Koukol 2010). Cladophialphora exuberans and $C$. mycetomatis differ from $C$. aquatica in having conidiogenous cells that sympodially proliferate with a few conidiogenous loci, and the presence of ramoconidia and conidia (Badali et al. 2008; Nascimento et al. 2017).

\section{Eurotiales G.W. Martin ex Benny \& Kimbr.}

Notes: We follow the latest treatment and updated accounts of Eurotiales in Hyde et al. (2019).

\section{Aspergillaceae Link (=Monascaceae J. Schröt.)}

Notes: We follow the latest treatment and updated accounts of Aspergillaceae in Hyde et al. (2019).

Aspergillus P. Micheli ex Haller.

Notes: Aspergillus is the largest genus in Aspergillaceae contains more than 700 accepted species and divided into 25 sections (Houbraken et al. 2020; Species Fungorum 2021). Aspergillus section Sparsi is typified by A. sparsus and comprised four species were isolated from soil substrate (Raper and Thom 1944). Members of Aspergillus section Sparsi are commonly found in soil and their characterized by produced large globose conidia and irregular split with age (Smith 1956; Mares et al. 2008; Chen et al. 2016). Most species in this section are identified by pigment ranging from light grey to olive-buff (Varga et al. 2010). We follow the latest treatment and update accounts of Aspergillus in (Houbraken et al. 2020).

Aspergillus lannaensis N. Suwannarach, S. Khuna \& S. Lumyong, sp. nov.

MycoBank number: MB838058; Facesoffungi number: FoF 09955; Fig. 104
Etymology: "lannaensis" referring to Lanna, the old name of the region including Chiang Mai Province, northern Thailand, where soil containing the new fungus was collected.

\section{Holotype: SDBR-CMUO8}

Culture characteristics: Colonies growing after 7 days at $25{ }^{\circ} \mathrm{C}$ on the following agar: CYA $45-48 \mathrm{~mm}$, MEA 46-49 mm, and CREA $41-43 \mathrm{~mm}$. Slow growth was observed on CYA, MEA, and CREA are 18-20, 12-14 and 13-15 mm, respectively after 7 days at $37^{\circ} \mathrm{C}$. On all agar media the colonies first white, gradually becoming light yellow from entre outwards, then conidiophores are produced, conidial areas are light yellow to olive drab on MEA and CREA; light yellow to dark yellow on CYA. Reverse yellowish-brown on CYA and CREA; light yellow on MEA. On CREA thin colonies with poor sporulation and no acid production. Conidiophores produced abundantly on MEA. Conidial heads 35-137 $\mu \mathrm{m}$ diam., globose, light yellow when young, olive drab in age, radiate, commonly splitting into columns with age. Stipes 190-800 $\times 7-15 \mu \mathrm{m}$ wide near vesicle, short, smooth, thick walled, hyaline (light yellowish-brown pigment on upper portion near vesicle). Vesicles globose or nearly so, 14-46 $\mu \mathrm{m}$ in diam.; uni-seriate. Phialides $8-15 \times 2-3 \mu \mathrm{m}$, pyriform and covering the entire surface of the vesicle. Conidia $3-6 \times 2-3 \mu \mathrm{m}$ wide, oval or ellipsoidal, light yellow, with smooth-walled, and arranged in long chains.

Material examined: THAILAND, Chiang Mai Province,

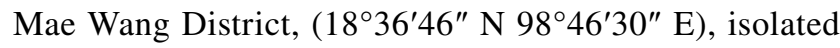
from soil of longan orchard, 8 August 2017, S. Khuna, dried culture: SDBR-CMUO8, holotype; Chiang Mai Province, Mae Wang District, $\left(18^{\circ} 36^{\prime} 46^{\prime \prime}\right.$ N 98 46'30" E), isolated from soil of longan orchard, 8 August 2017, S. Khuna, living culture, SDBR-CMUO6.

GenBank numbers: SDBR-CMUO6: Cam MW219780, $B e n A=$ MW219782, RPB2 = MW219784; SDBRCMUO8: $C a m=\mathrm{MW} 219781$, BenA $=$ MW219783, $\mathrm{RPB} 2=\mathrm{MW} 219785$.

Notes: A concatenated phylogenetic tree (cam, benA, and RPB2) revealed that Aspergillus lannaensis forms distinct lineages sister to A. funiculosus with $100 \%$ MLBS, 1.00 BYPP support (Fig. 105). However, A. lannaensis can be distinguished from A. funiculosus in shapes and sizes of conidia and phialides (Smith 1956). Therefore, we introduce a new species Aspergillus lannaensis (Fig. 104) based on morphology coupled with phylogenetic evidence.

\section{Sclerococcales Réblová, Unter. \& W. Gams}

Notes: Réblová et al. (2017) introduced Sclerococcales with Sclerococcum Fr. as type genus. Dactylosporaceae (=Sclerococcaceae) constitutes the order with five known genera: Cylindroconidiis, Fusichalara, Longimultiseptata, Rhopalophora and Sclerococcum (=Dactylospora) (Wijayawardene et al. 2020). Sclerococcales comprises ca. 80 species that dwell in terrestrial and aquatic environments as wood 


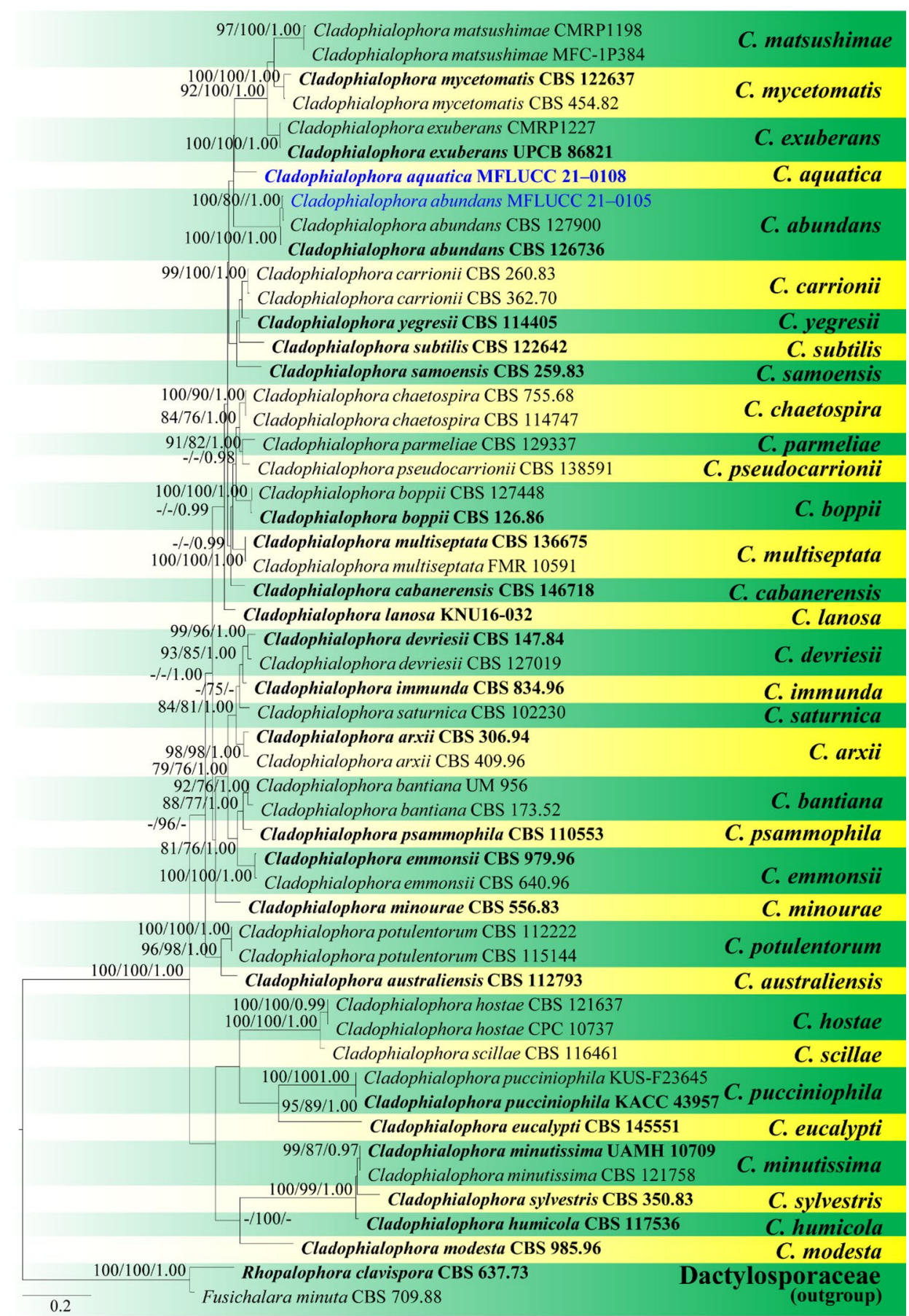

Fig. 103 Phylogram generated from maximum likelihood analysis based on combined LSU and ITS sequence data representing the species of Cladophialophora. Related sequences are taken from Kiyuna et al. (2018). Rhopalophora clavispora (CBS 637.73) and Fusichalara minuta (CBS 709.88) were used as the outgroup taxa. Fifty-three taxa are included in the combined analyses which comprised 1041 characters (502 characters for LSU, 539 characters for ITS) after alignment. The best scoring RAxML tree with a final likelihood value of - 9519.130038 is presented. The matrix had 524 distinct alignment patterns, with $16.53 \%$ of undetermined characters or gaps. Estimated base frequencies were as follows: $\mathrm{A}=0.236745, \mathrm{C}=0.249453, \mathrm{G}=0.269107, \mathrm{~T}=0.244695$; substitution rates: $\mathrm{AC}=1.891141, \mathrm{AG}=3.179450, \mathrm{AT}=1.427526$, $\mathrm{CG}=0.913317, \mathrm{CT}=6.628918, \mathrm{GT}=1.000000$; gamma distribution shape parameter $\alpha=0.260996$. The MP analysis resulted a single most parsimonious tree $(\mathrm{TL}=1789, \mathrm{CI}=0.455, \mathrm{RI}=0.710, \mathrm{RC}=0.323, \mathrm{HI}=0.545)$. Bootstrap support values for MLBS and MPBS equal to or greater than $75 \%$ and BYPP equal to or greater than 0.95 are given above the nodes. Ex-type strains are in bold and newly generated sequences are in blue 

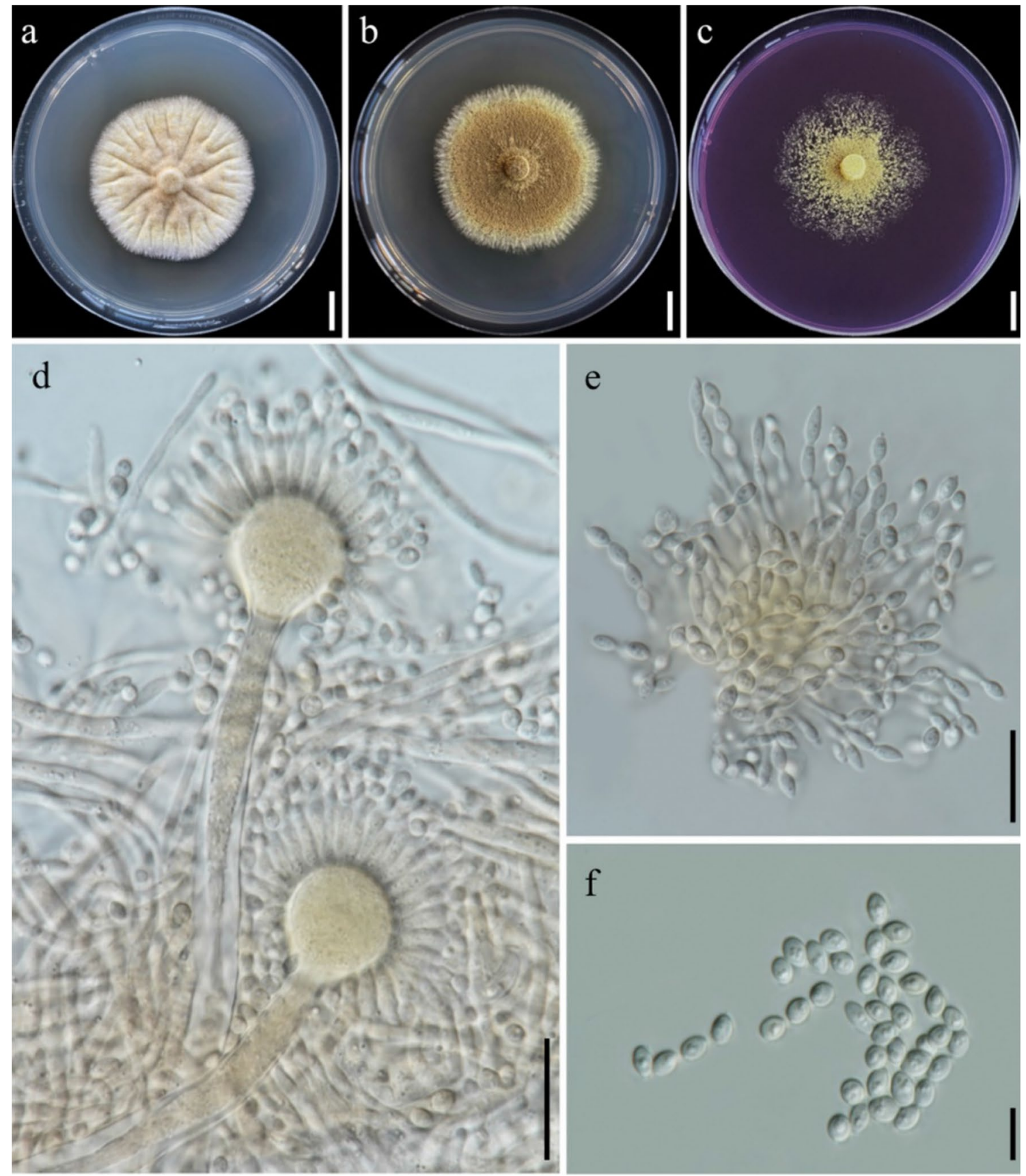

Fig. 104 Aspergillus lannaensis (SDBR-CMUO8, holotype). Colonies incubated at $25^{\circ} \mathrm{C}$ for 7 days. a Colony on CYA. b Colony on MEA. c Colony on CREA. d Conidiophores. e Conidia arranged in

saprobes, lichenized species on lichens, non-lichenized fungi or leafy liverworts, or associated with beetles as a part of intestinal microbiota (Diederich et al. 2018; Olariaga et al. 2019).

Dactylosporaceae Bellem. \& Hafellner (= Sclerococcaceae Réblová, Unter. \& W. Gams)

Notes: Dactylosporaceae was established by Bellemère and Hafellner (1982) with various placements based on long chains. f Oval or ellipsoidal conidia. Scale bars: $\mathbf{a}-\mathbf{c}=10 \mathrm{~mm}, \mathbf{d}$ $\mathbf{e}=20 \mu \mathrm{m}, \mathbf{f}=10 \mu \mathrm{m}$

morphology and phylogenetic studies: Eurotiomycetes (Schoch et al. 2009b; Diederich et al. 2013; Pang et al. 2014; Ekanayaka et al. 2019b; Wijayawardene et al. 2020), Lecanoromycetes (Miadlikowska et al. 2014; Pino-Bodas et al. 2017), Pezizomycotina incertae sedis (Jaklitsch et al. 2016) and Eurotiomycetes incertae sedis (Wijayawardene et al. 2020). Réblová et al. (2017) introduced Sclerococcaceae as type family of Sclerococcales but Diederich et al. (2018) 


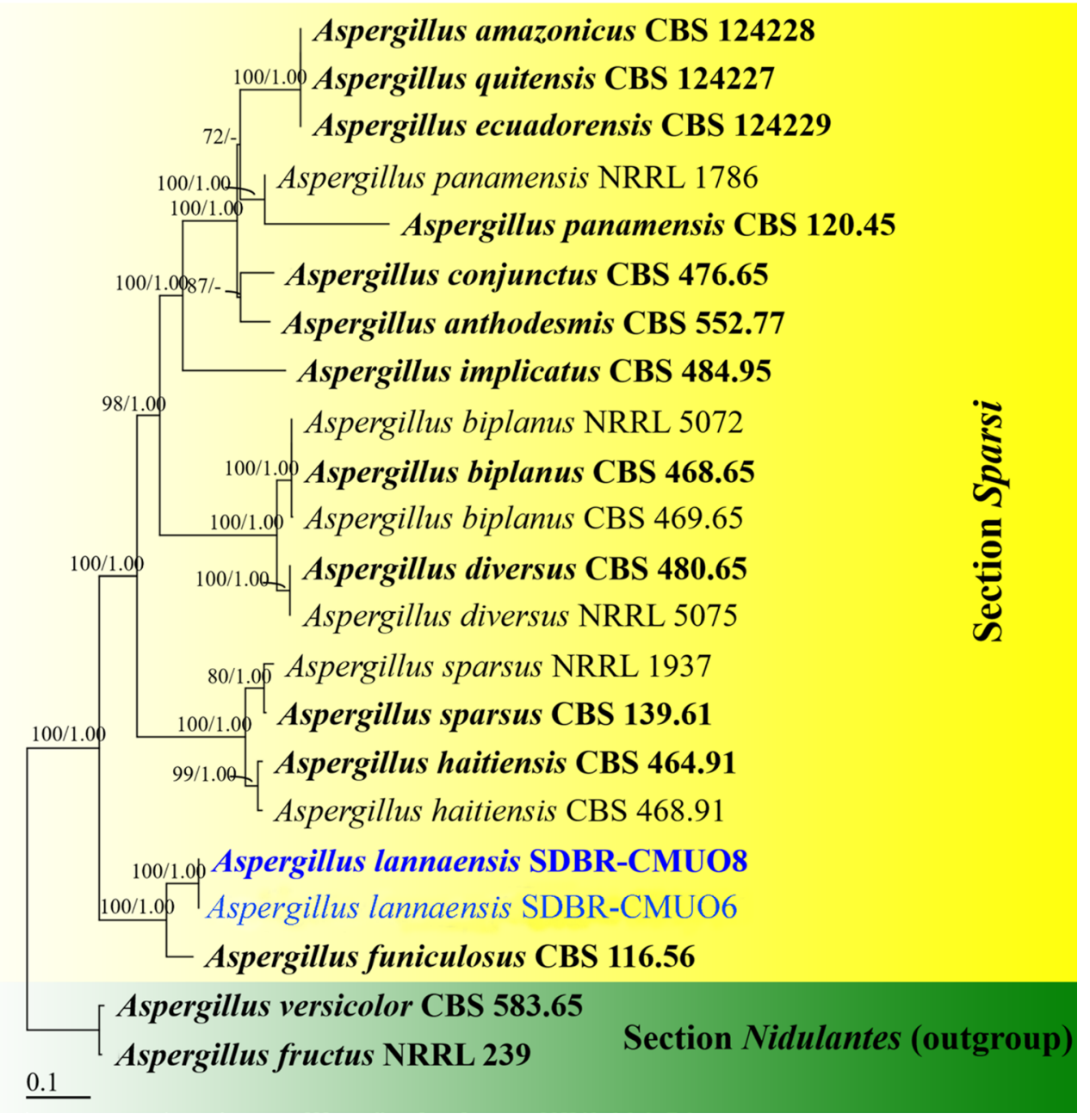

Fig. 105 Phylogenetic tree derived from maximum likelihood analysis of a combined Cam, BenA and RPB2 genes of 22 sequences and the aligned dataset was comprised of 2360 characters including gaps (Cam: 1-792, BenA: 793-1310 and RPB2: 1311-2360). The average standard deviation of the split frequencies of the BI analysis was 0.004276 . A best scoring RAxML tree was established with a final ML optimization likelihood value of -13462.8650 . The matrix had 1055 distinct alignment patterns with $21.67 \%$ undetermined characters or gaps. Estimated base frequencies were found to be: $\mathrm{A}=0.2500, \mathrm{C}=0.2468, \mathrm{G}=0.2390, \mathrm{~T}=0.2642$; substitution rates

reinstated Dactylosporaceae to replace Sclerococcaceae. Five genera are included in the family and species are mostly lignicolous, lichenicolous or associated with beetles as a part of intestinal microbiota (Pang et al. 2014; Jaklitsch et al. 2016; Réblová et al. 2017; Wijayawardene et al. 2020).

Pseudobactrodesmium H. Zhang, W. Dong \& K.D. Hyde

Notes: Pseudobactrodesmium was introduced by Dong et al. (2020a) with P. longisporum (三 Bactrodesmium longisporum) as the type species. Pseudobactrodesmium aquaticum, $P$. chiangmaiensis and $P$. longisporum were included in the genus. Pseudobactrodesmium longisporum was formerly
$\mathrm{AC}=1.0520, \mathrm{AG}=3.4446, \mathrm{AT}=1.0037, \mathrm{CG}=0.6646, \mathrm{CT}=5.3732$, $\mathrm{GT}=1.0000$; proportion of invariable sites $=0.1320$ and gamma distribution $=0.6870$. Aspergillus fructus NRRL 239 and Aspergillus versicolor CBS 583.65 were used as outgroup. Numbers above branches are the bootstrap statistics percentages (left) and Bayesian posterior probabilities (right). Branches with bootstrap values equal to or greater than $70 \%$ are shown at each branch and the bar represents 0.1 substitutions per nucleotide position. Hyphen (-) represents support values equal to or greater than 70\%/0.95. Ex-type strains are in bold and newly generated sequences are in blue

placed in Bactrodesmium (Dothideomycetes genera incertae sedis), but multigene phylogenetic analysis revealed it was unrelated to Bactrodesmium and clustered within Sclerococcales (Eurotiomycetes) (Ellis 1976; Pem et al. 2019b; Dong et al. 2020a; Wijayawardene et al. 2020).

Pseudobactrodesmium stilboideum (R. F. Castañeda \& G.R.W. Arnold) M.S. Calabon, Boonmee, E.B.G. Jones, K.D. Hyde, comb. nov.

三 Bactrodesmium stilboideum R. F. Castañeda \& G. R. W. Arnold, Revta Jardín bot. Nac., Univ. Habana 6(1): 48 (1985) 

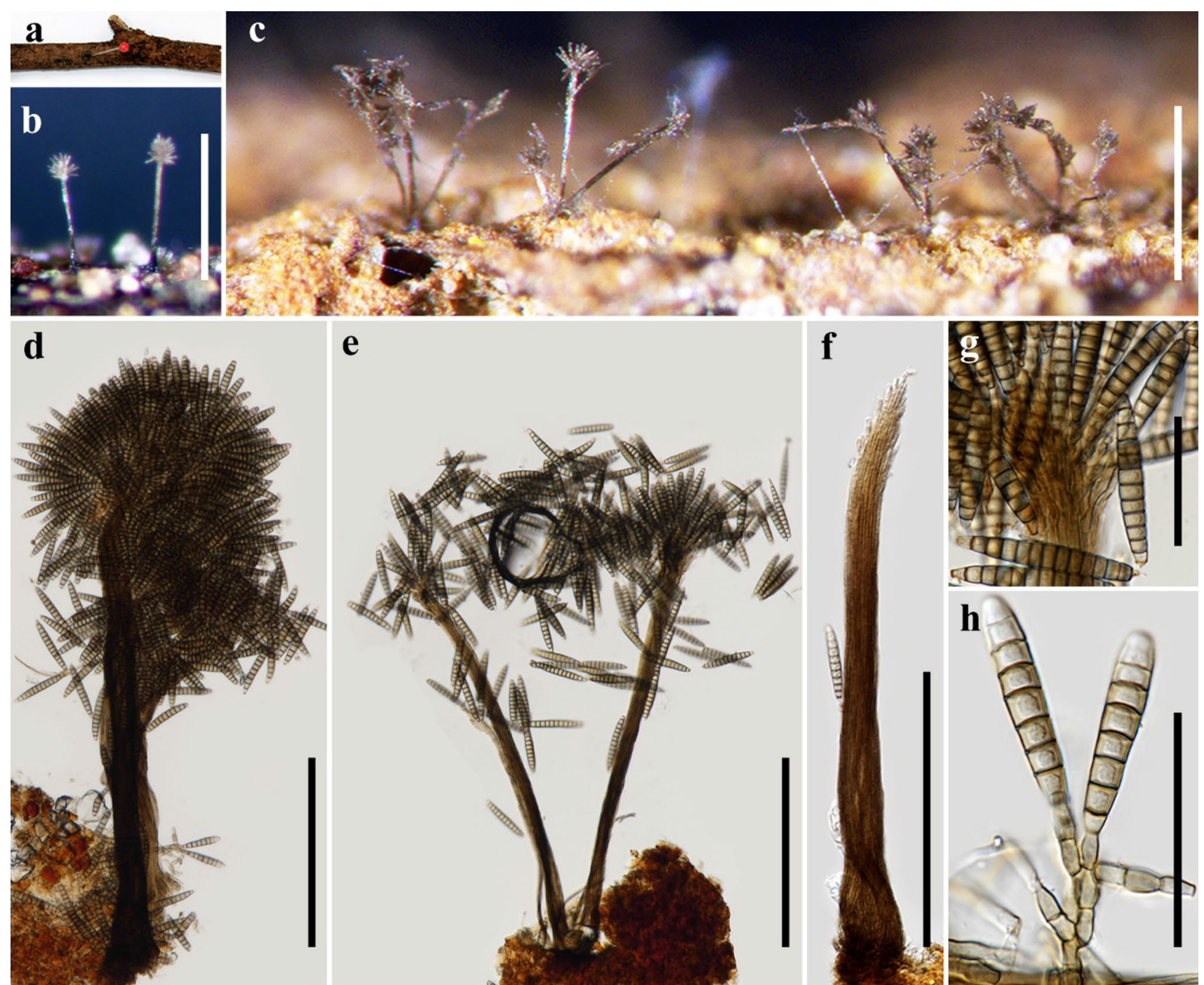

e

f
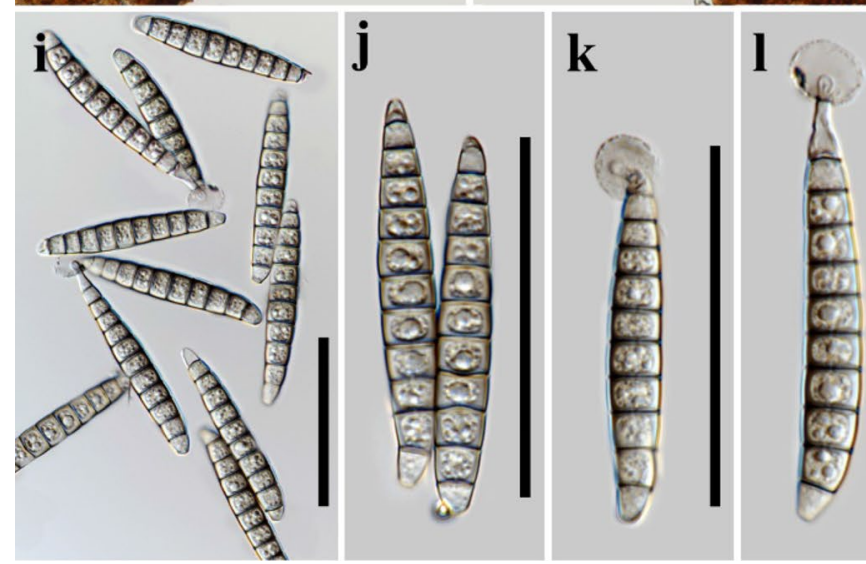

Fig. 106 Pseudobactrodesmium stilboideum (MFLU 21-0120). a Substrate. b-e Synnemata on submerged wood. $\mathbf{f}-\mathbf{h}$ Synnemata and densely branched conidiophores, conidiogenous cells and attached

$=$ Stigmina longispora var. stilboidea (R.F. Castañeda \& G.R.W. Arnold) J. Mena \& Mercado, Reporte de Investigacion del Instituto de Ecología y Sistemática 17: 10(1987).

conidia. i-l Conidia and mucilaginous cap at the apex. $\mathbf{m}$ Germinated conidium. $\mathbf{n}$ Culture on MEA from surface and reverse. Scale bars: $\mathbf{b}$, $\mathbf{c}=500 \mu \mathrm{m}, \mathbf{d}-\mathbf{f}=200 \mu \mathrm{m}, \mathbf{g}-\mathbf{m}=50 \mu \mathrm{m}$

Index Fungorum number: IF558643; Facesoffungi number: FoF 09154; Fig. 106 
Saprobic on submerged decaying wood in a freshwater habitat. Sexual morph Undetermined. Asexual morph Hyphomycetous. Colonies on natural substrate 415-635 × 14-40.5 $\mu \mathrm{m}(\bar{x}=494.5 \times 28 \mu \mathrm{m}, \mathrm{n}=15)$, synnematous, superficial, effuse, scattered, dark brown to dark olivaceous brown. Mycelium mostly immersed, composed of white, septate, branched and guttulate hyphae. Conidiophores $15-30 \times 2-4 \mu \mathrm{m}(\bar{x}=25 \times 3 \mu \mathrm{m}, \mathrm{n}=15)$ macronematous, fasciculate, synnematous, compact, erect, subcylindrical, septate, slightly constricted at the septa, unbranched or branched, brown, smooth. Conidiogenous cells monoblastic, terminal, discrete, subcylindrical, pale brown, elongating percurrently. Conidia $48-65 \times 7-8 \mu \mathrm{m}$ $(\bar{x}=55 \times 7.5 \mu \mathrm{m}, \mathrm{n}=50)$, solitary, dry, subcylindrical to narrowly fusiform, usually straight or slightly curved, euseptate, 9-13-phragmoseptate, thin and smooth-walled, slightly constricted and darker at septa, brown, paler towards both ends, obscurely guttulate, wedge-shaped at basal cell, with tapering apical cells, often enveloped by a hyaline, spherical, thin, mucilaginous cap at the apex, 8-10 $\mu \mathrm{m}$ diam. Apical cells elongated, up to $12 \mu \mathrm{m}$ long, tapering gradually toward apex, hyaline to subhyaline, with subglobose tuberculate ends, secession schizolytic.

Culture characteristics: Conidia germinating on malt extract agar (MEA) within $24 \mathrm{~h}$. Germ tubes produced from the basal and apical cell of conidia. Colonies growing on MEA, reaching $20-25 \mathrm{~mm}$ in 2 weeks at $25{ }^{\circ} \mathrm{C}$. Mycelia superficial, circular, with entire margin, flat, smooth, from above white, from below smoke grey.

Material examined: THAILAND, Tak Province, Tha Sing Yang, Ban Mae Ja Wang, on submerged decaying wood in a freshwater river, 17 October 2019, N. Padaruth, CC44 (MFLU 21-0120), living culture, MFLUCC 21-0101.

GenBank numbers: ITS $=$ MT864357, LSU $=$ MT860435.

Notes: Dong et al. (2020a) synonymized Pseudobactrodesmium stilboideum under $P$. longisporum but based on the phylogenetic analysis of LSU and ITS sequence dataset (Fig. 107), it did not group with strains of $P$. longisporum but clustered with $P$. stilboideum MHR 18017. The aggregation of conidiophores distinguished the two species wherein $P$. stilboideum has synnematous formation but in P. longisporum, it is sporodochial with mononematous, fasciculate conidiophores (Ellis 1976; Castañeda-Ruiz and Arnold 1985; Dong et al. 2020a). Moreover, P. stilboideum has shorter and narrower $(48-65 \times 7-8 \mu \mathrm{m})$ and 9-13-septate conidia (Fig. 106), while P. longisporum has longer and wider $(85-119 \times 8-9.5 \mu \mathrm{m})$ and 16-21-septate conidia (Ellis 1976). Pseudobactrodesmium stilboideum was isolated on decaying unidentified wood and dead leaves of Calyptronoma plumeriana and is distributed in Cuba, Puerto Rico, South Africa, Taiwan, Thailand and UK (Ellis 1976; Castañeda-Ruiz and Arnold 1985; Hu et al. 2010a; this study).

\section{Laboulbeniomycetes Engl.}

Laboulbeniales Lindau

Notes: see Hyde et al. (2019).

Laboulbeniaceae G. Winter

Notes: see Hyde et al. (2019).

\section{Hydrophilomyces Thaxt.}

Notes: This genus includes to date 16 species associated with aquatic beetles. It is characterized by a long "accessory" cell flanking the perithecial venter and by a multicellular axis extending above the insertion of the perithecium. A single sequence is available for species in this genus (Goldmann and Weir 2018).

Hydrophilomyces hydraenae W. Rossi \& M. Leonardi, sp. nov.

Index Fungorum number: IF557383; Facesoffungi number: FoF 07847; Fig. 108

Etymology: Named after the host insect.

Holotype: FI WR 2462

Thallus hyaline or nearly so. Receptacle consisting of 11-12 superposed cells, of which the basal is irregularly rhomboid, the following 2-3 cells, small, each producing laterally an elongate and variably curved buffer cell, the others gradually increasing in length from almost flattened to slightly longer than broad. Appendage usually curving outwards and downwards, consisting of 8-10 subequal cells, each separating a small corner cell on the anterior side; these corner cells give rise to short branchlets bearing distally 2-4 elongate antheridia soon displaced by long and slender sterile branchlets, which are repeatedly divided in the distal portion of the appendage. Perithecial venter ovoid, relatively short, tapering without abrupt transition to the broad neck; the latter almost isodiametric, up to three times longer than the venter, with an elongate and undistinguished tip ending in rounded apex. Total length from foot to perithecial apex 300-345 $\mu \mathrm{m}$; length of perithecium $205-250 \mu \mathrm{m}$; perithecial venter $35 \times 60 \mu \mathrm{m}$.

Material examined: USA, CA, Santa Barbara Co., W Camino Cielo (rd.) at Hwy. 154, 590 msl, 34³0.273' N $119^{\circ} 48.806^{\prime} \mathrm{W}$, edge of a stream in hardwood forest, 19 July 2000, A. Newton and M. Thayer, at the base of the legs of Hydraena vandykei Orchymont (Coleoptera, Hydraenidae) (FI WR2462, holotype; FI WR2477, paratype).

Notes: The species of Hydrophilomyces described so far are 16, of which 8 are reported on Hydraenidae (genera Ochtebius and Limnebius), the other 8 on Hydrophilidae. These species can be distinguished from Hydrophilomyces hydraenae (Fig. 108) by the following characteristics: $H$. gracilis, $H$. lumbricoides, $H$. major, $H$. reflexus and $H$. rhynchophorus (all parasitic on Hyrophilidae) lack any buffer 


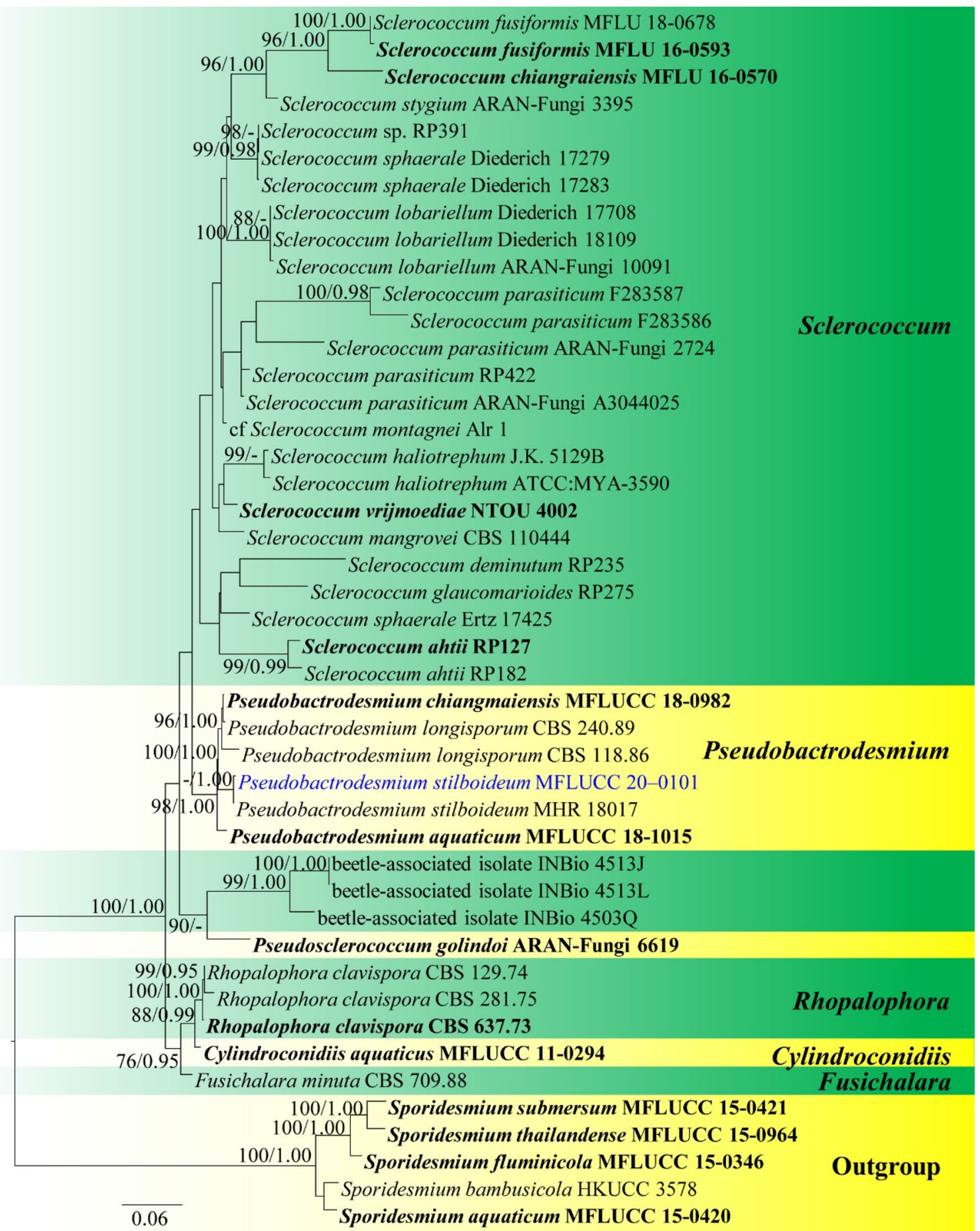

Fig. 107 Phylogenetic tree generated from maximum likelihood (ML) analysis based on combined LSU and ITS sequence data for the species from Dactylosporaceae. Five species of Sporidesmium aquaticum, S. bambusicola, S. fluminicola, S. submersum and $S$. thailandense (Sporidesmiaceae) are used as outgroup taxa. The dataset comprised and 1222 characters after alignment including gaps $(\mathrm{LSU}=793 \mathrm{bp}$, ITS $=429 \mathrm{bp}$ ). The RAxML analysis of the combined dataset yielded a best scoring tree with a final ML optimization likelihood value of -3662.794208 . The matrix had 1053 distinct align- ment patterns, with $51.86 \%$ undetermined characters or gaps. Estimated base frequencies were as follows: $\mathrm{A}=0.266384, \mathrm{C}=0.211364$, $\mathrm{G}=0.273019, \mathrm{~T}=0.249233 ;$ substitution rates $\mathrm{AC}=1.261100$, $\mathrm{AG}=2.304874, \quad \mathrm{AT}=1.337644, \quad \mathrm{CG}=1.220148, \quad \mathrm{CT}=5.907736$, GT $=1.000000$; gamma distribution shape parameter $\alpha=0.284689$. Support values for maximum likelihood (MLBS) above than $75 \%$ and Bayesian posterior probabilities (BYPP) greater than 0.95 are given at the nodes. Ex-type species are in bold and newly generated sequence is in blue 

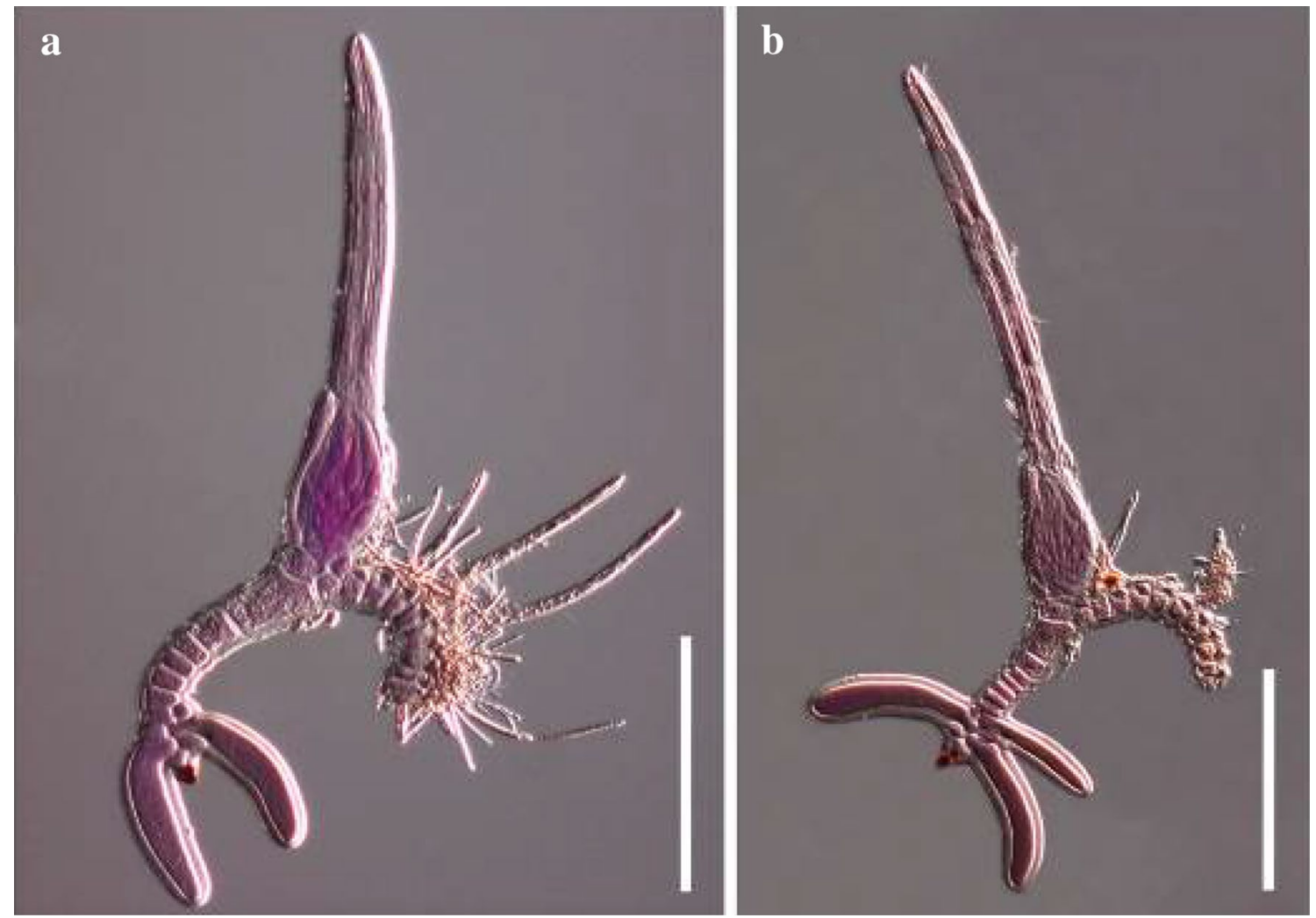

Fig. 108 Hydrophilomyces hydraenae (FI WR2462, holotype). a Thallus from the type slide stained with acid fuchsin. b Thallus from the paratype slide stained with acid fuchsin. Scale bars: $\mathbf{a}, \mathbf{b}=100 \mu \mathrm{m}$

cells and have a very elongate receptacle consisting of 20 or more cells; $H$. coneglianensis has the perithecial neck about as long as the venter; $H$. arcuatus, $H$. pusillus, $H$. rhytidopus have the perithecial neck much shorter than the venter; $H$. aduncus, $H$. deflexus, $H$. hamatus, $H$. limnebii have a distinctly curved perithecial neck; $H$. atroseptatus has a stout and tapering appendage, consisting of flattened cells bearing corner cells separated by constricted dark septa from antheridia or sterile branchlets; $H$. riberae has a shorter receptacle and a broader perithecial neck; $H$. digitatus has the receptacle consisting of more or less isodiametric cells, a more elongate perithecial venter, a slenderer and shorter perithecial neck distinctly broadened at the junction with the tapering and truncate tip (Spegazzini 1917; Thaxter 1908; Huldén 1983; Majewski 1994; Santamaria 2003, 2006; Santamaria et al. 2020).

\section{Laboulbenia Mont. \& C.P. Robin}

Notes: Laboulbenia is by far the largest genus among the Laboulbeniales with about 650 described species, which represent almost one third of all the known species. Besides the accepted species, several subspecific taxa have also been described as subspecies, varieties and forms. The species of Laboulbenia occur on various families of beetles (Coleoptera), but are also found on flies (Diptera), bugs (Hemiptera), ants, (Hymenoptera), termites (Isoptera), cockroaches (Blattodea), crickets (Orthoptera) and mites (Acarina) (Kong et al. 2020). Very few sequences are available, and is still lacking, among many others, are those of the type species.

Laboulbenia divisa W. Rossi \& M. Leonardi, sp. nov.

Index Fungorum number: IF557384; Facesoffungi number: FoF 07846; Fig. 109

Etymology: From Latin: divided, because of the lower portion of the receptacle unusually divided into many cells.

Holotype: FI WR3479a

Perithecium pale greyish brown; antheridia dark brown; appendages brown to blackish, with paler tips; the rest of the fungus is pale yellowish grey. Thallus very long and slender. Basal and suprabasal cells of the receptacle (cells I \& II) replaced by a series of 6-9 gradually longer cells originated by secondary divisions. Cell III + IV stout, about one and half times, or less, longer than its maximum width, with the outer margin straight or slightly concave. Cell V relatively large, irregularly trapezoidal or wedge-shaped. Insertion cell narrow and thick, free from the base of the perithecium. Outer appendage consisting of a large, erect 


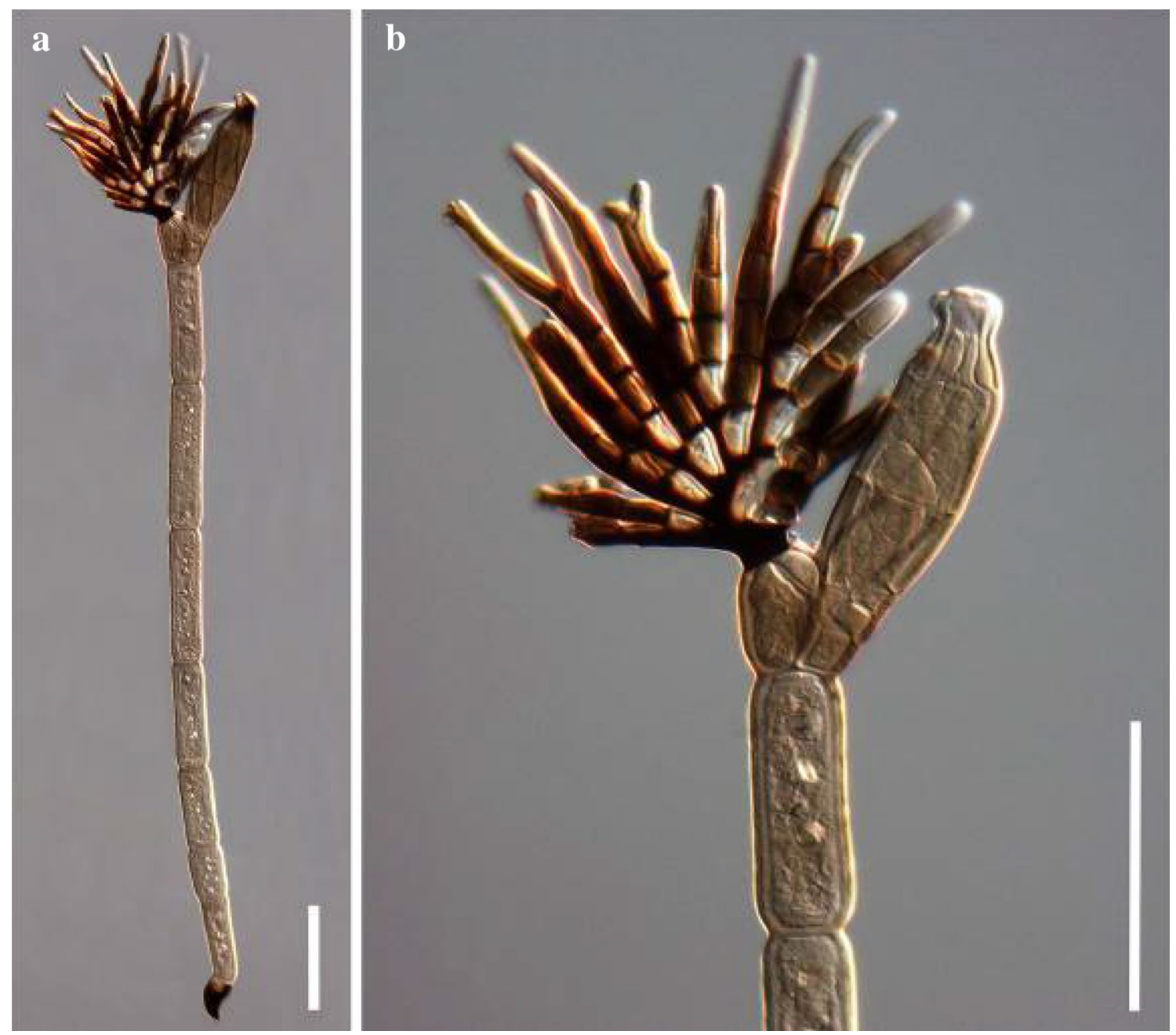

Fig. 109 Laboulbenia divisa (FI WR3479a, holotype). a Whole thallus from the type slide. b Upper portion of an immature thallus from the type slide. Scale bars: $\mathbf{a}, \mathbf{b}=100 \mu \mathrm{m}$

basal cell, blackish on the outer side and almost hyaline on the inner, from which arise outwardly five branches, each dividing dichotomously once or twice above the lower cell, which is hyaline and bordered with black on the outer side, the following cells being smaller and brownish; as whole the outer appendage appears as a dense, fan-shaped tuft of branchlets slightly exceeding in length the apex of the perithecium. Inner appendage consisting of a very small and hyaline basal cell distinctly shorter than its maximum width, from which arise two short bicellular branchlets, the lower cell of which is almost rounded, grayish brown, contrasting, separated from the smaller, paler, greyish and slenderer upper cell by a marked constriction and a thick, black septum; each of the two branchlets bear apically a tuft of (2-)3-4, elongate, bottle-shaped antheridia. Cell VI relatively small, rectangular in outline, about one and half times longer than broad. Perithecium almost entirely free, the venter slightly and evenly inflated, the wall-cells spirally twisted, describing a half turn from the base to the tip, which is rather abruptly distinguished, much darker, slightly bent inward, ending with four larges, rounded, subequal lips with hyaline margins. Length from foot to perithecial apex 880-1575 $\mu \mathrm{m}$; perithecium 45-65 $\times 140-170 \mu \mathrm{m}$; antheridia $35 \mu \mathrm{m}$; longest appendages $225 \mu \mathrm{m}$.

Material examined: COSTA RICA, Prov. Cartago, Turrialba, P. N. Barbilla, Sendero el Felino, LN-217000 N 595000E, alt. 400-500 msl, 18.VII.2002, E. Rojas, upper surface of the tip of the abdomen of a female specimen of Richardia telescopica Gerdstacker (Diptera, Richardiidae) (FI WR3479a, holotype; WR3479b in InBIO, isotype). Costa Rica, Prov. Cartago, P. N. Barbilla, Dantas River, LN-218100 N 593600E, alt. 500-600 msl, Malaise trap, 9 December 1999-8 January 2000, E. Rojas, tip of the abdomen of R. telescopica (FI WR2700a and FI WR2700b, paratypes). 
Notes: The division of the lower cells of the receptacle was observed so far in only two species of the large genus Laboulbenia: L. partita reported from various African and Asian countries on Chrysomelidae Alticini (Rossi and Bernardi 2018), and L. hingstonii described on a Carabidae from India (as Misgomyces hingstoni: Balazuc 1971) and known only from the type collection. Laboulbenia divisa is distinct from and not allied with these two latter species. Likely, the division of the cells of the receptacle is a rare feature arisen independently in species of Laboulbenia occurring on very different host-insects in different continents.

The species of Laboulbenia occurring on the Diptera are relatively few and clearly polyphyletic (Rossi and KirkSpriggs 2011). The new species seems to be allied with $L$. richardiana described on Richardia teevani Curran from French Guinea (Rossi and Kotrba 2004). Laboulbenia divisa shares with $L$. richardiana the undivided cells III and IV, the perithecium being wholly free and the inner appendage lacking sterile branches. L. richardiana has been described as a polymorphic species, but recent molecular analysis carried out on Laboulbeniales of other genera suggests that the different "morphotypes" may be different phylogenetic species (Goldmann and Weir 2012; Goldmann et al. 2013).

With a length greater than $1.5 \mathrm{~mm}$ in length, Laboulbenia divisa is one of the tallest species of Laboulbenia (Fig. 109). In this ranking is surpassed only by L. kunkelii, occurring on a large ground beetle from tropical Asia, and by L. caprae (which is likely a synonym of $L$. gigantea, occurring on large ground beetles from Europe).

Laboulbenia triarthronis W. Rossi \& M. Leonardi, sp. nov. Index Fungorum number: IF558362; Facesoffungi number: FoF 09956; Fig. 110

Etymology: Referring to the host genus, Triarthron.

Holotype: SYRF RKB 601a

Thallus tinged with pale yellow, rather stocky, regularly enlarging from below upwards. Basal cell of the receptacle (cell I) slender, twice (or slightly more) longer than broad. Cell II slightly longer and distinctly broader than cell I, divided from cell III by a short transverse septum and from cell VI by a much longer, oblique and concave septum. Cell III broadly quadrangular, distinctly longer than broad. Cell IV about as long as cell III but broader. Cell V relatively large, shaped like an inverted tringle. Insertion cell oblique, narrow and thick, distinctly free from the perithecium. Basal cell of the outer appendage relatively large, longer than broad, bearing two branches in mature thalli; one of these branches is erect, relatively short, consisting of a linear series of small cells subtended by a contrasting blackish, constricted and elongate collarette; the second branch is produced after the former from the outer, upper angle of the basal cell: it is usually longer than the former and its cells are gradually longer towards the tip. In immature thalli the inner appendage consists of two superimposed subequal cells slightly longer than broad bearing distally a single, short antheridium, which is later displaced laterally by the growth of a branch longer than the branches of the outer appendage; sometimes this latter branch divides in two from the base. Cell VI longer than broad, irregularly shaped. Perithecium adnate to the receptacle for $2 / 3$ to $3 / 4$ of its length, slightly inflated, almost three times longer than maximum width, tapering without abrupt constriction to the truncate, dark brown tip slightly oriented outwards and ending in an almost flattened, hyaline apex. Length from foot to perithecial apex 190-300 $\mu \mathrm{m}$; longest appendage $175 \mu \mathrm{m}$; perithecium $95-140 \times 35-60 \mu \mathrm{m}$.

Material examined: USA, Illinois, Champaign Co., Brownfield Woods, 3 mi NE of Urbana, 14 August 1950, R.K. Benjamin, on all parts of the body of Triarthron lecontei Horn (=T. pennsylvanicum Horn) (Coleoptera, Leiodidae) collected on Armillariella mellea., (SYRF RKB 601a, holotype; SYRF RKB 601b, 601c, 601d, 601e, and FI WR4485, paratypes).

Notes: The appendages of Laboulbenia triarthronis easily distinguish the new species from the many others described so far (Fig. 110). As to the rest of the thallus, it might be compared with L. madeirae, described on a ground beetle, which however bears a single, robust and elongate outer appendage (Thaxter 1908, Plate LIV; Fig. 7). Laboulbenia triarthronis is the first species of Laboulbenia reported on Coleoptera Leiodidae. The beetles of this family of insects are the hosts of the other 14 genera in the Laboulbeniales, 4 of which are found only on Leiodidae (Benjamin 1955; Rossi and Santamaria 2012; Haelewaters and Rossi 2017).

\section{Mimeomyces Thaxt.}

Notes: Most of the 16 species recognized at present in this genus were described as Corethromyces Thaxt. or Sphaleromyces Thaxt. One species made the move in both directions: Corethromyces bicolor Thaxt. was first transferred to Mimeomyces (Tavares 1985), but was later brought back to Corethromyces (Weir and Hughes 2002). Only two of the species described so far were reported outside the American continent. No DNA sequence is available for any of the species in Mimeomyces.

Mimeomyces digitatus W. Rossi \& M. Leonardi, sp. nov. Index Fungorum number: IF557385; Facesoffungi number: FoF 07845; Fig. 111

Etymology: From Latin: bearing a finger, because of the finger-like preapical outgrowth.

Holotype: FI WR3388

Receptacle almost wholly blackened, with the basal cell pale yellow to almost hyaline above the foot; the rest of the thallus is yellowish brown, darker just above the receptacle and paler in the distal portion of the branchlets of the 
Fig. 110 Laboulbenia triarthronis (SYRF RKB 601a, holotype). a Thallus from the type slide stained with cotton blue. b Immature thallus from a paratype slide stained with cotton blue. Scale bars: a, $\mathbf{b}=50 \mu \mathrm{m}$

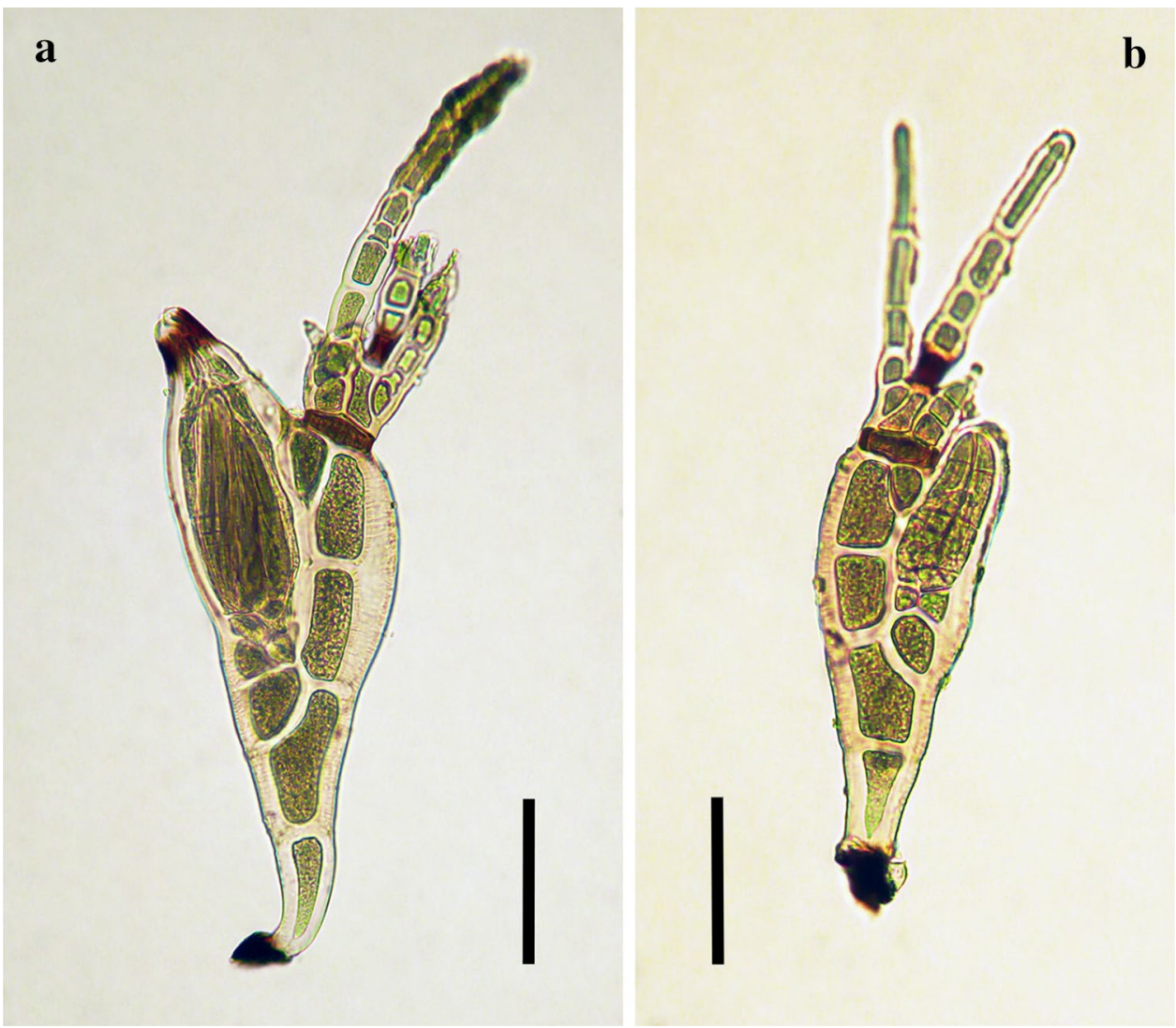

appendages; the preapical outgrowth of the perithecium is chestnut brown. Basal cell of the receptacle relatively large, obconical, slightly inflated above the foot. Suprabasal cell very short, irregularly pentagonal. Axis of the appendage consisting of five superimposed cells subequal in length but progressively narrower, bearing on the inner side short, erect, relatively stout branchlets, most of which are bifurcate from the base. Antheridia not seen. Stalk cell of the perithecium oblique, distinctly broader than long. Basal cells of the perithecium relatively large, subequal, distinctly longer than the stalk cell. Perithecium oblong, nearly symmetrical, slightly inflated below, the septa between the wall cell tiers marked by slight elevations, the tip rather abruptly tapering to a subtuncate apex bearing tiny hyaline lips and a subapical long, slender, straight outgrowth pointing obliquely upwards. Length from foot to perithecial apex 295-310 $\mu \mathrm{m}$; from foot to tip of longest appendages $160 \mu \mathrm{m}$; perithecium 35-37×195-205 $\mu \mathrm{m}$; perithecial outgrowth $35 \mu \mathrm{m}$.

Material examined: ECUADOR, Napo, near Papallacta, Rio Guango, 2714 msl, 00²2'35" S 7804'29" W, 29 July 2009, W. Rossi and J.A. Torres Celi, on styla of Philonthus sp. (Coleoptera, Staphylinidae, Staphilininae, Philonthini) (FI WR3388, holotype).

Notes: Because of the long, straight and nearly erect preapical outgrowth, the new species can be easily distinguished from the 16 others in the same genus, 9 of which bear no outgrowth at all (Fig. 111). To date, the only species of Mimeomyces reported from Ecuador were $M$. latonae (Proaño Castro and Rossi 2008) and M. gregarius (Rossi 2010), both of which lack any preapical outgrowth; the latter is also the only species associated with Philonthus, all the others being parasitic on Staphylininae Quediini, except $M$. latonae occurring on Paederinae (Tavares 1985).

\section{Synandromyces Thaxt.}

Notes: This genus includes ten species characterized mostly by a short appendage bearing a few antheridia forming a compact group and by the arrangement of cells II and III. A single sequence is available for species in this genus (Goldmann and Weir 2018).

Synandromyces makranczyi W. Rossi \& M. Leonardi, sp. nov.

Index Fungorum number: IF557386; Facesoffungi number: FoF 07844; Fig. 112

Etymology: Named after the Hungarian entomologist György Makranczy, who provided the material utilized for the description of the new species.

\section{Holotype: FI WR3154}

Receptacle, appendage and antheridia dark brown; stalk cell of perithecium and basal cells of the same light yellow; perithecium brownish red. Cell I elongate, distinctly tapering 
Fig. 111 Mimeomyces digitatus (FI WR3388, holotype). a, b Thalli from the type slide. Scale bars: $\mathbf{a}, \mathbf{b}=100 \mu \mathrm{m}$

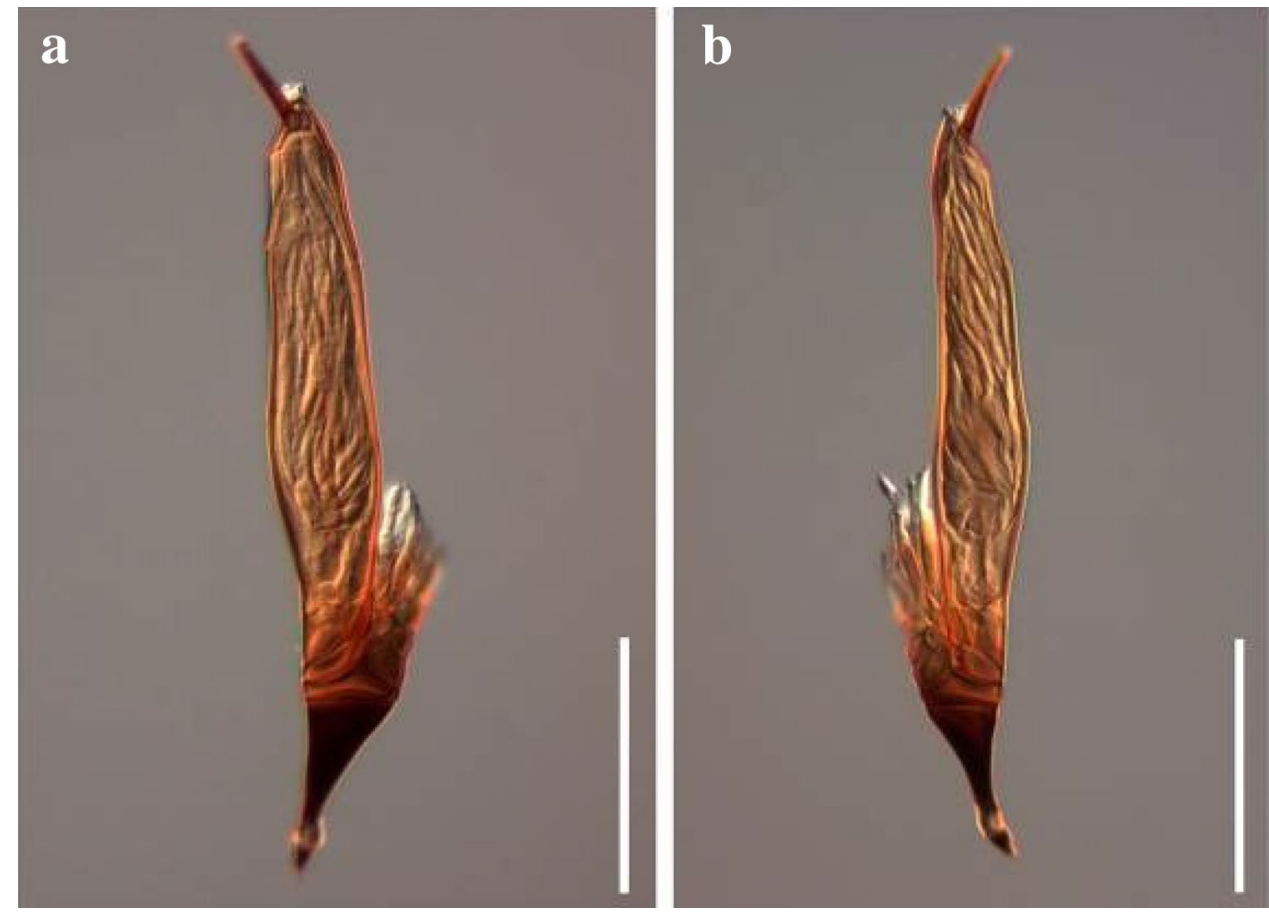

to the foot. The arrangement of cell II and III is hidden by the dark tinge: together with cell I they form a club-shaped structure. Antheridiiferous axis short, consisting of five superposed and gradually smaller cells: the lower two sterile, the others bearing on the inner side single antheridia with falcate and gradually shorter efferent necks, the apexes of which reach the same height. Stalk cell of the perithecium about as long as the free appendage, gradually broader from below upwards. Perithecial basal cells large and unequal: the lower two are longer then broad, distinctly longer than the upper, which bulge externally. Perithecial venter symmetrically inflated, passing without abrupt transition to the tapering tip and rounded apex. Total length from foot to perithecial apex 305-370 $\mu \mathrm{m}$; length from foot to apex of free appendage $120-140 \mu \mathrm{m}$; perithecium 50-55×135-175 $\mu \mathrm{m}$; ascospores about $32 \mu \mathrm{m}$.

Material examined: PERU, Cuzco Dept., Pillahuata, Manu rd. km 128, leaf litter, 22.IX.1982, L.E. Watrous \& G. Mazurek, on the upper side of the abdomen of a paratype of Parosus major Makranczy (Coleoptera, Staphylinidae, Oxytelinae) (FI WR3154, holotype).

Notes: Synandromyces makranczyi is the first species in the genus found occurring on Staphylinidae. It shares with S. platydemae, described from Cameroon on Platydema tomentosum (Tenebrionidae), by the dark tinge of receptacle, appendage and antheridia and the peculiar shape and arrangement of the latter (Fig. 112). However, S. platydemae differs from the new species by a shorter appendage, a much smaller stalk cell of the perithecium, and the very pale colour of the latter, which also bears a much shorter and less tapering tip (Thaxter 1931).

\section{Leotiomycetes O.E. Erikss. \& Winka}

Notes: The class Leotiomycetes was introduced by Eriksson and Winka (1997) to accommodate non-lichenized ascomycetes which initially included only the inoperculate apothecial discomycetes having unitunicate asci that released their ascospores by pore or apical perforation (Eriksson 2005; Ekanayaka et al. 2017, 2019a; Johnston et al. 2019). The taxonomic concept of the class has been revised from the last decade based on the molecular-phylogeny approach, resulting in the class to now be considered more diverse (Johnston et al. 2019). Updated taxonomic classification of Leotiomycetes have been proposed by Ekanayaka et al. (2019a) and Johnston et al. (2019) based on different morpho-molecular criteria. Wijayawardene et al. (2020) followed the Leotiomycetes classification provided by Johnston et al. (2019) and used the Leotiomycetes's classification of Ekanayaka et al. (2019a) as an alternative classification. They accepted 13 orders, with 52 families and 626 genera in Leotiomycetes (Wijayawardene et al. 2020).

\section{Erysiphales Gwynne-Vaughan}

Notes: See an updated account of Erysiphales in Wijayawardene et al. (2020).

\section{Erysiphaceae Tul. \& C. Tul.}

Notes: Erysiphaceae is a family with species that cause powdery mildew diseases on about 10,000 species of angiosperms including many cultivated crops, vegetables, fruits and ornamentals (Takamatsu et al. 2015). This family comprises about 873 species belonging to 17 genera (Braun and 

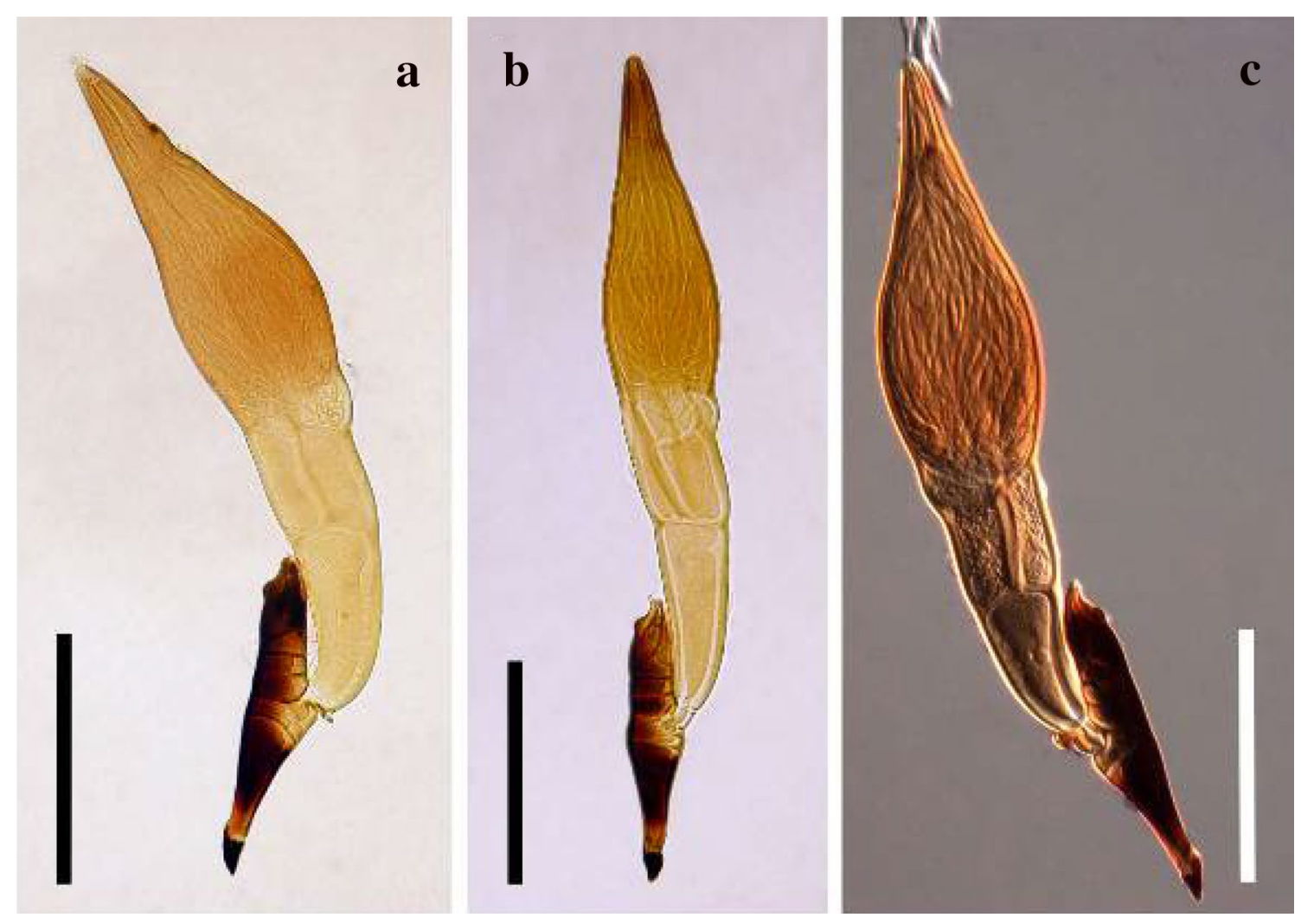

Fig. 112 Synandromyces makranczyi (FI WR3154, holotype). a, b Thalli from the type slide (BF). c Thallus from the type slide (DIC). Scale bars: $\mathbf{a}-\mathbf{c}=100 \mu \mathrm{m}$

Cook 2012). All species are obligate biotrophic parasites of plants (Takamatsu 2013).

\section{Erysiphe R. Hedw. ex DC.}

Notes: Erysiphe is the largest genus in the Erysiphaceae and contains 450 species (Takamatsu et al. 2015). It includes five tribes and two basal genera, which have been described by Mori et al. (2000) and Takamatsu et al. (2015). Some species cause serious diseases on cultivated plants, such as cereals, flowers, ornamentals (Glawe 2008; Braun and Cook 2012; Takamatsu et al. 2015). In this study, a new species of Erysiphe found on Salix gracilistyla var. melanostachys (black pussy willow) in Korea is described and illustrated based on morphological characters and molecular evidence (Figs. 113 and 114).

Erysiphe salicicola Hyang B. Lee, P.M. Kirk \& T.T.T. Nguyen, sp. nov.

Index Fungorum number: IF557324; Facesoffungi number: FoF 09197; Fig. 113

Etymology: Referring to the host plant.

Holotype: CNUFC PWS1

Sexual morph Chasmothecia scattered, 98.5-139.5(-152.5) $\mu \mathrm{m}$ diam., containing 3-13 asci. Each chasmothecium had 26-56 appendages. Appendages hyaline, aseptate, straight to curved, (0.85-)1-1.4 times as long as the cleistothecial diam., $4.5-6.5 \mu \mathrm{m}$ wide at the base, slightly increasing towards the apex, with single septum at the base, hyaline, sometimes slightly brown at the base, 6-7.5 $\mu \mathrm{m}$ wide at the apex, circinate at the near apex. Asci sessile or short-stalked, $48.5-68.5 \times 27.5-43 \mu \mathrm{m}$, obovoid or clavate, with 3-5 ascospores. Ascospores ellipsoidal to obovoid, 15-25(-26.5) × 10.5-15 $\mu \mathrm{m}$. Asexual morph Appressoria developed, nipple-shaped to slightly lobed, solitary or in opposite pairs. Conidiophores arising from the upper part of mother cells, short, cylindrical, 57-95.5×7.5-9.5 $\mu \mathrm{m}$. Foot cells cylindrical, straight to slightly curved near the base, followed by two shorter cells. Conidia oval, cylindrical, 27-35.5(-38.5) × 13.5-18.5(-21) $\mu \mathrm{m}$.

Material examined: REPUBLIC OF KOREA, Jeonnam Province, garden of the Chonnam National University located in Gwangju (35 $\left.10^{\prime} 20.3^{\prime \prime} \mathrm{N} 126^{\circ} 53^{\prime} 56.9^{\prime \prime} \mathrm{E}\right)$, on Salix gracilistyla var. melanostachys, 17 September 2018, collected by H.B. Lee (CNUFC PWS1, holotype).

GenBank numbers: ITS = MT192530, MT192531, $\mathrm{LSU}=\mathrm{MT} 192534, \mathrm{MT} 192535$.

Notes: Erysiphe salicicola belongs to Erysiphe sect. Uncinula and is phylogenetically related to E. adunca. 


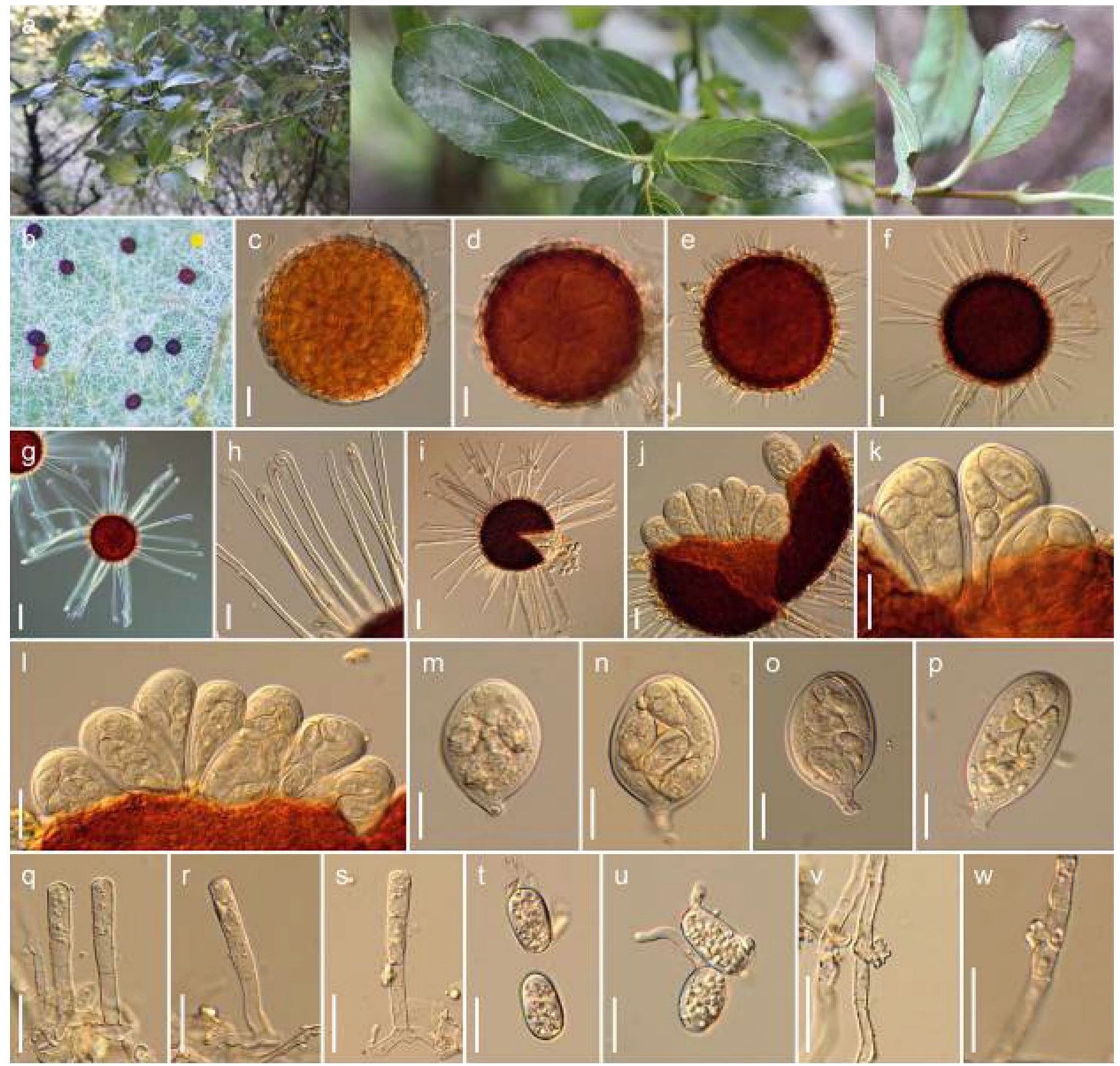

Fig. 113 Erysiphe salicicola (CNUFC PWS1, holotype). a Symptoms of powdery mildew on black pussy willow (Salix gracilistyla var. melanostachys). b Chasmothecia on the leaf-surface. $\mathbf{c}-\mathbf{g}, \mathbf{i}$ Young and mature chasmothecium with appendages. $\mathbf{h}$ Appendages.

However, E. salicicola differs from E. adunca in having smaller conidia and ascospores. The number of ascospores in the asci (chasmothecium), and appendages are less than E. adunca. Furthermore, phylogenetic analyses indicate that $E$. salicicola forms a separate branch distinct from $E$. adunca, confirming it represents a new species. A BLASTn search of the ITS sequences indicated that Erysiphe salicicola was closest to Erysiphe sp. (MH358357) and E. adunca (KY660862) with similarity values of $99.2 \%$ and $93.1 \%$, $\mathbf{j}-\mathbf{p}$ Asci containing ascospores. $\mathbf{q}-\mathbf{s}$ Conidiophores. $\mathbf{t}$, u Conidia and conidial germ tubes. v, w Appressoria (Fig. b: observed under a stereomicroscope; Figs. c-w: light microscope). Scale bars: $\mathbf{c}-\mathbf{f}, \mathbf{h}$, $\mathbf{j}-\mathbf{w}=20 \mu \mathrm{m}, \mathbf{g}, \mathbf{i}=50 \mu \mathrm{m}$

respectively. The species most closely related to E. salicicola, with $97.9 \%$ similarity based on LSU sequences was $E$. adunca (KR048118).

\section{Leotiales, genus incertae sedis}

Notes: Leotiales was introduced by Korf and Lizoň (2001) to accommodate a single family Leotiaceae. Based on multi-gene phylogenetic analyses, Ekanayaka et al. (2019a) included the other two families in this order viz. 

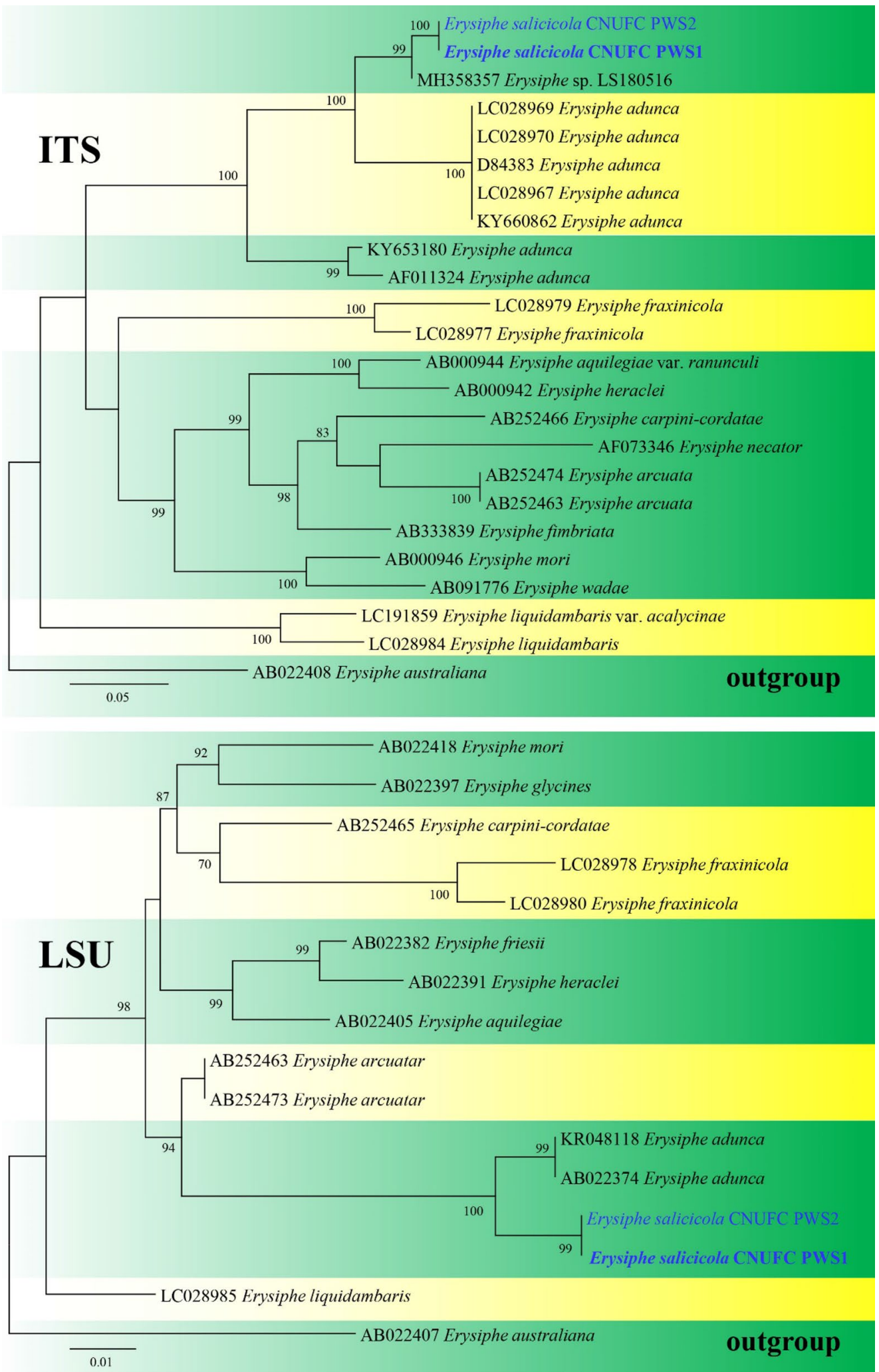

Fig. 114 Phylogram generated from maximum likelihood analysis based on ITS and LSU sequence data for species of Erysiphe. Bootstrap value equal to or greater than $70 \%$ for ML analyses is presented at the nodes. Erysiphe australiana was used as outgroup. Type strains are in bold and newly generated sequences are in blue 
Cochlearomycetaceae and Tympanidaceae as well as including Gelatinomyces in Leotiales genera incertae sedis. Johnston et al. (2019) introduced a new family Mniaeciaceae to accommodate Mniaecia and also treated Alatospora, Aotearoamyces, Claussenomyces, and Flagellospora in Leotiales. Johnston et al. (2019) also presumed that Gorgomyces, Miniancora, Mycosymbioses, and Satchmopsis possibly belong to Leotiales based on phylogenetic analyses of the ITS region. Wijayawardene et al. (2020) listed four families (Cochlearomycetaceae, Leotiaceae, Mniaeciaceae and Tympanidaceae) and three genera incertae sedis (Aotearoamyces, Alatospora and Flagellospora) in Leotiales. In this study, we introduce a monotypic genus Scelecoleotia to accommodate a coelomycetous asexual species, namely Scelecoleotia eriocamporesi sp. nov. in Leotiales based on multi-gene phylogenetic analyses coupled with morphological characteristics. However, the current phylogenetic status of the new genus could not be classified as any of the families in Leotiales. We, hence, tentatively place the genus in Leotiales genera incertae sedis pending further studies.

Scolecoleotia H.B. Jiang, Phookamsak \& K.D. Hyde, gen. nov.

Index Fungorum number: IF558192; Facesofungi number: FoF 09763.

Etymology: The generic epithet "Scolecoleotia" refers to taxa in Leotiales having scolecosporus conidia.

Saprobic on dead aerial fronds of Pteridium aquilinum. Sexual morph Undetermined. Asexual morph Coelomycetous, visible as black, raised, elongate area on the host. Conidiomata dull, black, pycnidial, solitary to gregarious, scattered, sometimes arranged in rows on host substrates, immersed, slightly raised, elongate, hemisphaerical to subconical, with wedge-shaped at the basal angles, uniloculate, glabrous, apapillate, with inconspicuous ostiolate. Pycnidial wall thin-wall of equally thickness, composed of 3-5 layers, of brown to dark brown pseudoparenchymatous cells, arranged in textura angularis to textura globulosa, difficult to distinguish from the hymenium. Hymenium composed of 2-3 strata, of hyaline polygonal cells. Conidiophores reduced to conidiogenous cells. Conidiogenous cells phialidic, determinate, discrete, hyaline, ampulliform to subcylindrical, aseptate, smooth-walled, arising from innermost later of the cavity of conidioma. Conidia acrogenous, scolecosporous, solitary, cylindrical to filiform, curved, hyaline, aseptate, smooth-walled.

Type species: Scolecoleotia eriocamporesi H.B. Jiang, Phookamsak \& K.D. Hyde,

Notes: The monotypic genus Scolecoleotia is introduced herein to accommodate the asexual taxon, Scolecoleotia eriocamporesi which was collected from Pteridium aquilinum (eagle fern) in Italy. The genus can be distinguished from the other genera in Leotiales in having elongate, hemisphaerical to subconical conidiomata, holoblastic, phialidic conidiogenous cells, with hyaline, scolecosporous, cylindrical to filiform aseptate conidia. Most genera in Leotiales have hyphomycetous asexual morphs (Wijayawardene et al. 2017; Ekanayaka et al. 2019a). Scolecoleotia has a pycnidial coelomycetous asexual morph and resembling Collophorina, Epithamnolia, Gelatinosporium, and Satchmopsis (Funk 1979; Suija et al. 2017; Wijayawardene et al. 2017; Li et al. 2020b). Scolecoleotia is most similar to the asexual morph of Gelatinosporium in having phialidic conidiogenous cells, with acrogenous, hyaline, cylindrical to filiform, aseptate conidia (Funk 1979). Species of Gelatinosporium are varied in terms of the shape of the conidiomata such as globose to discoid, elongate subsphaerical, or irregular-shaped which is also typical with Scolecoleotia in having elongate subsphaerical conidiomata (Funk 1979). Gelatinosporium has no molecular data to confirm their phylogenetic placements in Leotiales. Therefore, we introduced the new genus Scolecoleotia as a distinct genus from Gelatinosporium. Phylogenetic analyses of a combined LSU, SSU and ITS sequence dataset showed that Scolecoleotia forms an independent lineage basal to Leotiales and is not related to any families in Leotiales. Therefore, the genus is tentatively placed in Leotiales genus incertae sedis.

Scolecoleotia eriocamporesi H.B. Jiang, Phookamsak \& K.D. Hyde, sp. nov.

Index Fungorum number: IF558193; Facesofungi number: FoF 09764; Fig. 115

Etymology: Named after Erio Camporesi who has contributed many fungal collections from Italy.

Holotype: MFLU 16-2133

Saprobic on dead aerial fronds of Pteridium aquilinum. Sexual morph Undetermined. Asexual morph Coelomycetous, visible as black, raised, elongate area on the host. Conidiomata 75-145 $\mu \mathrm{m}$ high, 600-1100 $\mu \mathrm{m}$ long, black, pycnidial, solitary to gregarious, scattered, sometimes arranged in rows on host substrates, immersed, slightly raised, elongate, hemisphaerical to subconical, with wedge-shaped at the basal angles, uniloculate, glabrous, apapillate, with inconspicuous ostiolate. Pycnidial wall 10-30 $\mu \mathrm{m}$ wide, thin-wall of equally thickness, composed of 3-5 layers, of brown to dark brown pseudoparenchymatous cells, arranged in textura angularis to textura globulosa, difficult to distinguish from the hymenium. Hymenium composed of 2-3-strata, of hyaline polygonal cells, polygonal cells $3-6 \times 3-6 \mu \mathrm{m}(\bar{x}=5 \times 5 \mu \mathrm{m}, \mathrm{n}=30)$. Conidiophores reduced to conidiogenous cells. Conidiogenous cells $(5.5-) 7-10(-12) \times 2-3(-4) \mu \mathrm{m}(\bar{x}=9 \times 3 \mu \mathrm{m}, \mathrm{n}=50)$, holoblastic, phialidic, determinate, discrete, hyaline, ampulliform to subcylindrical, aseptate, smooth-walled, arising 

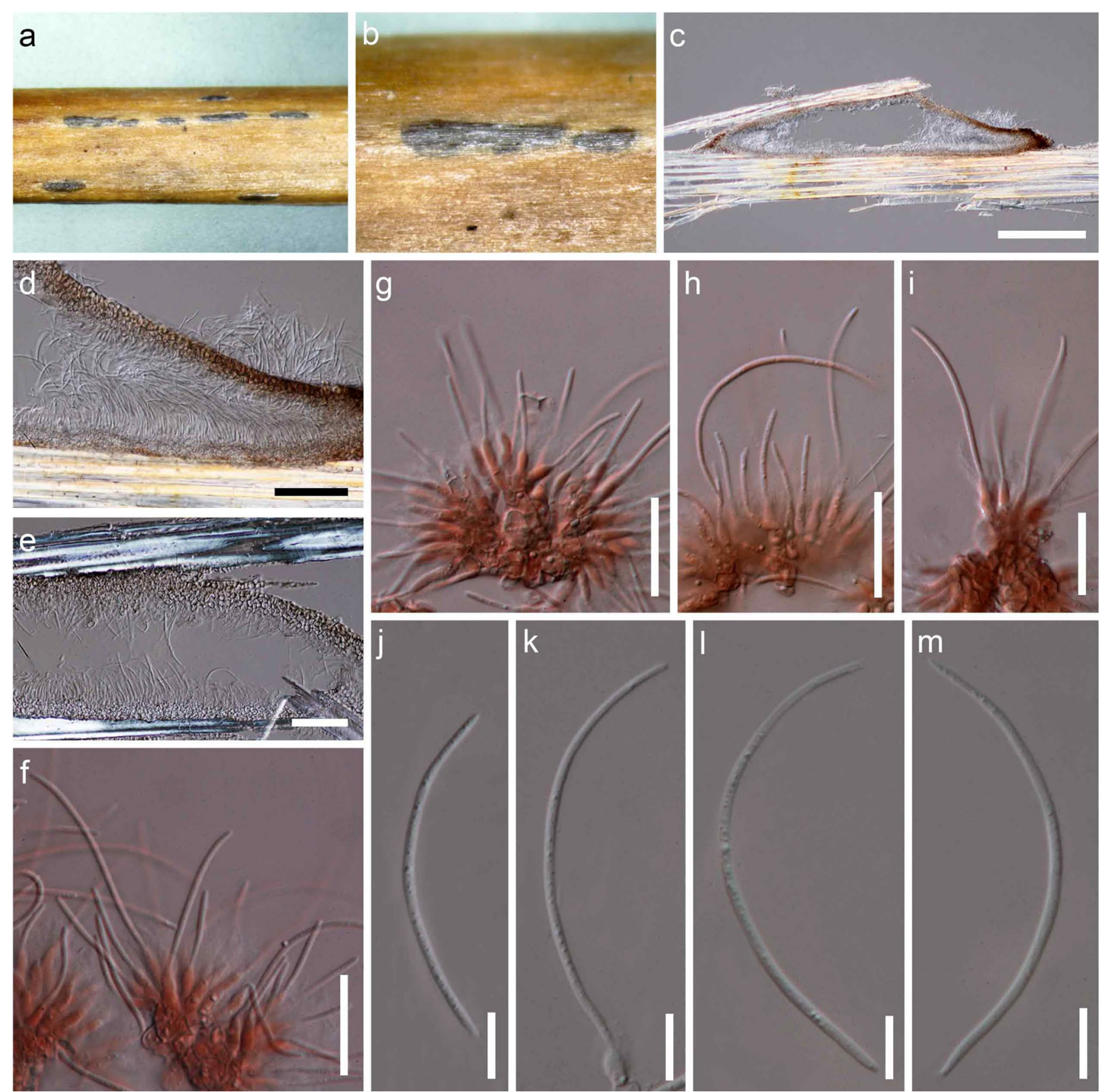

Fig. 115 Scolecoleotia eriocamporesi (MFLU 16-2133, holotype). a, b Appearance of conidiomata on host surface. c Section through conidioma. d, e Section through pycnidial wall. f-i Conidiogenous cells

with attached conidia stained in Congo red. $\mathbf{j}-\mathbf{m}$ Conidia. Scale bars: $\mathbf{c}=500 \mu \mathrm{m}, \mathbf{d}, \mathbf{e}=50 \mu \mathrm{m}, \mathbf{f}-\mathbf{i}=20 \mu \mathrm{m}, \mathbf{j}-\mathbf{m}=10 \mu \mathrm{m}$

from the innermost layer of cavity of conidioma. Conidia $(23-) 40-65(-75) \times 1.5-2.5 \mu \mathrm{m}(\bar{x}=57 \times 2 \mu \mathrm{m}, \mathrm{n}=50)$, acrogenous, scolecosporous, solitary, cylindrical to filiform, curved, hyaline, aseptate, smooth-walled.

Material examined: ITALY, Province of Arezzo, Montemezzano-Stia, on dead aerial fronds of Pteridium aquilinum, 7 July 2016, E. Camporesi, IT3027A (MFLU 16-2133, holotype); ibid., MFLU 16-2133 (isotype).

GenBank numbers: ITS = MW981448, MW981449, LSU $=$ MW981450, MW981451, SSU $=$ MW981452, MW981453.

Notes: Based on the NCBI BLASTn search results of the ITS, LSU and SSU sequences, Scolecoleotia eriocamporesi (strains IT3027A and IT3027B) showed 92\% similarity with Urceolella carestiana (CBS 319.71), 96.26\% similarity with Leotiomycetes sp. (BY-2018b) and $99.36 \%$ similarity with Dicephalospora rufocornea (MFLU 18-1827), respectively. 
Vu et al. (2019) obtained the sequence data of Unguicularia carestiana (=Urceolella carestiana strain CBS 319.71) from Müller's collection (ETH 755, Switzerland). However, this specimen is not the type specimen of Unguicularia carestiana and Vu et al. (2019) did not provide a description and illustration of $U$. carestiana in their study. Therefore, we could not compare the morphological characteristics of $S$. eriocamporesi (Fig. 115) with U. carestiana. Dicephalospora rufocornea (MFLU 18-1827) was obtained by Ekanayaka et al. (2019a). The asexual morph of D. rufocornea has not yet been determined; thus, the morphological characteristics of $S$. eriocamporesi could not be compared with D. rufocornea. Whereas Leotiomycetes sp. (BY-2018b) is unpublished. DNA sequences of two strains of S. eriocamporesi (strains IT3027A and IT3027B) were directly obtained from the fruiting bodies and the two strains share the same branch length on the phylogenetic tree with $100 \%$ MLBS, 1.00 PP support (Fig. 116).

\section{Sordariomycetes O.E. Erikss. \& Winka}

Notes: We follow the latest treatment and updated accounts of Sordariomycetes in Yuan et al. (2020).

\section{Diaporthales Nannf.}

Notes: Diaporthales, introduced by Nannfeldt (1932), forms a well-supported, monophyletic clade within Diaporthomycetidae (Senanayake et al. 2018). Based on molecular data, Senanayake et al. (2017a) accepted 21 families in the order, while Yang et al. (2017a), Braun et al. (2018) and Fan et al. (2018) introduced five new families. Senanayake et al. (2018) re-introduced Tirisporellaceae in Diaporthales after its exclusion by Jones et al. (2015). Thus, currently, Diaporthales comprises 27 families, namely Apiosporopsidaceae, Apoharknessiaceae, Asterosporiaceae, Auratiopycnidiellaceae, Coryneaceae, Cryphonectriaceae, Cytosporaceae, Diaporthaceae, Diaporthosporellaceae, Diaporthostomataceae, Erythrogloeaceae, Gnomoniaceae, Harknessiaceae, Juglanconidaceae, Lamproconiaceae, Macrohilaceae, Melanconidaceae, Melanconiellaceae, Prosopidicolaceae, Pseudomelanconidaceae, Pseudoplagiostomaceae, Schizoparmaceae, Stilbosporaceae, Sydowiellaceae, Synnemasporellaceae, Tirisporellaceae and Tubakiaceae.

\section{Coryneaceae Corda (=Pseudovalsaceae M.E. Barr)}

Notes: Coryneaceae includes two genera i.e., Coryneum and Hyaloterminalis, the most recent treatments for the family is Rathnayaka et al. (2020).

\section{Coryneum Nees}

Notes: Coryneum was the genus of Coryneaceae and formed a distinct phylogenetic lineage in Diaporthales (Senanayake et al. 2017a, 2018; Fan et al. 2018; Jiang et al.
2020). Members of this genus were easily distinguished from the other diaporthalean species in having black perithecia, often immersed in wood, asci that deliquesce at maturity, and an asexual morph with transversely distoseptate brown conidia (Senanayake et al. 2017a, 2018). Coryneum was recommended to be adopted as the generic name due to priority rather than Pseudovalsa (Rossman et al. 2015). Most Coryneum species were considered strongly host-specific and associated with tree branch cankers (Jiang et al. 2018, 2019; Senwanna et al. 2018).

Coryneum fagi C.M. Tian \& N. Jiang, sp. nov.

Index Fungorum number: IF558647; Facesoffungi number: FoF 09957; Fig. 117

Etymology: Named after the host genus, Fagus.

Holotype: BJFC-S1782

Saprobic or weak pathogenic branches of Fagus sp. Sexual morph Undetermined. Asexual morph Conidiomata $0.2-0.6 \mathrm{~mm}$ wide, $0.2-0.8 \mathrm{~mm}$ high, acervular, solitary, erumpent through the outer periderm layers of the host, scattered, surface tissues above slightly domed, formed of brown cell, thick-walled textura angularis. Conidiophores 60-125 $\mu \mathrm{m}$ long, 4-7 $\mu \mathrm{m}$ wide, cylindrical, apically pale brown, paler at the base, smooth, septate, unbranched, arising from basal stroma. Conidiogenous cells annellidic, integrated, terminal, cylindrical, expanding towards the apices, pale brown, smooth, with 1-3 slightly percurrent proliferations. Conidia $45-75 \times 10-15.5 \mu \mathrm{m}(\bar{x}=62.5 \times 12 \mu \mathrm{m}$, $\mathrm{n}=20$ ), variable in shape, curved, broadly fusiform to fusiform, cylindrical or clavate, dark brown, smooth-walled, 6-12-distoseptate, apical cell with a hyaline tip, truncate and black at the base.

Material examined: CHINA, Sichuan Province, Yaan City, on dead branches of Fagus sp., 10 September 2020, N. Jiang (BJFC-S1782, holotype), ibid. BJFC-S1783 (isotype).

GenBank numbers: BJFC-S1782: ITS = MW144761, LSU = MW 144953; BJFC-S1783: ITS = MW144762, $\mathrm{LSU}=\mathrm{MW} 144954$.

Notes: Coryneum species were mainly named based on host associations and morphology, especially conidial characters, due to the absence of molecular data for most species (Jiang et al. 2018). In the present study, we discovered Coryneum species from a new host genus, Fagus (Fig. 117). The DNA was extracted directly from the conidiomata on branches and ITS and LSU sequences were amplified, sequenced and compared with known species (Fig. 118). Coryneum fagi was phylogenetically close to $C$. ilicis, but they can be distinguished by the wider conidia of $C$. fagi $(C$. fagi $=45-75 \times 10-15.5 \mu \mathrm{m}$ vs. $82-105 \times 9.5-12.5 \mu \mathrm{m}$ in $C$. ilicis) (Jiang et al. 2019).

Diaporthaceae Höhn. ex Wehm. 


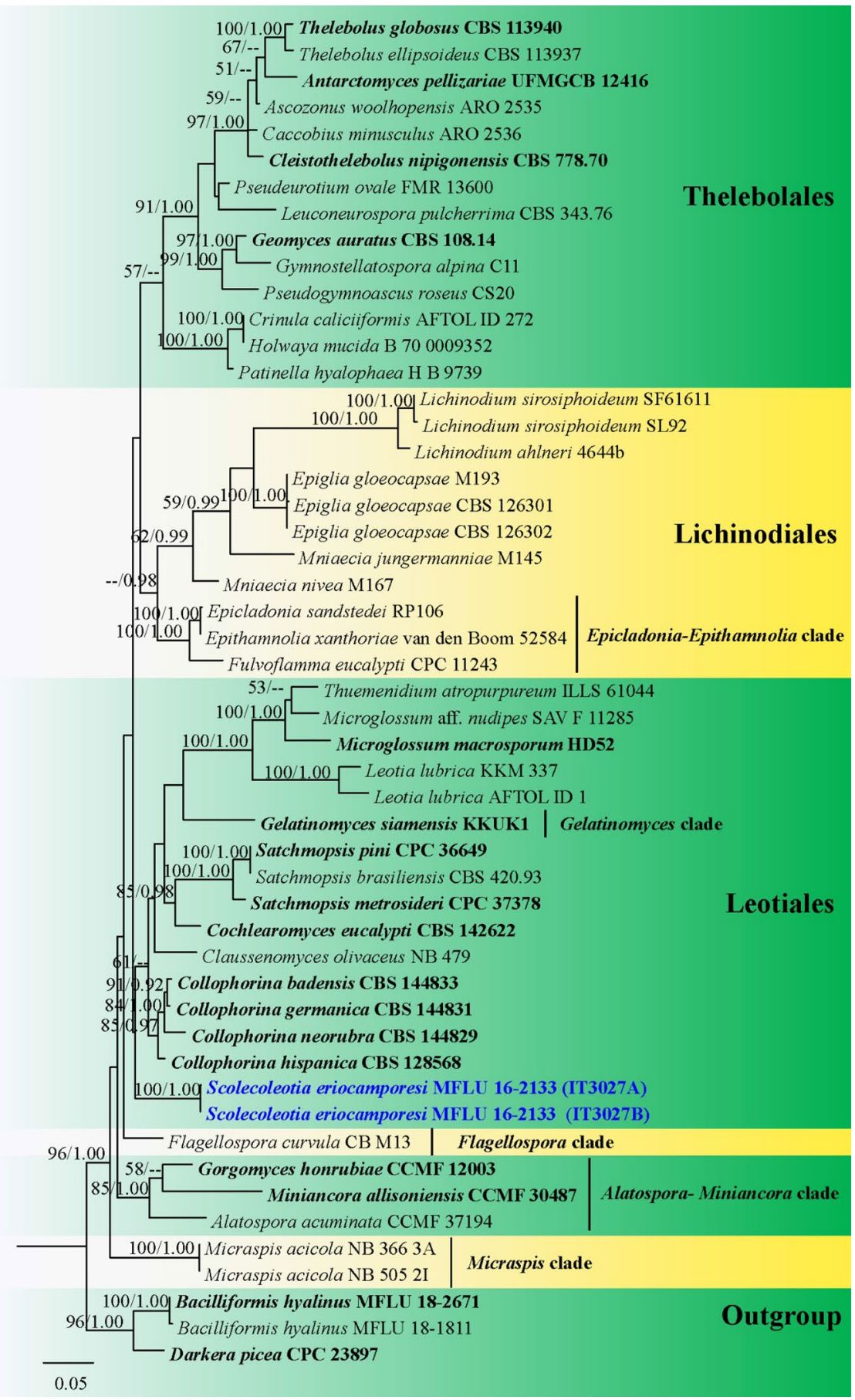

Fig. 116 Phylogram generated from maximum likelihood analysis based on combined ITS, LSU, SSU and RPB2 sequence data. Fifty-one strains are included in the combined gene analyses comprising 3907 characters after alignment (632 characters for ITS, 1019 characters for LSU, 1283 characters for SSU, 973 characters for RPB2). Bacilliformis hyalinus (MFLU 18-2671 and MFLU 18-1811) and Darkera picea (CPC 23897) are used as outgroup taxa. The tree topology of the Bayesian analysis was similar to the maximum likelihood analysis. The best RAxML tree with a final likelihood value of -19994.043154 is presented. The matrix had 1424 distinct alignment patterns. Estimated base frequencies were as follows: $\mathrm{A}=0.256835, \mathrm{C}=0.219258, \mathrm{G}=0.273899, \mathrm{~T}=0.250008$; substitution rates $\mathrm{AC}=2.087920, \mathrm{AG}=4.320672, \mathrm{AT}=2.285635$, $\mathrm{CG}=1.273283, \mathrm{CT}=9.026514, \mathrm{GT}=1.000000$; gamma distribution shape parameter $\alpha=0.216097$. The final average standard deviation of split frequencies at the end of total MCMC generations calculated as 0.006795 in BI analysis. Bootstrap values for maximum likelihood equal to or greater than $50 \%$ and Bayesian posterior probabilities equal or greater than $0.90 \mathrm{BYPP}$ are placed above or below the branches. Ex-type strains are in bold and newly generated sequences are in blue 

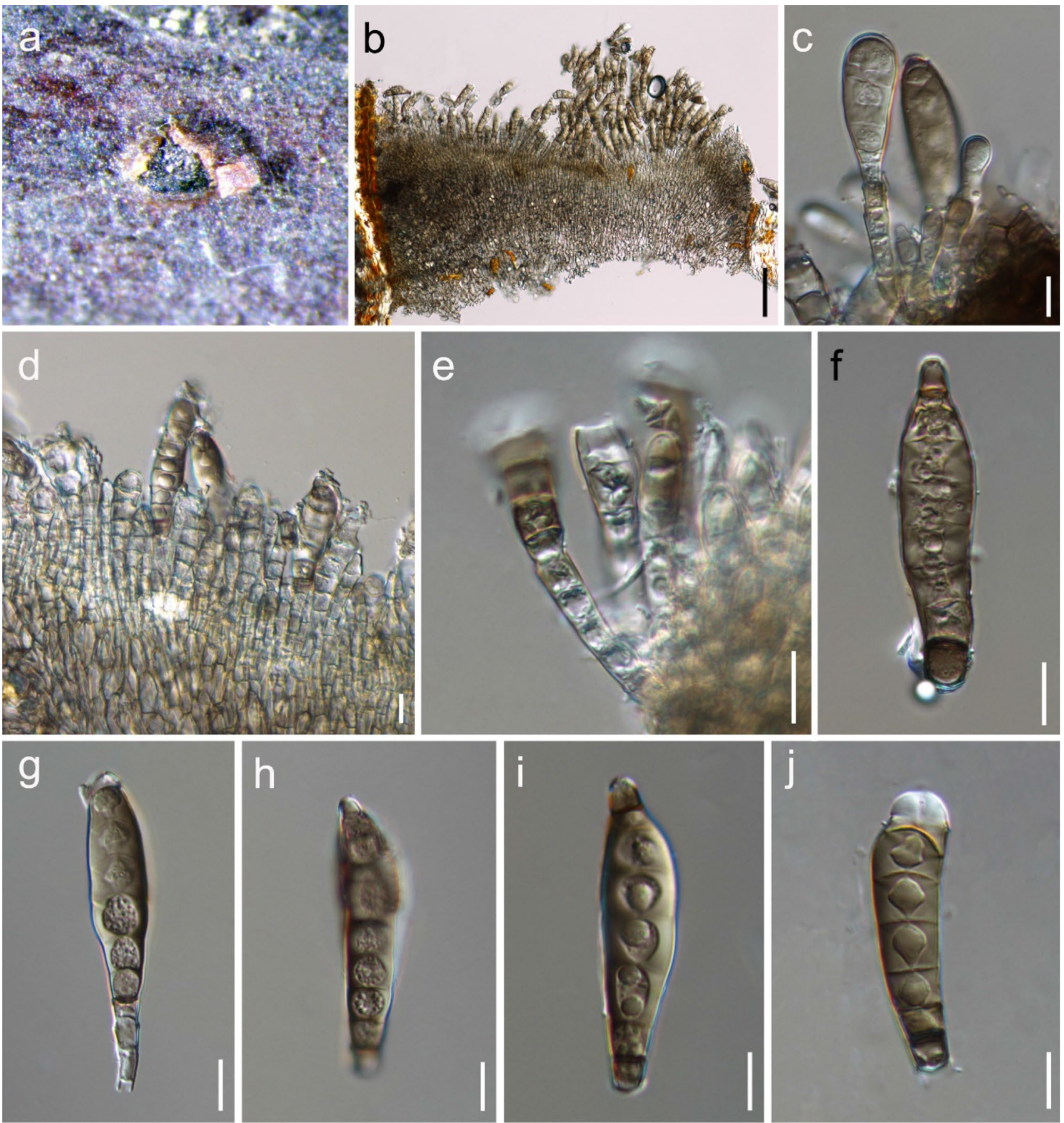

Fig. 117 Coryneum fagi (BJFC-S1782, holotype). a Conidiomata on the dead branch of Fagus sp. b Cross section of conidioma. c-e Developing conidia from conidiogenous cells. $\mathbf{f}-\mathbf{j}$ Conidia. Scale bars: $\mathbf{b}=100 \mu \mathrm{m}, \mathbf{c}-\mathbf{j}=10 \mu \mathrm{m}$

Notes: Diaporthaceae, introduced by von Höhnel (1917), is represented by species of saprobes, endophytes and phytopathogens (Dai et al. 2014; Udayanga et al. 2014a, b), including Diaporthe, which is the most species-rich genus (Liu et al. 2015a). The taxonomy of this family has been revised several times over the last decade based on both morphology and phylogeny (e.g., Maharachchikumbura et al. 2015, 2016; Senanayake et al. 2017b; Wanasinghe et al. 2018). Currently, Diaporthaceae comprises 14 genera. These include 10 genera accepted by Maharachchikumbura et al. (2016), namely Allantoporthe, Apioporthella, Clypeoporthella, Diaporthe, Diaporthella, Leucodiaporthe, Mazzantia, Mazzantiella, Ophiodiaporthe and Pustulomyces, three genera accepted by Senanayake et al. (2017a), namely 


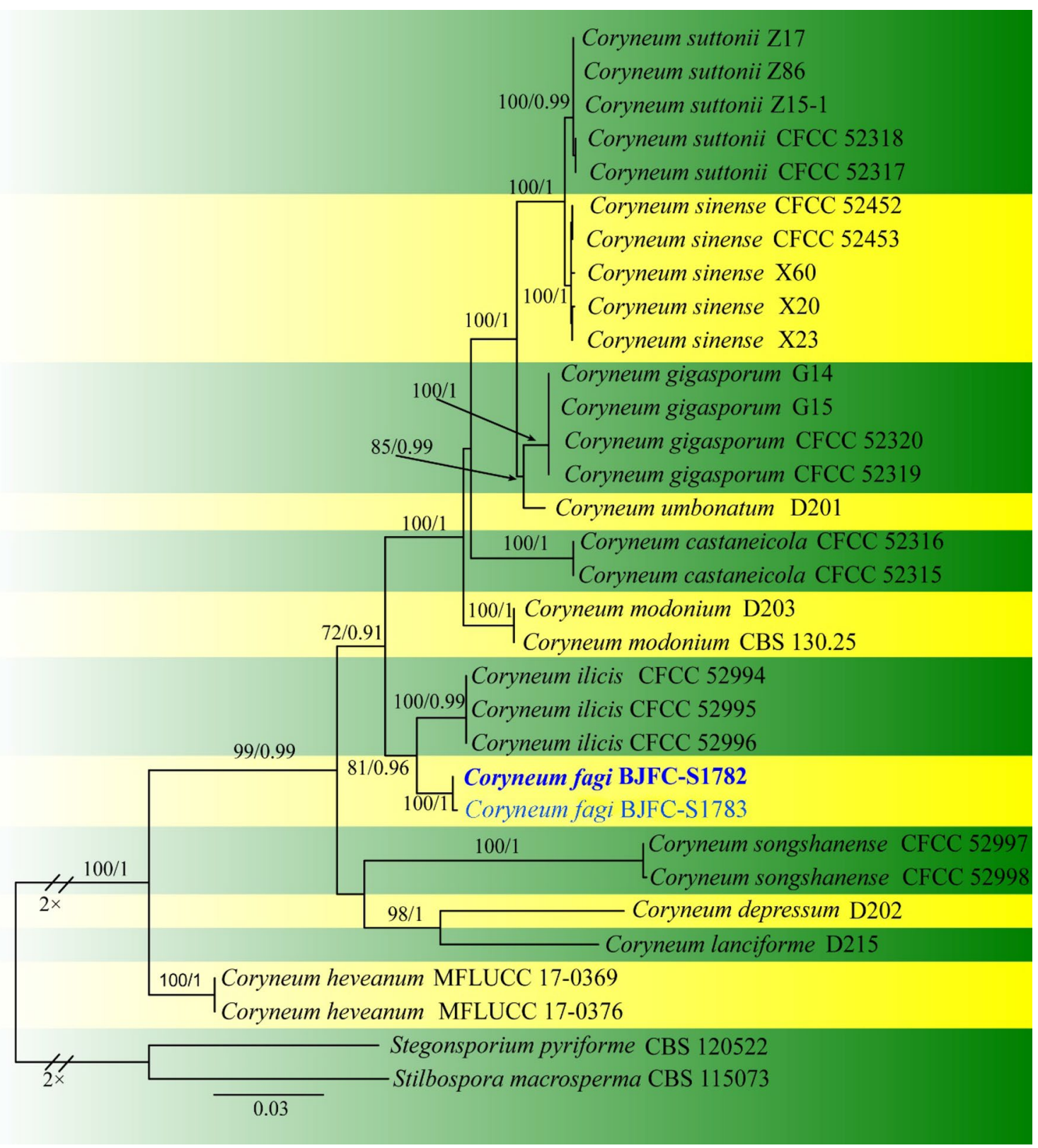

Fig. 118 Phylogram generated from RAxML analysis based on combined LSU, ITS, TEF1- $\alpha$ and RPB2 sequence data of Coryneum. Related sequences were obtained from GenBank. Thirty-two strains are included in the combined analyses, which comprise 4690 characters (1500 characters for ITS-LSU, 232 characters for TEF1- $\alpha, 1079$ characters for RPB2) after alignment. Stilbospora macrosperma (CBS 115073) and Stegonsporium pyriforme (CBS 120522) are used as the outgroup taxa. The tree topology of the ML analysis was simi-

Chiangraiomyces, Hyaliappendispora and Paradiaporthe, and Chaetoconis accepted by Senanayake et al. (2017b).

\section{Diaporthe Nitschke}

Notes: Diaporthe was introduced by Nitschke (1870) based on Diaporthe eres collected in Germany from Ulmus sp. This genus is represented by endophytes, saprobes and lar to the BI. The best scoring RAxML tree with a final likelihood value of -12745.413163 is presented. The matrix had 817 distinct alignment patterns, with $18.15 \%$ of undetermined characters or gaps. Maximum likelihood bootstrap support values equal to or greater than 50\% (MLBS, left), and Bayesian posterior probabilities equal to or greater than 0.90 (BYPP, right) are given at the nodes. The newly generated sequences are in blue bold

plant pathogens on a variety of economically important crops, ornamentals and trees (Santos et al. 2017; Dissanayake et al. 2015, 2017; Guarnaccia et al. 2018). Although revised several times (e.g., Gomes et al. 2013; Gao et al. 2017; Marín-Félix et al. 2019b; Hyde et al. 2019), the taxonomy of Diaporthe continues to be confused and the genus urgently needs to be reassessed. Circumscription of the Diaporthe species relies on 
molecular phylogenies based on ITS and partial sequences of TEF1, TUB2, CAL and HIS3 genes (Yang et al. 2018b; Hyde et al. 2019). Marín-Félix et al. (2019b) accepted 213 species in Diaporthe supported by ex-type cultures and molecular data. Hyde et al. (2019) introduced two new species, Diaporthe italiana and Diaporthe rumicicola. Thus, currently Diaporthe comprises 215 species. We introduce a new species, Diaporthe chamaeropicola, from palms (Arecaceae) in Lisbon, Portugal, based on morphological characters and phylogenetic analyses of ITS, TEF1, TUB2 and CAL sequence data. The phylogenetic tree is presented in Fig. 124. Four new records of Diaporthe species from palms are presented. These include the isolates of $D$. foeniculina and D. pyracanthae from Chamaerops humilis and $D$. foeniculina from Trachycarpus fortunei, along with the isolate of $D$. pseudophoenicicola from $C$. humilis.

A survey of the literature reveals that no intensive study supported by molecular data has been carried out to resolve the complex nature of Diaporthe species occurring on palms. Although several Diaporthe species have been recorded on palms, most of them were based mainly on their unique palm hosts but without molecular data to confirm their phylogenetic position. As a result, most of these species have not been transferred to Diaporthe and remain in Phomopsis. Fröhlich et al. (1997) provided a synopsis of Diaporthe (as Phomopsis) species known from palms and several other species that have been reported by Taylor and Hyde (2003). Herein, a new synopsis of Diaporthe species from palms is presented considering the currently accepted and phylogenetically validated Diaporthe names (Table 4). A search of the US National Fungus Collections Fungus-Host Database (Farr and Rossman 2019) revealed 31 species of Diaporthe/Phomopsis associated with hosts in the Arecaceae. These names were verified against MycoBank and Index Fungorum as well as the literature, which reduced the number to five Diaporthe species. Table 4 lists all currently accepted names of Diaporthe species associated with Arecaceae, their respective hosts and the countries from which they were recorded. Diaporthe arctii was described from Trachycarpus fortunei by Taylor and Hyde (2003) in Switzerland and the United Kingdom based solely on morphology. However, since morphology is of limited value in defining species in Diaporthe, the validity of this name for these isolates cannot be confirmed and therefore, the report was excluded from the list. Diaporthe eres was recorded from diseased leaves of Rhapis subtilis by Gao et al. (2016). Nevertheless, Gao et al. (2016) regarded its phylogenetic position as a species complex since many isolates evaluated revealed ambiguous clades with short branches and moderate bootstrap support. Thus, the correct name for the species recorded on $R$. subtilis needs to be clarified and this record was excluded. Udayanga et al. (2012a, b) recorded another Diaporthe species from Rhapis sp. in Thailand. Although molecular data support this species, no morphological information was included, the species remained unnamed and was simply regarded as Diaporthe sp. Consequently, this species was also disregarded from the present listing of Diaporthe names reported from Arecaceae.

Diaporthe chamaeropicola D.R.S. Pereira \& A.J.L. Phillips, sp. nov.

Index Fungorum number: IF557847; Facesoffungi number: FoF 09160; Fig. 119

Etymology: Named after the host genus from which it was collected, Chamaerops humilis.

Holotype: AVE-F-8

Associated with foliar lesions. Sexual morph Undetermined. Asexual morph Conidiomata pycnidial, solitary, occasionally aggregated, subglobose, dark-brown to black, thick-walled, up to $4 \mathrm{~mm}$ diam., covered with hyphal outgrows, superficial, lacking an ostiole, dehiscent by irregular fissures on pycnidial wall, exuding a creamy mucoid mass
Table 4 Accepted Diaporthe species associated with Arecaceae

\begin{tabular}{llll}
\hline Species & Host & Country & References \\
\hline Diaporthe arecae & Areca catechu & India & Gomes et al. (2013) \\
Diaporthe arengae & Arenga engleri & China & Gomes et al. (2013) \\
Diaporthe chamaeropis & Chamaerops humilis & Greece & Gomes et al. (2013) \\
Diaporthe chamaeropicola sp. nov & Chamaerops humilis & Portugal & Present study \\
Diaporthe eres & Rhapis subtilis & China & Gao et al. (2016) \\
Diaporthe foeniculina & Chamaerops humilis & Portugal & Present study \\
& Trachycarpus fortunei & Portugal & Present study \\
Diaporthe pseudophoenicicola & Chamaerops humilis & Portugal & Present study \\
& Phoenix canariensis & China & Gao et al. (2017) \\
& Phoenix dactylifera & Spain & Gomes et al. (2013) \\
& Phoenix dactylifera & Portugal & Present study \\
Diaporthe pyracanthae & Chamaerops humilis & Portugal & Present study \\
\hline
\end{tabular}

The Diaporthe species considered were only those described with both morphological and molecular data. Currently valid Diaporthe names that were described on palms based solely on morphological data were disregarded 

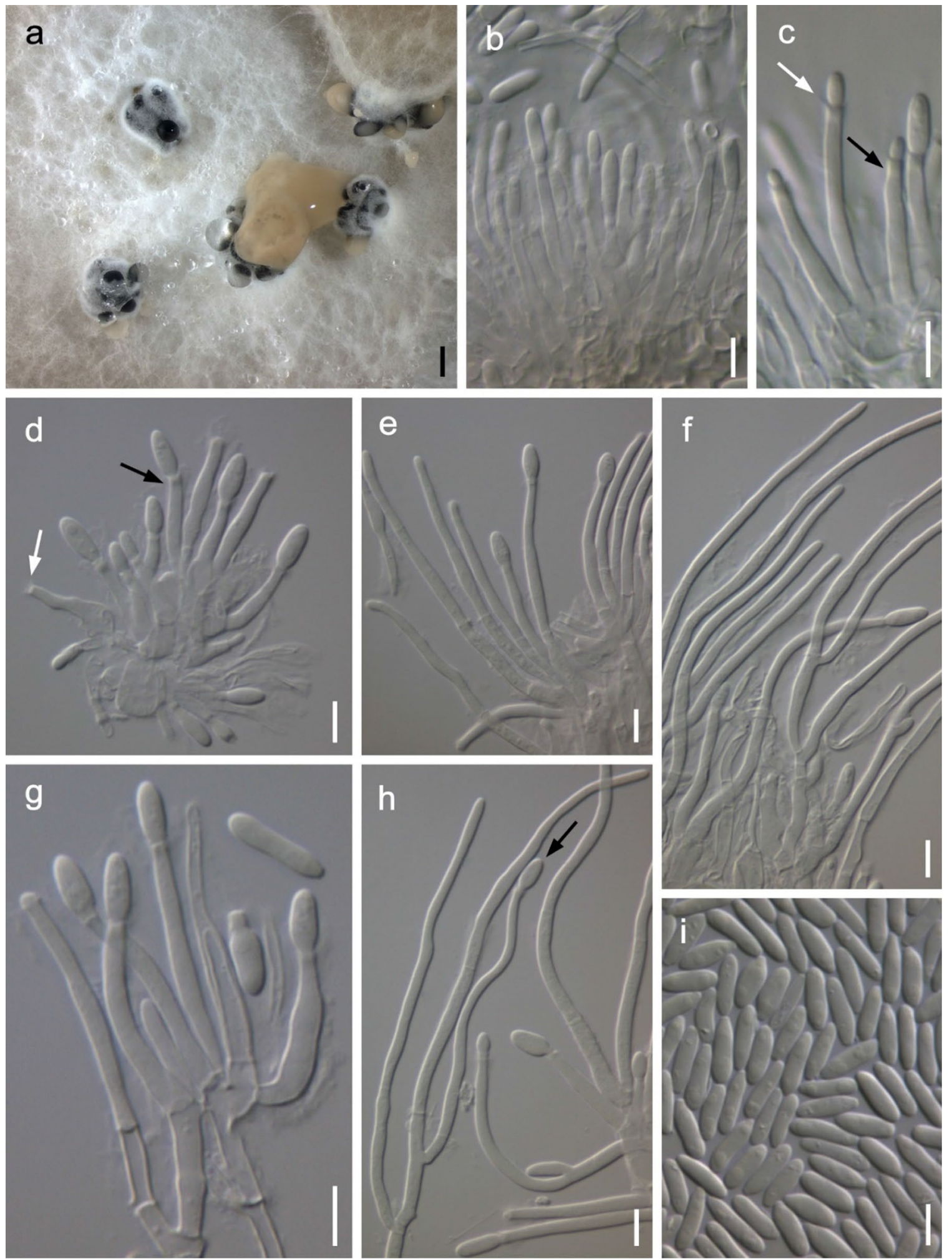

Fig. 119 Diaporthe chamaeropicola (AVE-F-8, holotype), a Conidiomata formed on 1/2 PDA with conidia oozing in creamy mucoid masses. b-d Short conidiogenous cells (white arrows point to collarettes, black arrows point at periclinal thickenings). e, $\mathbf{g}$ Long conid- iogenous cells. $\mathbf{f}, \mathbf{h}$ Paraphyses (black arrow indicates a branch that is functioning as a conidiogenous cell). i Conidia. Scale bars: $\mathbf{a}=1 \mathrm{~mm}$, $\mathbf{b}-\mathbf{i}=5 \mu \mathrm{m}$ 
of conidia. Pycnidial wall pseudoparenchymatous of darkbrown textura angularis, cells thick-walled in outer layers, becoming thin-walled and hyaline towards the inner layers. Conidiophores absent. Conidiogenous cells lining the entire cavity, straight, hyaline, smooth-walled, thin-walled, cylindrical, occasionally ampulliform, tapering towards the apex, aseptate or 1-3-septate, unbranched or branched, collarette up to $1 \mu \mathrm{m}$ long, variable in length, dimorphic, short conidiogenous cells, 4.9-19.4 $\times 0.9-2.6 \mu \mathrm{m}$ (mea $\mathrm{n} \pm \mathrm{SD}=13.66 \pm 3.68 \times 1.75 \pm 0.39 \mu \mathrm{m})$, long conidiogenous cells, $15.2-49.2 \times 1.1-2.7 \mu \mathrm{m}($ mean $\pm \mathrm{SD}=29.5$ $4 \pm 7.28 \times 1.75 \pm 0.36 \mu \mathrm{m}$ ), mostly phialidic, proliferating at the same level giving rise to periclinal thickenings, enteroblastic, occasionally proliferating percurrently giving rise to 1-2 annellations, often intermingled with paraphyses. Paraphyses straight, flexuous, hyaline, smooth- and thin-walled, cylindrical, tapering towards the apex, with 1-2-(3) basal septa, unbranched or branched below, often one of the branches later functioning as a conidiogenous cell, extending above conidiogenous cells, 26.6-78.8 $\mu \mathrm{m}$ (mean $\pm \mathrm{SD}=53.57 \pm 12.72 \mu \mathrm{m}$ ) long. Alpha conidia cylindrical to ellipsoidal, mostly with rounded apex and obtuse to truncate base, hyaline, smooth- and thin-walled, aseptate, bi-guttulate, with a conspicuous guttule at each end, occasionally with several minute scattered guttules, straight to slightly curved, 5.6-9.4 $\times 1.7-3 \mu \mathrm{m}$ (mean $\pm \mathrm{SD}=7.53 \pm$ $0.89 \times 2.31 \pm 0.30 \mu \mathrm{m}) ;$ mean $\pm \mathrm{SD}$ conidium length/width ratio $=3.33 \pm 0.73$. Beta and gamma conidia not seen.

Culture characteristics: Colonies on 1/2 PDA, reaching $60 \mathrm{~mm}$ diam. after $7 \mathrm{~d}$ at $20{ }^{\circ} \mathrm{C}$. Surface flat, sparse aerial mycelium, often growing with concentric zones, with filiform margin, circular, pearl white to dirty white, opaque to slightly translucent. Reverse luteous, pale brown towards the centre. No diffusible pigment. Conidiomata black, scattered over the surface of the colony.

Material examined: PORTUGAL, Lisbon, Parque das Nações, Jardins da Água, near Oceanário de Lisboa, on foliar lesions of segments of Chamaerops humilis (Arecaceae), 16 October 2018, D.R.S. Pereira, HDP 034 (AVE-F-8 a dried culture of CDP 460 , holotype), ex-type living culture, CBS $\mathrm{XXXX}=\mathrm{CDP} 460 / 01$.

GenBank Numbers: $\mathrm{CAL}=\mathrm{MT011068}$, ITS $=$ MT022111, TEF1- $\alpha=$ MT011074, TUB2 $=$ MT011080.

Distribution: Lisbon, Portugal.

Notes: Diaporthe chamaeropicola was found associated with foliar lesions of Chamaerops humilis (Fig. 123), but pathogenicity has not been tested. The phylogenetic position of D. chamaeropicola among accepted Diaporthe species has not clearly been resolved. Nevertheless, this species is phylogenetically related to but distinct from $D$. ceratozamiae, D. phyllanthicola and D. loropetali (Fig. 123). Diaporthe chamaeropicola is similar to D. ceratozamiae (Fig. 119), producing globose pycnidia, whose internal cavity is lined with cylindrical conidiogenous cells intermingled with long cylindrical, septate and branched paraphyses (Crous et al. 2011). However, D. chamaeropicola has larger conidiomata than D. ceratozamiae (up to $4 \mathrm{~mm}$ diam. vs. $300 \mu \mathrm{m}$ diam.), lacks conidiophores, and alpha conidia have a different shape (cylindrical/ellipsoidal vs. fusiform). In addition, the two species differ in 13 nucleotide positions in ITS. Diaporthe chamaeropicola differs from D. phyllanthicola and D. loropetali in 9 and 13 nucleotide positions in ITS, respectively. No TUB2, TEF1 and CAL sequences are available for $D$. ceratozamiae, D. phyllanthicola or D. loropetali.

Diaporthe foeniculina (Sacc.) Udayanga \& Castl., Persoonia 32: 95 (2014)

Index Fungorum number: IF803929; Facesoffungi number: FoF 02183; Fig. 120

三 Phoma foeniculina Sacc., Michelia 2: 95 (1880)

= Diaporthe foeniculacea Niessl, Instituto de Coimbra 27: 168 (1879)

= Phomopsis californica HS Fawc., Phytopathology 12: 419 (1922)

$=$ Diaporthe theicola Curzi, Atti dell'Istituto Botanico della Università e Laboratorio Crittogamico di Pavia 3: 60 (1927)

$=$ Phomopsis theicola Curzi, Atti dell'Istituto Botanico della Università e Laboratorio Crittogamico di Pavia 3: 64 (1927)

三 Phomopsis foeniculina (Sacc.) Sousa da Câmara, Agronomia Lusitanica 9: 104 (1947)

$=$ Diaporthe neotheicola AJL Phillips \& JM Santos, Fungal Diversity 34: 120 (2009)

= Diaporthe rhusicola Crous, Persoonia 26: 135 (2011)

Associated with foliar lesions. Sexual morph Undetermined. Asexual morph Conidiomata pycnidial, stromatic, unito multi-locular, solitary or aggregated, black, thick-walled, of variable morphology and dimensions, up to $1.3 \mathrm{~mm}$ diam., subglobose to pulvinate, glabrous or covered with hyphal outgrows, superficial, 1-3-ostiolate, mostly 3-ostiolate, exuding a creamy, yellowish mass or cirri of conidia. Pycnidial locules uni-chambered, often with a globose base and a long neck. Pycnidial wall pseudoparenchymatous, composed of oblong to isodiametric thin-walled cells, outer cell layers of textura epidermoidea, pigmented, dark-brown, inner cell layers of textura angularis, hyaline. Conidiophores arising all around the cavity of conidioma from the innermost wall layer, rarely absent, straight, sometimes curved, hyaline, smooth- and thinwalled, cylindrical, subcylindrical or doliiform, rarely with a globose base, aseptate, unbranched, rarely with a small branch, 3.7-13.2 $\times 1.3-4.12 \mu \mathrm{m}($ mean $\pm \mathrm{SD}=7.77 \pm 1.90 \times 2.17 \pm 0.5$ $2 \mu \mathrm{m})$. Conidiogenous cells lining the entire cavity, straight, hyaline, smooth- and thin-walled, dimorphic, short conidiogenous cells, on Populus sp. twigs 9.2-19.6×1.4-2.5 $\mu \mathrm{m}$ (me an $\pm \mathrm{SD}=12.91 \pm 1.88 \times 1.84 \pm 0.26 \mu \mathrm{m})$, producing mostly 

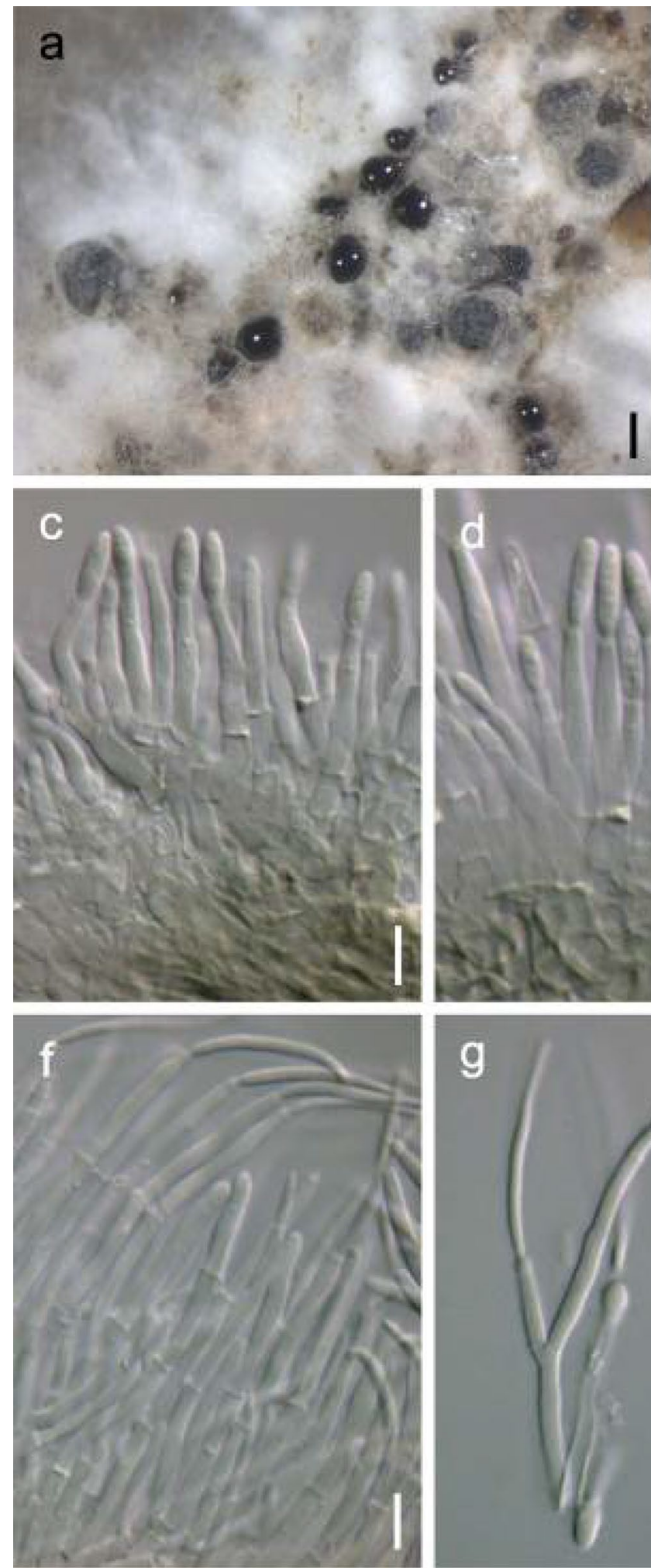
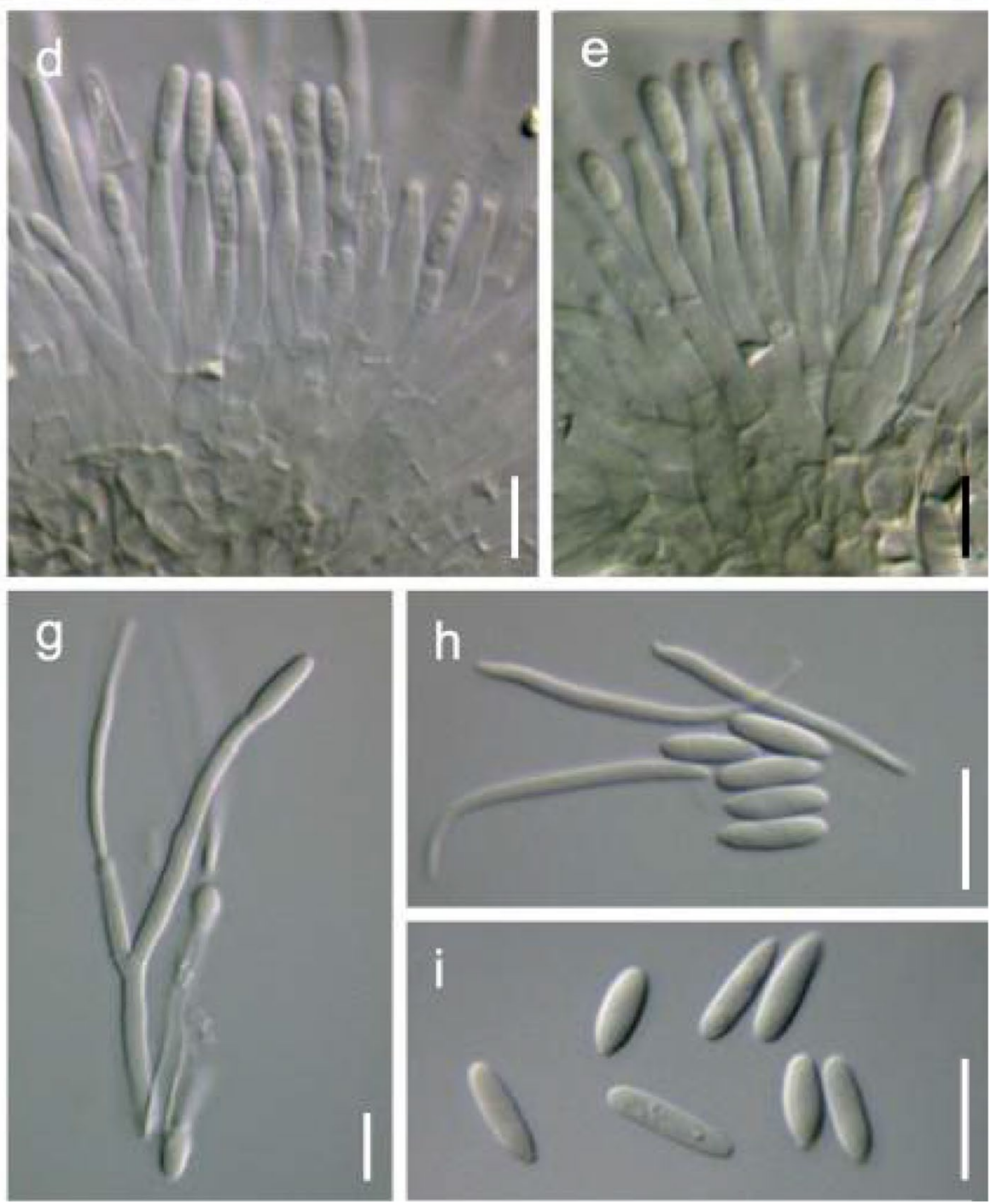

Fig. 120 Diaporthe foeniculina (CDP 022, new record). a, b Conidiomata formed on 1/2 PDA. Conidia are oozing in yellowish cirri. c-e Conidiophores and short conidiogenous cells. f, $\mathbf{g}$ Long conidiog- enous cells. h Alpha and beta conidia. i Alpha conidia. Scale bars: $\mathbf{a}=0.5 \mathrm{~mm}, \mathbf{b}=0.1 \mathrm{~mm}, \mathbf{c}-\mathbf{i}=5 \mu \mathrm{m}$ 
alpha conidia, cylindrical, subcylindrical, occasionally ampulliform, tapering towards the apex, aseptate, unbranched, rarely branched, small collarette rarely present, long conidiogenous cells, on Populus sp. twigs up to $60 \mu \mathrm{m}$ long, 1.1-2.1 $\mu \mathrm{m}$ (mean $\pm \mathrm{SD}=1.55 \pm 0.20 \mu \mathrm{m}$ ) wide, producing mostly beta conidia, filiform or cylindrical, mostly 3-septate, branched, phialidic, enteroblastic, proliferating at the same level giving rise to periclinal thickenings. Alpha conidia obovoid, with one end rounded and the other obtuse or truncate, hyaline, smooth- and thin-walled, aseptate, aguttulate or bi-guttulate, rarely with more than 2 guttules at each end, not produced on 1/2 PDA, on Populus sp. twigs 5.8-9×1.5-2.7 $\mu \mathrm{m}$ (mea $\mathrm{n} \pm \mathrm{SD}=7.18 \pm 0.66 \times 2.09 \pm 0.27 \mu \mathrm{m})$; mean $\pm \mathrm{SD}$ conidium length/width ratio $=3.49 \pm 0.62$. Beta conidia filiform, hamate, sometimes slightly sigmoid, base rounded or truncate, apex acute and tapered, hyaline, smooth- and thin-walled, aseptate, aguttulate, on Populus sp. twigs 19.5-24.6×1.1-1.7 $\mu \mathrm{m}$ (mea $\mathrm{n} \pm \mathrm{SD}=22.13 \pm 1.33 \times 1.33 \pm 0.22 \mu \mathrm{m}) ;$ mean $\pm \mathrm{SD}$ conidium length/width ratio $=17.04 \pm 2.77$. Gamma conidia infrequent, fusiform, with both ends acute or slightly truncate, hyaline, smooth- and thin-walled, aseptate, aguttulate or with several minute guttules.

Culture characteristics: Colonies on PDA, reaching $38 \mathrm{~mm}$ diam. after 7 at $20{ }^{\circ} \mathrm{C}$. Surface flat, glabrous to velvety, with sparse aerial mycelium, with filamentous or filiform margin, irregular, whitish, orange- to brownish, goldto brownish, opaque. Reverse yellowish to pale, becoming brownish towards the centre. No diffusible pigment. Conidiomata black, scattered over the surface of the colony, often exuding orange or black droplets.

Material examined: PORTUGAL, Lisbon, Parque das Nações, Jardins Garcia d'Orta, Talhão do Coloane, on foliar lesions of segments of Trachycarpus fortunei (Arecaceae), 5 October 2018, D.R.S. Pereira (specimen HDP 013/02, living culture CDP 022); PORTUGAL, Lisbon, Marvila, Ferreira de Castro Street, near Casa dos Direitos Sociais, on foliar lesions of segments of Chamaerops humilis (Arecaceae), 13 October 2018, D.R.S. Pereira (specimen HDP 025/02, living culture CDP 209); PORTUGAL, Lisbon, Parque das Nações, Jardins da Água, near Oceanário de Lisboa, on foliar lesions of segments of Chamaerops humilis (Arecaceae), 16 October 2018, D.R.S. Pereira (specimen HDP 035/02, living culture CDP 315).

Distribution: Argentina, Australia, Europe (Greece, Portugal, Spain, Italy), New Zealand, South Africa, USA (California) (Udayanga et al. 2014b; Lawrence et al. 2015; Annesi et al. 2016; Guarnaccia et al. 2016; present study).

Hosts: Acacia spp., Acer spp., Actinidia deliciosa, Aspalathus linearis, Bougainvillea spectabilis, Camellia sinensis, Castanea spp., Chamaerops humilis, Citrus limon, $C$. limonia, Crataegus spp., Diospyros spp., Foeniculum vulgare, Fuchsia spp., Hydrangea spp., Juglans spp., Malus spp., Olea spp., Persea americana, Prunus spp., Pyrus spp., Quercus spp., Rhus spp., Ribes spp., Salix sp., Trachycarpus fortunei, Vitis vinifera, Wisteria sinensis (Udayanga et al. 2014b; Lawrence et al. 2015; Annesi et al. 2016; Guarnaccia et al. 2016; present study).

GenBank number: CDP 022: ITS = MT000992, TEF $1-\alpha=$ MN990208, TUB2 = MN990209; CDP 209: $\quad$ ITS $=$ MT004913, TEF $1-\alpha=$ MT011071, TUB2 = MT011077; CDP 315: ITS = MT004914, TEF1- $\alpha=$ MT011072, TUB2 = MT011078.

Notes: Diaporthe foeniculina was first reported on Foeniculum vulgare from Madeira, Portugal (Phillips 2003) and has since then been recorded on more than 20 different hosts in nine countries around the world. The taxonomic and phylogenetic position of this species has been unclear in the past, but it is now well-resolved (see Udayanga et al. 2014 b). Three isolates of $D$. foeniculina were recorded from foliar lesions of palms (Fig. 123), but pathogenicity has not been tested. This is the first time this Diaporthe species is reported from Arecaceae, representing a new host record. Two of the isolates were recorded from Chamaerops humilis, another was recorded from Trachycarpus fortunei, thus giving new insight into the wide host range already reported for D. foeniculina (Fig. 120).

Diaporthe pseudophoenicicola R.R. Gomes, Glienke \& Crous, Persoonia 31: 30 (2013)

Index Fungorum number: IF803839; Facesoffungi number: FoF 09161; Fig. 121

Associated with foliar lesions. Sexual morph Undetermined. Asexual morph Conidiomata pycnidial, stromatic, uni- to multi-locular or convoluted, solitary, black, thick-walled, of variable morphology and dimensions, up to $3.5 \mathrm{~mm}$ diam., subglobose to pulvinate, sometimes conical or discoid, glabrous or covered with some hyphal outgrows, superficial to semi-immersed, inostiolate, dehiscent by irregular fissures on pycnidial wall, exuding a creamy, pale-luteous mucoid mass of conidia. Pycnidial locules unito multi-chambered, subdivided by invaginations of common pycnidial walls. Pycnidial wall pseudoparenchymatous, composed of oblong to isodiametric thin-walled cells, outer cell layers of textura globulosa, pigmented, dark-brown, inner cell layers of textura angularis, hyaline. Conidiophores absent. Conidiogenous cells lining the entire cavity, $11.7-49.7 \times 1.1-3.1 \mu \mathrm{m}($ mean $\pm \mathrm{SD}=21.99 \pm 9.09 \times$ $1.83 \pm 0.44 \mu \mathrm{m})$, intermingled with paraphyses, straight, hyaline, smooth- and thin-walled, cylindrical, ampulliform, aseptate to 1-septate at the base, unbranched, rarely with 1 branch below the septum, phialidic, collarette rarely present, enteroblastic, proliferating at the same level giving rise to periclinal thickenings, occasionally proliferating percurrently giving rise to 1-2 distinct annellations or enteroblastic proliferating percurrently after the formation of a new conidiogenous cell by apical wall-building. Paraphyses straight or curved at the apex, hyaline, smooth- and 

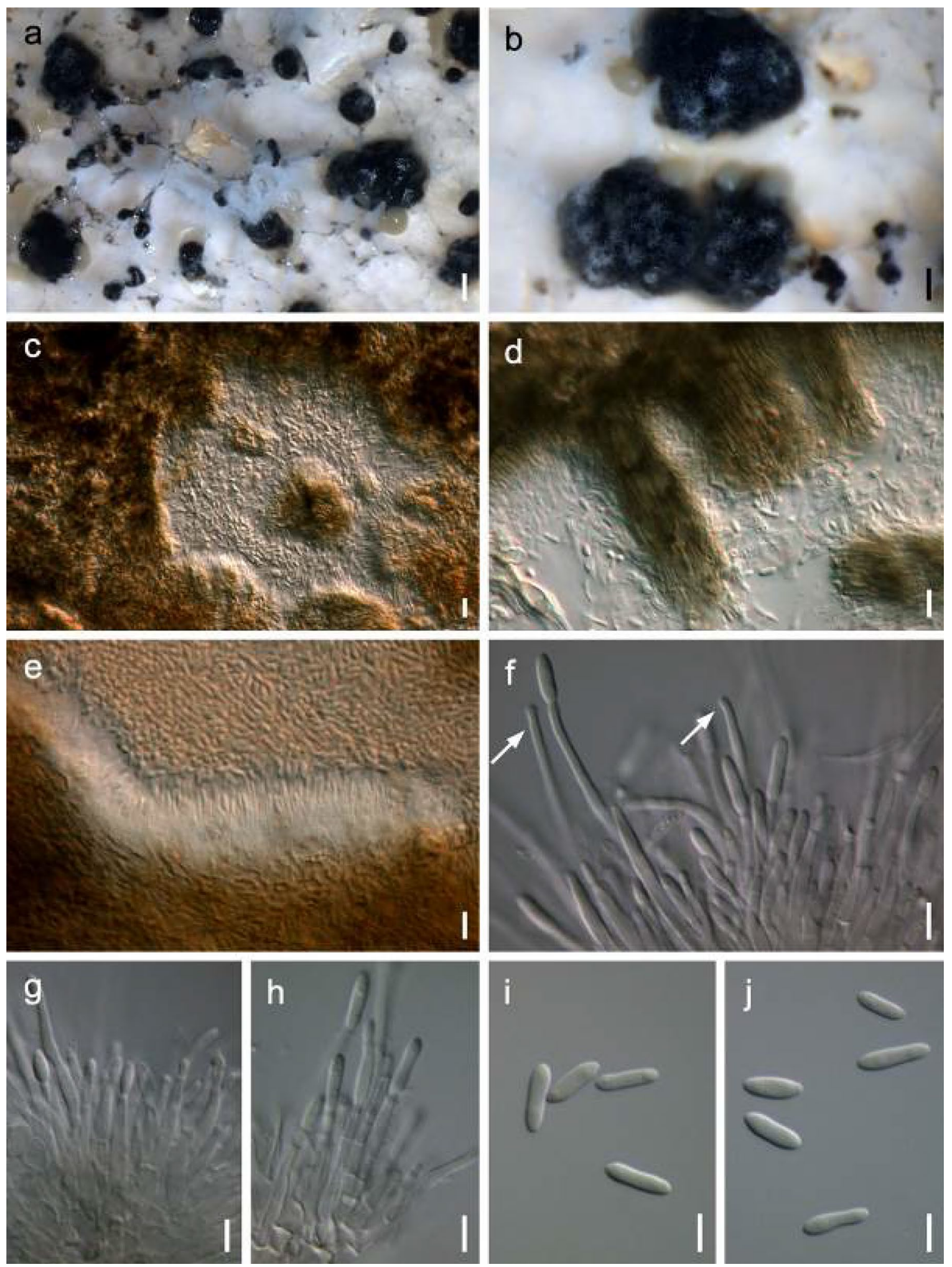

Fig. 121 Diaporthe pseudophoenicicola (CDP 047, new record). a, b Conidiomata formed on 1/2 PDA. Conidia are oozing in creamy mucoid masses. c Section through conidioma loculus. d Section through conidioma loculus showing wall invaginations. e Section through conidiogenous layer. $\mathbf{f}-\mathbf{h}$ Conidiogenous cells and paraphyses (white arrows). $\mathbf{i}, \mathbf{j}$ Conidia. Scale bars: $\mathbf{a}=1 \mathrm{~mm}, \mathbf{b}=0.5 \mathrm{~mm}$, $\mathbf{c}-\mathbf{e}=10 \mu \mathrm{m}, \mathbf{f}-\mathbf{j}=5 \mu \mathrm{m}$ 
thin-walled, aseptate to 1-2-septate, unbranched or rarely branched at the base, extending above conidiogenous cells, up to $60 \mu \mathrm{m}$ long. Alpha conidia cylindrical to allantoid, often mediumly narrowed, mostly with one end rounded and the other acute or slightly truncate, hyaline, smooth-walled, thin-walled, aseptate, bi-guttulate, with an inconspicuous guttule at each end, 6.4-9.4 $\times 2.9-1.5 \mu \mathrm{m}$ (mean $\pm \mathrm{SD}=8$ $.07 \pm 0.80 \times 2.11 \pm 0.31 \mu \mathrm{m})$; mean $\pm \mathrm{SD}$ conidium length/ width ratio $=3.91 \pm 0.70$. Beta and gamma conidia not seen.

Culture characteristics: Colonies on PDA, reaching $42 \mathrm{~mm}$ diam. after 7 at $20{ }^{\circ} \mathrm{C}$. Surface flat to velvety, with filiform margin, circular, whitish to pale, opaque to slightly translucent. Reverse yellowish to pale, becoming buff towards the centre. No diffusible pigment. Conidiomata black, formed in poorly defined concentric rings.

Material examined: PORTUGAL, Lisbon, Parque das Nações, Jardins da Água, Pomar do Mediterrâneo, on foliar lesions of segments of Chamaerops humilis (Arecaceae), 16 October 2018, D.R.S. Pereira (specimen HDP 039/02, living culture CDP 047); PORTUGAL, Lisbon, Parque das Nações, on foliar lesions of leaflets of Phoenix dactylifera (Arecaceae), 16 October 2018, D.R.S. Pereira (specimen HDP 044/01, living culture CDP 358).

Distribution: China (Gao et al. 2017), Iraq (Shalt El Arab), Spain (Mallorca) (Gomes et al. 2013), Portugal (Lisbon) (present study).

Hosts: Chamaerops humilis (present study), Mangifera indica, P. canariensis (Gao et al. 2017), Phoenix dactylifera (Gomes et al. 2013; present study).

GenBank number: CDP 047: $\mathrm{CAL}=\mathrm{MT} 011065$, ITS $=$ M T002357, TEF $1-\alpha=$ MT011069, TUB2 $=$ MT011075; CDP 358, CAL $=$ MT011067, ITS $=$ M T004743, TEF $1-\alpha=$ MT011073, TUB2 $=$ MT011079.

Notes: Diaporthe pseudophoenicicola was first reported on Phoenix dactylifera from Mallorca, Spain (Gomes et al. 2013). Two isolates of D. pseudophoenicicola were recorded from foliar lesions of palms (Fig. 123), but pathogenicity has not been tested (Figs. 121 and 123). This is the first report of this species from Portugal. One of the isolates was recorded from $C$. humilis and represented a new host record. The other isolate was recorded from $P$. canariensis, a host it has already been reported on in China (Gao et al. 2017).

Diaporthe pyracanthae L. Santos \& A. Alves, Mycosphere 8: 493 (2017)

Index Fungorum number: IF820224; Facesoffungi number: FoF 09198; Fig. 122

Associated with foliar lesions. Sexual morph Undetermined. Asexual morph Conidiomata pycnidial, stromatic, solitary, subglobose to pyriform, dark-brown to black, thickwalled, $600 \mu \mathrm{m}$ diam., mostly glabrous, but often covered with hyphal outgrows, superficial, opening via a central ostiole, internal cavity heart-shaped, exuding a pearl mucoid mass of conidia. Pycnidial wall pseudoparenchymatous of dark-brown, paler on the apex or around the ostiole, thinwalled textura angularis, becoming hyaline towards the inner layers. Conidiophores absent. Conidiogenous cells lining the entire cavity, lining the entire cavity, 5.9-22.6×1.5-3.4 $\mu \mathrm{m}$ $($ mean $\pm \mathrm{SD}=12.54 \pm 3.47 \times 2.09 \pm 0.42 \mu \mathrm{m})$, straight or curved, hyaline, smooth- and thin-walled, cylindrical, occasionally ampulliform, tapering towards the apex, aseptate, rarely 1 -septate, with a small branch below the septum, phialidic, collarette minute, enteroblastic, proliferating at the same level giving rise to periclinal thickenings. Alpha conidia fusiform to ellipsoidal, with both ends rounded or one end subobtuse, hyaline, smooth- and thin-walled, aseptate, mostly aguttulate, often with two inconspicuous guttules, not produced on PDA, on autoclaved palm leaf pieces $5-6.4 \times 1.8-2.9 \mu \mathrm{m}($ mean $\pm \mathrm{SD}=5.77 \pm 0.34 \times 2.39 \pm 0.22$ $\mu \mathrm{m}) ;$ mean $\pm \mathrm{SD}$ conidium length/width ratio $=2.43 \pm 0.26$. Beta conidia filiform, mostly hooked at the apex, base truncate, apex acute and tapered, hyaline, smooth- and thinwalled, aseptate, aguttulate, $21.6-32.1 \times 0.9-1.8 \mu \mathrm{m}$ (mean $\pm \mathrm{SD}=27.47 \pm 1.91 \times 1.28 \pm 0.17 \mu \mathrm{m})$; mean $\pm \mathrm{SD}$ conidium length/width ratio $=21.82 \pm 3.34$. Gamma conidia not seen.

Culture characteristics: Colonies on PDA, reaching $55 \mathrm{~mm}$ diam. after $7 \mathrm{~d}$ at $20{ }^{\circ} \mathrm{C}$. Surface flat, sparse aerial mycelium in raised concentric circles, with irregular margin, circular to irregular, white, pale-brown to yellowish towards the centre, opaque. Reverse luteous, buff to marron towards the centre. No diffusible pigment. Conidiomata black, scattered over the surface of the colony.

Material examined: PORTUGAL, Lisbon, Parque das Nações, Jardins Garcia d'Orta, Talhão do Coloane, on foliar lesions of segments of Chamaerops humilis (Arecaceae), 16 October 2018, D.R.S. Pereira (specimen HDP 039/02, living culture CDP 052).

Distribution: Portugal, Aveiro (Santos et al. 2017), Lisbon (present study).

Hosts: Pyracantha coccinea (Santos et al. 2017), Chamaerops humilis (present study).

GenBank number: $\mathrm{CAL}=\mathrm{MT011066}, \mathrm{ITS}=\mathrm{MT} 002843$, TEF1- $\alpha=$ MT011070, TUB2 = MT011076.

Notes: Diaporthe pyracanthae was introduced by Santos et al. (2017) for a collection on Pyracanthus coccinia in Portugal and since then it has not been reported on any other hosts. One isolate of $D$. pyracanthae was recorded from foliar lesions of palms, but pathogenicity has not been tested (Figs. 122 and 123). This is the first time that D. pyracanthae is reported from Arecaceae, namely Chamaerops humilis, representing a new host record.

\section{Phaeocytostroma Petr.}

Notes: Phaeocytostroma was introduced by Petrak (1921) with the type species $P$. istrica isolated from rotting culms 

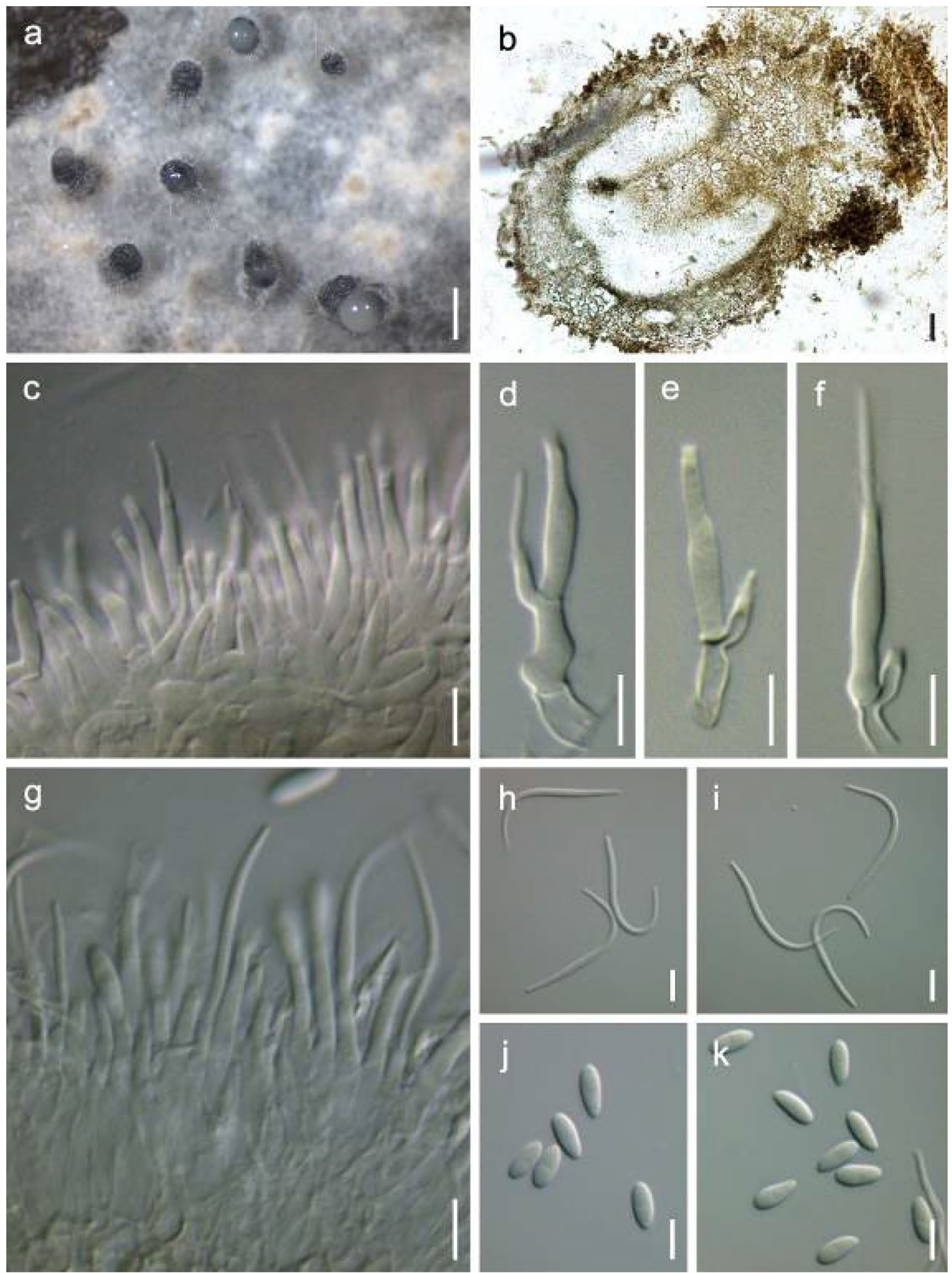

Fig. 122 Diaporthe pyracanthae (CDP 052, new record). a Conidiomata formed on 1/2 PDA. Conidia are oozing in pearl mucoid masses. b Vertical section of conidioma, showing its heart-shaped

internal cavity. $\mathbf{c}-\mathbf{g}$ Conidiogenous cells. $\mathbf{h}, \mathbf{i}$ Beta conidia. $\mathbf{j}, \mathbf{k}$ Alpha conidia. Scale bars: $\mathbf{a}=0.5 \mathrm{~mm}, \mathbf{b}=20 \mu \mathrm{m}, \mathbf{c}-\mathbf{k}=5 \mu \mathrm{m}$ 


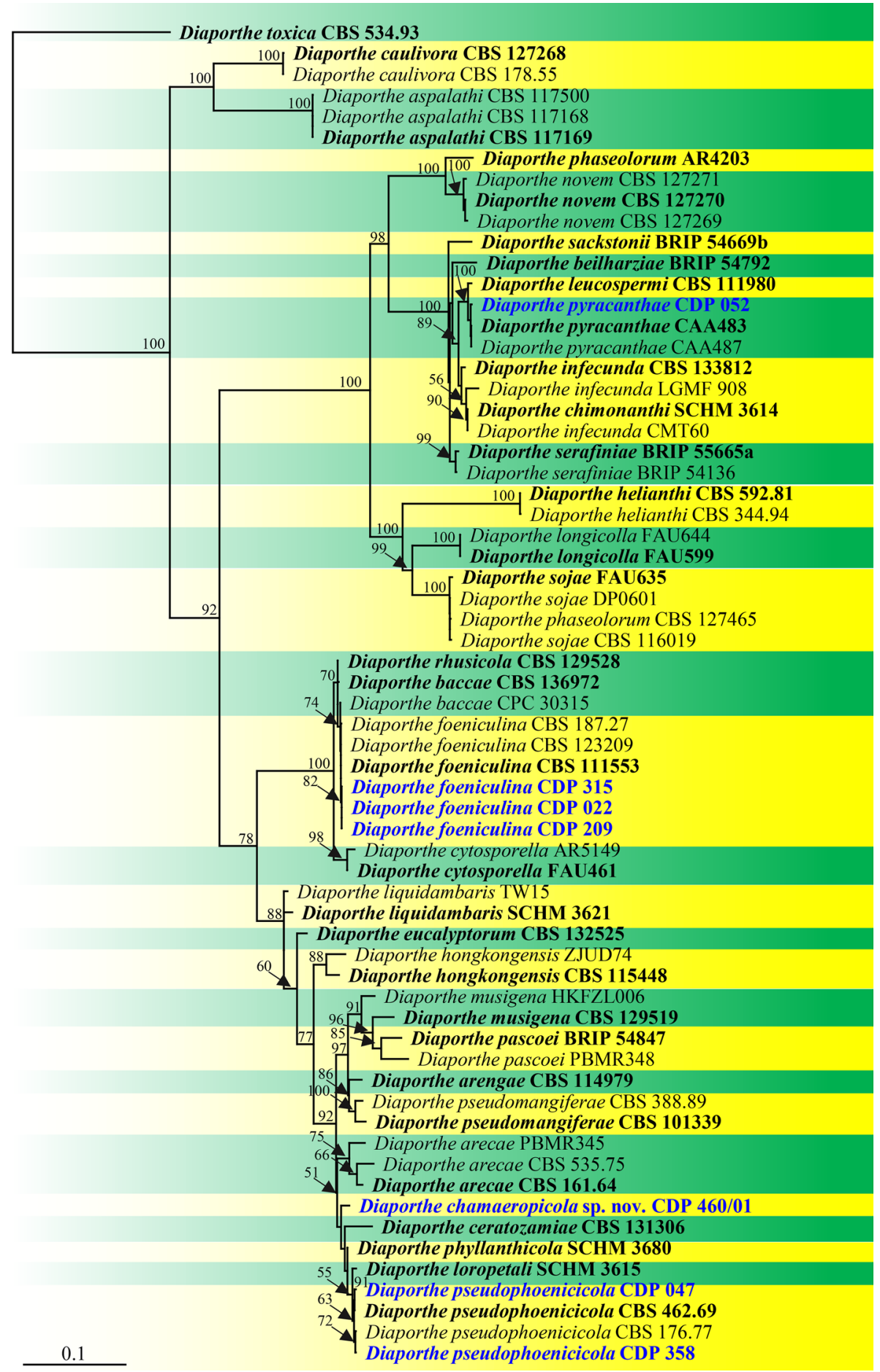

Fig. 123 Maximum Likelihood tree generated by RAxML with GTR + G+ I nucleotide substitution model using the combined four loci ITS, TEF1- $\alpha$, TUB and CAL. The sequences were retrieved from GenBank according to BLAST searches with the ITS locus and by reference to the recent literature. Sixty-four strains are included in the combined analyses that comprise 2248 characters (including gaps) (502 characters for ITS, 669 for TUB, 411 for TEF1- $\alpha$ and 554 for CAL) after alignment and manual adjustment. The final likelihood score for the ML tree was 15269.024241. The matrix had 1121 distinct alignment patterns, with $27.31 \%$ undetermined characters or gaps. ML bootstrap support values $(>50 \%)$ are shown above the branches. The scale bar represents the expected number of nucleotide changes per site. Diaporthe toxica (CBS 534.93) was included as an outgroup. Ex-type/ex-epitype/ex-isotype/ex-neotype cultures are in bold and newly generated sequences are in blue 
of Zea mays in Croatia. Sutton (1964) re-circumscribed Phaeocytostroma and synonymized Pleocyta and Phaeocytosporella under Phaeocytostroma. Lamprecht et al. (2011) provided additional collections and phylogenetic placement of species in Phaeocytostroma and included in Diaporthaceae. Our phylogenetic taxon sampling Phaeocytostroma were retrieved from Lamprecht et al. (2011) and the GenBank database. Six species have been included in Phaeocytostroma (Index Fungorum 2021). This study introduces a new species Phaeocytostroma yomense as a saprobic taxon isolated from decaying submerged wood in a freshwater habitat (Fig. 123).

Phaeocytostroma yomense Boonmee, Chandrasiri \& K.D. Hyde, sp. nov.

Index Fungorum number: IF558544; Facesoffungi number: FoF 09958; Fig. 124

Etymology: In reference to the Yom River where the holotype was collected.

\section{Holotype: MFLU 21-0070}

Saprobic on decaying submerged wood in the river. Sexual morph Undetermined. Asexual morph Coelomycetous. Conidiomata 337-452 $\mu \mathrm{m}$ high, 246-393 $\mu \mathrm{m}$ diam., pycnidial, white cream, superficial, scattered, gregarious, covered by massy brown hyphal mats, subglobose, uniloculate, transparent when fresh, simply fragile when dry, ostiole, dehiscence by breakdown of upper wall of conidiomata. Conidiomata walls easily fragile, difficult to observe, conidiophores and conidiogenous cells not seen. Conidia $14-23 \times 3-6 \mu \mathrm{m}(\bar{x}=18 \times 4.5 \mu \mathrm{m}, \mathrm{n}=20)$, cylindrical-ellipsoid, oblong allantoid, asymmetrical with obtuse ends, aseptate, pale brown, smooth-walled.

Culture characteristics: Conidium germinating on water agar within $12 \mathrm{~h}$ at room temperature. Colonies on PDA, fast growing, reaching $5 \mathrm{~cm}$ diam., in 2 weeks, slightly effuse, entire edge, brown to dark brown in surface, dark brown in reverse, mycelium superficial, partially immersed, brown, radiating outwards.

Material examined: THAILAND, Phayao Province, Pong, Yom River, on decaying submerged wood in the river shore, 18 December 2019, S. Boonmee and K.S.U. Chandrasiri, YR2 (MFLU 21-0070, holotype), ex-type living culture, MFLUCC 21-0093.

GenBank numbers: ITS = MZ538521, LSU = MZ538555.

Notes: Phaeocytostroma yomense shares similar conidial features such as cylindrical-ellipsoid to oblong shapes and pale brown colour to species Ph. sacchari (Sutton 1964, 1980), but $P$. yomense differs from $P h$. sacchari in conidiomatal features such as superficial, bright pigmented and covered by hyphal mats. Phylogenetically, Ph. yomense is position in a distinct lineage basal to the strain of $P h$. plurivorum (CBS 113835) with 98\% MLBS, 1.00 BYPP support (Fig. 125). A comparison of the ITS sequence between
Ph. yomense (MFLUCC 21-0093) and Ph. plurivorum (CBS 113835) showed $1.54 \%$ (9/581 bp with 7 gabs difference). However, Ph. yomense differs from Ph. plurivorum (Sutton 1980) in having white cream and smaller conidiomata (337-452 × 246-393 $\mu \mathrm{m}$ vs. up to $700 \times 300-400 \mu \mathrm{m}$ ). Phaeocytostroma yomense differs from other species by its current occurrence as an aquatic taxon in a freshwater habitat and having superficial, white cream pigmented, covered by massy hyphal mat conidiomata and pale brown conidia (Fig. 124). We therefore, introduce a new species $P h$. yomense based on its morphological distinctness and phylogenetic affinity.

Fuscosporellales Jing Yang, Bhat \& K.D. Hyde

Notes: Yang et al. (2016) introduced the new order Fuscosporellales based on the evidence of multigene phylogenetic analysis to accommodate a monotypic family Fuscosporellaceae.

Fuscosporellaceae Jing Yang, Bhat \& K.D. Hyde

Notes: Fuscosporellaceae was introduced by Yang et al. (2016) to accommodate six genera Bactrodesmiastrum, Fuscosporella, Mucispora, Parafuscosporella, Plagiascoma and Pseudoascotaiwania and 14 species all of which were described from lignicolous substrates in freshwater habitats. The type species of the family is Fuscosporella pyriformis Jing Yang, Bhat \& K.D. Hyde (Boonyuen et al. 2016; Yang et al. 2016). The family is characterized by macronematous, mononematous, branched, hyaline conidiophores; holoblastic, globose to clavate conidiogenous cells; obovate to obpyriform, with a septum near the base, smooth, brown to dark brown conidia. Based on morphology and the combined phylogenetic analyses of ITS and SSU sequence data, a novel species Parafuscosporella nilotica is introduced.

\section{Parafuscosporella Jing Yang \& K.D. Hyde}

Notes: Parafuscosporella currently comprises three species described from freshwater habitats in Thailand. The genus is characterized by sphaerical to cushion-shaped, erumpent to superficial, black, gelatinous sporodochia, with a jelly-like cover; semi-macronematous, mononematous, compact, flexuous, simple or branched, mostly moniliform, conidiophores with globose to subglobose, ellipsoidal or clavate cells; monoblastic, integrated, sometimes discrete, terminal, globose, subglobose, ellipsoidal or clavate conidiogenous cells and acrogenous, ellipsoidal to broadly obpyriform, smooth, dark brown to black conidia, with a septum near the base, sometimes with a small protuberance, with pale brown basal cells (Boonyuen et al. 2016; Yang et al. 2016). In this study, a new species Parafuscosporella nilotica is introduced based on evidence of morphology and phylogenetic placement (Figs. 126 and 127). 

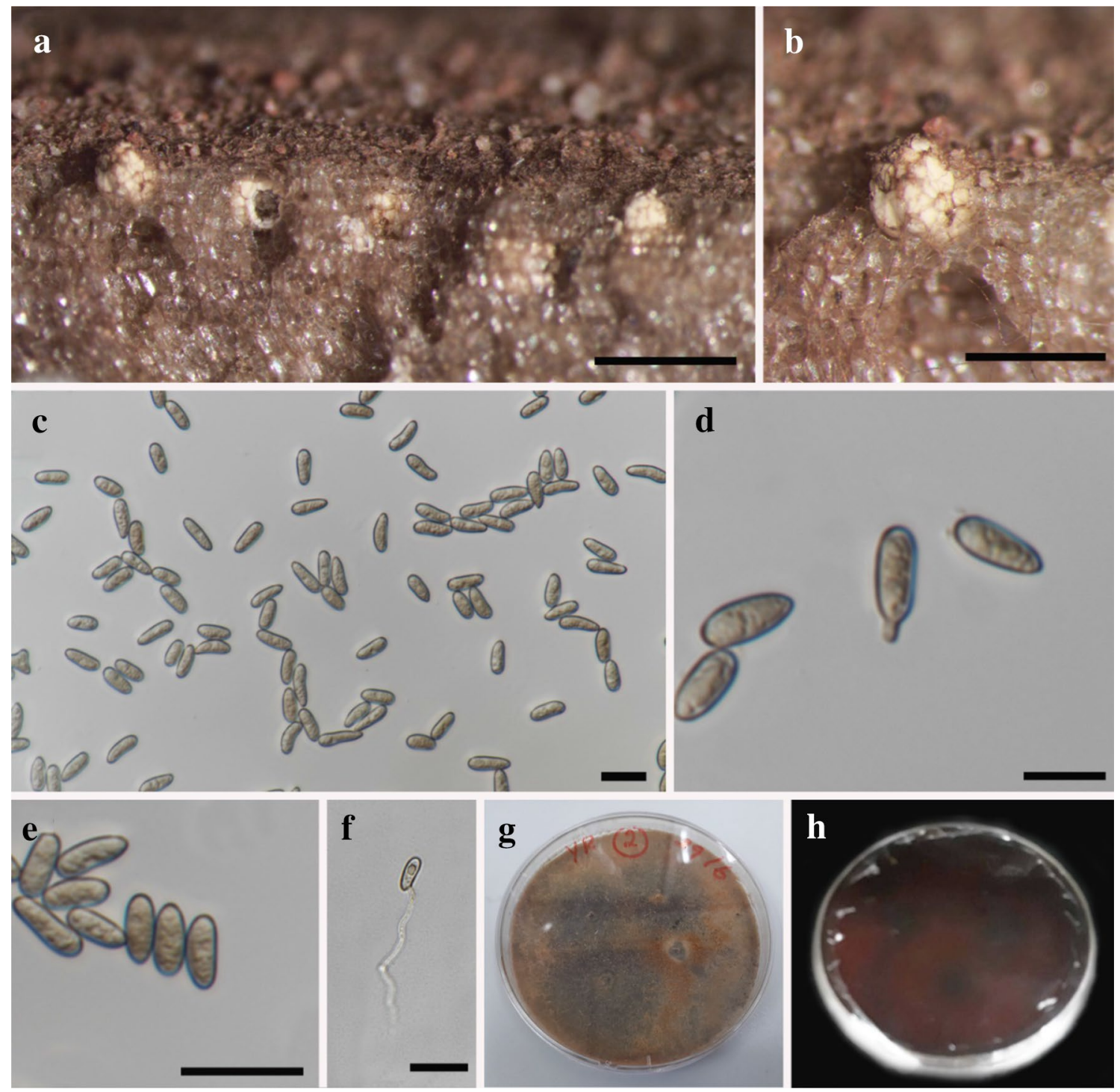

Fig. 124 Phaeocytostroma yomense (MFLU 21-0070, holotype). a, b Appearance of white conidiomata on decaying submersed wood substrate. $\mathbf{c}-\mathbf{e}$ Conidia. f Germinated conidium. $\mathbf{g}, \mathbf{h}$ Culture on PDA

\section{Parafuscosporella nilotica Abdel-Aziz sp. nov.}

Index Fungorum number: IF557838; Facesoffungi number: FoF 09199; Fig. 126

Etymology: Named after the River Nile, where this fungus was collected.

Holotype: CBS H-22128

Saprobic on the surface of decaying submerged wood in a freshwater river. Sexual morph Undetermined. Asexual morph Colonies on natural substrate, effuse, black. Mycelium partly immersed, partly superficial, composed of septate, hyaline hyphae, 1.5-3 $\mu \mathrm{m}$ wide. Conidiomata $300-600 \mu \mathrm{m}$ diam. $(x=421 \mu \mathrm{m}, \mathrm{n}=10)$, sporodochial, scattered, sphaerical to cushion-shaped, with jelly-like cover, gelatinous. Conidiophores micronematous, mononematous, from surface and reverse. Scale bars: $\mathbf{a}=100 \mu \mathrm{m}, \mathbf{b}=50 \mu \mathrm{m}, \mathbf{c}, \mathbf{e}$, $\mathbf{f}=20 \mu \mathrm{m}, \mathbf{d}=10 \mu \mathrm{m}$

either reduced to conidiogenous cells and conidia born directly on hyphae or when present unicellular, cylindrical to clavate, smooth-walled, hyaline, 3-7 $\mu \mathrm{m}$ long, 1.5-3 $\mu \mathrm{m}$ wide. Conidiogenous cells holoblastic, monoblastic, integrate. Conidial secession rhexolytic. Conidia $18-22 \times 11-15 \mu \mathrm{m}(\bar{x}=21 \times 13.5 \mu \mathrm{m}, \mathrm{n}=50)$, acrogenous, obpyriform, with a septum near the base, smooth, brown to dark brown, basal cell paler.

Culture characteristics: Conidia germinating on PDA within $24 \mathrm{~h}$ at $25{ }^{\circ} \mathrm{C}$ and germ tubes produced from both ends. Colonies on PDA reaching $1-1.5 \mathrm{~cm}$ diam. after 1 month at $25{ }^{\circ} \mathrm{C}$, dark-brown, same from below.

Material examined: EGYPT, Sohag City, the River Nile, on decaying submerged wood, 14 August 2012, F.A. 
Fig. 125 Maximum likelihood phylogenetic tree based on a combined ITS, LSU and TEF1- $\alpha$ sequence data of Phaeocytostroma taxa and some related genera in Diaporthaceae. The tree is rooted with Phomopsis viticola (UCD2009SB). Bootstrap support values for maximum likelihood (MLBS, left) equal to or greater than $70 \%$ is given above the nodes. Bayesian posterior probabilities (BYPP, right) equal to or greater than 0.95 are given above the nodes. The newly generated sequence is in blue bold

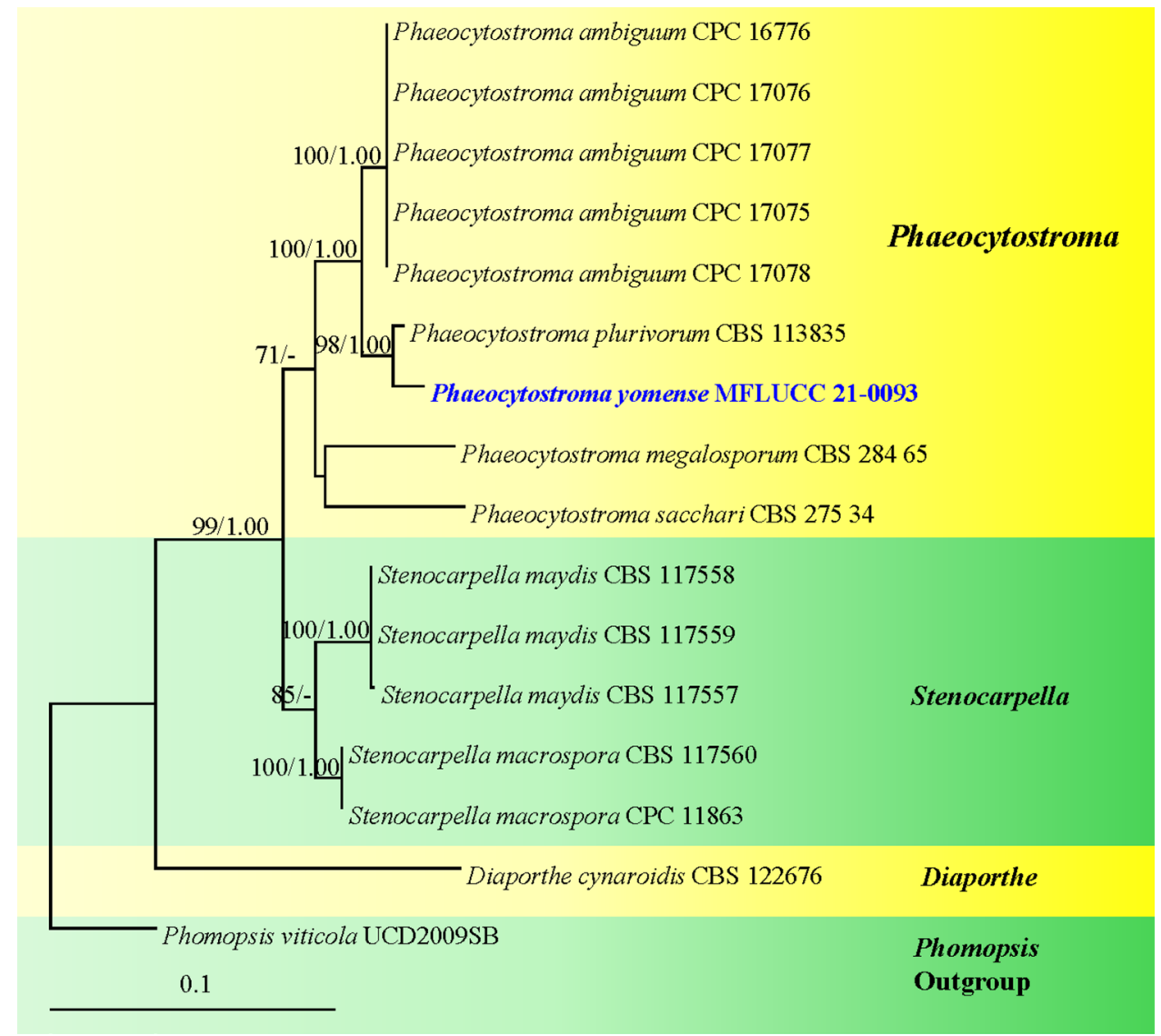

Abdel-Aziz (CBS H-22128, holotype), ex-type living culture (MF 1312).

GenBank numbers: ITS = MN921198, SSU = MN921199.

Notes: Phylogenetic analyses of ITS placed Parafuscosporella nilotica as distinct novel species within the Parafuscosporella clade with 87\% MLBS, 95\% MPBS, 0.98 BYPP support (Fig. 127). Parafuscosporella nilotica differs from the other Parafuscosporella species in having simple, micronematous, cylindrical to clavate conidiophores, whereas other species have macronematous, mostly moniliform, with globose to subglobose, ellipsoidal or clavate cells (Fig. 126). Parafuscosporella nilotica has much smaller conidial sizes than all species; $18-22 \times 11-15 \mu \mathrm{m}$ vs $20-29 \times 13-19 \mu \mathrm{m}$ for $P$. aquatica; $(37.5-) 40-47.5 \times$ (25-)27.5-42.5 $\mu \mathrm{m}$ for $P$. garethii; $28-37 \times 14-21 \mu \mathrm{m}$ for P. moniliformis; $26.5-36 \times 12-26 \mu \mathrm{m}$ for $P$. mucosa and 23-30 $\times 16-26 \mu \mathrm{m}$ for $P$. pyriformis (Boonyuen et al. 2016; Yang et al. 2016, 2020).

\section{Hypocreales Lindau}

Notes: The recent treatments for Hypocreales in Hyde et al. (2020d) and Yuan et al. (2020).

Nectriaceae Tul. \& C. Tul. [as 'Nectriei']
Notes: Nectriaceae was introduced by Tulasne and Tulasne (1865) and accepted as a family in Hypocreales, based on stromatic and perithecial characters (Seaver 1909; Petch 1938; Kreisel 1969; Rossman et al. 1999). Presently, Nectriaceae comprised 46 genera (Wijayawardene et al. 2020). Asexual morphs are mainly hyphomycetous, less commonly coelomycetous (Hyde et al. 2020b, d). Nectriaceae members are cosmopolitan in distribution as saprobes, endophytes or pathogens of various host species (Rossman et al. 1999; Lombard et al. 2015).

\section{Fusarium Link}

Notes: Fusarium species are economically important fungi including, many well-known plant pathogens, which cause blights, cankers, root and stalk rots and wilts of broadacre, horticultural, ornamental and forest plants, and cause human infections worldwide (Jayawardena et al. 2019; Summerell 2019). We describe a new species of Fusarium isolated from grain sorghum (Sorghum bicolor) in Queensland, Australia, and introduce new records of $F$. atrovinosum and F. clavum on $S$. bicolor based on morphological characters and multi-locus phylogenetic analyses.

Fusarium atrovinosum L. Lombard \& Crous, Fungal Systematics and Evolution 4: 190 (2019) 

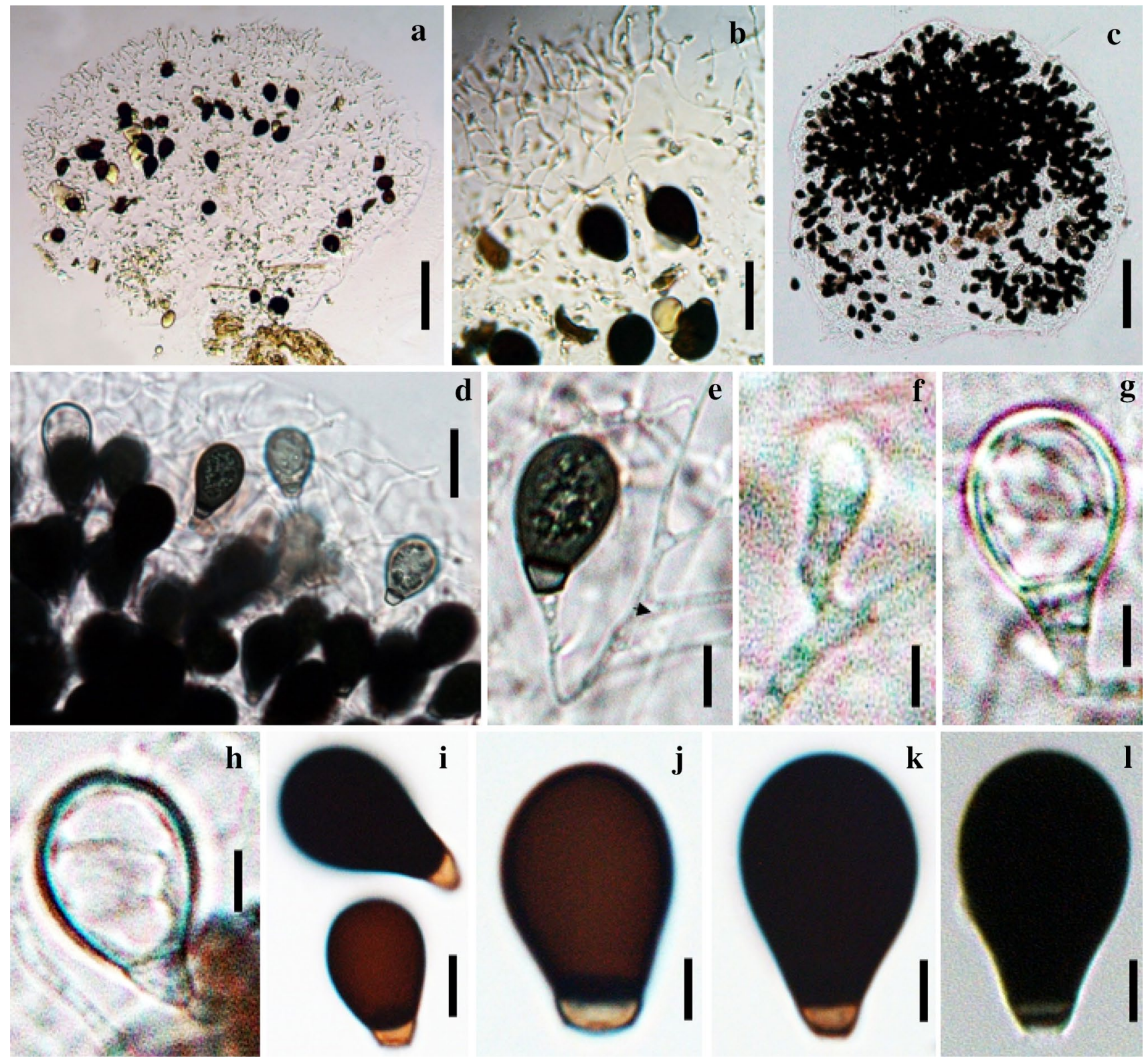

Fig. 126 Parafuscosporella nilotica (CBS H-22128, holotype). a, b Vertical section through sporodochium. c, d Squash of sporodochium. e-l Conidia at different stages of development. Scale bars: $\mathbf{a}, \mathbf{c}=120 \mu \mathrm{m}, \mathbf{b}, \mathbf{d}=20 \mu \mathrm{m}, \mathbf{e}, \mathbf{i}=10 \mu \mathrm{m}, \mathbf{f}-\mathbf{h}, \mathbf{j}-\mathbf{l}=5 \mu \mathrm{m}$

Index Fungorum number: IF831559; Facesoffungi number: FoF 09577; Fig. 128

Saprobic on dead stalk of Sorghum bicolor. Sexual morph Undetermined. Asexual morph Conidiophores 20-50 $\mu \mathrm{m}$ tall, carried on aerial mycelium, unbranched or irregularly or sympodially branched, bearing a terminal single phialide or whorl of 2-3 phialides. Conidiogenous cells 9-23 $\times 2-4 \mu \mathrm{m}$, polyphialides, subulate to subcylindrical, smooth. Conidia $4-14 \times 2-3 \mu \mathrm{m}$, formed in false heads on the phialide tips, hyaline, fusiform to ellipsoidal to obovoid, smooth and thin-walled, mostly 0 -septate, rarely 1-2-septate then larger. Chlamydospores 5-22 $\mu \mathrm{m}$ diam., abundant, globose to subglobose, smooth to slightly verrucose, formed terminally or intercalarily in chains of three or more; wall $1-1.5 \mu \mathrm{m}$.

Culture characteristics: Colonies on PDA reaching $90 \mathrm{~mm}$ at $24{ }^{\circ} \mathrm{C}$ after $28 \mathrm{~d}$ in the dark; surface vinaceous, mycelium dense to woolly, without odour; reverse livid red to dark vinaceous. On SNA colonies sparse, white to pale rosy buff in the centre, powdery due to abundant; reverse pale rosy buff. On CLA aerial mycelium abundant, white, lacking sporodochia on the carnation leaf pieces. On WA colonies sparse and powdery; pale rosy vinaceous in the centre; reverse pale rosy vinaceous. 


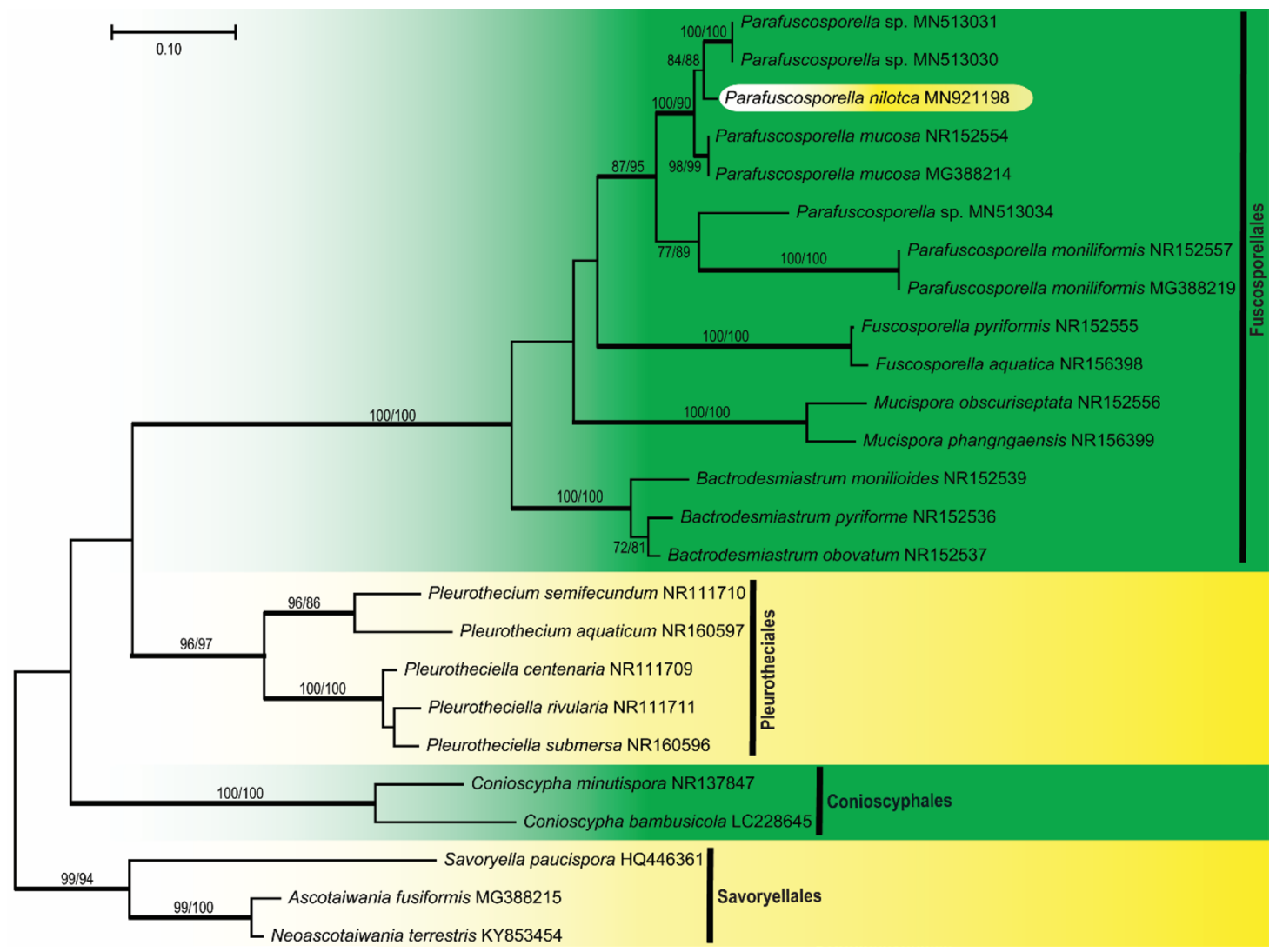

Fig. 127 Phylogenetic relationship of Parafuscosporella nilotica with related taxa in Fuscosporellaceae based on the nucleotide sequences of the ITS. The maximum likelihood (ML) tree (-In likelihood $=4928.50)$ was constructed in MEGA X (Kumar et al. 2018). The maximum parsimonious data set of the combined genes consisted of 25 taxa with 3 representatives of Savoryellales are used as the outgroup taxa. The dataset includes 503 total characters, of which 175 were constant, 30 parsimony-uninformative and 298 parsimony-informative. The parsimony analyses of the data

Material examined: AUSTRALIA, Goondiwindi, on dead stalk of Sorghum bicolor, 19 February 2018, N. Vaghefi, BRIP 70767a, new record.

GenBank numbers: $\mathrm{CAL}=\mathrm{MW} 403492$, RPB2 = MW403493, TEF1- $\alpha=$ MW403494.

Notes: Fusarium atrovinosum belongs to the Fusarium chlamydosporum species complex (O'Donnell et al. 2009; Lombard et al. 2019b). In a four-locus phylogeny (Fig. 129), the isolate (BRIP 70767a) was recovered in a well-supported clade (98\% MLBS, 1.00 BYPP) that contained the type strain of $F$. atrovinosum (CBS 445.67). This isolate (BRIP 70767a) was morphologically and culturally similar to the type strain of $F$. atrovinosum (CBS 445.67) described in Lombard et al. (2019b). Fusarium atrovinosum was described on Triticum aestivum (wheat) in Australia (Lombard et al. 2019b). matrix yielded 3 equally most parsimonious trees with a tree length of 1021 steps [consistency index $(\mathrm{CI})=0.6298$, homoplasy index $(\mathrm{HI})=0.3702$, retention index $(\mathrm{RI})=0.7816$, rescaled consistency index $(\mathrm{RC})=0.4923$ ]. Phylogenetic trees obtained from ML, maximum parsimony (MP) and Bayesian inference posterior probabilities (BIPP) were similar in topology. Bootstrap support on the nodes represents MLBS and MPBS equal to or greater than 50\%. Branches with a BYPP of equal to or greater than $95 \%$ are in bold. The new taxon is in yellow box

Subsequently, $F$. atrovinosum has been reported from Brazil in association with rice seed and other hosts (Costa 2020). These studies indicate that $F$. atrovinosum may have an association with grass (Poaceae) hosts. This is the first record of F. atrovinosum on Sorghum (Fig. 128).

Fusarium clavum J.W. Xia, L. Lombard, Sand.-Den., X.G. Zhang \& Crous, Persoonia 43: 199 (2019)

Index Fungorum number: IF833468; Facesoffungi number: FoF 09578; Fig. 130

Saprobic on dead stalk of Sorghum bicolor. Sexual morph Undetermined. Asexual morph Sporodochia salmon to orange, formed abundantly on carnation leaf pieces. Conidiophores densely and irregularly branched. Conidiogenous cells monophialides, subulate to subcylindrical, smooth, thin 

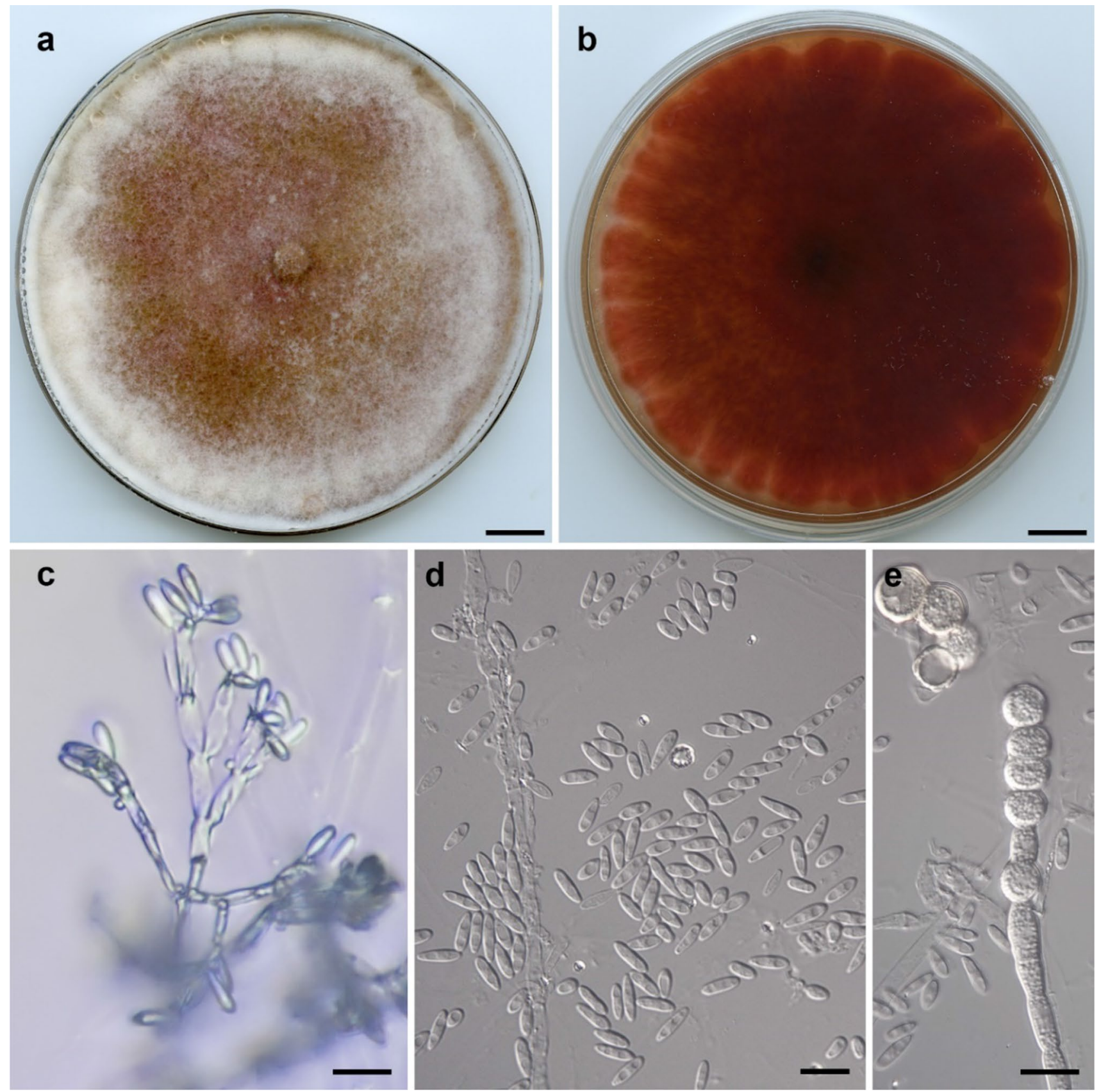

Fig. 128 Fusarium atrovinosum (BRIP 70767a, new record). a, b Culture on PDA from surface and reverse. c Conidiophores. d Conidia. e Chlamydospores. Scale bars: $\mathbf{a}, \mathbf{b}=1 \mathrm{~cm}, \mathbf{c}-\mathbf{e}=10 \mu \mathrm{m}$

walled. Conidia $25-35 \times 3-4 \mu \mathrm{m}$, falcate, slender, curved dorsiventrally, tapering towards both ends, with elongated or whip-like curved apical cells, mostly 3 -septate, hyaline, smooth-walled. Chlamydospores 6-12 $\mu$ m diam., abundant, globose, subglobose to oval, subhyaline to yellowish-brown, smooth-walled, terminal or intercalary, solitary, in pairs or forming chains.

Culture characteristics: Colonies on PDA reaching $90 \mathrm{~mm}$ at $24{ }^{\circ} \mathrm{C}$ after $28 \mathrm{~d}$; surface cinnamon, flat, velvety, with scant aerial mycelium; reverse cinnamon. On SNA and WA colonies sparse and flat, without colour, sporulation moderate on the surface of the agar and the carnation leaf pieces.

Material examined: AUSTRALIA, Brookstead, on dead stalk of Sorghum bicolor, 19 February 2018, N. Vaghefi, BRIP 70756a, new record. 


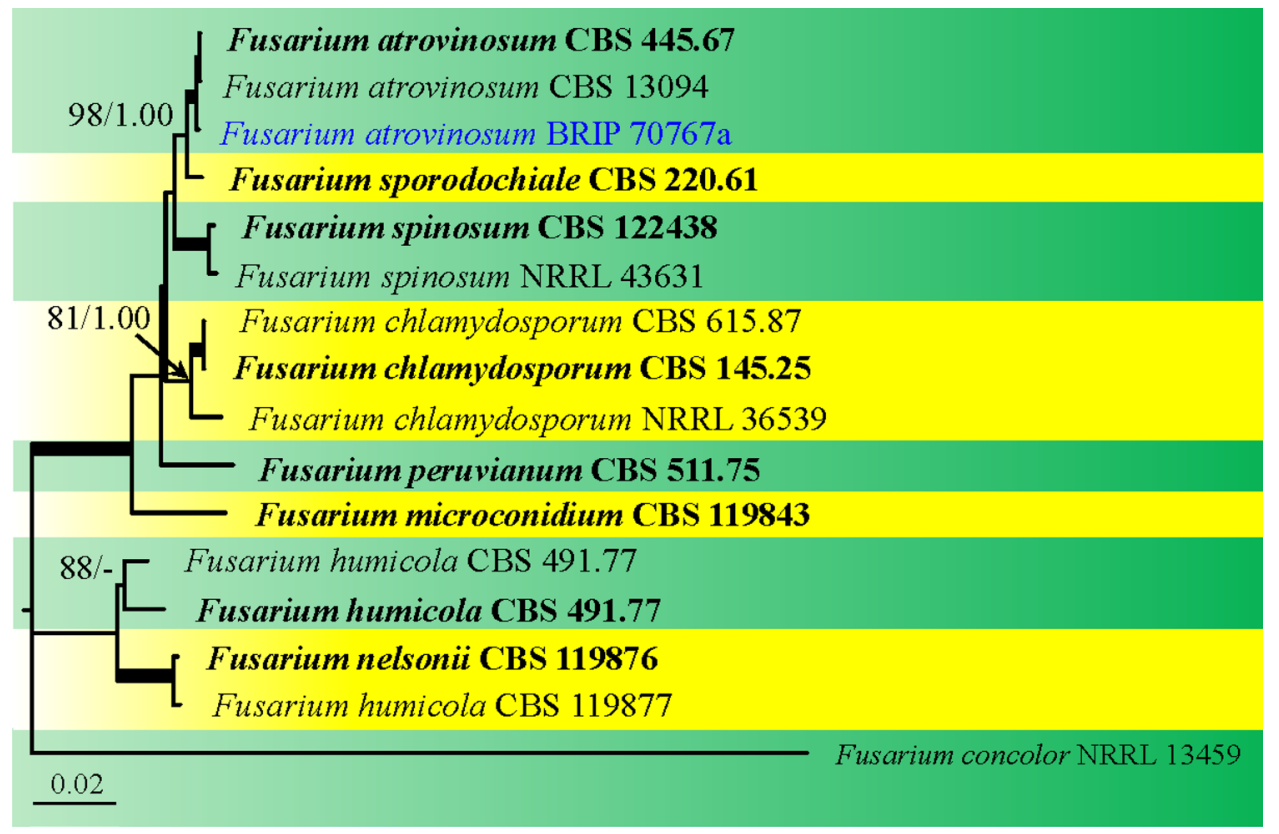

Fig. 129 Phylogram generated from Bayesian analysis based on combined CAL, RPB1, RPB2 and TEF1- $\alpha$ sequence data representing Fusarium chlamydosporum species complex and related taxa. The second measure of branch support was obtained through Maximum Likelihood (ML) analysis of the same alignment using RAxML v. 8 (Stamatakis 2014) based on the GTR substitution model with gamma-distribution rate variation for each partition. Reference strains were obtained from Lombard et al. (2019b). The tree is rooted to Fusarium concolor (NRRL 13459). The analysis was performed

GenBank numbers: $\mathrm{CAL}=\mathrm{MW} 403051$, RPB $1=$ MW403052, RPB 2= MW403053, TEF1- $\alpha=$ MW403054.

Notes: Fusarium clavum belongs to the Fusarium incarnatum-equiseti species complex (Wang et al. 2019a; Xia et al. 2019). In a three-locus phylogeny (Fig. 131), the isolate (BRIP 70756a) was recovered in a well-supported clade (99\% MLBS, 1.00 BYPP) with the type strain $F$. clavum (CBS 126202). Fusarium clavum has been reported from various plants, human organs, and soil in Africa, Europe, Asia, and the USA (Xia et al 2019; Matic et al. 2020). Although the isolate (BRIP 70756a) was isolated from stalk rot on S. bicolor, the pathogenicity of this isolate has not been established (Fig. 130). This is the first record of $F$. clavum on S. bicolor, and the first record of this fungus in Australia.

Fusarium queenslandicum T.B. Potter, A.H. Sparks, Vaghefi \& R.G. Shivas, sp. nov.

MycoBank number: MB837495; Facesoffungi number: FoF 09579; Fig. 132

Etymology. The name refers to the State of Queensland, from where this fungus was collected.

Holotype: BRIP 70769a using MrBayes v. 3.2 .4 (Ronquist et al. 2012) based on the K80, $\mathrm{K} 80+\mathrm{G}$, and $\mathrm{HKY}+\mathrm{G}$ nucleotide substitution models selected for CAL, RPB2, and TEF1- $\alpha$, respectively, using PAUP v. 4.0 (Swofford 2003) and MrModeltest v. 2.3. (Nylander 2009). Maximum likelihood bootstrap support values (MLBS) greater than $80 \%$ are placed above the nodes and Bayesian posterior probabilities (BYPP) equal to or greater than 0.95 . Branches with MLBS $=100 \%$ and $\mathrm{BYPP}=1.00$ are thickened. Scale bar indicates the number of substitutions per nucleotide. Ex-type strains are in bold and newly isolate is in blue

Saprobic on dead stalk of Sorghum bicolor. Sexual morph Undetermined. Asexual Morph Conidiophores abundant on aerial mycelium, unbranched, lateral or terminal. Conidiogenous cells monophialides, subcylindrical. Microconidia in false heads on aerial mycelium, $4-25 \times 3-4.5 \mu \mathrm{m}$, narrowly ellipsoidal or reniform, hyaline, aseptate. Sporodochia and macroconidia not seen. Chlamydospores $7-13 \times 7-9 \mu \mathrm{m}$, abundant, globose to ellipsoidal, intercalary or terminal, single or in pairs, rough-walled.

Culture characteristics: Colonies on PDA reaching $90 \mathrm{~mm}$ at $24{ }^{\circ} \mathrm{C}$ after $28 \mathrm{~d}$, surface appressed with velvety cream patches of aerial mycelium up to $1 \mathrm{~cm}$ diam., subhyaline on the agar; reverse pale buff; on SNA with sparse aerial mycelium, cream becoming pale violet in the central part; sporulation abundant on PDA, SNA, WA and carnation leaf pieces.

Material examined: AUSTRALIA, Goondiwindi, on dead stalk of Sorghum bicolor, 19 February 2018, N. Vaghefi, BRIP 70769a (holotype), includes an ex-type living culture.

GenBank numbers: RPB $1=\mathrm{MW} 038832$, RPB $2=\mathrm{MW} 038833, \quad$ TEF $1-\alpha=\mathrm{MW} 038834$, TUB2 $=$ MW403138.

Notes: Fusarium queenslandicum belongs to the $F$. oxysporum species complex (Lombard et al. 2019a) based 

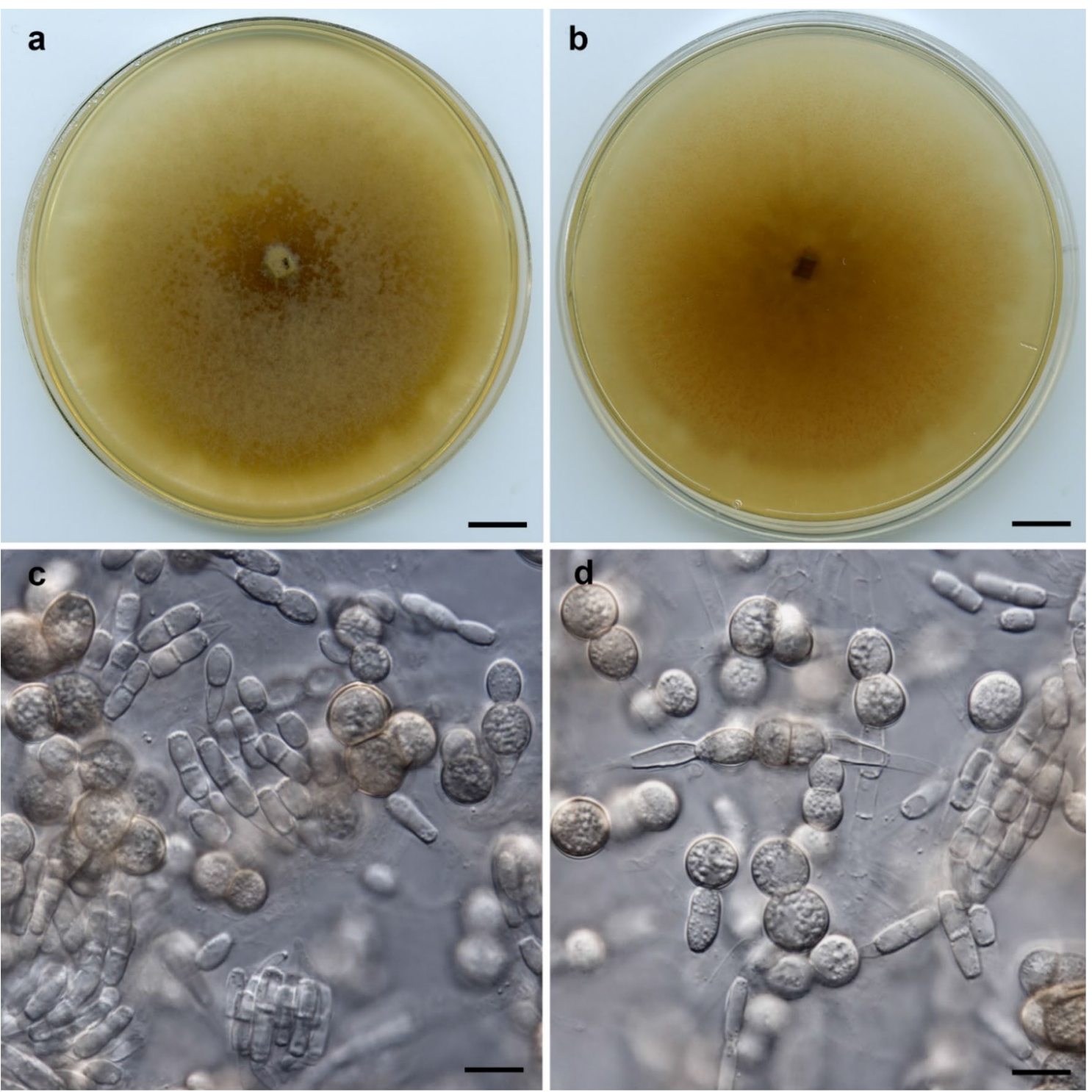

Fig. 130 Fusarium clavum (BRIP 70756a, new record). a, b Culture on PDA from surface and reverse. c, d Conidia and chlamydospores. Scale bars: $\mathbf{a}, \mathbf{b}=1 \mathrm{~cm}, \mathbf{c}, \mathbf{d}=10 \mu \mathrm{m}$

on sequence comparisons of the RPB1, RPB2, TEF1- $\alpha$ and TUB2 loci with those available in the Fusarium MLST database (http://www.cbs.knaw.nl/fusarium). The TEF1- $\alpha$ sequence of $F$. queenslandicum was identical to a culture (CBS $412.90=$ NRRL 36464) collected from tomato in Israel that was identified as $F$. languescens. A pairwise comparison revealed 10 nucleotide variations between the TEF1- $\alpha$ sequence of $F$. queenslandicum and that of the extype culture of $F$. languescens (CBS 64578=NRRL 36531). The four-locus phylogeny based on sequences of CAL, RPB2, TEF1- $\alpha$, and TUB2 clearly separated $F$. queenslandicum from $F$. languescens and other species within the FOSC (Fig. 133). Fusarium queenslandicum was isolated from an asymptomatic stalk of cultivated Sorghum bicolor (Fig. 132).

\section{Mariannaea G. Arnaud ex Samson}

Notes: We follow the latest treatment and updated accounts of Mariannaea in Hyde et al. (2020b).

Mariannaea camelliae N. Suwannarach \& J. Kumla, sp. nov.

Mycobank number: MB838938, Facesoffungi number: FoF 09959; Fig. 134

Etymology: The specific epithet "camelliae" refers to generic name of the host plant, Camellia. 


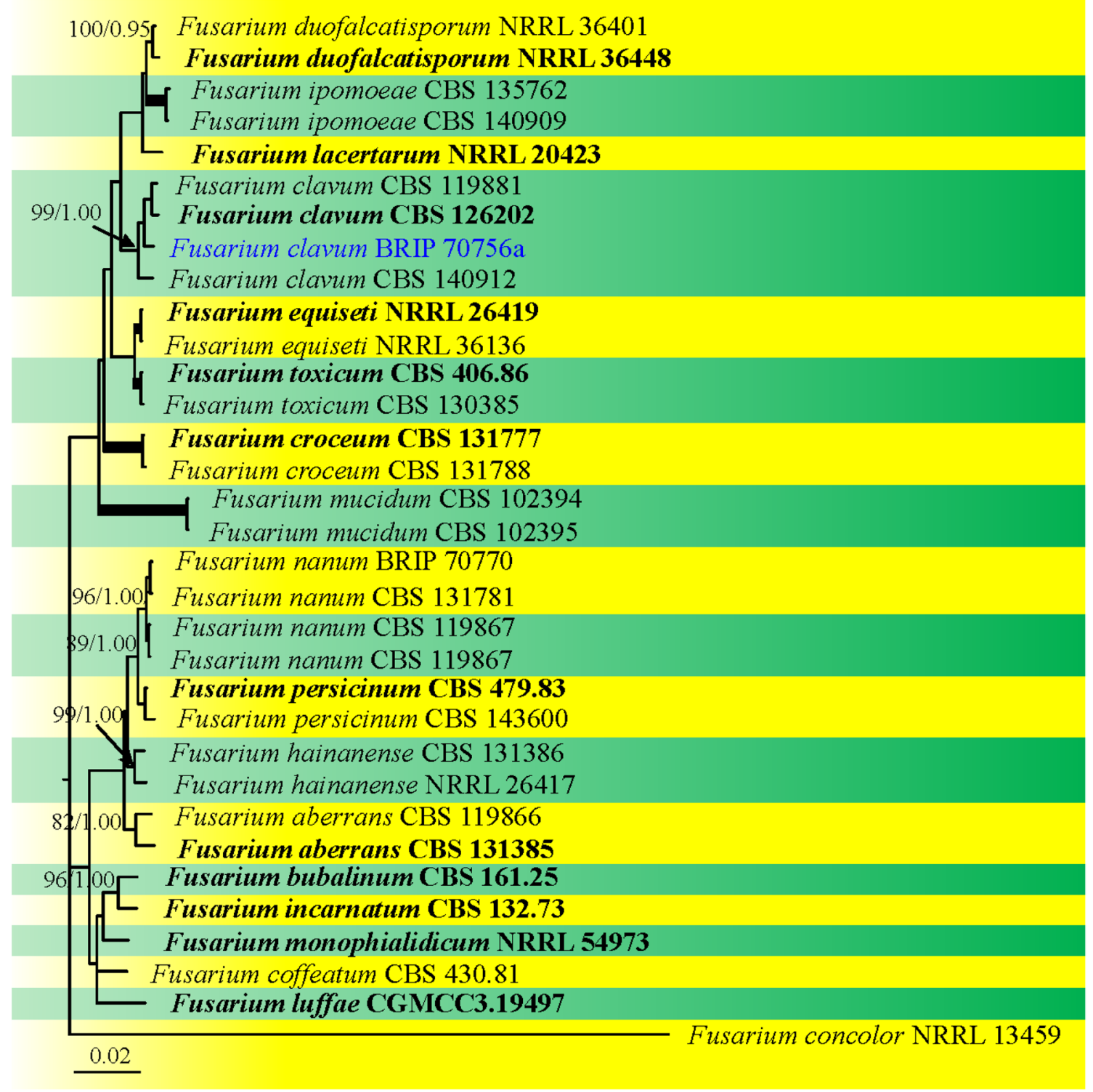

Fig. 131 Phylogram generated from Bayesian analysis based on combined CAL, RPB2, and TEF1- $\alpha$ sequence data representing Fusarium incarnatum-equiseti species complex and related taxa. The second measure of branch support was obtained through Maximum Likelihood (ML) analysis of the same alignment using RAxML v. 8 (Stamatakis 2014) based on the GTR substitution model with gamma-distribution rate variation for each partition. Reference sequence were obtained from Xia et al. (2019). The tree is rooted to Fusarium concolor (NRRL 13459). The analysis was performed

\section{Holotype: SDBR-CMU329}

Endophyte from leaves of Camellia sinensis var. assamica. Sexual morph Undetermined. Asexual morph Colonies growing on PDA growing moderately fast, $30-50 \mathrm{~cm}$ diam., within 1 week at $25{ }^{\circ} \mathrm{C}$, consisting of a matted felt with velutinous appearance, in age becoming funiculose to fasciculate, slightly powdery upon sporulation, surface of the colonies pale yellowish, with age brown, margin regular using MrBayes v. 3.2.4 (Ronquist et al. 2012) based on the K80, $\mathrm{K} 80+\mathrm{G}$, and $\mathrm{HKY}+\mathrm{G}$ nucleotide substitution models selected for CAL, RPB2, and TEF1- $\alpha$, respectively, using PAUP v. 4.0 (Swofford 2003) and MrModeltest v. 2.3. (Nylander 2009). Maximum likelihood bootstrap support values (MLBS) greater than $80 \%$ are placed above the nodes and Bayesian posterior probabilities (BYPP) equal to or greater than 0.95. Branches with MLBS $=100 \%$ and $\mathrm{BYPP}=1.00$ are thickened. Scale bar indicates the number of substitutions per nucleotide. Ex-type strains are in bold and newly isolate is in blue

or irregular; reverse yellowish brown. Colonies growing on MEA growing moderately fast, $30-4.7 \mathrm{~cm}$ diam., within 1 week at $25^{\circ} \mathrm{C}$, consisting of a flat, homogeneous, mycelium loose, surface of the colonies white, margin regular or irregular; reverse white. Colonies growing on OA growing moderately fast, $30-50 \mathrm{~cm}$ diam., within 1 week at $25^{\circ} \mathrm{C}$, consisting of a matted felt with velutinous appearance, slightly powdery upon sporulation, surface of the 

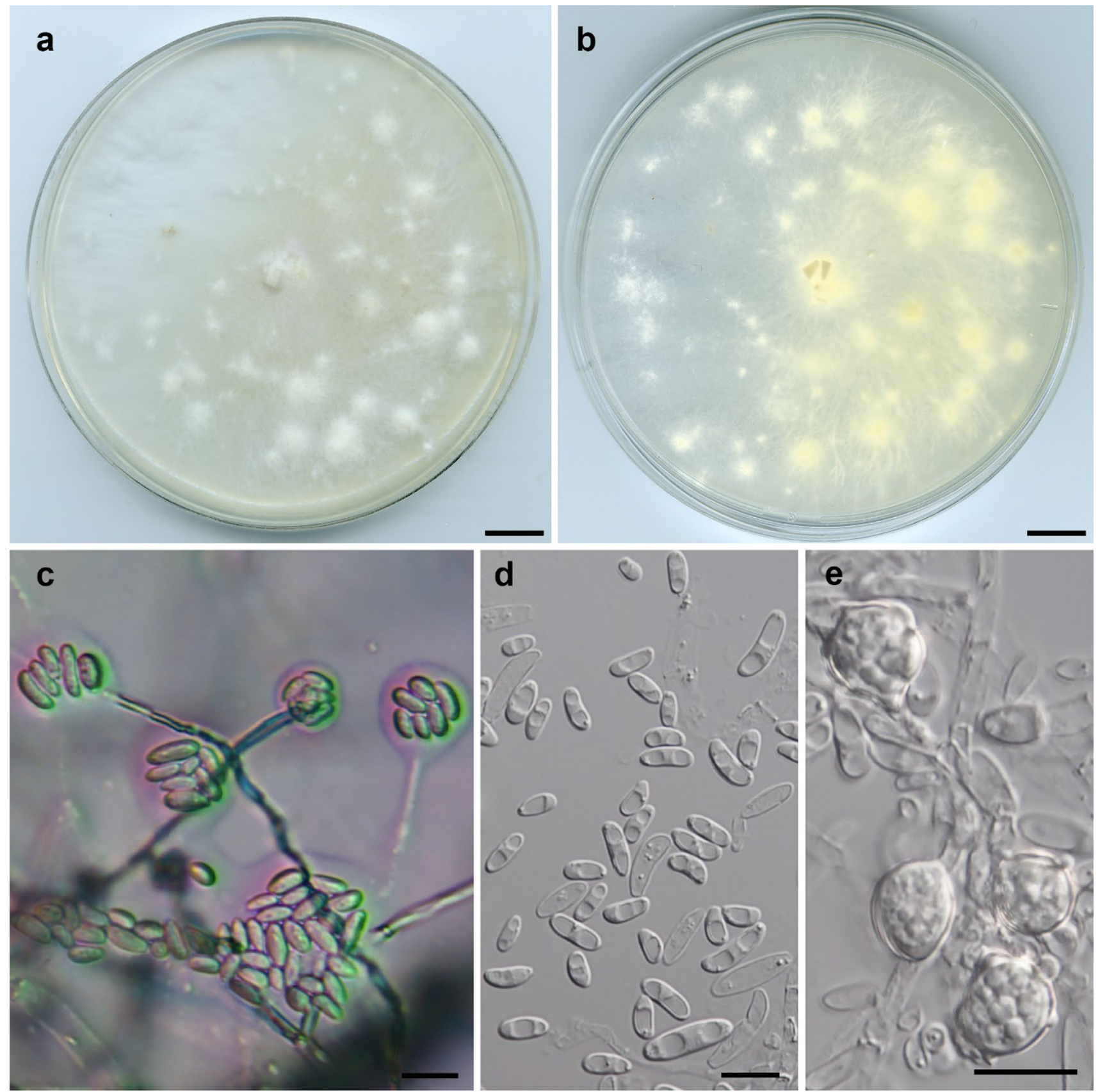

Fig. 132 Fusarium queenslandicum (BRIP 70769a, holotype). a, b Culture on PDA from surface and reverse. c Conidiophores with false heads. d Microconidia. e Chlamydospores. Scale bars: $\mathbf{a}, \mathbf{b}=1 \mathrm{~cm}, \mathbf{c}-\mathbf{e}=10 \mu \mathrm{m}$

colonies grayish white, margin regular or irregular; reverse grayish white. Hyphae hyaline to slightly pale brown, smooth-walled, up to $5 \mu \mathrm{m}$ wide, margin regular or irregular. Conidial formation was observed in PDA, MEA and OA. Conidiophores 105-225 $\mu \mathrm{m}$ long, axis 3.5-7.5 mm wide, usually macronematous, mononematous, erect, septate, bearing short branches with whorls of 1-4 phialides, or whorls of phialides in verticils along the main stalk; Axis and branches usually terminating into a longer phialide.
Phialides 13-17.5 ×2-4.5 $\mu \mathrm{m}$, flask-like, hyaline, smooth. Conidia 4-7 $\times 3-5 \mu \mathrm{m}$, globose to subglobose, aseptate, hyaline, smooth-walled, produced in imbricatechains. Chlamydospores present, $6-11 \times 4-9 \mu \mathrm{m}$, intercalary or terminal, produced in short chains, thick-walled, yellowish white, globose to ellipsoidal or lageniform.

Material exminated: THAILAND, Nan Province, Skad,

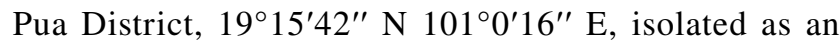
endophyte from leaves of Camellia sinensis var. assamica, 


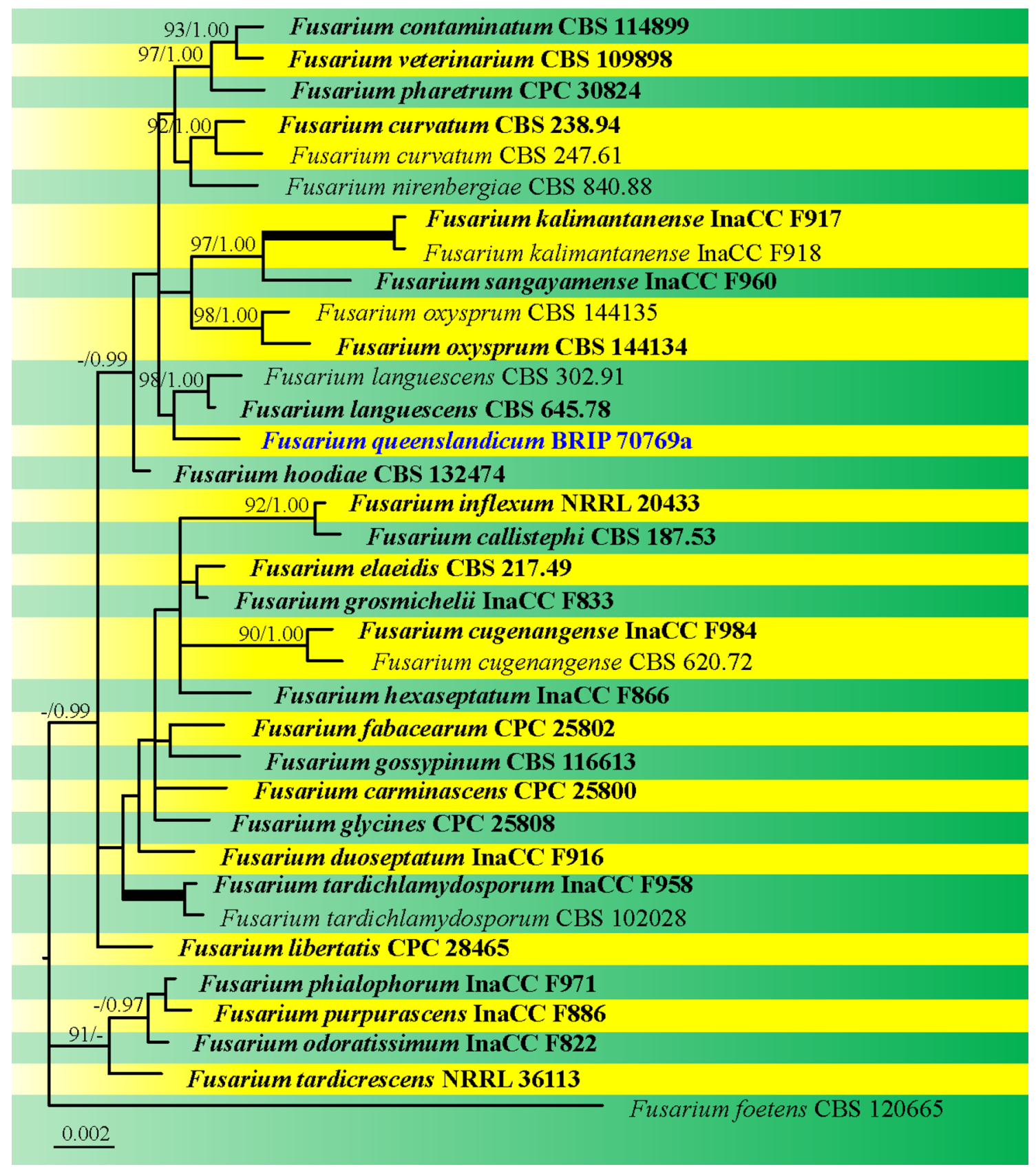

Fig. 133 Phylogram generated from Bayesian analysis based on combined CAL, RPB2, TEF1- $\alpha$, and TUB2 sequence data representing Fusarium oxysporum species complex and related taxa. The second measure of branch support was obtained through Maximum Likelihood (ML) analysis of the same alignment using RAxML v. 8 (Stamatakis 2014) based on the GTR substitution model with gamma-distribution rate variation for each partition. Reference sequence were obtained from Lombard et al. (2019a) and Maryani et al. (2019). The tree is rooted to Fusarium foetens (CBS 120665). The analysis was performed using MrBayes v. 3.2.4 (Ronquist et al.

September 2017, N. Suwannarach, SDBR-CMU329 (dried culture, holotype), ex-type living culture, TBRC13889.
2012) based on the K80, K80 + G, HKY + G, and SYM+ I+ G nucleotide substitution models selected for CAL, RPB2, TEF1- $\alpha$, and TUB2, respectively, using PAUP v. 4.0 (Swofford 2003) and MrModeltest v. 2.3. (Nylander 2009). Maximum likelihood bootstrap support values (MLBS) greater than $80 \%$ are placed above the nodes and Bayesian posterior probabilities (BYPP) equal to or greater than 0.95 . Branches with BYPP $=1.00$ and MLBS $=100 \%$ are thickened. Scale bar indicates the number of substitutions per nucleotide. Ex-type strains are in bold and newly sequence is in blue

GenBank number: ITS $=$ MH734517, LSU $=$ MH734518, TEF1- $\alpha=$ MH734520, TUB-2 = MH734519. 

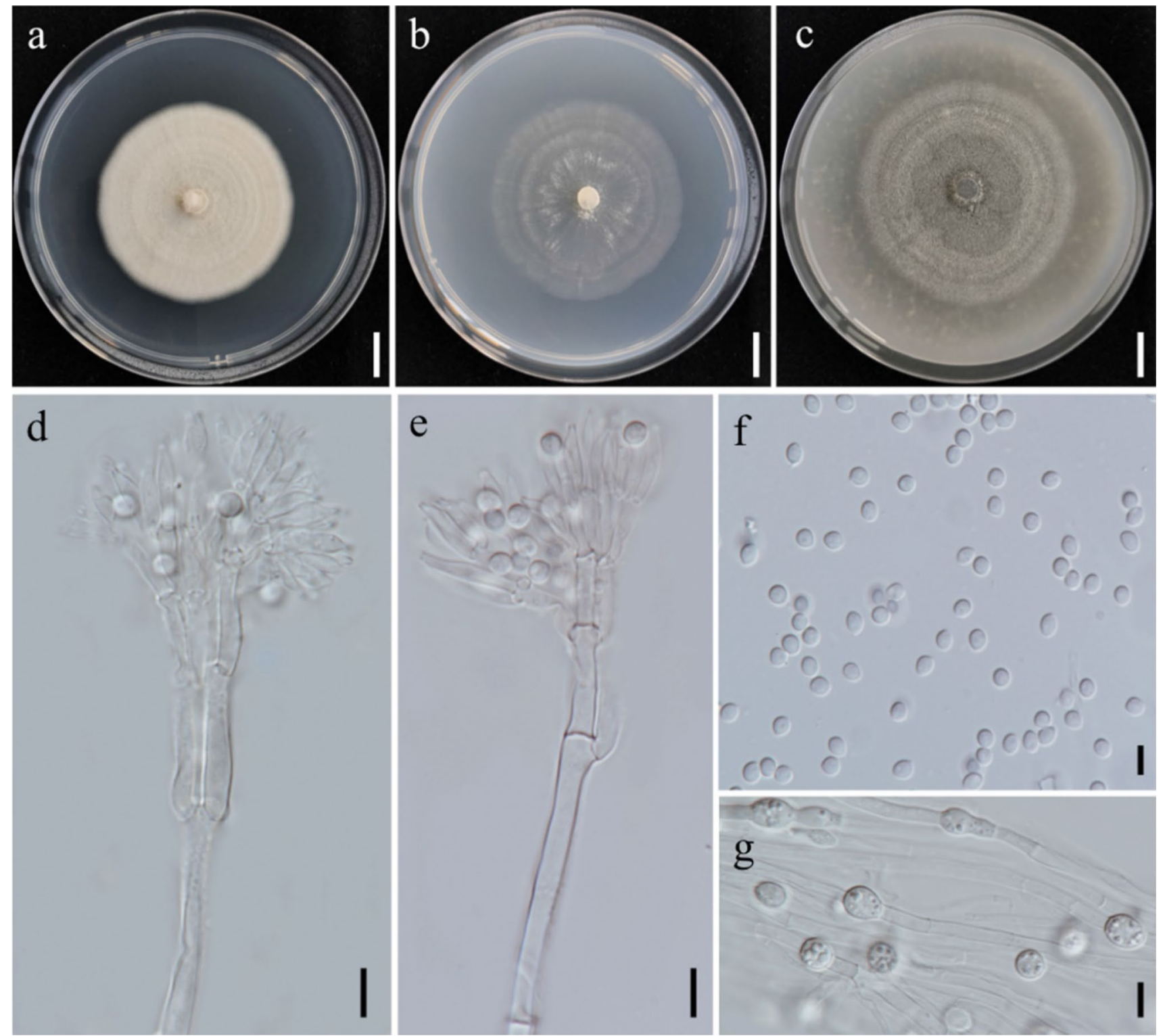

Fig. 134 Mariannaea camelliae (SDBR-CMU329, holotype). a Culture on PDA. b Culture on MEA. c Culture on OA. d, e Conidiophores and phialides. f Conidia. g Chlamydospores. Scale bars: $\mathbf{a}-\mathbf{c}=1 \mathrm{~cm}, \mathbf{d}-\mathbf{g}=10 \mu \mathrm{m}$

Notes: Mariannaea camelliae was isolated as an endophytic fungus from leaves of Camellia sinensis var. assamica collected in northern Thailand. Mariannaea camelliae is characterized by pale yellowish colonies on PDA, globose to subglobose conidia and presenting chlamydospores (Fig. 134), and shares resemblance to M. chlamydospora (Hu et al. 2016). However, M. camelliae differs from $M$. chlamydospora in size of conidia and chlamydospores. Phylogenetically, M. camelliae forms a well-supported lineage basal to M. atlantica, M. fusiformis and M. punicea $(100 \%$ MLBS, 1.00 BYPP; Fig. 135), the phylogeny also indicates that they are distinct species. Therefore, Mariannaea camelliae introduce as a new species based on morphology and phylogenetic evidence.

\section{Thyronectria Sacc.}

Notes: Thyronectria was introduced by Saccardo (1875) to accommodate $T$. patavina (Sacc.) as the type species. This species was later synonymized under Pleonectria (Hirooka et al. 2012). Therefore, Seeler (1940) introduced Thyronectria rhodochlora as the type species of Thyronectria. Recently, Pleonectria was synonymized with Thyronectria (Jaklitsch and Voglmayr 2014). Jaklitsch and Voglmayr (2014) accepted 42 Thyronectria species based on morphology and phylogenetic based evidence. In our 


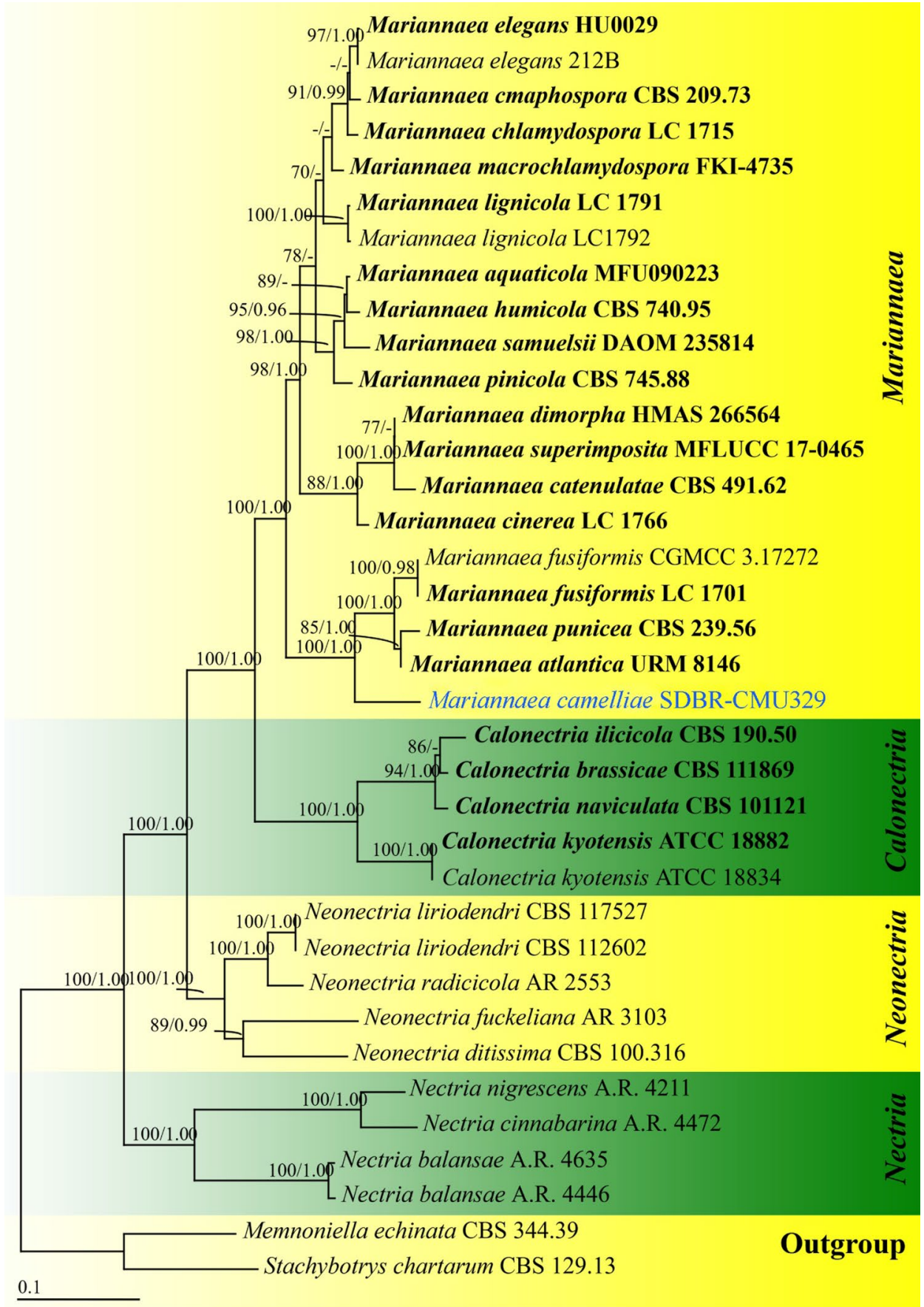

Fig. 135 Phylogenetic tree derived from maximum likelihood analysis of a combined ITS, LSU, TUB-2 and TEF1- $\alpha$ genes of 37 sequences and the aligned dataset was comprised of 2360 characters including gaps (ITS: 1-602, LSU: 603-1437, TUB-2: 1437-2061 and TEF1- $\alpha$ : 20622645). The average standard deviation of the split frequencies of the BI analysis was 0.002106. A best scoring RAxML tree was established with a final ML optimization likelihood value of -14705.9596 . The matrix had 1180 distinct alignment patterns with $33.94 \%$ undetermined characters or gaps. Estimated base frequencies were found to be: $\mathrm{A}=0.2219, \mathrm{C}=0.2777, \mathrm{G}=0.2538, \mathrm{~T}=0.2466$; substitution rates $\mathrm{AC}=1.4433$, $\mathrm{AG}=2.8971, \mathrm{AT}=1.5532, \mathrm{CG}=0.7904, \mathrm{CT}=5.2827, \mathrm{GT}=1.0000$; proportion of invariable sites $=0.4850$ and gamma distribution $=0.6770$. Memnoniella echinata CBS 344.39 and Stachybotry chartarum CBS 129.13 were used as outgroup. Numbers above branches are the bootstrap statistics percentages (left) and Bayesian posterior probabilities (right). Branches with bootstrap values equal to or greater than $70 \%$ are shown at each branch and the bar represents 0.1 substitutions per nucleotide position. Hyphen (-) represents support values equal to or less than $70 \% / 0.95$. Ex-type strains are in bold and newly generated sequence is in blue 
study, we introduce a record of Thyronectria caudata from Uzbekistan.

Thyronectria caudata (Malençon) Jaklitsch \& Voglmayr, Persoonia 33: 195 (2014)

Index Fungorum number: IF808296; Facesoffungi number: FoF 09960; Fig. 136

$\equiv$ Thyronectria lamyi var. caudata Malençon, Bull. Trimestriel Soc. Mycol. France 95: 99 (1979)

Saprobic on woody twigs. Sexual morph Ascomata 280-380 $\mu \mathrm{m}$ high, $300-400 \mu \mathrm{m}$ diam. $(\bar{x}=329 \times 356 \mu \mathrm{m}$, $\mathrm{n}=15$ ), superficial on the hypostroma, not surrounded by bark flaps, aggregated in round or elongated clusters, rarely solitary, globose to obovoid, rarely cupulate when dry, reddish brown. Ostiole central, slightly papillate or flat-umbilicate, periphysate. Peridium $30-60 \mu \mathrm{m}$ wide, widest at the apex, composed of several layers of yellowish-brown cells of textura angularis. Hamathecium comprising numerous, 1.5-3.5 $\mu \mathrm{m}$ wide, filamentous, rarely branched, hyaline, paraphyses. Asci $90-120 \times 15-25 \mu \mathrm{m}(\bar{x}=103.5 \times 19 \mu \mathrm{m}$, $\mathrm{n}=15$ ), 8-spored, unitunicate, cylindrical to clavate, pedicellate, rounded at the apex, mostly filled with ascoconidia when mature. Ascospores $20-28 \times 4-6 \mu \mathrm{m}(\bar{x}=24 \times 5 \mu \mathrm{m}$, $\mathrm{n}=30$ ), 1-2-seriate, overlapping, narrowly clavate, with 6-10 transverse septa and 1 longitudinal or oblique septum in 1 or few cells of, all cells budding to produce oblong, 1-celled, hyaline, straight to slightly curved ascoconidia $3.5-5 \times 1-1.5 \mu \mathrm{m}(\bar{x}=4 \times 1 \mu \mathrm{m}, \mathrm{n}=40)$. Asexual morph Undetermined.

Material examined: UZBEKISTAN, Jizzakh Province, Zaamin District, Zaamin National Nature Park, river of Uriklisoy, Turkestan range of Pamir-Alay Mountains, on dead stems of Berberis oblonga (Regel) C.K. Schneid (Berberidaceae), 13 July 2019, Yusufjon Gafforov YG-Z54-1 (TASM 6160, KUN-HKAS 115784, new record); ibid., 13 July 2019, Y. Gafforov and T. Kholmuradova, YG-Z54-2 (TASM 6161, KUN-HKAS 115785).

GenBank numbers: ACT $=$ MZ508400, MZ508401, ITS $=$ MZ493292, MZ493293, LSU $=$ MZ493306, MZ493307, TEF1- $\alpha=$ MZ508402, MZ508403.

Notes: Thyronectria caudata was initially introduced as Thyronectria lamyi var. caudate by Malençon (1979). Jaklitsch and Voglmayr (2014) synonymized this as the Thyronectria caudata. Morphological features (Fig. 136) of TASM 6160 and TASM 6161 are similar to those in the holotype of Thyronectria caudata. These collections were all made from the same host genus (Berberis) but in different locations. Phylogenetically, TASM 6160 and TASM 6161 are closely related to Thyronectria berberidicola (Ma et al. 2020) in the multi-gene concatenated tree (Fig. 137). However, this species does not deposit in MycoBank or Index Fungorum. Ma et al. (2020) introduced Thyronectria berberidicola from the Xinjiang Uygur Autonomous Region in China and the morphological features between Thyronectria caudata and this species results in no outstanding differences. We reckon the collections made by Ma et al. (2020) should also belong to Thyronectria caudata.

\section{Pleurotheciales Réblová \& Seifert}

Notes: We follow the latest treatment and updated accounts of Pleurotheciales in Réblová et al. (2016) and Hyde et al. (2019, 2020b, d).

\section{Pleurotheciaceae Réblová \& Seifert}

Notes: Réblová et al. (2016) introduced a single family Pleurotheciaceae in Pleurotheciales and introduced two genera in this family viz. Adelosphaeria and Melanotrigonum. Based on their multi-gene phylogenetic analyses, taxa in eleven genera formed six monophyletic clades within this family (Réblová et al. 2016). However, only eight genera were listed in this family by Wijayawardene et al. (2018) which was updated to 11 genera by Wijayawardene et al. (2020) and Hyde et al. (2020b). Pleurotheciaceae is known from both sexual and asexual morphs which are characterized by perithecial, immersed to superficial, ascomata, with papillate or with a central rarely eccentric neck, leathery to fragile, carbonaceous peridial walls, unitunicate, 8-spored, cylindrical or cylindrical-clavate asci, with a J-, apical ring, abundant paraphyses and ellipsoidal to fusiform, hyaline or versicolorous, septate ascospores, with polar cells, hyaline to brown at the central cell (Réblová et al. 2016; Luo et al. 2018; Hyde et al. 2020d). The asexual morphs of genera in this family have been reported as hyphomycetes forming loose fascicles or indeterminate synnemata. Conidiophores are macronematous or semi-macronematous, or sometimes forming percurrently conidiophores. Conidiogenous cells produce holoblastic conidia, with rhexolytic conidial secession on short denticles or extending polyblastically on a sympodial rachis, or schizolytic on monoblastic or solitary thallic conidiogenous cells. Conidia are hyaline to brown or versicolorous, varied in shape, aseptate or septate (Réblová et al. 2016; Luo et al. 2018; Hyde et al. 2020b, d). Taxa of this family can be found on a wide range of hosts and substrates from terrestrial and freshwater habitats (Farr and Rossman 2020).

\section{Phaeoisaria Höhn.}

Notes: Phaeoisaria was introduced by von Höhnel (1909) to accommodate a hyphomycetous taxon which was found on a bamboo substrate with Phaeoisaria bambusae as the type species. The genus was characterized by indeterminate synnemata with aseptate or septate ellipsoidal, obovoidal, fusiform-cylindrical to falcate, hyaline conidia (Réblová et al. 2016; Luo et al. 2019; Hyde et al. 2019). For the latest 

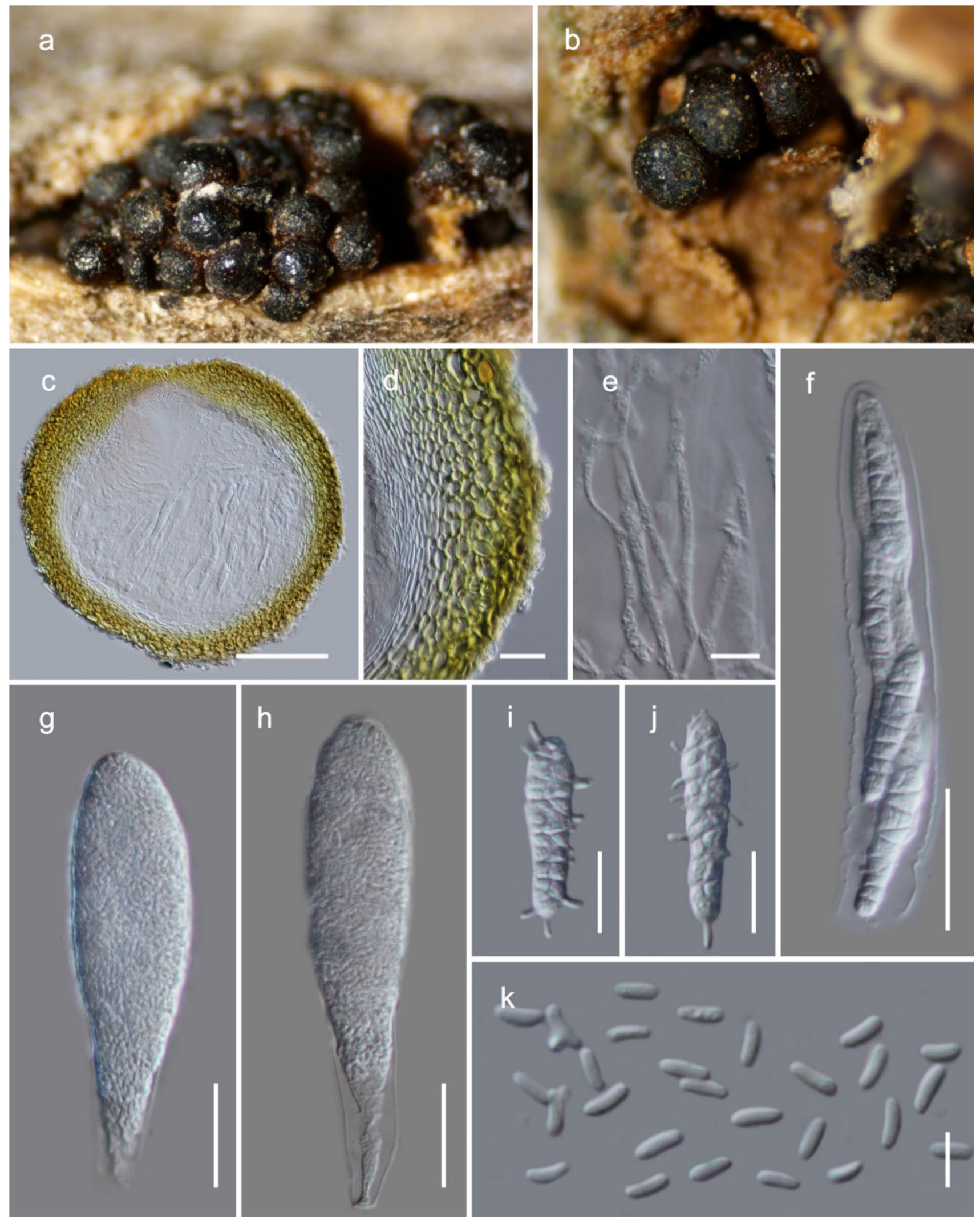

Fig. 136 Thyronectria caudata (TASM 6160, KUN-HKAS 115784, new record). a, b Perithecial aggregates on host surface. c Longitudinal section of a perithecium. d Section of the peridium cells. e Paraphyses. f-h Asci. i, j Ascospores. k Ascoconidia. Scale bars: $\mathbf{c}=100 \mu \mathrm{m}, \mathbf{d}, \mathbf{f}-\mathbf{h}=20 \mu \mathrm{m}, \mathbf{e}, \mathbf{i}, \mathbf{j}=10 \mu \mathrm{m}, \mathbf{k}=5 \mu \mathrm{m}$ 


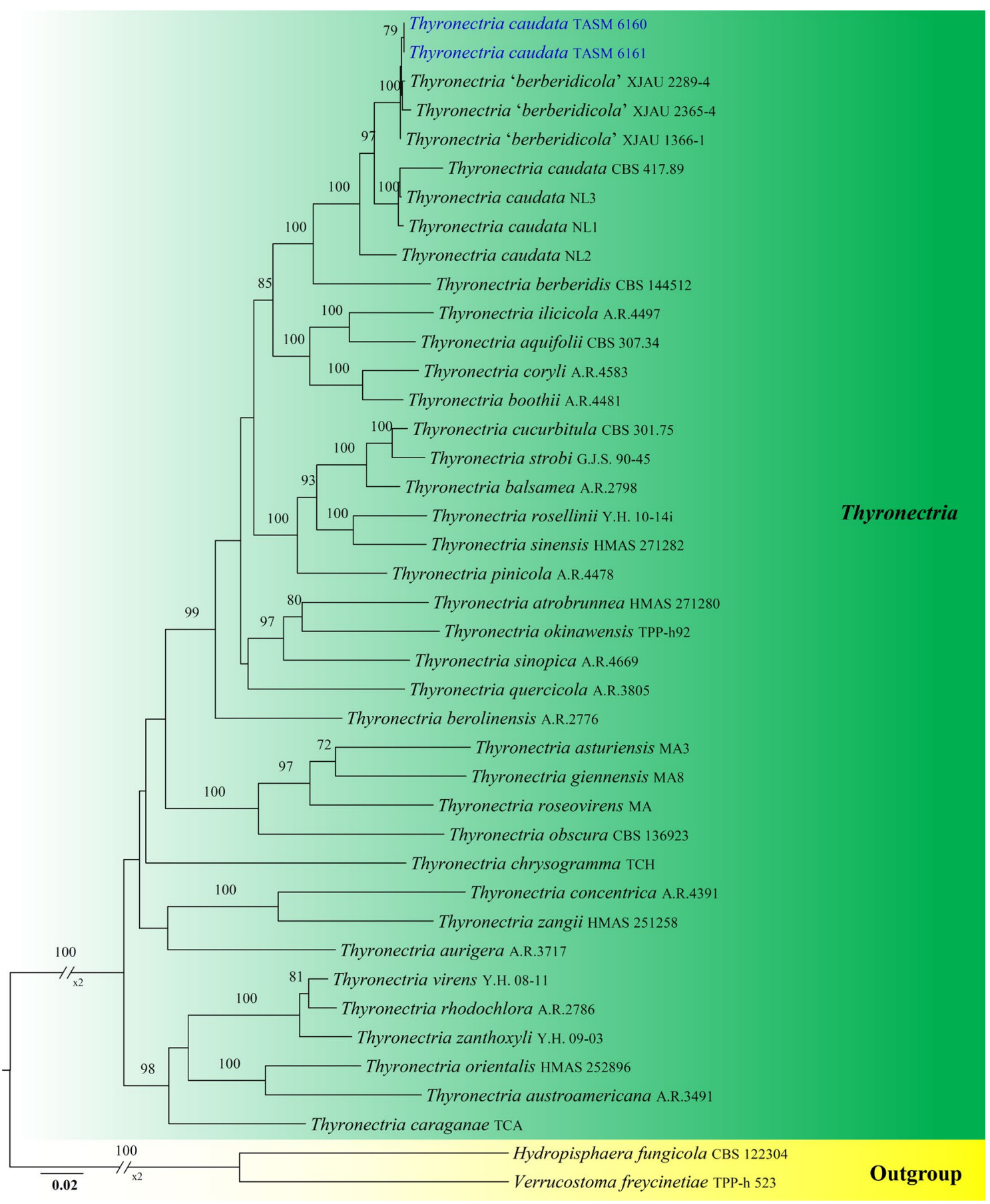

Fig. 137 Phylogram generated from the best scoring of the RAxML tree based on combined LSU, ITS, ACT, BTUB and TEF1- $\alpha$ sequence dataset to indicate the taxa in Thyronectria. Forty-one strains are included in the combined analyses which comprise a total of 4040 characters. Hydropisphaera fungicola (CBS 122304) and Verrucostoma freycinetiae (TPP-h 523) are selected as the outgroup taxa. The best RAxML tree with a final likelihood value of - 28737.934247 is presented. RAxML analysis yielded 1441 dis- tinct alignment patterns and $21.81 \%$ of undetermined characters or gaps. Estimated base frequencies were as follows: $\mathrm{A}=0.218868$, $\mathrm{C}=0.290291, \mathrm{G}=0.260083, \mathrm{~T}=0.230759$, with substitution rates $\mathrm{AC}=0.91049, \quad \mathrm{AG}=2.312893, \quad \mathrm{AT}=1.471029, \quad \mathrm{CG}=0.955498$, $\mathrm{CT}=5.294875, \mathrm{GT}=1.000000$; gamma distribution shape parameter alpha $=0.629127$. Bootstrap support values for maximum likelihood (MLBS) equal to or greater than $70 \%$ is given above the nodes. Newly generated sequences are in blue 
treatment and updated accounts of Phaeoisaria, we follow Luo et al. (2019), Hyde et al. (2020a) and Wijayawardene et al. (2020).

Phaeoisaria aquatica Z.L. Luo, X.J. Su \& K.D. Hyde, Mycol. Progr. 17(5): 514 (2018)

Index Fungorum number: IF821837; Facesoffungi number: FoF 03411; Fig. 138

Saprobic on submerged decaying wood. Sexual morph Undetermined. Asexual morph Hyphomycetous. Colonies effuse, solitary, dark brown to black, hairy, covered by white conidial mass. Mycelium immersed in the substrate. Synnemata $313-727 \times 11-23 \mu \mathrm{m}(\bar{x}=470 \times 16 \mu \mathrm{m}, \mathrm{n}=5)$, erect, straight to flexuous, dark brown to black, composed of compact parallel conidiophores, wide at the base, wide at the apical fertile region. Conidiophores macronematous, synematous, septate, branched, dark brown, smooth. Conidiogenous cell $12-21 \times 2-5 \mu \mathrm{m}(\bar{x}=17 \times 3.5 \mu \mathrm{m}, \mathrm{n}=10)$, polyblastic, integrated, terminal and intercalary, sympodial, cylindrical or attenuated towards tip, hyaline to light brown, denticulate, denticle conspicuously cylindrical. Conidia $4.5-8 \times 2-3 \mu \mathrm{m}(\bar{x}=5.5 \times 2 \mu \mathrm{m}, \mathrm{n}=30)$, ellipsoid to obovoid or sub-spatulate, straight, aseptate, hyaline, smooth-walled.

Culture characteristics: Conidia germinating on PDA within $24 \mathrm{~h}$ and germ tubes arising from both ends. Colonies on malt extract agar (MEA), reaching $2 \mathrm{~cm}$ diam. after 2 weeks at room temperature, flat, circular, surface slightly rough with crenated edge, white mist at the middle region, surrounded by greenish brown towards white grey at the margin, olivaceous dark green at the reverse with surrounded by white grey at the margin.

Material examined: THAILAND, Chiang Rai Province, Muang, Mae Yao Village, saprobic on submerged decaying wood, 23 September 2019, N. Huanraluek, MY01 (MFLU 21-0071, new record), living culture, MFLUCC 21-0094.

GenBank number: ITS $=$ MZ538522, LSU $=$ MZ538556, TEF1- $\alpha=$ MZ567098.

Notes: Phaeoisaria aquatica was introduced as a new species by Luo et al. (2018), which was found on decaying submerged wood in Jinsh River in China. The strain MFLUCC 21-0094 that was isolated and described in this study, is phylogenetically related to the type strain MFLUCC 16-1298 with 94\% MLBS, 1.00 BYPP support (Fig. 141). Furthermore, our strain (Fig. 138) shares similar features of dark brown synematous, compact parallel conidiophores and ellipsoid to obovoid, aseptate, hyaline conidia with Ph. aquatica (MFLUCC 16-1298), the type strain and both strains were found in freshwater habitats. Therefore, based on morphology and phylogenetic affinity, our strain MFLUCC 21-0094 is identified as Ph. aquatica and it is reported here as a new geographical record from Thailand.
Phaeoisaria synnematicus P.N. Singh \& S.K. Singh sp. nov. MycoBank number: MB830711; Facesoffungi number: FoF 06138; Fig. 139

Etymology: specific epithet 'synnematicus' refers to the formation of synnemata.

Holotype: AMH 10055

Color Codes Follow: Methuen Handbook of Colour (Kornerup and Wanscher 1978).

Saprobic on dead bark of Azadirachta indica Juss., in a terrestrial habitat. Sexual morph Undetermined. Asexual morph Vegetative hyphae septate, branched, smooth-walled, subhyaline to light olivaceous, up to $2.35 \mu \mathrm{m}$ wide. Chlamydospores $8.5-25.5 \times 6-10.5 \mu \mathrm{m}$, produced abundantly from submerged and superficial mycelium, intercalary, lateral to terminal, globose, sub-globose, clavate to ampulliform, solitary to catenate, dark brown, smooth walled, wall thickened and darkened, sessile, persistent to caducous, aseptate (rarely with one or two septa). Synnemata up to $960 \mu \mathrm{m}$ long and 12-30 $\mu \mathrm{m}$ diam., synnematal, erect, rigid, dark brown to olivaceous brown, composed of compact parallel appressed conidiophores, cylindrical to clavate. Conidiogenous cells $1.5-18 \times 0.5-2 \mu \mathrm{m}$, polyblastic, integrated, acropleurogenous, terminal or intercalary, sympodial, cylindrical to denticulate, hyaline, smooth walled, denticles simple to rarely branched, hyaline, up to $2 \mu \mathrm{m}$ long and $1 \mu \mathrm{m}$ wide. Secession schizolytic. Conidiophores 1.5-960 $\mu \mathrm{m}$ long, 1-3.5 $\mu \mathrm{m}$ wide, macronematous to semi-macronematous, highly geniculate, dark brown to olivaceous brown, synnematous, simple to dichotomously branched, emerging out at the apex and along the sides of the upper half or two thirds of each synnema, dark brown at the base, brown to pale brown towards the apex. Sometimes conidiophores reduced to a single conidiogenous cell, arising from aerial hyphae. Conidia $4-11 \times 2-5 \mu \mathrm{m}(\bar{x}=6 \times 2.5 \mu \mathrm{m}, \mathrm{n}=30)$, dimorphic, clavate to ellipsoidal, cylindrical to falcate, base narrowly truncate, tip obtuse, variable in size, sometimes constricted near septa, 1-2-guttulate, hyaline, aseptate to one septate, smooth-walled.

Culture characteristics: on MGYP (Malt Extract Glucose Yeast Peptone agar), slow growing, floccose, pale yellow (4A3), $37 \times 37$ diam. in 37 days at $25^{\circ} \mathrm{C}$, periphery light grey, floccose $(1 \mathrm{C} 1)$, margin regular, reverse dark brown (7F-4). Hyphae septate, unbranched to branched, smooth walled subhyaline to light olivaceous, $1.8-2.3 \mu \mathrm{m}$ wide.

Material examined: INDIA, Maharashtra, Pune District, dead bark of Azadirachta indica (Meliaceae), 4 June 2017, P.N. Singh, AMH 10055 (holotype), ex-type living culture, NFCCI 4479.

GenBank Numbers: ITS $=$ MK391494, LSU = MK391492.

Notes: The present taxon was isolated in pure culture from dead bark of Azadirchta indica. The overall morphological and cultural characteristics are distinct from other species in the genus. Though in having prominent synnemata 

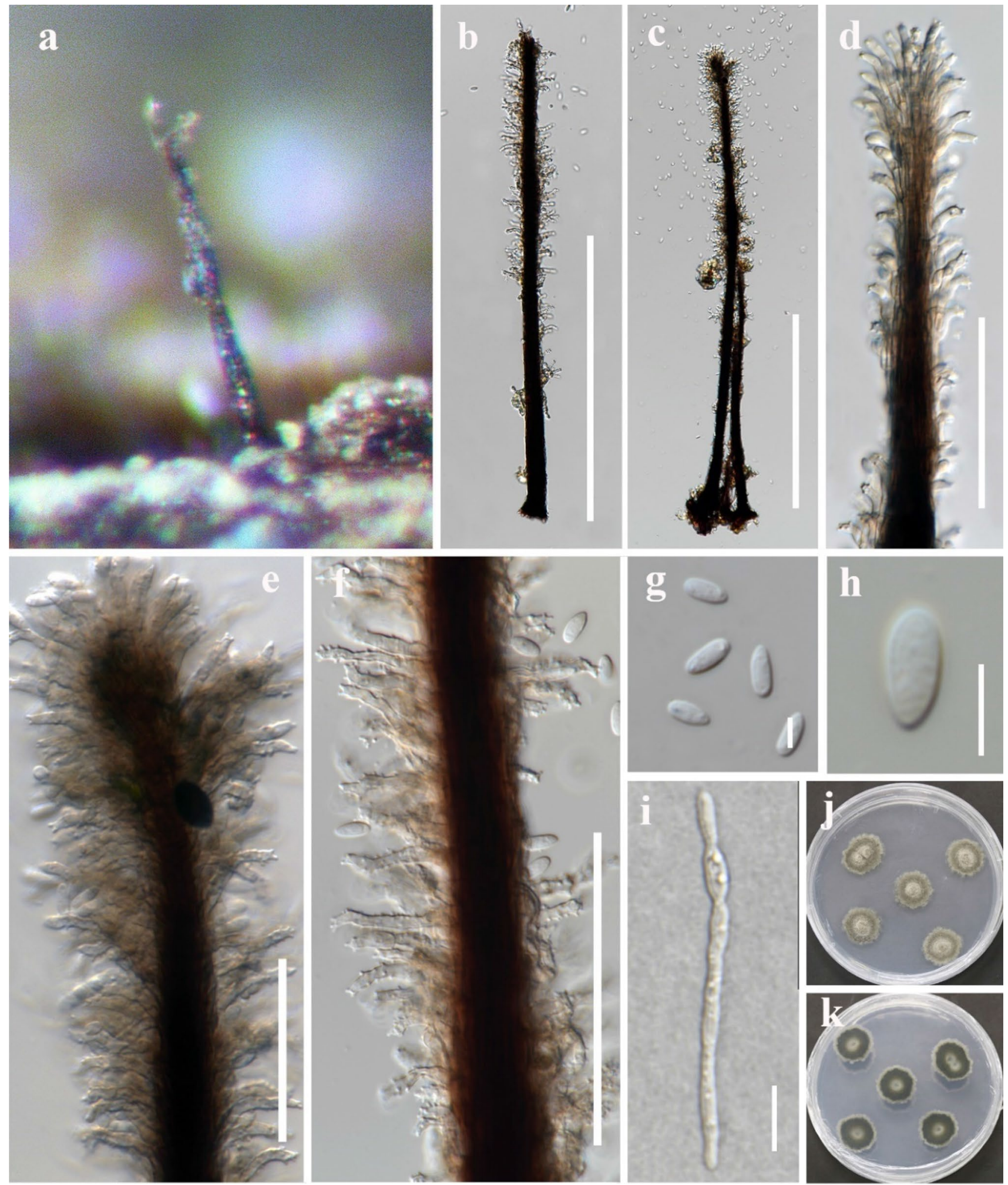

Fig. 138 Phaeoisaria aquatica (MFLU 21-0071, new record). a Conidiomata synnemata on wood. b, c Conidiophores. d-f Conon MEA from surface and reverse. Scale bars: $\mathbf{b}, \mathbf{c}=300 \mu \mathrm{m}, \mathbf{d}-$ idiogenous cells. g, h Conidia. i Germinated conidium. j, k Culture 

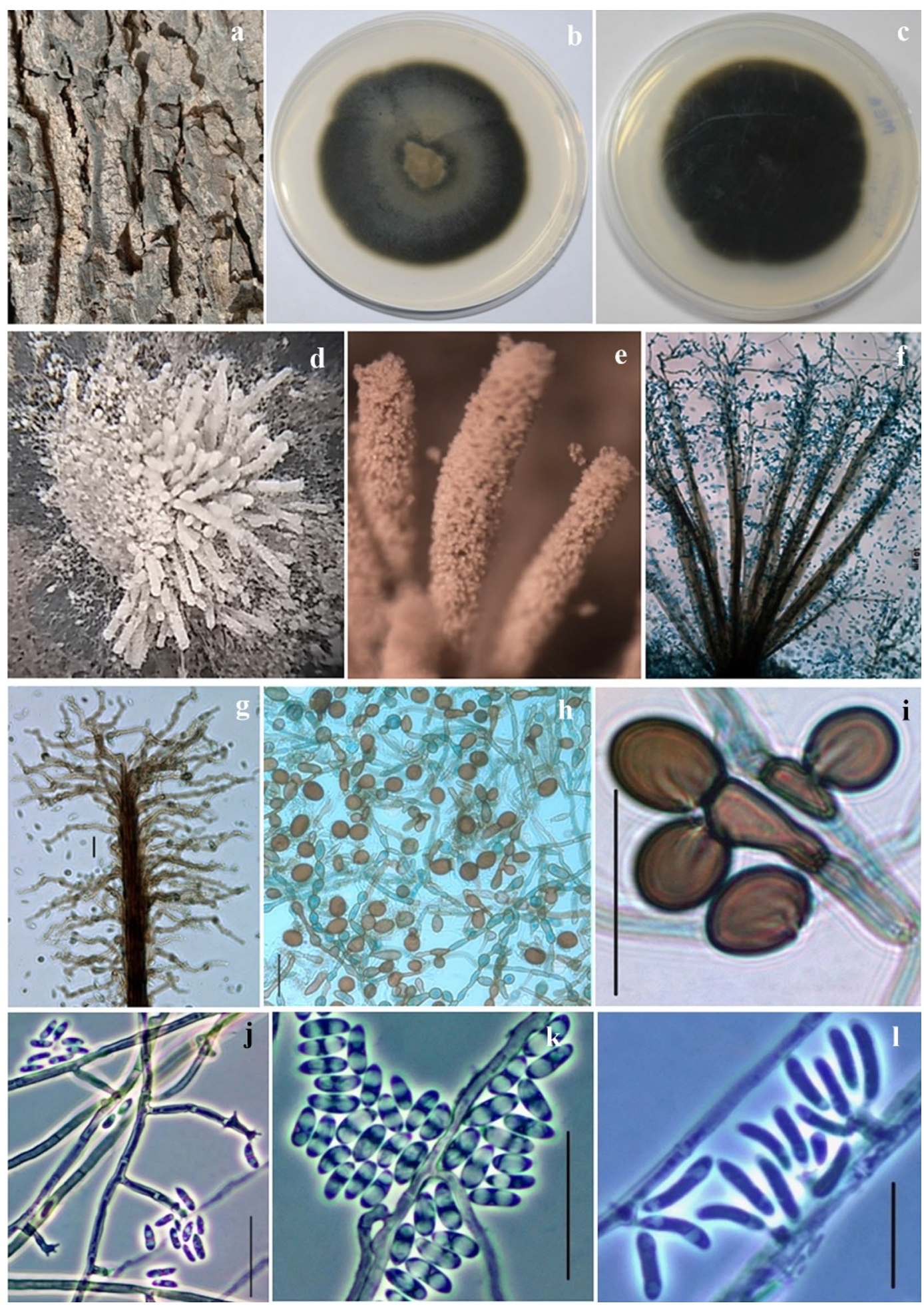

Fig. 139 Phaeoisaria synnematicus (AMH 10055, holotype). a Substrate (Bark of Azadirchta indica). b Colony morphology (front view). c Reverse view of colony. d, e Stereoscopic view of synnemata (in culture). f Numerous synnemata (slide culture microscopic view). g Synnema showing splaying conidiophores. h Numerous simple to catenate chlamydospores. i Persistent lateral to terminal produced sub-globose chlamydospores. $\mathbf{j}$ Enlarged view of conidiophores showing acropleurogenous conidiogenous cells and conidia. $\mathbf{k}$ Enlarged microscopic view of fusoid to clavate conidia and hyphae. 1 Curved and cylindrical guttulate conidia produced from small conidiophores. Scale bars: $\mathbf{g}-\mathbf{I}=20 \mu \mathrm{m}$ 
the morphological characters are comparable with some species, like Phaeoisaria clematidis (Hughes 1958) and $P$. aquatica (Luo et al. 2018). The new species is different in having smaller synnemata $(399-960 \times 12-30 \mu \mathrm{m})$ as against to larger $(1000-1500 \times 20-80 \mu \mathrm{m})$ in $P$. clematidis. Conidia in present taxon are significantly larger

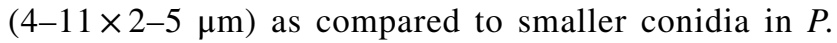
annesophiae $(4.5-9 \times 2-3.5 \mu \mathrm{m})$ (Crous et al. 2017), $P$. clematidis $(4-10 \times 1.5-2.5 \mu \mathrm{m})$, P. aquatica $(6.5-7.5 \mu \mathrm{m}$ long) and P. guttulata $(3.5-5.5 \times 2.5-4.8 \mu \mathrm{m})$ (Hyde et al. 2018a). Our new taxon is distinct from $P$. annesophieae (Crous et al. 2017) in the presence of synnemata, which is absent in the latter taxon. Similarly, the new taxon is also different from $P$. guttulata (Hyde et al. 2018a) in having larger synnemata $(480-700 \times 2-5$ vs. $399-960 \times 12.35-30 \mu \mathrm{m})$ and conidia $(3.5-5.5 \times 2.5-4.8$ vs. $4-11 \times 2-5 \mu \mathrm{m})$. As such, in overall morphological features, the present taxon is distinct from allied taxa.

Based on the sequence analysis, the proposed Phaeoisaria synnematicus is different from other known species. For the megablast analysis, the ITS sequence of $P$. synnematicus showed $97.58 \%$ (526/539) similarity and 2 gaps with P. annesophieae (CBS 143235, ex-type), 93.85\% (519/553) similarity and 12 gaps (2.17\%) with P. clematidis (CBS 113340), $92.67 \%$ (506/546) similarity and 15 gaps (2.74\%) with $P$. pseudoclematidis (MFLUCC 11-0393, type), 92.62\% (465/502) similarity and 14 gaps $(2.78 \%)$ with $P$. sedimenticola (CGMCC 3.14949, ex-type), 92.81\% (439/473) similarity and 11 (2.32\%) gaps with P. aquatica (MFLU 17-0918, ex-type) and $93.76 \%$ (511/545) similarity and 9 (1.65\%) gaps with $P$. guttulata (MFLUCC 17-1965, ex-type). The distinct morphological features and phylogenetic analysis confirm the proposed taxon to be a new species, Phaeoisaria synnematicus (Figs. 139 and 141).

\section{Pleurotheciella Réblová, Seifert \& J. Fourn.}

Notes: Pleurotheciella was introduced by Réblová et al. (2012) and is typified by P. rivularia Réblová, Seifert \& Fournier. The genus was introduced to accommodate pleurothecium-like taxa, which are characterized by non-stromatic, perithecial, semi-immersed to superficial, subglobose, heavily pigmented, glabrous ascomata, with bi-layered, leathery to fragile peridium, with abundant apically tapering paraphyses, unitunicate, 8 -spored, cylindrical-clavate asci with a J-, apical ring, and ellipsoidal-fusiform, hyaline to subhyaline, septate ascospores lacking mucilaginous sheath or appendages (Réblová et al. 2012). The hyphomycetous asexual morph is characterized by subhyaline unbranched to sparingly branched, septate conidiophores, often reduced to the conidiogenous cells, with integrated, subhyaline to hyaline, minute denticles, and ellipsoidal to obovoidal, hyaline, aseptate to septate conidia (Réblová et al. 2012).
Réblová et al. (2012) initially included two species in this genus. Subsequently, Réblová et al. (2016) included another new combination, Pleurotheciella uniseptata (Matsush.) Seifert in this genus. Luo et al. (2018) introduced other six lignicolous freshwater species of Pleurotheciella from the Greater Mekong Subregion. Hyde et al. (2018a) introduced Pleurotheciella krabiensis and P. tropica from submerged dead and decaying wood in Thailand. Currently, there are 11 species accommodated in this genus. Based on phylogenetic analyses coupled with morphological characteristics, we introduce a novel species $P$. dimorphospora occurring on dead branches of Malus sp. from a terrestrial environment in Yunnan, China.

Pleurotheciella dimorphospora H.B. Jiang, Phookamsak \& K.D. Hyde, sp. nov.

Index Fungorum number: IF558191; Facesoffungi number: FoF 09762; Fig. 140

Etymology: The specific epithet "dimorphospora" refers to the fungus forming two types of conidia.

Holotype: MFLU 20-0138

Saprobic on dead branches of Malus sp. Sexual morph Undetermined. Asexual morph Natural substrate visible as small, black, shiny dots on host substrate. Mycelium immersed to partly immersed, sparse, brown, composed of septate, branched hyphae. Conidiophores $9-15(-21) \times 2-4 \mu \mathrm{m}(\bar{x}=10.5 \times 2 \mu \mathrm{m}, \mathrm{n}=30)$, difficult to distinguish on host, macronematous, mononematous, subhyaline to brown, septate, unbranched to branched, erect on host surface. Conidiogenous cells holoblastic, terminal, integrated. Conidia (16.5-)20-27(-32) $\times(15-) 20-25(-28)$ $\mu \mathrm{m}(\bar{x}=23 \times 20.5 \mu \mathrm{m}, \mathrm{n}=30)$, dark brown to black, muriform, variedly shaped, ellipsoidal, subglobose, or irregular in shape, sectored, inconspicuously septate. In vitro: Dimorphic, with two types of conidial morphology. Type I: Conidiophores $4.5-6 \times 2.5-4 \mu \mathrm{m}(\bar{x}=5-3 \mu \mathrm{m}, \mathrm{n}=20)$, semi-macronematous or macronematous, mononematous, straight or flexuous, hyaline to brown, cylindrical, septate, unbranched. Conidiogenous cells holoblastic, terminal or intercalary, integrated, brown. Conidia 20-29×14-19 $\mu \mathrm{m}$ wide $(\bar{x}=24.5 \times 17 \mu \mathrm{m}, \mathrm{n}=30)$, brown, muriform, varied in shape, subglobose to cordiform, or irregular in shape, with a protuberant hilum. Type II: Conidiophores reduced to conidiogenous cells. Conidiogenous cells $6-8 \mu \mathrm{m}$ long $(\bar{x}=8 \mu \mathrm{m}, \mathrm{n}=20), 2-3 \mu \mathrm{m}$ wide $(\bar{x}=2 \mu \mathrm{m}, \mathrm{n}=20)$, holoblastic, terminal, hyaline, ellipsoidal, aseptate, unbranched. Conidia $8.5-10.8 \mu \mathrm{m}$ long $(\bar{x}=9.7 \mu \mathrm{m}, \mathrm{n}=20), 4-5 \mu \mathrm{m}$ wide ( $\bar{x}=4 \mu \mathrm{m}, \mathrm{n}=20$ ), hyaline, ellipsoidal, $0-1$-septate, 2-guttulate, smooth-walled.

Culture characteristics: Conidia germinating on WA within $24 \mathrm{~h}$ and germ tubes produced in all directions. Colonies on PDA, reaching 20-25 mm diam. after 14 days 


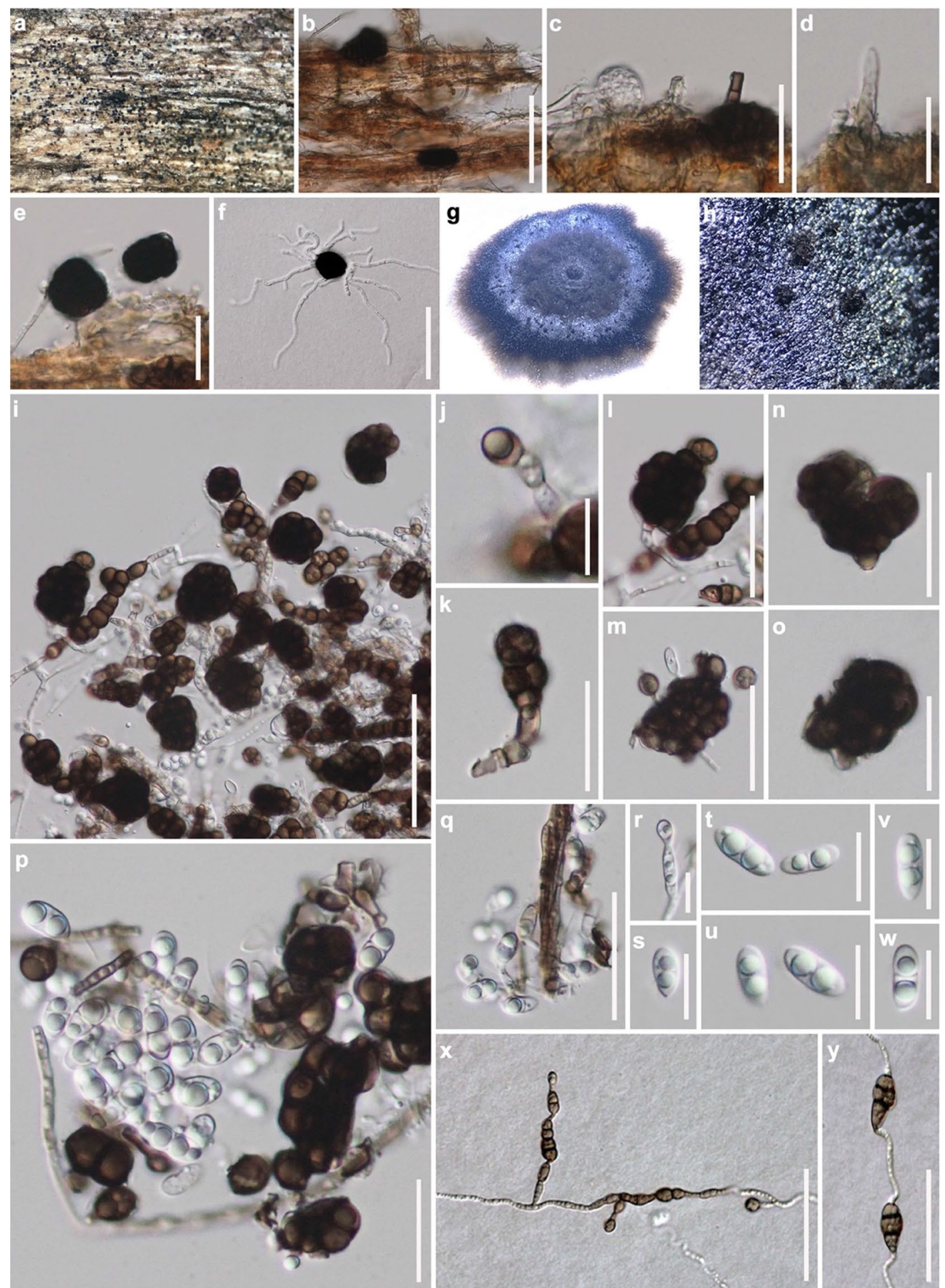

Fig. 140 Pleurotheciella dimorphospora (MFLU 20-0138, holotype; KUMCC 20-0185, ex-type). a Appearance of black, shiny conidia on host substrate. b Squash mount showing mycelia partly immersed in host substrate. c, d Conidiophores erect on host surface. e Black, muriform conidia on natural substrate. f Germinated conidium on WA. g Colony on PDA after 4 weeks. h Conidial masses induced on culture growing on PDA after 4 weeks. i, m-o Dark brown, muriform conidia on PDA. $\mathbf{j}-\mathbf{l}$ Conidiogenous cells with attached conidia. p Hyaline, didymosporous conidia associated with dark brown, muriform conidia. $\mathbf{q}, \mathbf{r}$ Conidiogenesis of hyaline conidia. $\mathbf{s}-\mathbf{w}$ Hyaline conidia. $\mathbf{x}, \mathbf{y}$ Lateral and intercalary conidia. Scale bars: $\mathbf{b}, \mathbf{f}, \mathbf{i}$, $\mathbf{x}=50 \mu \mathrm{m}, \mathbf{c}, \mathbf{l}-\mathbf{n}, \mathbf{q}, \mathbf{y}=30 \mu \mathrm{m}, \mathbf{d}, \mathbf{e}, \mathbf{k}, \mathbf{o}, \mathbf{p}=20 \mu \mathrm{m}, \mathbf{j}, \mathbf{r}-\mathbf{w}=10 \mu \mathrm{m}$ 
at room temperature, with subglobose, dense, humid, dark brown mycelium on the frontage, convex at the entre, dark brown to black in reverse. Mycelium $1.5-3 \mu \mathrm{m}(\bar{x}=2 \mu \mathrm{m}$, $\mathrm{n}=20$ ) wide, superficial to immersed in media, with dark brown, composed of septate, branched, smooth hyphae.

Material examined: CHINA, Yunnan Province, Kunming City, Kunming Institute of Botany Kunming Institute of Botany (102 44'19" E 25 ${ }^{\circ} 27^{\prime \prime} \mathrm{N}$; $1951 \mathrm{msl} \pm 3 \mathrm{msl}$ ), on dead branch of Malus sp. (Rosaceae), 18 August 2018, R. Phookamsak, KIB049 (MFLU 20-0138, holotype), ex-type living culture, KUMCC 20-0185.

GenBank Numbers: ITS = MW981446, MW981447, $\mathrm{LSU}=\mathrm{MW} 81444, \mathrm{MW} 91445, \mathrm{SSU}=\mathrm{MW} 981454$, MW981455, TEF $1-\alpha=$ MZ509663, MZ509664, RPB2 = MZ509665, MZ509666.

Notes: In this study, we observed a terrestrial fungus occurring on dead branches of Malus sp. in Kunming, Yunnan, China. The species forms dark brown to black, shiny conidia on the host tissue in nature. We obtained a pure culture of this fungus by single conidia isolation from these dark brown to black conidia and the species formed black conidial masses and hyaline conidial masses on PDA after 1 month. We re-isolated hyaline and brown conidia, and obtained DNA sequence data for both hyaline and brown conidia. Based on DNA sequence data analyses, the sample forming hyaline conidia (KUMCC 20-0185H) are supported to be conspecific to the sample forming brown conidia (KUMCC 20-0185).

Pleurotheciella dimorphospora can be distinguished from other Pleurotheciella species in forming two types of conidia and lacking sympodially conidia on conspicuous denticles (Fig. 140). The species formed a distinct clade, clustering with $P$. saprophytica with $100 \%$ MLBS, 1.00 BYPP (Fig. 141). Pleurotheciella saprophytica differs from $P$. dimorphospora in having polyblastic, sympodial, denticulate conidiogenous cells, with conspicuous denticles (Luo et al. 2018). A comparison of ITS and RPB2 nucleotide bases between $P$. dimorphospora and $P$. saprophytica shows that $P$. dimorphospora is different from $P$. saprophytica in $23 / 519$ bp (4.4\%) of ITS and 56/723 bp (7.7\%) of RPB2. According to the recommendation of Jeewon and Hyde (2016), we introduce $P$. dimorphospora as a new species from the terrestrial environment.

\section{Pseudodactylariales Crous}

Notes: We follow the latest treatment and updated accounts of Pseudodactylariales in Hyde et al. (2020b).

\section{Pseudodactylariaceae Crous}

Notes: Pseudodactylariaceae is a monotypic family introduced by Crous et al. (2017) to accommodate a monotypic genus Pseudodactylaria. We follow the latest treatment and updated accounts of Pseudodactylariaceae in Hyde et al. (2020b).

\section{Pseudodactylaria Crous}

Notes: Crous et al. (2017) introduced Pseudodactylaria to accommodate species $P$. hyalotunicata and $P$. xanthorrhoeae (type species). Pseudodactylaria is characterized by white erect conidiophores, fusoid-dllipsoid conidia that are surrounded by a thin mucilaginous sheath. Four species are presently included in Pseudodactylaria has presently included four species (Index Fungorum 2021).

Pseudodactylaria albicolonia R.J. Xu, Boonmee \& K.D. Hyde, sp. nov.

Index Fungorum number: IF558548; Facesoffungi number: FoF 09961; Fig. 142

Etymology: 'albicolonia' referring to the white colonies of this fungus.

\section{Holotype: MFLU 21-0095}

Saprobic on decaying submerged wood in a freshwater stream. Sexual morph Undetermined. Asexual morph Colonies effuse, gregarious, plentiful, white. Mycelium partly superficial, partly immersed in the substrate, consisting of branched, septate, smooth, hyaline hyphae. Conidiophores 43-85 $\times 3-5.5 \mu \mathrm{m}(\bar{x}=62 \times 4 \mu \mathrm{m}, \mathrm{n}=11)$, macronematous, mononematous, unbranched, solitary, erect, subcylindrical, straight, or slightly flexuous, hyaline, sometimes light brown at the base 1-4-septate, smooth, sometimes expands at the base and slightly constricted at the septa of the apex. Conidiogenous cells $18-51 \times 3-6 \mu \mathrm{m}(\bar{x}=34 \times 4 \mu \mathrm{m}, \mathrm{n}=14)$, holoblastic, polyblastic, integrated, terminal, sympodial, subcylindrical, straight or flexuous, hyaline, rough-walled, apical part forming a rachis with numerous, denticles, sometimes percurrently regenerating. Conidia $14-19.5 \times 2.5-4 \mu \mathrm{m}$ $(\bar{x}=17 \times 3 \mu \mathrm{m}, \mathrm{n}=30)$, acropleurogenous, solitary, fusiform, 0-1-septate, rarely slightly constricted at septum, hyaline, guttulate.

Culture characteristics: Conidia germinated on MEA media within $12 \mathrm{~h}$ and germ tubes were produced from both ends. Colony reached $3 \mathrm{~cm}$ at $28^{\circ} \mathrm{C}$ in the dark for 1 week, on MEA media, circular, flat, with fluffy, dense, white mycelium, edge entire.

Material examined: THAILAND, Chiang Rai Province, Mueang, Ban Du, (9949'18.12" E 2006'36" N), on decaying submerged wood in a freshwater stream, 18 August 2020, R.J. Xu, MD-85 (MFLU 21-0095, holotype), ex-type culture, MFLUCC 21-0073.

GenBank number: ITS = MW751848, LSU = MZ493341.

Notes: Pseudodactylaria albicolonia shares several characters in common with $P$. fusiformis and $P$. camporesiana such as unbranched conidiophores, holoblastic, polyblastic conidiogenous cells and fusiform, hyaline conidia (Lin et al. 2018; Lu et al. 2020; Hyde et al. 2020b). Phylogenetically, 


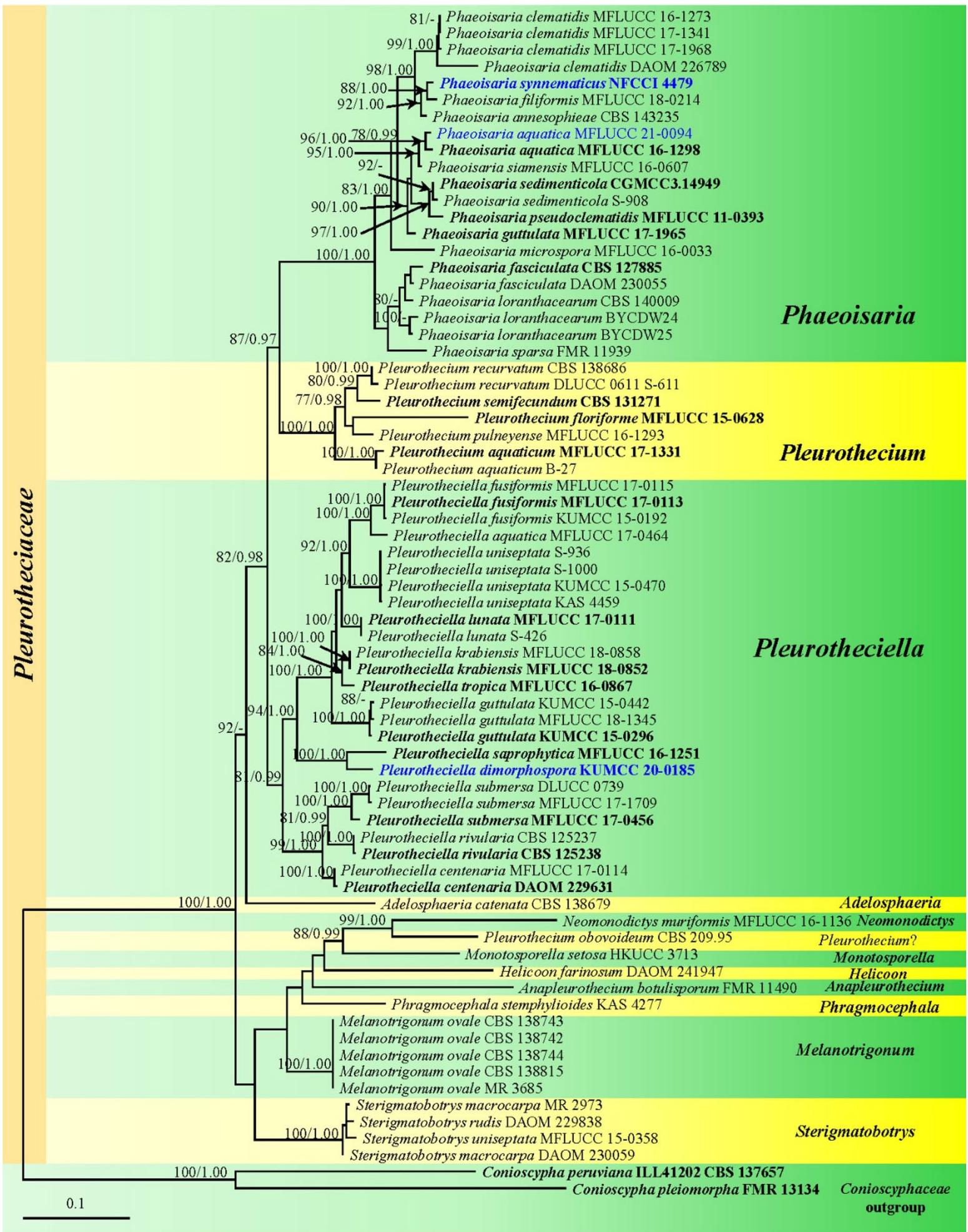

Fig. 141 Maximum likelihood phylogenetic tree based on a combined LSU, ITS, SSU and RPB2 sequence data of genera in Pleurotheciaceae. Conioscypha peruviana CBS 137657 and C. pleiomorpha FMR 13,134 were selected as the outgroup taxa. Bootstrap support values for maximum likelihood (MLBS, left) equal to or greater than $70 \%$ is given above the nodes. Bayesian posterior probabilities (BYPP, right) equal to or greater than $95 \%$ are given above the nodes. Ex-type strains are in bold and newly generated sequences are in blue 

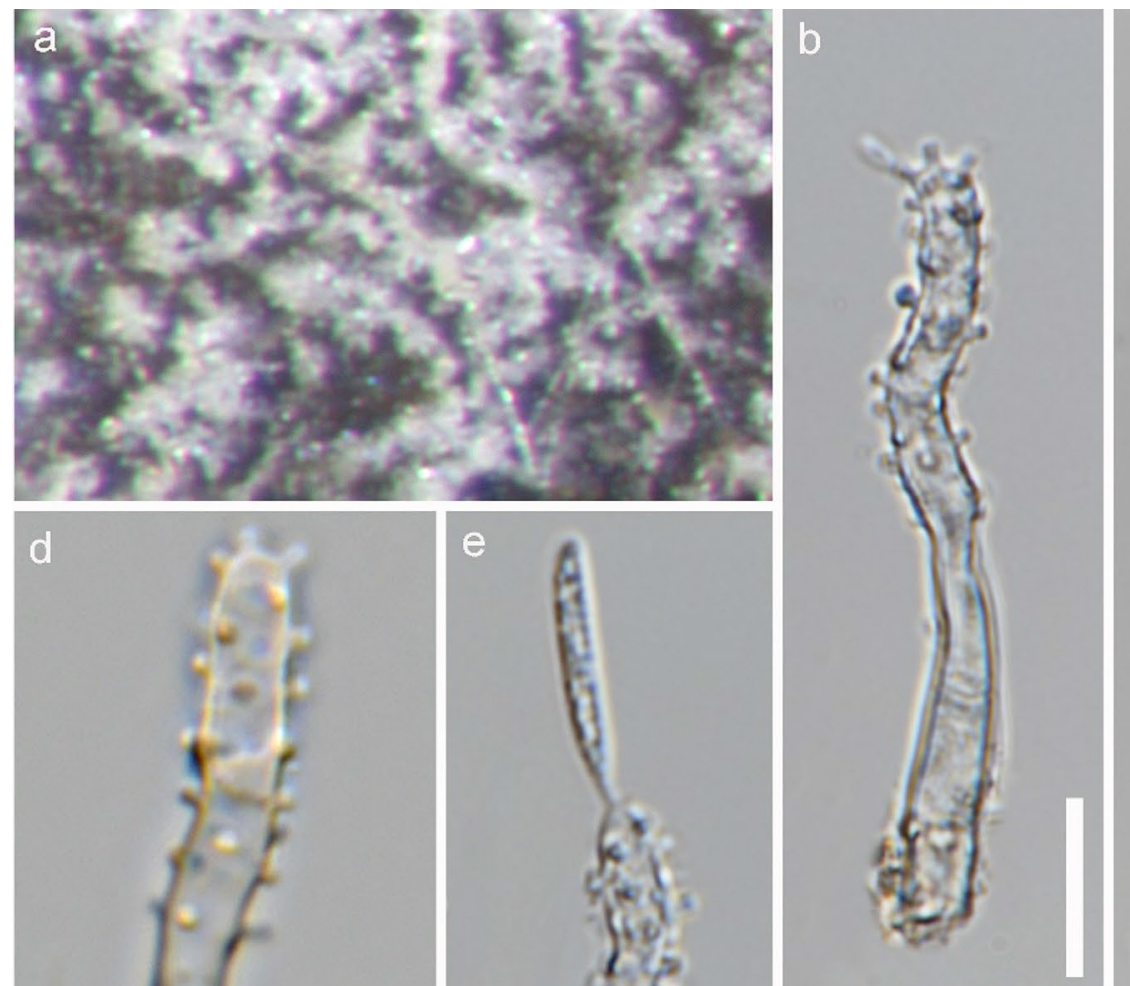

d

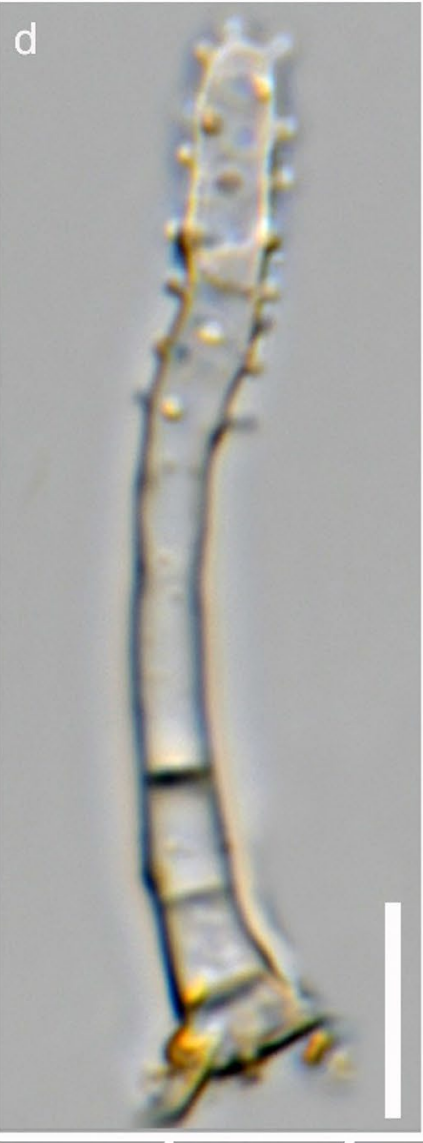

e

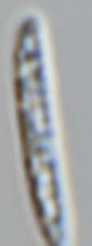

h
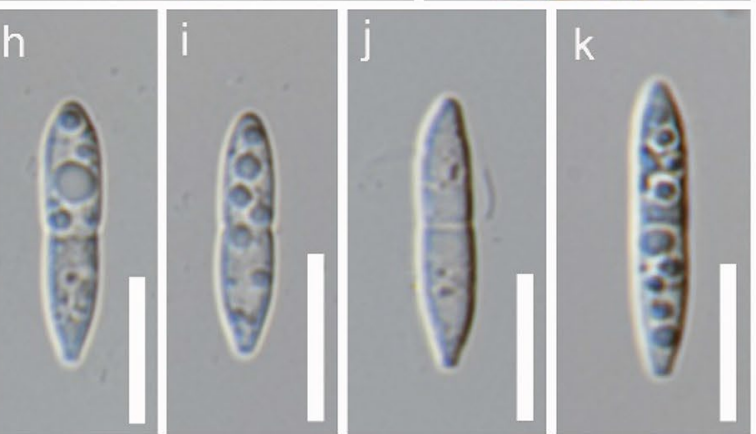

c.
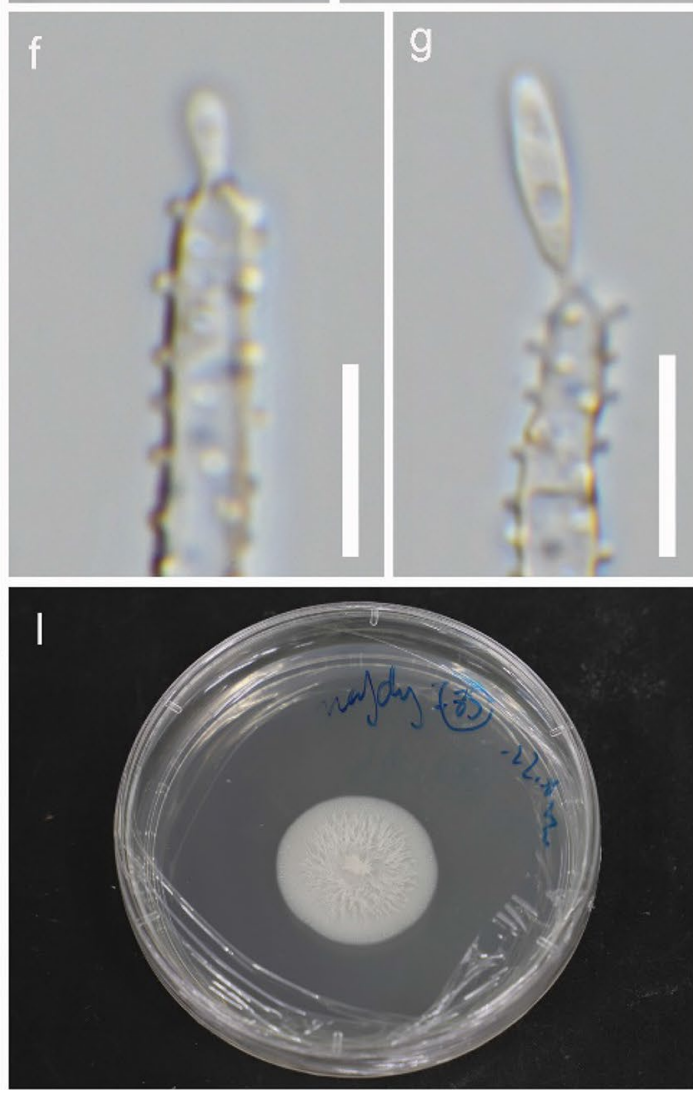

Fig. 142 Pseudodactylaria albicolonia (MFLU 21-0095, holotype). a Colony on decaying wood. b-d Conidiophores with conidia. e-g Conidiogenous cells with attached conidia. $\mathbf{h}-\mathbf{k}$ conidia. I Colony on MEA medium. Scale bars: $\mathbf{b}-\mathbf{k}=10 \mu \mathrm{m}$ 
$P$. albumcoloniam formed a distinct lineage basal to $P$. hyalotunicata and P. xanthorrhoeae with 86\% MLBS, 1.00 BYPP support (Fig. 143). Pseudodactylaria albicolonia differs from $P$. hyalotunicata and the type species $P$. xanthorrhoeae in having shorter conidia (14-19.5 $\times 2.5-4 \mu \mathrm{m}$ vs. $20-25 \times 2.5-3 \mu \mathrm{m}$ and (20-)22-27(-33) $\times(3-) 3.5(-4)$ $\mu \mathrm{m}$, respectively) and lacking a mucilaginous sheath (Tsui et al. 1997; Crous et al. 2017). Therefore, we introduce a new species $P$. albumcoloniam (Fig. 142) from a freshwater habitat in Thailand based on morphological differences and phylogenetic evidence.

Savoryellales Boonyuen, Suetrong, Sivichai, K.L. Pang \& E.B.G. Jones

Notes: Savoryellales (Boonyuen et al. 2011) comprises a single family Savoryellaceae. We follow the latest treatment and updated accounts of Savoryellales in Hyde et al. (2020b).

\section{Savoryellaceae Jaklitsch \& Réblová}

Notes: Savoryellaceae comprises five genera viz. Ascotaiwania, Canalisporium, Neoascotaiwania, Rhexoacrodictys and Savoryella (Wijayawardene et al. 2020). For the latest treatment and updated accounts of Savoryellales, we follow in Hyde et al. (2019, 2020b) and Réblová et al. (2020).

Canalisporium Nawawi \& Kuthub. (=Ascothailandia Sriindr., Boonyuen, Sivichai \& E.B.G. Jones)

Notes: Canalisporium was introduced to accommodate a taxon in having dark brown and muriform conidia and is typified by Canalisporium caribense (Sri-indrasutdhi et al.

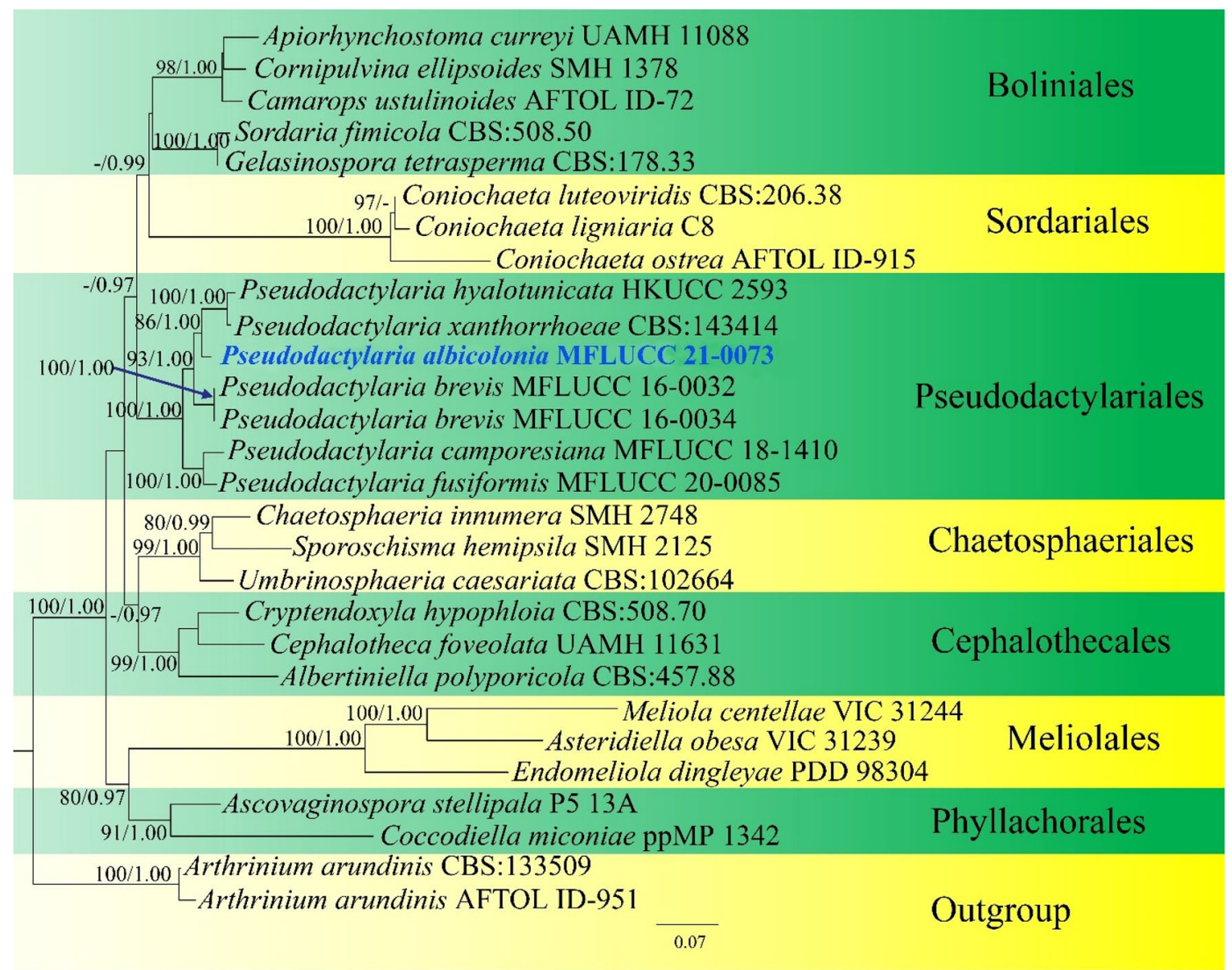

Fig. 143 Phylogram generated from the best scoring of the RAxML tree based on combined LSU, ITS, sequence dataset to indicate the new species Pseudodactylaria albicolonia and related genera in Pseudodactylariales. Twenty-eight strains are included in the combined analysis, which comprise a total of 1306 characters. Arthrinium arundinis CBS 133509 and A. montagnei AFTOL ID-951 are selected as the outgroup taxa. The best RAxML tree with a final likelihood value of -10010.943263 is presented. RAxML analysis yielded 664 distinct alignment patterns and $21.66 \%$ of undeter- mined characters or gaps. Estimated base frequencies were as follows: $\mathrm{A}=0.246125, \mathrm{C}=0.236142, \mathrm{G}=0.297752, \mathrm{~T}=0.219981$, with substitution rates $\mathrm{AC}=0.978864, \mathrm{AG}=2.183946, \mathrm{AT}=1.688622$, $\mathrm{CG}=0.686434, \mathrm{CT}=5.976862, \mathrm{GT}=1.000000$; gamma distribution shape parameter alpha $=0.403195$. Bootstrap support values for maximum likelihood (MLBS, left) equal to or greater than $70 \%$ is given above the nodes. Bayesian posterior probabilities (BYPP, right) equal to or greater than 0.95 are given above the nodes. Newly generated sequences are in blue and bold 
2010; Hyde et al. 2020b). Members of Canalisporium are saprobes commonly found on dead or decaying wood and dead plants in different habitats such as freshwater and terrestrial (Sri-indrasutdhi et al. 2010; Dayarathne et al. 2019; Hyde et al. 2019, 2020b; Index Fungorum 2021). Sixteen species are included in Canalisporium based on morphological and phylogenetic analyses (Hyde et al. 2020b).

Canalisporium caribense (Hol.-Jech. \& Mercado) Nawawi \& Kuthub., Mycotaxon 34(2): 479 (1989)

$\equiv$ Berkleasmium caribense Hol.-Jech. \& Mercado 1984

Index Fungorum number: IF125432; Facesoffungi number: FoF 05486; Fig. 144

Saprobic on submerged decaying wood in the river. Sexual morph Undetermined. Asexual morph Colonies on natural substrate sporodochial, scattered, punctiform, granular, black, shining. Mycelium immersed, composed of branched, septate, pale brown to brown. Conidiophores micronematous or semi-macronematous, mononematous, fasciculate, simple or sometimes branched, septate, hyaline to very pale brown. Conidiogenous cells monoblastic, integrated, terminal, determinate, hyaline or pale brown. Conidia $21-42 \times 18-26 \mu \mathrm{m}(\bar{x}=32.5 \times 24 \mu \mathrm{m}, \mathrm{n}=30)$, acrogenous, muriform, solitary, broadly ellipsoidal to obovoid, fusiform to obclavate, flattened, brown to dark brown, one longitudinal column septum, thick and dark at the septum, 9-13-transverse septate, constricted at the septa, smooth-walled.

Culture characteristics: Conidia germinating on water agar and germ tubes produced from basal conidium within $24 \mathrm{~h}$ at room temperature. Colonies growing on PDA, circular, umbonate, slightly fimbriate to entire edge, olivaceous grey in the middle, with dark brown at the margin, dark in reverse. Mycelium superficial and partially immersed, branched, septate, hyaline to pale brown.

Material examined: THAILAND, Phayao Province, Pong District, Yom River, on decaying submerged wood in the river shore, 18 December 2019, S.K.U. Chandrasiri and S. Boonmee, YR1 (MFLU 21-0072, new record), living culture, MFLUCC 21-0095.

GenBank numbers: ITS = MZ538501, LSU = MZ538535, TEF1- $\alpha$ : MZ567080.

Notes: A new collection of Canalisporium caribense (MFLUCC 21-0095) was collected from a freshwater river in northern Thailand (Fig. 144). Our collection shares identical morphology to the type species $C$. caribense (HolubováJechová and Mercado 1984; Nawawi and Kuthubutheen 1989). Further, phylogenetic analysis based on a combined LSU, ITS, SSU, TEF1- $\alpha$ and RPB2 sequences place our isolate MFLUCC 21-0095with strains of $C$. caribense (SS03683 and SS03839; Sri-indrasutdhi et al. 2010) with 99\% MLBS, 1.00 BYPP support (Fig. 146). Therefore, a new additional record of $C$. caribense is reported from northern Thailand.

Rhexoacrodictys W.A. Baker \& Morgan-Jones

Notes: Rhexoacrodictys was introduced to accommodate hyphomycetous taxa presence of acrodictys-like structures (Baker et al. 2002). The latest treatment and updated accounts of Rhexoacrodictys consists of five species (Luo et al. 2019; Wijayawardene et al. 2020).

Rhexoacrodictys nigrospora Boonmee, D.F. Bao \& K.D. Hyde, sp. nov.

Index Fungorum number: IF558545; Facesoffungi number: FoF 09200; Fig. 145

Etymology: The specific epithet "nigrospora" refers to the black conidia.

\section{Holotype: MFLU 21-0073}

Saprobic on decaying bark. Sexual morph Undetermined. Asexual morph Hyphomycetous. Colonies superficial, dark brown to black. Mycelium superficial and immersed, composed of branched, septate, pale brown to brown hyphae, 1-2 $\mu \mathrm{m}$ wide, verruculose or finely echinulate-walled. Conidiophores micronematous or semi-macronematous, mononematous, cylindrical filamentous, flexuous, scattered to grouped, simple, single or a loose cluster brown to dark brown, smooth-walled. Conidiogenous cells monoblastic, polyblastic, integrated, terminal and intercalary or discrete, determinate. Conidia $15-31 \times 16-33 \mu \mathrm{m}(\bar{x}=22 \times 23 \mu \mathrm{m}$, $\mathrm{n}=20$ ), holoblastic, solitary, dry, acrogenous, simple, globose to subglobose, truncate at the base, multi-septate, dark brown when immature, becoming black and faintly septate at maturity, thick and smooth-walled.

Culture characteristics: Conidia germinating on PDA within $24 \mathrm{~h}$ and germ tubes arising from terminal end cell. Colonies on malt extract agar (MEA), reaching $3.5 \mathrm{~cm}$ diam. after 2 weeks at room temperature, colonies circular, low convex, surface slightly rough with entire edge, around the white to greyish brown, dark green to black at the reverse.

Material examined: THAILAND, Phetchabun Province, Lom Sak, on decaying bark, 25 July 2019, S. Boonmee, LSP03 (MFLU 21-0073, holotype), ex-type living culture, MFLUCC 21-0096; Mukdahan Province, on decaying wood, 24 July 2019, S. Boonmee, WD09 (MFLU 21-0074), living culture MFLUCC 21-0097.

GenBank number: LSP03: ITS $=$ MZ538524, LSU $=$ MZ538558, TEF $1-\alpha=$ MZ567100; WD09: ITS $=$ MZ538525, LSU $=$ MZ538559, SSU $=$ MZ538574, RPB2 $=$ MZ567113, TEF1- $\alpha=$ MZ567101.

Notes: Rhexoacrodictys nigrospora differs from the type $R h$. erecta and other species in having cylindrical filamentous, flexuous conidiophores and globose to subglobose, faintly septate, dark brown to black conidia (Baker et al. 2002; Xiao et al. 2018). Phylogenetically, Rh. nigrospora 

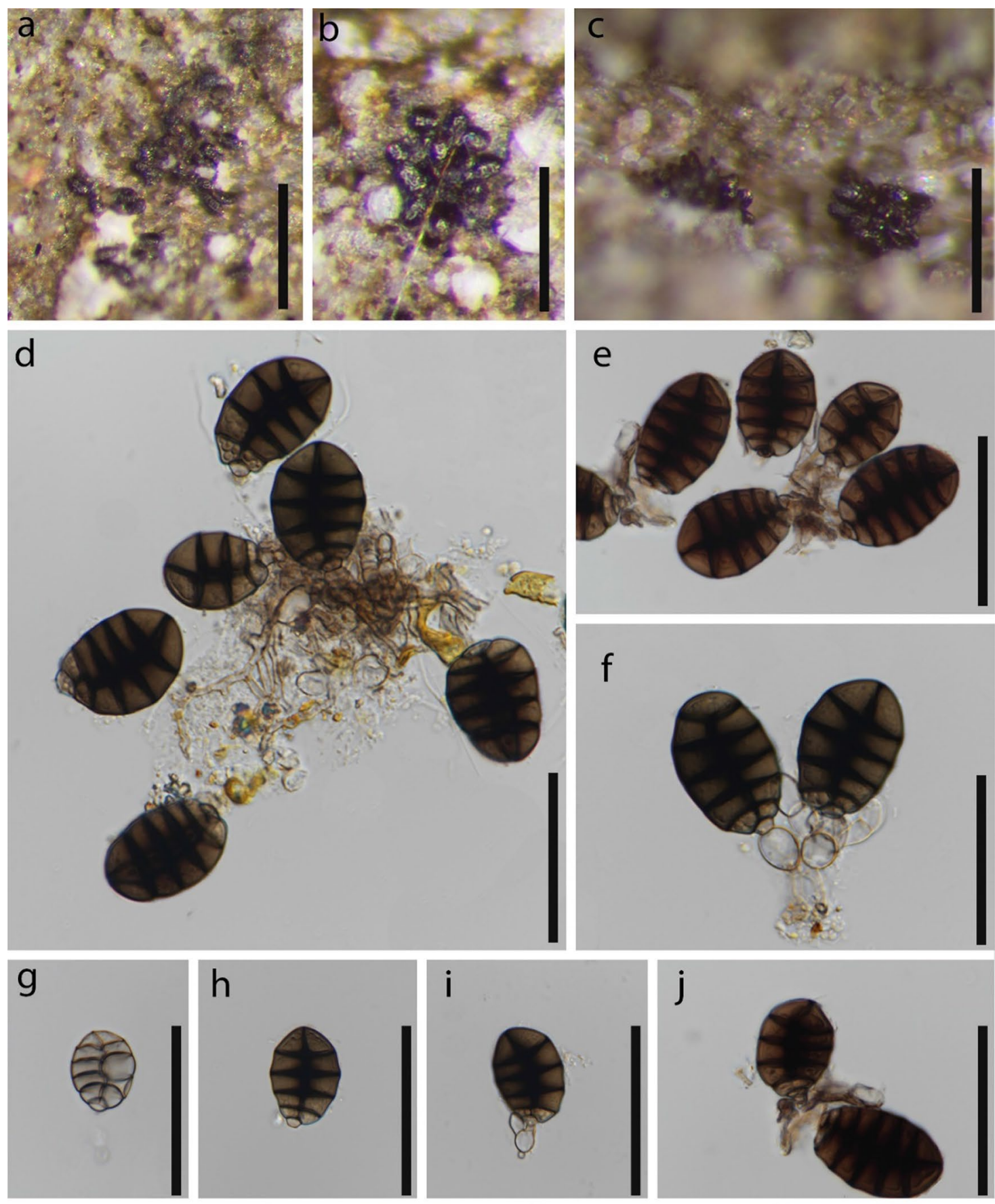

320.
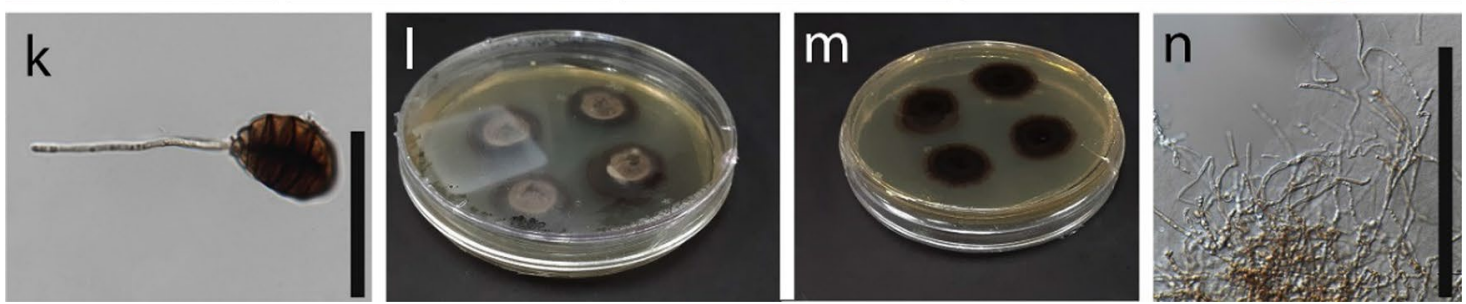

Fig. 144 Canalisporium caribense (MFLU 21-0072, new record) a-c Sporodochia on wood. d, e Squash mount of sporodochia. $\mathbf{f ~ C o n -}$ idiogenous cells with conidia. $\mathbf{g}-\mathbf{j}$ Conidia. $\mathbf{k}$ Germinated conidium.
I, $\mathbf{m}$ Culture on PDA from surface and reverse. $\mathbf{n}$ Mycelium on PDA. Scale bars: $\mathbf{a}-\mathbf{c}=20 \mu \mathrm{m}, \mathbf{d}-\mathbf{n}=5 \mu \mathrm{m}$ 

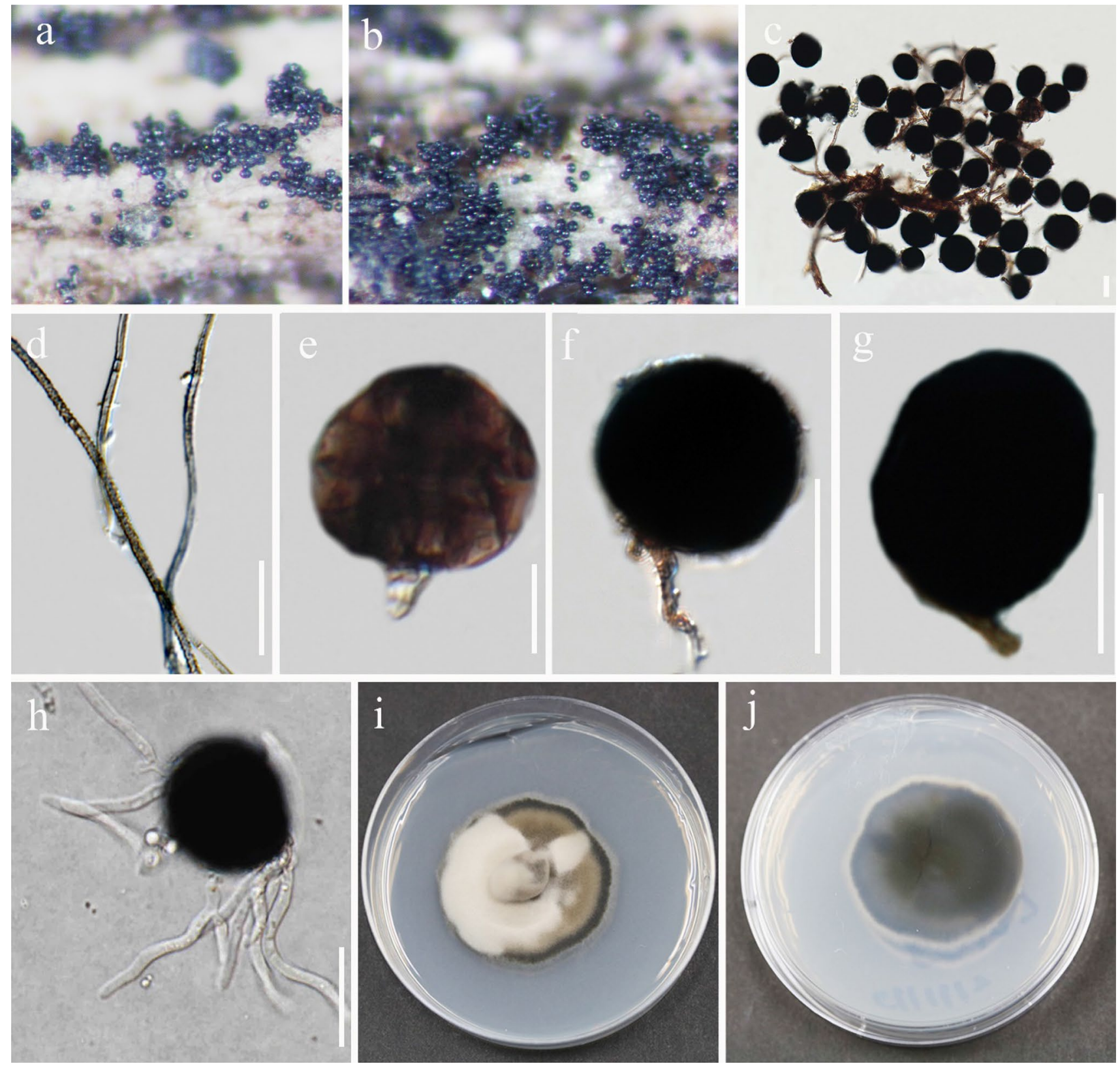

Fig. 145 Rhexoacrodictys nigrospora (MFLU 21-0073, holotype). a, b Conidiomata on the substrate. c Squash of mount of conidia. d Conidiophores with minutely conidiogenous cells. e-g Conidia. h

forms a distinct lineage basal to Rhexoacrodictys species with 100\% MSBS, 1.00 BYPP support (Fig. 146). Currently, only four species listed in this genus (Species Fungorum 2021). We, therefore introduce Rh. nigrospora (Fig. 145) as the fifth species in Rhexoacrodictys based on morphological and phylogenetic analyses.

\section{Lasiosphaeriaceae Nannf.}

Notes: Lasiosphaeriaceae, typified by Lasiosphaeria Ces. \& De Not, was introduced by Nannfeldt (1932). The
Germinated conidium. $\mathbf{i}, \mathbf{j}$ Culture on PDA from surface and reverse. Scale bars: $\mathbf{c}, \mathbf{e}, \mathbf{f}=10 \mu \mathrm{m}, \mathbf{d}, \mathbf{g}=20 \mu \mathrm{m}$

family is highly polyphyletic with several paraphyletic genera (Huhndorf et al. 2004; Miller and Huhndorf 2004, 2005; Cai et al. 2006; Kruys et al. 2015). Furthermore, Lasiosphaeriaceae consists of morphologically diverse taxa being the most diverse genera in Sordariales (Huhndorf et al. 2004; Miller and Huhndorf 2005). The latest treatment of the family followed Hyde et al. (2020d).

\section{Cercophora Fuckel}




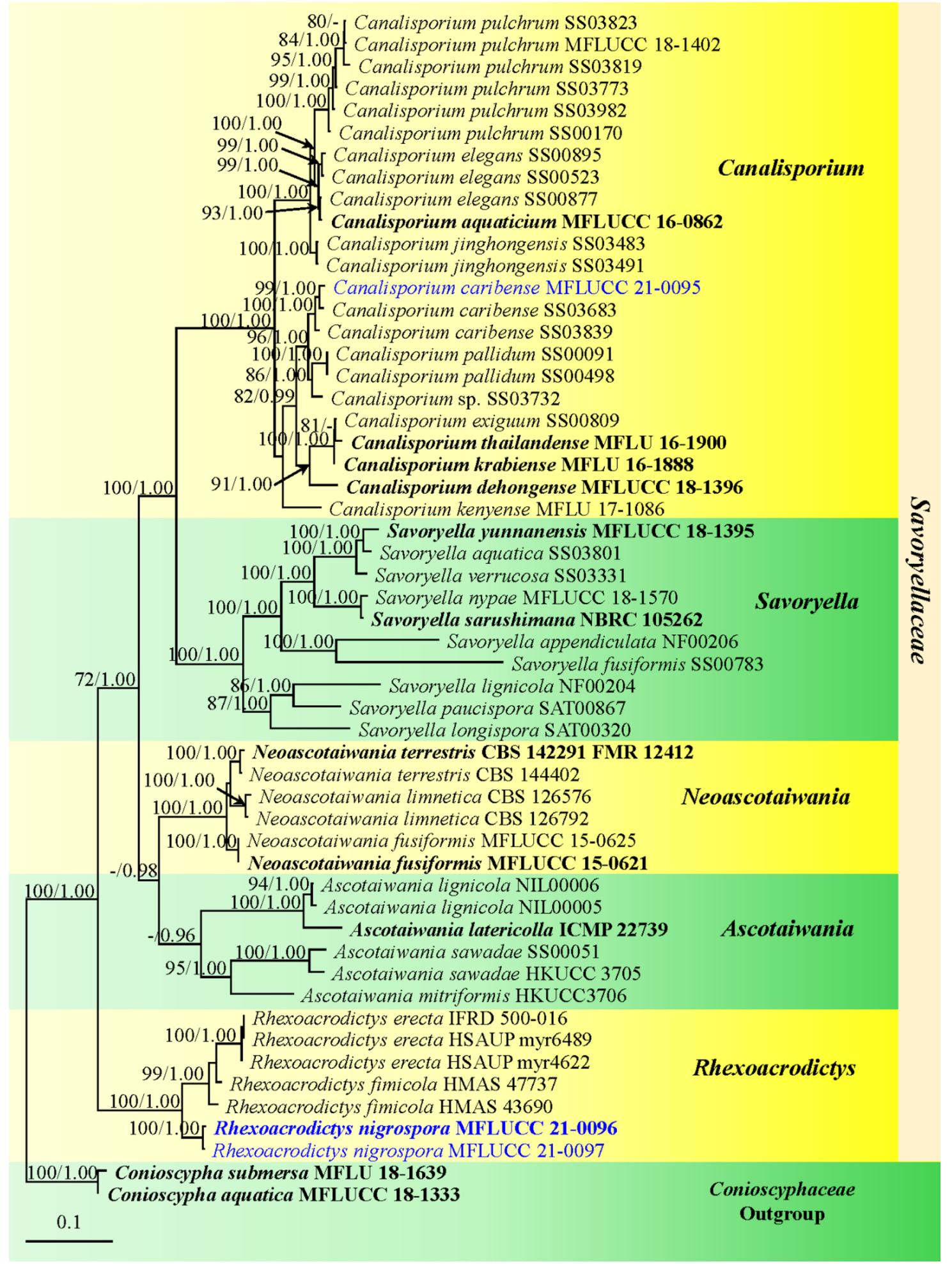

Fig. 146 Maximum likelihood phylogenetic tree based on a combined LSU, ITS, SSU, TEF1- $\alpha$ and RPB2 sequence data of genera in Savoryellaceae. Conioscypha aquatica MFLUCC 18-1333 and C. submersa MFLU 18-1639 are selected as the outgroup taxa. Bootstrap support values for maximum likelihood (MLBS, left) equal to or greater than $70 \%$ is given above the nodes. Bayesian posterior probabilities (BYPP, right) equal to or greater than 0.95 are given above the nodes. Ex-type strains are in bold and newly generated sequences are in blue 
Notes: Fuckel (2011) established Cercophora with three species: C. conica, C. fimiseda, and C. mirabilis (type species). Multi-loci phylogenetic analyses of Cercophora species clustered in the lasiosphaeriaceous complex (Huhndorf et al. 2004; Kruys et al. 2015; Hyde et al. 2020d). Sixty-four morphologically distinct Cercophora species are listed in Species Fungorum (2021) wherein 27 species have molecular data. The latest treatment of Cercophora is Hyde et al. (2020d).

Cercophora dulciaquae M.S. Calabon, E.B.G. Jones \& K.D. Hyde, sp.nov.

Index Fungorum number: IF558645; Facesoffungi number: FoF 09962; Fig. 147

Etymology: The specific epithet "dulciaquae" reflects the origin in a freshwater habitat.

Holotype: MFLU 21-0122

Saprobic on submerged decaying wood. Sexual morph Ascomata 390-700 $\mu \mathrm{m}$ high, 350-475 $\mu \mathrm{m}$ diam., perithecial, mostly solitary, superficial, with base immersed in the hyphae, subglobose to obpyriform with conical neck, black, centrally located ostiole, without periphyses. Peridium $30-40 \mu \mathrm{m}$ thick, slightly thicker at apex, 2-layers, outer layer composed of dark brown, thick-walled, irregular, brown cells of textura angularis; inner layer thin-walled, composed of elongated, flattened, light brown to hyaline cells. Hamathecium 2-3 $\mu \mathrm{m}$ wide, filiform, septate, unbranched, abundant, hyaline paraphyses. Asci $110-180 \times 15-25 \mu \mathrm{m}$ $(\bar{x}=135 \times 16 \mu \mathrm{m}, \mathrm{n}=20), 8$-spored, unitunicate, cylindrical with premature ascospores, cylindric-clavate at later stages, apex rounded, narrow, apical ring, thin-walled, with a short pedicel. Ascospores 35-60 $\times 6-8 \mu \mathrm{m}(\bar{x}=45 \times 7 \mu \mathrm{m}$, $\mathrm{n}=20$ ), 2-seriate, premature, hyaline, aseptate, cylindrical, slightly sigmoid to geniculate, numerous oil droplets, curved, rounded at both ends, with a lash-like appendage attached to both ends, $8-15 \mu \mathrm{m}$ long; becoming differentiated into a swollen head and pedicel, 1-transversely septate, head 10-20×9-11 $\mu \mathrm{m}(\bar{x}=17 \times 10 \mu \mathrm{m}, \mathrm{n}=30)$, conical at the apex, truncate at the base, hyaline to dark brown, with several oil globules, pedicel $19-27 \times 4-6 \mu \mathrm{m}(\bar{x}=23 \times 5 \mu \mathrm{m}$, $\mathrm{n}=30$ ), hyaline to pale brown, curved; appendages disappear with age. Asexual morph: Undetermined.

Culture characters: Conidia germinated on MEA within $24 \mathrm{~h}$. Colonies on MEA reaching 1-2 cm diam. after 4 weeks at room temperature, slow growing, colonies irregular, medium dense, flat with smooth and filiform margins; brown to dark brown in top view, reverse brown.

Material examined: THAILAND, Chiang Mai Province, Mushroom Research Center, on decaying wood submerged in a freshwater stream, 29 January 2019, S. Boonmee, SB1710 (MFLU 21-0122, holotype), ex-type living culture, MFLUCC 21-0104.

GenBank numbers: ITS $=$ MZ497331, LSU $=$ MZ497335.
Notes: Cercophora dulciaquae is the seventh species from Cercophora (C. appalachianensis, C. aquatica, C. caudata, C. costariensis, C. squamulosa, $C$. vinosa) observed from freshwater habitats (Luo et al. 2019; Calabon et al. 2020b). It closely resembles $C$. aquatica and $C$. costariensis. Still, it differs in ascomata (390-700 $\mu \mathrm{m}$ high vs. 400-600 $\mu \mathrm{m}$ high vs. $350-500 \mu \mathrm{m}$ high), asci $(110-180 \times 15-25 \mu \mathrm{m}$ vs. $180-250 \times 13-16 \mu \mathrm{m}$ vs. $135-186 \times 13-16 \mu \mathrm{m})$ and ascospore size $(35-60 \times 6-8 \mu \mathrm{m}$ vs. $36-51 \times 4-6 \mu \mathrm{m}$ vs. 40-49 $\times 4-5 \mu \mathrm{m})$ (Chaudhary et al. 2007; Catania et al. 2011). Furthermore, C. dulciaqaue (Fig. 147) has aseptate ascospores while $C$. aquatica has up to 5-septate ascospores after liberation from the ascus. Immature ascospores of $C$. dulciaquae have bipolar appendages, but these are absent in C. costariensis (Catania et al. 2011). Based on the phylogenetic analysis of combined LSU and ITS sequence data, $C$. dulciaquae clustered with Zopfiella marina (CBS 155.77) with low bootstrap support (Fig. 148). The two species are morphologically different and the novel species fits the generic description of Cercophora. Cercophora dulciaquae is phylogenetically close to $C$. tuberculata. The former species differs in ascomatal morphology (short neck vs. neck up to $150 \mu \mathrm{m}$ high, with basal tubercles) and ascospore size $(35-60 \times 6-8 \mu \mathrm{m}$ vs. $37.5-40 \times 2.5-5 \mu \mathrm{m})($ Chang and Wang 2005). Blast results of ITS sequence data revealed a $99.44 \%$ similarity to Apodus oryzae (E-219) while LSU sequence data showed a $99.88 \%$ similarity with Apodus oryzae (ATCC 38847), Triangularia mangenotii (CBS 419.67; ATCC 38847) and Zopfiella pilifera (CBS 413.73).

Torpedosporales E.B.G. Jones, Abdel-Wahab \& K.L. Pang Notes: see latest update account of Torpedosporales in Wijayawardene et al. (2020).

Juncigenaceae E.B.G. Jones, Abdel-Wahab \& K.L. Pang

Notes: Juncigenaceae was introduced by Jones et al. (2014) to accommodate three genera Fulvocentrum, Juncigena and Marinokulati with Juncigena adarca as the type species. Another two new genera Elbamycella and Khaleijomyces were described (Abdel-Wahab et al. 2018; Poli et al. 2019). The family is characterized by small-sized, coriaceous, immersed to superficial ascomata with apricot colour either in the peridium, neck or ascospores; periphysate neck; numerous, septate paraphyses that are embedded in gel and attached to the top and base of the ascomatal venter; cylindrical, clavate or fusiform asci and ellipsoidal, fusiform to clavate, septate, hyaline to pigmented ascospores, with or without sheath, smooth- to rough-walled. Currently, five genera i.e., Elbamycella, Fulvocentrum, Juncigena, Khaleijomyces, and Marinokulati and eight species are included in Juncigenaceae (Kohlmeyer et al. 1997; Jones et al. 2014; Abdel-Wahab et al. 2018, 2019; Poli et al. 2019). The eight species were described from lignicolous substrates in marine 

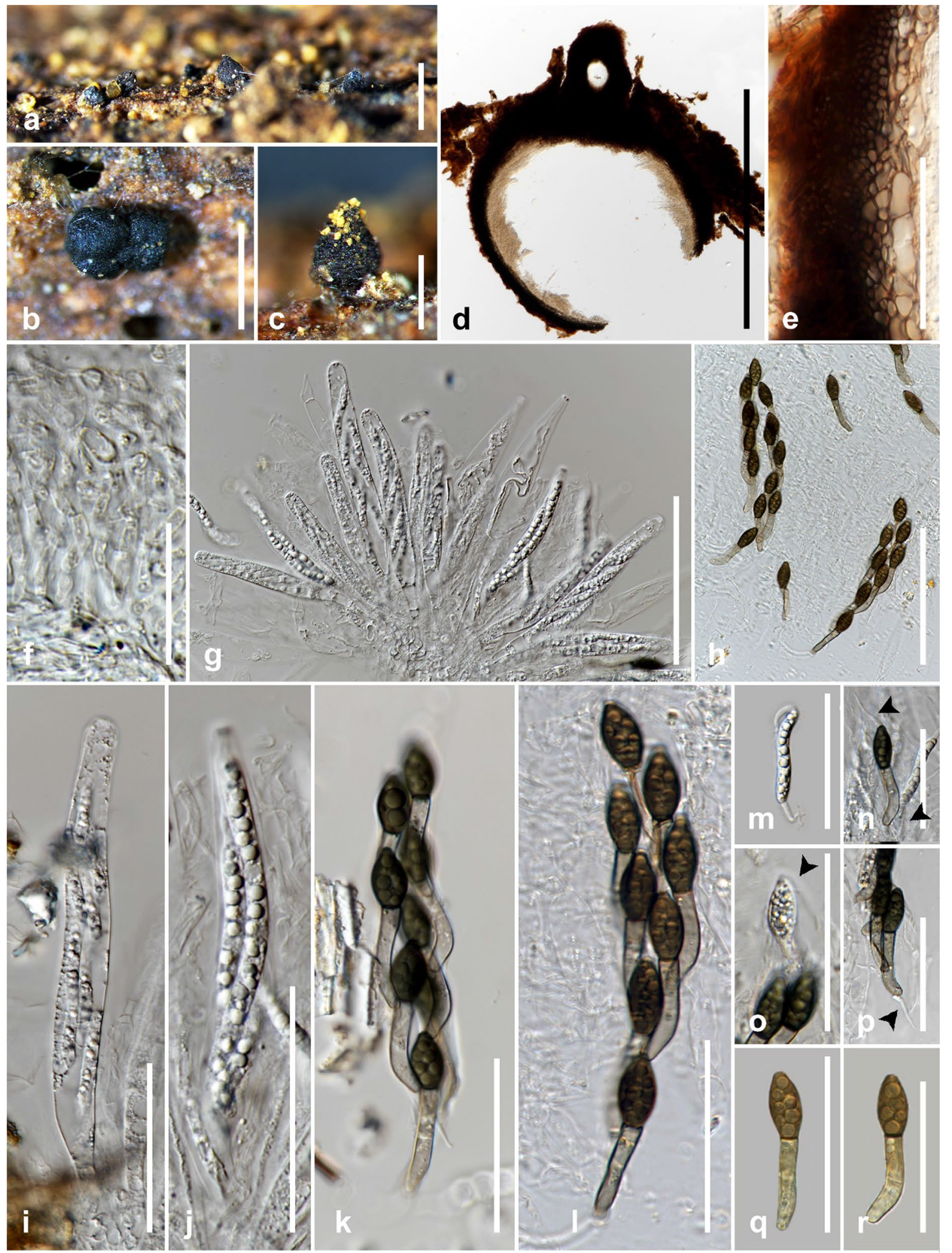

Fig. 147 Cercophora dulciaquae (MFLU 21-0122, holotype). a-c Appearance of ascomata on host substrate. d Vertical section through ascoma. e Section of pycnidial wall. f Paraphyses. g-l Asci. $\mathbf{m}-\mathbf{r}$ Ascospores with bipolar appendages (arrow). Scale bars: a $\mathbf{b}=1 \mathrm{~mm}, \mathbf{c}, \mathbf{d}=500 \mu \mathrm{m}, \mathbf{e}, \mathbf{i}-\mathbf{r}=50 \mu \mathrm{m}, \mathbf{f}=20 \mu \mathrm{m}, \mathbf{g}, \mathbf{h}=100 \mu \mathrm{m}$ 


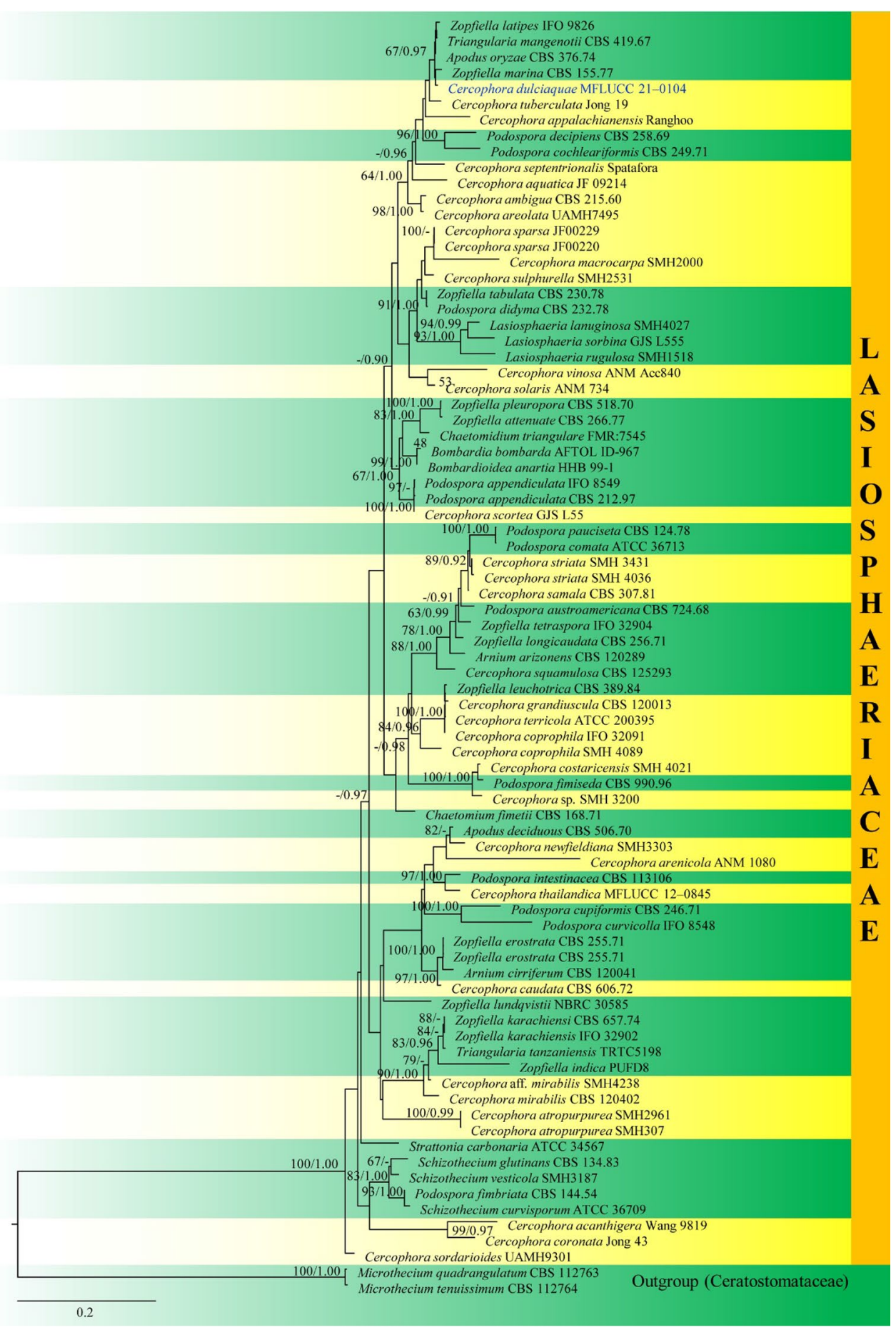

Fig. 148 Phylogenetic tree generated from maximum likelihood (ML) analysis based on combined LSU and ITS sequence data for the species from Lasiosphaeriaceae. Microthecium quadrangulatum (CBS 112763 ) and M. tenuissimum (CBS 112764) were used as outgroup taxa. The dataset comprised and 1243 characters after alignment including gaps ( $\mathrm{LSU}=828 \mathrm{bp}$ and $\mathrm{ITS}=415 \mathrm{bp}$ ). The RAxML analysis of the combined dataset yielded a best scoring tree with a final ML optimization likelihood value of -4540.010304 . The matrix had
520 distinct alignment patterns, with $17.16 \%$ undetermined characters or gaps. Estimated base frequencies were as follows: $A=0.243204$, $\mathrm{C}=0.239086, \quad \mathrm{G}=0.300179, \quad \mathrm{~T}=0.217531 ;$ substitution rates $\mathrm{AC}=1.598327, \quad \mathrm{AG}=1.651124, \mathrm{AT}=2.172105, \quad \mathrm{CG}=0.801233$, $\mathrm{CT}=6.709684, \mathrm{GT}=1.000000$; gamma distribution shape parameter $\alpha=0.254987$. Bootstrap support values for ML and MP equal to or greater than $60 \%$ and BYPP equal to or greater than 0.90 are given above the nodes. The newly generated sequence is in blue and bold 
habitats. Based on morphology and the combined phylogenetic analyses of SSU and LSU rDNA sequence data, a novel species Khaleijomyces umikazeanus is introduced.

\section{Khaleijomyces Abdel-Wahab}

Notes: Khaleijomyces is a monotypic, marine genus that was described from driftwood in the intertidal zone, Arabian Gulf, Saudi Arabia (Abdel-Wahab et al. 2018). The genus is characterized by apricot to reddish-brown, membranous to coriaceous, superficial to immersed ascomata, surrounded by dense brown, septate hyphae, with long, periphysate necks that are apricot in colour, a common character in members of Torpedosporales, cymbiform to fusiform, thinwalled asci, without an apical apparatus; hyaline to brown, unicellular to septate ascospores that are 1-3 seriate in the asci (Abdel-Wahab et al. 2018). In this study, a new species Khaleijomyces umikazeanus is introduced based on evidence of morphology and phylogenetic placement (Figs. 149 and 150).

\section{Khaleijomyces umikazeanus Abdel-Wahab sp. nov.}

Index Fungorum number: IF557837; Facesoffungi number: FoF 09201; Fig. 149

Etymology: Named after Umikaze Beach, where this fungus was collected.

\section{Holotype: CBS H-23860}

Saprobic on the surface of driftwood in the intertidal zone. Sexual morph Ascomata 110-200 $\mu \mathrm{m}$ diam. $(\bar{x}=144 \mu \mathrm{m}, \mathrm{n}=8)$, superficial, solitary, scattered on substrate, subglobose to globose, coriaceous, apricot reddishbrown. Necks 130-180 $\mu \mathrm{m}$ long, 40-50 $\mu \mathrm{m}$ wide, hyaline to apricot in colour, periphysate. Peridium 18-28 $\mu \mathrm{m}$ wide, forming textura angularis, two-layered; outer layer 11-16 $\mu \mathrm{m}$ wide, 4 to 11 layers of apricot to reddish-brown, thick-walled, polygonal to flattened cells; inner layer 9-13 $\mu \mathrm{m}$ wide, 4 to 10 layers of hyaline, thick-walled, polygonal to flattened cells. Paraphyses $2-4 \mu \mathrm{m}$ wide, septate with swollen cells, constricted at the septa, embedded in gel, attached to the top and the base of the ascomatal venter. Asci $73-83 \times 9-11 \mu \mathrm{m}(\bar{x}=81 \times 10 \mu \mathrm{m}, \mathrm{n}=20)$, 8 -spored, unitunicate, thin-walled, persistent, fusiform, curved, developing from ascogenous tissue at the base of the ascomata. Ascospores $19-24 \times 5-6 \mu \mathrm{m}(\bar{x}=22 \times 6 \mu \mathrm{m}$, $\mathrm{n}=40$ ), 1-3-seriate, fusiform to clavate, with rounded ends, 3 -septate, not constricted at the septa, hyaline to faint apricot in colour, surrounded by a deciduous amorphous material.

\section{Asexual morph Undetermined.}

Culture characteristics: Ascospores germinating on PDA within $24 \mathrm{~h}$ at $25^{\circ} \mathrm{C}$ and germ tubes produced from the ends of the ascospore. Colonies on PDA reaching $2 \mathrm{~cm}$ diam. after 3 weeks, at $25^{\circ} \mathrm{C}$, white to yellow-brown, white from below.
Material examined: JAPAN, Yokosuka City, Umikaze Beach, on decaying driftwood in the intertidal zone, 4 August 2008, M.A. Abdel-Wahab (CBS H-23860, holotype), ex-type living culture, NBRC 105287.

GenBank numbers: LSU = MN921253, $\mathrm{SSU}=\mathrm{MN} 921252$.

Notes: Combined phylogenetic analyses of SSU and LSU rDNA placed Khaleijomyces umikazeanus as a distinct species. It grouped with $K$. marinus in phylogenetic analysis but with low statistical support. Khaleijomyces umikazeanus differs from $K$. marinus in having 3-septate, hyaline ascospores, while those in the latter species are unicellular when young that become 1-4-septate and yellow-brown to brown in older specimens. The outer layer of the peridium in $K$. umikazeanus is textura angularis while K. marinus is textura epidermoidea. The hamathecium in K. umikazeanus forms persistent paraphyses, embedded in gel and attached to the top and the bottom of the ascomatal venter, while it is pseudoparenchymatous in $K$. marinus that fills the young ascomatal venter and breaks down into catenophyses that become deliquescent early (Abdel-Wahab et al. 2018). Khaleijomyces umikazeanus differs from Fulvocentrum species in having asci without an apical apparatus and wide and septate paraphyses (2-4 $\mu \mathrm{m}$ wide). The asci in Fulvocentrum species either have apical thickening in $F$. aegyptiacum and $F$. clavatisporum, and apical ring in $F$. rubrum. The hamathecium in Fulvocentrum species are narrow (1-2 $\mu \mathrm{m}$ wide), numerous and aseptate (Abdel-Wahab et al. 2001, 2019). Khaleijomyces umikazeanus also differs from Fulvocentrum species in the morphology of ascomata, asci and ascospores and are phylogenetically distant (Figs. 149 and 150).

\section{Xylariales Nannf.}

Notes: Xylariomycetidae consists of two main orders, namely Amphisphaeriales and Xylariales. Xylariales was considered the second largest order among the Sordariomycetes and a detailed description is provided by Maharachchikumbura et al. (2016) and Hyde et al. (2020b). This order has been revised based on both morphology and phylogenetic data and many families were either newly introduced or transferred (Hongsanan et al. 2017; Daranagama et al. 2018; Voglmayr et al. 2018; Wendt et al. 2018). Currently, 148 genera (Wijayawardane et al. 2020) are accepted in Xylariales, which are distributed in 22 families and 12 families incertae sedis. Similarly, Cainiaceae and Calceomyces were placed in Xylariomycetidae incertae sedis. Major contributions in the order Xylariales such as monographs, checklists and keys along with detailed descriptions and illustrations were provided by different mycologists (Miller 1961; Lowen 1991; Petrini 2013; Stadler et al. 2014; Shang et al. 2018; Niranjan and Sarma 2020; Hyde et al 2020a). In the recent times chemotaxonomic inputs have been widely 

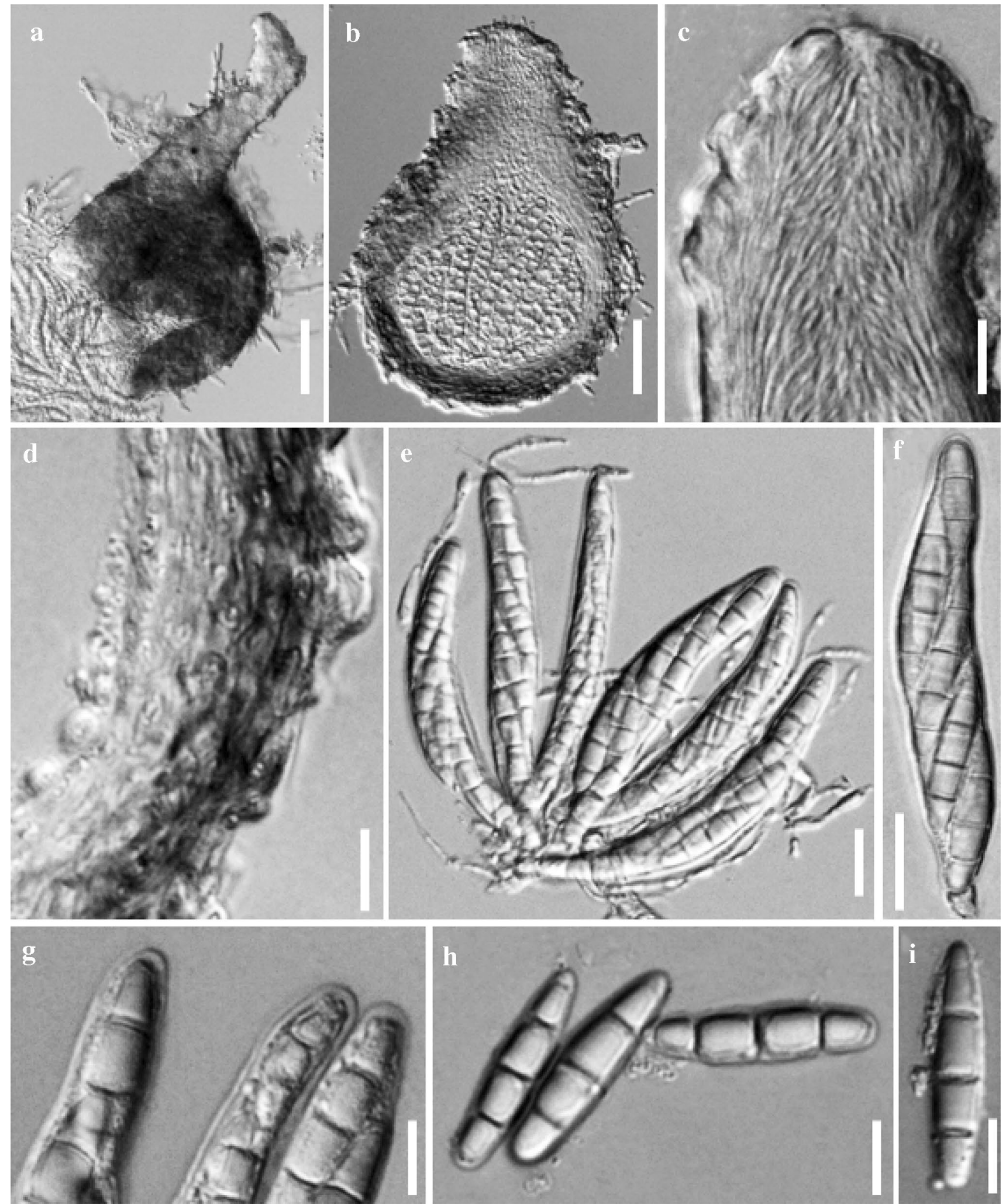

Fig. 149 Khaleijomyces umikazeanus (CBS H-23860, holotype). a Squash of ascoma. b Vertical section through ascoma. c Close up of neck show periphyses. d Close up of peridium. e, f Asci and para- physes. $\mathbf{g}$ Ascal tips. h, $\mathbf{i}$ Ascospores with amorphous material. Scale bars: $\mathbf{a}, \mathbf{b}=60 \mu \mathrm{m}, \mathbf{c}, \mathbf{d}, \mathbf{g}-\mathbf{i}=10 \mu \mathrm{m}, \mathbf{e}, \mathbf{f}=15 \mu \mathrm{m}$ 


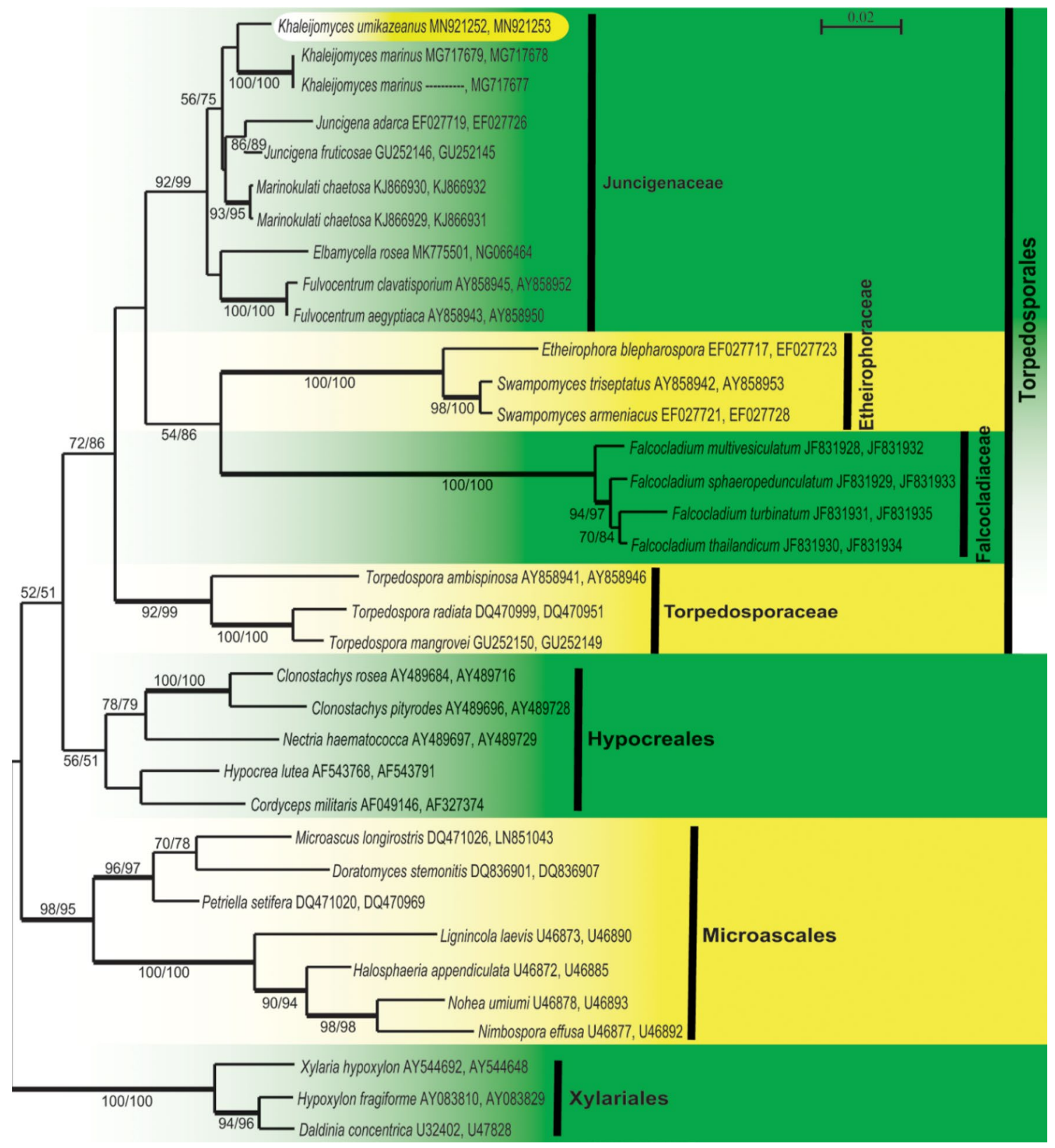

Fig. 150 Phylogenetic relationship of Khaleijomyces umikazeanus with related taxa in Juncigenaceae based on the nucleotide sequences of the combined SSU and LSU rDNA. The maximum likelihood (ML) tree $(-\ln$ likelihood $=14429.06)$ was constructed in PAUP 4 (Swofford 2002). The maximum parsimonious data set of the combined genes consisted of 35 taxa with 3 representatives of Xylariales used as outgroup. The combined dataset includes 1116 total characters, of which 712 were constant, 89 parsimony-uninformative and 315 parsimony-informative. The parsimony analyses of the data

used in Hypoxylaceae (Kuhnert et al. 2017a, b; Helaly et al. 2018; Wendt et al. 2018; Becker et al. 2020), while other mycologists made molecular taxonomy and morphological matrix yielded 2 equally most parsimonious trees with a tree length of 1084 steps [consistency index $(\mathrm{CI})=0.5424$, homoplasy index $(\mathrm{HI})=0.4576$, retention index $(\mathrm{RI})=0.7385$, rescaled consistency index $(\mathrm{RC})=0.4005]$. Phylogenetic trees obtained from ML, maximum parsimony (MP) and Bayesian inference posterior probabilities (BYPP) were similar in topology. Bootstrap support on the nodes represents MLBS and MPBS equal to or greater than 50\%. Branches with a BYPP of equal to or greater than 0.95 are in bold. The newly taxon is in yellow box

observations in the order Xylariales including the establishment of a web portal (Niranjan and Sarma 2018; Wijayawardene et al. 2017, 2018; Basnet et al. 2019; Bundhun et al. 2020; Hyde et al. 2020a; Stadler et al. 2020). 


\section{Diatrypaceae Nitschke}

Notes: Diatrypaceae was introduced by Nitschke (1869) and it is typified by Diatrype (Augusto et al. 2016; Hyde et al. 2020d). In the past, this family belonged to Diatrypales and in current classification Diatrypaceae belongs to Xylariales (Augusto et al. 2016; Hyde et al. 2020d; Wijayawardene et al. 2020). Diatrypaceous taxa are most likely colonise any kind of ecosystem with woody plants in both aquatic and terrestrial habitats and some are pathogens of economic crops as well as forest trees (Chacón et al. 2013; Senanayake et al. 2015; Dayarathne et al. 2016; Hyde et al. 2020d; Konta et al. 2020). There are 20 genera accounted to Diatrypaceae (Wijayawardene et al. 2020). In this study we discuss two Eutypa species collected from Italy.

\section{Eutypa Tul. \& C. Tul.}

Notes: Eutypa was established by Tulasne and Tulasne (1863) and typified by Eutypa lata which is a serious pathogen of grape cankers (Rolshausen et al. 2004, 2008). The genus consists of both pathogenic and saprobic members, and pathogens are reported as the causal agents for Eutypa dieback of apricots, cherries and grapevine (Camps et al. 2014; Hyde et al. 2020d; Lolas et al. 2020). There are 219 epithets listed in Index Fungorum (2021) however, 131 species are accounted for in the genus (Wijayawardene et al. 2020). We follow the latest treatment for Diatrypaceae in Konta et al. (2020) to resolve the taxonomic placements of our newly isolated strains. An updated phylogenetic tree is presented in Fig. 153. This study reports two new host records of Eutypa flavovirens and E. lata from two different Italian sites.

Eutypa flavovirens (Pers.) Tul. \& C. Tul., Select. fung. carpol. (Paris) 2: 57 (1863)

Index Fungorum number: IF122506; Facesoffungi number: FoF 00692; Fig. 151

Saprobic on dead land branch of Quercus sp. Sexual morph Stromata 0.7-1 mm diam., gregarious, aggregates are solitary to scattered, erumpent on the bark, black, outer thin carbonaceous layer, pustulate, with numerous ascomata in a single stroma. Ascomata 350-650 $\mu \mathrm{m}$ high, 300-450 $\mu \mathrm{m}$ diam., ( $\bar{x}=360 \times 490 \mu \mathrm{m}, \mathrm{n}=10)$ length with ostiole neck, immersed in the stroma, globose to broadly ovoid, narrowing towards the apex, very narrow at the base of papilla, ostiolate, papillate. Ostiole neck 100-130 × 250-275 $\mu \mathrm{m}$ $(\bar{x}=90 \times 260, \mathrm{n}=5)$, opening to outer surface, appearing as black spots, comprising outer dark brown and inner pale brown to yellowish parenchymatous cell layers, with periphyses oriented towards apical direction. Peridium 15-25 $\mu \mathrm{m}$ thick, with two layers, outer layer comprising 4-6 layers with thick-walled, dark brown to pale brown cells of textura angularis to textura prismatica cells, inner layer comprising 2-3 layers with thin-walled, hyaline cells of textura prismatica. Hamathecium 1-1.5 $\mu \mathrm{m}$ wide, filiform, longer than the asci, septate, unbranched, guttulate, hyaline paraphyses. Asci $80-120 \times 8-10 \mu \mathrm{m}(\bar{x}=105 \times 8.5 \mu \mathrm{m}$, $\mathrm{n}=20$ ), 8 -spored, unitunicate, thin-walled, clavate, with a $\mathrm{J}$ - apical ring, long, thin-walled pedicel, with cylindrical, thick-walled, swollen upper portion, apically flat. Ascospores $7-9.5 \times 2-2.5 \mu \mathrm{m}(\bar{x}=8.5 \times 1.5 \mu \mathrm{m}, \mathrm{n}=40)$, overlapping, 1-3-seriate, yellowish to brown, unicellular, ellipsoidal to cylindrical or elongate-allantoid, aseptate, straight to slightly curved, with or without guttules, smooth-walled. Asexual morph See Senanayake et al. (2015) and Niranjan et al. (2018).

Culture characteristics: Ascospores germinating on PDA within $24 \mathrm{~h}$ and germ tubes arising from one end of the ascospore. Colonies on PDA, reaching $4-5 \mathrm{~cm}$ diam. after 7 days at $25^{\circ} \mathrm{C}$, colonies medium dense, flat or effuse, slightly raised, cottony, white, margin rough.

Material examined: ITALY, Province of Bologna, Calistri-Porretta Terme, on the dead land branch of Quercus sp. (Fagaceae), 9 April 2019, E. Camporesi, IT 4287 (MFLU 19-0911, new record), living culture, MFLUCC 21-0069.

GenBank numbers: ITS = MZ456005, TUB2 $=$ MZ476771.

Notes: In the multigene phylogeny (ITS and TUB2) of this study, our isolate (MFLUCC 21-0069) and Eutypa flavovirens (E48C, CBS 272) clustered together with relatively high support (98\% MLBS, 1.00 BYPP; Fig. 153). Eutypa flavovirens strains MFLUCC 12-0052, MFLUCC 13-0625 and PUFNI 310 were studied by Senanayake et al. (2015) and Niranjan et al. (2018) and they did not provide TUB2 DNA sequences to support. However, in our phylogenetic analyses, Eutypa flavovirens strains are grouped within Allodiatrype clade (Fig. 153) introduced by Konta et al. (2020). In our study Eutypa shown to be polyphyletic genus which are presently reside in A, B, C and D clades. According to Niranjan et al. (2018) several strains of Eutypa flavovirens have been identified with few differences in size of the asci and ascospores. Also, Senanayake et al. (2015) reported, the specimen collection in Thailand (MFLUCC 13-0625) may not be identical to species collected from Europe. Likewise, our isolate collected from Italy having ascomata, ostiole neck, peridium thickness are larger than previously studied and differs in having thinner paraphyses (Fig. 151). However, our isolate showed that the distinct yellowish green stromatic tissues the same as other Eutypa flavovirens isolates from Senanayake et al. (2015) and Niranjan et al. (2018). Based on the morpho-molecular analyses we conclude that our new collection is another record of Eutypa flavovirens and also a new host record on Quercus sp. (Fagaceae) in Italy.

Eutypa lata (Pers.) Tul. \& C. Tul., Select. fung. carpol. (Paris) 2: 56 (1863) 

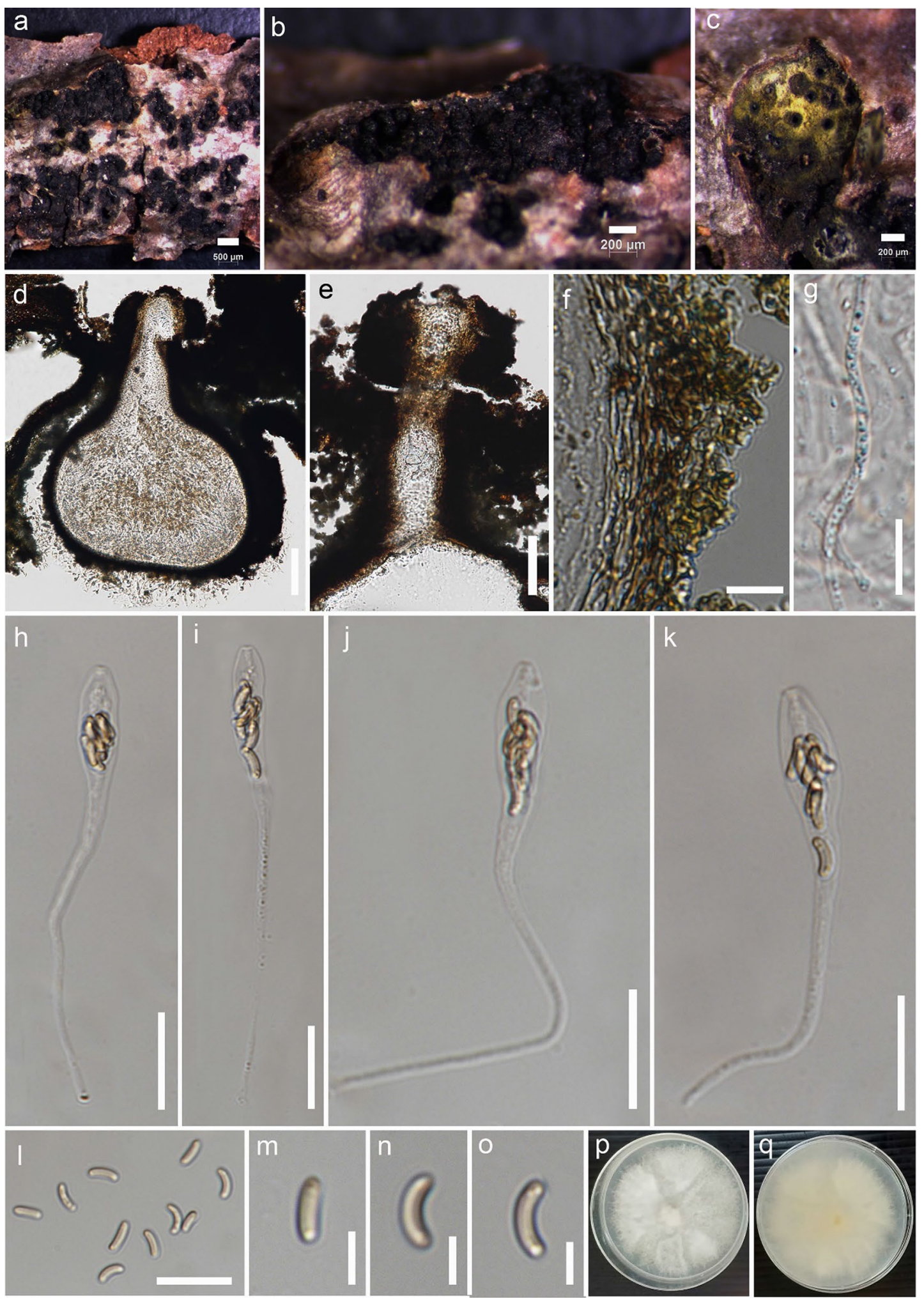

Fig. 151 Eutypa flavovirens (MFLU 19-0717, new record). a, b Stromata on substrate. c Cross-section of stroma. d, e Vertical section through stromata showing ostiolar canals. f Peridium. g Paraphy- ses. h-k Asci. l-o Ascospores. p, q Culture on PDA from above and below. Scale bars: $\mathbf{a}=500 \mu \mathrm{m}, \mathbf{b}, \mathbf{c}=200 \mu \mathrm{m}, \mathbf{d}=100 \mu \mathrm{m}, \mathbf{e}=50 \mu \mathrm{m}$, $\mathbf{h}-\mathbf{l}=20 \mu \mathrm{m}, \mathbf{f}-\mathbf{g}=10 \mu \mathrm{m}, \mathbf{m}-\mathbf{0}=5 \mu \mathrm{m}$ 


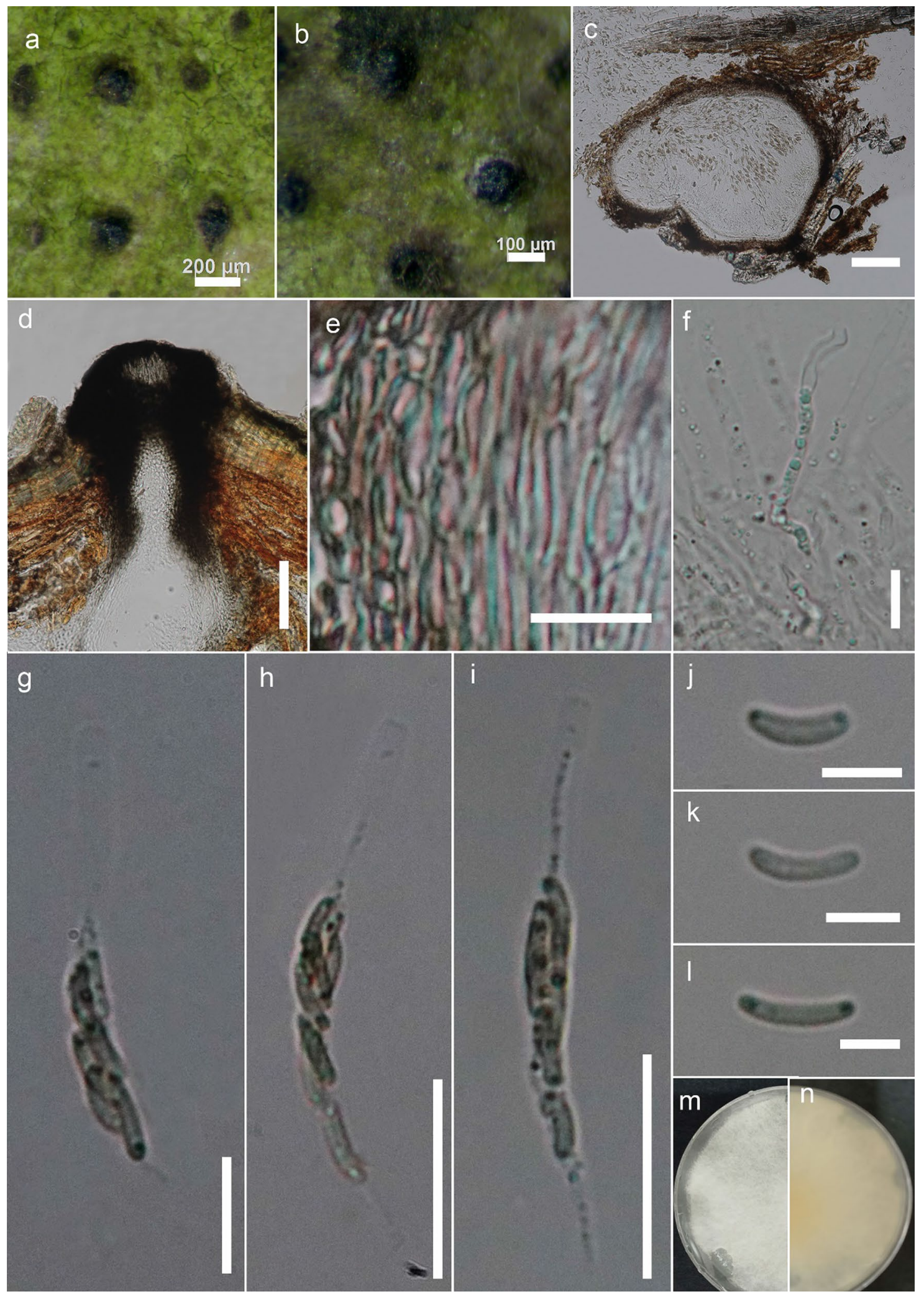

Fig. 152 Eutypa lata (MFLU 19-0501, new record). a, b Stromata on substrate. c Cross-section of ascoma. d Ostiole. e Peridium. f Paraphyses. $\mathbf{g}-\mathbf{i}$ Asci. $\mathbf{j}-\mathbf{l}$ Ascospores. m, $\mathbf{n}$ Culture on PDA from above and below. Scale bars: $\mathbf{a}=200 \mu \mathrm{m}, \mathbf{b}, \mathbf{c}=100 \mu \mathrm{m}, \mathbf{d}=50 \mu \mathrm{m}, \mathbf{f}, \mathbf{h}$, $\mathbf{i}=20 \mu \mathrm{m}, \mathbf{e}, \mathbf{g}=10 \mu \mathrm{m}, \mathbf{j}-\mathbf{l}=5 \mu \mathrm{m}$ 
Index Fungorum number: IF140211; Facesoffungi number: FoF 09963; Fig. 152

Saprobic on the dead aerial branch of Corylus avellana. Sexual morph Ascostromata 400-600 $\mu \mathrm{m}$ high, 350-450 $\mu \mathrm{m}$ diam. $(\bar{x}=380 \times 490 \mu \mathrm{m}, \mathrm{n}=10)$ length with ostiole neck, solitary, scattered semi-immersed, erumpent on the bark, subglobose to irregular, black, ostiolate. Ostiole individual, central, ostiole neck 150-200 $\mu \mathrm{m}$ high ( $\bar{x}=165 \mu \mathrm{m}, \mathrm{n}=5$ ). Peridium $20-30 \mu \mathrm{m}$ wide, composed of two layers, outer layer comprising 4-5 layers of thick-walled, brown to pale brown cells of textura angularis to textura prismatica, thin inner layer comprising 3-5 layers of thinwalled, hyaline cells of textura prismatica. Hamathecium 1.8-3 $\mu \mathrm{m}$ wide, filiform, longer than the asci, septate, guttulate, unbranched, hyaline paraphyses. Asci 40-65 $\times 4-5 \mu \mathrm{m}$ $(\bar{x}=53 \times 4.5 \mu \mathrm{m}, \mathrm{n}=10), 8$-spored, unitunicate, thin-walled, clavate, long pedicillate, apically truncate. Ascospores $5-9 \times 1-2 \mu \mathrm{m}(\bar{x}=7 \times 1.5 \mu \mathrm{m}, \mathrm{n}=30)$, overlapping bi-seriate, aseptate, hyaline, ellipsoidal to cylindrical or allantoid, slightly curved, smooth-walled, mostly with small guttules at the edges. Asexual morph sees (Moyo et al. 2018).

Culture characteristics: Ascospores germinating on PDA within $24 \mathrm{~h}$ and germ tubes arising from both ends of the ascospore. Colonies on PDA, reaching 4-5 cm diam. after 7 days at $25^{\circ} \mathrm{C}$, colonies medium dense, flat or effuse, slightly raised, cottony, white, margin rough.

Material examined: ITALY, Province of Forlì-Cesena, Teodorano-Meldola, on the dead aerial branch of Corylus avellana (Betulaceae), 20 January 2019, E. Camporesi, IT 4212 (MFLU 19-0501, new record), living culture, MFLUCC 21-0068.

GenBank numbers: ITS = MZ453405, TUB2 = MZ476772.

Notes: The multigene phylogeny (ITS and TUB2) of this study, our strain (MFLUCC 21-0068) and Eutypa lata (EP18, RGA01 and ATCC 28120) strains clustered together with $89 \%$ MLBS, 0.96 BYPP support (Clade B; Fig. 153). Comparing the ITS (ITS1-5.8S-ITS2) and TUB2 rDNA regions between EP18 and RGA01 strains, only 1 bp (0.19\%) difference was found from 526 nucleotides in the ITS and no base pair differences in the TUB2. Comparison of the same gene regions between our strain and RGA01 strain, there are only $1 \mathrm{bp}(0.18 \%)$ difference that could in the ITS and within the $350 \mathrm{bp}$ of the TUB2 region, there are $7 \mathrm{bp}(2.0 \%)$ difference. In comparison of the same gene regions between our strain and ATCC 28,120 strain there were no base pair differences in the ITS and 7 bp (1.88\%) of difference from $372 \mathrm{bp}$ in the TUB2. When morphological characters of our species were examined, they were similar with the genus description provided by Vasilyeva and Stephenson (2006) and Senanayake et al. (2015). The species description of Eutypa lata provided by Moyo et al. (2018) is morphologically similar to our isolate MFLUCC 21-0068 (Fig. 152).
According to morpho-molecular analyses we conclude that our new collection is another record of Eutypa lata and also a new host record on Corylus avellana (Betulaceae) in Italy.

Xylariaceae Tul. \& C. Tul.

Notes: Members of Xylariaceae are occur throughout the world as saprobes, pathogens or endophytes in dead and decaying logs, leaves and fruits, or insect vectors. The family has been covered extensively in several recent treatises including Maharachchikumbura et al. (2016); Daranagama et al. (2018) and Xie et al. (2020). Several new genera and new species have been added into this family in recent times (Tibpromma et al. 2017a, b; Heredia et al. 2020; Voglmayr and Beenken 2020) into this family. Maharachchikumbura et al. (2016) accepted more than 80 genera. The family has also been broadly divided into two sub families Xylaroideae and Hypoxyloideae, the latter became Hypoxylaceae (Wendt et al. 2018). After the separation of Hypoxylaceae, the number of genera in Xylariaceae reduced to 37 genera (Daranagama et al. 2018). In a recent paper only 32 genera were accepted (Wijayawardane et al. 2020). Xylariaceae is known to produce different kinds of secondary metabolites (Helaly et al. 2018) and these are used as markers by some authors (Song et al. 2014). For example, the following genera could be delineated through the chemotaxonomic data by incorporating the secondary metabolite profiles: Coniolariella, Entoleuca, Hypocopra, Kretzschmaria, Rosellinia and Xylotumulus (Shang et al. 2018; Helaly et al. 2018; Becker et al. 2020).

\section{Xylaria Hill ex Schrank}

Notes: Xylaria members are mostly macroscopic in nature in that they can be seen by the unaided eye, and consist of members with different lifestyles including saprobic, pathogenic or endophytic in plant tissues, fallen leaves, fruits, seeds, manure and insects. Xylaria species are characterized by superficial, massive or sometimes reduced stroma in different shapes and size, unitunicate, cylindrical and pedicellate asci with or without a $\mathrm{J}+$ apical ring and ellipsoidal, dark ascospores with germ pores or germ slits, as well as geniculosporium, nodulisporium-like or libertella-like asexual morphs (Maharachchikumbura et al. 2016; Hyde et al. 2020a, b). There are more than 600 species known in this family and this number has been increasing with recent additions from different parts of the world (Husbands et al. 2018; Ju et al. 2018; Fournier et al. 2018; Ma and Li 2018; Crous et al. 2019; Hsieh et al. 2020; Ibrahim et al. 2020).

Xylaria apiospora M. Niranjan \& V.V. Sarma, sp. nov.

Index Fungorum number: IF557848; Facesoffungi number: FoF 08683; Fig. 154

Etymology: The ascospores having the apical cell.

Holotype: AMH-10071 


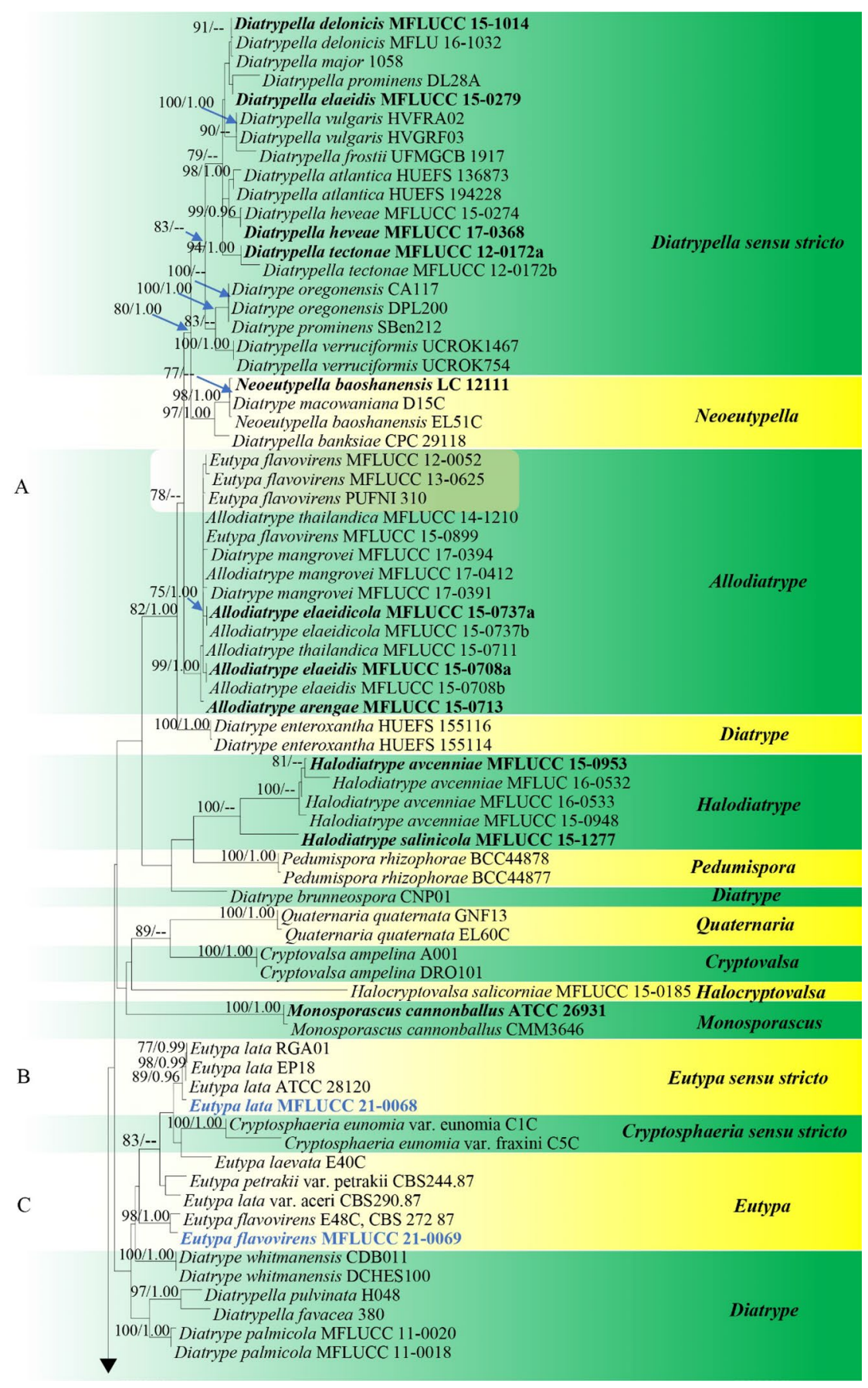

Fig. 153 Phylogram generated from maximum likelihood analysis based on combined ITS and TUB2 sequence data representing Diatrypaceae in Xylariales. Related sequences are taken from Konta et al. (2020) and additions according to the BLAST searches in NCBI. Hundred and twenty-nine strains are included in the combined analyses which comprised 1180 characters (665 characters for ITS and 515 characters for TUB2) after alignment. Kretzschmaria deusta (CBS 826.72) and Xylaria hypoxylon (CBS 122620) in Xylariaceae (Xylariales) were used as the outgroup taxa. The best scoring RAxML tree with a final likelihood value of -17362.853779 is presented. The matrix had 906 distinct alignment patterns, with $37.13 \%$ of undetermined characters or gaps. Estimated base frequencies were as follows: $\mathrm{A}=0.224946, \mathrm{C}=0.263658$, $\mathrm{G}=0.237000, \mathrm{~T}=0.274396$; substitution rates: $\mathrm{AC}=1.093989, \mathrm{AG}=3.037146, \mathrm{AT}=1.238822, \mathrm{CG}=0.814851, \mathrm{CT}=4.048082, \mathrm{GT}=1.000000$; gamma distribution shape parameter $\alpha=0.183977$. Bootstrap support values for ML equal to or greater than $75 \%$ are given above the nodes (left side). Bayesian posterior probabilities (BYPP) equal to or greater than 0.95 are given above the nodes (right side). Ex-type strains are in bold and newly generated sequences are in blue 


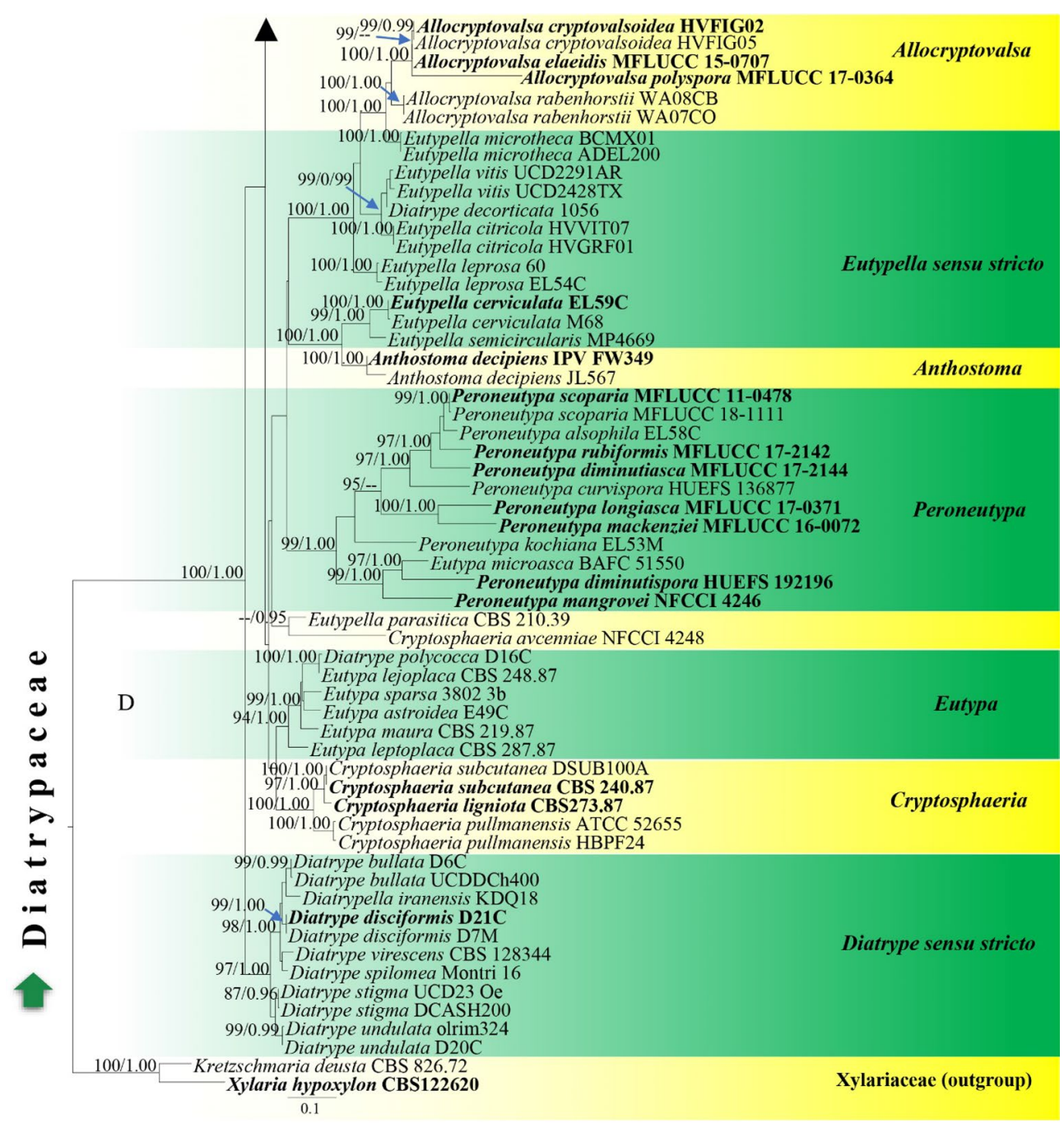

Fig. 153 (continued)

Saprobic on decaying twig. Sexual morph Ascostromata superficial, solitary, black, crust $95 \mu \mathrm{m}$ high, with brown hyphae on surface, loosely connected, hyaline, filamentous tissue and central brown tissue. Ascomata (193)285-460×(180-)215-260 $\mu \mathrm{m}(\bar{x}=242 \times 233 \mu \mathrm{m}, \mathrm{n}=8)$, globose, erumpent, pullvinate, clypeate, periphysate. Necks 82-120 $\mu \mathrm{m}$ high. Peridium $39 \mu \mathrm{m}$ wide, with brown to hyaline cell layers of textura porrecta. Hamathecium comprising $5.9 \mu \mathrm{m}$ wide, septate, branched, longer than asci, sparsely paraphyses. Asci $91-126 \times 4.5-6 \mu \mathrm{m}$ $(\bar{x}=108 \times 5 \mu \mathrm{m}, \mathrm{n}=25), 8-$ spored, unitunicate, cylindrical, apically rounded, cylindrical, with a $\mathrm{J}+$ an apical ring, rings $1.5-2 \times 1.5-2 \mu \mathrm{m}(\bar{x}=2 \times 2 \mu \mathrm{m}, \mathrm{n}=25)$, long pedicellate, persistent. Ascospores $12-14.5 \times 4-5 \mu \mathrm{m}(x=13 \times 4 \mu \mathrm{m}$, $\mathrm{n}=25$ ), overlapping uni-seriate, fusiform, hyaline to brown at maturity, germ slit curved, obtuse ends, with hyaline apical cell at one end, smooth walled. Asexual morph Undetermined.
Material examined: INDIA, Andaman and Nicobar Islands, South Andaman, Chidiya Tapu, Viewpoint Area $\left(11^{\circ} 48^{\prime} 10^{\prime \prime} \mathrm{N} 92^{\circ} 71^{\prime} 08^{\prime \prime} \mathrm{E}\right)$. Recorded on a decaying log, 9 December 2017, M. Niranjan and V.V. Sarma (PUFNI 1766). Herbarium specimen submitted to Ajrekar Mycological Herbarium-AMH (AMH-10071, holotype) and ex-type living culture (NFCCI-4370) deposited at National Fungal Culture Collection of India (NFCCI), Agarkhar Reserch Institute, Pune, Maharasthra, India.

GenBank numbers: ITS = MT572916.

Notes: Xylaria apiospora slightly differs from $X$. longipes (Rogers 1983) in having smaller ascomata $(285-460 \times 215-260$ vs. $450-650 \times 400-600 \mu \mathrm{m})$ and asci $(91-126 \times 4.5-6$ vs. $130-180 \times 7-9 \mu \mathrm{m})$, and larger ascospores $(12-14.5 \times 4-5$ vs. $13-15 \times 5-7 \mu \mathrm{m})$ with hyaline smaller, apical cells. While X. apiospora has fusiform ascospores with hyaline, smaller cells, $X$. longipes has ellipsoid-inequilateral, aseptate ascospores. Phylogenetically, 

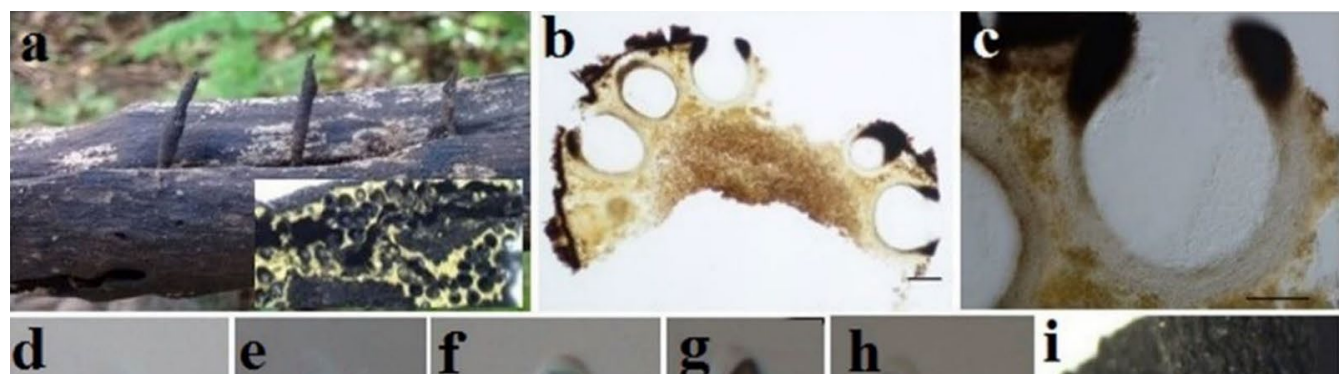

d

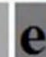

f
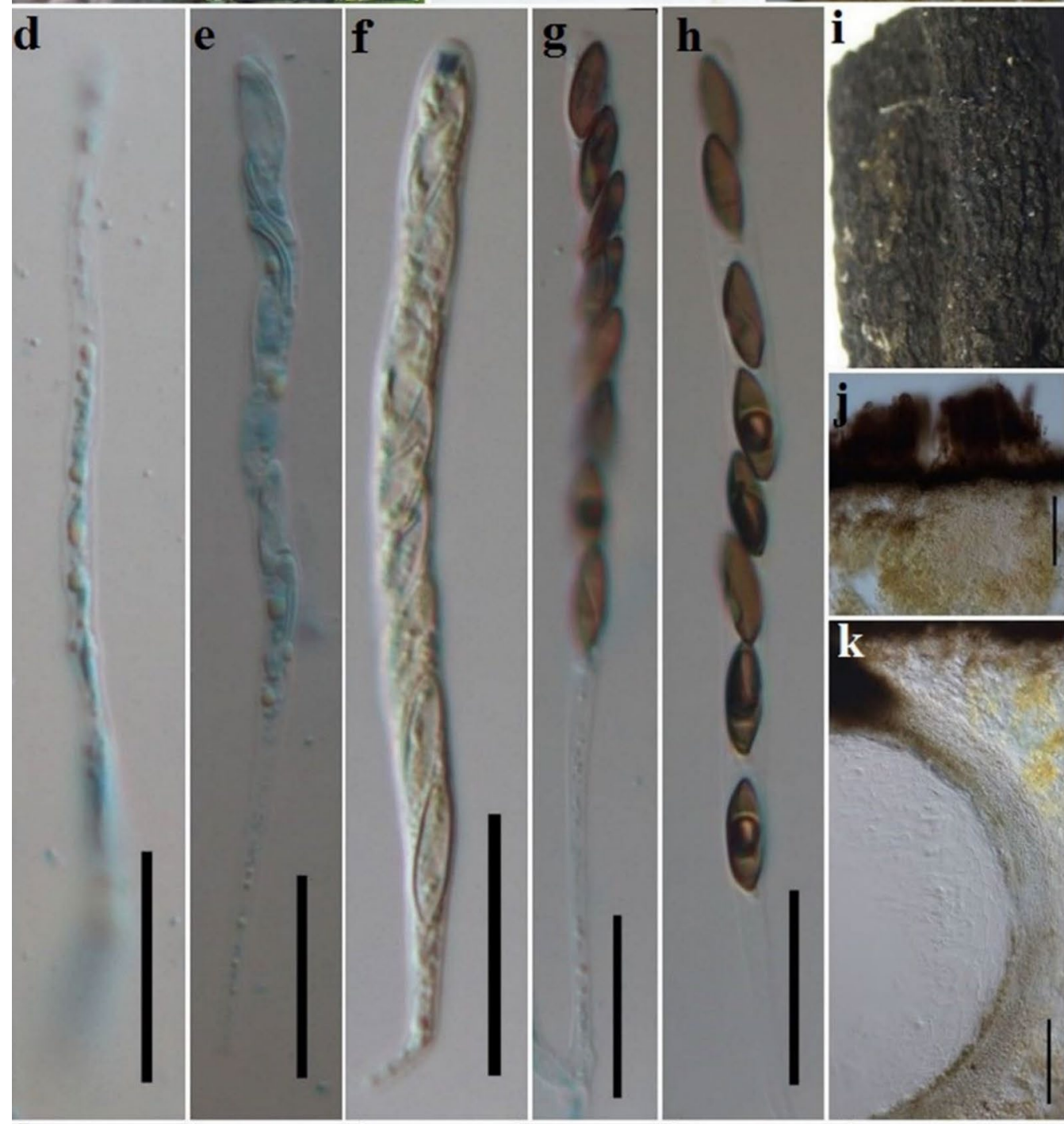

t
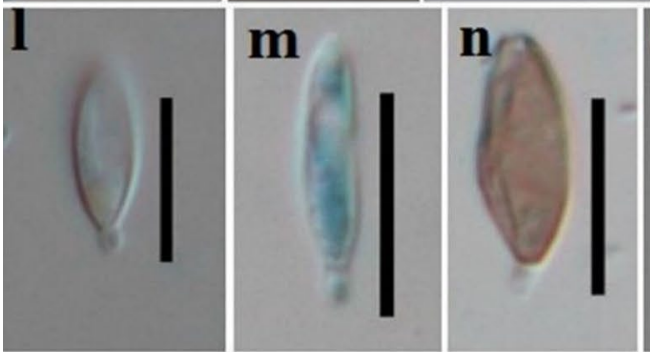

Fig. 154 Xylaria apiospora (NFCCI-4370, holotype). a Stromata on decaying host. b, c Horizontal section of stromata. d Paraphyses. e-h Asci. i Striations on stromatal surface. $\mathbf{j}$ Cross section of stroma. $\mathbf{k}$

Peridium. l-q Ascospores. Scale bars: $\mathbf{b}=100 \mu \mathrm{m}, \mathbf{c}, \mathbf{j}, \mathbf{k}=50 \mu \mathrm{m}$, $\mathbf{d}-\mathbf{h}=20 \mu \mathrm{m}, \mathbf{l}-\mathbf{q}=10 \mu \mathrm{m}$ 
$X$. apiospora constitutes a distinct basal lineage to Xylaria multiplex (1010). Therefore, we introduce $X$. apiospora as a new species based on morphology and phylogenetic evidence (Figs. 154 and 156).

Xylaria haemorrhoidalis Berk. \& Broome. Journal of the Linnean Society. Botany 14: 117 (1875).

Index Fungorum number: IF224410; Facesoffungi number: FoF 08682; Fig. 155

Saprobic on decaying twig. Sexual morph Stromata $1.2-1.5 \mathrm{~cm}$ long, $0.3 \mathrm{~cm}$ wide, 0.9 long at stipes, upright, aggregated, unbranched, obclavate, fertile apices, surface pulvinate, black, soft with scattered brown hairs emerging from grooves, internally has white thick hyphae, stalks long very distinct from stroma. Ascomata perithecial $478-630 \times 495-580 \mu \mathrm{m}(\bar{x}=567 \times 542 \mu \mathrm{m}, \mathrm{n}=5)$, globose, clypeate. Necks $152-210 \mu \mathrm{m}$ high $(\bar{x}=182.5 \mu \mathrm{m}$, $\mathrm{n}=5$ ), periphysate. Peridium 34-41 $\mu \mathrm{m}$ wide, with outer brown cell layers of textura porrecta, inner thin cell layers of textura angularis. Hamathecium comprising 8.5-12 $\mu \mathrm{m}$ wide, septate, branched, longer than asci, sparsely paraphyses. Asci $140-206 \times(10-) 12-18(-21) \mu \mathrm{m}(\bar{x}=167 \times 14 \mu \mathrm{m}$, $\mathrm{n}=25), 8$-spored, unitunicate, cylindrical, apically rounded, urn-shaped $\mathrm{J}+$ an apical ring 6-10 $\times 4-5(-6)$ $\mu \mathrm{m}(\bar{x}=8 \times 5 \mu \mathrm{m}, \mathrm{n}=25)$, long pedicellate, persistent. Ascospores (21-)23-26 $\times 10-12 \mu \mathrm{m}(\bar{x}=25 \times 10.5 \mu \mathrm{m}$, $\mathrm{n}=25$ ), overlapping uni-seriate, hyaline to brown at maturity, ovoid, straight germ slit, obtuse ends, smooth walled. Asexual morph Undetermined.

Material examined: INDIA, Andaman and Nicobar Islands, South Andaman, Mount Harriet, (11 ${ }^{\circ} 71^{\prime} 09.8^{\prime \prime} \mathrm{N}$ 92 $73^{\prime} 30.6^{\prime \prime}$ E). Recorded from a decaying log, 7 December 2017, M. Niranjan and V.V. Sarma (PUFNI 1765). Herbarium specimen submitted to Ajrekar Mycological Herbarium-AMH (AMH-10070, new record) and living culture (NFCCI-4369) deposited at National Fungal Culture Collection of India (NFCCI), Agarkhar Research Institute, Pune, Maharashtra, India.

GenBank numbers: ITS = MT572915.

Notes: Xylaria haemorrhoidalis is poorly known due to the fact that very few reports are available on its occurrence (Ju et al. 2009). Dade (1940) synonymized this fungus with $X$. allantoidea but recent molecular data show that it is distinct (Fig. 156). In the NCBI database, sequence data of only six accessions of $X$. haemorrhoidalis are available of which three are of ITS and one each of $\alpha$-actin, $\beta$-tubulin and RPB2 genes. It has earlier been reported from Taiwan and the present record from Andaman forests, India extends its geographical range (Fig. 155).

Xylariales, genus incertae sedis

Melanographium Sacc.
Notes: Melanographium was introduced by Saccardo (1913) to accommodate the hyphomycetous taxon and is typified by M. spleniosporum. Melanographium taxa are saprobes and commonly found on dead or decaying wood, leaves, stems, culms or petioles, mostly on palms (Ellis 1963; Goh and Hyde 1997; Somrithipol and Jones 2005). The genus is characterized by having superficial colonies, unbranched and erect brown conidiophores, polyblastic conidiogenous cells with sympodial proliferation, and holoblastic, reniform, aseptate, brown to dark brown conidia (Ellis 1963, 1971). Melanographium includes 14 accepted species mostly based on morphology, but only one species, namely Melanographium phoenicis (MFLUCC 18-1481), have sequence data (Hyde et al. 2020b; Index Fungorum 2021). In this study, we introduce a new species Melanographium smilacis based on morphological and phylogenetic analyses.

Melanographium smilacis Boonmee, Huanraluek \& K.D. Hyde, sp. nov.

Index Fungorum number: IF558546; Facesoffungi number: FoF 09964; Fig. 157

Etymology: The specific epithet "smilacis" reflects the host genus Smilax.

Holotype: MLFU 21-0075

Saprobic on dead stems of Smilax perfoliate. Sexual morph Undetermined. Asexual morph Colonies on natural substrate superficial, effuse, velvety, tufted, dark brown. Mycelium immersed, composed of branched, septate, smooth, brown hyphae. Conidiophores $270-561 \times 2-7 \mu \mathrm{m}$ $(\bar{x}=460 \times 5 \mu \mathrm{m}, \mathrm{n}=20$ ), macronematous, loosely or densely, fascicle, panicle or tufted, unbranched, multiseptate, straight below, slightly flexuous, geniculate near the apex, brown to dark brown below, brown to pale brown towards the apex, subhyaline at the tip, thick-walled, rarely with percurrent proliferations. Conidiogenous cells polyblastic, integrated, terminal, sympodial, cylindrical or slightly clavate, with minutely protruding conidiogenous scars. Conidia $11-17 \times 4-13 \mu \mathrm{m}(\bar{x}=14 \times 9 \mu \mathrm{m}, \mathrm{n}=50)$, holoblastic, acropleurogenous, solitary, reniform or broad obovoid to semicircular, slightly curved, olivaceous brown to dark brown, aseptate, large guttulate when immature, verrucose or smooth-walled. Conidial secession schizolytic.

Culture characteristics: Conidia germinated on MEA, colony circular and flat, reaching $3.5 \mathrm{~cm}$ in 2 weeks at $25^{\circ} \mathrm{C}$, dense, effuse, velvety, radially with fimbriate edge, white grey to pale brown in surface, reddish brown to brown in reverse, with orangish yellow pigmented in media, with radiating outwards mycelium with dull white margin.

Material examined: THAILAND, Chiang Rai, Muang, Doi Lan, on dead stems of Smilax perfoliate L. (Smilacaceae), 27 March 2019, S. Boonmee, DL1 (MFLU 21-0075, holotype), ex-type living culture, MFLUCC 21-0098. 

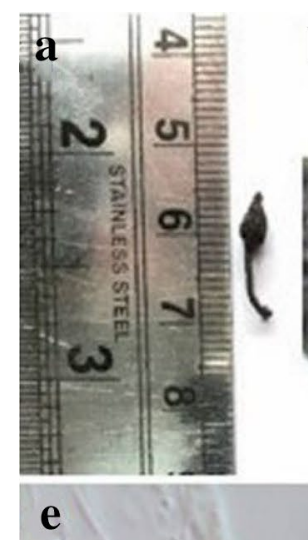

(
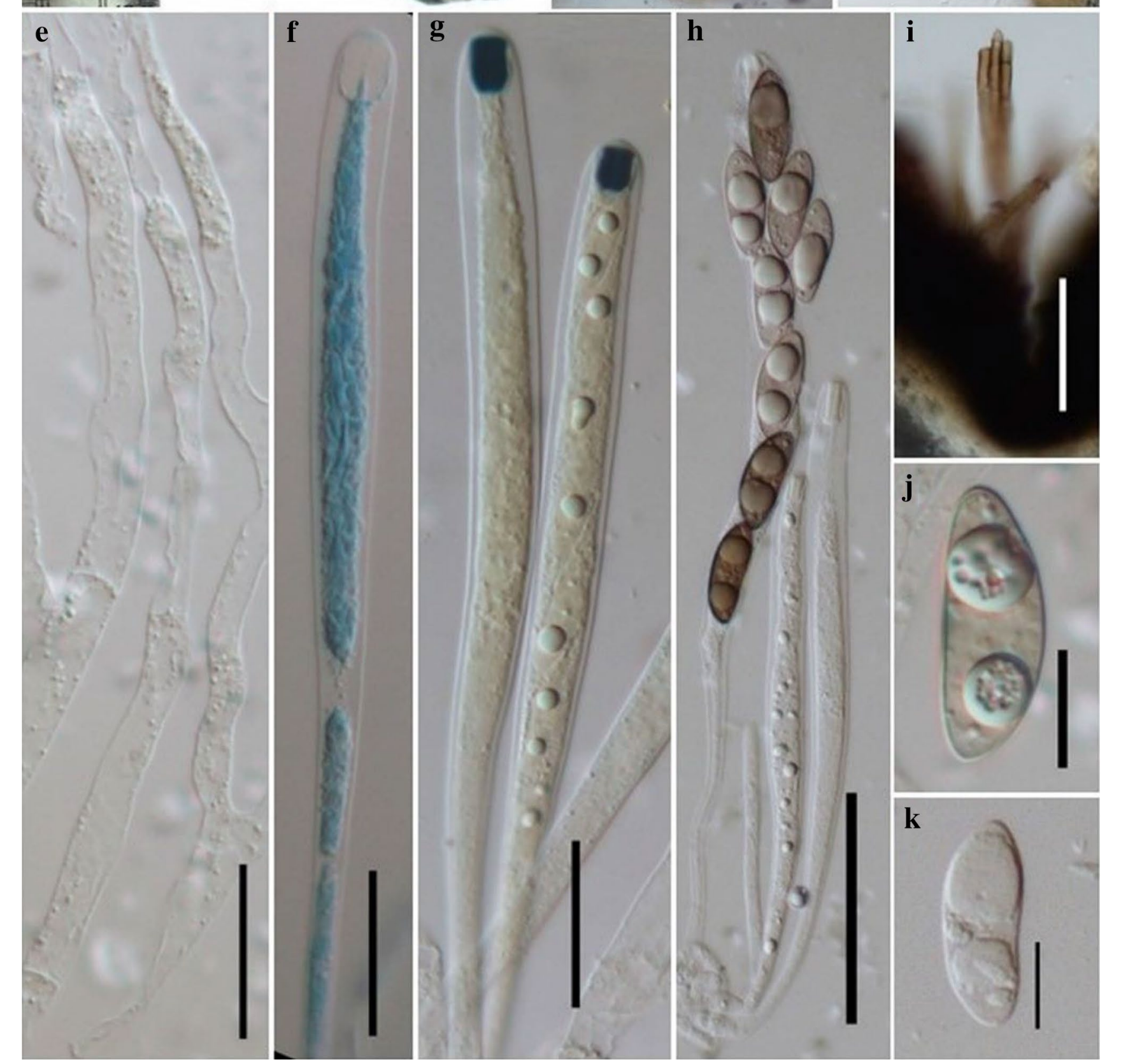

Fig. 155 Xylaria haemorrhoidalis (NFCCI-4369, new record) a Stromata on decaying host. b, c Horizontal section of ascoma. d Peridium. e Paraphyses. $\mathbf{f}-\mathbf{h}$ Asci. i Hyphae on stromata surface. j, $\mathbf{k}$ Ascospores. Scale bars: $\mathbf{c}=200 \mu \mathrm{m}, \mathbf{d}, \mathbf{h}, \mathbf{i}=50 \mu \mathrm{m}, \mathbf{e}-\mathbf{g}=20 \mu \mathrm{m}, \mathbf{k}, \mathbf{n}=10 \mu \mathrm{m}$ 


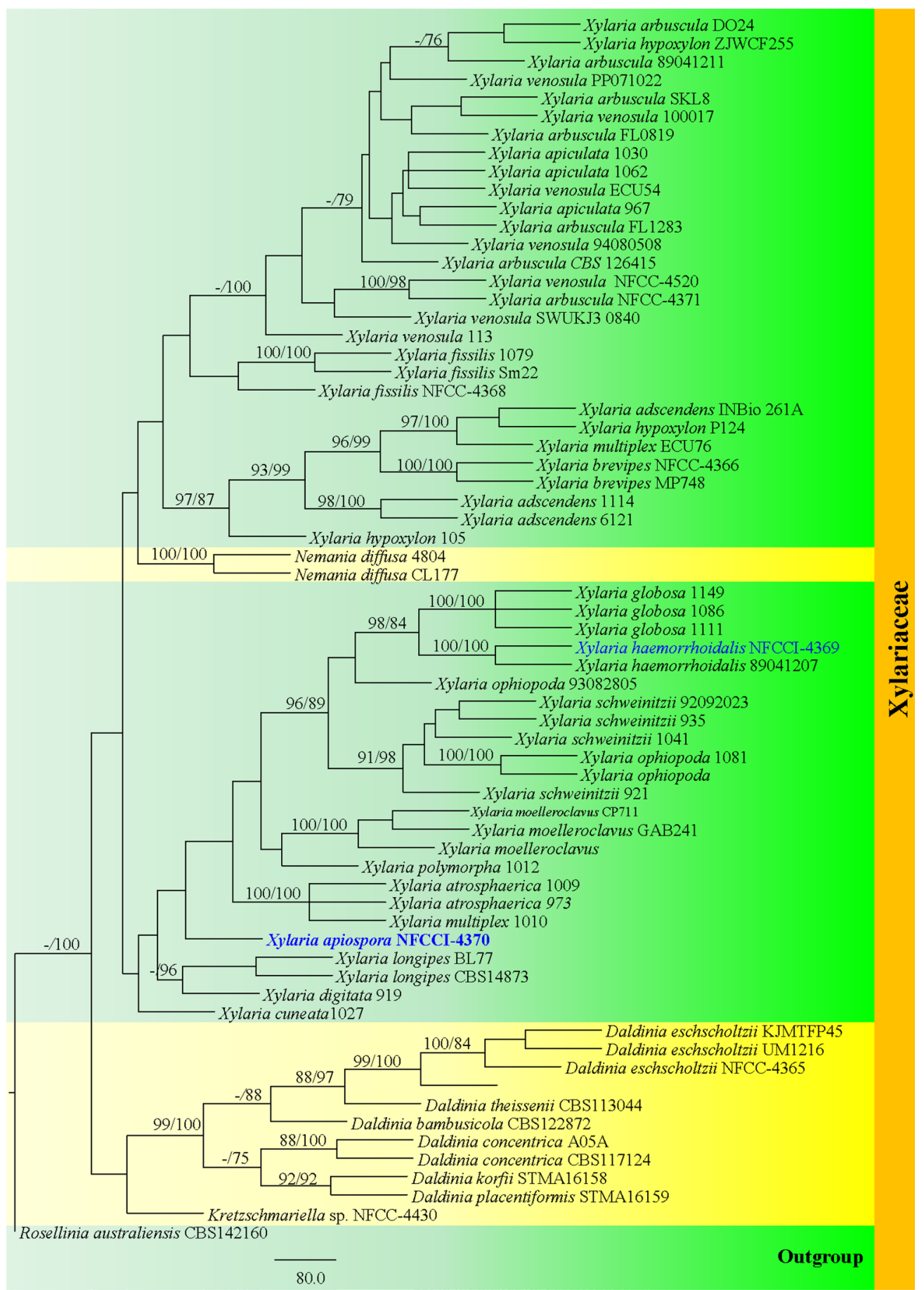

Fig. 156 Maximum parsimony tree of Xylariaceae constructed by using the ITS sequence. The tree includes both ML and MP values. Rosellinia australiensis is selected as an outgroup taxon. RAxML analysis yielded a minimum scoring tree with a final ML optimization likelihood value of - 5854.053127. The matrix had 393 distinct alignment patterns with $10.20 \%$ of undetermined characters or gaps. The maximum parsimonious dataset consists of 626 characters of which 278 were constant, 233 parsimony-informative and 115 parsimony-uninformative. The parsimony analysis of the data matrix resulted in one thousand equally parsimonious trees with a length of 1041 steps $(\mathrm{CI} 0.540, \mathrm{RI}=0.829, \mathrm{RC} 0.447, \mathrm{HI}=0.460)$ in the first tree. The overall topology of the phylogenetic trees resulted from ML and MP were similar and incongruent in with earlier studies. The phylogenetic analysis showed that Xylaria haemorrhoidalis NFCC-4369 nested with Xylaria haemorrhoidalis 89041207 with $100 \%$ MLBS and $100 \%$ MPBS support. Similarly, Xylaria apiospora NFCC-4370 branched with Xylaria multiplex 1010 with week bootstrap support of MPBS. The newly generated sequences are indicated in blue bold 


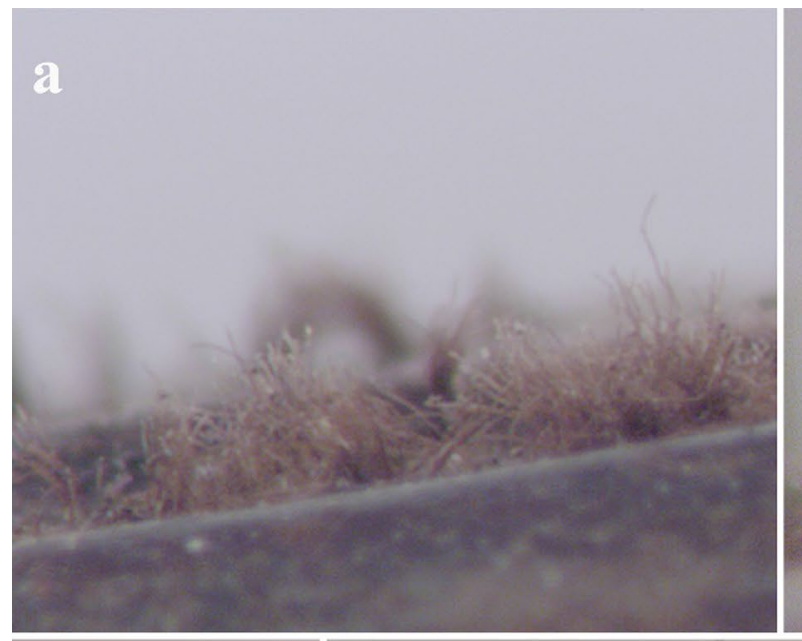

\section{b}
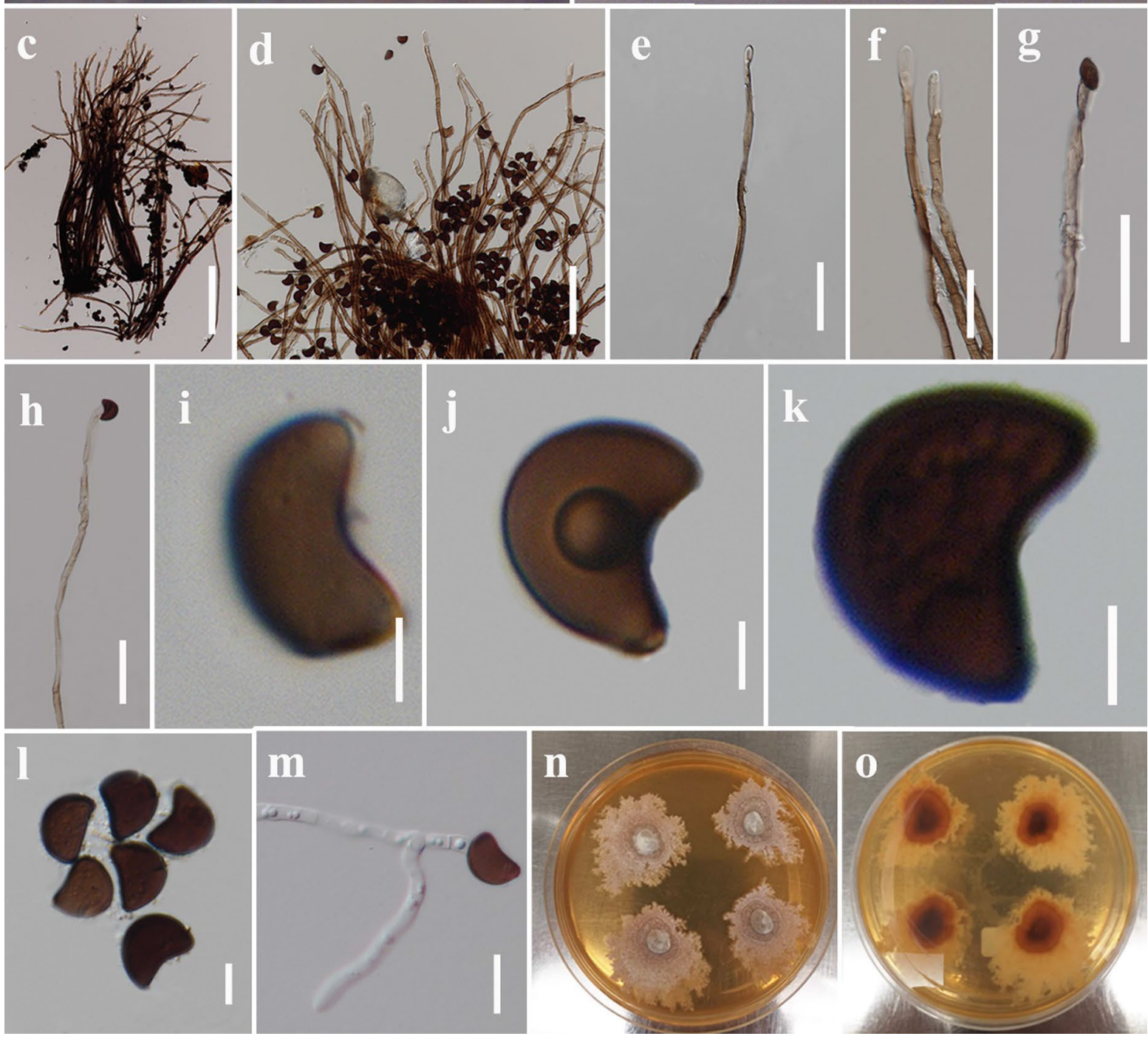

Fig. 157 Melanographium smilacis (MFLU 21-0075, holotype). a, b Appearance of colonies on natural substrate. c, $\mathbf{d}$ Squash mount of conidiophores with attached conidia. e, $\mathbf{f}$ Close-up of conidiophores showing septation with paler at the apex. $\mathbf{g}$, $\mathbf{h}$ Apex of conidiophores with developing conidia. $\mathbf{i}-\mathbf{l}$ Conidia with a basal scar. $\mathbf{m}$ Germinated conidium. $\mathbf{n}, \mathbf{o}$ Culture on MEA from surface and reverse. Scale bars: $\mathbf{b}=100 \mu \mathrm{m}, \mathbf{c}, \mathbf{d}=200 \mu \mathrm{m}, \mathbf{e}-\mathbf{h}=50 \mu \mathrm{m}, \mathbf{i}-\mathbf{l}=5 \mu \mathrm{m}, \mathbf{m}=10 \mu \mathrm{m}$ 
GenBank numbers: ITS $=$ MZ538514, LSU $=$ MZ538548, SSU $=$ MZ538572, TEF1- $\alpha=$ MZ567091.

Notes: Melanographium smilacis was found on dead stems of Smilax perfoliate (Smilacaceae). Melanographium smilacis shares common characters with species in Melanographium such as effused and tufted colonies, loosely or densely fascicle and brown conidiophores and reniform shape, aseptate with dark pigmented conidia (Saccardo
1913; Trotter 1931; Ellis 1963, 1971; Goh and Hyde 1997; Somrithipol and Jones 2005; Hyde et al. 2020b). Phylogenetically, Melanographium smilacis is as a sister lineage with the ex-type strain of M. phoenicis (MFLUCC 18-1481) with 100\% MLBS, 1.00 BYPP support (Fig. 158). However, $M$. smilacis differs from M. phoenicis and other species in terms of the conidial features such as reniform or broad obovoid to semicircular shapes and lacking a slit (Fig. 157). According

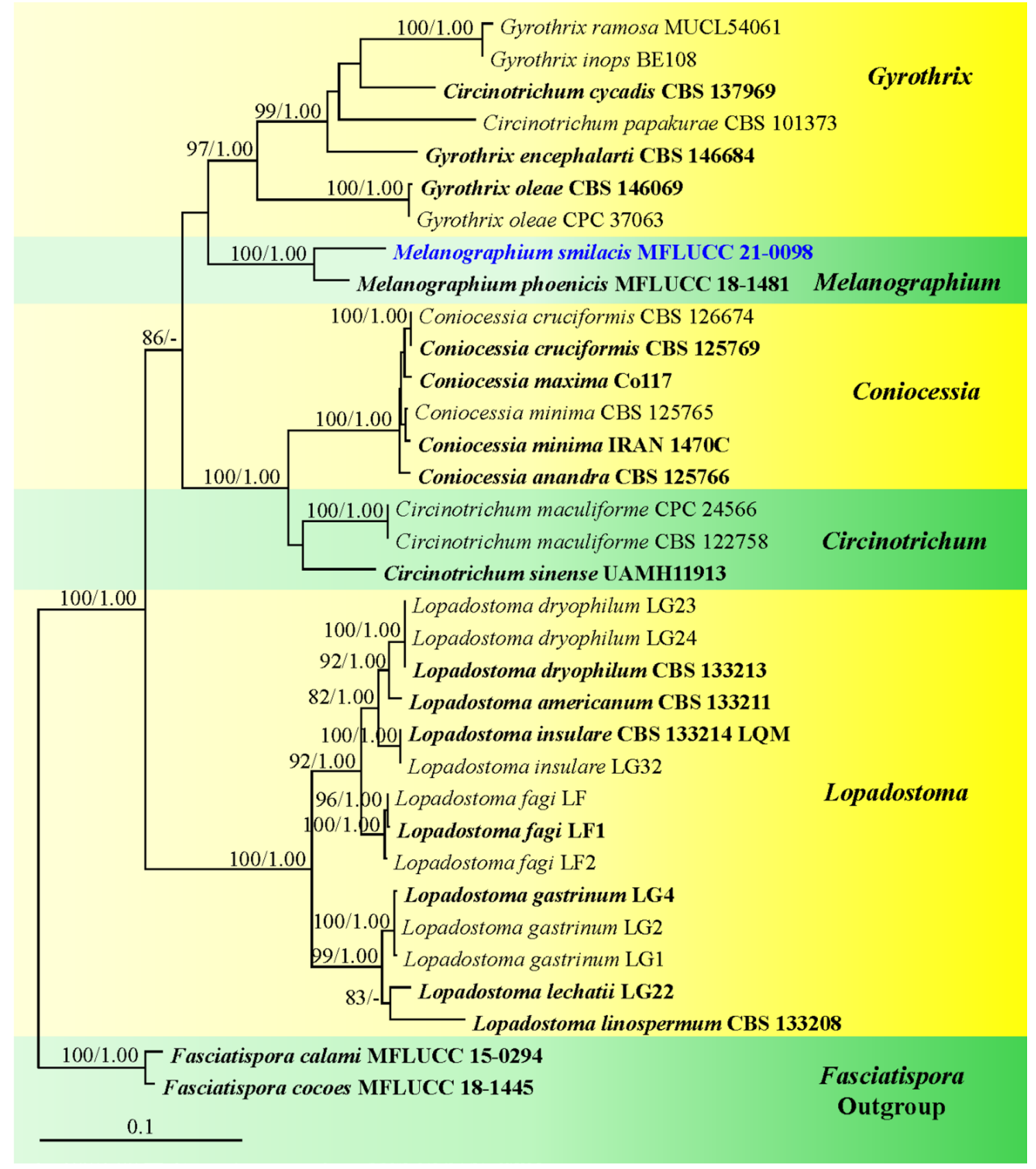

Fig. 158 Maximum likelihood phylogenetic tree based on a combined LSU and ITS sequence data of Melanographium taxa and related genera. The tree is rooted with Fasciatispora calami (MFLUCC 15-0294) and F. cocoes (MFLUCC 18-1445). Bootstrap support values for maximum likelihood (MLBS, left) equal to or greater than $70 \%$ is given above the nodes. Bayesian posterior probabilities (BYPP, right) equal to or greater than 0.95 are given above the nodes. Ex-type strains are in bold and newly generated sequence is in blue 
to the morphological and phylogenetic analyses, we introduce a new species, Melanographium smilacis.

\section{Basidiomycota R.T. Moore}

Notes: The modern classification of Basidiomycota is adopted in this note following Zhao et al. (2017) and He et al. (2019).

\section{Agaricomycetes Doweld}

Notes: The classification of the families in Agaricomycetes is adopted herein following Hibbett et al. (2014), Zhao et al. (2017) and He et al. (2019).

\section{Agaricales Underw.}

Notes: Agaricales includes six well-accepted clades, viz. Agaricoid, Tricholomatoid, Marasmioid, Pluteoid, Hygrophoroid and Plicaturopsidoid (Matheny et al. 2006). He et al (2019) listed around 17,291 species belonging to 508 genera of 38 families in Agaricales. Some genera in this order have uncertain positions at the family level. Several genera are even polyphyletic. Therefore, it is necessary to construct a comprehensive phylogenetic frame for Agaricales to properly establish the position of the taxa.

\section{Agaricaceae Chevall.}

Notes: According to the recent study of He et al. (2019), Agaricaceae is a large group of Basidiomycota consisting of approximately 54 genera. Several studies established the genus classification under different families based on the molecular data (Vellinga 2004; Vellinga et al. 2011; Matheny et al. 2006).

\section{Chlorophyllum Massee}

Notes: Chlorophyllum belongs to Agaricaceae comprising 19 species (Vellinga 2004; He et al. 2019) distributed in both tropical and temperate regions. This genus has six accepted sections namely Chlorophyllum, Ellipsoidospororum, Endoptychorum, Parvispororum, Rhacodium and Sphaerospororum (Ge et al. 2018). Chlorophyllum molybdites is the sole species recorded from Laos (Læssøe et al. 2019). In this study, we propose another three species of Chlorophyllum first recorded in Laos.

Chlorophyllum demangei (Pat.) Z.W. Ge \& Zhu L. Yang, MycoKeys 33: 80 (2018)

Index Fungorum number: IF823863; Facesoffungi number: FoF 07067; Figs. 159, 160

Pileus $40-80 \mathrm{~mm}$ diam., convex-hemispherical to umbonate, expanding to applanate when fully mature, with straight margin, center glabrous, with brown to brown (5D4, 6D7-8) calotte, with concolourous with tufted patches to suamules around umbo toward margin, on white background and becoming orange white (5A2) when mature,

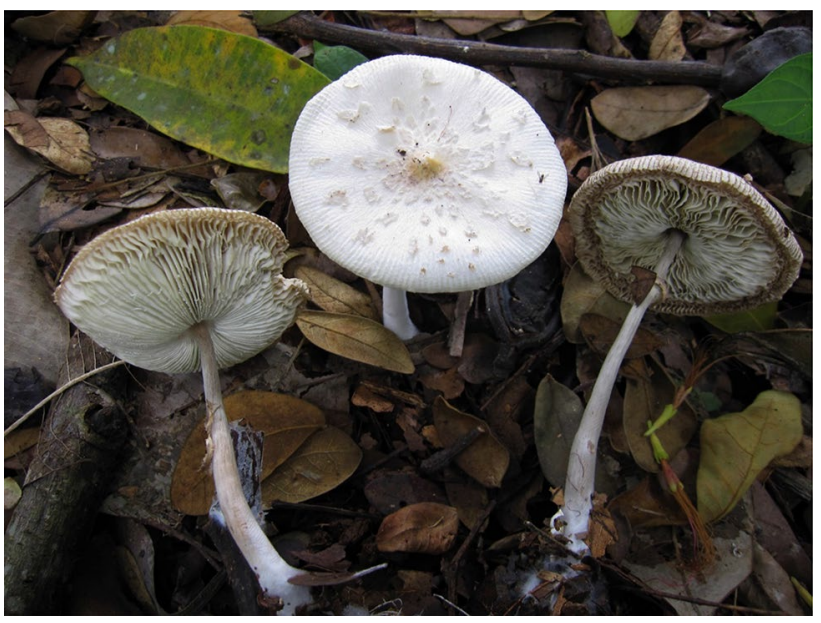

Fig. 159 Basidiomata of Chlorophyllum demangei (HNL502498, new record)

margin sulcate and striate, with exceeding lamellae when mature. Lamellae free, ventricose, 4-7 mm wide, white and becoming yellowish white (4A2) with age, crowded, with concolorous eroded lamella-edge. Stipe $80-130 \times 6-12 \mathrm{~mm}$, cylindrical to downward base; surface smooth, covered with white fibrils at base zone. Annulus superonate, moveable, with brown to brown (5D4, 6D7-8) surface. Context in pileus white, in stipe white and hollow, turning orange white (5A2) in both stipe and pileus context. Taste peanutliked. Smell mild. Spore print white. Basidiospores [50,2,2] 6-8 $\times 4-6.5 \mu \mathrm{m}$, avl $\times$ avw $=7 \times 5 \mu \mathrm{m}, \mathrm{Q}=1-1.5$, Qav $=1.5$, ellipsoid to oblong-amygdaliform in side-viewed, ellipsoid to oblong in frontal view, without germ pore, hyaline, thickwalled, dextrinoid, congophilous. Basidia 15-25×8-12 $\mu \mathrm{m}$, clavate, hyaline, thin-walled, 2-spored and 4-spored. Cheilocystidia abundant, $20-60 \times 5-11 \mu \mathrm{m}$, clavate to narrowly clavate, sometimes lageniform, cylindrical with papillate apex, hyaline, thick-walled. Pleurocystidia absent. Pileus covering a trichoderm made up of cylindrical to narrowly clavate elements with long stalk, $60-140 \times 5-16 \mu \mathrm{m}$, hyaline to pale brown parietal pigment, sometimes encrusted. Stipe covering a cutis made up of cylindrical hyphae and elements, up to 7-15 $\mu \mathrm{m}$ wide. Clamp connections absent.

Material examined: LAOS, Oudomxay Province, Xay, Houay Houm Village, 18 August 2014, P. Sysouphanthong, PS2014-827 (HNL502498); Oudomxay Province, Beng, Na Pa Village, 19 July 2014, P. Sysouphanthong, PS2014-467 (HNL502138, new record).

GenBank numbers: ITS = MW193055, MW040572.

Notes: Lao specimens of Chlorophyllum demangei are solitary to gregarious. Chlorophyllum demangei is saprotrophic, growing on rotten wood and decayed leaves. It is found in various habitats (Figs. 159 and 160). This species was described as Lepiota demangei Pat. in sect. 


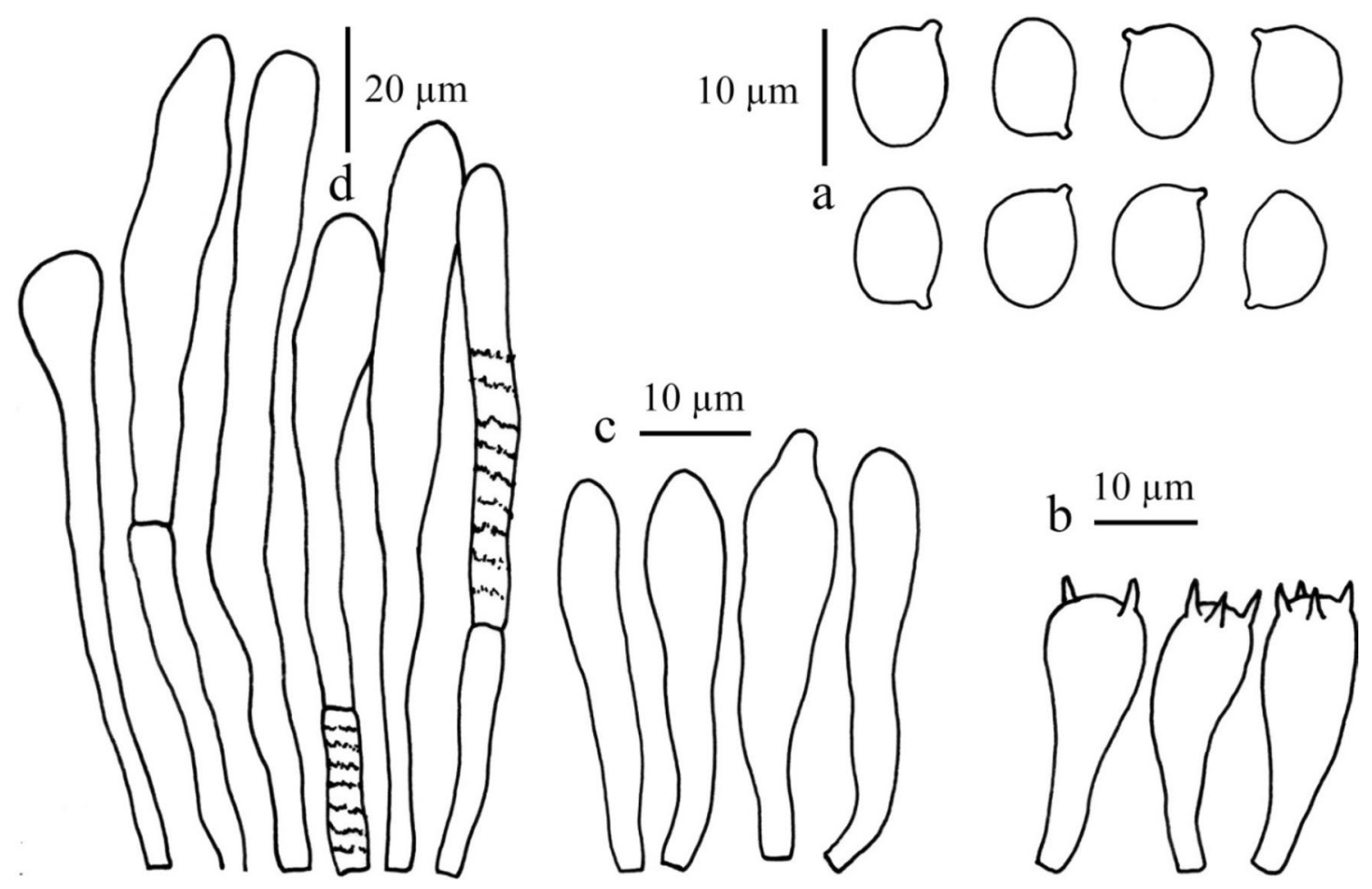

Fig. 160 Microcharacters of Chlorophyllum demangei (HNL502498). a Basidiospores. b Basidia. c Cheilocystidia. d Element cells at pileus covering. Scale bars: $\mathbf{a}-\mathbf{c}=10 \mu \mathrm{m}, \mathbf{d}=20 \mu \mathrm{m}$

Ellipsoidospororum, including other species with white basidiospores (Ge et al. 2018). Chlorophyllum demangei can be distinguished from $C$. hortense (Murrill) Vellinga by having 4-spored basidia while the latter has 2-spored basidia. Multigene phylogeny showed that they are two different species (Ge et al. 2018). ITS gene tree shows that the two Lao specimens clustered with other Chlorophyllum demangei collections in GenBank (Fig. 165). Chlorophyllum demangei in this study is a first record for Laos.

Chlorophyllum globosum (Mossebo) Vellinga, Mycotaxon 83: 416 (2002)

Index Fungorum number: IF823863; Facesoffungi number: FoF 07069; Figs. 161, 162

Pileus up to $150 \mathrm{~mm}$ diam., first subglobose, expanding to convex and plano-concave when mature, with straight margin, when young glabrous, brownish grey to light brown (7D3-8), soon surface broken and leaving concolorous glabrous at center, with concolorous patches spreading towards margin, on white to yellowish-white (4A2) fibrillose background, margin white, sulcate and exceeding lamellae when mature. Lamellae free, white to orangewhite (5A2) at first, becoming grayish green (29B4) with age, very crowded, ventricose, $10-16 \mathrm{~mm}$ wide, with eroded lamellae-edge. Stipe $140-180 \times 10-20 \mathrm{~mm}$, tapering to apex, with wide bulb at base, $25-30 \mathrm{~mm}$ wide; smooth and white background, becoming orange grey to greyish orange (6B23 ) with age. Annulus cuff-like, moveable, white in upper part, with brownish grey to light brown (7D3-8) squamules at underside. Context thick and white in pileus, white in stipe, turning pastel red (9A4) in both pileus and stipe when cut. Smell and oduor not observed. Spore print grayish-green (29D5-6). Basidiospores [25,1,1] 8.5-12.5 $\times 6.5-8.5 \mu \mathrm{m}$, avl $\times$ avw $=10-11.5 \times 7.5-8 \mu \mathrm{m}, \mathrm{Q}=1-1.5$, avQ $=1$, broadly ellipsoid to ellipsoid-amygdaliform in side-view, broadly ellipsoid or amygdaliform in frontal view, apex truncate and with germ pore, thick-walled, hyaline, dextrinoid, congophilous. Basidia $18-350 \times 8-16 \mu \mathrm{m}$, clavate, hyaline, slightly thick-walled, 4-spored. Cheilocystidia abundant, $35-62 \times 8-15 \mu \mathrm{m}$, clavate to narrowly clavate, slightly thick-walled, hyaline. Pleurocystidia absent. Pileus covering a hymenoderm made up of several layers of cylindrical hyphae, terminal elements cylindrical to narrowly clavate with rounded or attenuate apex 50-950×4-13 $\mu \mathrm{m}$, slightly thick-walled, hyaline or with pale yellow intracellular pigment. Clamp connections absent.

Material examined: LAOS, Vientiane Capital, Xaythany, Houay Yang Preserve Forest, 16 June 2017, P. Sysouphanthong, PS2017-7 (HNL503445, new record).

GenBank numbers: ITS $=$ MN317315.

Notes: We observed the specimens of Chlorophyllum globosum from young to mature stages. Chlorophyllum 

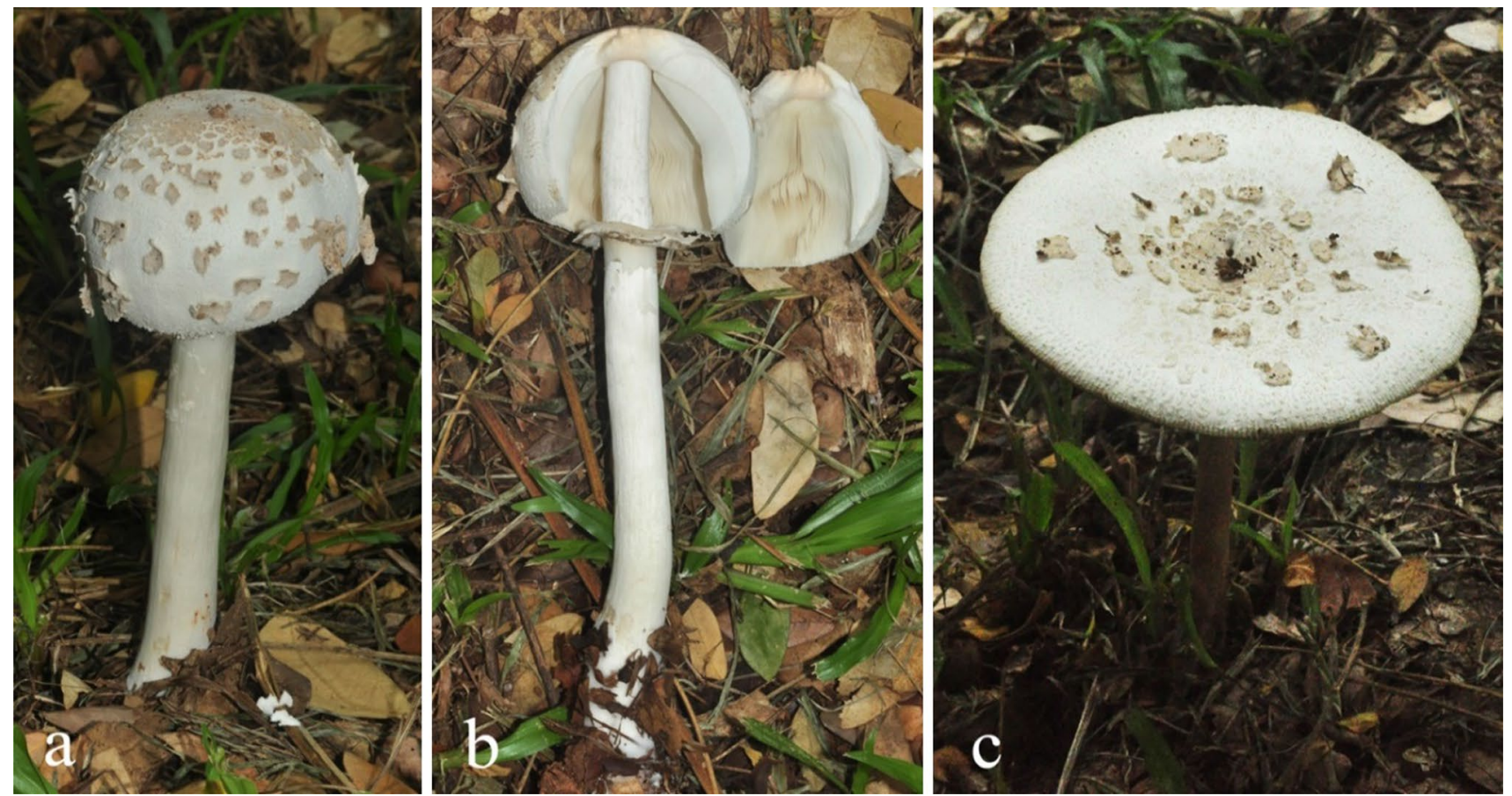

Fig. 161 Chlorophyllum globosum (HNL503445, new record). a-c Basidiomata
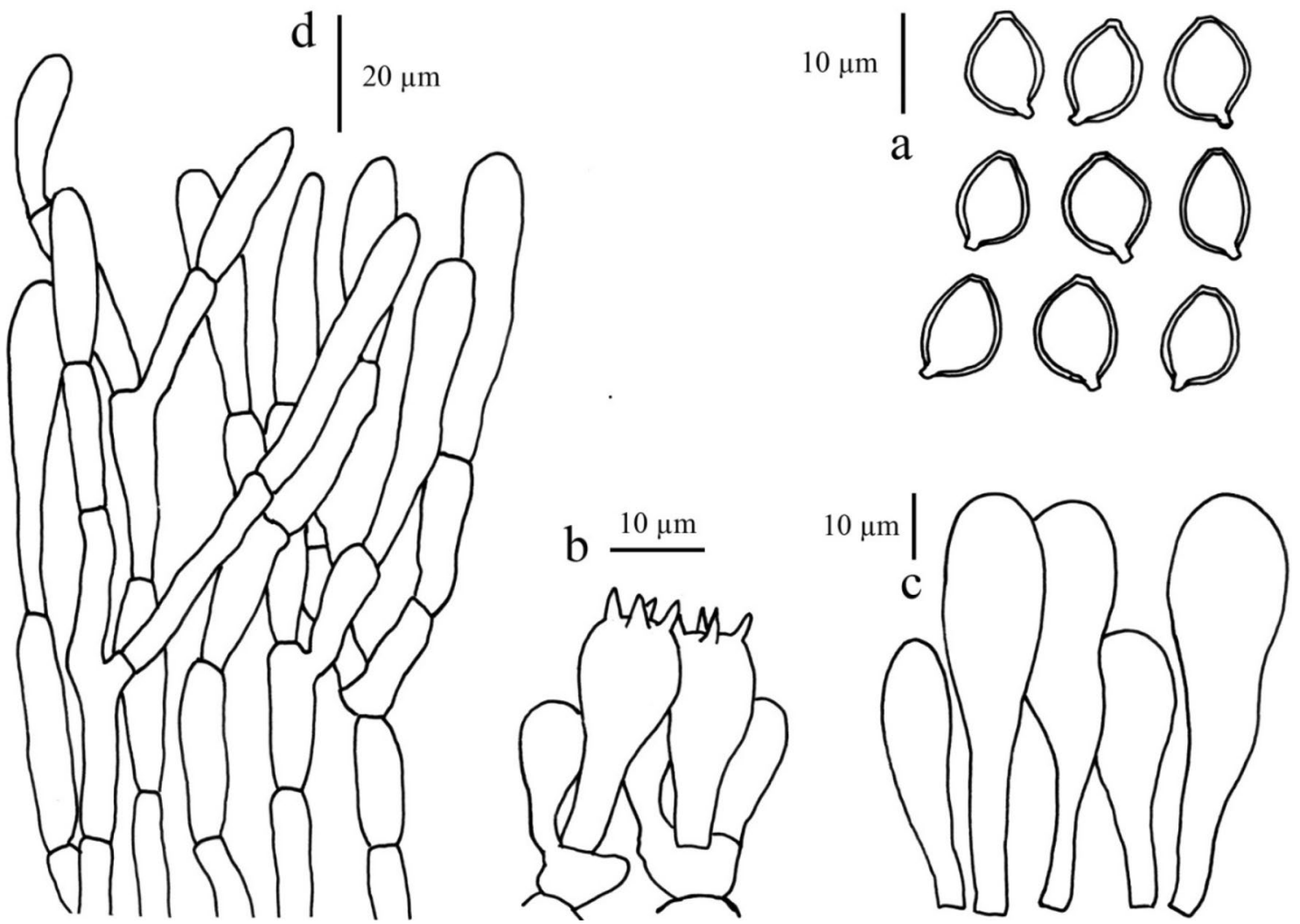

Fig. 162 Microcharacters of Chlorophyllum demangei (HNL503445). a Basidiospores. b basidia. c Cheilocystidia. d Element cells at pileus covering. Scale bars: $\mathbf{a}-\mathbf{c}=10 \mu \mathrm{m}, \mathbf{d}=20 \mu \mathrm{m}$ 

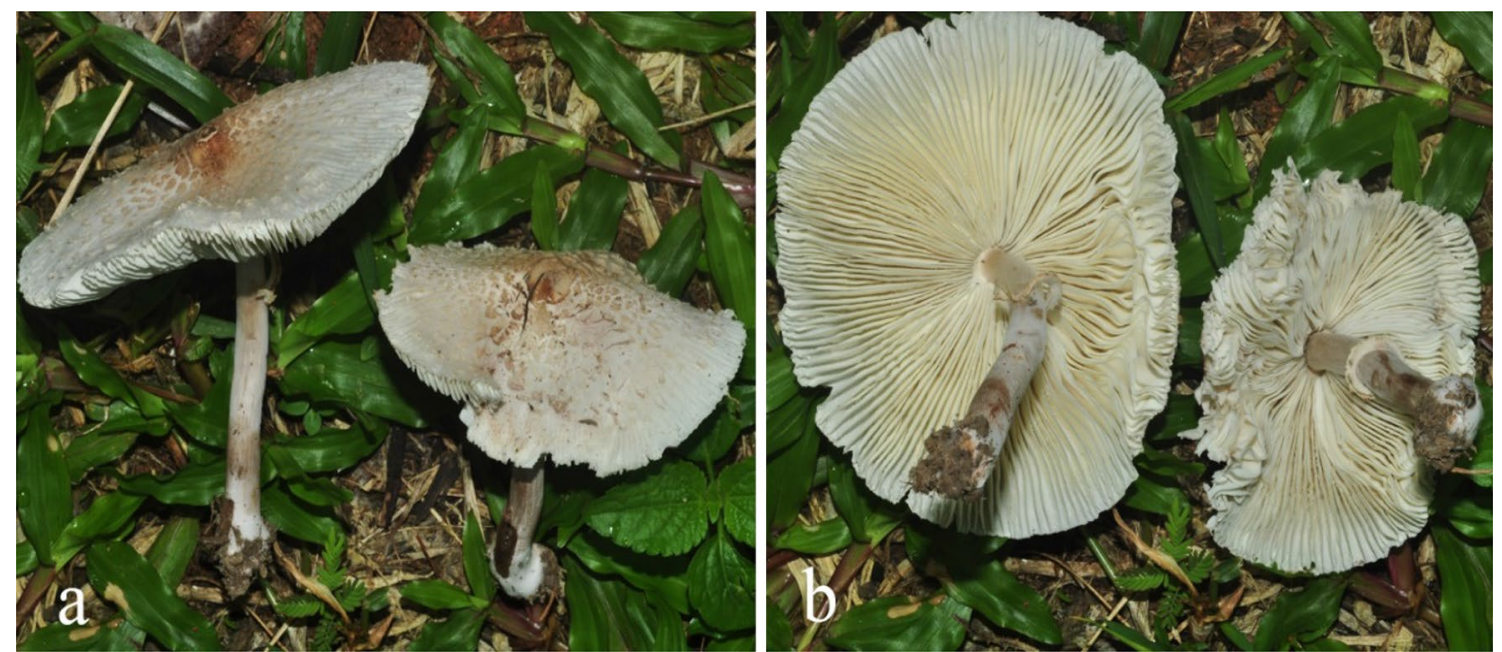

Fig. 163 Chlorophyllum hortense (HNL502149, new record). a, b Basidiomata

globosum is a tropical species originally described from Cameroon (Mossebo et al. 2000). It is distributed in other tropical countries such as China, India, South Africa and Thailand (Ge et al. 2018). It closely resembles $C$. molybdites in morphology, but the phylogenetic analysis showed that they are two different species (Vellinga 2003). This study suggests the first record of Chlorophyllum globosum for Laos (Figs. 161, 162 and 165).
Chlorophyllum hortense (Murrill) Vellinga, Mycotaxon 83: 416 (2002)

Index Fungorum number: IF374396; Facesoffungi number: FoF 03444; Figs. 163, 164

Pileus $72-98 \mathrm{~mm}$, convex, expanding to umbonate with distinctly umbo, with straight margin, surface covered with light brown to yellow brown (5D4-5) glabrous

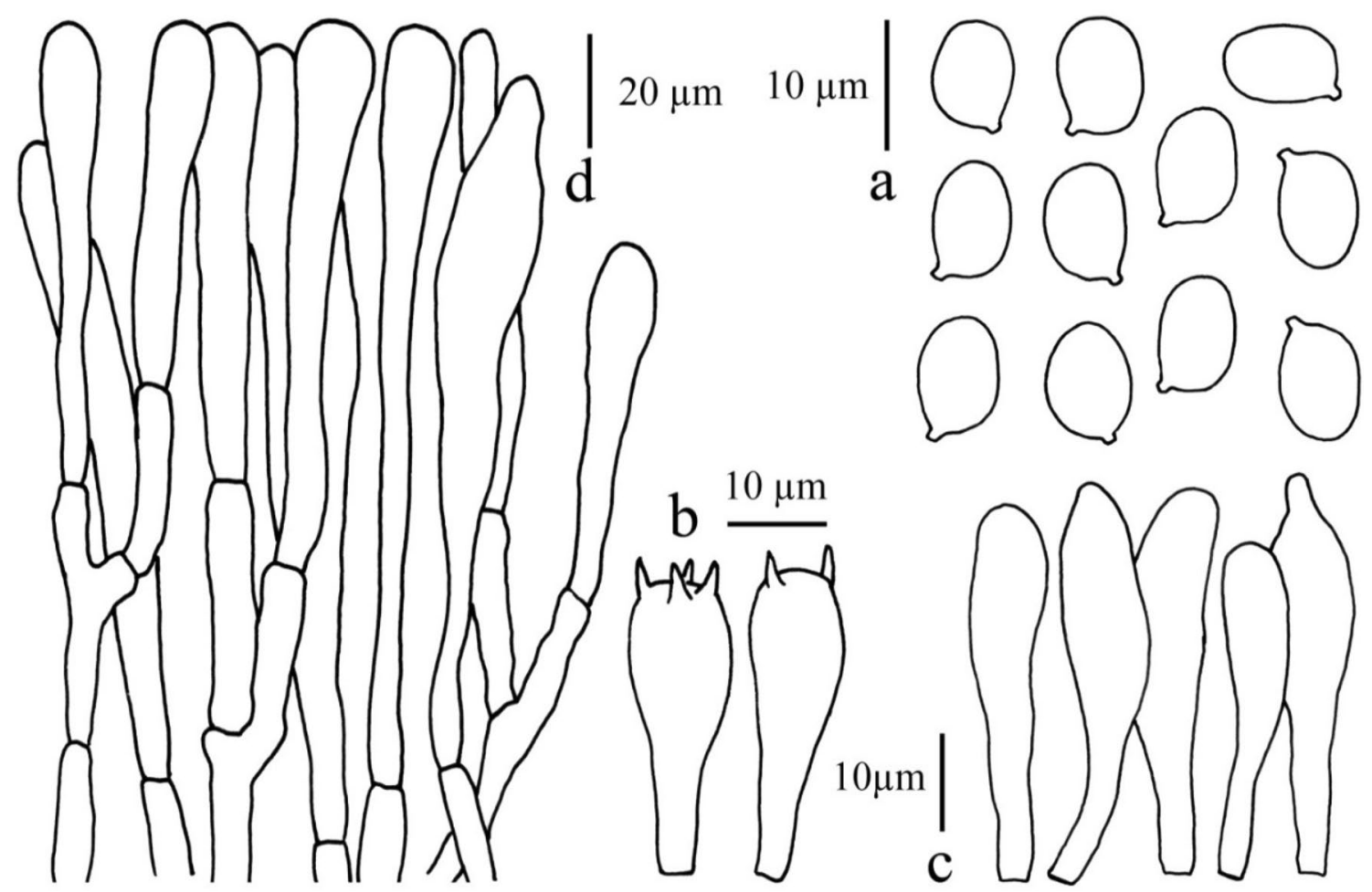

Fig. 164 Microcharacters of Chlorophyllum hortense (HNL502149). a Basidiospores. b Basidia. c Cheilocystidia. d Element cells at pilus covering. Scale bars: $\mathbf{a}-\mathbf{c}=10 \mu \mathrm{m}, \mathbf{d}=20 \mu \mathrm{m}$ 
calotte at center, with brownish yellow (5C7-8) irregular patches or squamules toward margin, on orang white to light orange (5A2-4) background, margin sulcate or slightly striate, white. Lamellae free, narrowly fusiform, up to $7 \mathrm{~mm}$ wide, white, crowded, lamella-edge eroded. Stipe $60-110 \times 6-7 \mathrm{~mm}$, cylindrical, slightly wider to base; surface smooth, white. Annulus superonate, moveable, white, with brownish yellow (5C7-8) on upper part. Context white in pileus, white in stipe and hollow. All parts of basidiomata turning orange-white (5A2) when touched. Taste peanutlike. Smell mild. Spore print white. Basidiospores [50,2,1] 6-8.5×5-6.5 $\mu \mathrm{m}$, avl $\times \mathrm{avw}=7.5 \times 6 \mu \mathrm{m}, \mathrm{Q}=1-1.5$, Qav $=1$, in side-view broadly ellipsoid to ellipsoid amygdaliform, in frontal view ellipsoid, oblong, without germ pore, hyaline, dextrinoid, congophilous, cyanophilous, metachromatic. Basidia 25-30 $\times 8-11.5 \mu \mathrm{m}$, clavate, hyaline, 2-spored, occasionally 1-spored. Lamella edge sterile, with abundant cheilocystidia. Cheilocystidia 35-50×7-9 $\mu \mathrm{m}$, narrowly clavate, cylindrical, sometimes with short apical excrescence or appendage, colorless. Pleurocystidia absent. Pileus covering of scales a trichoderm made up of cylindrical, narrowly clavate elements with long stalk, 30-155×6-16 $\mu \mathrm{m}$, colorless or with pale brown parietal pigment, with encrusted wall in some elements and lower hyphae. Stipe covering a cutis made up of cylindrical hyphae and elements, colorless, $10 \mu \mathrm{m}$ wide. Clamp connections not observed.

Material examined: LAOS, Oudomxay Province, Xay District, Houay Houm Village, 19 July 2014, P. Sysouphanthong, PS2014-478 (HNL502149, new record).

GenBank numbers: ITS = MW040573.

Notes: Two basidiomata of Chlorophyllum hortense were found in grasslands in Laos. Chlorophyllum hortense is characterized by white to yellowish brown pileus, free and white lamellae, and the presence of annulus (Fig. 163). Microcharacters are oblong ovoid and white basidiospores without a germ pore, basically 2 -spored basidia, clavate to cylindrical cheilocystidia, trichodermal pileus covering made up of narrowly clavate elements, without clamp-connection in all tissue (Fig. 164). Chlorophyllum hortense is distributed amongst tropical countries, mostly found on grassland, dung or compost (Vellinga 2004). This species is similar to $C$. demangei except that the latter has 4-spored basidia. Phylogenetic tree confirmed that they are different species (Fig. 165).

\section{Micropsalliota Höhn.}

Notes: Micropsalliota is a genus of Agaricaceae with 70 species distributed worldwide. This genus is highly diversity in tropical regions (Zhao et al. 2010; He et al. 2019). It is related to Xanthagaricus and has a tiny to medium sized, brown basidiospores (Zhao et al. 2010; Vellinga et al. 2011). Twenty-three species have been reported from Thailand, a neighboring country of Laos (Zhao et al. 2010). Based on morphology and molecular-phylogenetic analysis, we report the first records of M. globocystis Heinem. and M. gracilis Heinem. in Laos.

Micropsalliota globocystis Heinem., Bull. Jard. Bot. natn. Belg. 50(1-2): 57 (1980)

Index Fungorum number: IF113558; Facesoffungi number: FoF 09760; Figs. 166, 167

Pileus $30-70 \mathrm{~mm}$ diam., when young subglobose to broadly conical, expanding to convex or umbonate, applanate to plano-concave when mature, with straight margin, surface fibrillose to squamules fibrillose, reddish brown (9D4-8), darker at center, on white to pale yellow (5A3) fibrillose background, turning orange white (5A2) when touched, margin with sulcate marginal zone, with exceeding lamellae when mature. Lamellae free, ventricose, up to $5 \mathrm{~mm}$ wide, white to yellowish white (4A2) at first, becoming orange grey to greyish orange (6B2-3) when mature, crowded, with white eroded lamella-edge. Stipe $50-100 \times 5-8 \mathrm{~mm}$, cylindrical, or slightly wider at base; surface covered with white fibrillose, crowded, on white fibrillose background, turning orange-white (5A2) when touched. Annulus superior, persistent, white. Context in pileus white, in stipe white and hollow, when cut turning orange white (5A2) in both stipe and pileus context. Taste and oduor not observed. Spore print orange grey (6B2). Basidiospores $[50,2,2] 5.5-8.5 \times 3-4 \mu \mathrm{m}, \mathrm{avl} \times \mathrm{avw}=6 \times 4 \mu \mathrm{m}, \mathrm{Q}=1-2$, Qav $=1.5$, ellipsoid in side-view, ellipsoid to oblong in frontal view, without germ pore, slightly thick-walled, hyaline to pale brown. Basidia $15-23 \times 7-10 \mu \mathrm{m}$, clavate, hyaline and slightly thick-walled, 4-spored. Cheilocystidia abundant, $28-65 \times 7-16 \mu \mathrm{m}$, narrowly clavate to broadly clavate, sometimes with median constriction and 1-2-septate, hyaline, thick-walled. Pleurocystidia absent. Pileus covering a cutis made up of cylindrical elements, $60-110 \times 10-23 \mu \mathrm{m}$, encrusted, with reddish brown parietal and intracellular pigment, with encrusted hyphae in lower layer, hyaline or with reddish brown parietal and intracellular pigment. Stipe covering not observed.

Material examined: LAOS, Xiangkhouang Province, Koun District, Om Village, 27 May 2016, P. Sysouphanthong, PS2016-69 (HNL501440); Oudomxay Province, Beng District, Na Pa Village, 23 June 2014, P. Sysouphanthong, PS2014-106 (HNL501777, new record).

GenBank numbers: ITS = MW073388, MW073389.

Notes: Micropsalliota globocystis is a saprotrophic species found in the northern part of Laos (Figs. 166 and 167). Zhao et al. (2010) indicated that the species is very common in Thailand. Molecular analysis showed that M. globocystis is sister to M. megarubescens, but they are quite different in morphology. Another species, M. pseudoglobocystis also resembles to $M$. globocystis, but the cheilocystidia vary in shape. Molecular analysis confirmed that they are distinct 


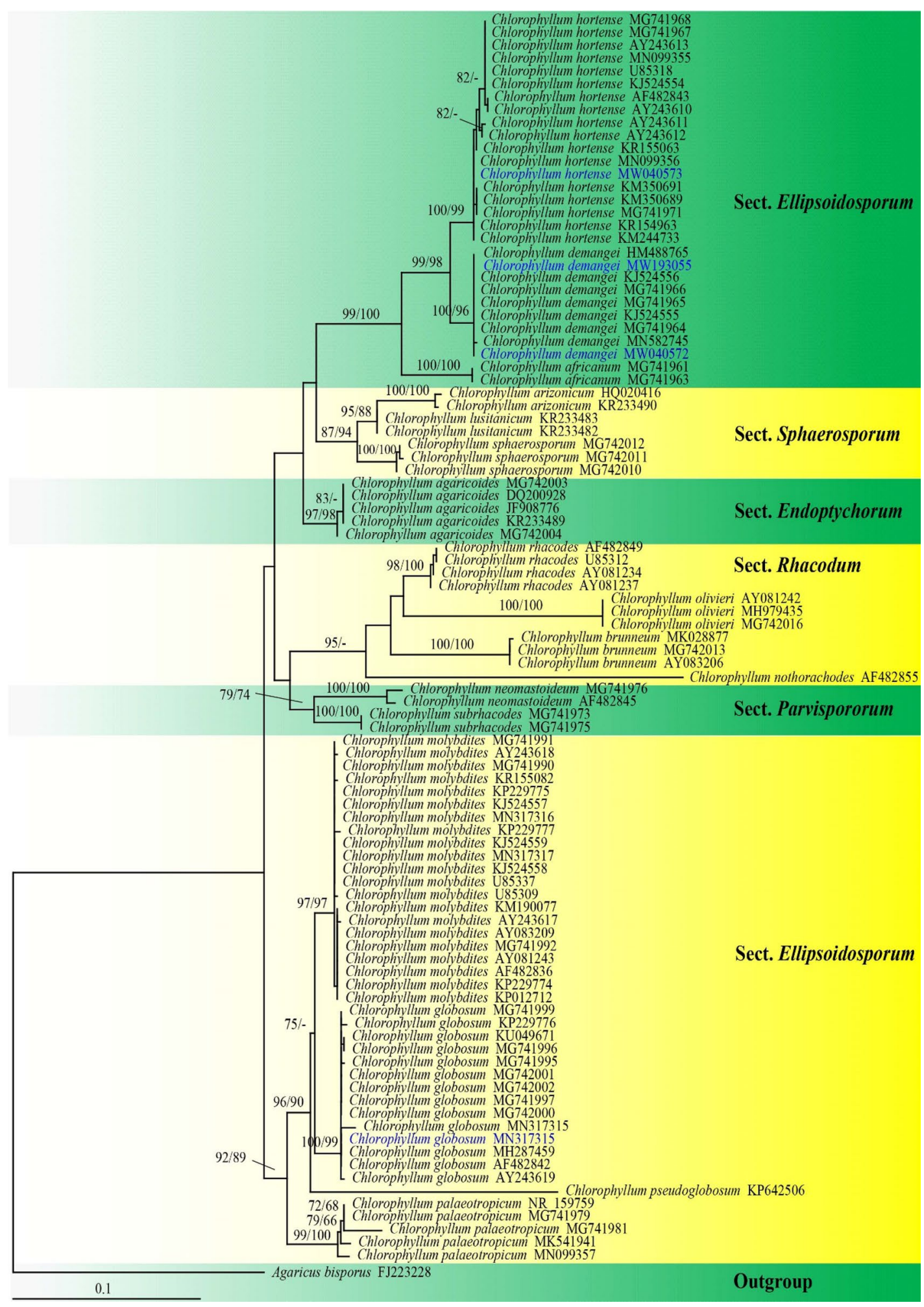

Fig. 165 Maximum likelihood phylogenetic tree of Chlorophyllum based on nrITS sequences. The maximum likelihood (ML) analysis was performed in RAxML 7.2.6 (Stamatakis et al. 2008) and maximum parsimony (MP) analysis was performed the program PAUP* 4.0 b10 (Swofford 2004). Bootstrap values of ML/MP equal to or greater than $70 \%$ are given above branches. GenBank accession number is indicated after species name. Agaricus bisporus (FJ223228) is an outgroup. The three shows six different sections. Newly sequences generated from Laos are in blue 

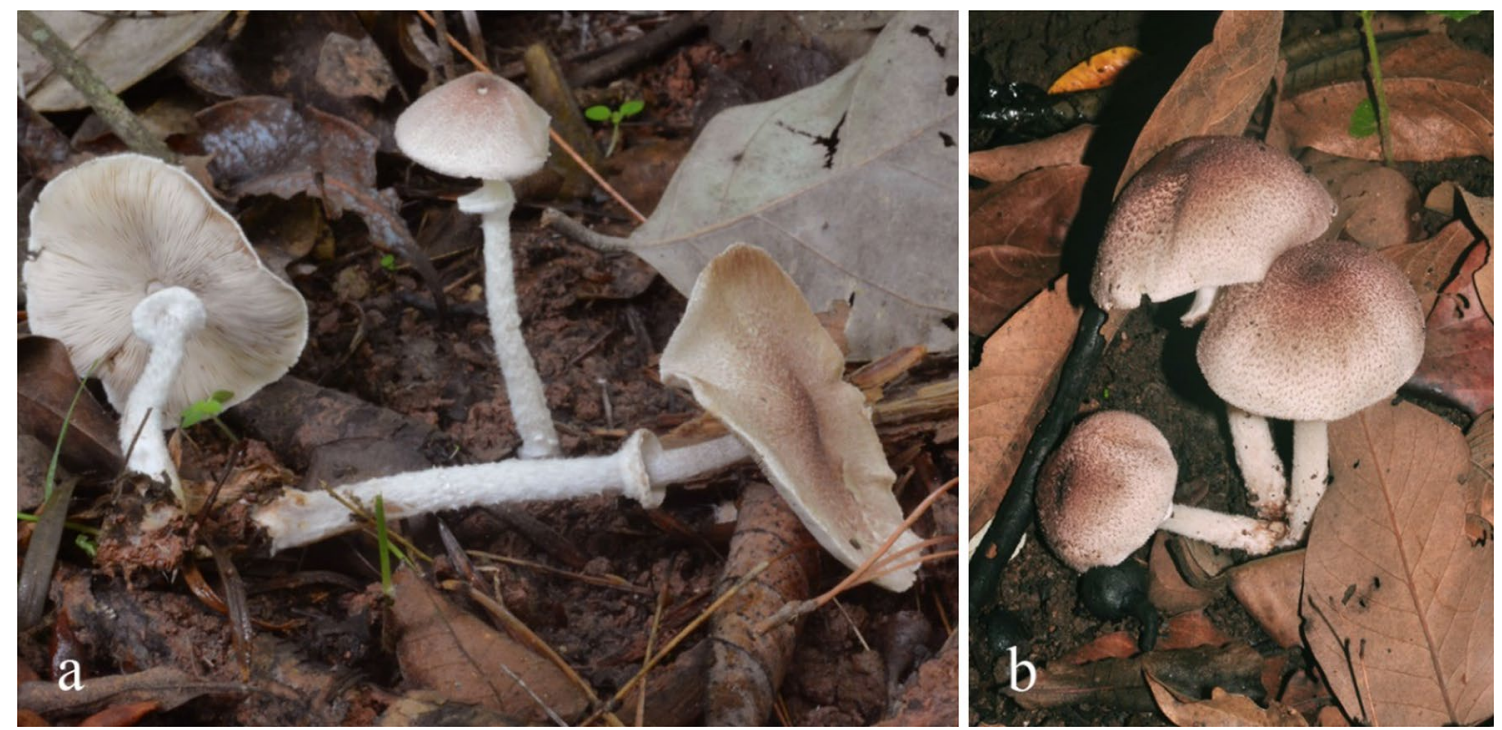

Fig. 166 Micropsalliota globocystis (HNL501777, new record). a, b Basidiomata

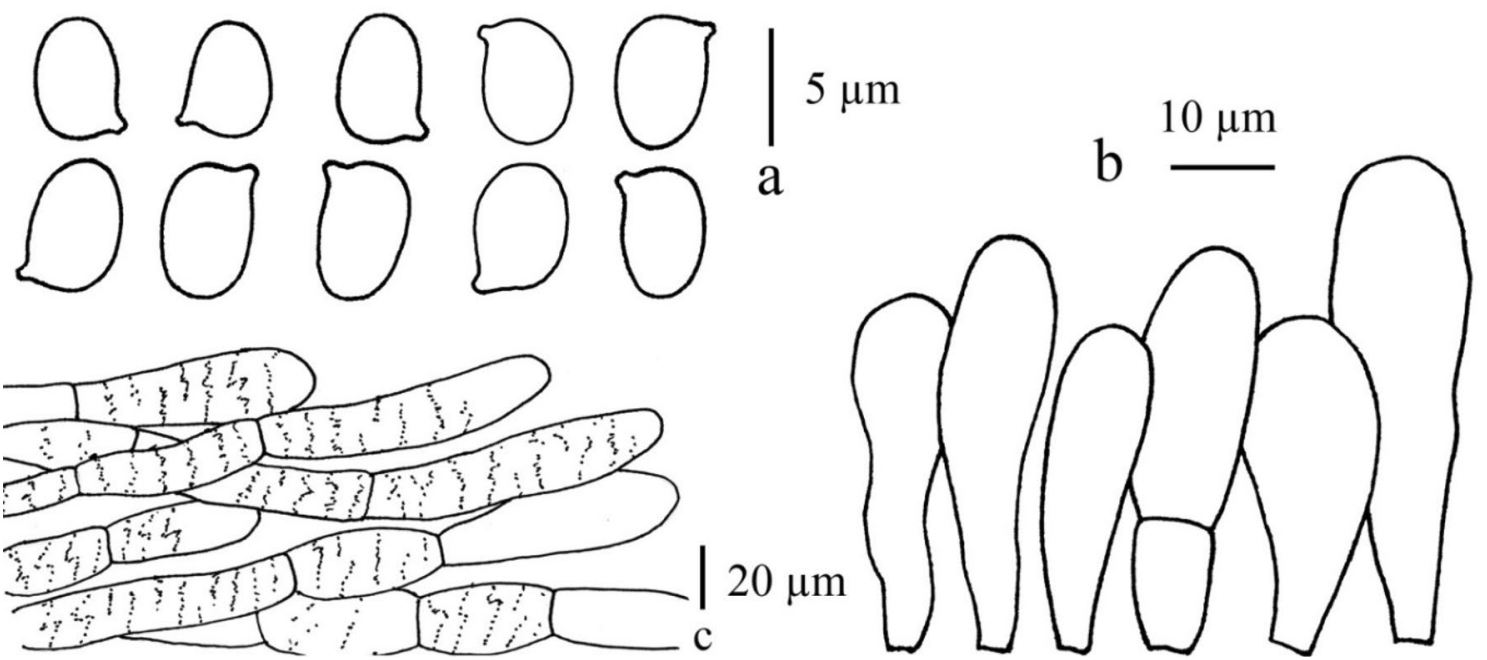

Fig. 167 Microcharacters of Micropsalliota globocystis (HNL501777). a Basidiospores. b Cheilocystidia. c Element cells at pileus covering. Scale bars: $\mathbf{a}=5 \mu \mathrm{m}, \mathbf{b}=10 \mu \mathrm{m}, \mathbf{c}=20 \mu \mathrm{m}$

species (Wei et al. 2015). According to the nrITS analysis, the $M$. globocystis complex includes M. globocystis, $M$. pseudoglobocystis and M. pseudoglobocystis. Lao specimens of M. globocystis grouped well with those from Thailand and China (Fig. 170). Both morphology and molecular data derived from Lao and Thai specimens indicate that they are conspecific.

Micropsalliota gracilis Heinem., Bull. Jard. Bot. natn. Belg. 50(1-2): 60 (1980)

Index Fungorum number: IF113559; Facesoffungi number: FoF 09761; Figs. 168, 169
Pileus 10-30 mm, first conical, expanding to umbonate, applanate when mature, completely covered with crowded fibrillose when young, brownish red to violet brown (10 D6-8 to (10E6-8), with paler colour when mature, fibrillose, sometimes striate, with white background, margin appendiculate, with white and short velar remnants, fragile when mature. Lamellae free, white when young, becoming orange grey to greyish orange (6B2-3) when mature, ventricose 4-6 mm wide, moderately crowded, with eroded lamella-edge. Stipe $25-60 \times 2-4 \mathrm{~mm}$, cylindrical, wider at base (5-7 mm wide); surface covered with white fibrillose, on white background, turning orange-white (5A2) when 

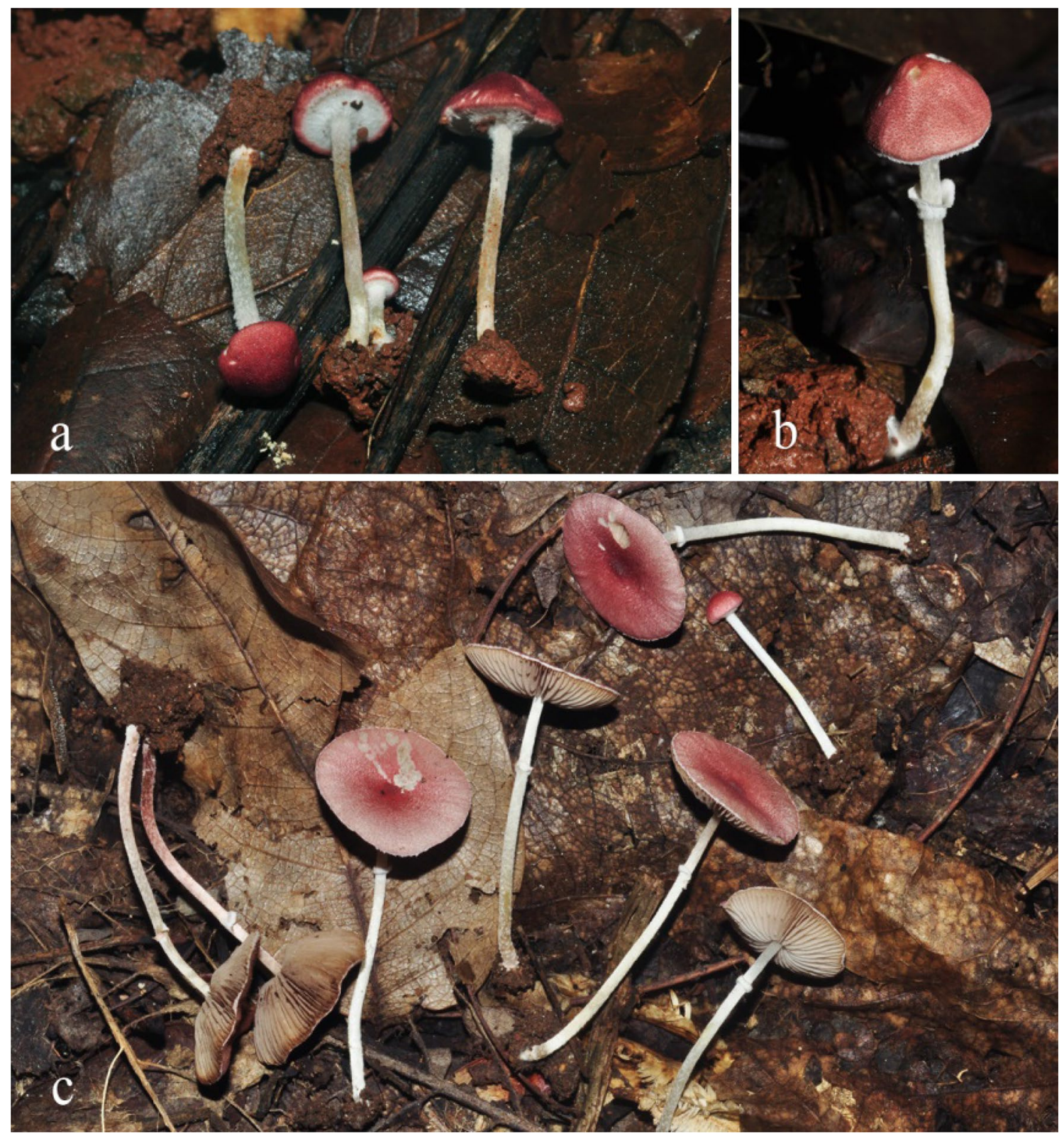

Fig. 168 Micropsalliota gracilis (HNL503436, new record). a-c Basidiomata

touched. Annulus superior, persistent, white. Context in pileus white and thin, in stipe white and hollow, turning orange white (5A2) in both stipe and pileus when touched. Taste and oduor not observed. Spore pint orange grey (6B2). Basidiospores 5-6.5 $\times 3-4.5 \mu \mathrm{m}$, avl $\times$ avw $=6 \times 3.5 \mu \mathrm{m}$, $\mathrm{Q}=1.5-2$, Qav $=1.6$, in side-view amygdaliform to ellipsoid, in frontal view ellipsoid to oblong, slightly thickwalled, hyaline to pale brown. Basidia $13-17 \times 6-8 \mu \mathrm{m}$, clavate, hyaline and thin-walled, 4 -spored. Cheilocystidia $25-50 \times 7-12 \mu \mathrm{m}$, various in shape, versiform, narrowly utriform, narrowly lageniform, lecythiform, with a long neck and capitulum at apex. Pleurocystidia absent. Pileus covering a cutis composed of cylindrical to oblong to fusiform elements, up to $25 \mu \mathrm{m}$, with brownish red parietal and intracellular pigment. Clamp connections absent.

Material examined: LAOS, Vientiane Capital, Xaythany District, Houay Yang Preserve Forest, 17 November 2015, P. Sysouphanthong, PS205 (HNL503432); Chiang Rai Province, Muang District, Campus of Mae Fah Luang University, 08 Jun 2019, P. Sysouphanthong, PS2019-45 (MFLU 19-2358); ibidem, 17 November 2015, P. Sysouphanthong, PS209 (HNL503436, new record).

GenBank numbers: ITS = MW192914, MW192915. 


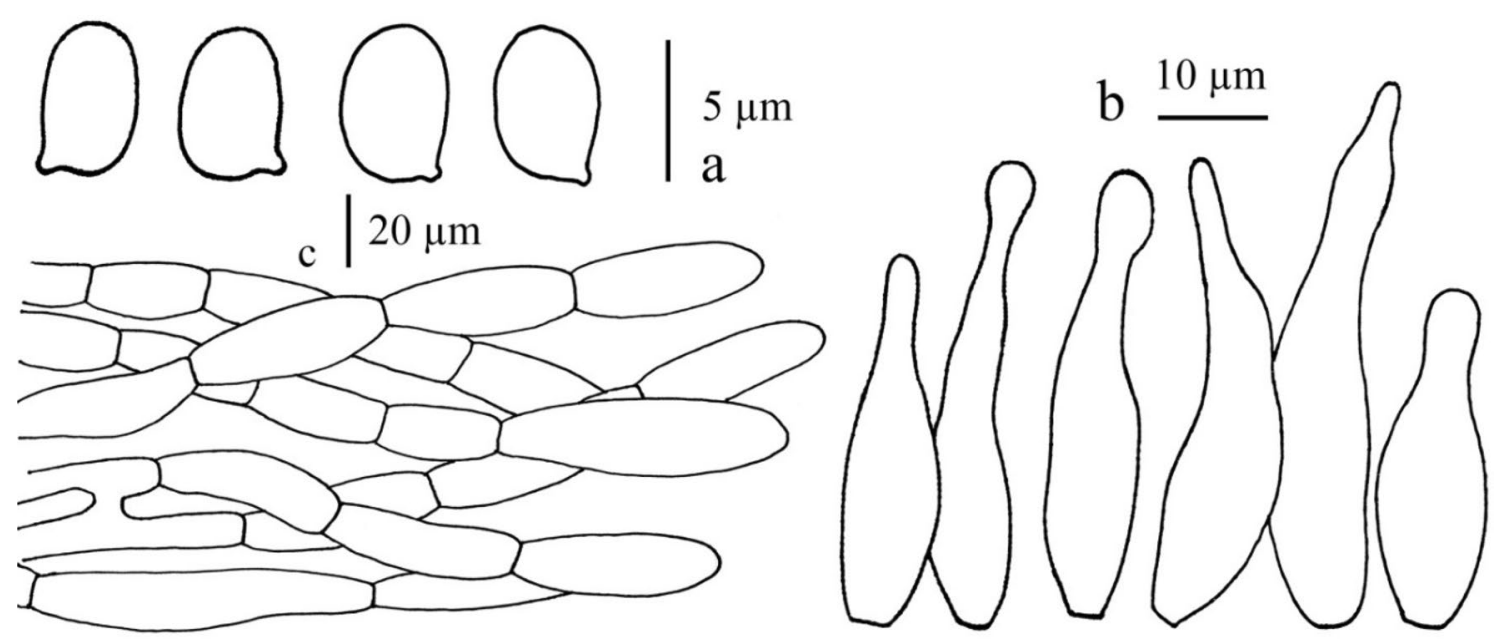

Fig. 169 Microcharacters of Micropsalliota grcilis (HNL503436). a Basidiospores. b Cheilocystidia. c Element cells at pileus covering. Scale bars: $\mathbf{a}=5 \mu \mathrm{m}, \mathbf{b}=10 \mu \mathrm{m}, \mathbf{c}=20 \mu \mathrm{m}$

Notes: Two specimens of saprotrophic Micropsalliota gracilis were found on rich humus soil of deciduous forest growing in solitary or in large groups from central Laos. This species has tiny basidiomata with brownish red to violet brown fibrillose pileus (Figs. 168 and 169). The taxonomic details have been well discussed by Zhao et al. (2010). Lao specimens are similar to Thai specimens based on morphology and nrITS sequence analysis (Fig. 170) indicating that they are conspecific. This is the first record of Micropsalliota gracilis in Laos.

Xanthagaricus (Heinem.) Little Flower, Hosag. \& T.K. Abraham

Xanthagaricus, a tropical genus of Agaricaceae, consists of 12 species with the type species X. flavidorufus (He et al. 2019). Molecular-phylogenetic analysis showed that Xanthagaricus is related to Micropsalliota (Villinga et al. 2011; Zhao et al. 2010). In this study showed that $X$. necopinatus is a new record from Laos.

Xanthagaricus necopinatus Iqbal Hosen, T.H. Li, \& G.M. Gates, MycoKeys 28: 9 (2017)

Index Fungorum number: IF822731; Facesoffungi number: FoF 07070; Figs. 171, 172

Pileus 08-18 mm diam., convex to plano-convex, sometimes umbonate, with straight margin; covered with greyish yellow (3B5-6) crowded squamules, with brownish orange to brownish yellow (5C6-8) at center, with yellowish white (3A2) background; margin covered with greyish orange (6B4) appendiculate velar remnants, sometimes absent. Lamellae free, 2-3 $\mathrm{mm}$ wide, yellowish white to orange white (4A2) at first, greyish orange (5B5) when mature, ventricose to broadly ventricose, with eroded edge. Stipe $25-50 \times 2-3 \mathrm{~mm}$, cylindrical, with wider at base $(4-5 \mathrm{~mm}$ wide), covered with greyish yellow (3B5-6) crowded squamules, with orange white (5B2) background, turning greyish red (7B5) when touched. Annulus membranous, greyish orange (6B4), fragile or absent with age. Context orange white to pale orange (5A2-3) in pileus, thin, up to $2 \mathrm{~mm}$, hollow in stipe, concolorous with surface, turning greyish red (7B5) when cut. Odour and taste not observed. Spore pint orange grey (6B2). Basidiospores $4-5.5 \times 2.5-3.7 \mu \mathrm{m}$, $\mathrm{Q}=1.40-1.78$, avQ $=1.64$, ellipsoid to oblong-ovoid in frontal view, slightly thick-walled, smooth, hyaline to pale brown. Basidia $18-22 \times 5-10 \mu \mathrm{m}$, short clavate to clavate, slightly thick-walled, hyaline, 4 -spored, rarely 2 -spored. Pleurocystidia absent. Cheilocystidia 15-23 ×6-12 $\mu \mathrm{m}$, short clavate to clavate, hyaline, thick-walled. Pileus irregular epithelium, element cells oblong to short clavate, 8-23 $\times 4-17 \mu \mathrm{m}$, thick-walled, encrusted, with pale brown to brown parietal and intracellular pigments. Stipe covering similar to pileus covering. Clamp connections absent.

Material examined: LAOS, Oudomxay Province, Xay District, Houay Houm Village, 16 July 2014, P. Sysouphanthong, PS2014-412 (HNL502083, new record); Oudomxay Province, Beng District, Na Pa Village, 19 July 2014, P. Sysouphanthong, PS2014-463 (HNL502134).

GenBank numbers: ITS = MW040548.

Notes: Xanthagaricus necopinatus is saprotrophic on humus-rich soil and normally grows in large groups in various forest types. Xanthagaricus necopinatus is recognized by small and pale yellow to brownish yellow basidiomata, abundant appendiculate velar remnants at the pileus margin, ellipsoid to oblong-ovoid and hyaline to pale brown basidiospores, clavate cheilocystidia, and irregular epithelium of pileus and stipe covering (Figs. 171 and 172). The species was first described from Bangladesh by Hosen et al. (2017). Lao specimens resembled with the type material in terms of 


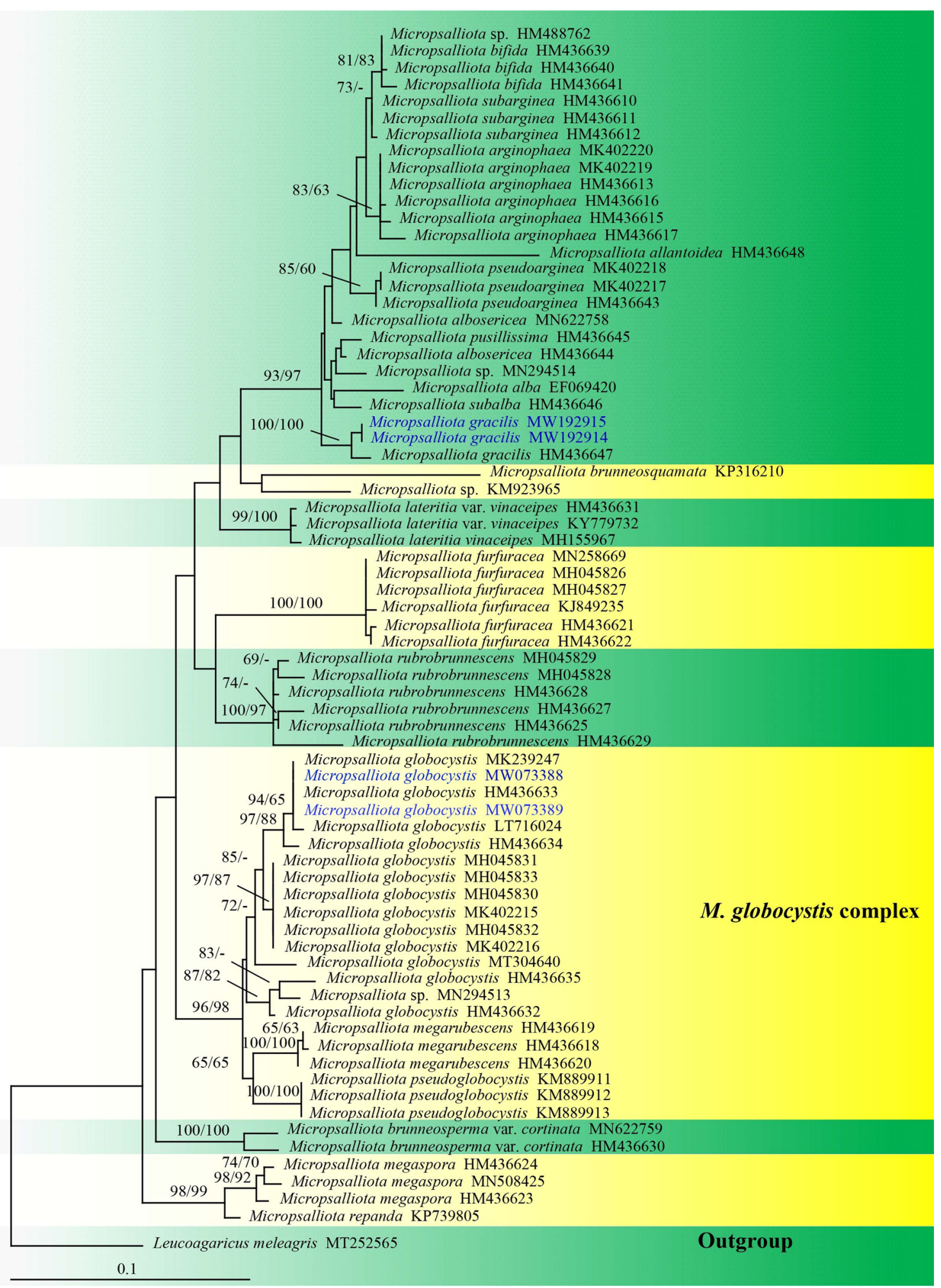

Fig. 170 Maximum likelihood phylogenetic tree of Micropsalliota based on nrITS sequences. The maximum likelihood (ML) analysis was performed in RAxML 7.2.6 (Stamatakis et al. 2008) and maximum parsimony (MP) analysis was performed the program PAUP* 4.0 b10 (Swofford 2004). Bootstrap values of ML/MP equal to or greater than $70 \%$ are given above branches. GenBank accession number is indicated after species name. Leucoagaricus meleagris (MT252565) is an outgroup. Newly sequences generated from Laos are in blue 


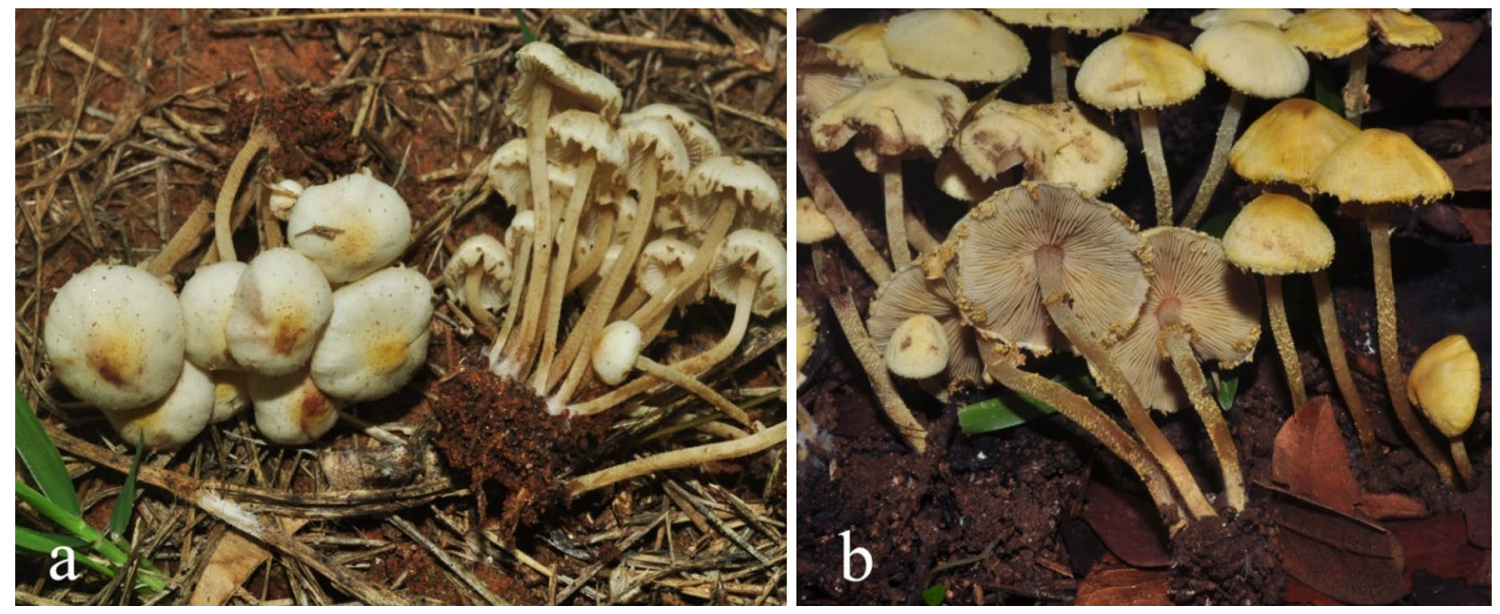

Fig. 171 Xanthagaricus necopinatus (HNL502083, new record). a, b Basidiomata

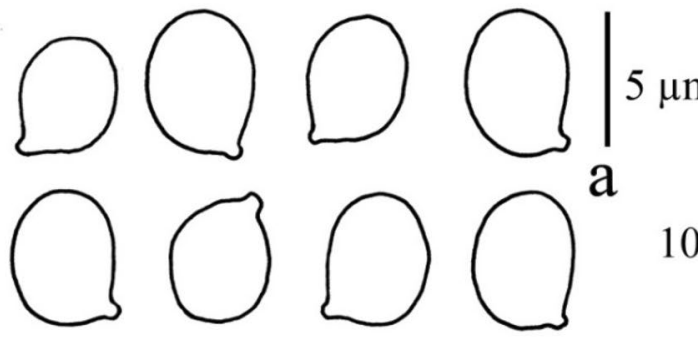

$\mu \mathrm{m}$

$10 \mu \mathrm{m}$

b
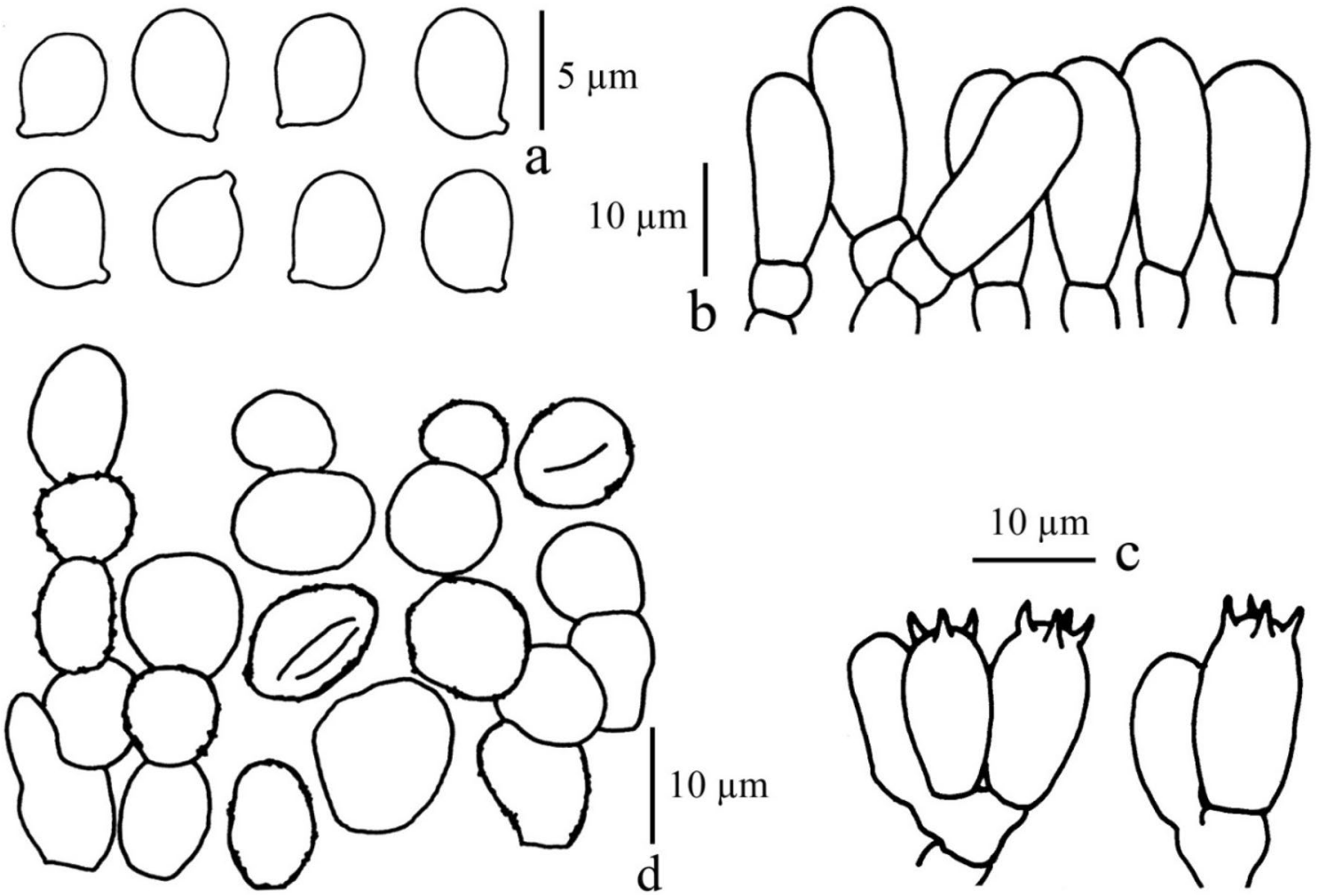

Fig. 172 Microcharacters of Xanthagaricus necopinatus (HNL502083). a Basidiospores. b Cheilocystidia. c Basidia. d Element cells at pileus covering. Scale bars: $\mathbf{a}=5 \mu \mathrm{m}, \mathbf{b}-\mathbf{d}=10 \mu \mathrm{m}$

morphology and nrITS sequences of Lao specimens grouped with the type specimens (Fig. 173), thus confirming they are conspecific. This is the first record of Xanthagaricus necopinatus in Laos and outside of Bangladesh.

Amanitaceae E.-J. Gilbert
Notes: Updated accounts are provided in Phookamsak et al. (2019).

Saproamanita Redhead, Vizzini, Drehmel \& Contu

Notes: Amanita has been divided into two monophyletic units, Amanita (ectomycorrhizal) and Aspidella (saprotrophic) (Gilbert 1940; Bas 1969; Vizzini et al. 2012; Wolfe 


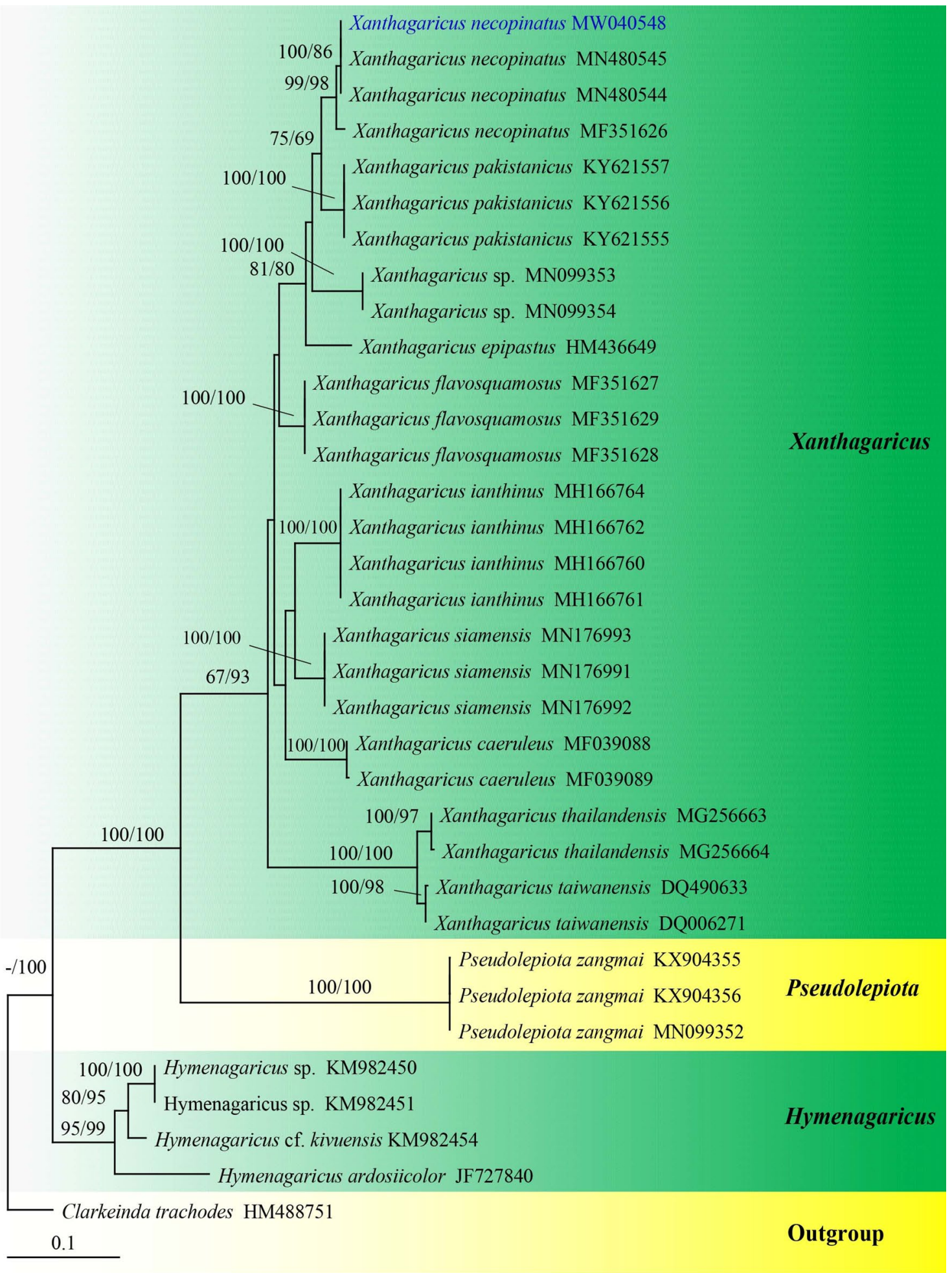

Fig. 173 Maximum likelihood phylogenetic tree of Xanthagaricus based on nrITS sequences. The maximum likelihood (ML) analysis was performed in RAxML 7.2.6 (Stamatakis et al. 2008) and maximum parsimony (MP) analysis was performed the program PAUP* 4.0 b10 (Swofford 2004). Bootstrap values of ML/MP equal to or greater than $70 \%$ are given above branches. GenBank accession number is indicated after species name. Clarkeinda trachodes (HM488751) is an outgroup. Newly sequences generated from Laos is in blue 
et al. 2012). Saproamanita was introduced by Redhead et al. (2016) to replace Aspidella and typified by S. vittadinii (syn. A. vittadinii). Based on the morphology, Saproamanita was placed in Amanitaceae (Agaricales, Agaricomycetes). Saproamanita is most similar to that of the Amanita subgenus Lepidella, characterized by volval elements dominated by cylindrical to slender clavate inflated cells together with elongated stipes in which these elements are mostly scattered mid-stipe. The species in this genus can grow in open fields and grass land steppes (Beauseigneur 1926; Bas 1969). The multi-locus phylogenetic studies also support the contention that Saproamanita should be placed in Amanita subgen. Lepidella (Davison et al. 2013; Cui et al. 2018; Yang et al. 2018c). Currently, 24 taxa of Saproamanita are listed in the species Fungorum (2021). However, only two species, $S$. praegraveolens and $S$. thiersii have been reported in Thailand (Chandrasrikul et al. 2011).

Saproamanita manicata (Berk. \& Broome) Redhead, Vizzini, Drehmel \& Contu IMA Fungus 7: 123 (2016)

Index Fungorum number: IF816358; Facesoffungi number: FoF 09965; Fig. 174

Pileus 5-12 cm wide, at first hemispherical then convexcampanulate; surface pale orange (5A3) to brownish orange (6C7), initially covered by universal veil which disrupts into soft, detessile, floccoso-verucose squamules; margin initially curved, appendiculate with large, vela floccose fragments, not striate, finally revolute. Lamellae free, up to $12 \mathrm{~mm}$ wide, whitish to pinkish tint, with 2-4 series of lamellulae. Stipe $8-15 \times 1-2 \mathrm{~cm}$, cylindic, sometimes slightly inflated at the base, solid; surface white (5A1) becoming pale orange (5A3) to brownish orange (6C7), densely floccoso-squamose above, white but bruising ochraceous buff. Annulus superior, densely floccoso-squamose. Volva reduced to remnants concentrated over the middle of stipe and forming tawny brown squamules, covering the friable annular zone of the partial veil. Context up to $10 \mathrm{~mm}$ thick, white, soft spongy, consisting of loosely woven, thin-wall hyphae, 5-20 $\mu \mathrm{m}$ in diam. Odour strong, sweet, and unpleasant. Basidiospores 7-8.5 $\times 5.5-8 \mu \mathrm{m}, \mathrm{Q}=1, \mathrm{Q}_{\mathrm{m}}=1 \pm 0.5$, subglobose, hyaline, amyloid. Basidia 35-50 $\times 9-13 \mu \mathrm{m}$, clavate, bearing four sterigmata. Lamell-edge not recovered. Hymenophoral trama bilateral, hyaline, with inflated, divergent hyphae 3-6 $\mu \mathrm{m}$ wide. Subhymenial layer pseudoparenchymatous. Pileipellis composed of radially arranged, filamentous hyphae 4-7 $\mu \mathrm{m}$ wide. Velar squamules formed by chains of detersile elements, up to $35 \mu \mathrm{m}$ in diameter, cylindric to fusoid, hyaline, thin-walled. Stipe trama composed of longitudinally arranged, clavate terminal cells, $85-125 \times 15-25 \mu \mathrm{m}$, filamentous hyphae scattered to abundant, 4-8 $\mu \mathrm{m}$ wide; vascular hyphae scarce. Clamp connections absent in all issue types.

Habitat: scattered on the ground in grassland and forest.
Distribution: Known from Dominican Republic, New Zealand Sri Lanka, Thailand, and USA (Hawaiian Islands) (Petch 1910; Pegler 1986; Hemmes and Desjardin 2008; Vizzini et al. 2016).

Material examined: THAILAND, Chiang Mai Province, Muang District, Chiang Mai University, $18^{\circ} 48^{\prime} 9^{\prime \prime} \mathrm{N}$ $98^{\circ} 57^{\prime} 5^{\prime \prime} \mathrm{E}, 339 \mathrm{msl}$, on the ground in grassland, 4 May 2019, J. Kumla and N. Suwannarach, SDBR-CMU-NK0356, new record.

GenBank number: ITS $=$ MW648326, LSU $=$ MW648589, TEF1- $\alpha=$ MW659711.

Notes: The multigene phylogenetic analysis of ITS, LSU and TEF1- $\alpha$ sequence data (Fig. 175) indicate that S. manicata was clearly distinguishable from other Saproamanita species forming a monophyletic clade with 100\% MLBS, 1.00 BYPP support. It formed a sister taxon to $S$. flavofloccosa and S. foetidissima with 88\% MLBS, 0.98 BYPP support. Saproamanita flavofloccosa (Fig. 174) can be distinguished from $S$. manicata by its subglobose to broadly ellipsoid basidiospores $\left(6.5-13 \times 5.5-9 \mu \mathrm{m}, \mathrm{Q}_{\mathrm{m}}=1\right)$ (Purushothama and Natarajan 1987; Senthilarasu 2014). Saproamanita foetidissima has broadly ellipsoid to ellipsoid basidiospores $\left(8-11.5 \times 7-9 \mu \mathrm{m}, \mathrm{Q}_{\mathrm{m}}=1\right)$ which is quite different from S. manicata (Reid and Eicker 1991).

\section{Cortinariaceae R. Heim ex Pouzar 1983}

Notes: The limits of the large, species-rich family Cortinariaceae remain unclear. Most species in this family belong to Cortinarius. Many genera formerly placed in the Cortinariaceae, e.g., Phaeocollybia, Hebeloma and Galerina have been moved to other families in the Agaricales. Conversely, the sequestrate genera, Thaxterogaster, Quadrispora, Protoglossum and Hymenogaster as well as Cuphocybe, Rapacea and species of Rozites, once thought to belong to the Cortinariaceae, are currently included in Cortinarius (Peintner et al. 2001, 2002).

\section{Cortinarius (Pers.) Gray}

Notes: Cortinarius is the largest genus of Agaricales, comprising over 3,000 species worldwide. They are important ectomycorrhizal partners of trees and shrubs from the families Fagaceae, Salicaceae, Caesalpiniaceae, Cistaceae, Dipterocarpaceae, Myrtaceae, Rhamnaceae, Rosaceae and Pinaceae, as well as some herbaceous plants from the Cyperaceae and Polygonaceae in boreal, temperate and subtropical ecosystems in both the northern and southern hemispheres (Frøslev et al. 2006; Willis 2018). The basidiomata range from agaricoid to sequestrate and usually have poorly to well-developed universal and partial veils. The basidiospore shape vary from subglobose to fusiform, they are weakly to strongly verrucose and rusty brown in deposit. Most of the Cortinarius species are difficult to identify based on macro- and micromorphological features and, usually, 

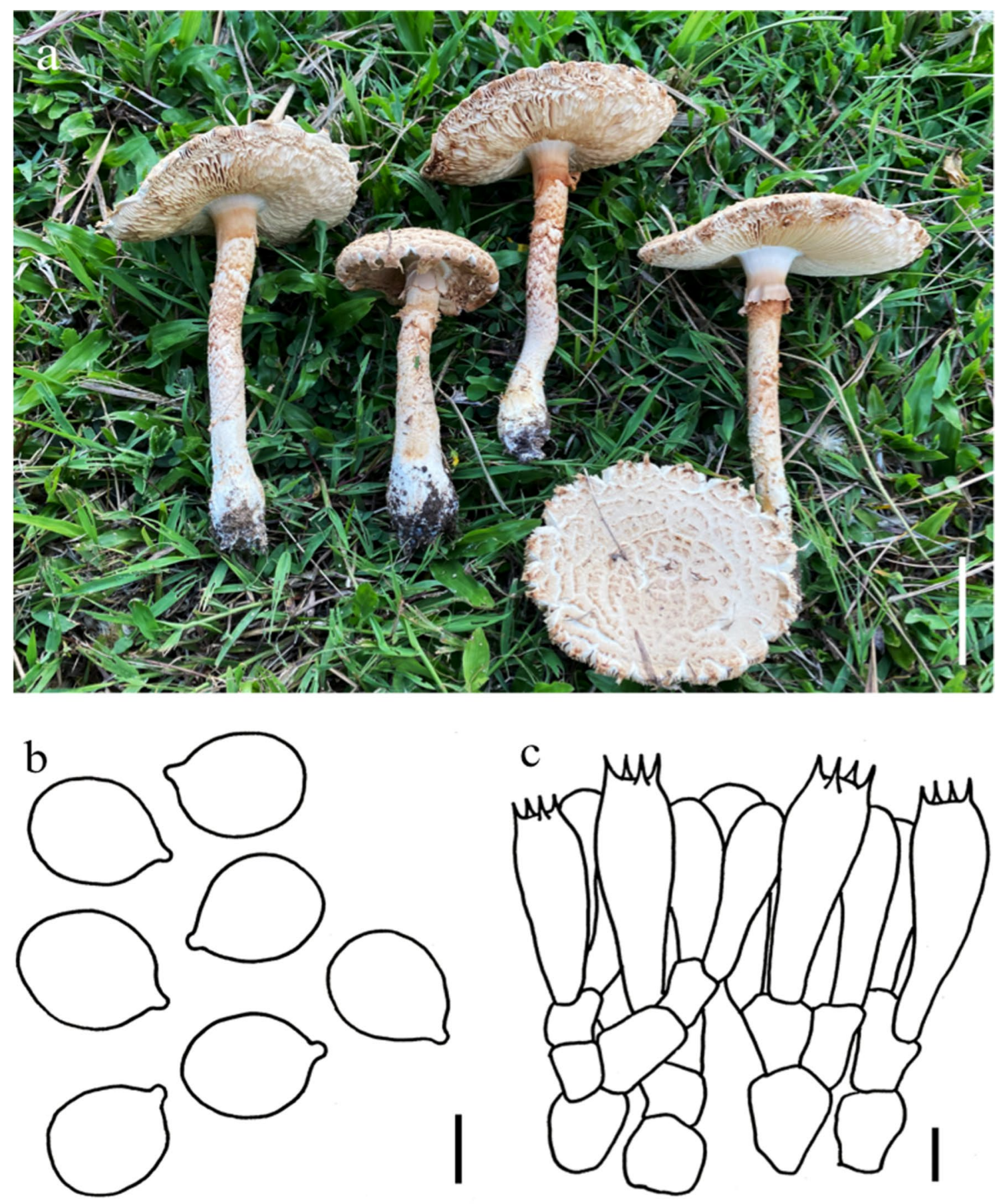

Fig. 174 Saproamanita manicata (SDBR-CMU-NK0356, new record). a Basidiomata. b Basidiospores. c Basidia. Scale bars: a=5 cm, $\mathbf{b}=5 \mu \mathrm{m}, \mathbf{c}=10 \mu \mathrm{m}$

molecular analyses are essential for species delimitation. Still, many species of Cortinarius remain undescribed. In this study, three new species of this genus are introduced based on evidence of morphology and phylogenetic placement (Figs. 176, 177, 178, 179 and 180); two of them were found in a specific habitat of reclamation forests on lignite mine spoil heaps in Europe, the third one is known to occur in boreal and mountainous coniferous forests of the northern hemisphere.

Cortinarius alutarius Kałucka \& Liimat., sp. nov.

Index Fungorum number: IF558552; Facesoffungi number: FoF 09966; Fig. 176 


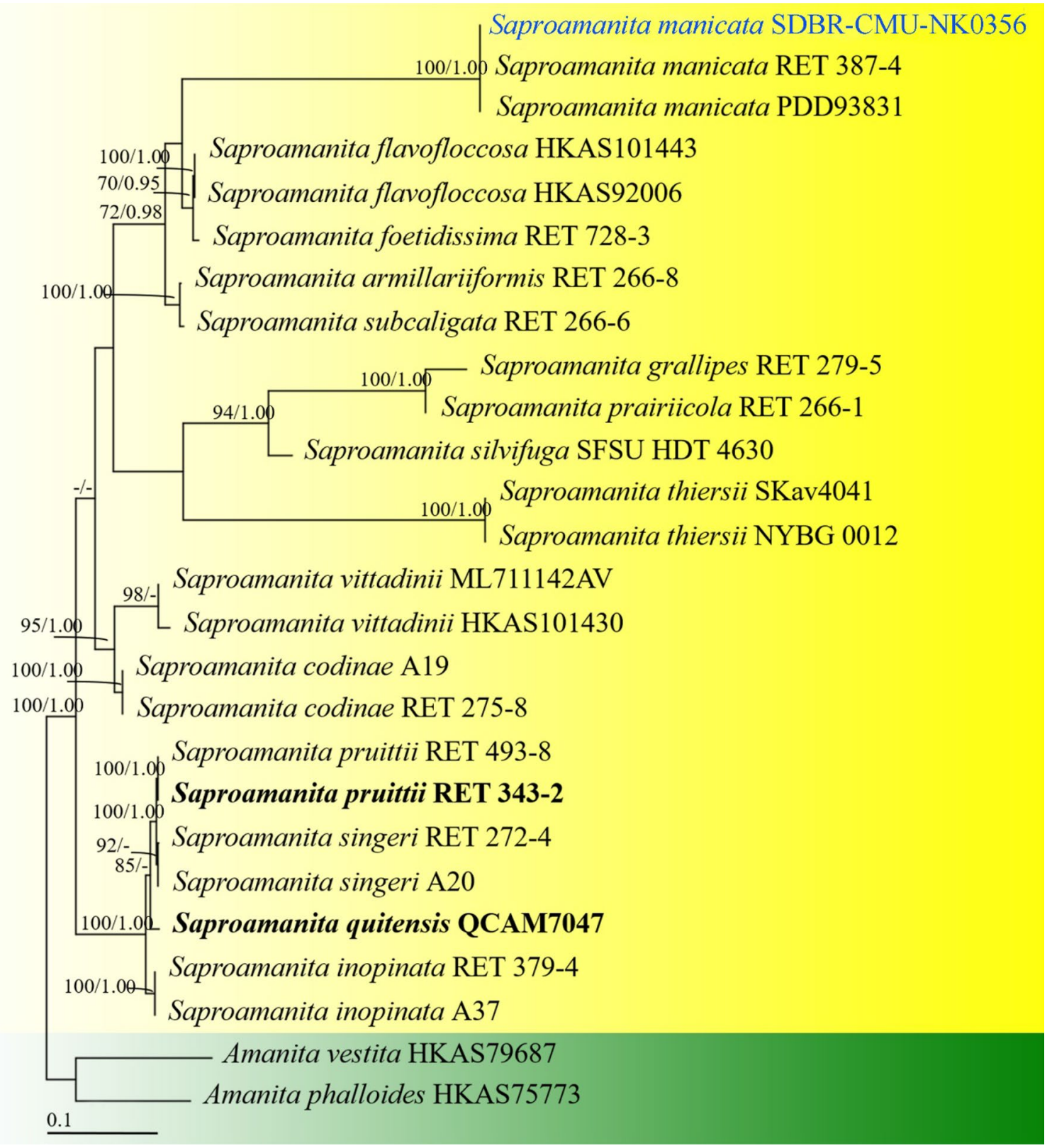

Fig. 175 Phylogenetic tree derived from maximum likelihood analysis of a combined ITS, LSU and TEF1- $\alpha$ genes of 26 sequences and the aligned dataset was comprised of 2352 characters including gaps (ITS: 1-923, LSU: $924-1788$ and TEF1- $\alpha$ : 1789-2352). The average standard deviation of the split frequencies of the BI analysis was 0.00535 . A best scoring RAxML tree was established with a final ML optimization likelihood value of -10465.4175 . The matrix had 949 distinct alignment patterns with $48.95 \%$ undetermined characters or gaps. Estimated base frequencies were found to

Etymology: The name is based on a Latin adjective alutarius (made of soft leather), referring to the suede-like cap surface.

Holotype: $\operatorname{LOD}(\mathrm{F}): 47001$

Pileus $1.5-2.5 \mathrm{~cm}$ in diam., at first conical, later low conical to almost plane, with \pm distinct umbo, tomentose, be: $\mathrm{A}=0.2711, \mathrm{C}=0.2011, \mathrm{G}=0.2422, \mathrm{~T}=0.2850$; substitution rates $\mathrm{AC}=1.2478, \mathrm{AG}=2.7197, \mathrm{AT}=1.6015, \mathrm{CG}=0.6534, \mathrm{CT}=3.9595$, $\mathrm{GT}=1.0000$; proportion of invariable sites $=0.0700$ and gamma distribution $=1.0010$. Amanita phalloides HKAS75773 and Amanita vestita HKAS75773 were used as outgroup. Numbers above branches are the bootstrap statistics percentages equal to or greater than $70 \%$ (MLBS, left) and Bayesian posterior probabilities equal to or greater than 0.95 (BYPP, right). Ex-type strains are in bold and newly generated sequence is in blue

floccose to finely scaly, dark rusty brown, cinnamon brown, usually darker at the centre and paler, ochraceous to buff, towards the margin, light brown or ochraceous when dry; hygrophanous. Lamellae fairly distant to medium spaced, adnate to emarginate, medium brown to dark brown, the edge may be paler, at least when young. Stipe $3-4 \mathrm{~cm}$ 

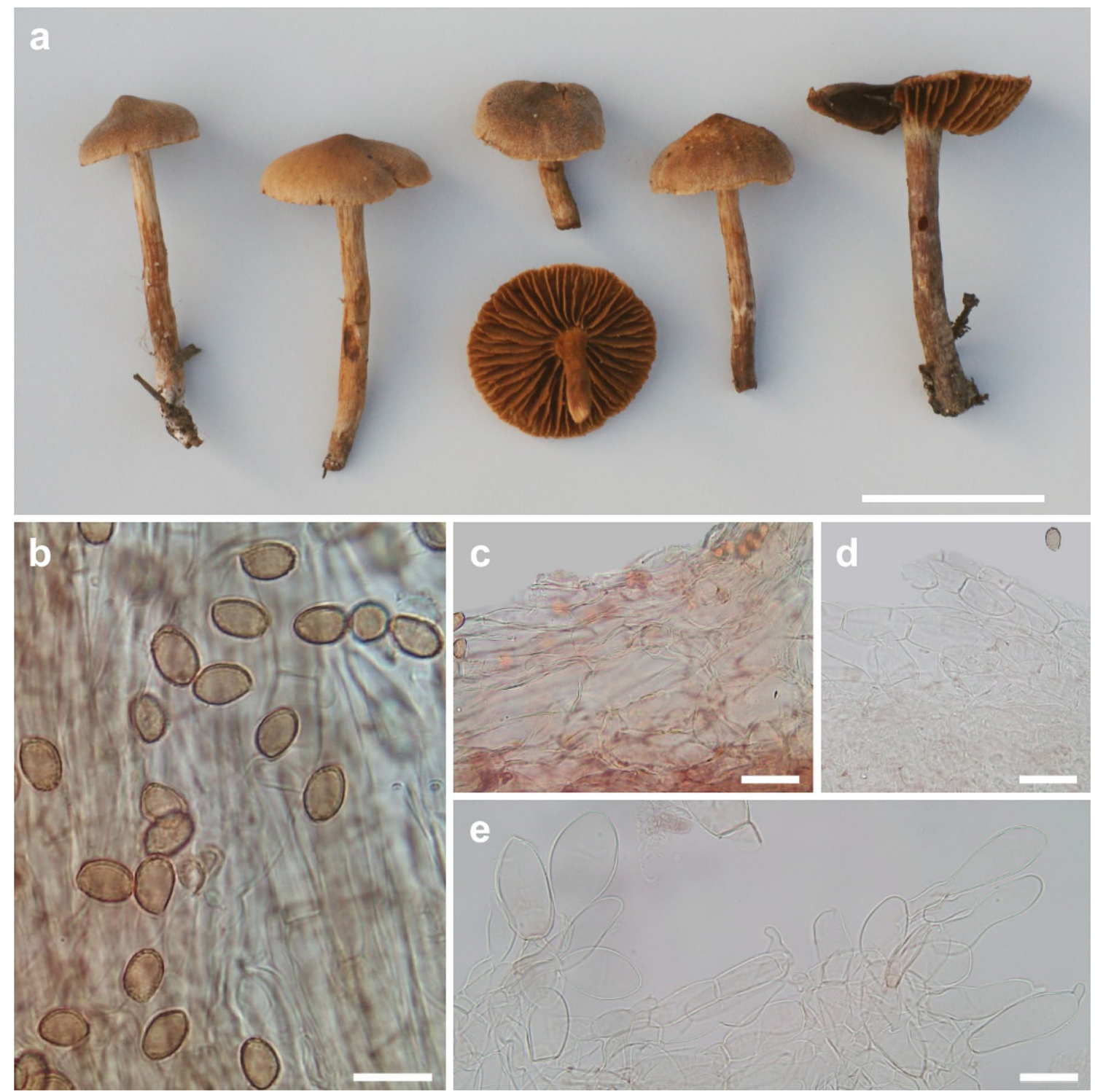

Fig. 176 Cortinarius alutarius (LOD(F):47001, holotype). a Basidiomata. b Basidiospores. c Pileipellis structure. d, e Ascending elements of pileipellis. Scale bars: $\mathbf{a}=20 \mathrm{~mm}, \mathbf{b}=10 \mu \mathrm{m}, \mathbf{c}-\mathbf{e}=20 \mu \mathrm{m}$

long, $0.2-0.4 \mathrm{~cm}$ thick, cylindrical, fibrillose, ochraceous buff-brown, becoming brownish to dark brown from base upwards with age or after handling. Context brown, darkening when old or bruised. Universal veil white to cream buff, distinct on the cap margin, forming fine stripes and incomplete girdles on the stipe. Odour not recorded. Basidiospores 6.5-7.5 $\times 4.5-5 \mu \mathrm{m}$, av. $=7 \times 5 \mu \mathrm{m}, \mathrm{Q}=1-1.5$, Qav. $=1.5$, ellipsoid to somewhat obovoid, finely to moderately verrucose, especially at the apex, indextrinoid to weakly dextrinoid (developing dextrinoid reaction very slowly to dextrinoid after a few hours), fulvous brown in $10 \% \mathrm{KOH}$, spore deposit rusty brown. Basidia 19-25×6-9 $\mu \mathrm{m}$, clavate, with four sterigmata; sterile cells distinct on the gill edge, clavate, apex 5-12 $\mu \mathrm{m}$ wide, av. $=8 \mu \mathrm{m}$. Lamellar trama hyphae light ochraceous brown, smooth, with parallel streaks of parietal to encrusting dark brown, amber-like pigment, at places with pigment lumps in the corners near septa. Pileipellis: Epicutis thin, of hyaline, pale, \pm parallel hyphae 4-11 $\mu \mathrm{m}$, av. $=7 \mu \mathrm{m}$, smooth, with yellowish parietal pigment, with frequent ascending bundles consisting of appressed thick-pigmented hyphae or/and thin-walled hyphae slightly constricted at septate, mixed with hypoderm elements, some with end cells swollen, 13-23 $\mu \mathrm{m}$ wide, av. $=16.5 \mu \mathrm{m}$ (bundles visible as fine flocs under the dissecting microscope). Hypoderm consisting of inflated elements $21-52 \times 10-21 \mu \mathrm{m}$, av. $=31 \times 14 \mu \mathrm{m}$, smooth, some thinwalled and some with parietal pigment, in deeper parts with fine, spot-like encrustations. Trama pale brown, consisting 
of parallel hyphae $3-7(-8) \mu \mathrm{m}$ wide, av. $=5 \mu \mathrm{m}$, smooth with numerous spots and streaks of parietal to encrusting dark brown, amber-like pigment and lumps of pigment near the septate, in deeper layers of subcellular elements $37-44 \times 13.5-23.5 \mu \mathrm{m}$, av. $=41 \times 18 \mu \mathrm{m}$, with much less distinct spot-like encrustations and yellowish pigment. ITS sequence (GenBank MZ648197, holotype) differing from the most similar sequences available with at least 9 substitutions and indels. Distinct from other members of Cortinarius sect. Rubricosi and deviating from them with at least 17 substitutions and indels.

Ecology and distribution: In temperate (possibly also boreal) pine forests on acid soil, growing in groups of sporocarps; basidiomata found in August. So far known from one locality and one collection only.

Material examined: POLAND, Mt Kamieńsk lignite mine spoil heap near Bełchatów, young Pinus sylvestris plantation on strongly to slightly acid loamy sand, $0-0.45 \% \mathrm{CaCO}_{3}$, 2 August 2011, leg. I.L. Kałucka 00220 (LOD(F):47001, holotype), $\mathrm{K}(\mathrm{M})$, isotype.

GenBank number: ITS = MZ648197.

Notes: This species is only known from one locality in Poland, on Mt Kamieńsk, the outer spoil heap of the Bełchatów opencast lignite mine, in ca. 20 year old pine plantation (Pinus sylvestris L.), on mineral soil among short mosses, lichens and pine needles, on strongly to slightly acid loamy sand, $0-0.45 \% \mathrm{CaCO}_{3}$. The presence of Betula or Salix in the vicinity cannot be excluded. As at the same locality many rare species of northern distribution have been found (Kałucka et al. 2016), we suspect that the species can occur also in Northern Europe. Cortinarius alutarius has finely tomentose-floccose dark rusty brown cap drying to light ochraceous brown, ochraceous brown stipe darkening from base upwards and ellipsoid-obovoid moderately verrucose spores (Fig. 176). It is distinct from known closely related species, like $C$. subscotoides, $C$. scotoides, C. minusculus, C. rubricosus and C. comptulus and can be relatively easily distinguished based on the combination of the basidiomata habit, spore measurements and habitat type. Cortinarius alutarius fits well in the C. sect. Rubricosi Bidaud, Moenne-Locc. \& Reumaux (Fig. 180). Based on the molecular and morphological data, the species is described here as new.

Cortinarius mammillatus Kałucka, Kytöv., Niskanen \& Liimat., sp. nov.

Index Fungorum number: IF558553; Facesoffungi number: FoF 09967; Figs. 177, 178

Etymology: The name is based on a Latin adjective mammillatus (having the form of a bluntly rounded protuberance) referring to a distinct nipple-like umbo.

Holotype: $\operatorname{LOD}(\mathrm{F}): 48001$
Pileus $1.5-3 \mathrm{~cm}$ in diam., distinctly umbonate, with pronounced, \pm acute to bluntly rounded umbo, glabrous, finely tomentose-fibrillose towards the margin, fulvous brown, ochraceous orange brown, drying mat with radial lighter stripes to pale grayish/yellowish/orange ochraceous; hygrophanous, striate. Lamellae medium spaced, adnate to emarginate, medium rusty brown, edge concolour or slightly paler, especially near the margin and when young. Stipe $5-9(-11) \mathrm{cm}$ long, $0.2-0.5 \mathrm{~cm}$ thick at apex, cylindrical, sometimes slightly wider at the base, whitish silky fibrillose to sparsely flocculose on ochraceous to rusty brown background. Context: in pileus brown, in stipe paler, ochraceous brown. Universal veil white, fibrous to floccose. Odour in lamellae of cedar wood, sometimes with raphanoid note; at the base of stem iodoform-like, best observed when slightly dried. Basidiospores 7.5-8.5(-9) $\times 4.5-5.5 \mu \mathrm{m}$, av. $=8 \times 5 \mu \mathrm{m}, \mathrm{Q}=1-2$, Qav. $=1.5$, narrowly ellipsoid to ellipsoid to ellipsoid-amygdaloid, finely to moderately evenly verrucose, dextrinoid to distinctly dextrinoid, olive yellowish brown in $10 \% \mathrm{KOH}$, spore deposit medium brown. Basidia $24-34 \times 6-7 \mu \mathrm{m}$, narrowly clavate, with four sterigmata. In $\mathrm{KOH}$, many basidia and basidioles yellowish olivaceous or amber brown. Lamellae edge mostly fertile, with sterile cells close to the cap margin; sterile cells broadly clavate to balloon-shaped, apex 7-13 $\mu \mathrm{m}$ wide, av. $=10 \mu \mathrm{m}$, usually on short basal cells. Lamellar trama hyphae pale olive yellowish, yellowish to orange brown in $\mathrm{KOH}$, yellowish to orange yellow parietally encrusted, with streaks and lumps of amber-like pigment, numerous hyphae densely finely scabrous. Pileipellis: Epicutis of hyaline, pale, parallel hyphae 5-11 $\mu \mathrm{m}, \mathrm{av} .=8 \mu \mathrm{m}$, smooth, some with yellowish parietal encrustation. Hypoderm consisting of inflated subcellular elements and inflated hyphae, $(24-) 28-58(-80) \times 13-25(-26) \mu \mathrm{m}$, av. $=41.5 \times 18.5 \mu \mathrm{m}$, smooth, some yellowish, yellowish ochraceous parietally encrusted, some with fine, spot-like encrustations to scabrous, and some with small lumps of olive ochraceous pigment, especially in deeper parts. Trama ochraceous brown, olive brown, consisting of \pm parallel hyphae, $6-14 \mu \mathrm{m}$ wide, av. $=10.2 \mu \mathrm{m}$, smooth to finely scabrous, with thin to thick parietal encrustation, some with olive brown to amber brown, spot-like encrustations or lumps of pigment, especially near the septa, in deeper layers of subcellular elements $45-68 \times 12-19 \mu \mathrm{m}$, av. $=56 \times 16 \mu \mathrm{m}$ with similar pigmentation. ITS sequence (GenBank MZ648201, holotype) differs from other sequences of C. sect. Obtusi known to occur in the Northern hemisphere with more than 20 substitutions and indels.

Ecology and distribution: In boreal and mountainous coniferous forests of the northern hemisphere, growing in groups of sporocarps; basidiomata found in September and October. 

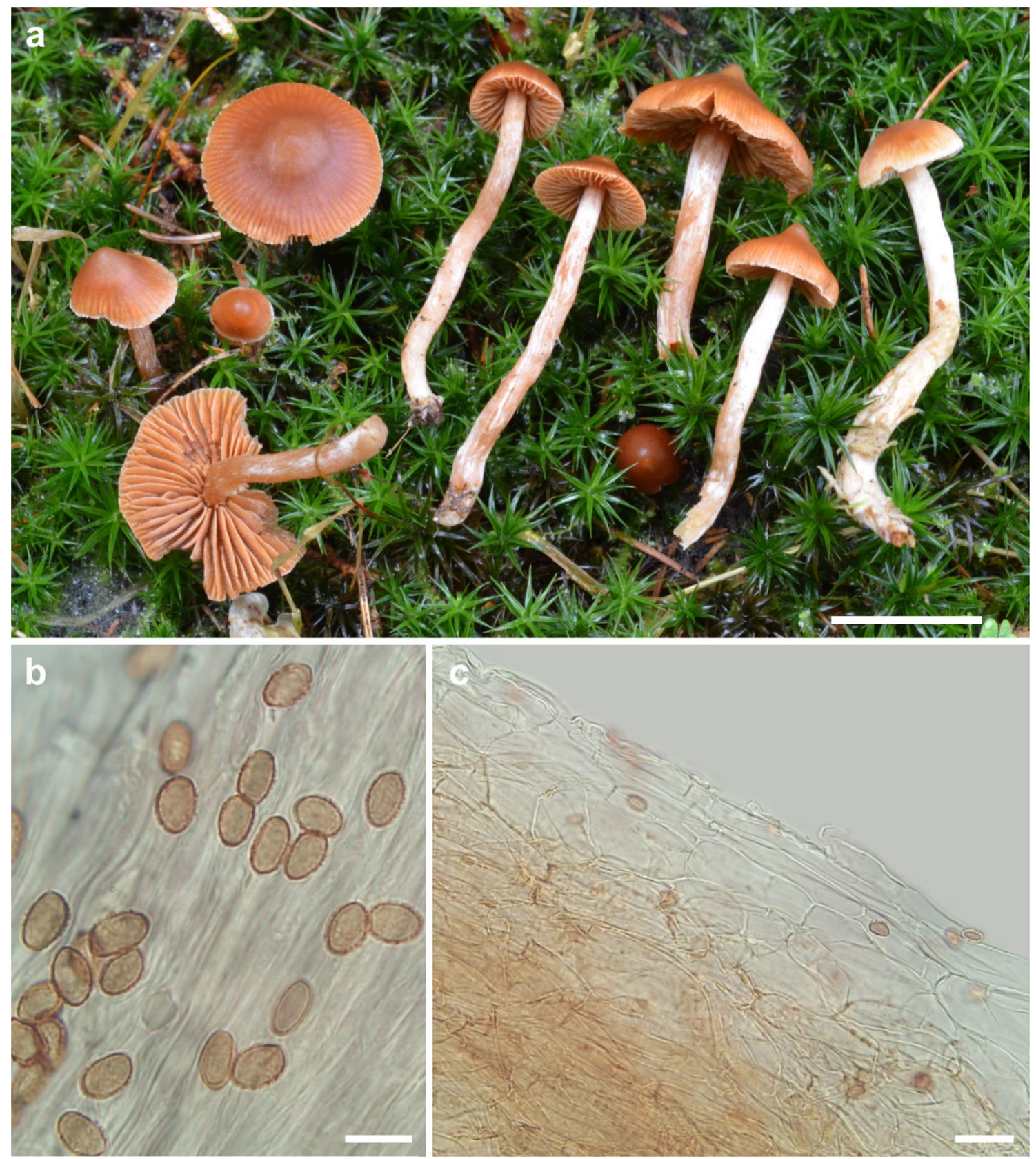

Fig. 177 Cortinarius mammillatus (LOD(F):48001, holotype). a Basidiomata. b Basidiospores. c Pileipellis structure. Scale bars: a=20 mm, $\mathbf{b}=10 \mu \mathrm{m}, \mathbf{c}=20 \mu \mathrm{m}$

Material examined: POLAND, Sudetes, Stołowe Mountains National Park, Czerwona Woda River valley, Picea abies old-growth forest, among Polytrichum moss, 15 September 2017, leg. I.L. Kałucka 17-140, (LOD(F):48001, holotype; K(M), isotype); loc. cit., among Sphagnum and Polytrichum mosses, 19 October 2017, leg. I.L. Kałucka 17-153 (LOD(F):48002); loc. cit., among Sphagnum and Polytrichum mosses, 18 September 2017, leg. I.L. Kałucka 17-159 (LOD(F):48003); loc. cit., among litter, grass and mosses, 7 September 2017, leg. I.L. Kałucka 17-160
(LOD(F):48004). FINLAND, Uusimaa, Sipoo, Paippinen, 14 Sep 2004, leg. K. Liimatainen and T. Niskanen 04-814 (H:6030110); Satakunta, Säkylä, Virttaankangas, Porsaanharju, pine (Pinus sylvestris) heath forest on sandy soil, 19 October 2006, leg. K. Liimatainen and T. Niskanen 06-249 (H:6029329). UK, Scotland, Woodcock Hill Plantation, in conifer plantation, under Picea, 21 October 2018, leg. D.J. Savage 488/18 (K(M):262938). CANADA, Ontario, Nipissing, Algonquin Provincial Park, Wildlife research station, chit lake trail, mixed forest of deciduous (e.g. Betula) and 

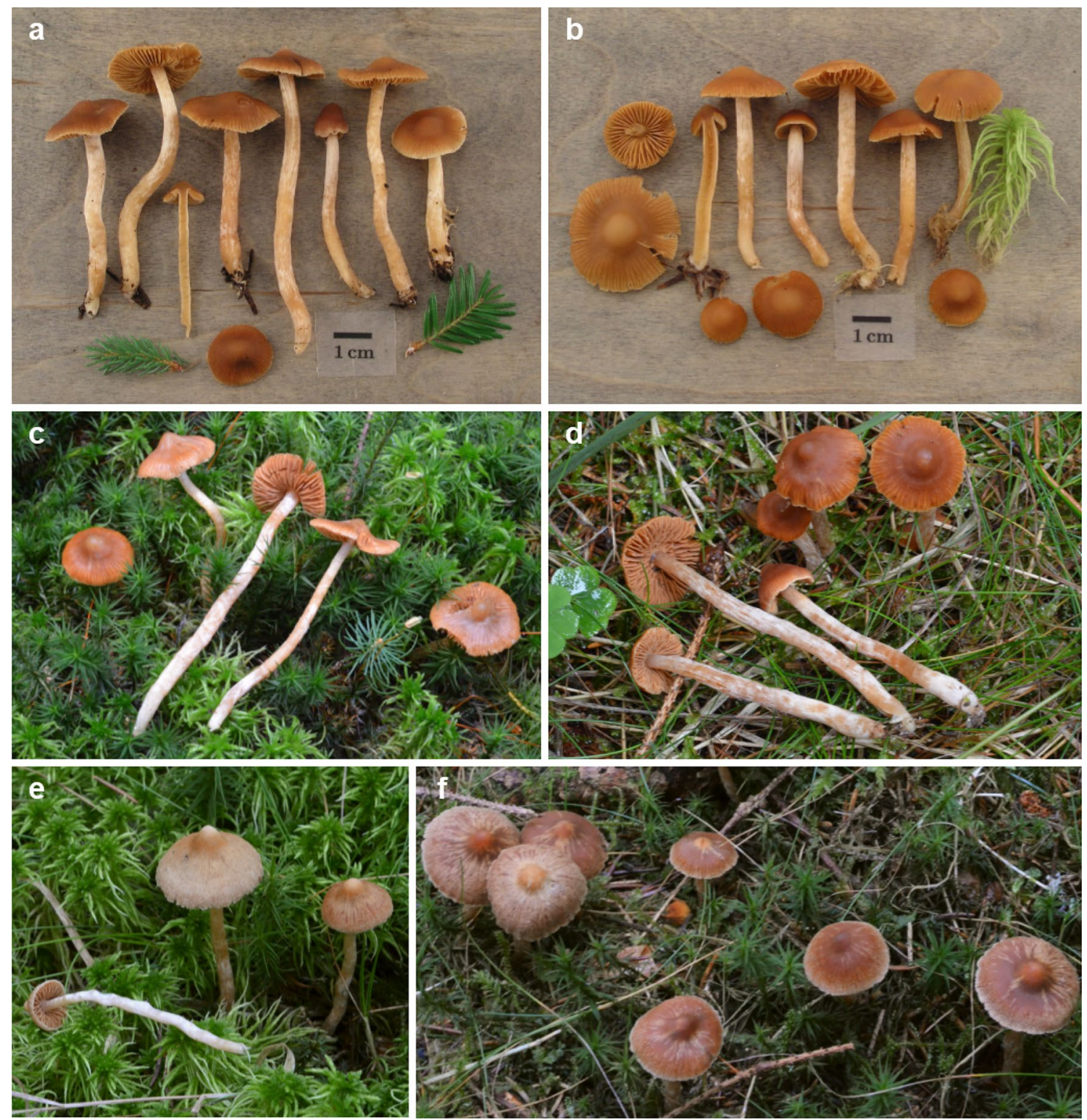

Fig. 178 Cortinarius mammillatus_additional collections (basidiomata). a H:7000935. b H:7000963. c LOD(F):48003. d LOD(F):48004. e LOD(F):48002. f LOD(F):48002a. Notes: Photographs: a, b K. Liimatainen, c-f I.L. Kałucka

coniferous trees (e.g. Abies balsamea), 13 September 2007, leg. K. Liimatainen and T. Niskanen 07-172 (H:7000834); Newfoundland, west coast Gros Morne National Park, N end of the Bonne Bay's Eastern Arm, James Callaghan hiking trail to Gros Morne Mountain, mesic to moist Abies balsamea dominated forest with some Picea, Alnus, and Betula, collected under Alnus, 21 September 2007, leg. K. Liimatainen and T. Niskanen 07-272 (H:7000935); Newfoundland, Avalon Peninsula, Salmonier Nature Park, mesic to moist Picea dominated forest with some Abies, Betula and Larix, 26 September 2007, leg. K. Liimatainen and T. Niskanen 07-299 (H:7000963); Newfoundland, 26 September 2007, leg. A. Voitk 2-101 (K(M)); Newfoundland, west coast Gros Morne National Park, $\mathrm{N}$ end of the Bonne Bay's Eastern Arm, James Callaghan hiking trail to Stuckless Pond, mesic to moist Abies balsamea dominated forest with some Picea, Alnus, and Betula, 19 September 2010, leg. K. Liimatainen and T. Niskanen 10-106 (H). USA. Washington, Snohomish county, Barlow past, Mt. Baker Snoqualmie National Forest, mixed coniferous forest, mainly Tsuga heterophylla with some Pseudotsuga menziesii and Abies amabilis, 10 October 2009, leg. K. Liimatainen and T. Niskanen 09-077 (H).

GenBank number: LOD(F):48001: ITS = MZ648201; LOD $(F): 48002:$ ITS = MZ648202; $(\operatorname{LOD}(\mathrm{F}): 48003)$ : ITS $=$ MZ648203; LOD(F):48004：ITS = MZ648204; 
H:6030110: ITS =MZ648205; H:6029329: ITS = MZ648206; H:7000834: ITS $=$ MZ648208; H:7000935: ITS =MZ648209; H:7000963: ITS = MZ648210; K(M): A. Voitk 2-101: MZ648211; H: K. Liimatainen and T. Niskanen 10-106: MZ648212; H: K. Liimatainen and T. Niskanen 09-077: MZ648213; K(M):262938: ITS = MZ648207.

Notes: The type collection and other three collections from Poland come from the mossy old-growth spruce (Picea abies) forest growing along the mountain stream (Pielech et al. 2018). In Finland, the species was found in pine (Pinus sylvestris) heath forest on sandy soil. All the North American collections are from mixed coniferous forests, with Abies, Picea and Betula on the east coast and Tsuga, Pseudotsuga and Abies on the west coast. A number of sequences available in GenBank and UNITE repositories, which do not differ from the type sequence by more than 3 substitutions and indels, come from the ECM roots of Pinus sylvestris (AY641464, Scotland) and Picea rubens (MK131478, MK131479, Canada), soil DNA from Cedar-Hemlock and Hemlock-Amabilis Fir Forests (KP889457, Canada) as well as sporocarps identified as C. obtusus from Picea abies forests (KY287715, KY287718, KY287698, Tatra Mts, Slovakia; AJ238035=UDB000127, Norway). Cortinarius mammillatus has distinctly umbonate, fulvous brown, striate hygrophanous cap, glabrous with tomentose margin, slender, whitish silky fibrillose stipe and narrowly ellipsoid to ellipsoid-amygdaloid, verrucose spores (Figs. 177 and 178). It most resembles $C$. obtusus and C. acutus. The former is somewhat fleshier and has more slender spores with coarser ornamentation; the latter has a usually more conical cap with pointed umbo, lighter gills and thinner, more brittle stipe; both species have a greater proportion of gill edge whitish fimbriate. C. fulvescens has darker cap centre, pale pink universal veil and slightly longer spores. Cortinarius mammillatus formed a well-supported clade (100\% MLBS; Fig. 180) and based on the molecular and morphological data is described here as new.

Cortinarius quercoflocculosus Kałucka \& Liimat., sp. nov. Index Fungorum number: IF558554; Facesoffungi number: FoF 09968; Fig. 179

Etymology: The name refers to the association of the species with oak (Quercus) as well as flocculose cap and stipe.

Holotype: $\mathrm{LOD}(\mathrm{F}): 47002$

Pileus $2-4 \mathrm{~cm}$ in diam., convex, not umbonate or with obtuse umbo, later broadly truncate or even with a shallow depression in the centre, with margin incurved downwards, finely fibrillose to sparsely flocculose, especially towards the margin, rusty medium brown, cinnamon brown, honey brown, beige brownish, ochraceous, frequently with darker centre; hygrophanous, margin striate, paler. Lamellae distant, adnate, rusty brown, bluish brown when young, with paler edge. Stipe $3.5-7 \mathrm{~cm}$ long, $0.4-0.8 \mathrm{~cm}$ thick at apex, $0.5-1 \mathrm{~cm}$ thick at the base, cylindric or frequently slightly wider at the base, solitary or cespitose, whitish silky fibrillose to sparsely flocculose, greyish white, light brownish, with bluish/lilac or rosy tinge (usually present more at the apex, but sometimes visible only at the base). Context: not checked. Universal veil white, fibrous to floccose, abundant. Odour in lamellae indistinct or of cedar wood. Basidiospores $(8-) 8.5-9.5(-10) \times 5-6 \mu \mathrm{m}$, av. $=9 \times 5.5 \mu \mathrm{m}, \mathrm{Q}=1.5-2$, Qav. $=1.5$, ellipsoid to somewhat amygdaloid, moderately verrucose, moderately to fairly strongly dextrinoid, ochre brown in $10 \% \mathrm{KOH}$, spore deposit relatively bright, light rusty brown. Basidia 26-32 $\times 8-9 \mu \mathrm{m}$, narrowly clavate, with four sterigmata. In $\mathrm{KOH}$, some basidia with dark brown amorphous pigment. Lamellar trama hyphae weakly pigmented, light yellowish ochre, smooth, with infrequent fine granular or spot-like encrustations. Pileipellis: Epicutis very thin, of 2-3 layers of parallel hyphae $4-10 \mu \mathrm{m}$, av. $=6.5 \mu \mathrm{m}$, smooth, with cementing yellow parietal pigment, with a few ascending, slightly inflated hyphae with ends 8-17 $\mu \mathrm{m}$, av. $=12.7 \mu \mathrm{m}$. Hypoderm elements pale, consisting of 5-6 rows of \pm parallel subcellular elements, $28-57(78) \times 14-25 \mu \mathrm{m}$, av. $=41.5 \times 19 \mu \mathrm{m}$, hyaline with yellowish cementing parietal pigment. Trama yellowish, consisting of parallel hyphae, 7-15(19) $\mu \mathrm{m}$ wide, av. $=11 \mu \mathrm{m}$, in the upper part tightly cemented with yellowish parietal pigment, with infrequent, scattered spot-like encrustations, parietal streaks and lumps of amber-like pigment, gradually transitioning to the more loose layer of subcellular elements, $42-74 \times 16-24 \mu \mathrm{m}, a v .=20 \times 57 \mu \mathrm{m}$, with very few fine spotlike encrustations. Oleiferous hyphae present. ITS sequence (GenBank MZ648198, holotype) different from the most similar sequence available in GenBank (FJ946918; Spain, Quercus ectomycorrhizal root) with only 1 substitution so probably represents the same species. C. quercoflocculosus is distinct from the closest known species, C. fulvopaludosus, and deviating with 12-14 substitutions and indels.

Ecology and distribution: In European oak forests, growing in groups of sporocarps and frequently cespitose; basidiomata found in October and November.

Material examined: POLAND, Mt Kamieńsk lignite mine spoil heap near Bełchatów, young Quercus robur plantation with some solitary Betula pendula and Pinus sylvestris, on slightly acid to slightly alkaline clay, $1.17-5.96 \% \mathrm{CaCO}_{3}, 12$ November 2011, leg. I.L. Kałucka 00260 (LOD(F):47002, holotype; K, isotype); loc. cit., 2 October 2013, leg. I.L. Kałucka 00270 (LOD(F):47003); Adamów lignite mine spoil heap near Turek, in Q. robur stand, 10 October 2012, leg. I.L. Kałucka 00523 (LOD(F):47004).

GenBank number: $\operatorname{LOD}(\mathrm{F}): 47002:$ ITS $=$ MZ648198; $\operatorname{LOD}(\mathrm{F}): 47003 ： \operatorname{ITS}=\mathrm{MZ648199} ; \operatorname{LOD}(\mathrm{F}): 47004$ : ITS $=$ MZ648200.

Notes: The two collections from Mt Kamieńsk, which is the outer spoil heap of the Bełchatów opencast lignite mine, including the holotype, come from the same young oak stand; the third Polish collection was found in oak 

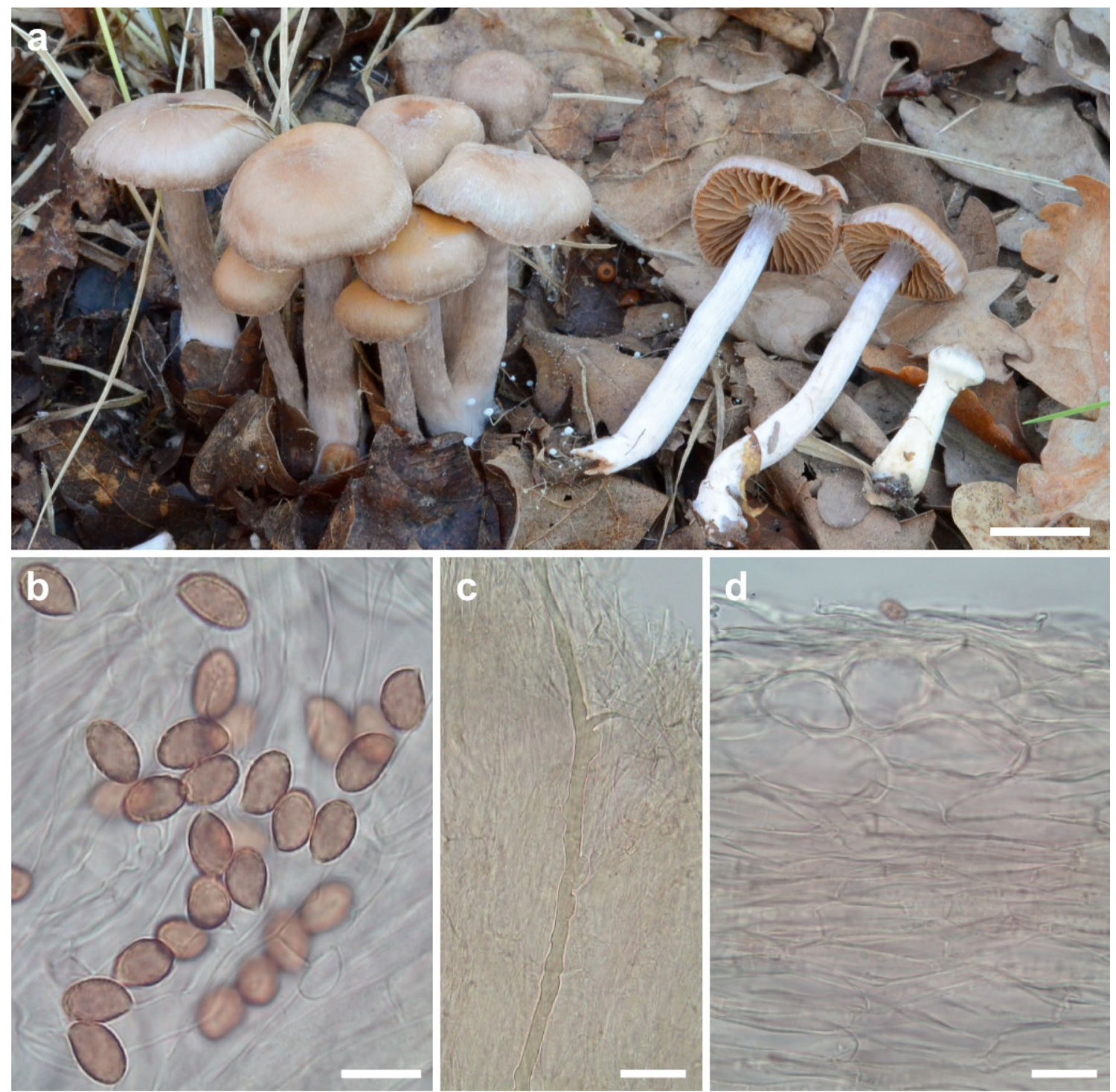

Fig. 179 Cortinarius quercoflocculosus (LOD(F):47002, holotype). a Basidiomata. b Basidiospores. c Oleiferous hypha in trama. d Pileipellis structure. Scale bars: $\mathbf{a}=20 \mathrm{~mm}, \mathbf{b}=10 \mu \mathrm{m}, \mathbf{c}, \mathbf{d}=20 \mu \mathrm{m}$

stand on a lignite mine spoil heap Adamów, $110 \mathrm{~km}$ apart (Kałucka et al. 2016). The ITS region of this species seems to match a single sequence known from a Quercus ectomycorrhizal root from Spain. Thus, the species is expected to occur at least throughout the oak forests of Europe. The closest known species, Cortinarius fulvopaludosus, differs in having acute umbo, slenderer stipe with very sparse veil fibrils, with a lack of blueish tinge in lamellae and stipe and narrower spores (Fig. 179). In our phylogenetic analysis, Cortinarius quercoflocculosus formed a well-supported clade (100\% MLBS; Fig. 180) within other telamonioid ITS sequences and based on the molecular and morphological data, the taxon is described here as new.

Hydnangiaceae Gäum. \& C.W. Dodge
Notes: Hydnangiaceae (Gäumann and Dodge 1928) is a family of Agaricales and currently comprises two genera, Hydnangium and Laccaria (Kirk et al. 2008). The family is characterized by basidiomata stipitate and pileate or gasteroid, epigeous or hypogeous basidiomata. When pileate, the fruitbodies are generally orange-brown or violet in coloration, lamellae thick and waxy; in gasteroid forms, fruit body shape is irregular, with or without columella, the peridium is sometimes evanescent and the hymenium is not gelatinized, and formed in locules. Hyphal system monomitic; hyphae with clamp-connections; pleurocystidia generally absent; basidia 4- or 2-spored; cheilocystidia sometimes present; basidiospores globose to cylindrical, rarely elongate with spines or slightly roughened. The spines are composed of 


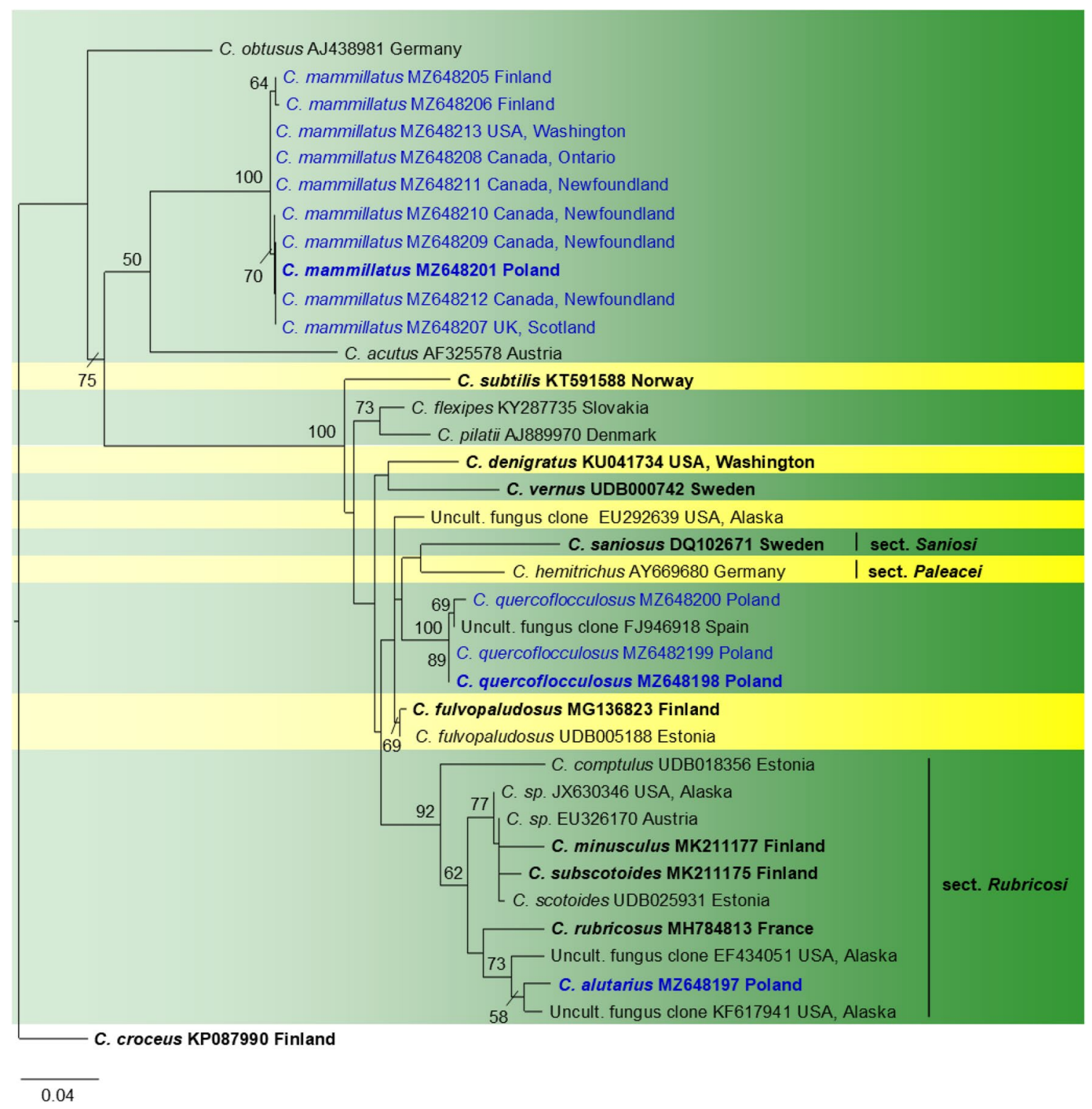

Fig. 180 Phylogram resulting from the RAxML (Stamatakis 2014) analysis of ITS region. Bootstrap values equal to or greater than 50\% are indicated above the nodes. The specimens in boldface represent the type specimens of the species. The newly generated sequences are in blue

parallel microtubules converging at the tip of the cones (Cannon and Kirk 2007).

\section{Laccaria Berk. \& Broome}

Notes: Laccaria currently comprises about 85 species (He et al. 2019), but numerous new species have been described in recent years (Wilson et al. 2013, 2016; Popa et al. 2014; Luo et al. 2016; Ramos et al. 2017; Vincenot et al. 2017; Cho et al. 2018; Wang et al. 2019b), a clear indication that the exact number of species in this genus is still undetermined. Laccaria species form mutualistic symbioses with many shrubs and forest tree species and are widely distributed over geographical areas from the tropics to the boreal regions (Gardes et al. 1990; Mueller 1992; Vincenot et al. 2011; Popa et al. 2014). Laccaria is easily recognized by having distinctive characters: basidiocarps are orange, purple, brown or flesh-coloured; pileus dry to subhygrophanous, lamellae rather thick and rather distant, from adnate to slightly decurrent; generally, with echinulate basidiospores; spore print white or pale violet (Singer 1986; Mueller 1992; Osmundson et al. 2005). In this study, a new species Laccaria populina is introduced based on morphological evidence and phylogenetic placement (Figs. 181 and 182).

Laccaria populina Dovana, sp. nov. 

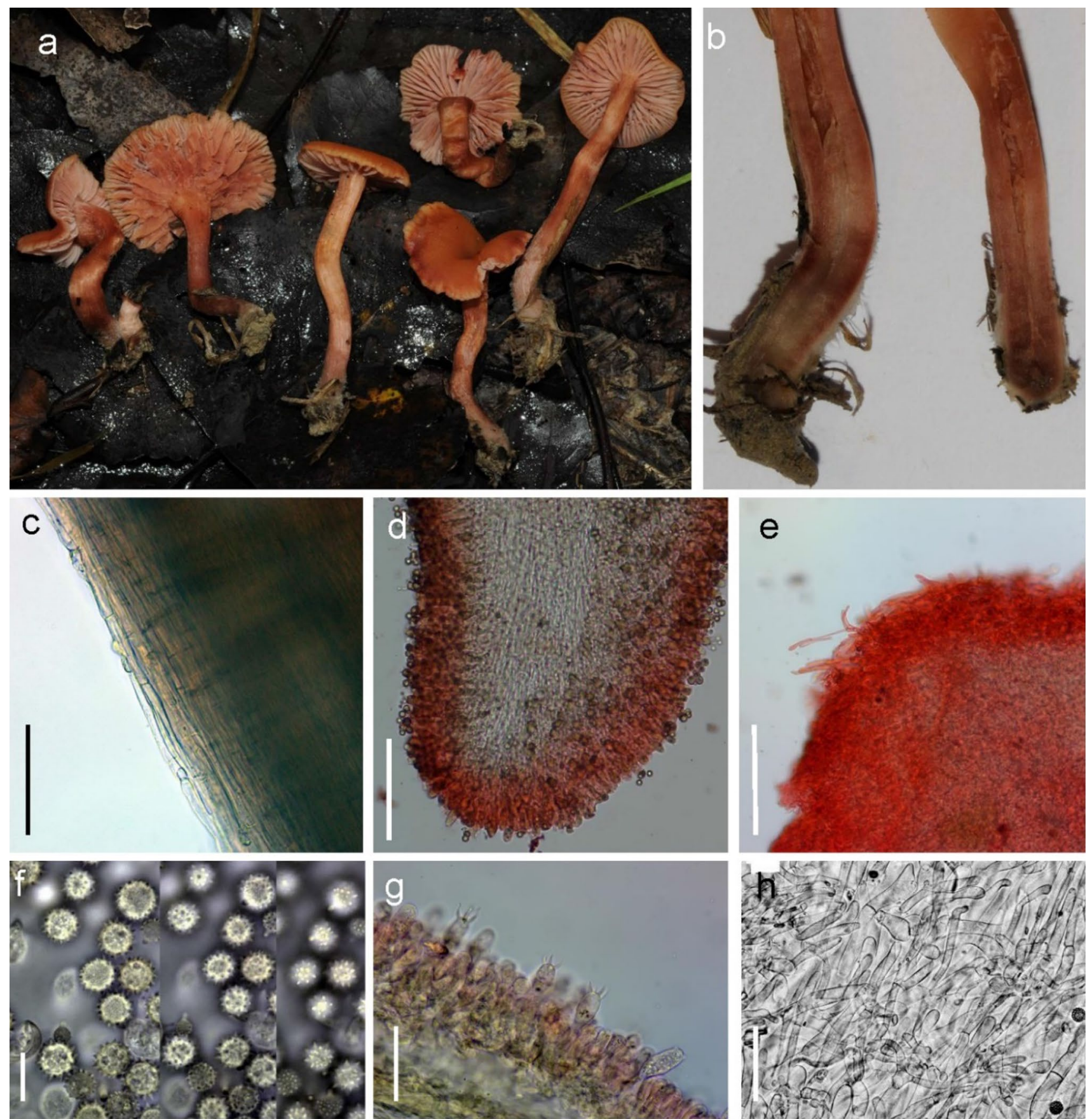

Fig. 181 Laccaria populina (GDOR 411, holotype). a Basidiomata in the field. b Section of stem. c Stipitipellis in KOH 3\%. d Section of gills in Phloxin B. e Pileipellis in Phloxin B. f Basidiospore in $\mathrm{KOH}$

Index Fungorum number: IF557832; Facesoffungi number: FoF 09202; Fig. 181

Etymology: The specific epithet populina is in reference to the genus Populus, because it was collected under poplar trees.

Holotype: GDOR411
3\%. g Basidia in Phloxin B. h terminal elements of pileipellis in $\mathrm{KOH}$ $3 \%$. Scale bars: $\mathbf{c}, \mathbf{e}, \mathbf{g}, \mathbf{h}=50 \mu \mathrm{m}, \mathbf{d}=100 \mu \mathrm{m}, \mathbf{f}=10 \mu \mathrm{m}$

Pileus 15-35 mm diam., convex to plane-convex centrally depressed to subumbilicate; surface glabrous when moist, on drying slightly squamulose at center, slightly translucentstriate, hygrophan, orange, brown-red at disk transitioning to pale orange toward margin, often with wine red areas; paler with age; margin striate and crenate. Lamellae up to $5 \mathrm{~mm}$ broad, moderately distant, emarginate, sinuate to adnate with 


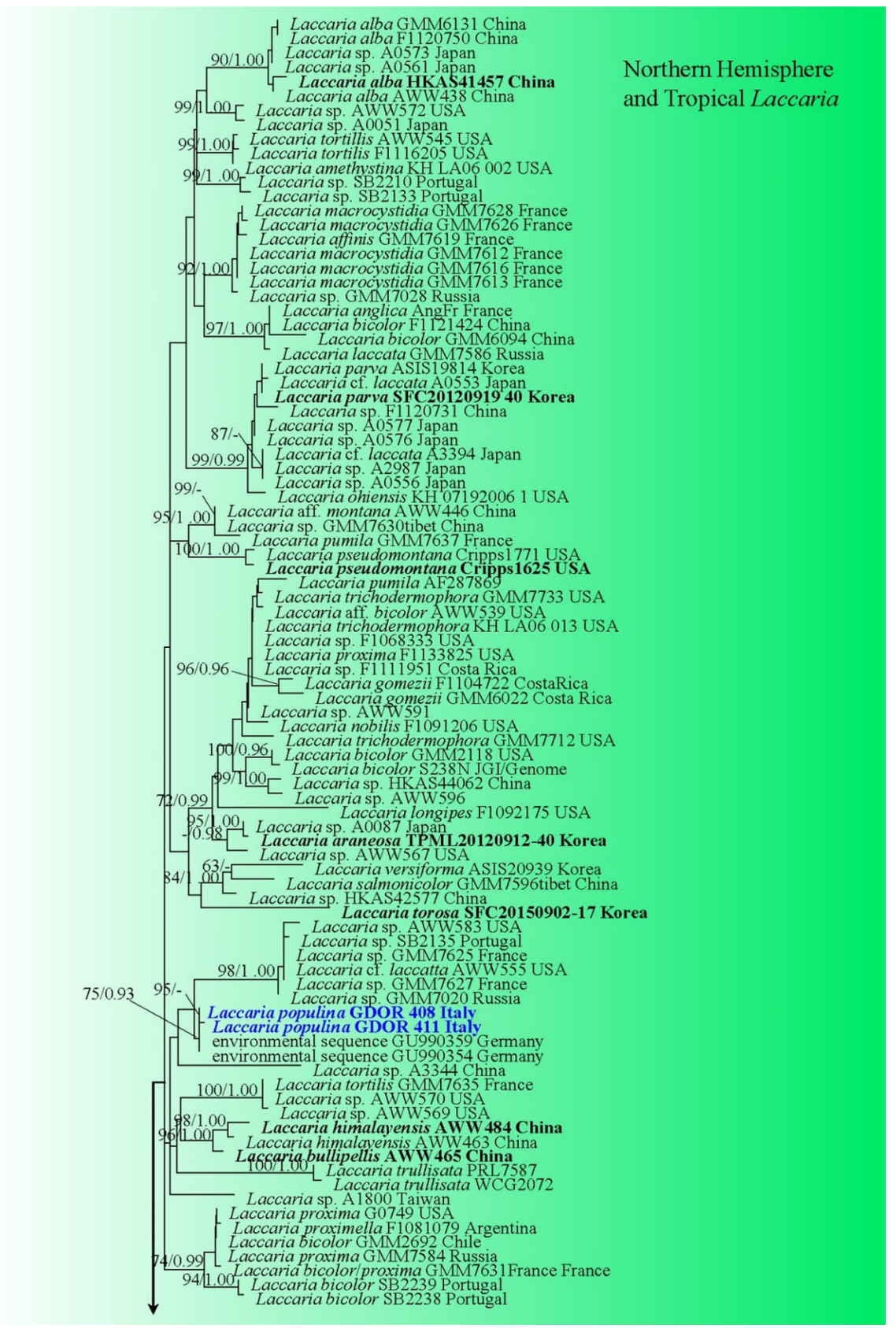

Fig. 182 Phylogram generated from maximum likelihood analysis based on LSU and ITS sequence data representing Laccaria of northern hemisphere and tropical areas. Related sequences aretaken from previous studies (Wilson et al. 2013; Popa et al. 2014; Luo et al. 2016; Ramos et al. 2017; Vincenot et al. 2017; Cho et al. 2018; Wang et al. 2019b). One hundred fifty-six strains are included in the combined analyses which comprise 1624 characters. Mythicomyces corneipes (AFTOL ID972) is used as the outgroup taxa. Single gene analyses were also performed to compare the topology and clade stability with combined gene analyses. Tree topology of the maximum likelihood analysis is similar to the Bayesian analysis. The best RaxML tree with a final likelihood values of - 9283.670199 is presented. Estimated base frequencies for the two partitions were as follows: the ITS partition $\mathrm{A}=0.248432, \mathrm{C}=0.203290, \mathrm{G}=0.210151$, $\mathrm{T}=0.338126 ;$ substitution rates $\mathrm{AC}=1.729861, \mathrm{AG}=6.506286$, $\mathrm{AT}=2.326472, \quad \mathrm{CG}=0.685905, \quad \mathrm{CT}=5.294619, \quad \mathrm{GT}=1.000000$, gamma distribution shape parameter $\alpha=0.357610$; the LSU partition $\mathrm{A}=0.269370, \mathrm{C}=0.185989, \mathrm{G}=0.290996, \mathrm{~T}=0.253645$; substitution rates $\mathrm{AC}=1.151721, \mathrm{AG}=25.007835, \mathrm{AT}=2.282933$, $\mathrm{CG}=0.751032, \mathrm{CT}=11.631350, \mathrm{GT}=1.000000$, gamma distribution shape parameter $\alpha=0.144016$. Bootstrap values for maximum likelihood (MLBS) equal to or greater than $70 \%$ and clade credibility values greater than 0.95 (the rounding of values to 2 decimal proportions) from Bayesian-inference analysis labeled on the nodes. Ex-type strains are in bold and newly generated sequences are in blue 


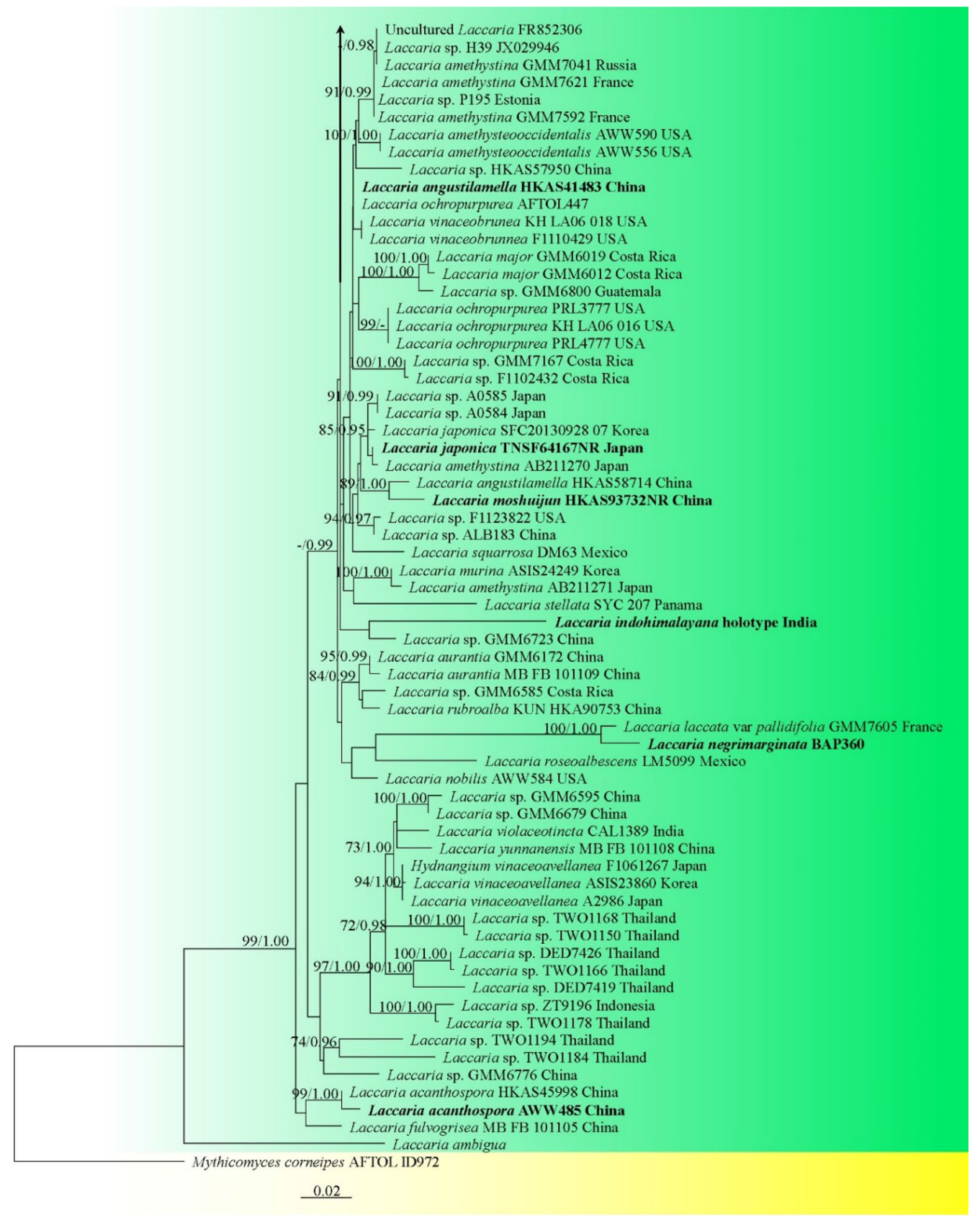

Fig. 182 (continued)

decurrent tooth, with 2-4(-5) lamellulae between two lamellae of different size, sometimes anastomosing or forked, from flesh-pink to dark orange, when old often with wine red tinge. Stipe 10-60×2-6 mm, cylindrical, centrally inserted, solid to fistulose with age, generally darker than the pileus, orange to red-brown, when moist from glabrous to fibrillose, on drying generally squamulose to squarrose overall, squamules white to ochraceous. Context concolorous with surface, sometimes with red-vinaceous tinge, generally vinaceous in the base of stipe. Taste mild and fungoid. Basal mycelium white. Basidiospores (6.6-)7.2-8.8(-9.6) $\times(6.4-) 7.1-8.8(-$ 9.6) $\mu \mathrm{m}, \mathrm{Q}=(0.98-) 0.99-1.03(-1.06) \mu \mathrm{m}$, globose, hyaline, moderately echinulate, $1-2 \mu \mathrm{m}$ long, $0.8-1.5 \mu \mathrm{m}$ broad at the base. Basidia 40-50×11-13 $\mu \mathrm{m}$, 4-spored, clavate, containing a yellow-brown pigmentation in $3 \% \mathrm{KOH}$. Pleurocystidia 40-50 $\times 3-7 \mu \mathrm{m}$, flexuous to narrowly-cylindrical. Cheilocystidia rare, similar to pleurocystidia. Hymenophoral trama, subregular, consisting of $15-56 \times 3-6 \mu \mathrm{m}$ subparallel hyphae. Pileipellis of interwoven hyphae with scattered fascicles of \pm perpendicular hyphae; terminal elements 
variable in shape, from cylindrical to clavate, sometimes with median constriction, rarely with lobed to coralloid terminal elements. Stipitipellis a cutis consisting of thin, parallel, 18-40×4-9 $\mu \mathrm{m}$ hyphae. Clamp-connections frequent everywhere.

Habitat: Associated with Populus alba, Populus nigra, Populus spp. and Salix spp.

Material examined: ITALY, Province of Alessandria, Mombello, locality Casalino, $220 \mathrm{~m}$ a.s.l., in a grassland with scattered Poplars (Populus alba, Populus nigra, Populus spp.) and Willows (Salix spp.) nearby, 11 November 2019, F. Dovana (GDOR 411, holotype); ibidem, 3 September 2010, F. Dovana (GDOR 408).

GenBank numbers: GDOR 411: ITS $=$ MN871895, $\mathrm{LSU}=\mathrm{MN} 873017 ;$ GDOR 408: $\mathrm{ITS}=\mathrm{MN} 871894$, $\mathrm{LSU}=\mathrm{MN} 873018$.

Notes: Macroscopically, Laccaria populina is characterized by its medium-sized basidiomata, with brown-red or orange pileus, crenulate at the margin, context concolour with pileus, with irregular red-wine spots especially near the base of stem and copious white basal mycelium (Fig. 181a, b). Laccaria populina observed appeared to be associated with Salicaceae. Microscopically, it can be recognized by the four-spored basidia, basidiospores echinulate with spines up to $2 \mu \mathrm{m}$ long, and pileipellis with terminal elements variable in shape and size (Fig. 181c-h). A megablast search of GenBank nucleotide database at NCBI (24 Dec. 2019) using LSU sequence of L. populina (holotype) showed that the best hits were L. tortilis (GMM7635), L. proximella (F1081079), L. proxima (GMM7631) and L. bicolor (GMM2692), all with $98.71 \%$ similarity and 6 gaps. As for megablast analysis (24 Dec. 2019), ITS sequence of L. populina showed $99.86 \%$ (708/709) similarity and no gaps with two german environmental sequences (GenBank GU990359 and GU990354) that come from Salix viminalis and Populus maximowiczii x Populus nigra root respectively that probably are the same species and $98.71 \%$ similarity, and 1 gap with two Laccaria amethystina samples (GMM7592 and GMM7621). Laccaria proximella and L. proxima differ from $L$. populina mainly by their larger ellipsoidal basidiospores (Mueller 1992). Laccaria bicolor differs mainly in its remarkable violet basal mycelium and presence of subglobose to broadly ellipsoid basidiospores with smaller echinulae (1-1.8 in length and $\leq 1 \mu \mathrm{m}$ wide at base) (Mueller 1992). Compared to L. populina, L. tortilis has a smaller basidiocarp, 2-sterigmate basidia and larger globose basidiospores $(9.2-) 10-14.5(-16) \times(8.3-) 10-14.5(-16) \mu \mathrm{m}$ (Mueller 1992). Laccaria amethystina is distinguished from L. populina by the bright grayish purple basidiomata and abundant filamentous, clavate or ventricose-rostrate conspicuous cheilocystidia (Mueller 1992). In the combined phylogenetic analysis of LSU and ITS sequences, the two collections of $L$. populina (holotype included) clustered in a supported clade (75\% MLBS, 0.93 BYPP; Fig. 182) with two environmental sequences (GenBank GU990359 and GU990354) sister to the L. cf. laccata clade but lacking in statistical support. Laccaria laccata differs from L. populina mainly by their subglobose to ellipsoid basidiospores (Mueller 1992); Laccaria laccata var. pallidifolia is very similar to L. populina from which it can be distinguished by its slightly larger globose to subglobose spores $(6.4-) 7.4-10(-13) \times(6-$ ) $7-10(-11.5) \mu \mathrm{m}$, with smaller echinulae $(\leq 1 \mu \mathrm{m}$ wide at base), context concolorous with pileus without a wine-red spot (Mueller 1992).

\section{Hygrophoraceae Lotsy}

Notes: Hygrophoraceae was validly established by Lotsy (1907). It is one of the larger families in the Agaricales and comprises 26 genera and more than 600 species (Lodge et al. 2014). It contains agarics, basidiolichens and corticoid fungi. We follow the treatment and updated accounts of Hygrophoraceae in Lodge et al. (2014). Based on morphological characteristics and phylogenetic analysis of ITS sequence data, a novel species, Hygrocybe boertmannii is introduced.

\section{Hygrocybe (Fr.) P. Kumm.}

Notes: Hygrocybe is distributed worldwide and characterized by brilliant colours of basidiomata with waxy, widely-spaced lamellae; absence of veil remnants on stipe; white, smooth, inamyloid basidiospores; presence of clampconnections; lack of true pleurocystidia and wide, regular to subregular or parallel lamellar trama (Boertmann 1995; Babos et al. 2011; Lodge et al. 2014; Hosen et al. 2016; Singh et al. 2017). In this study, a new species Hygrocybe boertmannii is introduced based on evidence of morphology and phylogenetic placement (Figs. 184 and 185).

\section{Hygrocybe boertmannii U. Singh \& R.P. Bhatt, sp. nov.}

Index Fungorum number: IF557840; Facesoffungi number: FoF 07737; Fig. 183

Etymology: In recognition to David Boertmann for his contribution to the wild mushrooms.

Holotype: CAL 1802

Pileus 12-52 mm in diam., convex when young, becoming planoconvex to applanate or slightly depressed with age, non-striated, non-perforate, dry, non-viscid, orange to deep orange (5A7-5A8), with maize yellow to sunflower yellow (4A6-4A7) fibrillose-squamules on the surface; margin decurved to plane, crenate; context up to $2 \mathrm{~mm}$ wide, fragile, concolorous towards cap surface and pastel yellow to light yellow (3A4-3A5) towards lamellae. Lamellae up to $7 \mathrm{~mm}$ wide, sub-decurrent to decurrent or sometimes sinuate, distant, white when young, becoming yellowish white (2A2-2A3), edges concolorous but white near the stipe attachment, with about 3 complete lamellae (at margin) 

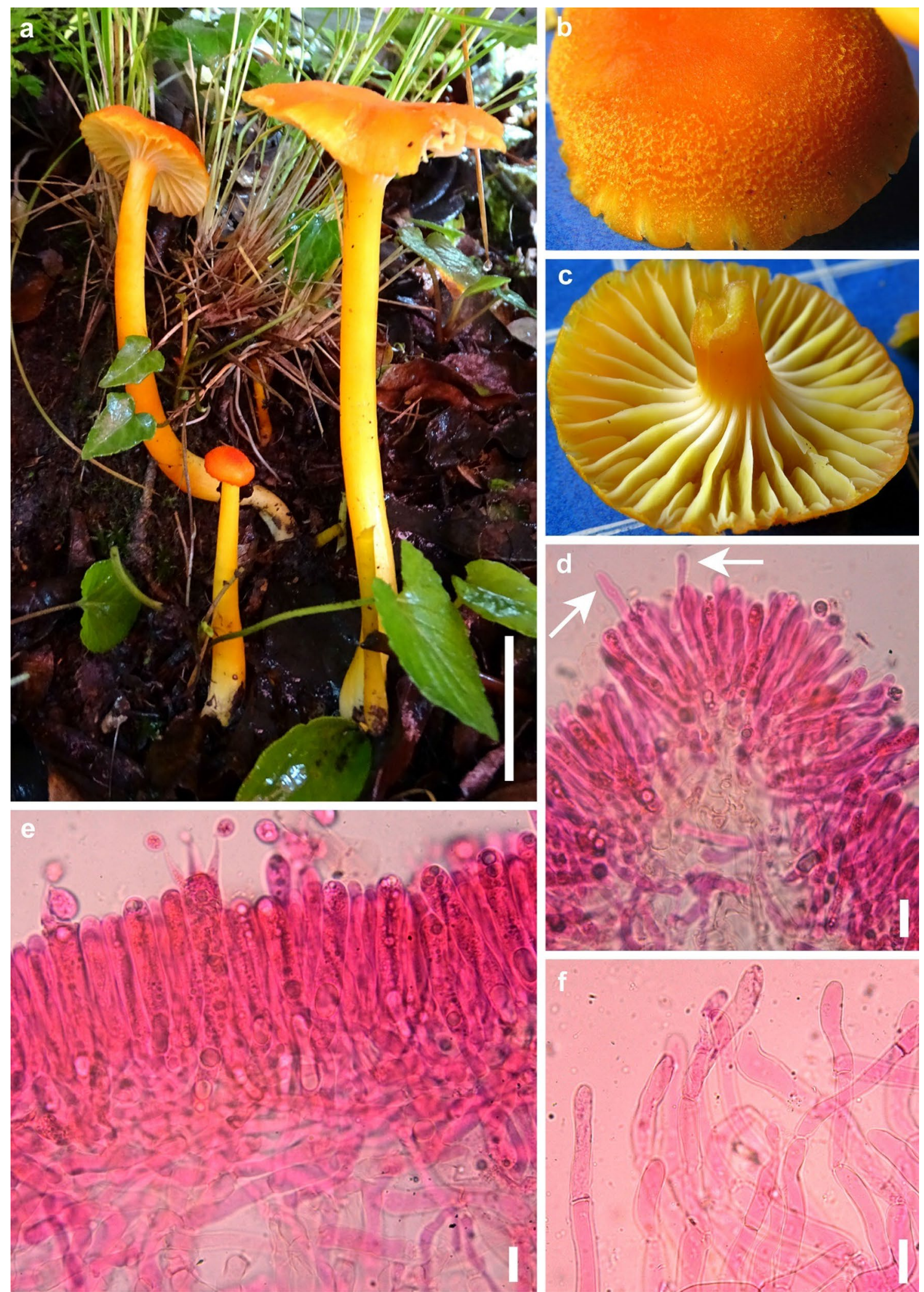

Fig. 183 Hygrocybe boertmannii (US 1552, holotype). a Fresh basidiomata in the field. b Pileus surface with fine squamules. c Lamellae with lamellulae d Cystidia-like hyphoid elements. e Trans- verse section through hymenium showing basidia and basidiospores. $\mathbf{f}$ Terminal elements of pileipellis. Scale bars: $\mathbf{a}=25 \mathrm{~mm}, \mathbf{d}, \mathbf{e}=10 \mu \mathrm{m}$, $\mathbf{f}=20 \mu \mathrm{m}$ 
per $\mathrm{cm}$, and usually with 3 lamellulae of different lengths between the two complete lamellae, often interveined. Stipe $51-116 \times 6-11 \mathrm{~mm}$, central, tapered towards apex, curved near base in mature specimen, smooth, maize yellow to sunflower yellow (4A6-4A7), yellowish white (2A2) at base, hollow; context thin, concolorous to surface, fragile. Spore deposit white. Taste and odour indistinct. Spore print not obtained. Basidiospores (8-)8.7-9.8-10.9(-11) $\times(6-) 6$. $8-7.6-8.4(-9) \mu \mathrm{m}(\mathrm{Q}=(1.1-) 1.2-1.3-1.4 \mu \mathrm{m}, \mathrm{n}=30)$, broadly ellipsoid to ellipsoid, rarely subglobose, smooth, thin-walled, hyaline, inamyloid, single to multi-guttulate, with apicules up to $1 \mu \mathrm{m}$ long. Basidia $62-78 \times 11-13 \mu \mathrm{m}$, clavate to narrowly clavate, thin-walled, turning light yellowish brown with $\mathrm{KOH}$, with a basal clamp connection, 4-spored; sterigmata up to $13 \times 4 \mu \mathrm{m}$. Lamellar edge nonfertile. Cystidia-like hyphoid elements 22-38 $\times 4-5 \mu \mathrm{m}$, emanating from the lamellar context, clavate to cylindro-clavate, fusiform, rarely ventricose; frequently clamped at bases. Hymenophoral trama subregular, composed of hyaline, thinwalled (up to $1 \mu \mathrm{m})$, cylindrical elements $(26-51 \times 5-7 \mu \mathrm{m})$ intermixed with inflated cells $(30-75 \times 10-16 \mu \mathrm{m})$, rarely with clamp connections. Pileipellis a trichoderm, made up of erect to semi-erect septate, thin-walled, unbranched hyphae; terminal elements $74-118 \times 10-20 \mu \mathrm{m}$, with round apex, rarely pointed apex, clamp connections absent; underlying hyphae repent, cylindrical, rarely clamped, devoid of any pigments. Stipitipellis composed of septate, thin-walled, unbranched hyphae, up to $21 \mu \mathrm{m}$ wide; clamp connection present.

Material examined: INDIA, Uttarakhand, Rudraprayag district, Baniyakund forest, growing scattered on ground under Quercus sp. in mixed temperate forest, $2653 \mathrm{msl}$, N30 28.998' E079 ${ }^{\circ} 10.658^{\prime}, 08$ August 2017, U. Singh, US 1552 (CAL 1802, holotype); $2617 \mathrm{msl}$, N30 28.926' E079 ${ }^{\circ} 10.615^{\prime}, 25$ August 2016, U. Singh, US 1358 (CAL 1803, paratype).

GenBank numbers: ITS = MT127548, MT127549.

Notes: Hygrocybe boertmannii is characterized by orange to deep orange pileus with yellowish fibrillose-squamules all over the pileus, crenate margin, white to yellowish distant lamellae, smooth yellow stipe, broadly ellipsoid to ellipsoid basidiospores, presence of cystidia-like hyphoid elements and a trichoderm nature of pileipellis (Fig. 183). Based on a combination of characters, e.g., dry basidiomata, squamulose pileus, smooth stipe, subregular hymenophoral trama and a trichoderm pattern of the pileipellis, it is referable to H. subsect. Squamulose (Borgen and Arnolds 2004; Boertmann 2010; Lodge et al. 2014). This placement was further confirmed by our ITS-based molecular phylogeny (Fig. 184). Hygrocybe boertmannii shares some similarities with $H$. cantharellus (originally described from USA) including having orange pileus with crenate margin, the presence of squamulose on pileus, yellow distant lamellae and a smooth stipe surface but the latter can be distinguished by smaller basidiomata (pileus 7-30 mm; stipe 18-67×1.8-4 mm), weakly to strongly arcuate decurrent lamellae, ellipsoid to ovoid basidiospores, smaller basidia $(35-54 \times 7.5-9.5 \mu \mathrm{m})$ and absence of cheilocystidia (Arnolds 1995).

Phylogenetically, Hygrocybe boertmannii appeared as sister to another Indian species, $H$. rajendrae, however our novel species of Hygrocybe is separated from $H$. rajendrae with very strong nodal support (100\% MLBS, 1.00 BYPP). Hygrocybe rajendrae is clearly distinct from $H$. boertmannii on the basis of larger basidiomata (pileus 37-69 mm diam.; stipe $109-218 \times 8-14 \mathrm{~mm}$ ), yellowish pileus and absence of cystidia-like hyphoid elements (Singh et al. 2017). The ITS dataset consisting of 41 sequences (including our isolates: US 1552, represented by GenBank acc. no. MT12631 and US 1358, represented by GenBank acc. no. MT12634) of Hygrocybe and Hygroaster was analysed (Fig. 184). Two sequences isolated from these Indian materials appear to be nested amongst other sequences of Hygrocybe subg. Pseudohygrocybe sect. Coccineae subsect. Squamulosae and form a distinct clade that is sister to $H$. rajendrae (another Indian species) with $100 \%$ MLBS, 1.00 BYPP support. The combination of morphological features and phylogenetic analysis corroborates the proposed taxon Hygrocybe boertmannii as a new species distinct from all the known taxa of Hygrocybe.

\section{Marasmiaceae Roze ex Kühner}

Notes: Marasmiaceae is one of the most diverse family consisting of ca. 54 genera and 1590 species (Kirk et al. 2008). Most members of the family are commonly found to grow on decayed leaves, twigs, branches, trunks and hence are saprophytic in nature. As a saprobe, these fungi play an important role for the sustenance of forest ecosystem by recycling of nutrients.

\section{Marasmius Fr.}

Notes: Marasmius was proposed by Fries (1836). This is a world-wide distributed genus which has been a topic of interest by researchers all around the globe. Presently, India hosts a record of ca. 75 species of Marasmius among which most of them have been reported from the southern Indian regions (Dutta et al. 2015). In this study, three new species of the Marasmius viz. M. benghalensis, M. jinfoshanensis and M. subtropicus are described from China and India based on evidence of morphology and phylogenetic analysis (Figs. 185, 186, 187, 188, 189, 190, 181, 192 and 193). The present study follows the latest treatment on Marasmius by Shay et al. (2017) and He et al. (2019).

Marasmius benghalensis A.K. Dutta \& K. Acharya, sp. nov. Index Fungorum number: IF557845; Facesoffungi number: FoF 07864; Figs. 185, 186 


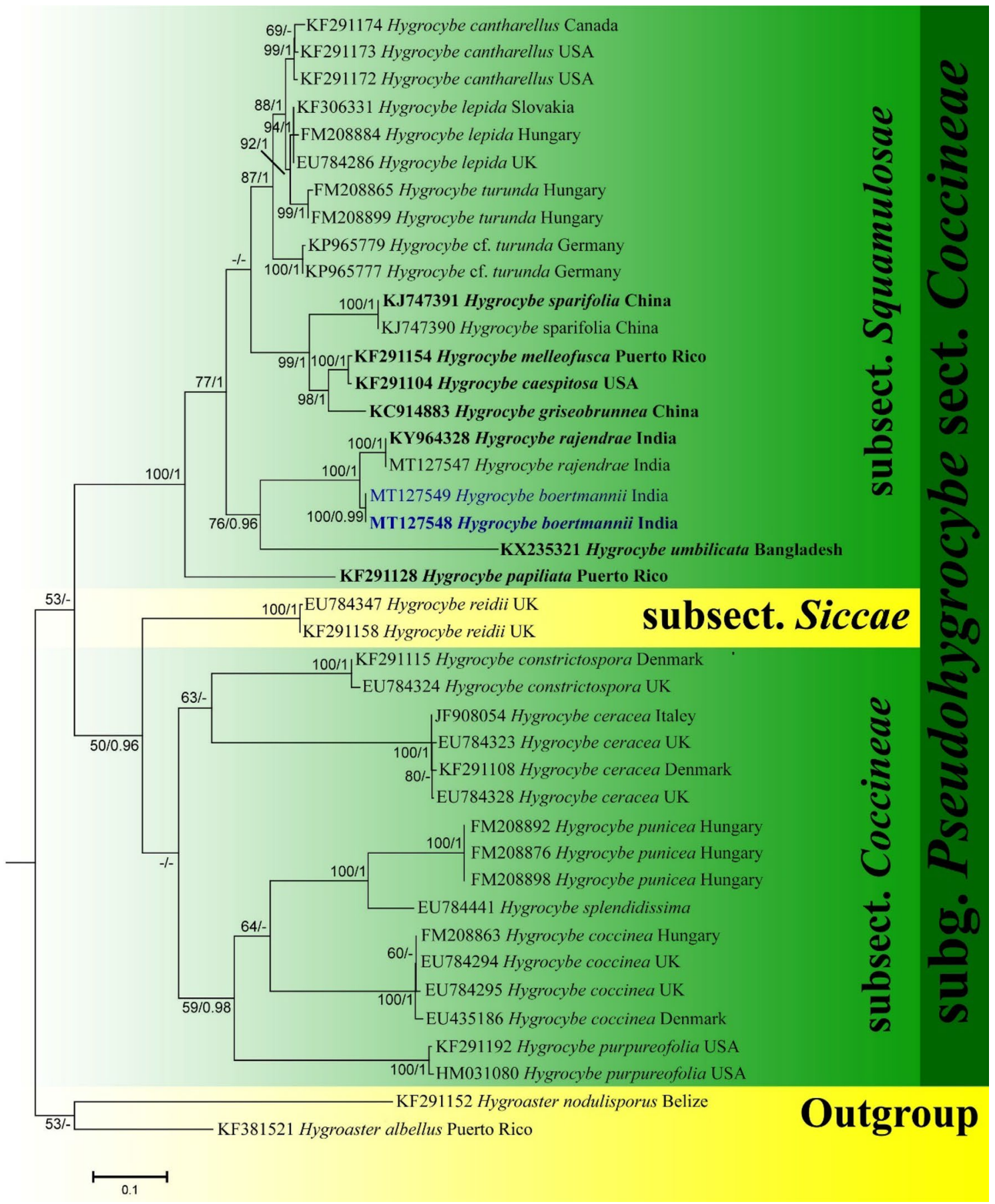

Fig. 184 Phylogenetic analyses were based on data set of nrITS sequences. Reference sequences were selected from relevant literature (Lodge et al 2014; Singh et al. 2017), BLAST searches (Altschul et al. 1997) and data retrieved from GenBank (Clark et al. 2016). Species of Hygroaster are selected as the outgroup taxa (Lodge et al 2014; Singh et al. 2017). Multiple sequence alignment was performed using MAFFT v.7 (Katoh and Standley 2013). Alignments were manually edited in BioEdit v 7.2.5 (Hall 1999). To change the multiple alignment format, Alignment Transformation Environment (ALTER) was used (Glez-Peña et al. 2010). Maximum Likelihood analysis was performed with the programme RAxML GUI 1.5 (Silvestro and Michalak 2012). One thousand bootstrap replicates were analysed to obtain nodal support values. Bayesian inference was computed inde- pendently twice in MrBayes v.3.2.2 (Ronquist et al. 2012), under different models. The best-fit substitution model of nucleotide evolution was carried out in MrModeltest 3.7 (Posada and Crandall 1998). Bayesian posterior probabilities (BPP) were calculated in two simultaneous runs with Markov chain Monte Carlo (MCMC) algorithm (Larget and Simon 1999). Markov chains were run for 10 million generations, saving a tree every 100th generation. Default settings in MrBayes were used for the incremental heating scheme for the chains ( 3 heated and 1 cold chain), unconstrained branch length [unconstrained: exponential (10.0)] and uniformative topology (uniform) priors. The first 25\% of trees was discarded as burnin (Hall 2004). Ex-type strains are in black bold and newly generated sequences are indicated in blue 
Fig. 185 Field pictures of the basidiomata. a, b Marasmius benghalensis (CUH AM214, holotype), habitats of basidiomata and lower side of the pileus showing lamellae features. Scale bars: $\mathbf{a}, \mathbf{b}=10 \mathrm{~mm}$
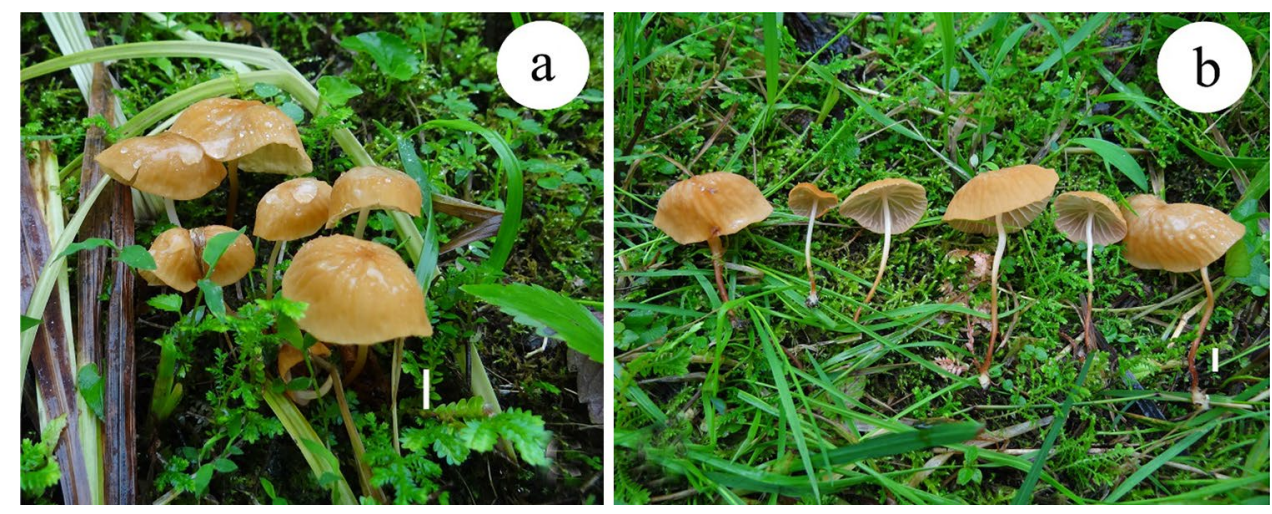

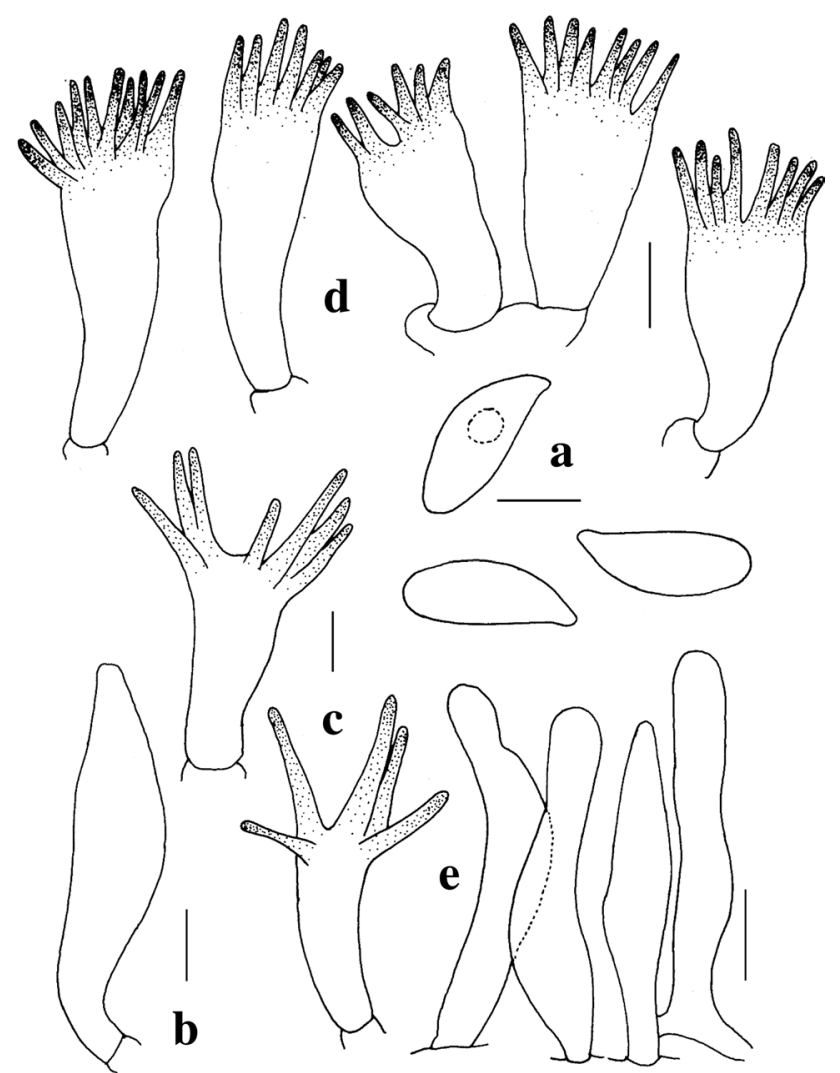

Fig. 186 Marasmius benghalensis (CUH AM214, holotype). a Basidiospores. b Basidioles. c Cheilocystidia. d Siccus-type pileipellis cells. e Caulocystidia. Scale bars: $\mathbf{a}-\mathbf{c}=5 \mu \mathrm{m}, \mathbf{d}-\mathbf{e}=10 \mu \mathrm{m}$

Etymology: 'benghalensis' refers to West Bengal, India, the region from where the type species was collected.

Holotype: CUH AM214

Pileus 21-56 mm diam., convex when young, becoming broadly convex to undulate when old, with or without a small central depression, wrinkled, glabrous, semidry to moist, light orange (5A5), orange (5A6) to greyish orange (5B5-6) or brownish orange (5C6) or golden yellow (5B7), margin greyish yellow (4B3-4) to pale orange (5A3) or light orange (5A4), becoming yellowish with $\mathrm{KOH}$, hygrophanous, non-striate. Context very thin, pale orange (5-6A3) to light orange (6A4) or orange grey (6B2) to greyish orange (6B3). Lamellae narrow, non-collariate, adnexed, subdistant ( $\mathrm{L}=11-14)$ with 2-4 series of lamellulae, vein-like, nonintervenose, sometimes forked from the middle towards margin, white, concolorous, smooth, even, often not reaching to the margin of pileus. Stipe well-developed, $52-75 \times 2-4 \mathrm{~mm}$, central, cylindrical, equal, hollow, dry, minutely pruinose, shiny, greyish orange (5B6) to golden yellow (5B7) or brownish orange (6C8) to brown (7D7-8) towards base, light yellow (3-4A5) to olive yellow (3C6-7) from the middle towards apex, non-insititious, strigose, basal mycelium white. Taste mild. Odour sweet.

Basidiospores $(7.2-) 8.5-10(-10.8) \times 3.5-4(-4.5) \mu \mathrm{m}$ $(\bar{x}=9.5 \times 3.8 \mu \mathrm{m}, \mathrm{n}=30), \mathrm{Q}=1.8-2.7 \mu \mathrm{m}(\bar{x}=2.5 \mu \mathrm{m}$, $\mathrm{n}=30$ ), ellipsoid to lacrymoid, hyaline, inamyloid, one guttate when viewed with $\mathrm{KOH}$, thin-walled. Basidia not observed. Basidioles 24-27(-29) ×5-6(-7.5) $\mu \mathrm{m}$, fusoid to clavate, hyaline, thin-walled. Pleurocystidia absent. Lamellae-edge sterile, with crowded cystidia. Cheilocystidia composed of Siccus-type broom cells; main-body (11-) 14-16(-18) $\times(3.5-) 4.5-5.5(-7.5) \mu \mathrm{m}$, cylindrical to clavate, hyaline, thin- to thick-walled; apical setulae (6-)9-10(-11.5) $\mu \mathrm{m}$ long, cylindrical, less dense, often with obtuse apex, pale yellowish, thick-walled. Pileipellis a hymeniform layer, consists of Siccus-type of broom cells; main-body (18-)21$25(-36) \times(5-) 7.5-9(-15) \mu \mathrm{m}$, cylindrical to clavate or subclavate, hyaline, thin- to thick-walled walled; apical setulae 3.5-6(-11) $\mu \mathrm{m}$ long, cylindrical, pale yellowish, acute to obtuse, thick-walled. Lamellae trama hyphae 3.5-6(-7.5) $\mu \mathrm{m}$ broad, interwoven, cylindrical, hyaline, smooth, dextrinoid, thin- to slightly thick-walled. Pileus trama hyphae 6.5-7(-9) $\mu \mathrm{m}$ broad, interwoven, cylindrical to irregularly cylindrical, sometimes branched, hyaline, dextrinoid, thinto slightly thick-walled. Stipitipellis hyphae 3.5-4.5(-6.5) $\mu \mathrm{m}$ broad, parallel to subparallel, cylindrical, hyaline, dextrinoid, moderately thick-walled. Stipe trama hyphae 5-7(-11) 
$\mu \mathrm{m}$ broad, parallel, cylindrical, hyaline, strongly dextrinoid, moderately thick-walled (up to $0.9 \mu \mathrm{m}$ ). Caulocystidia (21-) 39-60(-76) $\times(5-) 6.5-7.5(-8.5) \mu \mathrm{m}$, abundant, non-setulose, cylindrical to irregular in outline, hyaline, inamyloid, moderately thick-walled.

Material examined: INDIA, West Bengal, Darjeeling dis-

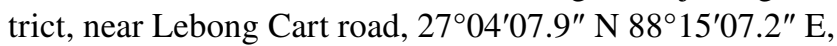
$1836 \mathrm{msl}$, on decomposed leaf litter among Gleichenia sp., 24 June 2016, S. Paloi (CUH AM214, holotype); Darjeeling

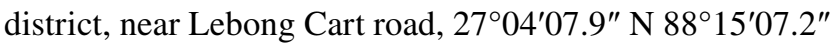
E, 1836 msl, 25 June 2016, S. Paloi (CUH AM221).

GenBank numbers: ITS = MF189043, MF189044, $\mathrm{LSU}=\mathrm{MT} 255002$, MT255001.

Notes: Diagnostic features of the present taxon include: a non-striate, wrinkled, greyish yellow to pale orange pileus with orange to brownish orange or golden yellow disc; narrow, vein-like, adnexed, subdistant (11-14), white lamellae that often do not reach the pileus margin; a minutely pruinose, strigose stipe with white basal mycelium; presence of a sweetish odour; ellipsoid to lacrymoid basidiospores with mean $9.5 \times 3.8 \mu \mathrm{m}$; absence of pleurocystidia; presence of Siccus-type broom cells in the pileipellis and form of cheilocystidia; and simple cylindrical caulocystidia ranging 21-76×5-8.5 $\mu \mathrm{m}$ (Figs. 185 and 186). These combinations of features undoubtedly place Marasmius benghalensis under ser. Atrorubentes of the sect. Sicci (Tan et al. 2009; Wannathes et al. 2009).

Among similar species within ser. Atrorubentes of the sect. Sicci, Marasmius xestocephalous Singer has a striate pileus, a greater number of lamellae (15-18), shorter stipe (18-35 mm long), much longer basidiospores $(10-15 \mu \mathrm{m})$, and caulocystidia that is forked or apically lobed (Wannathes et al. 2009). Marasmius inthanonensis described for the first time from Northern Thailand (Wannathes et al. 2009), differs by its pileus-coloured, dark olive to olive at disc, close and much broader lamellae $(3-6 \mathrm{~mm})$ coloured light brown at the edge, much longer stipe (up to $102 \mathrm{~mm}$ long), and relatively shorter caulocystidia (up to $48 \mu \mathrm{m}$ long).

Among other similar species within the sect. Sicci: Marasmius hypochroides, one of the frequently encountered taxon in India, primarily differs by the absence of caulocystidia and belongs to a different ser. Leonini of the sect. Sicci (Pegler 1986; Wannathes et al. 2009). The Brazilian taxon Marasmius cladophyllus (Singer 1976; Pegler 1983) has adnate, close reticulately interveined, crowded lamellae coloured pale buff, a stipe-coloured purplish brown towards base with pale buff apex, and less broad basidiospores (2.5-3.5 $\mu \mathrm{m}, \mathrm{Q}=2.0$ ). Marasmius ruber (Pegler 1983) differs by its sulcate pileus, crowded lamellae coloured yellow to orange with scarlet red towards edge, relatively shorter stipe $(15-50 \mathrm{~mm})$ with glabrous surface, and less broad basidiospores (2.5-3.5 $\mu \mathrm{m})$. The Mexican taxon, Marasmius leoninus Berk. has somewhat differently coloured pileus with white context, much broader lamellae (3-4.5 mm), glabrous stipe surface coloured white towards apex, absence of any sweetish odour, and presence of Siccus-type broom cells in the form of caulocystidia (Singer 1976).

In the phylogenetic tree, Marasmius benghalensis appears to be close to M. luteolus (60\% MLBS, 0.99 BYPP; Fig. 187). However, M. luteolus has minutely velutinous to pruinose pileus surface, adnate attachment of lamellae, presence of pleurocystidia of the Siccus-type, and two types viz. Siccus-type as well as non-setulose caulocystidia (Wannathes et al. 2009).

Marasmius jinfoshanensis Chun Y. Deng \& Gafforov sp. nov.

MycoBank number: MB836094; Facesoffungi Number: FoF 09969; Figs. 188, 189

Etymology: Referring to the Jinfoshan National Nature Reserve, the location where the type specimen was collected.

Holotype: HGASMF 01-4344

Pileus 3-5 mm, convex to broadly conical, umbonate; glabrous; greyish orange (5B4, 5B5, 6B5), light brown (7D5), brownish orange $(6 \mathrm{C} 6,7 \mathrm{C} 6,7 \mathrm{C} 4)$, at center with a surrounding white dot. Lamellae subdistant, $\mathrm{L}=12-16,1=0$, collariate, pale cream, with a concolorous edge. Stipe up to $60-80 \mathrm{~mm}$ long, filiform, insititious, smooth, glabrous, white at apex, reddish brown (7E8, $8 \mathrm{E} 8)$ to dark brown $(7 \mathrm{~F} 7,8 \mathrm{~F} 8)$ to black. Basidiospores (6.5-)7-8(-9.8) $\times 4-5 \mu \mathrm{m}, \mathrm{E}=1.6-2, \mathrm{Q}=1.7$, ellipsoid, sublacrymoid, ellipsoid-fusoid, thin-walled, smooth, hyaline, inamyloid. Basidia 19-27 ×9-11 $\mu \mathrm{m}$, 4-spored, clavate. Basidioles 12-28 $\times 4-9 \mu \mathrm{m}$, clavate, cylindrical, fusoid. Cheilocystidia in the form of broom-cells of the Rotalistype, 20-30 $\times 5-8 \mu \mathrm{m}$, clavate, subcylindrical, thin-walled, with \pm thin-walled or slightly thick-walled, projections. Pleurocystidia absent. Trama hyphae cylindrical to subinflated, thin-walled, hyaline, dextrinoid, up to $12 \mu \mathrm{m}$ wide. Pileipellis a hymeniderm consisting of broom-cells of the Rotalistype, 13-18 $\times 7-14 \mu \mathrm{m}$, clavate, subvesiculose, pyriform, thin-walled, sometimes with slightly thick-walled apex, with cylindrical to warty, up to $2 \times 1 \mu \mathrm{m}$ large projections. Stipitipellis a cutis composed of cylindrical, parallel, slightly thick-walled, smooth, up to $5 \mu \mathrm{m}$ wide hyphae with yellowish walls in $\mathrm{KOH}$; medulla hyphae slightly dextrinoid. Caulocystidia absent. Clamp connections present in all tissues.

Material examined: CHINA, Chongqing, Nanchuan district, Jinfoshan National Nature Reserve, N107.182222, E29.030152, 2037 m alt., 6 Apr. 2020, C.Y. Deng, DCY 2409 (HGASMF 01-4344, holotype); ibid., 6 Apr. 2020, C.Y. Deng, DCY 2413, (HGASMF 01-4345, paratype).

GenBank numbers: HGASMF 01-4344: ITS $=$ MT556448, LSU $=$ MW237220; HGASMF 01-4345: $\mathrm{ITS}=\mathrm{MT556449}, \mathrm{LSU}=\mathrm{MW} 237221$. 


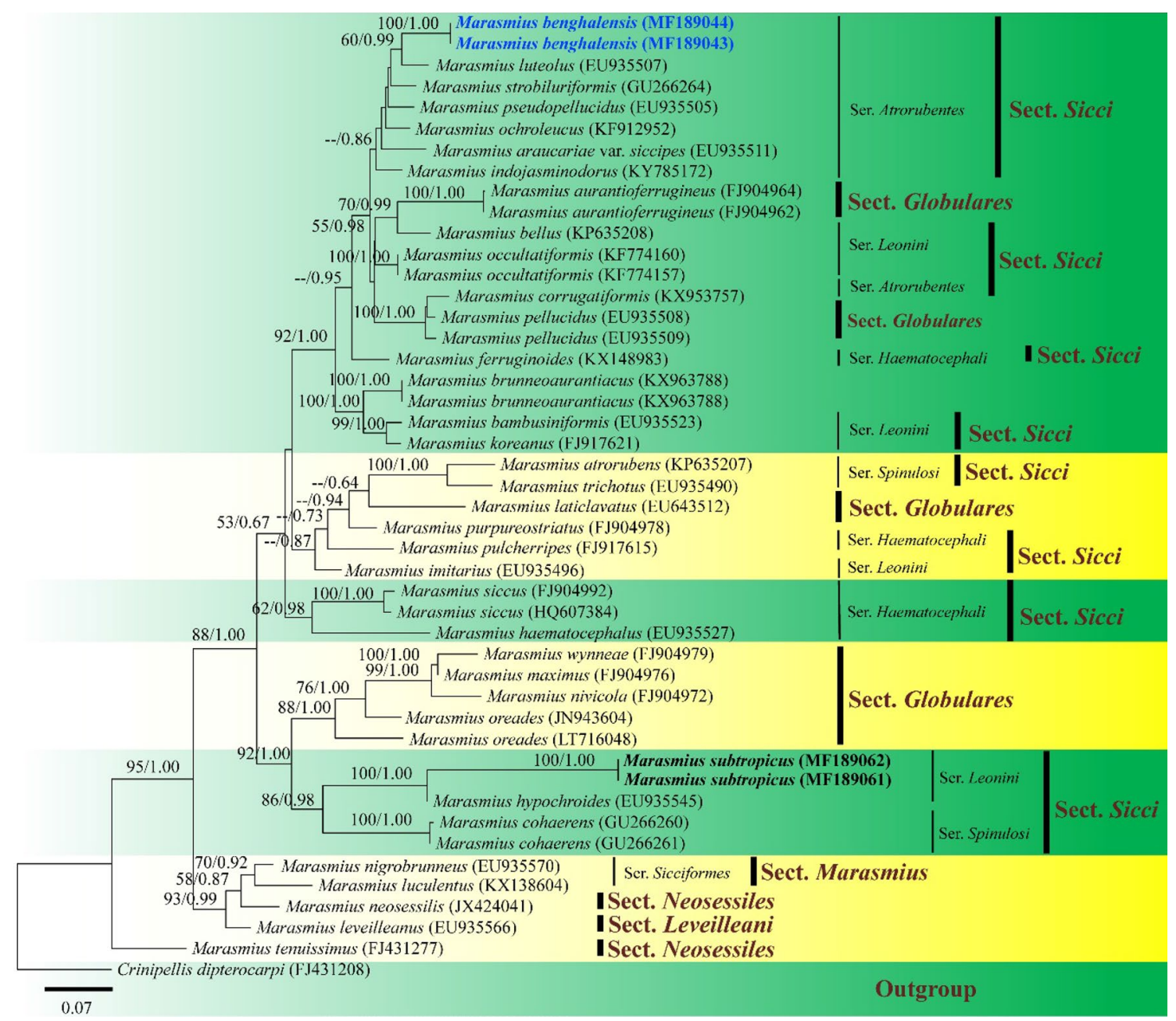

Fig. 187 Phylogram generated from maximum likelihood (ML) analysis based on nrDNA ITS sequence data of Marasmius species using best-fit model $(\mathrm{HKY}+\mathrm{G})$ with Bayesian Information Criterion (BIC) value of 14371.193718. Related sequences are mostly taken from Wannathes et al. (2009) and Antonín et al. (2012). Thirty-six taxa of Marasmius and one outgroup taxon are included in the analyses comprising 742 characters after alignment. Crinipellis dipterocarpi is used as the outgroup taxon following Wannathes et al. (2009). Bayesian analysis (BA) reached a standard deviation of split frequencies of 0.004984 after $10^{6}$ generations and produced 11210 credible set of trees after discarding initial 3,792 trees as burnin phase. Tree

Notes: Marasmius sect. Marasmius species are unique because they have collariate lamellae (Singer 1976, 1986; Desjardin 1989; Antonín 1991, 2007; Antonín and Noordeloos 2010). Based on nrITS sequences, subsect. Maramius is monophyletic while subsect. Sicciformes is paraphyletic (Tan et al. 2009; Wannathes et al. 2009). Marasmius subsect. Horriduli might have a wider range (de Oliveira et al. 2020). Marasmius jinfoshanensis is in Marasmius sect. Marasmius because it has collariate lamellae. ITS and LSU dataset of $M$. siccus were chosen as an outgroup for rooting purposes based on a study by Tan et al. (2009) and topology of the ML analysis is similar to the BA. The best RaxML tree with a final likelihood values of -6870.939953 is presented. The matrix had 483 distinct alignment patterns, with $17.46 \%$ proportion of gaps and completely undetermined characters. Estimated base frequencies were as follows: $\mathrm{A}=0.2461, \mathrm{C}=0.2043, \mathrm{G}=0.2006$, $\mathrm{T}=0.3490$; gamma distribution shape parameter $=0.4030$. ML Bootstrap values (MLBS) equal or greater than $50 \%$ (on the left of ' $\%$ ') and posterior probabilities (BYPP) values equal or greater than 0.50 (on the right of ' $/$ ') from BA are labeled above or below the nodes. The newly generated sequences are in blue bold. Representative sections and series of the Marasmius taxa are marked in the right side

Wannathes et al. (2009). Two sequences of M. jinfoshanensis constitutes a subclade on a long branch with $100 \%$ bootstrap support. Marasmius jinfoshanensis is sister to Marasmius wisteriae with high support. Marasmius wisteriae was described from Korea. It differs from other Maramius species by a pale colored pileus with a dark central dot and large, clavate-fusoid, fusoid, narrowly lacrymoid basidiospores (Antonín et al. 2013). Two sequences of Marasmius rotalis (KC415765 and JN003837) were likely misidentified. Marasmius capillaris is represented on two separate branches in our phylogeny (Fig. 190). Both species were 


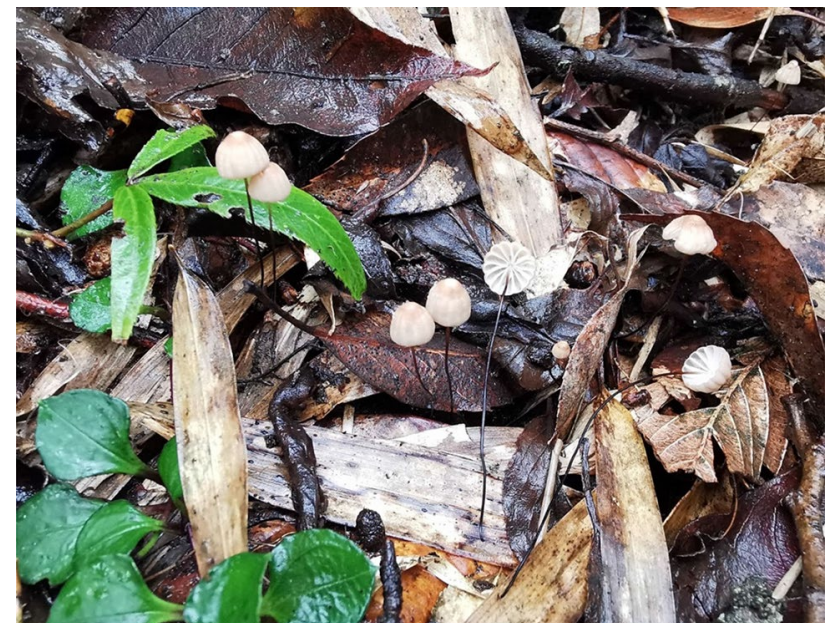

Fig. 188 Marasmius jinfoshanensis (HGASMF 01-4344, holotype): Basidiomata of on decaying leaves of dicotyledons in the litter in a subtropical-montane forest

collected in two different states in North America. At least one of these species must be misidentified.

Marasmius rotalis differs in having a campanulate to umbilicate, shallowly depressed pileus and distant lamella
(8-11). Marasmius rotula also differs by having a navel-like central depression in the white pileus. Marasmius somalomoensis differs in having a plicate pileus and non-marginate lamellae (Jackie et al. 2017). The distinct pallid zone surrounding a dot in the center of the pileus in M. jinfoshanensis is similar to the zone in M. avellaneus, M. vigintifolius and M. subvigintifolius. Marasmius jinfoshanensis (Figs. 188 and 189) differs from these species in the colour of the pileus and the size of the basidiospores $(7.8-11.5 \times 4.5-7$, $6.8-10.8 \times 3.3-6 \mu \mathrm{m}$ and $6.6-9 \times 3.8-5 \mu \mathrm{m}$, respectively) (de Oliveira et al. 2020; Singer 1976). Marasmius idroboi has a brown pileus with a white zone around the central dot, but differs in having a pileus with a shallow umbilicus and having much more numerous lamellae (20-23) (Singer 1976). Marasmius andasibensis is somewhat similar to $M$. jinfoshanensis. However, the former species differs in having a smaller grey orange pileus and distant lamellae (Antonín and Buyck 2006).

Marasmius subtropicus A.K. Dutta \& K. Acharya, sp. nov. Index Fungorum number: IF557846; Facesoffungi number: FoF 07865; Figs. 191, 192
Fig. 189 Marasmius jinfoshanensis (HGASMF 01-4344, holotype): Microscopic structures. a Basidiospores. b Basidia. c Cheilocystidia. d Pileipellis. Scale bar: $\mathbf{d}=10 \mu \mathrm{m}$

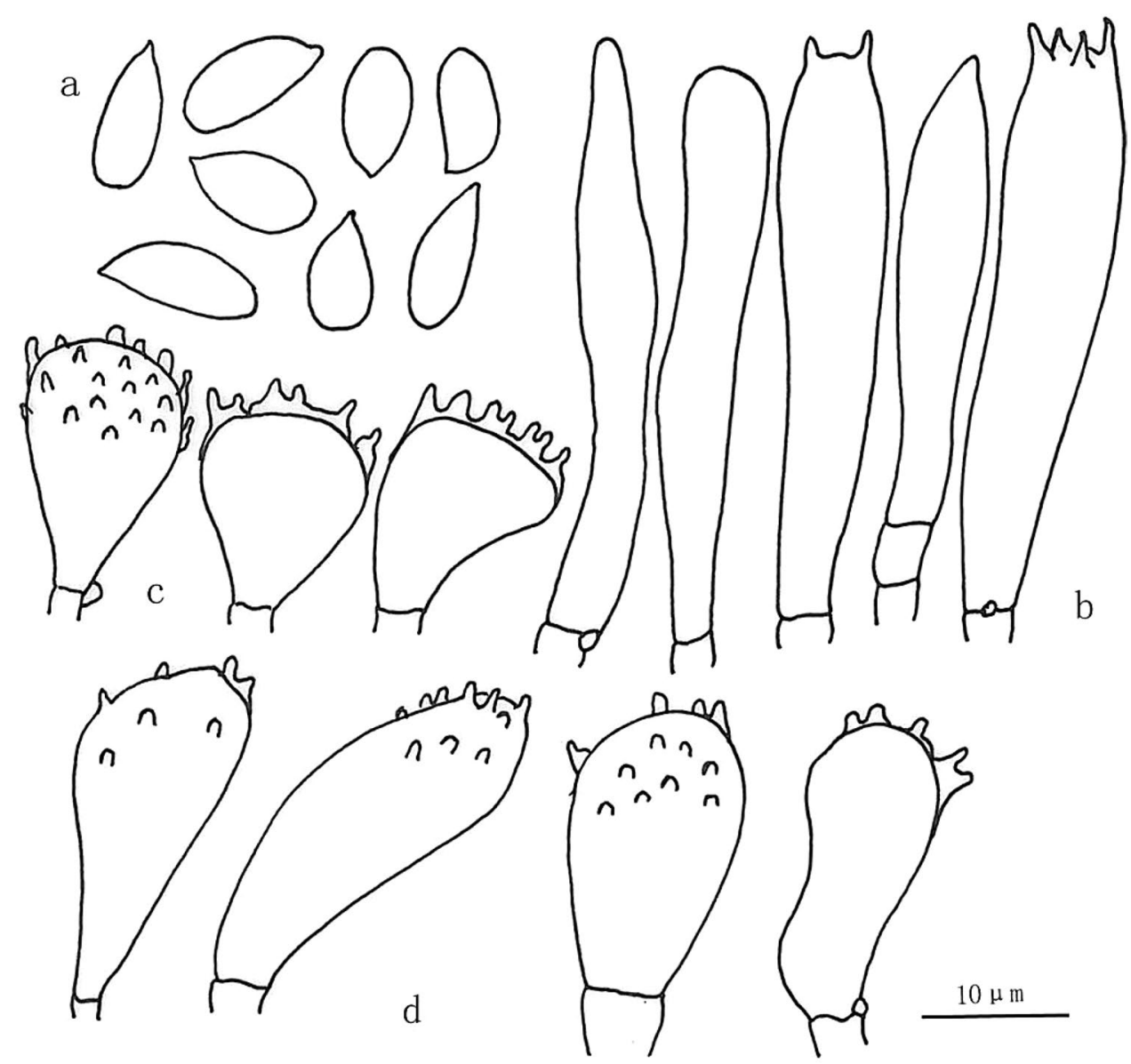




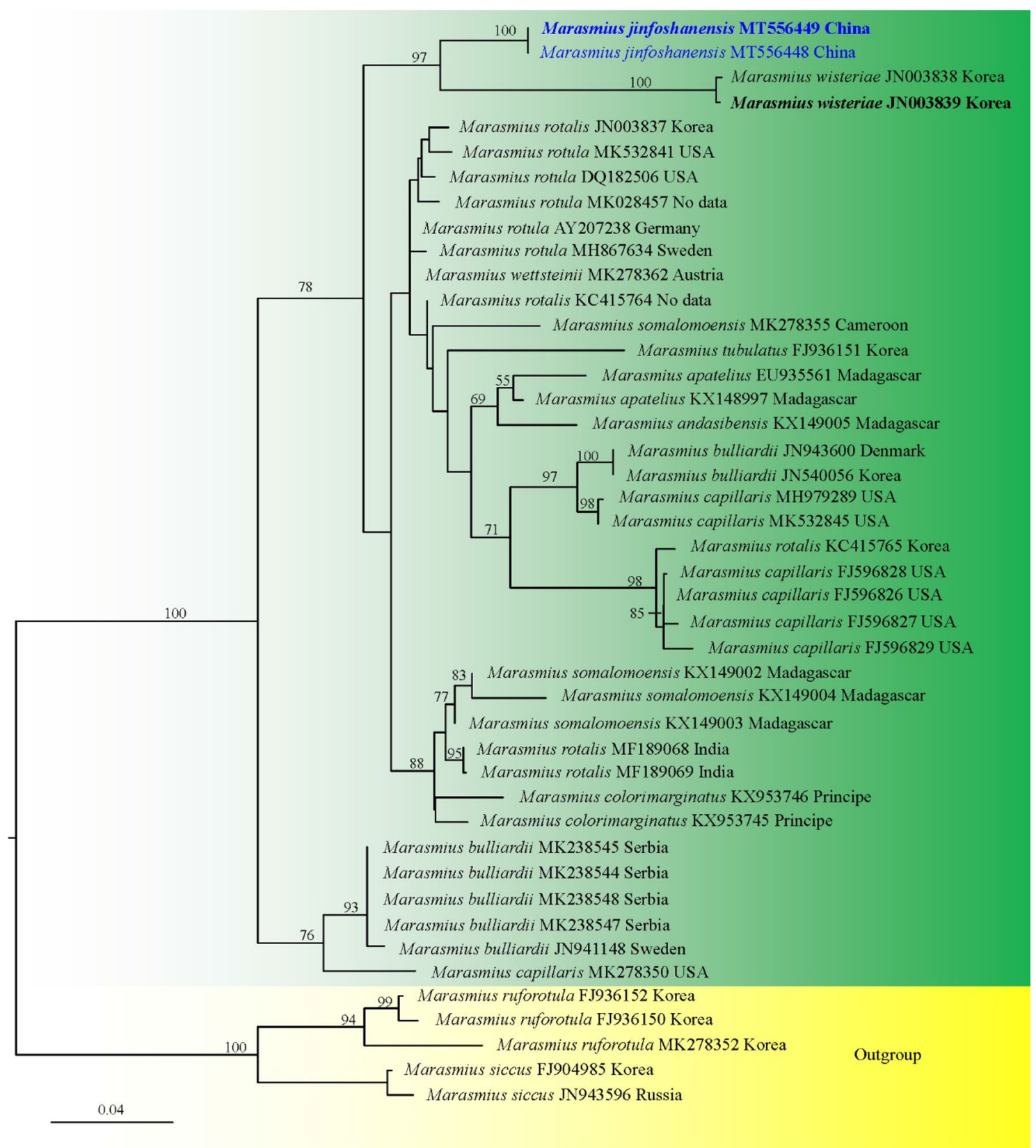

Fig. 190 Maximum likelihood tree inferred from ITS-LSU nrDNA, using RAxML GUI v. 2.0.0 (Edler et al. 2020), showing the placement of Marasmius jinfoshanensis. Support values ( $>50 \%$ with 1,000 replicates) are shown above branches. Marasmius ruforo-

Etymology: 'subtropicus' refers to the subtropical geographic region from where the taxon was collected.

\section{Holotype: CUH AM226}

Pileus 20-58 mm diam., convex when young, becoming broadly convex to plano-convex with a small umbo at maturity, margin often upturned, smooth to minutely pubescent, semi moist to dry, disc light brown (6D6) to brown (6E68 ), slightly rugulose, orange (5A6-7) to brownish yellow (5C7) towards middle, light yellow (1A5) to greyish yellow tula (FJ936150, FJ936152, MK278352) and M. siccus (FJ904985, JN943596) were chosen as outgroup. Ex-type strain is in bold and newly generated sequences are in blue

(1B6-7, 2-3B5, 4B6) towards extreme margin, on drying turns orange $(6 \mathrm{~A} 7,6 \mathrm{~B} 8)$ to deep orange $(6 \mathrm{~A} 8)$ or brownish orange (6C8) with brown (6E7-8) disc, translucent when young, becoming sulcate to often rimos at maturity, context thin, cream. Lamellae $2-3 \mathrm{~mm}$ broad, adnexed, distant to subdistant $(\mathrm{L}=19-22,1=4-5)$, white $(1 \mathrm{~A} 1)$ to cream, strongly intervenose, often forked from the middle, concolorous. Stipe $25-64 \times 2-4 \mathrm{~mm}$, central, cylindrical, noninsititious, equal with slightly broader at base (up to $6 \mathrm{~mm}$ ), 

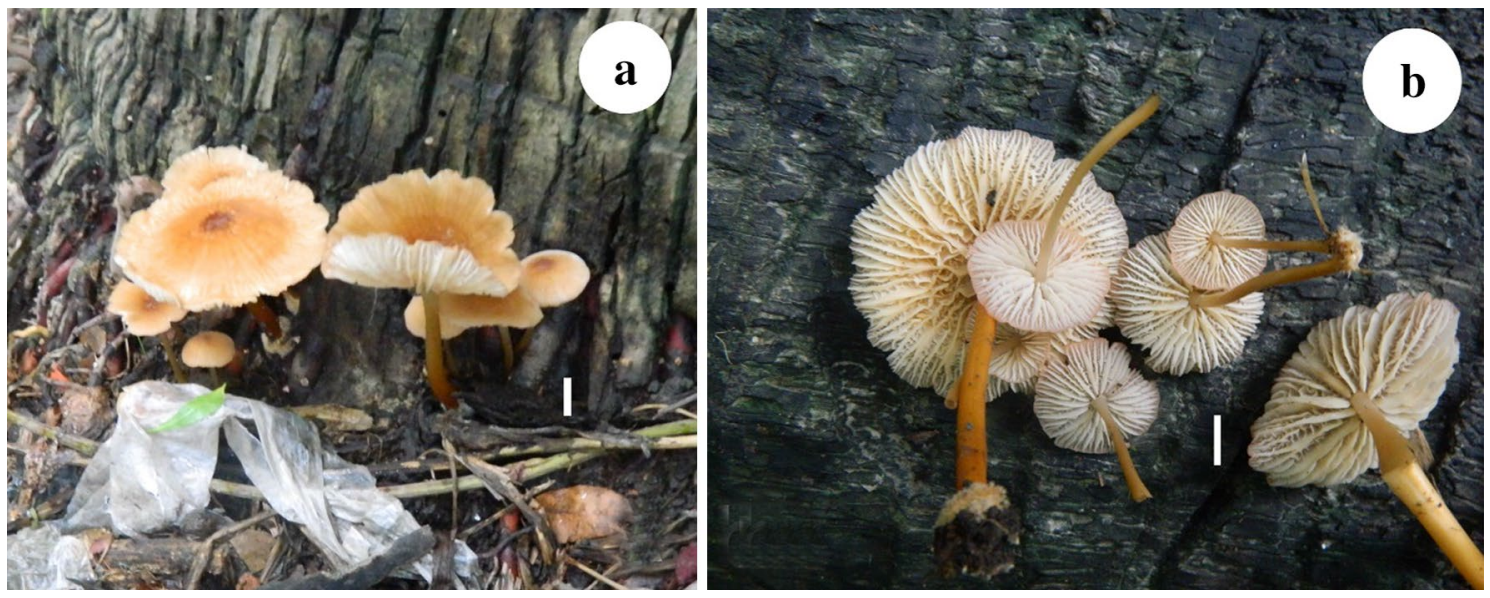

Fig. 191 Field pictures of the basidiomata. a, b Marasmius subtropicus (CUH AM226, holotype), habitats of basidiomata and lamellae showing intervenose nature. Scale bars: $\mathbf{a}, \mathbf{b}=10 \mathrm{~mm}$

Fig. 192 Marasmius subtropicus (CUH AM226, holotype). a Basidiospores. b basidia. c Siccus-type cheilocystidia. d Siccus-type pileipellis cells. Scale bars: $\mathbf{a}-\mathbf{d}=5 \mu \mathrm{m}$
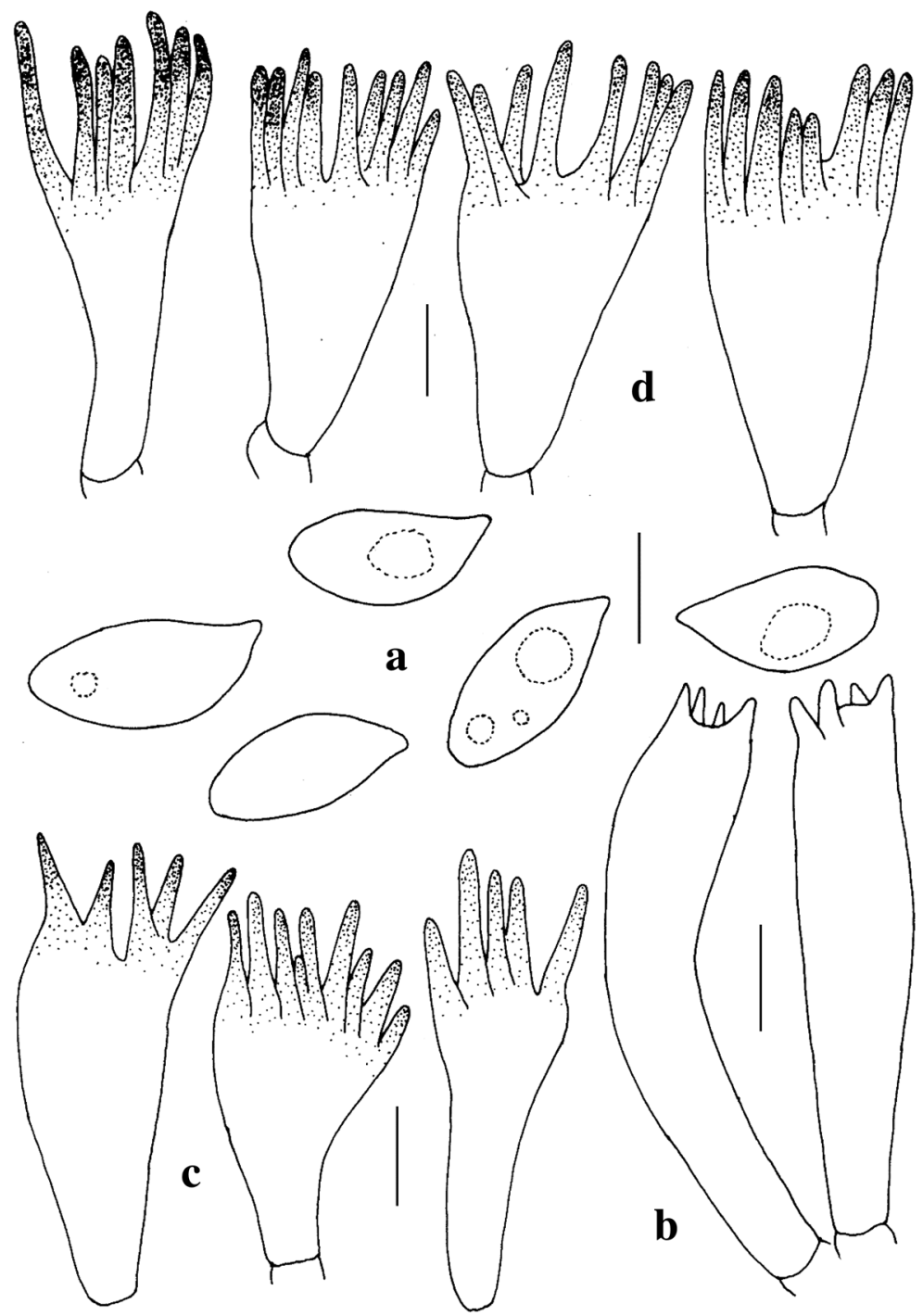
often cleft towards middle, brownish yellow (5C7) to orange or deep orange (6A8) with paler to cream towards apex, glabrous, hollow, basal mycelia well developed, orange. Odour and taste indistinctive. Basidiospores (7-)9-10.5(-11.5) $\times$ (4-)6-7(-7.2) $\mu \mathrm{m}(\bar{x}=10 \times 6.5 \mu \mathrm{m}, \mathrm{n}=30), \mathrm{Q}=1.2-1.7 \mu \mathrm{m}$ $(\bar{x}=1.5 \mu \mathrm{m}, \mathrm{n}=30)$, ellipsoid, hyaline, inamyloid, thinwalled. Basidia $25-29 \times 5-6.5(-7.5) \mu \mathrm{m}$, clavate, hyaline, thin-walled, 4-spored, sterigmata $2.5-4 \mu \mathrm{m}$ long. Basidioles $(21.5-) 28-31(-36) \times(5-) 6-6.5(-7.5) \mu \mathrm{m}$, cylindrical to clavate or fusoid, hyaline, thin-walled. Pleurocystidia absent. Lamellae-edge sterile, with well-developed cystidia. Cheilocystidia common, composed of Siccus-type broom cells; main body (10-)11-14(-18) $\times(3.5-) 4.5-6(-8$ .5) $\mu \mathrm{m}$, cylindrical to clavate or irregular in outline, often branched, hyaline, inamyloid, thin- to thick-walled; apical setulae (2.5-)6.5-7.5(-10.5) $\mu \mathrm{m}$ long, cylindrical, hyaline to pale yellow, inamyloid, acute to subacute, thick-walled. Pileipellis hymeniform, composed of Siccus-type of broom cell; main body (10.5-)14-15(-18) $\times(5.5-) 7-9(-11) \mu \mathrm{m}$, cylindrical to clavate, hyaline, inamyloid, thin- to thickwalled; apical setulae (3.5-)6-8(-10.5) $\mu \mathrm{m}$ long, cylindrical, yellowish to light brown, inamyloid, acute to subacute, thick-walled. Pileus trama hyphae interwoven, (4-)7-8(-11) $\mu \mathrm{m}$ broad, cylindrical to often inflated, hyaline, dextrinoid, thin-walled, non-gelatinous. Lamellae trama hyphae interwoven, 3.5-7(-7.5) $\mu \mathrm{m}$ broad, cylindrical, hyaline, dextrinoid, thin-walled, non-gelatinous. Stipitipellis hyphae 3.5-4.5(-5.5) $\mu \mathrm{m}$ broad, parallel to subparallel, cylindrical, thin-walled, pale yellowish in $\mathrm{KOH}$, nongelatinous. Stipe trama hyphae 7-8(-9.5) $\mu \mathrm{m}$ broad, parallel, cylindrical, hyaline, dextrinoid, thin- to moderately thick-walled, nongelatinous. Caulocystidia absent. Clamp-connections present in all the tissues.

Material examined: INDIA, West Bengal, South-24-Parganas district, Lakshmikantapur, 22 $2^{\circ} 06^{\prime} 35.9^{\prime \prime} \mathrm{N} 88^{\circ} 19^{\prime} 12.7^{\prime \prime}$ E, 6 msl, among decomposed leaf litter under Cocos nucifera tree, 26 June 2015, J. Sarkar (CUH AM226, holotype); South-24-Parganas district, Baruipur, 22 $21^{\prime} 35.3^{\prime \prime} \mathrm{N}$ $88^{\circ} 27^{\prime} 13.6^{\prime \prime} \mathrm{E}, 9 \mathrm{msl}$, among decomposed leaf litter, 23 June 2016, A.K. Dutta (CUH AM706).

GenBank numbers: ITS = MF189061, MF189062, $\mathrm{LSU}=\mathrm{MT254998}$, MT255000.

Notes: Distinctive feature of the specimen includes a medium to large, convex to plano-convex pileus coloured orange to brownish yellow with light brown to brown towards central small umbo, distant to subdistant, strongly intervenose, white lamellae, a central, non-insititious, glabrous, brownish yellow to orange or deep orange stipe with well-developed basal mycelia coloured orange, ellipsoid basidiospores with a mean of $10 \times 6.5 \mu \mathrm{m}(\mathrm{Q}$ mean 1.5$)$, presence of Siccus-type cheilocystidia with apical setulae measuring 2.5-10.5 $\mu \mathrm{m}$, and absence of pleurocystidia and caulocystidia. All these combination features undoubtedly place the present taxon to ser. Leonini of the sect. Sicci (Desjardin et al. 2000).

Based on the morphological features (Figs. 191 and 192) as well as phylogenetic analysis (Fig. 193), Marasmius hypochroides appears to be most close to M. subtropicus with 100\% MLBS, 1.00 BYPP support. However, $M$. hypochroides primarily differs from $M$. subtropicus by its pileus-coloured brown (Pegler 1986) or brownish orange to yellowish brown (Wannathes et al. 2009), lesser number of lamellae (12-16, Wannathes et al. 2009), and yellowish brown to reddish brown coloured stipe. Among other similar taxa belonging to the same section: Marasmius cladophyllus, originally described from Brazil, has smaller pileus (up to $20 \mathrm{~mm}$ diam.), adnate, narrow, reticulately interveined or poroid lamellae that possess lamellulae of two lengths, purplish brown stipe, and smaller spores with a mean of $9.2 \times 3 \mu \mathrm{m}$ (Pegler 1983; Singer 1976). Marasmius submarginatus Singer primarily differs by brownish orange pileus (Desjardin 1989), lesser number of lamellae (13-15), white coloured basal mycelium and less wide basidiospores (3.5-5 $\mu \mathrm{m}$, Singer 1989). The Brazilian taxa, Marasmius asemiformis has a non-striate pileus, crowded lamellae coloured white, chestnut to brown coloured stipe that is entirely pubescent, less broad basidiospores $(8-10 \times 3.2-3.7 \mu \mathrm{m})$, and presence of caulocystidia (Singer 1989). The Malaysian taxa, M. angustilamellatus (Tan et al. 2009), has yellowish brown to light brown pileus with blackish center, much crowded lamellae (24-40) coloured light brown, and basidiospores that is less broad (3.5-4.5 $\mu \mathrm{m}$ broad). Marasmius hygrocybiformis, described from China (Deng et al. 2012), has reddish orange to orange red pileus, adnate lamellae with lesser number of lamellulae ( $\mathrm{L}=12-16,1=1-2)$, greyish green coloured stipe, presence of pleurocystidia, pleurosetae, and cheilosetae.

Among previously reported taxa from India: Marasmius rhizophilus primarily differs by its much smaller pilei (10-13 mm diam.) coloured cream (Saccardo 1887; Patil 1978). Marasmius floriceps reported from Tamil Nadu (Natarajan and Manjula 1982), primarily differs by its brown to light brown pileus surface, rare occurrence of the intervenose lamellae, and smaller pileipellis Siccus-type cells measuring 6.6-8.8 $\times 3.3-6.6 \mu \mathrm{m}$.

\section{Mycenaceae Overeem}

Notes: Mycenaceae belongs to the order Agaricales. Members of this family are saprotrophic, distributed worldwide and are found in almost all ecological zones (Cannon and Kirk 2007). Currently, 19 genera and more than 1700 species are included in this family (Kirk et al. 2008; Species Fungorum 2021). We follow the treatment and updated accounts of Mycenaceae in Moncalvo et al. (2002) and Matheny et al. (2006). Based on morphological 


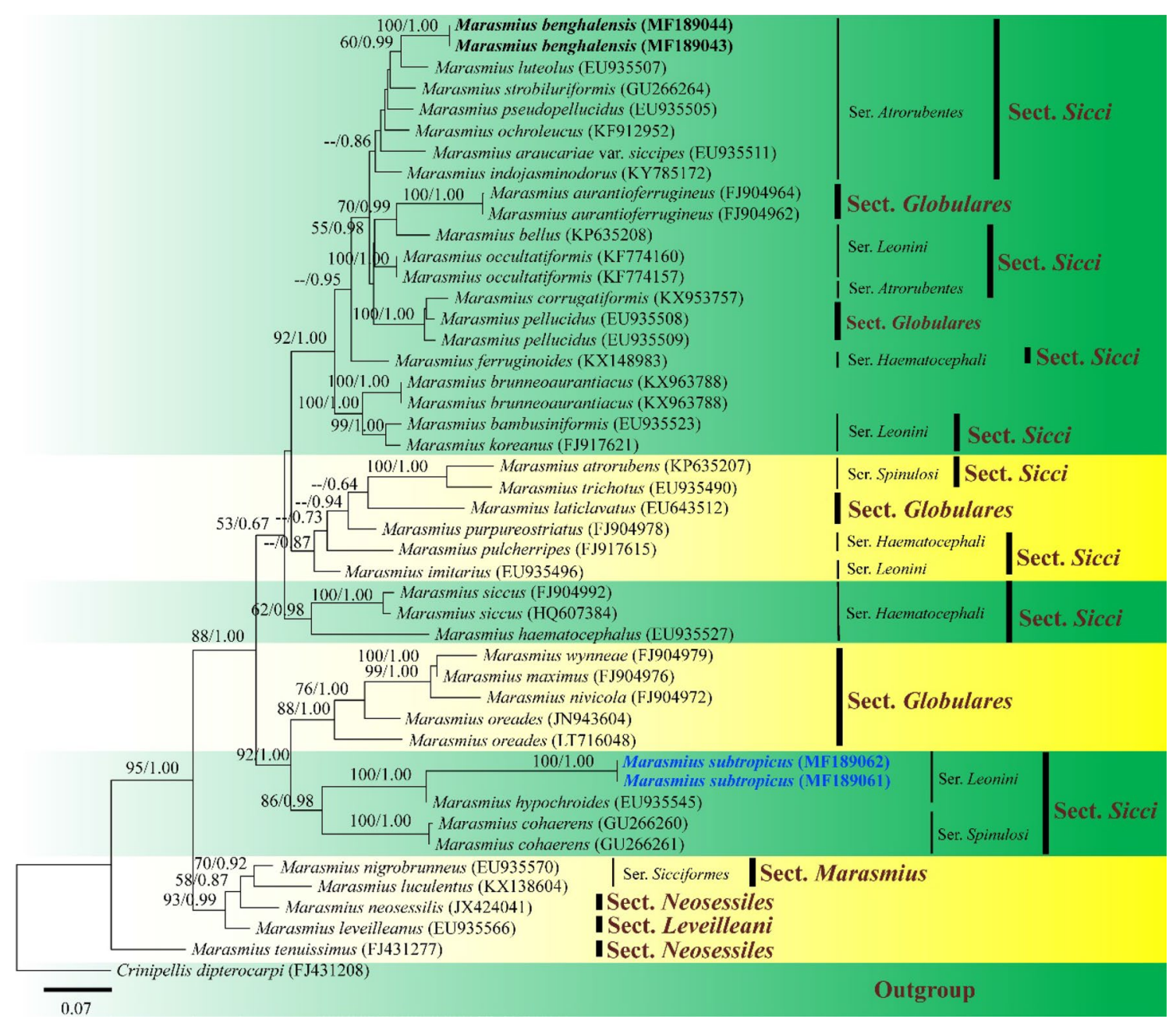

Fig. 193 Phylogram generated from maximum likelihood (ML) analysis based on nrDNA ITS sequence data of Marasmius species using best-fit model $(\mathrm{HKY}+\mathrm{G})$ with Bayesian Information Criterion (BIC) value of 14371.193718. Related sequences are mostly taken from Wannathes et al. (2009) and Antonín et al. (2012). Thirty-six taxa of Marasmius and one outgroup taxon are included in the analyses comprising 742 characters after alignment. Crinipellis dipterocarpi is used as the outgroup taxon following Wannathes et al. (2009). Bayesian analysis (BA) reached a standard deviation of split frequencies of 0.004984 after $10^{6}$ generations and produced 11210 credible set of trees after discarding initial 3,792 trees as burnin phase. Tree

characteristics and phylogenetic analysis of ITS sequence data, a novel species Cruentomycena uttarakhandina is introduced.

Cruentomycena R.H. Petersen, Kovalenko \& O.V. Morozova

Notes: Cruentomycena is established by Petersen et al. (2008). The genus is characterized by mycenoid or marasminoid basidiomata, viscid blood red pileus, deccurent lamellae, presence of cheilocystidia and elongated basidiospores (Petersen et al. 2008). Cruentomycena currently comprises topology of the ML analysis is similar to the BA. The best RAxML tree with a final likelihood values of 6870.939953 is presented. The matrix had 483 distinct alignment patterns, with $17.46 \%$ proportion of gaps and completely undetermined characters. Estimated base frequencies were as follows: $\mathrm{A}=0.2461, \mathrm{C}=0.2043, \mathrm{G}=0.2006$, $\mathrm{T}=0.3490$; gamma distribution shape parameter $=0.4030$. ML Bootstrap values (MLBS) equal or greater than $50 \%$ (on the left of ' $\%$ ') and posterior probabilities (BYPP) values equal or greater than 0.50 (on the right of ' $/$ ') from BA are labeled above or below the nodes. The newly generated sequences are in blue bold. Representative sections and series of the Marasmius taxa are marked in the right side

three species namely, C. kedrovayae, $C$. orientalis and $C$. viscidocruenta (Petersen et al. 2008; Terashima et al. 2016; Species Fungorum 2021). In this study, a new species $\mathrm{Cru}$ entomycena uttarakhandina is introduced based on evidence of morphology and phylogenetic placement (Figs. 194 and 195).

Cruentomycena uttarakhandina U. Singh \& R.P. Bhatt, sp. nov.

Index Fungorum number: IF557839; Facesoffungi number: FoF 07738; Fig. 194 

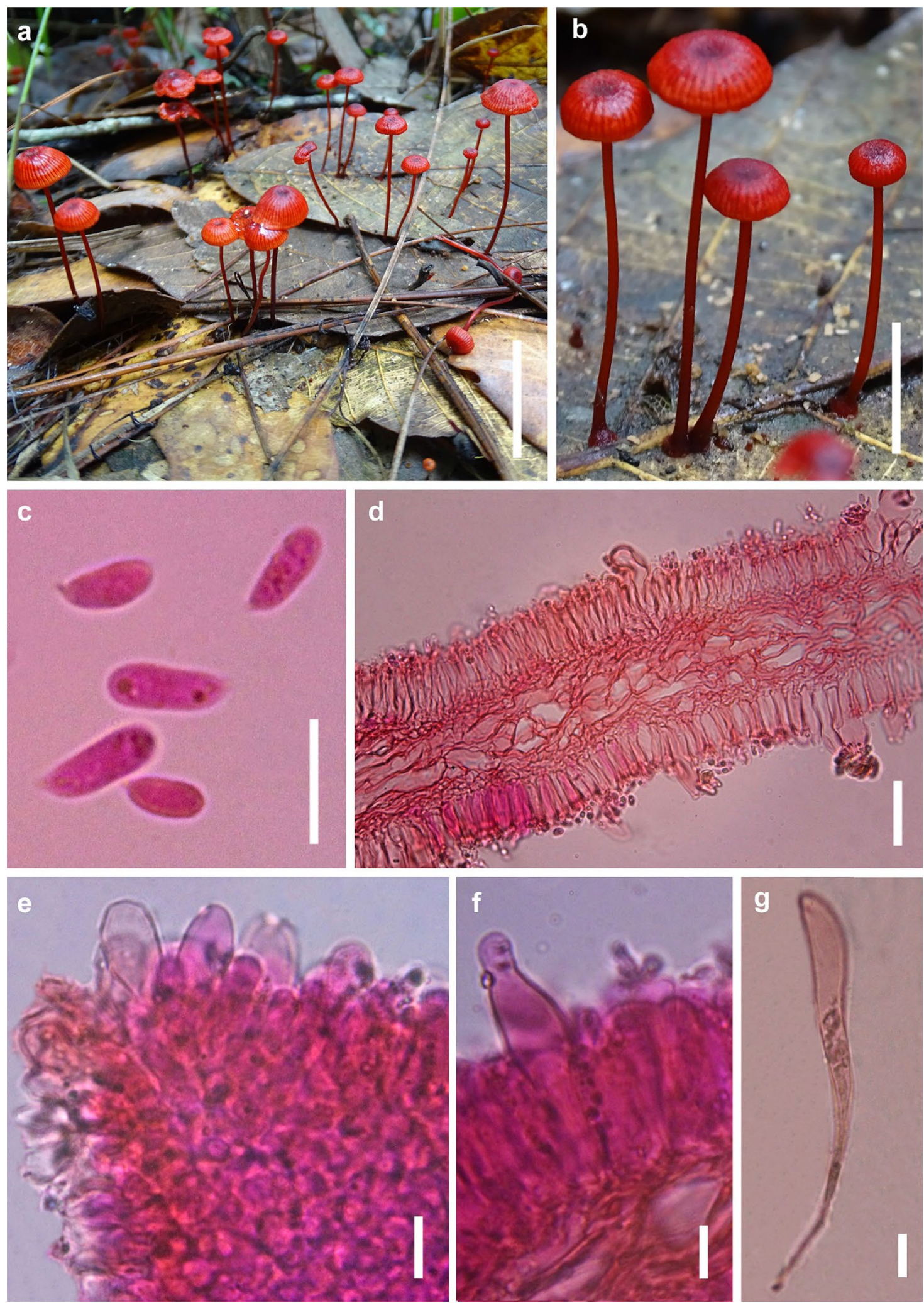

Fig. 194 Cruentomycena uttarakhandina (US 1510, holotype). a Habitat. b Basidiomata. c Basidiospores d Transverse section through hymenium showing basidia, pleurocystidia and lamellar

trama. e Cheilocystidia. f Pleurocystidia. g Caulocystidia. Scale bars: $\mathbf{a}=20 \mathrm{~mm}, \mathbf{b}=10 \mathrm{~mm}, \mathbf{c}, \mathbf{e}-\mathbf{g}=10 \mu \mathrm{m}, \mathbf{d}=25 \mu \mathrm{m}$ 


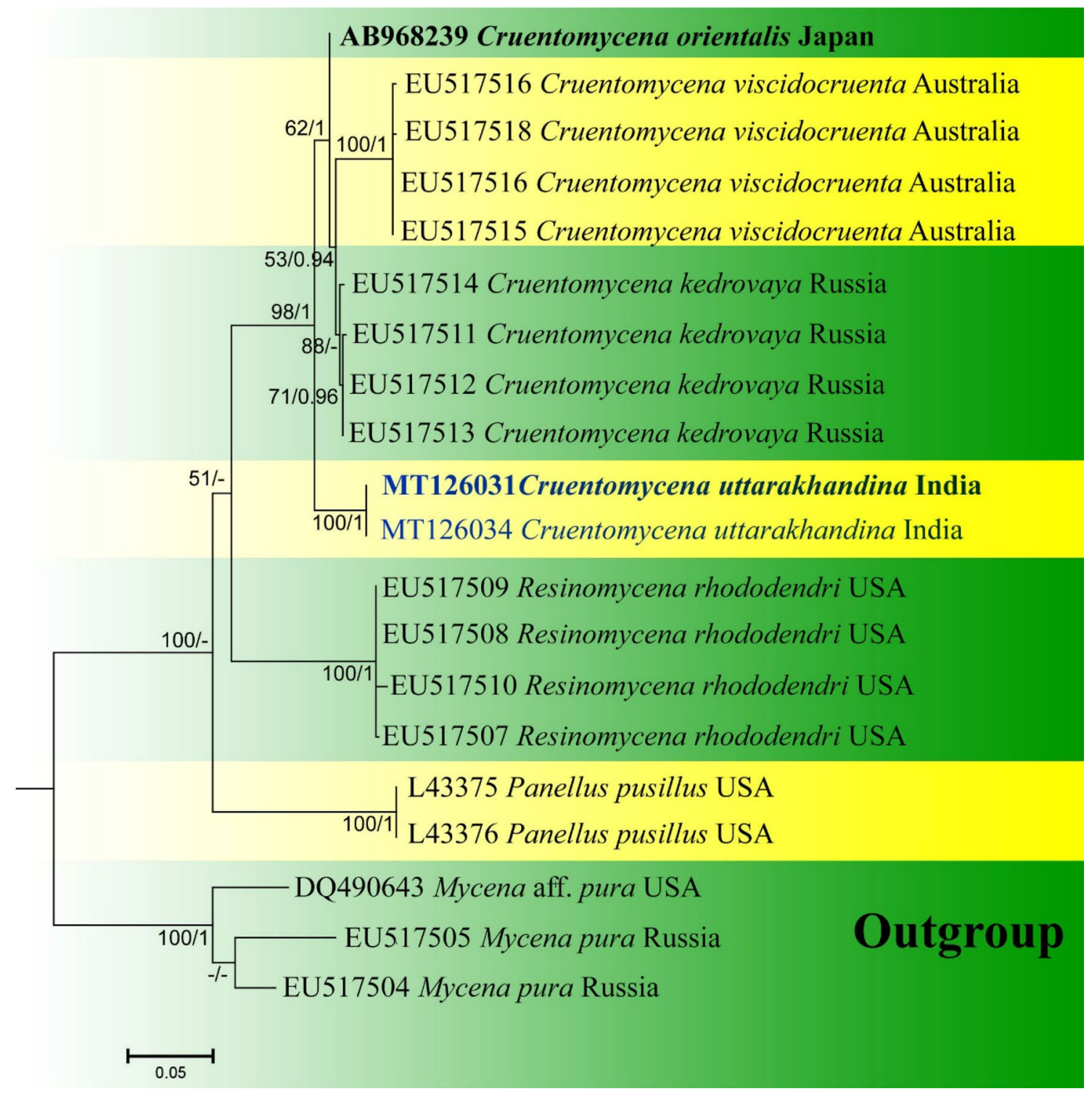

Fig. 195 Phylogenetic analyses were based on data set of nrITS sequences. Reference sequences were selected from relevant literature (Petersen et al. 2008), BLAST searches (Altschul et al. 1997) and data retrieved from GenBank (Clark et al. 2016). Species of Mycena are selected as the outgroup taxa (Petersen et al. 2008). Multiple sequence alignment was performed using MAFFT v.7 (Katoh and Standley 2013). Alignments were manually edited in BioEdit v 7.2.5 (Hall 1999). To change the multiple alignment format, Alignment Transformation Environment (ALTER) was used (Glez-Peña et al. 2010). Maximum Likelihood analysis was performed with the programme RAxML GUI 1.5 (Silvestro and Michalak 2012). One thousand bootstrap replicates were analysed to obtain nodal support values. Bayesian inference was computed independently twice in

Etymology: Named after Uttarakhand, from where this mushroom was collected.

Holotype: CAL 1800

Basidiomata small-sized, mycenoid, 16-42 ×2-10 mm. Pileus $2-10 \mathrm{~mm}$ in diam., convex, centrally depressed,
MrBayes v.3.2.2 (Ronquist et al. 2012), under different models. The best-fit substitution model of nucleotide evolution was carried out in MrModeltest 3.7 (Posada and Crandall 1998). Bayesian posterior probabilities (BYPP) were calculated in two simultaneous runs with Markov chain Monte Carlo (MCMC) algorithm (Larget and Simon 1999). Markov chains were run for 10 million generations, saving a tree every 100th generation. Default settings in MrBayes were used for the incremental heating scheme for the chains ( 3 heated and 1 cold chain), unconstrained branch length [unconstrained: exponential (10.0)] and uniformative topology (uniform) priors. The first $25 \%$ of trees was discarded as burnin (Hall 2004). Ex-type strain is in bold and newly generated sequences are in blue

surface viscid to glutinous when moist, maddor red (9A7) to vivid red or cinnabar red (9A8), smooth; margin entire, incurved to decurved, sulcate, concolorous; pileus context concolorous, fleshy. Lamellae distant, sub-decurrent, concolorous to pileus, with lamellulae in 4 series of different 
lengths; lamellar edges slightly lighter than lamellar face, entire. Stipe $14-40 \times 0.5-1 \mathrm{~mm}$, central, curved, equal to slightly tapered at base, slimy, concolorous to pileus, darker when handling, unchanged after bruising; stipe context concolorous, fibrous, hollow. Taste and odour indistinct. Spore print not obtained. Basidiospores 8-9.2-11 $\times 3-3.4-4 \mu \mathrm{m}$ $(\mathrm{Q}=2.25-2.69-3.14 \mu \mathrm{m}, \mathrm{n}=30)$, elongated, tapering asymmetrically toward hilar appendage, smooth, hyaline in $\mathrm{KOH}$, weakly amyloid. Basidia $20-30 \times 5-7 \mu \mathrm{m}$, sub-clavate to clavate, thin-walled, 2- to 4- spored; sterigmata up to $5 \mu \mathrm{m}$ long. Pleurocystidia $32-45 \times 9-14 \mu \mathrm{m}$, clavate to ventricose with rounded to acute apex, emergent up to $22 \mu \mathrm{m}$, arising from the trama. Cheilocystidia $22-30 \times 7-11 \mu \mathrm{m}$, clavate to broadly clavate. Lamellar trama sub-regular to irregular, of somewhat inflated cells (wide up to $48 \mu \mathrm{m}$ ). Pileipellis an ixocutis, composed of repent, cylindrical hyphae (wide up to $4 \mu \mathrm{m}$ ), rarely clamped; terminal hyphae $34-39 \times 3-4 \mu \mathrm{m}$, cylindrical with rounded apex; Pileus trama composed of inflated hyphae up to $34 \mu \mathrm{m}$ wide, thin-walled. Stipitipellis an ixocutis, with parallel hyphae, thin-walled, up to $6 \mu \mathrm{m}$ wide. Caulocystidia 52-79 $\times 7-10 \mu \mathrm{m}$, thin-walled, subcylindrical with tapered base.

Material examined: INDIA, Uttarakhand, Pauri district, Chaurikhal, growing solitary to gregarious, on leaf litters under Quercus sp. in mixed forest, $1981 \mathrm{msl}, \mathrm{N} 30^{\circ} 02.389^{\prime}$ E07903.406', 1 August 2017, U. Singh, US 1510 (CAL 1800, holotype); Phedkhal, 1913 msl, N3009.697' E07851.195', 24 August 2018, U. Singh, US 1667 (CAL 1801, paratype).

GenBank numbers: ITS = MT126031 (holotype), MT126034 (paratype).

Notes: Cruentomycena uttarakhandina can easily be characterized by its small size, red colour basidiomata with viscid to glutinous pileus, distant lamellae with lamellulae in 4 series of different lengths, thin stipe, elongated basidiospores, clavate to sub-clavate basidia, clavate to ventricose pleurocystidia, clavate to broadly clavate cheilocystidia, subregular to irregular lamellar trama, an ixocutis pileipellis without pilocystidia and an ixocutis stipitipellis with subcylindrical caulocystidia (Fig. 194).

Cruentomycena kedrovayae, C. viscidocruenta and C. orientalis may appear similar to C. uttarakhandina in terms of their small-size and red colour basidiomata. However, these three species all show morphological differences from C. uttarakhandina. Cruentomycena kedrovayae differs from present species by plane to subinfundibuliform pileus, thick (almost fold-like) lamellae, smaller stipe $(8-13 \times 0.5-0.8 \mathrm{~mm})$, ellipsoid and smaller basidiospores $(6-8(-9) \times 3.5-4 \mu \mathrm{m})$, and absence of pleurocystidia (Petersen et al. 2008). Cruentomycena viscidocruenta is distinguished from $C$. uttarakhandina by larger pileus (4-11(-14) $\mathrm{mm}$ broad), subdistant lamellae, smaller stipe (6-25 mm long, 0.4-1 mm broad), slightly smaller basidi-

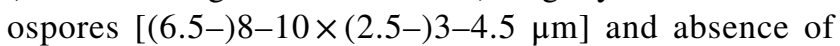

pleurocystidia (Petersen et al. 2008). Cruentomycena orientalis distinctively differs from $C$. uttarakhandina by its smaller pileus (4-8 $\mathrm{mm}$ diam.), sub-distant lamellae, smaller stipe (15-35 $\times 0.7-1 \mathrm{~mm})$, smaller and ellipsoid to oblong-ellipsoid basidiospores [(7.1-)7.9-9(-10.5) $\times(3.5-) 3.8-4.3(-5.1)$ $\mu \mathrm{m}]$ and absence of pleurocystidia (Terashima et al. 2016).

The ITS dataset consisting of 20 sequences (including our isolates: US 1510, represented by GenBank acc. no. MT126031 and US 1667, represented by GenBank acc. no. MT126034) of Cruentomycena, Resinomycena, Panellus and Mycena were analysed. Our isolated sequences were recovered as a distinct taxon (marked with blue font) in a strongly supported (98\% MLBS, 1.00 BYPP; Fig. 195) clade and clustered with other species of Cruentomycena. The combination of morphological features and phylogenetic analysis corroborates the proposed taxon Cruentomycena uttarakhandina as a new species being distinct from all the known taxa of Cruentomycena.

\section{Nidulariaceae Dumort.}

Notes: Species of Nidulariaceae are saprobic, growing on decomposing organic matter, on decaying wood and in soils enriched with wood chips or bark mulch; they have a widespread distribution in most ecological regions. Five genera, Crucibulum, Cyathus, Mycocalia, Nidula and Nidularia. These are distinguished from each other by differences in morphology and peridiole structures. In this study, phylogenetic analysis and comparison of DNA sequences reveal a new species, Cyathus uniperidiolus in Cyathus, Nidulariaceae.

\section{Cyathus Haller}

Notes: Cyathus was established by von Haller (1768) and is typified by Cyathus striatus (Huds.) Willd. The genus is characterized by having deeper or fluted, inverted bell-like fruiting bodies which is covered with shaggy or tomentose hairs on the outside. The inside peridium are filled with a number of small, hard lentil-shaped structures (peridioles) attached with flexible thread-like cords (funiculus). The peridioles bear basidiospores, that are hyaline, smooth or thick-walled and variable in shape and size (Lloyd 1906; Brodie 1975; Miller and Miller 1988).

Cyathus uniperidiolus P.N. Singh \& S.K. Singh, sp. nov.

Index Fungorum number: IF556766; Facesoffungi number: FoF 06586; Fig. 196

Etymology: specific epithet 'uniperidiolus' refers to single peridiole in basidiocarp.

Holotype: AMH10196

Colour codes follow: Methuen Handbook of Colour (Kornerup and Wanscher 1978).

Saprobic on dead bamboo grass of Muhlenbergia in a terrestrial habitat. Asexual morph Undetermined. Sexual 

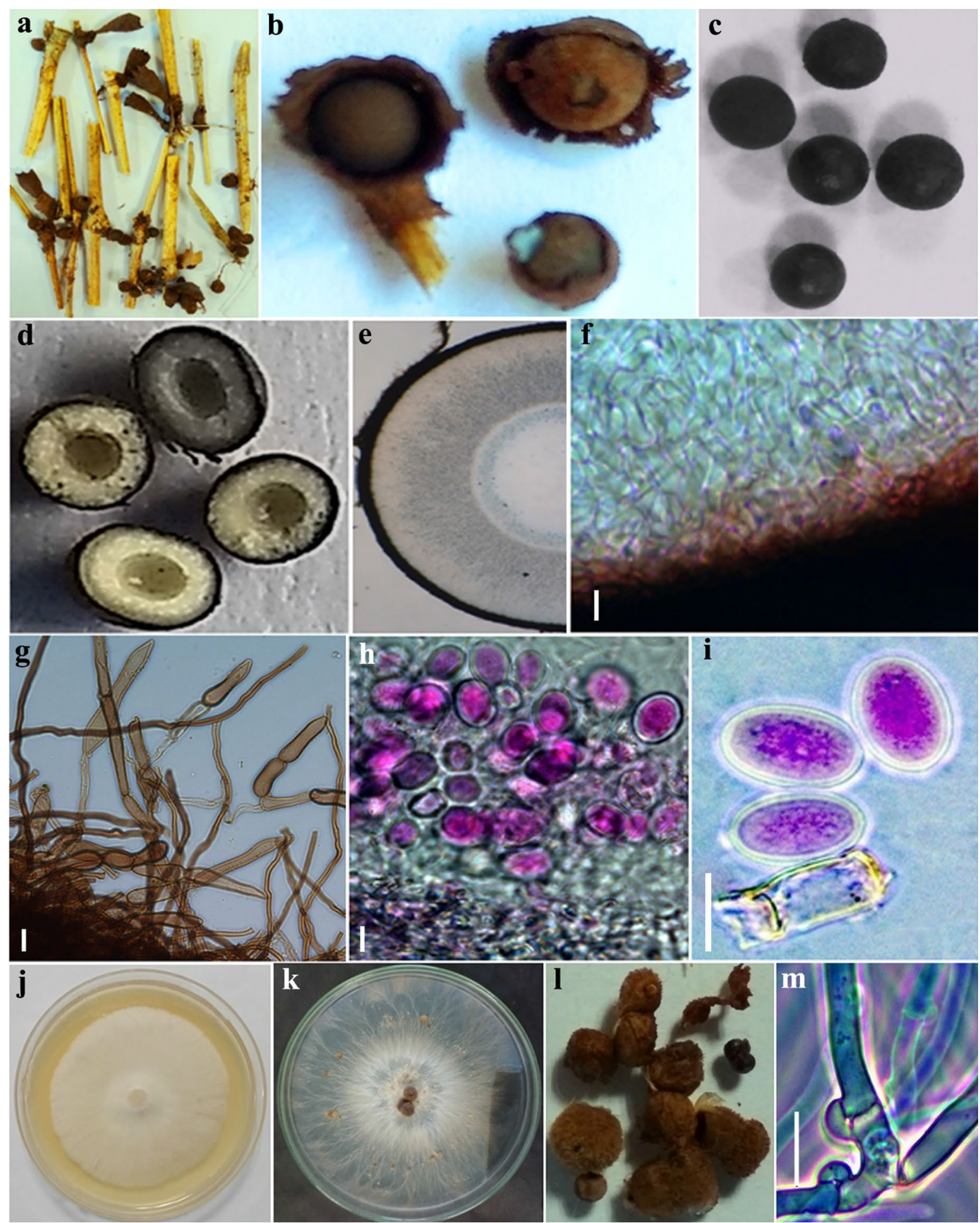

Fig. 196 Cyathus uniperidiolus (AMH-10196, holotype, NFCCI4697 ex-type culture). a Substrate (stem of Muhlenbergia sp. with attached basidiocarps). b Basidiocarps bearing single globose peridiole. $\mathbf{c}$ Globose peridioles with shiny surfaces. $\mathbf{d}$ Sections of peridioles showing emptied cavity inside. e Transverse section of basidiocarp showing different layers, innermost region showed emptied. f Transverse section of peridiole showing layer of cortex and sub-cortex (a part view). $\mathbf{g}$ Terminal and subterminal thick-walled encrusted loose and interwoven cells of basidiocarp. h Hymenial layer with hyphae and basidiospores. i Enlarged view of basidiospores. $\mathbf{j}$ Colony morphology on MEA (front view). k Colony with developing basidiocarps. I Harvested basidiocarps with velvety surfaces from culture. m Hyphae with clamps. Scale bars: $\mathbf{f}-\mathbf{i}, \mathbf{m}=10 \mu \mathrm{m}$ 
morph Basidiocarp (peridia) are of two types viz. globose to sub-globose, like small cannon balls, abundant and rarely fluted/deeper, sessile to short stalked, attached to substrate with brownish bulging pad, dark brown 2-12 $\mathrm{mm}$ high and $2-3.5 \mathrm{~mm}$ wide at mouth with serrate margin. Exterior wall of the peridium dark brown (6F8), with slightly velvety surfaces, non-plicate. Interior wall of the peridium, dark brown (6F8), nonplicate, smooth walled. Peridial wall up to $78 \mu \mathrm{m}$ thick, made up of terminal and subterminal thick and thin encrusted cells of peridial hairs, 2.7-7.15 $\mu \mathrm{m}$ wide. Peridioles, produced singly in each basidiocarp, sessile (funicular cord hard to observe), globose, resembling small cannonballs, hard, smooth, black with shiny surfaces, 2-2.5 mm diam. Transverse section of peridiole showed four layered: cortex, subcortex, hymenium and innermost layer is emptied. Cortex thick and black, compact, hard to observe the cells, up to $103.5 \mu \mathrm{m}$ thick. Subcortex layer is made up of compact pseudoparenchymatous cells, light olivaceous to subhyaline, up to $642.7 \mu \mathrm{m}$ thick. Hymenium (fertile layer) up to $275.9 \mu \mathrm{m}$ thick composed of hyaline hyphae and spore mass, the fourth inner layer is emptied as in coconut. Basidiopores broadly ellipsoid to ellipsoid-elongate, rarely oval to sub-globose, thick and smooth walled, hyaline, amyloid, $14.2-28.7 \times 11.7-23.7 \mu \mathrm{m}(\bar{x}=21.8 \times 16 \mu \mathrm{m}, \mathrm{n}=30)$.

Culture characteristics: on MEA (Malt Extract Agar), fast growing, floccose, white (1A1), $61 \times 61 \mu \mathrm{m}$ diam. in 8 days at $25^{\circ} \mathrm{C}$, margin irregular, reverse pale yellow (3A3). Exudates produced in the form of droplets, yellow. Hyphae septate, branched, clamped, smooth walled, sub hyaline to light olivaceous, $2.1-7 \mu \mathrm{m}$ wide. Globose to sub-globose yellowish brown (5E8) basidiocarps produced abundantly which bears single globose black peridiole in each basidiocarp which was entirely covered with thin velvety peridial wall.

Material examined: INDIA, Maharashtra, Pune District, dead stem of Muhlenbergia sp (Poaceae), 30 August 2017, P.N. Singh (AMH 10196, holotype), ex-type living culture (NFCCI 4697), National Fungal Culture Collection of India (WDCM932).

GenBank Numbers: ITS $=$ MN398297, LSU $=$ MN398298.

Notes: The present taxon was isolated in pure cultures from the basidiocarp associated with dead stem of
Muhlenbergia sp. (Bamboo grass). The macro and micromorphological characters such as basidiocarp and peridioles, multi-layered peridiolar wall, and globose blackish peridioles, broadly ellipsoid to ellipsoid-elongate, hyaline, smooth-walled basidiospores (Fig. 196), place this taxon under Cyathus (von Haller 1768; Baseia and Milanez 2001). Present species is distinct from its allied taxa in having unique shape of peridia and peridioles i.e., globose, small cannon ball-like, produced abundantly, deeper or fluted, are rarely produced. These characteristic features clearly separate present taxon from allied Cythus spp., viz. C. albinus, C. badius, C. renweii, C. poeppigii, $C$. thindii and C. striatus (type species) (Table 5) (von Willdenow 1787; Tulasne and Tulasne 1844; Kobayasi 1937; Zhou et al. 2004; Cruz et al. 2014, 2018; Das et al. 2015; Accioly et al. 2018). As such these are the distinct and unique features of the proposed species. The other morphological features are also compared in Table 5. The phylogenetic analysis reveals that C. uniperidiolus forms a distinct sister clade with $C$. badius which is statistically supported (Fig. 197). From morphological data and sequence analyses it is evident that $C$. uniperidiolus is distinct from all other species in the genus. Therefore, Cyathus uniperidiolus is described and illustrated here as a new species.

\section{Omphalotaceae Bresinsky}

Notes: The latest update accounts of Omphalotaceae see Haelewaters et al. (2020).

\section{Marasmiellus Murrill}

Notes: Marasmiellus is widely distributed throughout tropical and subtropical areas (Wilson and Desjardin 2005; Kirk et al. 2008). This genus is saprotrophic and rarely grows on living trees. Marasmiellus was previously assigned to Tricholomataceae (Singer 1986) but was later transferred to the Marasmiaceae (Kirk et al. 2008). However, phylogenetic analysis of ITS and nrLSU showed that it belonged to the Omphalotaceae (Moncalvo et al. 2002; Wilson and Desjardin 2005). Marasmiellus is characterized by basidiomes that are marasmioid-collybioid, collybioid-mycenoid or sometimes pleurotoid. Basidiomes are mostly whitish or occasionally

Table 5 Morphological comparison of Cyathus uniperidiolus with some allied taxa

\begin{tabular}{|c|c|c|c|c|c|c|}
\hline $\begin{array}{l}\text { Morpho-logical } \\
\text { features }\end{array}$ & $\begin{array}{l}\text { C. albinus Accioly } \\
\text { et al. } 2018\end{array}$ & $\begin{array}{l}\text { C. badius Kob- } \\
\text { ayasi } 1937\end{array}$ & $\begin{array}{l}\text { C. renweii Zhou } \\
\text { et al. } 2004\end{array}$ & $\begin{array}{l}\text { C. poeppigii } \\
\text { Tulasne and } \\
\text { Tulasne } 1844\end{array}$ & $\begin{array}{l}\text { C. thindii Das et al. } \\
2015\end{array}$ & $\begin{array}{l}\text { C. uniperidi-olus } \\
\text { sp. nov., in this } \\
\text { study }\end{array}$ \\
\hline Basidiocarps & $\begin{array}{l}\text { 6-8.5 mm high, } \\
5-6.5 \mathrm{~mm} \text { wide }\end{array}$ & $\begin{array}{l}\text { 4.5-9 } \mathrm{mm} \text { high, } \\
4-6 \mathrm{~mm} \text { wide }\end{array}$ & $\begin{array}{l}\text { 8-10 mm high, } \\
5-6 \mathrm{~mm} \text { wide }\end{array}$ & $\begin{array}{l}\text { 6-8 mm high, } \\
4-6 \mathrm{~mm} \text { wide }\end{array}$ & $\begin{array}{l}7-9.5 \mathrm{~mm} \text { high, } \\
5-9 \mathrm{~mm} \text { wide }\end{array}$ & $\begin{array}{l}\text { 2-12 mm high, } \\
2-3.5 \mathrm{~mm} \text { wide }\end{array}$ \\
\hline Peridioles & $\begin{array}{l}2.1-2.6 \times 1.8 \\
-2.2 \mathrm{~mm}\end{array}$ & $2 \times 1.5-2 \mathrm{~mm}$ & $2 \mathrm{~mm}$ & $2-2.5 \mathrm{~mm}$ & $1.5-3 \mathrm{~mm}$ & $2-2.5 \mathrm{~mm}$ \\
\hline Basidiospores & $\begin{array}{l}14.8-20 \times 10.4- \\
14.3 \mu \mathrm{m}\end{array}$ & $14-20 \times 11-15 \mu \mathrm{m}$ & $\begin{array}{l}21-31 \times 10.5- \\
13 \mu \mathrm{m}\end{array}$ & $25-47 \times 15-24 \mu \mathrm{m}$ & $10-16 \times 7-10 \mu \mathrm{m}$ & $\begin{array}{l}14-29 \times 11.5- \\
24 \mu \mathrm{m}\end{array}$ \\
\hline
\end{tabular}




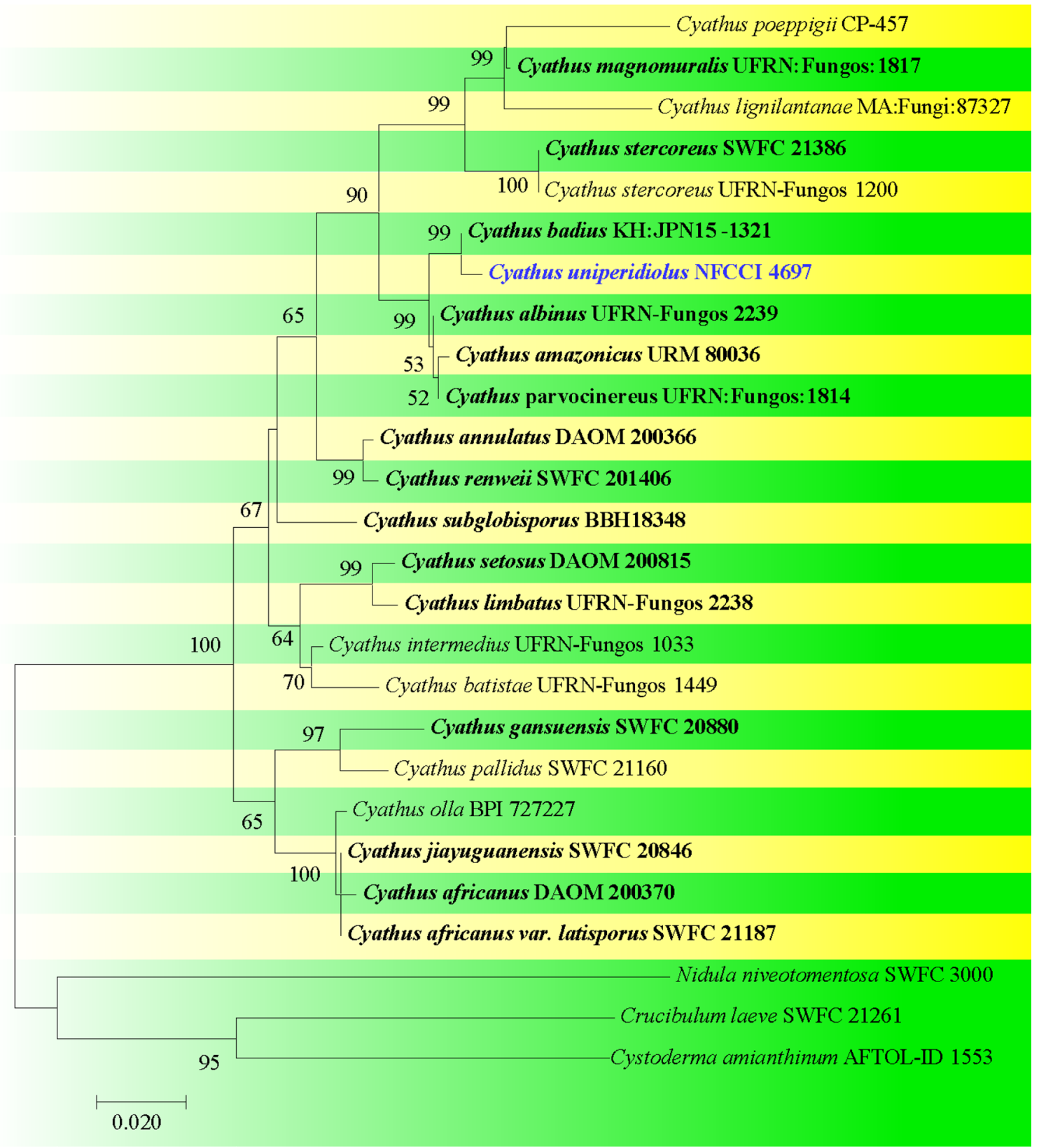

Fig. 197 Phylogram generated from maximum likelihood analysis for Cyathus uniperidiolus (NFCCI: 4697) using combined ITS and LSU sequence data based on the General Time Reversible model (Nei and Kumar 2000). A discrete Gamma distribution was used to model evolutionary rate differences among sites ( 5 categories $(+\mathrm{G}$, parameter $=0.2814)$ ). Bootstrap support values are indicated at the nodes

grayish, reddish brown or dark brown in colour with ellipsoid basidiospores and hyphae having clamp connections (Singer 1973; Moncalvo et al. 2002). There are more than 400 taxa listed in Species Fungorum (2021) for this genus.

Marasmiellus palmivorus (Sharples) Desjardin, Mycologia 97: 670 (2005) and values below 50\% are not shown. Phylogenetics analyses were conducted in MEGA7 (Kumar et al. 2016). Nidula niveotomentosa SWFC 3000, Crucibulum leave SWFC 21,261 and Cystoderma amianthinum AFTOL-ID 1553 were used as outgroup. Type specimens are in bold and newly sequence is in blue

Index Fungorum number: IF613706; Facesoffungi number: FoF 09970; Fig. 198

$\equiv$ Marasmius palmivorus Sharples, Malay. agric. Journal 16(nos 9-10): [1] (1928).

Pileus $10-25 \mathrm{~mm}$ in diam., hemispherical to convex, expanding to plano-convex, in age depressed to subumbilicate; margin initially smooth, soon striate to sulcate or 

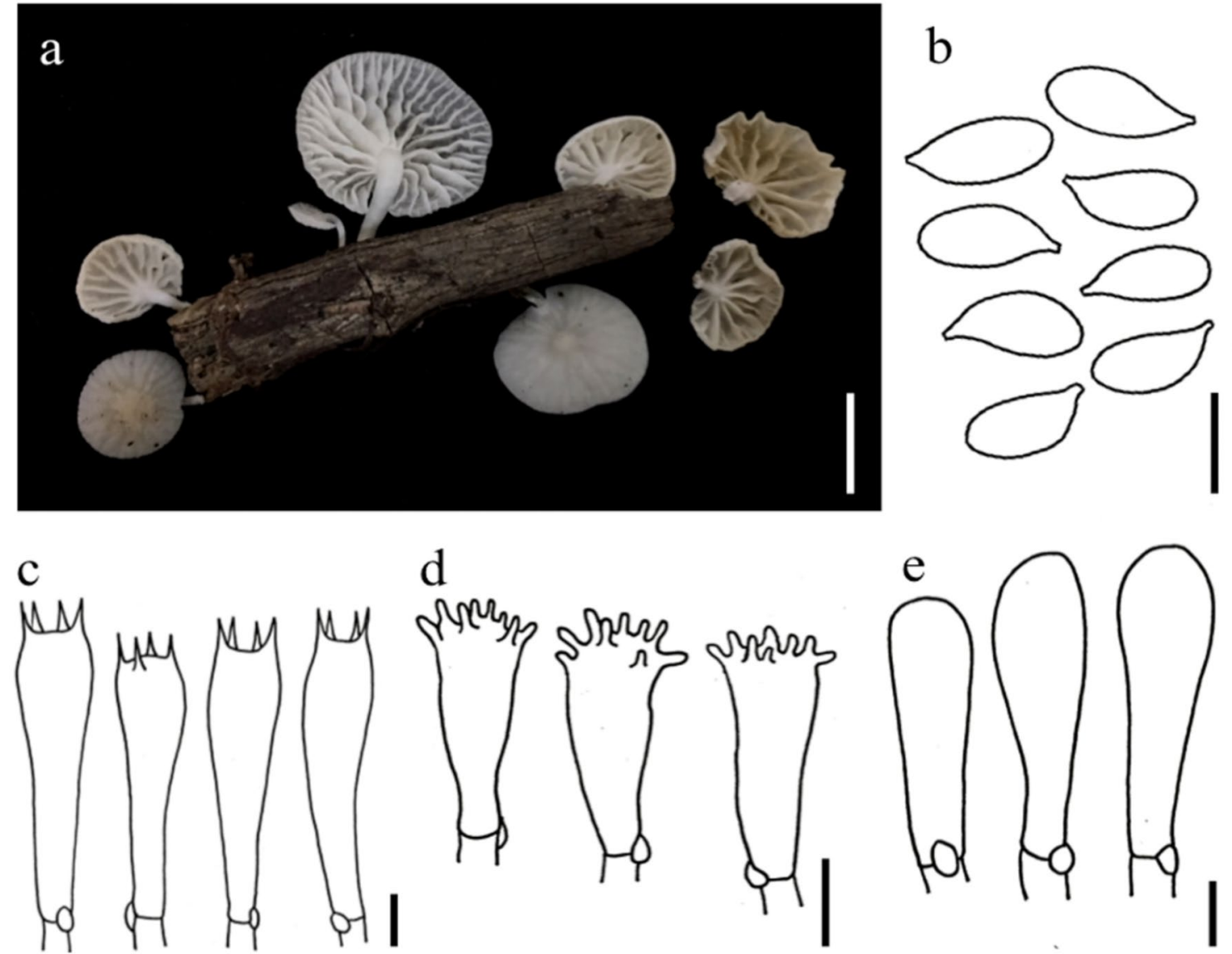

d
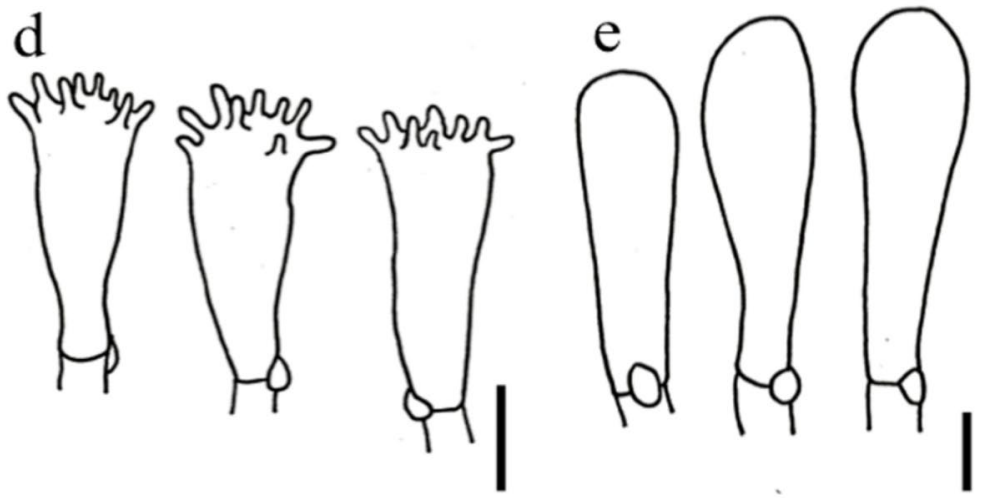

Fig. 198 Marasmiellus palmivorus (SDBR-CMU-NK076, new record). a Basidiomata. b Basidiospores. c Basidia. d Cheilocystidia. e Caulocystidia. Scale bars: $\mathbf{a}=1 \mathrm{~cm}, \mathbf{b}-\mathbf{e}=10 \mu \mathrm{m}$

rugulose-striate, sometimes wavy or cleft; surface dull, moist to dry, glabrous to felted or radially appressed-fibrillose, subhygrophanous; when young white (4A1) to pale yellow (4A2) or more commonly orange white (6A2), in age retaining pinkish white (7A2) or light orange (6A4) tones on the disc. Context up to $1 \mathrm{~mm}$ thick, soft, pliant, white (4A1). Lamellae horizontal, adnate, distant with 1-3 series of lamellulae, often intervenose in age, rarely forked, broad (2-4 mm), white (4A1), sometimes discoloring pinkish white (7A2) or light orange (6A4). Stipe $1.5-7 \times 1-1.5 \mathrm{~mm}$, central to eccentric, terete, cylindrical, base often enlarged, curved, solid, tough, subinsititious or arising from a pad of appressed white to pale orange mycelium; surface dull, dry, glabrous or minutely silky-pruinose, at first white overall, base darkening in age to greyish red (7B3), reddish orange (7B6-7B7) or reddish brown (8F5); often with fanlike white mycelium over substrate. Odour not distinctive. Basidiospores 8-12×4.5-6 $\mu \mathrm{m}, \mathrm{Q}=1.7-2.3, \mathrm{Q}_{\mathrm{m}}=1.95 \pm 0.1$, ellipsoid, smooth, hyaline, inamyloid, thin-walled. Basidia 20-34 $\times 6-10 \mu \mathrm{m}$, clavate, 2 to 4 -spored. Pleurocystidia absent. Cheilocystidia $17.5-24 \times 8-12 \mu \mathrm{m}$, clavate with numerous apical setulae, hyaline, inamyloid, thin-walled; setulae 1.5-3.5 $\times 1-3.2 \mu \mathrm{m}$, irregularly cylindrical or knoblike, sometimes forked, obtuse to acute, hyaline. Pileipellis a cutis of loosely interwoven, repent hyphae, not a hymeniform layer, not a Rameales-structure; hyphae 4-12 $\mu \mathrm{m}$ in diameter, cylindrical, non-diverticulate, smooth or incrusted with annular to helical hyaline or yellow pigments; walls hyaline, inamyloid, non-dextrinoid, non-gelatinous; thin-walled; terminal cells undifferentiated, cylindrical. Pileus trama interwoven, lamellar trama regular; hyphae 3-15 $\mu \mathrm{m}$ in diameter, cylindrical or inflated, smooth, hyaline, inamyloid, non-dextrinoid, non-gelatinous, thin-walled or with walls up to $1 \mu \mathrm{m}$ thick. Stipe trama 3-6.5 $\mu \mathrm{m}$ in diameter, cylindrical, parallel, nondiverticulate, smooth, hyaline, inamyloid, non-dextrinoid, non-gelatinous, thin-walled; medullary hyphae similar but many fusoid, up to $20 \mu \mathrm{m}$ in diameter, some with walls up to $1 \mu \mathrm{m}$ thick. Caulocystidia scattered, $25-30 \times 5-8.5 \mu \mathrm{m}$, cylindrical to clavate or fusoid, non-diverticulate, hyaline, thin-walled. Clamp connections present in all tissue type.

Habitat: scattered on rotten coconut palm wood or fruits, on leaves and stems of banana and Lagerstroemia tree, or on 
undetermined woody debris in coastal coconut groves and banana-cacao groves.

Distribution: Known from Africa (São Tomé), India, Indonesia (Java), Malaysia, Taiwan, Thailand and USA (Hawaiian Islands) (Wilson and Desjardin 2005; Desjardin and Perry 2017; Dutta and Achaya 2018; Pham et al. 2019).

Material examined: THAILAND, Chiang Mai Province, Muang District, Chiang Mai University, $18^{\circ} 48^{\prime} 10^{\prime \prime} \mathrm{N}$ 98 57'23" E, $334 \mathrm{msl}$, 15 June 2018, on the rotten wood, J. Kumla and N. Suwannarach, SDBR-CMU-NK076 (new record).

GenBank numbers: $\quad$ ITS $=$ MW647877, $\mathrm{LSU}=\mathrm{MW647892.}$

Notes: Marasmiellus palmivorus is characterized by small to moderate-sized basidiomes with white or pink to orange striate, plano-convex pileus that fade to white with age and possess distant, occasionally intervenose white lamellae (Fig. 198). They also have a central to eccentric white stipe that becomes reddish orange to reddish brown at the base, ellipsoid basidiospores, clavate cheilocystidia with apical setulae, a cutis-type pileipellis of non-diverticulate hyphae, clavate and thin-walled caulocystidia. Marasmiellus palmivorus is similar to Collybia purpureogrisea, which was originally described as M. purpureogriseus. However, C. purpureogrisea differs by the presence of its non-striate pileus and has a longer stipe (up to $4 \mathrm{~cm}$ long). Basidiome is brownish-white, sub-close to crowded lamellae and displays the presence of a rameales structure in the pileipellis (Petch 1947; Manimohan and Leelavathy 1989). Phylogenetic analysis of the combined ITS and LSU regions indicates that our strain of M. palmivorus clusters with other strains in a strongly supported subclade (100\% MLBS, 1.00 BYPP; Fig. 199).

Psathyrellaceae Vilgalys, Moncalvo \& Redhead

Notes: Psathyrellaceae was proposed by Redhead et al. (2001) based on molecular phylogenetic studies which circumscribed by Psathyrella and Lacrymaria, together with related species in the polyphyletic genus Coprinus sensu lato, which were transferred to the genera Coprinellus, Coprinopsis or Parasola. Afterwards, several other genera were included in Psathyrellaceae and Kirk et al. (2008) consider that this family comprises 12 genera.

\section{Coprinellus P. Karst.}

Notes: Coprinellus, consists of approximately 85 described species, characterized by independent lineages in Psathyrellaceae (Redhead et al. 2001; Walther et al. 2005; Padamsee et al. 2008; Vašutová et al. 2008; Nagy et al. 2011, 2012, 2013; Örstadius et al. 2015; Hussain et al. 2018). Mushrooms belonging to Coprienllus are common saprotrophs and are divided into three major sections on the basis of veil anatomy and the presence or absence of pileocystidia. Section Domestici (Singer) D.J. Schaf. is characterized by the veil on the pileus in the form of floccose scales, fusiform or subglobose cells in the form of chains. Section Micacei (Fr.) D.J. Schaf., is represented by presence of veil remnants in the form of scattered, granulose flocks, consisting of globose cells. Section Setulosi (J. Lange) D.J. Schaf., featured by the presence or absence of the veil on pileus, but the pileus and stipe are covered with thin-walled pileocystidia and caulocystidia, respectively (Schafer 2010). However, Nagy et al. (2012) found that these sections were not entirely consistent with the molecular phylogeny, in particular because clades corresponding to sections, Micacei and Domestici, each included some setulosi species.

\section{Coprinellus punjabensis Usman \& Khalid sp. nov.}

Index Fungorum number: IF557381; Facesoffungi number: FoF 07848; Fig. 200

Etymology: Named after Punjab Province, where this fungus was collected.

\section{Holotype: LAH35321}

The most important features of Coprinellus punjabensis: Pileus cylindrical to campanulate, white greyish, plicate from center to margins. Basidiospores 7.3-9 $\times 4.3-5.2 \mu \mathrm{m}$ ellipsoid to amygdaliform, brown to blackish, germ pore central up to $1.2 \mu \mathrm{m}$, with truncate base. Veil elements globose to sub globose hyaline cells up to $45 \mu \mathrm{m}$. Macroscopic characters: Pileus 5-12 mm diam., cylindrical when young campanulate at maturity, white to light greyish $(2.5 \mathrm{Y}$ 7/4), surface pruinose, plicate toward margin, edges striate to plicate. Lamellae sinuate to uncinate, distant with 1-3 lamellulae, dark greyish-brown. Stipe $25-40 \mu \mathrm{m}$ in length, equal, central, light greyish and white near base, hollow, surface pruinose to pulverulent. Annulus absent. Odour and taste not recorded. Microscopic characters: Basidiospores (7-)7.3-9(-9.3) $\times(4-) 4.3-5.2(-5.6) \mu \mathrm{m},(\bar{x}=8 \times 4.8 \mu \mathrm{m}$, $\mathrm{n}=50), \mathrm{Q}=1.68$; in face view, ellipsoid, in side view, amygdaliform, dark brown to blackish (5YR 3/1), in $\mathrm{KOH,}$ smooth, thick-walled, apex truncate, germ-pore central, up to $1.2 \mu \mathrm{m}$ wide. Basidia $20-30 \times 5.8-8.4 \mu \mathrm{m}$, cylindrical to clavate, hyaline with $2-4$ strigmata up to $6 \mu \mathrm{m}$. Cheilocystidia and pleurocystidia absent. Pileipellis consist of irregular epithelium, arranged by globose to sub-globose cells, $8-30 \times 10-25 \mu \mathrm{m}$, hyaline and smooth. Stipitipellis 3-10 $\mu \mathrm{m}$ diam., regular, septate and clamp connections not observed. Veil elements up to $45 \mu \mathrm{m}$, globose to sub-globose, smooth and hyaline.

Habitat and distribution: Solitary to gregarious on moist soil, rich in leaf litter under trees of Acacia modesta Wall.

Material examined: PAKISTAN, Punjab, Pabbi Forest Park, (32॰49'52.94" N 7350'12.04" E,) 286 msl, 11 August 2016, M. Usman and A.N. Khalid, MU30 (LAH35321, holotype). PAKISTAN, Punjab, Pabbi Forest Park, (32 ${ }^{\circ} 49^{\prime} 51.30^{\prime \prime}$ 


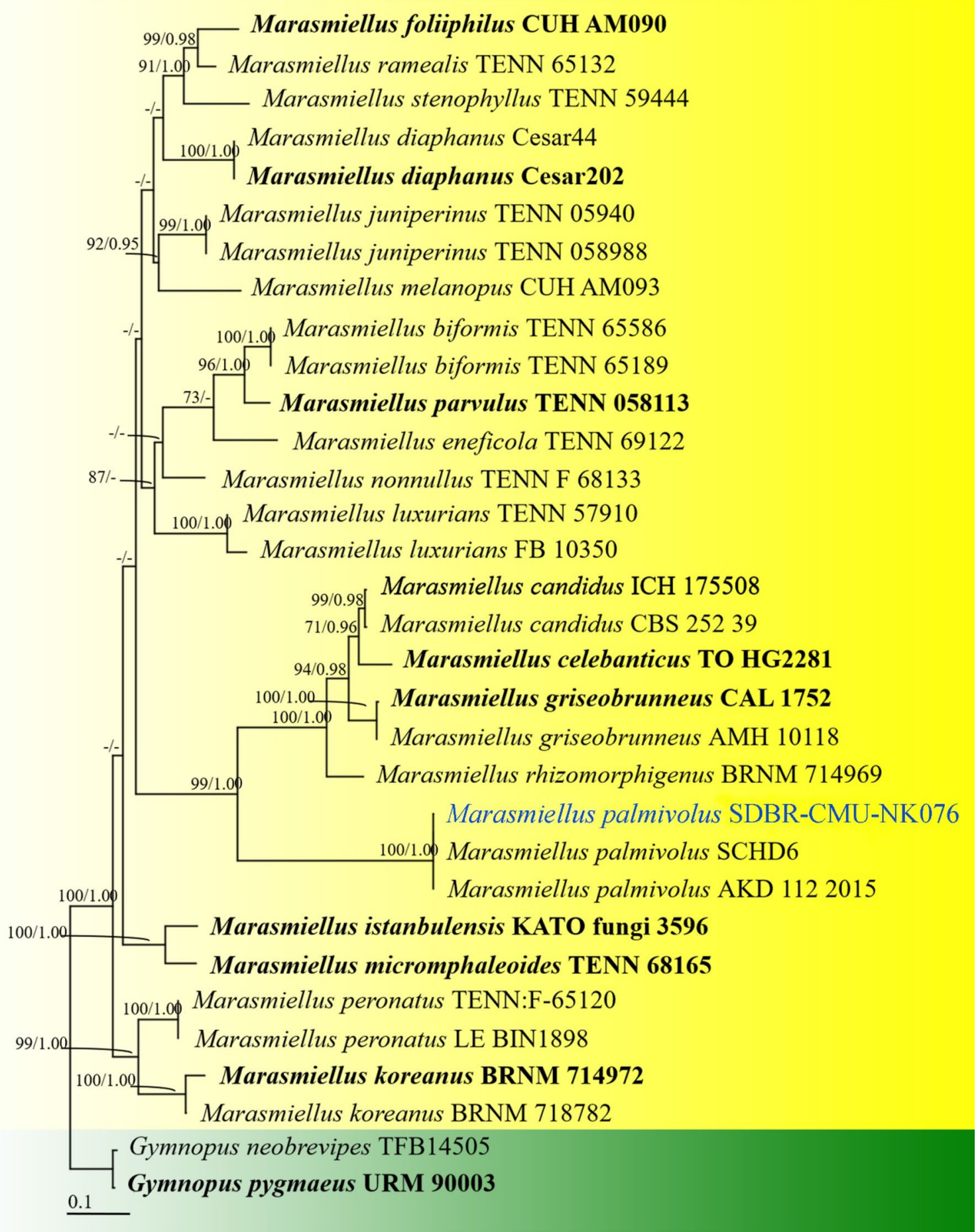

Fig. 199 Phylogenetic tree derived from maximum likelihood analysis of a combined ITS, and LSU genes of 22 sequences and the aligned dataset was comprised of 2070 characters including gaps (ITS: 1-1120 and LSU: 1121-2070). The average standard deviation of the split frequencies of the BI analysis was 0.00731. A best scoring RAxML tree was established with a final ML optimization likelihood value of - 10104.6944. The matrix had 806 distinct alignment patterns with $56.72 \%$ undetermined characters or gaps. Estimated base frequencies were found to be: $\mathrm{A}=0.2519, \mathrm{C}=0.1827$,
$\mathrm{G}=0.2462, \mathrm{~T}=0.3193$; substitution rates $\mathrm{AC}=1.0427, \mathrm{AG}=3.9637$, $\mathrm{AT}=1.7229, \mathrm{CG}=0.7530, \mathrm{CT}=4.8493, \mathrm{GT}=1.0000$; proportion of invariable sites $=0.0510$ and gamma distribution $=0.4750$. Gymnopus nebrevipes TFB14505 and Gynnopus pygmaeus URM90003 were used as outgroup. Numbers above branches are the bootstrap statistics percentages equal to or greater than $70 \%$ (MLBS, left) and Bayesian posterior probabilities equal to or greater than 0.95 (BYPP, right). Extype strains are in bold and newly generated sequence is in blue 

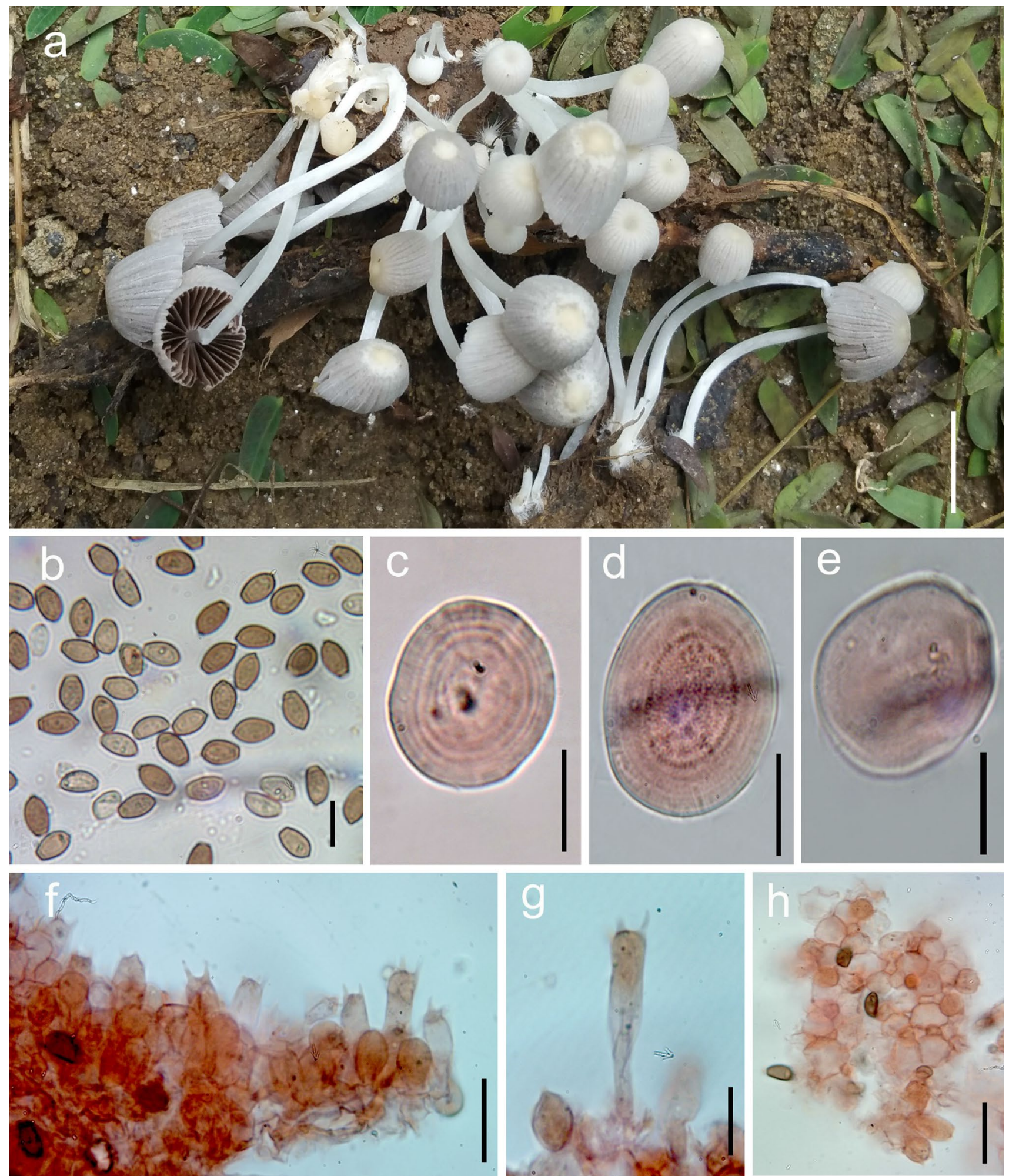

Fig. 200 Coprinellus punjabensis (LAH35321, holotype). a Fresh basidiomata. b Basidiospores. c-e Veil elements. f Hymenium having immature and mature basidia. $\mathbf{g}$ A mature basidium with sharp

strigmata. h Pileipellis. Microscopic structures were captured after stain with Congo red. Scale bars: $\mathbf{a}=10 \mathrm{~mm}, \mathbf{b}=\mathbf{1 0} \mu \mathrm{m}, \mathbf{c}-\mathbf{g}=20 \mu \mathrm{m}$, $\mathbf{h}=15 \mu \mathrm{m}$ 


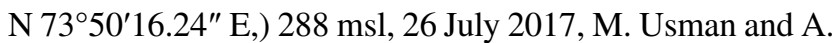
Hameed, MU58 (LAH36392).

GenBank numbers: ITS = MT180990, MT180991.

Notes: Closely related species, including Coprinellus disseminatisimilis Hussain mostly resemble $C$. punjabensis (Fig. 200) in having cylindrical to campanulate pileus, length of cylindrical stipe $20-40 \mu \mathrm{m}$, absence of annulus, ellipsoid to amygdamliform dark brownish basidiospores and hyaline cylindrical to clavate basidia (Hussain et al. 2018). Coprinellus disseminatus also resemble with our taxon in having whitish grey cylindrical stipe, lamellulae $0-3$, globose to subglobose veil elements up to $40 \mu \mathrm{m}$ (Uljé 2005). However, C. punjabensis differs from C. disseminatisimilis in smaller size of white greyish pileus up to $12 \mathrm{~mm}$ diam., lamellulae $0-3$, pileipellis irregular epithelium, veil elements hyaline globose to sub-globose up to $45 \mu \mathrm{m}$ while $C$. disseminatisimilis has larger greyish-brown to greyish-yellowish-brown pileus (15-20 $\mu \mathrm{m}$ diam.), lamellulae 0-2, pileipellis light brown to hyaline loosely euhymenoderm, veil elements greyish brown up to $40 \mu \mathrm{m}$ (Hussian et al. 2018). Coprinellus disseminates also differs from C. punjabensis in having larger pale-brown to yellow-brown, sometime almost white conical to convex pileus (up to $20 \mu \mathrm{m}$ diam.), ovoid to obconical red dark brown basidiospores (Uljé 2005). Furthermore, phylogenetic analyses indicate that $C$. punjabensis forms a basal lineage in the clade with 100\% MLBS support (Fig. 201). To provide the recommendation to justify our new species, we follow Hussain et al. (2018). Comparison of 625 nucleotides of the ITS sequences between $C$. punjabensis (MT180990) and C. disseminatisimilis (MH753670) reveals 32 substitutions and 6 deletions in our taxon $(6.08 \%$ nucleotide difference) while comparison of 643 nucleotides of ITS sequences between C. punjabensis (MT180990) and C. disseminates (JN159560) reveals 23 substitutions and 27 deletions in our taxon (7.77\% nucleotide differences), which we believe should be sufficient to delineate our new species.

Geastrales K. Hosaka \& Castellano

Notes: The latest update accounts of Geastrales see Wijayawardene et al. (2020).

\section{Geastraceae Corda}

Notes: Geastraceae [as Geastrideae] was proposed by Corda (1842) with Geastrum Pers. [as Geaster P. Micheli] as the generic type. Currently two genera are accepted in Geastraceae: Geastrum and Myriostoma. Traditionally, these genera have been considered close to puffballs (e.g., Bovista, Calvatia and Lycoperdon) in the obsolete class Gasteromycetes in the obsolete order Lycoperdales. Hibbett et al. (1997) based on molecular analyses, placed puffballs in the euagaric groups; while Geastrum is phylogenetically far in the gomphoid-phalloid clade, together with Sphaerobolus. Later, Hosaka et al. (2006) proposed the new order Geastrales (Phallomycetidae) to include four families: Geastraceae, Pyrenogastraceae, Sphaerobolaceae and Sclerogastraceae. Jeppson et al. (2013) followed this treatment in their systematic approach for European Geastraceae.

The family is characterized by globose to subglobose unexpanded basidiomata, with a multi-layer exoperidium, and an endoperidium enclosing the mature gleba. At maturity, the exoperidium splits in a stellate manner, revealing the globose endoperidium, which may have one or multiple ostioles; the endoperidium can be sessile, or with one or multiple pseudostipes. The mature spores are globose to subglobose and ornamented (verrucose to irregularly baculate or with crests).

\section{Geastrum Pers.}

Notes: Geastrum was described by Persoon (1794) to accommodate three species G. multifidum, G. quadrifidium and G. rufescens. Later, Persoon (1801) expanded the genus to include $G$. coliforme, G. coronatum, G. pectinatum, $G$. quadrifidum, G. rufescens and G. hygrometricum. Currently, two of these species are included in two different genera: Geastrum coliforme in Myriostoma and G. hygrometricum in Astraeus.

Clements and Shear (1931) made the first typification of Geastrum selecting G. pectinatum as the type of the genus; however, Demoulin (1984) selected G. multifidum as the generic type, and later, Zamora (2014) proposed that the generic name Geastrum should be conserved with G. coronatum as the conserved type. Currently, Index Fungorum (2021) and Mycobank (Robert et al. 2005) databases, both accessed on November 2019, maintain G. pectinatum as the generic type.

Geastrum comprises ca. 100-120 widely distributed species (Zamora et al. 2014), although this number is increasing every year. In the last five years, according to the databases mentioned above, 24 new species have been described. The main morphological characters to delimitate the Geastrum species or 'earthstar' fungi are the exoperidium, hygroscopic or not; the endoperidium persistent or sometimes evanescent, sessile or pseudostipitate, with a unique ostiole that can be delimitated or not, mammiform or broadly conical forming a peristome, fibrillose or sulcate (Calonge 1998; Douanla-Meli et al. 2005; Trierveiler-Pereira et al. 2011). The size and ornamentation of the spores, verrucose to irregularly baculate, are important characters to identify species; also, as indicated in Zamora et al. (2013), the presence and shapes of calcium oxalate crystals in the rhizomorphs are also good characters to delimitate species in Geastrum.

In this study, we introduce two new species, Geastrum gorgonicum and G. hansagiense based on morphology and molecular phylogenetic analyses (Figs. 202, 203, 204 and 205). 


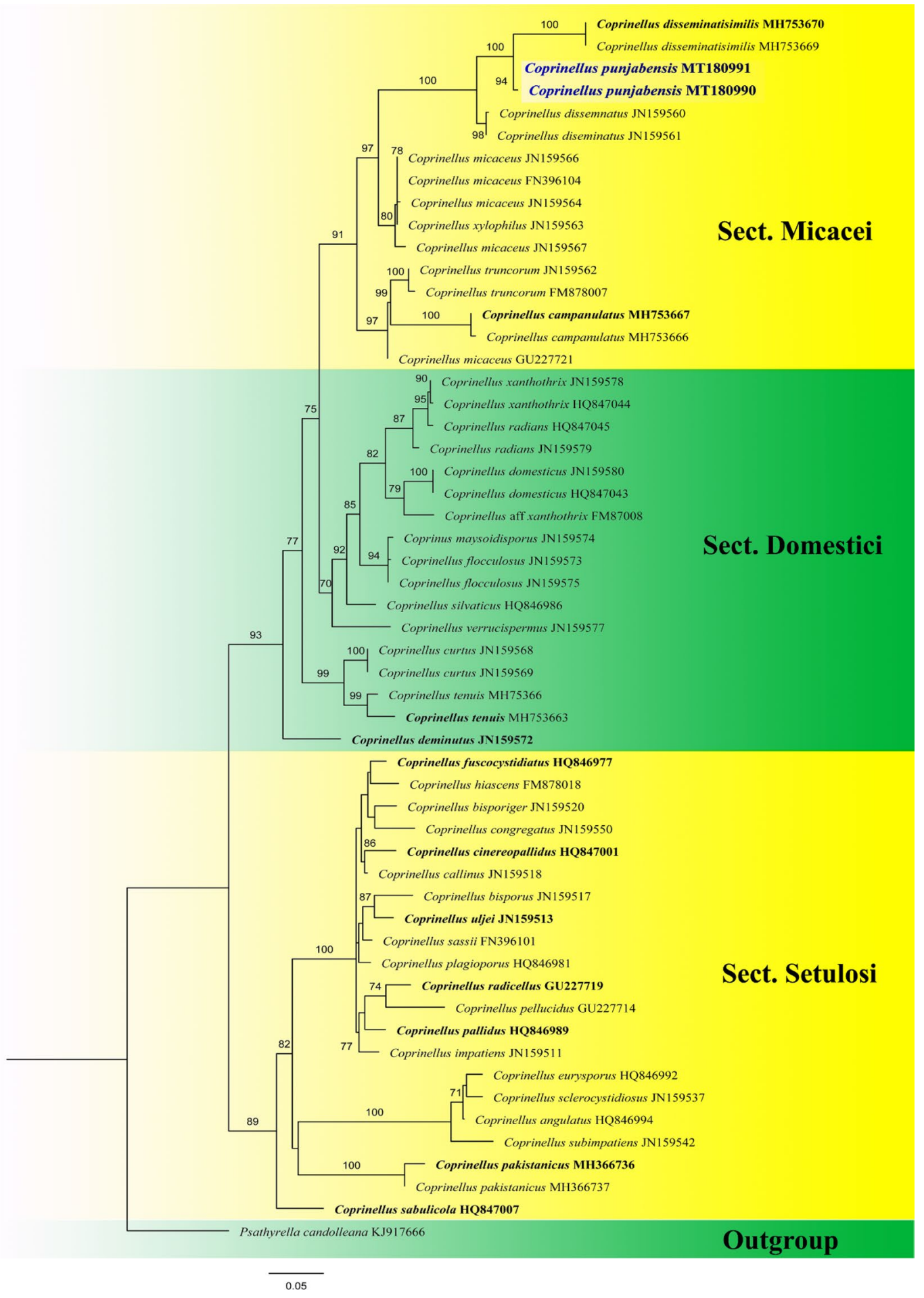

Fig. 201 Phylogram generated from maximum likelihood analysis based on ITS sequence data representing Coprinellus punjabensis (MT180990) and related species. Related sequences are taken from Hussain et al. (2018). Fifty-five sequences are included in the analysis which comprise 708 characters after alignement. Psathyrella candolleana (KJ917666) is used as the outgroup taxon. The best RAxML tree with a final likelihood values of -4869.810366 is presented. The matrix had 351 distinct alignment patterns, with $15.82 \%$ undetermined characters or gaps. Estimated base frequencies were as follows: $\mathrm{A}=0.233377, \mathrm{C}=0.241159, \mathrm{G}=0.233394, \mathrm{~T}=0.292070$; substitution rates $\mathrm{AC}=1.849754, \mathrm{AG}=2.784571, \mathrm{AT}=1.813146$, $\mathrm{CG}=1.135142, \mathrm{CT}=1.135142, \mathrm{GT}=1.000000$; gamma distribution shape parameter $\alpha=0.294349$. Bootstrap values for maximum likelihood (MLBS) equal to or greater than $75 \%$. The newly generated sequence is in blue bold 
Geastrum gorgonicum M.P. Martín, M. Dueñas \& Telleria, sp. nov.

Mycobank number: MB834616; Facesoffungi number: FoF 09203; Fig. 202

Etymology: Named after Gorgades, an ancient name for the Cape Verde Islands, Atlantic Ocean.

Holotype: MA-Fungi 92118

Colour code follow: XXX Code Universal des couleurs (Séguy 1936).
Unexpanded basidiomata 11-12 mm high, 7-11 mm diam., rounded with an umbo, and attached to the ground by a basal mycelium tuft, with many rhizomorphs. Peridium smooth to felt-like, pale brown (702); when the surface is damaged the fibrous layer appears dirty white (680). Expanded basidiomata $10-11 \mathrm{~mm}$ high $\times 11-23 \mathrm{~mm}$ diam. Exoperidium splitting in 8-10 more or less equal rays, not hygroscopic; the rays are often recurved under the exoperidial disk. Mycelial layer brownish (702) to dirty white (680), when peeling off. Fibrous layer dirty white (680),
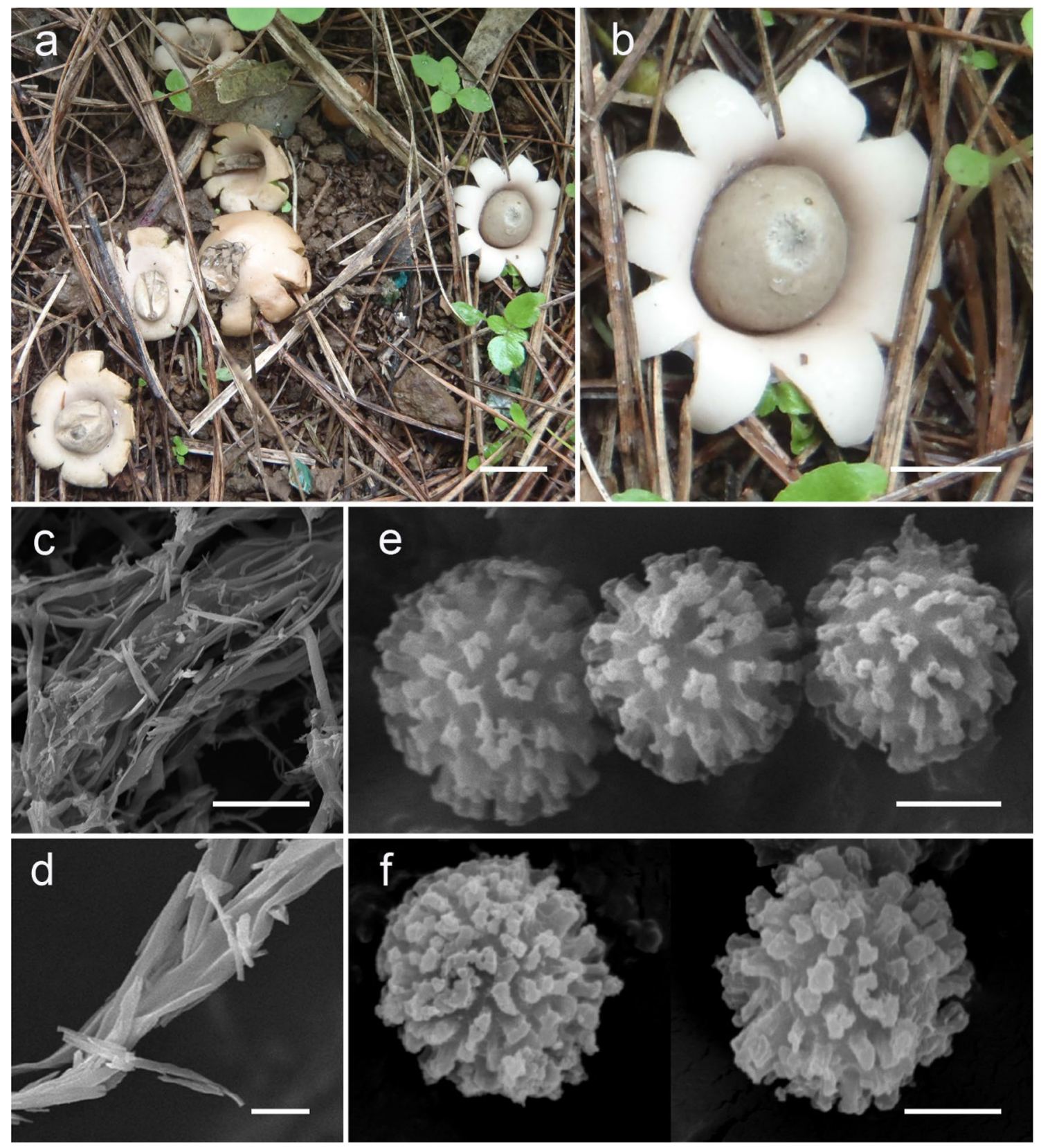

Fig. 202 Geastrum gorgonicum (MA-Fungi 92118, holotype). a, b Fresh basidiomata in field. c, d Rhizomorphs with acicular crystals. e, f Basidiospores. Scale bars: $\mathbf{a}=1 \mathrm{~cm}, \mathbf{b}=5 \mathrm{~mm}, \mathbf{c}=\mathbf{1 0} \mu \mathrm{m}, \mathbf{d}-\mathbf{f}=2 \mu \mathrm{m}$ 
papery when dry. Pseudoparenchymatous layer pale brown (190), peeling off when dry. Endoperidium globose, 7-11 mm diam., sessile, brown (702), without crystals. Peristome finely fibrillose, well delimited, conical, colour similar to the mycelial layer (190). Mature gleba brown $(697,702)$. Mycelial layer with the outer part formed by 2-3 $\mu \mathrm{m}$ diam., brownish, unbranched, aseptate hyphae, with walls $<0.2 \mu \mathrm{m}$ wide; and inner part with $1.5-2 \mu \mathrm{m}$ diam., hyaline, unbranched, aseptate hyphae, with some clamps, and with very thin walls $(<0.1 \mu \mathrm{m}$ wide). Fibrous layer $1.5-3 \mu \mathrm{m}$ diam., hyaline, thick-walled hyphae with narrow lumen. Pseudoparenchymatous layer with thin-walled, hyaline cells, variable in shape and size, about $18-58 \mu \mathrm{m}$ diam. Endoperidial layer with 2.5-4 $\mu \mathrm{m}$ diam., hyaline hyphae, some with thin walls $(<0.2 \mu \mathrm{m}$ wide) and others thickwalled with narrow lumen. Peristome with $2-3 \mu \mathrm{m}$ diam., yellowish, aseptate hyphae, thick-walled with narrow lumen and without clamps. Capillitium formed by 1.5-3 $\mu \mathrm{m}$ diam., brown, aseptate hyphae with walls $<0.2 \mu \mathrm{m}$ wide. Basidia not seen. Basidiospores globose to subglobose, brownish to yellowish brown, with an oil drop; basidiospores $4.5-5 \mu \mathrm{m}$ diam. including the baculate ornamentation $(0.3-0.5 \mu \mathrm{m}$ high).

Material examined: CAPE VERDE, Santiago, Parque Natural da Serra da Malagueta, Concejo de Sta. Catarina, $15^{\circ} 10^{\prime} 28^{\prime \prime} \mathrm{N} 28^{\circ} 40^{\prime} 37^{\prime \prime} \mathrm{W}, 914 \mathrm{msl}$, in a slope under Pinus sp., 20 November 2010, J. Cardoso, L.M. Caterino, L.M. Catarino, M. Dueñas, M.P. Martín, I. Melo, I. Salcedo and M.T. Telleria, 3242MPM (MA-Fungi 92118, holotype); idem, 3243MPM (MA-Fungi 92116); idem, 3244MPM (MA-Fungi 92114); idem, 13248MD (MA-Fungi 92112).

GenBank numbers: MA-Fungi 92118: ITS $=$ MN754045, LSU $=$ MN754083; MA-Fungi 92116: ITS $=$ MN754046, LSU $=$ MN754084; MA-Fungi 92114: ITS $=$ MN754047, $\mathrm{LSU}=\mathrm{MN} 754085$.

Notes: Based on ITS and LSU analyses (Fig. 203), Geastrum gorgonicum clustered in sect. Corollina J.C. Zamora in its own well-supported clade (100\% MPBS, 95\% MLBS, $1.00 \mathrm{BYPP})$ close to two collections under Geastrum aff. saccatum 1 in Zamora et al. (2014), one from Argentina

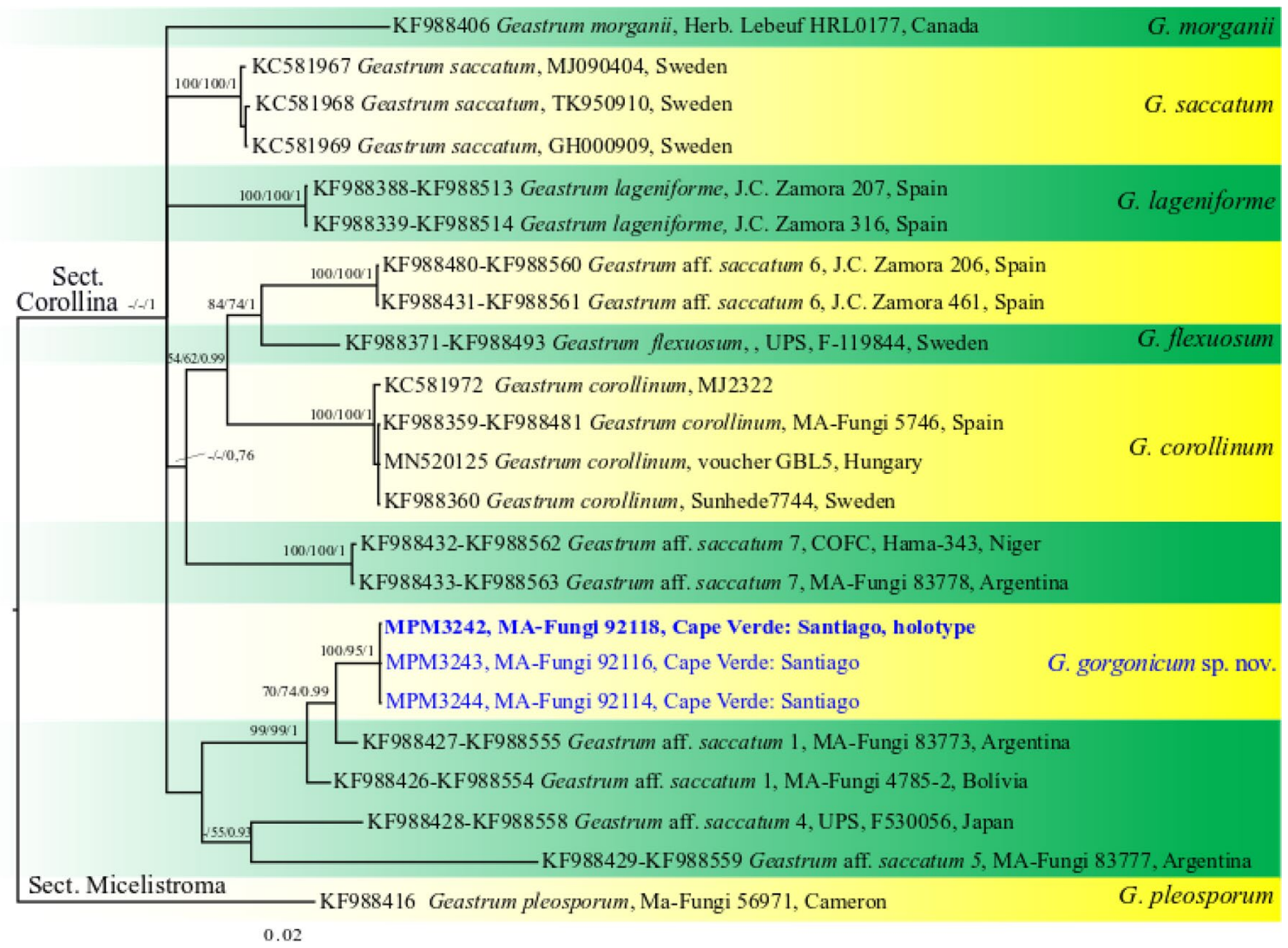

Fig. 203 Phylogram generated from Bayesian analysis based on ITS and LSU nrDNA sequence data representing Geastrum from Section Corollina, with G. pleosporum from Sect Micelistroma as outgroup. Related sequences are mainly taken from Zamora et al. (2014). Twenty-three sequences are included in the combined analyses, which comprise 1561 characters. Maximum parsimony (MP) and maximum likelihood (ML) analyses were undertaken; MP and ML tree topologies (not shown) were similar to the Bayesian one. Bootstrap values for MP and ML equal to or greater than $50 \%$, and clade credibility values greater than 0.90 (the rounding of values to 2 decimal places) from Bayesian-inference analysis are labeled on the nodes. The newly generated sequences are in blue bold 
(MA-Fungi 83775), and the other from Bolivia (MA-Fungi 47185-2). On the other hand, the G. gorgonicum clade grouped separately from other species of section Corollina: Geastrum corollinum, G. flexuosum, G. lageniforme, G. morganii and G. saccatum, as well as from Geastrum aff. saccatum 4, 5, 6 and 7 from Zamora et al. (2014).

Geastrum gorgonicum can be separated from G. saccatum by a number of characters (Fig. 202). The expanded basidiomata of $G$. saccatum are larger (10-40 mm diam.) with an endoperidium of 10-20 mm diam., beige to greyish brown; whereas in G. gorgonicum the endoperidium is brown, and the two types of hyphae (thin and thick-walled) are not wider than $11 \mu \mathrm{m}$ diam. The capillitium in $G$. saccatum is formed by hyphae up to $10 \mu \mathrm{m}$ wide, but in G. gorgonicum these hyphae are not wider than $3 \mu \mathrm{m}$. In general, in $G$. gorgonicum all the hyphae are narrower than those mentioned by Sunhede (1989) for G. saccatum.

Geastrum gorgonicum can be separated easily from $G$. lageniforme by the kind of rhizomorph crystals: acicular in G. gorgonicum (Fig. 202), and horn-like in G. lageniforme; as well as by the basidiospores that, according to Jeppson et al. (2013), are smaller (2.5-3.5 $\mu \mathrm{m})$ in G lageniforme. Geastrum morganii has larger fully expanded basidiomata (30-60 mm diam.) and an irregularly folded peristome; whereas in G. gorgonicum the basidiomata are not larger than $23 \mathrm{~mm}$ diam., and the peristome is fibrillose.

Geastrum hansagiense Bóna, Merényi, Boros, Stielow \& Bratek, sp. nov.

MycoBank number: MB834968; Facesoffungi number: FoF 08141; Fig. 204

Etymology: The name refers to a Hungarian area (Hanság), where the type specimen was collected.

Holotype: BP110893

Basidiomata epigeous at maturity. Unexpanded basidiomata subglobose, depressed, 6.6(4-10) $\mathrm{mm}$ high, the minimal width 10.4(6-16) mm, the maximal 12(8-19) mm. Expanded basidiomata subglobose, 9.3(5.5-12) $\mathrm{mm}$ high, the minimal width 24.5(11-37), the maximal 27.5(20-38) mm, including rays. The outer surface of exoperidium smooth, light brown, silvery shining, vinaceous buff (31) with shade of smoke grey (34), the inner surface of exoperidium darker, date brown (24) under dry condition (Colour Identification Chart of Royal Botanic Garden, Edinburgh (RBGE) 1969). Exoperidium strongly hygroscopic, splitting into 7-9(6-12) triangular rays. Endoperidial body subglobose, saccate, flatted, sessile, distinctly pruinose, 13.2(9.5-19) high and 15.2(11-23) mm wide, light brownish, vinaceous buff (31), clay buff (32), drab (33) (RBGE 1969). Mycelial layer not always visible or intact, white to pale yellow, encrusted with some debris, formed hyaline to quite pale yellow, sinuous hyphae, 4.8(3-9.1) $\mu \mathrm{m}$ diam., thin-walled $(<1 \mu \mathrm{m})$, lumen evident. Fibrous layer papery, compact, persistent, yellowish to brownish, formed yellowish, sinuous hyphae, 4.4(3-6.5) $\mu \mathrm{m}$ diam., thin-walled $(<1 \mu \mathrm{m})$, lumen evident. Pseudoparenchymatous layer persistent, yellowish, with irregular subglobose to ellipsoid hyphal cells, in various shapes and size, 21.2(12.5-37.1) $\mu \mathrm{m}$ long and 14.4(8-26.7) $\mu \mathrm{m}$ wide, with $1.5(0.5-2.5) \mu \mathrm{m}$ wall. Stalk absent. Apophysis absent. Peristome undelimited, almost flat to slightly elevated, mammiform, tenderly fibrillose, closed or opened with age, with irregularly shaped pores, similar or lighter than the endoperidium, milky coffee (28) or fawn (29) (RBGE 1969), but seems too darker because of the effused basidiospores. Columella central, pyramid shape, often imperceptible, light brown, clay buff (32) (RBGE 1969). Mature gleba forcibly dark brown to blackish, fuscous black (36) (RBGE 1969). Basidiospores globose, brownish or dark brown in 5\% $\mathrm{KOH}, 5.7(4.9-7.1) \mu \mathrm{m}$ diam. with verrucose ornamentation. Basidia not seen. Eucapillitium sinuous, 5.9(3.5-8) $\mu \mathrm{m}$ diam., rarely verrucosed, unbranched, thin-walled $(\leq 1 \mu \mathrm{m})$, lumen evident.

Habit and habitat: Hundreds of specimens were found in a small, few $\mathrm{m}^{2}$ area. The habitat of $G$. hansagiense is a ruderal nitrogen-rich grassland (dominant plant species Galium aparine, Conium maculatum, Urtica dioica) with nutritious soils.

Distribution: Specimens were found near to Mosonmagyaróvár (4750'22.3" N 17¹4'30.1" E), Hanság-area, Hungary. The formerly drained sweetwater turf marshland, Hanság-area is situated in the Little Hungarian Plain and closely connected with the westernmost Salt Lake of Eurasia, Lake Neusiedl. Until now the known distribution is restricted to the locus classicus.

Material examined: HUNGARY, Hanság-area, Moson-

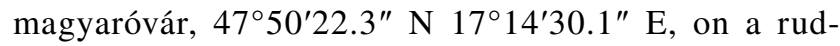
eral nitrogen-rich grassland, 17 October 2015, L. Boros (BP110893, holotype).

GenBank numbers: ITS = MN582753, MN582754, $\mathrm{LSU}=\mathrm{MN} 582739$.

Notes: Macromorphological descriptions based on ca. 30 fruitbodies in different developmental stages. Geastrum hansagiense is recognized by depressed, flatted, subglobose, usually wider than higher basidiomata, distinctly pruinose, sessile endoperidial body, tenderly fibrillose, mammiform, undelimited peristome, hygroscopic exoperidium, lacking of the stalk and apophysis and almost black gleba with globose, verrucose basidiospores (Fig. 204). One species with nearly identical characters is G. floriforme. Geastrum hansagiense is differentiated from $G$. floriforme by the size of the basidiomata, since the unexpanded G. floriforme basidiomata is $8.5 \mathrm{~mm}$ wide, the expanded basidiomata $21.5 \mathrm{~mm}$ wide and the endoperidial body $8.8 \mathrm{~mm}$ wide on average, based on our own mesurements and data from the literature (Hollós 1903; Jeppson et al. 2013; Sousa et al. 2014). Similarly, hygroscopic and sessile species are in genus Geastrum, G. kotlabae, G. corollinum and G. hungaricum, but their peristomes 

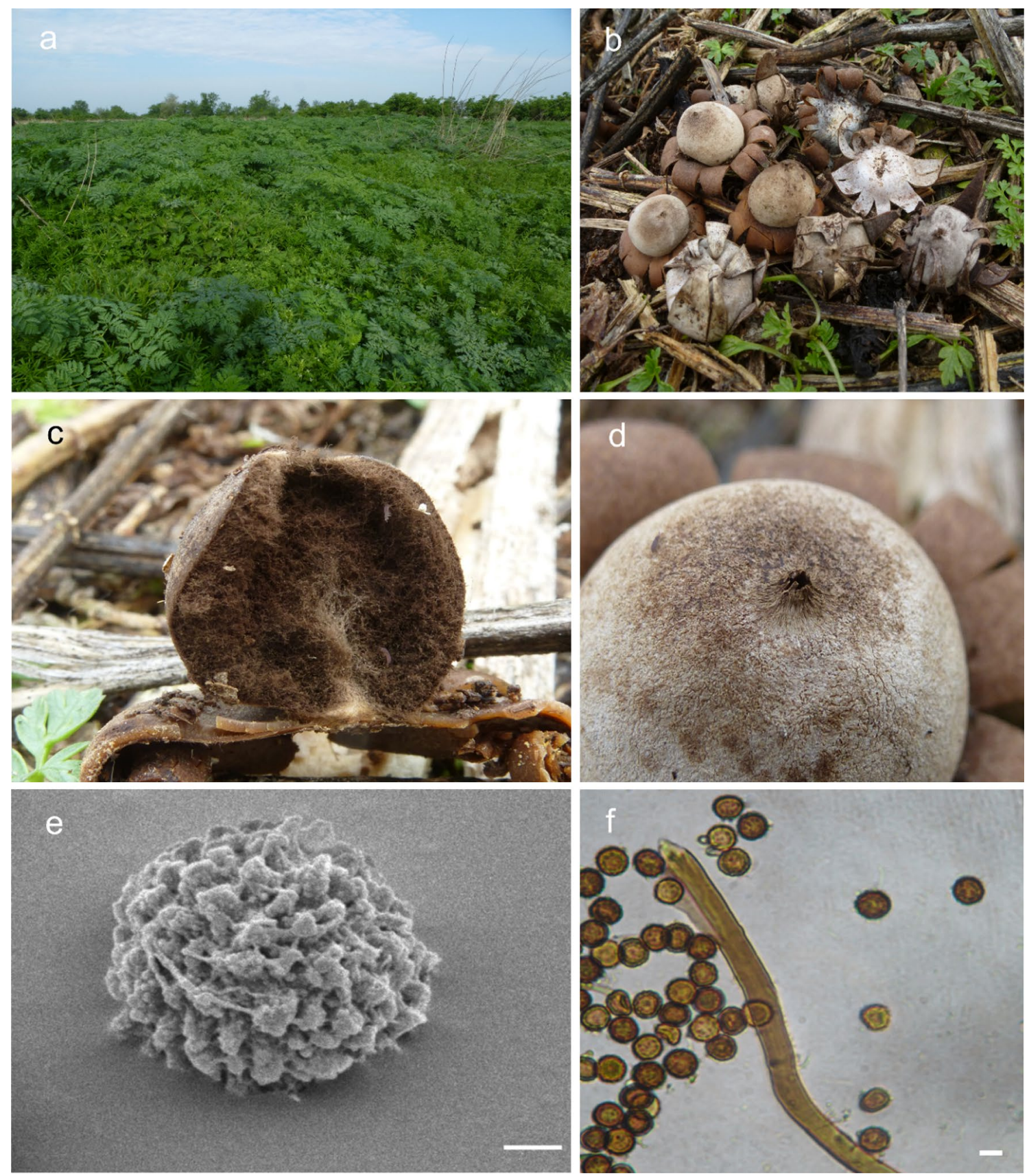

Fig. 204 Geastrum hansagiense (BP110893, holotype). a Habitat of G. hansagiense. b Basidiomata. c Columella. d Peristome. e Basidiospore under SEM f Basidiospores and eucapillitium under LM. Scale bars: $\mathbf{e}=1 \mu \mathrm{m}, \mathbf{f}=6 \mu \mathrm{m}$

are totally different. Geastrum hansagiense is presumably a pseudocryptic species in the absence of remarkable morphological differences from $G$. floriforme. However, $G$. hansagiense differs from $G$. floriforme in some unique fixed alleles of the ITS locus, based on the alignments of sequences (Fig. 205). Geastrum hansagiense's unique fixed alleles are: 14(T), 16(A), 17(G), 18(A), 19(T), 21(A), 22(G), 23(A), 26(G), 41(C), 43(gap), 46-48(gap), 62(T), 72(C), 111-112(A), 119(T), 125(C), 127-128(T), 152(A), 155-156(gap), 158(gap), 159(G), 160(T), 176-178(A), 179(T), 180(A), 184(T), 348(C), 362-363(T), 370(A), 375(G), 382(C), 387(A), 394(C), 395(G), 405(G), 411(C), 432(C), 463(gap), 468(T), 478(T), 492(A), 493(T), 505(C), 510(gap), 513-514(gap), 522(T), 534(G).

\section{Schizoporaceae Jülich}

Notes: Jülich (1981) proposed Schizoporaceae with Schizopora Velen. as the generic type. It is one of the most 
Fig. 205 Maximum likelihood (ML) phylogenetic tree of Geastrum species derived from concatenated internal transcribed spacer (ITS) and 28S rRNA (LSU) regions. Analysis was performed by RAxML under GTR + GAMMA model. Myriostoma coliforme (KC582020) is selected as the outgroup taxon. ML bootstrap values were obtained from 1000 replicates and values greater than $70 \%$ were indicated. The scale bar represents 0.007 expected nucleotide changes per site. The novel species are in blue, holotype specimen is in bold, herbarium numbers and NCBI Accession Numbers in brackets

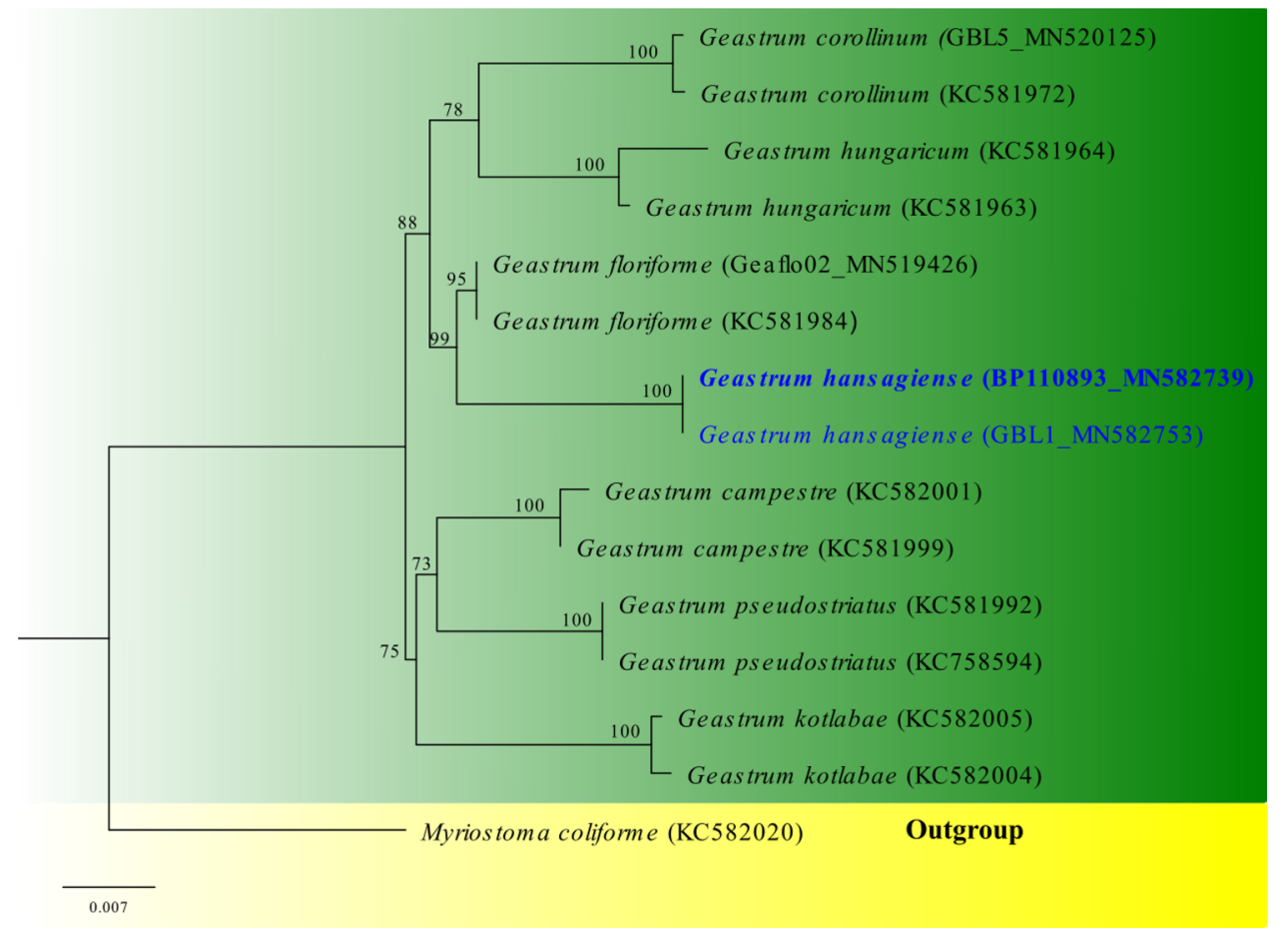

widespread and diverse families in Hymenochaetales. It includes species characterized by basidiomata with corticioid, hydnoid to poroid hymenophore, a monomitic or dimitic hyphal system, skeletal hyphae often poorly developed, generative hyphae bearing clamp connections, cystida usually capitate with encrustions; basidiospores ellipsoid, colorless, thin-walled, negative in Melzer's reagent and cyanophilous in Cotton Blue (Jülich 1981).

\section{Hyphodontia J. Erikss.}

Notes: This genus is one of the largest genera in Schizoporaceae and was proposed by Eriksson (1958) with Hyphodontia pallidula (Bres.) J. Erikss as the type species. Species in this widespread genus commonly occur in temperate to tropical areas causing white rot. Hyphodontia are characterized by a monomitic hyphal system with clamped generative hyphae, broadly-ellipsoid basidiospores (Eriksson 1958). In addition, the various shapes of cystidia elements are also important morphological characteristics for the identification in the genus.

Hyphodontia yunnanensis C.L. Zhao \& Y.C. Dai, sp. nov. Index Fungorum number: IF558551, Facesoffungi number: FoF 09971; Figs. 206, 207

Etymology: "yunnanensis" refers to collecting site.

Holotype: SWFC 00006804

Basidiomata: Annual, resupinate, adnate, without odour or taste when fresh, becoming fragile to slightly rigid upon drying, up to $12 \mathrm{~cm}$ long, $3 \mathrm{~cm}$ wide, $1.5 \mathrm{~mm}$ thick. Hymenial surface odontioid, vinaceous to brownish vinaceous when fresh, becoming pale brown upon drying, aculei conical, 2-4 per $\mathrm{mm}$, up to $1 \mathrm{~mm}$ long. Subiculum very thin to lacking, 50-100 $\mu \mathrm{m}$ thick. Margin pale brown, usually determinate and thinning out. Hyphal structure, hyphal system monomitic; generative hyphae bearing clamp connections, colorless, slightly thick-walled, frequently branched, IKI-, CB +; tissues unchanged in $\mathrm{KOH}$. Hymenium: Cystidia numerous, obviously projecting, cylindrical or tubular, colorless, encrusted, 26-37.5 ×2.5-4.5 $\mu \mathrm{m}$; basidia clavate, $13.5-20.5 \times 2.5-4.5 \mu \mathrm{m}$, four-spored and with a basal clamp connection; basidioles dominant, in shape similar to basidia, but slightly smaller. Basidiospores broadly ellipsoid to subglobose, colorless, thin-walled, IKI-, CB-, with a distinct apiculus, some bearing a guttule, $(3.3-) 3.5-4.5(-4.8) \times(2.7-) 3-3.6(-3.9) \mu \mathrm{m}$, $\mathrm{L}=4.04 \mu \mathrm{m}, \mathrm{W}=3.26 \mu \mathrm{m}, \mathrm{Q}=1.23-1.25(\mathrm{n}=90 / 3)$.

Material examined: CHINA, Yunnan Province, Chuxiong, Zixishan National Forestry Park, on angiosperm trunk, 30 June 2018, C.L. Zhao, CL Zhao 6804 (SWFC 00006804, holotype); Puer, Jingdong County, Huilianghe Village, on fallen branch of angiosperm, 5 January 2019, C.L. Zhao, CL Zhao 9570 (SWFC 00009570, paratype); Yuxi, Xinping County, Mopanshan National Forestry Park, on angiosperm trunk, 18 January 2018, C.L. Zhao, CL Zhao 6397 (SWFC 00006397, paratype).

GenBank numbers: ITS = MW020702, MW020701, MW020700.

Notes: Phylogenetically, Hyphodontia yunnanensis is sister to H. mollis Sheng H. Wu with 97\% MLBS, $100 \%$ 

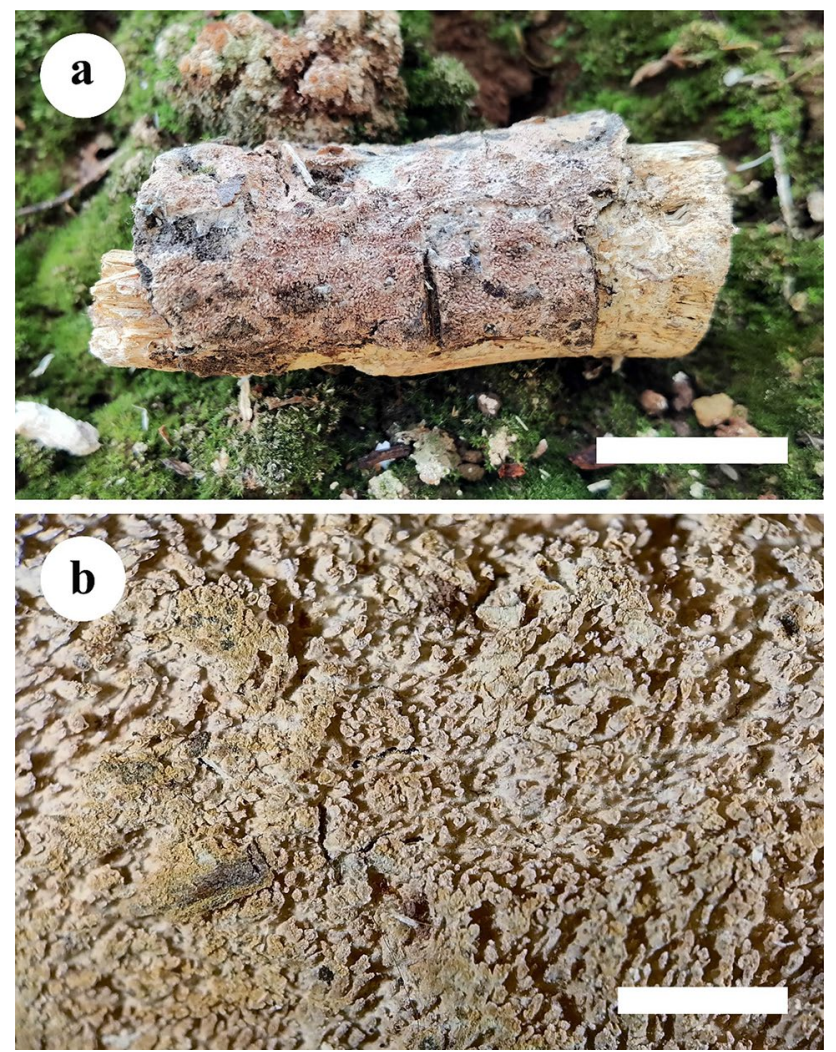

Fig. 206 Hyphodontia yunnanensis (CL Zhao 6804, holotype). a, b Basidiomata. Scale bars: $\mathbf{a}=2 \mathrm{~cm}, \mathbf{b}=3 \mathrm{~mm}$

Fig. 207 Hyphodontia yunnanensis (drawn from the holotype), microscopic structures. a Basidiospores. b Basidia and basidioles. c Cystidia. d A section of aculei trama. Scale bars: $\mathbf{a}-\mathbf{d}=10 \mu \mathrm{m}$
MPBS, 1.00 BYPP support (Fig. 208). However, H. mollis differs from H. yunnanensis (Figs. 206 and 207) in having the ochraceous, distinctly hydnoid hymenophore with a pink

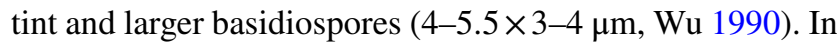
addition, the two species share less than $96 \%$ in common based on the ITS.

\section{Thelephoraceae Chevall.}

Notes: Thelephoraceae was built up by Chevallier (1826) based on the type genus Thelephora, and is composed of 34 genera. Most of the species of Thelephoraceae belong to ectomycorrhizal fungi which play an extremely important role in maintaining the balance of a terrestrial ecosystems by receiving energy from their host plants and, in return, transporting nutrients to them (Deckmyn et al. 2014).

\section{Odontia Pers.}

Notes: Odontia was introduced by Persoon (1794) with the type species $O$. ferruginea Pers. Odontia had been considered to be a synonym of Tomentella. However, recent phylogenetic analyses revealed that Odontia was in a monophyletic position close to Tomentella, and the species were generally collected in old forests possessing a saprotrophic lifestyle compared with an ectomycorrhizal one (Gorjón and Greslebin 2012; Tedersoo et al. 2014). The general characteristics of the genus are: resupinate and arachnoid basidiomata which are separable from the substrate, smooth to granulose or hydnoid hymenophoral surface, a dimitic hyphal system in the rhizomorphs, a monomitic or dimitic hyphal system in the
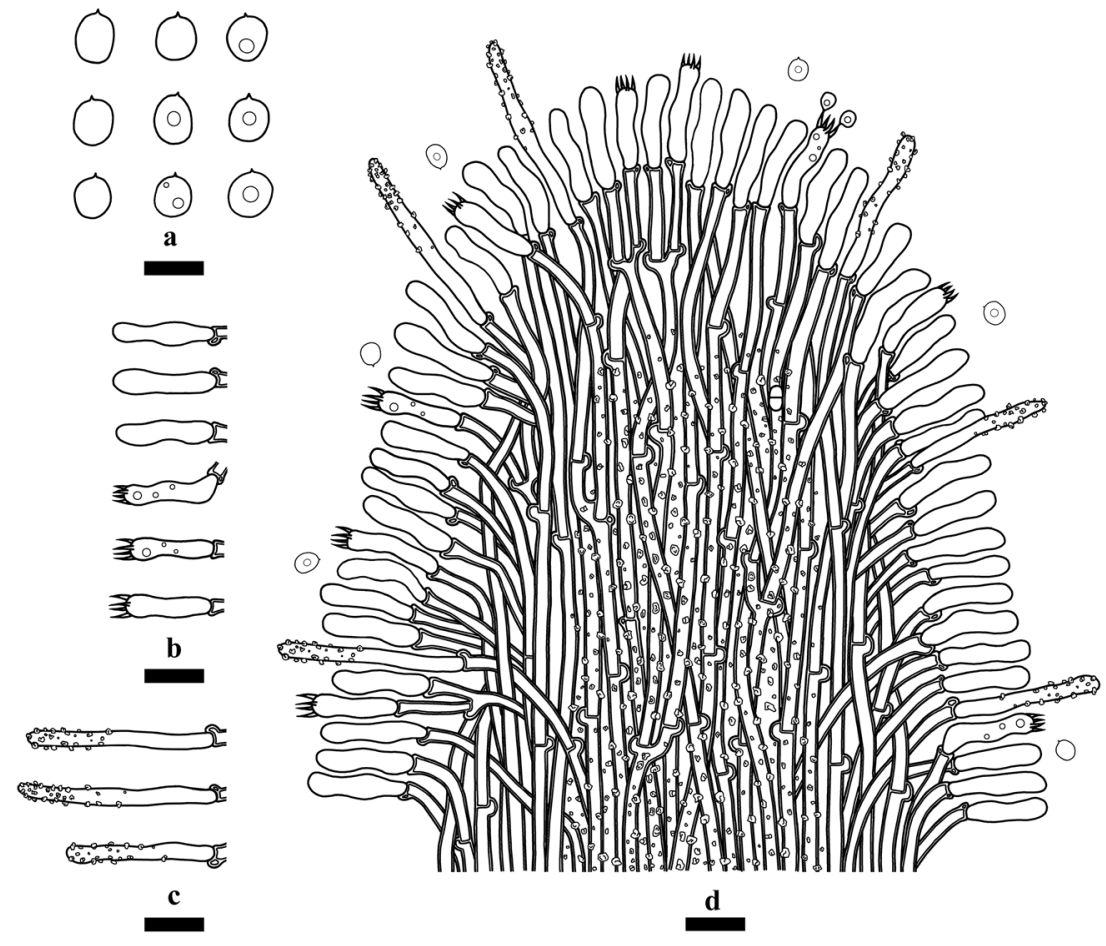


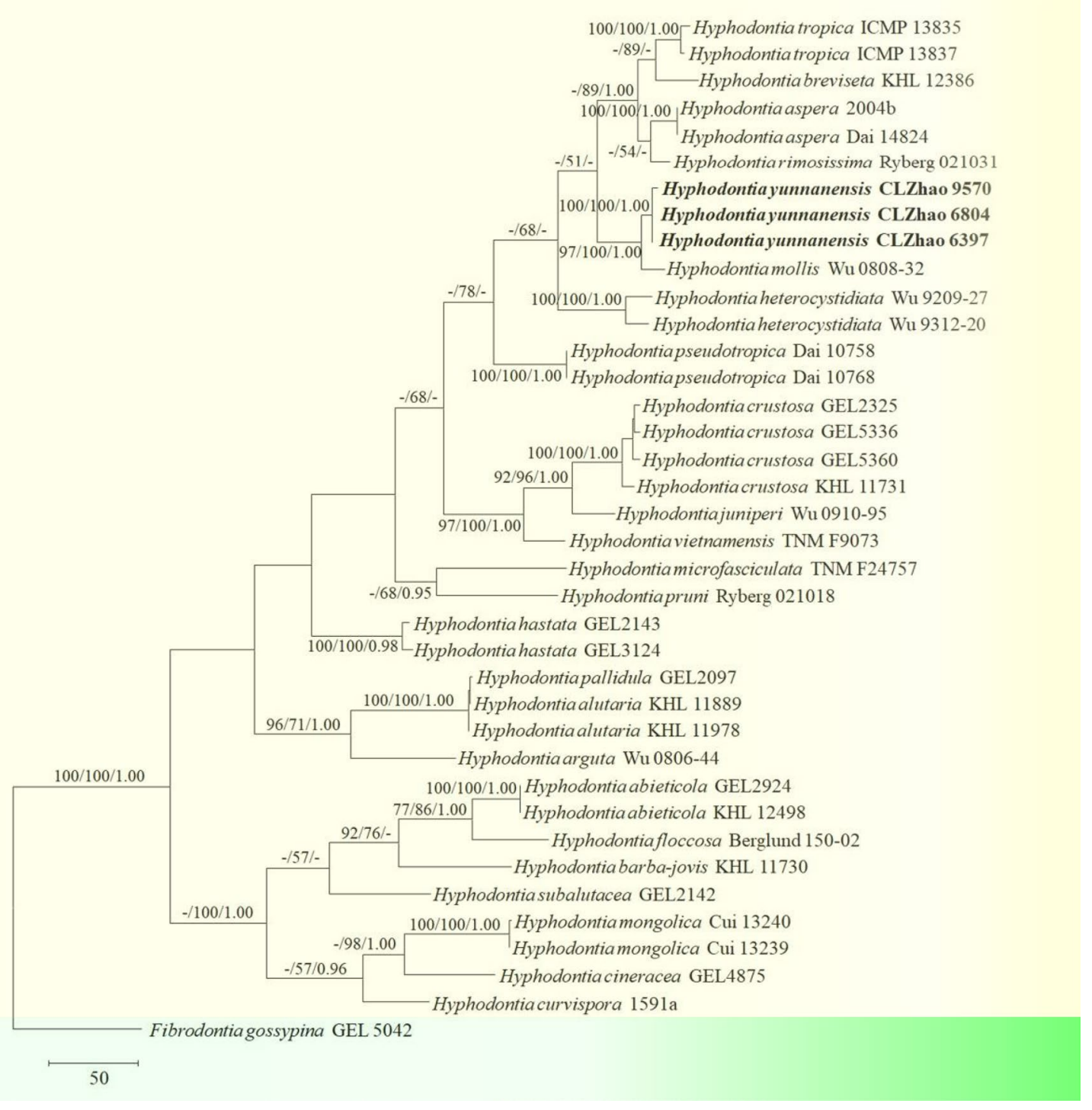

Fig. 208 Maximum parsimony strict consensus tree of Hyphodontia yunnanensis and related species in Hyphodontia based on ITS sequences. The ITS dataset included sequences from 38 fungal specimens representing 24 taxa. Fibrodontia gossypina (GEL 5042) was selected as an outgroup for phylogenetic analyses of ITS phylogenetic trees (Zhao et al. 2014). The PCR procedure and editing the DNA sequences and approaches to phylogenetic analysis from maximum parsimony, maximum Likelihood (ML) and Bayesian inference (BI) followed previous studies (Dai 2012; Zhao and Wu 2017; Cui et al. 2019). The dataset had an aligned length of 755 characters in the dataset, of which 307 characters are constant, 92 are variable and parsimony-uninformative, and 356 are parsimony-informative. Maximum

subiculum, clavate basidia and irregular ellipsoid to globose verruculose basidiospores (Kõljalg 1996; Yuan et al. 2018). The species of Odontia were reported to be spread over temperate Eurasia, North America, Africa, France, Australia, New Zealand, China and Turkey (Rodway 1897; Larsen 1974; Kõljalg 1996; Kõljalg and Larsson 1998; Gorjón and Greslebin 2012; Tedersoo et al. 2014; Yuan et al. 2018). parsimony analysis yielded 1 equally parsimonious tree $(\mathrm{TL}=1954$, CI $0.4417, \mathrm{HI}=0.5583, \mathrm{RI}=0.6329, \mathrm{RC}=0.2795$ ). Best model for ITS estimated and applied in the Bayesian analysis: GTR $+\mathrm{I}+\mathrm{G}$, 1set $n s t=6$, rates $=$ invgamma; prset statefreqpr $=$ dirichlet $(1,1,1,1)$. Bayesian analysis resulted in the similar topology with an average standard deviation of split frequencies $=0.009980$ (BI). The ITS region was amplified with primer pairs ITS5 and ITS4 (White et al. 1990). Branches are labeled with maximum likelihood bootstrap values greater than 70\% (MLBS), parsimony bootstrap values higher than 50\% (MPBS) and Bayesian posterior probabilities greater than 0.95 (BYPP), respectively

Odontia huanrenensis Y.H. Mu, H.S. Yuan \& Y.C. Dai, sp. nov.

MycoBank number: MB840455; Facesoffungi number: FoF 09972; Figs. 209, 210, 211

Etymology: The specific epithet "huanrenensis" refers to the Huanren County, where the specimen is found.

Holotype: Yuan 10663 


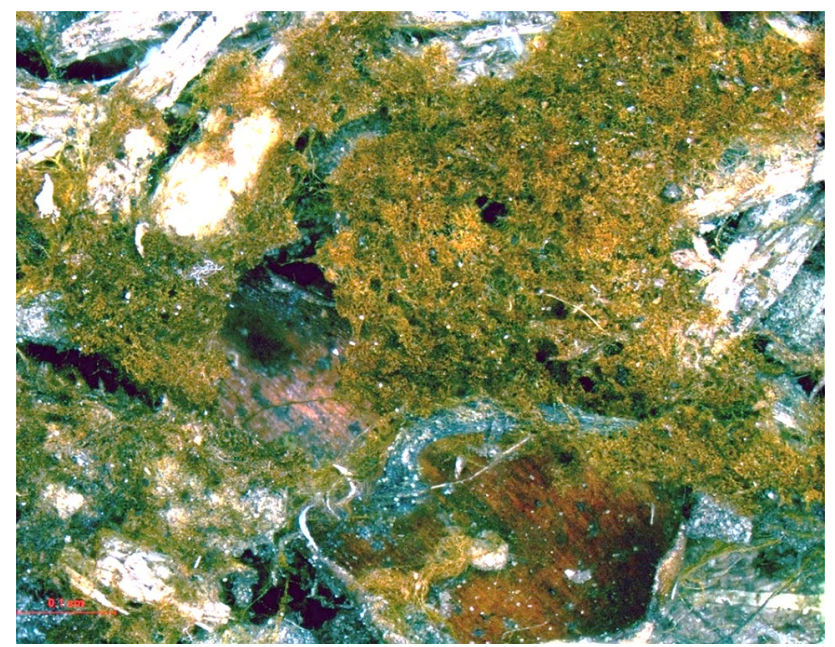

Fig. 209 Odontia huanrenensis (Yuan 10663, holotype): Basidiomata

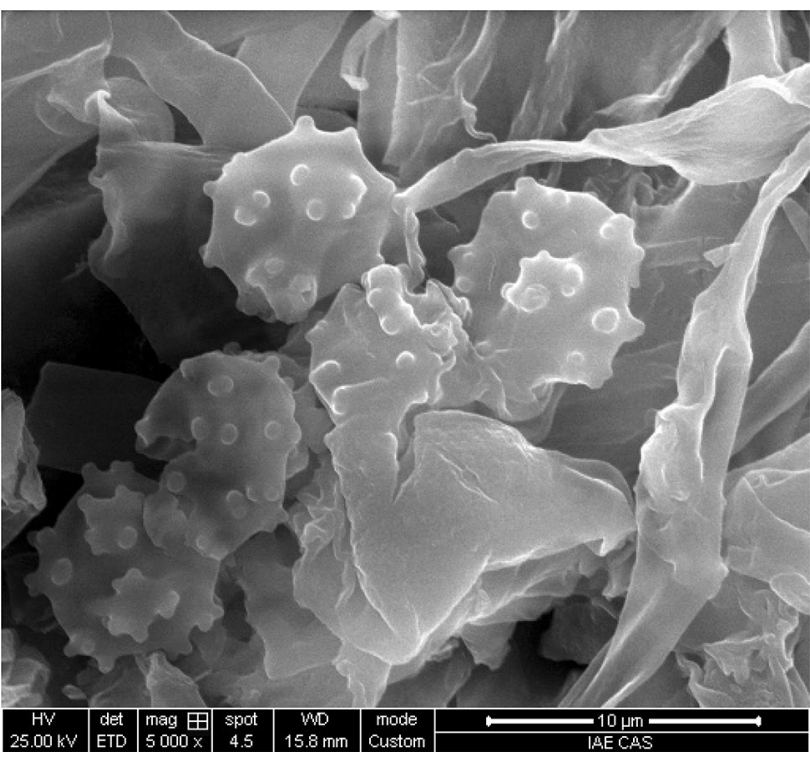

Fig. 210 Odontia huanrenensis (Yuan 10663, holotype): SEM of basidiospores

Basidiomata annual, resupinate, easily separable from the substrate, arachnoid, continuous, without odour or taste when fresh, becoming cottony upon drying, up to $0.25 \mathrm{~mm}$ thick. Hymenophoral surface light brown to brown (6D7-6E8) when dry, hydnoid; spines conical, up to $0.4 \mathrm{~mm}$ long, $0.1-0.2 \mathrm{~mm}$ wide at the base, evenly distributed, $2-4$ per $\mathrm{mm}$, turning darker or concolorous with subiculum. Subiculum mostly brown. Sterile margin determinate, byssoid, concolorous with hymenophore. Rhizomorphs present in subiculum and margins, 10-50 $\mu \mathrm{m}$ diam.; rhizomorph surface rather smooth; hyphal structure in rhizomorphs dimitic, differentiated, of type $\mathrm{C}$ (according to Agerer
1987-2008), compactly arranged; generative hyphae in center of rhizomorph simple-septate, thick-walled, 3-5 $\mu \mathrm{m}$ diam., colorless in $\mathrm{KOH}$; skeletal hyphae at outer part of rhizomorph thick-walled, $1-2 \mu \mathrm{m}$ diam., grayish yellow in $\mathrm{KOH}$, cyanophilous, inamyloid. Hyphal structure subicular hyphae monomitic, generative hyphae with clamp connections, thin- to slightly thick-walled, $2.5-4 \mu \mathrm{m}$ diam., occasionally collapsed, without encrustation, grayish yellow in $\mathrm{KOH}$, acyanophilous, inamyloid. Subhymenial hyphae with clamp connections, thin-walled, 3-4 $\mu \mathrm{m}$ diam.; hyphal cells more or less uniform, colorless in $\mathrm{KOH}$, acyanophilous, inamyloid. Spine tramal hyphae monomitic, generative hyphae with clamp connections, slightly thick-walled, $2.5-4(-5) \mu \mathrm{m}$ diam., grayish yellow in $\mathrm{KOH}$, cyanophilous, inamyloid.

Hymenium cystidia and cystidioles absent. Basidia 18-33 $\mu \mathrm{m}$ long and 5-9 $\mu \mathrm{m}$ diam. at apex, 3-4 $\mu \mathrm{m}$ at base, with a clamp connection at base, clavate, stalked, sinuous, without transverse septa, grayish yellow in $\mathrm{KOH}$ and in distilled water, bearing 4 sterigmata; sterigmata up to $3 \mu \mathrm{m}$ long, 1-2 $\mu \mathrm{m}$ diam. at base. Basidiospores (7-) 7.1-9.4(-9.9) $\times(6-) 6.1-8.3(-9) \mu \mathrm{m}$ in lateral and frontal face, $\mathrm{L}=8.48 \mu \mathrm{m}, \mathrm{W}=7.6 \mu \mathrm{m}, \mathrm{Q}=1.12-1.13(\mathrm{n}=60 / 2)$, irregular globose frontal and ellipsoid lateral face, oildrops absent, verruculose, light brown in $\mathrm{KOH}$ and in distilled water, acyanophilous, inamyloid; warts usually grouped in 2 or more, bi- to trifurcate alike, $1-1.6 \mu \mathrm{m}$ long. Chlamydospores absent.

Material examined: CHINA, Liaoning Province, Huanren County, Laotudingzi Nature Reserve, on fallen angiosperm trunk, 20 October 2015, H.S. Yuan (Yuan 10663, holotype); Qingyuan County, ecological observatory station, on fallen angiosperm trunk, 23 October 2015, H.S. Yuan (Yuan 10741).

GenBank numbers: Yuan 10663: ITS = MW033326; Yuan 10741: ITS = MW033327.

Notes: Odontia huanrenensis is closely related to $O$. aculeata and O. ferruginea in phylogeny (Fig. 215). Odontia huanrenensis is similar to $O$. aculeata in having resupinate, arachnoid, continuous basidiomata separable from the substrate, hydnoid hymenophoral surface, determinate and byssoid sterile margin, a dimitic hyphal system in the rhizomorphs, monomitic and clamped subicular hyphae, the absence of cystidia and irregular globose to ellipsoid and verruculose basidiospores (Figs. 209, 210 and 211). However, $O$. aculeata differs from $O$. huanrenensis in having thinner basidiomata (up to $0.15 \mathrm{~mm}$ vs. up to $0.25 \mathrm{~mm}$ in $O$. huanrenensis), longer spines (up to $1 \mathrm{~mm}$ vs. up to $0.4 \mathrm{~mm}$ in $O$. huanrenensis), clamped and thin-walled generative hyphae in the center of rhizomorph, thin-walled spinal tramal hyphae and shorter basidia (12-15 $\mu \mathrm{m}$ vs. $18-33 \mu \mathrm{m}$ in O. huanrenensis) (Yuan et al. 2018). Odontia huanrenensis shares features with $O$. ferruginea including hydnoid basidiomata, a dimitic hyphal system in the rhizomorphs, clamped 
Fig. 211 Odontia huanrenensis (drawn from the holotype), microscopic structures. a Section through basidiomata. b Section through rhizomorph. c Basidiospores in frontal and lateral view

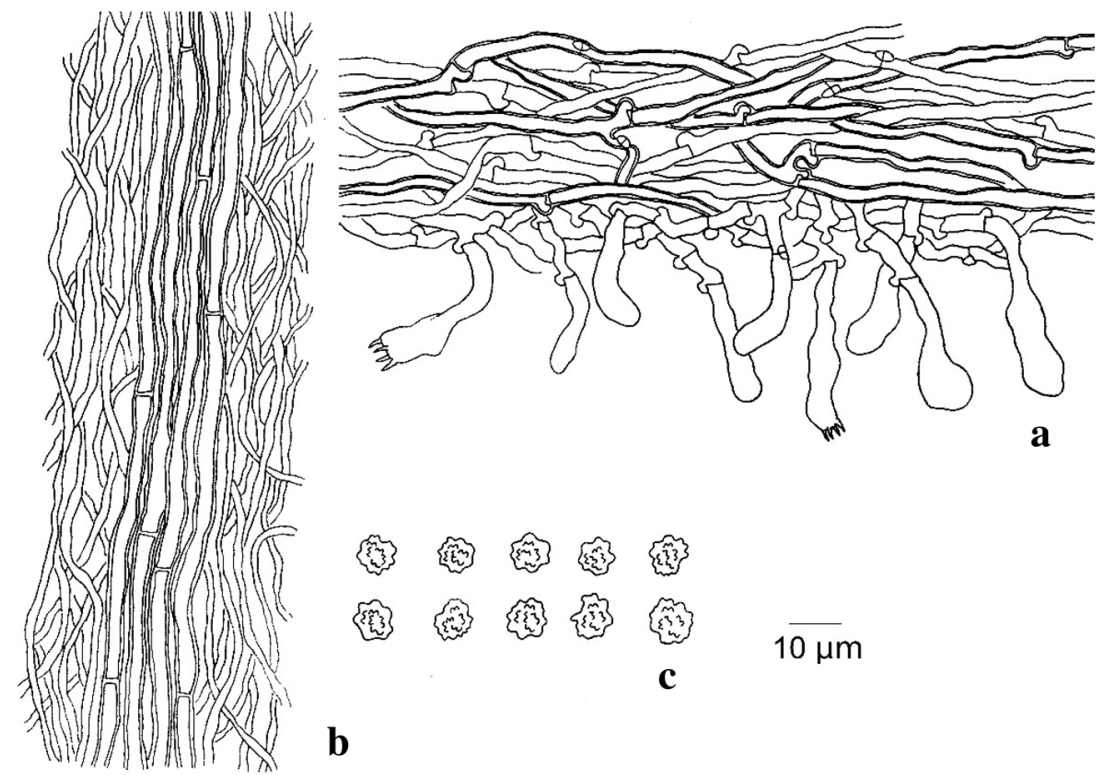

subicular hyphae and verruculose basidiospores of similar shape. However, $O$. ferruginea differs from $O$. huanrenensis in having longer spines (up to $1.75 \mathrm{~mm}$ vs. up to $0.4 \mathrm{~mm}$ in $O$. huanrenensis), the presence of simple-septate and pseudoskeletal hyphae in spine trama and clamped generative hyphae in rhizomorphs (Larsen 1967; Kõljalg 1996).

\section{Odontia parvispina Y.H. Mu, H.S. Yuan \& Y.C. Dai, sp.} nov.

MycoBank number: MB840456; Facesoffungi number: FoF 09973; Figs. 212, 213, 214

Etymology: The specific epithet "parvispina" refers to hymenophoral surface with very small spines.

Holotype: Yuan 10652

Basidiomata annual, resupinate, easily separable from the substrate, arachnoid, continuous, without odour or taste when fresh, becoming cottony upon drying, up to $0.25 \mathrm{~mm}$ thick. Hymenophoral surface yellowish brown to brown (5D8-6E8) when dry, smooth or hydnoid to the naked eye; spines conical, up to $0.06 \mathrm{~mm}$ long, $0.02-0.03 \mathrm{~mm}$ wide at the base, evenly distributed, 4-6 per mm, turning darker than subiculum. Subiculum mostly brown. Sterile margin determinate, byssoid, paler than hymenophore, brown. Rhizomorphs present in subiculum and margins, 10-60 $\mu \mathrm{m}$ diam.; rhizomorph surface rather smooth; hyphal structure in rhizomorphs dimitic, differentiated, of type C (according to Agerer 1987-2008), compactly arranged; generative hyphae in center of rhizomorph simple-septate, slightly thick-walled, 3-4 $\mu \mathrm{m}$ diam., colorless in $\mathrm{KOH}$; skeletal hyphae at outer part of rhizomorph thick-walled, 1-2 $\mu \mathrm{m}$ diam., grayish yellow in $\mathrm{KOH}$, cyanophilous, inamyloid.

Hyphal structure subicular hyphae monomitic, generative hyphae simple-septate, thin- to slightly thick-walled, 3-4 $\mu \mathrm{m}$ diam., occasionally collapsed, without encrustation, grayish yellow in $\mathrm{KOH}$, acyanophilous, inamyloid. Subhymenial hyphae simple-septate, thin-walled, 2-3 $\mu \mathrm{m}$ diam.; hyphal cells more or less uniform, colorless in $\mathrm{KOH}$, acyanophilous, inamyloid. Spine tramal hyphae monomitic, generative hyphae with clamp connections, thick-walled, 3-5(-6) $\mu \mathrm{m}$ diam., grayish yellow in $\mathrm{KOH}$, cyanophilous, inamyloid (Fig. 215).

Hymenium cystidia and cystidioles absent. Basidia 15-44 $\mu \mathrm{m}$ long and 3-8 $\mu \mathrm{m}$ diam. at apex, $2-4 \mu \mathrm{m}$ at base, simple-septate at base, clavate, stalked, sinuous, with transverse septa, grayish yellow in $\mathrm{KOH}$ and in distilled water, bearing 4 sterigmata; sterigmata up to $5 \mu \mathrm{m}$ long, $1-3 \mu \mathrm{m}$ diam at base. Basidiospores (5.5-)6-7(-8) $\times(4-) 5-6.4(-7)$ $\mu \mathrm{m}$ in lateral and frontal face, $\mathrm{L}=6.39 \mu \mathrm{m}, \mathrm{W}=5.68 \mu \mathrm{m}$, $\mathrm{Q}=1.13-1.15(\mathrm{n}=60 / 2)$, irregular subglobose frontal and ellipsoid lateral face, oil drops absent, verruculose, light brown in $\mathrm{KOH}$ and in distilled water, acyanophilous, inamyloid; warts usually grouped in 2 or more, bi- to trifurcate alike, 1-2 $\mu \mathrm{m}$ long. Chlamydospores absent.

Material examined: CHINA, Liaoning Province, Huanren County, Laotudingzi Nature Reserve, on fallen angiosperm trunk, 20 October 2015, H.S. Yuan (Yuan 10652, holotype); Kuandian County, Baishilazi Nature Reserve, on fallen angiosperm branch, 22 October 2015, H.S. Yuan (Yuan 10716).

GenBank numbers: Yuan 10652: ITS = MW033328, Yuan 10716: ITS $=$ MW033329.

Notes: Odontia parvispina is closely related to $O$. fibrosa in the phylogenetic tree (Fig. 215) and share similar morphological and anatomical characteristics: annual, resupinate, arachnoid basidiomata, smooth or hydnoid hymenophoral surface, brown subiculum, the presence of rhizomorphs with 


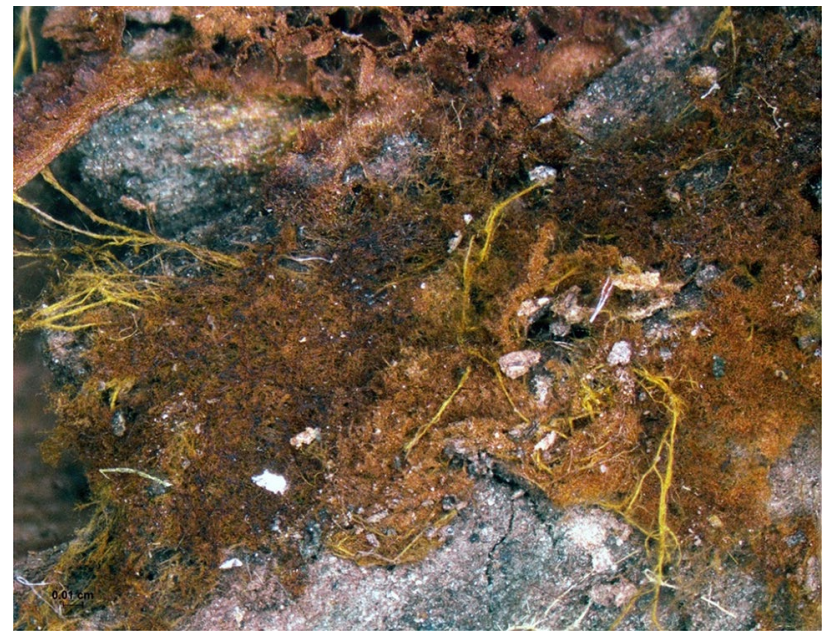

Fig. 212 Odontia parvispina (Yuan 10652, holotype): Basidiomata

dimitic hyphal system, thin- to slightly thick-walled and simple-septate generative hyphae in the subiculum and clavate basidia of similar size (Figs. 212, 213 and 214). However, $O$. fibrosa can be differentiated by thicker basidiomata (up to $0.4 \mathrm{~mm}$ vs. up to $0.25 \mathrm{~mm}$ in $O$. parvispina), dark brown and granulose hymenophoral surface when dry, longer spines (up to $1 \mathrm{~mm}$ vs. up to $0.06 \mathrm{~mm}$ in $O$. parvispina), simple-septate and wider hyphae (5-7 $\mu \mathrm{m}$ vs. 3-5 $\mu \mathrm{m}$ in $O$. parvispina) in spine trama and larger basidiospores $(7.1-9 \times 6.1-8 \mu \mathrm{m}$ vs. 6-7 $\times 5-6.4$ in $O$. parvispina). Odontia sparsa resembles $O$. parvispina in having resupinate and arachnoid basidiomata, a dimitic system in the rhizomorphs with simple-septate and slightly thick-walled generative hyphae and thick-walled skeletal hyphae and verruculose basidiospores. However, the former species differs by thin and clamped generative hyphae, the presence of skeletal hyphae in the subicular and reddish brown basidiospores in $\mathrm{KOH}$ with shorter warts (0.3-0.8 $\mu \mathrm{m}$ vs. $1-2 \mu \mathrm{m}$ in O. parvispina) (Yuan et al. 2018).

\section{Bartheletiomycetes Thines}

\section{Polyporales Gäum.}

Notes: The latest treatments and updated accounts of Polyporales in Phookamsak et al. (2019) and Wijayawardene et al (2020).

\section{Hyphodermataceae Jülich}

Notes: Jülich (1981) described Hyphodermataceae with Hyphoderma as the generic type, also including the genera Athelidium, Atheloderma, Basidioradulum, Bulbillomyces, Conohypha, Granulobasidium, Hyphoderma, Hyphodermella, Hypochnicium, Intextomyces and Metulodontia. Currently, according to the phylogenetic study of Polyporales at the family-level carried out by Justo et al. (2017), only Hyphoderma is considered in this family which includes corticioid species with monomitic hyphal systems, hyphae with clamp-connections; cystidia present in most species; spores thin-walled, smooth, and hyaline.

\section{Hyphoderma Wallr.}

Notes: Hyphoderma is one of the largest genera of corticioid fungi, with 105 recognized species (Index Fungorum 2021). All species of Hyphoderma produce white rot and their main habitat is decaying wood of deciduous and coniferous trees. From a morphological point of view, the species included in this genus are characterized by their basidioma, resupinate and
Fig. 213 Odontia parvispina

(Yuan 10652, holotype): SEM

of basidiospores

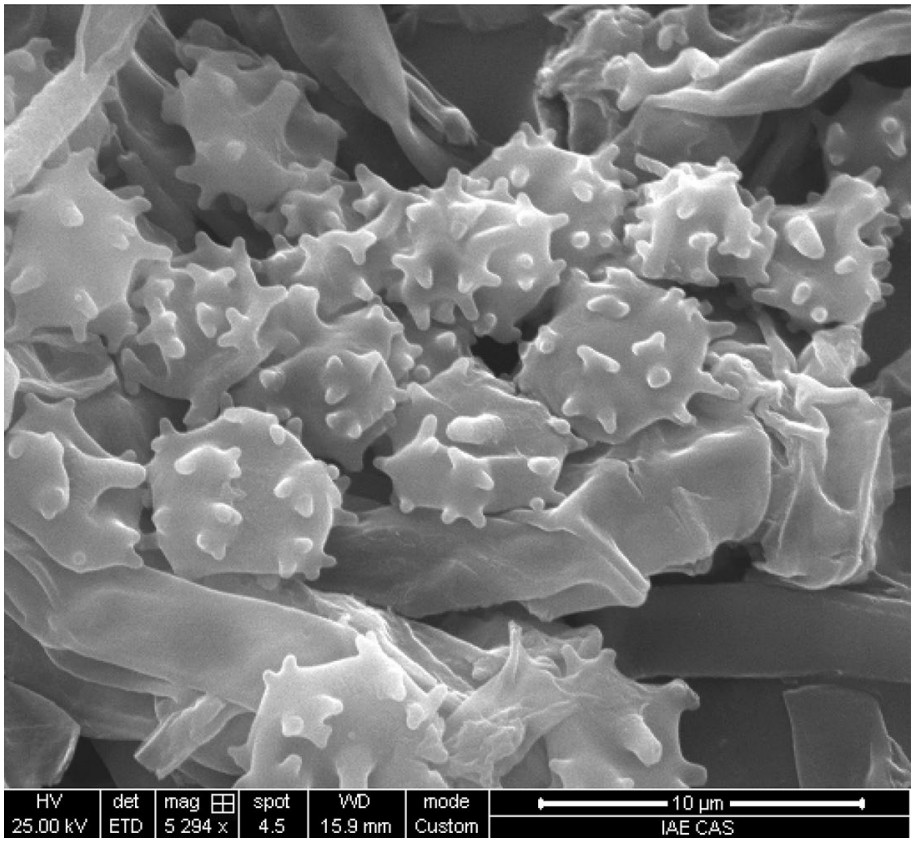


Fig. 214 Odontia parvispina (drawn from the holotype), microscopic structures. a Section through basidiomata. b Section through rhizomorph. c Basidiospores in frontal and lateral view
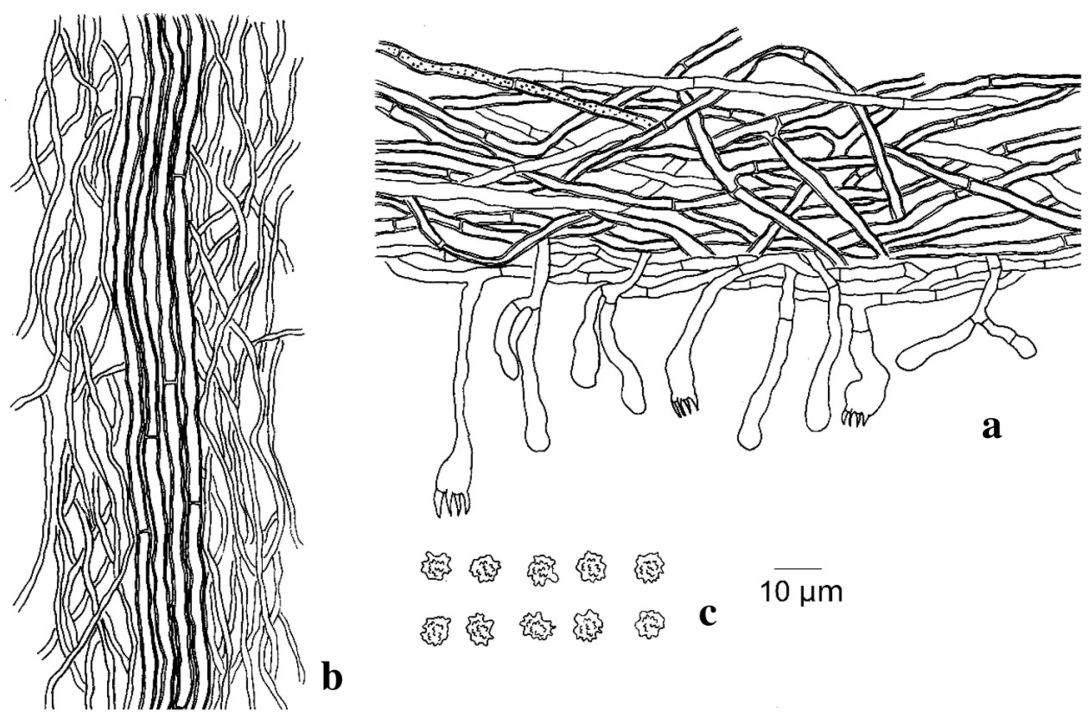

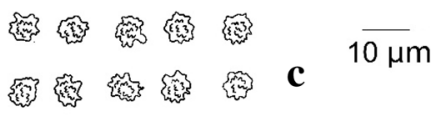

b effused, with hymenophore smooth, tuberculate or hydnoid; a monomitic hyphal system and the hyphae with clamps; cystidia are frequent; basidia are suburniform to subcylindrical, with two or four sterigmata and basal clamps; spores are subglobose, ellipsoid, cylindrical to allantoid, thin-walled, smooth, with oily contents, and non-amyloid (Larsson 2007).

Hyphoderma was described by Wallroth (1833) to accommodate eight species namely Hyphoderma aspera, $H$. fibrillosa, H. granulosa, H. lintaceum, H. spiculosum, H. sulphurea and $H$. terrestre. Long time neglected, this genus was recovered and emended by Donk $(1949,1957)$ who designated $H$. spiculosum Wallr. [= Hyphoderma setigerum (Fr.) Donk] as the generic type. Parmasto (1968) divided it into three sections i.e., Hyphoderma sect. Hyphoderma, Hyphoderma sect. Stephanocystis, and Hyphoderma sect. Mutatoderma, and, several years after, Eriksson and Ryvarden (1975) recognized twelve groups: Hyphoderma albocremeum-group, $H$. argillaceum-group, $H$. capitatum-group, $H$. cremeoalbum-group, $H$. guttuliferum-group, $H$. mutatumgroup, $H$. orphanellum-group, $H$. praetermissum-group, $H$. puberum-group, $H$. radula-group, $H$. roseocremeum-group and $H$. setigerum-group.

Hyphoderma setigerum-group was characterized by its cystidia, which are multi-septate, clamped and more or less encrusted (Eriksson and Ryvarden 1975) and was represented by only one species, $H$. setigerum, widely distributed with great variation in both macro and microscopic characters. Based on molecular, morphological and crossing test analyses, Nilsson et al. (2003) concluded that H. setigerum is a species complex and nine taxa exist inside it. Moreover, a succession of studies (Gilbertson and Blackwell 1988; Wu 1997; Boidin and Gilles 2003; Yurchenko and Wu 2014) described four more species with this type of cystidia: Hyphoderma nudicephalum from Louisiana, USA, Hyphoderma subsetigerum from Taiwan, Hyphoderma bisetigerum from Madagascar, and Hyphoderma pinicola from China.

Hyphoderma australosetigerum M. Dueñas, Telleria \& M.P. Martín, sp. nov.

MycoBank number: MB834614; Facesoffungi number: FoF 09204; Fig. 216

Etymology: From Latin "australis" which means southern and "setigerum" refers to morphological similarity of this species with Hyphoderma setigerum (Fr.) Donk.

Holotype: 13970MD, MA-Fungi 92235

Colour codes follow: ISCC-NBS Centroid Colour Charts (Kelly and Judd 1976)

Basidiomata resupinate, orbicular to confluent, porosereticulate under the lens, adnate; hymenophore smooth, under the lens pilose by the projecting cystidia; white (92. Y White) to greyish (264. L Gray). Margin thin, fimbriate, not differentiated. Hyphal system monomitic. Hyphae thin- to thick-walled, 3-4 $\mu \mathrm{m}$ wide, with clamps, loosely interwoven in the subiculum and more densely interwoven in the subhymenium. Cystidia cylindrical, multi-septate, with clamps, thick-walled except for the apical cell, at first naked then encrusted, apex obtuse, 108-160×10-11 $\mu \mathrm{m}$. Basidia subclaviform to claviform, four sterigmata, $18-30 \times 5-6 \mu \mathrm{m}$, with basal clamp. Spores narrowly ellipsoid to cylindrical, 9-11 $(-12) \times 4.5-5(-5.5) \mu \mathrm{m},(\bar{x}=10 \times 5 \mu \mathrm{m})$, hyaline, thin-walled, smooth, with oil drops in the protoplasm, $\mathrm{Q}$ $(\mathrm{L} / \mathrm{W})=2$.

Habitat and distribution: Frequent in the Chilean Patagonian region, both on typical species of the Valdivian temperate rainforest such as Lophosoria quadripinnata, Eucryphia cordifolia, and in plantations of Eucalyptus globulus or Pinus radiata.

Material examined: CHILE, Los Lagos, Palena, Hornopirén, comuna Hualaihué, Huinay Biological Reserve, 


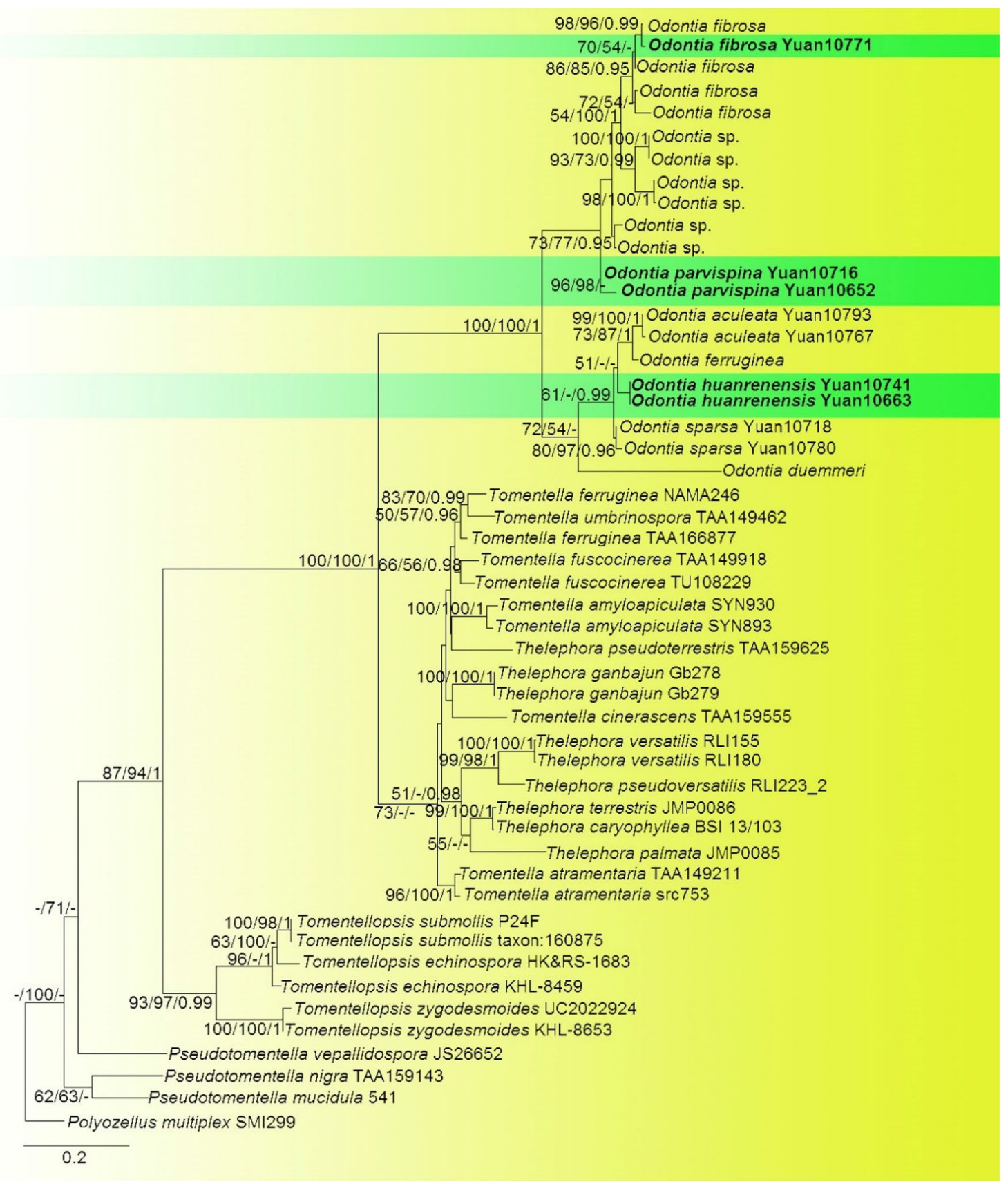

Fig. 215 Maximum likelihood tree illustrating the phylogeny of Odontia huanrenensis, $O$. parvispina and related taxa based on ITS sequence dataset. Fifty sequences are included in the analyses. Polyzellus multiplex SMI299 was selected as the outgroup taxa. Branches

path to Cerro del Tambor, $42^{\circ} 22^{\prime} 50.9^{\prime \prime} \mathrm{S} 72^{\circ} 24^{\prime} 54.7^{\prime \prime} \mathrm{W}$, $34 \mathrm{msl}$, on Eucryphia cordifolia, 25 April 2012, M. Dueñas, M.P. Martín and M.T. Telleria, 13970MD (MA-Fungi 92235, holotype); 13995MD (MA-Fungi 92240); on fallen branches, 13983MD (MA-Fungi 92234). Los Lagos, Palena, Hornopirén, comuna Hualaihué, Huinay Biological Reserve, path to Cerro del Tambor, $42^{\circ} 22^{\prime} 50.6^{\prime \prime} \mathrm{S} 72^{\circ} 24^{\prime} 50.6^{\prime \prime} \mathrm{W}$, are labeled with maximum likelihood bootstrap support greater than 50\% (MLBS), parsimony bootstrap proportions greater than $50 \%$ (MPBS) and Bayesian posterior probabilities greater than 0.95 (BYPP). The new species and new record are in bold
202 msl, on Lophosoria quadripinnata, 7 May 2013, M. Dueñas, M.P. Martín and M.T. Telleria, 20070Tell. (MAFungi 92236); on unidentified wood, 20089Tell. (MAFungi 92233). Los Lagos, Palena, Hornopirén, comuna Hualaihué, Huinay Biological Reserve, path to Cerro del Tambor, behind the hydroelectric power station, $42^{\circ} 22^{\prime} 54.2^{\prime \prime}$ S 72 $24^{\prime} 53.5^{\prime \prime} \mathrm{W}, 202 \mathrm{msl}$, on Lophosoria quadripinnata, 


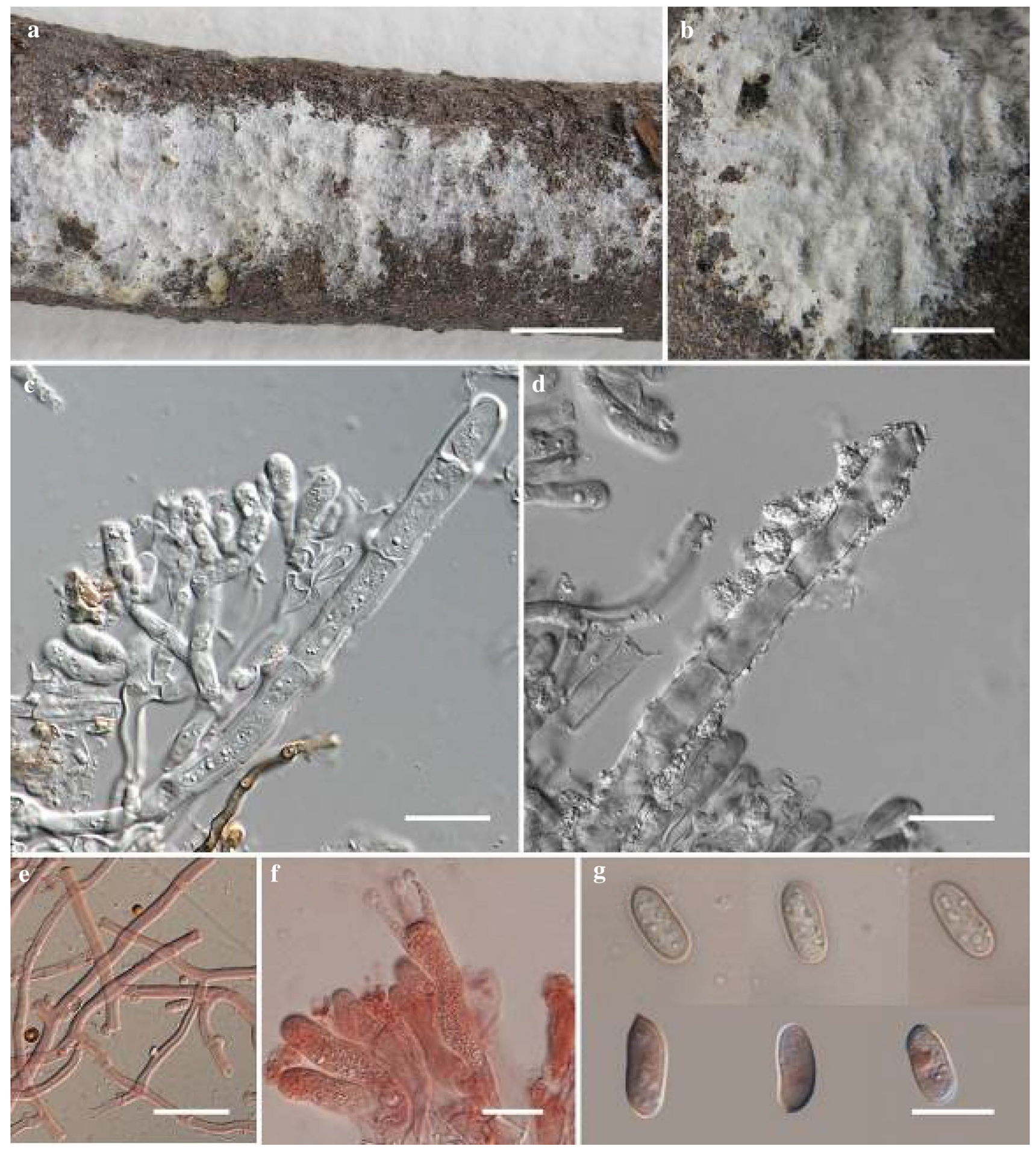

Fig. 216 Hyphoderma australosetigerum (13970MD, MA-Fungi 92235, holotype). a Basidiomata dry specimen. b Hymenophore. c Septate cystidium. d Septate and encrusted cystidium. e Basal hyphae

8 May 2013, M. Dueñas, M.P. Martín and M.T. Telleria, 14580MD (MA-Fungi 92239). Los Lagos, Palena, Hornopirén, comuna Hualaihué, Huinay Biological Reserve, "Cementerio de los Alerces" experimental plot, $42^{\circ} 22^{\prime} 01.5^{\prime \prime}$ thick-walled, with clamps. $\mathbf{f}$ Basidium and probasidia. g Spores. Scale bars: $\mathbf{a}, \mathbf{b}=5 \mathrm{~mm}, \mathbf{c}-\mathbf{e}=20 \mu \mathrm{m}, \mathbf{f}-\mathbf{g}=10 \mu \mathrm{m}$

S 72 $24^{\prime} 57.8^{\prime \prime} \mathrm{W}, 50 \mathrm{msl}$, on unidentified wood, 10 May 2013, M. Dueñas, M.P. Martín and M.T. Telleria, 3636MPM (MA-Fungi 92241). Los Ríos, Ranco, comuna de La Unión,

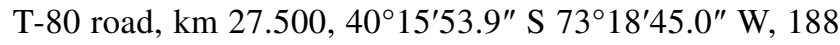


msl, on Pinus radiata, 6 November 2017, M. Dueñas, J. Fernández López, M.P. Martín, S. Nogal-Prata and M.T. Telleria, 15624MD (MA-Fungi 92238). Los Ríos, Ranco, comuna de La Unión, T-80 road, km 18, Putraro detour, $40^{\circ} 18^{\prime} 12.0^{\prime \prime} \mathrm{S} 73^{\circ} 14^{\prime} 15.0^{\prime \prime} \mathrm{W}, 82 \mathrm{msl}$, on Eucalyptus globulus, 6 November 2017, M. Dueñas, J. Fernández López, M.P. Martín, S. Nogal-Prata and M.T. Telleria, 508JFL (MAFungi 92237).

GenBank numbers: 13970MD, MA-Fungi 92235 (holotype): ITS $=$ MN963764; 13995MD, MA-Fungi 92240: ITS $=$ MN963760; 13983MD, MA-Fungi 92234: ITS $=$ MN963763; 20070Tell., MA-Fungi 92236: ITS $=$ MN963765; 20089Tell., MA-Fungi 92233: ITS $=$ MN963762; 14580MD, MA-Fungi 92239: ITS $=$ MN963759; 3636MPM, MA-Fungi 92241: ITS $=$ MN96376; 15624MD, MA-Fungi 92238: ITS $=$ MN963758; 508JFL, MA-Fungi 92237: ITS $=$ MN963757.

Notes: Based on ITS analysis (Fig. 217), 11 sequences of Hyphoderma australosetigerum clustered together in their own clade (68\% MPBS, 88\% MLBS, 1.00 BYPP); nine obtained from our Chilean specimens and two from GenBank sequences from Argentina. Two sequences from GenBank under $H$. setigerum from South Africa are quite close to those of the new species. Hyphoderma australosetigerum grouped close to different clades from Nilsson et al. (2003) under H. setigerum: 1A (Germany, Norway, Romania and Russia), 1B (Greenland), 2 (Canada), 3A (Canada), 3B (USA), 4 (Norway, UK), and 6 (Canada). As well as with two sequences from GenBank under $H$. setigerum from the USA, and clade 5 (Nilsson et al. 2003) from China and Japan under $H$. nudicephalum. On the other hand, $H$. setigerum s.str and $H$. subsetigerum (clades 7A and 7B) from Nilsson et al. (2003) grouped close to $H$. pinicola described from China by Yurchenko and $\mathrm{Wu}$ (2014).

From a morphological point of view, $H$. australosetigerum (Fig. 216) differs from $H$. bisetigerum and H. pinicola in that they have bisporic basidia; from $H$. nudicephalum by the cystidia with swollen apex, characteristic of this species; from $H$. subsetigerum by the size of spores, 6-8 $\times 2.8-3.2 \mu \mathrm{m}$ in H. substigerum, while $H$. australosetigerum has spores $9-11(-12) \times 4.5-5(-5.5) \mu \mathrm{m}$. This species is difficult to separate from $H$. setigerum s. str.; molecular characters and their geographic origin are useful for its identification. Hyphoderma australosetigerum is frequent in Patagonia region, where it probably has been confused with $H$. setigerum reported by Gorjón and Hallenberg (2013) from Chile and by Greslebin and Rajchenberg (2003) from Argentina.

Irpicaceae Spirin \& Zmitr.

Notes: Irpicaceae (Polyporales) was described by Spirin and Zmitovich in Spirin (2003), with Irpex as the generic type, to accommodate Antrodiella, Fabisporus, and Phyllotopsis. Currently, according to Justo et al. (2017), the family includes the following genera Byssomerulius, Ceriporia, Cytidiella, Efibula, Emmia, Flavodon, Gloeoporus, Hydnopolyporus, Leptoporus, Meruliopsis and Trametopsis, as well as several more additional species. These taxa include a great assortment of basidiomata and hymenophore types; microscopically, they are characterized by a hyphal system that is monomitic, rarely dimitic, and most hyphae are without clamps; cystidia are usually absent and spores thin-walled, smooth and hyaline. All species are saprobes, growing on decayed wood, and most of them are associated with white rot.

\section{Efibula Sheng $\mathrm{H}$. Wu}

Notes: Efibula was described by Wu (1990) to accommodate species previously included in Phlebia (Ph. deflectens) and Phanerochaete (Ph. avellanea and Ph. pallidovirens, in addition, two new species Efibula lutea and Efibula tropica were also described and proposing E. tropica as the generic type. Two more species were accommodated in the genus by Kotiranka and Saarenoska (1993) i.e., Efibula rosea and E. verruculosa. Zmitrovich et al. (2006) enlarged the genus with eight more species, E. aurata, E. bubalina, E. cordylines, E. corymbata, E. ginnsii, E. subodontoidea, E. subquercina and E. tuberculata. Subsequent phylogenetic analysis (Wu et al. 2010) showed that Efibula sensu Zmitrovich et al. (2006) was polyphyletic and, according to Floudas and Hibbett (2015), only five species were accepted in the genus namely E. americana, E. clarki, E. gracilis, E. tropica and E. tuberculata.

From a morphological point of view, the species included in this genus are characterized by their basidiomata resupinate, effused, subceraceous to ceraceous; smooth hymenophore; compact subiculum, with hyphae densely interwoven; monomitic hyphal system, hyphae mostly without clamps; cystidia absent; basidia with four sterigmata; spores ellipsoid to broadly ellipsoid, thin-walled, smooth and hyaline, Melzer (-), and acyanophilous (Wu 1990; Floudas and Hibbett 2015).

Efibula rodriguezarmasiae Telleria, M. Dueñas, BeltránTej., Melo, Salcedo \& M.P. Martín, sp. nov.

MycoBank number: MB834615; Facesoffungi number: FoF 09205; Fig. 218

Etymology: Named after the Canary mycologist J. Laura Rodríguez-Armas, "Susi", colleague and friend.

Holotype: 17681Tell., MA-Fungi 86635

Colour codes follow: ISCC-NBS Centroid Colour Charts (Kelly and Judd 1976)

Basidiomata resupinate, effuse, ceraceous, when dry specimens pale to dark orange yellow or light yellowish brown (73. p. OY - 72. d. OY, 76. L. y Br); hymenophore 


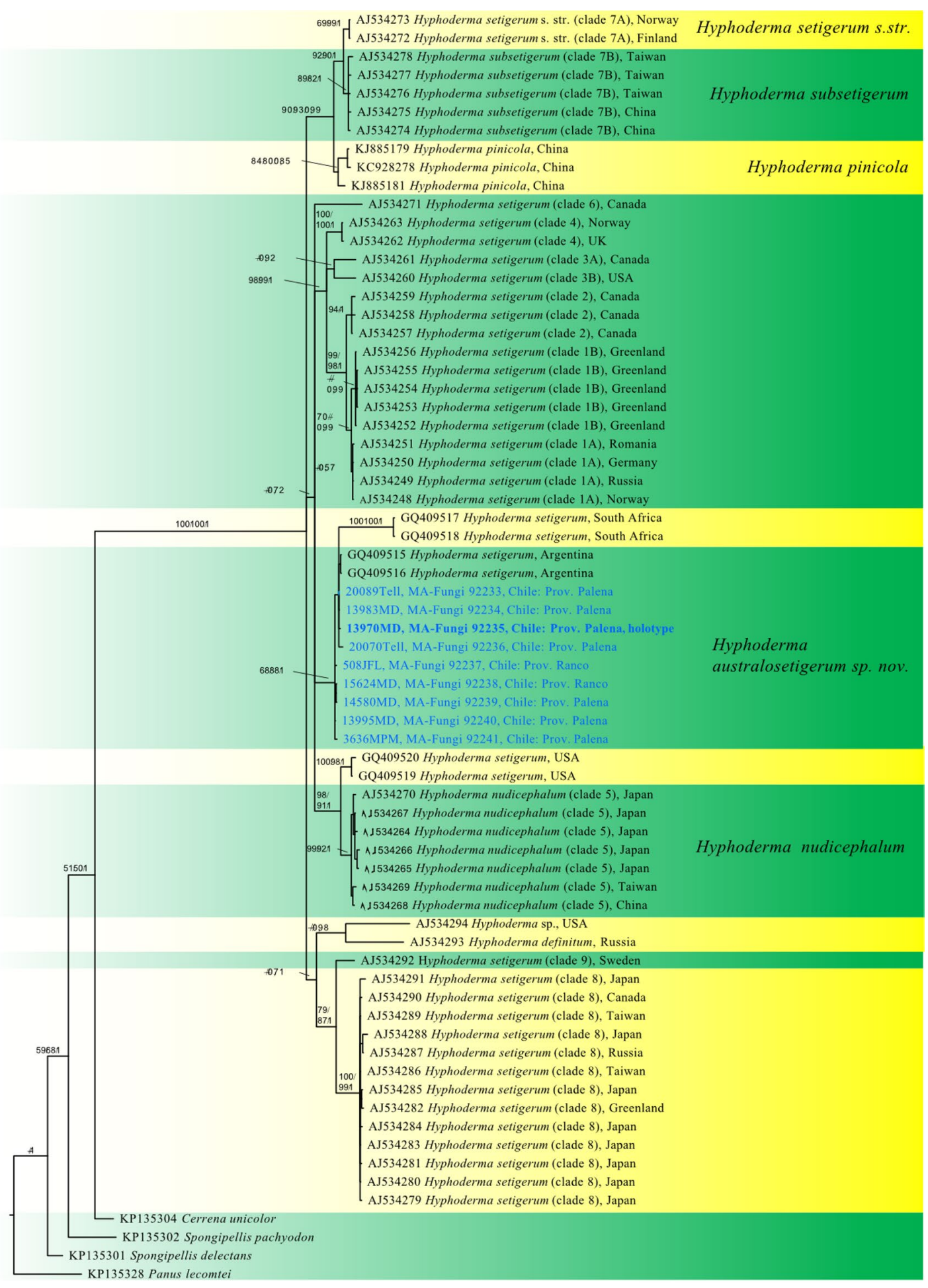

02

Fig. 217 Phylogram generated from Bayesian analysis based on newly-generated ITS sequence data and including sequences mainly from Nilsson et al. (2003) and Yurchenko and Wu (2014); Cerrena unicolor, Panus lecomtei, Spongipellis delectans and S. pachyodon are included as outgroup. The alignment comprises 701 characters.
Maximum parsimony (MP) and maximum likelihood (ML) analyses were also performed; the tree topologies were similar to the Bayesian one. Bootstrap values for MP and ML analyses equal to or greater than $50 \%$, and posterior probabilities of the Bayesian analysis are labeled on the nodes. The newly generated sequence is in blue bold 

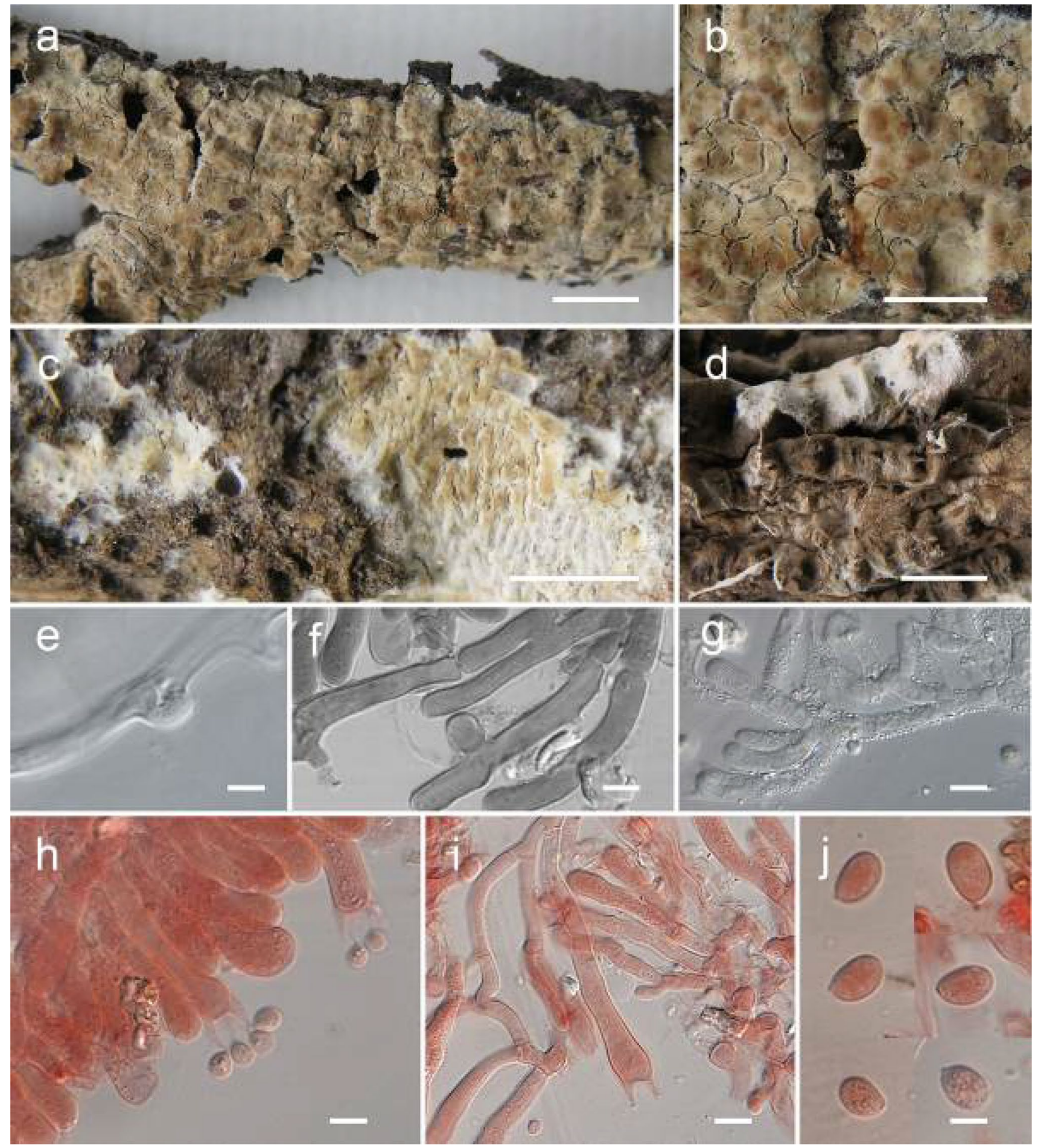

Fig. 218 Efibula rodriguezarmasiae (17681Tell., MA-Fungi 86635, holotype). a Basidiomata dry specimen. b Hymenophore. c, d Basidiomata young and dry specimen. e Basal hyphae with clamp. $\mathbf{f}$ Hyphae with a vesicle. g Basidia, probasidia and subhymenial hyphae with thin crystals. h, i Basidia. j Spores. Efibula rodriguezarmasiae (18761Tell., MA-Fungi 86652). d Basidiomata with little strands. Scale bars: $\mathbf{a}-\mathbf{d}=5 \mathrm{~mm}, \mathbf{e}-\mathbf{j}=5 \mu \mathrm{m}$

with occasional clamps, and small vesicles; subhymenial hyphae $4 \mu \mathrm{m}$ wide, without clamps, short celled, sometimes with very thin crystals scattered on the hyphae. Cystidia tinct, little strands present in some specimens. Hyphal system monomitic, subicular hyphae 4-6 $\mu$ m wide, thin walled, 
absent. Basidia claviform, 35-48 $\times 6-8 \mu \mathrm{m}$, with four sterigmata, basal clamp absent. Spores ellipsoid, 6-7×4-5 $\mu \mathrm{m}$ $(\mathrm{L} / \mathrm{W}=1.4)$, thin-walled, smooth, colorless.

Habitat and distribution: This species is distributed throughout the Macaronesian region: Canary Archipelago and West coast of Morocco, growing on debris and decayed wood of Pericallis murrayi, Euphorbia regis-jubae, Euphorbia balsamifera, Euphorbia officinarum and Kleinia neriifolia, among others.

Material examined: SPAIN, Canary Islands: El Hierro, Frontera, Sabinar de la Dehesa, 2744'43.97" N 18 $07^{\circ} 2.01^{\prime \prime}$ W, 610 msl, 26 January 2007, on Pericallis murrayi, E. Beltrán-Tejera, J. Cardoso, M. Dueñas, J. Leal, I. Melo, J.L. Rodríguez-Armas and M.T. Telleria, 11400MD (MAFungi 86626). Gran Canaria, Aldea de San Nicolás, barranco de Tasarte, $672 \mathrm{msl}$, on Euphorbia regis-jubae, 6 December 2007, E. Beltrán-Tejera, M. Dueñas, J.L. Rodríguez-Armas and M.T. Telleria, 17681Tell. (MA-Fungi 86635, holotype). Lanzarote, Haría, Órzola, 29² 12'54.50" N 13²7'3.92" W, 30 msl, 2 December 2007, on Nicotiana glauca, E. Beltrán-Tejera, M. Dueñas, J.L. RodríguezArmas and M.T. Telleria, 12233MD (MA-Fungi 86638). Lanzarote, Teguise, Arrieta, $30 \mathrm{msl}$, on Euphorbia balsamifera, 2 December 2007, E. Beltrán-Tejera, M. Dueñas, J.L. Rodríguez-Armas and M.T. Telleria, 17629Tell. (MA-Fungi 86633); on Kleinia neriifolia, 2 December 2007, 17633Tell. (MA-Fungi 86632). Lanzarote, Parque Nacional de Timanfaya, Yaiza, El Mojón, 2902'38.86" N $13^{\circ} 45^{\prime} 08.66^{\prime \prime}$ W, 150 msl, 3 December 2007, on Euphorbia balsamifera, E. Beltrán-Tejera, M. Dueñas, J.L. Rodríguez-Armas and M.T. Telleria, 12238MD (MA-Fungi 86637). Lanzarote, Parque Nacional de Timanfaya, Yaiza, Tinajo, next to Montaña Bermeja, $114 \mathrm{msl}$, on Nicotiana glauca, 3 December 2007, E. Beltrán-Tejera, M. Dueñas, J.L. Rodríguez-Armas and M.T. Telleria, 17657Tell. (MAFungi 86631). Fuerteventura, Betancuria, 357 msl, on Pinus sp., 4 December 2007, E. Beltrán-Tejera, M. Dueñas, J.L. Rodríguez-Armas and M.T. Telleria, 17665Tell. (MA-Fungi 86634). MOROCCO, Souss-Massa-Daraa, road Tiznit to Anglou Plage, 29 45'29.3" N 09 46'42.2" W, 158 msl, on Euphorbia sp., 20 April 2010, J. Cardoso, M. Dueñas, I. Melo, M.P. Martín, I. Salcedo and M.T. Telleria, 18699Tell. (MA-Fungi 86648). Souss-Massa-Daraa, road N1, Agadir to Essaouira, next to Tamri, 30³7'36.9" N 09 $50^{\prime} 57.4^{\prime \prime} \mathrm{W}, 23$ msl, on Euphorbia officinarum, 21 April 2010, J. Cardoso, M. Dueñas, I. Melo, M.P. Martín, I. Salcedo and M.T. Telleria, 18761Tell. (MA-Fungi 86652). Souss-Massa-Daraa, road R104, Tiznit to Mirleft, 29³7'21.7" N 09 $53^{\circ}$ '05.3" W, 278 msl, on Euphorbia sp., 21 April 2010, J. Cardoso, M. Dueñas, I. Melo, M.P. Martín, I. Salcedo and M.T. Telleria, 18704Tell. (MA-Fungi 86650); 18743Tell., MA-Fungi 86651. Souss-Massa-Daraa, road R104, Mirleft to Sidi Ifni, $29^{\circ} 32^{\prime} 15.4^{\prime \prime} \mathrm{N} 10^{\circ} 03^{\prime} 24.1^{\prime \prime} \mathrm{W}, 50 \mathrm{msl}$, on Euphorbia sp., 20
April 2010, J. Cardoso, M. Dueñas, I. Melo, M.P. Martín, I. Salcedo and M.T. Telleria, 18701Tell. (MA-Fungi 86649).

Other material examined: Phanerochaete sp.: SPAIN, Canary Islands, Lanzarote, Haría, Órzola, $29^{\circ} 12^{\prime} 54.50^{\prime \prime} \mathrm{N}$ $13^{\circ} 27^{\prime} 3.92^{\prime \prime}$ W, 30 msl, on Nicotiana glauca, 2 December 2007, E. Beltrán-Tejera, M. Dueñas, J.L. Rodríguez-Armas and M.T. Telleria, 12234MD (MA-Fungi 86625). Efibula tuberculata: SPAIN, Canary Islands, La Palma, Santa Cruz de La Palma, Parque Natural de Las Nieves, road to Roque de los Muchachos, next to César Manrique monument, $28^{\circ} 45^{\prime} 05.36^{\prime \prime} \mathrm{N} 17^{\circ} 50^{\prime} 06.70^{\prime \prime} \mathrm{W}, 2,137 \mathrm{msl}, 20 \mathrm{Janu}-$ ary 2007, on Adenocarpus viscosus subsp. spartioides $\mathrm{E}$. Beltrán-Tejera, J. Cardoso, J. Leal, J.L. Rodríguez-Armas and M.T. Telleria, 16871Tell. (MA-Fungi 86621). Corticium tuberculatum P. Karst. [三 Efibula tuberculata]: FINLAND, Etelä - Maine (EH/Ta). Temmela, Mustiala, in populo, 7 October 1895, P.A. Karsten 1503 (H6003416, lectotype). Phanerochaete xerophila Burdsall: USA, Arizona, Cochise County, San Pedro River Rd., between Redington and Benson, on Prosopis velutina, 26 February 1971, R.L. Gilbertson, RLG-10082 (CFMR, holotype); idem (MA-Fungi 63223, isotype).

GenBank numbers: Efibula rodriguezarmasiae, 11400MD, MA-Fungi 86626: ITS = KF483015, LSU $=$ KF528106; 17657Tell., MA-Fungi 86631: ITS $=$ KF483020, LSU = KF528111; 17633Tell., MA-Fungi 86632: ITS $=$ KF483021, LSU $=$ KF528112; 17629Tell., MA-Fungi 86633: ITS $=$ KF483022, LSU $=$ KF528113; 17665Tell., MA-Fungi 86634: ITS $=$ KF483023, LSU $=$ KF528114; 17681Tell., MA-Fungi 86,635 (holotype): $\mathrm{ITS}=\mathrm{KF} 483024$, LSU $=\mathrm{KF} 528115 ; 12238 \mathrm{MD}$, MA-Fungi 86,637: ITS $=$ KF483026, LSU $=$ KF528117; 12233MD, MA-Fungi 86,638: ITS = KF483027, LSU $=$ KF528118; 18699Tell., MA-Fungi 86648: ITS $=$ KF483041, LSU = KF528132; 18701 Tell., MA-Fungi 86649: ITS $=$ KF483039, LSU = KF528130; 18704Tell., MA-Fungi 86650: ITS $=$ KF483040, LSU $=$ KF528131; 18743Tell., MA-Fungi 86651: ITS $=$ KF483042, LSU $=$ KF528133; 18761Tell., MA-Fungi 86652: ITS $=$ KF483038, LSU $=$ KF528129. Efibula tubercu lata, 16871Tell., MA-Fungi 86621: ITS = KF483011, $\mathrm{LSU}=\mathrm{KF} 528102$. Phanerochaete sp, 12234MD, MA-Fungi 86625: ITS $=$ KF483014, LSU $=$ KF528105. Phanerochaete xerophila (isotype), MA-Fungi 63223: ITS $=$ KF483056, $\mathrm{LSU}=\mathrm{KF} 528147$.

Notes: Based on ITS and LSU analysis (Fig. 219), 13 sequences of Efibula rodriguezarmasiae clustered together in its own clade (93\% MPBS, 73\% MLBS, 1.00 BYPP) and appear as sister species of a clade formed by nine sequences of Efibula americana (85\% MPBS, 84\% MLBS, 1.00 BYPP). From a morphological point of view, these species can be separated by the shape and size of basidia and spores (Fig. 218). Efibula americana has cylindrical to clavate 


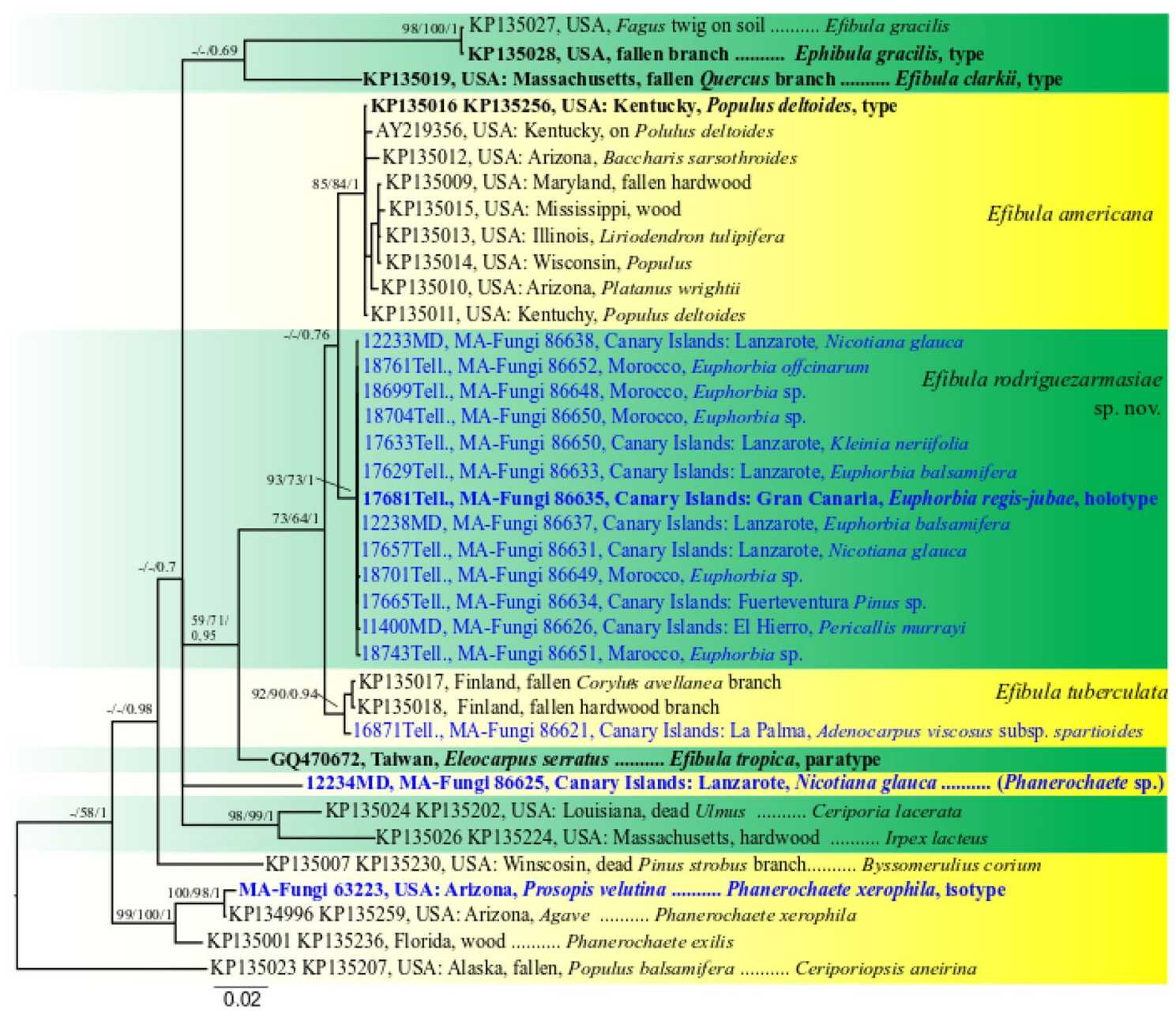

Fig. 219 Phylogram generated from Bayesian analysis based on new generated ITS + LSU sequences data and including sequences from clades 5 (Phanerochaete s.l.) and 6 (Efibula) in Floudas and Hibbett (2015). The sequence of Efibula tropica (paratype) is included (Floudas and Hibbett 2015: Fig. S5B, 1) and Ceriporiopsis aneirina as outgroup. The alignment comprises 2061 characters. Maximum par-

basidia, 20-32 $\times 5-8 \mu \mathrm{m}$, and ellipsoid to cylindrical spores, $(4.5-) 5.3-6.5(-7.3) \times(2.3-) 3-3.8(-4.5) \mu \mathrm{m}, \mathrm{L} / \mathrm{W}=1.71$ (Floudas and Hibbett 2015), while E. rodriguezarmasiae has clavate and longer basidia, 35-48 $\times 6-8 \mu \mathrm{m}$, and ellipsoid and wider spores, $6-7 \times 4-5 \mu \mathrm{m}, \mathrm{L} / \mathrm{W}=1.4$. Furthermore, the phylogenetic analysis indicated that these two species grouped with three sequences of Efibula tuberculata (73\% MPBS, 64\% MLBS, 1.00 BYPP) that, according to Floudas and Hibbett (2015), can be confused with E. americana without characters to easily separate one from the other. The thirteen collections of the new species have been reported from arid, semiarid or dry zones of the Canary Islands and Morocco where it is frequent and, probably, has been confused with Phanerochaete xerophila (Beltrán-Tejera and Rodríguez-Armas 1999; Beltrán-Tejera et al. 2013). simony (MP) and maximum likelihood (ML) analyses were also performed; the tree topologies were similar to the Bayesian one. Bootstrap values for MP and ML analyses equal to or greater than $50 \%$, and posterior probabilities to the Bayesian analysis are labeled on the nodes. The newly generated sequences are in blue bold

\section{Phanerochaetaceae Jülich}

Notes: Phanerochaetaceae is one of the three largest subclades of the phleboid clade of Polyporales, Basidiomycota (Miettinen et al. 2016; Justo et al. 2017). The family includes about 17 known genera, most of which are corticioid fungi with Phanerochaete, Phlebiopsis and Rhizochaete. as the three largest genera in the family (Floudas and Hibbett 2015; Miettinen et al. 2016; Nakasone et al. 2017). Although several new genera were described recently by different mycologists (Miettinen et al. 2016; Yuan et al. 2017; Chen et al. 2018; Ma and Zhao 2019), the phylogeny of the family still needs further study. All species in the family are saprobes and cause a white rot on many kinds of substrates.

Phanerochaete P. Karst. 

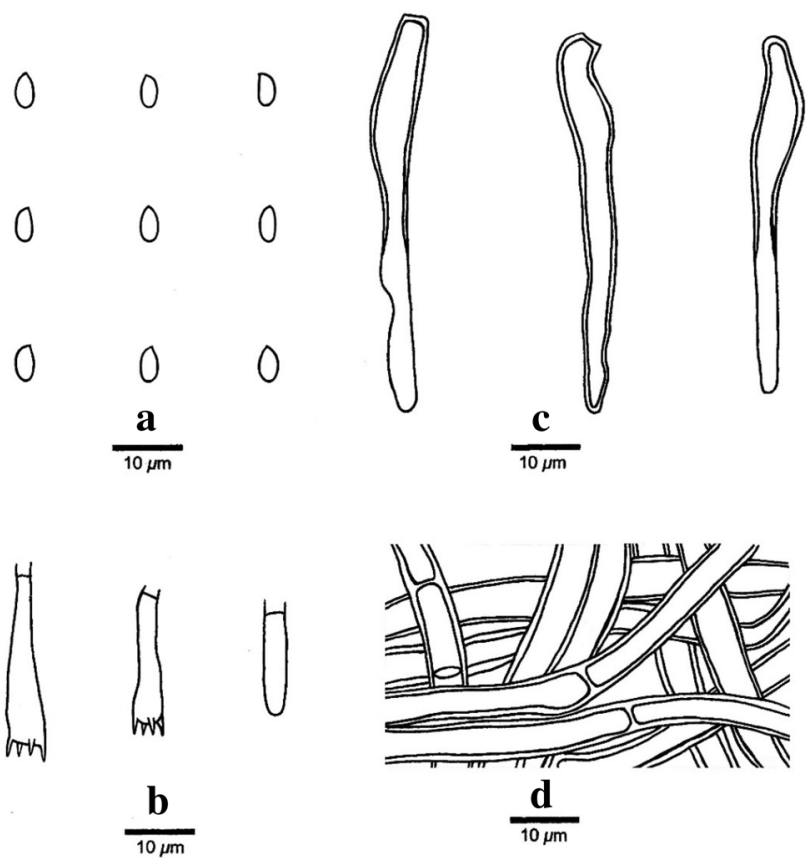

Fig. 220 Phanerochaete hainanensis (BJFC 022063, holotype). a Basidiospores. b Basidia and basidiole. c Cystidia. d Hyphae from subiculum

Notes: Recent molecular studies demonstrate that Phanerochaete s.l. is polyphyletic and distributed across several lineages in the phlebioid clade of the Polyporales and also the Hymenochaetales (De Koker et al. 2003; Greslebin et al. 2004; Wu et al. 2010; Floudas and Hibbett 2015; Miettinen et al. 2016; Justo et al. 2017; Chen et al. 2018). In several studies, Phanerochaete s.s. which includes the type, P. alnea (Fr.) P. Karst., formed a strongly supported clade in Phanerochaetaceae (Floudas and Hibbett 2015; Miettinen et al. 2016; Spirin et al. 2017). Many new species were described in Phanerochaete s.s. based on both morphological and molecular evidence in recent years (Ghobad-Nejhad et al. 2015; Volobuev et al. 2015; Liu and He 2016; Sádlíková and Kout 2017; Spirin et al. 2017; Wu et al. 2018a, b; Phookamsak et al. 2019; Xu et al. 2020).

Phanerochaete hainanensis S.H. He \& Y.C. Dai, sp. nov. MycoBank number: MB837508; Facesoffungi number: FoF 09974; Fig. 220

Etymology: The specific epithet "hainanensis" refers to the Hainan Province, southern tropical China where the type was collected.

\section{Holotype: BJFC 022063}

Fruiting body: Basidiomata annual, resupinate, broadly effused, closely adnate, inseparable from substrate, coriaceous to soft corky, first as small patches, later confluent up to $20 \mu \mathrm{m}$ long, $8 \mathrm{~cm}$ wide. Hymenophore smooth, pale orange (5A3), light orange [5A (4-5)] to greyish orange [5B (3-6)], darkening in $\mathrm{KOH}$, uncracked; margin thinning out, distinct, lighter than hymenophore surface, white (5A1) to orange white [5A2], up to $0.2 \mathrm{~cm}$ wide. Context greyish orange, up to $500 \mu \mathrm{m}$ thick.

Microscopic structures-Hyphal system monomitic; all hyphae without clamp connections. Subiculum thin; hyphae colorless, distinctly thick-walled with a wide lumen, smooth, rarely branched, moderately septate, interwoven, $4-9 \mu \mathrm{m}$ in diam. Subhymenium thickening; hyphae colorless, thinwalled, smooth, vertically arranged, more or less agglutinated, 3-7 $\mu \mathrm{m}$ in diam. Cystidia subulate to subcylindrical, slight tapered toward the apex, sometimes with one or two secondary simple septa, colorless, slightly thick-walled or thin-walled at the tip part, smooth, projecting above the hymenium, with a basal simple septum, 35-70×3-7 $\mu \mathrm{m}$. Basidia clavate, colorless, thin-walled, with a basal simple septum and four sterigmata, $16-25 \times 4-5.5 \mu \mathrm{m}$; basidioles numerous, similar to basidia but smaller. Basidiospores ellipsoid, colorless, thin-walled, smooth, neither dextrinoid nor amyloid in Melzer's agent, acyanophilous in cotton blue, (4.3-) 4.5-5 (-5.3) $\times 2.2-2.8(-3) \mu \mathrm{m}, \mathrm{L}=4.9 \mu \mathrm{m}$, $\mathrm{W}=2.5 \mu \mathrm{m}, \mathrm{Q}=1.96(\mathrm{n}=30 / 1)$.

Material examined: CHINA, Hainan Province, Lingshui County, Diaoluoshan Nature Reserve, on fallen angiosperm trunk, 17 March 2016, He 3562 (BJFC 022063, holotype). Guangxi Autonomous Region, Shangsi County, Shiwandashan Forest Park, on fallen angiosperm trunk, 26 July 2012, He20120726-1 (BJFC 014522), BJM (isotype).

GenBank numbers: ITS $=$ MT235692, LSU $=$ MT248179.

Notes: Phanerochaete hainanensis is characterized by the large basidiomata with pale orange hymenophore, thickened subhymenium with a compact texture, smooth leptocystida and small basidiospores (Fig. 220). The new species is similar to $P$. argillacea in that it shares the compact texture and similar size of leptocystidia and basidiospores but differs in having lighter hymenophore darkening in $\mathrm{KOH}$ (Wu 1998). Phanerochaete hainanensis is somewhat similar to P. metuloidea and $P$. sordida in that they share the thick and tough basidiomata, which, however, differs in having encrusted cystidia and a well-developed subiculum (Burdsall 1985; Xu et al. 2020). In the phylogenetic tree, P. hainanensis forms a distinct lineage and is not closely related to other species of Phanerochaete s.s. (Fig. 221).

\section{Polyporaceae Fr. ex Corda}

Notes: Polyporaceae [as 'Polyporei'] belongs to polyporoid fungi, which was established by Corda (1839). The fruit bodies may be soft or tough having fertile layer with vertical pores on the underside of the elongated pores which constitute a corky labyrinth. They form brackets with a small or definite stipe. 


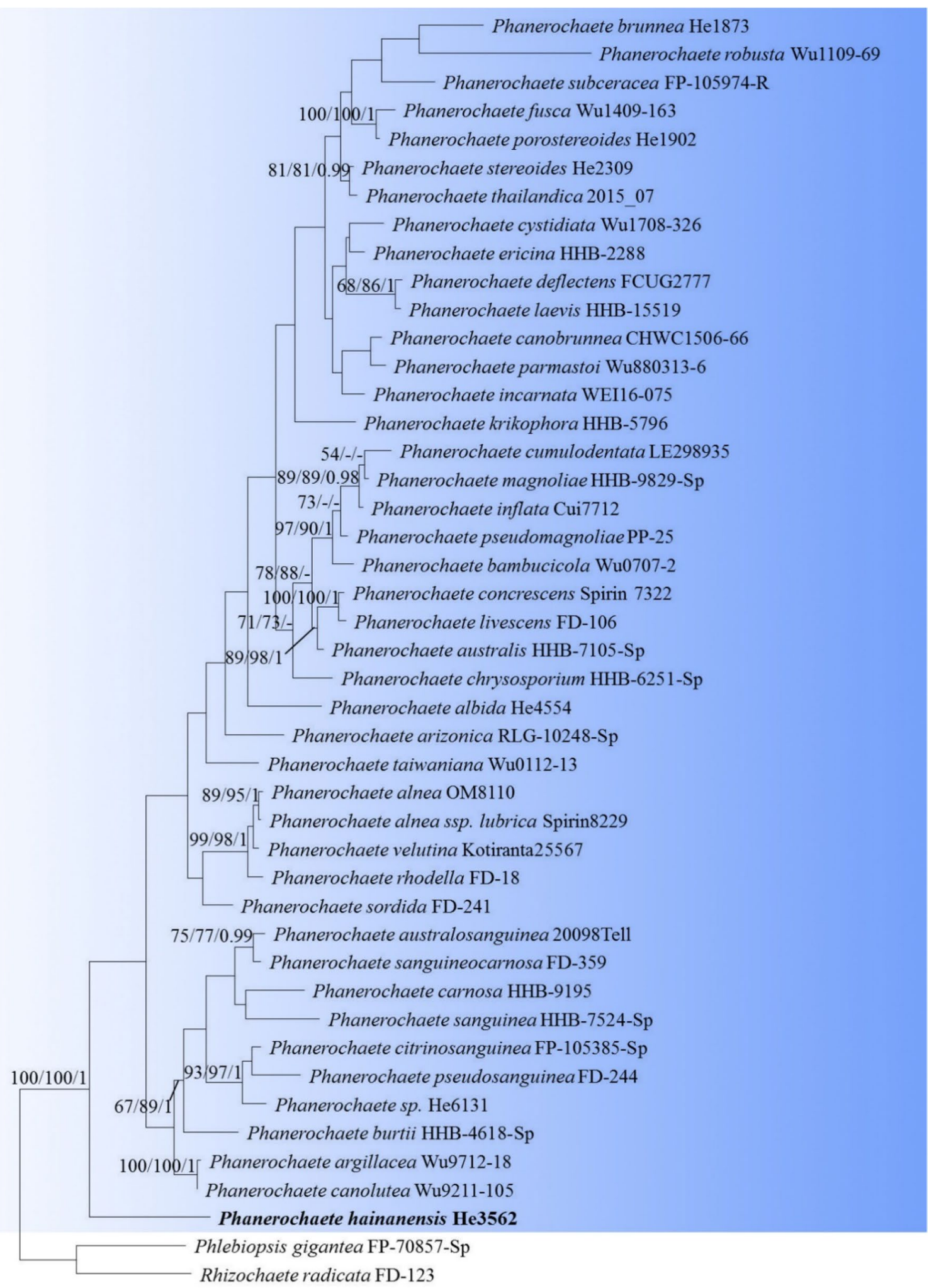

10

Fig. 221 Phylogenetic tree obtained from maximum parsimony analysis of a concatenated ITS and nrLSU sequence data of Phanerochaete s.s. Branches are labelled with parsimony bootstrap values equal to or greater than $50 \%$ (front), likelihood bootstrap values equal to or greater than $50 \%$ (middle) and Bayesian posterior probabilities equal to or greater than 0.95 (back). The new species is in bold 


\section{Favolus Fr.}

Notes: Favolus was established by Fries (1828) with the type species, F. brasiliensis. Species of this genus are considered as wood decaying fungi. The basidiocarps of Favolus species are fleshy with radially arranged pores on the underside of the cap that are angular and deeply pitted, somewhat resembling a honeycomb. Earlier, Favolus was treated as a synonym of Polyporus (Nunez and Ryvarden 1995). Sotome et al. (2013) proposed new genus, Favolus based on morphological and molecular phylogenetic analysis.

Favolus septatus J.L. Zhou \& B.K. Cui, Mycologia 109(5): 773 (2017)

Index Fungorum number: IF817942; Faces of fungi number: FoF 08019; Fig. 222

Colour codes follow: Methuen Handbook of Colour (Kornerup and Wanscher 1978)

Fruit bodies associated with dead wood of Dalbergia melanoxylon Guill. \& Perr. (Fabaceae). Basidiocarps solitary or 1-2 in group, tough or leathery and flexible when fresh, fragile when dry, persistently attached with dead branches in the form of brackets, stipitate. Stipe short, up to $5 \mathrm{~mm}$ long and $7 \mathrm{~mm}$ diam. Pileus circular to flabellate, sometimes notched, fibrillose with flattened, radially striate, azonate, from base to tip up to $5.5 \mathrm{~cm}$ long $\times 3.5 \mathrm{~cm}$ wide. Pilial surface wet, buff to yellowish, smooth-walled. Pores surface buff when fresh, buff brown when dry, pores hexangular, radially elongated, 4-5 mm long, 1-2 mm wide near stipe to 1 per $\mathrm{mm}$ at margin. Context (internal tissue of the fruit body) has a tough and fleshy to leathery texture when fresh, buff brown, corky, brittle when dry, buff brown, up to $4 \mathrm{~mm}$ thick. Tube continuous with the context, up to 5-6 mm thick. Spore prints white. Hyphal system dimitic. Generative hyphae without clamp connection, hyaline in $\mathrm{KOH}$, thin and smooth-walled, 1.8-5 $\mu \mathrm{m}$. Skeletal-binding hyphae thick walled, aseptate, highly branched, hyaline, 2.5-6.5 $\mu \mathrm{m}$. Cystidial cells absent. Basidia persistant, clavate, sometimes bulged, constricted near septa, septate, pigmented, 11.5-11.8 $\times 5.9-6.3 \mu \mathrm{m}$. Sterigmata persistent, straight to incurved, tip sub-obtuse, $2-4$ in numbers per basidium, $1-2.4 \mu \mathrm{m}(\bar{x}=14.3 \times 6 \mu \mathrm{m}, \mathrm{n}=6)$. Basidiospores, cylindric to oblong, pigmented, smooth and thin walled, hyaline, $6.5-9.5 \times 3.3-4.3 \mu \mathrm{m}$.

Culture characteristics: Colonies on PDA (Potato Dextrose Agar), center pale yellow (4A3), periphery white (1A1), floccose, $7.5 \mathrm{~cm}$ diam. in 24 days. Margin irregular, reverse pale yellow (4A3). Hyphae septate, unbranched to branched, smooth and thin walled, subhyaline to light olivacous, sometimes coroloid, rope like, twisted, forming loose and compact parallel bundles of hyphae, pigmented or granulated. Chlamydospores produced from superficial hyphae, terminal to lateral, solitary, bulbus, stalked, up to $8.9 \times 8.6 \mu \mathrm{m}$ wide. Clamp connections in hyphae absent.
Material examined: INDIA, Maharashtra, Pune District, on dead wood of Dalbergia melanoxylon (Fabaceae), 17 July 2019, D.K. Maurya (AMH 10219, new record), living culture (NFCCI 4776).

GenBank numbers: ITS $=$ MT372798, LSU $=$ MT372843.

Notes: Phylogenetic analysis based on a combined ITS and LSU sequence dataset of 35 taxa shows that the Indian taxon (NFCCI 4776) clusters with type of Favolus septatus. A Megablast search of the ITS sequence shows $99.48 \%$ similarity (569/572) with Favolus septatus (Zhou 287, Type) (Fig. 223). In our observation, the morphological features, especially length and width of basidiocarps is somewhat larger in the present collection than originally described in Favolus septatus (Fig. 222). The pilial surface is buff to yellowish in the present collection, while it is pinkish buff to yellowish in the original description of $F$. septatus. However, based on similarity regarding other morphological characteristics, and phylogenetic analysis, similarity of the present collection is confirmed as Favolus septatus (Zhou and Cui 2017). As per our understanding, this is the first report of Favolus septatus being reported from India. Therefore, described and illustrated here.

Russulales Kreisel ex P.M. Kirk, P.F. Cannon \& J.C. David Notes: We follow the latest treatment of Russulales (He et al. 2019).

\section{Russulaceae Losty}

Notes: Russulaceae was introduced to accommodate three genera Lactarius, Russula and Russulina with Russula as the type genus (Donk 1971). The family is characterized by convex to funnel-shaped pileus, stipes without annulus and volva, granular fragile context, spiny basidiospores, and sphaerocytes intermixed in filamentous hyphae. Currently, seven genera, Boidinia, Gloeopeniophorella, Lactarius, Lactifluus, Multifurca, Pseudoxenasma and Russula are known as members of Russulaceae. Approximately 1,900 known species are included in this family (He et al. 2019; Species Fungorum 2021). We follow the latest treatment of the genera in Russulaceae (He et al. 2019). Two new species Lactarius pallidozonarius and Russula paravioleipes are introduced with support from morphological and phylogenetic analyses.

\section{Lactarius Pers.}

Notes: Lactarius currently contains roughly 450 agaricoid and gasteroid species. Despite the establishments of Lactifluus and Multifurca, it is still one of the major groups in family Russulaceae based on morphological characters and phylogenic topology (Buyck et al. 2010; Verbeken et al. 2011, 2012; Stubbe 2012). In this study, one new species Lactarius pallidozonarius is introduced new to science. The morphology and phylogenetic placement of this new species are provided below (Figs. 224 and 225). 

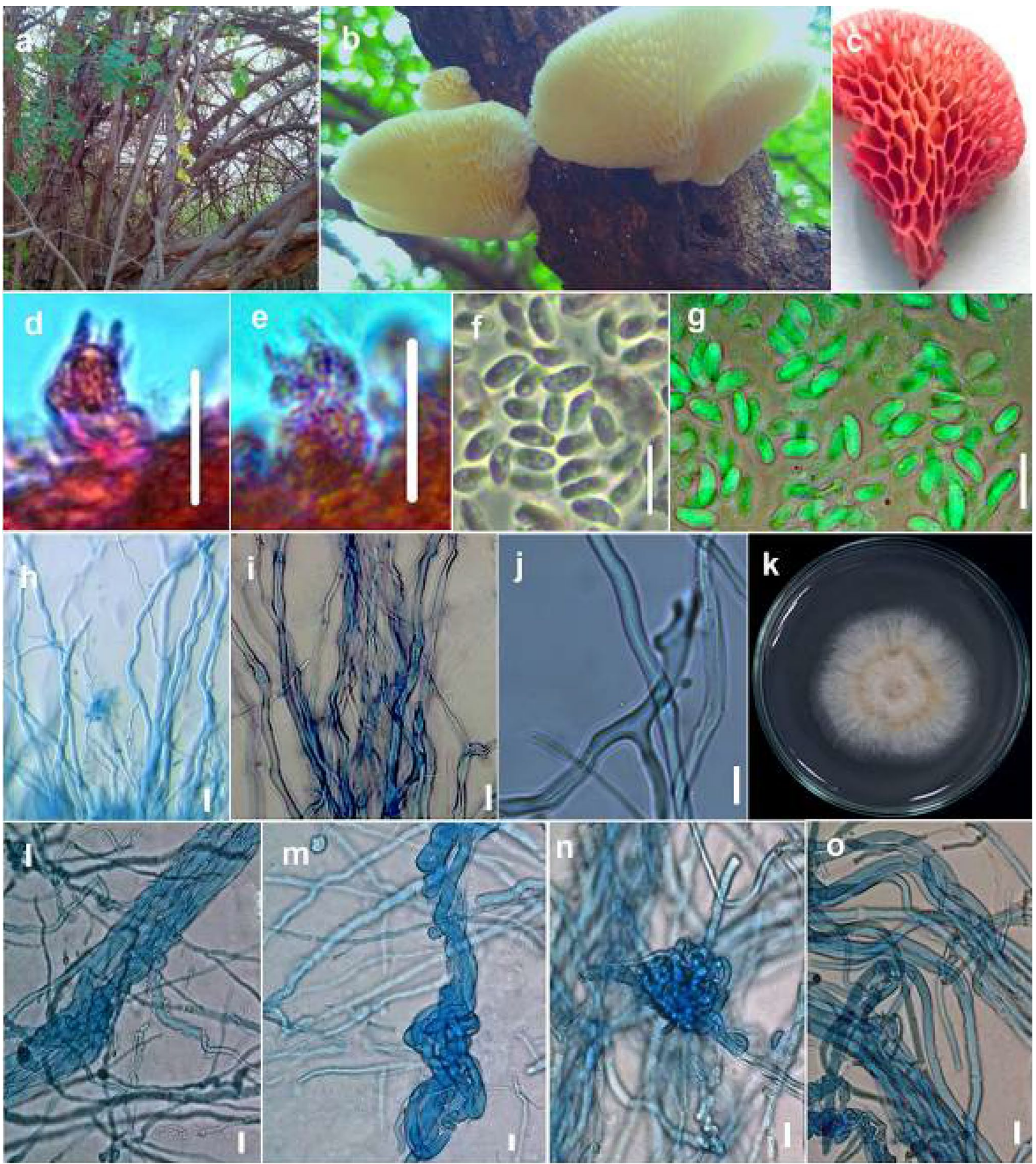

Fig. 222 Favolus septatus (AMH 10219, new record). a Habitat. b Basidiomata growing on substrate. c Basidioma showing hexagonal pores on lower surfaces. d, e Basidia with sterigmata. f, $\mathbf{g}$ Basidiospores. h, i Generative hyphae. j Skeletal binding hyphae. k Culture on PDA after 24 days (surface view). 1 Simple and compact hyphal bundle. m, n Simple and rope like twisted hyphae. o Parallel loose bundles of hyphae. Scale bars: $\mathbf{d}-\mathbf{o}=10 \mu \mathrm{m}$ 


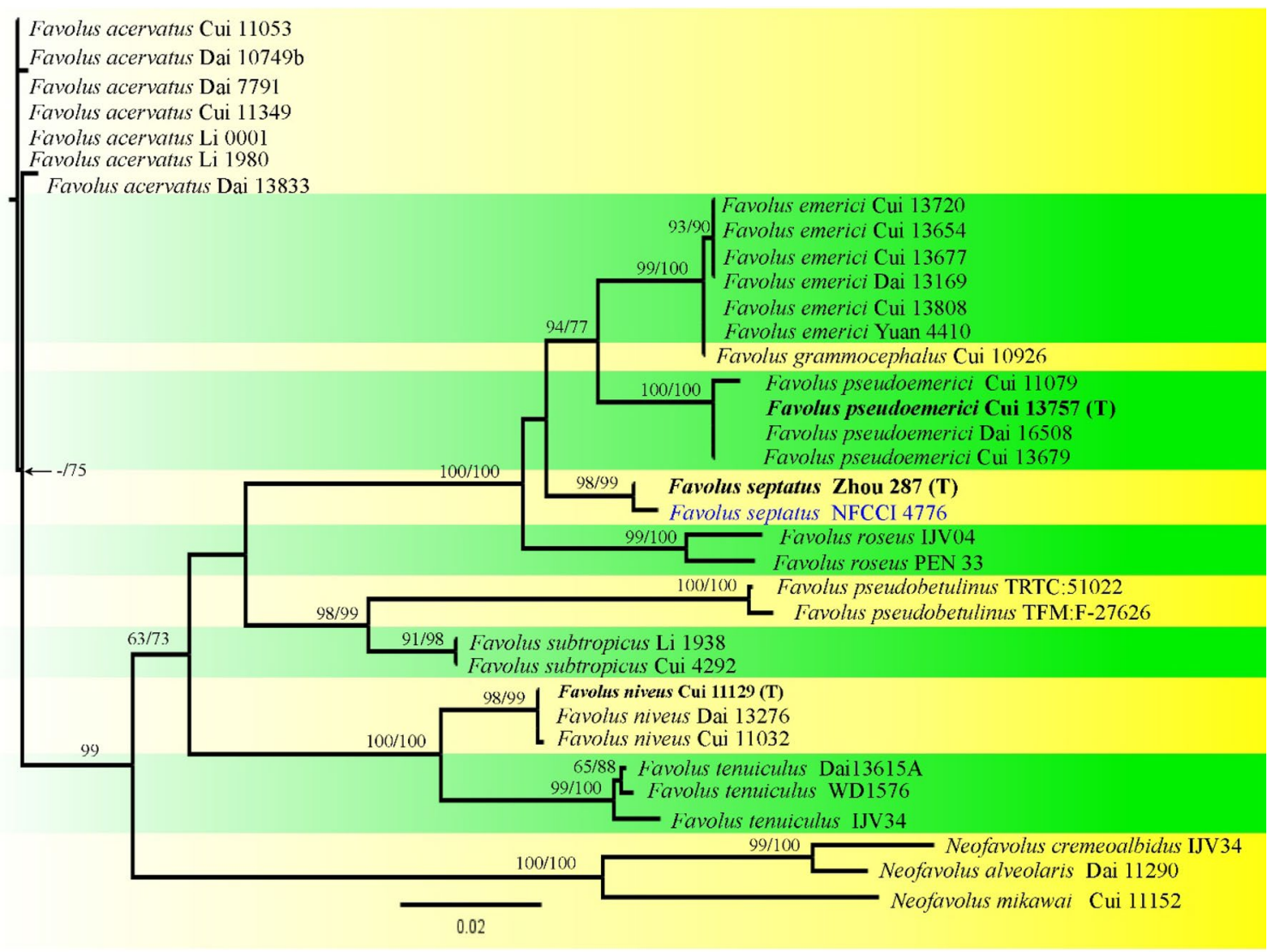

Fig. 223 The phylogenetic tree has been generated using maximum likelihood analysis based on LSU and ITS sequence data. Thirty-five strains are included in the LSU and ITS combined analyses comprising 1532 sites of which 1202 were the conserved and 320 were the variable sites, and 264 parsimony-informative sites. Neofavolus species are used as the outgroup taxa. The optimal tree with a final likelihood value of -5057.256 is presented. Estimated base frequencies

\section{Lactarius pallidozonarius G.J. Li \& W.F. Lin, sp. nov.}

Index Fungorum number: IF55864; Facesoffungi number: FoF 09206; Fig. 224

Etymology: Refers to the pale zonary pileus.

\section{Holotype: HBAU 15004}

Basidiomata medium sized. Pileus $42-58 \mathrm{~mm}$ in diam., convex to hemispheric when young, then applanate to shallow funneled, depressed at center when old, a pale erythrinus tinge of army brown (XL13"'i), vinaceous fawn (XL13"'b), fawn colour (XL13"') to light vinaceous-fawn (XL13"'d) at center, fade to vinaceous buff (XL17"'d), avellaneous (XL17"'b) to pale vinaceous-fawn (XL13"'f) towards the margin, densely zonate, dull, smooth, not viscid when wet; margin decurved first, expanded to partly curled upwards when mature, even, not striate, rarely cracked. Lamellae adnate to slightly decurrent, dense, 16-20 pieces per centimeter in the edge, interveined, rarely forked near stipe, $4-5 \mathrm{~mm}$ in height, white (LIII), turning a pale brownish tinge of yellow ocher (XV17') were as follows: $\mathrm{A}=0.250, \mathrm{C}=0.250, \mathrm{G}=0.250, \mathrm{~T}=0.250$; substitution rates $\mathrm{AC}=1.00000, \mathrm{AG}=4.63563, \mathrm{AT}=1.00000, \mathrm{CG}=1.00000$, $\mathrm{CT}=8.90564, \mathrm{GT}=1.00000$; gamma distribution shape parameter $\alpha=0.961$. Bootstrap values for maximum likelihood (ML) equal to or greater than $50 \%$ are labeled on the nodes. Type sequences are in bold and newly generated sequence of Favolus septatus NFCCI 4776 is in blue bold

to buckthorn brown $(\mathrm{XV17}$ 'i) when injured, lamellulae numerous. Stipes $25-34 \times 12-17 \mathrm{~mm}$, central, cylindrical, tapered towards the base, white (LIII), turning a brownish tinge of tawny-olive (XXXIX17"i) to isabella colour (XXX19"i) when bruised, dull, smooth, not viscid when wet, first stuffed, partly cavernous when mature, annulus absent. Context 3-5 mm thick at the pileus center, White (LIII), same colour changes as the lamellae, taste mild, smell indistinct. Latex watery white, unchanging. Spore print cream to pale ocher.

Basidiospores [200/4/4] (6.2)6.5-8.1(-8.4) $\times 4.8-6.0(-$ 6.3) $\mu \mathrm{m}, \mathrm{Q}=1.01-1.30(1.34) \mu \mathrm{m}, \mathrm{Q}=1.17 \pm 0.08 \mu \mathrm{m})$, hyaline, subglobose to broad ellipsoid, rarely globose and ellipsoid, ornamentations amyloid, up to $1 \mu \mathrm{m}$ in height, composed of incompletely to completely reticulated long ridges, isolated warts and short crests also exist, suprahilar area plage distinct and amyloid. Basidia 40-58 $\times 10-12 \mu \mathrm{m}$, hyaline, subcylindrical to clavate, four-spored, projecting 15-30 $\mu \mathrm{m}$ beyond hymenium, sterigmata 5-6 $\mu \mathrm{m}$ long. Hymenial cystidia not observed. Marginal cells 

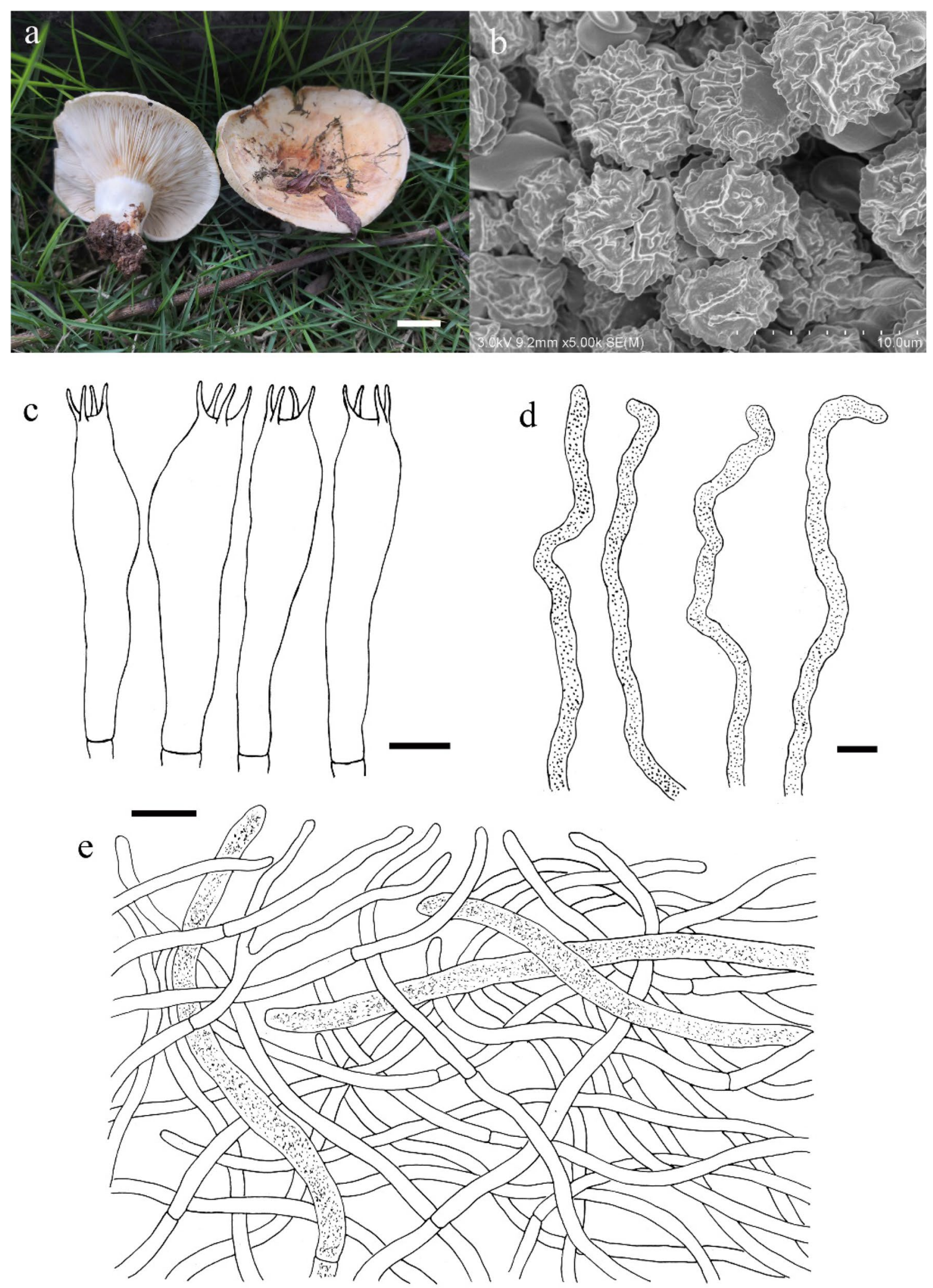

Fig. 224 Lactarius pallidozonarius (HBAU 15004, holotype). a Appearance of basidiomata on ground. b SEM photo of basidiospores. c Basidia. d Pseudocystidia. e Pileipellis. Scale bars: $\mathrm{a}=10 \mathrm{~mm}, \mathbf{c}-\mathbf{e}=10 \mu \mathrm{m}$ 
22-33×8-11 $\mu \mathrm{m}$, subclavate to clavate, hyaline. Pseudocystidia abundant, aseptate, mostly embedded in subhymenium, rarely emergent, not brached, content granular; apex obtuse. Pileipellis a cutis, 100-300 $\mu \mathrm{m}$ thick, hyphae densely interwoven, rarely shriveled, 3-5 $\mu \mathrm{m}$ wide, hyaline, apex obtuse, rarely attenuated. Stipipellis an ixocutis, composed of interwoven, pale yellowish hyphae 3-5 $\mu \mathrm{m}$ wide.

Habit and habitat: scattered in broad-leaved forest of Salix spp. and Cinnamomum camphora.

Material examined: CHINA, Zhejiang Province, Hangzhou City, Xihu District, Zhejiang University, Zijingang Campus, on ground, 28 June 2019, W.F. Lin, 20190016 (HBAU 15004, holotype); ibid, 20190701 (HBAU 15006); ibid, 26 June 2019, 20190005 (HBAU 15005); ibid, 20190753 (HBAU 15007).

GenBank numbers: ITS = MN658520, MN658521, MN658522, MN658523.

Notes: A new species, Lactarius pallidozonarius (Figs. 224 and 225) resembles L. inquinatus for their pale reddish brownish tinge near pileus center, lamellae turning reddish brown when injured, and watery white latex. Lactarius inquinatus however, has larger basidiospores $7.8-11 \times 6.5-8.2 \mu \mathrm{m}$ with lower ornamentations up to $0.5 \mu \mathrm{m}$ in height, absence of hymenial pleurocystidia, and a habitat of forest dominated by Quercus (Lee et al. 2019). The other two closely related European species, L. evosmus and $L$. zonarius can be differentiated as following: L. evos$m u s$ has basidiocarps of fruity smell, and lower and less reticulated basidiospore ornamentations; $L$. zonarius has pale yellowish tinged pileus and subreticulate basidiospores (Heilmann-Clausen et al. 1998).

\section{Russula Pers.}

Notes: Russula consists of ca. 800 agaricoid and gasteroid species which contain the most members of species in Russulaceae based on evidence of morphology and molecular data. In this study, one new species Russula paravioleipes is described based on morphological evidence and phylogenetic placement (Figs. 226 and 227).

\section{Russula paravioleipes G.J. Li \& W.F. Lin, sp. nov.}

Index Fungorum number: IF558649; Facesoffungi number: FoF 09207; Fig. 226

Etymology: Named after the close phylogenetic relationship with $R$. violeipes.

\section{Holotype: HBAU 15001}

Basidiomata small to medium sized. Pileus $28-46 \mathrm{~mm}$ diam., first hemisphere, then convex, applanate when mature, a tinge of brightly purplish red, daphne red (XXXVIII69"), spinal red (XXVI71'), to eosine pink (I1d), sometimes faded to a paler orange reddish tinge of peach red (I5b) to strawberry pink (I5d), smooth, glabrous, slightly viscid when wet; margin not striate, rarely cracked, peeling
1/3-1/2 from the edge, rose colour (XII71b) to spinal pink (XXVI71'b). Lamellae adnate, 3-4 mm in height, 13-16 pieces per centimeter at edge, not forked, interveined, white (LIII), unchanging when bruised, lamellulae not observed. Stipes $29-48 \times 10-13 \mathrm{~mm}$, central to subcentral, cylindrical, rugulose longitudinally, White (LIII), unchanging when injured, smooth, dull, not viscid when wet, slightly attenuate downward the base, first stuffed, hollow when old, annulus absent. Context 2-3 mm thick at pileus center, White (LIII), unchanging, taste mild, smell indistinct. Spore print pale cream (Romagnesi IIa-IIb).

Basidiospores [150/3/3] 5.5-7.1(-7.4) $\times 5.5-7.2 \mu \mathrm{m}$, $\mathrm{Q}=1.02-1.27(1.31) \mu \mathrm{m}, \mathrm{Q}=1.17 \pm 0.07) \mu \mathrm{m}$, hyaline, subglobose to broad ellipsoid, rarely globose and ellipsoid, ornamentations amyloid, up to $0.7 \mu \mathrm{m}$ in height, composed of long ridges interconnected as incomplete reticulum, isolated warts and short crests rare, suprahilar area plage distinct but inamyloid. Basidia 35-45×8-12 $\mu \mathrm{m}$, cylindrical, subcylindrical to subclavate, rarely clavate four-spored, projecting $10-20 \mu \mathrm{m}$ beyond hymenium, sterigmata $4-6 \mu \mathrm{m}$ long. Hymenial cystidia $91-110 \times 8-10 \mu \mathrm{m}$, hyaline, fusiform to subulate, sometimes clavate, unchanging in sulfovanillin (SV), apex acute. Pileipellis two layered; epipellis pseudoparenchymatous, ca. 150-200 $\mu \mathrm{m}$ deep, not clearly delimited from the spherocytes in context, terminal cells hyaline, subulate to bayonet-shaped, $57-85 \times 5-8 \mu \mathrm{m}$, subapical cells $7-11 \mu \mathrm{m}$ wide, pileocystidia absent; subpellis composed of somewhat gelatinized, densely interlaced, rarely branched and septate, hyaline hypha 3-6 $\mu \mathrm{m}$ wide. Stipitipellis a cutis, composed of parallel hyaline hypha 3-5 $\mu \mathrm{m}$ wide.

Habit and habitat: scattered in broad-leaved forest of Salix spp. and Cinnamomum camphora.

Material examined: CHINA, Zhejiang Province, Hangzhou City, Xihu District, Zhejiang University, Zijingang Campus, 26 June 2019, W.F. Lin, 20190001 (HBAU 15001, holotype); ibid, 20190688 (HBAU 15002); ibid, 20190699 (HBAU 15003).

GenBank numbers: ITS = MN658517, MN658518, MN658519.

Notes: Russula paravioleipes is a member of subsection Amoeninae Buyck, subgenus Heterophyllidia Romagn., section Heterophyllae Fr. because of its absence of pileocystidia, long and subulate terminal hyphal cells of pileipellis, and empty hymenial cystidia not turning grey or black in Sulfovanillin (Sarnari 1998). The reddish tinged pileus is the remnants of " $R$. violeipes Quél." in Park et al. (2013). The Korean " $R$. violeipes" can be differentiated from this new species for its areolate pileus surfaces and flushed stipes. However, the ITS phylogenetic analyses of this study cannot distinguish them (Fig. 227). Whether or not these Chinese and Korean specimens are of the same species still needs further analysis. The other members of subsection 


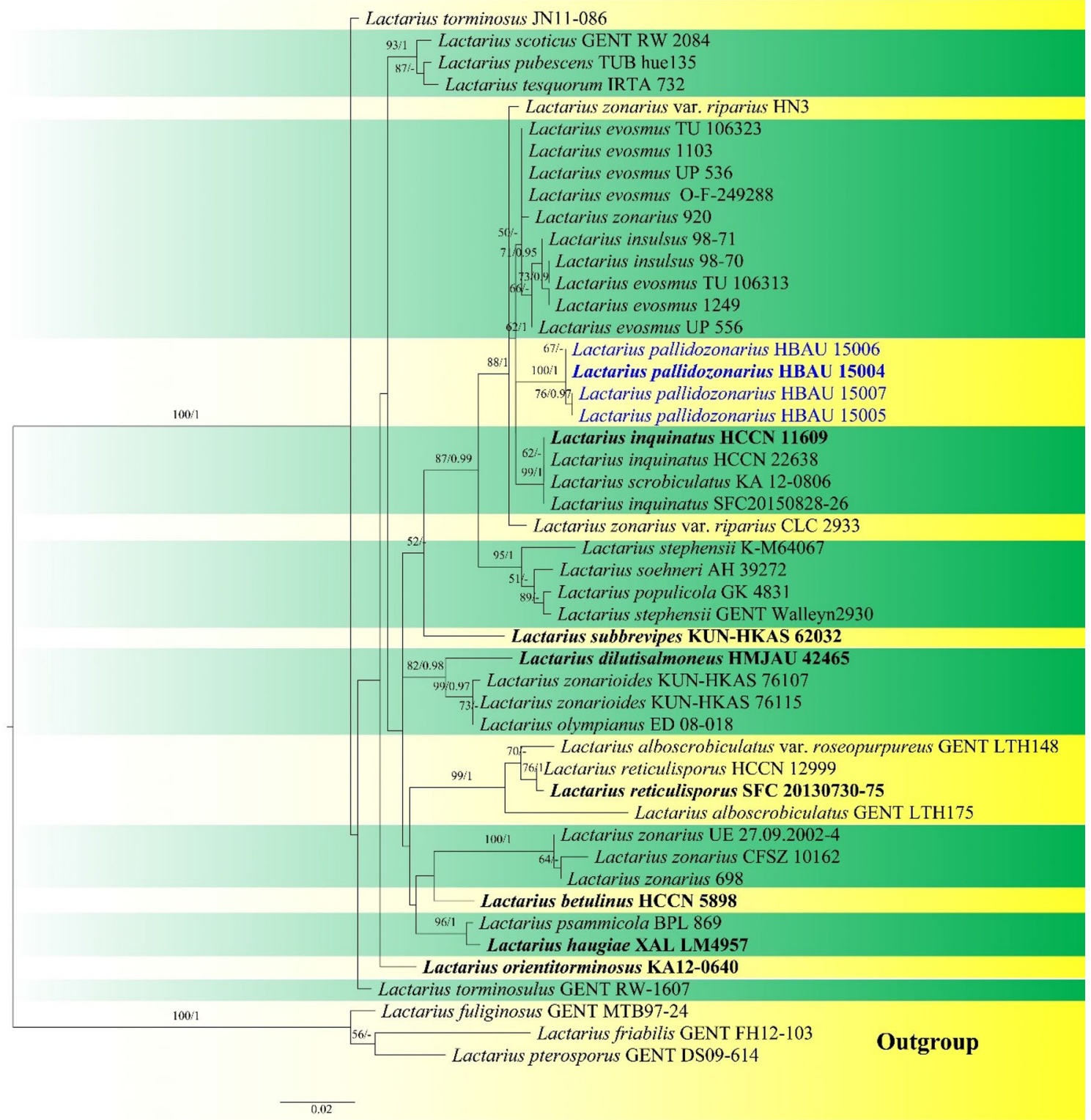

Fig. 225 Phylogram generated from maximum likelihood analysis based and ITS sequence data representing Lactarius pallidozonarius and closely related species. Related sequences are taken from Lee et al. (2019), UNITE and GenBank databases. Forty-nine sequences are included in the combined analysis which comprises 2505 characters 546 characters for ITS after alignment. Lactarius friabilis (GENT FH12-103), L. fuliginosus (GENT MTB97-24) and L. pterosporus (GENT DS09-614) in subg. Plinthogalus are used as the outgroup taxa. Tree topology of the maximum likelihood analysis is similar to the Bayesian analysis. The best RAxML tree with a final likelihood values of -2161.573832 is presented. The matrix had

Amoeninae can be distinguished from $R$. paravioleipes (Fig. 226) by the following morphological characteristics: $R$. violeipes in Europe differs in its violaceous pileus and conspicuous hymenial cystidia $10-20 \mu \mathrm{m}$ wide; $R$. amoenicolor Romagn. has purple, green, or variegated pileus, dark cream basidiospore print (IIc-IId), and a habitat of Quercus
158 distinct alignment patterns, with $0.71 \%$ undetermined characters or gaps. Estimated base frequencies were as follows: $\mathrm{A}=0.229615$, $\mathrm{C}=0.259476, \quad \mathrm{G}=0.226053, \mathrm{~T}=0.284855 ; \quad$ substitution rates $\mathrm{AC}=0.965485, \quad \mathrm{AG}=9.458565, \quad \mathrm{AT}=0.865989, \quad \mathrm{CG}=0.392267$, $\mathrm{CT}=8.367348, \mathrm{GT}=1.000000$; gamma distribution shape parameter $\alpha=0.217720$. Bootstrap values for maximum likelihood (ML) greater than $50 \%$ and posterior probability values greater than 0.90 (the rounding of values to 2 decimal proportions) from Bayesian-inference analysis are shown above the nodes. Sequences of type specimens are presented in bold and newly generated sequences are in blue

forest; $R$. amoena can be differentiated by its violaceous purple to olive greenish tinged pileus, and longer basidia 60-70×9-12 $\mu \mathrm{m}$ (Sarnari 1998); R. mariae differs in its soft pruinose pileus surface, and wider hymenial cystidia up to $12.5 \mu \mathrm{m}$ (Adamčík et al. 2018). 


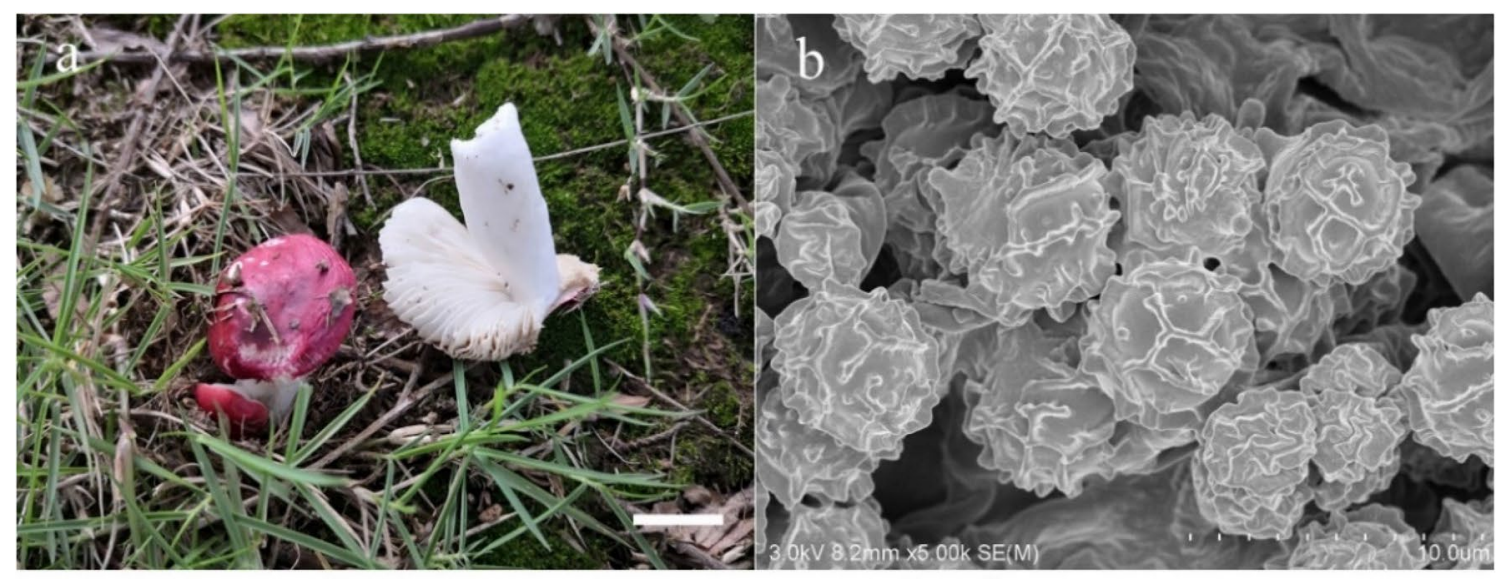

$\mathrm{C}$

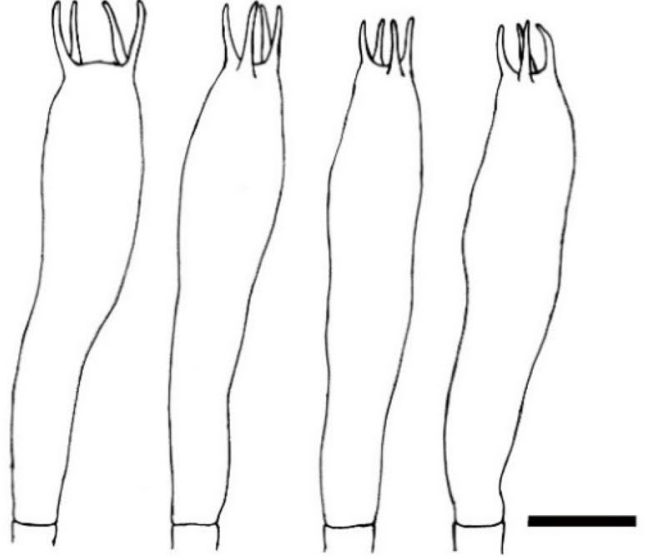

d
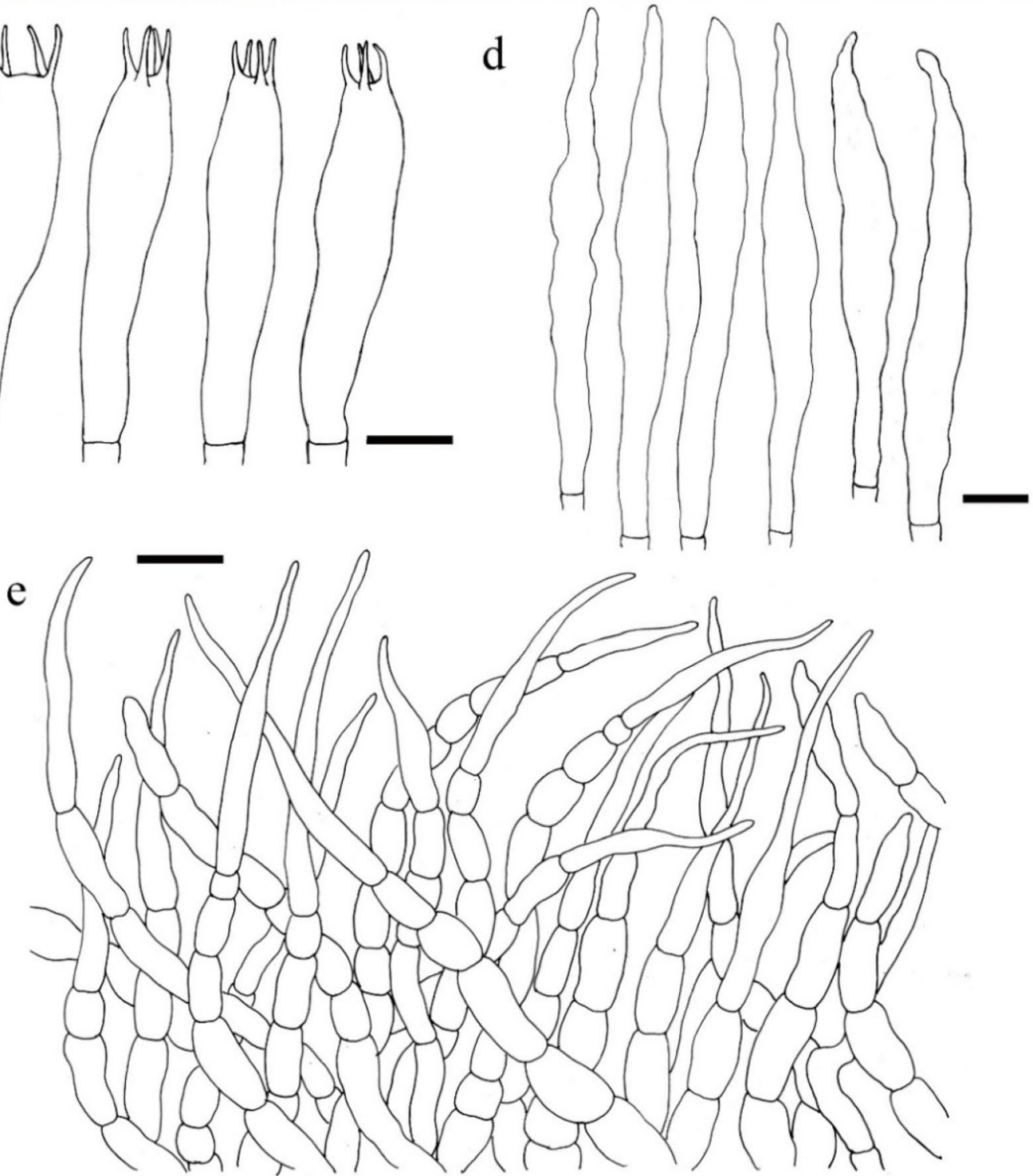

Fig. 226 Russula paravioleipes (HBAU 15001, holotype). a Appearance of basidiomata on ground. b SEM photo of basidiospores. c Basidia. d Pseudocystidia. e Pileipellis. Scale bars: $\mathbf{a}=10 \mathrm{~mm}, \mathbf{c}-\mathbf{e}=10 \mu \mathrm{m}$ 


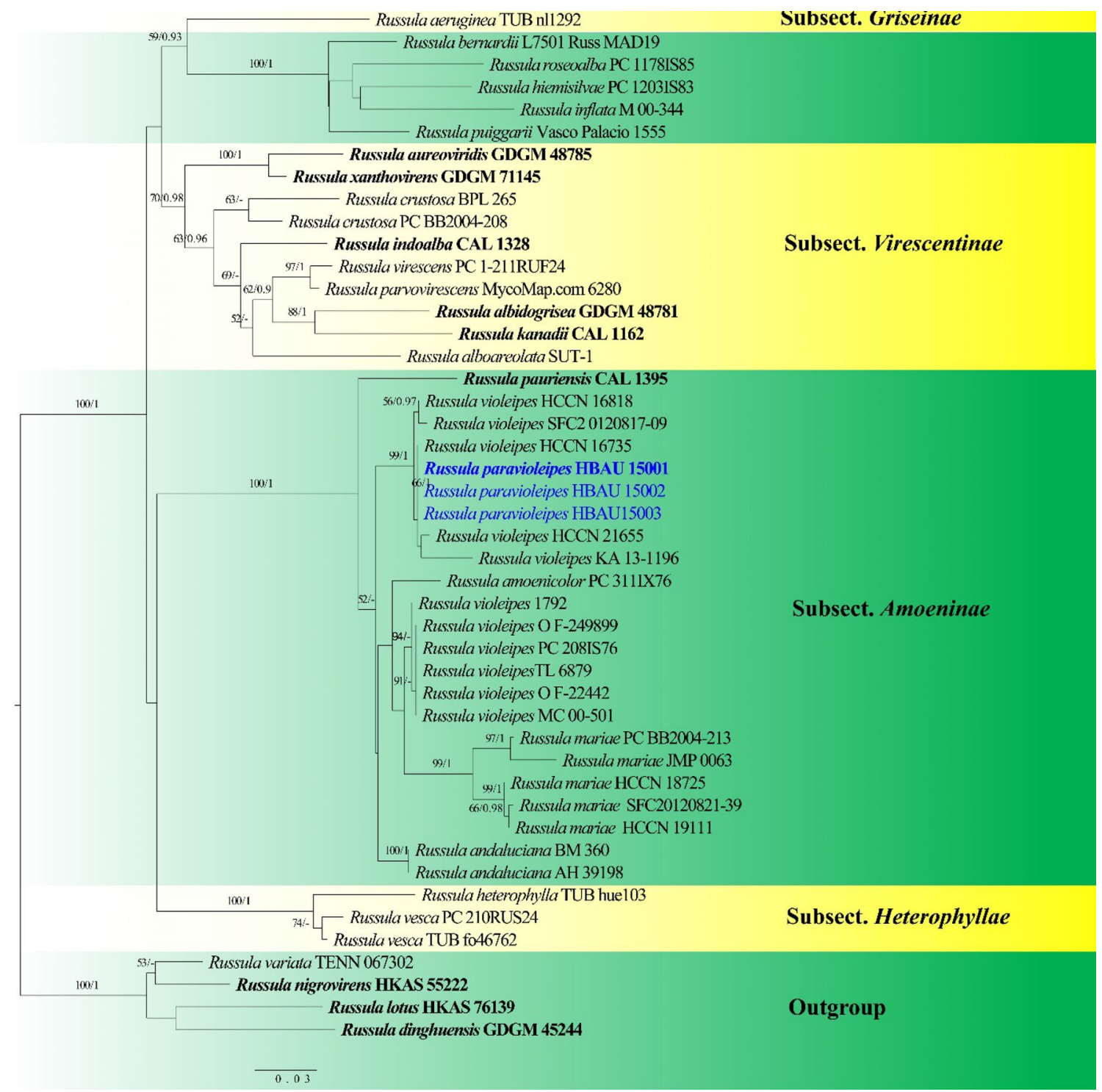

Fig. 227 Phylogram generated from maximum likelihood analysis based and ITS sequence data representing Russula paravioleipe and related species in Sect. Heterophyllae. Related sequences are retrieved from GenBank and UNITE databases. Forty-seven ITS sequences are included in the analysis comprises 546 characters after alignments. Russula dinghuensis (GDGM 45244), R. lotus (HKAS 76139), R. nigrovirens (HKAS 55222) and $R$. variata (TENN 067302) in Sect. Cyanoxanthinae (Subg. Heterophyllidia) are used as the outgroup taxa. Tree topology of the maximum likelihood analysis is similar to the Bayesian analysis. The best RAxML tree with a final likelihood values of -4326.910274 is pre-

Microbotryomycetes R. Bauer et al.

Notes: The classification of the orders in Microbotryomycetes follows Begerow and McTaggart (2018).

\section{Microbotryales R. Bauer \& Oberw}

Notes: There are two families in this order: Microbotryaceae and Ustilentylomataceae (Vánky 2013; He et al. 2019). sented. The matrix had 281 distinct alignment patterns, with $2.78 \%$ undetermined characters or gaps. Estimated base frequencies were as follows: $\mathrm{A}=0.217569, \mathrm{C}=0.256487, \mathrm{G}=0.225771, \mathrm{~T}=0.300173$; substitution rates $\mathrm{AC}=1.359519, \mathrm{AG}=4.554062, \mathrm{AT}=0.588669, \mathrm{CG}=0.728567$, $\mathrm{CT}=5.548012, \mathrm{GT}=1.000000$; gamma distribution shape parameter $\alpha=0.379017$. Bootstrap values for maximum likelihood (ML) greater than $50 \%$ and posterior probability values greater than 0.90 (the rounding of values to 2 decimal proportions) from Bayesian-inference analysis are shown above the nodes. Type and ex-type are indicated in bold and the newly generated sequences are indicated in blue

\section{Microbotryaceae R.T. Moore}

Notes: Microbotryaceae was introduced by Moore (1996). Five genera are currently recognized in this family (He et al. 2019; Denchev et al. 2020).

\section{Microbotryum Lév.}




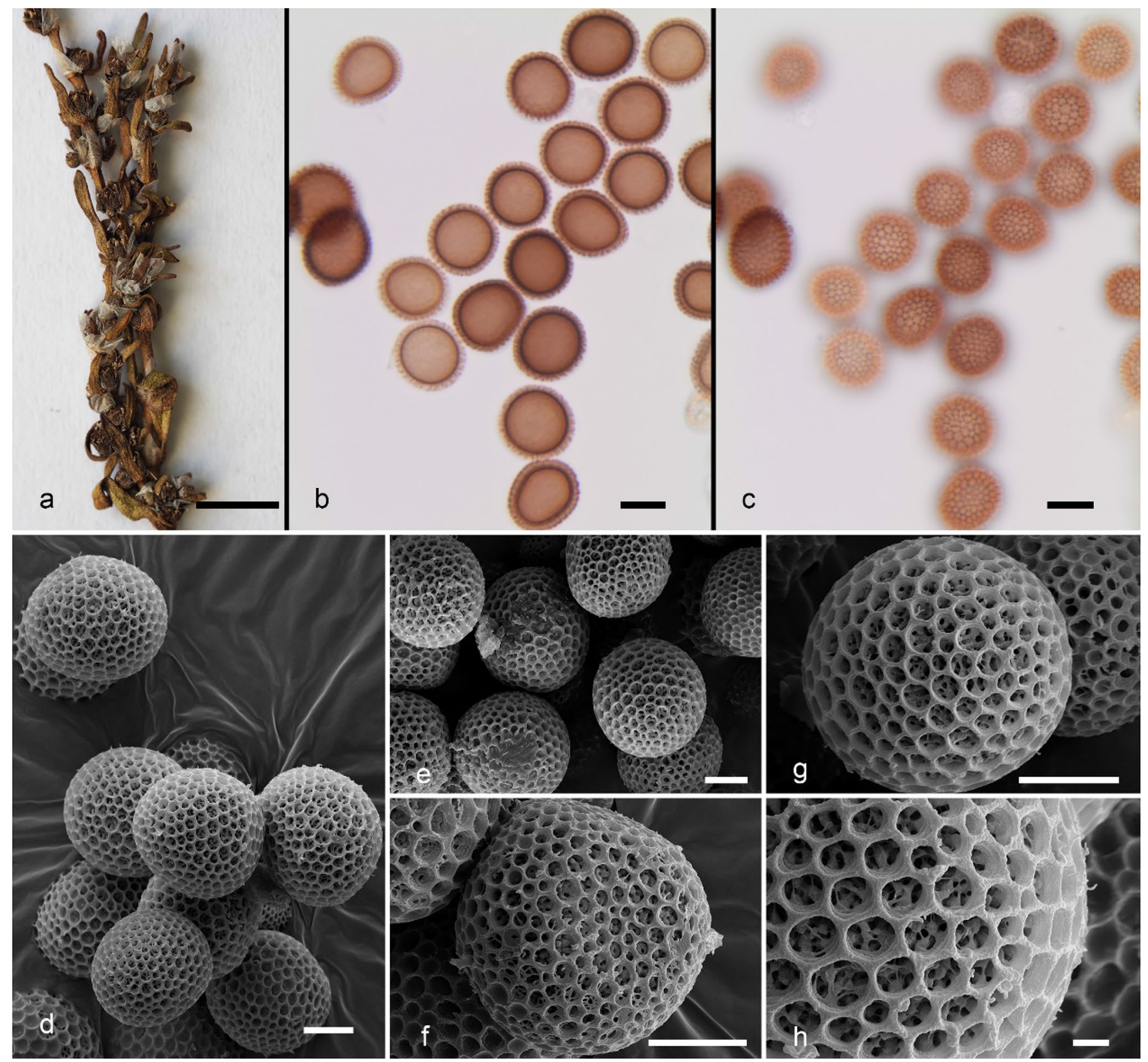

Fig. 228 Microbotryum polycnemoides (SOMF 30200, holotype). a Habit. b, c Spores in LM (in median and surface view, respectively). d-h Spores in SEM. Scale bars: $\mathbf{a}=0.5 \mathrm{~cm}, \mathbf{b}, \mathbf{c}=10 \mu \mathrm{m}, \mathbf{d}-\mathbf{g}=5 \mu \mathrm{m}, \mathbf{h}=1 \mu \mathrm{m}$

Notes: In its broad circumscription, Microbotryum comprises 99 species (Denchev and Denchev 2011; Vánky 2011; Piątek et al. 2012, 2013; Denchev et al. 2019; Ziegler et al. 2018; Kemler et al. 2020). During an examination of Polygonum specimens in the herbarium of the Meise Botanic Garden, Belgium (BR), a smut fungus belonging to Microbotryum was found on a specimen of Polygonum polycnemoides (BR, s.n.) from Turkey. Based on distinct morphology (Table 6; Fig. 228) and phylogenetic evidence (Fig. 229), this fungus is introduced here as a novel species.
Microbotryum polycnemoides T. Denchev, Denchev, Kemler \& Begerow, sp. nov.

Index Fungorum number: IF557222; Facesoffungi number: FoF 07517; Fig. 228

Etymology: The specific epithet refers to the host species.

Holotype: SOMF 30200

Parasitic on Polygonum polycnemoides. Infection systemic, all flowers of an inflorescence affected. Sori in perianth tube, hypanthium, and filaments of each flower, and in achenes, swelling considerably the affected organs and 
filling them with pulverulent, blackish brown spore mass. Ovaries, anthers, and perianth lobes remain intact. Spores subglobose, broadly ellipsoidal, ovoid, globose, slightly irregular or sometimes ellipsoidal, (14-)15-19(-21) $\times(12$ $.5-) 13.5-17(-18.5)(16.6 \pm 1.1 \times 15.2 \pm 0.9) \mu \mathrm{m}(\mathrm{n}=300)$, medium reddish brown; wall reticulate, $(1.7-) 2-2.7(-3) \mu \mathrm{m}$ thick (including reticulum), meshes (8-)9-12(-13) per spore diameter, 0.4-2.5(-3.5) $\mu \mathrm{m}$ wide, muri 1-1.9(-2.3) $\mu \mathrm{m}$ high. In SEM meshes mostly rounded, interspaces often perforate with up to 4(-6) holes per interspace, sometimes with 2(-4) warts.

Material examined: TURKEY, the Anti-Taurus Mts (Aladağlar), Niğde Province, near Çamardı, Mt. Küçük Denizli, alt. 2300-2500 mls, on Polygonum polycnemoides Jaub. \& Spach (Polygonaceae), 11 August 1992, V. Vašák s.n. (SOMF 30200, holotype).

GenBank numbers: ITS = MN989380, LSU = MN989381.

Additional material examined: Microbotryum aviculare (Liro) Vánky—SWEDEN, Uppland, near Uppsala, on Polygonum aviculare L., 10 August 1978, Sz., T. \& K. Vánky (as 'Ustilago avicularis', H.U.V. 7439, kept at BRIP); $M$. shastense (Zundel) Vánky—USA, California, Siskiyou Co., Mt. Shasta, $41^{\circ} 22^{\prime} \mathrm{N} 122^{\circ} 12^{\prime} \mathrm{W}$, ca $2700 \mathrm{msl}$, on Polygonum shastense W.H. Brewer, 8 August 1988, F. Oberwinkler et al., 3207 (Vánky, Ustilag. 717, in SOMF 19825; topotype).

Notes: Polygonum polycnemoides is an Irano-Turanian species, distributed in Armenia, Nakhichevan, Turkey, Syria, Lebanon, Israel, North Iraq, Iran, the Central Asian Republics, Xinjiang, Mongolia, Afghanistan, and West Himalaya (Davis 1967; Li et al. 2003; Uotila 2017). Microbotryum polycnemoides is known only from the type collection.

Microbotryum contains species that parasitize plants from many different lineages of euasterids, with host-specificity of individual parasite species in general being exceptionally high (Kemler et al. 2020). On hosts in Polygonum, only two species of Microbotryum have been previously known: M. aviculare on Polygonum aviculare, $P$. norvegicum and $P$. oxyspermum subsp. raii (from Europe, Asia, and North America), and M. shastense on Polygonum shastense (from North America) (Vánky 2011). Microbotryum polycnemoides (Fig. 228) can be easily distinguished from $M$. aviculare and M. shastense in having a different colour of the spore mass, larger spores, higher number of meshes per spore diameter, and higher spore wall muri (Table 6).

Microbotryum polycnemoides is closely related to $M$. parlatorei (97\% MLBS support) and does not form a monophylum with $M$. shastense. The topology of our phylogenetic tree additionally indicates that $M$. polycnemoides is not closely related to $M$. shastense, but there is no statistical support for this relationship. Further analyses are warranted to understand the relationship between the clade of $M$. polycnemoides/M. parlatorei and M. shastense (Fig. 229).

Mortierellomycota Tedersoo, Sanchez-Ramirez, Kõljalg, Bahram, M. Döring, Schigel, T.W. May, M. Ryberg \& Abarenkov

\section{Mortierellomycetes Doweld}

\section{Mortierellales Caval.-Sm.}

Notes: We follow the latest treatments and updated accounts of Mortierellales in Wagner et al. (2013) and Spatafora et al. (2016).

\section{Mortierellaceae A. Fisch.}

Notes: We follow the updated concept of Mortierellaceae as presented in Wagner et al. (2013).

\section{Mortierella Coem.}

Notes: Mortierella (phylum Mortierellomycota) was described by Coemans (1863) based on the type $M$. polycephala. Mortierella is widespread in the temperate zone, where it is almost cosmopolitan and occurs in a wide range of habitats; Mortierella sp. have been reported from bat dung (Degawa and Gams 2004), anthropods (Hyde et al. 2017), freshwaters (Nguyen et al. 2019), and from the recently deglaciated barren ground in glacier forefields (Kuhnert et al. 2012; Dresch et al. 2019). Numerous pure culture isolates of Mortierella spp. were obtained during a study focusing on the seasonal dynamics of soil fungi in a glacier forefield, among them also Mortierella gamsii. Moreover, an undescribed species was repeatedly isolated. Micromorphological studies elucidated clear distinctive
Table 6 Diagnostic morphological characters of the smut fungi of Microbotryum on Polygonum

\begin{tabular}{|c|c|c|c|}
\hline Character & M. aviculare & M. polycnemoides & M. shastense \\
\hline Spore mass (colour) & Cinnamon & Blackish brown & Dark reddish brown \\
\hline Spore length $(\mu \mathrm{m})$ & $(9.5-) 10.5-14.5(-16)$ & $(14-) 15-19(-21)$ & $(7-) 7.5-10(-11)$ \\
\hline Spore mean length $(\mu \mathrm{m})$ & $12.4 \pm 0.9$ & $16.6 \pm 1.1$ & $8.8 \pm 0.6$ \\
\hline $\begin{array}{l}\text { Meshes per spore diameter } \\
\text { (number) }\end{array}$ & $(4-) 5-8(-9)$ & $(8-) 9-12(-13)$ & $6-9$ \\
\hline Muri (height, $\mu \mathrm{m}$ ) & $0.6-1.1(-1.4)$ & $1-1.9(-2.3)$ & $0.5-0.8$ \\
\hline
\end{tabular}




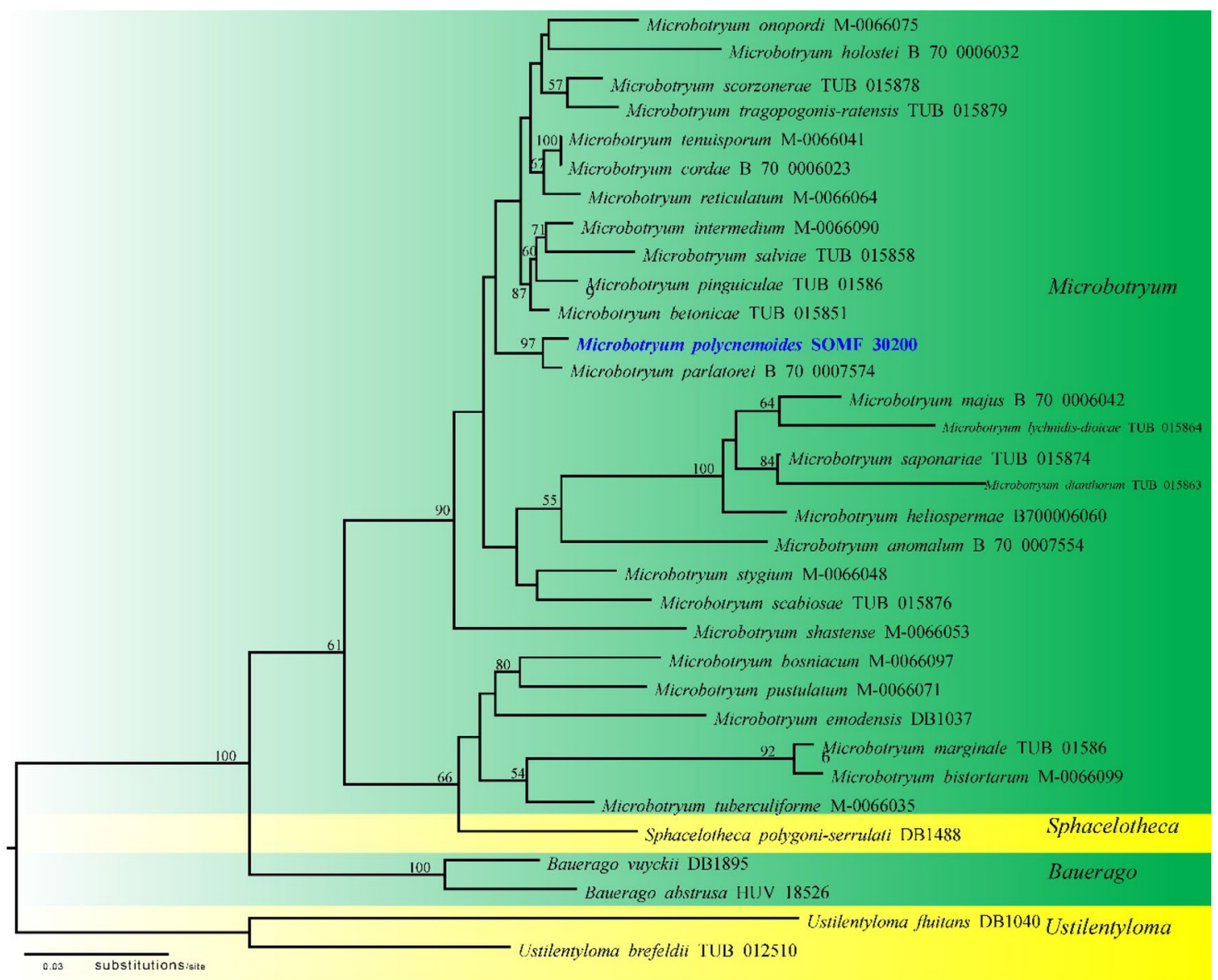

Fig. 229 Most likely tree inferred by maximum likelihood analysis via RAxML version 8.2.11 (Stamatakis 2014) based on concatenated MAFFT v7.450 (Katoh and Standley 2013) alignments of ITS and LSU dataset for Microbotryum species and several closely related genera in Microbotryales. The tree is based on the same dataset as in

morphological characters. Together with phylogenetic analyses of ITS and LSU sequence data, a novel species Mortierella solitaria is introduced (Figs. 230, 231, 232, 233 and 234).

Mortierella solitaria Telagathoti, M. Probst \& Peintner $\boldsymbol{s p}$. nov.

Index Fungorum number: IF557446; Facesoffungi number: FoF 09208; Figs. 230, 231, 232

Etymology: Latin solitaria means solitary, living / acting on its own. This refers to i) the unbranched sporangiophores, which usually appear solitary or in small groups on the mycelium. ii) The phylogenetic position without a clear sister-group. iii) The solitary status of people in quarantine during the COVID 19 pandemic.

\section{Holotype: OAS3}

Saprobic barren or poorly vegetated ground in alpine areas. Asexual morph Colonies rapidly forming characteristic concentrically zonate pattern consisting of broad lobes is produced on LCA media, whereas this pattern is
Kemler et al. (2009), but was pruned to only show one specimen per fungal species. Values at nodes indicate bootstrap values inferred by 100 replicates; only values greater than $50 \%$ are shown. The newly generated sequence is in blue bold

inconspicuous under the thick cottony layer of mycelium, which readily appears on PDA from day 1 . A pronounced smell of garlic at $10{ }^{\circ} \mathrm{C}$ changes to a faded slightly fruity odour when incubated at the higher temperatures. Colonies exhibiting faster growth rates at temperatures $>10^{\circ} \mathrm{C}$, forming thick mycelial layers, but no or very few sporangia. Sporangia hyaline, many-spored, globose with a smooth-walled surface. They are 15-22 $\mu \mathrm{m}$ diam. with a deliquescent wall. A conspicuous collarette is left after spore liberation. Sporangiophores arise from both the aerial and substrate mycelium. They are simple and unbranched, 50-200 $\mu \mathrm{m}$ $(-500 \mu \mathrm{m})$ tall, non-septate. Sporangiophores are tapering from $4-9 \mu \mathrm{m}$ at the base to $3-4 \mu \mathrm{m}$ near the tip. One of the isolates (OAS4) formed part of the sporangiophores with a swollen base similar to those formed by M. alpina. Sporangiophores typically awl-like, sometimes slightly widening to an inconspicuous 6-8 $\mu \mathrm{m}$ wide apophysis-like inflation. The columella is slightly bulged, but without spinose outgrowing typical for M. gamsii. Sporangiospores are subglobose 

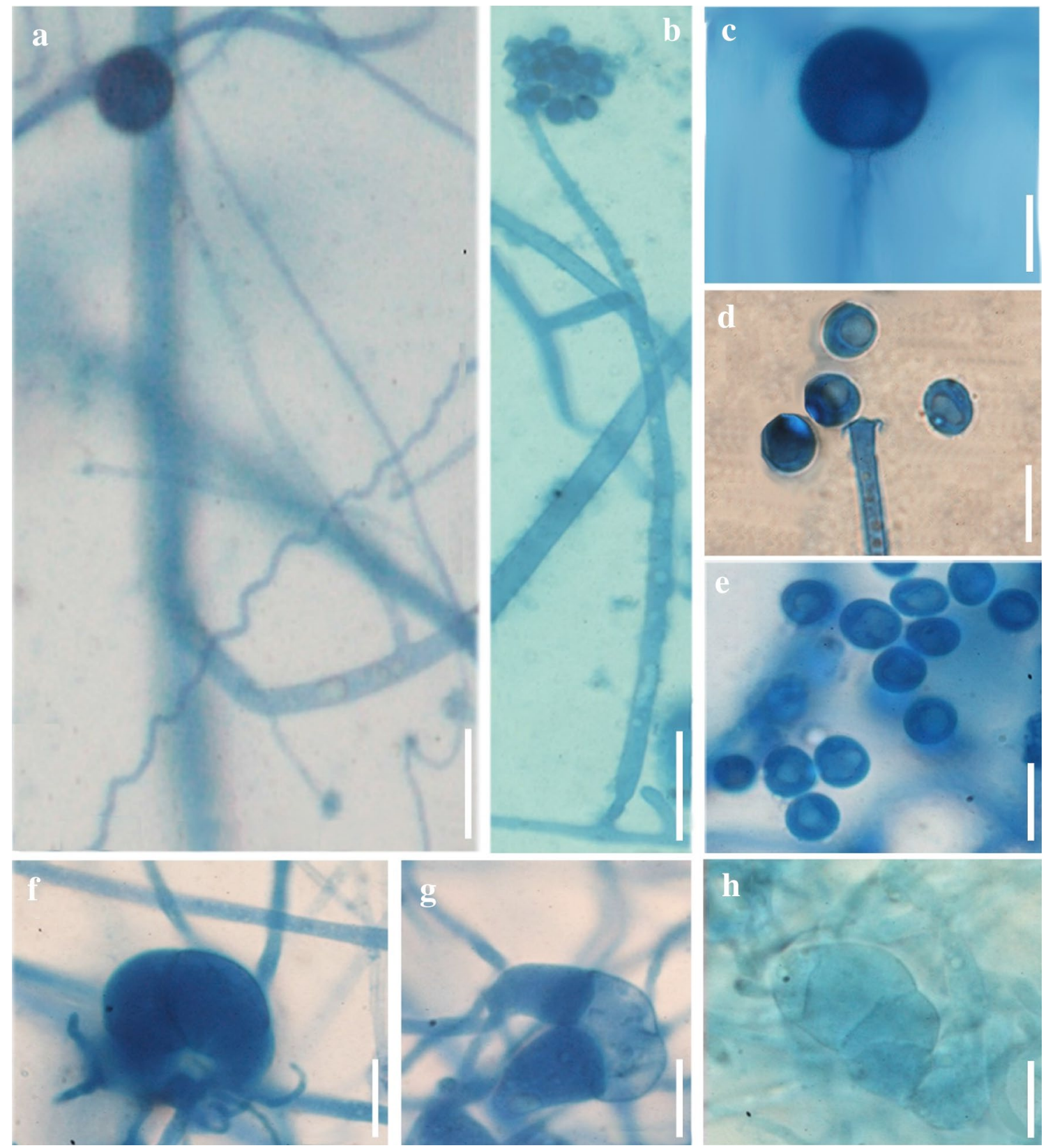
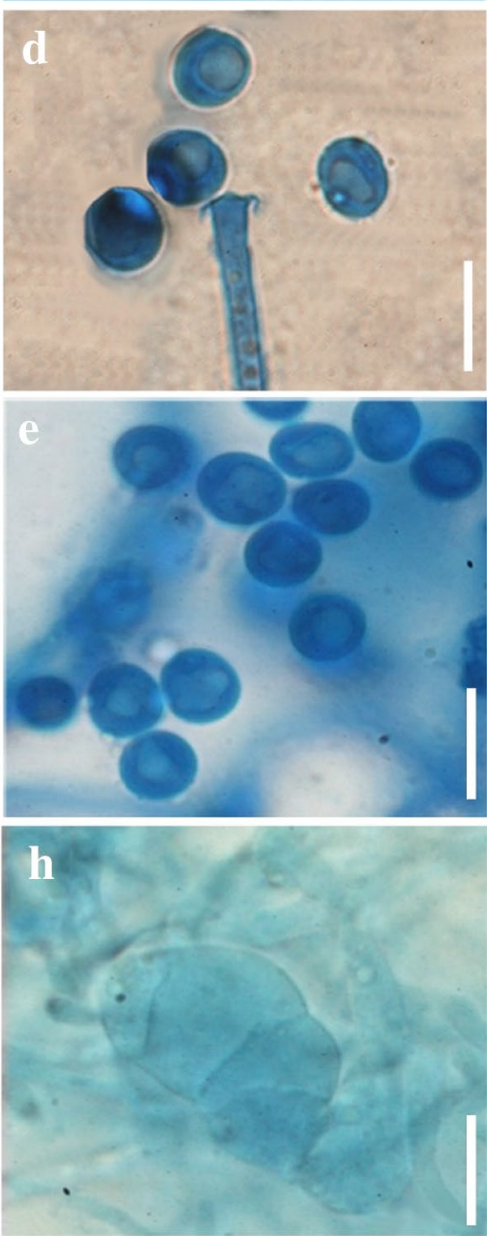

Fig. 230 Mortierella solitaria (OAS3, holotype). a, b Sporangiophores formed on aerial or basal hyphae. c Sphaerical sporangial head with intact smooth peridium and small apophysis-like widening of the sporangiophore. $\mathbf{d}$ Tip of a sporangiophore with collarette and

to cylindrical-ovoid, smooth-walled, (6-) 7-8 (-10) $\mu \mathrm{m} \times(5.5-) 6-8 \mu \mathrm{m}(\overline{x=} 7.8 \times 6.9 \mu \mathrm{m}, \mathrm{n}=30)$, often with an oil drop. Gemme with 5.3-5.7 $\mu \mathrm{m}$ diam., appears macroscopically as white-spotted clusters. They were observed in the substrate mycelium on Hempseed agar at $25^{\circ} \mathrm{C}$. Chlamydospores and stylospores undetermined. Sexual morph Undetermined. The formation of zygospores is initiated, but aborted on PDA and SNA at $20^{\circ} \mathrm{C}$ : suspensor cells and the progametangia are formed but do not further develop: empty hyphal compartment remained after plasmogamy. spores. e Sporangiospores released from the sporangium. f-h Initial stages of gametangiogamy with empty, aborted zygospore. Scale bars: $\mathbf{a}, \mathbf{b}=20 \mu \mathrm{m}, \mathbf{c}-\mathbf{h}=10 \mu \mathrm{m}$

Culture characteristics: Colonies rapidly growing, white, and filling the agar plate $(8 \mathrm{~cm})$ within $5-10 \mathrm{~d}$ of incubation (Fig. 231).

Material examined: AUSTRIA, Obergurgl, Rotmoos glacier forefield 2300 msl., barren ground with scattered Saxifraga aizoides and Persicaria vivipara, 10 September 2016, A. Telagathoti, J. Falbesoner P. Dresch (OAS3, holotype), ex-type living culture JMRC: SF013920; OAS4 (JMRC: SF013921) and OAS5 (JMRC: SF013922) are from the same origin, but different sampling sites. 


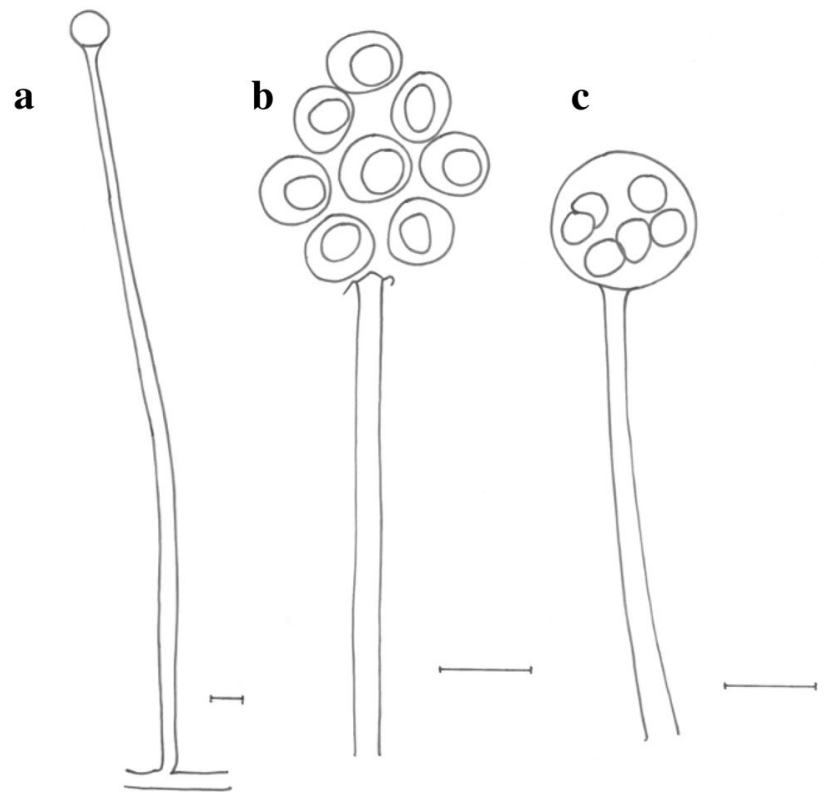

Fig. 231 Sporangia of Mortierella solitaria. a Length of the sporangia $(-500 \mu \mathrm{m}$ tall). b Sporangiophore tips showing collarette and the spores. $\mathbf{c}$ Details of the peridial wall of the sporangia. Scale bars: $\mathbf{a}-\mathbf{c}=10 \mu \mathrm{m}$

GenBank numbers: ITS $=$ MT279272, MT279273, MT279274, LSU = MT279275, MT279276.

Notes: The rDNA ITS sequences generated from our Mortierella solitaria isolates showed $<95 \%$ sequence similarity to the sequences generated from the type materials of $M$. gamsii and of M. fluviae. The phylogenetic analysis (maximum likelihood, parsimony bootstrap analysis, and Bayesian inference analysis; Fig. 233) indicates that M. solitaria could belong to $M$. gamsii species complex. However, M. solitaria lineage is clearly distinct from $M$. gamsii, M. fluviae, $M$. exigua, M. elongata, M. alpina, M. schmuckeri, M. cogitans and M. sclerotiella (Fig. 232). An analysis of the concatenated ITS and LSU sequences confirmed that $M$. solitaria constitutes a distinct lineage of Mortierella (Fig. 234), but relationships have not yet been further resolved.

Mortierella solitaria differs in several morphological features from similar species. Based on the classical concepts (Linnemann 1941), M. solitaria species could either be placed in M. simplex or M. alpina morpho-groups. However, species in $M$. simplex group usually have sporangiophores longer than $200 \mu \mathrm{m}$, and typical representative species of M. alpina morpho-group have sporangiophores with a typically widening base. All currently described species of M. alpina group differ due to e.g., presence of chlamydospores or different spore morphology. According to our studies, only one (OAS4) of the three isolates occasionally forms sporangiophores with a swollen base. However, the sporangiophore base is only slightly widened. In M. simplex group, $M$. rostafinskii could be similar, but it differs by larger sporangiophores and smaller spores 5-6.5 $\times 3-5 \mu \mathrm{m}$. Sequences of these species (including sequences from type strains) are available in public databases, further confirming that $M$. solitaria is a morphologically and phylogenetically distinct species.

Mortierella solitaria is clearly distinct from all species in $M$. gamsii complex: M. gamsii differs by its branched sporangiophores (Figs. 230 and 231). Mortierella fluviae differs clearly by branched sporangiophores with a bell-shaped apophysis, M. humilis differs by warted sporangiophores and M. exigua has pronounced acrotonous branching of larger sporangiophores and has amoeba-like chlamydospores. Two of M. solitaria isolates (MT279272 and MT279274) were found to be associated with endohyphal bacteria of Burkholderiaceae. The isolates were cured with antibiotics before performing the experiments in this current study.

\section{Mucoromycota Doweld}

\section{Mucoromycetes Doweld}

\section{Mucorales Dumort}

Notes: We follow the latest treatments and updated accounts of Mucorales in Phookamsak et al. (2019) and Wanasinghe et al. (2018).

\section{Mucoraceae Fr.}

Notes: We follow the latest treatments and updated accounts of Mucoraceae in Spatafora et al. (2016), Wanasinghe et al. (2018), and Phookamsak et al. (2019).

\section{Mucor Fresen.}

Notes: Mucor was described by Fresenius (1850), and contains the largest number of species within the Mucorales (Benny et al. 2014; Walther et al. 2013). Species of Mucor are characterized by the formation of sporangia, equally and unequally shaped suspensors, as well as the presence of apophysis, rhizoids, and sporangiola (Walther et al. 2013; Wagner et al. 2019). Mucor species are known to be saprobes that are usually isolated from dung, soil, water, food products, other plant debris, or human clinical specimens (Benny 2008; Álvarez et al. 2011; Walther et al. 2013; Wanasinghe et al. 2018). Some species have positive or negative impacts on human activities. Several species of Mucor have important biotechnological applications and are used in Asia for the production of fermented foods and beverages (Batra and Millner 1974; Nout and Aidoo 2010). Mucor belongs to the main genera causing mucormycoses (Jeong et al. 2019). To date, 12 species are known to be involved in infections (Wagner et al. 2019). Mucor contains 76 accepted species (Walther et al. 2019). 

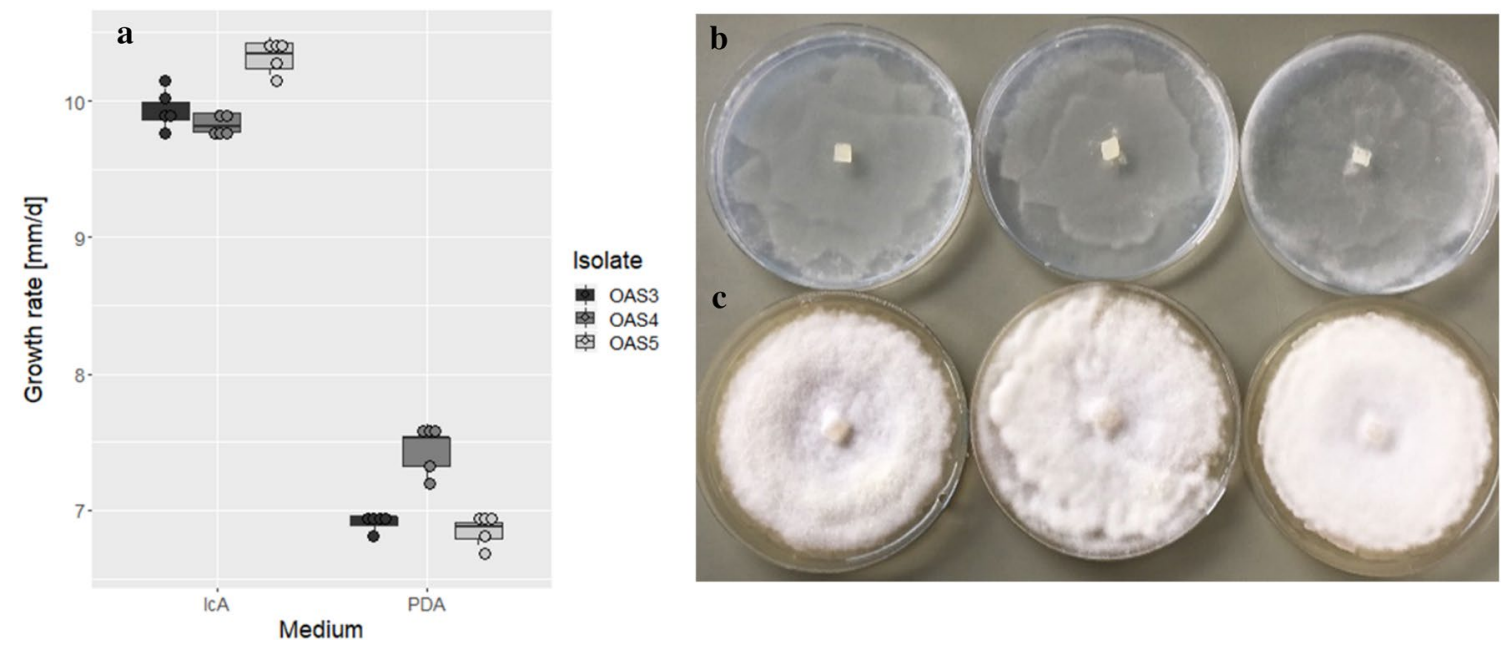

Fig. 232 Growth rates of Mortierella solitaria as boxplots. a Boxplot displaying the mean 25th and 75th percentiles as box and outside values as dots. b, c Seven days old culture of the isolates on LcA and PDA

During an investigation of fungi of the order Mucorales from insects in the Jeonnam Province, Korea, a new species Mucor harpali was isolated from the head of a bombardier beetle (Harpalus sp.). The new species is described and illustrated herein based on morphological characters and molecular phylogenetic analyses (Figs. 235, 236 and 237).

\section{Mucor harpali Hyang B. Lee, P.M. Kirk \& T.T.T. Nguyen sp. nov.}

Index Fungorum number: IF557325; Facesoffungi number: FoF 09209; Fig. 235

Etymology: Referring to the name of a bombardier beetle, Harpalus sp.

Holotype: CNUFC HT 18012

Colonies on MEA at $25{ }^{\circ} \mathrm{C}$ in darkness moderate reddish-brown $(8.1 \mathrm{R} / 3.1)$, the central part with mature sporangia and the margin with immature sporangia, reaching 72-75 mm diam. after 4 days incubation; reverse vivid yellow (1.3Y/7.3). Sporangiophores arising directly from the hyphae immersed in the substrate, tall, 8-19.5 $\mu \mathrm{m}$ diam., usually unbranched or with few secondary branches, septate or coenocytic; one or two septa may be formed below the columella; branches commonly bearing a sterile sporangium. Sporangia non-apophysate, hyaline to yellow when young, becoming reddish-brown, globose, subglobose, 36.5-103.5 ×35-100.5 $\mu \mathrm{m}$, wall deliquescent. Columellae often globose to subglobose, but also obovoid with truncate base, $27.5-55.5 \times 26.5-52.5 \mu \mathrm{m}$, with distinct colar; a few reniform columellae also found. Sporangiospores mostly ellipsoidal, subglobose, reniform, or some irregular, $5.5-11.5 \times 3-5.5 \mu \mathrm{m}$, smooth-walled. Oidia often observed. Zygospores not observed. Substrate mycelium branched, containing inflated regions terminating in thin rhizoid-like filaments and yellow droplets.

Culture characteristics: The isolate grew over a wide range of temperatures with varying growth rates on SMA (synthetic mucor agar), PDA (potato dextrose agar) and MEA (malt extract agar) of $13 \mathrm{~mm}, 15 \mathrm{~mm}$, and $18.5 \mathrm{~mm}$ per $24 \mathrm{~h}$, respectively. Optimal growth was observed around $25^{\circ} \mathrm{C}$, and slow growth was observed at below $10^{\circ} \mathrm{C}$. Maximum growth temperature was $35^{\circ} \mathrm{C}$. The fungus did not grow at $36^{\circ} \mathrm{C}$.

Material examined: REPUBLIC OF KOREA, Jeonnam Province, garden of the Chonnam National University located in Gwangju (35 $\left.10^{\prime} 20.3^{\prime \prime} \mathrm{N} 126^{\circ} 53^{\prime} 56.9^{\prime \prime} \mathrm{E}\right)$, from head of a bombardier beetle (Harpalus sp.), 30 July 2018, collected by H.B. Lee (CNUFC HT18012, holotype), extype living culture (CNUFC ICT18001).

GenBank numbers: ITS = MT192528, MT192529, LSU $=$ MT192532, MT192533.

Notes: Phylogenetic analyses (Figs. 236 and 237) clearly placed Mucor harpali among other Mucor species within the Mucor racemosus group previously suggested by Walther et al. (2013). Mucor harpali and Mucor sp. CBS 608.78 (former: M. circinelloides $\mathrm{f}$. griseocyanus) are the phylogenetically nearest species in the ITS tree (Fig. 236). Mucor harpali (Fig. 235) shared some similarities with M. circinelloides f. griseocyanus (current name: Mucor griseocyanus) such as ellipsoidal sporangiospores, and maximum temperature growth (Schipper 1978; Wagner et al. 2019). However, sporangia (up to $60 \mu \mathrm{m})$ and collumella $(38 \times 35 \mu \mathrm{m})$ of $M$. griseocyanus reported in the literature (Schipper 1978; Wagner et al. 2019) were smaller than those of M. harpali. Mucor harpali can be distinguished from the other species in this 


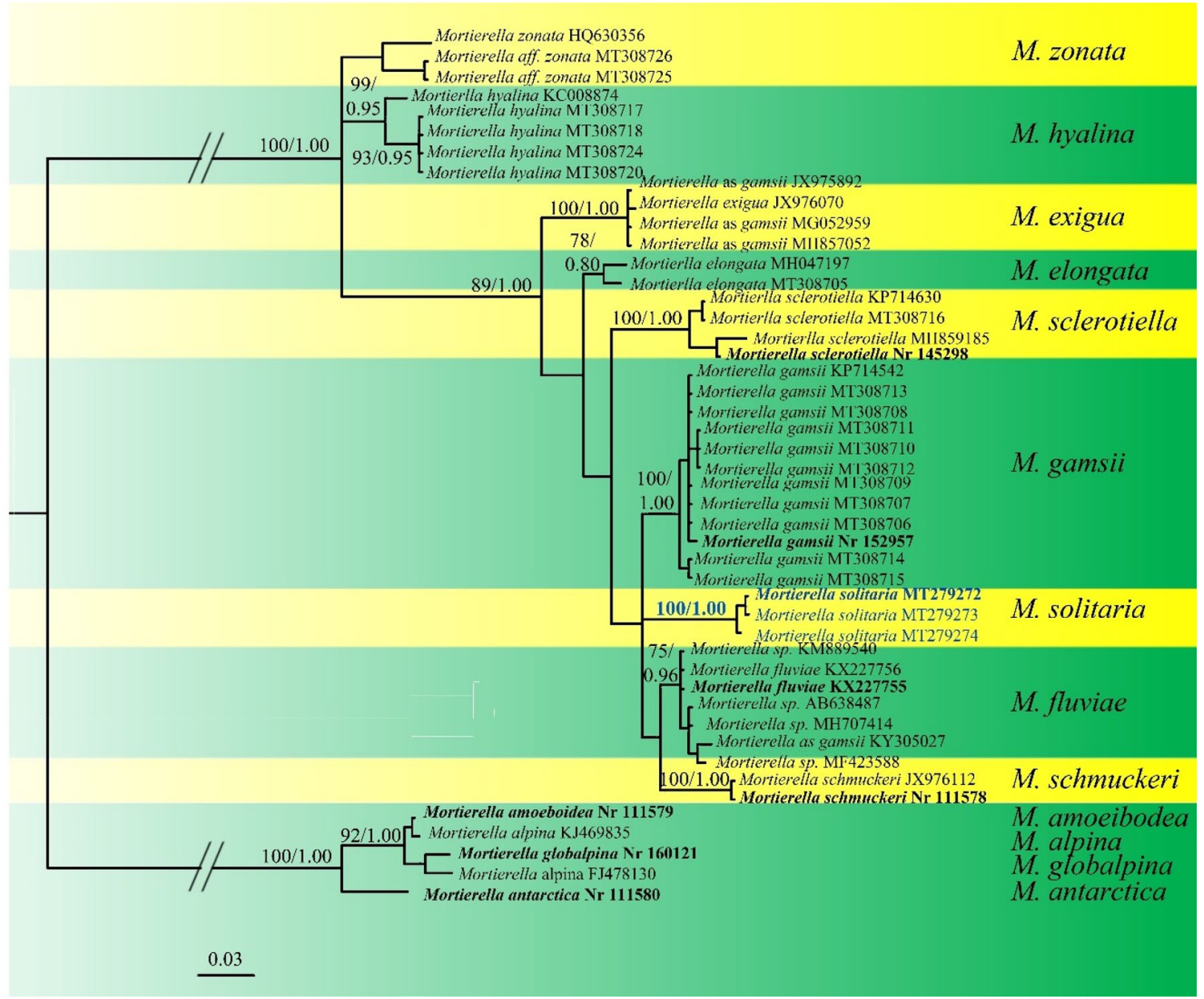

Fig. 233 Phylogenetic relationship of Mortierella solitaria with related taxa based on the nucleotide sequences of the ITS. The maximum likelihood (ML) tree (-ln likelihood=1899.6675) was constructed in MEGA X (Kumar et al. 2018). Five representatives of Mortierella alpina complex are used as the outgroup taxa. The data-

group, including $M$. racemosus, $M$. plumbeus, $M$. racemosus f. sphaerosporus, M. brunneogriseus, and M. mousanensis. Schipper (1978) has mentioned that M. plumbeus produces columellae usually with apical projections, whereas in $M$. harpali apical projections are absent. Mucor racemosus f. sphaerosporus produces mainly subglobose sporangiospores (Schipper 1978), while M. harpali produces variable set includes 494 total characters. Phylogenetic trees obtained from ML and Bayesian inference posterior probabilities (BYPP) were similar in topology. Bootstrap support on the nodes represents MPBS/ BYPP. The new species $M$. solitaria is highlighted in blue in the yellow box

in shape, mostly ellipsoidal. Mucor mousanensis produces columellae applanate elongated conical (up to $70 \times 50 \mu \mathrm{m}$ ) (Schipper 1978), while M. harpali does not produce this structure. Based on the phylogenetic analyses and morphological comparison with similar species of Mucor, M. harpali is a distinct species. 


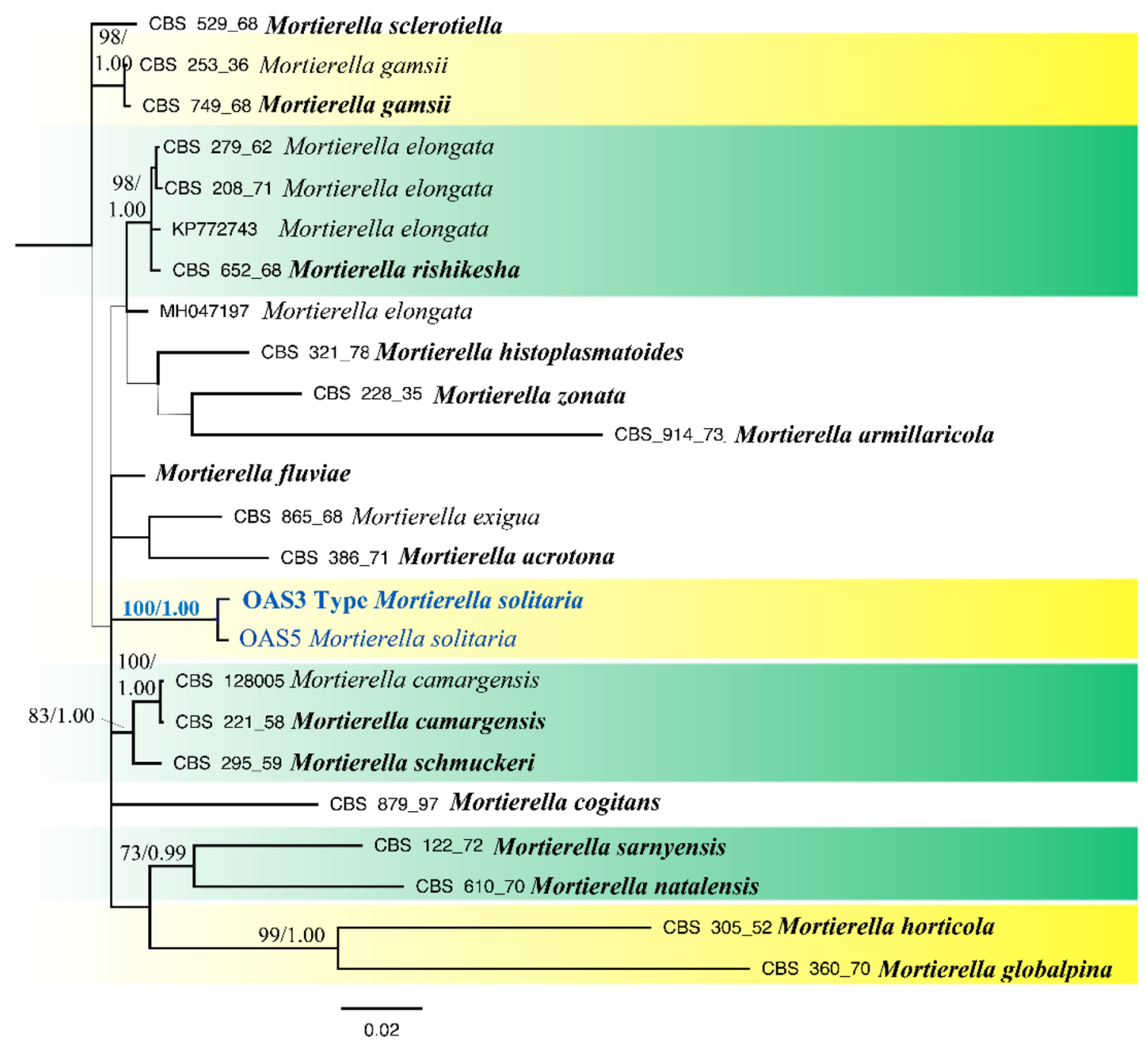

Fig. 234 Phylogenetic relationship of Mortierella solitaria with related taxa based on the concatenated nucleotide sequences of the ITS and LSU. The maximum likelihood (ML) tree (-ln likelihood $=5141.7516$ ) was constructed in MEGA X (Kumar et al. 2018). Mortierella horticola and M. globalpina are used as the outgroup taxa. The dataset includes 1439 total characters. Phylogenetic trees obtained from ML, and Bayesian inference posterior probabilities (BYPP) were similar in topology. Bootstrap support on the nodes represents MPBS/BYPP. The new species Mortierella solitaria is highlighted in blue in the yellow box 

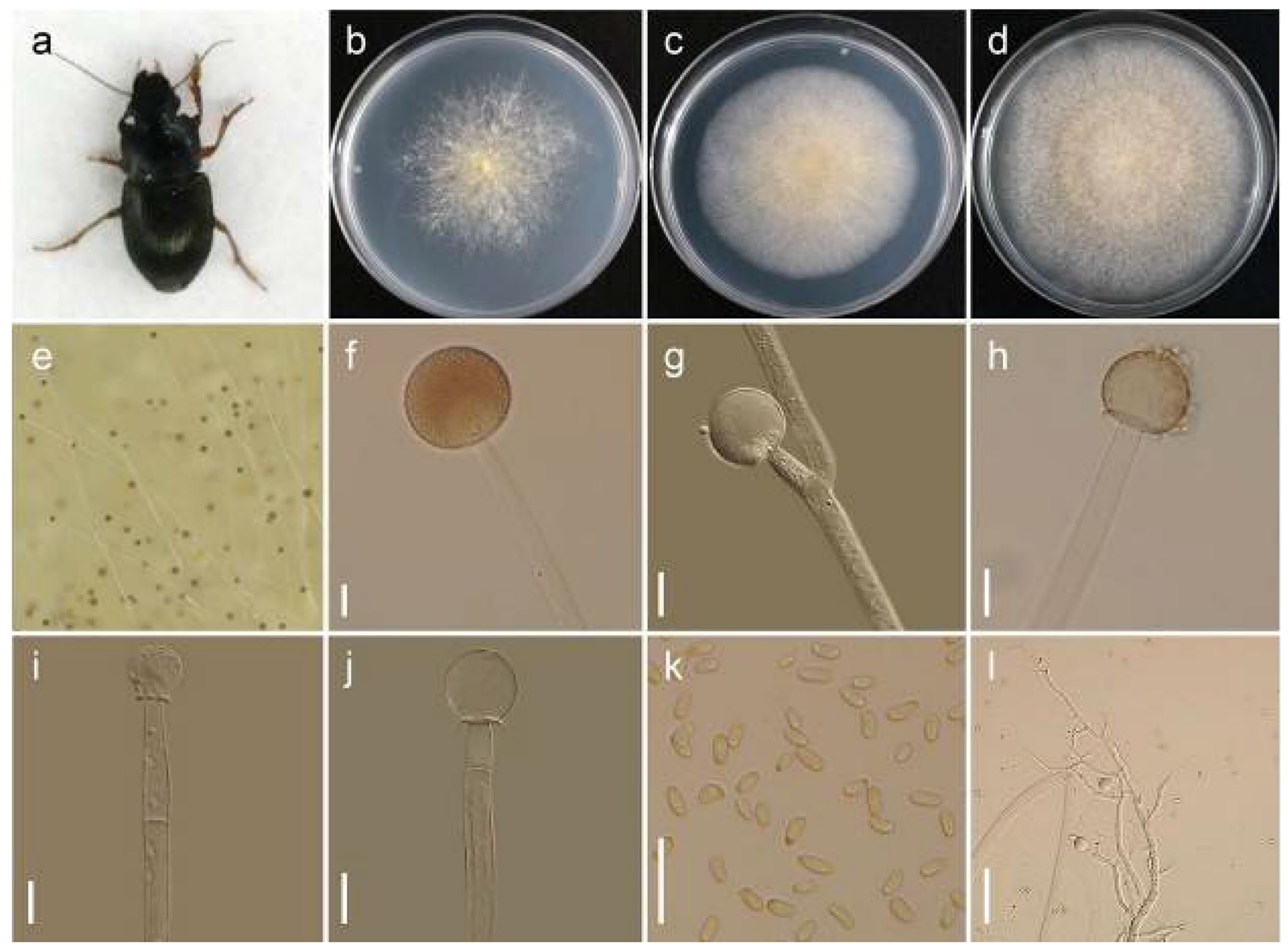

Fig. 235 Mucor harpali (CNUFC ICT18001, ex-type living culture). a A bombardier beetle of Harpalus sp. belonging to Coleoptera. b Colony on synthetic mucor agar (SMA). c Colony on potato dextrose agar (PDA). d Colony on malt extract agar (MEA). e, f Spo-

rangiophores and sporangia. g Sterile sporangium. h-j Typical columellae. k Sporangiospores. I Rhizoids (Fig. e: observed under a stereomicroscope; Figs. f-l: light microscope). Scale bars: $\mathbf{f}-\mathbf{l}=20 \mu \mathrm{m}$ 


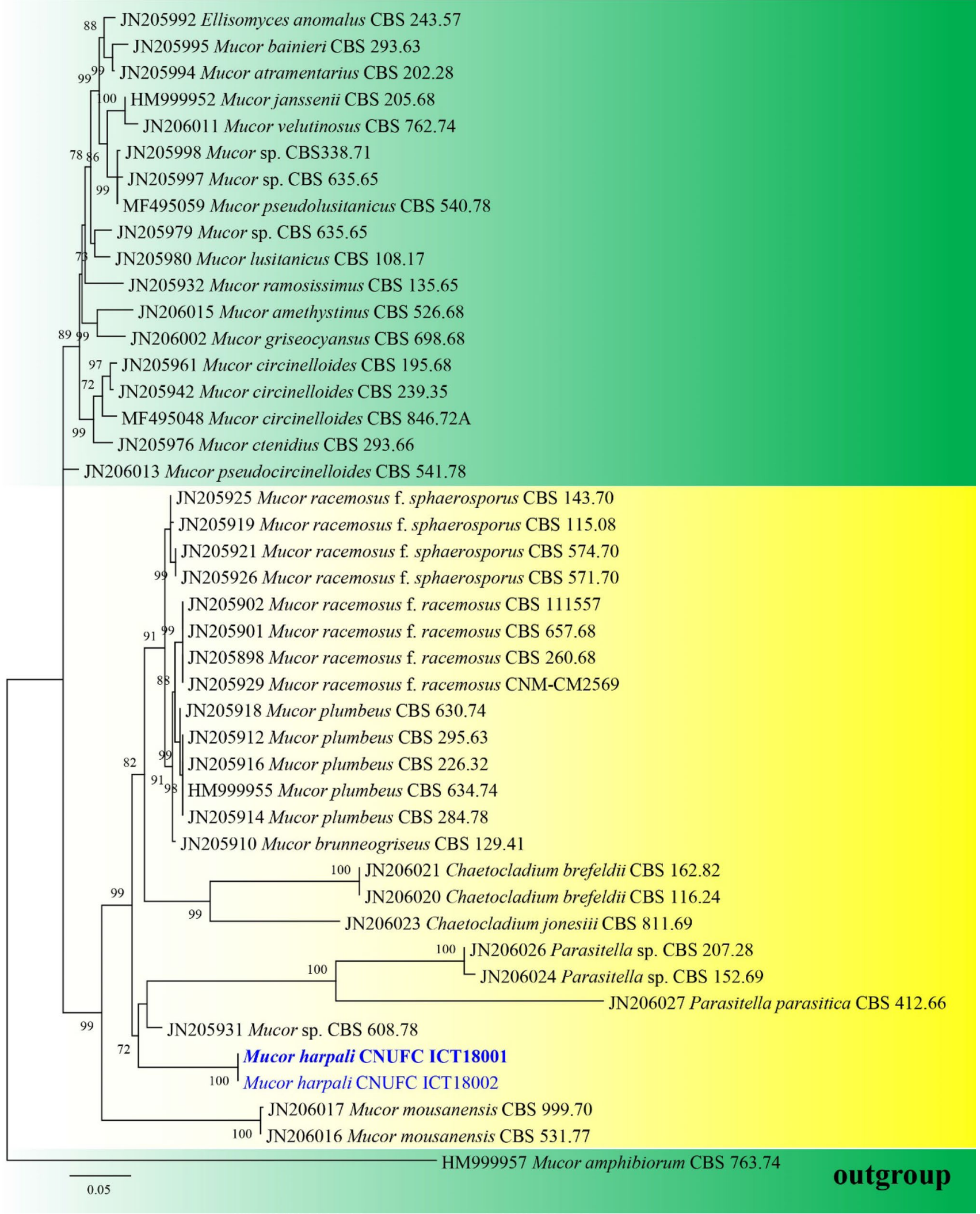

Fig. 236 Phylogram generated from maximum likelihood analysis based on ITS sequence data for the species of Mucor. Bootstrap values equal to or greater than $70 \%$ for ML analyses is presented at the nodes. Mucor amphibiorum is used as the outgroup taxon. Type strains are in bold and newly generated sequences are in blue 


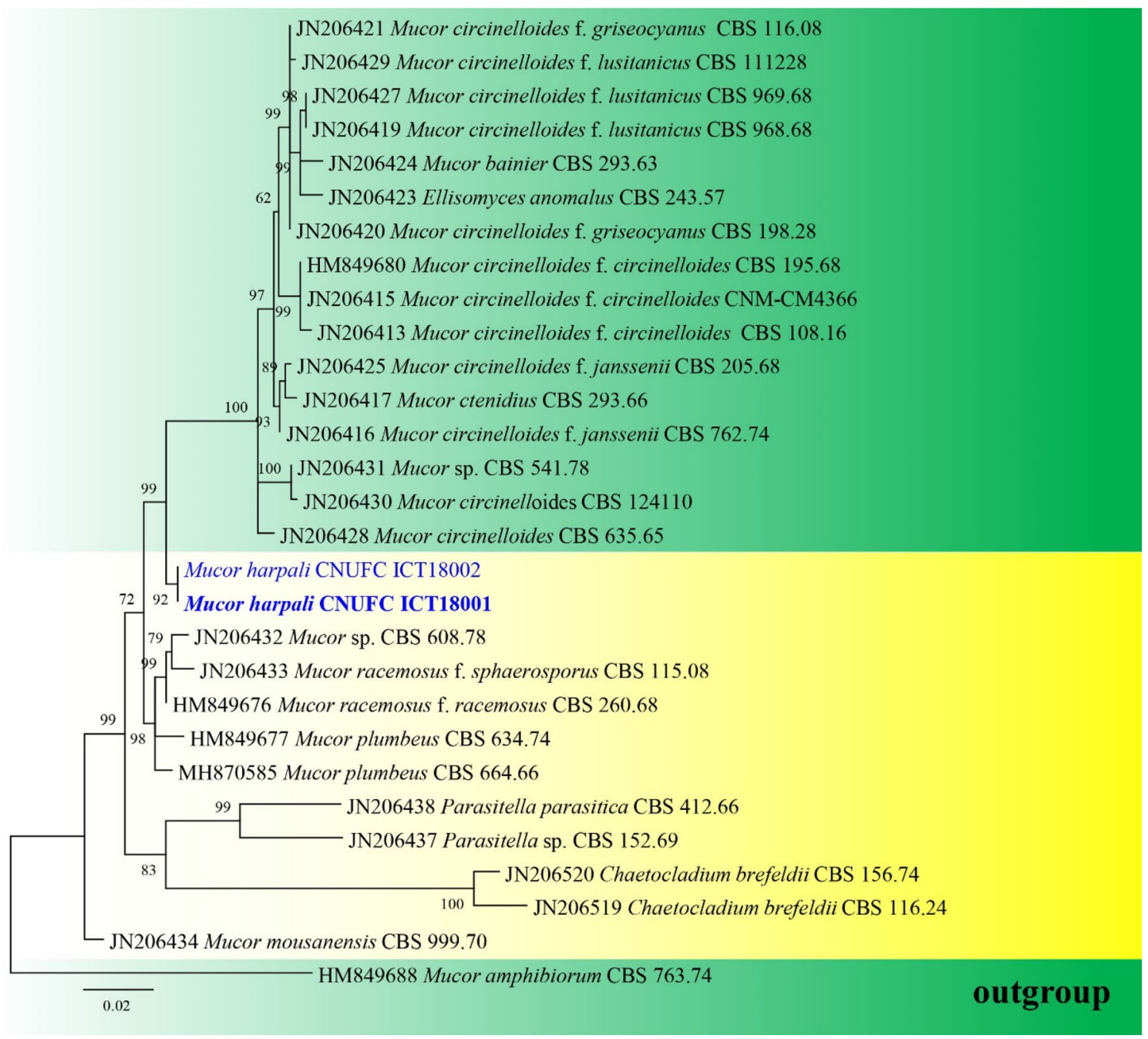

Fig. 237 Phylogram generated from maximum likelihood analysis based on LSU sequence data for the species of Mucor. Bootstrap values equal to or greater than $70 \%$ for ML analyses is presented at

Acknowledgements Saranyaphat Boonmee would like to thank the Thailand Research Fund (Grant No. TRG6180001), the Mae Fah Luang University Fund (Grant No. 631C15001) and Plant Genetic Conservation Project under the Royal Initiation of Her Royal Highness Princess Maha Chakri Sirindhorn-Mae Fah Luang University. Saranyaphat Boonmee also thanks Mingkwan Doilom, Chun-Fang Liao and Er-Fu Yang for helping with the molecular DNA sequencing of the samples and thanks Shaun Pennycook for his advice on fungal names and an essential nomenclatural review. Sajini K.U. Chandrasiri would like to thank the Mushroom Research Foundation. Kevin D. Hyde thanks the 2019 high-end foreign expert introduction plan to Kunming Institute of Botany (Granted by the Ministry of Science and Technology of the People's Republic of China, Grant No. G20190139006), the future of specialist fungi in a changing climate: baseline data for generalist and specialist fungi associated with ants, Rhododendron species and Dracaena species (Grant No. DBG6080013), Impact of climate change on fungal diversity and biogeography in the Greater Mekong Subregion (Grant No. RDG6130001). Kevin D. Hyde also thanks Chiang Mai University for the award of visiting Professor. Dhanushka Wanasinghe thanks CAS President's International Fellowship Initiative (PIFI) for the nodes. Mucor amphibiorum is used as the outgroup taxon. Type strains are in bold and newly generated sequences are in blue

funding his postdoctoral research (Grant No. 2021FYB0005) and the Postdoctoral Fund from Human Resources and Social Security Bureau of Yunnan Province. Mark S. Calabon is grateful to Mushroom Research Foundation and Department of Science and TechnologyScience Education Institute (Philippines). Gareth Jones is supported under the Distinguished Scientist Fellowship Program (DSFP), King Saud University, Kingdom of Saudi Arabia. Walter Rossi and Marco Leonardi thank the entomologists who supplied or identified the insects bearing Laboulbeniales: V. Assing, G. Makranczy, M. Kotrba, A.F. Newton, M.A. Zumbado; they also wish to thank S. Santamaria (Universitat Autònoma de Barcelona) for the photos with DIC optics. Paras Nath Singh, Sanjay K. Singh and Deepak K. Maurya thank Director MACS' Agharkar Research Institute, Pune, DST, Govt. of India and Savitribai Phule Pune University (SPPU) for providing research facilities, financial and laboratory support on their contribution. Shiwali Rana, A.C. Lagashetti and Deepika Choudhary thank Director MACS' Agharkar Research Institute, Pune for providing research facilities, University Grant Commission (UGC), CSIR, New Delhi and Savitribai Phule Pune University (SPPU) for granting Research Fellowships and for registration to Ph.D., and also thank to Mr. S.B. Gaikwad for 
providing photoplates on their contribution. Chang-Lin Zhao, YuCheng Dai, Yan-Hong Mu, Hai-Sheng Yuan and Shuang-Hui He by the National Natural Science Foundation of China (Nos. 31870011, 31750001, 31770028 and 31970017). Rungtiwa Phookamsak would like to thank CAS President's International Fellowship Initiative (PIFI) for young staff (Grant No. Y9215811Q1), Provincial Science and Technology Department (grant no. 202003AD150004), Yunnan Provincial Key Programs of Yunnan Eco-friendly Food International Cooperation Research Center (Grant No. 2019ZG00908), Key Research Program of Frontier Sciences "Response of Asian mountain ecosystems to global change", CAS, Grant No. QYZDY-SSWSMC014", thank the director Jun-Bo Yang and Plant Germplasm and Genomics Center in Germplasm Bank of Wild Species, Kunming Institute of Botany for the molecular laboratory support and Er-Fu Yang at Chiang Mai University for general assistance. María P. Martín, Margarita Dueñas and M. Teresa Telleria would like to thank the Agreement ENDESA and San Ignacio de Huinay Foundations and Consejo Superior de Investigaciones Científicas, CSIC (Projects No. 2011HUIN10, 2013CL0012) and DGICYT projects CGL2005-01192/BOS, CGL2009-07231, CGL2015-67459-P, CSIC project PIE202030E059 for financial support and also thank to Reinhard Fitzek (San Ignacio del Huinay Foundation, Chile) for his invaluable help during fieldwork, Marian Glenn (Seton Hall University, US) for checking the English, and curators of $\mathrm{H}$ and CFMR herbaria for their assistance. Izabela L. Kałucka, Andrzej M. Jagodziński and Kare Liimatainen would like to thank Royal Botanic Gardens, Kew, its Department of Identification and Naming and Fungarium for providing their facilities for the new species analyses. Izabela L. Kałucka, Andrzej M. Jagodziński and Kare Liimatainen are also acknowledge the support from the Polish Ministry of Science and Higher Education (grant No. N N305 2996 40), the General Directorate of State Forests, Warsaw, Poland (research project "Environmental and genetic factors affecting productivity of forest ecosystems in forest and post-industrial habitats"; the Polish Forest Fund research project "Recognition and assessment of the current state of natural habitats in the vicinity of the Czerwona Woda River") and the University of Lodz: Faculty of Biology and Environmental Protection (grant for international scientific visits) and Department of Algology and Mycology (MSHE subsidy). Diana S. Pereira and Alan J.L. Phillips acknowledge the support from UIDB/04046/2020 and UIDP/04046/2020 Centre grants from FCT, Portugal (to BioISI). Nakarin Suwannarach also thanks Chiang Mai University for financial and facility support. Tarynn B. Potter, Roger G. Shivas, Adam H. Sparks and Niloofar Vaghefi would like to thank the University of Southern Queensland and the Grains Research and Development Corporation projects DAQ00186 and DAQ00194. Tarynn B. Potter, Roger G. Shivas, Adam H. Sparks and Niloofar Vaghefi are grateful to Ms. Encarnacion Adorada and Dr. Precila Gonzales for help with fungal isolations. M.A. Abdel-Wahab would like to thank the Japan Society for the Promotion of Science (JSPS) for the award of post-doctoral fellowship and the research grants (No. 185701000001 and No. 18-06620). M. A. Abdel-Wahab and Faten A. Abdel-Aziz thank Prof. Shaun Pennycook for revising the Latin names. Guo-Jie Li and Wen-Fei Lin would like to thank the National Natural Science Foundation of China (Nos. 31500013, 30770013), Talent Introduction Scientific Research Special Project of Hebei Agricultural University (YJ201849), and the Earmarked Fund for Hebei Edible Fungi Innovation Team of Modern Agro-industry Technology Research System (Project ID: HBCT2018050205). Upendra Singh and R.P. Bhatt are grateful to the Head, Department of Botany \& Microbiology, HNB Garhwal University, Srinagar Garhwal for providing all kinds of facilities during the present study. Upendra Singh received financial assistance from Govind Ballabh Pant National Institute of Himalayan Environment and Sustainable Development (GBPNIHESD), Kosi-Katarmal, Almora, Uttarakhand is gratefully acknowledged. Upendra Singh and R.P. Bhatt are also grateful to Dr. Kanad Das for his support, useful discussions and helpful suggestions, while Dr. Priyanka Uniyal, Dr. Tahir Mehmood,
Dr. Aniket Ghosh HNB Garhwal University and Dr. M.E. Hembrom (BSI) are duly acknowledged for their assistance in field. Hyang Burm Lee is supported by the Graduate Program for the Undiscovered Taxa of Korea, and the Project on Survey and Discovery of Indigenous Fungal Species of Korea funded by National Institute of Biological Resources of the Ministry of Environment (MOE), Korea. V.V. Sarma would like to thank SERB, Department of Science and Technology, Government of India, for funding a project (SERB/SB/SO/PS/18/2014 dt.19.5.2015) the Department of Biotechnology, Pondicherry University for facilities; forest departments of Andaman and Nicobar Islands and Tamil Nadu, India are thanked for providing permission to collect samples. M Niranjan thanks SERB, Govt. of India for a fellowship. Kunhiraman C. Rajeshkumar thanks SERB, Department of Science and Technology, Government of India for providing financial support under the project YSS/2015/001590 and Dr. P. K. Dhakephalkar, Director, ARI for providing the facility. KC Rajeshkumar also thank Mr. Atul Dwivedi for support in SEM photography. Rong-Ju Xu would like to acknowledge the Second Tibetan Plateau Scientific Expedition and Research (STEP) Program [Grant No. 2019QZKK0503], the open research project of "Cross Cooperative Team" of the Germplasm Bank of Wild Species, Kunming Institute of Botany, Chinese Academy of Sciences [Grant No. 292019312511043], Science and Technology Service Network Initiative, Chinese Academy of Sciences [KFJ-STSQYZD-171]. S.N. Wijesinghe would like to acknowledge Mae Fah Luang University, National Science Foundation of China (NSFC) project code 31851110759 and National Natural Science Foundation of China (No. 31972222, 31560489), Program of Introducing Talents of Discipline to Universities of China (111 Program, D20023), Talent Project of Guizhou Science and Technology Cooperation Platform ([2017]5788-5, [2019]5641 and [2020]5001), Guizhou Science, Technology Department International Cooperation Basic project ([2018]5806). Hong-Wei Shen would like to thank the National Natural Science Foundation of China (Project ID: 31970021 and 32060005), and Fungal Diversity Conservation and Utilization Innovation Team of Dali University (ZKLX2019213) for financial support. Hongwei Shen thanks Qishan Zhou, Qingxiong Ruan, Songming Tang, and Xiu $\mathrm{He}$ for their help on sample collection, and Yanmei Zhang, Longli Li, and Wenli Li for their kindly help on DNA extraction and PCR amplification. Jing-Yi Zhang would like to thank the National Natural Science Foundation of China (NSFC 32060013) and Youth Science and Technology Talent Development Project from Guizhou Provincial Department of Education (QJHKYZ [2021]263). Dan-Feng Bao would like to thank the National Natural Science Foundation of China (Project ID: 31660008 and 31860006), Fungal diversity conservation and utilization innovation team (ZKLX2019213) and the Thailand Research Fund grant "impact of climate change on fungal diversity and biogeography in the Greater Mekong Sub-region (RDG6130001)" for financial and laboratory support. JafarAbdollahzadeh was supported by University of Kurdistan-Sanandaj, Iranian Research Institute of Plant Protection-Tehran and Tarbiat ModaresUniversity, Tehran, Iran. F. Dovana would like to thank Alfredo Justo (New Brunswick Museum) for checking the English and suggestions on their contribution. Muhammad Usman and Abdul Nasir Khalid would like to thank Higher Education Commission, Pakistan for financial support through NRPU research project no. 20-3383/NRPU/R\&D/HEC/14/184. Lilla Bóna, Zsolt Merényi, Lajos Boros, J. Benjamin Stielow and Bratek Zoltán are greatly appreciate to János Patonai for discovering the habitat of Geastrum hansagiense to Csaba Locsmándi (curator of the Macrofungi Collection of the Hungarian Natural History Museum) for kindly providing the G. floriforme aff. specimens and to Ferenc Mészáros for support in LM photography and also thank to the Széchenyi 2020 Programme (Grant No. GINOP 2.2.1-15-2017-00042). Anusha Telagathoti, Maraike Probst and Ursula Peintner would like to thank the FWF and the Land Tirol for funding the MICINSNOW project (P31038), and also thank Philipp Dresch and Johannes Falbesoner for assistance with fieldwork. Dr. Kerstin Voigt is acknowledged for valuable suggestions. 
Isaac Garrido-Benavent would like to thank Asunción de los Ríos and Esther Rodríguez (MNCN-CSIC, Spain) for logistical support and help at the laboratory, and Reinhard Berndt (Z+ZT curator) for providing useful information about the type collection of Chaetoscutula juniperi. The study of Cvetomir M. Denchev and Teodor T. Denchev received support from the SYNTHESYS Plus project (www.synthesys.info), which is financed by the H2020 Research Infrastructures Programme at the Botanic Garden Meise, Belgium (Grant no. BE-TAF-2445). Chun Y. Deng acknowledges the Biodiversity Survey and Assessment Project of the Ministry of Ecology and Environment of China (Project No. 2019HJ2096001006), and the Science and Technology Support Project of Guizhou Province (Project No.20192451-2) for research support. Yusufjon Gafforov acknowledges Ministry of Innovative Development of the Republic of Uzbekistan (Project no. P3-2014-0830174425 and P3-20170921183), CAS President's International Fellowship Initiative (PIFI) for a Visiting Scientist grant (no.: 2018VBB0021).

\section{Declarations}

Conflict of interest The authors declare that they have no conflict of interest.

\section{References}

Abdel-Wahab MA, El-Sharouny HM, Jones EBG (2001) Two new intertidal lignicolous Swampomyces species from Red Sea mangroves in Egypt. Fungal Divers 8:35-40

Abdel-Wahab MA, El-Samawaty AMA, El Gorban AM, Yassin MA, Alsaadi MH (2018) Khaleijomyces marinus gen. et sp. nov. (Juncigenaceae, Torpedosporales) a new lignicolous marine fungus from Saudi Arabia. Phytotaxa 340:277-285

Abdel-Wahab MA, Jones EBG, Bahkali AHA, El-Gorban AM (2019) Marine fungi from Red Sea mangroves in Saudi Arabia with Fulvocentrum rubrum sp. nov. (Torpedosporales, Ascomycota). Nova Hedwig 108:365-377

Abdollahzadeh J (2015) Diplodia bulgarica, as a new pathogen and potential threat to the apple industry in Iran. Phytopathol Mediterr 54:128-132

Abdollahzadeh J, Zolfaghari S (2014) Efficiency of rep-PCR fingerprinting as a useful technique for molecular typing of plant pathogenic fungal species: Botryosphaeriaceae species as a case study. FEMS Microbiol Lett 361:144-157

Accioly T, Cruz RHSF, Assis NM, Ishikawa NK, Hosaka K, Martín MP, Baseia IG (2018) Amazonian bird's nest fungi (Basidiomycota): current knowledge and novelties on Cyathus species. Mycoscience 59:331-342

Adamčík S, Jančovičová S, Buyck B (2018) The Russulas described by Charles Horton Peck. Cryptog Mycol 39:3-108

Alcorn JL, Irwin JAG (1987) Acrocalymma medicaginis gen. et. sp. nov. causing root and crown rot of Medicago sativa in Australia. Trans Br Mycol Soc 88:163-167

Altschul SF, Madden TL, Schäffer AA, Zhang J, Zhang Z, Miller W, Lipman DJ (1997) Gapped BLAST and PSI-BLAST: a new generation of protein database search programs. Nucleic Acids Res 25:3389-3402

Álvarez E, Cano J, Stchigel AM, Sutton DA, Fothergill AW, Salas V, Rinaldi MG, Guarro J (2011) Two new species of Mucor from clinical samples. Med Mycol 49:62-72

Ahmad S (1978) Ascomycetes of Pakistan part II. Biol Soc Pak Monogr 8:1-144

Ahmad S, Iqbal SH, Khalid AN (1997) Fungi of Pakistan. Sultan Ahmad Mycological Society of Pakistan, p 248
Ahmed SA, van de Sande WW, Stevens DA, Fahal A, Van Diepeningen AD, Menken SB, De Hoog GS (2014) Revision of agents of black-grain eumycetoma in the order Pleosporales. Persoonia 33:141-154

Ahmed SA, Desbois N, Quist D, Miossec C, Atoche C, Bonifaz A, De Hoog GS (2015) Phaeohyphomycosis caused by a novel species, Pseudochaetosphaeronema martinelli. J Clin Microbiol 53:2927-2934

Andreasen M, Skrede I, Jaklitsch MW, Voglmayr H, Nordén B (2021) Multi-locus phylogenetic analysis of lophiostomatoid fungi motivates a broad concept of Lophiostoma and reveals nine new species. Persoonia 46:240-271

Annesi T, Luongo L, Vitale S, Galli M, Belisario A (2016) Characterization and pathogenicity of Phomopsis theicola anamorph of Diaporthe foeniculina causing stem and shoot cankers on sweet chestnut in Italy. J Phytopathol 164:412-416

Annotated checklist for larger fungi on vancouver island (2020) http:// azkurs.org/annotated-checklist-for-larger-fungi-on-vancouverisland.html?page $=3$

Antonín V (1991) Studies in marasmioid fungi-VI. A new subsection Sicciformes within Marasmius section Marasmius and a key to the European species of Marasmius section Marasmius. Acta Mus Moraviae Sci Nat 76:145-147

Antonín V (2007) Monograph of Marasmius, Gloiocephala, Palaeocephala and Setulipes in tropical Africa. Fung Fl Trop Afr $1: 1-164$

Antonín V, Buyck B (2006) Marasmius (Basidiomycota, Marasmiaceae) in Madagascar and the Mascarenes. Fungal Divers 23:17-50

Antonín V, Noordeloos ME (2010) A monograph of marasmioid and collybioid fungi in Europe. IHW-Verlag, Eching

Antonín V, Ryoo R, Shin HD (2012) Marasmioid and gymnopoid fungi of the Republic of Korea. 4. Marasmius Sect Sicci. Mycol Prog 11:615-638

Antonín V, Ryoo R, Ka KH, Shin HD (2013) Marasmioid and gymnopoid fungi of the Republic of Korea. 6. Marasmius Sect Marasmius. Mycoscience 55:149-157

Ariyawansa HA, Camporesi E, Thambugala KM, Mapook A, Kang JC, Alias SA, Chukeatirote E, Thines M, Mckenzie EHC, Hyde KD (2014a) Confusion surrounding Didymosphaeria-phylogenetic and morphological evidence suggest Didymosphaeriaceae is not a distinct family. Phytotaxa 176:102-119

Ariyawansa HA, Tanaka K, Thambugala KM, Phookamsak R, Tian Q, Camporesi E, Hongsanan S, Monkai J, Wanasinghe DN, Mapook A, Chukeatirote E, Kang JC, Xu JC, McKenzie EHC, Jones EBG, Hyde KD (2014b) A molecular phylogenetic reappraisal of the Didymosphaeriaceae (= Montagnulaceae). Fungal Divers 68:69-104

Ariyawansa HA, Hyde KD, Jayasiri SC, Buyck B, Chethana KWT, Dai DQ, Dai YC, Daranagama DA, Jayawardena RS, Lücking R, Ghobad-Nejhad M, Niskanen T, Thambugala KM, Voigt K, Zhao RL, Li GJ, Doilom M, Boonmee S, Yang ZL, Cai Q, Cui YY, Bahkali AH, Chen J, Cui BK, Chen JJ, Dayarathne MC, Dissanayake AJ, Ekanayaka AH, Hashimoto A, Hongsanan S, Jones EBG, Larsson E, Li WJ, Li QR, Liu JK, Luo ZL, Maharachchikumbura SSN, Mapook A, McKenzie EHC, Nor-phanphoun C, Konta S, Pang KL, Perera RH, Phookamsak R, Phukhamsakda C, Pinruan U, Randrianjohany E, Singtripop C, Tanaka K, Tian CM, Tibpromma S, Abdel-Wahab MA, Wanasinghe DN, Wijayawardene NN, Zhang JF, Zhang H, Abdel-Aziz FA, Wedin M, Westberg M, Ammirati JF, Bulgakov TS, Lima DX, Callaghan TM, Callac P, Chang CH, Coca LF, Dal-Forno M, Dollhofer V, Fliegerová K, Greiner K, Griffith GW, Ho HM, Hofstetter V, Jeewon R, Kang JC, Wen TC, Kirk PM, Kytövuori I, Lawrey JD, Xing J, Li H, Liu ZY, Liu XZ, Liimatainen K, Thorsten Lumbsch H, Matsumura M, Moncada B, Nuankaew S, Parnmen 
S, Santiago ALCMA, Sommai S, Song Y, de Souza CAF, de Souza-Motta CM, Su HY, Suetrong S, Wang Y, FongWS YH, Zhou LW, Réblová M, Fournier J, Camporesi E, Luangsaard JJ, Tasanathai K, Khonsanit A, Thanakitpipattana D, Somrithipol S, Diederich P, Millanes AM, Common RS, Stadler M, Yan JY, Li XH, Lee HW, Nguyen TTT, Lee HB, Battistin E, Marsico O, Vizzini A, Vila J, Ercole E, Eberhardt U, Simonini G, Wen HA, Chen XH, Miettinen O, Spirin V (2015a) Fungal diversity notes 111-252 taxonomic and phylogenetic contributions to fungal taxa. Fungal Divers 75:1-274

Ariyawansa HA, Thambugala KM, Manamgoda DS, Jayawardena RS, Camporesi E, Boonmee S, Wanasinghe DN, Phookamsak R, Hongsanan S, Singtripop C, Chukeatirot E, Kang JC, Jones EBG, Hyde KD (2015b) Towards a natural classification and backbone tree for Pleosporaceae. Fungal Divers 71:85-139

Ariyawansa HA, Tsai I, Thambugala KM, Chuang WY, Lin SR, Hozzein WN, Cheewangkoon R (2020) Species diversity of pleosporalean taxa associated with Camellia sinensis (L.) Kuntze in Taiwan. Sci Rep 10:1-20

Arnolds E (1995) Hygrophoraceae (Agaricales) in New York State and adjacent areas. 1. Introduction and Hygrocybe Subsection Squamulosae. Mycotaxon 53:1-27

Augusto D, de Almeida C, Fernando L, Gusmão P, Miller AN (2016) Taxonomy and molecular phylogeny of Diatrypaceae (Ascomycota, Xylariales) species from the Brazilian semi-arid region, including four new species. Mycol Prog 15:53

Babos M, Halász K, Zagyva T, Zöld-Balogh A, Szegő D, Bratek Z (2011) Preliminary notes on dual relevance of ITS sequences and pigments in Hygrocybe taxonomy. Persoonia 26:99-107

Badali H, Gueidan C, Najafzadeh MJ, Bonifaz A, Gerrits van den Ende AHG, De Hoog GS (2008) Biodiversity of the genus Cladophialophora. Stud Mycol 61:175-191

Baker WA, Partridge EC, Morgan-Jones G (2002) Notes on Hyphomycetes LXXXVII. Rhexoacrodictys, a new segregate genus to accommodate four species previously classified in Acrodictys. Mycotaxon 82:95-113

Balazuc J (1971) Laboulbéniales nouvelles, parasites d'Insectes. Bull Soc Entomol France 76:226-235

Bao DF, Hyde KD, Luo ZL, Su HY, Nalumpang S (2019a) Minutisphaera aquaticum sp. nov. increases the known diversity of Minutisphaeraceae. Asian J Mycol 2:306-314

Bao DF, Su HY, Maharachchikumbura SSN, Liu JK, Nalumpang S, Luo ZL, Hyde KD (2019b) Lignicolous freshwater fungi from China and Thailand: multi-gene phylogeny reveals new species and new records in Lophiostomataceae. Mycosphere 10:1080-1099

Barr ME (1972) Preliminary studies on the Dothideales in temperate North America. Contr Univ Michigan Herb 9:523-638

Barr ME (1977) Acanthostigmella (Herpotrichiellaceae). Mycotaxon $7: 17-23$

Barr ME (1987) New taxa and combinations in the Loculoascomycetes. Mycotaxon 29:501-505

Barr ME (1990) Melanommatales (Loculoascomycetes). N Am Fl 13:1-129

Barr ME (1994) Notes on the Amphisphaeriaceae and related families. Mycotaxon 51:191-224

Bas C (1969) Morphology and subdivision of Amanita and a monograph of its section Lepidella. Persoonia 5:285-579

Baseia IG, Milanez AI (2001) Cyathus (Gasteromycetes) in areas of the Brazilian Cerrado Region, Sao Paulo state. Mycotaxon $80: 493-502$

Basnet BB, Chen B, Suleimen YM, Ma K, Guo S, Bao L, Huang Y, Liu H (2019) Cytotoxic secondary metabolites from the endolichenic fungus Hypoxylon fuscum. Planta Med 13:1088-1097

Batra LR, Millner PD (1974) Some Asian fermented foods and beverages. Mycologia 66:942-950
Beauseigneur A (1926) Contribution à l'étude de la Flore Mycologique des Landes (Basidiomycètes et Ascomycètes). J Glize, SaintSever sur Adour, p 244

Becker K, Wessel AC, Luangsa-ard JJ, Stadler M (2020) Viridistratins $\mathrm{A}-\mathrm{C}$, Antimicrobial and Cytotoxic Benzo [j] fluoranthenes from Stromata of Annulohypoxylon viridistratum (Hypoxylaceae, Ascomycota). Biomolecules 10:1-11

Begerow D, McTaggart A (2018) Pucciniomycotina. In: Begerow D et al (eds) Syllabus of plant families-A. Engler's Syllabus der Pflanzenfamilien, Part 1/3: Basidiomycota and Entorrhizomycota. Schweizerbart Science Publishers, Stuttgart, pp 17-91

Bellemère A, Hafellner J (1982) L'ultrastructure des asques de genre Dactylospora (Discomycetes) et son interet taxonomique. Cryptog Mycol 3:71-93

Beltrán-Tejera E, Rodríguez-Armas JL (1999) Aphyllophorales (Basidiomycotina) of arid habitats of Canary Islands. Preliminary Data. Mycotaxon 70:111-125

Beltrán-Tejera E, Rodríguez-Armas JL, Telleria MT, Dueñas M, Melo I, Díaz-Armas MJ, Salcedo I, Cardoso J (2013) Corticioid fungi from arid and semiarid zones of the Canary Islands (Spain) Additional data. 2. Mycotaxon 123:491-492

Benjamin RK (1955) New genera of Laboulbeniales. Aliso 3:183-197

Benny GL (2008) Methods used by Dr. R. K. Benjamin, and other mycologists, to isolate Zygomycetes. Aliso 26:37-61

Benny GL, Humber RA, Voigt K (2014) Zygomycetous fungi: Phylum Entomophthoromycota and subphyla Kickxellomycotina, Mortierellomycotina, Mucoromycotina, and Zoopagomycotina. In: McLaughlin DJ, Spatafora JW (eds) Mycota VII, part A, Systematics and evolution. Springer, New York, pp 209-250

Bensch K, Braun U, Groenewald JZ, Crous PW (2012) The genus Cladosporium. Stud Mycol 72:1-401

Boertmann D (1995) The genus Hygrocybe. In: Fungi of Northern Europe, vol 1. Greve, Denmark

Boertmann D (2010) The genus Hygrocybe 2nd revised edn. Fungi of Northern Europe 1. Danish Mycological Soc 1-200

Boidin J, Gilles G (2003) Homobasidiomycètes Aphyllophorales non porés à basides dominantes à 2(3) stérigmates. Bull Soc Mycol France 119:1-17

Boonmee S, Rossman AY, Liu JK, Li WJ, Dai DQ, Bhat JD, Jones EG, McKenzie EH, Xu JC, Hyde KD (2014) Tubeufiales, ord. nov., integrating sexual and asexual generic names. Fungal Divers 68:239-298

Boonmee S, Dsouza MJ, Luo ZL, Pinruan U, Tanaka K, Su HY, Bhat DJ, McKenzie EHC, Jones EBG, Taylor JE (2016) Dictyosporiaceae Fam. Nov. Fungal Divers 80:457-482

Boonmee S, Sorvongxay T, Huanraluek N, Hyde KD (2019) Wicklowia submersa sp. nov. (Wicklowiaceae, Pleosporales), a second species in a monotypic family. Phytotaxa 411:73-83

Boonyuen N, Chuaseeharonnachai C, Suetrong S, Sri-indrasutdhi V, Sivichai S, Jones EBG, Pang KL (2011) Savoryellales (Hypocreomycetidae, Sordariomycetes): a novel lineage of aquatic ascomycetes inferred from multiple-gene phylogenies of the genera Ascotaiwania, Ascothailandia, and Savoryella. Mycologia 103:1351-1371

Boonyuen N, Chuaseeharonnachai C, Suetrong S, Sujinda S, Somrithipol S (2016) Parafuscosporella garethii sp. nov. (Fuscosporellales) from a rivulet in a community-based northern forest, in Thailand. Mycosphere 7:1265-1272

Borelli D (1980) Causal agents of chromoblastomycosis (Chromomycetes). In: Proceedings of the 5th International Conference on Mycoses, pp 335-340

Borgen T, Arnolds E (2004) Taxonomy, ecology and distribution of Hygrocybe (Fr.) P. Kumm. and Camarophyllopsis Herink (Fungi, Basidiomycota, Hygrocybeae) in Greenland. Meddelelser Om Grønland. Bioscience 54:1-68 
Brahamanage RS, Lu YZ, Bhat DJ, Wanasinghe DN, Yan JY, Hyde KD, Boonmee S (2017) Phylogenetic investigations on freshwater fungi in Tubeufiaceae (Tubeufiales) reveals the new genus Dictyospora and new species Chlamydotubeufia aquatica and Helicosporium flavum. Mycosphere 8:917-933

Braun U, Cook RTA (2012) Taxonomic manual of Erysiphales (powdery mildews). In: CBS Biodiversity Series No 11. CBS, Utrecht

Braun U, Feiler U (1995) Cladophialophora and its teleomorph. Microbiol Res 150:81-91

Braun U, Nakashima C, Crous PW, Groenewald JZ, Moreno-Rico O, Rooney-Latham S, Blomquist CL, Haas J, Marmolejo J (2018) Phylogeny and taxonomy of the genus Tubakia s. lat. Fungal Syst Evol 1:41-99

Brodie HJ (1975) The bird's nest fungi. Univ. of Toronto Press, Toronto

Bundhun D, Maharachchikumbura SS, Jeewon R, Senanayake IC, Jayawardena RS, Hongsanan S (2020) a platform for the identification, ranking. Asian J Mycol 3:13-21

Burdsall HH Jr (1985) A contribution to the taxonomy of the genus Phanerochaete. Mycol Mem 10:1-165

Buyck B, Hofsetter V, Verbeken A, Walleyn R (2010) Proposal 1919: to conserve Lactarius nom. Cons. (Basidiomycota) with a conserved type. Taxon 59:295-296

Cai L, Jeewon R, Hyde KD (2006) Phylogenetic investigations of Sordariaceae based on multiple gene sequences and morphology. Mycol Res 110:137-150

Calabon MS, Hyde KD, Gentekaki E, Jones EBG, Phookamsak R, Doilom M, Boonmee S, Promputtha I (2020a) Wicklowia phuketensis (Wicklowiaceae, Pleosporales), a novel freshwater taxon from Thailand. Phytotaxa 452:55-64

Calabon MS, Hyde KD, Jones EBG, Chandrasiri S, Dong W, Fryar SC, Yang J, Luo ZL, Lu YZ, Bao DF, Boonmee S (2020b) www. freshwaterfungi.org, an online platform for the taxonomic classification of freshwater fungi. Asian J Mycol 3:419-445

Calonge FD (1998) Gasteromycetes I. Lycoperdales, Nidulariales, Phallales, Sclerodermatales, Tulostomatales. Flora Mycol Iberica 3:1-271

Câmara MP, Ramaley AW, Castlebury LA, Palm ME (2003) Neophaeosphaeria and Phaeosphaeriopsis, segregates of Paraphaeosphaeria. Mycol Res 107:516-522

Campbell J, Ferrer A, Raja HA, Sivichai S, Shearer CA (2007) Phylogenetic relationships among taxa in the Jahnulales inferred from $18 \mathrm{~S}$ and $28 \mathrm{~S}$ nuclear ribosomal DNA sequences. Can J Bot 85:873-882

Camps C, Kappel C, Lecomte P, Léon C, Coutos-Thévenot P, Delrot $\mathrm{S}$, Gomès $\mathrm{E}$ (2014) Identification of grapevine marker genes for early, non-destructive Eutypa lata infection diagnosis. Plant Pathol 63:323-333

Cannon PF, Kirk PM (2007) Fungal families of the world, 7th edn. CABI, Wallingford

Cannon PF, Hawksworth DL, Sherwood-Pike MA (1985) The British Ascomycotina. An Annotated Checklist. Commonwealth Mycological Institute, Kew, Surrey, England, p 302

Castañeda Ruiz RF, Arnold GR (1985) Deuteromycotina de Cuba I. Hyphomycetes. Revista Jard Bot Nac 6:47-67

Chacón S, Dorge D, Weisenborn J, Piepenbring M (2013) A new species and a new record of Diatrypaceae from Panama. Mycologia 105:681-688

Chandrasrikul A, Suwanarit P, Sangwanit U, Lumyong S, Payapanon A, Sanoamuang N, Pukahuta C, Petcharat V, Sardsud U, Duengkae K, Klinhom U, Thongkantha S, Thongklam S (2011) Mushroom (basidiomycetes) in Thailand. Office of Natural Resources and Environmental Policy and Planning, Bangkok

Chang JH, Wang YZ (2005) The genus Cercophora (Lasiosphaeriaceae) in Taiwan. Fungal Sci 20:19-25

Chaudhary P, Fournier J, Miller AN (2007) Cercophora aquatica sp. nov. from a streambed in southern France. Sydowia 59:217-225
Checa J (2004) Dictyosporic Dothideales. Fl Mycol Iberica 6:1-162

Chen JL, Tzean SS (1993) Megacapitula villosa gen. et sp. nov. from Taiwan. Mycol Res 97:347-350

Chen C, Li W, Gao L, Zhang R, Sun G, Gleason ML (2014) First report of Pseudoveronaea ellipsoidea causing sooty blotch and flyspeck in China. Mycotaxon 129:247-253

Chen AJ, Frisvad JC, Sun BD, Varga J, Kocsubé S, Dijksterhuis J, Kim DH, Hong SB, Houbraken J, Samson RA (2016) Aspergillus section Nidulantes (formerly Emericella): Polyphasic taxonomy, chemistry and biology. Stud Mycol 84:1-118

Chen Q, Hou LW, Duan WJ, Crous PW, Cai L (2017) Didymellaceae revisited. Stud Mycol 87:105-159

Chen CC, Wu SH, Chen CY (2018) Hydnophanerochaete and Odontoefibula, two new genera of phanerochaetoid fungi (Polyporales, Basidiomycota) from East Asia. MycoKeys 39:75-96

Chen Q, Jiang JR, Zhang GZ, Cai L, Crous PW (2015) Resolving the Phoma enigma. Stud Mycol 82:137-217

Chethana KWT, Niranjan M, Dong W, Samarakoon MC, Bao DF, Calabon MS, Chaiwan N, Chuankid B, Dayarathne MC, de Silva NI, Devadatha B, Dissanayake AJ, Dong W, Goonasekara ID, Jayawardena RS, Karunarathna A, Luo ZL, Marasinghe DS, Ma XY, Norphanphoun C, Pem D, Perera RH, Rathnayaka AR, Samarakoon BC, Sarma VV, Senwanna C, Sun YR, Tang X, Thiyagaraja V, Tennakoon DS, Zeng M, Zeng XY, Zhang JY, Zhang SN, Bulgakov TS, Wang Y, Bhat DJ, Hyde KD (2021) AJOM new records and collections of fungi: 101-150. Asian J Mycol 4:113-260

Chevallier FF (1826) Flore Générale des Environs de Paris [General flora of the area around Paris], vol 1. Ferra Jeune, France, pp $1-674$

Cho HJ, Park MS, Lee H, Oh SY, Wilson AW, Mueller GM, Lim YW (2018) A systematic revision of the ectomycorrhizal genus Laccaria from Korea. Mycologia 110:948-961

Chomnunti P, Bhat DJ, Jones EBG, Chukeatirote E, Bahkali AH, Hyde KD (2012) Trichomeriaceae, a new sooty mould family of Chaetothyriales. Fungal Divers 56:63-76

Clark K, Karsch-Mizrachi I, Lipman DJ, Ostell J, Sayers EW (2016) GenBank. Nucleic Acids Res 44:D67-D72

Clements FE (1909) The genera of fungi. Genera of Fungi, 1st edn. HW Wilson Company, Minneapolis, pp 1-227

Clements FE, Shear CL (1931) The genera of fungi. HW Wilson Co., New York

Coemans E (1863) Quelques hyphomyce`tes nouveaux. 1. notice: $M o r-$ tierella polycephala et Martinella pectinata. Bull Cl SciAcad R Belg 15:536-540

Cooke WB (1983) The 1979 Oklahoma foray. Mycologia 75:752-755

Corda ACJ (1836) Mykologische Beobachtungen. Weitenweber's Beitrage zur gesammtem Natur- und Heilwissenschaften Prague

Corda ACJ (1839) Icones Fungorum Hucusque Cognitorum 3:1-55

Corda ACJ (1842) Icones fungorum hucusque cognitorum. JG Calve, Prague, pp 1-92

Costa MM (2020) Fusarium species associated with tropical grasses. Portal de Programas de Pós-Graduação (UFLA)

Crane JL, Schoknecht JD (1977) Revision of Torula species. Rutola, a new name for Torula graminis. Can J Bot 55:3013-3019

Crous PW, Groenewald JZ (2016) They seldom occur alone. Fungal Biol 120:1392-1415

Crous PW, Groenewald JZ (2017) The genera of fungi-G 4: Camarosporium and Dothiora. IMA Fungus 8:131-152

Crous PW, Schubert K, Braun U, De Hoog GS, Hocking AD, Shin HD, Groenewald JZ (2007) Opportunistic, human-pathogenic species in the Herpotrichiellaceae are phenotypically similar to saprobic or phytopathogenic species in the Venturiaceae. Stud Mycol 58:185-217 
Crous PW, Schoch CL, Hyde KD, Wood AR, Gueidan C, De Hoog GS, Groenewald JZ (2009) Phylogenetic lineages in the Capnodiales. Stud Mycol 64:17-47

Crous PW, Summerell BA, Shivas RG, Romberg M, Mel'nik VA, Verkley GJM, Groenewald JZ (2011) Fungal Planet description sheets: 92-106. Persoonia 27:130-162

Crous PW, Shivas RG, Quaedvlieg W, van der Bank M, Zhang Y, Summerell BA, Guarro J, Wingfield MJ, Wood AR, Alfenas AC, Braun U, Cano-Lira JF, García D, Marin-Felix Y, Alvarado P, Andrade JP, Armengol J, Assefa A, den Breeÿen A, Camele I, Cheewangkoon R, De Souza JT, Duong TA, Esteve-Raventós F, Fournier J, Frisullo S, García-Jiménez J, Gardiennet A, Gené J, Hernández-Restrepo M, Hirooka Y, Hospenthal DR, King A, Lechat C, Lombard L, Mang SM, Marbach PAS, Marincowitz S, Marin-Felix Y, Montaño-Mata NJ, Moreno G, Perez CA, Pérez Sierra AM, Robertson JL, Roux J, Rubio E, Schumacher RK, Stchigel AM, Sutton DA, Tan YP, Thompson EH, van der Linde E, Walker AK, Walker DM, Wickes BL, Wong PTW, Groenewald JZ (2014a) Fungal Planet description sheets: 214-280. Persoonia 32:184-306

Crous PW, Wingfield MJ, Schumacher RK, Summerell BA, Giraldo A, Gené J, Guarro J, Wanasinghe DN, Hyde KD, Camporesi E, Gareth Jones EB, Thambugala KM, Malysheva EF, Malysheva VF, Acharya K, Álvarez J, Alvarado P, Assefa A, Barnes CW, Bartlett JS, Blanchette RA, Burgess TI, Carlavilla JR, Coetzee MP, Damm U, Decock CA, den Breeÿen A, de Vries B, Dutta AK, Holdom DG, Rooney-Latham S, Manjón JL, Marincowitz S, Mirabolfathy M, Moreno G, Nakashima C, Papizadeh M, Shahzadeh Fazeli SA, Amoozegar MA, Romberg MK, Shivas RG, Stalpers JA, Stielow B, Stukely MJ, Swart WJ, Tan YP, van der Bank M, Wood AR, Zhang Y, Groenewald JZ (2014b) Fungal Planet description sheets: 281-319. Persoonia 33:212-289

Crous PW, Carris LM, Giraldo A, Groenewald JZ, Hawksworth DL, Hernández-Restrepo M, Wood AR (2015) The genera of fungifixing the application of the type species of generic names-G2: Allantophomopsis, Latorua, Macrodiplodiopsis, Macrohilum, Milospium, Protostegia, Pyricularia, Robillarda, Rotula, Septoriella, Torula and Wojnowicia. IMA Fungus 6:163-198

Crous PW, Wingfield MJ, Burgess TI, Carnegie AJ, Hardy G, Smith D, Summerell BA, Cano-Lira JF, Guarro J, Houbraken J, Lombard L, Martin MP, Sandoval-Denis M, Alexandrova AV, Barnes CW, Baseia IG, Bezerra JDP, Guarnaccia V, May TW, HernandezRestrepo M, Stchigel AM, Miller AN, Ordonez ME, Abreu VP, Accioly T, Agnello C, Agustin Colman A, Albuquerque CC, Alfredo DS, Alvarado P, Araujo-Magalhaes GR, Arauzo S, Atkinson T, Barili A, Barreto RW, Bezerra JL, Cabral TS, Camello Rodriguez F, Cruz R, Daniels PP, da Silva BDB, de Almeida DAC, de Carvalho Junior AA, Decock CA, Delgat L, Denman S, Dimitrov RA, Edwards J, Fedosova AG, Ferreira RJ, Firmino AL, Flores JA, Garcia D, Gene J, Giraldo A, Gois JS, Gomes AAM, Goncalves CM, Gouliamova DE, Groenewald M, Gueorguiev BV, Guevara-Suarez M, Gusmao LFP, Hosaka K, Hubka V, Huhndorf SM, Jadan M, Jurjevic Z, Kraak B, Kucera V, Kumar TKA, Kusan I, Lacerda SR, Lamlertthon S, Lisboa WS, Loizides M, Luangsa-Ard JJ, Lyskova P, Mac Cormack WP, Macedo DM, Machado AR, Malysheva EF, Marinho P, Matocec N, Meijer M, Mesic A, Mongkolsamrit S, Moreira KA, Morozova OV, Nair KU, Nakamura N, Noisripoom W, Olariaga I, Oliveira RJV, Paiva LM, Pawar P, Pereira OL, Peterson SW, Prieto M, Rodriguez-Andrade E, Rojo De Blas C, Roy M, Santos ES, Sharma R, Silva GA, Souza-Motta CM, Takeuchi-Kaneko Y, Tanaka C, Thakur A, Smith MT, Tkalcec Z, Valenzuela-Lopez N, van der Kleij P, Verbeken A, Viana MG, Wang XW, Groenewald JZ (2017) Fungal Planet description sheets: 625-715. Persoonia 39:270-467
Crous PW, Wingfeld MJ, Burgess TI, Hardy GESJ, Gené J, Guarro J, Baseia IG, García D, Gusmão LFP, Souza-Motta CM, Thangavel R, Adamčík S, Barili A, Barnes CW, Bezerra JDP, Bordallo JJ, Cano-Lira JF, de Oliveira RJV, Ercole E, Hubka V, IturrietaGonzález I, Kubátová A, Martín MP, Moreau PA, Morte A, Ordoñez ME, Rodríguez A, Stchigel AM, Vizzini A, Abdollahzadeh J, Abreu VP, Adamčíková K, Albuquerque GMR, Alexandrova AV, Álvarez Duarte E, Armstrong-Cho C, Banniza S, Barbosa RN, Bellanger JM, Bezerra JL, Cabral TS, Caboň M, Caicedo E, Cantillo T, Carnegie AJ, Carmo LT, CastañedaRuiz RF, Clement CR, Čmoková A, Conceição LB, Cruz RHSF, Damm U, da Silva BDB, da Silva GA, da Silva RMF, Santiago ALCMdA, de Oliveira LF, de Souza CAF, Déniel F, Dima B, Dong G, Edwards J, Félix CR, Fournier J, Gibertoni TB, Hosaka K, Iturriaga T, Jadan M, Jany JL, Jurjević Ž, Kolařík M, Kušan I, Landell MF, Leite Cordeiro TR, Lima DX, Loizides M, Luo S, Machado AR, Madrid H, Magalhães OMC, Marinho P, Matočec N, Mešić A, Miller AN, Morozova OV, Neves RP, Nonaka K, Nováková A, Oberlies NH, Oliveira-Filho JRC, Oliveira TGL, Papp V, Pereira OL, Perrone G, Peterson SW, Pham THG, Raja HA (2018) Fungal Planet description sheets: 716-784. Persoonia 40:239-393

Crous PW, Wingfield MJ, Lombard L, Roets F, Swart WJ, Alvarado $\mathrm{P}$, Carnegie AJ, Moreno G, Luangsaard J, Thangavel R, Alexandrova AV, Baseia IG, Bellanger JM, Bessette AE, Bessette AR, De la Peña-Lastra S, García D, Gené J, Pham THG, Heykoop M, Malysheva E, Malysheva V, Martín MP, Morozova OV, Noisripoom W, Overton BE, Rea AE, Sewall BJ, Smith ME, Smyth CW, Tasanathai K, Visagie CM, Adamčík S, Alves A, Andrade JP, Aninat MJ, Araújo RVB, Bordallo JJ, Boufleur T, Baroncelli R, Barreto RW, Bolin J, Cabero J, Caboň M, Cafà G, Caffot MLH, Cai L, Carlavilla JR, Chávez R, de Castro RRL, Delgat L, Deschuyteneer D, Dios MM, Domínguez LS, Evans HC, Eyssartier G, Ferreira BW, Figueiredo CN, Liu F, Fournier J, Galli-Terasawa LV, Gil-Durán C, Glienke C, Gonçalves MFM, Gryta H, Guarro J, Himaman W, Hywel-Jones N, IturrietaGonzález I, Ivanushkina NE, Jargeat P, Khalid AN, Khan J, Kiran M, Kiss L, Kochkina GA, Kolařík M, Kubátová A, Lodge DJ, Loizides M, Luque D, Manjón JL, Marbach PAS, Massola NS Jr, Mata M, Miller AN, Mongkolsamrit S, Moreau PA, Morte A, Mujic A, Navarro-Ródenas A, Németh MZ, Nóbrega TF, Nováková A, Olariaga I, Ozerskaya SM, Palma MA, Petters-Vandresen DAL, Piontelli E, Popov ES, Rodríguez A, Requejo Ó, Rodrigues ACM, Rong IH, Roux J, Seifert KA, Silva BDB, Sklenář F, Smith JA, Sousa JO, Souza HG, De Souza JT, Švec K, Tanchaud P, Tanney JB, Terasawa F, Thanakitpipattana D, Torres-Garcia D, Vaca I, Vaghefi N, van Iperen AL, Vasilenko OV, Verbeken A, Yilmaz N, Zamora JC, Zapata M, Jurjević Ž, Groenewald JZ (2019) Fungal Planet description sheets: 951-1041. Persoonia 43:223-425

Crous PW, Schumacher RK, Wood AR, Groenewald JZ (2020a) The genera of fungi-G5: Arthrinium, Ceratosphaeria, Dimerosporiopsis, Hormodochis, Lecanostictopsis, Lembosina, Neomelanconium, Phragmotrichum, Pseudomelanconium, Rutola, and Trullula. Fungal Syst Evol 5:77-98

Crous PW, Wingfield MJ, Chooi YH, Gilchrist CLM, Lacey E, Pitt JI, Roets F, Swart WJ, Cano-Lira JF, Valenzuela-Lopez N, Hubka V, Shivas RG, Stchigel AM, Holdom DG, Jurjević Ž, Kachalkin AV, Lebel T, Lock C, Martín MP, Tan YP, Tomashevskaya MA, Vitelli JS, Baseia IG, Bhatt VK, Brandrud TE, De Souza JT, Dima B, Lacey HJ, Lombard L, Johnston PR, Morte A, Papp V, Rodríguez A, Rodríguez-Andrade E, Semwal KC, Tegart L, Abad ZG, Akulov A, Alvarado P, Alves A, Andrade JP, Arenas $\mathrm{F}$, Asenjo C, Ballarà J, Barrett MD, Berná LM, Berraf-Tebbal A, Bianchinotti MV, Bransgrove K, Burgess TI, Carmo FS, Chávez R, Čmoková A, Dearnaley JDW, de Santiago ALCM, Freitas-Neto JF, Denman S, Douglas B, Dovana F, Eichmeier A, 
Esteve-Raventós F, Farid A, Fedosova AG, Ferisin G, Ferreira RJ, Ferrer A, Figueiredo CN, Figueiredo YF, Reinoso-Fuentealba CG, Garrido-Benavent I, Cañete-Gibas CF, Gil-Durán C, Glushakova AM, Gonçalves MFM, González M, Gorczak M, Gorton C, Guard FE, Guarnizo AL, Guarro J, Gutiérrez M, Hamal P, Hien LT, Hocking AD, Houbraken J, Hunter GC, Inácio CA, Jourdan M, Kapitonov VI, Kelly L, Khanh TN, Kisło K, Kiss L, Kiyashko A, Kolařík M, Kruse J, Kubátová A, Kučera V, Kučerová I, Kušan I, Lee HB, Levicán G, Lewis A, Liem NV, Liimatainen K, Lim HJ, Lyons MN, Maciá-Vicente JG, Magaña-Dueñas V, Mahiques R, Malysheva EF, Marbach PAS, Marinho P, Matočec N, McTaggart AR, Mešić A, Morin L, Muñoz-Mohedano JM, Navarro-Ródenas A, Nicolli CP, Oliveira RL, Otsing E, Ovrebo CL, Pankratov TA, Paños A, Paz-Conde A, Pérez-Sierra A, Phosri C, Pintos Á, Pošta A, Prencipe S, Rubio E, Saitta A, Sales LS, Sanhueza L, Shuttleworth LA, Smith J, Smith ME, Spadaro D, Spetik M, Sochor M, Sochorová Z, Sousa JO, Suwannasai N, Tedersoo L, Thanh HM, Thao LD, Tkalčec Z, Vaghefi N, Venzhik AS, Verbeken A, Vizzini A, Voyron S, Wainhouse M, Whalley AJS, Wrzosek M, Zapata M, Zeil-Rolfe I, Groenewald JZ (2020b) Fungal Planet description sheets: 1042-1111. Persoonia 44:301-459

Cui YY, Cai Q, Tang LP, Liu JW, Yang ZL (2018) The family Amanitaceae: molecular phylogeny, higher-rank taxonomy and the species in China. Fungal Divers 91:5-230

Cui BK, Li HJ, Ji X, Zhou JL, Song J, Si J, Yang ZL, Dai YC (2019) Species diversity, taxonomy and phylogeny of Polyporaceae (Basidiomycota) in China. Fungal Divers 97:137-302

Cruz RHSF, Assis NM, Silva MA, Baseia IG (2014) Revision of the genus Cyathus (Basidiomycota) from the herbaria of northeast Brazil. Mycosphere 5:531-540

Cruz RHSF, Baseia IG, Hosaka K (2018) Rediscovery of Cyathus badius, an 'extinct' species from the Bonin Islands, Japan. Mycoscience 59:193-199

Dade HA (1940) A revised list of Gold Coast fungi and plant diseases. Bull Misc Inf 1940:205-247

Dai YC (2012) Polypore diversity in China with an annotated checklist of Chinese polypores. Mycoscience 53:49-80

Dai DQ, Wijayawardene NN, Bhat DJ, Chukeatirote E, Bahkali AH, Zhao RL, Xu JC, Hyde KD (2014) Pustulomyces gen. nov. accommodated in Diaporthaceae, Diaporthales, as revealed by morphology and molecular analyses. Cryptog Mycol 35:63-72

Dai DQ, Phookamsak R, Wijayawardene NN, Li WJ, Bhat DJ, Xu JC, Taylor JE, Hyde KD, Chukeatirote E (2017) Bambusicolous Fungi. Fungal Divers 82:1-105

Daranagama DA, Hyde KD, Sir EB, Thambugala KM, Tian Q, Samarakoon MC, McKenzie EHC, Jayasiri SC, Tibpromma S, Bhat JD, Liu XZ, Stadler M (2018) Towards a natural classification and backbone tree for Graphostromataceae, Hypoxylaceae, Lopadostomataceae and Xylariaceae. Fungal Divers 88:1-165

Das K, Hembrom ME, Parihar A, Zhao RL (2015) A new species of Cyathus (Agaricaeae) from India. Turk J Bot 39:1-7

Davis PH (ed) (1967) Flora of Turkey and the East Aegean Islands, vol 2. Edinburgh University Press, Edinburgh, pp 1-581

Davison EM, McGurk LE, Bougher NL, Syme K, Watkin EL (2013) Amanita lesueurii and A. wadjukiorum (Basidiomycota), two new species from Western Australia, and an expanded description of A. fibrillopes. Nuytsia 23:589-606

Dayarathne MC, Phookamsak R, Hyde KD, Manawasinghe IS, Toanun C, Jones EBG (2016) Halodiatrype, a novel diatrypaceous genus from mangroves with $H$. salinicola and $H$. avicenniae sp. nov. Mycosphere 7:612-627

Dayarathne MC, Maharachchikumbura SSN, Jones EBG, Wei D, Devadatha B, Yang J, Ekanayake H, De Silva W, Sarma VV, AlSadi AM, Khongphinitbunjong K, Hyde KD, Zhao RL (2019)
Phylogenetic revision of Savoryellaceae and evidence for its ranking as a subclass. Front Microbiol 10:840

Dayarathne MC, Jones E, Maharachchikumbura S, Devadatha B, Sarma V, Khongphinitbunjong K, Chomnunti P, Hyde K (2020) Morpho-molecular characterization of microfungi associated with marine based habitats. Mycosphere 11:1-188

de Gruyter J, Woudenberg JHC, Aveskamp MM, Verkley GJM, Groenewald JZ, Crous PW (2012) Rediposition of phoma-like anamorphs in Pleosporales. Stud Mycol 75:1-36

De Hoog GS (2014) Ecology and phylogeny of black yeast-like fungi: diversity in unexplored habitats. Fungal Divers 65:1-28

De Hoog GS, Guarro J, Gené J, Figueras MJ (2000) Atlas of clinical fungi, 2nd edn. Centraalbureau voor Schimmelcultures, Utrecht, p 1126

De Hoog GS, Nishikaku AS, Fernandez-Zeppenfeldt G, PadínGonzález C, Burger E, Badali H, Richard-Yegres N, Gerrits Van Den Ende AHG (2007) Molecular analysis and pathogenicity of the Cladophialophora carrionii complex, with the description of a novel species. Stud Mycol 58:219-234

De Koker T, Nakasone KK, Haarhof J, Burdsall HH Jr, Janse BJH (2003) Phylogenetic relationships of the genus Phanerochaete inferred from the internal transcribed spacer region. Mycol Res 107:1032-1040

De Notaris G (1862) Erbario Crittogamico Italiano. Fasc 16 no. 875

De Oliveira JS, Moncalvo JM, Margaritescu S, Capelari M (2020) Phylogenetic and morphological analyses of species of Marasmius sect. Marasmius from the Atlantic Rainforest, Brazil. Plant Syst Evol 306:31

Deckmyn G, Meyer A, Smits MM, Ekblad A, Grebenc T, Komarov A, Kraigher H (2014) Simulating ectomycorrhizal fungi and their role in carbon and nitrogen cycling in forest ecosystems. Can J for Res 44:535-553

Degawa Y, Gams W (2004) A new species of Mortierella, and an associated sporangiiferous mycoparasite in a new genus, Nothadelphia. Stud Mycol 50:567-572

del Catania VM, Romero AI, Huhndorf SM, Miller AN (2011) A new species and new records of Cercophora from Argentina. Mycologia 103:1372-1383

Delgado G (2010) South Florida microfungi: Kalamarospora multiflagellata gen. et sp. nov. (hyphomycetes), with additional new records from USA. Mycotaxon 114:231-246

Demoulin V (1984) Typification of Geastrum Pers.:Pers. and its orthographic variant Geaster (Gasteromycetes). Taxon 33:498-501

Denchev CM, Denchev TT (2011) New records of smut fungi. 4. Microbotryum coronariae comb nov. Mycotaxon 118:53-56

Denchev TT, Denchev CM, Kemler M, Begerow D (2020) Kalmanago gen. nov. (Microbotryaceae) on Commelina and Tinantia (Commelinaceae). Mycobiota 10:21-37

Denchev TT, Kemler M, Begerow D, Denchev CM (2019) Molecular and morphological evidence reveals a new smut fungus, Microbotryum arcticum (Microbotryaceae), on Silene uralensis (Caryophyllaceae) from Greenland and Canada. Willdenowia 49:241-255

Deng C, Li T, Li T, Antonín V (2012) New species and new records in Marasmius sect. Sicci from China. Cryptog Mycol 33:439-451

Dennis RWG (1978) British Ascomycetes. J Cramer Vaduz, p 585

Desjardin DE (1989) The genus Marasmrus from the southern Appalachian Mountains. Ph.D. Dissertation. University of Tennessee, Knoxville

Desjardin DE, Perry BA (2017) The gymnopoid fungi (Basidiomycota, Agaricales) from the Republic of São Tomé and Príncipe, West Africa. Mycosphere 8:1317-1391

Desjardin DE, Retnowati A, Horak E (2000) Agaricales of Indonesia: 2. A preliminary monograph of Marasmius from Java and Bali. Sydowia 52:92-193 
Devadatha B, Sarma VV, Wanasinghe DN, Hyde KD, Jone EBG (2017) Introducing the new Indian mangrove species, Vaginatispora microarmatispora (Lophiostomataceae) based on morphology and multigene phylogenetic analysis. Phytotaxa 329:139-149

Diaz Arias MM, Batzer JC, Harrington TC, Wong AW, Bost SC, Cooley DR, Ellis MA, Hartman JR, Rosenberger DA, Sundin GW, Sutton TB, Travis JW, Wheeler MJ, Yoder KS, Gleason ML (2010) Diversity and biogeography of sooty blotch and flyspeck fungi on apple in the eastern and midwestern United States. Phytopathology 100:345-355

Diederich P, Ertz D, Lawrey JD, Sikaroodi M, Untereiner WA (2013) Molecular data place the hyphomycetous lichenicolous genus Sclerococcum close to Dactylospora (Eurotiomycetes) and $S$. parmeliae in Cladophialophora (Chaetothyriales). Fungal Divers 58:61-72

Diederich P, Lawrey JD, Ertz D (2018) The 2018 classification and checklist of lichenicolous fungi, with 2000 non-lichenized, obligately lichenicolous taxa. Bryologist 121:340-425

Dissanayake AJ, Liu M, Zhang W, Chen Z, Udayanga D, Chukeatirote E, Li X, Yan J, Hyde KD (2015) Morphological and molecular characterization of Diaporthe species associated with grapevine trunk disease in China. Fungal Biol 119:283-294

Dissanayake AJ, Phillips AJL, Hyde KD, Li XH (2016) Botryosphaeriaceae: current status of genera and species. Mycosphere 7:1001-1073

Dissanayake AJ, Camporesi E, Hyde KD, Zhang W, Yan JY, Li X (2017) Molecular phylogenetic analysis reveals seven new Diaporthe species from Italy. Mycosphere 8:853-877

Doilom M, Dissanayake AJ, Wanasinghe DN, Boonmee S, Liu JK, Bhat DJ, Taylor JE, Bahkali AH, McKenzie EHC, Hyde KD (2016) Microfungi on Tectona grandis (teak) in Northern Thailand. Fungal Divers 82:107-182

Doilom M, Hyde KD, Phookamsak R, Dai DQ, Tang LZ, Hongsanan S, Chomnunti P, Boonmee S, Dayarathne MC, Li WJ, Thambugala KM, Perera RH, Daranagama DA, Norphanphoun C, Konta S, Dong W, Ertz D, Phillips AJL, McKenzie EHC, Vinit K, Ariyawansa HA, Jones EBG, Mortimer PE, Xu JC, Promputtha I (2018) Mycosphere Notes 225-274: types and other specimens of some genera of Ascomycota. Mycosphere 9:647-754

Donk MA (1949) New and revised nomina generica conservanda proposed for Basidiomycetes (Fungi). Bull Jard Bot Buitenzorg 18:83-168

Donk MA (1957) Notes on Resupinate Hymenomycetes IV. Fungus 27:1-29

Donk MA (1971) Progress in the study of the classification of the higher Basidiomycetes. In: Petersen RH (ed) Evolution in the higher Basidiomycetes. Knoxville, p 3-25

Dong W, Hyde KD, Jayarama Bhat D, Zhang H (2018) Introducing Aculeata aquatica gen. et sp. nov., Minimelanolocus thailandensis sp. nov. and Thysanorea aquatica sp. nov. (Herpotrichiellaceae, Chaetothyriales) from freshwater in northern Thailand. Mycol Prog 17:617-629

Dong W, Hyde KD, Doilom M, Yu XD, Bhat DJ, Jeewon R, Boonmee S, Wang GN, Nalumpang S, Zhang H (2020a) Pseudobactrodesmium (Dactylosporaceae, Eurotiomycetes, Fungi) a novel lignicolous genus. Front Microbiol 11:456

Dong W, Wang B, Hyde KD, McKenzie EHC, Raja HA, Tanaka K, Abdel-Wahab MA, Abdel-Aziz FA, Doilom M, Phookamsak R, Hongsanan S, Wanasinghe DN, Yu XD, Wang GN, Yang H, Yang J, Thambugala KM, Tian Q, Luo ZL, Yang JB, Miller AN, Fournier J, Boonmee S, Hu DM, Nalumpang S, Zhang H (2020b) Freshwater Dothideomycetes. Fungal Divers 105:319-575

Douanla-Meli C, Langer E, Calonge FD (2005) Geastrum pleosporus sp. nov., a new species of Geastraceae identified by morphological and molecular phylogenetic data. Mycol Prog 4:239-250
Dresch P, Falbesoner J, Ennemoser C, Hittorf M, Kuhnert R, Peintner U (2019) Emerging from the ice-fungal communities are diverse and dynamic in earliest soil developmental stages of a receding glacier. Environ Microbiol 21:1864-1880

Dudka IO, Heluta VP, Tykhonenko YY, Andrianova TV, Hayova VP, Prydiuk MP, Dzhagan VV, Isikov VP (2004) Fungi of the Crimean Peninsula (Translated from Russian). M.G. Kholodny Institute of Botany, National Academy of Sciences of Ukraine, p 452

Dutta AK, Achaya K (2018) A new host for the parasitic macrofungus Marasmius palmivorus Sharples (Marasmiaceae). Sci Corres 2018:1400-1402

Dutta AK, Das K, Acharya K (2015) A new species of Marasmius sect. Globulares from Indian Himalaya with tall basidiomata. Mycosphere 6:560-567

Edler D, Klein J, Antonelli A, Silvestro D (2020) raxmlGUI 2.0: a graphical interface and toolkit for phylogenetic analyses using RAxML. Methods Ecol Evol 1-5

Ekanayaka AH, Ariyawansa HA, Hyde KD, Jones EBG, Daranagama DA, Phillips AJL, Hongsanan S, Jayasiri SC, Zhao Q (2017) Discomycetes: theapothecial representatives of the phylum Ascomycota. Fungal Divers 87:237-298

Ekanayaka AH, Hyde KD, Gentekaki E, McKenzie EHC, Zhao Q, Bulgakov TS, Camporesi E (2019a) Preliminary classification of leotiomycetes. Mycosphere 10:310-489

Ekanayaka AH, Jones EBG, Hyde KD, Zhao Q (2019b) A stable phylogeny for Dactylosporaceae. Cryptog Mycol 40:23-44

Ellis MB (1963) Dematiaceous hyphomycetes. V. Mycol Pap 93:1-33

Ellis MB (1971) Dematiaceous hyphomycetes. Commonwealth Mycological Institute, Kew

Ellis MB (1976) More dematiaceous hyphomycetes. Commonwealth Mycological Institute, Kew

Eriksson J (1958) Studies in the Heterobasidiomycetes and Homobasidiomycetes- Aphyllophorales of Muddus National Park in North Sweden. Symbolae Botanicae Upsalienses 16:1-172

Eriksson OE (1992) The non-lichenized pyrenomycetes of Sweden. Btjtryck, Lund, p 208

Eriksson OE (2005) Outline of ascomycota. Myconet 11:1-113

Eriksson J, Ryvarden L (1975) The Corticiaceae of North Europe, vol 3. Coronicium-Hyphoderma. Fungiflora, Oslo

Eriksson OE, Winka W (1997) Supraordinal taxa of Ascomycota. Myconet 1:1-16

Fan XL, Bezerra JDP, Tian CM, Crous PW (2018) Families and genera of diaporthalean fungi associated with canker and dieback of tree hosts. Persoonia 40:119-134

Farr DF, Rossman AY (2019) Fungal databases, US National Fungus Collections, ARS, USDA. https://nt.ars-grin.gov/fungaldata bases. Accessed 22 Nov 2019

Farr DF, Rossman AY (2020) Fungal databases, Systematic Mycology and Microbiology Laboratory, ARS, USDA. http://nt.ars-grin gov/fungaldatabases/. Accessed $10 \mathrm{Feb} 2020$

Farr DF, Rossman AY (2021) Fungal databases, U.S. National Fungus Collections, ARS, USDA. https://nt.ars-grin.gov/fungaldata bases/. Accessed 2 April 2021

Feng PY, De Hoog GS, Najafzadeh MJ, Gerrits van den Ende AHG, Stielow JB, Badali H, Boeger WA, Vicente VA (2014) Cladophialophora abundans, a novel species of Chaetothyriales isolated from the natural environment. Mycol Prog 13:381-391

Ferrer A, Miller AN, Shearer CA (2011) Minutisphaera and Natipusilla: two new genera of freshwater Dothideomycetes. Mycologia 103:411-423

Floudas D, Hibbett D (2015) Revisiting the taxonomy of Phanerochaete (Polyporales, Basidiomycota) using a four gene dataset and extensive ITS sampling. Fungal Biol 119:679-719

Foister CE (1961) The economic plant diseases of Scotland. Techn Bull Dept Agric Fish Scotland 1:1-210 
Fournier J, Ju YM, Hsieh HM, Lindemann U (2018) Xylaria aethiopica sp. nov. a new pod-inhabiting species of Xylaria (Xylariaceae) from Ethiopia. Ascomycete 10:209-215

French AM (1989) California plant disease host index. Calif Dept Food Agric, Sacramento, p 394

Fresenius G (1850) Beiträge zur Mykologie. Hefte 1. Frankfurt 1:1-38

Fries EM (1828) Elenchus Fungorum. Greifswald 1:1-238

Fries EM (1836) Corpus Florarum provincialium suecicae I. Floram Scan, p 349

Fries EM (1849) Summa vegetabilium scandinaviae. Part 2. Bonnier, Leipzig

Fröhlich J, Hyde KD, Guest DI (1997) Fungi associated with leaf spots of palms in north Queensland, Australia. Mycol Res 101:721-732

Frøslev TG, Jeppesen TS, Lassøe T (2006) Seven new calochroid and fulvoid species of Cortinarius. Mycol Res 110:1046-1058

Fuckel L (1865) Fungi Rhenani Exsiccati Cent. XV-XVI:1401-1600

Fuckel KWGL (1869) Sytnbolae mycologicae. Symbolae mycologicae. Beiträge zur Kenntniss der Rheinischen Pilze. Jb Nassau Ver Naturk 23-24:1-459

Fuckel L (2011) Symbolae mycologicae. Beiträge zur Kenntniss der Rheinischen Pilze. Jb Nassau Ver Naturk 23-24:1-459

Funk A (1979) New species of Gelatinosporium on western conifers. Can J Bot 57:765-767

Gao Y, Liu F, Cai L (2016) Unravelling Diaporthe species associated with Camellia. Syst Biodivers 14:102-117

Gao Y, Liu F, Duan W, Crous PW, Cai L (2017) Diaporthe is paraphyletic. IMA Fungus 8:153-187

Gardes M, Fortin J, Mueller G, Kropp B (1990) Restriction fragment length polymorphisms in the nuclear ribosomal DNA of four Laccaria spp.: L. bicolor, L. laccata L. Proxima and L. Amethystina. Phytopathology 80:1312-1317

Gäumann EA, Dodge CW (1928) Comparative morphology of fungi, p 1-701

Ge ZW, Jacobs A, Vellinga EC, Sysouphanthong P, van der Walt R, Lavorato C, An YF, Yang ZL (2018) A multi-gene phylogeny of Chlorophyllum (Agaricaceae, Basidiomycota): new species, new combination and infrageneric classification. MycoKeys 32:65-90

Ghobad-Nejhad M, Liu SL, Langer E, Dai YC (2015) Molecular and morphological evidence reveal a new non-cystidiate species belonging to the core Phanerochaete (Polyporales). Mycol Prog 14:68

Gilbert EJ (1940) Amanitaceae BRESADOLA. J Iconogr Mycol 27(Suppl. 1):1-200

Gilbertson RL, Blackwell M (1988) Some new or unusual corticioid fungi from the Gulf Coast region. Mycotaxon 33:375-386

Glawe DA (2008) The powdery mildews: a review of the world's most familiar (yet poorly known) plant pathogens. Annu Rev Phytopathol 46:27-51

Glez-Peña D, Gòmez-Blanco D, Reboiro-Jato M, Fdez-Riverola F, Posada D (2010) ALTER: program-oriented conversion of DNA and protein alignments. Nucleic Acids Res 38:W14-W18

Goh TK, Hyde KD (1997) Melanographium palmicolum sp. nov. from Hong Kong, and a key to the genus. Mycol Res 101:1097-1100

Goh TK, Hyde KD, Ho WH (1999) A revision of the genus Dictyosporium, with descriptions of three new species. Fungal Divers 2:65-100

Goldmann L, Weir A (2012) Position specificity in Chitonomyces (Ascomycota, Laboulbeniomycetes) on Laccophilus (Coleoptera, Dytiscidae): a molecular approach resolves a century-old debate. Mycologia 104:1143-1158

Goldmann L, Weir A (2018) Molecular phylogeny of the Laboulbeniomycetes (Ascomycota). Fungal Biol 122:87-100

Goldmann L, Weir A, Rossi W (2013) Molecular analysis reveals two new dimorphic species of Hesperomyces (Ascomycota,
Laboulbeniomycetes) parasitic on the ladybird Coleomegilla maculata (Coleoptera, Coccinellidae). Fungal Biol 117:807-813

Gomes RR, Glienke C, Videira SIR, Lombard L, Groenewald JZ, Crous PW (2013) Diaporthe: a genus of endophytic, saprobic and plant pathogenic fungi. Persoonia $31: 1-41$

Gorjón SP, Greslebin AG (2012) Type studies of the species of Odontia described by GH Cunningham. NZ J Bot 50:289-301

Gorjón SP, Hallenberg N (2013) Some new species and a first checklist of corticioid fungi (Basidiomycota) from Chile. Mycol Prog $12: 185-192$

Green FM (1931) Observations on Cucurbitaria laburni. Trans Br Mycol Soc 16:289-303

Greslebin AG, Rajchenberg M (2003) Diversity of Corticiaceae sens. lat. in Patagonia, Southern Argentina. NZ J Bot 41:437-446

Greslebin AG, Nakasone KK, Rajchenberg M (2004) Rhizochaete, a new genus of phanerochaetoid fungi. Mycologia 96:260-271

Guarnaccia V, Vitale A, Cirvilleri G, Aiello D, Susca A, Epifani F, Perrone G, Polizzi G (2016) Characterisation and pathogenicity of fungal species associated with branch cankers and stem-end rot of avocado in Italy. Eur J Plant Pathol 146:963-976

Guarnaccia V, Groenewald JZ, Woodhall J, Armengol J, Cinelli T, Eichmeier A, Ezra D, Fontaine F, Gramaje D, Gutierrez-Aguirregabiria A, Kaliterna J, Kiss L, Larignon P, Luque L, Mugnai L, Naor V, Raposo R, Sándor E, Váczy KZ, Crous PW (2018) Diaporthe diversity and pathogenicity revealed from a broad survey of grapevine diseases in Europe. Persoonia 40:135-153

Gueidan C, Villaseñor CR, De Hoog GS, Gorbushina AA, Untereiner WA, Lutzoni F (2008) A rock-inhabiting ancestor for mutualistic and pathogen-rich fungal lineages. Stud Mycol 61:111-119

Gueidan C, Aptroot A, da Silva Cáceres ME, Badali H, Stenroos S (2014) A reappraisal of orders and families within the subclass Chaetothyriomycetidae (Eurotiomycetes, Ascomycota). Mycol Prog 13:1027-1039

Haase G, Sonntag L, Melzer-Krick B, De Hoog GS (1999) Phylogenetic inference by SSU-gene analysis of members of the Herpotrichiellaceae with special reference to human pathogenic species. Stud Mycol 43:80-97

Haelewaters D, Rossi W (2017) Laboulbeniales parasitic on American small carrion beetles: new species of Corethromyces, Diphymyces, and Rodaucea. Mycologia 109:142-148

Haelewaters D, Dima B, Abdel-Hafiz BII, Abdel-Wahab MA, AbulEzz SR, Acar I, Aguirre-Acosta E, Aime MC, Aldemir S, Ali M, Ayala-Vásquez O, Bakhit MS, Bashir H, Battistin E, Bendiksen E, Castro-Rivera R, Çolak ÖF, De Kesel A, de la Fuente JI, Dizkırıcı A, Hussain S, Jansen GM, Kaygusuz O, Khalid AN, Khan J, Kiyashko AA, Larsson E, MartínezGonzález CR, Morozova OV, Niazi AR, Noordeloos ME, Pham THG, Popov ES, Psurtseva NV, Schoutteten N, Sher H, Türkekul I, Verbeken A, Ahmad H, Afshan NS, Christe P, Fiaz M, Glaizot O, Liu J, Majeed J, Markotter W, Nagy A, Nawaz H, Papp V, Péter Á, Pfliegler WP, Qasim T, Riaz M, Sándor AD, Szentiványi T, Voglmayr H, Yousaf N, Krisai-Greilhuber I (2020) Fungal systematics and evolution 6. Sydowia 72:231-356

Hall TA (1999) BioEdit: a user-friendly biological sequence alignment editor and analysis program for Windows 95/98/NT. Nucleic Acids Symp Ser 41:95-98

Hall BG (2004) Phylogenetic trees made easy: a how-to manual, 2nd edn. Sinauer Associates, Sunderland

Hashimoto A, Matsumura M, Hirayama K, Yonezawa H, Tanaka K (2016) Taxonomy and phylogeny of Cryptocoryneum (Pleosporales, Dothideomycetes). Mycol Prog 15:45

Hashimoto A, Matsumura M, Hirayama K, Tanaka K (2017) Revision of Lophiotremataceae sensu lato (Pleosporales, Dothideomycetes): establishment of Aquasubmersaceae, Cryptocoryneaceae, and Hermatomycetaceae fam. nov. Persoonia 39:51-73 
Hashimoto A, Hirayama K, Takahashi H, Matsumura M, Okada G, Chen CY, Huang JW, Kakishima M, Ono T, Tanaka K (2018) Resolving the Lophiostoma bipolare complex: generic delimitations within Lophiostomataceae. Stud Mycol 90:161-189

Hatakeyama S, Tanaka K, Harada Y (2008) Bambusicolous fungi in Japan (7): a new coelomycetous genus, Versicolorisporium. Mycoscience 49:211-214

Hawksworth DL (1979) Ascospore sculpturing and generic concepts in the Testudinaceae (syn. Zopfiaceae). Can J Bot 57:91-99

Hawksworth DL, Kirk PM, Sutton BC, Pegler DN (1995) Dictionary of the fungi. 8th edn, p 616

He MQ, Zhao RL, Hyde KD, Begerow D, Kemler M, Yurkov A, McKenzie EHC, Raspé O, Kakishima M, Sánchez-Ramírez S, Vellinga EC, Halling R, Papp V, Zmitrovich IV, Buyck B, Ertz D, Wijayawardene NN, Cui BK, Schoutteten N, Liu XZ, Li TH, Yao YJ, Zhu XY, Liu AQ, Li GJ, Zhang MZ, Ling ZL, Cao B, Antonín V, Boekhout T, da Silva BDB, De Crop E, Decock C, Dima B, Dutta AK, Fell JW, Geml J, Ghobad-Nejhad M, Giachini AJ, Gibertoni TB, Gorjón SP, Haelewaters D, He SH, Hodkinson BP, Horak E, Hoshino T, Justo A, Lim YW, Menolli N Jr, Mešić A, Moncalvo JM, Mueller GM, Nagy LG, Nilsson RH, Noordeloos M, Nuytinck J, Orihara T, Ratchadawan C, Rajchenberg M, Silva-Filho AGS, Sulzbacher MA, Tkalčec Z, Valenzuela R, Verbeken A, Vizzini A, Wartchow F, Wei TZ, Weiß M, Zhao CL, Kirk PM (2019) Notes, outline and divergence times of Basidiomycota. Fungal Divers 99:105-367

Heilmann-Clausen J, Verbeken A, Vesterholt J (1998) The genus Lactarius: fungi of Northern Europe, vol 2. Svampetryk, Mundelstrup

Helaly SE, Thongbai B, Stadler M (2018) Diversity of biologically active secondary metabolites from endophytic and saprotrophic fungi of the ascomycete order Xylariales. Nat Prod Rep 35:992-1014

Hemmes DE, Desjardin DE (2008) Annotated list of Boletes and Amanita in the Hawaiian Islands. N Am Fungi 3:165-174

Heredia G, Li DW, Wendt L, Réblová M, Arias RM, Gamboa-Angulo M, Štěpánek V, Stadler M, Castañeda-Ruíz RF (2020) Natonodosa speciosa gen. et sp. nov. and rediscovery of Poroisariopsis inornata: neotropical anamorphic fungi in Xylariales. Mycol Prog 19:15-30

Hibbett DS, Pine EM, Langer G, Langer E, Donoghue MJ (1997) Evolution of gilled mushrooms and puffballs inferred from ribosomal DNA sequences. Proc Natl Acad Sci USA 94:12002-12006

Hibbett DS, Bauer R, Binder M, Giachini AJ, Hosaka K, Justo A, Larsson E, Larsson KH, Lawrey JD, Miettinen O, Nagy LG, Nilsson RH, Weiss M, Thorn RG (2014) 14 Agaricomycetes. In: McLaughlin D, Spatafora J (eds) Systematics and evolution. The Mycota (A comprehensive treatise on fungi as experimental systems for basic and applied research), vol 7A. Springer, Berlin, pp 373-429

Hirayama K, Tanaka K (2011) Taxonomic revision of Lophiostoma and Lophiotrema based on reevaluation of morphological characters and molecular analyses. Mycoscience 52:401-412

Hirooka Y, Rossman AY, Samuels GJ, Lechat C, Chaverri P (2012) A monograph of Allantonectria, Nectria, and Pleonectria (Nectriaceae, Hypocreales, Ascomycota) and their pycnidial, sporodochial, and synnematous anamorphs. Stud Mycol 71:1-210

Ho MHM, Castañeda RF, Dugan FM, Jong SC (1999) Cladosporium and Cladophialophora in culture: descriptions and an expanded key. Mycotaxon 72:115-157

Ho WH, Yanna HKD, Hodgkiss IJ (2002) Seasonality and sequential occurrence of fungi on wood submerged in Tai Po Kau Foreststream, Hong Kong. Fungal Divers 10:21-43

Hollós L (1903) Magyarorszag Gasteromycetai. Franklin Társulat, Budapest
Holm L, Holm K (1988) Studies in the Lophiostmataceae with emphasis on the Swedish species. Symb Bot Upsal 28:1-50

Holubová-Jechová V, Mercado Sierra A (1984) Studies on Hyphomycetes from Cuba II. Hyphomycetes from the Isla de la Juventud. Česká Mykol 38:96-120

Hongsanan S, Tian Q, Hyde KD, Hu DM (2016) The asexual morph of Trichomerium gloeosporum. Mycosphere 7:1473-1479

Hongsanan S, Maharachchikumbura SSN, Hyde KD, Samarakoon MC, Jeewon R, Zhao Q, Al-Sadi AM, Bahkali AH (2017) An updated phylogeny of Sordariomycetes based on phylogenetic and molecular clock evidence. Fungal Divers 84:25-41

Hongsanan S, Hyde KD, Phookamsak R, Wanasinghe DN, McKenzie EHC, Sarma VV, Boonmee S, Lücking R, Bhat DJ, Liu NG, Tennakoon DS, Pem D, Karunarathna A, Jiang SH, Jones EBG, Phillips AJL, Manawasinghe IS, Tibpromma S, Jayasiri SC, Sandamali DS, Jayawardena RS, Wijayawardene NN, Ekanayaka AH, Jeewon R, Lu YZ, Dissanayake AJ, Zeng XY, Luo ZL, Tian Q, Phukhamsakda C, Thambugala KM, Dai DQ, Chethana KWT, Samarakoon MC, Ertz D, Bao DF, Doilom M, Liu JK, Pérez-Ortega S, Suija A, Senwanna C, Wijesinghe SN, Konta S, Niranjan M, Zhang SN, Ariyawansa HA, Jiang HB, Zhang JF, Norphanphoun C, de Silva NI, Thiyagaraja V, Zhang H, Bezerra JDP, Miranda-González R, Aptroot A, Kashiwadani H, Harishchandra D, Sérusiaux E, Aluthmuhandiram JVS, Abeywickrama PD, Devadatha B, Wu HX, Moon KH, Gueidan C, Schumm F, Bundhun D, Mapook A, Monkai J, Chomnunti P, Suetrong S, Chaiwan N, Dayarathne MC, Yang J, Rathnayaka AR, Bhunjun CS, Xu JC, Zheng JS, Liu G, Feng Y, Xie N (2020a) Refined families of Dothideomycetes: Dothideomycetidae and Pleosporomycetidae. Mycosphere 11:1553-2107

Hongsanan S, Hyde KD, Phookamsak R, Wanasinghe DN, McKenzie EHC, Sarma VV, Lücking R, Boonmee S, Bhat DJ, Liu NG, Tennakoon DS, Pem D, Karunarathna A, Jiang SH, Jones EBG, Phillips AJL, Manawasinghe IS, Tibpromma S, Jayasiri SC, Sandamali DS, Jayawardena RS, Wijayawardene NN, Ekanayaka AH, Jeewon R, Lu YZ, Phukhamsakda C, Dissanayake AJ, Zeng XY, Luo ZL, Tian Q, Thambugala KM, Dai DQ, Samarakoon MC, Chethana KWT, Ertz D, Doilom M, Liu JK, Pérez-Ortega S, Suija A, Senwanna C, Wijesinghe SN, Niranjan M, Zhang SN, Ariyawansa HA, Jiang HB, Zhang JF, Norphanphoun C, de Silva NI, Thiyagaraja V, Zhang H, Bezerra JDP, Miranda-González R, Aptroot A, Kashiwadani H, Harishchandra D, Sérusiaux E, Abeywickrama PD, Bao DF, Devadatha B, Wu HX, Moon KH, Gueidan C, Schumm F, Bundhun D, Mapook A, Monkai J, Bhunjun CS, Chomnunti P, Suetrong S, Chaiwan N, Dayarathne MC, Yang J, Rathnayaka AR, Xu JC, Zheng JS, Liu G, Feng Y, Xie N (2020b) Refined families of Dothideomycetes: orders and families incertae sedis. Fungal Divers 105:17-318

Hosaka K, Bates ST, Beever RE, Castellano MA, Colgan W 3rd, Domínguez LS, Nouhra ER, Geml J, Giachini AJ, Kenney SR, Simpson NB, Spatafora JW, Trappe JM (2006) Molecular phylogenetics of the gomphoid-phalloid fungi with an establishment of the new subclass Phallomycetidae and two new orders. Mycologia 98:949-959

Hosen MI, Li TH, Aminuzzaman FM, Islam MR (2016) Hygrocybe umbilicata sp. nov., with first generic report for Bangladesh and its phylogenetic placement. Phytotaxa 280:70-76

Hosen MI, Song ZP, Gates G, Karunarathna SC, Chowdhury MSM, Li TH (2017) Two new species of Xanthagaricus and some notes on Heinemannomyces from Asia. MycoKeys 28:1-18

Houbraken J, Kocsubé S, Visagie CM, Yilmaz N, Wang XC, Meijer M, Kraak B, Hubka V, Bensch K, Samson RA, Frisvad JC (2020) Classification of Aspergillus, Penicillium, Talaromyces and related genera (Eurotiales): an overview of families, genera, subgenera, sections, series and species. Stud Mycol 95:5-169 
Hsieh HM, Chou JC, Ju YM (2020) Xylaria insolita and X. subescharoidea: two newly described species collected from a termite nesting site in Hua-lien, Taiwan. Bot Stud 61:1-9

Hu DM, Cai L, Chen H, Bahkali AH, Hyde KD (2010a) Fungal diversity on submerged wood in a tropical stream and an artificial lake. Biodivers Conserv 19:3799-3808

Hu HL, Jacques F, Rajesh J, Bahkali AH, Hyde KD (2010b) Revisiting the taxonomy of Daruvedia bacillata. Mycotaxon 114:135-144

Hu DM, Wang M, Cai L (2016) Phylogenetic assessment and taxonomic revision of Mariannaea. Mycol Prog 16:271-283

Hughes SJ (1958) Revisiones hyphomycetum aliquot cum appendice de nominibus rejiciendis. Can J Bot 36:727-836

Huhndorf SM, Miller AN, Fernández FA (2004) Molecular systematics of the Sordariales: the order and the family Lasiosphaeriaceae redefined. Mycologia 96:368-387

Huldén L (1983) Laboulbeniales (Ascomycetes) of Finland and adjacent parts of the U.S.S.R. Karstenia 23:31-136

Husbands DR, Urbina H, Lewis SM, Aime MC (2018) Xylaria karyophthora: a new seed-inhabiting fungus of Greenheart from Guyana. Mycologia 110:434-447

Hussain S, Usman M, Afshan NS, Ahmad H, Khan J, Khalid AN (2018) The genus Coprinellus (Basidiomycota; Agaricales) in Pakistan with the description of four new species. MycoKeys 39:41-61

Hyde KD (1992) The genus Saccardoella from intertidal mangrove wood. Mycologia 84:803-810

Hyde KD (1995) Tropical Australasian fungi. IX. Vaginatispora aquatica gen. et sp. nov. Nova Hedwig 61:233-241

Hyde KD, Aptroot A (1998) Tropical freshwater species of the genera Massarina and Lophiostoma (ascomycetes). Nova Hedwig 66:489-502

Hyde KD, Jones EBG, Liu JK, Ariyawansa H, Boehm E, Boonmee S, Braun U, Chomnunti P, Crous PW, Dai DQ, Diederich P, Dissanayake A, Doilom M, Doveri F, Hongsanan S, Jayawardena R, Lawrey JD, Li YM, Liu YX, Lücking R, Monkai J, Muggia L, Nelsen MP, Pang KL, Phookamsak R, Senanayake IC, Shearer CA, Suetrong S, Tanaka K, Thambugala KM, Wijayawardene NN, Wikee S, Wu HX, Zhang Y, Aguirre-Hudson B, Alias SA, Aptroot A, Bahkali AH, Bezerra JL, Bhat DJ, Camporesi E, Chukeatirote E, Gueidan C, Hawksworth DL, Hirayama K, De Hoog S, Kang JC, Knudsen K, Li WJ, Li XH, Liu ZY, Mapook A, McKenzie EHC, Miller AN, Mortimer PE, Phillips AJL, Raja HA, Scheuer C, Schumm F, Taylor JE, Tian Q, Tibpromma S, Wanasinghe DN, Wang Y, Xu JC, Yacharoen S, Yan JY, Zhang M (2013) Families of Dothideomycetes. Fungal Divers 63:1-313

Hyde KD, Hongsanan S, Jeewon R, Bhat DJ, McKenzie EHC, Jones EBG, Phookamsak R, Ariyawansa HA, Boonmee S, Zhao Q, Abdel-Aziz FA, Abdel-Wahab MA, Banmai S, Chomnunti P, Cui BK, Daranagama DA, Das K, Dayarathne MC, de Silva NI, Dissanayake AJ, Doilom M, Ehanayaka AH, Gibertoni TB, GoesNeto A, Huang SK, Jayasiri SC, Jayawardena RS, Konta S, Lee HB, Li WJ, Lin CG, Liu JK, Lu YZ, Luo ZL, Manawasinghe IS, Manimohan P, Mapook A, Niskanen T, Norphanphoun C, Papizadeh M, Perera RH, Phukhamsakda C, Richter C, de Santiago ALCM, Drechsler-Santos ER, Senanayake IC, Tanaka K, Tennakoon TMDS, Thambugala KM, Tian Q, Tibpromma S, Thongbai B, Vizzini A, Wanasinghe DN, Wijayawardene NN, Wu HX, Yang J, Zeng XY, Zhang H, Zhang JF, Bulgakov TS, Camporesi E, Bahkali AH, Amoozegar MA, Araujo-Neta LS, Ammirati JF, Baghela A, Bhatt RP, Bojantchev D, Buyck B, de Silva GA, de Lima CLF, de Oliveira RJV, de Souza CAF, Dai YC, Dima B, Duong TT, Ercole E, Mafalda-Freire F, Ghosh A, Hashimoto A, Kamolhan S, Kang JC, Karunarathna SC, Kirk PM, Kytovuori I, Lantieri A, Liimatainen K, Liu ZY, Liu XZ, Lucking R, Medardi G, Mortimer PE, Nguyen TTT, Promputtha I, Raj KNA, Reck MA, Lumyong S, Shahzadeh-Fazeli SA,
Stadler M, Soudi MR, Su HY, Takahashi T, Tangthirasunun N, Uniyal P, Wang Y, Wen TC, Xu JC, Zhang ZK, Zhao YC, Zhou JL, Zhu L (2016) Fungal diversity notes 367-490: taxonomic and phylogenetic contributions to fungal taxa. Fungal Divers 80:1-270

Hyde KD, Norphanphoun C, Abreu VP, Bazzicalupo A, Chethana KWT, Clericuzio M, Dayarathne MC, Dissanayake AJ, Ekanayaka AH, He MQ, Hongsanan S, Huang SK, Jayasiri SC, Jayawardena RS, Karunarathna A, Konta S, Kus`an I, Lee H, Li J, Lin CG, Liu NG, Lu YZ, Luo ZL, Manawasinghe IS, Mapook A, Perera RH, Phookamsak R, Phukhamsakda C, Siedlecki I, Soares AM, Tennakoon DS, Tian Q, Tibpromma S, Wanasinghe DN, Xiao YP, Yang J, Zeng XY, Abdel-Aziz FA, Li WJ, Senanayake IC, Shang QJ, Daranagama DA, De Silva NI, Thambugala KM, Abdel-Wahab MA, Bahkali AH, Berbee ML, Boonmee S, Bhat DJ, Bulgakov TS, Buyck B, Camporesi E, Castañeda-Ruiz RF, Chomnunti P, Doilom M, Dovana F, Gibertoni TB, Jadan M, Jeewon R, Jones GEB, Kang JC, Karunarathna SC, Lim YW, Liu JK, Liu ZY, Plautz HL Jr, Lumyong S, Maharachchikumbura SSN, Matočec N, Mckenzie EHC, Meśič A, Miller D, Pawłowska J, Pereira OL, Promputtha I, Romero AI, Ryvarden L, Su HY, Suetrong S, Tkalčec Z, Vizzini A, Wen TC, Wisitrassameewong K, Wrzosek M, Xu JC, Zhao Q, Zhao RL, Mortimer PE (2017) Fungal diversity notes 603-708: taxonomic and phylogenetic notes on genera and species. Fungal Divers 87:1-235

Hyde KD, Chaiwan N, Norphanphoun C, Boonmee S, Camporesi E, Chethana KWT, Dayarathne MC, de Silva NI, Dissanayake AJ, Ekanayaka AH, Hongsanan S, Huang SK, Jayasiri SC, Jayawardena RS, Jiang HB, Karunarathna A, Lin CG, Liu JK, Liu NG, Lu YZ, Luo ZL, Maharachchimbura SSN, Manawasinghe IS, Pem D, Perera RH, Phukhamsakda C, Samarakoon MC, Senwanna C, Shang QJ, Tennakoon DS, Thambugala KM, Tibpromma S, Wanasinghe DN, Xiao YP, Yang J, Zeng XY, Zhang JF, Zhang SN, Bulgakov TS, Bhat DJ, Cheewangkoon R, Goh TK, Jones EBG, Kang JC, Jeewon R, Liu ZY, Lumyong S, Kuo CH, McKenzie EHC, Wen TC, Yan JY, Zhao Q (2018a) Mycosphere notes 169-224. Mycosphere 9:271-430

Hyde KD, Norphanphoun C, Chen J, Dissanayake AJ, Doilom M, Hongsanan S, Jayawardena RS, Jeewon R, Perera RH, Thongbai B, Wanasinghe DN, Wisitrassameewong K, Tibpromma S, Stadler M (2018b) Thailand's amazing diversity—up to $96 \%$ of fungi in northern Thailand are novel. Fungal Divers 93:215-239

Hyde KD, Danushka S, Tennakoon DS, Jeewon R, Bhat DJ, Maharachchikumbura SSN, Rossi W, Leonardi M, Lee HB, Mun HY, Houbraken J, Nguyen TTT, Jeon SJ, Frisvad JC, Dhanushka N, Wanasinghe DN, Luücking R, Aptroot A, Cáceres MES, Karunarathna SC, Hongsanan S, Phookamsak R, de Silva NI, Thambugala KM, Jayawardena RS, Senanayake IC, Boonmee S, Chen J, Luo ZL, Phukhamsakda C, Pereira OL, Abreu VP, Rosado AWC, Bart B, Randrianjohany E, Hofstetter V, Gibertoni TB, da Silva Soares AM, Plautz HL Jr, Sotão HMP, Xavier WKS, Bezerra JDP, de Oliveira TGL, de Souza Motta CM, Magalhães OMC, Bundhun D, Harishchandra D, Manawasinghe IS, Dong W, Zhang SN, Bao DF, Samarakoon MC, Pem D, Karunarathna A, Lin CG, Yang J, Perera RH, Kumar V, Huang SK, Dayarathne MC, Ekanayaka AH, Jayasiri SC, Xiao YP, Konta S, Niskanen T, Liimatainen K, Dai YC, Ji XH, Tian XM, Mešić A, Singh SK, Phutthacharoen K, Cai L, Sorvongxay T, Thiyagaraja V, Norphanphoun C, Chaiwan N, Lu YZ, Jiang HB, Zhang JF, Abeywickrama PD, Aluthmuhandiram JVS, Brahmanage RS, Zeng M, Chethana T, Wei DP, Réblová M, Fournier J, Nekvindová J, do Nascimento Barbosa R, dos Santos JEF, de Oliveira NT, Li GJ, Ertz D, Shang QJ, Phillips AJL, Kuo CH, Camporesi E, Bulgakov TS, Lumyong S, Jones EBG, Chomnunti P, Gentekaki E, Bungartz F, Zeng XY, Fryar S, Tkalčec Z, Liang J, Li GS, Wen TC, Singh PN, Gafforov Y, Promputtha I, Yasanthika E, 
Goonasekara ID, Zhao RL, Zhao Q, Kirk PM, Liu JK, Yan JY, Mortimer PE, Xu JC (2019) Fungal diversity notes 1036-1150: taxonomic and phylogenetic contributions on genera and species of fungal taxa. Fungal Divers 96:1-242

Hyde KD, de Silva NI, Jeewon R, Bhat DJ, Phookamsak R, Doilom M, Boonmee S, Jayawardena RS, Maharachchikumbura SSN, Senanayake IC, Manawasinghe IS, Liu NG, Abeywickrama PD, Chaiwan N, Karunarathna A, Pem D, Lin CG, Sysouphanthong P, Luo ZL, Wei DP, Wanasinghe DN, Norphanphoun C, Tennakoon DS, Samarakoon MC, Jayasiri SC, Jiang HB, Zeng XY, Li JF, Wijesinghe SN, Devadatha B, Goonasekara ID, Brahmanage RS, Yang EF, Aluthmuhandiram JVS, Dayarathne MC, Marasinghe DS, Li WJ, Dissanayake LS, Dong W, Huanraluek N, Lumyong S, Liu JK, Karunarathna SC, Jones EBG, Al-Sadi AM, Xu JC, Harishchandra D, Sarma VV (2020a) AJOM new records and collections of fungi: 1-100. Asian J Mycol 3:22-294

Hyde KD, Dong Y, Phookamsak R, Jeewon R, Bhat DJ, Jones EBG, Liu NG, Abeywickrama PD, Mapook A, Wei DP, Perera RH, Manawasinghe IS, Pem D, Bundhun D, Karunarathna A, Ekanayaka AH, Bao DF, Li JF, Samarakoon MC, Chaiwan N, Lin CG, Phutthacharoen K, Zhang SN, Senanayake IC, Goonasekara ID, Thambugala KM, Phukhamsakda C, Tennakoon DS, Jiang HB, Yang J, Zeng M, Huanraluek N, Liu JK, Wijesinghe SN, Tian Q, Tibpromma S, Brahmanage RS, Boonmee S, Huang SK, Thiyagaraja V, Lu YZ, Jayawardena RS, Dong W, Yang EF, Singh SK, Singh SM, Rana S, Lad SS, Anand G, Devadatha B, Niranjan M, Sarma VV, Liimatainen K, Aguirre- Hudson B, Niskanen T, Overall A, Alvarenga RLM, Gibertoni TB, Pfliegler WP, Horváth E, Imre A, Alves AL, da Silva Santos AC, Tiago PV, Bulgakov TS, Wanasinghe DN, Bahkali AH, Doilom M, Elgorban AM, Maharachchikumbura SSN, Rajeshkumar KC, Haelewaters D, Mortimer PE, Zhao Q, Lumyong S, Xu JC, Sheng J (2020b) Fungal diversity notes 1151-1276: taxonomic and phylogenetic contributions on genera and species of fungal taxa. Fungal Divers 100:5-277

Hyde KD, Jeewon R, Chen YJ, Bhunjun CS, Calabon MS, Jiang HB, Lin CG, Norphanphoun C, Sysouphanthong P, Pem D, Tibpromma S, Zhang Q, Doilom M, Jayawardena RS, Liu JK, Maharachchikumbura SSN, Phukhamsakda C, Phookamsak R, Al-Sadi AM, Naritsada Thongklang N, Wang Y, Gafforov Y, Jones EBG, Lumyong $S$ (2020c) The numbers of fungi: is the descriptive curve flattening? Fungal Divers 103:219-271

Hyde KD, Norphanphoun C, Maharachchikumbura SSN, Bhat DJ, Jones EBG, Bundhun D, Chen YJ, Bao DF, Boonmee S, Calabon MS, Chaiwan N, Chethana KWT, Dai DQ, Dayarathne MC, Devadatha B, Dissanayake AJ, Dissanayake LS, Doilom M, Dong W, Fan XL, Goonasekara ID, Hongsanan S, Huang SK, Jayawardena RS, Jeewon R, Karunarathna A, Konta S, Kumar V, Lin CG, Liu JK, Liu NG, Luangsa-ard J, Lumyong S, Luo ZL, Marasinghe DS, McKenzie EHC, Niego AGT, Niranjan M, Perera RH, Phukhamsakda C, Rathnayaka AR, Samarakoon MC, Samarakoon SMBC, Sarma VV, Senanayake IC, Shang QJ, Stadler M, Tibpromma S, Wanasinghe DN, Wei DP, Wijayawardene NN, Xiao YP, Yang J, Zeng XY, Zhang SN, Xiang MM (2020d) Refined families of sordariomycetes. Mycosphere 11:305-1059

Ibrahim A, Tanney JB, Fei F, Seifert KA, Cutler GC, Capretta A, Miller JD, Sumarah MW (2020) Metabolomic-guided discovery of cyclic nonribosomal peptides from Xylaria ellisii sp. nov., a leaf and stem endophyte of Vaccinium angustifolium. Sci Rep 10:1-7

Inderbitzin P, Landvik S, Abdel-Wahab MA, Berbee ML (2001) Aliquandostipitaceae, a new family for two new tropical ascomycetes with unusually wide hyphae and dimorphic ascomata. Am J Bot 88:52-61

Index Fungorum (2021) Index Fungorum. http://www.indexfungorum. org/
Jackie ES, Dennis ED, Brian AP, Chris LG, Danny SN (2017) Biodiversity and phylogeny of Marasmius (Agaricales, Basidiomycota) from Madagascar. Phytotaxa 292:101-149

Jaklitsch WM, Voglmayr H (2014) Persistent hamathecial threads in the Nectriaceae, Hypocreales: Thyronectria revisited and reinstated. Persoonia 33:182-211

Jaklitsch WM, Voglmayr H (2016) Hidden diversity in Thyridaria and a new circumscription of the Thyridariaceae. Stud Mycol 85:35-64

Jaklitsch WM, Baral HO, Lücking R, Lumbsch HT (2016) Ascomycota. In: Frey W (ed) Syllabus of plant families-Adolf Engler's syllabus der pflanzenfamilien. Borntraeger, Stuttgart, pp 1-322

Jayasiri SC, Hyde KD, Jeewon R, Bhat JD, Camporesi E, Kang JC (2016) Neooccultibambusa jonesii, a novel taxon within Occultibambusaceae. Mycosphere 7:1458-1472

Jayasiri SC, Hyde KD, Jones EBG, McKenzie EHC, Jeewon R, Phillips AJL, Bhat DJ, Wanasinghe DN, Liu JK, Lu YZ, Kang JC, Xu J, Karunarathna SC (2019) Diversity, morphology and molecular phylogeny of Dothideomycetes on decaying wild seed pods and fruits. Mycosphere 10:1-186

Jayawardena RS, Hyde KD, McKenzie EH, Jeewon R, Phillips AJ, Perera RH, de Silva NI, Maharachchikumburua SS, Samarakoon MC, Ekanayake AH, Tennakoon DS (2019) One stop shop III: taxonomic update with molecular phylogeny for important phytopathogenic genera: 51-75. Fungal Divers 98:77-160

Jeewon R, Hyde KD (2016) Establishing species boundaries and new taxa among fungi: recommendations to resolve taxonomic ambiguities. Mycosphere 7:1669-1677

Jeong W, Keighley C, Wolfe R, Lee W, Slavin MA, Kong DCM, Chen CA (2019) The epidemiology and clinical manifestations of mucormycosis: a systematic review and meta-analysis of case reports. Clin Microbiol Infect 25:26-34

Jeppson M, Nilsson RH, Larsson E (2013) European earthstars in Geastraceae (Geastrales, Phallomycetidae) - a systematic approach using morphology and molecular sequence data. Syst Biodivers 11:437-465

Jiang N, Voglmayr H, Tian CM (2018) New species and records of Coryneum from China. Mycologia 110:1172-1188

Jiang N, Voglmayr H, Tian CM (2019) Morphology and phylogeny reveal two novel Coryneum species from China. MycoKeys 56:67-80

Jiang N, Fan X, Tian C, Crous PW (2020) Reevaluating Cryphonectriaceae and allied families in Diaporthales. Mycologia 112:267-292

Johnston PR, Quijada L, Smith CA, Baral HO, Hosoya T, Baschien C, Pärtel K, Zhuang WY, Haelewaters D, Park D, Carl S, LópezGiráldez F, Wang Z, Townsend JP (2019) A multigene phylogeny toward a new phylogenetic classification for the Leotiomycetes. IMA Fungus 10:1

Jones EBG, Suetrong S, Cheng WH, Rungjindamai N, Sakayaroj J, Boonyuen N, Somrothipol S, Abdel-Wahab MA, Pang KL (2014) An additional fungal lineage in the Hypocreomycetidae (Falcocladium species) and the taxonomic re-evaluation of Chaetosphaeria chaetosa and Swampomyces species, based on morphology, ecology and phylogeny. Cryptog Mycol 35:119-138

Jones EBG, Suetrong S, Sakayaroj J, Bahkali AH, Abdel-Wahab MA, Boekhout T, Pang KL (2015) Classification of marine Ascomycota, Basidiomycota, Blastocladiomycota and Chytridiomycota. Fungal Divers 73:1-72

Ju YM, Hsieh HM, Vasilyeva L, Akulov A (2009) Three new Xylaria species from Russian Far East. Mycologia 101:548-553

Ju YM, Rogers JD, Hsieh HM (2018) Xylaria species associated with fallen fruits and seeds. Mycologia 110:726-749

Jülich W (1981) Higher taxa of Basidiomycetes. Biblthca Mycol 85:1-485 
Justo A, Miettinen O, Floudas D, Ortiz-Santana B, Sjökvist E, Lindner D, Nakasone K, Niemelä T, Larsson KH, Ryvarden L, Hibbet DS (2017) A revised family-level classification of the Polyporales (Basidiomycota). Fungal Biol 121:798-824

Kałucka IL, Jagodziński AM, Nowiński M (2016) Biodiversity of ectomycorrhizal fungi in open-cast mine spoil restoration stands in Poland-first time recorded, rare and red-listed species. Acta Mycol 51:1080

Katoh K, Standley DM (2013) MAFFT multiple sequence alignment software version 7: improvements in performance and usability. Mol Biol Evol 30:772-780

Kelly KL, Judd DB (1976) Color: Universal Language and Dictionary of Names. U.S. National Bureau of Standards, Special Publication 440. U.S. Government Printing Office, Washington

Kemler M, Lutz M, Göker M, Oberwinkler F, Begerow D (2009) Hidden diversity in the non-caryophyllaceous plant-parasitic members of Microbotryum (Pucciniomycotina: Microbotryales). Syst Biodivers 7:297-306

Kemler M, Denchev TT, Denchev CM, Begerow D, Piątek M, Lutz M (2020) Host preference and sorus location correlate with parasite phylogeny in the smut fungal genus Microbotryum (Basidiomycota, Microbotryales). Mycol Progr 19:481-493

Kirk PM (1983) New or interesting microfungi. X. Hyphomycetes on Laurus nobilis leaf litter. Mycotaxon 18:259-298

Kirk PM, Cannon PE, Minter DW, Stalpers JA (2008) Dictionary of the Fungi, 10th edn. CABI, Wallingford

Kiyuna T, An KD, Kigawa R, Sano C, Sugiyama J (2018) Two new Cladophialophora species, C. tumbae sp. nov. and C. tumulicola sp. nov., and chaetothyrialean fungi from biodeteriorated samples in the Takamatsuzuka and Kitora Tumuli. Mycoscience 59:75-84

Kobayasi Y (1937) Fungi Austro-Japoniae et Micronesiae I. The Botanical Magazine. Tokyo 51:749-758

Kohlmeyer J, Volkmann-Kohlmeyer B, Eriksson OE (1997) Fungi on Juncus roemerianus.9. New obligate and facultative marine ascomycotina. Bot Mar 40:291-300

Kolařík M (2018) New taxonomic combinations in endophytic representatives of the genus Nigrograna. Czech Mycol 70:123-126

Kõljalg U (1996) Tomentella (Basidiomycota) and related genera in temperate Eurasia. Synopsis Fungorum 9:1-213

Kõljalg U, Larsson E (1998) Pseudotomentella ochracea sp. nov., based on morphological and molecular data. Folia Cryptog Estonica 33:53-56

Kong V, Yorn T, Rossi W (2020) New species and new records of Laboulbenia (Ascomycota, Laboulbeniales) from Cambodia. Phytotaxa 474:119-131

Konta S, Maharachchikumbura SS, Senanayake IC, McKenzie EH, Stadler M, Boonmee S, Phookamsak R, Jayawardena RS, Senwanna C, Hyde KD, Elgorban AM (2020) A new genus Allodiatrype, five new species and a new host record of diatrypaceous fungi from palms (Arecaceae). Mycosphere 11:239-268

Korf RP, Lizoň P (2001) The status of the ordinal name Leotiales. Czech Mycol 52:255-257

Kornerup A, Wanscher JH (1978) Methuen handbook of colour, 3rd edn. Eyre Methuen Ltd., London

Kotiranka H, Saarenoksa R (1993) Rare finnish Aphyllophorales (Basidiomycetes) plus two new combinations in Efibula. Ann Bot Fenn 30:211-249

Koukol O (2010) Revision of "Septonema ochraceum" revealed three new species of Venturiaceae and Herpotrichiellaceae. Mycol Prog 9:369-378

Koukol O, Delgado G (2019) Do not forget Africa—revision of fungarium collections at Kew revealed a new species of Hermatomyces (Hermatomycetaceae, Pleosporales). Nova Hedwig 109:413-423

Koukol O, Delgado G, Hofmann TA, Piepenbring M (2018) Panama, a hot spot for Hermatomyces (Hermatomycetaceae, Pleosporales) with five new species, and a critical synopsis of the genus. IMA Fungus 9:107-141

Kreisel H (1969) Grunzüge eines natürlichen Systems der Pilze J Cramer Lehre 1-245

Kruys A, Huhndorf SM, Miller AN (2015) Coprophilous contributions to the phylogeny of Lasiosphaeriaceae and allied taxa within Sordariales (Ascomycota, Fungi). Fungal Divers 70:101-113

Kuhnert R, Oberkofler I, Peintner U (2012) Fungal growth and biomass development is boosted by plants in snow-covered soil. Microb Ecol 64:79-90

Kuhnert E, Sir EB, Lambert C, Hyde KD, Hladki AI, Romero AI, Rohde M, Stadler M (2017a) Phylogenetic and chemotaxonomic resolution of the genus Annulohypoxylon (Xylariaceae) including four new species. Fungal Divers 85:1-43

Kuhnert E, Surup F, Halecker S, Stadler M (2017b) Minutellins A-D, azaphilones from the stromata of Annulohypoxylon minutellum (Xylariaceae). Phytochemistry 137:66-71

Kumar S, Stecher G, Tamura K (2016) MEGA7: molecular evolutionary genetics analysis version 7.0 for bigger datasets. Mol Biol Evol 33:1870-1874

Kumar S, Stecher G, Li M, Knyaz C, Tamura K (2018) MEGA X: molecular evolutionary genetics analysis across computing platforms. Mol Biol Evol 35:1547-1549

Lamprecht SC, Crous PW, Groenewald JZ, Tewoldemedhin YT, Marasas WFO (2011) Diaporthaceae associated with root and crown rot of maize. IMA Fungus 2:13-24

Larget B, Simon DL (1999) Markov chain Monte Carlo algorithms for the Bayesian analysis of phylogenetic trees. Mol Biol Evol 16:750-759

Larios JM, Honrubia M, Gallego E (1988) Notas sobre Ascomycotina en al S.E. de Espana Peninsular. V. Pirenomicetos y Loculoascomicetos saprofitos. Int J Mycol Lichenol 3:303-317

Larsen MJ (1967) Tomentella and related genera in North America 4. Taxonomy and Nomenclature of Caldesiella. Taxon 16:510-511

Larsen MJ (1974) A contribution to the taxonomy of the genus Tomentella. Mycologia Memoir, p 145

Larsson KH (2007) Molecular phylogeny of Hyphoderma and the reinstatement of Peniophorella. Mycol Res 111:186-195

Læssøe T, Pedersen OS, Sysouphanthong P (2019) An introduction to the edible, poisonous and medicinal fungi of northern Laos. Privately published, printed in Bangkok, p 192

Lawrence DP, Travadon R, Baumgartner K (2015) Diversity of Diaporthe species associated with wood cankers of fruit and nut crops in northern California. Mycologia 107:926-940

Lee H, Wissitrassameewong K, Park MS, Verbeken A, Eimes J, Lim YW (2019) Taxonomic revision of the genus Lactarius (Russulales, Basidiomycota) in Korea. Fungal Divers 95:275-335

Li AJ, Grabovskaya-Borodina AE, Hong SP, McNeill J, Ohba H, Park CW (2003) Polygonum Linnaeus. In: Wu ZY et al (eds) Flora of China, vol 5. Science Press (Beijing) \& Missouri Botanical Garden Press (St. Louis), pp 75-78

Li HY, Sun GY, Zhai XR, Batzer JC, Mayfield DA, Crous PW, Groenewald JZ, Gleason ML (2012) Dissoconiaceae associated with sooty blotch and flyspeck on fruits in China and the United States. Persoonia 28:113-125

Li GJ, Hyde KD, Zhao RN, Hongsanan S, Abdel-Aziz FA, AbdelWahab MA, Alvarado P, Alves-Silva G, Ammirati JF, Ariyawansa HA, Baghela A, Bahkali AH, Beug M, Bhat DJ, Bojantchev D, Boonpratuang T, Bulgakov TS, Camporesi E, Boro MC, Ceska O, Chakraborty D, Chen JJ, Chethana KWT, Chomnunti P, Consiglio G, Cui BK, Dai DQ, Dai YC, Daranagama DA, Das K, Dayarathne MC, Crop ED, De Oliveira RJV, de Souza CAF, de Souza JI, Dentinger BTM, Dissanayake AJ, Doilom M, Drechsler-Santos ER, GhobadNejhad M, Gilmore SP, Góes-Neto A, Gorczak M, Haitjema GH, Hapuarachchi KK, Hashimoto A, He MQ, Henske JK, Hirayama K, 
Iribarren MJ, Jayasiri SC, Jayawardena RS, Jeon SJ, Jerónimo GH, Jesus AL, Jones EBG, Kang JC, Karunarathna SC, Kirk PM, Konta S, Kuhnert E, Langer E, Lee HS, Lee HB, Li WJ, Li XH, Liimatainen K, Lima DX, Lin CG, Liu JK, Liu XZ, Liu ZY, Luangsa-ard JJ, Lücking R, Lumbsch HT, Lumyong S, Leaño EM, Marano AV, Matsumura M, McKenzie EHC, Mongkolsamrit S, Mortimer PE, Nguyen TTT, Niskanen T, Norphanphoun C, O’Malley MA, Parnmen S, Pawłowska J, Perera RH, Phookamsak R, Phukhamsakda C, Pires-Zottarelli CLA, Raspe O, Reck MA, Rocha SCO, de Santiago ALCMA, Senanayake IC, Setti L, Shang QJ, Singh SK, Sir EB, Solomon KV, Song J, Srikitikulchai P, Stadler M, Suetrong S, Takahashi H, Takahashi T, Tanaka K, Tang LP, Thambugala KM, Thanakitpipattana D, Theodorou MK, Thongbai B, Thummarukcharoen T, Tian Q, Tibpromma S, Verbeken A, Vizzini A, Vlasák J, Voigt K, Wanasinghe DN, Wang Y, Weerakoon G, Wen HA, Wen TC, Wijayawardene NN, Wongkanoun S, Wrzosek M, Xiao YP, Xu JC, Yan JY, Yang J, Yang SD, Hu Y, Zhang JF, Zhao J, Zhou LW, Peršoh D, Phillips AJL, Maharachchikumbura SSN (2016a) Fungal divers notes 253-366: taxonomic and phylogenetic contributions to fungal taxa. Fungal Divers 78:1-237

Li JF, Bhat DJ, Phookamsak R, Mapook A, Lumyong S, Hyde KD (2016b) Sporidesmioides thailandica gen. et sp. nov. (Dothideomycetes) from northern Thailand. Mycol Prog 15:1169-1178

Li JF, Phookamsak R, Jeewon R, Bhat DJ, Mapook A, Camporesi E, Shang QJ, Chukeatirote E, Bahkali AH, Hyde KD (2017) Molecular taxonomy and morphological characterization reveal new species and new host records of Torula species (Torulaceae, Pleosporales). Mycol Prog 16:447-461

Li JF, Jeewon R, Mortimer PE, Doilom M, Phookamsak R, Promputtha I (2020a) Multigene phylogeny and taxonomy of Dendryphion hydei and Torula hydei spp. nov. from herbaceous litter in northern Thailand. PLoS ONE 15(2):e0228067

Li WJ, McKenzie EHC, Liu JK, Bhat DJ, Dai DQ, Camporesi E, Tian Q, Maharachchikumbura SSN, Luo ZL, Shang QJ, Zhang JF, Tangthirasunun N, Karunarathna SC, Xu JC, Hyde KD (2020b) Taxonomy and phylogeny of hyaline-spored coelomycetes. Fungal Divers 100:279-801

Lin CG, McKenzie EHC, Bhat DJ, Liu JK, Hyde KD, Lumyong S (2018) Pseudodactylaria brevis sp. nov. from Thailand confirms the status of Pseudodactylariaceae. Phytotaxa 369:241-250

Linnemann G (1941) Pflanzenforschung: Die Mucorineen-Gattung Mortierella Coemans, Heft 23. Gustav Fischer, Jena

Liu SL, He SH (2016) Phanerochaete porostereoides, a new species in the core clade with brown generative hyphae from China. Mycosphere 7:648-655

Liu JK, Phookamsak R, Doilom M, Wikee S, Li YM, Ariyawansha H, Boonmee S, Chomnunti P, Dai DQ, Bhat JD, Romero AI (2012) Towards a natural classification of Botryosphaeriales. Fungal Divers 57:149-210

Liu JK, Hyde KD, Jones EBG, Ariyawansa HA, Bhat DJ, Boonmee S, Maharachchikumbura SSN, McKenzie EHC, Phookamsak R, Phukhamsakda C, Shenoy BD, Abdel-Wahab MA, Buyck B, Chen J, Chethana KWT, Singtripop C, Dai DQ, Dai YC, Daranagama DA, Dissanayake AJ, Doilom M, D'souza MJ, Fan XL, Goonasekara ID, Hirayama K, Hongsanan S, Jayasiri SC, Jayawardena RS, Karunarathna SC, Li WJ, Mapook A, Norphanphoun C, Pang KL, Perera RH, Peršoh D, Pinruan U, Senanayake IC, Somrithipol S, Suetrong S, Tanaka K, Thambugala KM, Tian Q, Tibpromma S, Udayanga D, Wijayawardene NN, Wanasinghe D, Wisitrassameewong K, Zeng XY, Abdel-Aziz FA, Adamčík S, Bahkali AH, Boonyuen N, Bulgakov T, Callac P, Chomnunti P, Greiner K, Hashimoto A, Hofstetter V, Kang JC, Lewis D, Li XH, Liu XZ, Liu ZY, Matsumura M, Mortimer PE, Rambold G, Randrianjohany E, Sato G, SriIndrasutdhi V, Tian CM, Verbeken A, Brackel WV, Wang Y, Wen TC, Xu JC, Yan JY, Zhao RL,
Camporesi E (2015a) Fungal diversity notes 1-110: taxonomic and phylogenetic contributions to fungal species. Fungal Divers 72:1-197

Liu XY, Udayanga D, Luo ZL, Chen LJ, Zhou DQ, Su HY, Hyde KD (2015b) Backbone tree for Chaetothyriales with four new species of Minimelanolocus from aquatic habitats. Fungal Biol 119:1046-1062

Liu JK, Hyde KD, Jeewon R, Phillips AJ, Maharachchikumbura SS, Ryberg M, Liu ZY, Zhao Q (2017) Ranking higher taxa using divergence times: a case study in Dothideomycetes. Fungal Divers 84:75-99

Liu NG, Lin CG, Liu JK, Samarakoon MC, Hongsanan S, Bhat DJ, Hyde KD, McKenzie EHC, Jumpathong J (2018) Lentimurisporaceae, a new Pleosporalean family with divergence times estimates. Cryptog Mycol 39:259-283

Liu NG, Hyde KD, Bhat DJ, Jumpathong J, Liu JK (2019) Morphological and phylogenetic studies of Pleopunctum gen. nov. (Phaeoseptaceae, Pleosporales) from China. Mycosphere 10:757-775

Lloyd CG (1906) The Nidulariaceae or "bird's nest fungi". Cincinnati, pp 1-32

Locquin M (1984) Mycologie générale et structurale. Masson, Paris, pp 1-551

Lodge DJ, Padamsee M, Matheny PB, Aime MC, Cantrell SA, Boertmann D, Kovalenko A, Vizzini A, Dentinger BTM, Kirk PM, Ainsworth AM, Moncalvo JM, Vilgalys R, Larsson E, Lucking R, Griffith GW, Smith ME, Norvell LL, Desjardin DE, Redhead SA, Ovrebo CL, Lickey EB, Ercole E, Hughes KW, Courtecuisse R, Young A, Binder M, Minnis AM, Lindner DL, Ortiz-Santana B, Haight J, Lassoe T, Baroni TJ, Geml J, Hattori T (2014) Molecular phylogeny, morphology, pigment chemistry and ecology in Hygrophoraceae (Agaricales). Fungal Divers 64:1-99

Lombard L, Van der Merwe NA, Groenewald JZ, Crous PW (2015) Generic concepts in Nectriaceae. Stud Mycol 80:189-245

Lombard L, Sandoval-Denis M, Lamprecht SC, Crous PW (2019a) Epitypification of Fusarium oxysporum-clearing the taxonomic chaos. Persoonia 43:1-47

Lombard L, van Doorn R, Crous PW (2019b) Neotypification of Fusarium chlamydosporum - a reappraisal of a clinically important species complex. FUSE 4:183-200

Lolas MA, Castro A, Polanco R, Gainza-Cortés F, Ferrada E, Sosnowski MR, Díaz GA (2020) First report of Eutypa lata causing dieback of grapevines (Vitis vinifera) in Chile. Plant Dis 104:2024

Lotsy JP (1907) Vorträge über botanische Stammesgeschichte. Erster Band: Algen und Pike Gustav Fisher, Jena, pp 1-828

Lowen R (1991) A monograph of the genera Nectriella, nitschke and Pronectria clements with reference to Charonectria, Cryptonectriella, Hydronectria and Pseudonectria. Ph.D. Thesis, City University of New York

Lu YZ, Boonmee S, Bhat DJ, Hyde KD, Kang JC (2017) Helicosporium luteosporum sp. nov. and Acanthohelicospora aurea (Tubeufiaceae, Tubeufiales) from terrestrial habitats. Phytotaxa 319:241-253

Lu YZ, Boonmee S, Liu JK, Hyde KD, McKenzie EHC, Eungwanichayapant PD, Kang JC (2018a) Multi-gene phylogenetic analyses reveals Neohelicosporium gen. nov. and five new species of helicosporous hyphomycetes from aquatic habitats. Mycol Prog 17:631-646

Lu YZ, Liu JK, Hyde KD, Jeewon R, Kang JC, Fan C, Boonmee S, Bhat DJ, Luo ZL, Lin CG, Eungwanichayapant PD (2018b) A taxonomic reassessment of Tubeufiales based on multi-locus phylogeny and morphology. Fungal Divers 92:131-344

Lu YZ, Zhang JY, Lin CG, Luo ZL, Liu JKJ (2020) Pseudodactylaria fusiformis sp. nov. from freshwater habitat in China. Phytotaxa 446:95-102

Lücking R, Aime MC, Robbertse B, Miller AN, Aoki T, Ariyawansa HA, Cardinali G, Crous PW, Druzhinina IS, Geiser DM, 
Hawksworth DL, Hyde KD, Laszlo Irinyi L, Jeewon R, Johnston PR, Kirk PM, Elaine Malosso E, May TW, Meyer W, Nilsson HR, Öpik M, Robert V, Stadler M, Thines M, Vu D, Yurkov AM, Zhang N, Schoch CL (2021) Fungal taxonomy and sequencebased nomenclature. Nat Microbiol 6:540-548

Lumbsch HT, Huhndorf SM (2010) Myconet Volume 14. Part One. Outline of ascomycota-2009. Fieldiana Life Earth Sci 1:1-922

Luo X, Ye L, Chen J, Karunarathna SC, Xu J, Hyde KD, Mortimer PE (2016) Laccaria rubroalba sp. nov. (Hydnangiaceae, Agaricales) from Southwestern China. Phytotaxa 284:41-50

Luo ZL, Hyde KD, Bhat DJ, Jeewon R, Maharachchikumbura SSN, Bao DF, Li WL, Su XJ, Yang XY, Su HY (2018) Morphological and molecular taxonomy of novel species Pleurotheciaceae from freshwater habitats in Yunnan, China. Mycol Prog 17:511-530

Luo ZL, Hyde KD, Liu JK, Maharachchikumbura SSN, Jeewon R, Bao DF, Bhat DJ, Lin CG, Li WL, Yang J, Liu NG, Lu YZ, Jayawardena RS, Li JF, Su HY (2019) Freshwater sordariomycetes. Fungal Divers 99:451-660

Ma HX, Li Y (2018) Xylaria crinalis and X. betulicola from China-two new species with thread-like stromata. Sydowia 70:37-49

Ma X, Zhao CL (2019) Crepatura ellipsospora gen. et sp. nov. in Phanerochaetaceae (Polyporales, Basidiomycota) bearing a tuberculate hymenial surface. Mycol Prog 18:785-793

Ma R, Li SN, Zhao Y, Wang M, Michailides TJ, Tian CM (2020) New species of Nectria and Thyronectria from Xinjiang, China. Phytotaxa 433:253-264

Maharachchikumbura SSN, Hyde KD, Jones EBG, McKenzie EHC, Huang SK, AbdelWahab MA, Daranagama DA, Dayarathne M, D'souza MJ, Goonasekara ID, Hongsanan S, Jayawardena RS, Kirk PM, Konta S, Liu J-K, Liu ZY, Norphanphoun C, Pang KL, Perera RH, Senanayake IC, Shang QJ, Shenoy BD, Xiao YP, Bahkali AH, Kang JC, Somrothipol S, Suetrong S, Wen TC, Xu JC (2015) Towards a natural classification and backbone tree for Sodariomycetes. Fungal Divers 72:199-301

Maharachchikumbura SSN, Hyde KD, Jones EBG, McKenzie EHC, Bhat JD, Dayarathne MC, Huang SK, Norphanphoun C, Senanayake IC, Perera RH, Shang QJ, Xiao Y, D'souza MJ, Hongsanan S, Jayawardena RS, Daranagama DA, Konta S, Goonasekara ID, Zhuang WY, Jeewon R, Phillips AJL, Abdel-Wahab MA, AlSadi AM, Bahkali AH, Boonmee S, Boonyuen N, Cheewangkoon R, Dissanayake AJ, Kang J, Li QR, Liu JK, Liu XZ, Liu ZY, Luangsa-ard JJ, Pang KL, Phookamsak R, Promputtha I, Suetrong S, Stadler M, Wen T, Wijayawardene NN (2016) Families of sordariomycetes. Fungal Divers 79:1-317

Majewski T (1994) The Laboulbeniales of Poland. Polish Bot Stud 7:3-466

Malençon G (1979) Nouvelles contributions a la flore mycologique du Maroc-I. Bull Trimest Soc Mycol Fr 95:89-118

Manimohan P, Leelavathy KM (1989) Some agarics new to India. Sydowia 41:200-208

Mapook A, Hyde KD, McKenzie EHC, Jones EBG, Bhat DJ, Jeewon R, Stadler M, Samarakoon MC, Malaithong M, Tanunchai B, Buscot F, Wubet T, Purahong W (2020) Taxonomic and phylogenetic contributions to fungi associated with the invasive weed Chromolaena odorata (Siam weed). Fungal Divers 101:1-175

Mares D, Andreotti E, Maldonado ME, Pedrini P, Colalongo C, Romagnoli C (2008) Three new species of Aspergillus from Amazonian forest soil (Ecuador). Curr Microbiol 57:222-229

Maryani N, Lombard L, Poerba YS, Subandiya S, Crous PW (2019) Phylogeny and genetic diversity of the banana Fusarium wilt pathogen Fusarium oxysporum f. sp. cubense in the Indonesian centre of origin. Stud Mycol 92:155-194

Marín-Félix Y, Hernández-Restrepo M, Iturrieta-González I, García D, Gené J, Groenewald JZ, Cai L, Chen Q, Quaedvlieg W, Schumacher RK, Taylor PWJ, Ambers C, Bonthond B, Edwards J, Krueger-Hadfield SA, Luangsa-ard JJ, Morton L, Moslemi A,
Sandoval-Denis M, Tan YP, Thangavel R, Vaghefi N, Cheewangkoon R, Taylor PWJ (2019a) Genera of phytopathogenic fungi: GOPHY 3. Stud Mycol 94:1-124

Marín-Félix Y, Hernández-Restrepo M, Wingfield MJ, Akulov A, Carnegie AJ, Cheewangkoon R, Gramaje D, Groenewald JZ, Guarnaccia V, Halleen F, Lombard L, Luangsa-ard J, Marincowitz S, Moslemi A, Mostert L, Quaedvlieg W, Schumacher RK, Spies CFJ, Thangavel R, Taylor PWJ, Wilson AM, Wingfield BD, Wood AR, Crous PW (2019b) Genera of phytopathogenic fungi: GOPHY 2. Stud Mycol 92:47-133

Matheny PB, Curtis JM, Hofstetter V, Aime MC, Moncalvo J-M, Ge Z-W, Yang Z, Slot JC, Ammirati JF, Baroni TJ, Bougher NL, Hughes KW, Lodge DJ, Kerrigan RW, Seidl MT, Aanen DK, DeNotis M, Danielle GM, Desjardin DE, Kropp BR, Norvell LL, Parker A, Vellinga EC, Vilgalys R, Hibbet DS (2006) Major clades of Agaricales: a multilocus phylogenetic overview. Mycologia 98:982-995

Matic S, Tabone G, Guarnaccia V, Gullino ML, Garibaldi A (2020) Emerging leafy vegetable crop diseases caused by the Fusarium incarnatum-equiseti species complex. Phytopathol Medit 59:303-317

Matsushima T (1996) Matsushima mycological memoirs 9. Matsush Mycol Mem 9:1-30

Miadlikowska J, Kauff F, Högnabba F, Oliver JC, Molnár K, Fraker E, Gaya E, Hafellner J, Hofstetter V, Gueidan C, Otálora MAG, Hodkinson B, Kukwa M, Lücking R, Björk C, Sipman HJM, Burgaz AR, Thell A, Passo A, Myllys L, Goward T, FernándezBrime S, Hestmark G, Lendemer J, Lumbsch HT, Schmull M, Schoch CL, Sérusiaux E, Maddison DR, Arnold AE, Lutzoni F, Stenroos S (2014) A multigene phylogenetic synthesis for the class Lecanoromycetes (Ascomycota): 1307 fungi representing 1139 infrageneric taxa, 317 genera and 66 families. Mol Phylogenet Evol 79:132-168

Miettinen O, Spirin V, Vlasák J, Rivoire B, Stenroos S, Hibbett DS (2016) Polypores and genus concepts in Phanerochaetaceae (Polyporales, Basidiomycota). MycoKeys 17:1-46

Miller JH (1961) A monograph of the world species of Hypoxylon. University of Georgia Press, Athens, p 158

Miller OK Jr, Miller HH (1988) Gasteromycetes: morphological and development features with keys to the orders, families, and genera. Mad River Press, Eureka

Miller AN, Huhndorf SM (2004) A natural classification of Lasiosphaeria based on nuclear LSU rDNA sequences. Mycol Res 108:26-34

Miller AN, Huhndorf SM (2005) Multi-gene phylogenies indicate ascomal wall morphology is a better predictor of phylogenetic relationships than ascospore morphology in the Sordariales (Ascomycota, Fungi). Mol Phylogenet Evol 35:60-75

Mirza F (1968) Taxonomic investigations on the ascomycetous genus Cucurbitaria S.F. Gray Nova Hedwig 16:161-213

Moncalvo JM, Vilgalys R, Redhead SA, Johnson JE, James TY, Aime MC, Hofstetter V, Verduin SJ, Larsson E, Baroni TJ, Thorn RG, Jacobssong S, Clémençon H, Miller OK Jr (2002) One hundred and seventeen clades of euagarics. Mol Phylogenet Evol 23:357-400

Montagne JFC (1834) Notice sur les plantes cryptogames récemment dé- couvertes en France contenant aussi l'indication précis des localités de quelques espèces les plus rares de la flore française. Ann Sci Nat Bot 2:295-307

Monteiro JS, Leão-Ferreira SM, Gusmão LF, Kirk PM, CastañedaRuiz RF (2017) Distoceratosporella digitiformis gen. \& sp. nov. from Brazil, Alcornia sessilispora gen. \& comb. nov., and three new Distoceratosporella combinations. Mycotaxon 132:485-493

Moore RT (1996) An inventory of the phylum Ustomycota. Mycotaxon 59:1-31 
Mori Y, Sato Y, Takamatsu S (2000) Evolutionary analysis of the powdery mildew fungi using nucleotide sequences of the nuclear ribosomal DNA. Mycologia 92:74-93

Mortimer PE, Jeewon R, Xu J-C, Lumyong S, Wanasinghe DN (2021) Morpho-phylo taxonomy of novel dothideomycetous fungi associated with dead woody twigs in Yunnan Province, China. Front Microbiol 12:654683

Mossebo DC, Akoa A, Eteme RA (2000) Macrolepiota globosa et Nothopanus nsimalenensis, deux nouvelles espèces fongiques du Cameroun. Mycotaxon 76:267-278

Moyo P, Mostert L, Spies CF, Damm U, Halleen F (2018) Diatrypaceae species associated with dieback of grapevines in South Africa, with the description of Eutypa cremea sp. nov. Plant Dis 102:220-230

Mueller GM (1992) Systematics of Laccaria (Agaricales) in the continental United States and Canada, with discussions on extralimital taxa and descriptions of extant types. Fieldiana Bot Ns 30:1-158

Mulenko W, Majewski T, Ruszkiewicz-Michalska M (2008) A preliminary checklist of Micromycetes in Poland, vol 9. W. Szafer Institute of Botany, Polish Academy of Sciences, Kraków, p 752

Müller E (1958) Über zwei neue Ascomyceten auf Juniperus-Arten. Sydowia 12:189-196

Müller E (1977) Zur Pilzflora der Aletschwaldreservats (Kt Wallis, Schweiz). Beitr Kryptogamenfl Schweiz 15:1-126

Müller E, von Arx JA (1962) Die Gattungen der didymosporen Pyrenomyceten. Beitr KryptFl Schweiz 11:1-922

Müller E, Petrini O, Fisher PJ, Samuels GJ, Rossman AY (1987) Taxonomy and anamorphs of the Herpotrichiellaceae with notes on generic synonymy. Trans Br Mycol Soc 88:63-74

Munk A (1953) The system of the Pyrenomycetes. Dansk Bot Ark $15: 1-163$

Munk A (1957) Danish Pyrenomycetes. A preliminary flora. Dansk Bot Ark 17:1-491

Nagy LG, Walther G, Hazi J, Vágvölgyi C, Papp T (2011) Understanding the evolutionary processes of fungal fruiting bodies: correlated evolution and divergence times in the Psathyrellaceae. Syst Biol 60:303-317

Nagy LG, Hazi J, Vágvölgyi C, Papp T (2012) Phylogeny and species delimitation in the genus Coprinellus with special emphasis on the haired species. Mycologia 104:254-275

Nagy LG, Vágvölgyi C, Papp T (2013) Morphological characterization of clades of the Psathyrellaceae (Agaricales) inferred from a multigene phylogeny. Mycol Prog 12:505-517

Nakasone KK, Draeger KR, Ortiz-Santana B (2017) A contribution to the taxonomy of Rhizochaete (Polyporales, Basidiomycota). Cryptog Mycol 38:81-99

Nannfeldt JA (1932) Studien über die Morphologie und Systematik der nichtlichenisierten inoperculaten Discomyceten. Nova Acta R Soc Scient Upsal 8:1-368

Nascimento MMF, Vicente VA, Bittencourt JVM, Gelinski JML, Prenafeta-Boldú FX, Romero-Güiza M, Fornari G, Gomes RR, Santos GD, Gerrits Van Den Ende AHG, de Azevedo CDMPS, De Hoog GS (2017) Diversity of opportunistic black fungi on babassu coconut shells, a rich source of esters and hydrocarbons. Fungal Biol 121:488-500

Natarajan K, Manjula B (1982) South Indian Agaricales-XVIII-Marasmius. Kavaka 10:13-28

Nawawi A, Kuthubutheen AJ (1989) Canalisporium, a new genus of lignicolous hyphomycetes from Malaysia. Mycotaxon $34: 475-487$

Nei M, Kumar S (2000) Molecular evolution and phylogenetics. Oxford University Press, New York

Nguyen TTT, Park SW, Pangging M, Lee HB (2019) Molecular and morphological confirmation of three undescribed species of Mortierella from Korea. Mycobiology 47:31-39
Nilsson RH, Hallenbeg N, Nordén B, Maekawa N, Wu SH (2003) Phylogeography of Hyphoderma setigerum (Basidiomycota) in the Northern Hemisphere. Mycol Res 107:645-652

Niranjan M, Sarma VV (2018) Twelve new species of Ascomycetous fungi from Andaman Islands. Kavaka 50:84-97

Niranjan M, Sarma VV (2020) Diatrype: new records from Andaman Islands and a checklist from India. MycoAsia 2020/02

Niranjan M, Tiwari S, Baghela A, Sarma VV (2018) New records of Ascomycetous fungi from Andaman Islands, India and their molecular sequence data. Curr Res Environ Appl Mycol $8: 331-350$

Nitschke TRJ (1869) Grundlage eines Systems der Pyrenomyceten. Verhandlungen des Naturhistorischen Vereins der Preussischen Rheinlande. Westfalens Und Des Regierungsbezirks Osnabrück 26:70-77

Nitschke T (1870) Pyrenomycetes germanici: die Kernpilze Deutschlands 2:161-320

Nout MJR, Aidoo KE (2010) Asian fungal fermented food. In: Hofrichter M (ed) Industrial applications. The Mycota, vol 10, 2nd edn. Springer, Berlin, pp 30-58

Nuankaew S, Suetrong S, Wutikhun T, Pinruan U (2019) Hermatomyces trangensis $\mathrm{sp}$. nov., a new dematiaceous hyphomycete (Hermatomycetaceae, Pleosporales) on sugar palm in Thailand. Phytotaxa 391:277-288

Nunez M, Ryvarden L (1995) Polypores new to Japan 1. Species of Polyporus, with a note on $P$. hartmanni. Mycoscience 36:61-65

Nylander JAA (2009) MrModeltest 2.3. Program distributed by the author. Evolutionary Biology Centre, Uppsala University

Obase K, Douhan GW, Matsuda Y, Smith ME (2016) Cladophialophora floridana and Cladophialophora tortuosa, new species isolated from sclerotia of Cenococcum geophilum in forest soils of Florida, USA. Mycoscience 57:26-34

O'Donnell K, Sutton DA, Rinaldi MG, Gueidan C, Crous PW, Geiser DM (2009) Novel multilocus sequence typing scheme reveals high genetic diversity of human pathogenic members of the Fusarium incarnatum- $F$. equiseti and $F$. chlamydosporum species complexes within the United States. J Clin Microbiol 47:3851-3861

Olariaga I, Teres J, Martín J, Prieto M, Baral HO (2019) Pseudosclerococcum golindoi gen. et sp. nov., a new taxon with apothecial ascomata and a Chalara-like anamorph within the Sclerococcales (Eurotiomycetes). Mycol Prog 18:895-905

Osmundson TW, Cripps CL, Mueller GM (2005) Morphological and molecular systematics of Rocky Mountain alpine Laccaria. Mycologia 97:949-972

Örstadius L, Ryberg M, Larsson E (2015) Molecular phylogenetics and taxonomy in Psathyrellaceae (Agaricales) with focus on psathyrelloid species: introduction of three new genera and 18 new species. Mycol Prog 14:25

Padamsee M, Matheny PB, Dentinger BT, McLaughlin DJ (2008) The mushroom family Psathyrellaceae: evidence for largescale phylogeny of the genus Psathyrella. Mol Phylogenet Evol 46:415-429

Pang KL, Abdel-Wahab MA, Sivichai S, El-Sharouney HM, Jones EB (2002) Jahnulales (Dothideomycetes, Ascomycota): a new order of lignicolous freshwater ascomycetes. Mycol Res 106:1031-1042

Pang KL, Hyde KD, Alias SA, Suetrong S, Jones EBG (2013) Dyfrolomycetaceae, a new family in the Dothideomycetes, Ascomycota. Cryptog Mycol 34:223-232

Pang KL, Guo SY, Alias SA, Hafellner J, Jones EBG (2014) A new species of marine Dactylospora and its phylogenetic affinities within the Eurotiomycetes, Ascomycota. Bot Mar 57:315-321

Park MS, Fong JJ, Lee H, Oh SY, Jung PE, Min YJ, Seok SJ, Lim YW (2013) Delimitation of Russula subgenus Amoenula in Korea using three molecular markers. Mycobiology 41:191-201 
Parmasto E (1968) Conspectus systematis Corticiacearum. Institute of Zoology and Botany, Estonian Academic of Sciences, Tartu

Patil MS (1978) Some fleshy fungi from Maharashtra-III. Indian Phytopathol 31:32-35

Pegler DN (1983) Agaric flora of the Lesser Antilles. Kew Bull Adit Ser 9. HMSO, London

Pegler DN (1986) Agaric flora of Sri Lanka. Kew Bull Adit Ser 12. HMSO, London

Peintner U, Bougher NL, Castellano MA, Moncalvo JM, Moser MM, Trappe JM, Vilgalys R (2001) Multiple origins of sequestrate fungi related to Cortinarius (Cortinariaceae). Am J Bot 88:2168-2179

Peintner U, Horak E, Moser M, Vilgalys R (2002) Phylogeny of Rozites, Cuphocybe and Rapacea inferred from ITS and LSU rDNA sequences. Mycologia 94:620-629

Pem D, Hongsanan S, Doilom M, Tibpromma S, Wanasinghe DN, Dong W, Ningguo L, Phookamsak R, Phillips AJL, Jeewon R, Hyde KD (2019a) An online taxonomic resource for the classification, identification, and nomenclature of Dothideomycetes. Asian J Mycol 2:287-297

Pem D, Jeewon R, Bhat DJ, Doilom M, Boonmee S, Hongsanan S, Promputtha I, Xu JC, Hyde KD (2019b) Mycosphere notes 275-324: A morpho-taxonomic revision and typification of obscure Dothideomycetes genera (incertae sedis). Mycosphere 10:1115-1246

Persoon CH (1794) Neuer Versuch einer systematischen Einteilung der Schwämme. Neues Mag Bot 1:63-128

Persoon CH (1795) Observationes mycologicae. Ann Bot 15:1-39

Persoon CH (1801) Sypnosis Mehtodica Fungorum. Göttingen: apud Henricum Dieterich

Petch T (1910) Revisions of ceylon fungi 2. Ann Roy Bot Gard Peradeniya 4:373-444

Petch T (1938) British Hypocreales. Trans Br Mycol Soc 21:243-301

Petch T (1947) A revision of ceylon Marasmii. Trans Br Mycol Soc 31:19-44

Petersen RH, Hughes K, Lickey EB, Kovalenko AE, Morozova OV, Psurtseva NV (2008) A new genus, Cruentomycena, with Mycena viscidocruenta as type species. Mycotaxon 105:119-136

Petrak F (1921) Mykologische Notizen. II. Annls Mycol 19:17-128

Petrini LE (2013) Rosellinia-a World Monograph. Bibl Mycol 205:1-410

Pham MT, Huang CM, Kirschner R (2019) First report of the oil palm disease fungus Marasmius palmivorus from Taiwan causing stem rot disease on native Formosa palm Arenga engleri as new host. Lett Appl Microbiol 70:143-150

Phillips AJL (2003) Morphological characterization of Diaporthe foeniculacea and related species on Foeniculum vulgaris. Sydowia 54:274-285

Phillips AJL, Lopes J, Abdollahzadeh J, Bobev S, Alves A (2012) Resolving the Diplodia complex on apple and other Rosaceae hosts. Persoonia 29:29-38

Phillips AJL, Alves A, Abdollahzadeh J, Slippers B, Wingfield MJ, Groenewald JZ, Crous PW (2013) The Botryosphaeriaceae: genera and species known from culture. Stud Mycol 76:51-167

Phillips AJL, Hyde KD, Alves A, Liu JK (2019) Families in Botryosphaeriales: a phylogenetic, morphological and evolutionary perspective. Fungal Divers 94:1-22

Phookamsak R, Liu JK, McKenzie EHC, Manamgoda DS, Ariyawansa HA, Thambugala KM, Dai DQ, Camporesi E, Chukeatirote E, Wijayawardene NN, Bahkali AH, Mortimer PE, Xu JC, Hyde KD (2014) Revision of Phaeosphaeriaceae. Fungal Divers 68:159-238

Phookamsak R, Manamgoda DS, Li WJ, Dai DQ, Singtripop C, Hyde KD (2015) Poaceascoma helicoides gen et sp. nov., a new genus with scolecospores in Lentitheciaceae. Cryptog Mycol $36: 225-236$
Phookamsak R, Phukhamsakda C, Konta S, Jayasiri SC, Norphanphoun C, Tennakoon DS, Li JF, Dayarathne MC, Perera RH, Xiao YP, Wanasinghe DN, Senanayake IC, Goonasekara ID, de Silva NI, Mapook A, Jayawardena RS, Dissanayake AJ, Manawasinghe IS, Chethana KWT, Luo ZL, Hapuarachchi KK, Baghela A, Soares AM, Vizzini A, Meiras-Ottoni A, Mešić A, Dutta AK, de Souza CAF, Richter C, Lin CG, Chakrabarty D, Daranagama DA, Lima DX, Chakraborty D, Ercole E, Wu F, Simonini G, Vasquez G, da Silva GA, PlautzJr HL, Ariyawansa HA, Lee H, Kušan I, Song J, Sun JZ, Karmakar J, Hu KF, Semwal KC, Thambugala KM, Voigt K, Acharya K, Rajeshkumar KC, Ryvarden L, Jadan M, Hosen MI, Mikšk M, Samarakoon MC, Wijayawardene NN, Kim NK, Matočec N, Singh PN, Tian Q, Bhatt RP, de Oliveira RJV, Tulloss RE, Aamir S, Kaewchai S, Marathe SD, Khan S, Hongsanan S, Adhikari S, Mehmood T, Bandyopadhyay TK, Svetasheva TY, Nguyen TTT, Antonn V, Li WJ, Wang Y, Indoliya Y, Tkalčec Z, Elgorban AM, Bahkali AH, Tang AMC, Su HY, Zhang H, Promputtha I, Luangsa-Ard J, Xu JC, Yan JY, Kang JC, Stadler M, Mortimer PE, Chomnunti P, Zhao Q, Phillips AJL, Nontachaiyapoom S, Wen TC, Karunarathna SC (2017a) Fungal diversity notes 491-602: taxonomic and phylogenetic contributions to fungal taxa. Fungal Divers 83:1-261

Phookamsak R, Wanasinghe DN, Hongsanan S, Phukhamsakda C, Huang SK, Tennakoon DS, Norphanphoun C, Camporesi E, Bulgakov TS, Promputa I, Mortimer PE, Xu JC (2017b) Towards a natural classification of Ophiobolus and ophiobolus-like taxa; introducing three novel genera Ophiobolopsis, Paraophiobolus and Pseudoophiobolus in Phaeosphaeriaceae (Pleosporales). Fungal Divers 87:299-339

Phookamsak R, Hyde KD, Jeewon R, Bhat DJ, Jones EBG, Maharachchikumbura SSN, Raspé O, Karunarathna SC, Wanasinghe DN, Hongsanan S, Doilom M, Tennakoon DS, Machado AR, Firmino AL, Ghosh A, Karunarathna A, Mešić A, Dutta AK, Thongbai B, Devadatha B, Norphanphoun C, Senwanna C, Wei D, Pem D, Ackah FK, Wang GN, Jiang HB, Madrid H, Lee HB, Goonasekara ID, Manawasinghe IS, Kušan I, Josep Cano J, Gené J, Li JF, Das K, Acharya K, Raj KNA, Latha KPD, Thilini Chethana KWT, He MQ, Dueñas M, Jadan M, Martín MP, Samarakoon MC, Dayarathne MC, Raza M, Park MS, Telleria MT, Chaiwan N, Matočec N, de Silva NI, Pereira OL, Singh PN, Manimohan P, Uniyal P, Shang QJ, Bhatt RP, Perera RH, Alvarenga RLM, Nogal-Prata S, Singh SK, Vadthanarat S, Oh SY, Huang SK, Rana S, Konta S, Paloi S, Jayasiri SC, Jeon SJ, Mehmood T, Gibertoni TB, Nguyen TTT, Singh U, Thiyagaraja V, Sarma VV, Dong W, Yu XD, Lu YZ, Lim YW, Chen Y, Tkalčec Z, Zhang ZF, Luo ZL, Daranagama DA, Thambugala KM, Tibpromma S, Camporesi E, Bulgakov TS, Dissanayake AJ, Senanayake IC, Dai DQ, Tang LZ, Khan S, Zhang H, Promputtha I, Cai L, Chomnunti P, Zhao RL, Lumyong S, Boonmee S, Wen TC, Mortimer PE, Xu JC (2019) Fungal diversity notes 929-1035: taxonomic and phylogenetic contributions on genera and species of fungi. Fungal Divers 95:1-273

Phukhamsakda C, Hongsanan S, Ryberg M, Ariyawansa HA, Chomnunti P, Bahkali AH, Hyde KD (2016) The evolution of Massarineae with Longipedicellataceae fam. nov. Mycosphere 7:1713-1731

Phukhamsakda C, McKenzie EHC, Phillips AJL, Jones EBG, Bhat DJ, Stadler M, Bhunjun CS, Wanasinghe DN, Thongbai B, Camporesi E, Ertz D, Jayawardena RS, Perera RH, Ekanayake AH, Tibpromma S, Doilom M, Xu JC, Hyde KD (2020) Microfungi associated with Clematis (Ranunculaceae) with an integrated approach to delimiting species boundaries. Fungal Divers 102:1-203

Piątek M, Lutz M, Ronikier A, Kemler M, Swiderska-Burek U (2012) Microbotryum heliospermae, a new anther smut fungus parasitic 
on Heliosperma pusillum in the mountains of the European Alpine System. Fungal Biol 116:185-195

Piątek M, Lutz M, Kemler M (2013) Microbotryum silenes-saxifragae sp. nov. sporulating in the anthers of Silene saxifraga in southern European mountains. IMA Fungus 4:29-40

Pie M, Boeger W, Patella L, Vicente VA, Orélis-Ribeiro R, Ostrensky A (2011) Specific primers for the detection of the black-yeast fungus associated with lethargic crab disease (LCD). Dis Aquat Org 94:73-75

Pielech R, Malicki M, Smoczyk M, Jagodziński AM, Dyderski MK, Horodecki P, Wierzcholska S, Skorupski M, Kamczyc J, Kałucka IL, Hachułka M, Gazda A (2018) Plant communities of the Czerwona Woda River Valley (Stołowe Mountains National Park). For Res Pap 79:181-197

Pinnoi A, Lumyong S, Hyde KD, Jones EBG (2006) Biodiversity of fungi on the palm Eleiodoxa conferta in Sirindhorn peat swamp forest, Narathiwat, Thailand. Fungal Divers 22:205-218

Pino-Bodas R, Zhurbenko MP, Stenroos S (2017) Phylogenetic placement within Lecanoromycetes of lichenicolous fungi associated with Cladonia and some other genera. Persoonia 39:91-117

Pinruan U, Hyde KD, Lumyong S, McKenzie EHC, Jones EBG (2007) Occurrence of fungi on tissues of the peat swamp palm Licuala longicalycata. Fungal Divers 25:157-173

Poli A, Bovio E, Verkley G, Prigione V, Varese GC (2019) Elbamycella rosea gen. et sp. nov. (Juncigenaceae, Torpedosporales) isolated from the Mediterranean Sea. MycoKeys 55:15-28

Popa F, Rexer KH, Donges K, Yang ZL, Kost G (2014) Three new Laccaria species from Southwest China Yunnan. Mycol Prog 13:1105-1117

Posada D, Crandall KA (1998) MODELTEST: testing the model of DNA substitution. Bioinformatics 14:817-818

Prabhugaonkar A, Bhat DJ (2011) New record of Megacapitula villosa and Paradictyoarthrinium diffractum from India. Mycosphere 2:463-467

Proaño Castro AC, Rossi W (2008) New records of Laboulbeniales (Fungi, Ascomycota) from Ecuador. Biodiversity of South America. I Memoirs on Biodiversity 1:11-18

Punithalingam E (1979) Sphaeropsidales in culture from humans. Nova Hedwig 31:119-158

Purushothama KB, Natarajan K (1987) Amanita flavofloccosa-an addition to Indian agaric flora. Curr Sci 56:1073-1074

Quaedvlieg W, Verkley GJM, Shin H-D, Barretto RW, Alfenas AC, Swart WJ, Groenewald JZ, Crous PW (2013) Sizing up Septoria. Stud Mycol 75:307-390

Raja HA, Ferrer A, Shearer CA (2005) Aliquandostipite crystallinus, a new ascomycete species from wood submerged in freshwater habitats. Mycotaxon 91:207-215

Raja HA, Schmit J, Shearer C (2009) Latitudinal, habitat and substrate distribution patterns of freshwater ascomycetes in the Florida Peninsula. Biodivers Conserv 18:419-455

Raja HA, Ferrer A, Miller AN, Shearer CA (2010) Freshwater Ascomycetes: Wicklowia aquatica, a new genus and species in the Pleosporales from Florida and Costa Rica. Mycoscience 51:208-214

Raja HA, Oberlies NH, Figueroa M, Tanaka K, Hirayama K, Hashimoto A, Miller AN, Zelski SE, Shearer CA (2013) Freshwater ascomycetes: Minutisphaera (Dothideomycetes) revisited, including one new species from Japan. Mycologia 105:959-976

Raja HA, El-Elimat T, Oberlies NH, Shearer CA, Miller AN, Tanaka K, Hashimoto A, Fournier J (2015) Minutisphaerales (Dothideomycetes, Ascomycota): a new order of freshwater ascomycetes including a new family, Minutisphaeraceae, and two new species from North Carolina, USA. Mycologia 107:845-862

Ramos A, Bandala VM, Montoya L (2017) A new species and a new record of Laccaria (Fungi, Basidiomycota) found in a relict forest of the endangered Fagus grandifolia var. mexicana. MycoKeys 27:77-94

Raper KB, Thom C (1944) New aspergilli from soil. Mycologia 36:555-575

Rathnayaka AR, Dayarathne MC, Maharachchikumbura SSN, Liu JK, Tennakoon DS, Hyde KD (2019) Introducing Seriascoma yunnanense sp. nov. (Occultibambusaceae, Pleosporales) based on evidence from morphology and phylogeny. Asian J Mycol $2: 245-253$

Rathnayaka AR, Wanasinghe DN, Dayarathne MC, Chethana KWT, Bhat DJ, Kuo CH, Mortimer PE, Lumyong S, Hyde KD (2020) Hyaloterminalis, a novel genus of Coryneaceae in order Diaporthales. Phytotaxa 474:132-144

Réblová M, Hernández-Restrepo M, Fournier J, Nekvindová J (2020) New insights into the systematics of Bactrodesmium and its allies and introducing new genera, species and morphological patterns in the Pleurotheciales and Savoryellales (Sordariomycetes). Stud Mycol 95:415-466

Réblová M, Seifert KA, Fournier J, Štěpánek V (2012) Phylogenetic classification of Pleurothecium and Pleurotheciella gen. nov. and its dactylaria-like anamorph (Sordariomycetes) based on nuclear ribosomal and protein-coding genes. Mycologia 104:1299-1314

Réblová M, Untereiner WA, Réblová K (2013) Novel evolutionary lineages revealed in the Chaetothyriales (Fungi) based on multigene phylogenetic analyses and comparison of its secondary structure. PLoS ONE 8:e63547

Réblová M, Seifert KA, Fournier J, Štěpánek V (2016) Newly recognized lineages of perithecial ascomycetes: the new orders Conioscyphales and Pleurotheciales. Persoonia 37:57-81

Réblová M, Untereiner WA, Stepanek V, Gams W (2017) Disentangling Phialophora section Catenulatae: disposition of taxa with pigmented conidiophores and recognition of a new subclass, Sclerococcomycetidae (Eurotiomycetes). Mycol Prog 16:27-46

Redhead SA, Vilgalys R, Moncalvo JM, Johnson J, Hopple JS Jr (2001) Coprinus Pers. and the disposition of Coprinus species sensu lato. Taxon 50:203-241

Redhead SA, Vizzini A, Drehmel DC, Contu M (2016) Saproamanita, a new name for both Lepidella E.J. Gilbert and Aspidella E.J. Gilbert (Amaniteae, Amanitaceae). IMA Fungus 7:119-129

Reid DA, Eicker A (1991) South African fungi: The genus Amanita. Mycol Res 95:80-95

Robert V, Stegehuis G, Stalpers J (2005) The MycoBank engine and related databases. http://www.mycobank.org

Rodway L (1897) Tasmanian fungi. Paper presented at the papers \& Proceedings of the Royal Society of Tasmania

Rogers JD (1983) Xylaria bulbosa, Xylaria curta, and Xylaria longipes in continental United States. Mycologia 75:457-467

Rolshausen PE, Trouillas F, Gubler WD (2004) Identification of Eutypa lata by PCR-RFLP. Plant Dis 88:925-929

Rolshausen PE, Greve LC, Labavitch JM, Mahoney NE, Molyneux RJ, Gubler WD (2008) Pathogenesis of Eutypa lata in grapevine: identification of virulence factors and biochemical characterization of cordon dieback. Phytopathology 98:222-229

Ronquist F, Teslenko M, Van der Mark P, Ayres DL, Darling A, Höhna S, Larget B, Liu L, Suchard MA, Huelsenbeck JP (2012) MrBayes 3.2: efficient Bayesian phylogenetic inference and model choice across a large model space. Syst Biol 61:539-542

Rossi W (2010) New Laboulbeniales (Ascomycota) parasitic on Staphylinidae from Ecuador. Mycol Prog 9:407-415

Rossi W, Kotrba M (2004) A new polymorphic species of Laboulbenia parasitic on a South American fly. Mycol Res 108:1315-1319

Rossi W, Kirk-Spriggs AH (2011) A new species of Laboulbenia (Ascomycota) parasitic on an African fly (Diptera: Curtonotidae), with a brief review of Diptera-associated species of the genus. Afr Invertebr 52:211-216 
Rossi W, Santamaria S (2012) Rodaucea, a new genus of the Laboulbeniales. Mycologia 104:785-788

Rossi W, Bernardi M (2018) New species and new records of Laboulbeniales (Ascomycota) from Thailand. Mycosphere 9:1151-1172

Rossman AY, Samuels GJ, Rogerson CT, Lowen R (1999) Genera of Bionectriaceae, Hypocreaceae and Nectriaceae (Hypocreales, Ascomycetes). Stud Mycol 42:1-248

Rossman AY, Adams GC, Cannon PF, Castlebury LA, Crous PW, Gryzenhout M, Jaklitsch WM, Mejia LC, Stoykov D, Udayanga D, Voglmayr H, Walker DM (2015) Recommendations of generic names in Diaporthales competing for protection or use. IMA Fungus 6:145-154

Saccardo PA (1875) Nova ascomycetum genera. Grevillea 4:21-22

Saccardo PA (1880) Conspectus generum fungorum Italiae inferiorum nempe ad Sphaeropsideas, Melanconieas et Hyphomyceteas pertinentium systemate sporologico dispositorum. Michelia 2:1-38

Saccardo PA (1883) Sylloge Pyrenomycetum. Syll Fung 2:1-813

Saccardo PA (1887) Sylloge Fungorum V:535

Saccardo PA (1913) Notae Mycologicae Series XVII. Ann Mycol 11:546-568

Sádlíková M, Kout J (2017) A new Phanerochaete (Polyporales, Basidiomycota) with brown subicular hyphae from Thailand. Mycosphere 8:1024-1030

Samarakoon BC, Phookamsak R, Wanasinghe DN, Chomnunti P, Hyde KD, McKenzie EHC, Promputtha I, Xu JC, Li YJ (2020) Taxonomy and phylogenetic appraisal of Spegazzinia musae sp. nov. and S. deightonii (Didymosphaeriaceae, Pleosporales) on Musaceae from Thailand. MycoKeys 70:19-37

Santamaria S (2003) Laboulbeniales, II. Acompsomyces-Iliomyces. Flora Mycol Iberica 5:1-344

Santamaria S (2006) New or interesting Laboulbeniales (Fungi, Ascomycota) from Spain, V. Nova Hedwig 82:349-363

Santamaria S, Cuesta-Segura AD, Guardia L (2020) New and remarkable species of Laboulbeniales (Ascomycota) from Spain. Nova Hedwig 110:347-367

Santos L, Phillips AJL, Crous PW, Alves A (2017) Diaporthe species on Rosaceae with descriptions of $D$. pyracanthae sp. nov. and $D$. malorum sp. nov. Mycosphere 8:485-511

Sarnari M (1998) Monografia illustrate de genere Russula in Europa, Tomo Primo. AMB, Centro Studi Micologici, Trento

Schafer DJ (2010) Keys to sections of Parasola, Coprinellus, Coprinopsis and Coprinus in Britain. Field Mycol 11:44-51

Schipper MAA (1978) On certain species of Mucor with a key to all accepted species. Stud Mycol 17:1-52

Schoch CL, Shoemaker RA, Seifert KA, Hambleton S (2006) A multigene phylogeny of the Dothideomycetes using four nuclear loci. Mycologia 98:1041-1052

Schoch CL, Crous PW, Groenewald JZ, Boehm EW, Burgess TI, de Gruyter J, De Hoog GS, Dixon LJ, Grube M, Gueidan C, Harada Y, Hatakeyama S, Hirayama K, Hosoya T, Huhndorf SM, Hyde KD, Jones EB, Kohlmeyer J, Kruys A, Li YM, Lücking R, Lumbsch HT, Marvanová L, Mbatchou JS, McVay AH, Miller AN, Mugambi GK, Muggia L, Nelsen MP, Nelson P, Owensby CA, Phillips AJ, Phongpaichit S, Pointing SB, Pujade-Renaud V, Raja HA, Plata ER, Robbertse B, Ruibal C, Sakayaroj J, Sano T, Selbmann L, Shearer CA, Shirouzu T, Slippers B, Suetrong S, Tanaka K, Volkmann-Kohlmeyer B, Wingfield MJ, Wood AR, Woudenberg JH, Yonezawa H, Zhang Y, Spatafora JW (2009a) A class-wide phylogenetic assessment of Dothideomycetes. Stud Mycol 64:1-15

Schoch CL, Sung GH, López-Giráldez F, Townsend JP, Miadlikowska J, Hofstetter V, Robbertse B, Matheny PB, Kauff F, Wang Z, Gueidan C, Andrie RM, Trippe K, Ciufetti LM, Wynns A, Fraker E, Hodkinson BP, Bonito G, Groenewald JZ, Arzanlou M, Sybren De Hoog G, Crous PW, Hewitt D, Pfister DH, Peterson K, Gryzenhout M, Wingfield MJ, Aptroot A, Suh SO, Blackwell
M, Hillis DM, Griffith GW, Castlebury LA, Rossman AY, Lumbsch HT, Lücking R, Büdel B, Rauhut A, Diederich P, Ertz D, Geiser DM, Hosaka K, Inderbitzin P, Kohlmeyer J, VolkmannKohlmeyer B, Mostert L, O'Donnell K, Sipman H, Rogers JD, Shoemaker RA, Sugiyama J, Summerbell RC, Untereiner W, Johnston PR, Stenroos S, Zuccaro A, Dyer PS, Crittenden PD, Cole MS, Hansen K, Trappe JM, Yahr R, Lutzoni F, Spatafora JW (2009b) The ascomycota tree of life: a phylum-wide phylogeny clarifies the origin and evolution of fundamental reproductive and ecological traits. Syst Biol 58:224-239

Schoknecht JD, Crane JL (1977) Revision of Torula and Hormiscium species. Torula occulta, T. diversa, T. elasticae, T. bigemina and Hormiscium condensatum reexamined. Mycologia 69:533-546

Seaver FJ (1909) The Hypocreales of North America. I. Mycologia $1: 41-76$

Seeler EV (1940) A monographic study of the genus Thyronectria. J Arnold Arbor 21:429-460

Séguy EA (1936) Code universel des couleurs. P Lechevalier, Paris

Senanayake IC, Al-Sadi AM, Bhat JD, Camporesi E, Dissanayake AJ, Lumyong S, Maharachchikumbura SSN, Hyde KD (2016) Phomatosporales ord. nov. and Phomatosporaceae fam. nov., to accommodate Lanspora, Phomatospora and Tenuimurus, gen. nov. Mycosphere 7:628-641

Senanayake IC, Maharachchikumbura SS, Hyde KD, Bhat JD, Jones EG, McKenzie EH, Dai DQ, Daranagama DA, Dayarathne MC, Goonasekara ID, Konta S (2015) Towards unraveling relationships in Xylariomycetidae (Sordariomycetes). Fungal Divers 73:73-144

Senanayake IC, Crous PW, Groenewald JZ, Maharachchikumbura SSN, Jeewon R, Phillips AJL, Bhat JD, Perera RH, Li QR, Li WJ, Tangthirasunun N, Norphanphoun C, Karunarathna SC, Camporesi E, Manawasighe IS, Al-Sadi AM, Hyde KD (2017a) Families of Diaporthales based on morphological and phylogenetic evidence. Stud Mycol 86:217-296

Senanayake IC, Maharachchikumbura SSN, Jeewon R, Promputtha I, Al-Sadi AM, Camporesi E, Hyde KD (2017b) Morphophylogenetic study of Sydowiellaceae reveals several new genera. Mycosphere 8:172-217

Senanayake IC, Jeewon R, Chomnunti P, Wanasinghe DN, Norphanphoun C, Karunarathna A, Pem D, Perera RH, Camporesi E, McKenzie EHC, Karunarathna SC (2018) Taxonomic circumscription of Diaporthales based on multigene phylogeny and morphology. Fungal Divers 93:241-443

Senanayake IC, Rathnayaka AR, Marasinghe DS, Calabon MS, Gentekaki E, Lee HB, Hurdeal VG, Pem D, Dissanayake LS, Wijesinghe SN, Bundhun D, Nguyen TT, Goonasekara ID, Abeywickrama PD, Bhunjun CS, Jayawardena RS, Wanasinghe DN, Jeewon R, Bhat DJ, Xiang MM (2020) Morphological approaches in studying fungi: collection, examination, isolation, sporulation and preservation. Mycosphere 11:2678-2754

Senthilarasu G (2014) Diversity of agarics (gilled mushrooms) of Maharashtra, India. Curr Res Environ Appl Mycol 4:58-78

Senwanna C, Hyde KD, Phookamsak R, Jones EG, Cheewangkoon $\mathrm{R}$ (2018) Coryneum heveanum sp. nov. (Coryneaceae, Diaporthales) on twigs of Para rubber in Thailand. MycoKeys 43:75-90

Shang QJ, Hyde KD, Jeewon R, Khan S, Promputtha I, Phookamsak R (2018) Morpho-molecular characterization of Peroneutypa (Diatrypaceae, Xylariales) with two novel species from Thailand. Phytotaxa 356:1-8

Shay JE, Desjardin DE, Perry BA, Grace CL, Newman DS (2017) Biodiversity and phylogeny of Marasmius (Agaricales, Basidiomycota) from Madagascar. Phytotaxa 292:101-149

Shearer CA, Raja HA, Miller AN, Nelson P, Tanaka K, Hirayama K, Marvanová L, Hyde KD, Zhang Y (2009) The molecular phylogeny of freshwater Dothideomycetes. Stud Mycol 64:145-153 
Shoemaker RA, Babcock CE (1992) Applanodictyosporous Pleosporales: Clathrospora, Comoclathris, Graphyllium, Macrospora, and Platysporoides. Can J Bot 70:1617-1658

Shoemaker RA, Babcock CE, Irwin JAG (1991) Massarina walker $\mathrm{n}$. sp., the teleomorph of Acrocalymma medicaginis from Medicago sativa contrasted with Leptosphaeria pratensis, L. weimeri $\mathrm{n}$. sp., and L. viridella. Can J Bot 69:569-573

Silvestro D, Michalak I (2012) raxmlGUI: A graphical front-end for RAxML. Org Divers Evol 12:335-337

Singer R (1973) The genera Marasmiellus, Crepidotus and Simocybe in the neotropics. Nova Hedwig Beih 44:1-517

Singer R (1976) Marasmieae (Basidiomycetes-Tricholomataceae). Flora Neotropica 17:1-347

Singer R (1986) The Agaricales in modern taxonomy, 4th edn. Koeltz Scientific Books, Königstein, p 981

Singer R (1989) New taxa and new combinations of Agaricales (Diagnoses fungorum novorum Agaricalium 4). Fieldiana Bot 21:1-133

Singh U, Das K, Uniyal P, Mehmood T (2017) Hygrocybe rajendrae (Hygrophoraceae), a new species of subsection Squamulosae from Indian Himalaya. Nordic J Bot 36(1-2):1-6

Sivanesan A (1984) The bitunicate ascomycetes and their anamorphs. Vaduz, Liechtenstein: J Cramer, pp 1-701

Slippers B, Boissin E, Phillips AJL, Groenewald JZ, Lombard L, Wingfield MJ, Postma A, Burgess T, Crous PW (2013) Phylogenetic lineages in the Botryosphaeriales: a systematic and evolutionary framework. Stud Mycol 76:31-49

Smith G (1956) Some new species of soil moulds. Trans Brit Mycol Soc 39:111-114

Somrithipol S, Jones EBG (2005) An addition to the hyphomycete genus Melanographium from Thailand. Fungal Divers 19:137-144

Song F, Wu S, Zhai Y, Xuan QC, Wang T (2014) Secondary metabolites from the genus Xylaria and their bioactivities. Chem Biodivers 11:673-694

Sotome K, Akagi Y, Lee SS, Ishikawa NK, Hattori T (2013) Taxonomic study of Favolus and Neofavolus gen. nov. segregated from Polyporus (Basidiomycota, Polyporales). Fungal Divers 58:245-266

Sousa JO, Morais LA, Nascimento YM, Baseia IG (2014) Updates on the geographic distribution of three Geastrum species from Brazilian semi-arid region. Mycosphere 5:467-474

Spatafora JW, Chang Y, Benny GL, Lazarus K, Smith ME, Berbee ML, Bonito G, Corradi N, Grigoriev I, Gryganskyi A, James TY, O'Donnell K, Roberson RW, Taylor TN, Uehling J, Vilgalys R, White MM, Stajich JE (2016) A phylum-level phylogenetic classification of zygomycete fungi based on genome-scale data. Mycologia 108:1028-1046

Species Fungorum (2021) http://www.speciesfungorum.org/

Spegazzini C (1917) Revisión de las Laboulbeniales argentinas. An Mus Nac Hist Nat Buenos Aires 29:445-688

Spaulding P (1961) Foreign diseases of forest trees of the world. USDA Agric Handb 197:1-361

Spirin WA (2003) Antrodiella romellii (Irpicaceae, Basidiomycetes) in Russia. Mycena 3:47-52

Spirin V, Volobuev S, Okun M, Miettinen O, Larsson KH (2017) What is the type species of Phanerochaete (Polyporales, Basidiomycota)? Mycol Prog 16:171-183

Sri-indrasutdhi V, Boonyuen N, Suetrong S, Chuaseeharonnachai C, Sivichai S, Jones EBG (2010) Woodinhabiting freshwater fungi from Thailand: Ascothailandia grenadoidia gen. et sp. nov., Canalisporium grenadoidia sp. nov. with a key to Canalisporium species (Sordariomycetes, Ascomycota). Mycoscience $51: 411-420$
Stadler M, Læssøe T, Fournier J, Decock C, Schmieschek B, Tichy HV, Peršoh D (2014) A polyphasic taxonomy of Daldinia (Xylariaceae). Stud Mycol 77:1-143

Stadler M, Lambert C, Wibberg D, Kalinowski J, Cox RJ, Kolařík M, Kuhnert E (2020) Intragenomic polymorphisms in the ITS region of high-quality genomes of the Hypoxylaceae (Xylariales, Ascomycota). Mycol Prog 19:235-245

Stamatakis A (2014) RAxML version 8: a tool for phylogenetic analysis and post-analysis of large phylogenies. Bioinformatics 30:1312-1313

Stamatakis A, Hoover P, Rougemont J (2008) A rapid bootstrap algorithm for the RAxML webservers. Syst Biol 75:758-771

Stubbe D (2012) Systematics and phylogeny of Lactarius subgenus Plinthogalus sensu lato. Ph.D. thesis, Ghent University, Gent, Belgium

Sturm J (1829) Deutschlands Flora Abt III. Die Pilze Deutschlands 2:1-136

Su HY, Hyde KD, Maharachchikumbura SSN, Ariyawansa HA, Luo ZL, Promputtha I, Tian Q, Lin CG, Shang QJ, Zhao YC, Chai HM, Liu XY, Bahkali AH, Bhat DJ, McKenzie EHC, Zhou DQ (2016) The families Distoseptisporaceae fam. nov., Kirschsteiniotheliaceae, Sporidesmiaceae and Torulaceae, with new species from freshwater in Yunnan Province, China. Fungal Divers 80:375-409

Su XJ, Luo ZL, Jeewon R, Bhat DJ, Bao DF, Li WL, Hao YE, Su HY, Hyde KD (2018) Morphology and multigene phylogeny reveal new genus and species of Torulaceae from freshwater habitats in northwestern Yunnan, China. Mycol Prog 17:531-545

Subramanian CV (1957) Two new species of Petrakia. Beih Sydowia $1: 14-15$

Subramanian CV (1994) Hyphomycetes from South East Asia-novelties from Singapore and Malaysia. Kavaka 22(23):52-76

Suetrong S, Schoch CL, Spatafora JW, Kohlmeyer J, VolkmannKohlmeyer B, Sakayaroj J, Phongpaichit S, Tanaka K, Hirayama K, Jones EBG (2009) Molecular systematics of the marine Dothideomycetes. Stud Mycol 64:155-173

Suetrong S, Boonyuen N, Pang KL, Ueapattanakit J, Klaysuban A, Sri-indrasutdhi V, Sivichai S, Jones EBG (2011) A taxonomic revision and phylogenetic reconstruction of the Jahnulales (Dothideomycetes), and the new family Manglicolaceae. Fungal Divers 51:163-188

Suija A, van den Boom P, Zimmermann E, Zhurbenko MP, Diederich P (2017) Lichenicolous species of Hainesia belong to Phacidiales (Leotiomycetes) and are included in an extended concept of Epithamnolia. Mycologia 109:882-899

Summerell BA (2019) Resolving Fusarium: Current status of the genus. Ann Rev Phytopathol 57:323-339

Sunhede S (1989) Geastraceae (Basidiomycotina): Morphology, ecology and systematics with special emphasis on the North European species. Syn Fungorum 1:1-534

Sutton BC (1964) Coelomycetes III. Annellolacinia gen. nov., Aristastoma, Phaeocytostroma Seimatosporium etc. Mycol Pap 97:1-42

Sutton BC (1980) The Coelomycetes. Fungi imperfecti with pycnidia, acervuli and stromata. Commonwealth Mycological Institute, Kew, pp 1-696

Suwannarach N, Kumla J, Lumyong S (2021) Spegazzinia camelliae sp. nov. (Didymosphaeriaceae, Pleosprales), a new endophytic fungus from northern Thailand. Phytotaxa 483:117-128

Swofford DL (2002) PAUP* 4.0: phylogenetic analysis using parsimony (*and other methods). Sinauer, Sunderland

Swofford DL (2003) PAUP phylogenetic analysis using parsimony (and other methods), version 4. Sinauer Associates, Sunderland

Swofford DL (2004) PAUP*: phylogenetic analysis using parsimony, version 4.0b10. Sinauer Associates, Sunderland

Takamatsu S (2013) Origin and evolution of the powdery mildews (Ascomycota, Erysiphales). Mycoscience 54:75-86 
Takamatsu S, Ito H, Shiroya Y, Kiss L, Heluta V (2015) First comprehensive phylogenetic analysis of the genus Erysiphe (Erysiphales, Erysiphaceae) I. The Microsphaera Lineage. Mycologia 107:475-489

Talbot PHB (1952) Dispersal of fungus spores by small animals inhabiting wood and bark. Trans Br Mycol Soc 35:123-128

Tan YS, Desjardin DE, Perry BA, Vikineswary S, Noorlidah A (2009) Marasmius sensu stricto in Peninsular Malaysia. Fungal Divers 37:9-100

Tanaka K, Hirayama K, Yonezawa H, Hatakeyama S, Harada Y, Sano T, Shirouzu T, Hosoya T (2009) Molecular taxonomy of bambusicolous fungi: Tetraplosphaeriaceae, a new pleosporalean family with Tetraploa-like anamorphs. Stud Mycol 64:175-209

Tanaka K, Hirayama K, Yonezawa H, Sato G, Toriyabe A, Kudo H, Hashimoto A, Matsumura M, Harada Y, Kurihara Y, Shirouzu $\mathrm{T}$ (2015) Revision of the Massarineae (Pleosporales, Dothideomycetes). Stud Mycol 82:75-136

Tavares II (1985) Laboulbeniales (Fungi, Ascomycetes). Mycol Mem 9:1-627

Taylor JE, Hyde KD (2003) Microfungi of tropical and temperate palms. Fungal Divers Res Ser 12:1-495

Tedersoo L, Harend H, Buegger F, Pritsch K, Saar I, Kõljalg U (2014) Stable isotope analysis, field observations and synthesis experiments suggest that Odontia is a non-mycorrhizal sister genus of Tomentella and Thelephora. Fungal Ecol 11:80-90

Teixeira MM, Moreno LF, Stielow BJ, Muszewska A, Hainaut M, Gonzaga L, Abouelleil A, Patané JSL, Priest M, Souza R, Young S, Ferreira KS, Zeng Q, da Cunha MML, Gladki A, Barker B, Vicente VA, de Souza EM, Almeida S, Henrissat B, Vasconcelos ATR, Deng S, Voglmayr H, Moussa TAA, Gorbushina A, Felipe MSS, Cuomo CA, De Hoog GS (2017) Exploring the genomic diversity of black yeasts and relatives (Chaetothyriales, Ascomycota). Stud Mycol 86:1-28

Terashima Y, Takahashi H, Taneyama Y (2016) The fungal flora in southwestern Japan: Agarics and boletes. Tokai University Press, Tokyo

Thambugala KM, Ariyawansa HA, Li YM, Boonmee S, Hongsanan S, Tian Q, Singtripop C, Bhat DJ, Camporesi E, Jayawardena R, Liu ZY, Xu JC, Chukeatirote E, Hyde KD (2014a) Dothideales. Fungal Divers 68:105-158

Thambugala KM, Camporesi E, Ariyawansa HA, Phookamsak R, Zy L, Hyde KD (2014b) Phylogeny and morphology of Phaeosphaeriopsis triseptata sp. nov., and Phaeosphaeriopsis glaucopunctata. Phytotaxa 176:238-250

Thambugala KM, Hyde KD, Tanaka K, Tian Q, Wanasinghe DN, Ariyawansa HA, Jayasiri SC, Boonmee S, Camporesi E, Hashimoto A, Hirayama K (2015) Towards a natural classification and backbone tree for Lophiostomataceae, Floricolaceae, and Amorosiaceae fam. nov. Fungal Divers 74:199-266

Thambugala KM, Wanasinghe DN, Phillips AJL, Camporesi E, Bulgakov TS, Phukhamsakda C, Ariyawansa HA, Goonasekara ID, Phookamsak R, Dissanayake A, Tennakoon DS, Tibpromma S, Chen YY, Liu ZY, Hyde KD (2017) Mycosphere notes 1-50: Grass (Poaceae) inhabiting Dothideomycetes. Mycosphere 8:697-796

Thaxter R (1908) Contribution toward a monograph of the Laboulbeniaceae. Part II. Mem Am Acad Arts Sci 13:217-269

Thaxter R (1931) Contribution towards a monograph of the Laboulbeniaceae. Part V. Mem Am Acad Arts Sci 16:1-435

Tian Q, Chomnunti P, Bhat JD, Alias SA, Mortimer PE, Hyde KD (2014) Towards a natural classification of Dothideomycetes 5: the genera Ascostratum, Chaetoscutula, Ceratocarpia, Cystocoleus, and Colensoniella (Dothideomycetes incertae sedis). Phytotaxa 176:42-54
Tian Q, Li WJ, Hyde KD, Camporesi E, Bhat DJ, Chomnunti P, Xu JC (2017) Molecular taxonomy of five species of microfungi on Alnus spp. from Italy. Mycol Prog 17:255-274

Tibpromma S, Daranagama DA, Boonmee S, Promputtha I, Nontachaiyapoom S, Hyde KD (2017a) Anthostomelloides krabiensis gen. et sp. nov. (Xylariaceae) from Pandanus odorifer (Pandanaceae). Turkish J Bot 41:107-116

Tibpromma S, Hyde KD, Jeewon R, Maharachchikumbura SSN, Liu JK, Bhat DJ, Jones EBG, McKenzie EHC, Camporesi E, Bulgakov TS, Doilom M, de Azevedo SALCM, Das K, Manimohan P, Gibertoni TB, Lim YW, Ekanayaka AH, Thongbai B, Lee HB, Yang JB, Kirk PM, Sysouphanthong P, Singh SK, Boonmee S, Dong W, Raj KNA, Latha KPD, Phookamsak R, Phukhamsakda C, Konta S, Jayasiri SC, Norphanphoun C, Tennakoon DS, Li JF, Dayarathne MC, Perera RH, Xiao Y, Wanasinghe DN, Senanayake IC, Goonasekara ID, de Silva NI, Mapook A, Jayawardena RS, Dissanayake AJ, Manawasinghe IS, Chethana KWT, Luo ZL, Hapuarachchi KK, Baghela A, Soares AM, Vizzini A, Meiras-Ottoni A, Mešić A, Dutta AK, de Souza CAF, Richter C, Lin CG, Chakrabarty D, Daranagama DA, Lima DX, Chakraborty D, Ercole E, Wu F, Simonini G, Vasquez G, da Silva GA, Plautz HL Jr, Ariyawansa HA, Lee H, Kušan I, Song J, Sun J, Karmakar J, Hu K, Semwal KC, Thambugala KM, Voigt K, Acharya K, Rajeshkumar KC, Ryvarden L, Jadan M, Hosen MI, Mikš́́k M, Samarakoon MC, Wijayawardene NN, Kim NK, Matocec N, Singh PN, Tian Q, Bhatt RP, de Oliveira RJV, Tulloss RE, Aamir S, Kaewchai S, Svetasheva STY, Nguyen TTT, Antonın V, Li WJ, Wang Y, Indoliya Y, Tkalčec Z, Elgorban AM, Bahkali AH, Tang AMC, Su HY, Zhang H, Promputtha I, Luangsa-ard J, Xu JC, Yan J, Chuan KJ, Stadler M, Mortimer PE, Chomnunti P, Zhao Q, Phillips AJL, Nontachaiyapoom S, Wen TC, Karunarathna SC (2017b) Fungal diversity notes 491-602: taxonomic and phylogenetic contributions to fungal taxa. Fungal Divers 83:1-261

Tibpromma S, Hyde KD, McKenzie EH, Bhat DJ, Phillips AJ, Wanasinghe DN, Samarakoon MC, Jayawardena RS, Dissanayake AJ, Tennakoon DS, Doilom M, Phookamsak R, Tang AMC, Xu J, Mortimer PE, Promputtha I, Maharachchikumbura SSN, Khan S, Karunarathna SC (2018) Fungal diversity notes 840-928: microfungi associated with Pandanaceae. Fungal Divers 93:1-60

Trakunyingcharoen T, Lombard L, Groenewald JZ, Cheewangkoon R, Toanun C, Alfenas AC, Crous PW (2014) Mycoparasitic species of Sphaerellopsis, and allied lichenicolous and other genera. IMA Fungus 5:391-414

Trierveiler-Pereira L, Calonge FDD, Baseia IG (2011) New distributional data on Geastrum (Geastraceae, Basidiomycota) from Brazil. Acta Bot Bras 25:577-585

Trotter A (1931) Supplementum Universale, Pars X. Myxomycetae, Myxobacteriaceae, Deuteromycetae, Mycelia sterilia. Syll Fung 25:1-1093

Tsang CC, Chan JFW, Trendell-Smith NJ, Ngan AHY, Ling IWH, Lau SKP, Woo PCY (2014) Subcutaneous phaeohyphomycosis in a patient with IgG4-related sclerosing disease caused by a novel ascomycete, Hongkongmyces pedis gen. et sp. nov.: first report of human infection associated with the family Lindgomycetaceae. Med Mycol 52:736-747

Tsui KM, Goh TK, Hyde KD (1997) A new species of Dactylaria from Hong Kong. Sydowia 49:182-186

Tsui KM, Hyde KD, Hodgkiss IJ, Goh TK (1998) A new freshwater species of Saccardoella from Hong Kong and South Africa. Mycologia 90:701-704

Tulasne LR, Tulasne C (1844) Recherches sur l'organisation et le mode de fructification des champignons de la tribu des Nidulariées, suivies d'un essai monographique. Annls Sci Nat Bot 1:41-107

Tulasne LR, Tulasne C (1863) Selecta Fungorum Carpologia, Tomus Secundus. Xylariei-Valsei-Sphaeriei. 2:1-319 
Tulasne LR, Tulasne C (1865) Selecta Fungorum Carpologia: NectrieiPhacidiei- Pezizei 3

Udayanga D, Liu X, Crous PW, McKenzie EHC, Chukeatirote E, Hyde KD (2012a) A multi-locus phylogenetic evaluation of Diaporthe (Phomopsis). Fungal Divers 56:157-171

Udayanga D, Liu X, McKenzie EHC, Chukeatirote E, Hyde KD (2012b) Multi-locus phylogeny reveals three new species of Diaporthe from Thailand. Cryptog Mycol 33:295-309

Udayanga D, Castlebury LA, Rossman AY, Chukeatirote E, Hyde KD (2014a) Insights into the genus Diaporthe: phylogenetic species delimitation in the $D$. eres species complex. Fungal Divers 67:203-229

Udayanga D, Castlebury LA, Rossman AY, Hyde KD (2014b) Species limits in Diaporthe: molecular re-assessment of D. citri, D. cytosporella, D Foeniculina and $d$ Rudis. Persoonia 32:83-101

Uljé CB (2005) Coprinus. In: Noordeloos ME, Kuyper TW, Vellinga EC (eds) Flora Agaricina Neerl, vol 6, pp 22-109

Unamuno PLM (1941) Enumeracion y distribucion geografica de los ascomicetos de la Peninsula Iberica y de las Islas Baleares. Mem Real Acad, Ci Exact Madrid 8:1-403

Uotila P (2017) Polygonaceae. In: Euro+Med Plantbase-the information resource for Euro-Mediterranean plant diversity. http:// ww2.bgbm.org/EuroPlusMed/PTaxonDetail.asp?NameId=55710 \&PTRefFk $=7300000$. Accessed 6 Jan 2020

Vánky K (2011) ['2012'] Smut fungi of the world. APS Press, St. Paul, Minnesota

Vánky K (2013) Illustrated genera of smut fungi, 3rd edn. American Phytopathological Society Press, St. Paul

Varga J, Frisvad JC, Samson RA (2010) Polyphasic taxonomy of Aspergillus section Sparsi. IMA Fungus 1:187-195

Vasilyeva LN, Stephenson SL (2006) Pyrenomycetes of the Great Smoky Mountains National Park. III. Cryptosphaeria, Eutypa and Eutypella (Diatrypaceae). Fungal Divers 22:243-254

Vašutová M, Antonin V, Urban A (2008) Phylogenetic studies in Psathyrella focusing on section Pennatae and Spadiceae-new evidence for the paraphyly of the genus. Mycol Res 112:1153-1164

Vellinga EC (2003) Chlorophyllum and Macrolepiota (Agaricaceae) in Australia. Aust Syst Bot 16:361-370

Vellinga EC (2004) Genera in the family Agaricaceae-evidence from nrITS and nrLSU sequences. Mycol Res 1108:354-377

Vellinga EC, Sysouphanthong P, Hyde KD (2011) The family Agaricaceae: phylogenies and two new white-spored genera. Mycologia 103:494-509

Verbeken A, Nuytink J, Buyck B (2011) New combinations in Lactifluus. 1. L. subgenera Edules. Lactariopsis and Russulopsis. Mycotaxon 118:447-453

Verbeken A, Van de Putte K, De Crop E (2012) New combinations in Lactifluus. 3. L. Subgenera Lactifluus and Piperati. Mycotaxon 120:443-450

Verkley GJM, da Silva M, Wicklow DT, Crous PW (2004) Paraconiothyrium, a new genus to accommodate the mycoparasite Coniothyrium minitans, anamorphs of Paraphaeosphaeria, and four new species. Stud Mycol 50:323-335

Verkley GJM, Dukik K, Renfurm R, Göker M, Stielow JB (2014) Novel genera and species of coniothyrium-like fungi in Montagnulaceae (Ascomycota). Persoonia 32:25-51

Vincenot L, Kazuhide N, Schulz C, Labbé J, Dubois MP, Tendersoo L, Martin F, Selosse MA (2011) Extensive gene flow over Europe and possible speciation over Eurasia in the ectomycorrhizal basidiomycete Laccaria amethystina complex. Mol Ecol 21:281-299

Vincenot L, Popa F, Laso F, Donges K, Rexer KH, Kost G, Yang ZL, Nara K, Selosse MA (2017) Out of Asia: biogeography of fungal populations reveals Asian origin of diversification of the Laccaria amethystina complex, and two new species of violet Laccaria. Fungal Biol 121:939-955
Vizzini A, Contu M, Ercole E, Voyron S (2012) Rivalutazione e delimitazione del genere Aspidella (Agaricales, Amanitaceae), nuovamente separato da Amanita. Micol Veget Medit 27:75-90

Vizzini A, Angelini C, Bizzi A (2016) Saproamanita manicata in Repubblica Dominicana. RMR Boll AMER Anno XXXII 2:33-44

Voglmayr H, Beenken L (2020) Linosporopsis, a new leaf-inhabiting scolecosporous genus in Xylariaceae. Mycol Prog 19:205-222

Voglmayr H, Jaklitsch WM (2017) Corynespora, Exosporium and Helminthosporium revisited - new species and generic reclassification. Stud Mycol 87:43-76

Voglmayr H, Friebes G, Gardiennet A, Jaklitsch WM (2018) Barrmaelia and Entosordaria in Barrmaeliaceae (fam. nov., Xylariales) and critical notes on Anthostomella-like genera based on multigene phylogenies. Mycol Prog 17:155-177

Volobuev S, Okun M, Ordynets A, Spirin V (2015) The Phanerochaete sordida group (Polyporales, Basidiomycota) in temperate Eurasia, with a note on Phanerochaete pallida. Mycol Prog 14:80

von Arx JA, Müller E (1975) A re-evaluation of the bitunicate ascomycetes with keys to families and genera. Stud Mycol 9:1-159

von Haller A (1768) Historia stirpium indigenarum. Helvetiae Inchoate $3: 1-250$

von Höhnel F (1909) Fragmente zur Mykologie: VI. Mitteilung (Nr. 182 bis 288). Sitzungsberichte der Kaiserlichen Akademie der Wissenschaften Math.-naturw Klasse Abt I 118:275-452

von Höhnel F (1917) System der Diaportheen. Ber Dtsch Bot Gesellschaft 35:631-638

von Willdenow CL (1787) Florae Berolinensis Prodromus. Wilhelm Vieweg, Berlin

Vu D, Groenewald M, De Vries M, Gehrmann T, Stielow B, Eberhardt U, Al-Hatmi A, Groenewald JZ, Cardinali G, Houbraken J, Boekhout T, Crous PW, Robert V, Verkley GJM (2019) Largescale generation and analysis of filamentous fungal DNA barcodes boosts coverage for kingdom fungi and reveals thresholds for fungal species and higher taxon delimitation. Stud Mycol 92:135-154

Wagner L, Stielow B, Hoffmann K, Petkovits T, Papp T, Vágvölgyi C, De Hoog GS, Verkley G, Voigt K (2013) A comprehensive molecular phylogeny of the Mortierellales (Mortierellomycotina) based on nuclear ribosomal DNA. Persoonia 30:77-93

Wagner L, De Hoog S, Alastruey-Izquierdo A, Voigt K, Kurzai O, Walther G (2019) A revised species concept for opportunistic Mucor species reveals species-specific antifungal susceptibility profiles. Antimicrob Agents Chemother 63:1-8

Wallroth CFW (1833) Flora Cryptogamica Germaniae 2: ii-lvi:1-923. Nürnberg JL Schrag, Germany

Walther G, Garnica S, Weiss M (2005) The systematic relevance of conidiogenesis modes in the gilled Agaricales. Mycol Res 109:525-544

Walther G, Pawlowska J, Alastruey-Izquierdo A, Wrzosek M, Rodriguez-Tudela JL, Dolatabadi S, Chakrabarti A, De Hoog GS (2013) DNA barcoding in Mucorales: an inventory of biodiversity. Persoonia 30:11-47

Walther G, Wagner L, Kurzai O (2019) Updates on the taxonomy of Mucorales with an emphasis on clinically important taxa. J Fungi 5:106

Wanasinghe DN, Jones EBG, Dissanayake AJ, Hyde KD (2016) Saprobic Dothideomycetes in Thailand: Vaginatispora appendiculata sp. nov. (Lophiostomataceae) introduced based on morphological and molecular data. Stud Fung 1:56-68

Wanasinghe DN, Hyde KD, Jeewon R, Crous PW, Wijayawardene N, Jones EBG, Bhat DJ, Phillips AJL, Groenewald JZ, Dayarathne MC, Phukhamsakda C, Thambugala KM, Bulgakov TS, Camporesi E, Gaforov Y, Mortimer PE, Karunarathna SC (2017) Phylogenetic revision of Camarosporium (Pleosporineae, Dothideomycetes) and allied genera. Stud Mycol 87:207-256 
Wanasinghe DN, Phukhamsakda C, Hyde KD, Jeewon R, Lee HB, Jones EBG, Tibpromma S, Tennakoon DS, Dissanayake AJ, Jayasiri SC, Gafforov Y, Camporesi E, Bulgakov TS, Ekanayake AH, Perera RH, Samarakoon MC, Goonasekara ID, Mapook A, Li WJ, Senanayake IC, Li JF, Norphanphoun C, Doilom M, Bahkali AH, Xu JC, Mortimer PE, Tibell L, Savic ST, Karunarathna SC (2018) Fungal diversity notes 709-839: taxonomic and phylogenetic contributions to fungal taxa with an emphasis on fungi on Rosaceae. Fungal Divers 89:1-236

Wanasinghe DN, Mortimer PE, Senwanna C, Cheewangkoon R (2020a) Saprobic Dothideomycetes in Thailand: Phaeoseptum hydei $\mathrm{sp}$. nov., a new terrestrial ascomycete in Phaeoseptaceae. Phytotaxa 449:149-163

Wanasinghe DN, Wijayawardene NN, Xu JC, Cheewangkoon R, Mortimer PE (2020b) Taxonomic novelties in Magnolia-associated pleosporalean fungi in the Kunming Botanical Gardens (Yunnan, China). PLoS ONE 15:e0235855

Wang MM, Chen Q, Diao YZ, Duan WJ, Cai L (2019a) Fusarium incarnatum-equiseti complex from China. Persoonia 43:70-89

Wang XH, Das K, Bera I, Chen YH, Bhatt RP, Ghosh A, Hembrom ME, Hofstetter V, Parihar A, Vizzini A, Xu TM, Zhao CL, Buyck B (2019b) Fungal biodiversity profiles 81-90. Cryptog Mycol 40:57-95

Wannathes N, Desjardin DE, Hyde KD, Perry BA, Lumyong S (2009) A monograph of Marasmius (Basidiomycota) from Northern Thailand based on morphological and molecular (ITS sequences) data. Fungal Divers 37:209-306

Watson W (1929) The classification of lichens. II. New Phytol 28:85-116

Wei L, Li YH, Hyde KD, Zhao RL (2015) Micropsalliota pseudoglobocystis, a new species from China. Mycotaxon 130:555-561

Weir A, Hughes M (2002) The taxonomic status of Corethromyces bicolor from New Zealand, as inferred from morphological, developmental, and molecular studies. Mycologia 94:483-493

Wendt L, Sir EB, Kuhnert E, Heitkamper S, Lambert C, Hladki AI, Romero AI, Luangsa-ard JJ, Srikitikulchai P, Peršoh D, Stadler M (2018) Resurrection and emendation of the Hypoxylaceae, recognised from a multigene phylogeny of the Xylariales. Mycol Prog 17:115-154

White TJ, Bruns T, Lee S, Taylor J (1990) Amplification and direct sequencing of fungal ribosomal RNA genes for phylogenetics. In: Innis M, Gelfand D, Shinsky J, White T (eds) PCR protocols: a guide to methods and applications. Academic Press, San Diego, pp 315-322

Whitton SR, McKenzie EHC, Hyde KD (2012) Fungi associated with Pandanaceae. Springer, Dordecht

Wijayawardene NN, Hyde KD, Rajeshkumar KC, Hawksworth DL, Madrid H, Kirk PM, Braun U, Singh RV, Crous PW, Kukwa M, Lucking R, Kurtzman CP, Yurkov A, Haelewaters D, Aptroot A, Lumbsch HT, Timdal E, Ertz D, Etayo J, Phillips AJL, Groenewald JZ, Papizadeh M, Selbmann L, Dayarathne MC, Weerakoon G, Jones EBG, Suetrong S, Tian Q, Castanéda-Ruiz RF, Bahkali AH, Pang KL, Tanaka K, Dai DQ, Sakayaroj J, Hujslová M, Lombard L, Shenoy BD, Suija A, Maharachchikumbura SSN, Thambugala KM, Wanasinghe DN, Sharma BO, Gaikwad S, Pandit G, Zucconi L, Onofri S, Egidi E, Raja HA, Kodsueb R, Caceres MES, Perez-Ortega S, Fiuza PO, Monteiro JS, Vasilyeva LN, Shivas RG, Prieto M, Wedin M, Olariaga I, Lateef AA, Agrawal Y, Fazeli SAS, Amoozegar MA, Zhao GZ, Pfliegler WP, Sharma G, Oset M, Abdel MA, Takamatsu S, Bensch K, Silva NI, De Kesel A, Karunarathna A, Boonmee S, Pfister DH, Lu YZ, Luo ZL, Boonyuen N, Daranagama DA, Senanayake IC, Jayasiri SC, Samarakoon MC, Zeng XY, Doilom M, Quijada L, Rampadarath S, Heredia G, Dissanayake AJ, Jayawardana RS, Perera PH, Tang LZ, Phukhamsakda C, HernándezRestrepo M, Ma XY, Tibpromma S, Gusmao LFP, Weerahewa D,
Karunarathna SC (2017) Notes for Genera: Ascomycota. Fungal Divers 86:1-594

Wijayawardene NN, Hyde KD, Lumbsch HT, Liu JK, Maharachchikumbura SSN, Ekanayaka AH, Tian Q, Phookamsak R (2018) Outline of Ascomycota: 2017. Fungal Divers 88:167-263

Wijayawardene NN, Hyde KD, Al-Ani LKT, Tedersoo L, Haelewaters D, Rajeshkumar KC, Zhao RL, Aptroot A, Leontyev DV, Saxena RK, Tokarev YS, Dai DQ, Letcher PM, Stephenson SL, Ertz D, Lumbsch HT, Kukwa M, Issi IV, Madrid H, Phillips AJL, Selbmann L, Pfliegler WP, Horváth E, Bensch K, Kirk PM, Kolař́ková K, Raja HA, Radek R, Papp V, Dima V, Ma J, Malosso E, Takamatsu S, Rambold G, Gannibal PB, Triebel D, Gautam AK, Avasthi S, Suetrong S, Timdal E, Fryar SC, Delgado G, Réblová M, Doilom M, Dolatabadi S, Pawłowska J, Humber RA, Kodsueb R, Sánchez-Castro I, Goto BT, Silva DKA, de Souza FA, Oehl F, da Silva GA, Silva IR, Błaszkowski J, Jobim K, Maia LC, Barbosa FR, Fiuza PO, Divakar PK, Shenoy BD, Castañeda-Ruiz RF, Somrithipol S, Lateef AA, Karunarathna SC, Tibpromma S, Mortimer PE, Wanasinghe DN, Phookamsak R, Xu J, Wang Y, Tian F, Alvarado P, Li DW, Kušan I, Matočec N, Maharachchikumbura SSN, Papizadeh M, Heredia G, Wartchow F, Bakhshi M, Boehm E, Youssef N, Hustad VP, Lawrey JD, Santiago ALCMA, Bezerra JDP, Souza-Motta CM, Firmino AL, Tian Q, Houbraken J, Hongsanan S, Tanaka K, Dissanayake AJ, Monteiro JS, Grossart HP, Suija A, Weerakoon G, Etayo J, Tsurykau A, Vázquez V, Mungai P, Damm U, Li QR, Zhang H, Boonmee S, Lu YZ, Becerra AG, Kendrick B, Brearley FQ, Motiejūnaite J, Sharma B, Khare R, Gaikwad S, Wijesundara DSA, Tang LZ, He MQ, Flakus A, Rodriguez-Flakus P, Zhurbenko MP, McKenzie EHC, Stadler M, Bhat DJ, Liu JK, Raza M, Jeewon R, Nassonova ES, Prieto M, Jayalal RGU, Erdoğdu M, Yurkov A, Schnittler M, Shchepin ON, Novozhilov YK, SilvaFilho AGS, Liu P, Cavender JC, Kang Y, Mohammad S, Zhang LF, Xu RF, Li YM, Dayarathne MC, Ekanayaka AH, Wen TC, Deng CY, Pereira OL, Navathe S, Hawksworth DL, Fan XL, Dissanayake LS, Kuhnert E, Grossart HP, Thines M (2020) Outline of Fungi and fungus-like taxa. Mycosphere 11:1060-1456

Wijesinghe SN, Camporesi E, Wanasinghe DN, Maharachchikumbura SSN, Senanayake IC, Phookamsak R, Hongsanan S, Tibpromma S, Thambugala KM, Luangharn T, McKenzie EHC, Hyde KD (2021) A dynamic online documentation of Italian ascomycetes with hosts and substrates: www.italianmicrofungi.org. Asian J Mycol 4:10-18

Willis KJ (2018) State of the World's Fungi 2018 (ed). Report. Royal Botanic Gardens, Kew

Wilson AW, Desjardin DE (2005) Phylogenetic relationships in the gymnopoid and marasmioid fungi (Basidiomycetes, euagarics clade). Mycologia 97:667-679

Wilson AW, Hosaka K, Perry BA, Mueller GM (2013) Laccaria (Agaricomycetes, Basidiomycota) from Tibet (Xizang Autonomous Region, China). Mycoscience 54:406-419

Wilson AW, Hosaka K, Mueller GM (2016) Evolution of ectomycorrhizas as a driver of diversification and biogeographic patterns in the model mycorrhizal mushroom genus Laccaria. New Phytol 213(4):1862-1873

Wolfe BE, Kuo M, Pringle A (2012) Amanita thiersii is a saprotrophic fungus expanding its range in the United States. Mycologia 104:22-33

Wu SH (1990) The Corticiaceae (Basidiomycetes) subfamilies Phlebioideae, Phanerochaetoideae and Hyphodermoideae in Taiwan. Acta Bot Fenn 142:1-123

Wu SH (1997) New species of Hyphoderma from Taiwan. Mycologia 89:132-140

Wu SH (1998) Nine new species of Phanerochaete from Taiwan. Mycol Res 102:1126-1132 
Wu SH, Nilsson HR, Chen CT, Yu SY, Hallenberg N (2010) The whiterotting genus Phanerochaete is polyphyletic and distributed throughout the phlebioid clade of Polypores (Basidiomycota). Fungal Divers 47:107-118

Wu SH, Chen CC, Wei CL (2018a) Three new species of Phanerochaete (Polyporales, Basidiomycota). MycoKeys 41:91-106

Wu SH, Chen YP, Wei CL, Floudas D, Dai YC (2018b) Two new species of Phanerochaete (Basidiomycota) and redescription of $P$. robusta. Mycol Prog 17:425-435

Wu N, Dissanayake AJ, Manawasinghe IS, Rathnayaka AR, Liu JK, Phillips AJL, Promputtha I, Hyde KD (2021) https://botryospha eriales.org/, an up-to-date classification and account of taxa of Botryosphaeriales. Database (in press)

Xia JW, Sandoval-Denis M, Crous PW, Zhang XG, Lombard L (2019) Numbers to names-restyling the Fusarium incarnatum-equiseti species complex. Persoonia 43:186-221

Xiao ZJ, Li XX, Wang HD, Song PY, Tang L (2018) Rhexoacrodictys broussonetiae sp. nov. from Guizhou, China. Mycotaxon 133:149-152

Xie X, Liu LL, Shen XC, Kang YQ, Hyde KD, Kang JC, Li QR (2020) Contributions to species of Xylariales in China-3. Collodiscula tubulosa (Xylariaceae). Phytotaxa 428:122-130

Xu YL, Cao YF, Nakasone KK, Chen CC, He SH (2020) Taxonomy and phylogeny of Phanerochaete sensu stricto (Polyporales, Basidiomycota) with emphasis on Chinese collections and descriptions of nine new species. Mycosphere 11:1527-1552

Yang J, Maharachchikumbura SSN, Bhat DJ, Hyde KD, Mckenzie EHC, Jones EBG, Al-Sadi AM, Lumyong S (2016) Fuscosporellales, a new order of aquatic and terrestrial Hypocreomycetidae (Sordariomycetes). Cryptog Mycol 37:449-475

Yang Q, Fan XL, Du Z, Tian CM (2017a) Diaporthosporellaceae, a novel family of Diaporthales (Sordariomycetes, Ascomycota). Mycoscience 59:229-235

Yang T, Groenewald JZ, Cheewangkoon R, Jami F, Abdollahzadeh J, Lombard L, Crous PW (2017b) Families, genera, and species of Botryosphaeriales. Fungal Biol 121:322-346

Yang J, Liu JK, Hyde KD, Jones EBG, Liu ZY (2018a) New species in Dictyosporium, new combinations in Dictyocheirospora and an updated backbone tree for Dictyosporiaceae. MycoKeys 36:83-105

Yang Q, Fan XL, Guarnaccia V, Tian CM (2018b) High diversity of Diaporthe species associated with dieback diseases in China, with twelve new species described. MycoKeys 39:97-149

Yang ZL, Cai Q, Cui YY (2018c) Phylogeny, diversity and morphological evolution of Amanitaceae. Biochem Syst Ecol 34:359-380

Yang H, Dong W, Yu XD, Bhat DJ, Boonmee S, Zhang H (2020) Four freshwater dematiaceous hyphomycetes in Sordariomycetes with two new species of Parafuscosporella. Phytotaxa 441:19-34

Yuan Y, Chen JJ, He SH (2017) Geliporus exilisporus gen. et. comb. nov., a xanthochroic polypore in Phanerochaetaceae from China. Mycoscience 58:197-203

Yuan Y, Wu F, Dai YC, Qin WM, Yuan HS (2018) Odontia aculeata and $O$. sparsa, two new species of tomentelloid fungi (Thelephorales, Basidiomycota) from the secondary forests of northeast China. Phytotaxa 372:183-192

Yuan HS, Lu X, Dai YU, Hyde KD, Kan YH, Kušan I, He SH, Liu N, Sarma VV, Zhao CL, Cui BK, Yousaf N, Sun G, Liu SY, Wu F, Lin CG, Dayarathne MC, Gibertoni TB, Conceição LB, GaribayOrijel R, Villegas-Ríos M, Salas-Lizana R, Wei TZ, Qiu JZ, Yu ZF, Phookamsak R, Zeng M, Paloi S, Bao DF, Abeywickrama PD, Wei DP, Jing Y, Manawasinghe IS, Harishchandra D, Brahmanage RS, de Silva NI, Tennakoon DS, Karunarathna A, da Silva GA, Gafforov Y, Pem D, Zhang S, de Santiago ALCM, Bezerra JDP, Dima B, Acharya K, Alvarez-Manjarrez J, Bahkali AH, Bhatt VK, Brandrud TE, Bulgakov TS, Camporesi E, Cao T, Chen YX, Chen YY, Devadatha B, Elgorban AM, Fan LF,
Du X, Gao L, Gonçalves CM, Gusmão LFP, Huanraluek N, Jadan M, Jayawardena RS, Khalid AN, Langer E, Lima DX, de Lima-Júnior NC, de Lira CRS, Liu JK, Liu S, Lumyong S, Luo ZL, Matočec N, Niranjan M, Oliveira-Filho JRC, Papp V, PérezPazos E, Phillips AJL, Qiu PL, Ren Y, Ruiz RFC, Semwal KC, da Silva RMF, Soop K, de Souza CAF, Souza-Motta CM, Sun LH, Xie ML, Yao YJ, Zhao Q, Zhou LW (2020) Fungal diversity notes 1277-1386: taxonomic and phylogenetic contributions to fungal taxa. Fungal Divers 104:1-266

Yurchenko E, Wu SH (2014) Hyphoderma pinicola sp. nov. of H. setigerum complex (Basidiomycota) from Yunnan, China. Bot Stud 55:71-78

Zamora JC (2014) Proposal to conserve the name Geastrum (Basidiomycota: Geastrales) with a conserved type. Taxon 63:679-680

Zamora JC, Calonge FD, Martín MP (2013) New sources of taxonomic information for earthstars (Geastrum, Geastraceae, Basidiomycota): phenoloxidases and rhizomorph crystals. Phytotaxa $132: 1-20$

Zamora JC, Calonge FD, Hosaka K, Martín MP (2014) Systematics of the genus Geastrum (Basidiomycota, Fungi) revisited. Taxon 63:477-497

Zhang Y, Schoch CL, Fournier J, Crous PW, De Gruyter J, Woudenberg JHC, Hirayama K, Tanaka K, Pointing SB, Spatafora JW, Hyde KD (2009) Multi-locus phylogeny of Pleosporales: a taxonomic, ecological and evolutionary re-evaluation. Stud Mycol 64:85-102

Zhang H, Dong W, Hyde KD, Bahkali AH, Liu JK, Zhou DQ, Zhang D (2016a) Molecular data shows Didymella aptrootii is a new genus in Bambusicolaceae. Phytotaxa 247:99-108

Zhang T, Deng X, Yu Y, Zhang M, Zhang Y (2016b) Pseudochaetosphaeronema ginkgonis sp. nov., an endophyte isolated from Ginkgo biloba. Int J Syst Evol Microbiol 66:4377-4381

Zhang JF, Liu JK, Hyde KD, Yang W, Liu ZY (2017) Fungi from Asian Karst formations II. Two new species of Occultibambusa (Occultibambusaceae, Dothideomycetes) from karst landforms of China. Mycosphere 8:550-559

Zhang JF, Liu JK, Ran HY, Khongphinitbunjong K, Liu ZY (2018) A new species and new record of Lophiotrema (Lophiotremataceae, Dothideomycetes) from karst landforms in southwest China. Phytotaxa 379:169-179

Zhang JF, Liu JK, Thambugala KM, Yang J, Meng ZH, Liu ZY (2020) Two new species and a new record of Nigrograna (Nigrogranaceae, Pleosporales) from China and Thailand. Mycol Prog 19:1365-1375

Zhang W, Groenewald JZ, Lombard L, Schumacher RK, Phillips AJL, Crous PW (2021) Evaluating species in Botryosphaeriales. Persoonia 46:63-115

Zhao CL, Wu ZQ (2017) Ceriporiopsis kunmingensis sp. nov. (Polyporales, Basidiomycota) evidenced by morphological characters and phylogenetic analysis. Mycol Prog 16:93-100

Zhao CL, Cui BK, Dai YC (2014) Morphological and molecular identification of two new species of Hyphodontia (Schizoporaceae, Hymenochaetales) from southern China. Cryptog Mycol 35:87-97

Zhao N, Luo ZL, Hyde KD, Su HY, Bhat DJ, Liu JK, Bao DF, Hao YE (2018a) Helminthosporium submersum sp. nov. (Massarinaceae) from submerged wood in north-western Yunnan Province. China Phytotaxa 348:269-278

Zhao YZ, Zhang ZF, Cai L, Peng JW, Liu F (2018b) Four new filamentous fungal species from newly-collected and hive stored bee pollen. Mycosphere 9:1089-1116

Zhao RL, Li GJ, Sánchez-Ramírez S, Stata M, Yang ZL, Wu G, Dai YC, He SH, Cui BK, Zhou JL, Wu F, He MQ, Moncalvo JM, Hyde KD (2017) A six-gene phylogenetic overview of Basidiomycota and allied phyla with estimated divergence times of 
higher taxa and a phyloproteomics perspective. Fungal Divers 84:43-74

Zhao RL, Desjardin DE, Soytong K, Perry BA, Hyde KD (2010) A monograph of Micropsalliota in Northern Thailand based on morphological and molecular data. Fungal Divers 45:33-79

Zhou JL, Cui BK (2017) Phylogeny and taxonomy of Favolus (Basidiomycota). Mycologia 109:766-779
Zhou TX, Zhao LZ, Zhao RL, Chen YH (2004) Bird's nest fungi from China. Fungal Divers 17:243-251

Ziegler R, Lutz M, Piątek J, Piątek M (2018) Dismantling a complex of anther smuts (Microbotryum) on carnivorous plants in the genus Pinguicula. Mycologia 110:361-374

Zmitrovich IV, Malysheva VF, Spirin WA (2006) A new morphological arrangement of Polyporales. I. Phanerochaetineae. Mycena $6: 4-56$

\section{Authors and Affiliations}

Saranyaphat Boonmee ${ }^{1,2} \cdot$ Dhanushka N. Wanasinghe $^{3,4,5} \cdot$ Mark S. Calabon $^{1,2} \cdot$ Naruemon Huanraluek $^{1}$. Sajini K. U. Chandrasiri ${ }^{1,2}$. Gareth E. B. Jones ${ }^{6}$. Walter Rossi ${ }^{7}$. Marco Leonardi ${ }^{7}$. Sanjay K. Singh ${ }^{8}$. Shiwali Rana ${ }^{8}$. Paras N. Singh ${ }^{8}$. Deepak K. Maurya ${ }^{8} \cdot$ Ajay C. Lagashetti $^{8} \cdot$ Deepika Choudhary $^{8}$. Yu-Cheng Dai ${ }^{9}$. Chang-Lin Zhao ${ }^{10}$. Yan-Hong Mu ${ }^{11,12}$. Hai-Sheng Yuan ${ }^{11}$. Shuang-Hui He ${ }^{9}$. Rungtiwa Phookamsak ${ }^{3,4,5,13} \cdot$ Hong-Bo Jiang ${ }^{1,2,3} \cdot$ María P. Martín $^{14} \cdot$ Margarita Dueñas $^{14} \cdot$ M. Teresa Telleriaa $^{14}$. Izabela L. Kałucka ${ }^{15}$. Andrzej M. Jagodziński ${ }^{16} \cdot$ Kare Liimatainen $^{17}$. Diana S. Pereira ${ }^{18}$ • Alan J. L. Phillips ${ }^{18}$. Nakarin Suwannarach ${ }^{19,20}$. Jaturong Kumla ${ }^{19,20}$. Surapong Khuna ${ }^{19,20}$. Saisamorn Lumyong ${ }^{19,20,21}$. Tarynn B. Potter ${ }^{22}$ - Roger G. Shivas 22,23 - Adam H. Sparks ${ }^{22,24}$ - Niloofar Vaghefi' ${ }^{22}$ - Mohamed A. Abdel-Wahab ${ }^{25}$. Faten A. Abdel-Aziz ${ }^{25} \cdot$ Guo-Jie $\mathrm{Li}^{26} \cdot$ Wen-Fei $\mathrm{Lin}^{27}$. Upendra Singh ${ }^{28} \cdot$ Rajendra P. Bhatt $^{28} \cdot$ Hyang Burm Lee $^{29}$. Thuong T. T. Nguyen ${ }^{29}$. Paul M. Kirk ${ }^{30}$. Arun Kumar Dutta ${ }^{31,32} \cdot$ Krishnendu Acharya $^{32}$ • V. Venkateswara Sarma ${ }^{33}$. M. Niranjan ${ }^{33,34} \cdot$ Kunhiraman C. Rajeshkumar $^{8} \cdot$ Nikhil Ashtekar $^{8} \cdot$ Sneha Lad $^{8} \cdot$ Nalin N. Wijayawardene $^{35}$. Darbe J. Bhat ${ }^{36}$ - Rong-Ju Xu ${ }^{1,2,3}$. Subodini N. Wijesinghe ${ }^{1,2} \cdot$ Hong-Wei Shen ${ }^{1,2,37}$. Zong-Long Luo ${ }^{37}$. Jing-Yi Zhang ${ }^{1,2,38} \cdot$ Phongeun Sysouphanthong ${ }^{1,2,39} \cdot$ Naritsada Thongklang ${ }^{1,2}$. Dan-Feng Bao ${ }^{1,37,40}$. Janith V. S. Aluthmuhandiram ${ }^{1,2,41}$. Jafar Abdollahzadeh ${ }^{42}$. Alireza Javadi ${ }^{43}$. Francesco Dovana ${ }^{44}$. Muhammad Usman ${ }^{45}$. Abdul Nasir Khalid ${ }^{45}$. Asha J. Dissanayake ${ }^{46}$. Anusha Telagathoti ${ }^{47}$. Maraike Probst ${ }^{47}$. Ursula Peintner $^{47}$. Isaac Garrido-Benavent ${ }^{48}$. Lilla Bóna ${ }^{49} \cdot$ Zsolt Merényi $^{50}$. Lajos Boros $^{51}$ • Bratek Zoltán ${ }^{49}$. J. Benjamin Stielow ${ }^{52,53} \cdot$ Ning Jiang $^{54}$. Cheng-Ming Tian ${ }^{54} \cdot$ Esmaeil Shams $^{55} \cdot$ Farzaneh Dehghanizadeh $^{56}$. Adel Pordel ${ }^{57}$. Mohammad Javan-Nikkhah ${ }^{55}$. Teodor T. Denchev ${ }^{58}$. Cvetomir M. Denchev ${ }^{58} \cdot$ Martin Kemler $^{59}$. Dominik Begerow ${ }^{59}$. Chun-Ying Deng ${ }^{60}$. Emma Harrower ${ }^{61}$. Tohir Bozorov ${ }^{62}$. Tutigul Kholmuradova ${ }^{63}$.

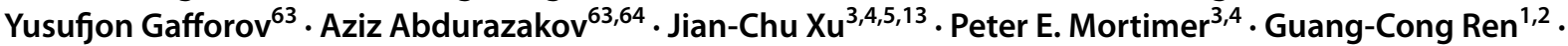
Rajesh Jeewon ${ }^{66}$. Sajeewa S. N. Maharachchikumbura ${ }^{46}$. Chayanard Phukhamsakda ${ }^{67}$. Ausana Mapook ${ }^{1}$. Kevin D. Hyde 1 1,3,19,20,65

Kevin D. Hyde

kdhyde3@gmail.com

1 Center of Excellence in Fungal Research, Mae Fah Luang University, Chiang Rai 57100, Thailand

2 School of Science, Mae Fah Luang University, Chiang Rai 57100, Thailand

3 CAS Key Laboratory for Plant Biodiversity and Biogeography of East Asia (KLPB), Kunming Institute of Botany, Chinese Academy of Science, Kunming 650201, Yunnan, People's Republic of China

4 CIFOR-ICRAF China Program, World Agroforestry (ICRAF), Kunming 650201, Yunnan, People's Republic of China

5 Honghe Center for Mountain Futures, Kunming Institute of Botany, Honghe County, Kunming 654400, Yunnan, People's Republic of China
6 Department of Botany and Microbiology, College of Science, King Saud University, P.O. Box 2455, Riyadh 11451, Saudi Arabia

7 Section Environmental Sciences, Department MeSVA, University of L'Aquila, 67100 Coppito, AQ, Italy

8 National Fungal Culture Collection of India (NFCCI), Biodiversity and Palaeobiology Group, MACS Agharkar Research Institute, G.G. Agarkar Road, Pune 411 004, India

9 Institute of Microbiology, School of Ecology and Nature Conservation, Beijing Forestry University, Beijing 100083, People's Republic of China

10 College of Biodiversity Conservation, Southwest Forestry University, Kunming 650224, People's Republic of China

11 CAS Key Laboratory of Forest Ecology and Management, Institute of Applied Ecology, Chinese Academy of Sciences, Shenyang 110164, People's Republic of China 
12 University of the Chinese Academy of Sciences, Beijing 100049, People's Republic of China

13 Centre for Mountain Futures (CMF), Kunming Institute of Botany, Kunming 650201, Yunnan, People's Republic of China

14 Department of Mycology, Real Jardín Botánico-CSIC, Plaza de Murillo 2, 28014 Madrid, Spain

15 Department of Algology and Mycology, Faculty of Biology and Environmental Protection, University of Lodz, Banacha 12/16, 90-237 Łódź, Poland

16 Institute of Dendrology, Polish Academy of Sciences, Parkowa 5, 62-035 Kórnik, Poland

17 Jodrell Laboratory, Royal Botanic Gardens, Kew, Richmond TW9 3DS, Surrey, UK

18 Faculdade de Ciências, Biosystems and Integrative Sciences Institute (BioISI), Universidade de Lisboa, Campo Grande, 1749-016 Lisbon, Portugal

19 Department of Biology, Faculty of Science, Chiang Mai University, Chiang Mai 50200, Thailand

20 Research Center of Microbial Diversity and Sustainable Utilization, Faculty of Science, Chiang Mai University, Chiang Mai 50200, Thailand

21 Academy of Science, The Royal Society of Thailand, 10300 Bangkok, Thailand

22 Centre for Crop Health, University of Southern Queensland, Toowoomba, QLD 4350, Australia

23 Department of Agriculture and Fisheries, Dutton Park, QLD 4102, Australia

24 Department of Primary Industries and Regional Development, Bentley Delivery Centre, Locked Bag 4, Bentley, WA 6983, Australia

25 Department of Botany and Microbiology, Faculty of Science, Sohag University, Sohag 82524, Egypt

26 Key Laboratory of Vegetable Germplasm Innovation and Utilization of Hebei, Collaborative Innovation Center of Vegetable, College of Horticulture, Hebei Agricultural University, No 2596 South Lekai Rd, Lianchi District, Baoding 071001, Hebei, China

27 Institute of Edible and Medicinal Fungi, College of Life Science, Zhejiang University, 866 Yuhangtang Rd, Xihu District, Hangzhou 310058, Zhejiang, China

28 Department of Botany \& Microbiology, HNB Garhwal University, Uttarakhand 246174 Srinagar Garhwal, India

29 Environmental Microbiology Lab, Department of Agricultural Biological Chemistry, College of Agriculture \& Life Sciences, Chonnam National University, Gwangju 61186, Korea

30 Biodiversity Informatics and Spatial Analysis, Royal Botanic Gardens Kew, Richmond TW9 3DS, Surrey, UK

31 Department of Botany, West Bengal State University, North-24-Parganas, Barasat, West Bengal PIN- 700126, India

32 Molecular and Applied Mycology and Plant Pathology Laboratory, Department of Botany, University of Calcutta, 35, Ballygunge Circular Road, Kolkata, West Bengal 700019, India
33 Fungal Biotechnology Laboratory, Department of Biotechnology, Pondicherry University, Kalapet, Puducherry 605014, India

34 Department of Botany, Rajiv Gandhi University, Rono Hills, Doimukh, Itanagar, Arunachal Pradesh 791112, India

35 Center for Yunnan Plateau Biological Resources Protection and Utilization, College of Biological Resource and Food Engineering, Qujing Normal University, Qujing 655011, Yunnan, People's Republic of China

36 Azad Housing Society, No. 128/1-J, Goa Velha, Curca, Goa, India

37 College of Agriculture and Biological Sciences, Dali University, Dali 671003, People's Republic of China

38 School of Food and Pharmaceutical Engineering, Guizhou Institute of Technology, Guiyang 550003, People's Republic of China

39 Biotechnology and Ecology Institute, Ministry of Agriculture and Forestry, P.O. Box: 811, Vientiane Capital, Lao People's Democratic Republic

40 Department of Entomology and Plant Pathology, Faculty of Agriculture, Chiang Mai University, Chiang Mai 50200, Thailand

41 Beijing Key Laboratory of Environment Friendly Management On Fruit Diseases and Pests in North China, Institute of Plant and Environment Protection, Beijing Academy of Agriculture and Forestry Sciences, Beijing 100097, People's Republic of China

42 Department of Plant Protection, Agriculture Faculty, University of Kurdistan, P.O. Box 416, Sanandaj, Iran

43 Department of Botany, Iranian Research Institute of Plant Protection, P.O. Box 1454, 19395 Tehran, Iran

44 Via Quargnento, 17, Solero, 15029 Alessandria, Italy

45 Fungal Biology and Systematics Research Laboratory, Department of Botany, University of the Punjab, Quaid-e-Azam Campus, Lahore 54590, Pakistan

46 School of Life Science and Technology, University of Electronic Science and Technology of China, Chengdu 611731, People's Republic of China

47 Institute of Microbiology, University of Innsbruck, Technikerstrasse 25, 6020 Innsbruck, Austria

48 Department of Botany and Geology (Fac. CC. Biológicas) \& Institut Cavanilles de Biodiversitat I Biologia Evolutiva (ICBIBE), Universitat de València, C/ Dr. Moliner 50, Burjassot, 46100 València, Spain

49 Department of Plant Physiology and Molecular Plant Biology, Eötvös Loránd University, Budapest 1117, Hungary

50 Institute of Biochemistry, Synthetic and Systems Biology Unit, Biological Research Centre, Szeged 6726, Hungary

51 Ócsa 2364, Hungary

52 Centre of Expertise in Mycology of Radboud University Medical Centre/Canisius Wilhelmina Hospital, Nijmegen, The Netherlands

53 Thermo Fisher Diagnostics, Specialty Diagnostics Group, Landsmeer, The Netherlands 
54 The Key Laboratory for Silviculture and Conservation of the Ministry of Education, Beijing Forestry University, Beijing 100083, People's Republic of China

55 Department of Plant Protection, College of Agriculture and Natural Resources, University of Tehran, Karaj, Iran

56 Department of Agricultural Biotechnology, College of Agriculture Engineering, Isfahan University of Technology, Isfahan, Iran

57 Plant Protection Research Department, Baluchestan Agricultural and Natural Resources Research and Education Center, AREEO, Iranshahr, Iran Institute of Biodiversity and Ecosystem Research, Bulgarian Academy of Sciences, 2 Gagarin St., 1113 Sofia, Bulgaria

59 Evolution der Pflanzen und Pilze, Ruhr-Universität Bochum, ND 03, Universitätsstraße 150, 44801 Bochum, Germany

60 Guizhou Institute of Biology, Guizhou Academy of Sciences, Shanxi Road No. 1, Yunyan district, 550001 Guiyang, People's Republic of China

612914 West 28th Ave, Vancouver, BC V6L 1X2, Canada
62 Institute of Genetics and Plant Experimental Biology, Academy of Sciences of Republic of Uzbekistan, Yukori-Yuz, Kubray Ds, Tashkent, Uzbekistan 111226

63 Laboratory of Mycology, Institute of Botany, Academy of Sciences of Republic of Uzbekistan, 32 Durmon Yuli Street, Tashkent, Uzbekistan 100125

64 Department of Ecology and Botany, Faculty of Natural Sciences, Andijan State University, 12 University Street, Andijan, Uzbekistan 170100

65 Innovative Institute of Plant Health, Zhongkai University of Agriculture and Engineering, Haizhu District, Guangzhou 510225, People's Republic of China

66 Department of Health Sciences, Faculty of Medicine and Health Sciences, University of Mauritius, Réduit, Republic of Mauritius

67 Engineering Research Center of Chinese Ministry of Education for Edible and Medicinal Fungi, Jilin Agricultural University, Changchun 130118, China 